\title{
MIXED WASTE MANAGEMENT FACILITY GROUNDWATER MONITORING REPORT-SECOND QUARTER 1994
}

by

J A Chase

Westinghouse Savannah River Company

Savannah River Site

Aiken, South Carolina 29808

This paper was prepared in connection with work done under the above contract number with the U. S. Department of Energy. By acceptance of this paper, the publisher and/or recipient acknowledges the U. S. Government's right to retain a nonexclusive, royalty-free license in and to any copyright covering this paper. along with the right to reproduce and to authorize others to reproduce all or part of the copyrighted paper. 
WSRC-TR-94-0345

Unclassified

\section{MIXED WASTE MANAGEMENT FACILITY GROUNDWATER MONITORING REPORT (U)}

\section{SECOND QUARTER 1994}

Publication Date: September 1994

Authorized Derivative Classifier:

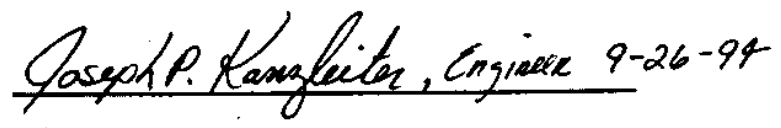

UNCLASSIEIED

Does Not Contain Unclassified

Controlled Nuclear Information

Westinghouse Savannah River Company

Savannah River Site

Aiken, SC 29808

Prepared for the U.S. Department of Energy under Control Contract No. DE-AC09-89SR18035 
WSRC-TR-94-0345

Unclassified

\section{MIXED WASTE MANAGEMENT FACILITY GROUNDWATER MONITORING \\ REPORT (U)}

\section{SECOND QUARTER 1994}

Publication Date: September 1994

Authorized Derivative Classifier:

UNCLASSIFIED

Does Not Contain Unclassified

Controlled Nuclear Information

Westinghouse Savannah River Company

Savannah River Site

Aiken, SC 29808

Prepared for the U.S. Department of Energy under Control Contract No. DE-AC09-89SR18035 


\section{DISCLAIMER}

This report was prepared by Westinghouse Savannah River Company (WSRC) for the United States Department of Energy under Contract No. DE-AC09-89SR18035 and is an account of work performed under that contract. Neither the United States Department of Energy, nor WSRC, nor any of their employees makes any warranty, expressed or implied, or assumes any legal liability or responsibility for the accuracy, completeness, or usefulness of any information, apparatus, product, or process disclosed herein or represents that its use will not infringe on privately owned rights. Reference herein to any specific commercial product, process, or service by trademark, name, manufacturer, or otherwise does not necessarily constitute or imply endorsement, recommendation, or favoring of same by WSRC or by the United States Government or any agency thereof. The views and opinions of the authors expressed herein do not necessarily state or reflect those of the United States Government or any agency thereof. 
WSRC-TR-94-0345

Unclassified

\title{
MIXED WASTE MANAGEMENT FACILITY GROUNDWATER MONITORING REPORT (U)
}

\author{
SECOND QUARTER 1994
}

Publication Date: September 1994

Key Words

aluminum

BGO wells

FSS wells

trichloroethylene

tritium

Westinghouse Savannah River Company

Savannah River Site

Aiken, SC 29808

Prepared for the U.S. Department of Energy under Control Contract No. DE-AC09-89SR18035 
WSRC-TR-94-0345

Unclassified

THIS PAGE LEFT BLANK INTENTIONALLY. 


\section{Abstract}

During second quarter 1994, seven constituents exceeded final Primary Drinking Water Standards (PDWS) in groundwater samples from downgradient monitoring wells at the Mixed Waste Management Facility, the Old Burial Ground, the E-Area Vaults, the proposed Hazardous Waste/ Mixed Waste Disposal Vaults, and the F-Area Sewage Sludge Application Site. Only one constituent, tritium, exceeded PDWS in samples from the upgradient monitoring wells.

As in previous quarters, tritium and trichloroethylene were the most widespread elevated constituents. Chloroethene (vinyl chloride), 1,1-dichloroethylene, gross alpha, lead, or tetrachloroethylene also exceeded standards in one or more wells. Elevated constituents were found in numerous Aquifer Zone IIB 2 (Water Table) and Aquifer Zone IIB (Barnwell/McBean) wells. No elevated constituents were found in Aquifer Unit IIA (Congaree) wells.

The groundwater flow directions and rates in the three hydrostratigraphic units were similar to those of previous quarters. 
Unclassified

THIS PAGE LEFT BLANK INTENTIONALLY. 


\section{Contents}

Page

Abstract $\ldots \ldots \ldots \ldots \ldots \ldots \ldots \ldots \ldots \ldots \ldots \ldots \ldots \ldots \ldots \ldots \ldots \ldots \ldots$

List of Figures $\ldots \ldots \ldots \ldots \ldots \ldots \ldots \ldots \ldots \ldots \ldots \ldots \ldots \ldots \ldots$

List of Tables $\ldots \ldots \ldots \ldots \ldots \ldots \ldots \ldots \ldots \ldots \ldots \ldots \ldots \ldots \ldots \ldots \ldots$

Executive Summary $\ldots \ldots \ldots \ldots \ldots \ldots \ldots \ldots \ldots \ldots \ldots \ldots \ldots$

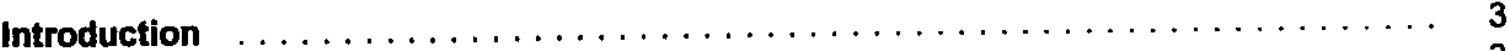

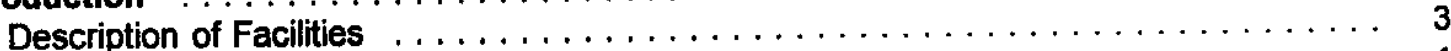

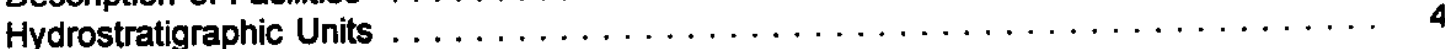

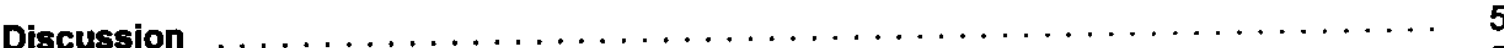

Groundwater Monitoring Data $\ldots \ldots \ldots \ldots \ldots \ldots \ldots \ldots \ldots \ldots \ldots \ldots \ldots$

Integrity of the Monitoring Well Network $\ldots \ldots \ldots \ldots \ldots \ldots \ldots \ldots \ldots \ldots, \ldots \ldots \ldots$

Analytical Results Exceeding Standards $\ldots \ldots \ldots \ldots \ldots \ldots \ldots \ldots \ldots \ldots \ldots, \ldots \ldots \ldots$

Trichloroethylene and Tritium Time-Trend Data $\ldots \ldots \ldots \ldots \ldots \ldots \ldots \ldots \ldots, 8$

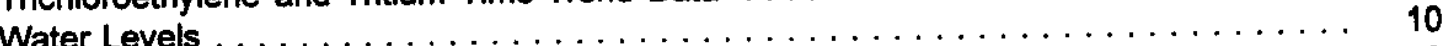

Groundwater Flow Rates and Directions $\ldots \ldots \ldots \ldots \ldots \ldots \ldots \ldots \ldots \ldots \ldots$

Upgradient Versus Downgradient Results $\ldots \ldots \ldots \ldots \ldots \ldots \ldots \ldots \ldots \ldots \ldots \ldots, \ldots \ldots \ldots$

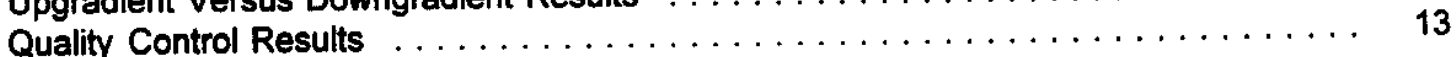

Conclusions $\ldots \ldots \ldots \ldots \ldots \ldots \ldots \ldots \ldots \ldots \ldots \ldots \ldots \ldots \ldots$

References Cited $\ldots \ldots \ldots \ldots \ldots \ldots \ldots \ldots \ldots \ldots \ldots \ldots \ldots \ldots \ldots \ldots$

Errata $\ldots \ldots \ldots \ldots \ldots \ldots \ldots \ldots \ldots$

Appendix A-Final Primary Drinking Water Standards $\ldots \ldots \ldots \ldots \ldots \ldots \ldots$ A-1

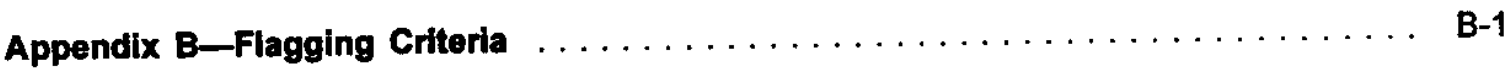

Appendix C-Figures $\ldots \ldots \ldots \ldots \ldots \ldots \ldots \ldots \ldots \ldots \ldots \ldots \ldots \ldots, \ldots \ldots$

Appendix D-Groundwater Monitoring Results Tables $\ldots \ldots \ldots \ldots \ldots \ldots \ldots$ D-1

Appendix E-Data Quality/Useability Assessment . . . . . . . . . . . . E-1

Appendix F-Time Series Plots $\ldots \ldots \ldots \ldots \ldots \ldots \ldots \ldots \ldots \ldots \ldots$

Appendix G-Hydrographs $\ldots \ldots \ldots \ldots \ldots \ldots \ldots \ldots \ldots \ldots \ldots \ldots \ldots$, G-1 


\section{List of Figures}

Page

1. Location of the Mixed Waste Management Facility at the Savannah River Site ... . C-3

2. Location of the Groundwater Monitoring Wells at the Mixed Waste Management

Facility, the Old Burial Ground, the E-Area Vaults, and the Hazardous Waste/ Mixed Waste Disposal Vaults (the Burial Ground Complex), and the F-Area Sewage Sludge Application Site $\ldots \ldots \ldots \ldots \ldots \ldots \ldots \ldots \ldots \ldots$ C-4

3. Hydrostratigraphic Nomenclature $\ldots \ldots \ldots \ldots \ldots \ldots \ldots \ldots \ldots \ldots \ldots \ldots$

4. Regional Correlation of Hydrostratigraphic and Lithostratigraphic Nomenclature . . . C -6

5. Location of Aquifer Zone $\|_{3} B_{2}$ (Water Table) Wells at the Burial Ground Complex . . . C-7

6. Location of Aquifer Zone IIB, (Barnwell/McBean) Wells at the Burial Ground Complex ............................ C-8

7. Location of Aquifer Unit IIA (Congaree) Wells at the Burial Ground Complex . . . . . C C-9

8. Lead Concentrations in Aquifer Zone $\|_{2}$ (Water Table) at the Burial Ground Complex, Second Quarter $1994 \ldots \ldots \ldots \ldots \ldots \ldots \ldots \ldots$. . . . . . . . . . . . . . .

9. Lead Concentrations in Aquifer Zone IIB

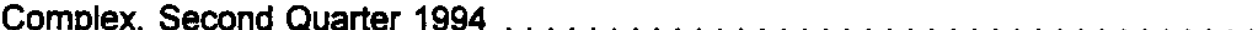

10. Lead Concentrations in Aquifer Unit IIA (Congaree) at the Burial Ground Complex,

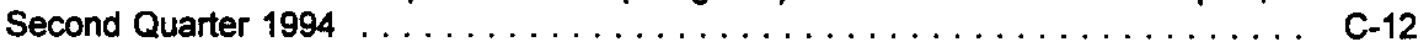

11. $\mathrm{pH}$ Values in Aquifer Zone $\| \mathrm{B}_{\mathbf{2}}$ (Water Table) at the Burial Ground Complex, Second Quarter $1994 \ldots \ldots \ldots \ldots \ldots \ldots \ldots \ldots \ldots \ldots \ldots \ldots \ldots$ C-13

12. pH Values in Aquifer Zone IIB, (Barnwell/McBean) at the Burial Ground Complex, Second Quarter $1994 \ldots \ldots \ldots \ldots \ldots \ldots \ldots \ldots$. . . . . . . . . . . . . . . . . . .

13. pH Values in Aquifer Unit IIA (Congaree) at the Burial Ground Complex, Second Quarter $1994 \ldots \ldots \ldots \ldots \ldots$. . . . . . . . . . . . . . . . . . . . . . .

14. Specific Conductance in Aquifer Zone $\|_{2}$ (Water Table) at the Burial Ground Complex, Second Quarter $1994 \ldots \ldots \ldots \ldots \ldots \ldots \ldots \ldots \ldots$ C-16

15. Specific Conductance in Aquifer Zone IIB, (Barnwell/McBean) at the Burial Ground Complex, Second Quarter $1994 \ldots \ldots \ldots \ldots \ldots \ldots \ldots \ldots \ldots \ldots$ C-17

16. Specific Conductance in Aquifer Unit IIA (Congaree) at the Burial Ground Complex, Second Quarter $1994 \ldots \ldots \ldots \ldots \ldots \ldots \ldots \ldots \ldots \ldots \ldots$ C-18 
17. Trichloroethylene Concentrations in Aquifer Zone $\mathrm{IB}_{2}$ (Water Table) at the Burial Ground Complex, Second Quarter $1994 \ldots \ldots \ldots \ldots$. . . . . . . . . . . C-19

18. Trichloroethylene Concentrations in Aquifer Zone IIB, (Barnwell/McBean) at the Burial Ground Complex, Second Quarter 1994

19. Trichloroethylene Concentrations in Aquifer Unit IIA (Congaree) at the Burial Ground Complex, Second Quarter $1994 \ldots \ldots \ldots \ldots \ldots \ldots \ldots \ldots$. . . . . . . . . . . . . . . . . . .

20. Tritium Activities in Aquifer Zone $\|_{2} B_{2}$ (Water Table) at the Burial Ground Complex, Second Quarter 1994

21. Tritium Activities in Aquifer Zone IIB, (Barnwell/McBean) at the Burial Ground Complex, Second Quarter 1994

22. Tritium Activities in Aquifer Unit IIA (Congaree) at the Burial Ground Complex,

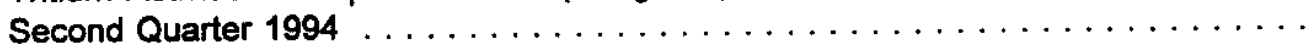

23. Piezometric Surface Map of Aquifer Zone $\| \mathrm{B}_{2}$ (Water Table) at the Burial Ground Complex

24. Potentiometric Surface Map of Aquifer Zone IIB, (Barnwell/McBean) at the Burial Ground Complex . .

25. Potentiometric Surface Map of Aquifer Unit IIA (Congaree) at the Burial Ground Complex

\section{List of Tables}

1. Maximum Levels of Constituents Exceeding the Final Primary Drinking Water Standards

2. Maximum Levels of Constituents Exceeding Other Flag 2 Criteria $\ldots \ldots \ldots \ldots$ D-11

3. Groundwater Monitoring Results for Individual Wells $\ldots \ldots \ldots \ldots \ldots \ldots \ldots$ 
WSRC-TR-94-0345

Unclassified

THIS PAGE LEFT BLANK INTENTIONALLY. 


\section{Executive Summary}

Currently, 125 wells monitor groundwater quality in the uppermost aquifer beneath the Mixed Waste Management Facility (MWMF) at the Savannah River Site as required by the South Carolina Hazardous Waste Management Regulations and settlement agreements 87-52-SW and 9151-SW. Samples from the wells are analyzed for selected heavy metals, indicator parameters, radionuclides, volatile organic compounds, and other constituents.

During second quarter 1994, chloroethene (vinyl chloride), 1,1-dichloroethylene, gross alpha, lead, tetrachloroethylene, trichloroethylene, or tritium exceeded final Primary Drinking Water Standards (PDWS) in approximately half of the downgradient wells at the MWMF. Consistent with historical trends, elevated constituent levels were found primarily in Aquifer Zone $\| B_{2}$ (Water Table) and Aquifer Zone IIB, (Barnwell/McBean). No elevated constituents occurred in the Aquifer Unit IIA (Congaree) wells.

As in previous quarters, tritium and trichloroethylene were the most widespread elevated constituents during second quarter 1994. Sixty-two $(50 \%)$ of the 125 monitoring wells contained elevated tritium activities. Trichloroethylene concentrations exceeded the final PDWS in $23(18 \%)$ wells. Chloroethene, 1,1-dichloroethylene, lead, and tetrachloroethylene, elevated in one or more wells during second quarter 1994, also occurred in elevated levels during first quarter 1994. These constituents generally were elevated in the same wells during both quarters. Gross alpha, which was not elevated in any well during first quarter 1994, was elevated in one well during second quarter. Copper, mercury, and nonvolatile beta were elevated during first quarter 1994 but not during second quarter.

Groundwater flow directions and rates in Aquifer Zone $\|_{2}$ (Water Table), Aquifer Zone $\| B_{1}$ (Barnwell/McBean), and Aquifer Unit IIA (Congaree) were generally similar to those of previous quarters. 
WSRC-TR-94-0345

Unclassified

THIS PAGE LEFT BLANK INTENTIONALLY. 


\section{Introduction}

\section{Description of Facilities}

The Mixed Waste Management Facility (MWMF) is part of the Burial Ground Complex, which also includes the Old Burial Ground, the Low-Level Radioactive Waste Disposal Facility (LLRWDF), the E-Area Vaults, and the proposed Hazardous Waste/Mixed Waste Disposal Vaults (HWMWDV). The Burial Ground Complex is located in the central part of the Savannah River Site (SRS) (Figures 1 and 2, Appendix C) within the General Separations Area. The following description outlines important events in the history of the MWMF:

- In 1972, the MWMF began receiving various radioactive and nonradioactive wastes (Heffner and Exploration Resources, 1991).

- A closure plan for the MWMF was filed November 23, 1985, with the South Carolina Department of Health and Environmental Control (SCDHEC) (Jaegge et al., 1987). The closure plan included placing a low permeability cap and final vegetative cover over the facility.

- The MWMF became inactive in 1986 (WSRC, 1993b).

- A consent decree between the U.S. Department of Energy and the Natural Resources Defense Council et al. (Civil Action 1:85-2583-6, U.S. District Court, District of South Carolina, Aiken Division), signed May 26, 1988, and effective June 1, 1988, identified the MWMF as subject to the requirements of Subtitle $\mathrm{C}$ of the Resource Conservation and Recovery Act (RCRA).

- SCDHEC approved an interim status closure plan for the MWMF in December 1990 and accepted the closure certification in April 1991 in accordance with South Carolina Hazardous Waste Management Regulations (SCHWMR), Subpart G (SCDHEC, 1993).

- A RCRA Part B post-closure care permit application renewal was submitted to SCDHEC in November 1992 (WSRC, 1992). This permit application includes the 58-acre MWMF and the 13-acre Solvent Rag Portions of the LLRWDF as agreed upon by SRS and SCDHEC in settlement agreements 87-52-SW (May 10, 1991) and 91-51-SW (August 26, 1991).

- A closure plan for the Solvent Rag Portions was submitted to SCDHEC in August 1993 (WSRC, 1993b).

- A revised RCRA Part B post-closure care permit application renewal, containing a groundwater corrective-action plan, was submitted to SCDHEC on November 30, 1993 (WSRC, 1993c).

- Revision 1 of the field investigation plan (FIP) for the Burial Ground Complex (WSRC, 1993a) was submitted to SCDHEC in September 1993. Phases 1A, 1B, and 4D were approved January 31, 1994; Phase 1C was approved March 8, 1994; and Phase 2A was approved on August 11, 1994. The FIP addresses data uncertainties that were identified during the preparation of the MWMF RCRA Part B post-closure care permit application renewal. 
- Phase 1 of the FIP field work has been divided in the following subphases: $1 A$-installation of 12 groundwater monitoring wells; 1B-exploratory work in the southwest corner of the Burial Ground Complex using direct-push technology and exploratory borings; $1 \mathrm{C}$-exploratory work in the northeast corner of the Burial Ground Complex using direct-push technology and exploratory borings; 1D-soil gas survey. Subphases $1 \mathrm{~B}$ and $1 \mathrm{C}$ will determine the horizontal and vertical extent of groundwater contamination away from the Burial Ground Complex. Phase 1A well drilling began May 3, 1994.

- Beginning first quarter 1994, wells FSS 1D, 2D, 3D, and 4D, located at the F-Area Sewage Sludge Application Site, were added to the MWMF monitoring well network. As a conditional requirement for the closure of the F-Area Sewage Sludge Application Site, the FSS monitoring wells are to be monitored for radionuclides related to the Burial Ground Complex.

Currently, the Environmental Protection Department/Environmental Monitoring Section (EPD/ EMS) conducts quarterly sampling of the 125 wells monitoring the groundwater beneath the MWMF as part of the SRS Groundwater Monitoring Program. The Environmental Restoration Department provides a quarterly report to SCDHEC describing the monitoring results to meet the requirements of SCHWMR (SCDHEC, 1993).

\section{Hydrostratigraphic Units}

Historically, groundwater quality assessment reports for the MWMF have used the lithostratigraphic nomenclature Water Table, Barnwell, McBean, and Congaree to identify hydrologic units. However, an interim alphanumeric system developed by Aadland and Bledsoe (1990) (Figure 3, Appendix $C$ ) defines the aquifer and aquitard units at SRS using hydrostratigraphic designations. Figure 4 (Appendix $C$ ) shows a correlation of these designations. This report uses both nomenclatures. The November 1992 MWMF RCRA Part B post-closure care permit application renewal includes an in-depth explanation of this nomenclature and a detailed description of the geologic and hydrogeologic systems at the Burial Ground Complex (WSRC, 1992).

The MWMF well network monitors three distinct hydrostratigraphic units in the uppermost aquifer beneath the facility: Aquifer Zone ${\| B_{2}}_{2}$ (Water Table), which is underlain by Confining Zone $\left\|_{1} B_{1}-\right\|_{2}$ (Tan Clay); the semi-confined Aquifer Zone IIB, (Barnwell/McBean); and the semiconfined Aquifer Unit IIA (Congaree), which is separated from the overlying Aquifer Zone IIB, by Confining Unit IIA-IIB (Green Clay). The boundary between the uppermost aquifer and the principal confining unit is the uppermost confining bed of Confining System I-1l (Ellenton Formation), which lies approximately $300 \mathrm{ft}$ below the surface of the Burial Ground Complex. 
WSRC-TR-94-0345

Unclassified

\section{Discussion}

\section{Groundwater Monitoring Data}

The EPD/EMS sampling procedure (EPD/EMS, 1992) requires evacuation of a minimum of two well volumes and stabilization of $\mathrm{pH}$, specific conductance, and turbidity prior to sample collection. Stability is established when a minimum of three successive measurements, taken within a given time period, are within a specified tolerance range. If a well pumps dry before two well volumes are purged or before stabilization is achieved, it must be revisited within 24 hours for the data to be considered from a single sampling event. On the second visit within 24 hours, samples are taken without purging or stability measurements; thus, these samples may not be representative of groundwater quality.

All of the wells in the BGO, BGX, FSS, and HMD series and in cluster HSB 85 have single-speed centrifugal downhole pumps.

During second quarter 1994, groundwater samples from the MWMF were analyzed for selected indicator parameters, heavy metals, radionuclides, and other constituents. This report describes the results that equaled or exceeded the Safe Drinking Water Act final Primary Drinking Water Standards (PDWS) or drinking water screening levels, as established by the U.S. Environmental Protection Agency (EPA) (Appendix A); the South Carolina final PDWS for lead (Appendix A); or SRS flagging criteria based on PDWS, Secondary Drinking Water Standards, or method detection limits (Appendix B). For simplicity, results that equaled or exceeded standards are described as exceeding or above standards or as elevated.

Both field and laboratory pH results are provided in this report. The field measurements are considered more reflective of actual groundwater conditions; however, laboratory pH measurements are required by current regulations. Because SCDHEC allows only 15 minutes to elapse between sampling and analysis of $\mathrm{pH}$, laboratory $\mathrm{pH}$ measurements always exceed the holding time (the time between sample collection and analysis) prescribed in the analytical method.

The final PDWS for individual analytes provided in Appendix A may not always match the SRS flagging criteria provided in Appendix B. The final PDWS are used as guidelines in this compliance report to meet regulatory requirements; the flagging criteria are used by EPD/EMS to identify relative levels of constituents in the groundwater and as guides for scheduling groundwater sampling.

\section{Integrity of the Monitoring Well Network}

The current groundwater monitoring well network at the MWMF (Figure 2, Appendix C) is composed of the following:

- 55 Aquifer Zone $\mathrm{IIB}_{2}$ (Water Table) wells (Figure 5, Appendix C):

BGo 1D, 2D, 3D, 4D, 5D, 6D, 7D, 8D, 9D, 10DR, 11D, 12D, 14DR, 15D, 16D, 17DR, 18D, 19D, 20D, 21D, 22DR, 23D, 24D, 26D, 27D, 28D, 29D, 30D, 31D, 32D, 33D, 34D, 35D, 36D, 
37D, 38D, 39D, 40D, 44D, 45D, 46D, 47D, 48D, 49D, 50D; BGX 1D, 9D, 10D, 11D, 12D; FSS $1 D, 2 D, 3 D, 4 D ;$ HSB $85 C$

- 46 Aquifer Zone IIB, (Barnwell/McBean) wells (Figure 6, Appendix C):

BGO 5C, 6B, 6C, 8C, 10B, 10C, 12CR, 13DR, 14CR, 16B, 27C, 29C, 30C, 31C, 33C, 35C, $37 \mathrm{C}, 42 \mathrm{C}, 43 \mathrm{CR}, 43 \mathrm{D}, 44 \mathrm{~B}, 44 \mathrm{C}, 45 \mathrm{~B}, 45 \mathrm{C}, 46 \mathrm{~B}, 46 \mathrm{C}, 47 \mathrm{C}, 48 \mathrm{C}, 49 \mathrm{C}, 50 \mathrm{C}$; BGX 1C, 2B, 2D, 3D, 4C, 4D, 5D, 6D, 7D, 8DR, 12C; HMD 1D, 2D, 3D, 4D; HSB 85B

- 24 Aquifer Unit IIA (Congaree) wells (Figure 7, Appendix C):

BGO 6A, 8AR, 9AA, 10AA, 10AR, 12AR, 14AR, 16AR, 18A, 25A, 26A, 29A, 41A, 43A, 43AA, 44A, 44AA, 45A, 47A, 49A, 50A; BGX 1A, 4A; HSB 85A

SRS has a program in place to rehabilitate and replace wells that do not produce representative samples from the units being monitored. A complete record of well installations, replacements, and abandonments at the MWMF is found in the EPD/EMS well inventory (EPD/EMS, 1994).

The designated background wells for Aquifer Zone ${\| B_{2}}_{2}$ (Water Table) are $B G O$ ID and $2 D$, and HSB 85C; HSB 85B is the background well for Aquifer Zone IIB, (Barnwell/McBean); and HSB $85 \mathrm{~A}$ is the background well for Aquifer Unit IIA (Congaree).

Table 3 (Appendix D) lists the number of well volumes purged from each of the BGO, BGX, FSS, and HMD series wells and the HSB 85 well cluster during second quarter 1994 and provides statements that describe unusual sampling events. Unusual sampling events occurred as follows.

- Wells that went dry during purging:

BGo 1D, 3D, 5C, 5D, 6B, 6D, 9AA, 10B, 10C, 10DR, 12CR, 12D, 13DR, 14CR, 16B, 16D, 17DR, 20D, 21D, 22DR, 24D, 25A, 26D, 27D, 28D, 29A, 29C, 29D, 30C, 30D, 31C, 31D, 32D, 33D, 34D, 35D, 36D, 37D, 38D, 39D, 40D, 42C, 43CR, 44B, 44C, 45B, 45C, 46C, 49D, 50A, 50C; BGX 1C, 1D, 2B, 2D, 10D, 11D, 12D; FSS 1D, 2D, 3D, 4D; HMD 1D, 4D; HSB 85B

- Wells that could not be sampled because they were inaccessible to the samplers:

BGO 4D

- Wells that could not be sampled because they had mechanical problems:

BGO 47C

- Wells that could not be sampled because they are in the Purge Water Containment (PWC) Program. The PWC program was instituted at SRS in 1991 to contain and dispose of purge water from wells that exhibits constituents 100 times their standards. Currently, MWMF does not have a method for containing and disposing of purged water; thus, these wells have only water-level measurements taken during sampling. 
Wells in the Purge Water Containment Program

\begin{tabular}{|lllll|}
\hline Well & $\begin{array}{l}\text { Quarter Last } \\
\text { Sampled }\end{array}$ & $\begin{array}{l}\text { Constituent } \\
\text { Exceeding Limit }\end{array}$ & $\begin{array}{l}\text { Level at } \\
\text { Last Sample }\end{array}$ & $\begin{array}{l}\text { Completion Date } \\
\text { of Next Evaluation }\end{array}$ \\
\hline BGO 26A & $3 Q 91$ & $\mathrm{pH}$ & $11.6 \mathrm{pH}$ units & June 30, 1994 \\
BGO 37C & $1 Q 91$ & trichloroethylene & $690 \mathrm{pg} /$ & June 30, 1994 \\
BGO 41A & $2 \mathrm{Q} 93$ & $\mathrm{pH}$ & $12.3 \mathrm{pH}$ units & June 30, 1994 \\
BGO 43A & $2 \mathrm{QH}$ & $\mathrm{pH}$ & $12.6 \mathrm{pH}$ units & June 30, 1994 \\
BGX 1A & $1 Q 93$ & $\mathrm{pH}$ & $12.4 \mathrm{pH}$ units & June 30, 1994 \\
\hline
\end{tabular}

a Field measurements.

\section{Analytical Results Exceeding Standards}

Results for analytes that exceeded the final PDWS during second quarter 1994 are summarized in Table 1 (Appendix D) and described below. In the text description, the maximum level for each constituent is indicated in parentheses following the well in which it was detected.

Aquifer Zone $\mathrm{IIB}_{2}$ (Water Table): $\mathbf{4 0}$ of the $\mathbf{5 4}$ wells sampled contained elevated constituents during second quarter 1994.

- Tritium was elevated in 39 wells: BGO 2D, 3D, 6D, 7D, 9D, 10DR, 11D, 12D, 15D, 16D, 19D, 20D, 21D, 22DR, 23D, 27D, 28D (maximum activity at 1.6E+05 pCi/mL), 29D, 30D, 32D, 33D, 34D, 35D, 36D, 37D, 38D, 39D, 44D, 45D, 46D, 47D, 48D, 49D, and 50D; BGX 1D, 11D, and 12D; and FSS 2D and 3D.

- Trichloroethylene was elevated in 13 wells: BGo 6D, 7D, 12D, 14DR, 15D, 16D, 28D (maximum concentration at $208 \mu \mathrm{g} / \mathrm{L}$ ), 30D, 32D, 46D, 47D, 48D, and 50D.

- Tetrachloroethylene was elevated in 5 wells: BGO 7D, 15D, 32D, 46D, and 48D (maximum concentration at $68 \mu \mathrm{g} / \mathrm{L}$ ).

- Chloroethene (vinyl chloride) was elevated in 3 wells: BGO 28D (maximum concentration at $96 \mu \mathrm{g} / \mathrm{L}), 30 \mathrm{D}$, and $46 \mathrm{D}$.

- 1,1-Dichloroethylene was elevated in well BGO 30D at $13 \mu \mathrm{g} / \mathrm{L}$.

- Lead was elevated in well FSS 3D at $956 \mu \mathrm{g} / \mathrm{L}$.

- Gross alpha was elevated in well BGO 32D at 1.5E+01 pCi/l.

Aquifer Zone $I I B_{1}$ (Barnwell/McBean): 25 of the 44 wells sampled contained elevated constituents during second quarter 1994.

- Tritium was elevated in 23 wells: BGO 5C, 6B, 6C, 14CR, 27C, 30C, 31C, 33C (maximum activity at $8.8 \mathrm{E}+03 \mathrm{pCi} / \mathrm{mL}$ ) $35 \mathrm{C}, 44 \mathrm{~B}, 44 \mathrm{C}, 45 \mathrm{C}, 46 \mathrm{C}, 48 \mathrm{C}, 49 \mathrm{C}$, and $50 \mathrm{C}$; BGX 1C, 2D, 3D, 5D, 7D, and 8DR; and HMD $1 D$. 
- Trichloroethylene was elevated in 10 wells: BGO 12CR (maximum concentration at $82 \mu \mathrm{g} / \mathrm{L}), 14 \mathrm{CR}, 27 \mathrm{C}, 30 \mathrm{C}, 33 \mathrm{C}, 35 \mathrm{C}, 42 \mathrm{C}, 46 \mathrm{C}$, and 50C; and BGX $2 \mathrm{D}$.

- Tetrachloroethylene was elevated in well BGO $33 C$ at $5.2 \mu \mathrm{g} / \mathrm{L}$.

Aquifer Unit IIA (Congaree): None of the $\mathbf{2 0}$ wells sampled contained elevated constituents during second quarter 1994.

Results for analytes that exceeded other SRS flagging criteria during second quarter 1994 are summarized in Table 2 (Appendix D).

Table 3 (Appendix D) shows the results for all of the constituents and indicates the analytical laboratories that conducted the analyses, the dilution factors used in the analyses, and the analyses that received modifiers (which help identify laboratory accuracy and precision) or that exceeded the EPA-approved holding times during second quarter 1994. Constituent results in Table 3 that appear to equal the final PDWS but are not marked in the $D$ column (exceeded final PDWS or screening level) are below the final PDWS in the database. Database results, the results that are compared to the final PDWS, are entered with more significant digits than the results given in this report. Apparent discrepancies are the result of the rounding of reported results.

In addition to the results tables, Appendix $\mathrm{D}$ provides definitions of the abbreviations and the modifiers used in the results tables as well as descriptions of holding times, data rounding, and data qualification practices. Appendix $E$ provides a general assessment of the quality and useability of the data.

Isoconcentration maps of lead, $\mathrm{pH}$, specific conductance, trichloroethylene, and tritium in Aquifer

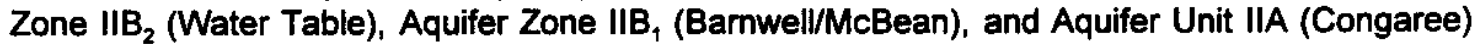
wells during second quarter 1994 appear in Figures 8 through 22 (Appendix C).

\section{Trichloroethylene and Tritium Time-Trend Data}

Time series plots from first quarter 1990 through second quarter 1994 for wells containing trichloroethylene and tritium are presented in Appendix F. Statistical analyses of these data have not been conducted; thus, statements concerning changes in constituent concentrations or activities over time are not included in this report. Statements concerning relative concentrations or activities of the constituents are provided below.

Trichloroethylene concentrations have exhibited the following trends since first quarter 1992:

- Trichloroethylene concentrations in wells BGO 6A, 6C, 8AR, 8C, 27D, 29D, 31D, 33D, $44 \mathrm{~A}, 44 \mathrm{AA}, 44 \mathrm{~B}, 44 \mathrm{D}, 47 \mathrm{~A}$, and 50A have been consistently below or near detection limits.

- Trichloroethylene concentrations in wells BGO 8D, 13DR, 14AR, 29A, 29C, 31C, 32D, $40 D, 44 C, 46 B, 47 C$, and $48 C$ and BGX $2 B$ have been consistently near or below the final PDWS.

- Wells BGO 6D, 7D, 12AR, 12CR, 12D, 14CR, 14DR, 15D, 16D, 27C, 28D, 30C, 30D, $33 C, 42 C, 46 C, 46 D, 47 D, 48 D$, and 50D and BGX 2D have exhibited concentrations of trichloroethylene that consistently exceeded the final PDWS. 
- Well BGO 28D, screened in Aquifer Zone $\mathrm{IIB}_{2}$ (Water Table), has consistently exhibited the highest trichloroethylene concentrations, usually ranging from approximately $190 \mu \mathrm{g} / \mathrm{L}$ to $340 \mu \mathrm{g} / \mathrm{L}$.

- Other wells have exhibited somewhat erratic activities, ranging from relatively high above the final PDWS to below the final PDWS (i.e., wells BGO 50C and BGX 3D). Data are insufficient to determine trends for other wells. However, trichloroethylene concentrations in wells BGO 6B, 16AR, 16B, and 41A have not exceeded the final PDWS since sampling began several quarters ago.

Tritium activities have exhibited the following trends since first quarter 1992:

- Tritium activities in wells BGo 8AR, 8C, 10AR, 12AR, 12CR, 18A, 25A, 43AA, 44A, 44AA, 45A, 47A, 49A, and 50A; BGX 4A and 12C; and HSB 85C have been consistently near or below detection limits.

- Tritium activities have not exceeded the final PDWS in upgradient wells BGO $1 D$ and HSB $85 \mathrm{~A}$ and $85 \mathrm{~B}$.

- Tritium activities in wells BGO 2D, 5C, 5D, 8D, 12D, 14AR, 17DR, 18D, 20D, 24D, 26D 29A, 33D, 35C, 40D, 42C, 43CR, 43D, and 45B; BGX 2B, 4C, 6D, 9D, 10D, 11D, and 12D; FSS $1 D$ and 4D; and HMD 2D, 3D, and 4D have been consistently near or below the final PDWS.

- Wells BGO 3D, 6C, 6D, 7D, 10DR, 11D, 14CR, 15D, 16D, 19D, 21D, 22DR, 23D, 27C 27D, 28D, 30C, 30D, 31C, 32D, 33C, 34D, 35D, 36D, 37D, 38D, 39D, 44B, 44C, 44D, 45C, 45D, 46C, 46D, 47C, 47D, 48C, 48D, 49C, 49D, 50C, and 50D; BGX 1C, 1D, 2D, $3 D$, and $8 D R$; and FSS $2 D$ and $3 D$ have exhibited tritium levels that have consistently exceeded the final PDWS.

- Well BGO 28D, located in Aquifer Zone $\|_{1} B_{2}$ (Water Table) at the west edge of the Old Burial Ground, has consistently exhibited the highest tritium activities, exceeding $1.0 \mathrm{E}+05 \mathrm{pCi} / \mathrm{mL}$ during most quarters.

- Some wells have exhibited erratic activities, ranging from relatively high above the final PDWS to below the final PDWS (e.g., wells BGO 9D, 10C, 13DR, and 46B and BGX 7D). Data are still insufficient to determine trends for other wells. However, tritium activities in wells BGO 9AA, 10AA, 16AR and 17DR have been consistently below the final PDWS since sampling began several quarters ago.

Tritium activities in wells BGO 12D, 14AR, 26D, 29A, 34D, 35C, 36D,37D, 38D, and 39D appear to have been anomalously high during fourth quarter 1991 as compared to preceding and more recent quarters.

Time series plots of $\mathrm{pH}$ for selected wells also are provided in Appendix $\mathrm{F}$. 


\section{Water Levels}

Hydrographs for selected wells and well clusters at the MWMF are provided in Appendix G. Average water elevations for all of the wells in each of the three hydrostratigraphic units beneath the MWMF for the past four quarters are shown in the following table.

Average Water Elevations (ft msl) in the Hydrostratigraphic Units beneath the MWMF

\begin{tabular}{|lllll|}
\hline Unit & 3093 & $4 Q 93$ & 1094 & 2094 \\
\hline Aquifer Zone IIB 2 (Water Table) & 233.07 & 231.13 & $230.70^{\mathrm{a}}$ & 230.48 \\
Aquifer Zone IIB, (BarnwellMcBean) & 220.34 & 219.14 & 218.15 & 218.79 \\
Aquifer Unit IIA (Congaree) & 160.60 & 160.29 & 159.45 & 159.85 \\
\hline
\end{tabular}

a Average includes water elevations for wells FSS 1D, 2D, and $3 D$ for the first time.

During second quarter 1994, the water level in Aquifer Zone $\| B_{2}$ (Water Table) decreased an average of $0.22 \mathrm{ft}$ compared to first quarter 1994, the water level in Aquifer Zone IIB, (Barnwell/ McBean) rose an average of $0.64 \mathrm{ft}$, and the water level in Aquifer Unit IIA (Congaree) rose an average of $0.4 \mathrm{ft}$.

A consistent vertical head relationship exists among the hydrostratigraphic units monitored at the MWMF. Flow potential is downward from Aquifer Zone $\|_{1} B_{2}$ (Water Table) to Aquifer Zone IIB (Barnwell/McBean) and downward from Aquifer Zone IIB (Barnwell/McBean) to Aquifer Unit IIA (Congaree). This relationship, which was first noted in 1988, exists at all BGO well clusters.

\section{Groundwater Flow Rates and Directions}

The groundwater in Aquifer Zone $\|_{2}$ (Water Table) and Aquifer Zone IIB $_{1}$ (Barnwell/McBean) diverges beneath the Burial Ground Complex. Historically the groundwater in Aquifer Zone $\|_{2}$ (Water Table) has discharged either to the north toward Upper Three Runs Creek or to the southwest toward Fourmile Branch (using universal transverse Mercator coordinates [UTM]); the groundwater in Aquifer Zone IIB, (Barnwell/McBean) has discharged either to the northwest or to the southwest. Near Upper Three Runs Creek, the upper portion of the saturated zone lies beneath Confining Zone $\left\|_{1} B_{1}-\right\| B_{2}$ (Tan Clay) in Aquifer Zone IIB (Barnwell/McBean). The historical horizontal groundwater flow direction in Aquifer Unit IIA (Congaree) has been consistently northwest toward Upper Three Runs Creek.

Using universal transverse Mercator coordinates, the second quarter 1994 flow directions in Aquifer Zone $\mathrm{IIB}_{2}$ (Water Table) were to the west-southwest (flow path A) and north (flow path B). Flow directions in Zone IIB, (Barnwell/McBean) were to the west-southwest (flow path A) and north (flow path B), and the flow direction in Aquifer Unit IIA (Congaree) was to the northwest (Figures 23, 24, and 25, Appendix $C$ ). Horizontal flow rate estimates for the three hydrostratigraphic units during the past four quarters are provided in the following table. 
Estimated Horizontal Groundwater Flow Rates (ft/yr) in the Hydrostratigraphic Units beneath the MWMF

\begin{tabular}{|lllll|}
\hline Unit & $3 Q 93$ & $4 Q 93$ & $1 \mathrm{Q} 94$ & $2 \mathrm{Q} 94$ \\
\hline Aquifer Zone IIB 2 (Water Table) & $27-51$ & $17-35$ & $20-31$ & $18-28$ \\
Aquifer Zone IIB, (BarnwellMcBean) & $5.1-15$ & $8.8-16$ & $8.8-16$ & $8.8-15$ \\
Aquifer Unit IIA (Congaree) & 190 & 180 & 150 & 220 \\
\hline
\end{tabular}

Horizontal flow rate calculations provide estimates of the transport rate of constituents originating from the MWMF. Flow rates in Aquifer Zone $\|_{1} B_{2}$ (Water Table) and Aquifer Zone IIB (Barnwell McBean) are calculated along two flow paths (designated flow paths $A$ and $B$ ) to characterize the divergent groundwater flows toward Upper Three Runs Creek and Fourmile Branch. The flow rate for Aquifer Unit IIA (Congaree) is calculated along a single flow path because flow directions within this unit are generally more uniform than in the overlying units. Flow rates are estimated using the following equation:

$$
\text { Flow }(\mathrm{ft} / \text { day })=\frac{\text { Hydraulic Conductivity }(\mathrm{ft} / \mathrm{day})}{\text { Porosity (unitless) }} \times \frac{d h(\mathrm{ft})}{d l(\mathrm{ft})}
$$

Hydraulic conductivity constants of $3.03 \mathrm{ft} /$ day, $1.50 \mathrm{ft} /$ day, and $45 \mathrm{ft} /$ day are used for Aquifer Zone IIB ${ }_{2}$ (Water Table), Aquifer Zone IIB (Barnwell/McBean), and Aquifer Unit IIA (Congaree), respectively (WSRC, 1992). An effective porosity value of $20 \%$ is used for the two upper units, and an effective porosity value of $25 \%$ is used for Aquifer Unit IIA (Congaree) (WSRC, 1992). The value $d h$ is the difference in head, and $d l$ is the length of the flow path.

Flow path lengths are calculated to the nearest $50 \mathrm{ft}$. Flow rate per day is calculated to two significant figures, then multiplied by 365 and rounded to two significant figures for the flow rate per year. Flow rate estimates vary depending on the vertical gradient between wells, the size of the area under consideration, the number of data points, and the length and location of the flow path. Because these are based on inferred or estimated parameters, flow rate estimates should be considered accurate to an order of magnitude only.

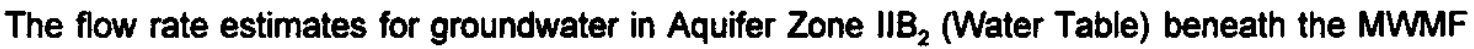
during second quarter 1994 are as follows (Figure 23, Appendix C):

- Flow path A (toward Fourmile Branch)

$\frac{3.03}{0.20} \times \frac{8}{2,450}=0.049 \mathrm{ft} / \mathrm{day}$

$0.049 \mathrm{ft} /$ day $\times 365$ days $\approx 18 \mathrm{ft} / \mathrm{yr}$

- Flow path B (toward Upper Three Runs Creek)

$$
\frac{3.03}{0.20} \times \frac{12}{2,400} \approx 0.076 \mathrm{ft} / \mathrm{day}
$$

$0.076 \mathrm{ft} /$ day $\times 365$ days $\approx 28 \mathrm{ft} / \mathrm{yr}$ 
The flow rate estimates for groundwater in Aquifer Zone IIB, (Barnwell/McBean) beneath the MWMF are as follows (Figure 24, Appendix C):

- Flow path A (toward Fourmile Branch)

$$
\frac{1.50}{0.20} \times \frac{8}{2,550} \approx 0.024 \mathrm{ft} / \mathrm{day}
$$

$0.024 \mathrm{ft} /$ day $\times 365$ days $\approx 8.8 \mathrm{ft} / \mathrm{yr}$

- Flow path B (toward Upper Three Runs Creek)

$$
\begin{aligned}
& \frac{1.50}{0.20} \times \frac{12}{2,200} \approx 0.041 \mathrm{ft} / \text { day } \\
& 0.041 \mathrm{ft} / \text { day } \times 365 \text { days } \approx 15 \mathrm{ft} / \mathrm{yr}
\end{aligned}
$$

The flow rate estimate for groundwater in Aquifer Unit IIA (Congaree) beneath the MWMF is as follows (Figure 25, Appendix C):

$$
\frac{45}{0.25} \times \frac{5}{1,500} \approx 0.60 \mathrm{ft} / \mathrm{day}
$$

$$
0.60 \mathrm{ft} / \text { day } \times 365 \text { days } \approx 220 \mathrm{ft} / \mathrm{yr}
$$

\section{Upgradient Versus Downgradient Results}

Wells BGO 1D, 2D, and HSB 85C are upgradient wells in Aquifer Zone $\| B_{2}$ (Water Table); wells HSB $85 \mathrm{~A}$ and 85B are upgradient wells in Aquifer Unit IIA (Congaree) and Aquifer Zone IIB, (Barnwell/McBean), respectively. The remaining wells in these hydrostratigraphic units monitor downgradient water quality.

Upgradient well BGO 2D contained tritium activity that exceeded the final PDWS during second quarter 1994. No elevated constituents were detected in the remaining upgradient wells. Downgradient wells in the three hydrostratigraphic units contained elevated levels of chloroethene, 1,1-dichloroethylene, gross alpha, lead, tetrachloroethylene, trichloroethylene, or tritium. 


\section{Quality Control Results}

Wells BGO $8 A R, 35 C, 46 B$, and BGX $4 A$ were selected to receive duplicate and blind replicate analyses during second quarter 1994 . Blind replicate analyses, representing approximately $5 \%$ of the quarter's total groundwater samples, are performed by the analytical laboratories each quarter for wells selected by EPD/EMS as part of the EPD/EMS quality assurance program (see Appendix E). The results of the analyses are used for both intralaboratory and interlaboratory comparisons. As a part of intralaboratory quality assurance procedures, certain analyses were duplicated by the laboratory. The results of duplicate and replicate analyses are reported in Table 3 (Appendix D).

The Savannah River Site's Groundwater Monitoring Program, Second Quarter 1994 (U) (EPD/EMS, 1994) provides a full evaluation of data quality control and an explanation of analytical results for the quarter. 


\section{Conclusions}

The groundwater at the MWMF contains elevated levels of heavy metals, radionuclides, volatile organic compounds, and other constituents resulting from 40 years of operations at the Burial Ground Complex. During second quarter 1994, chloroethene, 1,1-dichloroethylene, gross alpha, lead, tetrachloroethylene, trichloroethylene, and tritium exceeded their final PDWS in one or more wells at the MWMF. All of these constituents, except gross alpha, also occurred in elevated levels during first quarter 1994. Copper, mercury, and nonvolatile beta, which were elevated in one or two wells during first quarter, were not above standards during second quarter. Elevated lead occurred only in well FSS 3D during the quarter; nearby BGO wells do not have a history of elevated lead concentrations.

As in previous quarters, tritium and trichloroethylene were the most widespread constituents. Sixty-two (50\%) of the 125 monitoring wells contained elevated tritium activities, with maximum activity (1.6E+05 pCi/mL) occurring in well BGO 28D in Aquifer Zone $1 / B_{2}$ (Water Table). Trichloroethylene concentrations exceeded the final PDWS in $24(19 \%)$ of the wells, with the maximum concentration found at $208 \mu \mathrm{g} / \mathrm{L}$ in well BGO 28D in Aquifer Zone IIB ${ }_{2}$ (Water Table).

Elevated constituent levels were found primarily in Aquifer Zone $\mathrm{IBB}_{2}$ (Water Table) and Aquifer Zone IIB, (Barnwell/McBean), consistent with historical data. No elevated constituents occurred in wells in Aquifer Unit IIA (Congaree), but past quarters' results have indicated that vertical pathways into this deeper water-bearing unit exist. A current groundwater flow model for the General Separations Area indicates that the vertical component beneath this area is important (GeoTrans, Inc., 1992).

Constituents exceeding the final PDWS were found in upgradient well BGO 2D and in numerous downgradient wells. Generally, elevated levels of constituents found in downgradient wells but not in upgradient wells at a waste management unit are considered products of the waste management unit.

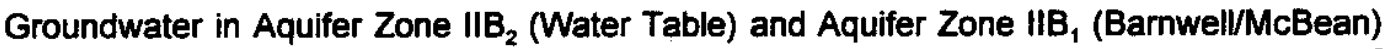
diverges beneath the Burial Ground Complex and discharges toward Upper Three Runs Creek or toward Fourmile Branch. During second quarter 1994, flow in these units was to the north toward Upper Three Runs Creek or to the west-southwest toward Fourmile Branch (UTM coordinates). The groundwater flow direction in Aquifer Unit IIA (Congaree) is consistently northwest toward Upper Three Runs Creek.

Groundwater flow rate estimates were in the same order of magnitude as previous quarters. Estimates ranged from $18 \mathrm{ft} / \mathrm{yr}$ to $28 \mathrm{ft} / \mathrm{yr}$ in Aquifer Zone $\| \mathrm{B}_{2}$ (Water Table) and from $8.8 \mathrm{ft} / \mathrm{yr}$ to $15 \mathrm{ft} / \mathrm{yr}$ in Aquifer Zone $\|_{1} \mathrm{~B}_{1}$ (Barnwell/McBean); the flow rate estimate. in Aquifer Unit IIA (Congaree) was $220 \mathrm{ft} / \mathrm{yr}$. 
WSRC-TR-94-0345

Unclassified

\section{References Cited}

Aadland, R. K., and H. W. Bledsoe, 1990. Classification of Hydrostratigraphic Units at the Savannah River Site, South Carolina, WSRC-RP-90-987. Westinghouse Savannah River Company, Savannah River Site, Aiken, SC.

EPD/EMS (Environmental Protection Department/Environmental Monitoring Section), 1992. Hydrogeologic Data Collection Procedures and Specifications: Sampling Groundwater Monitoring Wells, Manual 3Q5, Chapter 15, Revision 0. Westinghouse Savannah River Company, Savannah River Site, Aiken, SC.

EPD/EMS (Environmental Protection Department/Environmental Monitoring Section), 1994. Environmental Protection Department's Well Inventory (through the fourth quarter of 1993), ESH-EMS-930262. Westinghouse Savannah River Company, Savannah River Site, Aiken, SC.

EPD/EMS (Environmental Protection Department/Environmental Monitoring Section), 1994. The Savannah River Site's Groundwater Monitoring Program, Second Quarter 1994 (U), ESHEMS-940515. Westinghouse Savannah River Company, Savannah River Site, Aiken, SC (in preparation).

GeoTrans, Inc., 1992. Groundwater Flow Model for the General Separations Area, Savannah River Site. Prepared for Westinghouse Savannah River Company, Environmental Restoration Department, Savannah River Site, Aiken, SC.

Heffner, J. D., and Exploration Resources, inc., 1991. Technical Summary of Groundwater Quality Protection Program at the Savannah River Site (1952-1986), Volume I-Site Geohydrology and Waste Sites, DPSP-88-1002. Westinghouse Savannah River Company, Aiken, SC.

Jaegge, W. J., N. L. Kolb, B. B. Looney, I. W. Marine, O. A. Towler, and J. R. Cook, 1987. Environmental Information Document: Radioactive Waste Burial Grounds, DPST-85-694. Savannah River Laboratory, E. I. du Pont de Nemours \& Company, Aiken, SC.

SCDHEC (South Carolina Department of Health and Environmental Control), 1993. South Carolina Hazardous Waste Management Regulations; R.61-79.124, .260 through .266, .268, and .270. Columbia, SC.

WSRC (Westinghouse Savannah River Company), 1992. 1992 RCRA Part B Permit Renewal Application. Mixed Waste Management Facility (MWMF) Post-Closure, Volume VII, Revision 0. November 1992, WSRC-IM-91-53. Savannah River Site, Aiken, SC.

WSRC (Westinghouse Savannah River Company), 1993a. A Field Investigation Plan for the Burial Ground Complex. September 1993, WSRC-RP-93-848. Savannah River Site, Aiken, SC. 
WSRC (Westinghouse Savannah River Company), 1993b. Mixed Waste Management Facility Closure Plan (LLRWDF), Volume II, August 1993, Q-CLP-E-00001. Savannah River Site, Aiken, SC.

WSRC (Westinghouse Savannah River Company), 1993c. 1992 RCRA Part B Permit Application, Mixed Waste Management Facillty Postclosure, Volume VII, Revision 2, November 1993, WSRC-IM-91-53. Savannah River Site, Aiken, SC. 


\section{Errata}

In tables with four quarters of data, some values for earlier quarters may differ from values for those same quarters presented in earlier reports because some reanalyses may have been performed by the laboratories after the reports were printed.

Second Quarter 1993:

- No errata have been reported.

Third Quarter 1993:

- Page D-5, Table 1: The correct result for trichloroethylene in well BGO 12D during second quarter is $83 \mu \mathrm{g} / \mathrm{L}$.

- Page F-14, Trichloroethylene concentrations, well cluster BGO 6: The symbols were incorrect. The open triangle should be a black square; the black square should be an open triangle.

Fourth Quarter 1993:

- No errata have been reported.

First Quarter 1994:

- No errata have been reported. 
WSRC-TR-94-0345

Unclassified

THIS PAGE LEFT BLANK INTENTIONALLY. 


\section{Appendix A}

Final Primary Drinking Water Standards 
WSRC-TR-94-0345

Unclassified

THIS PAGE LEFT BLANK INTENTIONALLY. 
Final Primary Drinking Water Standards

\begin{tabular}{|c|c|c|c|c|}
\hline Analyte & Unit & Level & Status & Source \\
\hline Alachlor & $\mu g \Omega$ & 2 & Final & EPA, 1993 \\
\hline Aldicarb $^{a}$ & $\mu g / L$ & 3 & Final & $\begin{array}{l}\text { EPA, } 1993 \\
\text { EPA, } 1993\end{array}$ \\
\hline Aldicarb sulfone $e^{a}$ & $\mu g / L$ & 2 & Final & $\begin{array}{l}\text { EPA, } 1993 \\
\text { EPA, } 1993\end{array}$ \\
\hline Aldicarb sulfoxide ${ }^{a}$ & $\mu g / L$ & $\begin{array}{l}4 \\
6\end{array}$ & $\begin{array}{l}\text { Final } \\
\text { Final }\end{array}$ & EPA, 1993 \\
\hline Antimony & $\mu g / 2$ & $\begin{array}{l}6 \\
6\end{array}$ & Final & EPA, 1993 \\
\hline & $\lg / \mathrm{L}$ & $\begin{array}{l}6 \\
6\end{array}$ & Final & EPA, 1993 \\
\hline $\begin{array}{l}\text { Antimony, total recoverable } \\
\text { Arsenic }\end{array}$ & & $\begin{array}{l}6 \\
50\end{array}$ & Final & EPA, 1993 \\
\hline $\begin{array}{l}\text { Arsenic } \\
\text { Arsenic, dissolved }\end{array}$ & $\mu \mu_{\mu g h}^{\mu}$ & 50 & Final & EPA, 1993 \\
\hline $\begin{array}{l}\text { Arsenic, dissolved } \\
\text { Arsenic, total recoverable }\end{array}$ & $\mu g h$ & 50 & Final & EPA, 1993 \\
\hline Asbestos & Fibers $/$ & $7,000,000$ & Final & EPA, 1993 \\
\hline $\begin{array}{l}\text { Atrazine } \\
\text { Barium }\end{array}$ & $\mu g h$ & $\begin{array}{l}3 \\
2,000\end{array}$ & & $\begin{array}{l}\text { EPA, } 1993 \\
\text { EPA, } 1993\end{array}$ \\
\hline $\begin{array}{l}\text { Barium } \\
\text { Barium, dissolved }\end{array}$ & $\mu g / L$ & $\begin{array}{l}2,000 \\
2,000\end{array}$ & $\begin{array}{l}\text { Final } \\
\text { Final }\end{array}$ & EPA, 1993 \\
\hline $\begin{array}{l}\text { Barium, dissolved } \\
\text { Barium, total recoverable }\end{array}$ & $\begin{array}{l}\mu g / L \\
\mu g h\end{array}$ & 2,000 & Final & EPA, 1993 \\
\hline Benzene & $\mu g /$ & $\begin{array}{l}5 \\
0.2\end{array}$ & Final & $\begin{array}{l}\text { EPA, } 1993 \\
\text { EPA, } 1993\end{array}$ \\
\hline $\begin{array}{l}\text { Benzo[a]pyrene } \\
\text { Beryllium }\end{array}$ & $\mu g h /$ & $\begin{array}{l}0.2 \\
4\end{array}$ & $\begin{array}{l}\text { Final } \\
\text { Final }\end{array}$ & $\begin{array}{l}\text { EPA, } 1993 \\
\text { EPA, } 1993\end{array}$ \\
\hline $\begin{array}{l}\text { Beryllium } \\
\text { Beryllium, dissolved }\end{array}$ & $\begin{array}{l}\rho g / L \\
\mu g h L\end{array}$ & 4 & Final & EPA, 1993 \\
\hline $\begin{array}{l}\text { Beryllium, dissolved } \\
\text { Beryllium, total recoverable }\end{array}$ & $\mu g / 2$ & 4 & Final & EPA, 1993 \\
\hline $\begin{array}{l}\text { Beryllium, total recoverable } \\
\text { Bis(2-ethylhexyl) phthalate }\end{array}$ & $\mu g h$ & 6 & Final & EPA, 1993 \\
\hline $\begin{array}{l}\text { Bis(2-ethylhexyl) phthalate } \\
\text { Bromodichloromethane }\end{array}$ & $\mu g h$ & 100 & Final & EPA, 1993 \\
\hline $\begin{array}{l}\text { Bromodichloromethane } \\
\text { Bromoform }\end{array}$ & $\mu g h$ & 100 & Final & EPA, 1993 \\
\hline $\begin{array}{l}\text { Bromoform } \\
\text { 2-sec-Butyl-4.6-dinitrophenol }\end{array}$ & $\mu g / 2$ & 7 & Final & EPA, 1993 \\
\hline & $\mu g h$ & 5 & Final & EPA, 1993 \\
\hline $\begin{array}{l}\text { Cadmium } \\
\text { Cadmium, dissolved }\end{array}$ & $\mu g h$ & 5 & Final & EPA, 1993 \\
\hline & $\mu g / 2$ & 5 & Final & EPA, 1993 \\
\hline $\begin{array}{l}\text { Cadmium, total recoverable } \\
\text { Carbofuran }\end{array}$ & $\mu g / 2$ & 40 & Final & EPA, 1993 \\
\hline Carbon tetrachloride & $\mu g / \Omega$ & 5 & Final & EPA, 1993 \\
\hline Chlordane & $\mu g h$ & 2 & Final & $\begin{array}{l}\text { EPA, } 1993 \\
\text { FPA } 1993\end{array}$ \\
\hline Chlorobenzene & $\mu g h$ & 100 & Final & $\begin{array}{l}\text { EPA, } 1993 \\
\text { EPA, } 1993\end{array}$ \\
\hline Chloroethene (Vinyl chloride) & $\mu g / 2$ & $\begin{array}{l}2 \\
100\end{array}$ & $\begin{array}{l}\text { Final } \\
\text { Final }\end{array}$ & EPA, 1993 \\
\hline Chloroform & $\operatorname{mgh}$ & $\begin{array}{l}100 \\
100\end{array}$ & Final & EPA, 1993 \\
\hline Chromium & $\mu g h /$ & 100 & Final & EPA, 1993 \\
\hline Chromium, dissolved & $\mu g / 2$ & 100 & Final & EPA, 1993 \\
\hline Chromium, total recoverable & 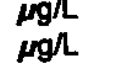 & 1,300 & Final & EPA, 1993 \\
\hline Copper & $\mu g / \mathrm{L}$ & 1,300 & Final & EPA, 1993 \\
\hline Copper, dissolved & $\mu g h$ & 1,300 & Final & EPA, 1993 \\
\hline Copper, total recoverable & $\mu g /$ & 200 & Final & EPA, 1993 \\
\hline Cyanide & $\mu g / L$ & 200 & Final & EPA, 1993 \\
\hline Dalapon ${ }^{\mathrm{a}}$ & $\mu g \Omega$ & 100 & Final & EPA, 1993 \\
\hline $\begin{array}{l}\text { Dibromochloromethane } \\
\text { 12-Dibromo-3-chloropropane }\end{array}$ & $\mu g h$ & 0.2 & Final & EPA, 1993 \\
\hline $\begin{array}{l}\text { 1,2-Dibromo-3-chloropropane } \\
\text { 1,2-Dibromoethane }\end{array}$ & $\mu g h$ & 0.05 & Final & EPA, 1993 \\
\hline $\begin{array}{l}\text { 1,2-Dibromoethane } \\
\text { 12-Dichlorobenzene }\end{array}$ & $\mu g \Omega$ & 600 & Finat & EPA, 1993 \\
\hline 1,2-Dichlorobenzene & & 75 & Final & EPA, 1993 \\
\hline $\begin{array}{l}\text { 1,4-Dichlorobenzene } \\
\text { 12-Dichloroethane }\end{array}$ & $\mu g h$ & 5 & Final & EPA, 1993 \\
\hline 1,2-Dichloroethane & $\mu \mathrm{g} / \mathrm{L}$ & 7 & Final & EPA, 1993 \\
\hline $\begin{array}{l}\text { 1,1-Dichloroethylene } \\
\text { 12-Dichloroethylene }\end{array}$ & $\mu g h$ & 50 & Final & EPA, 1993 \\
\hline & $\mu g h$ & 70 & Final & EPA, 1993 \\
\hline $\begin{array}{l}\text { cis-1,2-Dichloroethylene } \\
\text { trans-1,2-Dichloroethylene }\end{array}$ & & 100 & Final & EPA, 1993 \\
\hline Dichloromethane (Methylene chloride & $\mu \mathrm{g} / \mathrm{L}$ & $\begin{array}{l}5 \\
70\end{array}$ & $\begin{array}{l}\text { Final } \\
\text { Final }\end{array}$ & EPA, 1993 \\
\hline 2,4-Dichlorophenoxyacetic acid & & 70 & Final & EPA, 1993 \\
\hline
\end{tabular}


Analivte

1,2-Dichloropropane

Di(2-ethylhexyl) adipate ${ }^{a}$

Diquat dibromide ${ }^{a}$

Endothall ${ }^{\mathrm{a}}$

Endrin

Ethylbenzene

Fluoride

Glyphosate $^{a}$

Gross alphab

Heptachlor

Heptachlor epoxide

Hexachlorobenzene

Hexachlorocyclopentadiene

Lead

Lead, dissolved

Lead, total recoverable

Lindane

Mercury

Mercury, dissolved

Mercury, total recoverable

Methoxychlor

Nickel

Nickel, dissolved

Nickel, total recoverable

Nitrate as nitrogen

Nitrate-nitrite as nitrogen

Nitrite as nitrogen

Nonvolatile beta

Oxamyl ${ }^{a}$

PCB 1016

PCB 1221

PCB 1232

PCB 1242

PCB 1248

PCB 1254

PCB 1260

PCB 1262

Pentachlorophenol

Picloram $^{\text {a }}$

Selenium

Selenium, dissolved

Selenium, total recoverable

Simazine ${ }^{a}$

Strontium-89/90

Strontium-90

Styrene

2,3,7,8-TCDD

Tetrachloroethylene

Thallium

Thallium, dissolved

Thallium, total recoverable

Toluene

Toxaphene

2,4,5-TP (Silvex)

1,2,4-Trichlorobenzene

\begin{tabular}{|c|c|c|}
\hline Unit & Level & $\underline{\text { Status }}$ \\
\hline$\mu g /$ & 5 & Final \\
\hline$\mu g /$ & 400 & Final \\
\hline$\mu g / L$ & 20 & Final \\
\hline$\mu g /$ & 100 & Final \\
\hline$\mu g /$ & 2 & Final \\
\hline$\mu g / L$ & 700 & Final \\
\hline$\mu g /$ & 4,000 & Final \\
\hline$\mu g / L$ & 700 & Final \\
\hline $\mathrm{pCi} / \mathrm{L}$ & $1.5 E+01$ & Final \\
\hline$\mu g / L$ & 0.4 & Final \\
\hline$\mu g / L$ & 0.2 & Final \\
\hline$\mu g / h$ & 1 & Final \\
\hline$\mu g / L$ & 50 & Final \\
\hline$\mu g /$ & 50 & Final \\
\hline$\mu g / L$ & 50 & Final \\
\hline$\mu g /$ & 50 & Final \\
\hline$\mu g h$ & 0.2 & Final \\
\hline$\mu g /$ & 2 & Final \\
\hline$\mu g h$ & 2 & Final \\
\hline$\mu g / L$ & 2 & Final \\
\hline$\mu g / L$ & 40 & Final \\
\hline$\mu g / L$ & 100 & Final \\
\hline$\mu g /$ & 100 & Final \\
\hline$\mu g /$ & 100 & Final \\
\hline$\mu g /$ & 10,000 & Final \\
\hline$\mu g /$ & 10,000 & Final \\
\hline$\mu g / 2$ & 1,000 & Final \\
\hline pCin & $5 E+01$ & Interim Final \\
\hline$\mu g /$ & 200 & Final \\
\hline$\mu g / L$ & 0.5 & Final \\
\hline$\mu g / L$ & 0.5 & Final \\
\hline$\mu g / L$ & 0.5 & Final \\
\hline$\mu g / L$ & 0.5 & Final \\
\hline$\mu g / h$ & 0.5 & Final \\
\hline $\mathrm{\mu g} / \mathrm{L}$ & 0.5 & Final \\
\hline$\mu g / L$ & 0.5 & Final \\
\hline$\mu g /$ & 0.5 & Final \\
\hline$\mu g /$ & 1 & Final \\
\hline$\mu g /$ & 500 & Final \\
\hline$\mu g /$ & 50 & Final \\
\hline$\mu g /$ & 50 & Final \\
\hline$\mu g /$ & 50 & Final \\
\hline$\mu g /$ & 4 & Final \\
\hline $\mathrm{pCi}$ & $8 E+00$ & Final \\
\hline pCir & $8 E+00$ & Final \\
\hline$\mu g h$ & 100 & Final \\
\hline$\mu g /$ & 0.00003 & Final. \\
\hline$\mu g /$ & 5 & Final \\
\hline$\mu g /$ & 2 & Final \\
\hline$\mu g / L$ & 2 & Final \\
\hline$\mu g / L$ & 2 & Final \\
\hline$\mu g / L$ & 1,000 & Final \\
\hline$\mu g / 2$ & 3 & Final \\
\hline $\operatorname{mgh}$ & 50 & Final \\
\hline$\mu g /$ & 70 & Final \\
\hline
\end{tabular}

Source

EPA, 1993

EPA, 1993

EPA, 1993

EPA, 1993

EPA, 1993

EPA, 1993

EPA, 1993

EPA, 1993

EPA, 1993

EPA, 1993

EPA, 1993

EPA, 1993

EPA, 1993

SCDHEC, 1981

SCDHEC, 1981

SCDHEC, 1981

EPA, 1993

EPA, 1993

EPA, 1993

EPA, 1993

EPA, 1993

EPA, 1993

EPA, 1993

EPA, 1993

EPA, 1993

EPA, 1993

EPA, 1993

EPA, 1977

EPA, 1993

EPA, 1993

EPA, 1993

EPA, 1993

EPA, 1993

EPA, 1993

EPA, 1993

EPA, 1993

EPA, 1993

EPA, 1993

EPA, 1993

EPA, 1993

EPA, 1993

EPA, 1993

EPA, 1993

EPA, 1993

EPA, 1993

EPA, 1993

EPA, 1993

EPA, 1993

EPA, 1993

EPA, 1993

EPA, 1993

EPA, 1993

EPA, 1993

EPA, 1993

EPA, 1993 
Analyte

1,1,1-Trichloroethane

1,1,2-Trichloroethane

Trichloroethylene

Tritium

Xylenes
Unit

$\mu g / L \quad 200$

$\mu \mathrm{g} / \mathrm{L}$

$\mu g / L$ pCi/mL $\mu g / L$
Level

5

5

$2 \mathrm{E}+01$

10,000
Status

Final

Final

Final

Final

Final
Source

EPA, 1993

EPA, 1993

EPA, 1993

EPA, 1993

EPA, 1993

Note: Final PDWS were assigned to alachlor, aldicarb, aldicarb sulfone, aldicarb sulfoxide, atrazine, carbofuran, dalapon, di(2-ethylhexyl) adipate, diquat dibromide, endothall, glyphosate, oxamyl, picloram, and simazine in the SRS Groundwater Monitoring Program for the first time beginning first quarter 1994.

a At present, EMS does not perform this analysis because the constituent is not in the current contract.

b The standard given is for gross alpha including radium-226 but excluding radon and uranium.

c For double radionuclide analyses where each separate radionuclide has its own standard, the more stringent standard is used.

\section{References}

EPA (U.S. Environmental Protection Agency), 1977. National Interim Primary Drinking Water Regulations, EPA-570/9-76-003. Washington, DC.

EPA (U.S. Environmental Protection Agency), 1993. National Primary Drinking Water Regulations, Code of Federal Regulations, Section 40, Part 141, pp. 592-732. Washington, DC.

SCDHEC (South Carolina Department of Health and Environmental Control), 1981. State Primary Drinking Water Regulations, R.61-58.5. Columbia, SC. 
WSRC-TR-94-0345

Unclassified

THIS PAGE LEFT BLANK INTENTIONALLY. 


\section{Appendix B}

\section{Flagging Criteria}


WSRC-TR-94-0345

Unclassified

THIS PAGE LEFT BLANK INTENTIONALLY. 


\section{Flagging Criteria}

The Savannah River Site Environmental Protection Department/Environmental Monitoring Section (EPD/EMS) flagging criteria are as follows:

- Flag 2 criteria for constituents equal the Safe Drinking Water Act (SDWA) final Primary Drinking Water Standard (PDWS), the SDWA proposed PDWS, or the SDWA Secondary Drinking Water Standard (SDWS). If a constituent does not have a drinking water standard, the Flag 2 criterion equals 10 times the method detection limit (MDL) calculated as the 90th percentile detection limit obtained recently by one of the primary analytical laboratories.

- Flag 1 criteria for constituents equal one-half of the final PDWS, one-half the proposed PDWS, or one-half the SDWS. If a constituent does not have a drinking water standard, the Flag 1 criterion equals 5 times the MDL calculated as the 90th percentile detection limit obtained recently by one of the primary analytical laboratories.

- Flag 0 criteria are assigned to constituent levels below Flag 1 criteria, constituent levels below the sample detection limits, or constituents having no flagging criteria.

The following parameters are exceptions to the flagging rules:

- EPD/EMS sets flagging criteria for specific conductance and pH. No flags are set for alkalinity, calcium, carbonate, magnesium, potassium, silica, sodium, total dissolved solids, total phosphates (as P), and total phosphorus. Analyses for these parameters are conducted as part of the biennial comprehensive analyses or by special request.

- Aesthetic parameters such as color, corrosivity, Eh, odor, surfactants, and turbidity are not assigned flagging criteria but are analyzed by special request.

- Common laboratory contaminants and cleaners such as dichloromethane (methylene chloride), ketones, phthalates, and toluene are not assigned flagging criteria unless they have primary drinking water standards. These constituents are analyzed by special request.

\begin{tabular}{|c|c|c|c|c|}
\hline Analyte & Unit & Flag 1 & Flag 2 & Source $^{\mathrm{a}}$ \\
\hline Acenaphthene & $\mu g / L$ & 50 & 100 & EPA Method 8270 \\
\hline Acenaphthylene & $\mu g / L$ & 50 & 100 & EPA Method 8270 \\
\hline Acetone & $\mu g / L$ & 500 & 1,000 & EPA Method 8240 \\
\hline Acetonitrile (Methyl cyanide) & $\mu g / L$ & 500 & 1,000 & EPA Method 8240 \\
\hline Acetophenone & $\mu g / L$ & 50 & 100 & EPA Method 8270 \\
\hline 2-Acetylaminofluorene & $\mu g / L$ & 50 & 100 & EPA Method 8270 \\
\hline Acrolein & $\mu g / L$ & 100 & 200 & EPA Method 8240 \\
\hline Acrylonitrile & $\mu g / L$ & 100 & 200 & EPA Method 8240 \\
\hline Actinium-228 & $\mathrm{pCi} / \mathrm{L}$ & $1.64 E+03$ & $3.27 E+03$ & Proposed PDWS (EPA, 1991) \\
\hline Alachlor & $\mu g / L$ & 1 & 2 & Final PDWS (EPA, 1993a) \\
\hline Aldicarb ${ }^{b}$ & $\mu g / L$ & 1.5 & 3 & Final PDWS (EPA, 1993a) \\
\hline Aldicarb sulfone ${ }^{b}$ & $\mu g / L$ & 1 & 2 & Final PDWS (EPA, 1993a) \\
\hline Aldicarb sulfoxide ${ }^{b}$ & $\mu g / L$ & 2 & 4 & Final PDWS (EPA, 1993a) \\
\hline Aldrin & $\mu g / L$ & 0.25 & 0.5 & EPA Method 8080 \\
\hline Alkalinity (as $\mathrm{CaCO}_{3}$ ) & & No flag & No flag & Set by EPD/EMS \\
\hline Allyl chloride & $\mu g / L$ & 250 & 500 & EPA Method 8240 \\
\hline Aluminum & $\mu g / L$ & 25 & 50 & SDWS (EPA, 1993b) \\
\hline Aluminum, dissolved & $\mu g / L$ & 25 & 50 & SDWS (EPA, 1993b) \\
\hline Aluminum, total recoverable & $\mu g / L$ & 25 & 50 & SDWS (EPA, 1993b) \\
\hline
\end{tabular}




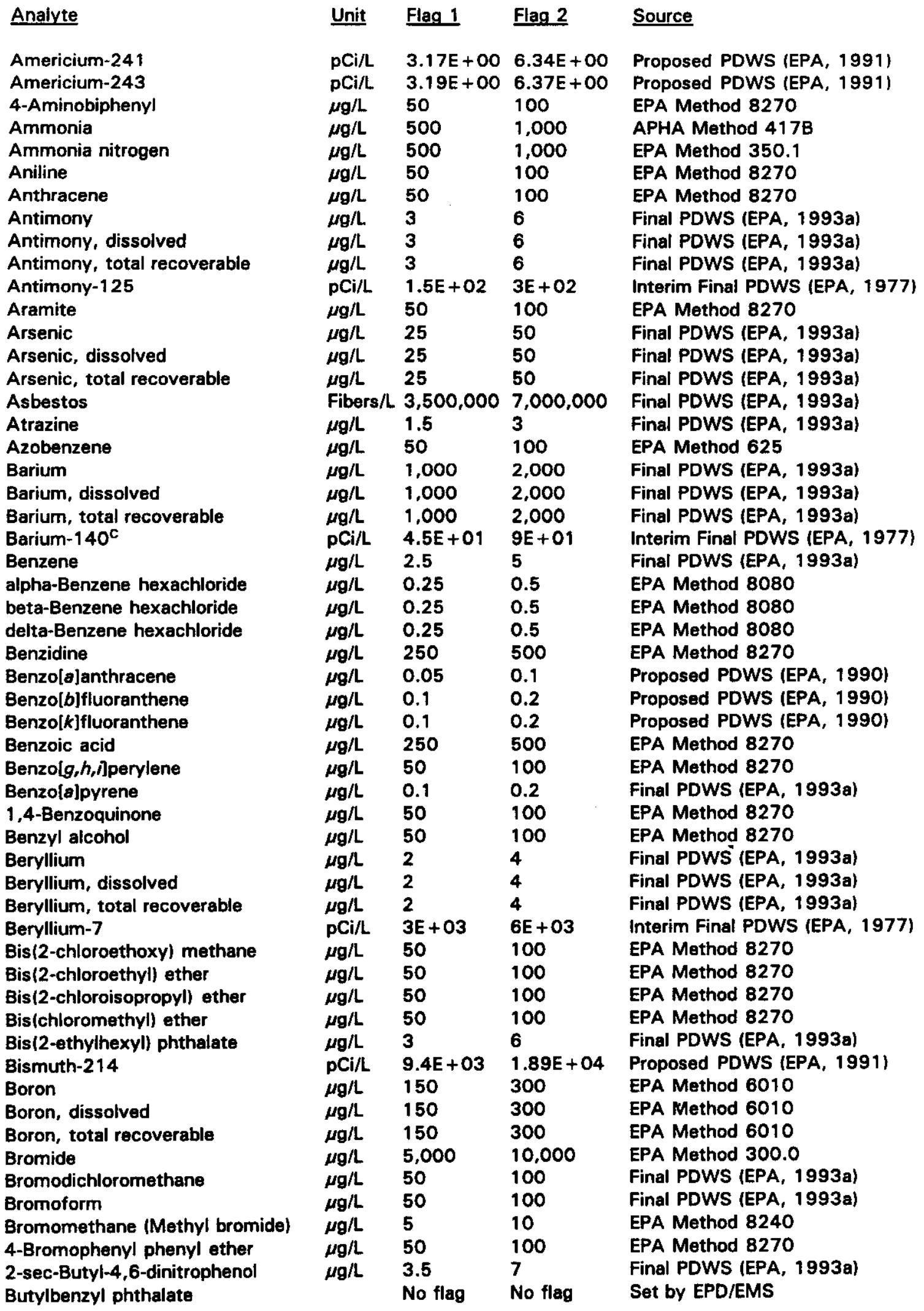




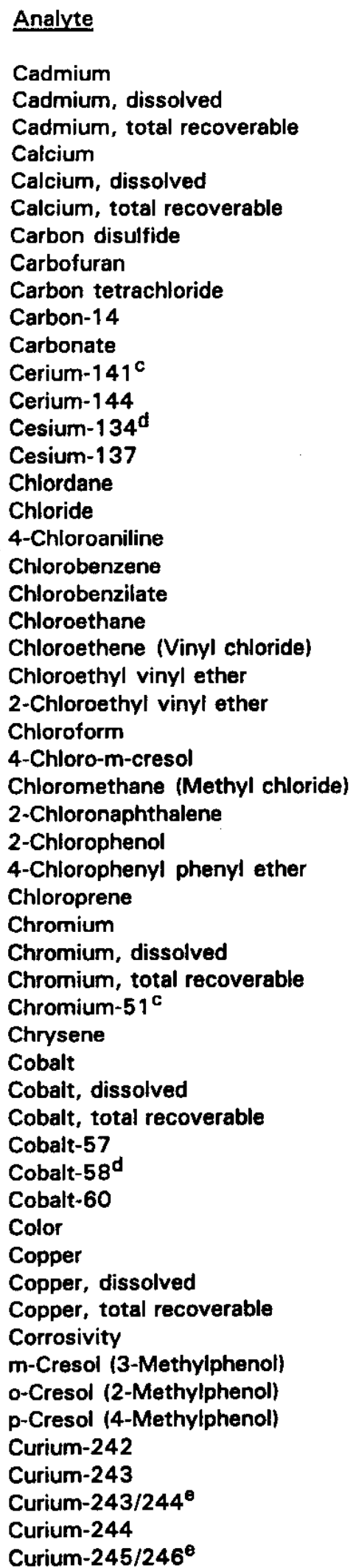

\begin{tabular}{|c|c|c|c|}
\hline Unit & Flag 1 & Flag 2 & Source \\
\hline$\mu g / L$ & 2.5 & 5 & Final PDWS (EPA, 1993a) \\
\hline$\mu g / L$ & 2.5 & 5 & Final PDWS (EPA, 1993a) \\
\hline$\mu g / L$ & 2.5 & 5 & Final PDWS (EPA, 1993a) \\
\hline & No flag & No flag & Set by EPD/EMS \\
\hline & No flag & No flag & Set by EPD/EMS \\
\hline & No flag & No flag & Set by EPD/EMS \\
\hline$\mu g / L$ & 5 & 10 & EPA Method 8240 \\
\hline$\mu g / L$ & 20 & 40 & Final PDWS (EPA, 1993a) \\
\hline$\mu g / L$ & 2.5 & 5 & Final PDWS (EPA, 1993a) \\
\hline $\mathrm{pCi} / \mathrm{L}$ & $1 E+03$ & $2 E+03$ & Interim Final PDWS (EPA, 1977) \\
\hline & No flag & No flag & Set by EPD/EMS \\
\hline $\mathrm{pCi} / \mathrm{L}$ & $1.5 \mathrm{E}+02$ & $3 E+02$ & Interim Final PDWS (EPA, 1977) \\
\hline $\mathrm{pCi} / \mathrm{L}$ & $1.31 E+02$ & $2.61 E+02$ & Proposed PDWS (EPA, 1991) \\
\hline $\mathrm{pCi} / \mathrm{L}$ & 4.07E+01 & $8.13 E+01$ & Proposed PDWS (EPA, 1991) \\
\hline $\mathrm{pCi} / \mathrm{L}$ & $1 E+02$ & $2 E+02$ & Interim Final PDWS (EPA, 1977) \\
\hline$\mu g / L$ & 1 & 2 & Final PDWS (EPA, 1993a) \\
\hline$\mu g / L$ & 125,000 & 250,000 & SDWS (EPA, 1993b) \\
\hline$\mu g / L$ & 50 & 100 & EPA Method 8270 \\
\hline$\mu \mathrm{g} / \mathrm{L}$ & 50 & 100 & Final PDWS (EPA, 1993a) \\
\hline$\mu \mathrm{g} / \mathrm{L}$ & 50 & 100 & EPA Method $\mathbf{8 2 7 0}$ \\
\hline$\mu g / L$ & 5 & 10 & EPA Method 8240 \\
\hline$\mu \mathrm{g} / \mathrm{L}$ & 1 & 2 & Final PDWS (EPA, 1993a) \\
\hline$\mu \mathrm{g} / \mathrm{L}$ & 5 & 10 & EPA Method 8240 \\
\hline$\mu g / L$ & 5 & 10 & EPA Method 8240 \\
\hline$\mu g / L$ & 50 & 100 & Final PDWS (EPA, 1993a) \\
\hline$\mu g / L$ & 50 & 100 & EPA Method 8270 \\
\hline$\mu g / L$ & 5 & 10 & EPA Method 8240 \\
\hline$\mu g / L$ & 50 & 100 & EPA Method 8240 \\
\hline$\mu g / L$ & 50 & 100 & EPA Method 8270 \\
\hline$\mu \mathrm{g} / \mathrm{L}$ & 50 & 100 & EPA Method 8270 \\
\hline$\mu \mathrm{g} / \mathrm{L}$ & 1,000 & 2,000 & EPA Method 8240 \\
\hline$\mu g / L$ & 50 & 100 & Final PDWS (EPA, 1993a) \\
\hline$\mu g / L$ & 50 & 100 & Final PDWS (EPA, 1993a) \\
\hline$\mu g / L$ & 50 & 100 & Final PDWS (EPA, 1993a) \\
\hline $\mathrm{pCi} / \mathrm{L}$ & $3 E+03$ & $6 E+03$ & Interim Final PDWS (EPA, 1977) \\
\hline$\mu g / L$ & 0.1 & 0.2 & Proposed PDWS (EPA, 1990) \\
\hline$\mu g / L$ & 20 & 40 & EPA Method 6010 \\
\hline$\mu \mathrm{g} / \mathrm{L}$ & 20 & 40 & EPA Method 6010 \\
\hline$\mu g / L$ & 20 & 40 & EPA Method 6010 \\
\hline $\mathrm{pCi} / \mathrm{L}$ & $5 E+02$ & $1 E+03$ & Interim Final PDWS (EPA, 1977) \\
\hline $\mathrm{pCi} / \mathrm{L}$ & $4.5 E+03$ & $9 E+03$ & Interim Final PDWS (EPA, 1977) \\
\hline pCi/L & $5 E+01$ & $1 E+02$ & Interim Final PDWS (EPA, 1977) \\
\hline & No flag & No flag & Set by EPD/EMS \\
\hline$\mu \mathrm{g} / \mathrm{L}$ & 500 & 1,000 & Final PDWS (SCDHEC, 1981) \\
\hline$\mu g / L$ & 500 & 1,000 & Final PDWS (SCDHEC, 1981) \\
\hline$\mu g / L$ & 500 & 1,000 & Final PDWS (SCDHEC, 1981) \\
\hline & No flag & No flag & Set by. EPD/EMS \\
\hline$\mu \mathrm{g} / \mathrm{L}$ & 50 & 100 & EPA Method 8270 \\
\hline & 50 & 100 & EPA Method 8270 \\
\hline$\mu g / L$ & 50 & 100 & EPA Method 8270 \\
\hline $\mathrm{pCi} / \mathrm{L}$ & $6.65 E+01$ & $1.33 E+02$ & Proposed PDWS (EPA, 1991) \\
\hline $\mathrm{pCi} / \mathrm{L}$ & $4.15 E+00$ & $8.3 E+00$ & Proposed PDWS (EPA, 1991) \\
\hline $\mathrm{pCi} / \mathrm{L}$ & $4.15 E+00$ & $8.3 E+00$ & Proposed PDWS (EPA, 1991) \\
\hline $\mathrm{pCi} / \mathrm{L}$ & $4.92 E+00$ & $9.84 E+00$ & Proposed PDWS (EPA, 1991) \\
\hline $\mathrm{pCi} / \mathrm{L}$ & $3.12 E+00$ & $6.23 E+00$ & Proposed PDWS (EPA, 1991) \\
\hline
\end{tabular}


Analyte

Curium-246

Cyanide

Dalapon $^{\mathrm{b}}$

$p, p^{\prime}-D D D$

$p, p^{\prime}-\mathrm{DDE}$

$p, p^{\prime}-D D T$

Di-n-butyl phthalate

Di-n-octyl phthalate

Diallate

Dibenz $[a, h]$ anthracene

Dibenzofuran

Dibromochloromethane

1,2-Dibromo-3-chloropropane

1,2-Dibromoethane

Dibromomethane

(Methylene bromide)

1,2-Dichlorobenzene

1,3-Dichlorobenzene

1,4-Dichlorobenzene

3,3'-Dichlorobenzidine

trans-1,4-Dichloro-2-butene

Dichlorodifluoromethane

1,1-Dichloroethane

1,2-Dichloroethane

1,1-Dichloroethylene

1,2-Dichloroethylene

cis-1,2-Dichloroethylene

trans-1,2-Dichloroethylene

Dichloromethane

(Methylene chloride)

2,4-Dichlorophenol

2,6-Dichlorophenol

2,4-Dichlorophenoxyacetic acid

1,2-Dichloropropane

cis-1,3-Dichloropropene

trans-1,3-Dichloropropene

Di(2-ethylhexyl) adipate

Dieldrin

Diethyl phthalate

Dimethoate

p-Dimethylaminoazobenzene

p-(Dimethylamino)ethylbenzene

7,12-Dimethylbenz[a]anthracene

3,3'-Dimethylbenzidine

a,a-Dimethylphenethylamine

2,4-Dimethyl phenol

Dimethyl phthalate

1,3-Dinitrobenzene

2,4-Dinitrophenol

2,4-Dinitrotoluene

2,6-Dinitrotoluene

Diquat dibromide $b$

1,4-Dioxane

Diphenylamine

1,2-Diphenylhydrazine

\begin{tabular}{|c|c|c|c|}
\hline Unit & Flag 1 & Flag 2 & Source \\
\hline $\begin{array}{l}\mathrm{pCi} / \mathrm{L} \\
\mu \mathrm{g} / \mathrm{L}\end{array}$ & $\begin{array}{l}3.14 E+00 \\
100\end{array}$ & $\begin{array}{l}6.27 E+00 \\
200\end{array}$ & $\begin{array}{l}\text { Proposed PDWS (EPA, } 1991 \\
\text { Final PDWS (EPA, 1993a) }\end{array}$ \\
\hline$\mu g / L$ & 100 & 200 & Final PDWS (EPA, 1993a) \\
\hline$\mu g / L$ & 0.5 & 1 & EPA Method 8080 \\
\hline$\mu \mathrm{g} / \mathrm{L}$ & 0.5 & 1 & EPA Method 8080 \\
\hline$\mu \mathrm{g} / \mathrm{L}$ & 0.5 & 1 & EPA Method $\mathbf{8 0 8 0}$ \\
\hline & No flag & No flag & Set by EPD/EMS \\
\hline$\mu g / L$ & $\begin{array}{l}\text { No flag } \\
50\end{array}$ & $\begin{array}{l}\text { No flag } \\
100\end{array}$ & $\begin{array}{l}\text { Set by EPD/EMS } \\
\text { EPA Method } 8270\end{array}$ \\
\hline$\mu g / L$ & 0.15 & 0.3 & $\begin{array}{l}\text { EPA Method } 8270 \\
\text { Proposed PDWS (EPA, } 1990\end{array}$ \\
\hline$\mu \mathrm{g} / \mathrm{L}$ & 50 & 100 & EPA Method 8270 \\
\hline$\mu \mathrm{g} / \mathrm{L}$ & 50 & 100 & Final PDWS (EPA, 1993a) \\
\hline$\mu g / L$ & 0.1 & 0.2 & Final PDWS (EPA, 1993a) \\
\hline$\mu g / L$ & 0.025 & 0.05 & Final PDWS (EPA, 1993a) \\
\hline$\mu g / L$ & 5 & 10 & EPA Method 8240 \\
\hline$\mu g / L$ & 300 & 600 & Final PDWS (EPA, 1993a) \\
\hline$\mu g / L$ & 50 & 100 & EPA Method 8270 \\
\hline$\mu g / L$ & 37.5 & 75 & Final PDWS (EPA, 1993a) \\
\hline$\mu g / L$ & 50 & 100 & EPA Method 8270 \\
\hline$\mu g / L$ & 150 & 300 & EPA Method 8240 \\
\hline$\mu g / L$ & 5 & 10 & EPA Method 8240 \\
\hline$\mu g / L$ & 5 & 10 & EPA Method 8240 \\
\hline$\mu \mathrm{g} / \mathrm{L}$ & 2.5 & 5 & Final PDWS (EPA, 1993a) \\
\hline$\mu \mathrm{g} / \mathrm{L}$ & 3.5 & 7 & Final PDWS (EPA, 1993a) \\
\hline$\mu g / L$ & 25 & 50 & Final PDWS (EPA, 1993a) \\
\hline$\mu g / L$ & 35 & 70 & Final PDWS (EPA, 1993a) \\
\hline$\mu g / L$ & 50 & 100 & Final PDWS (EPA, 1993a) \\
\hline$\mu \mathrm{g} / \mathrm{L}$ & 2.5 & 5 & Final PDWS (EPA, 1993a) \\
\hline$\mu g / L$ & 50 & 100 & EPA Method 8270 \\
\hline$\mu g / L$ & 50 & 100 & EPA Method 8270 \\
\hline$\mu \mathrm{g} / \mathrm{L}$ & 35 & 70 & Final PDWS (EPA, 1993a) \\
\hline$\mu \mathrm{g} / \mathrm{L}$ & 2.5 & 5 & Final PDWS (EPA, 1993a) \\
\hline$\mu g / L$ & 5 & 10 & EPA Method 8240 \\
\hline$\mu g / L$ & 5 & 10 & EPA Method 8240 \\
\hline$\mu g / L$ & 200 & 400 & Final PDWS (EPA, 1993a) \\
\hline$\mu g / L$ & 2.5 & 5 & EPA Method 8080 \\
\hline & No flag & No flag & Set by EPD/EMS \\
\hline$\mu g / L$ & 50 & 100 & EPA Method 8270 \\
\hline$\mu g / L$ & 50 & 100 & EPA Method 8270 \\
\hline $\begin{array}{l}\mu \mathrm{g} / \mathrm{L} \\
\mu \mathrm{g} / \mathrm{L}\end{array}$ & 50 & 100 & EPA Method 8270 \\
\hline $\begin{array}{l}\mu g / L \\
\mu g / L\end{array}$ & 50 & 100 & EPA Method 8270 \\
\hline$\mu \mathrm{g} / \mathrm{L}$ & 50 & 100 & EPA Method 8270 \\
\hline$\mu g / L$ & 50 & 100 & EPA Method 8270 \\
\hline$\mu \mathrm{g} / \mathrm{L}$ & 50 & 100 & EPA Method 8270 \\
\hline & No flag & No flag & Set by EPD/EMS \\
\hline $\begin{array}{l}\mu g / L \\
\mu g / L\end{array}$ & 50 & 100 & EPA Method 8270 \\
\hline $\begin{array}{l}\mu g / L \\
\mu g / L\end{array}$ & 250 & 500 & EPA Method 8270 \\
\hline$\mu g / \mathrm{L}$ & 50 & 100 & EPA Method 8270 \\
\hline$\mu g / L$ & 50 & 100 & EPA Method 8270 \\
\hline$\mu g / L$ & 10 & 20 & Final PDWS (EPA, 1993a) \\
\hline$\mu g / L$ & 50 & 100 & EPA Method 8270 \\
\hline$\mu \mathrm{g} / \mathrm{L}$ & 50 & 100 & EPA Method 8270 \\
\hline$\mu \mathrm{g} / \mathrm{L}$ & 50 & 100 & EPA Method 8270 \\
\hline
\end{tabular}


Analyte

Dissolved organic carbon Disulfoton

Eh

Endosulfan I

Endosulfan II

Endosulfan sulfate

Endothall ${ }^{b}$

Endrin

Endrin aldehyde

Endrin ketone

Ethylbenzene

Ethyl methacrylate

Ethyl methanesulfonate

Europium-152

Europium-154

Europium-155

Famphur

Fluoranthene

Fluorene

Fluoride

Glyphosate $^{b}$

Gross alpha

Heptachlor

Heptachlor epoxide

Heptachlorodibenzo-p-dioxin isomers

$1,2,3,4,6,7,8-H P C D D$

Heptachlorodibenzo-p-furan isomers

1,2,3,4,6,7,8-HPCDF

Hexachlorobenzene

Hexachlorobutadiene

Hexachlorocyclopentadiene

Hexachlorodibenzo-p-dioxin isomers

$1,2,3,4,7,8-H X C D D$

Hexachlorodibenzo-p-furan isomers

$1,2,3,4,7,8-H \times C D F$

Hexachloroethane

Hexachlorophene

Hexachloropropene

2-Hexanone

Indenol 1,2,3-c, d]pyrene

lodine

lodine-129

lodine-131 $\mathrm{c}$

lodomethane (Methyl iodide)

Iron

Iron, dissolved

Iron, total recoverable

Iron $-55^{\mathrm{c}}$

Iron-59 ${ }^{\mathrm{C}}$

Isobutyl alcohol

Isodrin

\begin{tabular}{|c|c|c|}
\hline Unit & Flag 1 & Flag 2 \\
\hline $\begin{array}{l}\mu g / L \\
\mu g / L\end{array}$ & $\begin{array}{l}5,000 \\
50 \\
\text { No flag }\end{array}$ & $\begin{array}{l}10,000 \\
100 \\
\text { No flag }\end{array}$ \\
\hline$\mu g / L$ & 0.5 & 1 \\
\hline$\mu \mathrm{g} / \mathrm{L}$ & 0.5 & 1 \\
\hline$\mu \mathrm{g} / \mathrm{L}$ & 0.5 & 1 \\
\hline$\mu \mathrm{g} / \mathrm{L}$ & 50 & 100 \\
\hline$\mu \mathrm{g} / \mathrm{L}$ & 1 & 2 \\
\hline$\mu g / L$ & 0.5 & 1 \\
\hline & No flag & No flag \\
\hline$\mu g / L$ & 350 & 700 \\
\hline$\mu \mathrm{g} / \mathrm{L}$ & 50 & 100 \\
\hline $\begin{array}{l}\mu g / L \\
\mathrm{pCi} / \mathrm{L}\end{array}$ & $\begin{array}{l}50 \\
3 E+01\end{array}$ & $\begin{array}{l}100 \\
6 E+01\end{array}$ \\
\hline $\mathrm{pCi} / \mathrm{L}$ & $1 E+02$ & $2 E+02$ \\
\hline $\mathrm{pCi} / \mathrm{L}$ & $3 E+02$ & $6 E+02$ \\
\hline$\mu \mathrm{g} / \mathrm{L}$ & 50 & 100 \\
\hline$\mu \mathrm{g} / \mathrm{L}$ & 50 & 100 \\
\hline$\mu \mathrm{g} / \mathrm{L}$ & 50 & 100 \\
\hline$\mu g / L$ & 2,000 & 4,000 \\
\hline$\mu g / L$ & 350 & 700 \\
\hline $\mathrm{pCi} / \mathrm{L}$ & $7.5 E+00$ & $1.5 E+C$ \\
\hline$\mu \mathbf{g} / \mathbf{L}$ & 0.2 & 0.4 \\
\hline$\mu g / L$ & 0.1 & 0.2 \\
\hline$\mu g / L$ & 0.00325 & 0.0065 \\
\hline$\mu g / L$ & 0.00325 & 0.0065 \\
\hline$\mu \mathrm{g} / \mathrm{L}$ & 0.00225 & 0.0045 \\
\hline$\mu \mathrm{g} / \mathrm{L}$ & 0.00225 & 0.0045 \\
\hline$\mu g / L$ & 0.5 & 1 \\
\hline$\mu \mathrm{g} / \mathrm{L}$ & 50 & 100 \\
\hline$\mu g / L$ & 25 & 50 \\
\hline$\mu \mathrm{g} / \mathrm{L}$ & 0.00225 & 0.0045 \\
\hline$\mu g / L$ & 0.00225 & 0.0045 \\
\hline$\mu g / L$ & 0.002 & 0.004 \\
\hline$\mu \mathrm{g} / \mathrm{L}$ & 0.002 & 0.004 \\
\hline$\mu g / L$ & 50 & 100 \\
\hline$\mu \mathrm{g} / \mathrm{L}$ & 250 & 500 \\
\hline$\mu g / L$ & 50 & 100 \\
\hline$\mu g / L$ & 50 & 100 \\
\hline$\mu g / L$ & 50 & 100 \\
\hline $\begin{array}{l}\mu \mathrm{g} / \mathrm{L} \\
\mathrm{pCi} / \mathrm{L}\end{array}$ & $\begin{array}{l}250 \\
5 E-01\end{array}$ & $\begin{array}{l}500 \\
1 E+00\end{array}$ \\
\hline $\mathrm{pCi} / \mathrm{L}$ & $1.5 E+00$ & $3 E+00$ \\
\hline$\mu g / L$ & 75 & 150 \\
\hline$\mu \mathrm{g} / \mathrm{L}$ & 150 & 300 \\
\hline$\mu g / L$ & 150 & 300 \\
\hline$\mu g / L$ & 150 & 300 \\
\hline $\mathrm{pCi} / \mathrm{L}$ & $1 E+03$ & $2 E+03$ \\
\hline $\mathrm{pCi} / \mathrm{L}$ & $1 E+02$ & $2 E+02$ \\
\hline & 500 & 1,000 \\
\hline 1 & 50 & 100 \\
\hline
\end{tabular}

Source

EPA Method 9060

EPA Method 8270

Set by EPD/EMS

EPA Method 8080

EPA Method 8080

EPA Method 8080

Final PDWS (EPA, 1993a)

Final PDWS (EPA, 1993a)

EPA Method 8080

Set by EPD/EMS

Final PDWS (EPA, 1993a)

EPA Method 8270

EPA Method 8270

Interim Final PDWS (EPA, 1977)

Interim Final POWS (EPA, 1977)

Interim Final PDWS (EPA, 1977)

EPA Method 8270

EPA Method 8270

EPA Method 8270

Final PDWS (EPA, 1993a)

Final PDWS (EPA, 1993a)

Final PDWS (EPA, 1993a)

Final PDWS (EPA, 1993a)

Final PDWS (EPA, 1993a)

EPA Method 8280

EPA Method 8280

EPA Method 8280

EPA Method 8280

Final PDWS (EPA, 1993a)

EPA Method 8270

Final PDWS (EPA, 1993a)

EPA Method 8280

EPA Method 8280

EPA Method 8280

EPA Method 8280

EPA Method 8270

EPA Method 8270

EPA Method 8270

EPA Method 8240

EPA Method 8270

APHA Method 415A

Interim Final PDWS (EPA, 1977)

Interim Final PDWS (EPA, 1977)

EPA Method 8240

SDWS (EPA, 1993b)

SDWS (EPA, 1993b)

SDWS (EPA, 1993b)

Interim Final PDWS (EPA, 1977)

Interim Final PDWS (EPA, 1977)

EPA Method 8240

EPA Method 8270 


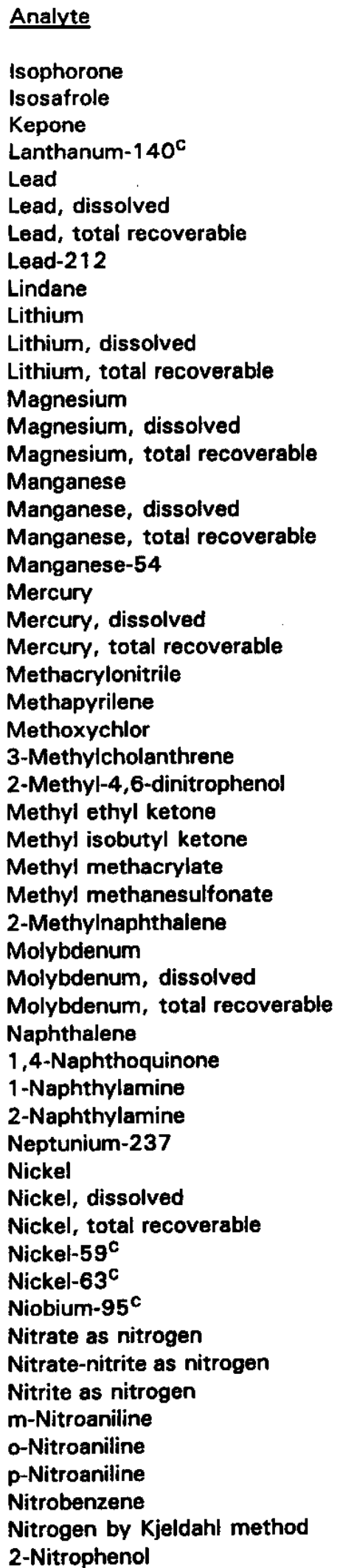

\begin{tabular}{|c|c|c|c|}
\hline Unit & Flag 1 & Flag 2 & Source \\
\hline$\mu g / L$ & 50 & 100 & EPA Method 8270 \\
\hline$\mu \mathrm{g} / \mathrm{L}$ & 50 & 100 & EPA Method 8270 \\
\hline$\mu g / L$ & 50 & 100 & EPA Method 8270 \\
\hline $\mathrm{pCi} / \mathrm{L}$ & $3 E+01$ & $6 E+01$ & Interim Final PDWS (EPA, 1977) \\
\hline$\mu g / L$ & 25 & 50 & Final PDWS (SCDHEC, 1981) \\
\hline$\mu g / L$ & 25 & 50 & Final PDWS (SCDHEC, 1981) \\
\hline$\mu g / L$ & 25 & 50 & Final PDWS (SCDHEC, 1981) \\
\hline $\mathrm{pCi} / \mathrm{L}$ & $6.2 E+01$ & $1.23 E+02$ & Proposed PDWS (EPA, 1991) \\
\hline$\mu g / L$ & 0.1 & 0.2 & Final PDWS (EPA, 1993a) \\
\hline$\mu \mathrm{g} / \mathrm{L}$ & 25 & 50 & EPA Method 6010 \\
\hline$\mu \mathrm{g} / \mathrm{L}$ & 25 & 50 & EPA Method 6010 \\
\hline$\mu \mathrm{g} / \mathrm{L}$ & 25 & 50 & EPA Method 6010 \\
\hline & No flag & No flag & Set by EPD/EMS \\
\hline & No flag & No flag & Set by EPD/EMS \\
\hline & No flag & No flag & Set by EPD/EMS \\
\hline$\mu g / L$ & 25 & 50 & SDWS (EPA, 1993b) \\
\hline$\mu g / L$ & 25 & 50 & SDWS (EPA, 1993b) \\
\hline$\mu g / L$ & 25 & 50 & SDWS (EPA, 1993b) \\
\hline $\mathrm{pCi} / \mathrm{L}$ & $1.5 E+02$ & $3 E+02$ & Interim Final PDWS (EPA, 1977) \\
\hline$\mu g / L$ & 1 & 2 & Final PDWS (EPA, 1993a) \\
\hline$\mu g / L$ & 1 & 2 & Final PDWS (EPA, 1993a) \\
\hline$\mu \mathrm{g} / \mathrm{L}$ & 1 & 2 & Final PDWS (EPA, 1993a) \\
\hline$\mu g / L$ & 250 & 500 & EPA Method 8240 \\
\hline$\mu g / L$ & 50 & 100 & EPA Method 8270 \\
\hline$\mu g / L$ & 20 & 40 & Final PDWS (EPA, 1993a) \\
\hline$\mu g / L$ & 50 & 100 & EPA Method 8270 \\
\hline$\mu g / L$ & 250 & 500 & EPA Method 8270 \\
\hline & No flag & No flag & Set by EPD/EMS \\
\hline & No flag & No flag & Set by EPD/EMS \\
\hline$\mu g / L$ & 50 & 100 & EPA Method 8270 \\
\hline$\mu g / L$ & 50 & 100 & EPA Method 8270 \\
\hline$\mu g / L$ & 50 & 100 & EPA Method 8270 \\
\hline$\mu g / L$ & 250 & 500 & EPA Method 6010 \\
\hline$\mu g / L$ & 250 & 500 & EPA Method 6010 \\
\hline$\mu g / L$ & 250 & 500 & EPA Method 6010 \\
\hline$\mu g / L$ & 50 & 100 & EPA Method 8270 \\
\hline$\mu g / L$ & 50 & 100 & EPA Method 8270 \\
\hline$\mu g / L$ & 50 & 100 & EPA Method 8270 \\
\hline$\mu g / L$ & 50 & 100 & EPA Method 8270 \\
\hline $\mathrm{pCi} / \mathrm{L}$ & $3.53 E+00$ & $7.06 \mathrm{E}+00$ & Proposed PDWS (EPA, 1991) \\
\hline$\mu g / L$ & 50 & 100 & Final PDWS (EPA, 1993a) \\
\hline$\mu g / L$ & 50 & 100 & Final PDWS (EPA, 1993a) \\
\hline$\mu \mathrm{g} / L$ & 50 & 100 & Final PDWS (EPA, 1993a) \\
\hline $\mathrm{pCi} / \mathrm{L}$ & $1.5 E+02$ & $3 E+02$ & Interim Final PDWS (EPA, 1977) \\
\hline $\mathrm{pCi} / \mathrm{L}$ & $2.5 E+01$ & $5 E+01$ & Interim Final PDWS (EPA, 1977) \\
\hline $\mathrm{pCi} / \mathrm{L}$ & $1.5 E+02$ & $3 . E+02$ & Interim Final PDWS (EPA, 1977) \\
\hline$\mu g / L$ & 5,000 & 10,000 & Final PDWS (EPA, 1993a) \\
\hline$\mu g / L$ & 5,000 & 10,000 & Final PDWS (EPA, 1993a) \\
\hline$\mu g / L$ & 500 & 1,000 & Final PDWS (EPA, 1993a) \\
\hline$\mu g / L$ & 50 & 100 & EPA Method 8270 \\
\hline$\mu g / L$ & 50 & 100 & EPA Method 8270 \\
\hline$\mu g / \mathrm{L}$ & 50 & 100 & EPA Method 8270 \\
\hline$\mu g / L$ & 50 & 100 & EPA Method $\mathbf{8 2 7 0}$ \\
\hline & 500 & 1,000 & EPA Method 351.2 \\
\hline$\mu g / L$ & 50 & 100 & EPA Method 8270 \\
\hline
\end{tabular}


Analyte

4-Nitrophenol

4-Nitroquinoline-1-oxide

$\mathrm{N}$-Nitrosodi-n-butylamine

N-Nitrosodiethylamine

$\mathrm{N}$-Nitrosodimethylamine

$\mathrm{N}$-Nitrosodiphenylamine

$\mathrm{N}$-Nitrosodipropylamine

$\mathrm{N}$-Nitrosomethylethylamine

$\mathrm{N}$-Nitrosomorpholine

$\mathrm{N}$-Nitrosopiperidine

$\mathrm{N}$-Nitrosopyrrolidine

5-Nitro-o-toluidine

Nonvolatile beta

Octachlorodibenzo-p-dioxin isomers

Octachlorodibenzo-p-furan isomers

Odor

Oil \& Grease

Oxamyl

Parathion

Parathion methyl

PCB 1016

PCB 1221

PCB 1232

PCB 1242

PCB 1248

PCB 1254

PCB 1260

PCB 1262

Pentachlorobenzene

Pentachlorodibenzo-p-dioxin isomers

1,2,3,7,8-PCDD

Pentachlorodibenzo-p-furan isomers

1,2,3,7,8-PCDF

Pentachloroethane

Pentachloronitrobenzene

Pentachlorophenol

pH

pH

Phenacetin

Phenanthrene

Phenol

Phenols

p-Phenylenediamine

Phorate

Picloramb

2-Picoline

Plutonium-238

Plutonium-239

Plutonium-239/240

Plutonium-240

Plutonium-241 $\mathrm{c}$

\begin{tabular}{|c|c|c|}
\hline Unit & Flag 1 & Flag 2 \\
\hline$\mu g / L$ & 50 & 100 \\
\hline$\mu \mathrm{g} / \mathrm{L}$ & 50 & 100 \\
\hline$\mu \mathrm{g} / \mathrm{L}$ & 50 & 100 \\
\hline$\mu g / L$ & 50 & 100 \\
\hline$\mu g / L$ & 50 & 100 \\
\hline$\mu g / L$ & 50 & 100 \\
\hline$\mu g / L$ & 50 & 100 \\
\hline$\mu g / L$ & 50 & 100 \\
\hline$\mu g / L$ & 50 & 100 \\
\hline$\mu g / L$ & 50 & 100 \\
\hline$\mu g / L$ & 50 & 100 \\
\hline$\mu g / L$ & 50 & 100 \\
\hline $\begin{array}{l}\mathrm{pCi} / \mathrm{L} \\
\mu \mathrm{g} / \mathrm{L}\end{array}$ & $\begin{array}{l}2.5 E+01 \\
0.005\end{array}$ & $\begin{array}{l}5 E+01 \\
0.01\end{array}$ \\
\hline$\mu g / L$ & 0.005 & 0.01 \\
\hline & No flag & No flag \\
\hline & $\begin{array}{l}5,000 \\
100\end{array}$ & $\begin{array}{l}10,000 \\
200\end{array}$ \\
\hline$\mu \mathrm{g} / \mathrm{L}$ & 0.25 & 0.5 \\
\hline$\mu g / L$ & 0.25 & 0.5 \\
\hline$\mu g / L$ & 0.25 & 0.5 \\
\hline$\mu g / L$ & 0.25 & 0.5 \\
\hline$\mu g / L$ & 0.25 & 0.5 \\
\hline$\mu g / L$ & 0.25 & 0.5 \\
\hline$\mu g / L$ & 0.25 & 0.5 \\
\hline$\mu \mathrm{g} / L$ & 0.25 & 0.5 \\
\hline$\mu g / L$ & 0.25 & 0.5 \\
\hline$\mu g / L$ & 0.25 & 0.5 \\
\hline$\mu g / L$ & 50 & 100 \\
\hline$\mu g / L$ & 0.00275 & 0.0055 \\
\hline $\begin{array}{l}\mu g / L \\
\mu g / L\end{array}$ & $\begin{array}{l}0.00275 \\
0.00275\end{array}$ & $\begin{array}{l}0.0055 \\
0.0055\end{array}$ \\
\hline$\mu g / L$ & 0.00275 & 0.0055 \\
\hline$\mu g / L$ & 50 & 100 \\
\hline$\mu g / L$ & 50 & 100 \\
\hline$\mu g / L$ & 0.5 & 1 \\
\hline $\mathrm{pH}$ & 8 & 10 \\
\hline pH & 4 & 3 \\
\hline$\mu g / L$ & 50 & 100 \\
\hline$\mu g / L$ & 50 & 100 \\
\hline$\mu g / L$ & 50 & 100 \\
\hline$\mu g / L$ & 25 & 50 \\
\hline$\mu g / L$ & 50 & 100 \\
\hline$\mu g / L$ & 0.5 & 1 \\
\hline$\mu g / L$ & 250 & 500 \\
\hline$\mu g / L$ & 50 & 100 \\
\hline $\mathrm{pCi} / \mathrm{L}$ & $3.51 E+00$ & $7.02 E+00$ \\
\hline $\mathrm{pCi} / \mathrm{L}$ & $3.11 E+01$ & $6.21 E+01$ \\
\hline $\mathrm{pCi} / \mathrm{L}$ & $3.11 E+01$ & $6.21 E+01$ \\
\hline $\mathrm{pCi} / \mathrm{L}$ & $3.11 E+01$ & $6.22 E+01$ \\
\hline $\mathrm{pCi} / \mathrm{L}$ & $3.13 E+01$ & $6.26 E+01$ \\
\hline
\end{tabular}

Source

EPA Method 8270

EPA Method 8270

EPA Method 8270

EPA Method 8270

EPA Method 8270

EPA Method 8270

EPA Method 8270

EPA Method 8270

EPA Method 8270

EPA Method 8270

EPA Method 8270

EPA Method 8270

Interim Final PDWS (EPA, 1977)

EPA Method 8280

EPA Method 8280

Set by EPD/EMS

EPA Method 413.1

Final PDWS (EPA, 1993a)

EPA Method $\mathbf{8 0 8 0}$

EPA Method 8080

Final PDWS (EPA, 1993a)

Final PDWS (EPA, 1993a)

Final PDWS (EPA, 1993a)

Final PDWS (EPA, 1993a)

Final PDWS (EPA, 1993a)

Final PDWS (EPA, 1993a)

Final PDWS (EPA, 1993a)

Final PDWS (EPA, 1993a)

EPA Method 8270

EPA Method 8280

EPA Method 8280

EPA Method $\mathbf{8 2 8 0}$

EPA Method 8280

EPA Method 8270

EPA Method 8270

Final PDWS (EPA, 1993a)

Set by EPD/EMS

Set by EPD/EMS

EPA Method 8270

EPA Method 8270

EPA Method 8270

EPA Method 420.1

EPA Method 8270

EPA Method 8080

Final PDWS (EPA, 1993a)

EPA Method 8270

Proposed PDWS (EPA, 1991)

Proposed PDWS (EPA, 1991)

Proposed PDWS (EPA, 1991)

Proposed PDWS (EPA, 1991)

Proposed PDWS (EPA, 1991) 
Analyte

Plutonium-242 ${ }^{c}$

Potassium

Potassium, dissolved

Potassium, total recoverable

Potassium-40

Promethium-144

Promethium-146

Promethium-147

Pronamid

Propionitrile

Pyrene

Pyridine

Radium (alpha-emitting) ${ }^{f}$

Radium-226

Radium-228

Radon-222

Ruthenium-103

Ruthenium-106

Safrole

Selenium

Selenium, dissolved

Selenium, total recoverable

Silica

Silica, dissolved

Silica, total recoverable

Silver

Silver, dissolved

Silver, total recoverable

Simazine ${ }^{b}$

Sodium

Sodium, dissolved

Sodium, total recoverable

Sodium-22

Specific conductance

Strontium-89

Strontium-89/90

Strontium-90

Styrene

Sulfate

Sulfide

Sulfotepp

Surfactants

2,3,7,8-TCDD

$2,3,7,8-T C D F$

Technetium-99

$1,2,4,5$-Tetrachlorobenzene

Tetrachlorodibenzo-p-dioxin isomers

Tetrachlorodibenzo-p-furan isomers

$1,1,1,2$-Tetrachloroethane

1,1,2,2-Tetrachloroethane

Tetrachloroethylene

2,3,4,6-Tetrachlorophenol

Thallium

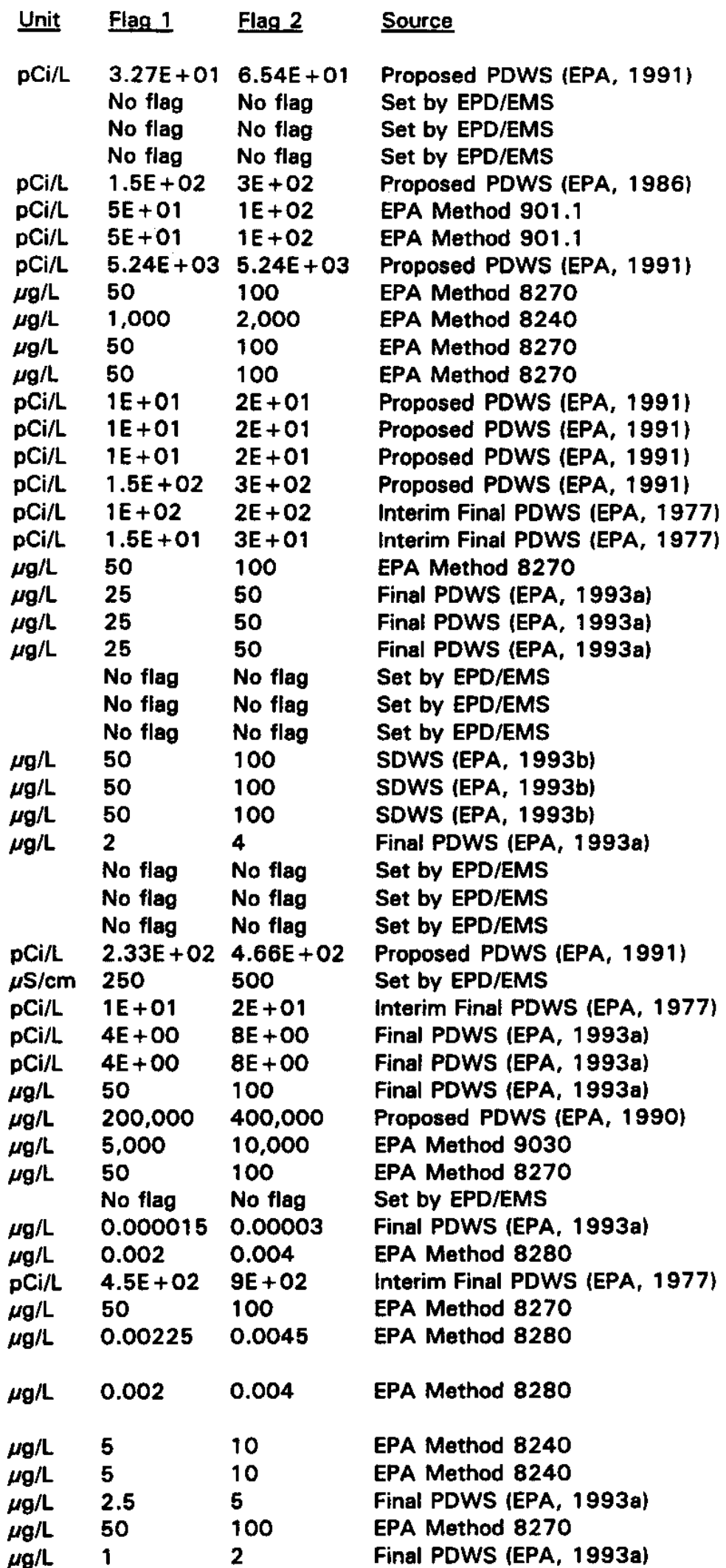


Analyte

Thallium, dissolved

Thallium, total recoverable

Thionazin

Thorium-228

Thorium-230

Thorium-232

Thorium-234

Tin

Tin, dissolved

Tin, total recoverable

Tin-113 $3^{c}$

Toluene

o-Toluidine

Total carbon

Total coliform

Total dissolved solids

Total hydrocarbons

Total inorganic carbon

Total organic carbon

Total organic halogens

Total organic nitrogen

Total petroleum hydrocarbons

Total phosphates (as P)

Total phosphorus

Toxaphene

2,4,5-TP (Silvex)

Tributyl phosphate

1,2,4-Trichlorobenzene

1,1,1-Trichloroethane

1,1,2-Trichloroethane

Trichloroethylene

Trichlorofluoromethane

2,4,5-Trichlorophenol

2,4,6-Trichlorophenol

2,4,5-Trichlorophenoxyacetic acid

1,2,3-Trichloropropane

$0,0,0$-Triethyl phosphorothioate

1,3,5-Trinitrobenzene

Tritium

Turbidity 9

Uranium

Uranium, dissolved

Uranium, total recoverable

Uranium alpha activity

Uranium-233/234 ${ }^{\circ}$

Uranium-234

Uranium-235

Uranium-238

Vanadium

Vanadium, dissolved

Vanadium, total recoverable

Vinyl acetate

\begin{tabular}{|c|c|c|}
\hline Unit & Flag 1 & Flag 2 \\
\hline$\mu g / L$ & 1 & 2 \\
\hline$\mu g / L$ & 1 & 2 \\
\hline$\mu \mathrm{g} / \mathrm{L}$ & 50 & 100 \\
\hline $\mathrm{pCi} / \mathrm{L}$ & $6.25 E+01$ & $1.25 E+.02$ \\
\hline $\mathrm{pCi} / \mathrm{L}$ & $3.96 \mathrm{E}+01$ & $7.92 E+01$ \\
\hline $\mathrm{pCi} / \mathrm{L}$ & $4.4 E+01$ & $8.8 E+01$ \\
\hline $\mathrm{pCi} / \mathrm{L}$ & $2 E+02$ & $4.01 E+02$ \\
\hline$\mu g / L$ & 10 & 20 \\
\hline$\mu g / L$ & 10 & 20 \\
\hline$\mu g / L$ & 10 & 20 \\
\hline $\mathrm{pCi} / \mathrm{L}$ & $1.5 E+02$ & $3 E+02$ \\
\hline$\mu g / L$ & 500 & 1,000 \\
\hline$\mu \mathrm{g} / \mathrm{L}$ & 50 & 100 \\
\hline$\mu \mathrm{g} / \mathrm{L}$ & 5,000 & 10,000 \\
\hline & & \\
\hline & No flag & No flag \\
\hline$\mu g / L$ & 5,000 & 10,000 \\
\hline$\mu g / L$ & 5,000 & 10,000 \\
\hline$\mu g / L$ & 5,000 & 10,000 \\
\hline$\mu g / L$ & 25 & 50 \\
\hline$\mu g / L$ & 500 & 1,000 \\
\hline$\mu g / L$ & 5,000 & 10,000 \\
\hline & No flag & No flag \\
\hline & No flag & No flag \\
\hline$\mu g / L$ & 1.5 & 3 \\
\hline$\mu g / L$ & 25 & 50 \\
\hline$\mu g / L$ & 50 & 100 \\
\hline$\mu g / L$ & 35 & 70 \\
\hline$\mu g / L$ & 100 & 200 \\
\hline$\mu g / L$ & 2.5 & 5 \\
\hline$\mu g / L$ & 2.5 & 5 \\
\hline$\mu g / L$ & 5 & 10 \\
\hline$\mu g / L$ & 50 & 100 \\
\hline$\mu g / L$ & 50 & 100 \\
\hline$\mu g / L$ & 2.5 & 5 \\
\hline$\mu g / L$ & 5 & 10 \\
\hline$\mu \mathrm{g} / \mathrm{L}$ & 50 & 100 \\
\hline$\mu \mathrm{g} / \mathrm{L}$ & 50 & 100 \\
\hline $\mathrm{pCi} / \mathrm{mL}$ & $1 E+01$ & $2 E+01$ \\
\hline & No flag & No flag \\
\hline$\mu g / L$ & 10 & 20 \\
\hline$\mu \mathrm{g} / \mathrm{L}$ & 10 & 20 \\
\hline$\mu \mathrm{g} / \mathrm{L}$ & 10 & 20 \\
\hline $\mathrm{pCi} / \mathrm{L}$ & $1.5 E+01$ & $3 E+01$ \\
\hline $\mathrm{pCi} / \mathrm{L}$ & $6.9 E+00$ & $1.38 E+01$ \\
\hline $\mathrm{pCi} / \mathrm{L}$ & $6.95 E+00$ & $1.39 E+01$ \\
\hline $\mathrm{pCi} / \mathrm{L}$ & $7.25 E+00$ & $1.45 E+01$ \\
\hline $\mathrm{pCi} / \mathrm{L}$ & $7.3 E+00$ & $1.46 \mathrm{E}+01$ \\
\hline$\mu g / L$ & 40 & 80 \\
\hline$\mu \mathrm{g} / \mathrm{L}$ & 40 & 80 \\
\hline$\mu \mathrm{g} / \mathrm{L}$ & 40 & 80 \\
\hline$\mu \mathrm{g} / \mathrm{L}$ & 5 & 10 \\
\hline
\end{tabular}

Source

Final PDWS (EPA, 1993a)

Final PDWS (EPA, 1993a)

EPA Method 8270

Proposed PDWS (EPA, 1991)

Proposed PDWS (EPA, 1991)

Proposed PDWS (EPA, 1991)

Proposed PDWS (EPA, 1991)

EPA Method 282.2

EPA Method 282.2

EPA Method 282.2

Interim Final PDWS (EPA, 1977)

Final PDWS (EPA, 1993a)

EPA Method 8270

EPA Method 9060

Final PDWS (EPA, 1993a)

Set by EPD/EMS

EPA Method 418.1

EPA Method 9060

EPA Method 9060

EPA Method 9020

APHA Method 420

EPA Method 418.1

Set by EPD/EMS

Set by EPD/EMS

Final PDWS (EPA, 1993a)

Final PDWS (EPA, 1993a)

EPA Method 8270

Final PDWS (EPA, 1993a)

Final PDWS (EPA, 1993a)

Final PDWS (EPA, 1993a)

Final PDWS (EPA, 1993a)

EPA Method 8240

EPA Method 8270

EPA Method 8270

EPA Method 8150

EPA Method 8240

EPA Method 8270

EPA Method 8270

Final PDWS (EPA, 1993a)

Set by EPD/EMS

Proposed PDWS (EPA, 1991)

Proposed PDWS (EPA, 1991)

Proposed PDWS (EPA, 1991)

Proposed PDWS (EPA, 1991)

Proposed PDWS (EPA, 1991)

Proposed PDWS (EPA, 1991)

Proposed PDWS (EPA, 1991)

Proposed PDWS (EPA, 1991)

EPA Method 6010

EPA Method 6010

EPA Method 6010

EPA Method 8240 


\begin{tabular}{|c|c|c|c|c|}
\hline Analyte & Unit & Flag 1 & Flag 2 & Source \\
\hline $\begin{array}{l}\text { Xylenes } \\
\text { Yttrium-88 } \\
\text { Zinc } \\
\text { Zinc, dissolved } \\
\text { Zinc, total recoverable } \\
\text { Zinc-65 } \\
\text { Zirconium-95c } \\
\text { Zirconium/Niobium-95c }\end{array}$ & $\begin{array}{l}\mu g / L \\
\mathrm{pCi} / \mathrm{L} \\
\mu \mathrm{g} / \mathrm{L} \\
\mu \mathrm{g} / \mathrm{L} \\
\mu \mathrm{g} / \mathrm{L} \\
\mathrm{pCi} / \mathrm{L} \\
\mathrm{pCi} / \mathrm{L} \\
\mathrm{pCi} / \mathrm{L}\end{array}$ & $\begin{array}{l}5,000 \\
5 E+01 \\
2,500 \\
2,500 \\
2,500 \\
1.5 E+02 \\
1 E+02 \\
1 E+02\end{array}$ & $\begin{array}{l}10,000 \\
1 E+02 \\
5,000 \\
5,000 \\
5,000 \\
3 E+02 \\
2 E+02 \\
2 E+02\end{array}$ & $\begin{array}{l}\text { Final PDWS (EPA, 1993a) } \\
\text { EPA Method 901.1 } \\
\text { SDWS (EPA, 1993b) } \\
\text { SDWS (EPA, 1993b) } \\
\text { SDWS (EPA, 1993b) } \\
\text { Interim Final PDWS (EPA, 1977) } \\
\text { Interim Final PDWS (EPA, 1977) } \\
\text { Interim Final PDWS (EPA, 1977) }\end{array}$ \\
\hline
\end{tabular}

a References for methods are in Appendix E; references for dated sources are at the end of this appendix.

b EMS is currently unable to perform this analysis.

c EMS discontinued monitoring this radionuclide because it is inappropriate for the SRS Groundwater Monitoring Program.

d EPD/EMS set this flagging criterion using the 1991 proposed PDWS because the final PDWS in 1977 may have been in error.

- For double radionuclide analyses where each separate radionuclide has its own standard, the more stringent standard is used.

The applied standard is for radium-226.

9 The primary maximum contaminant level range for turbidity is 1-5 NTU, which is inappropriate for the SRS Groundwater Monitoring Program.

\section{References}

EPA fU.S. Environmental Protection Agencyl, 1977. National Interim Primary Drinking Water Regulations, EPA-570/9-76-003. Washington, DC.

EPA (U.S. Environmental Protection Agency), 1986. Water Pollution Control; National Primary Drinking Water Regulations, Radionuclides (Proposed). Federal Register, September 30, 1986, pp. 3483534862. Washington, DC.

EPA (U.S. Environmental Protection Agency), 1990. National Primary and Secondary Drinking Water Regulations; Synthetic Organic Chemicals and Inorganic Chemicals (Proposed Rule). Federal Register, July 25, 1990, pp. 30369-30448. Washington, DC.

EPA (U.S. Environmental Protection Agencyl, 1991. National Primary Drinking Water Regulations; Radionuclides; Proposed Rule. Federal Register, July 18, 1991, pp. 33052-33127. Washington, DC.

EPA (U.S. Environmental Protection Agency), 1993a. National Primary Drinking Water Regulations. Code of Federal Regulations, Section 40, Part 141, pp. 592-732. Washington, DC.

EPA (U.S. Environmental Protection Agency), 1993b. National Secondary Drinking Water Regulations. Code of Federal Regulations, Section 40, Part 143, pp. 774-777. Washington, DC.

SCDHEC (South Carolina Department of Health and Environmental Control), 1981. State Primary Drinking Water Regulations, R.61-58.5. Columbia, SC. 
WSRC-TR-94-0345

Unclassified

\section{Appendix C}

\section{Figures}


WSRC-TR-94-0345

Unclassified

THIS PAGE LEFT BLANK INTENTIONALLY. 


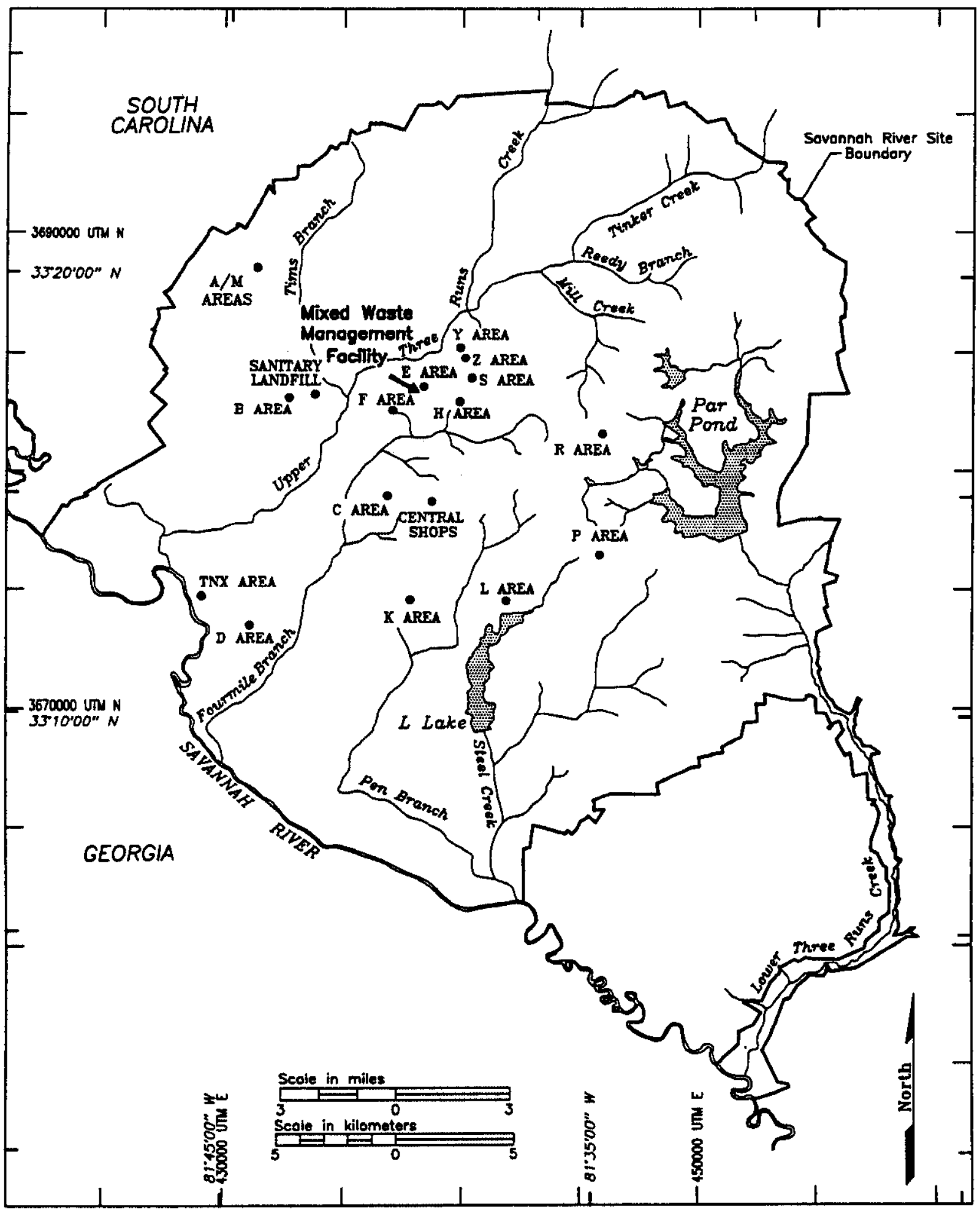

Figure 1. Location of the Mixed Waste Management Facility at the Savannah River Site 


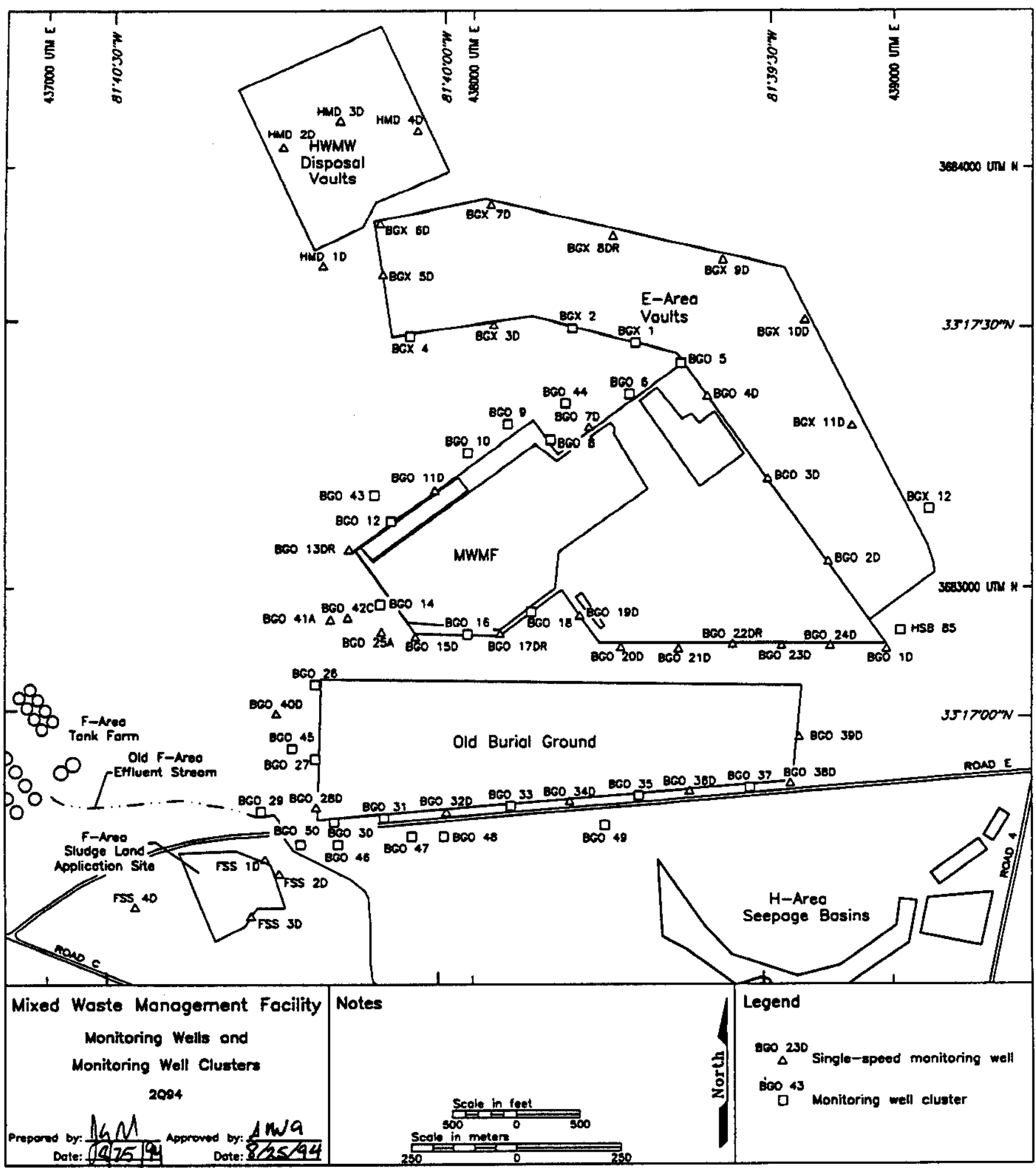

Figure 2. Location of the Groundwater Monitoring Wells at the Mixed Waste Management Facility, the Old Burial Ground, the E-Area Vaults, the Hazardous Waste/Mixed Waste Disposal Vaults (the Burial Ground Complex), and the F-Area Sewage Sludge Application Site 


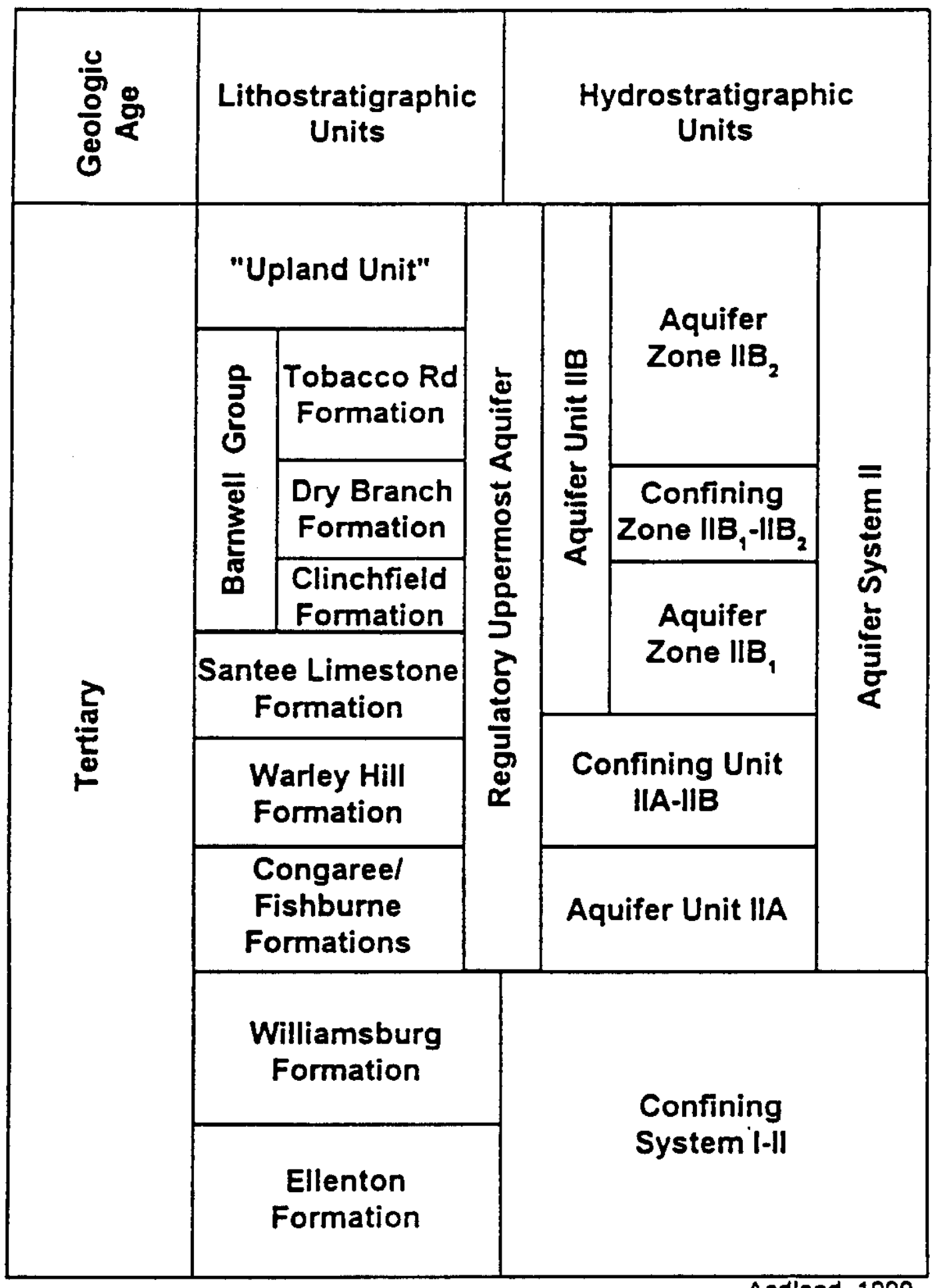

Aadland, 1990

Figure 3. Hydrostratigraphic Nomenclature 


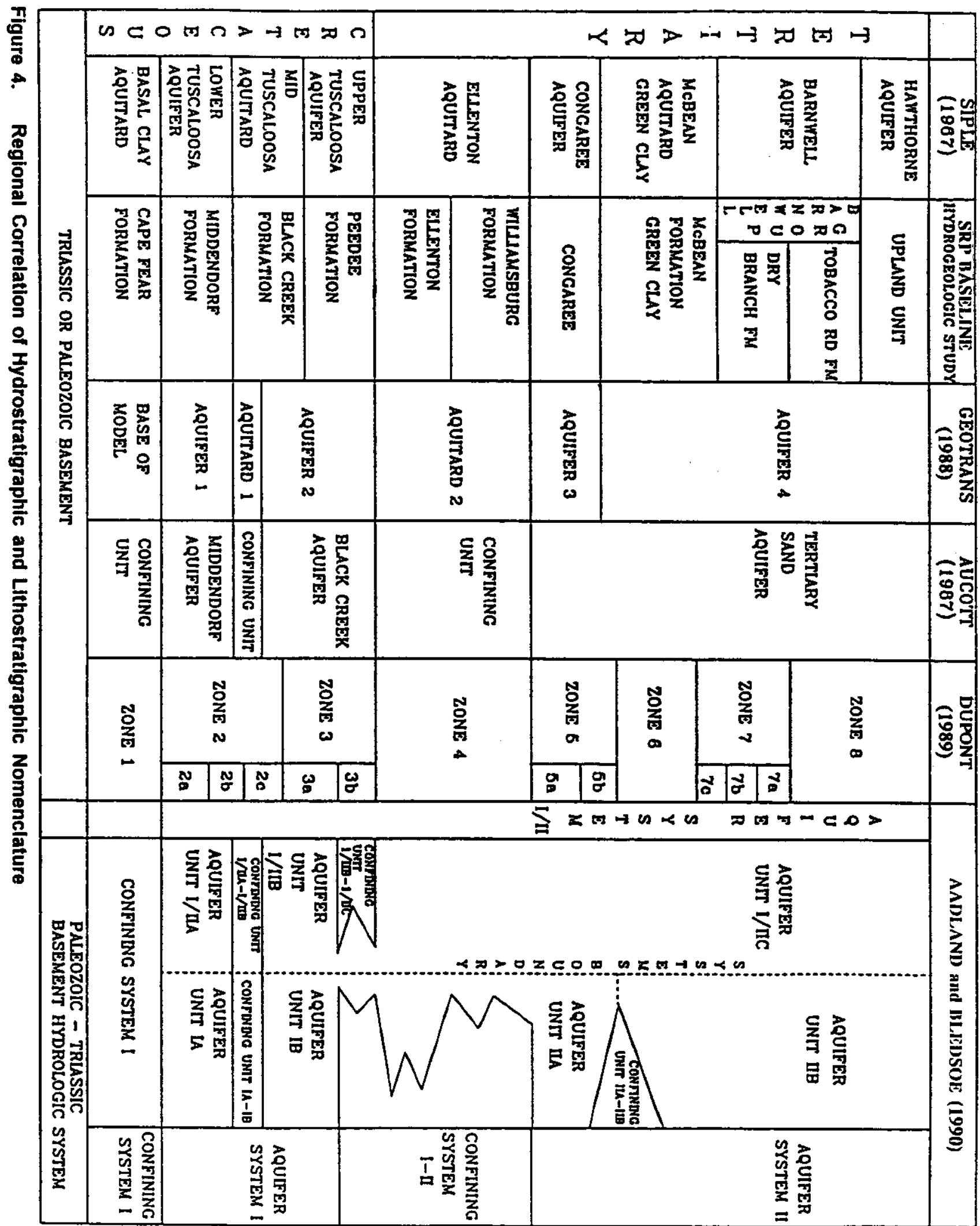




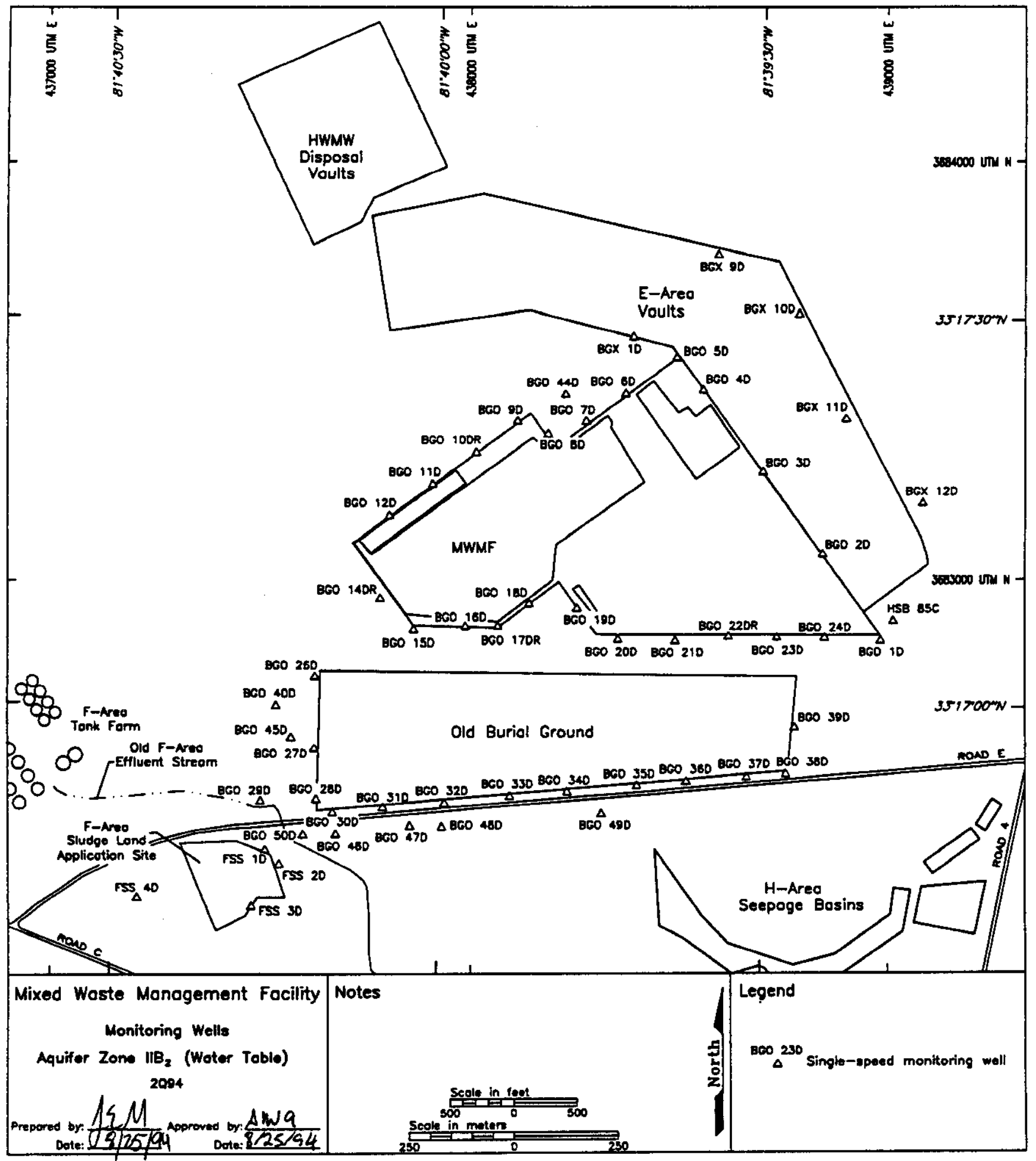

Figure 5. Location of Aquifer Zone $\|_{2}$ (Water Table) Wells at the Burial Ground Complex 


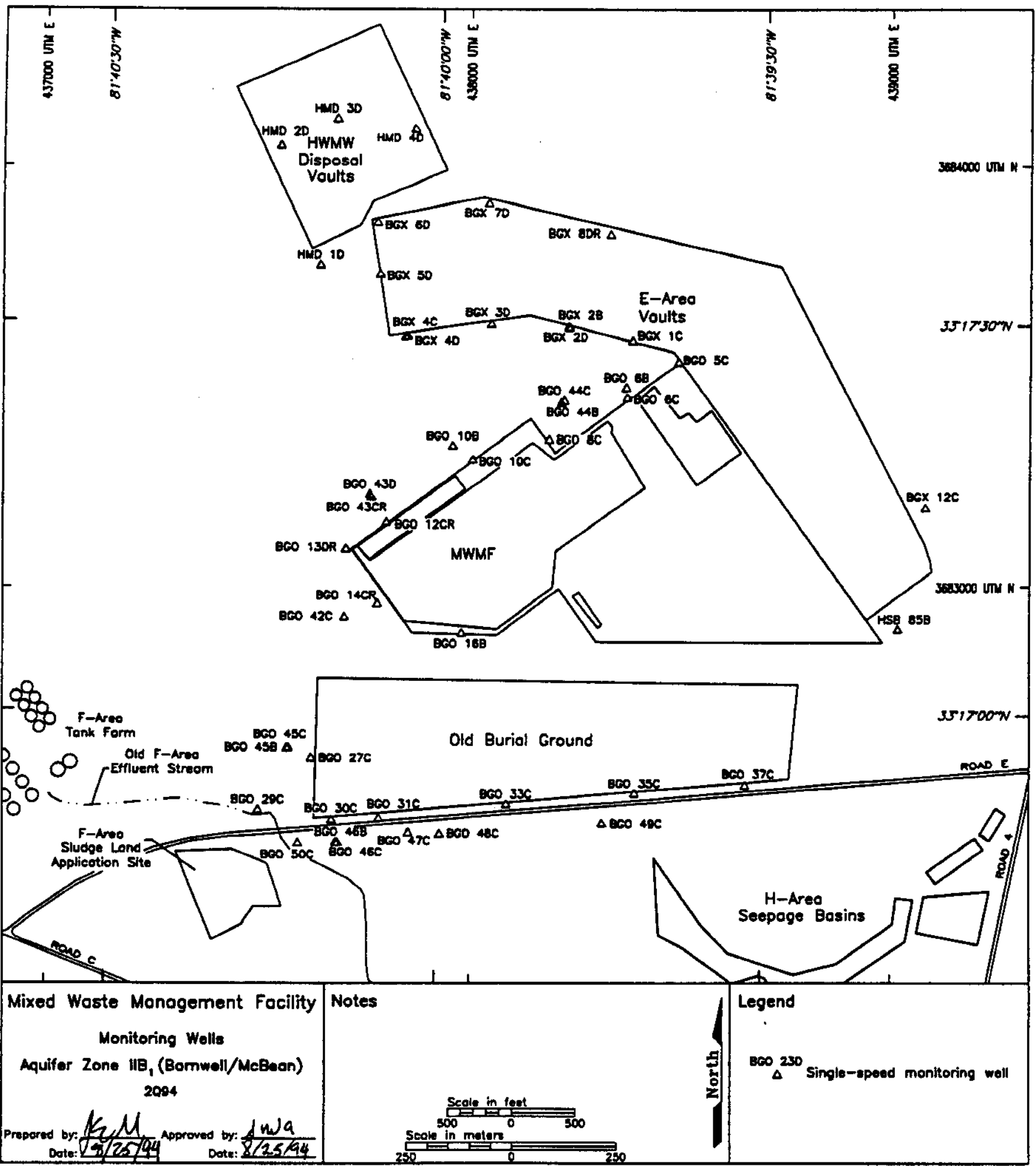

Figure 6. Location of Aquifer Zone IIB, (Barnwell/McBean) Wells at the Burial Ground Complex 


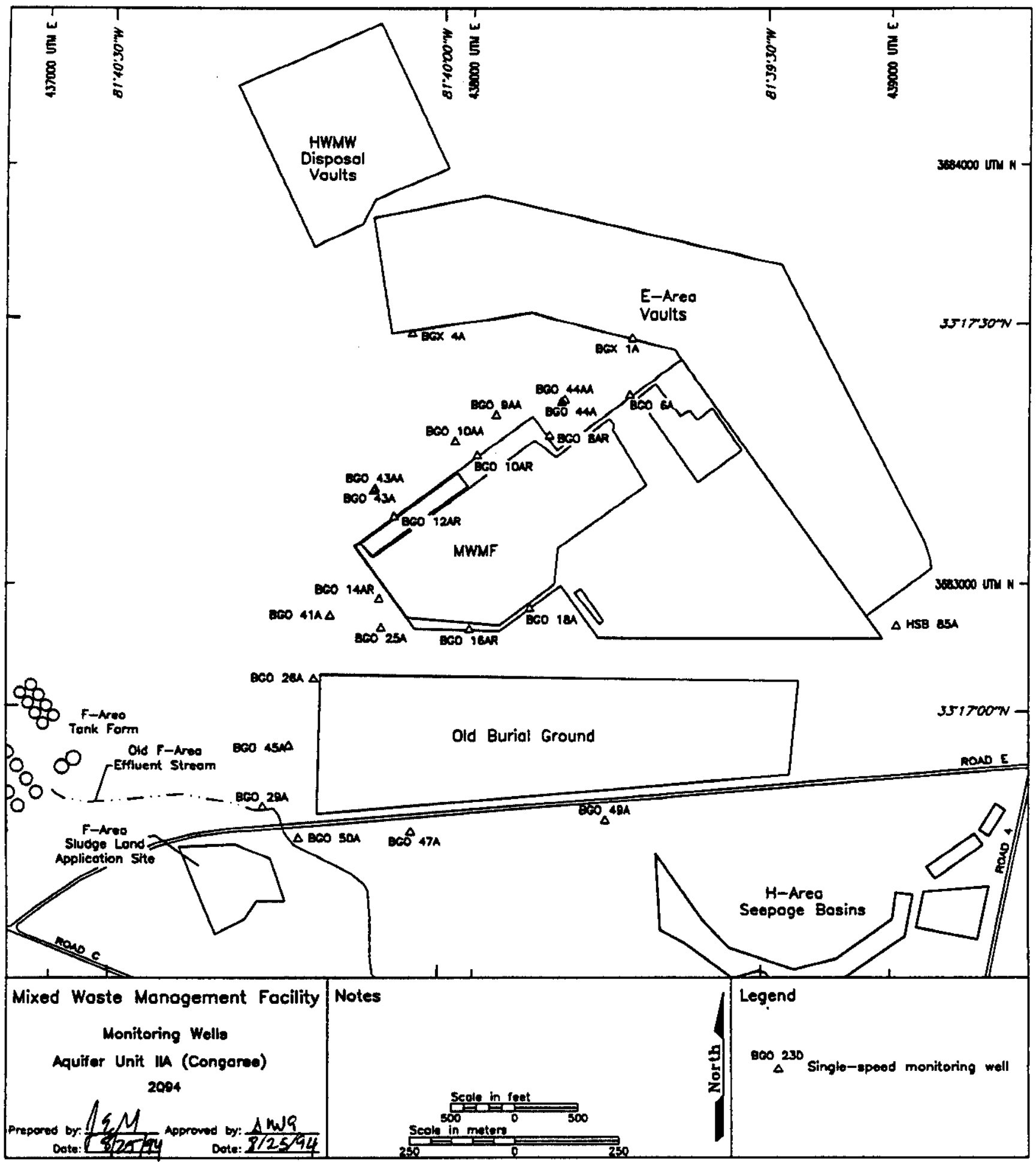

Figure 7. Location of Aquifer Unit IIA (Congaree) Wells at the Burial Ground Complex 


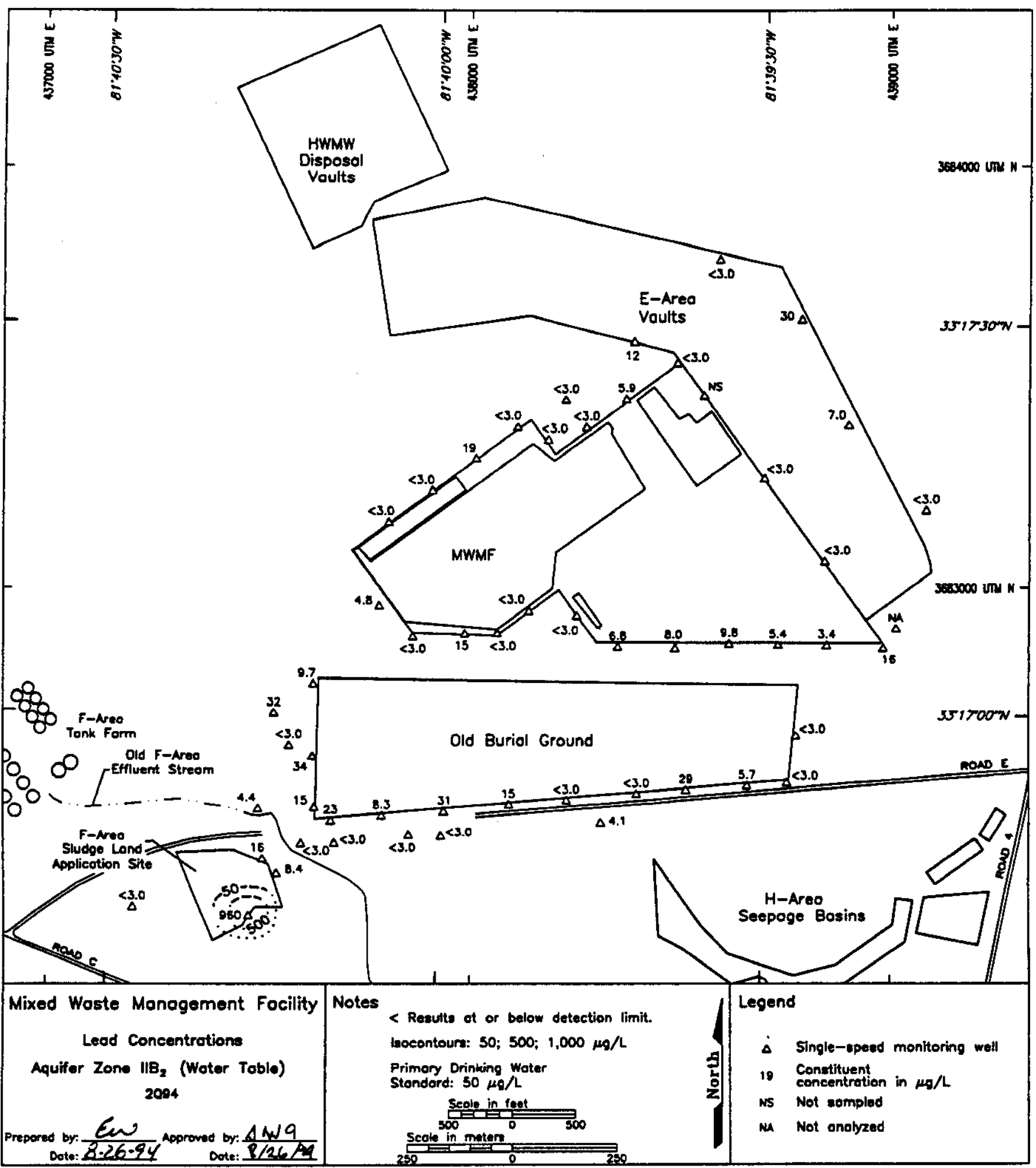

Figure 8. Lead Concentrations in Aquifer Zone ${\| B_{2}}_{2}$ (Water Table) at the Burial Ground Complex, Second Quarter 1994 


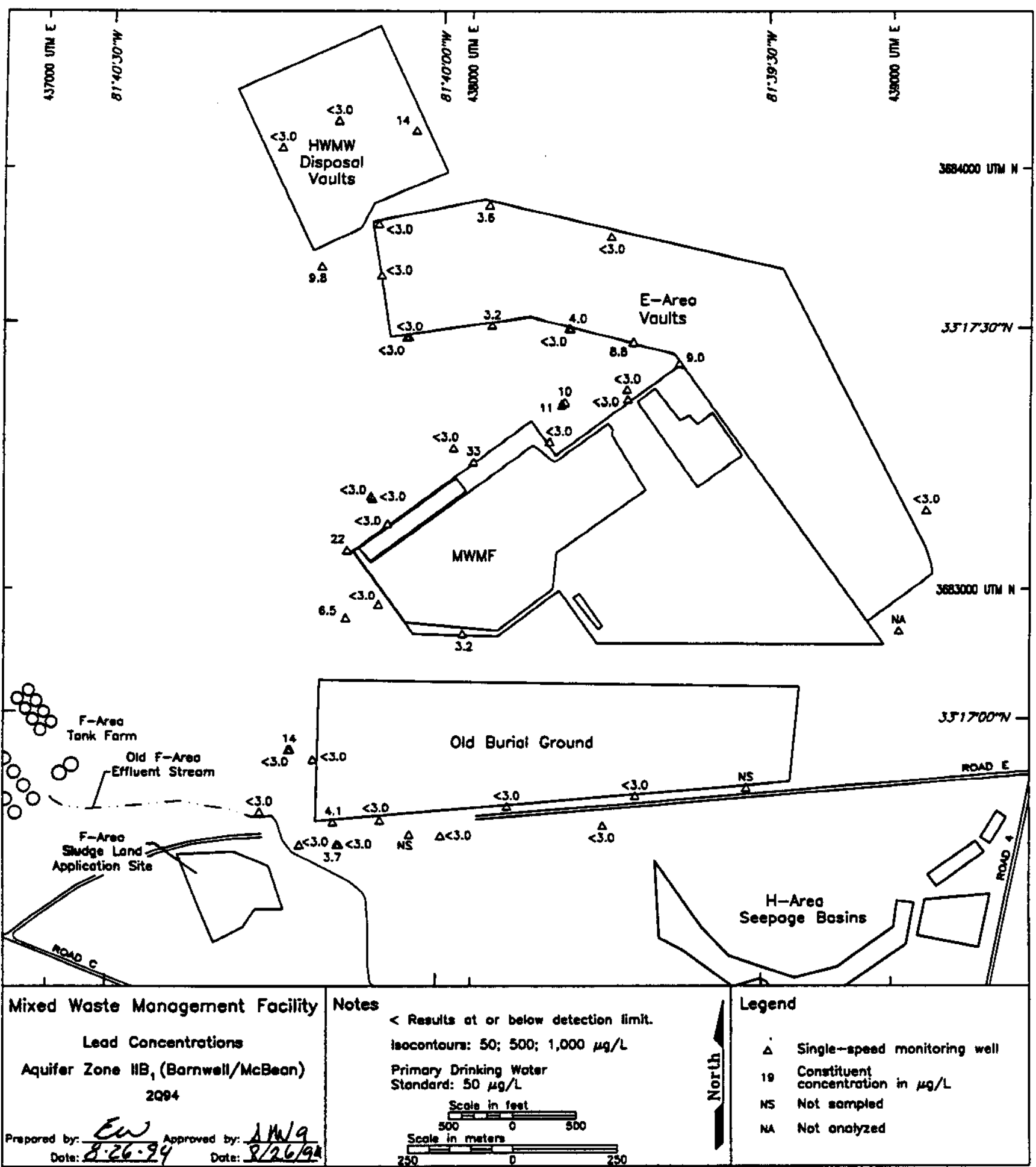

Figure 9. Lead Concentrations in Aquifer Zone IIB, (Barnwell/McBean) at the Burial Ground Complex, Second Quarter 1994 


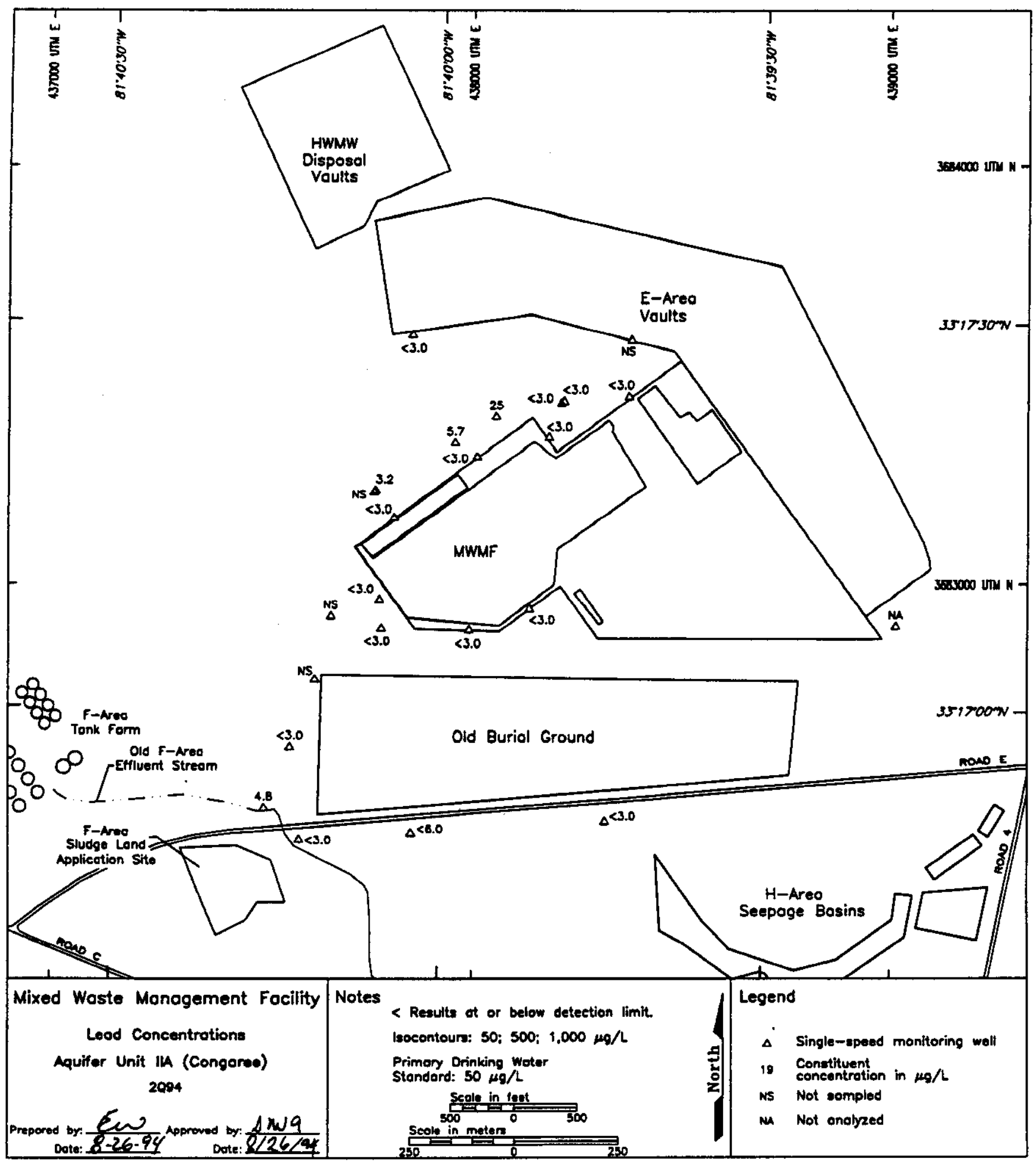

Figure 10. Lead Concentrations in Aquifer Unit IIA (Congaree) at the Burial Ground Complex, Second Quarter 1994 


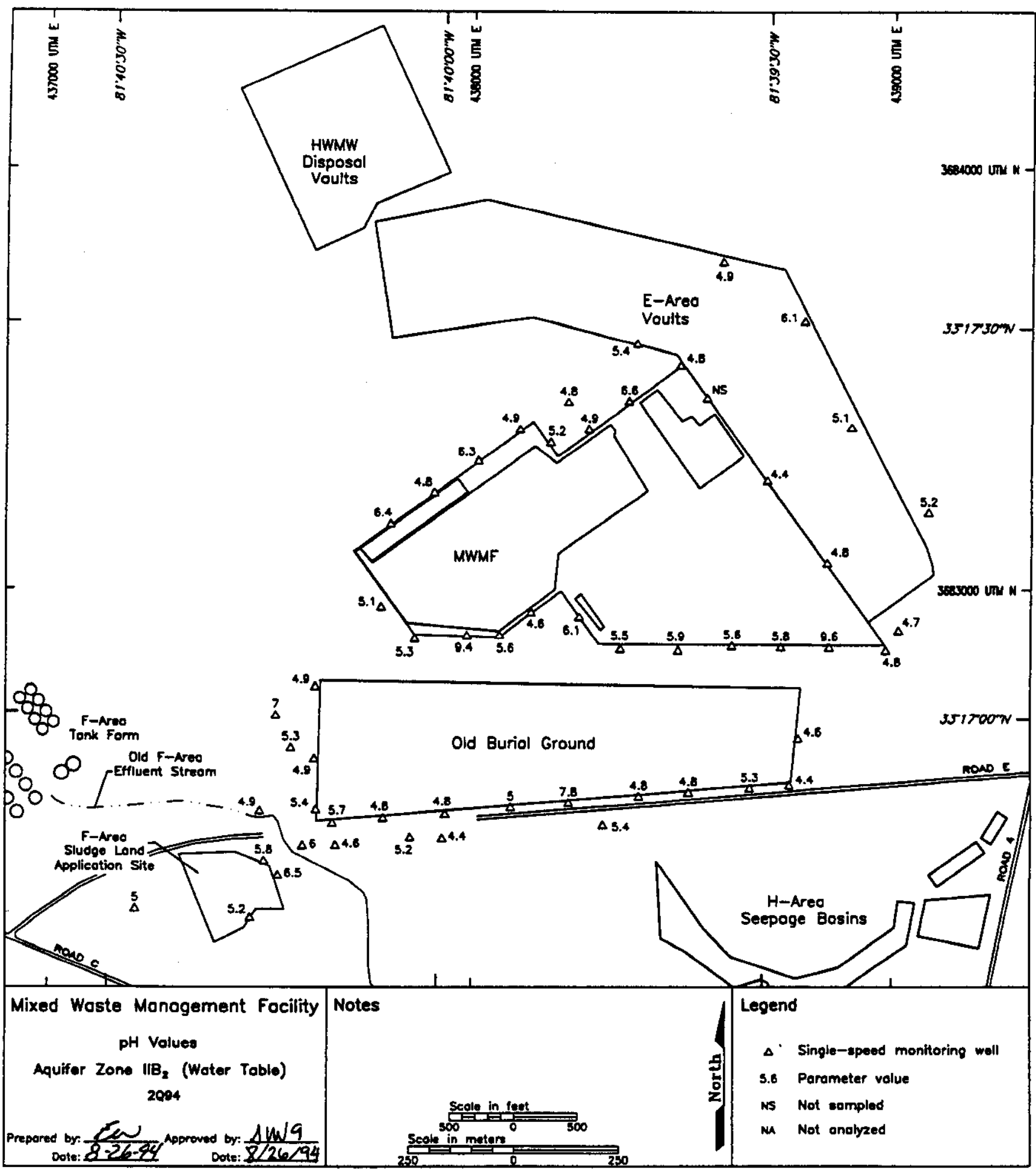

Figure 11. $\mathrm{pH}$ Values in Aquifer Zone ${\| B_{2}}_{2}$ (Water Table) at the Burial Ground Complex, Second Quarter 1994 


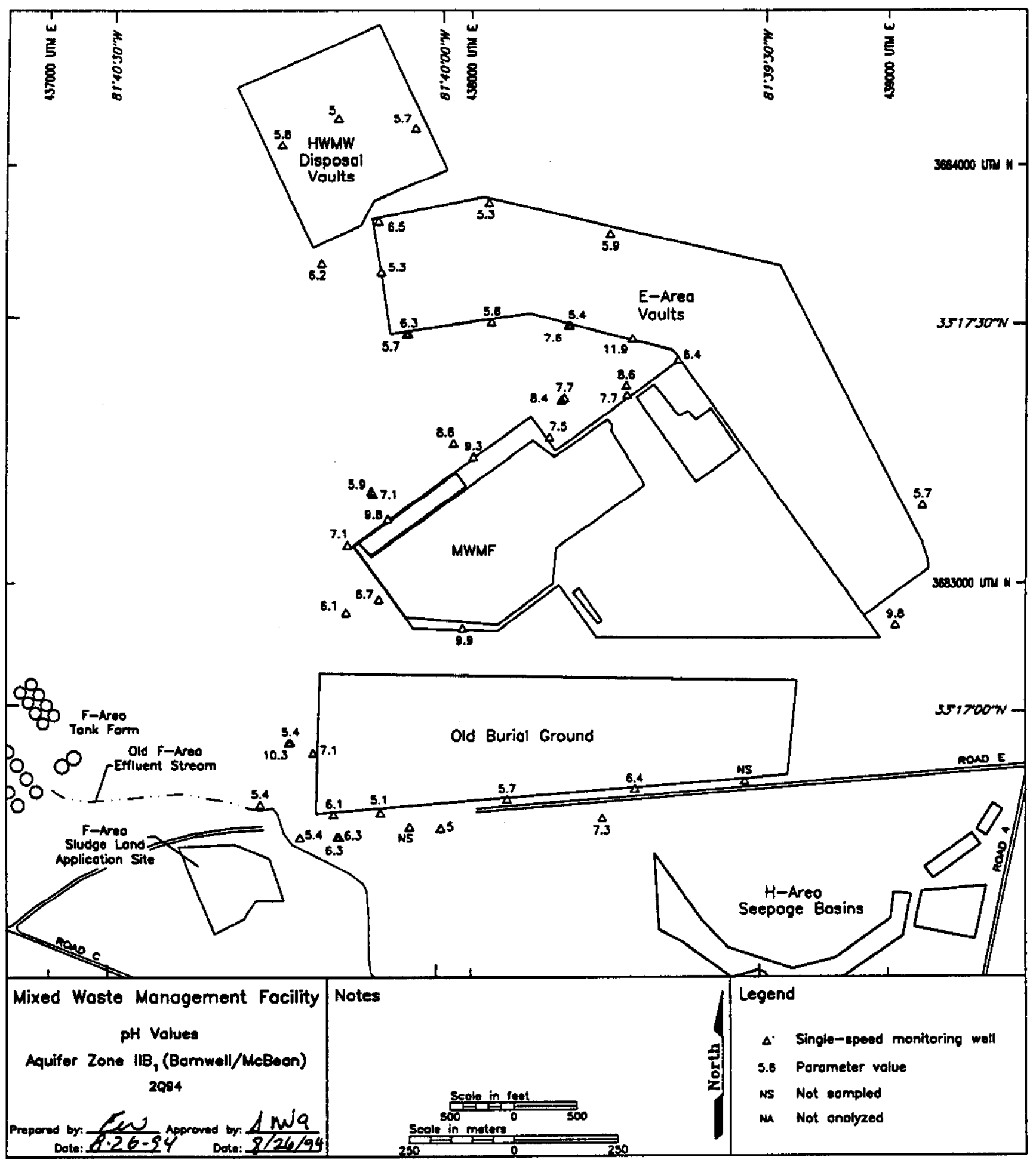

Figure 12. pH Values in Aquifer Zone IIB, (Barnwell/McBean) at the Burial Ground Complex, Second Quarter 1994 


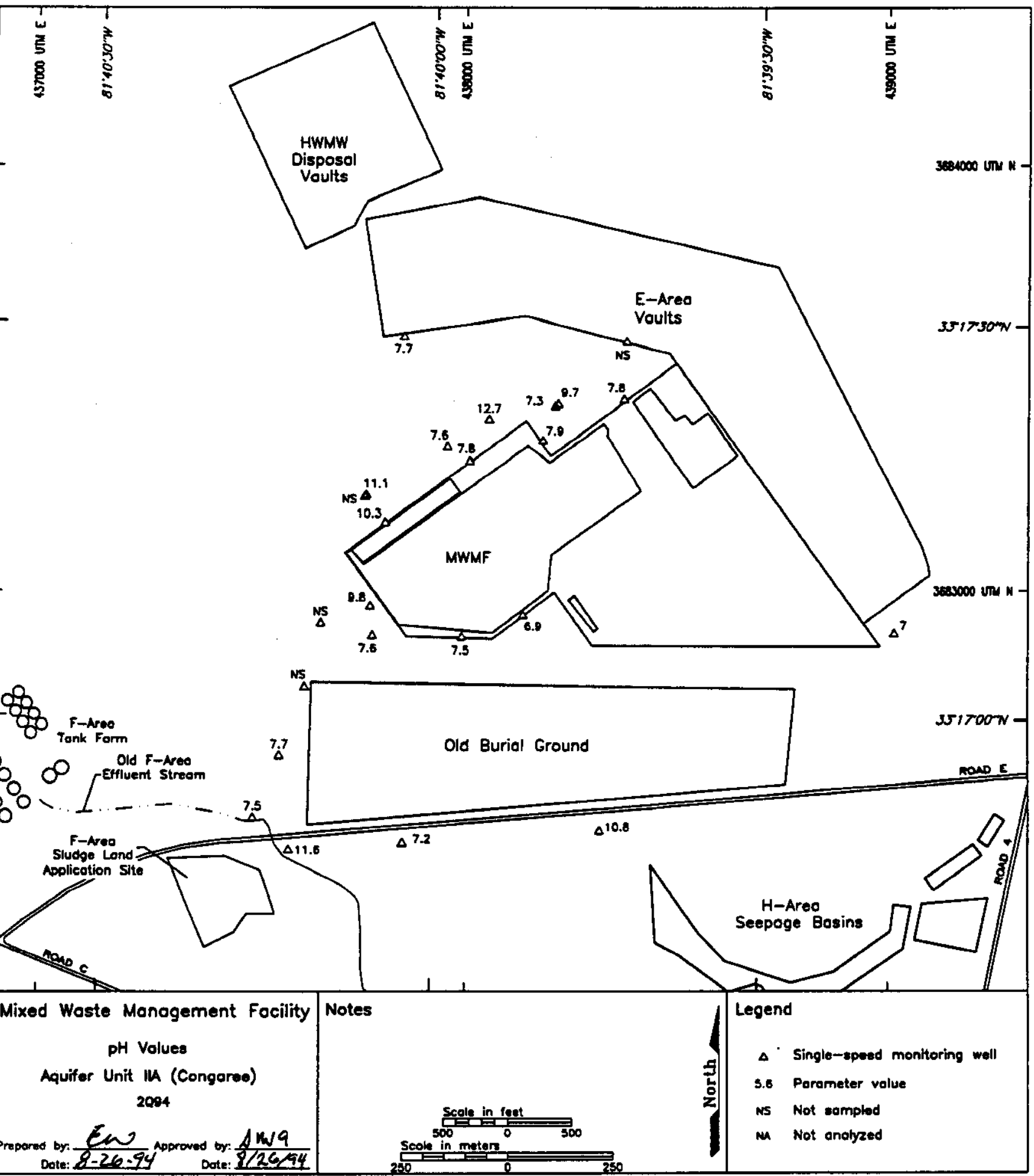

Figure 13. pH Values in Aquifer Zone IIA (Congaree) at the Burial Ground Complex, Second Quarter 1994 


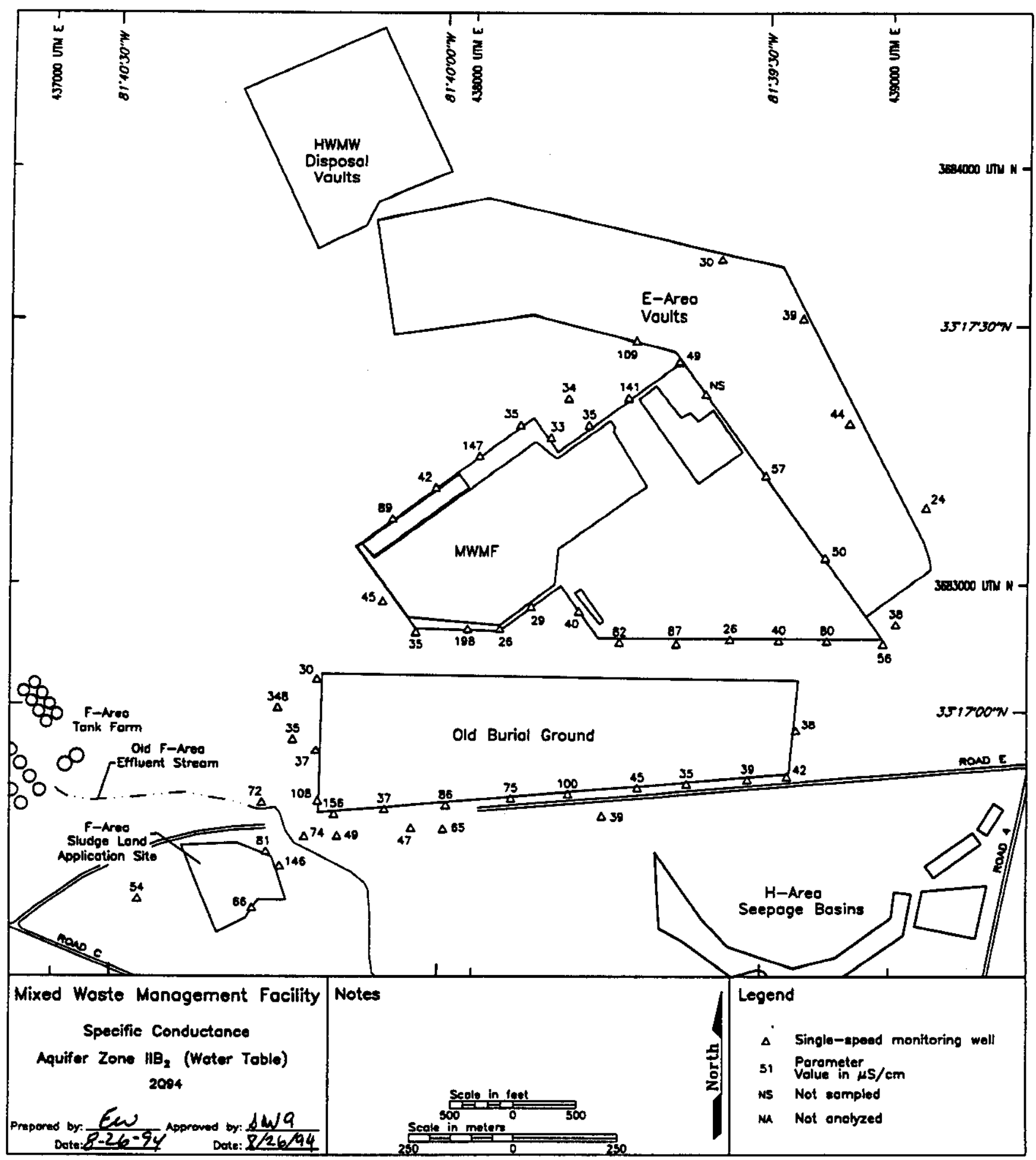

Figure 14. Specific Conductance in Aquifer Zone $\mathrm{IIB}_{2}$ (Water Table) at the Burial Ground Complex, Second Quarter 1994 


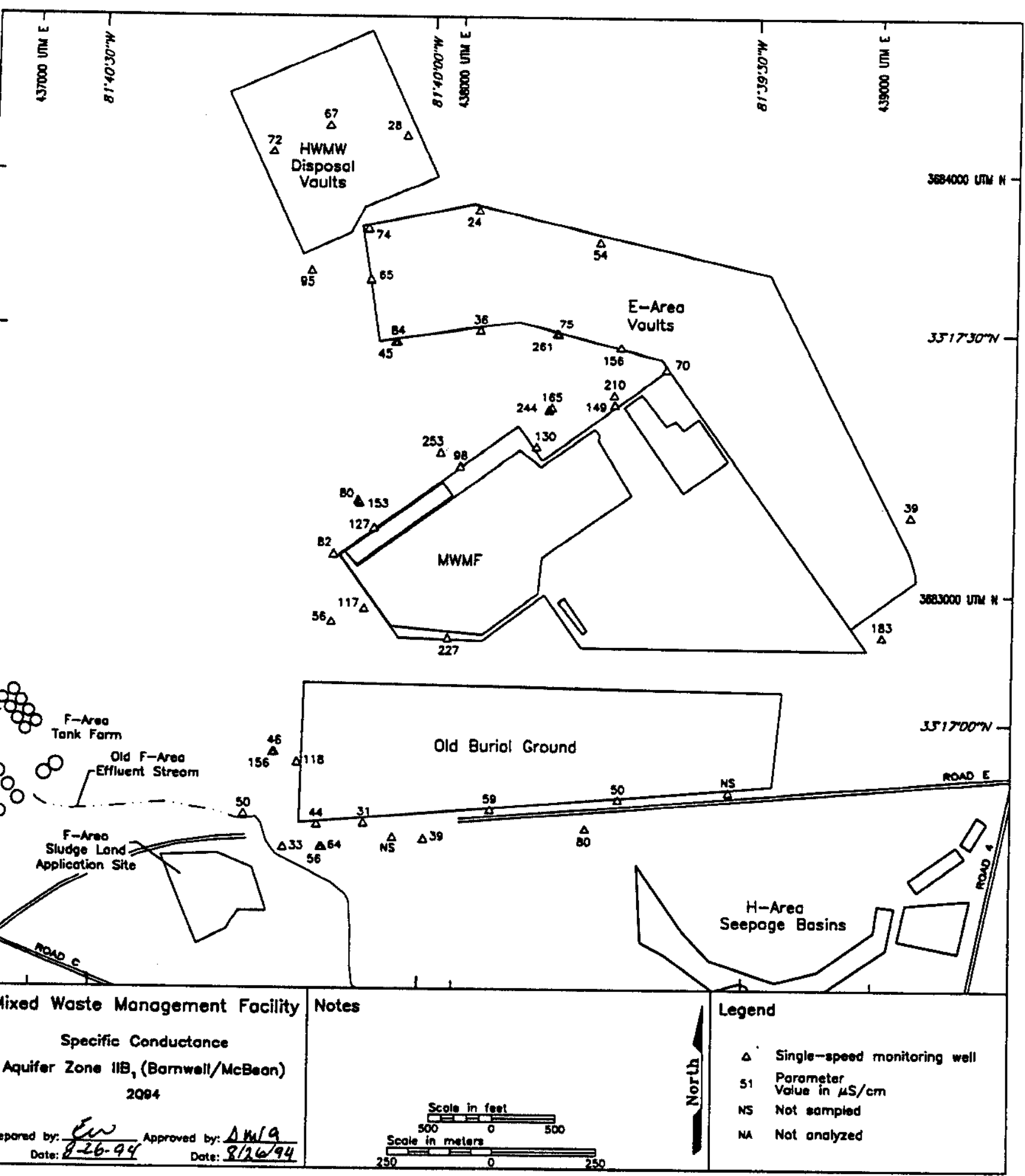

Figure 15. Specific Conductance in Aquifer Zone IIB, (Barnwell/McBean) at the Burial Ground Complex, Second Quarter 1994 


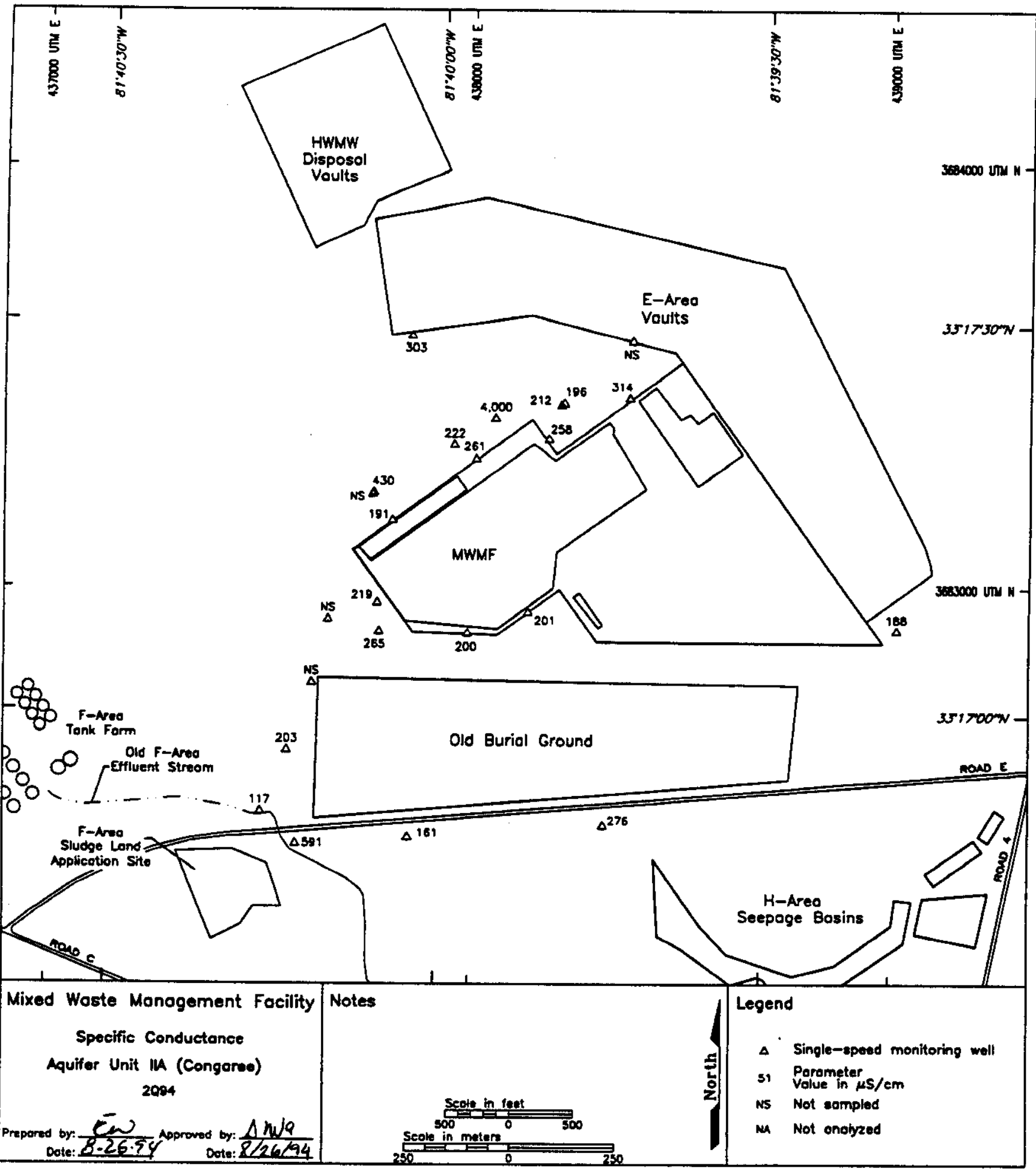

Figure 16. Specific Conductance in Aquifer Unit IIA (Congaree) at the Burial Ground Complex, Second Quarter 1994 


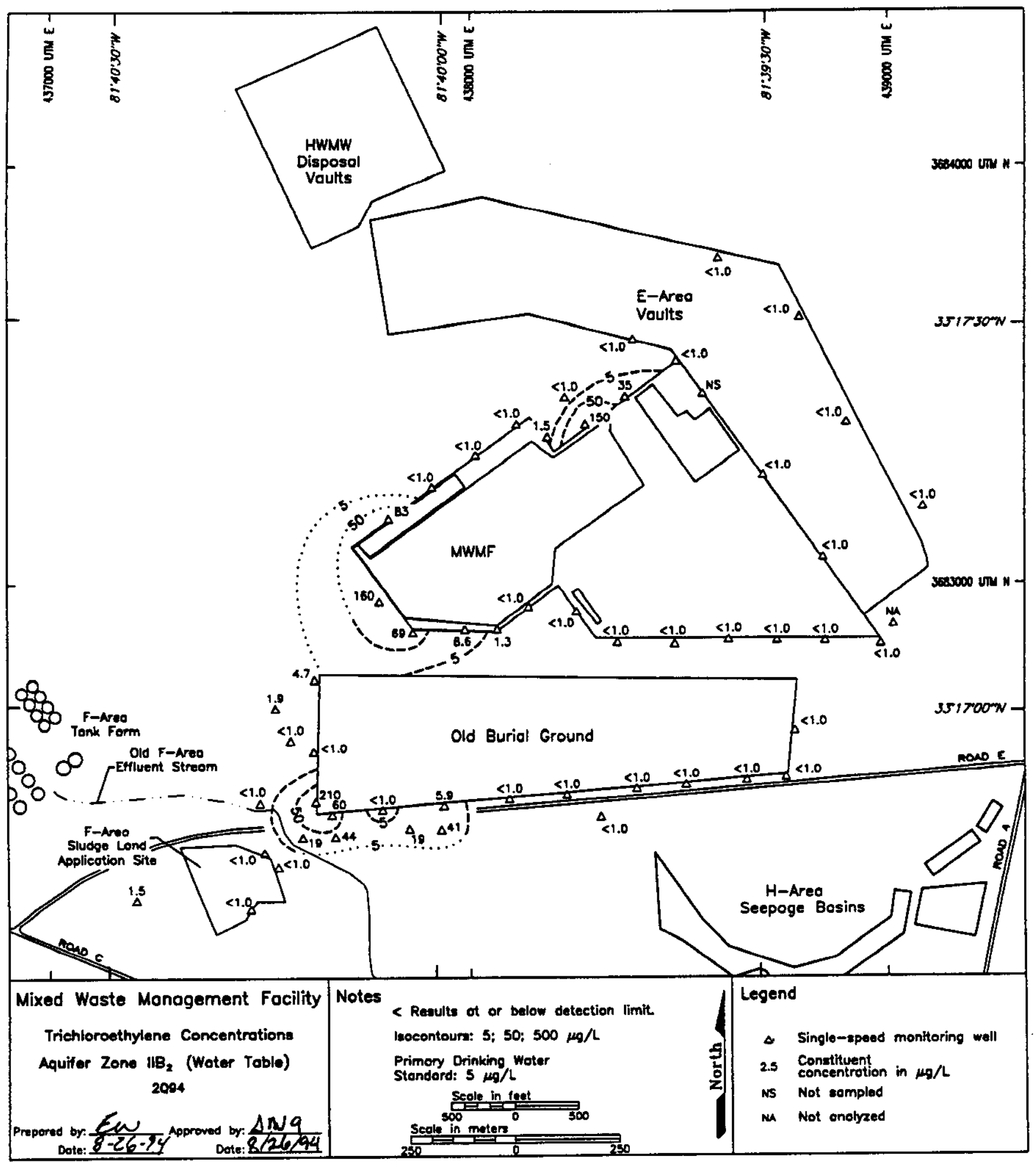

Figure 17. Trichloroethylene Concentrations in Aquifer Zone $\mathrm{IIB}_{2}$ (Water Table) at the Burial Ground Complex, Second Quarter 1994 


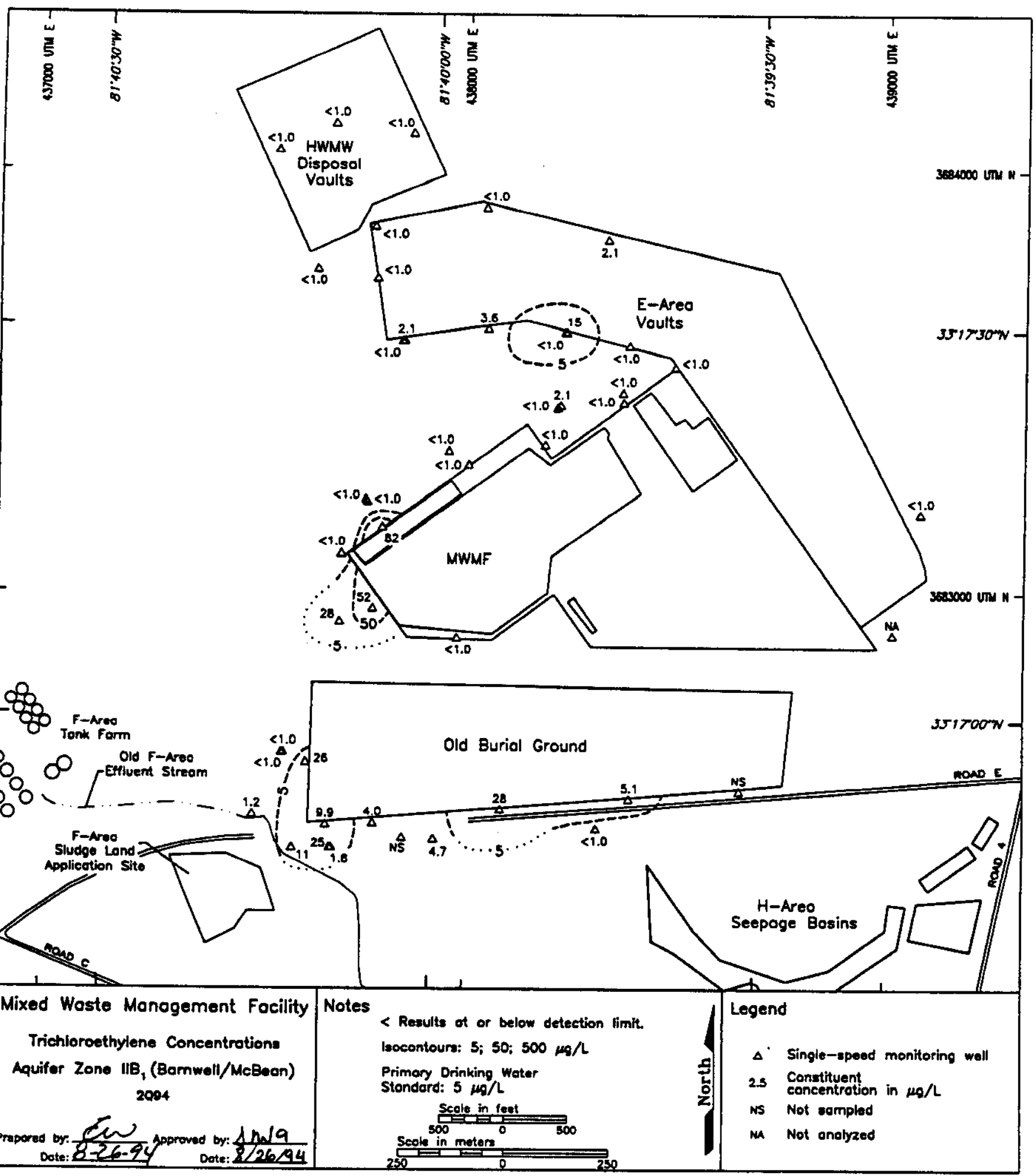

Figure 18. Trichloroethylene Concentrations in Aquifer Zone IIB, (Barnwell/McBean) at the Burial Ground Complex, Second Quarter 1994 


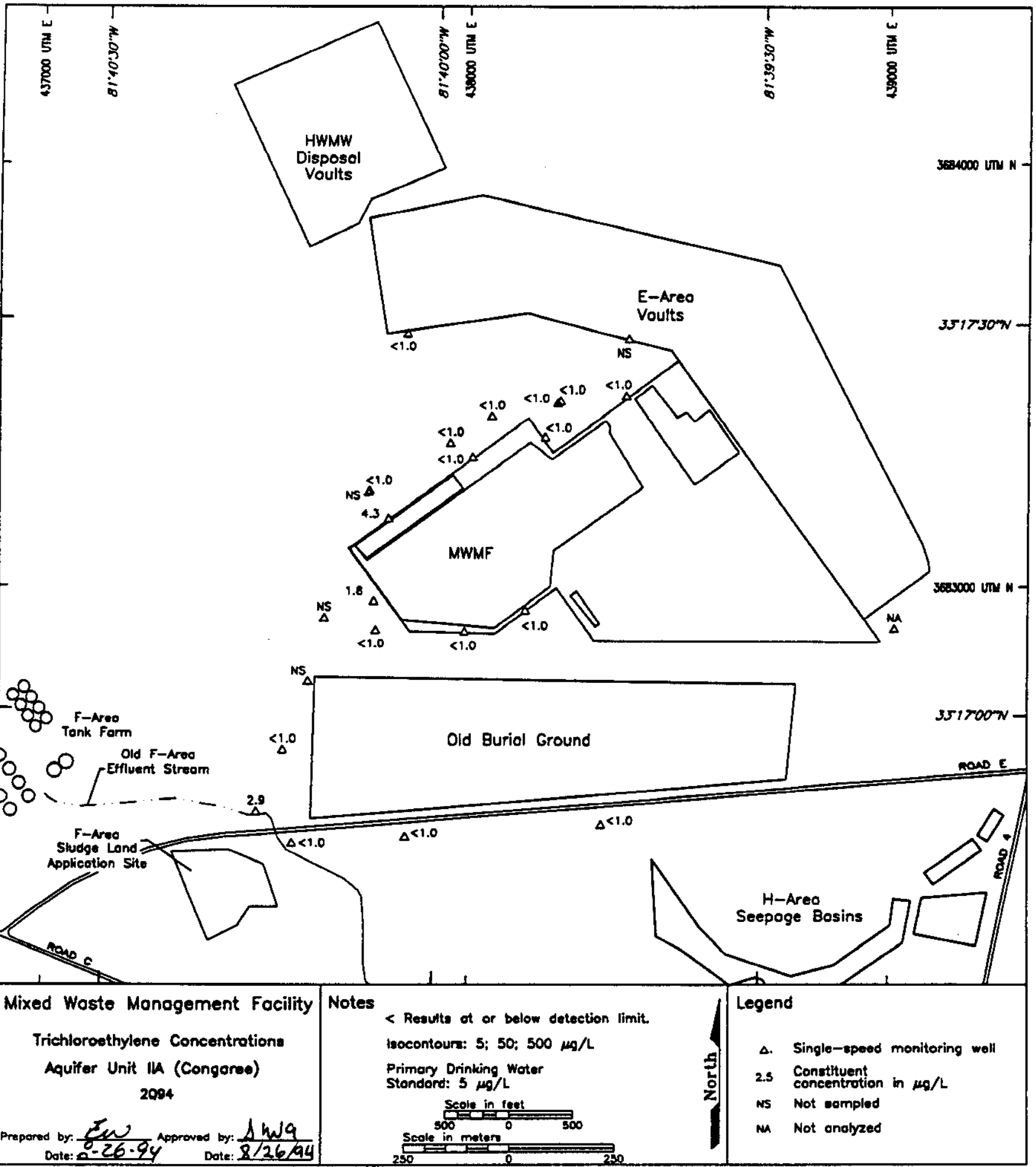

Figure 19. Trichloroethylene Concentrations in Aquifer Unit IIA (Congaree) at the Burial Ground Complex, Second Quarter 1994 


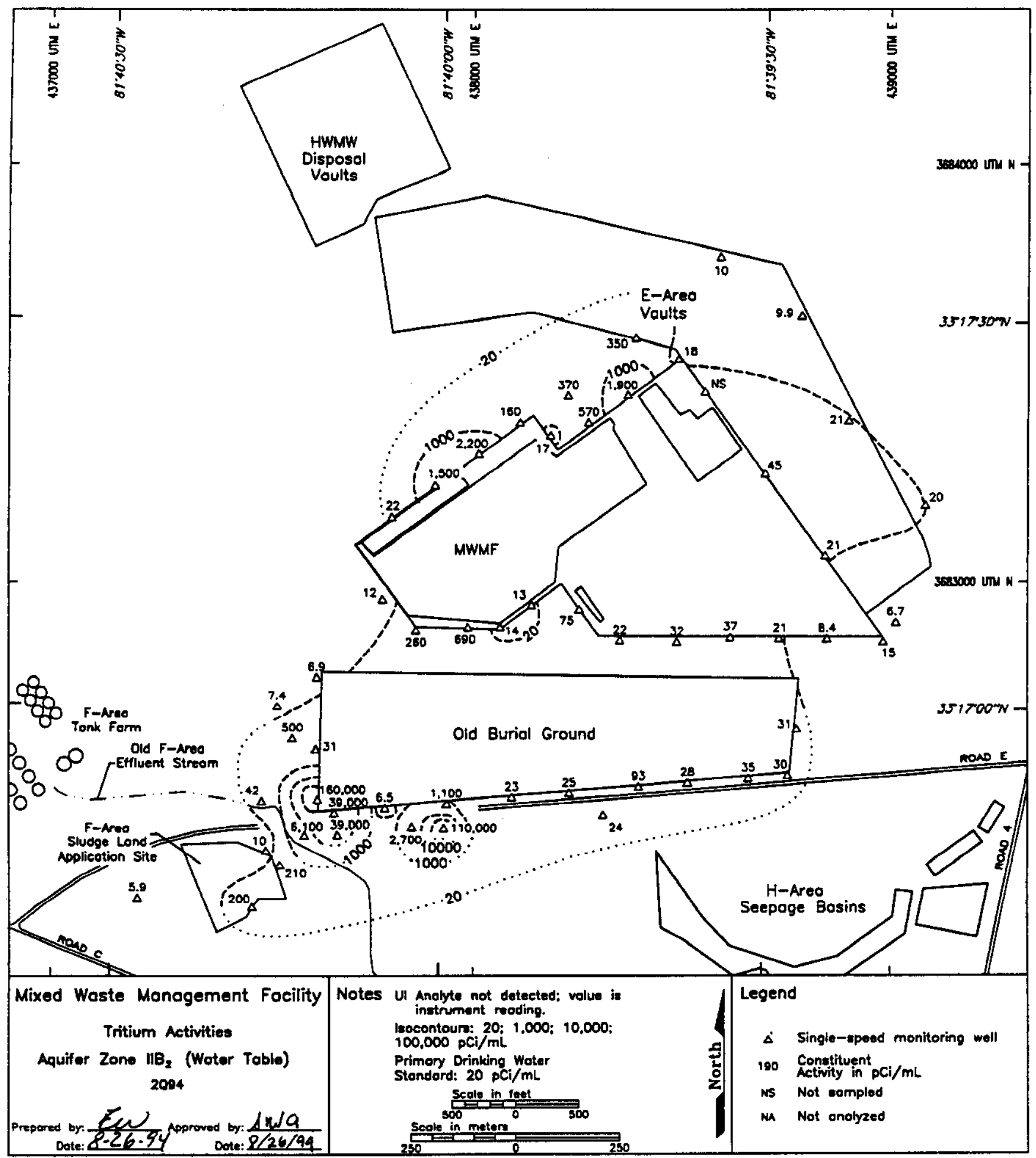

Figure 20. Tritium Activities in Aquifer Zone $\mathrm{HB}_{2}$ (Water Table) at the Burial Ground Complex, Second Quarter 1994 


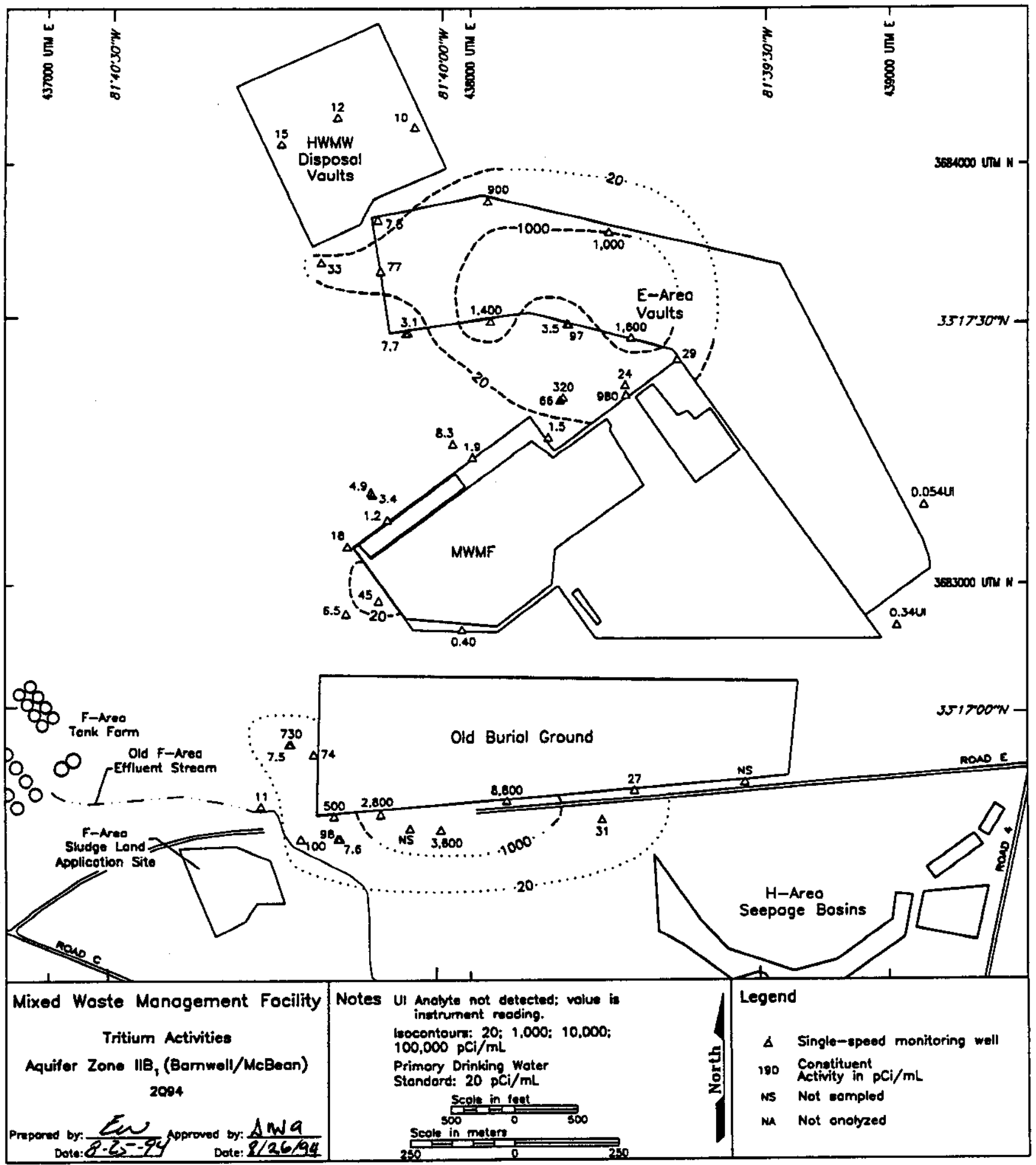

Figure 21. Tritium Activities in Aquifer Zone IIB, (Barnwell/McBean) at the Burial Ground Complex, Second Quarter 1994 


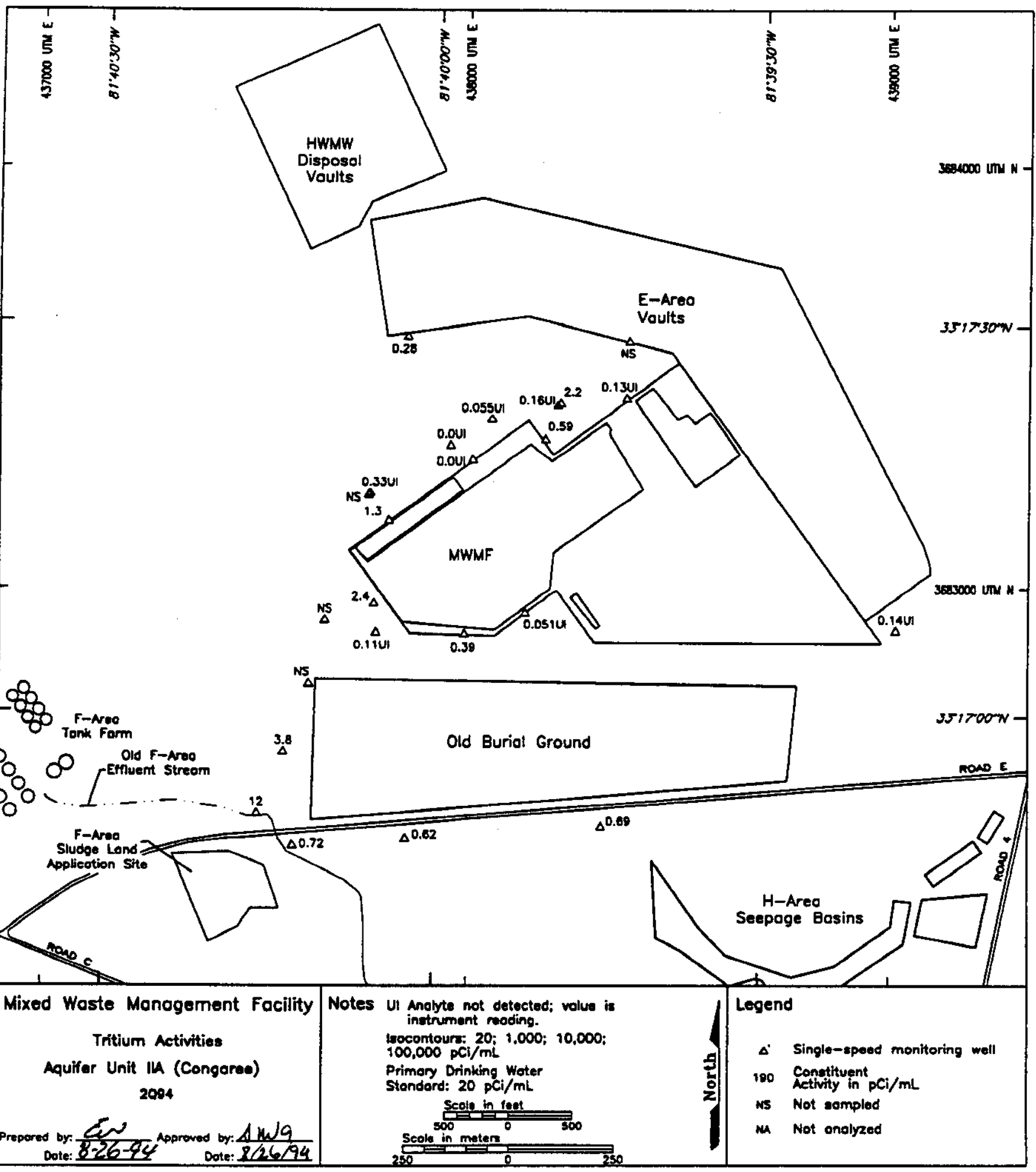

Figure 22. Tritium Activities in Aquifer Unit IIA (Congaree) at the Burial Ground Complex, Second Quarter 1994 


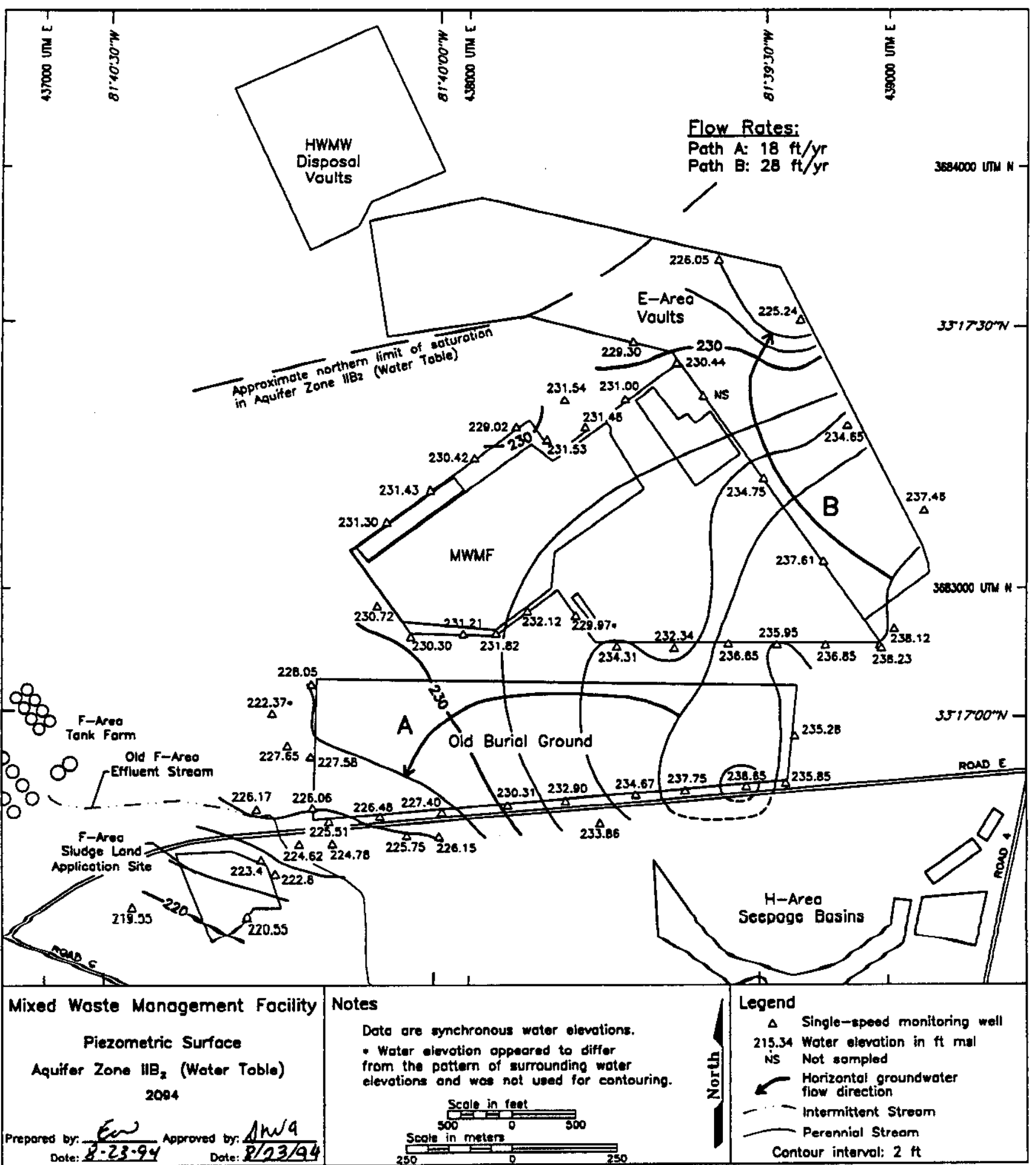

Figure 23. Piezometric Surface Map of Aquifer Zone $\mathrm{IBB}_{2}$ (Water Table) at the Burial Ground Complex 


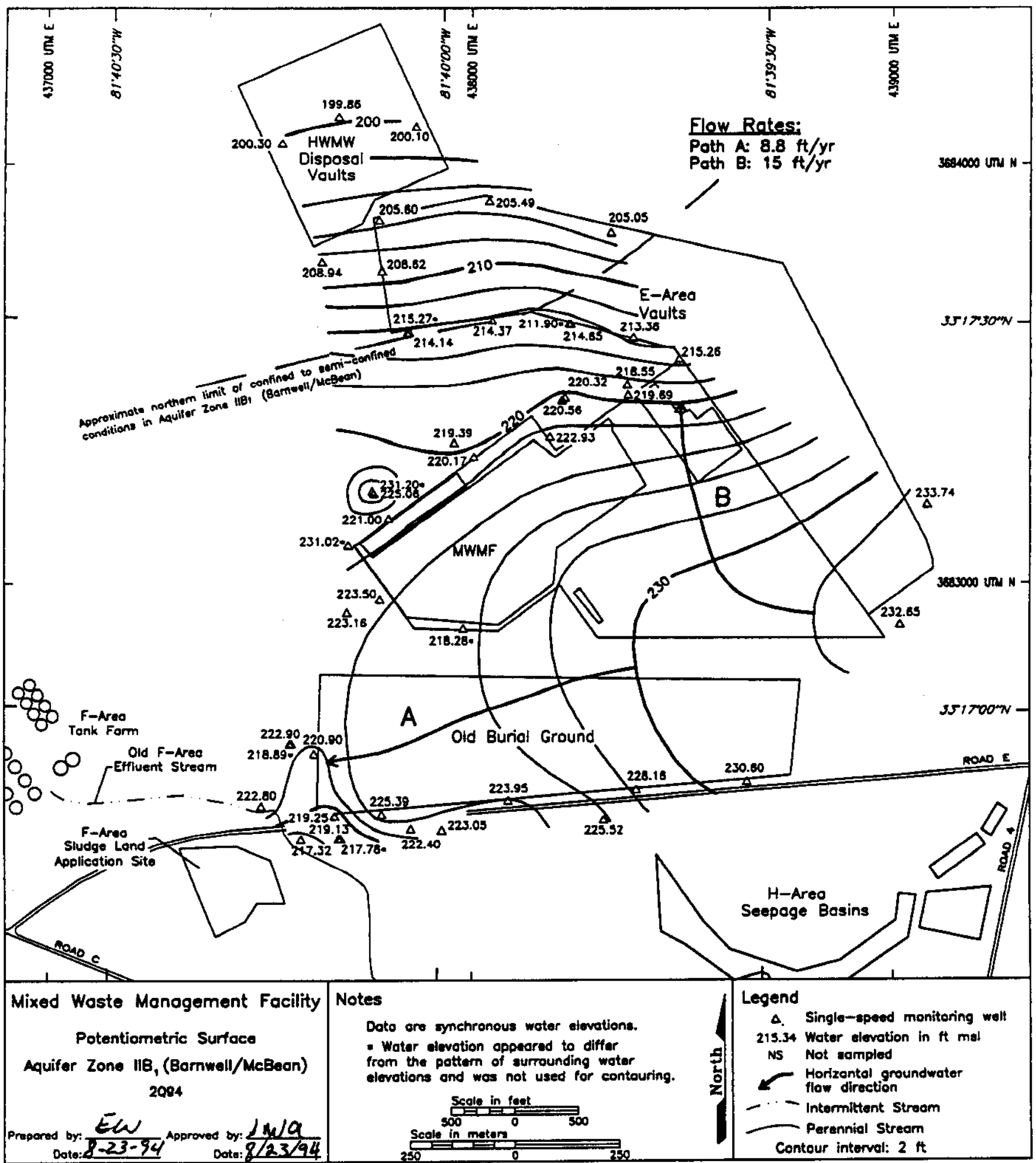

Figure 24. Potentiometric Surface Map of Aquifer Zone IIB, (Barnwell/McBean) at the Burial Ground Complex 


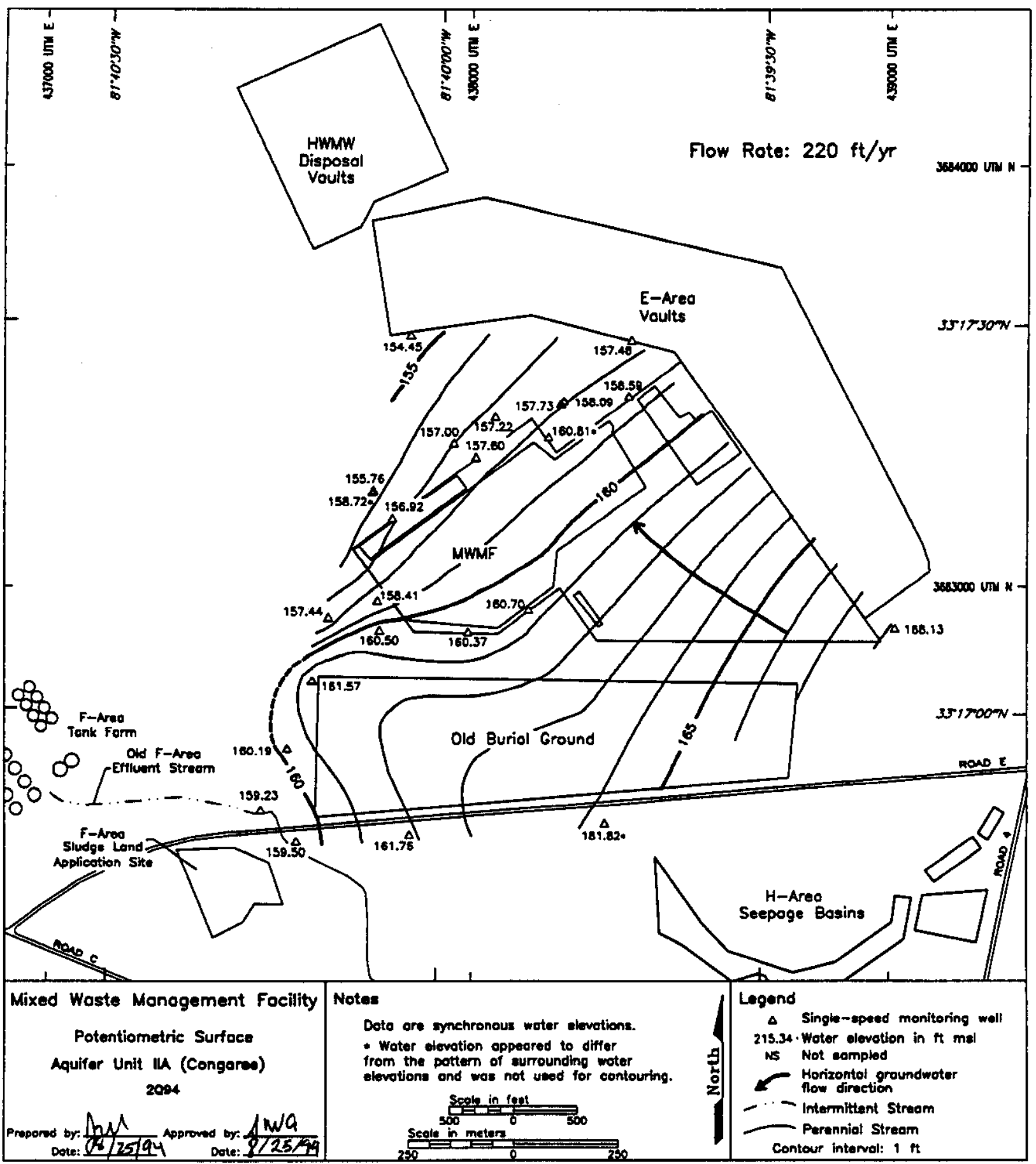

Figure 25. Potentiometric Surface Map of Aquifer Unit IIA (Congaree) at the Burial Ground Complex 


\section{References}

Aadland, R. K., and H. W. Bledsoe, 1990. Classification of Hydrostratigraphic Units at the Savannah River Site, South Carolina, WSRC-RP-90-987. Westinghouse Savannah River Company, Savannah River Site, Aiken, SC.

Aucott, W. R., M. E. Davis, and G. K. Speiran, 1987. Geohydrologic Framework of the Coastal Plain Aquifers of South Carolina, U.S. Geological Survey Water-Resources Investigations Report 85-4271.

Du Pont (E. l. du Pont de Nemours \& Company), 1989. Final Safety Analysis Report of the Defense Waste Processing Facility, DPSTSA-200-10. Savannah River Plant, Aiken, SC.

GeoTrans, Inc., 1988. A Numerical Model of the Hydrogeological System Underlying the Savannah River Plant, Final report submitted to Savannah River Laboratory. GeoTrans, Inc., Sterling, VA.

Siple, G. E., 1967. Geology and Ground Water of the Savannah River Plant and Vicinity, South Carolina. Geological Survey Water-Supply Paper 1841, Reston, VA. 
WSRC-TR-94-0345

Unclassified

\section{Appendix D}

\section{Groundwater Monitoring Results Tables}


WSRC-TR-94-0345

Unclassified

THIS PAGE LEFT BLANK INTENTIONALLY. 


\section{Key to Reading the Tables}

The following abbreviations may appear in the data tables:

\section{Constituents}

\section{1,2,3,4,6,7,8-HPCDD \\ $1,2,3,4,6,7,8$-HPCDF \\ $1,2,3,4,7,8-H X C D D$ \\ $1,2,3,4,7,8-H X C D F$ \\ Lindane \\ PCB \\ $1,2,3,7,8-P C D D$ \\ 1,2,3,7,8-PCDF \\ Sp. conductance \\ TCDD \\ TCDF}

\section{Laboratories}

\section{CN \\ EM}

GE and GP

SC

$S P$

TM

WA and WS

\section{Sampling Codes}

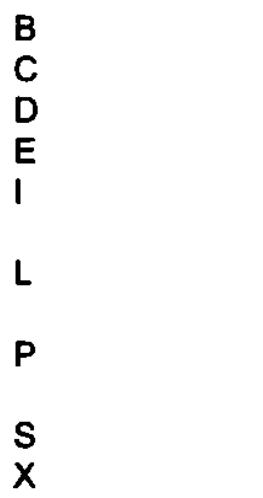

Sampling Methods
B
$P$
$S$
V

\author{
$1,2,3,4,6,7,8$-heptachlorodibenzo-p-dioxin \\ 1,2,3,4,6,7,8-heptachlorodibenzo-p-furan \\ $1,2,3,4,7,8$-hexachlorodibenzo-p-dioxin \\ $1,2,3,4,7,8$-hexachlorodibenzo-p-furan \\ gamma-benzene hexachloride \\ polychlorinated biphenyl \\ 1,2,3,7,8-pentachlorodibenzo-p-dioxin \\ 1,2,3,7,8-pentachlorodibenzo-p-furan \\ specific conductance \\ tetrachlorodibenzo-p-dioxin \\ tetrachlorodibenzo-p-furan
}

Clemson Technical Center, Inc.

Environmental Protection Department/Environmental

Monitoring Section (EPD/EMS) Laboratory

General Engineering Laboratories

Savannah River Technology Center

Spencer Testing Services, Inc.

TMAEberline

Roy F. Weston, Inc.

blank sample was collected

well was pumping continuously

well was dry

equipment blank was collected

well went dry during sampling; insufficient water to collect all samples

well went dry before sampling began; only depth to water can be determined

inaccessibility or mechanical failure prevented sample

collection and field analysis of the water

no water in standpipe; for water level events only

well went dry during purging; samples collected after well

recovered

sample collected using an open-bucket bailer

sample collected using a bladder pump

sample collected using a single-speed centrifugal

downhole pump

sample collected using a variable-speed pump 
Units

E

$\mathrm{mg} / \mathrm{L}$

ms!

MSL

NTU

$\mathrm{pCi} / \mathrm{L}$

$\mathrm{pCi} / \mathrm{mL}$

$\mathrm{pH}$

$\mu \mathrm{g} / \mathrm{L}$

$\mu \mathrm{S} / \mathrm{cm}$

exponential notation (e.g., $1.1 E-09=1.1 \times 10^{-9}=$ 0.0000000011 )

milligrams per liter

mean sea level

million structures per liter

turbidity unit

picocuries per liter

picocuries per milliliter

pH unit

micrograms per liter

microsiemens per centimeter

Other

CS
D
DF
GS
H
Mod
PDWS
PVC
TOC

carbon steel

exceeded final Primary Drinking Water Standard

(PDWS) or screening level column in data tables

dilution factor column in data tables

groundwater protection standard column in data tables

holding time column in data tables

modifier column in data tables

primary drinking water standard

polyvinyl chloride

top of casing

\section{Holding Times}

Standard analytical methods include a limit, called holding time, on the maximum elapsed time between sample collection and extraction or analysis by the laboratory. In the data tables, a large bullet $(\cdot)$ in the $H$ (holding time) column indicates that holding time was exceeded.

Analyses performed beyond holding times may not yield valid results.

The South Carolina Department of Health and Environmental Control allows only 15 minutes to elapse between sampling and analysis for $\mathrm{pH}$. Thus, only field pH measurements can meet the holding time criterion; laboratory $\mathrm{pH}$ analyses always will exceed it.

The laboratory procedure used for the determination of specific conductance allows one day to elapse between sampling and analysis. Thus, laboratory specific conductance measurements may exceed the holding time criterion.

\section{Data Rounding}

Constituent results in analytical results tables that appear to equal the final PDWS but are not marked in the $D$ (exceeded the final PDWS or screening level) column are below the final PDWS in the database. Values stored in the database contain more significant digits than the reported results. Apparent discrepancies in the tables are due to the rounding of reported results. 


\section{Data Qualification}

The contract laboratories continually assess their own accuracy and precision according to U.S. Environmental Protection Agency (EPA) guidelines. They submit sample- or batch-specific quality assurance/quality control information either at the same time as analytical results or in a quarterly summary. Properly defined and used result modifiers (also referred to as qualifiers) can be a key component in assessing data useability. Result modifiers designed by the Environmental Protection Department/Environmental Monitoring Section and provided to the primary laboratories are defined below. These modifiers appear in the data tables under the column Mod. The lettered modifiers are based on EPA's STORET codes.

\section{Result modifier}

Data are not qualified. Numbers should be interpreted exactly as reported.

J

1

L

M

$\mathbf{R}$

T

U

V

Y

1

2

3

4

6
Value is estimated because quantitation in the sample or in associated quality control samples did not meet specifications.

The value in the result field is the instrument reading, not the sample quantification limit. Always used with the result qualifier $U$.

Value is off-scale high. The actual value is not known but is known to be greater than the value shown.

Presence of the analyte is verified but not quantified.

Result was rejected because performance requirements in the sample analysis or associated quality control analyses were not met.

Analyte was not detected; if present, it was below the criteria for detection.

Material analyzed for but not detected. Analytical result reported is less than the sample quantitation limit.

Analyte was detected in an associated method blank.

Result was obtained from an unpreserved or improperly preserved sample. Data may not be accurate.

Result may be an underestimation of the true value due to analytical bias.

Result may be an overestimation of the true value due to analytical bias.

The associated result may be of poor precision (high variability) due to analytical bias.

Result is associated with QA results indicating matrix interference.

The associated result is from a reanalysis performed out of holding time due to problems with an earlier analysis. 
Table 1. Maximum Levels of Constituents Exceeding the Final Primary Drinking Water Standards Aquifer Zone IIB ${ }_{2}$ (Water Table)

\begin{tabular}{|c|c|c|c|c|c|c|c|}
\hline Well & & Constituent & Unit & $\underline{3093}$ & $\underline{4093}$ & $\underline{1094}$ & 2094 \\
\hline BGO & $2 D$ & Tritium & $\mathrm{pCi} / \mathrm{mL}$ & $-{ }^{a}$ & $2.0 E+01$ & $2.1 E+01$ & 2.1E+01 \\
\hline BGO & $3 D$ & Tritium & $\mathrm{pCi} / \mathrm{mL}$ & $4.0 \mathrm{E}+01$ & $3.9 E+01$ & $3.7 E+01$ & $4.5 \mathrm{E}+01$ \\
\hline BGO & $5 \mathrm{D}$ & Tritium & $\mathrm{pCi} / \mathrm{mL}$ & $2.7 E+01$ & - & $2.4 E+01$ & - \\
\hline BGO & $6 D$ & $\begin{array}{l}\text { Tetrachloroethylene } \\
\text { Trichloroethylene } \\
\text { Tritium }\end{array}$ & $\begin{array}{l}\mu g / L \\
\mu g / L \\
p C i / m L\end{array}$ & $\begin{array}{l}5.2 \\
40 \\
1.4 E+03\end{array}$ & $\begin{array}{l}- \\
23 \\
1.2 E+03\end{array}$ & $\begin{array}{l}- \\
23 \\
1.0 \mathrm{E}+03\end{array}$ & $\begin{array}{l}- \\
35 \\
1.9 E+03\end{array}$ \\
\hline BGO & 7D & $\begin{array}{l}\text { Dichloromethane } \\
\text { Tetrachloroethylene } \\
\text { Trichloroethylene } \\
\text { Tritium }\end{array}$ & $\begin{array}{l}\mu g / L \\
\mu g / L \\
\mu g h / \\
p C i / m L\end{array}$ & $\begin{array}{l}13 \\
- \\
55 \\
1.6 E+02\end{array}$ & $\begin{array}{l}\overline{7.0} \\
98 \\
5.2 E+02\end{array}$ & $\begin{array}{l}- \\
13 \\
175 \\
6.5 E+02\end{array}$ & $\begin{array}{l}\overline{9} \\
9.8 \\
153 \\
5.7 \mathrm{E}+02\end{array}$ \\
\hline BGO & 8D & Tritium & $\mathrm{pCi} / \mathrm{mL}$ & $2.9 \mathrm{E}+01$ & - & - & - \\
\hline BGO & 9D & Tritium & $\mathrm{pCi} / \mathrm{mL}$ & 2.7E+02 & $2.1 \mathrm{E}+02$ & $2.4 E+02$ & $1.6 \mathrm{E}+02$ \\
\hline BGO & 10DR & Tritium & $\mathrm{pCi} / \mathrm{mL}$ & $8.6 E+02$ & $6.7 \mathrm{E}+02$ & $1.0 E+03$ & $2.2 E+03$ \\
\hline BGO & 11D & Tritium & $\mathrm{pCi} / \mathrm{mL}$ & $1.4 E+03$ & $1.5 E+03$ & $1.7 E+03$ & $1.5 E+03$ \\
\hline BGO & 12D & $\begin{array}{l}\text { Dichloromethane } \\
\text { Trichloroethylene } \\
\text { Tritium }\end{array}$ & $\begin{array}{l}\mu g h / \\
\rho g C i / m L\end{array}$ & $\begin{array}{l}15 \\
139 \\
2.6 E+01\end{array}$ & $\begin{array}{l}\overline{100} \\
3.1 E+01\end{array}$ & $\begin{array}{l}\overline{127} \\
2.9 E+01\end{array}$ & $\begin{array}{l}- \\
83 \\
2.2 E+01\end{array}$ \\
\hline BGO & 14DR & $\begin{array}{l}\text { Dichloromethane } \\
\text { Trichloroethylene }\end{array}$ & $\begin{array}{l}\mu g / L \\
\mu g / L\end{array}$ & $\overline{86}$ & $\begin{array}{l}6.6 \\
252\end{array}$ & $\overline{61}$ & $\overline{162}$ \\
\hline BGO & 15D & $\begin{array}{l}\text { Tetrachloroethylene } \\
\text { Trichloroethylene } \\
\text { Tritium }\end{array}$ & $\begin{array}{l}\mu g / L \\
\mu g / L \\
p C i / m L\end{array}$ & $\begin{array}{l}8.2 \\
105 \\
4.6 E+02\end{array}$ & $\begin{array}{l}5.0 \\
54 \\
3.2 E+02\end{array}$ & $\begin{array}{l}\overline{67} \\
2.7 E+02\end{array}$ & $\begin{array}{l}5.8 \\
69 \\
2.6 E+02\end{array}$ \\
\hline BGO & 16D & $\begin{array}{l}\text { Trichloroethylene } \\
\text { Tritium }\end{array}$ & $\stackrel{\mu \mathrm{g} / \mathrm{L}}{\mathrm{pCi} / \mathrm{mL}}$ & $\begin{array}{l}16 \\
1.1 E+03\end{array}$ & $\begin{array}{l}10 \\
6.9 E+02\end{array}$ & $\begin{array}{l}22 \\
6.3 E+02\end{array}$ & $\begin{array}{l}8.6 \\
6.9 E+02\end{array}$ \\
\hline BGO & 19D & Tritium & $\mathrm{pCi} / \mathrm{mL}$ & $N A^{b}$ & $4.0 \mathrm{E}+01$ & $5.6 E+01$ & $7.5 E+01$ \\
\hline BGO & 20D & Tritium & $\mathrm{pCi} / \mathrm{mL}$ & 2.1E+01 & $2.2 E+01$ & $2.3 E+01$ & $2.2 E+01$ \\
\hline BGO & 21D & Tritium & $\mathrm{pCi} / \mathrm{mL}$ & $3.1 E+01$ & $3.2 E+01$ & $3.0 E+01$ & $3.2 E+01$ \\
\hline BGO & 22DR & Tritium & pCi/mL & $4.6 E+01$ & $4.4 E+01$ & $4.2 E+01$ & $3.7 E+01$ \\
\hline BGO & 23D & Tritium & $\mathrm{pCi} / \mathrm{mL}$ & $2.3 E+01$ & $2.4 \mathrm{E}+01$ & $2.0 \mathrm{E}+01$ & 2.1E+01 \\
\hline BGO & 26D & Lead & $\mu g /$ & - & 73 & - & - \\
\hline
\end{tabular}




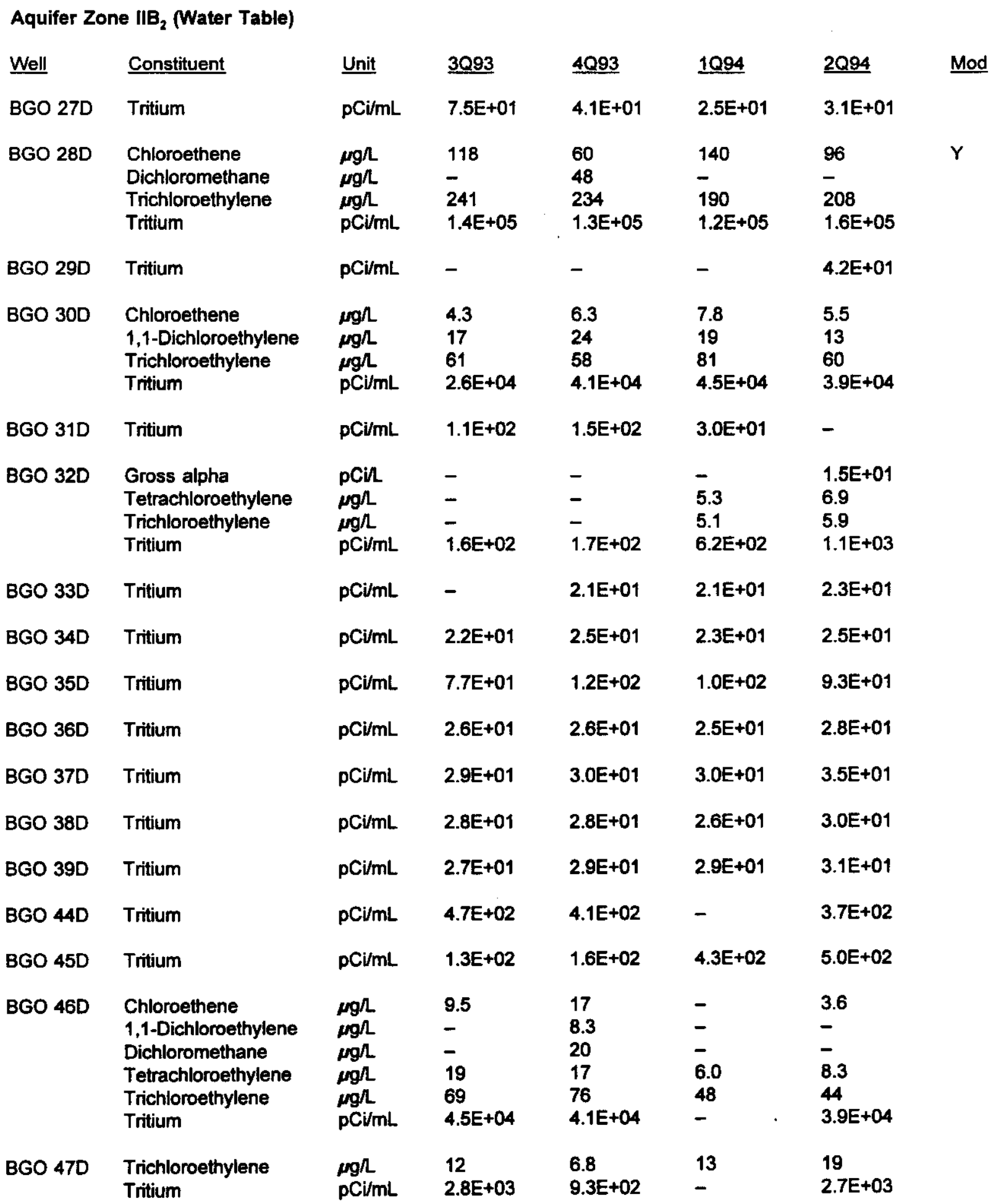


Aquifer Zone $\|_{2}$ (Water Table)

\begin{tabular}{|c|c|c|c|c|c|c|}
\hline Well & Constituent & Unit & $\underline{3 Q 93}$ & $\underline{4 Q 93}$ & 1094 & $\underline{2094}$ \\
\hline BGO 48D & $\begin{array}{l}\text { Carbon tetrachloride } \\
\text { Tetrachloroethylene } \\
\text { Trichloroethylene } \\
\text { Tritium }\end{array}$ & $\begin{array}{l}\mu g / L \\
\mu g / L \\
\mu g / L \\
\mathrm{pC} / \mathrm{mL}\end{array}$ & $\begin{array}{l}- \\
29 \\
125 \\
4.8 E+04\end{array}$ & $\begin{array}{l}5.5 \\
304 \\
300 \\
8.8 E+04\end{array}$ & $\begin{array}{l}- \\
68 \\
36 \\
-\end{array}$ & $\begin{array}{l}- \\
68 \\
41 \\
1.1 E+05\end{array}$ \\
\hline BGO 49D & Tritium & $\mathrm{pCi} / \mathrm{mL}$ & 2.1E+01 & 2.1E+01 & 2.1E+01 & 2.4E+01 \\
\hline BGO 50D & $\begin{array}{l}\text { Trichloroethylene } \\
\text { Tritium }\end{array}$ & $\begin{array}{l}\mu g / L \\
p C i / m L\end{array}$ & $\begin{array}{l}17 \\
4.5 E+03\end{array}$ & $\begin{array}{l}11 \\
3.1 E+03\end{array}$ & $\begin{array}{l}12 \\
-\end{array}$ & $\begin{array}{l}19 \\
6.1 E+03\end{array}$ \\
\hline$B G X$ 1D & Tritium & pCi/mL. & $3.3 E+02$ & $3.5 E+02$ & - & $3.5 E+02$ \\
\hline BGX 10D & Lead & $\mu g /$ & 59 & 53 & - & - \\
\hline BGX 11D & Tritium & pCi/mL & - & - & - & 2.1E+01 \\
\hline$B G X$ 12D & Tritium & pCi/mL & - & - & - & $2.0 E+01$ \\
\hline FSS 1D & $\begin{array}{l}\text { Copper } \\
\text { Lead }\end{array}$ & $\begin{array}{l}\mu g / L \\
\mu g / L\end{array}$ & $\begin{array}{l}\text { NA } \\
58\end{array}$ & $\begin{array}{l}\text { NA } \\
\text { NA }\end{array}$ & $\begin{array}{l}1,400 \\
119\end{array}$ & - \\
\hline FSS 2D & Tritium & pCi/mL & 7.7E+01 & NA & $1.8 E+02$ & 2.1E+02 \\
\hline FSS 3D & $\begin{array}{l}\text { Lead } \\
\text { Tritium }\end{array}$ & $\begin{array}{l}\mu g / L \\
p C i / m L\end{array}$ & $\begin{array}{l}329 \\
4.3 E+01\end{array}$ & $\begin{array}{l}\text { NA } \\
\text { NA }\end{array}$ & $\overline{9} .7 E+01$ & $\begin{array}{l}956 \\
2.0 \mathrm{E}+02\end{array}$ \\
\hline
\end{tabular}

Aquifer Zone IIB, (Barnwell/McBean)

\begin{tabular}{|c|c|c|c|c|c|c|}
\hline Well & Constituent & Unit & 3Q993 & 4093 & 1094 & $\underline{2094}$ \\
\hline BGO 5C & Tritium & $\mathrm{pCi} / \mathrm{mL}$ & $2.5 E+01$ & $2.5 E+01$ & $2.9 E+01$ & $2.9 E+01$ \\
\hline BGO 6B & Tritium & pCi/mL & - & $2.5 E+01$ & 2.1E+01 & $2.4 E+01$ \\
\hline BGO $6 \mathrm{C}$ & Tritium & pCi/mL & $9.2 E+02$ & $8.8 E+02$ & $8.9 E+02$ & $9.8 E+02$ \\
\hline BGO 10B & Tritium & pCi/mL & $1.9 E+02$ & $7.8 \mathrm{E}+01$ & 1.1E+02 & - \\
\hline BGO $10 \mathrm{C}$ & Tritium & $\mathrm{pCi} / \mathrm{mL}$ & $1.4 E+02$ & - & $1.1 E+02$ & - \\
\hline BGO 12CR & Trichloroethylene & $\mu g h$ & 81 & 71 & 79 & 82 \\
\hline BGO 13DR & Tritium & pCi/mL & $2.6 E+01$ & $2.1 E+01$ & - & - \\
\hline BGO 14CR & $\begin{array}{l}\text { Trichloroethylene } \\
\text { Tritium }\end{array}$ & $\begin{array}{l}\mu g / L \\
p C i / m L\end{array}$ & $\begin{array}{l}52 \\
3.4 E+01\end{array}$ & $\begin{array}{l}45 \\
3.0 E+01\end{array}$ & $\begin{array}{l}56 \\
3.0 E+01\end{array}$ & $\begin{array}{l}52 \\
4.5 E+01\end{array}$ \\
\hline BGO 27C & $\begin{array}{l}\text { Trichloroethylene } \\
\text { Tritium }\end{array}$ & $\begin{array}{l}\mu g / L \\
p C i / m L\end{array}$ & $\begin{array}{l}25 \\
7.8 E+01\end{array}$ & $\begin{array}{l}21 \\
6.5 E+01\end{array}$ & $\begin{array}{l}22 \\
6.6 E+01\end{array}$ & $\begin{array}{l}26 \\
7.4 E+01\end{array}$ \\
\hline
\end{tabular}


Aquifer Zone IIB, (Barnwell/McBean)

\begin{tabular}{|c|c|c|c|c|c|c|}
\hline Well & Constituent & Unit & 3093 & 4093 & 1094 & $\underline{2 Q 94}$ \\
\hline BGO 29C & Tritium & $\mathrm{pCi} / \mathrm{mL}$ & $1.2 \mathrm{E}+02$ & $1.5 E+02$ & $1.9 \mathrm{E}+02$ & - \\
\hline BGO 30C & $\begin{array}{l}\text { Trichloroethylene } \\
\text { Tritium }\end{array}$ & $\begin{array}{l}\mu g / L \\
\mathrm{pCi} / \mathrm{mL}\end{array}$ & $\begin{array}{l}11 \\
6.2 E+02\end{array}$ & $\begin{array}{l}11 \\
7.3 E+02\end{array}$ & $\begin{array}{l}11 \\
6.2 E+02\end{array}$ & $\begin{array}{l}9.9 \\
5.0 \mathrm{E}+02\end{array}$ \\
\hline BGO 31C & Tritium & pCi/mL & $2.4 E+03$ & $3.4 E+03$ & $3.1 E+03$ & $2.8 E+03$ \\
\hline BGO 33C & $\begin{array}{l}\text { Mercury } \\
\text { Tetrachloroethylene } \\
\text { Trichloroethylene } \\
\text { Tritium }\end{array}$ & $\begin{array}{l}\mu g / L \\
\mu g / L \\
\mu g / L \\
\mathrm{pCi} / \mathrm{mL}\end{array}$ & $\begin{array}{l}2.2 \\
\overline{28} \\
8.2 \mathrm{E}+03\end{array}$ & $\begin{array}{l}2.4 \\
- \\
22 \\
8.0 E+03\end{array}$ & $\begin{array}{l}2.8 \\
5.1 \\
26 \\
8.2 E+03\end{array}$ & $\begin{array}{l}\overline{5.2} \\
28 \\
8.8 E+03\end{array}$ \\
\hline BGO 35C & $\begin{array}{l}\text { Trichloroethylene } \\
\text { Tritium }\end{array}$ & $\begin{array}{l}\mu \mathrm{g} / \mathrm{L} \\
\mathrm{pCi} / \mathrm{mL}\end{array}$ & - & - & - & $\begin{array}{l}5.1 \\
2.7 E+01\end{array}$ \\
\hline BGO 42C & $\begin{array}{l}\text { Dichloromethane } \\
\text { Trichloroethylene }\end{array}$ & $\begin{array}{l}\mu g / L \\
\mu g / L\end{array}$ & $\overline{61}$ & $\begin{array}{l}10 \\
47\end{array}$ & $\frac{-}{36}$ & $\overline{28}$ \\
\hline BGO 44B & $\begin{array}{l}\text { Lead } \\
\text { Tritium }\end{array}$ & $\begin{array}{l}\mu \mathrm{g} / \mathrm{L} \\
\mathrm{pCi} / \mathrm{mL}\end{array}$ & $\overline{4.7 E}+02$ & $\overline{2.8 E}+02$ & $\begin{array}{l}55 \\
-\end{array}$ & $\overline{6} 6 E+01$ \\
\hline BGO 44C & Tritium & $\mathrm{pCi} / \mathrm{mL}$ & 4.1E+02 & $2.9 E+02$ & - & $3.2 E+02$ \\
\hline BGO 45C & Tritium & $\mathrm{pCi} / \mathrm{mL}$ & $6.9 E+02$ & $7.5 E+02$ & $7.4 E+02$ & $7.3 E+02$ \\
\hline BGO 46B & Tritium & $\mathrm{pCi} / \mathrm{mL}$ & $2.2 E+01$ & $4.0 E+01$ & - & - \\
\hline BGO 46C & $\begin{array}{l}\text { Trichloroethylene } \\
\text { Tritium }\end{array}$ & $\begin{array}{l}\mu g / L \\
p C i / m L\end{array}$ & $\begin{array}{l}26 \\
7.9 E+01\end{array}$ & $\begin{array}{l}22 \\
9.0 E+01\end{array}$ & $\begin{array}{l}24 \\
-\end{array}$ & $\begin{array}{l}25 \\
9.8 E+01\end{array}$ \\
\hline BGO 48C & $\begin{array}{l}\text { Trichloroethylene } \\
\text { Tritium }\end{array}$ & $\begin{array}{l}\mu g h \\
p C i / m L\end{array}$ & $\overline{3.1 E}+03$ & $\begin{array}{l}5.5 \\
3.2 E+03\end{array}$ & - & $\overline{3.6 E}+03$ \\
\hline BGO 49C & Tritium & $\mathrm{pCi} / \mathrm{mL}$ & $2.6 \mathrm{E}+01$ & $3.5 E+01$ & $2.5 E+01$ & $3.1 E+01$ \\
\hline BGO 50C & $\begin{array}{l}\text { Trichloroethylene } \\
\text { Tritium }\end{array}$ & $\begin{array}{l}\mu \mathrm{g} / \mathrm{h} \\
\mathrm{pCi} / \mathrm{mL}\end{array}$ & $\begin{array}{l}12 \\
7.9 E+01\end{array}$ & $\overline{8} .5 E+01$ & - & $\begin{array}{l}11 \\
1.0 E+02\end{array}$ \\
\hline$B G X \quad 1 C$ & Tritium & $\mathrm{pCi} / \mathrm{mL}$ & 8.7E+02 & $1.2 E+03$ & $1.5 E+03$ & $1.6 E+03$ \\
\hline BGX 2D & $\begin{array}{l}\text { Trichloroethylene } \\
\text { Tritium }\end{array}$ & $\begin{array}{l}\mu \mathrm{g} / \mathrm{L} \\
\mathrm{pCi} / \mathrm{mL}\end{array}$ & $\begin{array}{l}12 \\
8.7 E+01\end{array}$ & $\begin{array}{l}12 \\
8.6 E+01\end{array}$ & $\begin{array}{l}13 \\
-\end{array}$ & $\begin{array}{l}15 \\
9.7 E+01\end{array}$ \\
\hline$B G \times \quad 3 D$ & $\begin{array}{l}\text { Trichloroethylene } \\
\text { Tritium }\end{array}$ & $\underset{\rho \mathrm{g} / \mathrm{C} / \mathrm{mL}}{\mathrm{p}}$ & $\overline{1} .2 E+03$ & $\overline{1.2 E}+03$ & $\begin{array}{l}36 \\
1.3 E+03\end{array}$ & $\overline{1.4 E}+03$ \\
\hline$B G \times \quad 5 D$ & $\begin{array}{l}\text { Nonvolatile beta } \\
\text { Tritium }\end{array}$ & $\begin{array}{l}\mathrm{pCi} / \\
\mathrm{pCi} / \mathrm{mL}\end{array}$ & $\overline{5.3 E}+01$ & $\overline{6} .1 E+01$ & $\begin{array}{l}1.5 E+02 \\
7.1 E+01\end{array}$ & $\overline{7} .7 E+01$ \\
\hline$B G \times 7 D$ & Tritium & $\mathrm{pCi} / \mathrm{mL}$ & $1.6 E+03$ & $1.4 E+03$ & - & $9.0 E+02$ \\
\hline BGX 8DR & Tritium & $\mathrm{pCi} / \mathrm{mL}$ & $9.2 \mathrm{E}+02$ & $9.7 E+02$ & - & $1.0 \mathrm{E}+03$ \\
\hline
\end{tabular}


WSRC-TR-94-0345

Unclassified

Aquifer Zone IIB, (Barnwell/McBean)

\begin{tabular}{|c|c|c|c|c|c|c|c|}
\hline Well & Constituent & $\underline{\text { Unit }}$ & $\underline{3093}$ & $\underline{4 Q 93}$ & $\underline{1 Q 94}$ & $\underline{2 Q 94}$ & Mod \\
\hline HMD 1D & Tritium & $\mathrm{pCi} / \mathrm{mL}$ & - & $3.1 E+01$ & 3.7E+01 & $3.3 E+01$ & \\
\hline HMD 4D & Lead & $\mu g / L$ & - & 53 & - & - & \\
\hline \multicolumn{8}{|c|}{ Aquifer Unit IIA (Congaree) } \\
\hline Well & Constituent & Unit & $\underline{3093}$ & $\underline{4093}$ & $\underline{1094}$ & $\underline{2094}$ & Mod \\
\hline BGO 9AA & Nonvolatile beta & pCin & - & - & $7.8 \mathrm{E}+01$ & - & \\
\hline BGO 12AR & Trichloroethylene & $\mu g h$ & - & 5.2 & - & - & \\
\hline BGO 50A & Chloroform & $\mu g /$ & - & 191 & - & - & \\
\hline
\end{tabular}

Notes: The groundwater samples are unfiltered. Thus, the results for metals are for total recoverable metals. The modifier column applies to second quarter 1994 results only.

a $-=$ analyzed but not above final PDWS.

b NA = not analyzed or result was rejected because performance requirements in the sample analysis or associated quality control analyses were not met. 
Table 2. Maximum Levels of Constituents Exceeding Other Flag 2 Criteria

Aquifer Zone $\mathrm{II}_{2}$ (Water Table)

\begin{tabular}{|c|c|c|c|c|}
\hline Well & Constituent & $\underline{\text { Unit }}$ & $\underline{2 Q 94}$ & Mod \\
\hline BGO 1D & Aluminum & $\mu g / L$ & 297 & \\
\hline BGO 2D & Aluminum & $\mu g / L$ & 70 & \\
\hline BGO 3D & Aluminum & $\mu g h$ & 199 & \\
\hline BGO 5D & Aluminum & $\mu g / L$ & 78 & \\
\hline$B G O 6 D$ & Aluminum & $\mu g /$ & 277 & \\
\hline BGO $7 D$ & Total organic halogens & $\mu g /$ & 82 & \\
\hline BGO 10DR & Iron & $\mu g /$ & 322 & \\
\hline BGO 14DR & Total organic halogens & $\mu g /$ & 91 & \\
\hline BGO 15D & Total organic halogens & $\mu g /$ & 53 & \\
\hline BGO 16D & Aluminum & $\mu g /$ & 216 & \\
\hline BGO 17DR & Aluminum & $\lg / 2$ & 93 & \\
\hline BGO 22DR & Aluminum & $\mu g /$ & 238 & \\
\hline BGO 24D & Aluminum & $\mu g /$ & 438 & \\
\hline BGO 26D & Aluminum & $\mu g / L$ & 223 & \\
\hline BGO 27D & $\begin{array}{l}\text { Aluminum } \\
\text { Iron }\end{array}$ & $\begin{array}{l}\mu g / L \\
\mu g / L\end{array}$ & $\begin{array}{l}4,000 \\
1,810\end{array}$ & \\
\hline BGO 28D & $\begin{array}{l}\text { Aluminum } \\
\text { 1,1-Dichloroethane } \\
\text { Iron } \\
\text { Manganese } \\
\text { Total organic halogens }\end{array}$ & $\begin{array}{l}\mu g / L \\
\mu g / L \\
\mu g / L \\
\mu g / L \\
\mu g / L\end{array}$ & $\begin{array}{l}482 \\
50 \\
538 \\
73 \\
468\end{array}$ & $\mathbf{Y}$ \\
\hline BGO 29D & $\begin{array}{l}\text { Aluminum } \\
\text { Iron } \\
\text { Manganese }\end{array}$ & $\begin{array}{l}\mu g / L \\
\mu g / L \\
\mu g / L\end{array}$ & $\begin{array}{l}1,820 \\
1,090 \\
66\end{array}$ & \\
\hline BGO 30D & $\begin{array}{l}\text { Aluminum } \\
\text { 1,1-Dichloroethane } \\
\text { Manganese } \\
\text { Total organic halogens }\end{array}$ & $\begin{array}{l}\mu g / L \\
\mu g h / \\
\mu g / L\end{array}$ & $\begin{array}{l}249 \\
67 \\
71 \\
420\end{array}$ & $\mathrm{~J} 1$ \\
\hline BGO 31D & $\begin{array}{l}\text { Aluminum } \\
\text { Iron }\end{array}$ & $\underset{\mu g /}{\mu g /}$ & $\begin{array}{l}1,220 \\
685\end{array}$ & \\
\hline
\end{tabular}


Aquifer Zone $\|_{2} B_{2}$ (Water Table)

\begin{tabular}{|c|c|c|c|c|}
\hline Well & Constituent & Unit & $\underline{2 Q 94}$ & Mod \\
\hline BGO 32D & $\begin{array}{l}\text { Aluminum } \\
\text { Iron }\end{array}$ & $\begin{array}{l}\mu g / L \\
\mu g / L\end{array}$ & $\begin{array}{l}855 \\
393\end{array}$ & \\
\hline BGO 33D & Aluminum & $\boldsymbol{\mu} \boldsymbol{g} / \mathrm{h}$ & 332 & \\
\hline BGO 34D & Aluminum & $\mu g \Omega$ & 146 & \\
\hline BGO 35D & Aluminum & $\mu g /$ & 184 & \\
\hline BGO 36D & $\begin{array}{l}\text { Aluminum } \\
\text { Iron }\end{array}$ & $\begin{array}{l}\mu g h \\
\mu g h\end{array}$ & $\begin{array}{l}1,050 \\
356\end{array}$ & \\
\hline BGO 37D & Aluminum & $\mu g / L$ & 179 & \\
\hline BGO 38D & Aluminum & $\mu g /$ & 263 & \\
\hline BGO 39D & Aluminum & $\mu g /$ & 108 & \\
\hline BGO 40D & $\begin{array}{l}\text { Aluminum } \\
\text { Iron } \\
\text { Tin }\end{array}$ & $\begin{array}{l}\mu g / L \\
\mu g / L \\
\mu g / L\end{array}$ & $\begin{array}{l}619 \\
497 \\
74\end{array}$ & $\mathbf{J 3}$ \\
\hline BGO 46D & $\begin{array}{l}\text { 1,1-Dichloroethane } \\
\text { Total organic halogens }\end{array}$ & $\begin{array}{l}\mu g / h \\
\mu g / L\end{array}$ & $\begin{array}{l}22 \\
199\end{array}$ & \\
\hline BGO 48D & $\begin{array}{l}\text { Aluminum } \\
\text { Total organic halogens }\end{array}$ & $\begin{array}{l}\mu g h \\
\mu g / L\end{array}$ & $\begin{array}{l}121 \\
120\end{array}$ & \\
\hline BGO 49D & Aluminum & $\mu g /$ & 76 & \\
\hline BGO 50D & $\begin{array}{l}\text { Aluminum } \\
\text { 1,1-Dichloroethane } \\
\text { Total organic halogens }\end{array}$ & $\begin{array}{l}\mu g / L \\
\mu g / L \\
\mu g / L\end{array}$ & $\begin{array}{l}63 \\
10 \\
227\end{array}$ & J1 \\
\hline$B G X \quad 10$ & Aluminum & $\mu g /$ & 165 & \\
\hline$B G X 10 D$ & $\begin{array}{l}\text { Aluminum } \\
\text { Manganese }\end{array}$ & $\begin{array}{l}\mu g h \\
\mu g h\end{array}$ & $\begin{array}{l}159 \\
59\end{array}$ & \\
\hline$B G X 11 D$ & $\begin{array}{l}\text { Aluminum } \\
\text { Iron }\end{array}$ & $\begin{array}{l}\mu g / h \\
\mu g / L\end{array}$ & $\begin{array}{l}1,460 \\
828\end{array}$ & \\
\hline$B G X 12 D$ & Aluminum & $\mu g /$ & 68 & \\
\hline FSS 10 & $\begin{array}{l}\text { Aluminum } \\
\text { Iron }\end{array}$ & $\begin{array}{l}\mu g / \\
\mu g / L\end{array}$ & $\begin{array}{l}737 \\
845\end{array}$ & $\cdot$ \\
\hline FSS $2 D$ & $\begin{array}{l}\text { Aluminum } \\
\text { Iron }\end{array}$ & $\underset{\mu g / L}{\mu g h}$ & $\begin{array}{l}555 \\
1,370\end{array}$ & \\
\hline
\end{tabular}


Aquifer Zone IIB $_{2}$ (Water Table)

\begin{tabular}{|c|c|c|c|}
\hline Well & Constituent & Unit & 2Q94 \\
\hline FSS 3D & $\begin{array}{l}\text { Aluminum } \\
\text { Iron } \\
\text { Manganese }\end{array}$ & $\begin{array}{l}\mu g / h \\
\mu g / h \\
\mu g / h\end{array}$ & $\begin{array}{l}16,800 \\
33,200 \\
391\end{array}$ \\
\hline FSS 4D & $\begin{array}{l}\text { Aluminum } \\
\text { Iron }\end{array}$ & $\underset{\mu g / L}{\mu g / L}$ & $\begin{array}{l}418 \\
758\end{array}$ \\
\hline
\end{tabular}

Aquifer Zone IIB, (Barnwell/McBean)

\begin{tabular}{|c|c|c|c|c|}
\hline Well & Constituent & Unit & 2094 & Mod \\
\hline BGO 5C & $\begin{array}{l}\text { Aluminum } \\
\text { Iron } \\
\text { Manganese }\end{array}$ & $\begin{array}{l}\mu g / \\
\mu g / L \\
\mu g / L\end{array}$ & $\begin{array}{l}1,920 \\
338 \\
108\end{array}$ & \\
\hline BGO 10B & Aluminum & $\mu g / L$ & 60 & \\
\hline BGO 10C & $\begin{array}{l}\text { Aluminum } \\
\text { Iron } \\
\text { Manganese }\end{array}$ & $\begin{array}{l}\mu g / L \\
\mu g h / \\
\mu g / L\end{array}$ & $\begin{array}{l}223 \\
1,240 \\
67\end{array}$ & \\
\hline BGO 12CR & Aluminum & $\mu g / L$ & 289 & \\
\hline BGO 13DR & Manganese & $\mu g h$ & 73 & \\
\hline BGO 14CR & Aluminum & $\mu g /$ & 229 & \\
\hline BGO 16B & $\begin{array}{l}\text { Aluminum } \\
\text { Iron }\end{array}$ & $\begin{array}{l}\mu \mathrm{g} / \mathrm{h} \\
\mathrm{mg} / \mathrm{L}\end{array}$ & $\begin{array}{l}82 \\
332\end{array}$ & \\
\hline BGO 29C & Aluminum & $\mu g h$ & 99 & \\
\hline BGO 30C & Aluminum & $\mu g / L$ & 222 & \\
\hline BGO 33C & Total organic halogens & $\mu g /$ & 134 & \\
\hline BGO 42C & Aluminum & $\mu g /$ & 51 & \\
\hline BGO 43CR & Aluminum & $\mu g /$ & 61 & \\
\hline BGO 44B & $\begin{array}{l}\text { Aluminum } \\
\text { Manganese }\end{array}$ & $\frac{\operatorname{mg} h}{\mu g h}$ & $\begin{array}{l}250 \\
171\end{array}$ & \\
\hline BGO 44C & $\begin{array}{l}\text { Aluminum } \\
\text { Manganese }\end{array}$ & $\begin{array}{l}\mu g / L \\
\mu g / L\end{array}$ & $\begin{array}{l}391 \\
338\end{array}$ & . \\
\hline BGO 45B & $\begin{array}{l}\text { Aluminum } \\
\mathrm{pH}\end{array}$ & $\underset{\mathrm{pH}}{\mu \mathrm{g} / \mathrm{L}}$ & $\begin{array}{l}173 \\
11\end{array}$ & J1 \\
\hline BGO 50C & Aluminum & $\mu g /$ & 60 & \\
\hline
\end{tabular}


Aquifer Zone $11 B_{1}$ (Barnwell/McBean)

\begin{tabular}{|c|c|c|c|c|}
\hline Well & & Constituent & Unit & $\underline{2094}$ \\
\hline$B G X$ & $1 \mathrm{C}$ & $\begin{array}{l}\text { Aluminum } \\
\text { pH } \\
\text { Specific conductance }\end{array}$ & $\begin{array}{l}\mu \mathrm{g} / \mathrm{L} \\
\mathrm{pH} \\
\mu \mathrm{S} / \mathrm{cm}\end{array}$ & $\begin{array}{l}665 \\
12 \\
1,740\end{array}$ \\
\hline$B G X$ & 5D & $\begin{array}{l}\text { Aluminum } \\
\text { Manganese }\end{array}$ & $\begin{array}{l}\mu \mathrm{g} / \mathrm{L} \\
\mu \mathrm{g} / \mathrm{L}\end{array}$ & $\begin{array}{l}97 \\
335\end{array}$ \\
\hline$B G X$ & 8DR & Aluminum & $\mu \mathrm{gg} n$ & 85 \\
\hline HMD & 1D & $\begin{array}{l}\text { Aluminum } \\
\text { Iron }\end{array}$ & $\begin{array}{l}\mu \mathrm{g} / \\
\mu \mathrm{g} \Omega\end{array}$ & $\begin{array}{l}590 \\
642\end{array}$ \\
\hline HMD & 4D & Aluminum & $\mu g / L$ & 152 \\
\hline
\end{tabular}

Aquifer Unit IIA (Congaree)

\begin{tabular}{|c|c|c|c|c|}
\hline Well & Constituent & Unit & $2 \mathrm{Q94}$ & Mod \\
\hline BGO 9AA & $\begin{array}{l}\text { Aluminum } \\
\text { pH } \\
\text { Specific conductance } \\
\text { Tin }\end{array}$ & $\begin{array}{l}\mu \mathrm{g} / \mathrm{L} \\
\mathrm{pH} \\
\mu \mathrm{S} / \mathrm{cm} \\
\mu \mathrm{g} / \mathrm{L}\end{array}$ & $\begin{array}{l}1,210 \\
12 \\
1,430 \\
127\end{array}$ & J1 \\
\hline BGO 12AR & $\begin{array}{l}\text { Aluminum } \\
\text { pH }\end{array}$ & $\begin{array}{l}\mu \mathrm{g} / \mathrm{h} \\
\mathrm{pH}\end{array}$ & $\begin{array}{l}264 \\
10\end{array}$ & J1 \\
\hline BGO 14AR & Aluminum & $\mu g / h$ & 309 & \\
\hline BGO 29A & $\begin{array}{l}\text { Aluminum } \\
\text { Iron }\end{array}$ & $\begin{array}{l}\mu g / L \\
\mu g / L\end{array}$ & $\begin{array}{l}643 \\
408\end{array}$ & \\
\hline BGO 43AA & $\begin{array}{l}\text { Aluminum } \\
\text { pH } \\
\text { Tin }\end{array}$ & $\begin{array}{l}\mu g / h \\
\mathrm{pH} \\
\mu g / L\end{array}$ & $\begin{array}{l}148 \\
11 \\
20\end{array}$ & $\mathrm{~J} 1$ \\
\hline BGO 44AA & Aluminum & $\mu g /$ & 125 & \\
\hline BGO 49A & $\begin{array}{l}\text { Aluminum } \\
\text { pH }\end{array}$ & $\begin{array}{l}\mu g / L \\
\mathrm{pH}\end{array}$ & $\begin{array}{l}505 \\
11\end{array}$ & J1 \\
\hline BGO 50A & $\begin{array}{l}\text { Aluminum } \\
\text { pH } \\
\text { Specific conductance } \\
\text { Tin } \\
\text { Total organic halogens }\end{array}$ & $\begin{array}{l}\mu g / \\
\mathrm{pH} \\
\mu \mathrm{S} / \mathrm{cm} \\
\mu g / L \\
\mu g / L\end{array}$ & $\begin{array}{l}501 \\
12 \\
666 \\
23 \\
73\end{array}$ & J1 \\
\hline
\end{tabular}

Notes: These results do not include field data.

The groundwater samples are unfittered. Thus, the results for metals are for total recoverable metals.

Flags are established by EPD/EMS and are based on final PDWS, Secondary Drinking Water Standards, or method detection limits (see Appendix B). 
Table 3. Groundwater Monitoring Results for Individual Wells

\section{WELL BGO 1D}

\begin{tabular}{|c|c|c|c|c|c|c|}
\hline SRS Coord. & Lat/Longitude & Screen Zone Elevation & Top of Casing & Casing & Pump & Formation \\
\hline $\begin{array}{l}\text { N73737.9 } \\
\text { E58779.3 }\end{array}$ & $\begin{array}{l}33.284765^{\circ} \mathrm{N} \\
81.655257^{\circ} \mathrm{W}\end{array}$ & $245.0-225.0 \mathrm{ft} \mathrm{msl}$ & $295.1 \mathrm{ft} \mathrm{msl}$ & 4" PVC & $\mathbf{s}$ & Water Table (IIB ${ }_{2}$ ) \\
\hline
\end{tabular}

\section{FIELD MEASUREMENTS}

Sample date: $04 / 14 / 94$

Depth to water: $64.73 \mathrm{ft}(19.73 \mathrm{~m})$ below TOC

Water elevation: $230.37 \mathrm{ft}(70.22 \mathrm{~m}) \mathrm{msl}$

Sp. conductance: $56 \mu \mathrm{S} / \mathrm{cm}$

Turbidity: 73.3 NTU

Water evacuated before sampling: $9 \mathrm{gal}$

The well went dry during purging.

\section{LABORATORY ANALYSES}

\section{H D Analyte}

pH

Specific conductance

Specific conductance

Turbidity

Acetophenone

Aldrin

Aluminum, total recoverable

Antimony, total recoverable

Arsenic, total recoverable

Barium, total recoverable

Benzene

Bromodichloromethane

Bromoform

Bromomethane (Methyl bromide)

Cadmium, total recoverable

Calcium, total recoverable

Carbon tetrachloride

Chloride

Chloride

Chlorobenzene

Chloroethane

Chloroethene (Vinyl chloride)

2-Chloroethyl vinyl ether

Chloroform

Chloromethane (Methyl chloride)

Chromium, total recoverable

Copper, total recoverable

Cyanide

p.p'-DDT

Dibromochloromethane

1,1-Dichloroethane

1,2-Dichloroethane

1,1-Dichloroethylene

trans-1,2-Dichloroethylene

Dichloromethane (Methylene chloride)

2,4-Dichlorophenoxyacetic acid

1,2-Dichloropropane

$\begin{array}{ll}\quad \text { Result } & \text { DF } \\ 5.3 & 1 \\ 43 & 1 \\ 45 & 1 \\ 201 & 10 \\ <10 & 1 \\ <0.053 & 1 \\ 297 & 1 \\ <2.0 & 1 \\ <2.0 & 1 \\ 13 & 1 \\ <1.0 & 1 \\ <1.0 & 1 \\ <1.0 & 1 \\ <1.0 & 1 \\ <2.0 & 1 \\ 102 & 1 \\ <1.0 & 1 \\ 1.690 & 1 \\ <1.750 & 1 \\ <1.0 & 1 \\ <1.0 & 1 \\ <1.0 & 1 \\ <1.0 & 1 \\ <1.0 & 1 \\ <1.0 & 1 \\ <4.0 & 1 \\ 29 & 1 \\ <5.0 & 1 \\ <0.11 & 1 \\ <1.0 & 1 \\ <1.0 & 1 \\ <1.0 & 1 \\ <1.0 & 1 \\ <1.0 & 1 \\ <1.0 & 1 \\ <0.0015 & 1 \\ <1.0 & 1\end{array}$

Time: 10:03

pH: 4.8

Alkalinity: $0 \mathrm{mg} / \mathrm{L}$

Water temperature: $19.3^{\circ} \mathrm{C}$

Volumes purged: 2.6 well volumes

\begin{tabular}{|c|c|c|c|c|}
\hline DF & Mod & Unit & Flag & $\underline{\text { Lab }}$ \\
\hline 1 & J1 & pH & 0 & GE \\
\hline 1 & & $\mu \mathrm{S} / \mathrm{cm}$ & 0 & $\mathrm{GE}$ \\
\hline 1 & & $\mu \mathrm{s} / \mathrm{cm}$ & 0 & $\mathrm{GE}$ \\
\hline 10 & & NTU & 0 & GE \\
\hline$i$ & & $\mu g / L$ & 0 & $\mathrm{GE}$ \\
\hline 1 & & $\mu g / L$ & 0 & GE \\
\hline 1 & & $\mu g / L$ & 2 & GE \\
\hline 1 & & $\mu \mathrm{g} / \mathrm{L}$ & 0 & GE \\
\hline 1 & & $\mu g / L$ & 0 & GE \\
\hline 1 & & $\mu \mathrm{g} / \mathrm{L}$ & 0 & GE \\
\hline 1 & & $\mu \mathrm{g} / \mathrm{L}$ & 0 & GE \\
\hline 1 & & $\mu g / L$ & 0 & GE \\
\hline 1 & & $\mu g / L$ & 0 & GE \\
\hline 1 & & $\mu g / L$ & 0 & $\mathrm{GE}$ \\
\hline 1 & & $\mu \mathrm{g} / \mathrm{L}$ & 0 & GE \\
\hline 1 & & $\mu g / L$ & 0 & GE \\
\hline 1 & & $\mu g / L$ & 0 & GE \\
\hline 1 & & $\mu g / L$ & 0 & GE \\
\hline 1 & & $\mu g / L$ & 0 & GE \\
\hline 1 & & $\mu g / L$ & 0 & GE \\
\hline 1 & & $\mu g / L$ & 0 & GE \\
\hline 1 & & $\mu \mathrm{g} / \mathrm{L}$ & 0 & GE \\
\hline 1 & & $\mu \mathfrak{g} / \mathrm{L}$ & 0 & GE \\
\hline 1 & & $\mu g / L$ & 0 & GE \\
\hline 1 & & $\mu \mathrm{g} / \mathrm{L}$ & 0 & GE \\
\hline 1 & & $\mu g / L$ & 0 & GE \\
\hline 1 & & $\mu \mathrm{g} / \mathrm{L}$ & 0 & GE \\
\hline 1 & & $\mu \mathrm{g} / L$ & 0 & GE \\
\hline 1 & & $\mu \mathrm{g} / \mathrm{L}$ & 0 & GE \\
\hline 1 & & $\mu \mathrm{g} / \mathrm{L}$ & 0 & GE \\
\hline 1 & & $\mu \mathrm{g} / \mathrm{L}$ & 0 & GE \\
\hline 1 & & $\mu \mathrm{g} / \mathrm{L}$ & 0 & GE \\
\hline 1 & & $\mu \mathrm{g} / \mathrm{L}$ & 0 & GE \\
\hline 1 & & $\mu \mathrm{g} / \mathrm{L}$ & 0 & $\mathrm{GE}$ \\
\hline 1 & & $\mu \mathrm{g} / \mathrm{L}$ & 0 & GE \\
\hline 1 & & $\mu \mathrm{g} / \mathrm{L}$ & 0 & GE \\
\hline 1 & & $\mu \mathrm{g} / \mathrm{L}$ & 0 & GE \\
\hline
\end{tabular}

- = exceeded holding time. = exceeded screening level or final primary drinking water standard. 
WELL BGO 1D collected on 04/14/94, laboratory analyses (cont.)

H $\underline{\text { Analyte }}$

cis-1,3-Dichloropropene

trans-1,3-Dichloropropene

Dieldrin

Endrin

Ethylbenzene

Fluoride

Fluoride

Heptachlor

Iron, total recoverable

Lead, total recoverable

Lindane

Magnesium, total recoverable

Manganese, total recoverable

Mercury, total recoverable

Methoxychlor

Naphthalene

Nickel, total recoverable

Nitrate-nitrite as nitrogen

Phenols

Potassium, total recoverable

Selenium, total recoverable

Silica, total recoverable

Silver, total recoverable

Sodium, total recoverable

Sulfate

Sulfate

1,1,2,2-Tetrachloroethane

Tetrachloroethylene

Tin, total recoverable

Toluene

Total dissolved solids

Total organic carbon

Total organic halogens

Total phosphates (as P)

Toxaphene

2,4,5-TP (Silvex)

1,1,1-Trichloroethane

1,1,2-Trichloroethane

Trichloroethylene

Trichlorofluoromethane

2,4,5-T

Vanadium, total recoverable

Xylenes

Zinc, total recoverable

Carbon-14

Gross alpha

Nonvolatile beta

Radium, total alpha-emitting

Radium, total alpha-emitting

Tritium

Tritium

Uranium-233/234

Uranium-235

Uranium-238

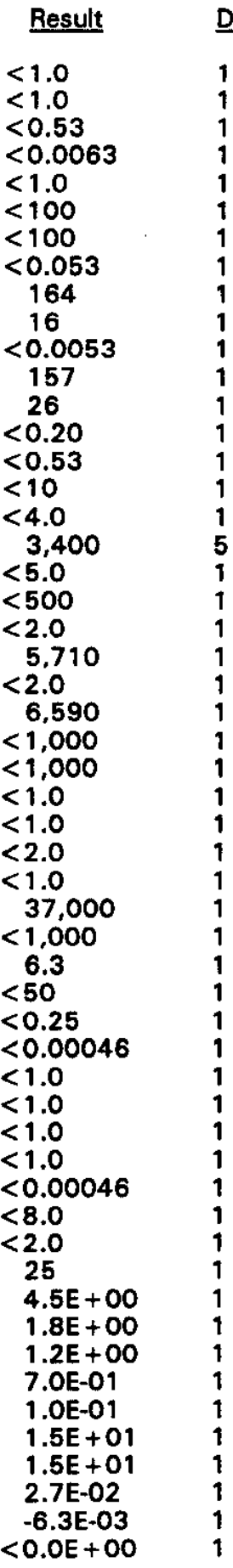

Mod Unit Flag Lab

$\mu g / L \quad 0 \quad G E$

$\mu \mathrm{g} / \mathrm{L} \quad 0 \quad \mathrm{GE}$

$\mu \mathrm{g} / \mathrm{L} \quad \mathrm{O} \quad \mathrm{GE}$

$\mu \mathrm{g} / \mathrm{L} \quad 0 \quad \mathrm{GE}$

$\mu g / L \quad O \quad$ GE

$\mu g / L \quad 0 \quad G E$

$\mu \mathrm{g} / \mathrm{L} \quad 0 \quad \mathrm{GE}$

$\mu \mathrm{g} / \mathrm{L} \quad 0 \quad \mathrm{GE}$

$\mu \mathrm{g} / \mathrm{L} \quad 1 \quad \mathrm{GE}$

$\mu g / L \quad 0 \quad G E$

$\mu g / L \quad 0 \quad G E$

$\mu g / L \quad 0 \quad G E$

$\mu \mathrm{g} / \mathrm{L} \quad 1 \quad \mathrm{GE}$

$\mu g / L \quad 0 \quad G E$

$\mu \mathrm{g} / \mathrm{L} \quad \mathrm{O} \quad \mathrm{GE}$

$\mu \mathrm{g} / \mathrm{L} \quad 0 \quad \mathrm{GE}$

$\mu g / L \quad 0 \quad$ GE

$\mu g / L \quad 0 \quad$ GE

$\mu g / L \quad 0 \quad$ GE

$\mu g / L \quad 0 \quad$ GE

$\mu g / L \quad 0 \quad$ GE

$\mu \mathrm{g} / \mathrm{L} \quad 0 \quad \mathrm{GE}$

$\mu \mathrm{g} / \mathrm{L} \quad 0 \quad \mathrm{GE}$

$\mu \mathrm{g} / \mathrm{L} \quad \mathrm{O} \quad \mathrm{GE}$

$\mu g / L \quad 0 \quad$ GE

$\mu g / L \quad 0 \quad$ GE

$\mu g / L \quad 0 \quad$ GE

$\mu g / L \quad 0 \quad$ GE

$\mu \mathrm{g} / \mathrm{L} \quad 0 \quad \mathrm{GE}$

$\mu g / L \quad 0 \quad$ GE

$\mu \mathrm{g} / \mathrm{L} \quad 0 \quad \mathrm{GE}$

$\mu g / L \quad 0 \quad$ GE

$\mu g / L \quad 0 \quad$ GE

$\mu g / L \quad 0 \quad$ GE

$\mu g / L \quad 0 \quad$ GE

$\mu g / L \quad 0 \quad G E$

$\mu \mathrm{g} / \mathrm{L} \quad \mathrm{O} \quad \mathrm{GE}$

$\mu g / L \quad 0 \quad G E$

$\mu \mathrm{g} / \mathrm{L} \quad 0 \quad \mathrm{GE}$

$\mu g / L \quad 0 \quad$ GE

$\mu \mathrm{g} / \mathrm{L} \quad 0 \quad \mathrm{GE}$

$\mu \mathrm{g} / \mathrm{L} \quad \mathrm{O} \quad \mathrm{GE}$

$\mu \mathrm{g} / \mathrm{L} \quad 0 \quad \mathrm{GE}$

$\mu g / L \quad 0 \quad G E$

$\mathrm{pCi} / \mathrm{L} \quad \mathrm{O} \quad \mathrm{GP}$

$\mathrm{pCi} / \mathrm{L} \quad \mathrm{G} \quad \mathrm{GP}$

$\mathrm{pCi} / \mathrm{L} \quad 0$

$\mathrm{pCi} / \mathrm{L} \quad 0 \quad \mathrm{GP}$

pCi/L $\quad 0 \quad$ GP

$\mathrm{pCi} / \mathrm{mL} \quad 1 \quad$ GP

$\mathrm{pCi} / \mathrm{mL} \quad 1 \quad$ GP

pCi/L $\quad 0 \quad$ GP

$\mathrm{pCi} / \mathrm{L} \quad \mathrm{O} \quad \mathrm{GP}$

$\mathrm{pCi} / \mathrm{L} \quad 0 \quad$ GP

- = exceeded holding time. $\|=$ exceeded screening level or final primary drinking water standard. 


\section{WELL BGO 2D}

\begin{tabular}{|c|c|c|c|c|c|c|}
\hline SRS Coord. & Lat/Longitude & Screen Zone Elevation & Top of Casing & Casing & Pump & Formation \\
\hline$\sqrt{ } 74$ & $\begin{array}{l}33.286617^{\circ} \mathrm{N} \\
81.656760^{\circ} \mathrm{W}\end{array}$ & & & & $\mathbf{s}$ & \\
\hline
\end{tabular}

\section{FIELD MEASUREMENTS}

Sample date: 04/18/94

Depth to water: $59.05 \mathrm{ft}(18.00 \mathrm{~m})$ below TOC

Water elevation: $237.85 \mathrm{ft}(72.50 \mathrm{~m}) \mathrm{msl}$

Sp. conductance: $50 \mu \mathrm{S} / \mathrm{cm}$

Turbidity: 0.4 NTU

Water evacuated before sampling: $39 \mathrm{gal}$

\section{LABORATORY ANALYSES}

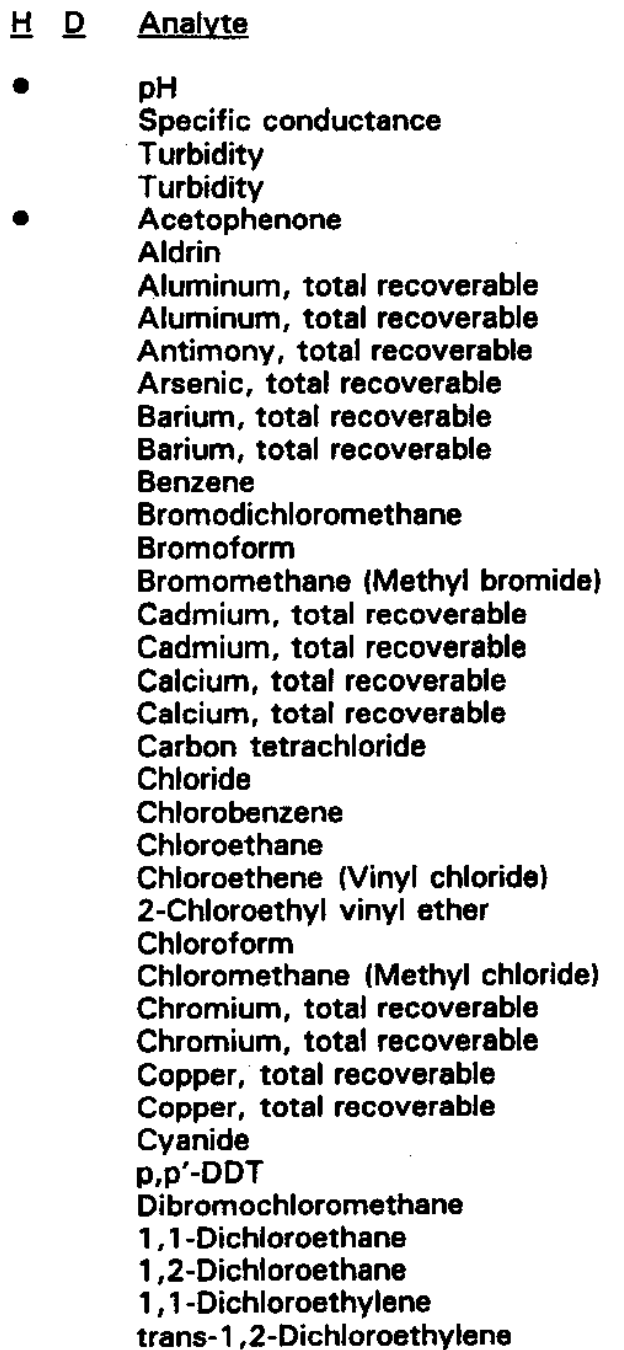

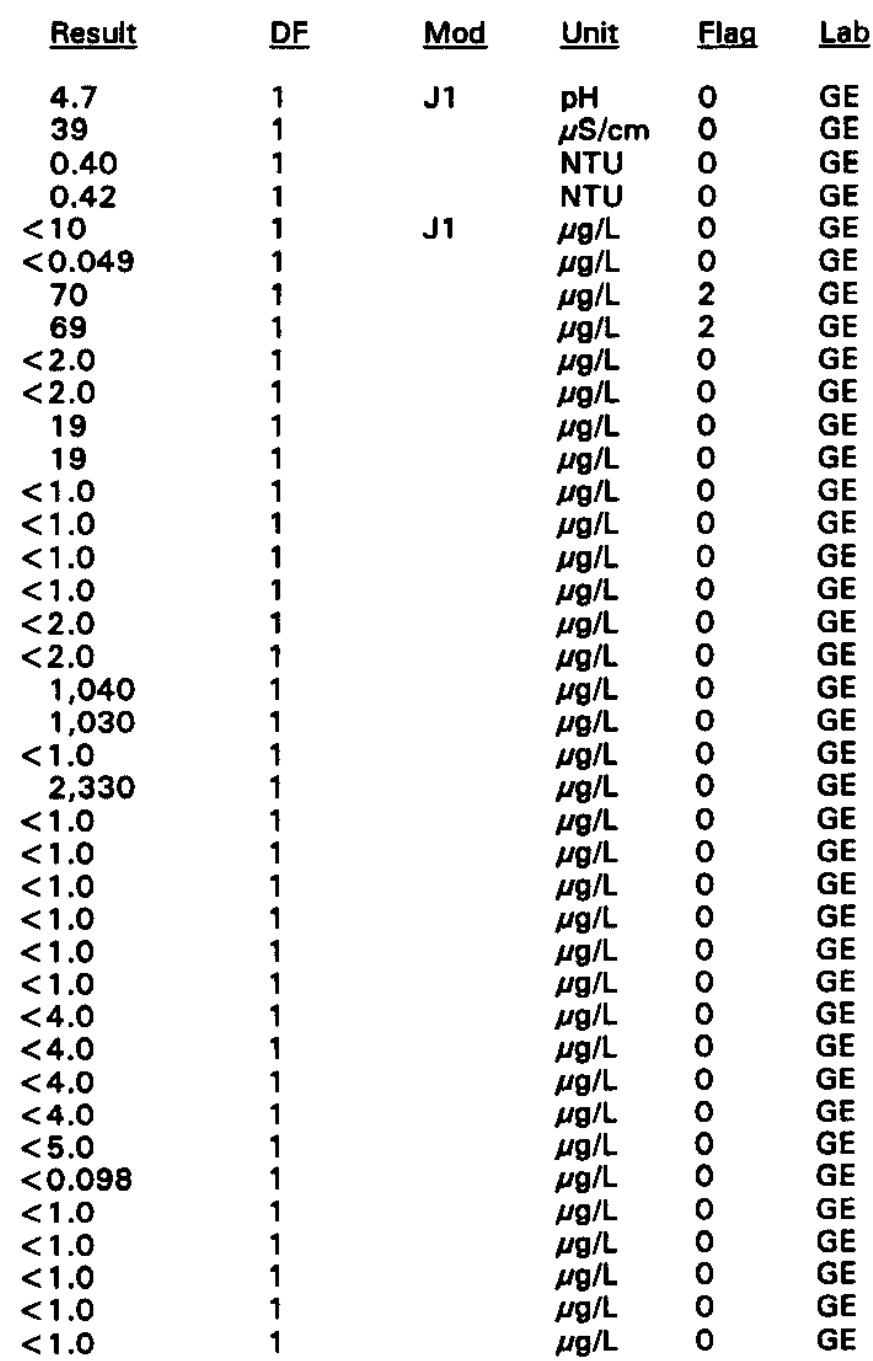

Time: 10:00

pH: 4.8

Alkalinity: $0 \mathrm{mg} / \mathrm{L}$

Water temperature: $20.2{ }^{\circ} \mathrm{C}$

Volumes purged: $\mathbf{3 . 1}$ well volumes 
WELL BGO 2D collected on $04 / 18 / 94$, laboratory analyses (cont.)

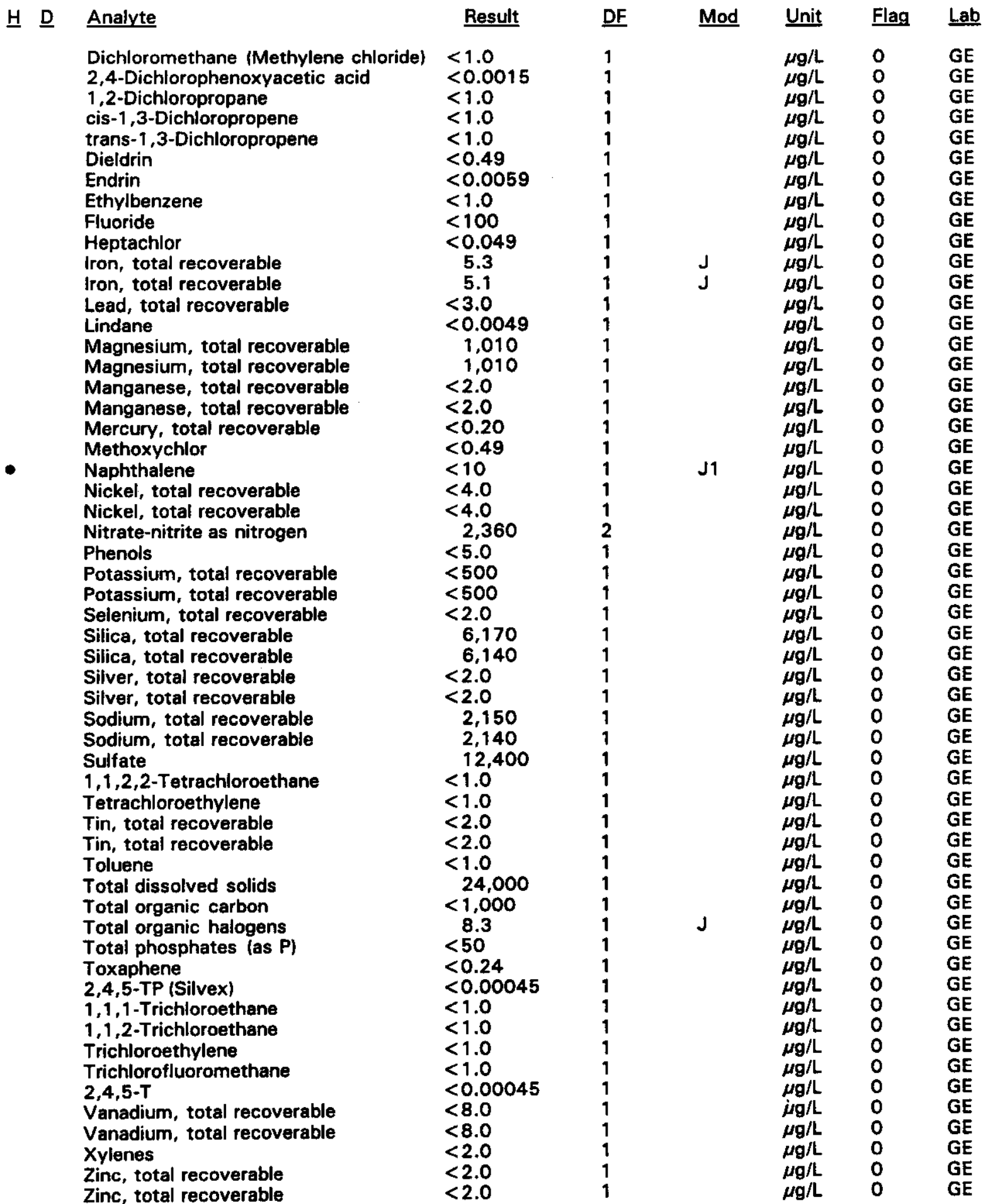

- exceeded holding time. $=$ exceeded screening level or final primary drinking water standard. 
WELL BGO 2D collected on 04/18/94, laboratory analyses (cont.)

\begin{tabular}{|c|c|c|c|c|c|c|}
\hline$\underline{H} \quad \underline{D}$ & Analyte & Result & $\underline{\mathrm{DF}}$ & Mod & Unit & Flag \\
\hline - & $\begin{array}{l}\text { Carbon-14 } \\
\text { Gross alpha } \\
\text { Nonvolatile beta } \\
\text { Radium, total alpha-emitting } \\
\text { Tritium } \\
\text { Uranium-233/234 } \\
\text { Uranium-235 } \\
\text { Uranium-238 }\end{array}$ & $\begin{array}{l}2.6 E+00 \\
3.0 E+00 \\
2.1 E+00 \\
1.5 E+00 \\
2.1 E+01 \\
1.2 E-01 \\
6.3 E-02 \\
8.4 E-02\end{array}$ & $\begin{array}{l}1 \\
1 \\
1 \\
1 \\
1 \\
1 \\
1 \\
1\end{array}$ & $\begin{array}{l}\text { UI } \\
\text { J } \\
\text { UI }\end{array}$ & $\begin{array}{l}\mathrm{pCi} / \mathrm{L} \\
\mathrm{pCi} / \mathrm{L} \\
\mathrm{pCi} / \mathrm{L} \\
\mathrm{pCi} / \mathrm{L} \\
\mathrm{pCi} / \mathrm{mL} \\
\mathrm{pCi} / \mathrm{L} \\
\mathrm{pCi} / \mathrm{L} \\
\mathrm{pCi} / \mathrm{L}\end{array}$ & $\begin{array}{l}0 \\
0 \\
0 \\
0 \\
2 \\
0 \\
0 \\
0\end{array}$ \\
\hline
\end{tabular}

\section{WELL BGO 3D}

\begin{tabular}{|c|c|c|c|c|c|c|}
\hline SRS Coord. & Lat/Longitude & Screen Zone Elevation & Top of Casing & Casing & Pump & Formation \\
\hline $\begin{array}{l}N 75351.3 \\
\text { E58809.2 }\end{array}$ & $\begin{array}{l}33.288382^{\circ} \mathrm{N} \\
81.658312^{\circ} \mathrm{W}\end{array}$ & $247.6-227.6 \mathrm{ft} \mathrm{msl}$ & $292.7 \mathrm{ft} \mathrm{msl}$ & 4" PVC & $\mathbf{S}$ & Water Table (IIB ${ }_{2}$ ) \\
\hline
\end{tabular}

FIELD MEASUREMENTS

Sample date: 04/19/94

Depth to water: $57.65 \mathrm{ft}(17.57 \mathrm{~m})$ below TOC

Water elevation: $235.05 \mathrm{ft}(71.64 \mathrm{~m}) \mathrm{msl}$

Sp. conductance: $57 \mu \mathrm{S} / \mathrm{cm}$

Turbidity: 23.0 NTU

Water evacuated before sampling: 2 gal

The well went dry during purging.

\section{LABORATORY ANALYSES}

\section{H D Analyte}

$\mathrm{pH}$

Specific conductance

Turbidity

Acetophenone

Aldrin

Aluminum, total recoverable

Aluminum, total recoverable

Antimony, total recoverable

Arsenic, total recoverable

Barium, total recoverable

Barium, total recoverable

Benzene

Bromodichloromethane

Bromoform

Bromomethane (Methyl bromide)

Cadmium, total recoverable

Cadmium, total recoverable

Calcium, total recoverable

Calcium, total recoverable

Carbon tetrachloride

Chloride

Chlorobenzene

Chloroethane

Chloroethene (Vinyl chloride)

2-Chloroethyl vinyl ether

Chloroform
Time: $8: 50$

$\mathrm{pH}: 4.4$

Alkalinity: $0 \mathrm{mg} / \mathrm{L}$

Water temperature: $16.6^{\circ} \mathrm{C}$

Volumes purged: 0.4 well volumes

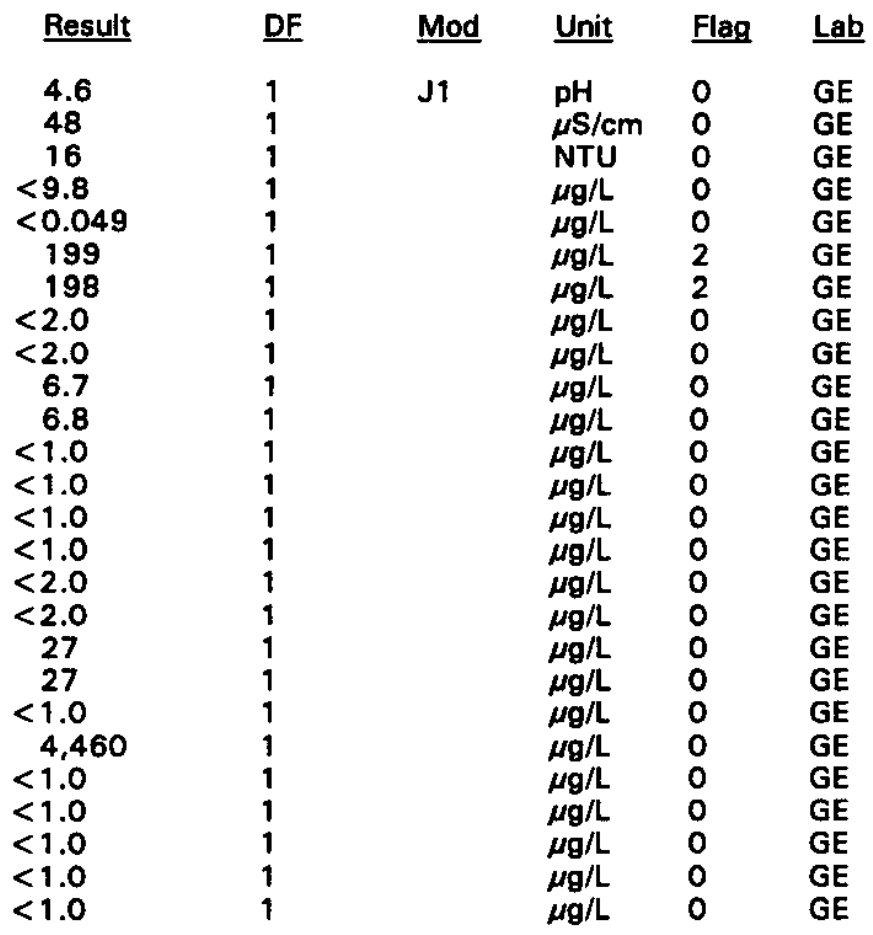

- = exceeded holding time. $=$ exceeded screening level or final primary drinking water standard. 
WELL BGO 3D collected on 04/19/94, laboratory analyses (cont.)

$\underline{H}$ D Analyte

Chloromethane (Methyl chloride)

Chromium, total recoverable

Chromium, total recoverable

Copper, total recoverable

Copper, total recoverable

Cyanide

p,p'-DDT

Dibromochloromethane

1,1-Dichloroethane

1,2-Dichloroethane

1,1-Dichloroethylene

trans-1,2-Dichloroethylene

Dichloromethane (Methylene chloride)

2,4-Dichlorophenoxyacetic acid

1,2-Dichloropropane

cis-1,3-Dichloropropene

trans-1,3-Dichloropropene

Dieldrin

Endrin

Ethylbenzene

Fluoride

Heptachlor

Iron, total recoverable

Iron, total recoverable

Lead, total recoverable

Lindane

Magnesium, total recoverable

Magnesium, total recoverable

Manganese, total recoverable

Manganese, total recoverable

Mercury, total recoverable

Methoxychlor

Naphthalene

Nickel, total recoverable

Nickel, total recoverable

Nitrate-nitrite as nitrogen

Phenols

Potassium, total recoverable

Potassium, total recoverable

Selenium, total recoverable

Silica, total recoverable

Silica, total recoverable

Silver, total recoverable

Silver, total recoverable

Sodium, total recoverable

Sodium, total recoverable

Sulfate

1,1,2,2-Tetrachloroethane

Tetrachloroethylene

Tin, total recoverable

Tin, total recoverable

Toluene

Total dissolved solids

Total organic carbon

Total organic carbon

Total organic halogens

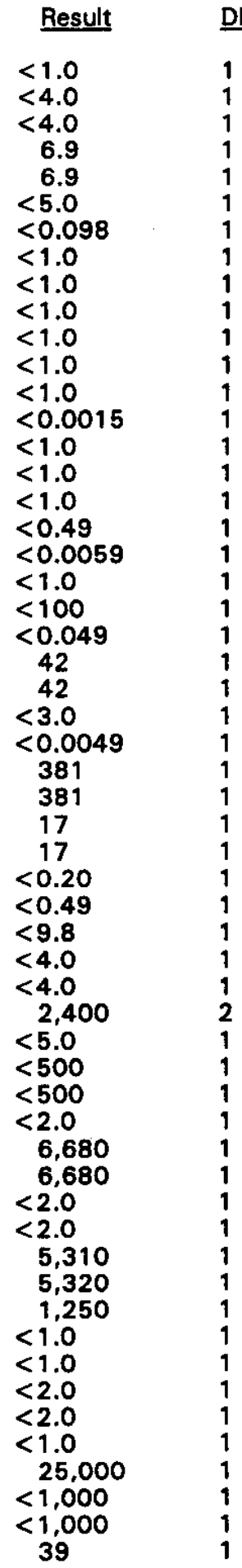

Mod Unit Flag Lab

$\mu g / L \quad 0 \quad$ GE

$\mu \mathrm{g} / \mathrm{L} \quad \mathrm{O} \quad \mathrm{GE}$

$\mu g / L \quad 0 \quad G E$

$\mu g / L \quad 0 \quad$ GE

$\mu g / L \quad 0 \quad G E$

$\mu g / L \quad 0$

$\mu g / L \quad 0 \quad G E$

$\mu g / L \quad 0$

$\mu g / L \quad 0 \quad G E$

$\mu g / L \quad 0$

$\mu g / L \quad 0 \quad$ GE

$\mu g / L \quad 0 \quad$ GE

$\mu g / L \quad 0 \quad \mathrm{GE}$

$\mu g / L \quad 0 \quad \mathrm{GE}$

$\mu \mathrm{g} / \mathrm{L} \quad 0 \quad \mathrm{GE}$

$\mu g / L \quad 0 \quad \mathrm{GE}$

$\mu g / \mathrm{L} \quad 0 \quad \mathrm{GE}$

$\mu g / L \quad 0 \quad$ GE

$\mu g / L \quad 0 \quad$ GE

$\mu g / L \quad 0 \quad G E$

$\mu g / L \quad 0 \quad$ GE

$\mu g / L \quad 0 \quad$ GE

$\mu \mathrm{g} / \mathrm{L} \quad 0 \quad \mathrm{GE}$

$\mu g / L \quad 0 \quad$ GE

$\mu \mathrm{g} / \mathrm{L} \quad \mathrm{O}$ GE

$\mu g / L \quad 0 \quad$ GE

$\mu \mathrm{g} / \mathrm{L} \quad 0 \quad \mathrm{GE}$

$\mu g / L \quad 0 \quad$ GE

$\mu \mathrm{g} / \mathrm{L} \quad \mathrm{O} \quad \mathrm{GE}$

$\mu g / L \quad 0 \quad$ GE

$\mu \mathrm{g} / \mathrm{L} \quad 0 \quad \mathrm{GE}$

$\mu \mathrm{g} / \mathrm{L} \quad 0 \quad \mathrm{GE}$

$\mu g / L \quad O \quad G E$

$\mu g / L \quad 0 \quad G E$

$\mu g / L \quad 0 \quad$ GE

$\mu g / L \quad 0 \quad G E$

$\mu \mathrm{g} / \mathrm{L} \quad 0 \quad \mathrm{GE}$

$\mu \mathrm{g} / \mathrm{L} \quad 0 \quad \mathrm{GE}$

$\mu \mathrm{g} / \mathrm{L} \quad 0 \quad \mathrm{GE}$

$\mu \mathrm{g} / \mathrm{L} \quad 0 \quad \mathrm{GE}$

$\mu g / L \quad 0 \quad G E$

$\mu g / L \quad 0 \quad G E$

$\mu g / L \quad 0 \quad G E$

$\mu g / L \quad 0 \quad G E$

$\mu g / L \quad 0 \quad G E$

$\mu g / L \quad 0 \quad G E$

$\mu g / L \quad 0 \quad$ GE

$\mu g / L \quad 0 \quad G E$

$\mu g / L \quad 0 \quad G E$

$\mu g / L \quad 0 \quad G E$

$\mu \mathrm{g} / \mathrm{L} \quad 0 \quad \mathrm{GE}$

$\mu g / L \quad 0 \quad G E$

$\mu g / L \quad 0 \quad G E$

$\mu g / L \quad 0 \quad$ GE

$\mu g / L \quad 0 \quad G E$

$\mu g / L \quad 1 \quad G E$

- = exceeded holding time. = exceeded screening level or final primary drinking water standard. 
WELL BGO 3D collected on 04/19/94, laboratory analyses (cont.)

H D $\quad$ Analyte
Total phosphates (as P)
Toxaphene
$2,4,5-T P$ (Silvex)
$1,1,1-T$ Trichloroethane
$1,1,2-$ Trichloroethane
Trichloroethylene
Trichlorofluoromethane
2,4,5-T
Vanadium, total recoverable
Vanadium, total recoverable
Xylenes
Zinc, total recoverable
Zinc, total recoverable
Carbon-14
Gross alpha
Nonvolatile beta
Radium, total alpha-emitting
Tritium
Tritium
Uranium-233/234
Uranium-235
Uranium-238

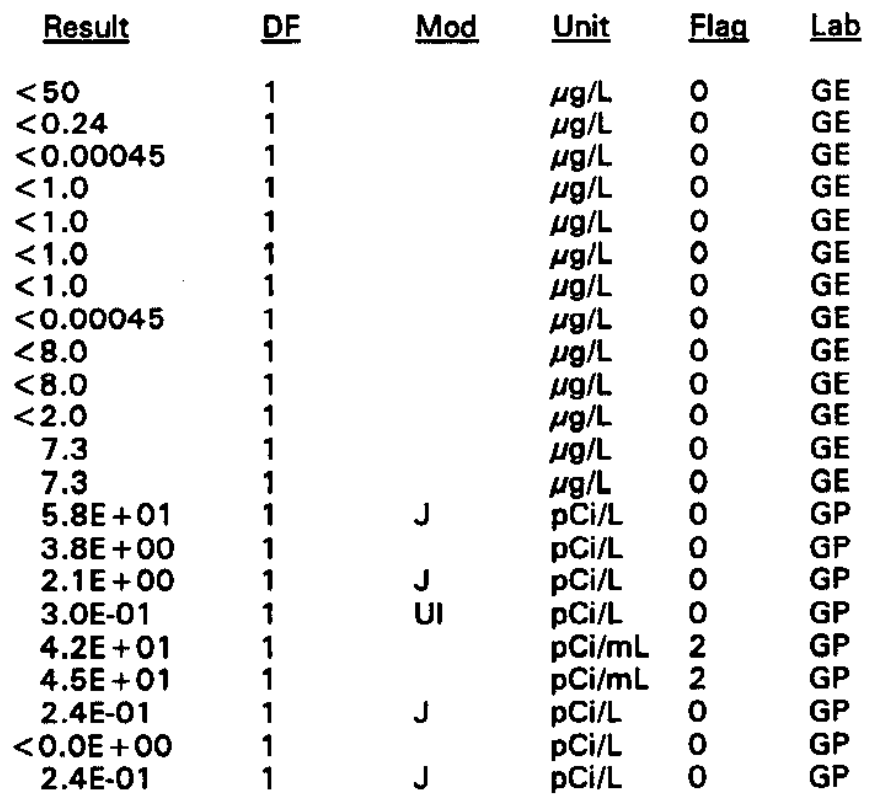

\section{WELL BGO 4D}

\begin{tabular}{|c|c|c|c|c|c|c|}
\hline SRS Coord. & Lat/Longitude & Screen Zone Elevation & Top of Casing & Casing & Pump & Formation \\
\hline $\begin{array}{l}N 76150.1 \\
E 58803.7\end{array}$ & $\begin{array}{l}33.290140^{\circ} \mathrm{N} \\
81.659878^{\circ} \mathrm{W}\end{array}$ & $240.6-220.6 \mathrm{ft} \mathrm{msl}$ & $297.5 \mathrm{ft} \mathrm{msl}$ & 4" PVC & & Water Table $\left(\mathrm{IIB}_{2}\right)$ \\
\hline
\end{tabular}

\section{FIELD MEASUREMENTS}

Sample date: $04 / 19 / 94$

Time: 16:00

No water evacuated before sampling.

Inaccessibility or pump failure prevented sample collection.

- = exceeded holding time. = exceeded screening level or final primary drinking water standard. 


\section{WELL BGO 5C}

$\begin{array}{lllllll}\text { SRS Coord. } & \text { Lat/Longitude } & \text { Screen Zone Elovation } & \text { Top of Casing } & \text { Casing } & \text { Pump Formation } & \text { Sum } \\ \text { N76476.9 } & 33.290848^{\circ} \mathrm{N} & 193.2-183.2 \mathrm{ft} \mathrm{msl} & 296.1 \mathrm{ft} \mathrm{msl} & 4^{\prime \prime} \text { PVC } & \text { S } & \text { McBean (IIB,) } \\ \text { E58794.5 } & 81.660537^{\circ} \mathrm{W} & & & \end{array}$

\section{FIELD MEASUREMENTS}

Sample date: 04/19/94

Depth to water: $80.47 \mathrm{ft}(24.53 \mathrm{~m})$ below TOC

Water elevation: $215.63 \mathrm{ft}(65.72 \mathrm{~m}) \mathrm{msl}$

Sp. conductance: $70 \mu \mathrm{S} / \mathrm{cm}$

Turbidity: 20.2 NTU

Water evacuated before sampling: 20 gal

The well went dry during purging.

\section{LABORATORY ANALYSES}

\section{H D Analyte}

- $\quad$ pH

Specific conductance

Specific conductance

Turbidity

Acetophenone

Aldrin

Aluminum, total recoverable

Antimony, total recoverable

Arsenic, total recoverable

Barium, total recoverable

Benzene

Bromodichloromethane

Bromoform

Bromomethane (Methyl bromide)

Cadmium, total recoverable

Calcium, total recoverable

Carbon tetrachloride

Chloride

Chlorobenzene

Chloroethane

Chloroethene (Vinyl chloride)

2-Chloroethyl vinyl ether

Chioroform

Chloromethane (Methyl chloride)

Chromium, total recoverable

Copper, total recoverable

Cyanide

$p, p^{\prime}-D D T$

Dibromochloromethane

1,1-Dichloroethane

1,2-Dichloroethane

1,1-Dichloroethylene

trans-1,2-Dichloroethylene

Dichloromethane (Methylene chloride)

2,4-Dichlorophenoxyacetic acid

1,2-Dichloropropane

cis-1,3-Dichloropropene

trans-1,3-Dichloropropene

Dieldrin

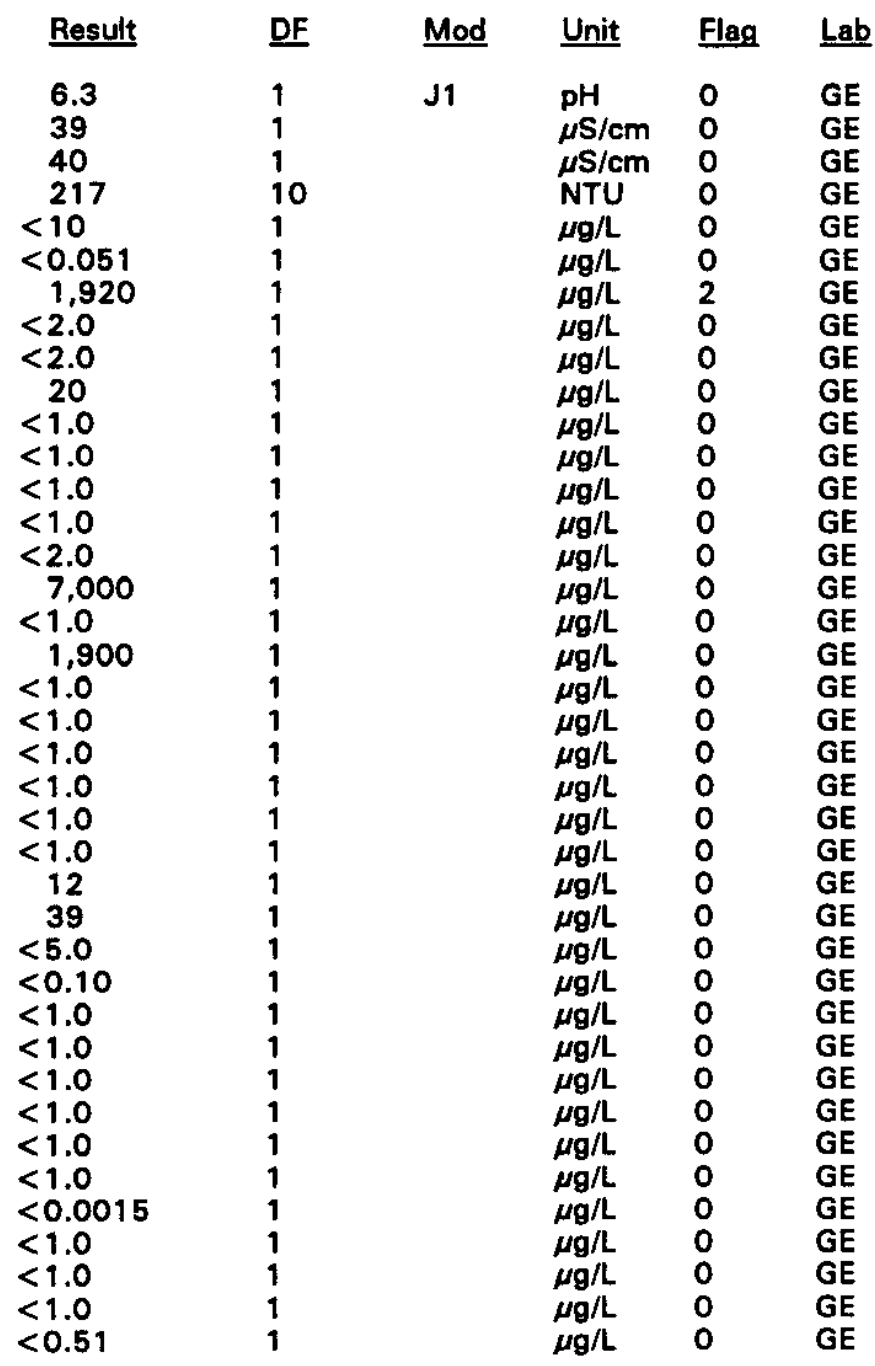

Time: 9:35

$\mathrm{pH}: 6.4$

Alkalinity: $12 \mathrm{mg} / \mathrm{L}$

Water temperature: $19.9^{\circ} \mathrm{C}$

Volumes purged: 0.9 well volumes 
WELL BGO $5 \mathrm{C}$ collected on $04 / 19 / 94$, laboratory analyses (cont.)

H $\underline{\text { Analyte }}$

Endrin

Ethylbenzene

Fluoride

Heptachlor

Iron, total recoverable

Lead, total recoverable

Lindane

Magnesium, total recoverable

Manganese, total recoverable

Mercury, total recoverable

Methoxychlor

Naphthalene

Nickel, total recoverable

Nitrate-nitrite as nitrogen

Phenols

Potassium, total recoverable

Selenium, total recoverable

Silica, total recoverable

Silver, total recoverable

Sodium, total recoverable

Sulfate

1, 1,2,2-Tetrachloroethane

Tetrachloroethylene

Tin, total recoverable

Toluene

Total dissolved solids

Total organic carbon

Total organic halogens

Total phosphates (as P)

Toxaphene

2,4,5-TP (Silvex)

$1,1,1$-Trichloroethane

1,1,2-Trichloroethane

Trichloroethylene

Trichlorofluoromethane

2,4,5-T

Vanadium, total recoverable

Xylenes

Zinc, total recoverable

Carbon-14

Gross alpha

Nonvolatile beta

Radium, total alpha-emitting

- Tritium

Uranium-233/234

Uranium-235

Uranium-238

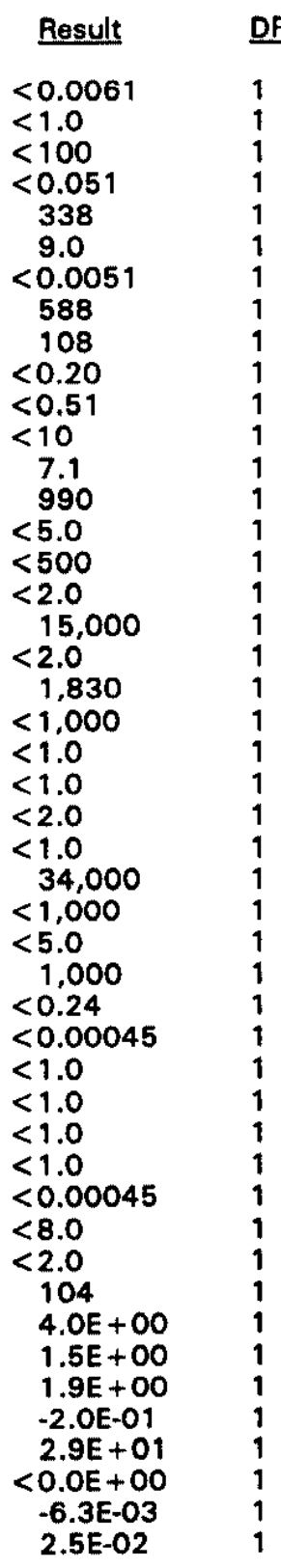

DF

Mod

Unit Flag Lab

$\mu g / L$

$\mu \mathrm{g} / \mathrm{L}$

$\mu g / L$

$\mu g / L$

$\mu g / L$

$\mu \mathrm{g} / \mathrm{L}$

$\mu g / L$

$\mu g / L$

$\mu \mathrm{g} / L$

$\mu \mathrm{g} / \mathrm{L}$

$\mu g / L$

$\mu g / L$

$\mu \mathrm{g} / \mathrm{L}$

$\mu g / L$

$\mu g / L$

$\mu g / L$

$\mu g / L$

$\mu g / L$

$\mu g / L$

$\mu g / L$

$\mu g / L$

$\mu g / L$

$\mu g / L$

$\mu g / L$

$\mu g / L$

$\mu g / L$

$\mu g / L$

$\mu \mathrm{g} / \mathrm{L}$

$\mu g / L$

$\mu g / L$

$\mu g / L$

$\mu g / L$

$\mu g / L$

$\mu \mathrm{g} / \mathrm{L}$

$\mu g / L$

$\mu g / L$

$\mu g / L$

$\mu \mathrm{g} / \mathrm{L}$

$\mu g / L$

UI $\quad \mathrm{pCi} / \mathrm{L}$

J $\mathrm{pCi} / \mathrm{L}$

UI

pCi/L

$\mathrm{pCi} / \mathrm{mL}$

$\mathrm{pCi} / \mathrm{L}$

U1

pCi/L

pCi/L
O GE

GE

GE

GE

GE

GE

GE

GE

GE

GE

GE

GE

GE

GE

GE

GE

GE

GE

GE

GE

GE

GE

GE

GE

GE

GE

GE

GE

GE

GE

GE

GE

GE

GE

GE

GE

GE

GE

GP

GP

GP

GP

GP

GP

GP

- = exceeded holding time. = exceeded screening level or final primary drinking water standard. 
WELL BGO 5D

\begin{tabular}{|c|c|c|c|c|c|c|}
\hline SRS Coord. & Lat/Longitude & Screen Zone Elevation & Top of Casing & Casing & Pump & Formation \\
\hline $\begin{array}{l}\text { N76477.5 } \\
\text { E58784.8 }\end{array}$ & $\begin{array}{l}33.290833^{\circ} \mathrm{N} \\
81.660564^{\circ} \mathrm{W}\end{array}$ & $239.3-219.3 \mathrm{ft} \mathrm{msl}$ & $296.3 \mathrm{ft} \mathrm{msl}$ & 4" PVC & $\mathbf{S}$ & Water Table $\left(\mathrm{IIB}_{2}\right)$ \\
\hline
\end{tabular}

FIELD MEASUREMENTS

Sample date: $04 / 19 / 94$

Depth to water: $65.64 \mathrm{ft}(20.01 \mathrm{~m})$ below TOC

Water elevation: $230.66 \mathrm{ft}(70.31 \mathrm{~m}) \mathrm{ms}$ l

Sp. conductance: $49 \mu \mathrm{S} / \mathrm{cm}$

Turbidity: 4.3 NTU

Water evacuated before sampling: $4 \mathrm{gal}$

The well went dry during purging.

Time: $9: 17$

pH: 4.8

Alkalinity: $0 \mathrm{mg} / \mathrm{L}$

Water temperature: $17.8^{\circ} \mathrm{C}$

Volumes purged: 0.5 well volumes

\section{LABORATORY ANALYSES}

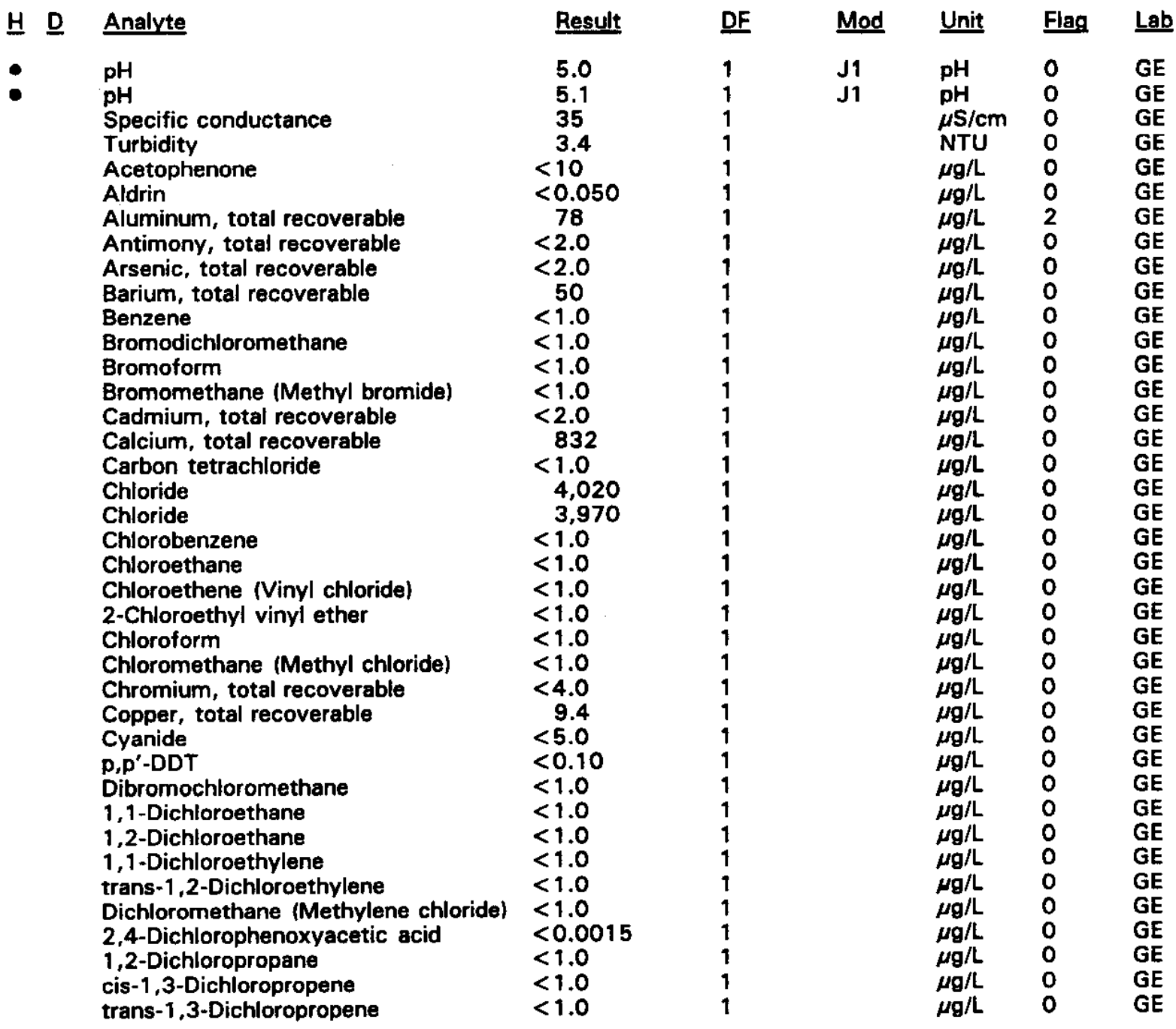

- =xceeded holding time. $\quad=$ exceeded screening level or final primary drinking water standard. 
WELL BGO 5D collected on 04/19/94, laboratory analyses (cont.)

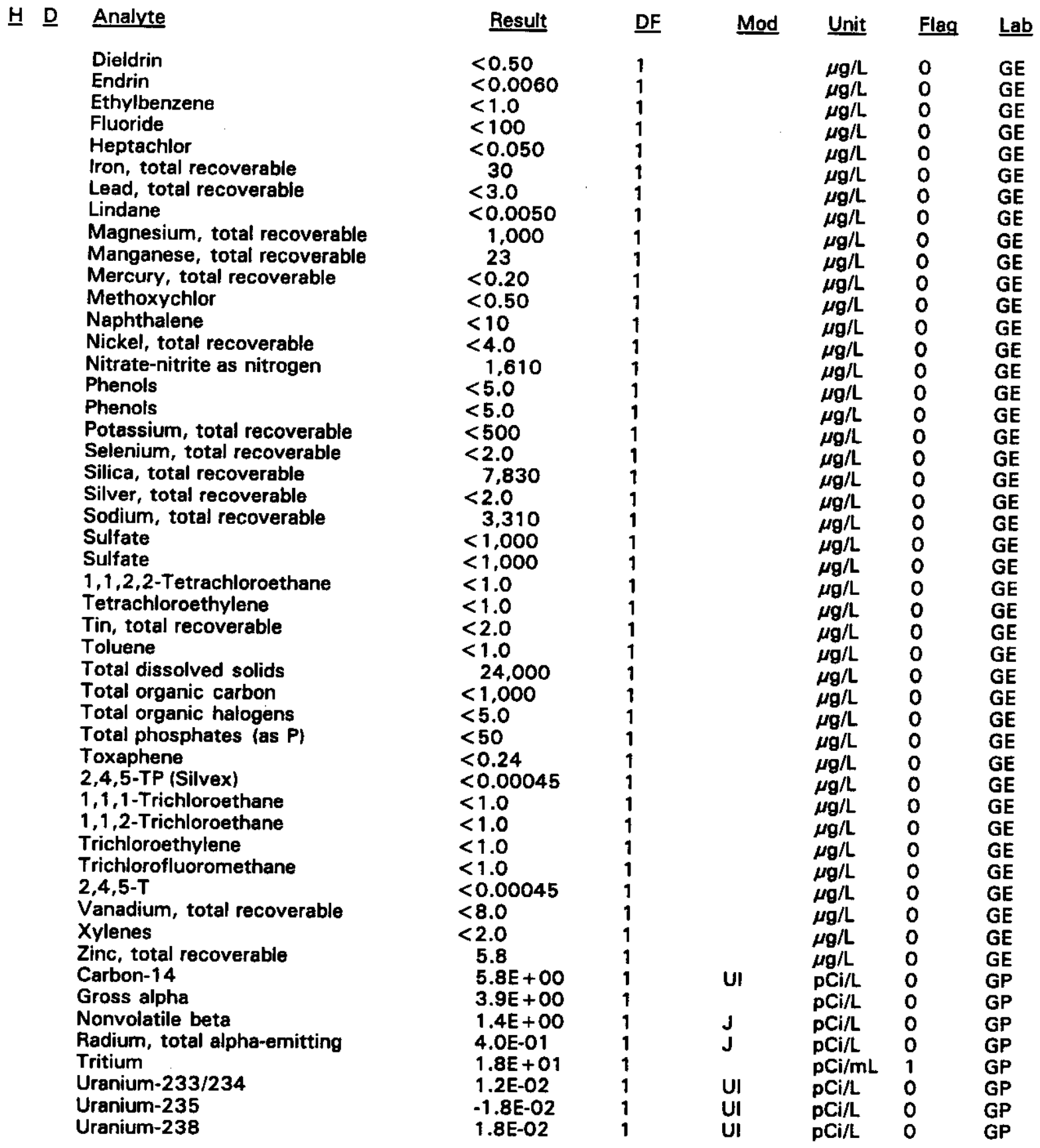

- = exceeded holding time. - exceeded screening level or final primary drinking water standard. 
WELL BGO 6A

$\begin{array}{lllllll}\text { SRS Coord. } & \text { Lat/Longitude } & \text { Screen Zone Elevation } & \text { Top of Casing } & \text { Casing } & \text { Pump } & \text { Formation } \\ \text { N76487.2 } & 33.290091^{\circ} \mathrm{N} & 117.5-107.5 \mathrm{ft} \mathrm{msl} & 285.6 \mathrm{ft} \mathrm{msl} & \text { 4" PVC } & \text { S } & \text { U. Congaree (IIA) } \\ \text { E58316.8 } & 81.661815^{\circ} \mathrm{W} & & & & \end{array}$

\section{FIELD MEASUREMENTS}

Sample date: 04/04/94

Depth to water: $126.34 \mathrm{ft}(38.51 \mathrm{~m})$ below TOC

Water elevation: $159.26 \mathrm{ft}(48.54 \mathrm{~m}) \mathrm{msl}$

Sp. conductance: $314 \mu \mathrm{S} / \mathrm{cm}$

Turbidity: 0.6 NTU

Water evacuated before sampling: $105 \mathrm{gal}$

Time: 13:02

pH: 7.8

Alkalinity: $127 \mathrm{mg} / \mathrm{L}$

Water temperature: $19.9^{\circ} \mathrm{C}$

Volumes purged: 3.1 well volumes

\section{LABORATORY ANALYSES}

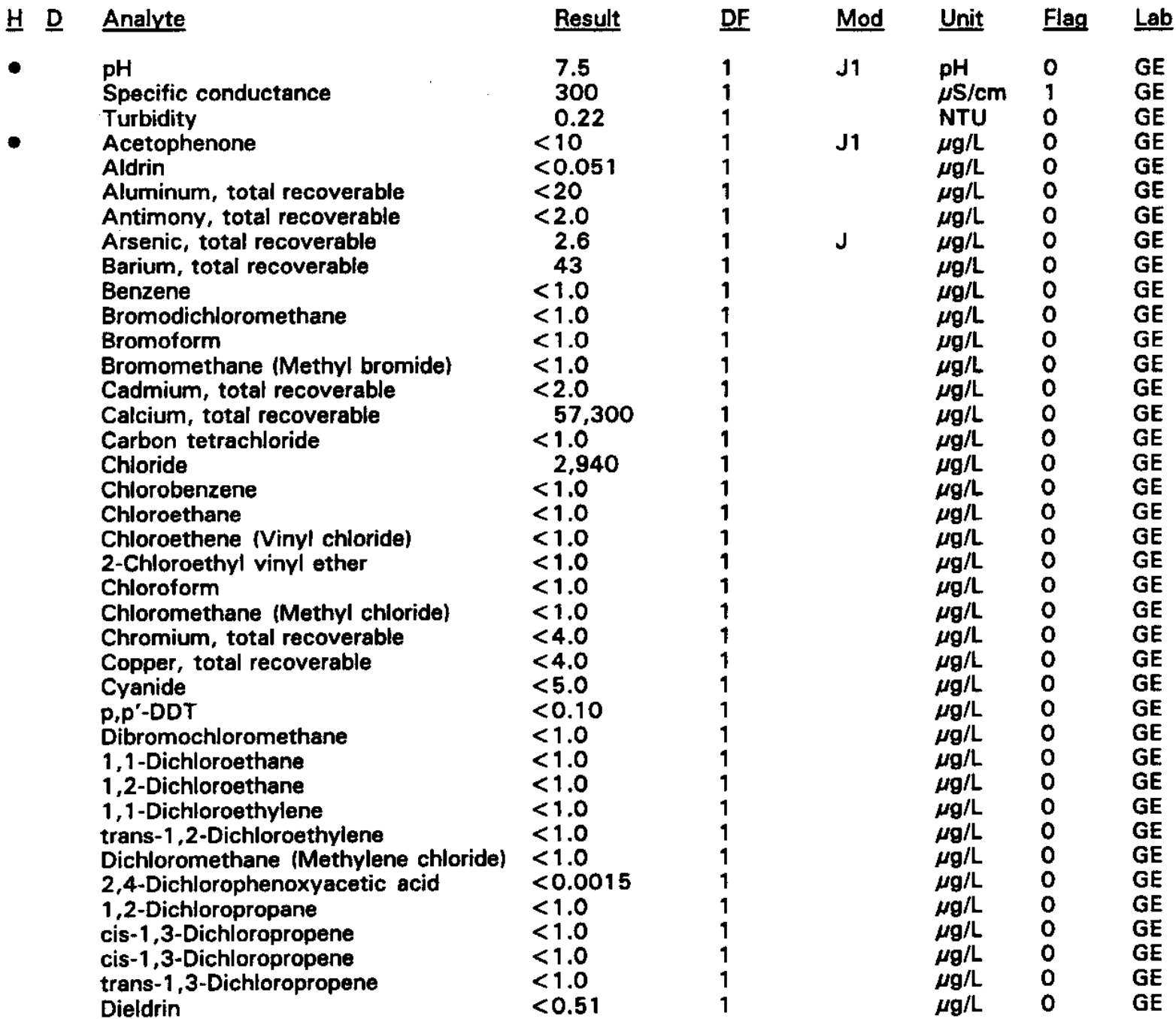

$\overline{0}$ = exceeded holding time. $\square=$ exceeded screening level or final primary drinking water standard. 
WELL BGO 6A collected on 04/04/94, laboratory analyses (cont.)

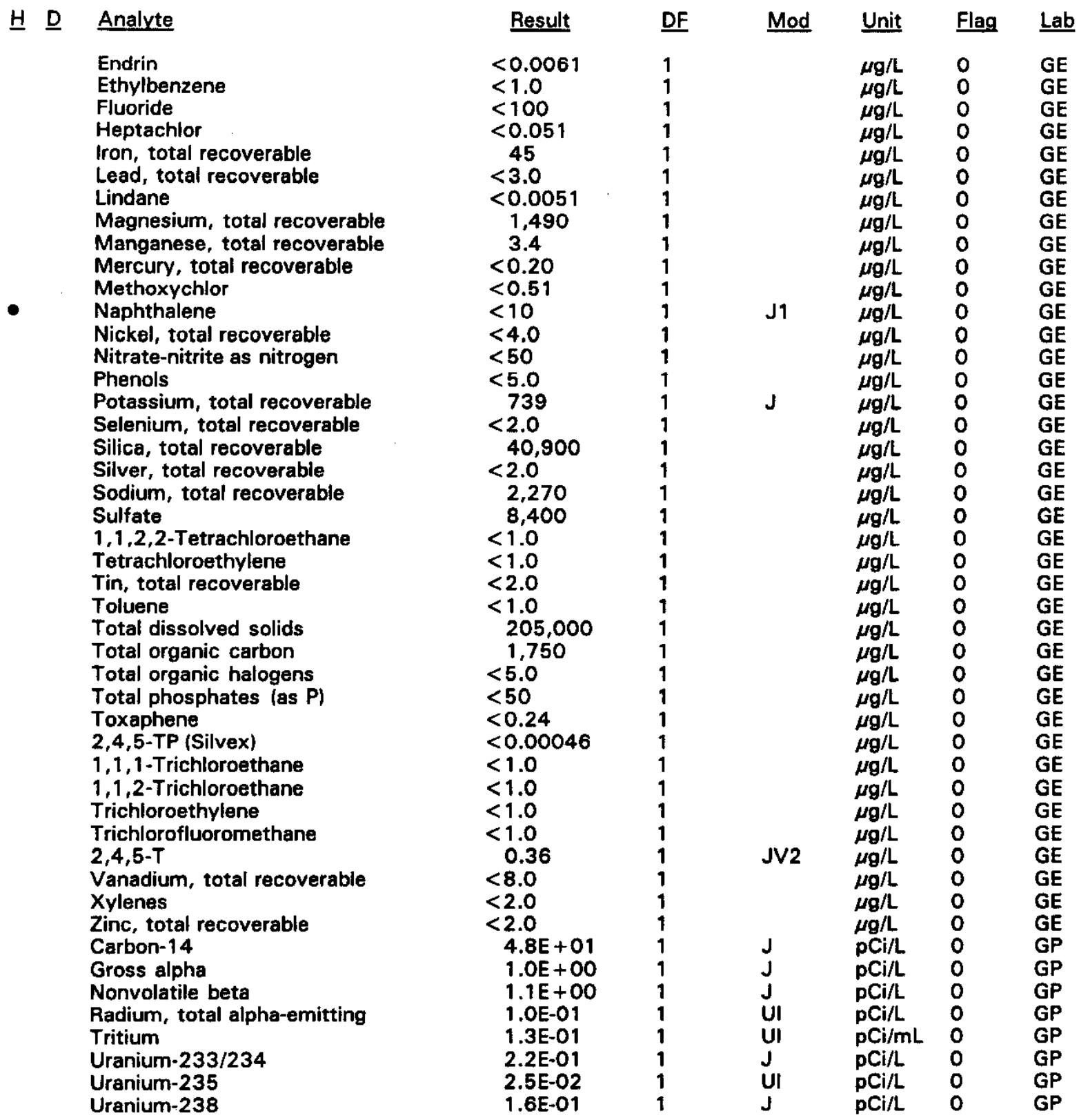

- = exceeded holding time. $\quad$ = exceeded screening level or final primary drinking water standard. 
WELL BGO 6B

\begin{tabular}{|c|c|c|c|c|c|c|}
\hline SRS Coord. & Lat/Longitude & Screen Zone Elevation & Top of Casing & Casing & Pump & Formation \\
\hline $\begin{array}{l}N 76553.2 \\
\text { E58346.5 }\end{array}$ & $\begin{array}{l}33.290286^{\circ} \mathrm{N} \\
81.661865^{\circ} \mathrm{W}\end{array}$ & $149.7-139.7 \mathrm{ft} \mathrm{msl}$ & $286.8 \mathrm{ft} \mathrm{msl}$ & 4" PVC & $\mathbf{S}$ & M. B/McB \\
\hline
\end{tabular}

\section{FIELD MEASUREMENTS}

Sample date: 04/05/94

Depth to water: $68.47 \mathrm{ft}(20.87 \mathrm{~m})$ below TOC

Water elevation: $218.33 \mathrm{ft}(66.55 \mathrm{~m}) \mathrm{ms}$

Sp. conductance: $210 \mu \mathrm{S} / \mathrm{cm}$

Turbidity: 2.4 NTU

Water evacuated before sampling: 42 gal

The well went dry during purging.

\section{LABORATORY ANALYSES}

H D Analyte

$\mathrm{pH}$

Specific conductance

Turbidity

Acetophenone

Aldrin

Aluminum, total recoverable

Antimony, total recoverable

Antimony, total recoverable

Arsenic, total recoverable

Arsenic, total recoverable

Barium, total recoverable

Benzene

Bromodichloromethane

Bromoform

Bromomethane (Methyl bromide)

Cadmium, total recoverable

Calcium, total recoverable

Carbon tetrachloride

Chloride

Chlorobenzene

Chloroethane

Chloroethene (Vinyl chloride)

2-Chloroethyl vinyl ether

Chloroform

Chloromethane (Methyl chloride)

Chromium, total recoverable

Copper, total recoverable

Cyanide

p,p'-DDT

Dibromochloromethane

1,1-Dichloroethane

1,2-Dichloroethane

1,1-Dichloroethylene

trans-1,2-Dichloroethylene

Dichloromethane (Methylene chloride)

2,4-Dichlorophenoxyacetic acid

1,2-Dichloropropane

cis-1,3-Dichloropropene

cis-1,3-Dichloropropene

\begin{tabular}{|c|c|c|c|c|}
\hline Result & DF & Mod & Unit & Flag \\
\hline $\begin{aligned} & 8.5 \\
& 193 \\
& 0.60 \\
&<10 \\
&<0.053 \\
&<20 \\
&<2.0 \\
&<2.0 \\
&<2.0 \\
&<2.0 \\
& 33 \\
&<1.0 \\
&<1.0 \\
&<1.0 \\
&<1.0 \\
&<2.0 \\
& 32.800 \\
&<1.0 \\
& 2,490 \\
&<1.0 \\
&<1.0 \\
&<1.0 \\
&<1.0 \\
&<1.0 \\
&<1.0 \\
&<4.0 \\
& 4.2 \\
&<5.0 \\
&<0.11 \\
&<1.0 \\
&<1.0 \\
&<1.0 \\
&<1.0 \\
&<1.0 \\
&<1.0 \\
&<0.0016 \\
&<1.0 \\
&<1.0 \\
&<\end{aligned}$ & $\begin{array}{l}1 \\
1 \\
1 \\
1 \\
1 \\
1 \\
1 \\
1 \\
1 \\
1 \\
1 \\
1 \\
1 \\
1 \\
1 \\
1 \\
1 \\
1 \\
1 \\
1 \\
1 \\
1 \\
1 \\
1 \\
1 \\
1 \\
1 \\
1 \\
1 \\
1 \\
1 \\
1 \\
1 \\
1 \\
1 \\
1 \\
1 \\
1 \\
1\end{array}$ & J1 & $\begin{array}{l}p H \\
\mu S / \mathrm{l} \\
N{ }^{2} \\
\mu g / L \\
\mu g / L \\
\mu g / L \\
\mu g / L \\
\mu g / L \\
\mu g / L \\
\mu g / L \\
\mu g / L \\
\mu g / L \\
\mu g / L \\
\mu g / L \\
\mu g / L \\
\mu g / L \\
\mu g / L \\
\mu g / L \\
\mu g / L \\
\mu g / L \\
\mu g / L\end{array}$ & $\begin{array}{l}1 \\
0 \\
0 \\
0 \\
0 \\
0 \\
0 \\
0 \\
0 \\
0 \\
0 \\
0 \\
0 \\
0 \\
0 \\
0 \\
0 \\
0 \\
0 \\
0 \\
0 \\
0 \\
0 \\
0 \\
0 \\
0 \\
0 \\
0 \\
0 \\
0 \\
0 \\
0 \\
0 \\
0 \\
0 \\
0 \\
0 \\
0 \\
0\end{array}$ \\
\hline
\end{tabular}

Time: 9:29

pH: 8.6

Alkalinity: $73 \mathrm{mg} / \mathrm{L}$

Water temperature: $18.3^{\circ} \mathrm{C}$

Volumes purged: 0.8 well volumes 
WELL BGO 6B collected on 04/05/94, laboratory analyses (cont.)

\section{H $\underline{\text { Analyte }}$}

trans-1,3-Dichloropropene

Dieldrin

Endrin

Ethylbenzene

Fluoride

Heptachlor

Iron, total recoverable

Lead, total recoverable

Lead, total recoverable

Lindane

Magnesium, total recoverable

Manganese, total recoverable

Mercury, total recoverable

Methoxychlor

Naphthalene

Nickel, total recoverable

Nitrate-nitrite as nitrogen

Phenols

Potassium, total recoverable

Selenium, total recoverable

Selenium, total recoverable

Silica, total recoverable

Silver, total recoverable

Sodium, total recoverable

Sulfate

1,1,2,2-Tetrachloroethane

Tetrachloroethylene

Tin, total recoverable

Toluene

Total dissolved solids

Total organic carbon

Total organic halogens

Total phosphates (as P)

Toxaphene

2,4,5-TP (Silvex)

$1,1,1$-Trichloroethane

1,1,2-Trichloroethane

Trichloroethylene

Trichlorofluoromethane

2,4,5-T

Vanadium, total recoverable

Xylenes

Gross alpha

Nonvolatile beta

Radium, total alpha-emitting

- Tritium

Uranium-233/234

Uranium-235

Uranium-238

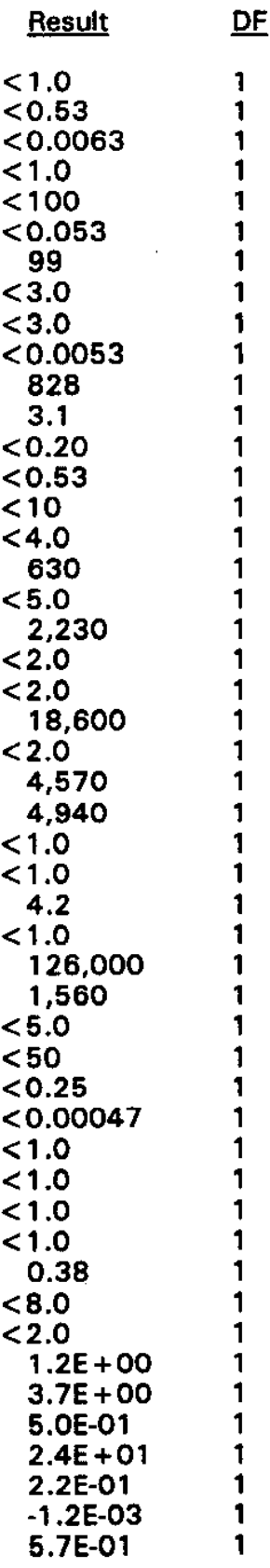

Mod Unit Flag Lab

$\mu \mathrm{g} / \mathrm{L} \quad \mathrm{O} \quad \mathrm{GE}$

$\mu \mathrm{g} / \mathrm{L} \quad \mathrm{O} \quad \mathrm{GE}$

$\mu \mathrm{g} / \mathrm{L} \quad \mathrm{O} \quad \mathrm{GE}$

$\mu g / L \quad 0 \quad G E$

$\mu \mathrm{g} / \mathrm{L} \quad \mathrm{O} \quad \mathrm{GE}$

$\mu \mathrm{g} / \mathrm{L} \quad 0 \quad \mathrm{GE}$

$\mu \mathrm{g} / \mathrm{L} \quad 0 \quad \mathrm{GE}$

$\mu \mathrm{g} / \mathrm{L} \quad \mathrm{O} \quad \mathrm{GE}$

$\mu g / L \quad 0 \quad G E$

$\mu g / L \quad 0 \quad$ GE

$\mu g / L \quad 0 \quad G E$

J $\mu g / L \quad 0 \quad$ GE

$\mu \mathrm{g} / \mathrm{L} \quad 0 \quad \mathrm{GE}$

$\mu g / L \quad 0 \quad$ GE

$\mu g / L \quad 0 \quad$ GE

$\mu \mathrm{g} / \mathrm{L} \quad \mathrm{O} \quad \mathrm{GE}$

$\mu \mathrm{g} / \mathrm{L} \quad \mathrm{O} \quad \mathrm{GE}$

$\mu g / L \quad 0 \quad$ GE

$\mu \mathrm{g} / \mathrm{L} \quad 0 \quad \mathrm{GE}$

J3 $\mu g / L \quad 0 \quad$ GE

$\mu g / L \quad 0 \quad G E$

$\mu g / L \quad 0 \quad G E$

$\mu \mathrm{g} / \mathrm{L} \quad 0$

$\mu g / L \quad 0 \quad$ GE

$\mu \mathrm{g} / \mathrm{L} \quad 0 \quad \mathrm{GE}$

$\mu \mathrm{g} / \mathrm{L} \quad 0 \quad \mathrm{GE}$

$\mu \mathrm{g} / \mathrm{L} \quad \mathrm{O} \quad \mathrm{GE}$

$\mu \mathrm{g} / \mathrm{L} \quad 0 \quad \mathrm{GE}$

$\mu \mathrm{g} / \mathrm{L} \quad 0 \quad \mathrm{GE}$

JV2 $\mu g / L \quad O \quad$ GE

J $\mu g / L \quad 0 \quad$ GE

$\mu g / L \quad 0 \quad$ GE

$\mu g / L \quad 0 \quad G E$

$\mu g / L \quad 0 \quad$ GE

$\mu g / L \quad 0 \quad$ GE

$\mu g / L \quad 0 \quad$ GE

$\mu g / L \quad 0 \quad$ GE

$\mu \mathrm{g} / \mathrm{L} \quad 0 \quad \mathrm{GE}$

$\mu g / L \quad 0 \quad$ GE

JV2 $\mu g / L \quad 0 \quad$ GE

$\mu g / L \quad 0 \quad$ GE

$\mu g / L \quad 0 \quad$ GE

$\mathrm{pCi} / \mathrm{L} \quad 0 \quad \mathrm{GP}$

PCi/L $0 \quad$ GP

$\mathrm{pCi} / \mathrm{L} \quad 0 \quad \mathrm{GP}$

$\mathrm{pCi} / \mathrm{mL} 2 \quad \mathrm{GP}$

$\mathrm{pCi} / \mathrm{L} \quad 0 \quad \mathrm{GP}$

PCi/L 0 GP

$\mathrm{pCi} / \mathrm{L} \quad 0 \quad$ GP

$\overline{- \text { = exceeded holding time. }}$ = exceeded screening level or final primary drinking water standard. 


\section{WELL BGO 6C}

\begin{tabular}{|c|c|c|c|c|c|c|}
\hline SRS Coord. & Lat/Longitude & Screen Zone Elevation & Top of Casing & Casing & Pump & Formation \\
\hline $\begin{array}{l}\text { N76487.1 } \\
\text { E58307.0 }\end{array}$ & $\begin{array}{l}33.290075^{\circ} \mathrm{N} \\
81.661841^{\circ} \mathrm{W}\end{array}$ & $168.0-158.0 \mathrm{ft} \mathrm{msl}$ & $285.6 \mathrm{ft} \mathrm{msl}$ & 4" PVC & $\mathbf{S}$ & McBean (IIB $)_{1}$ \\
\hline
\end{tabular}

\section{FIELD MEASUREMENTS}

Sample date: 04/04/94

Depth to water: $65.49 \mathrm{ft}(19.96 \mathrm{~m})$ below TOC

Water elevation: $220.11 \mathrm{ft}(67.09 \mathrm{~m}) \mathrm{msl}$

Sp. conductance: $149 \mu \mathrm{S} / \mathrm{cm}$

Turbidity: 0.1 NTU

Water evacuated before sampling: $115 \mathrm{gal}$

\section{LABORATORY ANALYSES}

H D Analyte

$\mathrm{pH}$

Specific conductance

Turbidity

Acetophenone

Aldrin

Aluminum, total recoverable

Antimony, total recoverable

Arsenic, total recoverable

Barium, total recoverable

Benzene

Benzene

Bromodichloromethane

Bromodichloromethane

Bromoform

Bromoform

Bromomethane (Methyl bromide)

Bromomethane (Methyl bromide)

Cadmium, total recoverable

Calcium, total recoverable

Carbon tetrachloride

Carbon tetrachloride

Chloride

Chlorobenzene

Chlorobenzene

Chloroethane

Chloroethane

Chloroethene (Vinyl chloride)

Chloroethene (Vinyl chloride)

2-Chloroethyl vinyl ether

2-Chloroethyl vinyl ether

2-Chloroethyl vinyl ether

Chloroform

Chloroform

Chloromethane (Methyl chloride)

Chloromethane (Methyl chloride)

Chromium, total recoverable

Copper, total recoverable

Cyanide

p.p'-DDT
Time: 13:28

pH: 7.7

Alkalinity: $52 \mathrm{mg} / \mathrm{L}$

Water temperature: $20.5^{\circ} \mathrm{C}$

Volumes purged: $\mathbf{2 . 8}$ well volumes

\begin{tabular}{|c|c|c|c|c|c|}
\hline Result & DF & Mod & Unit & Flag & Lal \\
\hline $\begin{array}{l}7.4 \\
131\end{array}$ & 1 & J1 & pH & 0 & GE \\
\hline $\begin{array}{r}137 \\
<0.10\end{array}$ & 1 & & $\begin{array}{l}\mu \mathrm{s} / \mathrm{cm} \\
\text { NTU }\end{array}$ & $\begin{array}{l}0 \\
0\end{array}$ & $\begin{array}{l}\mathrm{GE} \\
\mathrm{GE}\end{array}$ \\
\hline$<10$ & 1 & & $\mu g / L$ & 0 & $\mathrm{GE}$ \\
\hline$<0.051$ & 1 & & $\mu g / L$ & 0 & $\mathrm{GE}$ \\
\hline$<20$ & 1 & & $\mu g / L$ & 0 & GE \\
\hline$<2.0$ & 1 & & $\mu g / L$ & 0 & \\
\hline$<2.0$ & 1 & & $\mu \mathrm{g} / \mathrm{L}$ & 0 & GE \\
\hline 10 & 1 & & $\mu g / L$ & 0 & \\
\hline$<1.0$ & 1 & & $\mu g / L$ & 0 & $\mathrm{GE}$ \\
\hline$<1.0$ & 1 & & $\mu g / L$ & 0 & GE \\
\hline$<1.0$ & 1 & & $\mu g / L$ & 0 & \\
\hline$<1.0$ & 1 & & $\mu \mathrm{g} / \mathrm{L}$ & 0 & \\
\hline$<1.0$ & 1 & & $\mu g / L$ & 0 & \\
\hline$<1.0$ & 1 & & $\mu \mathrm{g} / \mathrm{L}$ & 0 & GE \\
\hline$<1.0$ & 1 & & $\mu g / L$ & 0 & \\
\hline$<1.0$ & 1 & & $\mu g / L$ & 0 & GE \\
\hline$<2.0$ & 1 & & $\mu g / L$ & 0 & GE \\
\hline 23,400 & 1 & & $\mu g / L$ & 0 & \\
\hline$<1.0$ & 1 & & $\mu g / L$ & 0 & GE \\
\hline$<1.0$ & 1 & & $\mu \mathrm{g} / \mathrm{L}$ & 0 & \\
\hline 2,130 & 1 & & $\mu \mathrm{g} / \mathrm{L}$ & 0 & \\
\hline$<1.0$ & 1 & & $\mu \mathrm{g} / \mathrm{L}$ & 0 & \\
\hline$<1.0$ & 1 & & $\mu g / L$ & 0 & \\
\hline$<1.0$ & $i$ & & $\mu \mathrm{g} / \mathrm{L}$ & 0 & \\
\hline$<1.0$ & 1 & & $\mu g / L$ & 0 & \\
\hline$<1.0$ & 1 & & $\mu g / L$ & 0 & GE \\
\hline$<1.0$ & 1 & & $\mu g / L$ & 0 & \\
\hline$<1.0$ & 1 & & $\mu g / L$ & 0 & \\
\hline$<1.0$ & 1 & & $\mu g / L$ & 0 & GE \\
\hline$<1.0$ & 1 & & $\mu g / L$ & 0 & $G E$ \\
\hline$<1.0$ & 1 & & $\mu \mathrm{g} / \mathrm{L}$ & 0 & \\
\hline$<1.0$ & 1 & & $\mu g / L$ & 0 & GI \\
\hline$<1.0$ & 1 & & $\mu g / L$ & 0 & \\
\hline$<1.0$ & 1 & & $\mu g / L$ & 0 & \\
\hline$<4.0$ & 1 & & $\mu \mathrm{g} / \mathrm{L}$ & 0 & G \\
\hline$<4.0$ & 1 & & $\mu g / L$ & 0 & \\
\hline$<5.0$ & 1 & & $\mu g / L$ & 0 & \\
\hline$<0.10$ & 1 & & $\mu g / L$ & 0 & \\
\hline
\end{tabular}

- = exceeded holding time. $\square=$ exceeded screening level or final primary drinking water standard. 
WELL BGO 6C collected on 04/04/94, laboratory analyses (cont.)

H $\underline{\text { Analyte }}$

Dibromochloromethane

Dibromochloromethane

1,1-Dichloroethane

1,1-Dichloroethane

1,1-Dichloroethane

1,2-Dichloroethane

1,2-Dichloroethane

1,2-Dichloroethane

1,1-Dichloroethylene

1,1-Dichloroethylene

1,1-Dichloroethylene

trans-1,2-Dichloroethylene

trans-1,2-Dichloroethylene

Dichloromethane (Methylene chloride)

Dichloromethane (Methylene chloride)

2,4-Dichlorophenoxyacetic acid

1,2-Dichloropropane

1,2-Dichloropropane

1,2-Dichloropropane

cis-1,3-Dichloropropene

cis-1,3-Dichloropropene

cis-1,3-Dichloropropene

trans-1,3-Dichloropropene

trans-1,3-Dichloropropene

trans-1,3-Dichloropropene

Dieldrin

Endrin

Ethylbenzene

Ethylbenzene

Fluoride

Heptachlor

Iron, total recoverable

Lead, total recoverable

Lindane

Magnesium, total recoverable

Manganese, total recoverable

Mercury, total recoverable

Methoxychlor

Naphthalene

Nickel, total recoverable

Nitrate-nitrite as nitrogen

Phenols

Potassium, total recoverable

Selenium, total recoverable

Silica, total recoverable

Silver, total recoverable

Sodium, total recoverable

Sulfate

1,1,2,2-Tetrachloroethane

1,1,2,2-Tetrachloroethane

Tetrachloroethylene

Tetrachloroethylene

Tin, total recoverable

Toluene

Toluene

Total dissolved solids
Result DF

$<1.0$

$<1.0$

$<1.0$

$<1.0$

$<1.0$

$<1.0$

$<1.0$

$<1.0$

$<1.0$

$<1.0$

$<1.0$

$<1.0$

$<1.0$

$<1.0$

$<1.0$

$<0.0016$

$<1.0$

$<1.0$

$<1.0$

$<1.0$

$<1.0$

$<1.0$

$<1.0$

$<1.0$

$<1.0$

$<0.51$

$<0.0061$

$<1.0$

$<1.0$

$<100$

$<0.051$

$<4.0$

$<3.0$

$<0.0051$

509

$<2.0$

$<0.20$

$<0.51$

$<10$

$<4.0$

1,040

$<5.0$

$<500$

$<2.0$

11,300

$<2.0$

1,940

$<1,000$

$<1.0$

$<1.0$

$<1.0$

$<1.0$

$<2.0$

$<1.0$

$<1.0$

92,000
Mod Unit Flag Lab

$\mu \mathrm{g} / \mathrm{L} \quad \mathrm{O} \quad \mathrm{GE}$

$\mu \mathrm{g} / \mathrm{L} \quad \mathrm{O} \quad \mathrm{GE}$

$\mu g / L \quad 0 \quad$ GE

$\mu g / L \quad 0 \quad G E$

$\mu \mathrm{g} / \mathrm{L} \quad 0 \quad \mathrm{GE}$

$\mu g / L \quad 0 \quad G E$

$\mu \mathrm{g} / \mathrm{L} \quad 0 \quad \mathrm{GE}$

$\mu g / L \quad 0 \quad$ GE

$\mu g / L \quad O \quad G E$

$\mu g / L \quad 0 \quad G E$

$\mu g / L \quad O \quad G E$

$\mu g / L \quad 0 \quad$ GE

$\mu \mathrm{g} / \mathrm{L} \quad \mathrm{O} \quad \mathrm{GE}$

$\mu g / L \quad 0 \quad$ GE

$\mu g / L \quad 0 \quad$ GE

$\mu \mathrm{g} / \mathrm{L} \quad 0 \quad \mathrm{GE}$

$\mu \mathrm{g} / \mathrm{L} \quad 0 \quad \mathrm{GE}$

$\mu \mathrm{g} / \mathrm{L} \quad 0 \quad \mathrm{GE}$

$\mu g / L \quad 0 \quad$ GE

$\mu \mathrm{g} / \mathrm{L} \quad 0 \quad \mathrm{GE}$

$\mu \mathrm{g} / \mathrm{L} \quad \mathrm{O} \quad \mathrm{GE}$

$\mu g / L \quad 0 \quad G E$

$\mu \mathrm{g} / \mathrm{L} \quad \mathrm{O} \quad \mathrm{GE}$

$\mu g / L \quad 0 \quad G E$

$\mu g / L \quad 0 \quad$ GE

$\mu g / L \quad 0 \quad G E$

$\mu g / L \quad 0 \quad$ GE

$\mu g / L \quad 0 \quad G E$

$\mu g / L \quad 0 \quad$ GE

$\mu \mathrm{g} / \mathrm{L} \quad 0 \quad \mathrm{GE}$

$\mu g / L \quad 0 \quad$ GE

$\mu g / L \quad 0 \quad G E$

$\mu \mathrm{g} / \mathrm{L} \quad 0 \quad \mathrm{GE}$

$\mu g / L \quad 0 \quad \mathrm{GE}$

$\mu \mathrm{g} / \mathrm{L} \quad 0 \quad \mathrm{GE}$

$\mu \mathrm{g} / \mathrm{L} \quad \mathrm{O} \quad \mathrm{GE}$

$\mu \mathrm{g} / \mathrm{L} \quad \mathrm{O} \quad \mathrm{GE}$

$\mu \mathrm{g} / \mathrm{L} \quad \mathrm{O} \quad \mathrm{GE}$

$\mu \mathrm{g} / \mathrm{L} \quad \mathrm{O} \quad \mathrm{GE}$

$\mu g / L \quad 0 \quad$ GE

$\mu \mathrm{g} / \mathrm{L} \quad \mathrm{O} \quad \mathrm{GE}$

$\mu g / L \quad 0 \quad G E$

$\mu g / L \quad 0 \quad$ GE

$\mu \mathrm{g} / \mathrm{L} \quad \mathrm{O} \quad \mathrm{GE}$

$\mu \mathrm{g} / \mathrm{L} \quad 0 \quad \mathrm{GE}$

$\mu \mathrm{g} / \mathrm{L} \quad \mathrm{O} \quad \mathrm{GE}$

$\mu g / L \quad 0 \quad G E$

$\mu \mathrm{g} / \mathrm{L} \quad \mathrm{O} \quad \mathrm{GE}$

$\mu \mathrm{g} / \mathrm{L} \quad \mathrm{O} \quad \mathrm{GE}$

$\mu g / L \quad 0 \quad$ GE

$\mu g / L \quad 0 \quad G E$

$\dot{\mu g} / \mathrm{L} \quad 0 \quad \mathrm{GE}$

$\mu \mathrm{g} / \mathrm{L} \quad \mathrm{O} \quad \mathrm{GE}$

$\mu g / L \quad 0 \quad$ GE

$\mu g / L \quad 0 \quad$ GE

JV2 $\mu \mathrm{g} / \mathrm{L} \quad \mathrm{O} \quad \mathrm{GE}$

$\overline{- \text { Exceeded holding time. }}$ = exceeded screening level or final primary drinking water standard. 
WELL BGO 6C collected on 04/04/94, laboratory analyses (cont.)

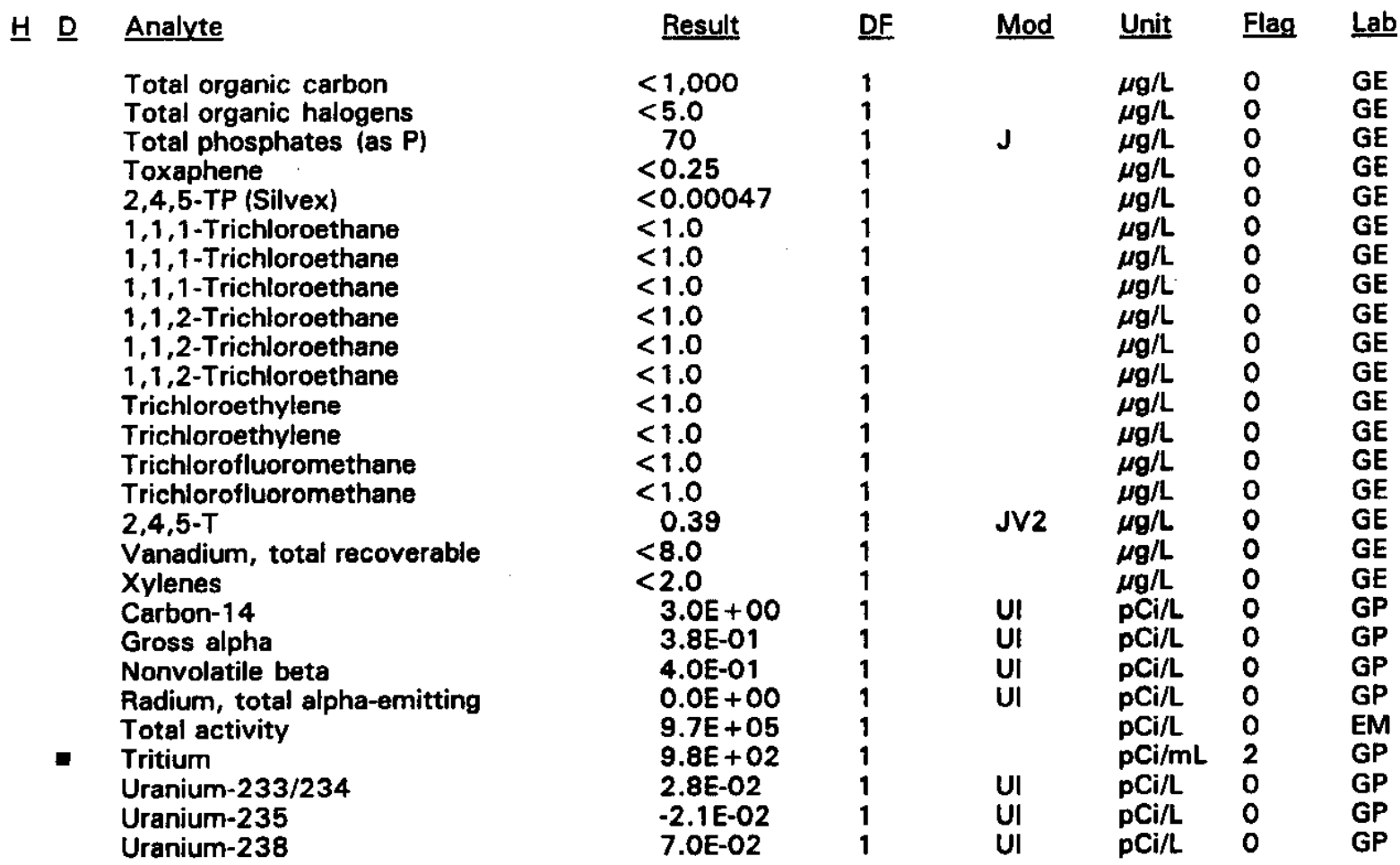

\section{WELL BGO 6D}

\begin{tabular}{|c|c|c|c|c|c|c|}
\hline SRS Coord. & Lat/Longitude & Screen Zone Elevation & Top of Casing & Casing & Pump & Formation \\
\hline $\begin{array}{l}\text { N76487.3 } \\
\text { E58297.1 }\end{array}$ & $\begin{array}{l}33.290059^{\circ} \mathrm{N} \\
81.661867^{\circ} \mathrm{W}\end{array}$ & $237.2-217.2 \mathrm{ft} \mathrm{msl}$ & $285.5 \mathrm{ft} \mathrm{msl}$ & 4" PVC & $\mathbf{s}$ & Water Table $\left(I I B_{2}\right)$ \\
\hline
\end{tabular}

\section{FIELD MEASUREMENTS}

Sample date: 04/05/94

Depth to water: $54.09 \mathrm{ft}(16.49 \mathrm{~m})$ below TOC

Water elevation: $231.41 \mathrm{ft}(70.53 \mathrm{~m}) \mathrm{msl}$

Sp. conductance: $141 \mu \mathrm{S} / \mathrm{cm}$

Turbidity: 4.7 NTU

Water evacuated before sampling: $8 \mathrm{gal}$

The well went dry during purging.

\section{LABORATORY ANALYSES}

$\begin{array}{ll}\text { H } D \quad \text { Analyte } \\ \text { - } & \text { pH } \\ & \text { Specific conductance } \\ \text { Turbidity } \\ \text { Acetophenone } \\ \text { Aldrin } \\ \text { Aluminum, total recoverable } \\ \text { Antimony, total recoverable }\end{array}$

- =xceeded holding time. = exceeded screening level or final primary drinking water standard.
Time: 9:00

$\mathrm{pH}: 6.6$

Alkalinity: $44 \mathrm{mg} / \mathrm{L}$

Water temperature: $17.7^{\circ} \mathrm{C}$

Volumes purged: 0.9 well volumes

$\begin{aligned} & \text { Result } \\ & 6.7 \\ & 144 \\ & 12 \\ < & 10 \\ < & 0.050 \\ & 277 \\ < & 2.0\end{aligned}$

$$
\begin{aligned}
& \text { DF } \\
& 1 \\
& 1 \\
& 1 \\
& 1 \\
& 1 \\
& 1
\end{aligned}
$$

\section{Mod}

$\begin{array}{lll}\text { Unit } & \text { Flag } & \text { Lab } \\ \text { pH } & 0 & \text { GE } \\ \mu \text { S/cm } & 0 & \text { GE } \\ \text { NTU } & 0 & \text { GE } \\ \mu g / L & 0 & \text { GE } \\ \mu g / L & 0 & \text { GE } \\ \mu g / L & 2 & \text { GE } \\ \mu g / L & 0 & \text { GE }\end{array}$

\section{max}


WELL BGO $6 D$ collected on $04 / 05 / 94$, laboratory analyses (cont.)

H $\underline{\text { Analyte }}$

Arsenic, total recoverable

Barium, total recoverable

Benzene

Bromodichloromethane

Bromoform

Bromomethane (Methyl bromide)

Cadmium, total recoverable

Calcium, total recoverable

Carbon tetrachloride

Chloride

Chlorobenzene

Chloroethane

Chloroethene (Vinyl chloride)

2-Chloroethyl vinyl ether

2-Chioroethyl vinyl ether

Chloroform

Chloromethane (Methyl chloride)

Chromium, total recoverable

Copper, total recoverable

Cyanide

p,p'-DDT

Dibromochloromethane

1,1-Dichloroethane

1,1-Dichloroethane

1,2-Dichloroethane

1,2-Dichloroethane

1,1-Dichloroethylene

1,1-Dichloroethylene

trans-1,2-Dichloroethylene

Dichloromethane (Methylene chloride)

2,4-Dichlorophenoxyacetic acid

1,2-Dichloropropane

1,2-Dichloropropane

cis-1,3-Dichloropropene

cis-1,3-Dichloropropene

trans-1,3-Dichloropropene

trans-1,3-Dichloropropene

Dieldrin

Endrin

Ethylbenzene

Fluoride

Heptachlor

Iron, total recoverable

Lead, total recoverable

Lindane

Magnesium, total recoverable

Manganese, total recoverable

Mercury, total recoverable

Methoxychlor

Naphthalene

Nickel, total recoverable

Nitrate-nitrite as nitrogen

Phenols

Potassium, total recoverable

Selenium, total recoverable

Silica, total recoverable
$<2.0$

27

$<1.0$

$<1.0$

$<1.0$

$<1.0$

$<2.0$

20,300

$<1.0$

1,920

$<1.0$

$<1.0$

$<1.0$

$<1.0$

$<1.0$

$<1.0$

$<1.0$

$<4.0$

$<4.0$

$<5.0$

$<0.10$

$<1.0$

1.1

1.1

$<1.0$

$<1.0$

$<1.0$

$<1.0$

$<1.0$

$<1.0$

$<0.0015$

$<1.0$

$<1.0$

$<1.0$

$<1.0$

$<1.0$

$<1.0$

$<0.50$

$<0.0060$

$<1.0$

$<100$

$<0.050$

74

5.9

$<0.0050$

1,130

18

$<0.20$

$<0.50$

$<10$

$<4.0$

670

$<5.0$

564

$<2.0$

10,400
Mod Unit Flag Lab

$\mu \mathrm{g} / \mathrm{L} \quad 0 \quad \mathrm{GE}$

$\mu g / L \quad 0 \quad G E$

$\mu g / L \quad 0 \quad G E$

$\mu \mathrm{g} / \mathrm{L} \quad 0 \quad \mathrm{GE}$

$\mu g / L \quad 0 \quad G E$

$\mu \mathrm{g} / \mathrm{L} \quad 0 \quad \mathrm{GE}$

$\mu g / L \quad 0 \quad G E$

$\mu \mathrm{g} / \mathrm{L} \quad \mathrm{O} \quad \mathrm{GE}$

$\mu g / L \quad 0 \quad G E$

$\mu \mathrm{g} / \mathrm{L} \quad 0 \quad \mathrm{GE}$

$\mu g / L \quad 0 \quad G E$

$\mu g / L \quad 0 \quad G E$

$\mu g / L \quad 0 \quad G E$

$\mu g / L \quad 0 \quad G E$

$\mu g / L \quad 0 \quad G E$

$\mu g / L \quad O \quad G E$

$\mu g / L \quad 0 \quad G E$

$\mu g / L \quad 0 \quad G E$

$\mu g / L \quad 0 \quad$ GE

$\mu g / L \quad 0 \quad \mathrm{GE}$

$\mu g / L \quad 0 \quad$ GE

$\mu \mathrm{g} / \mathrm{L} \quad 0 \quad \mathrm{GE}$

$\mu \mathrm{g} / \mathrm{L} \quad 0 \quad \mathrm{GE}$

$\mu g / L \quad 0 \quad$ GE

$\mu g / L \quad 0 \quad G E$

$\mu g / L \quad 0 \quad G E$

$\mu \mathrm{g} / \mathrm{L} \quad 0 \quad \mathrm{GE}$

$\mu \mathrm{g} / \mathrm{L} \quad 0 \quad \mathrm{GE}$

$\mu g / L \quad 0 \quad G E$

$\mu g / L \quad 0 \quad G E$

$\mu g / L \quad 0 \quad G E$

$\mu g / L \quad 0 \quad G E$

$\mu g / L \quad O \quad G E$

$\mu g / L \quad 0 \quad G E$

$\mu g / L \quad 0 \quad G E$

$\mu g / L \quad 0 \quad G E$

$\mu g / L \quad 0 \quad$ GE

$\mu g / L \quad 0 \quad G E$

$\mu g / L \quad 0 \quad G E$

$\mu g / L \quad 0 \quad G E$

$\mu g / L \quad 0 \quad G E$

$\mu g / L \quad 0 \quad G E$

$\mu g / L \quad 0 \quad G E$

$\mu g / L \quad 0 \quad G E$

$\mu g / L \quad 0 \quad G E$

$\mu g / L \quad O \quad G E$

$\mu g / L \quad 0 \quad G E$

$\mu \mathrm{g} / \mathrm{L} \quad 0 \quad \mathrm{GE}$

$\mu g / L \quad 0 \quad \mathrm{GE}$

$\mu g / L \quad 0 \quad G E$

$\mu g / L \quad 0 \quad G E$

$\mu g / L \quad 0 \quad G E$

$\mu g / L \quad 0 \quad$ GE

$\mu g / L \quad O \quad G E$

$\mu g / L \quad 0 \quad \mathrm{GE}$

$\mu g / L \quad 0 \quad G E$

- = exceeded holding time. $\square=$ exceeded screening level or final primary drinking water standard. 
WELL BGO 6D collected on 04/05/94, laboratory analyses (cont.)

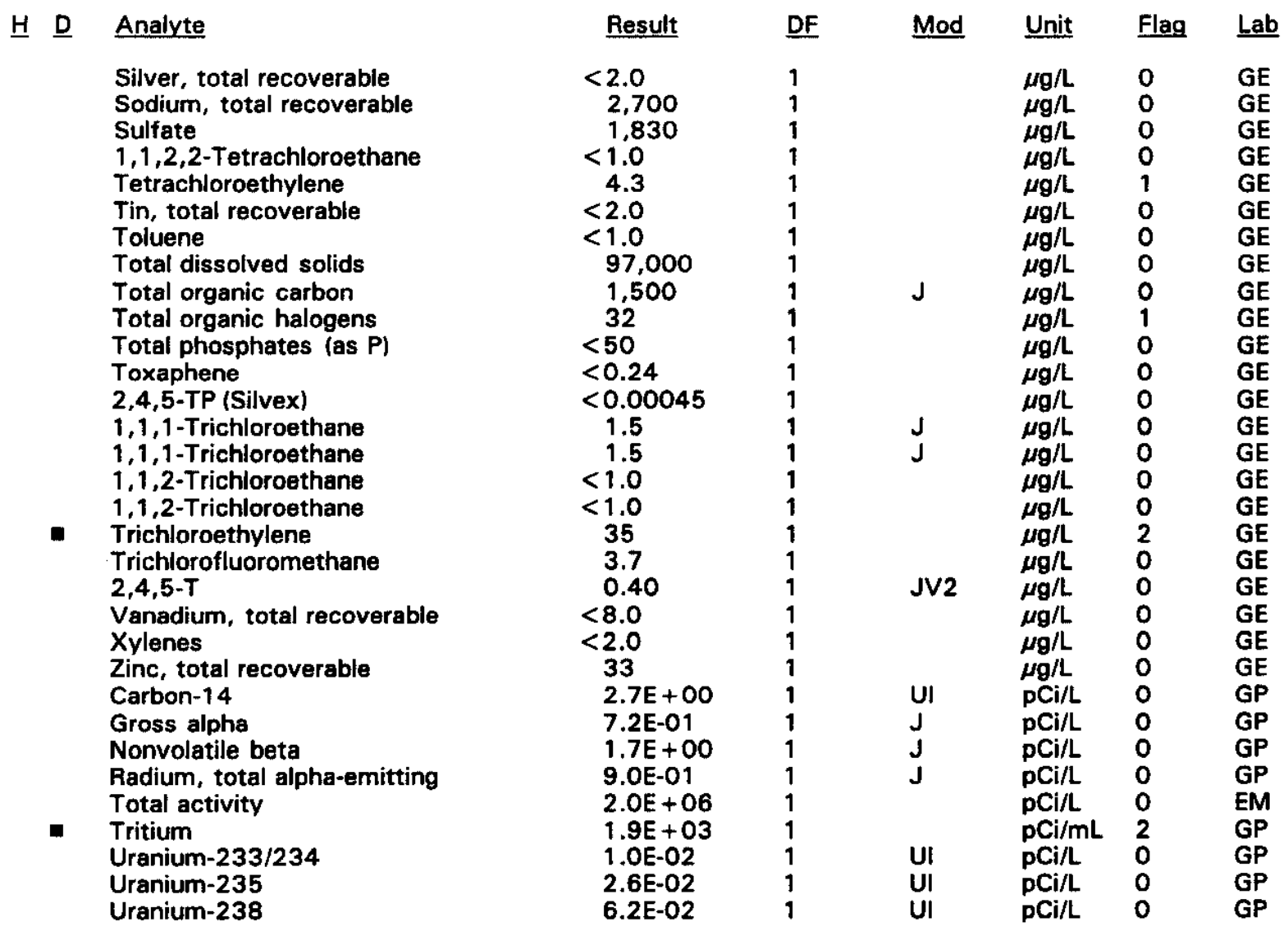

\section{WELL BGO 7D}

$\begin{array}{lllllll}\text { SRS Coord. } & \text { Lat/Longitude } & \text { Screen Zone Elevation } & \text { Top of Casing } & \text { Casing } & \text { Pump } & \text { Formation } \\ \text { N76494.5 } & 33.289455^{\circ} \mathrm{N} & 240.2-220.2 \mathrm{ft} \mathrm{msl} & 287 \mathrm{ft} \mathrm{msl} & 4^{*} \text { PVC } & \text { S } & \text { Water Table (IIB }{ }_{2} \text { ) } \\ \text { E57917.2 } & 81.662882^{\circ} \mathrm{W} & & & & & \end{array}$

\section{FIELD MEASUREMENTS}

Sample date: 04/04/94

Depth to water: $54.87 \mathrm{ft}(16.72 \mathrm{~m})$ below TOC

Water elevation: $232.13 \mathrm{ft}(70.75 \mathrm{~m}) \mathrm{msl}$

Sp. conductance: $35 \mu \mathrm{S} / \mathrm{cm}$

Turbidity: 1.1 NTU

Water evacuated before sampling: $129 \mathrm{gal}$
Time: $14: 13$

pH: 4.9

Alkalinity: $0 \mathrm{mg} / \mathrm{L}$

Water temperature: $19.8^{\circ} \mathrm{C}$

Volumes purged: 16.5 well volumes

\section{LABORATORY ANALYSES}

\begin{tabular}{|c|c|c|c|c|c|c|}
\hline 브 D & Analyte & Result & $\underline{\mathrm{DF}}$ & Mod & Unit & Flag \\
\hline - & $\begin{array}{l}\text { pH } \\
\text { Specific conductance }\end{array}$ & $\begin{array}{l}5.1 \\
28\end{array}$ & $\begin{array}{l}1 \\
1\end{array}$ & $\mathrm{~J} 1$ & $\begin{array}{l}\mathrm{pH} \\
\mu \mathrm{S} / \mathrm{cm}\end{array}$ & $\begin{array}{l}0 \\
0\end{array}$ \\
\hline
\end{tabular}

- exceeded holding time. $\square=$ exceeded screening level or final primary drinking water standard. 
WELL BGO 7D collected on 04/04/94, laboratory analyses (cont.)

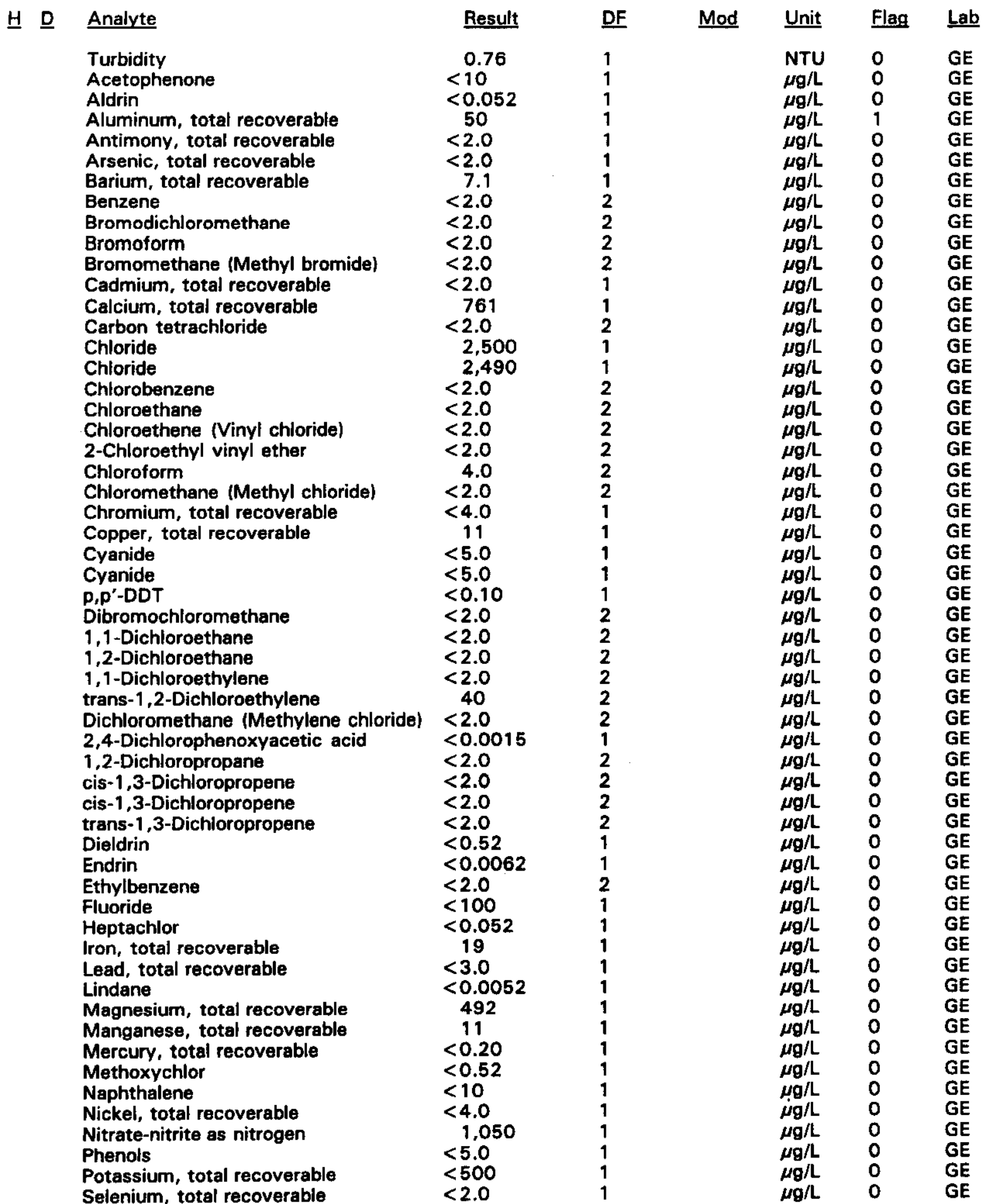

$\overline{-=\text { exceeded holding time. }}-$ = exceeded screening level or final primary drinking water standard. 
WELL BGO 7D collected on 04/04/94, laboratory analyses (cont.)

H D Analyte

Silica, total recoverable

Silver, total recoverable

Sodium, total recoverable

Sulfate

Sulfate

$1,1,2,2-T e t r a c h l o r o e t h a n e$

- Tetrachloroethylene

Tin, total recoverable

Toluene

Total dissolved solids

Total organic carbon

Total organic halogens

Total phosphates (as P)

Toxaphene

2,4,5-TP (Silvex)

1,1,1-Trichloroethane

1,1,2-Trichloroethane

- Trichloroethylene

Trichlorofluoromethane

2,4,5-T

Vanadium, total recoverable

Xylenes

Zinc, total recoverable

Carbon-14

Gross alpha

Nonvolatile beta

Radium, total alpha-emitting

Total activity

- Tritium

Uranium-233/234

Uranium-235

Uranium-238

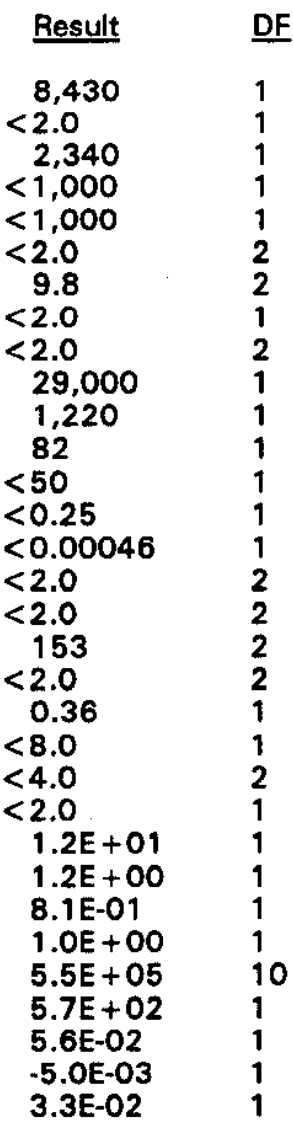

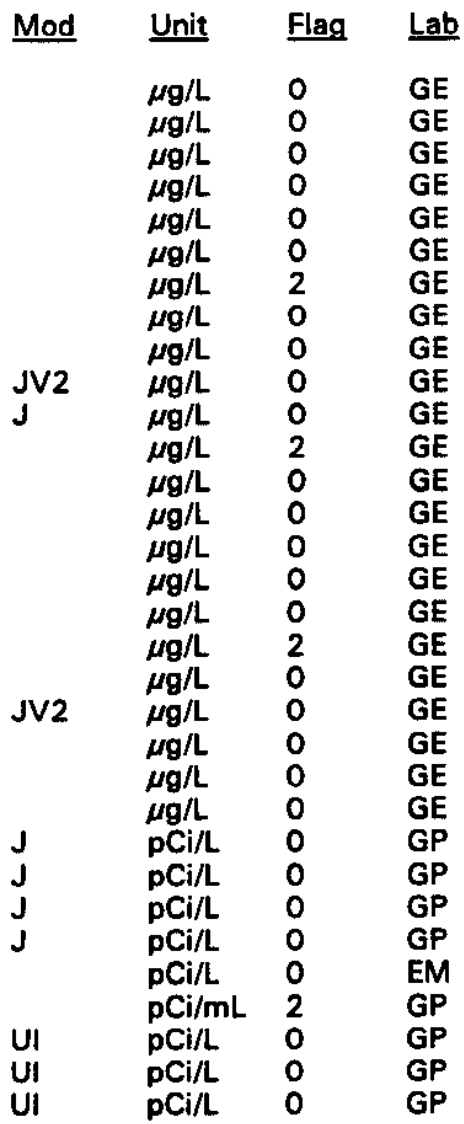

\section{WELL BGO 8AR}

$\begin{array}{lllllll}\text { SRS Coord. } & \text { Lat/Longitude } & \text { Screen Zone Elevation } & \text { Top of Casing } & \text { Casing } & \text { Pump Formation } & \text { Ful } \\ \text { N76598.8 } & 33.289197^{\circ} \mathrm{N} & 104.6-94.6 \mathrm{ft} \mathrm{msl} & 286.6 \mathrm{ft} \mathrm{msl} & 4^{\text {" PVC }} & \text { S } & \text { U. Congaree (IIA) } \\ \text { E57617.5 } & 81.663874^{\circ} \mathrm{W} & & & \end{array}$

\section{FIELD MEASUREMENTS}

Sample date: 04/04/94

Depth to water: $125.56 \mathrm{ft}(38.27 \mathrm{~m})$ below TOC

Water elevation: $161.04 \mathrm{ft}(49.09 \mathrm{~m}) \mathrm{msl}$

Sp. conductance: $258 \mu \mathrm{S} / \mathrm{cm}$

Turbidity: 0.2 NTU

Water evacuated before sampling: 179 gal

\section{LABORATORY ANALYSES}

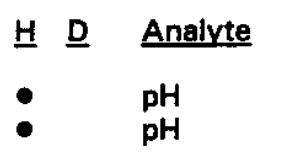

Time: 10:32

pH: 7.9

Alkalinity: $105 \mathrm{mg} / \mathrm{L}$

Water temperature: $19.7^{\circ} \mathrm{C}$

Volumes purged: 4.1 well volumes

$\overline{- \text { = exceeded holding time. }} \mathbf{a}=$ exceeded screening level or final primary drinking water standard. 
WELL BGO 8AR collected on 04/04/94, laboratory analyses (cont.)

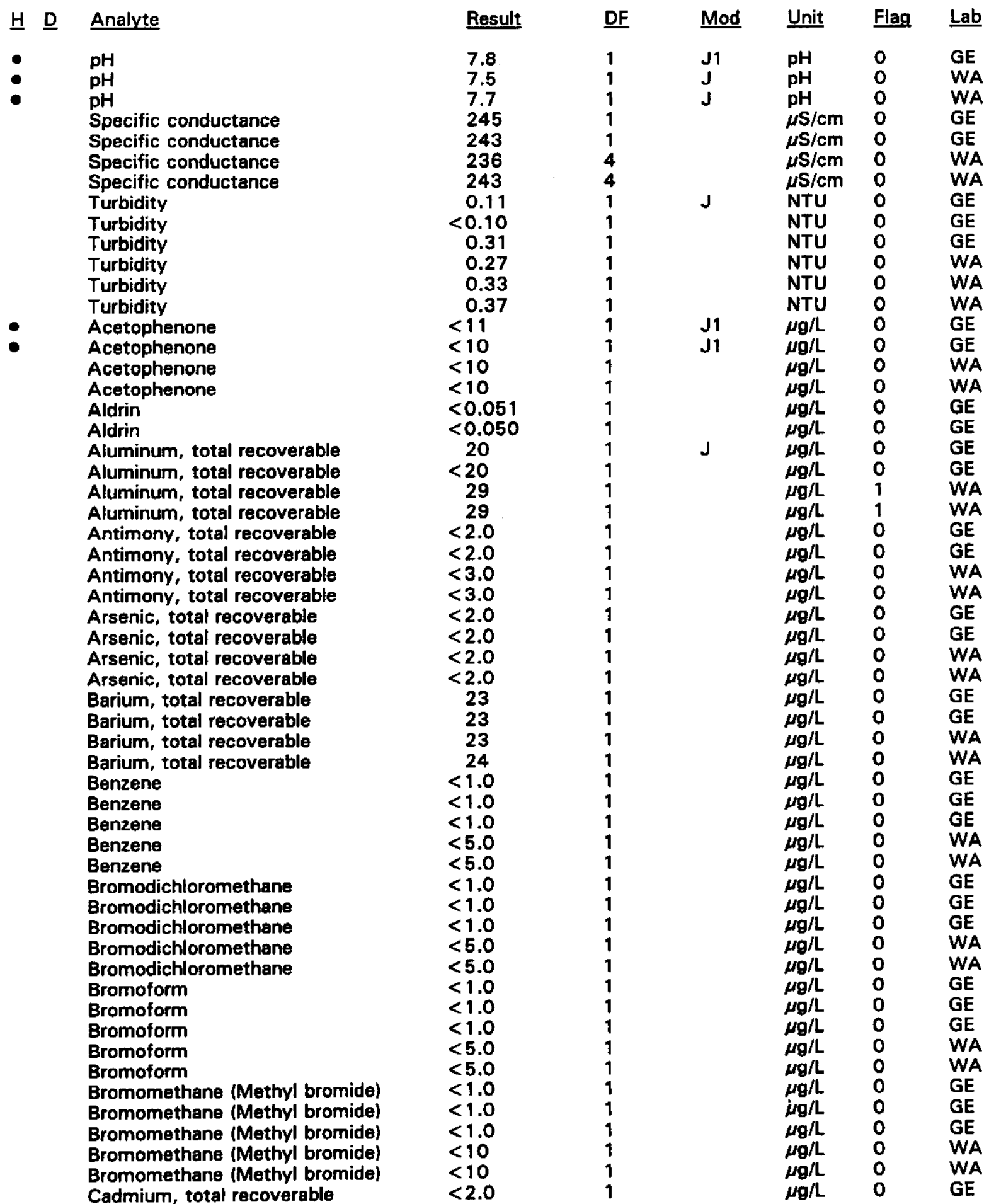

\footnotetext{
- = exceeded holding time. $\square=$ exceeded screening level or final primary drinking water standard.
} 
WELL BGO 8AR collected on 04/04/94, laboratory analyses (cont.)

$\underline{H}$ D Analyte

Cadmium, total recoverable

Cadmium, total recoverable

Cadmium, total recoverable

Calcium, total recoverable

Calcium, total recoverable

Calcium, total recoverable

Calcium, total recoverable

Carbon tetrachloride

Carbon tetrachloride

Carbon tetrachloride

Carbon tetrachloride

Carbon tetrachloride

Chloride

Chloride

Chloride

Chloride

Chloride

Chlorobenzene

Chlorobenzene

Chlorobenzene

Chlorobenzene

Chlorobenzene

Chloroethane

Chloroethane

Chloroethane

Chloroethane

Chloroethane

Chloroethene (Vinyl chloride)

Chloroethene (Vinyl chloride)

Chloroethene (Vinyl chloride)

Chloroethene (Vinyl chloride)

Chloroethene (Vinyl chloride)

2-Chloroethyl vinyl ether

2-Chloroethyl vinyl ether

2-Chioroethyl vinyl ether

2-Chloroethyl vinyl ether

2-Chloroethyl vinyl ether

Chloroform

Chloroform

Chioroform

Chloroform

Chloroform

Chloromethane (Methyl chloride)

Chloromethane (Methyl chloride)

Chloromethane (Methyl chloride)

Chloromethane (Methyl chloride)

Chloromethane (Methyl chloride)

Chromium, total recoverable

Chromium, total recoverable

Chromium, total recoverable

Chromium, total recoverable

Copper, total recoverable

Copper, total recoverable

Copper, total recoverable

Copper, total recoverable

Cyanide

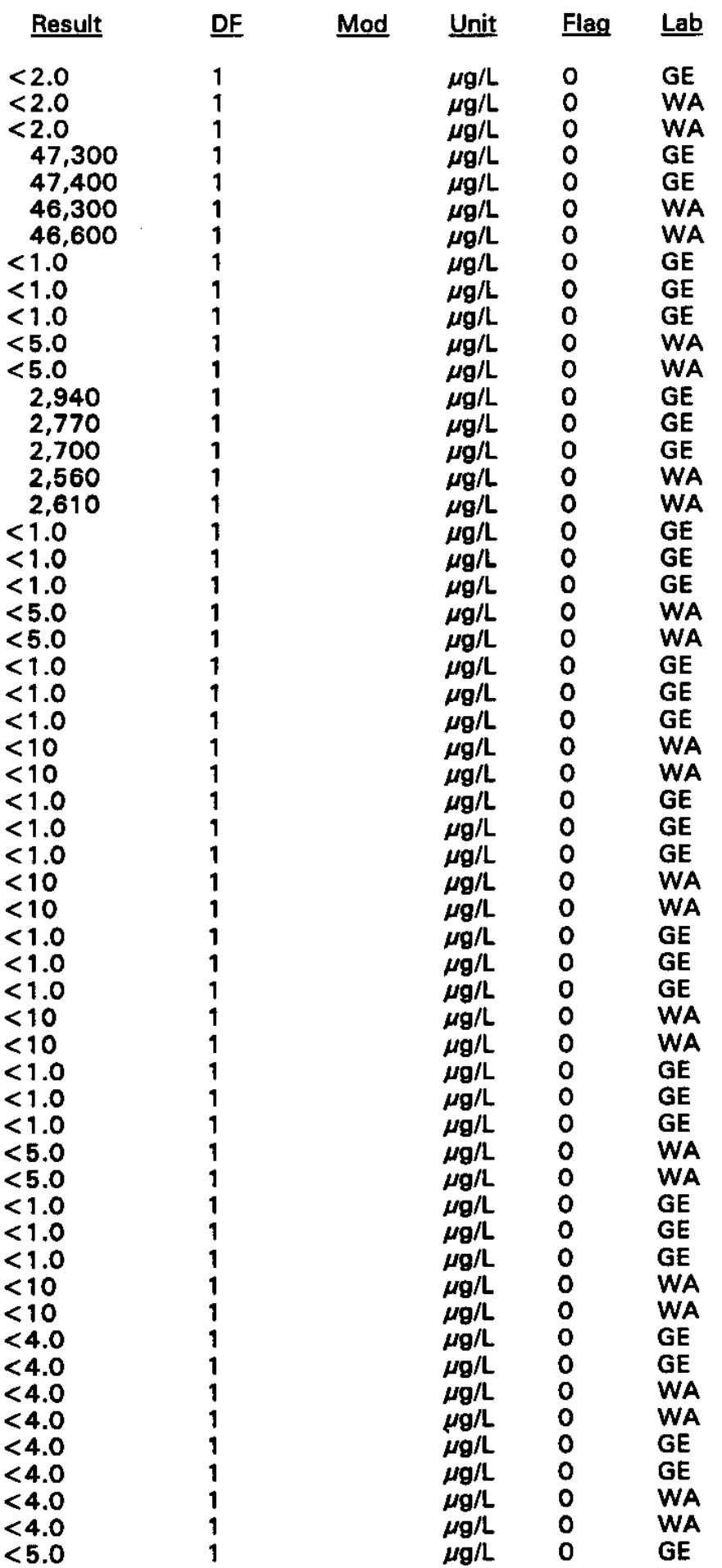

- = exceeded holding time. $=$ exceeded screening level or final primary drinking water standard. 
WELL BGO BAR collected on 04/04/94, laboratory analyses (cont.)

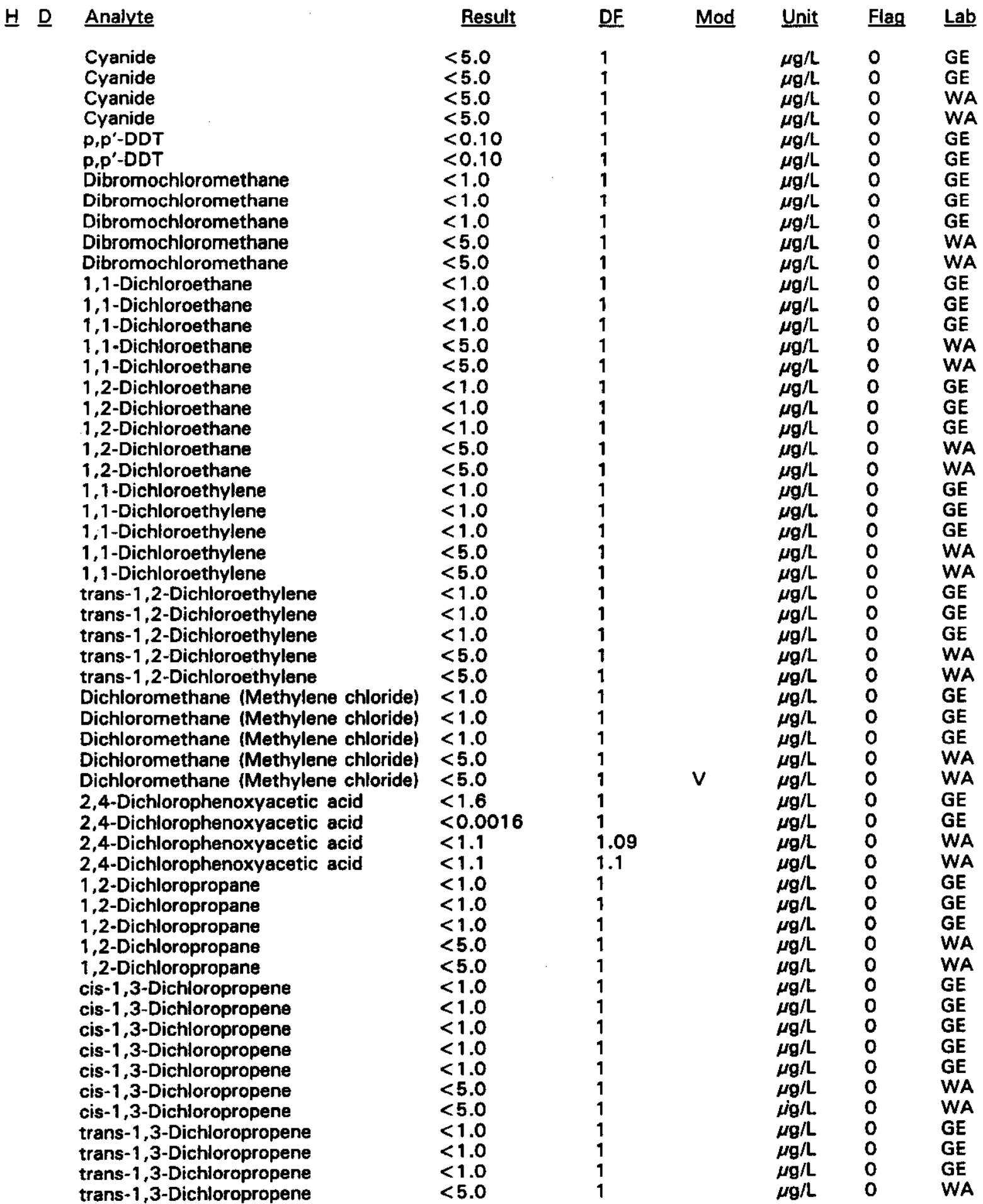

- exceeded holding time. = exceeded screening level or final primary drinking water standard. 
WELL BGO 8AR collected on 04/04/94, laboratory analyses (cont.)

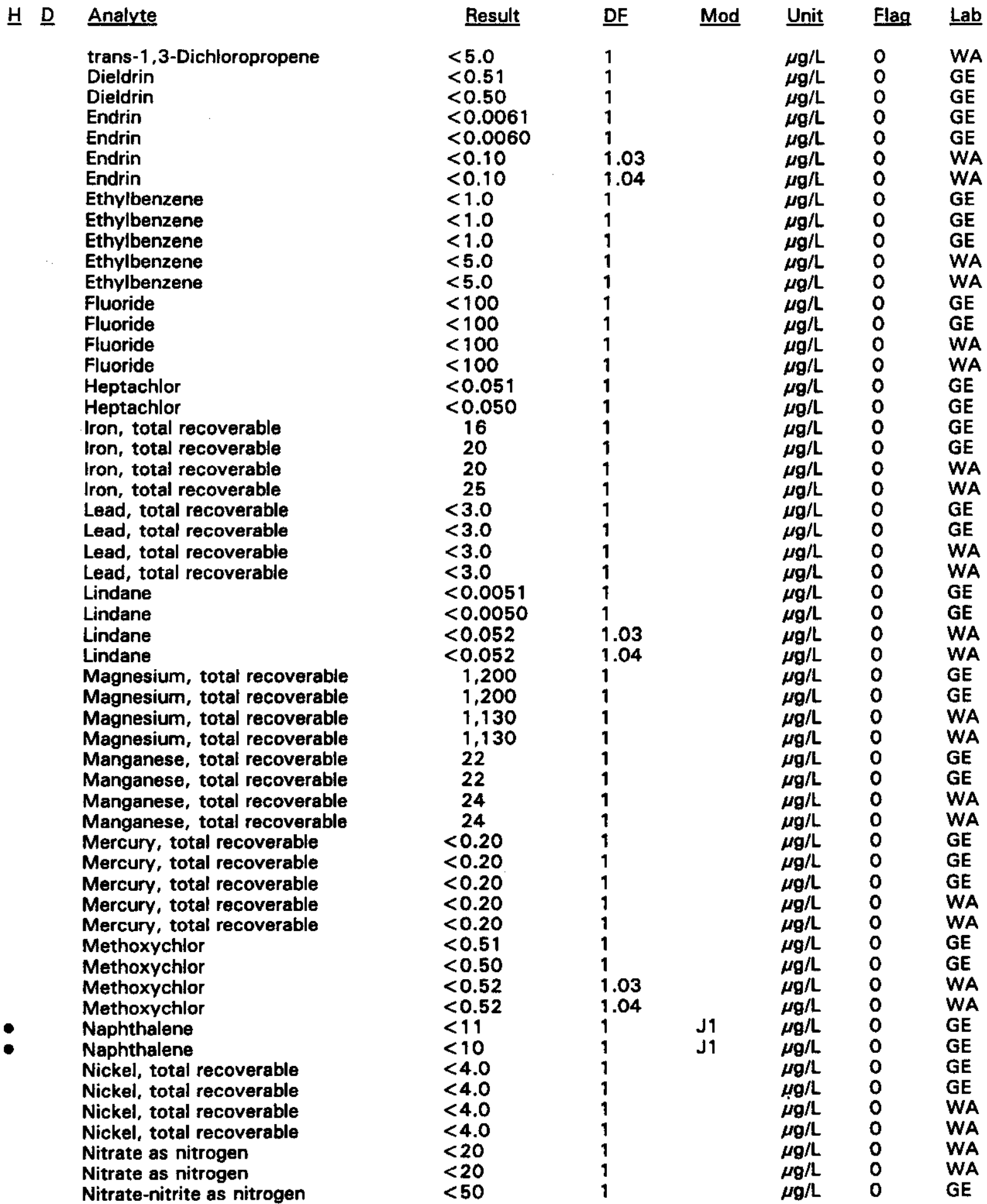

= exceeded holding time. $\square=$ exceeded screening level or final primary drinking water standard. 
WELL BGO 8AR collected on 04/04/94, laboratory analyses (cont.)

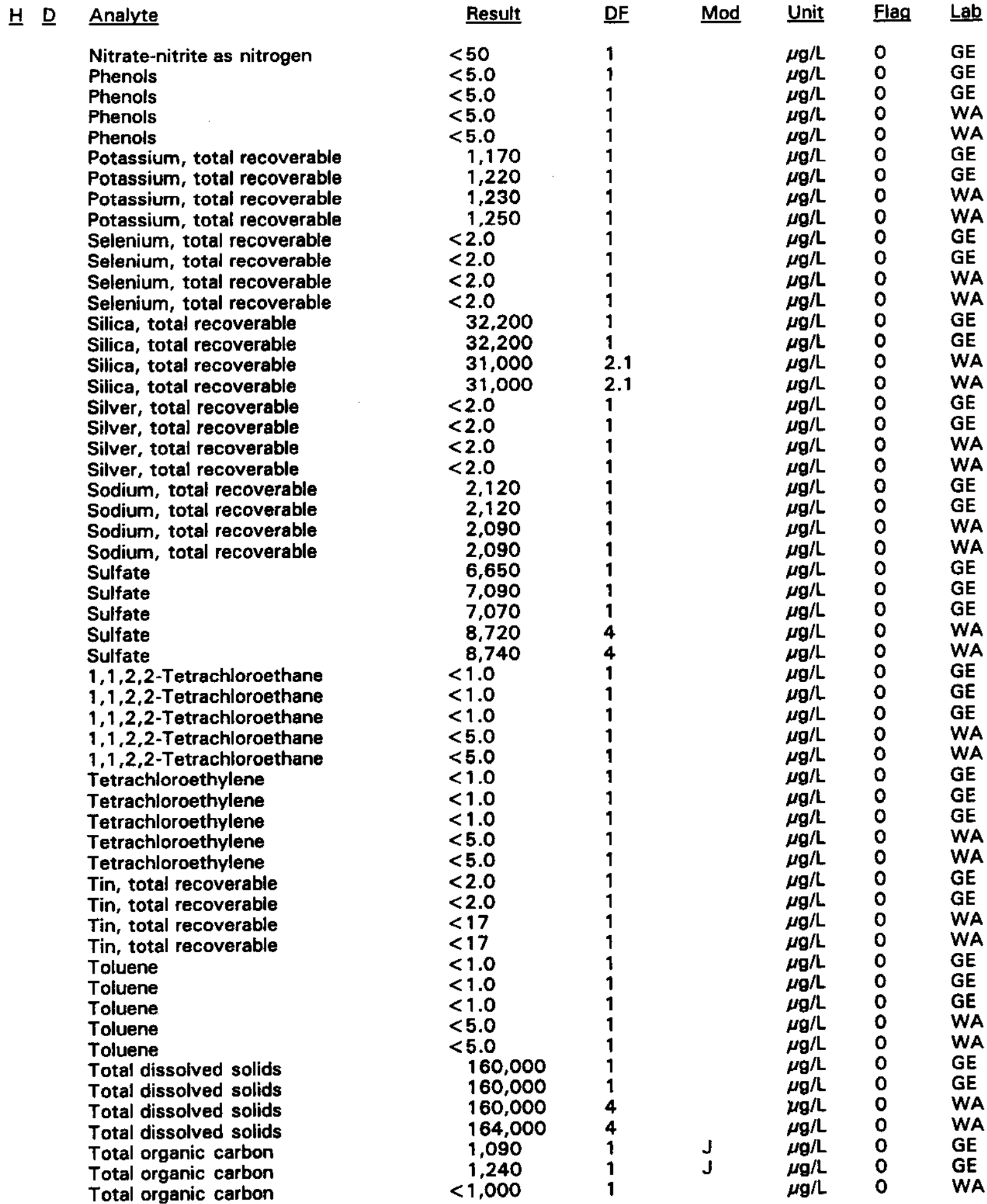

$\overline{-}=$ exceeded holding time. $\quad$ = exceeded screening level or final primary drinking water standard. 
WELL BGO 8AR collected on 04/04/94, laboratory analyses (cont.)

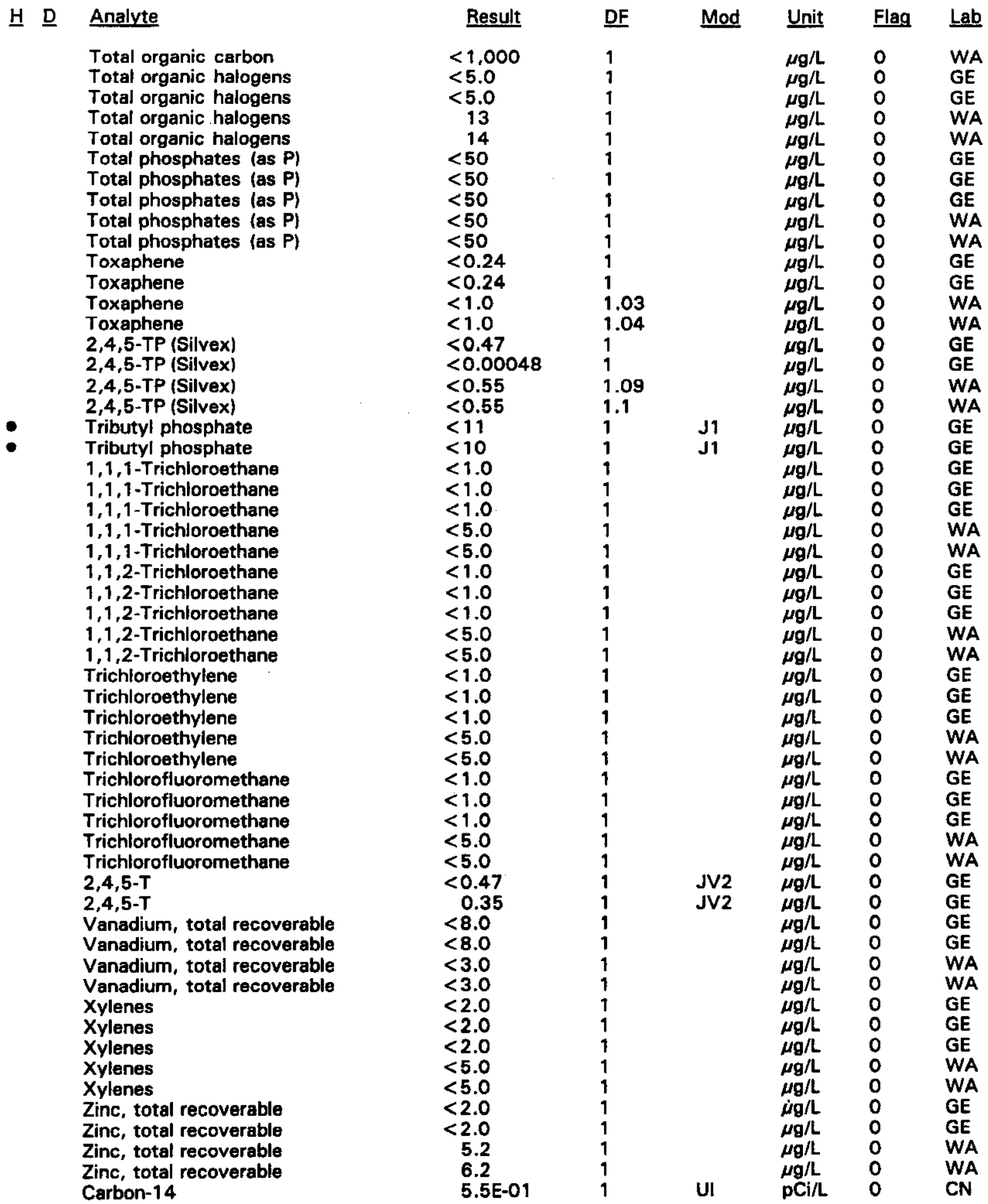

- = exceeded holding time. $=$ exceeded screening level or final primary drinking water standard. 
WELL BGO 8AR collected on 04/04/94, laboratory analyses (cont.)

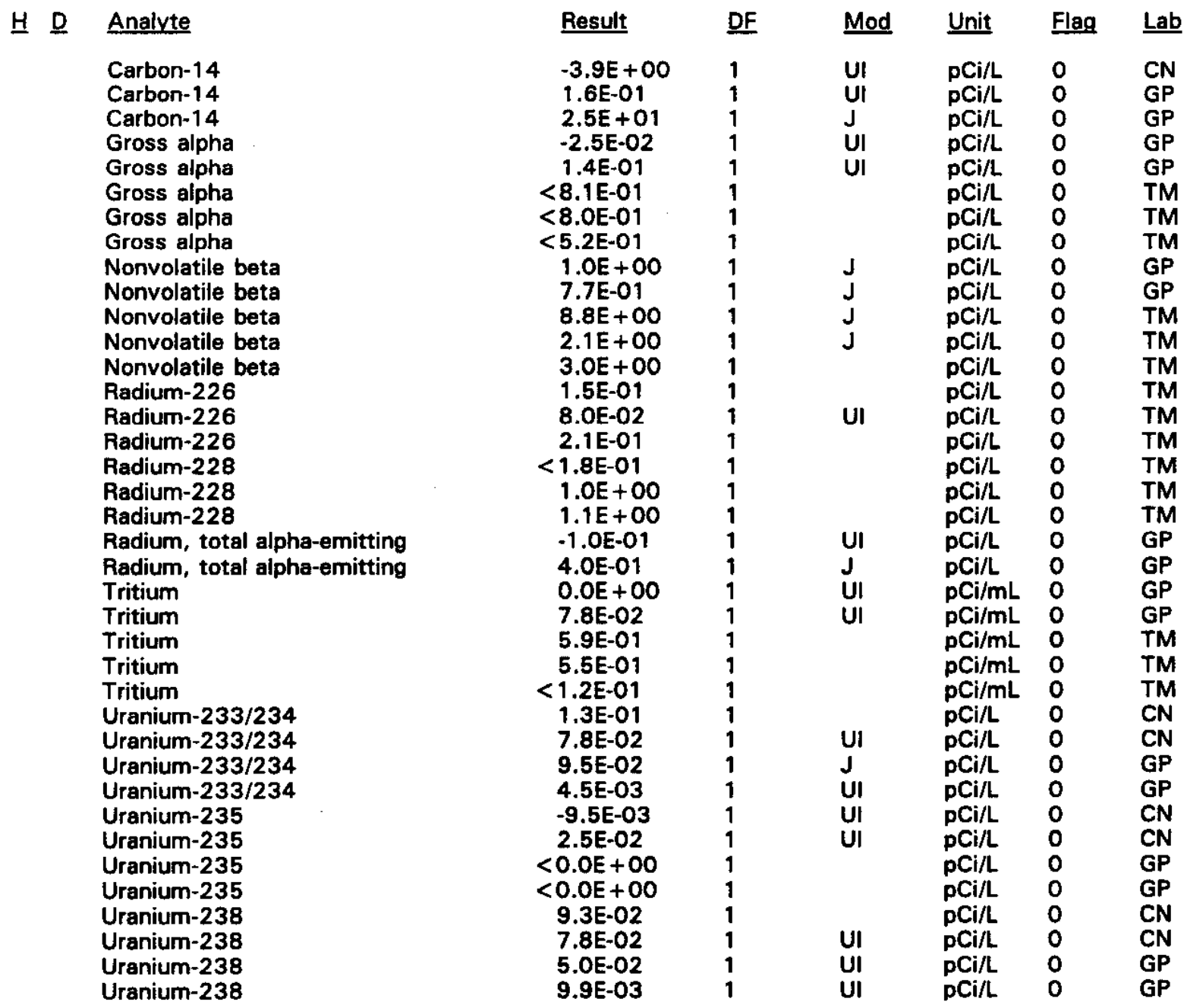

- = exceeded holding time. = exceeded screening level or final primary drinking water standard. 
WELL BGO 8C

\begin{tabular}{|c|c|c|c|c|c|c|}
\hline SRS Coord. & Lat/Longitude & Screen Zone Elevation & Top of Casing & Casing & Pump & Formation \\
\hline $\begin{array}{l}\text { N76579.2 } \\
\text { E57618.7 }\end{array}$ & $\begin{array}{l}33.289156^{\circ} \mathrm{N} \\
81.663832^{\circ} \mathrm{W}\end{array}$ & $184.3-174.3 \mathrm{ft} \mathrm{msl}$ & $287.9 \mathrm{ft} \mathrm{msl}$ & 4" PVC & $\mathbf{S}$ & $n\left(I \mid B_{1}\right)$ \\
\hline
\end{tabular}

\section{FIELD MEASUREMENTS}

Sample date: 04/04/94

Depth to water: $64.46 \mathrm{ft}(19.65 \mathrm{~m})$ below TOC

Water elevation: $223.44 \mathrm{ft}(68.11 \mathrm{~m}) \mathrm{msl}$

Sp. conductance: $130 \mu \mathrm{S} / \mathrm{cm}$

Turbidity: 0.3 NTU

Water evacuated before sampling: $138 \mathrm{gal}$

\section{LABORATORY ANALYSES}

H D Analyte

pH

Specific conductance

Turbidity

Acetophenone

Aldrin

Aluminum, total recoverable

Antimony, total recoverable

Arsenic, total recoverable

Barium, total recoverable

Benzene

Bromodichloromethane

Bromoform

Bromomethane (Methyl bromide)

Cadmium, total recoverable

Calcium, total recoverable

Carbon tetrachloride

Chloride

Chlorobenzene

Chloroethane

Chloroethene (Vinyl chloride)

2-Chloroethyl vinyl ether

Chloroform

Chloromethane (Methyl chloride)

Chromium, total recoverable

Copper, total recoverable

Cyanide

p,p'-DDT

Dibromochloromethane

1,1-Dichloroethane

1,2-Dichloroethane

1,1-Dichloroethylene

trans-1,2-Dichloroethylene

Dichloromethane (Methylene chloride)

2,4-Dichlorophenoxyacetic acid

1,2-Dichloropropane

cis-1,3-Dichloropropene

cis-1,3-Dichloropropene

trans-1,3-Dichloropropene

Dieldrin
Time: 12:05

pH: 7.5

Alkalinity: $39 \mathrm{mg} / \mathrm{h}$

Water temperature: $20.2{ }^{\circ} \mathrm{C}$

Volumes purged: $\mathbf{4 . 3}$ well volumes

\begin{tabular}{|c|c|c|c|c|c|}
\hline Result & DF & Mod & Unit & Flag & ㄴat \\
\hline $\begin{array}{l}7.2 \\
11.4\end{array}$ & 1 & J1 & $\mathrm{pH}$ & 0 & \\
\hline$<0.10$ & $\begin{array}{l}1 \\
1\end{array}$ & & $\begin{array}{l}\mu \mathrm{s} / \mathrm{cm} \\
\text { NTU }\end{array}$ & $\begin{array}{l}0 \\
0\end{array}$ & $\begin{array}{l}\mathrm{GE} \\
\mathrm{GE}\end{array}$ \\
\hline$<10$ & 1 & J1 & $\mu g / L$ & 0 & GE \\
\hline$<0.052$ & 1 & & $\mu \mathrm{g} / \mathrm{L}$ & 0 & GE \\
\hline 25 & 1 & $J$ & $\mu \mathrm{g} / \mathrm{L}$ & & GE \\
\hline$<2.0$ & 1 & & $\mu \mathrm{g} / \mathrm{L}$ & 0 & GE \\
\hline$<2.0$ & 1 & & $\mu \mathrm{g} / \mathrm{L}$ & 0 & GE \\
\hline 9.7 & 1 & & $\mu g / L$ & 0 & GE \\
\hline$<1.0$ & 1 & & $\mu g / L$ & 0 & GE \\
\hline$<1.0$ & 1 & & $\mu \mathrm{g} / \mathrm{L}$ & 0 & GE \\
\hline$<1.0$ & 1 & & $\mu g / L$ & 0 & GE \\
\hline$<1.0$ & 1 & & $\mu \mathrm{g} / \mathrm{L}$ & 0 & GE \\
\hline$<2.0$ & 1 & & $\mu \mathrm{g} / \mathrm{L}$ & 0 & \\
\hline 18,900 & 1 & & $\mu g / L$ & 0 & E \\
\hline$<1.0$ & 1 & & $\mu \mathrm{g} / \mathrm{L}$ & 0 & GE \\
\hline 2,390 & 1 & & $\mu \mathrm{g} / \mathrm{L}$ & 0 & GE \\
\hline$<1.0$ & 1 & & $\mu g / L$ & 0 & GE \\
\hline$<1.0$ & 1 & & $\mu g / L$ & 0 & $\mathrm{E}$ \\
\hline$<1.0$ & 1 & & $\mu g / L$ & 0 & GE \\
\hline$<1.0$ & 1 & & $\mu g / L$ & 0 & \\
\hline$<1.0$ & 1 & & $\mu g / L$ & 0 & \\
\hline$<1.0$ & 1 & & $\mu g / L$ & 0 & GE \\
\hline$<4.0$ & 1 & & $\mu \mathrm{g} / \mathrm{L}$ & 0 & \\
\hline$<4.0$ & 1 & & $\mu g / L$ & 0 & \\
\hline$<5.0$ & 1 & & $\mu \mathrm{g} / \mathrm{L}$ & 0 & \\
\hline$<0.10$ & 1 & & $\mu \mathrm{g} / \mathrm{L}$ & 0 & GE \\
\hline$<1.0$ & 1 & & $\mu g / L$ & 0 & GE \\
\hline$<1.0$ & 1 & & $\mu \mathrm{g} / \mathrm{L}$ & 0 & \\
\hline$<1.0$ & 1 & & $\mu g / L$ & 0 & GE \\
\hline$<1.0$ & 1 & & $\mu \mathrm{g} / \mathrm{L}$ & 0 & GE \\
\hline$<1.0$ & 1 & & $\mu g / L$ & 0 & \\
\hline$<1.0$ & 1 & & $\mu \mathrm{g} / \mathrm{L}$ & 0 & 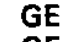 \\
\hline$<0.0016$ & 1 & & $\mu g / L$ & 0 & \\
\hline$<1.0$ & 1 & & $\mu g / L$ & 0 & \\
\hline$<1.0$ & 1 & & $\mu g / L$ & 0 & G \\
\hline$<1.0$ & 1 & & $\mu g / L$ & 0 & \\
\hline$<1.0$ & 1 & & $\mu g / L$ & 0 & \\
\hline$<0.52$ & 1 & & $\mu g / L$ & 0 & \\
\hline
\end{tabular}

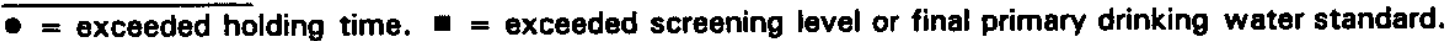


WELL BGO $8 \mathrm{C}$ collected on 04/04/94, laboratory analyses (cont.)

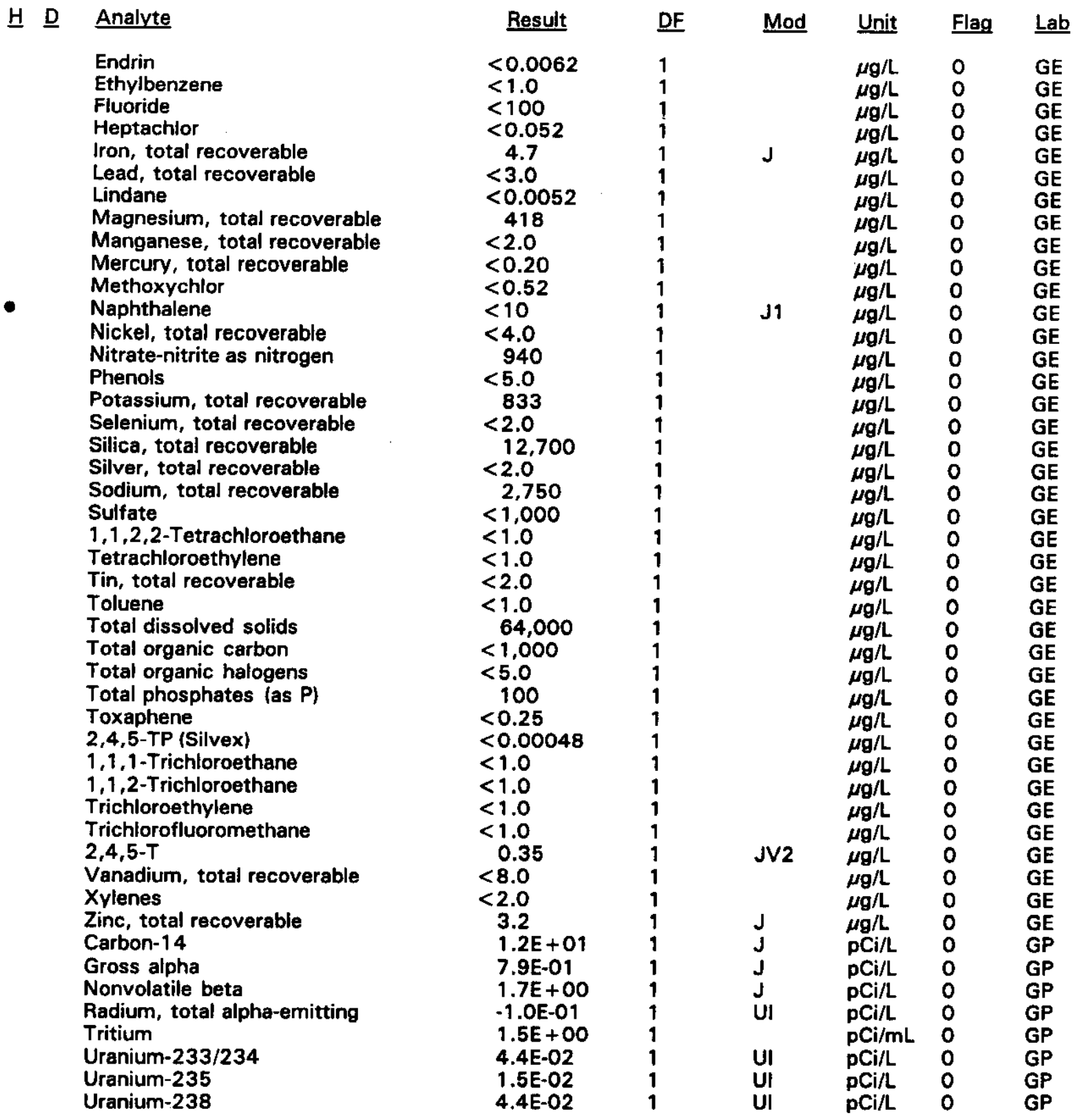

$\overline{-}=$ exceeded holding time. - = exceeded screening level or final primary drinking water standard. 
WELL BGO 8D

\begin{tabular}{|c|c|c|c|c|c|c|}
\hline SRS Coord. & Lat/Longitude & Screen Zone Elevation & Top of Casing & Casing & Pump & Formation \\
\hline $\begin{array}{l}\text { N76588.8 } \\
\text { E57617.8 }\end{array}$ & $\begin{array}{l}33.289175^{\circ} \mathrm{N} \\
81.663853^{\circ} \mathrm{W}\end{array}$ & $240.6-220.6 \mathrm{ft} \mathrm{msl}$ & $287.8 \mathrm{ft} \mathrm{msl}$ & 4" PVC & $\mathbf{S}$ & Water Table $\left(\mathrm{IIB}_{2}\right)$ \\
\hline
\end{tabular}

\section{FIELD MEASUREMENTS}

Sample date: 04/04/94

Depth to water: $55.56 \mathrm{ft}(16.93 \mathrm{~m})$ below TOC

Water elevation: $232.24 \mathrm{ft}(70.79 \mathrm{~m}) \mathrm{msl}$

Sp. conductance: $33 \mu \mathrm{S} / \mathrm{cm}$

Turbidity: 5.2 NTU

Water evacuated before sampling: $241 \mathrm{gal}$

Time: 11:08

pH: 5.2

Alkalinity: $0 \mathrm{mg} / \mathrm{L}$

Water temperature: $19.7^{\circ} \mathrm{C}$

Volumes purged: 31.6 well volumes

\section{LABORATORY ANALYSES}

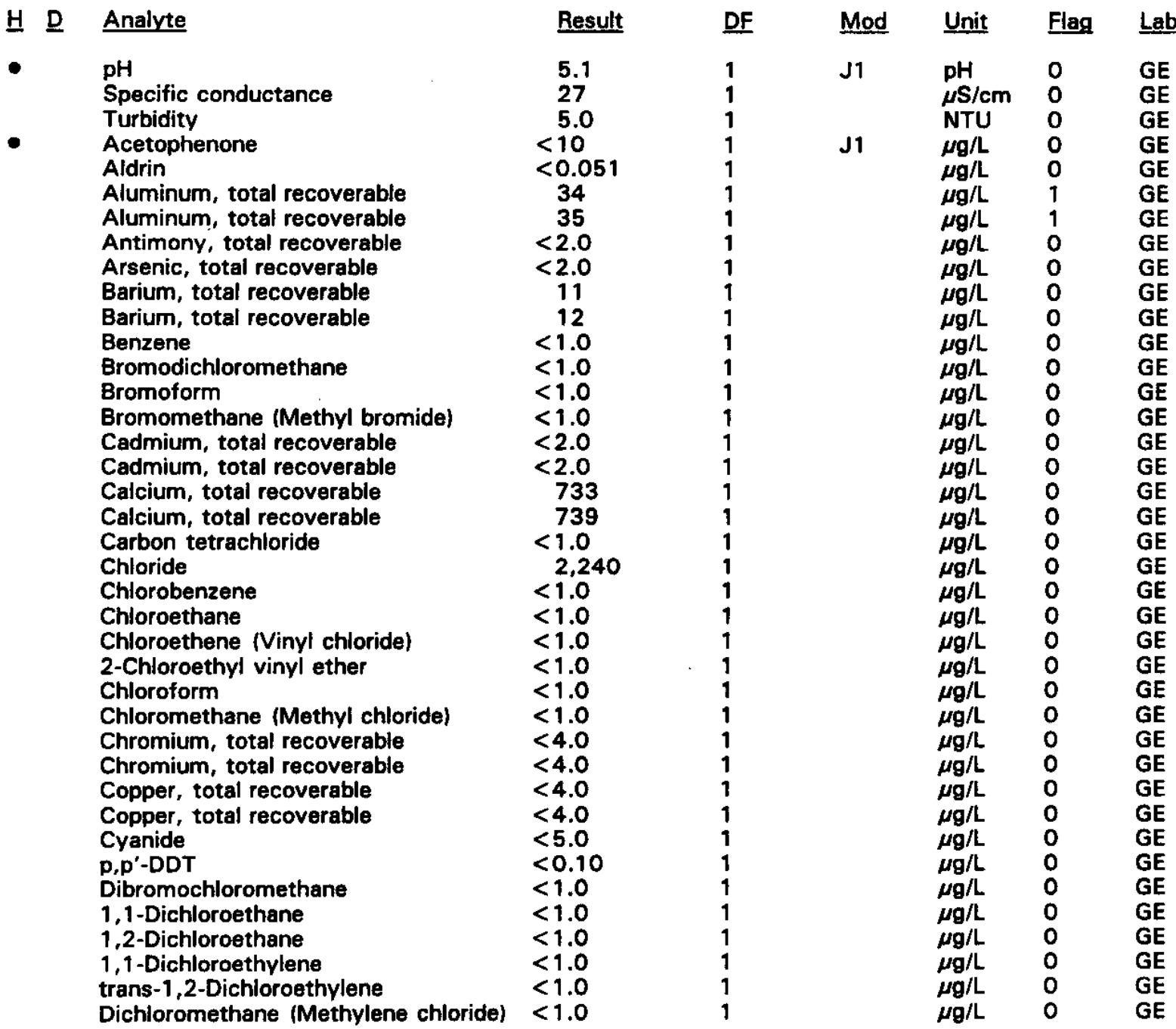

- = exceeded holding time. = =xceeded screening level or final primary drinking water standard. 
WELL BGO 8D collected on 04/04/94, laboratory analyses (cont.)

H $\underline{\text { Analyte }}$

2,4-Dichlorophenoxyacetic acid

1,2-Dichloropropane

cis-1,3-Dichloropropene

cis-1,3-Dichloropropene

trans-1,3-Dichloropropene

Dieldrin

Endrin

Ethylbenzene

Fluoride

Heptachlor

Iron, total recoverable

Iron, total recoverable

Lead, total recoverable

Lindane

Magnesium, total recoverable

Magnesium, total recoverable

Manganese, total recoverable

Manganese, total recoverable

Mercury, total recoverable

Methoxychlor

- Naphthalene

Nickel, total recoverable

Nickel, total recoverable

Nitrate-nitrite as nitrogen

Phenols

Potassium, total recoverable

Potassium, total recoverable

Selenium, total recoverable

Silica, total recoverable

Silica, total recoverable

Silver, total recoverable

Silver, total recoverable

Sodium, total recoverable

Sodium, total recoverable

Sulfate

1,1,2,2-Tetrachloroethane

Tetrachloroethylene

Tin, total recoverable

Tin, total recoverable

Toluene

Total dissolved solids

Total organic carbon

Total organic halogens

Total organic halogens

Total phosphates (as P)

Toxaphene

2,4,5-TP (Silvex)

1,1,1-Trichloroethane

1,1,2-Trichloroethane

Trichioroethylene

Trichlorofluoromethane

2,4,5-T

Vanadium, total recoverable

Vanadium, total recoverable

Xylenes

Zinc, total recoverable
Result

DF

$\underline{\operatorname{Mod}}$

Unit

Flag Lab

$<0.0015$

$<1.0$

$<1.0$

$<1.0$

$<1.0$

$<0.51$

$<0.0061$

$<1.0$

$<100$

$<0.051$

34

35

$<3.0$

$<0.0051$

473

477

11

11

$<0.20$

$<0.51$

$<10$

$<4.0$

$<4.0$

1,220

5.8

$<500$

$<500$

$<2.0$

7,510

7,570

$<2.0$

$<2.0$

2,410

2,420

1,190

$<1.0$

$<1.0$

$<2.0$

$<2.0$

$<1.0$

14,000

$<1,000$

7.3

$<5.0$

$<50$

$<0.24$

$<0.00046$

$<1.0$

$<1.0$

1.5

7.5

0.37

$<8.0$

$<8.0$

$<2.0$

$<2.0$

\section{1}

$\mu g / L \quad 0 \quad G E$

$\mu g / L \quad O \quad G E$

$\mu g / L \quad 0 \quad \mathrm{GE}$

$\mu \mathrm{g} / \mathrm{L} \quad \mathrm{O} \quad \mathrm{GE}$

$\mu \mathrm{g} / \mathrm{L} \quad \mathrm{O} \quad \mathrm{GE}$

$\mu g / L \quad O \quad G E$

$\mu \mathrm{g} / \mathrm{L} \quad 0 \quad \mathrm{GE}$

$\mu \mathrm{g} / \mathrm{L} \quad \mathrm{O} \quad \mathrm{GE}$

$\mu \mathrm{g} / \mathrm{L} \quad 0 \quad \mathrm{GE}$

$\mu g / L \quad 0 \quad G E$

$\mu g / \mathrm{L} \quad 0 \quad \mathrm{GE}$

$\mu g / L \quad 0 \quad$ GE

$\mu g / L \quad 0 \quad G E$

$\mu g / \mathrm{L} \quad 0 \quad \mathrm{GE}$

$\mu \mathrm{g} / \mathrm{L} \quad 0 \quad \mathrm{GE}$

$\mu g / L \quad 0 \quad G E$

$\mu g / L \quad 0 \quad$ GE

$\mu g / L \quad 0 \quad \mathrm{GE}$

$\mu \mathrm{g} / \mathrm{L} \quad 0 \quad \mathrm{GE}$

J1 $\underset{\mu g / L}{\mu g / L} \quad 0 \quad \mathrm{GE}$

$\mu g / L \quad 0 \quad$ GE

$\mu \mathrm{g} / \mathrm{L} \quad 0 \quad \mathrm{GE}$

$\mathrm{J} \quad \mu \mathrm{g} / \mathrm{L} \quad \mathrm{O} \quad \mathrm{GE}$

$\mu \mathrm{g} / \mathrm{L} \quad 0 \quad \mathrm{GE}$

$\mu g / L \quad 0 \quad G E$

$\mu g / \mathrm{L} \quad 0 \quad \mathrm{GE}$

$\mu g / L \quad 0 \quad G E$

$\mu \mathrm{g} / \mathrm{L} \quad 0 \quad \mathrm{GE}$

$\mu \mathrm{g} / \mathrm{L} \quad \mathrm{O} \quad \mathrm{GE}$

$\mu \mathrm{g} / \mathrm{L} \quad 0 \quad \mathrm{GE}$

$\mu \mathrm{g} / \mathrm{L} \quad 0 \quad \mathrm{GE}$

$\mu g / L \quad 0 \quad G E$

J $\mu g / L \quad 0 \quad G E$

$\mu g / L \quad 0 \quad$ GE

$\mu g / L \quad 0 \quad G E$

$\mu \mathrm{g} / \mathrm{L} \quad 0 \quad \mathrm{GE}$

$\mu g / L \quad 0 \quad G E$

$\mu g / L \quad 0 \quad$ GE

$\mu \mathrm{g} / \mathrm{L} \quad 0 \quad \mathrm{GE}$

$\mu \mathrm{g} / \mathrm{L} \quad \mathrm{O} \quad \mathrm{GE}$

$J \quad \mu g / L \quad 0 \quad G E$

$\mu \mathrm{g} / \mathrm{L} \quad 0 \quad \mathrm{GE}$

$\mu g / L \quad 0 \quad G E$

$\mu g / L \quad O \quad G E$

$\mu \mathrm{g} / \mathrm{L} \quad \mathrm{O} \quad \mathrm{GE}$

$\mu \mathrm{g} / \mathrm{L} \quad 0 \quad \mathrm{GE}$

$\mu \mathrm{g} / \mathrm{L} \quad \mathrm{O} \quad \mathrm{GE}$

$J \quad \mu g / L \quad O \quad G E$

JV2 $\underset{\mu g / L}{\mu g} \quad 0 \quad \mathrm{GE}$

$\mu g / L \quad 0 \quad G E$

$\mu g / L \quad O \quad G E$

$\mu \mathrm{g} / \mathrm{L} \quad 0 \quad \mathrm{GE}$

$\mu g / L \quad 0 \quad$ GE

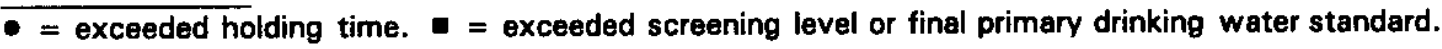


WELL BGO 8D collected on 04/04/94, laboratory analyses (cont.)

\begin{tabular}{|c|c|c|c|c|c|}
\hline Analyte & Result & DF & Mod & Unit & Flag \\
\hline $\begin{array}{l}\text { Zinc, total recoverable } \\
\text { Carbon-14 } \\
\text { Gross alpha } \\
\text { Nonvolatile beta } \\
\text { Radium, total alpha-emitting } \\
\text { Tritium } \\
\text { Uranium-233/234 } \\
\text { Uranium-235 } \\
\text { Uranium-238 }\end{array}$ & $\begin{array}{r}<2.0 \\
6.7 E+01 \\
2.0 E+00 \\
1.6 E+00 \\
1.6 E+00 \\
1.7 E+01 \\
1.4 E-01 \\
<0.0 E+00 \\
1.6 E-01\end{array}$ & $\begin{array}{l}1 \\
1 \\
1 \\
1 \\
1 \\
1 \\
1 \\
1 \\
1\end{array}$ & $\begin{array}{l}\mathbf{J} \\
\mathbf{J} \\
\mathbf{J}\end{array}$ & $\begin{array}{l}\mu \mathrm{g} / \mathrm{L} \\
\mathrm{pCi} / \mathrm{L} \\
\mathrm{pCi} / \mathrm{L} \\
\mathrm{pCi} / \mathrm{L} \\
\mathrm{pCi} / \mathrm{L} \\
\mathrm{pCi} / \mathrm{mL} \\
\mathrm{pCi} / \mathrm{L} \\
\mathrm{pCi} / \mathrm{L} \\
\mathrm{pCi} / \mathrm{L}\end{array}$ & $\begin{array}{l}0 \\
0 \\
0 \\
0 \\
0 \\
1 \\
0 \\
0 \\
0\end{array}$ \\
\hline
\end{tabular}

\section{WELL BGO 9AA}

$\begin{array}{llllllll}\text { SRS Coord. } & \text { Lat/Longitude } & \text { Screen Zone Elevation } & \text { Top of Casing } & \text { Casing } & \text { Pump Formation } \\ \text { N76975.7 } & 33.289630^{\circ} \mathrm{N} & 83.8-73.8 \mathrm{ft} \mathrm{msl} & 284.8 \mathrm{ft} \mathrm{msl} & 4^{\text {" PVC }} & \text { S } & \text { L. Congaree (IIA) } \\ \text { E57371.9 } & 81.665252^{\circ} \mathrm{W} & & & & \end{array}$

\section{FIELD MEASUREMENTS}

Sample date: 04/05/94

Depth to water: $126.88 \mathrm{ft}(38.67 \mathrm{~m})$ below TOC

Water elevation: $157.92 \mathrm{ft}(48.13 \mathrm{~m}) \mathrm{ms}$ l

Sp. conductance: $4000 \mu \mathrm{S} / \mathrm{cm}$

Turbidity: 2.6 NTU

Water evacuated before sampling: $\mathbf{4 5}$ gal

The well went dry during purging.

\section{LABORATORY ANALYSES}

H D Analyte

pH

Specific conductance

Turbidity

Acetophenone

Aldrin

Aluminum, total recoverable

Antimony, total recoverable

Arsenic, total recoverable

Barium, total recoverable

Benzene

Bromodichloromethane

Bromoform

Bromomethane (Methyl bromide)

Cadmium, total recoverable

Calcium, total recoverable

Carbon tetrachloride

Chloride

Chloride

Chlorobenzene

Chloroethane

Chloroethene (Vinyl chloride)

2-Chioroethyl vinyl ether

Chloroform

Chloromethane (Methyl chloride)

Chromium, total recoverable

$\begin{aligned} & \text { Result } \\ & 12 \\ & 1,430 \\ & 0.53 \\ &<9.8 \\ &<0.050 \\ & 1,210 \\ &<2.0 \\ &<2.0 \\ & 306 \\ &<1.0 \\ &<1.0 \\ &<1.0 \\ &<1.0 \\ &<10 \\ & 174,000 \\ &<1.0 \\ & 2.630 \\ & 2,660 \\ &<1.0 \\ &<1.0 \\ &<1.0 \\ &<1.0 \\ &<1.0 \\ &<1.0 \\ &<20\end{aligned}$

Time: 10:13

pH: 12.7

Alkalinity: $806 \mathrm{mg} / \mathrm{L}$

Water temperature: $19.0^{\circ} \mathrm{C}$

Volumes purged: 0.8 well volumes

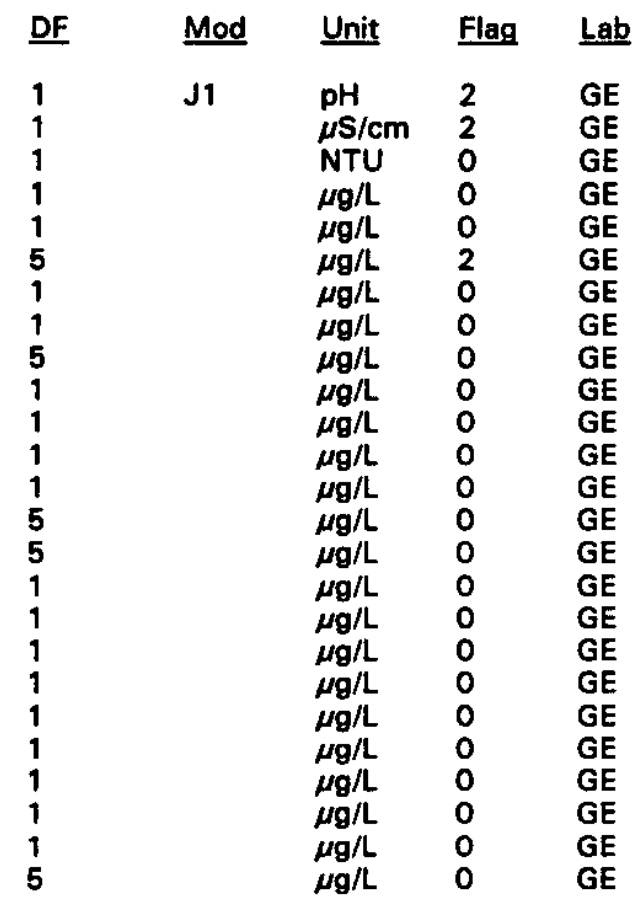

$\overline{-~=~ e x c e e d e d ~ h o l d i n g ~ t i m e . ~}-$ = exceeded screening level or final primary drinking water standard. 
WELL BGO 9AA collected on 04/05/94, laboratory analyses (cont.)

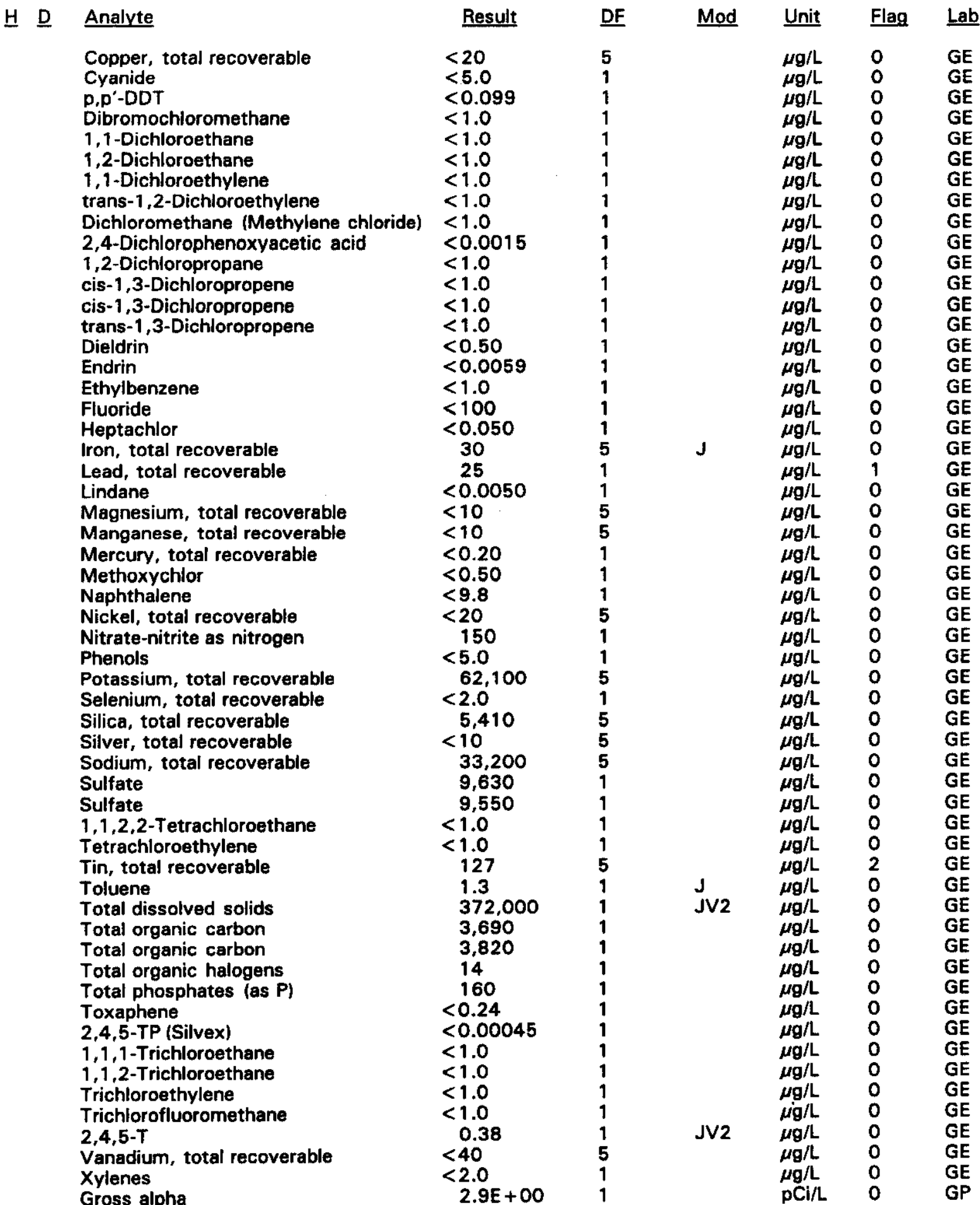

$\overline{0}$ = exceeded holding time. $\square=$ exceeded screening level or final primary drinking water standard. 
WELL BGO 9AA collected on 04/05/94, laboratory analyses (cont.)

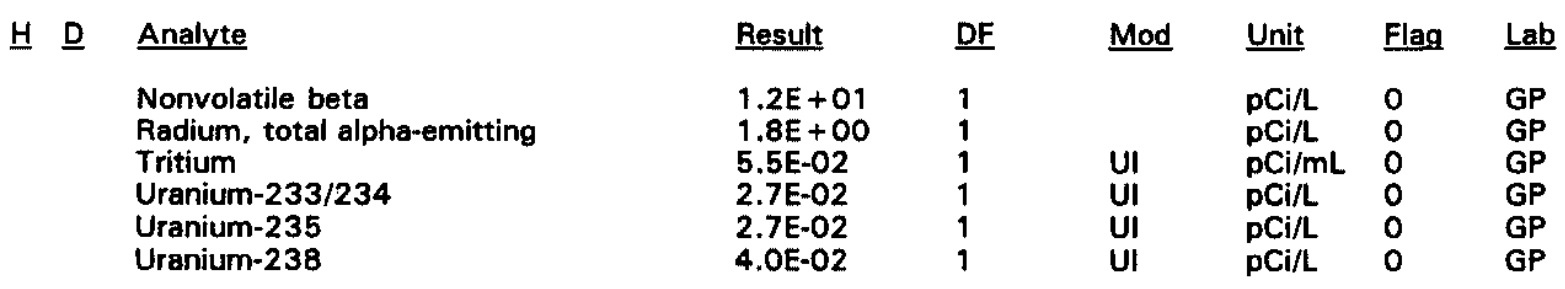

\section{WELL BGO 9D}

\begin{tabular}{|c|c|c|c|c|c|c|}
\hline SRS Coord. & Lat/Longitude & Screen Zone Elevation & Top of Casing & Casing & Pump & Formation \\
\hline $\begin{array}{l}N 76811.6 \\
E 57478.9\end{array}$ & $\begin{array}{l}33.289442^{\circ} \mathrm{N} \\
81.664652^{\circ} \mathrm{W}\end{array}$ & $229.2-209.2 \mathrm{ft} \mathrm{msl}$ & $285.1 \mathrm{ft} \mathrm{msl}$ & 4" PVC & $\mathbf{S}$ & Water Table $\left(\mathrm{IIB}_{2}\right)$ \\
\hline
\end{tabular}

\section{FIELD MEASUREMENTS}

Sample date: 04/04/94

Depth to water: $54.96 \mathrm{ft}(16.75 \mathrm{~m})$ below TOC

Water elevation: $230.14 \mathrm{ft}(70.15 \mathrm{~m}) \mathrm{msl}$

Sp. conductance: $35 \mu \mathrm{S} / \mathrm{cm}$

Turbidity: 0.1 NTU

Water evacuated before sampling: $34 \mathrm{gal}$

\section{LABORATORY ANALYSES}

H D Analyte

- pH

Specific conductance

Turbidity

Acetophenone

Aldrin

Aluminum, total recoverable

Antimony, total recoverable

Arsenic, total recoverable

Barium, total recoverable

Benzene

Bromodichloromethane

Bromoform

Bromomethane (Methyl bromide)

Cadmium, total recoverable

Calcium, total recoverable

Carbon tetrachloride

Chloride

Chlorobenzene

Chloroethane

Chloroethene (Vinyl chloride)

2-Chloroethyl vinyl ether

Chloroform

Chloromethane (Methyl chloride)

Chromium, total recoverable

Copper, total recoverable

Cyanide

p, $p^{\prime}$-DDT

Dibromochloromethane
Time: 16:34

pH: 4.9

Alkalinity: $0 \mathrm{mg} / \mathrm{L}$

Water temperature: $20.5^{\circ} \mathrm{C}$

Volumes purged: 2.5 well volumes

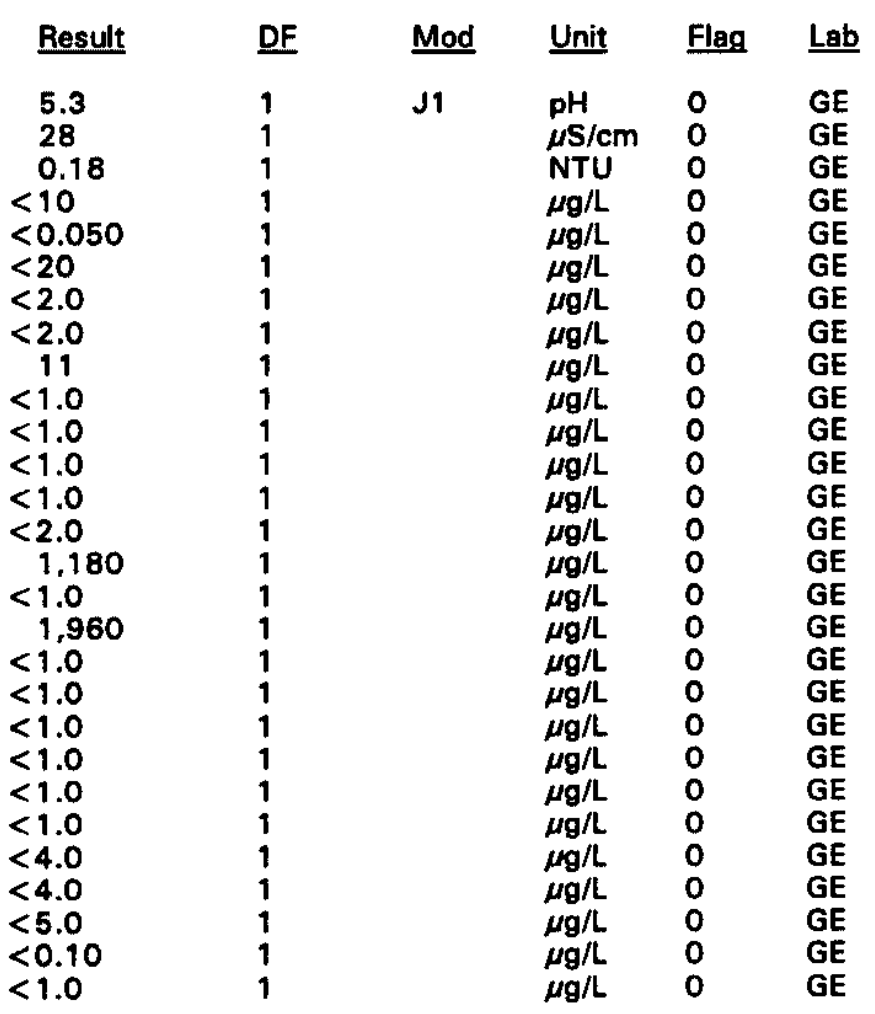

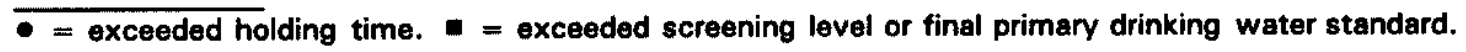


WELL BGO 9D collected on 04/04/94, laboratory analyses (cont.)

H D Analyte

1,1-Dichloroethane

1,2-Dichloroethane

1,1-Dichloroethylene

trans-1,2-Dichloroethylene

Dichloromethane (Methylene chloride)

2,4-Dichlorophenoxyacetic acid

1,2-Dichloropropane

cis-1,3-Dichloropropene

cis-1,3-Dichloropropene

trans-1,3-Dichloropropene

Dieldrin

Endrin

Ethylbenzene

Fluoride

Heptachlor

Iron, total recoverable

Lead, total recoverable

Lindane

Magnesium, total recoverable

Manganese, total recoverable

Mercury, total recoverable

Methoxychlor

Naphthalene

Nickel, total recoverable

Nitrate-nitrite as nitrogen

Phenols

Potassium, total recoverable

Selenium, total recoverable

Silica, total recoverable

Silver, total recoverable

Sodium, total recoverable

Sulfate

$1,1,2,2$-Tetrachloroethane

Tetrachloroethylene

Tin, total recoverable

Toluene

Total dissolved solids

Total organic carbon

Total organic halogens

Total organic halogens

Total phosphates (as P)

Total phosphates (as P)

Toxaphene

2,4,5-TP (Silvex)

1,1,1-Trichloroethane

1,1,2-Trichloroethane

Trichloroethylene

Trichlorofluoromethane

2,4,5-T

Vanadium, total recoverable

Xylenes

Zinc, total recoverable

Carbon-14

Gross aipha

Nonvolatile beta

Radium, total alpha-emitting
Result DF

$<1.0$

$<1.0$

$<1.0$

$<1.0$

$<1.0$

$<0.0015$

$<1.0$

$<1.0$

$<1.0$

$<1.0$

$<0.50$

$<0.0060$

$<1.0$

$<100$

$<0.050$

6.2

$<3.0$

$<0.0050$

527

20

$<0.20$

$<0.50$

$<10$

$<4.0$

1,450

$<5.0$

$<500$

$<2.0$

8,470

$<2.0$

2,390

$<1,000$

$<1.0$

$<1.0$

$<2.0$

$<1.0$

34,000

$<1,000$

$<5.0$

$<5.0$

$<50$

$<50$

$<0.24$

$<0.00045$

$<1.0$

$<1.0$

$<1.0$

$<1.0$

0.41

$<8.0$

$<2.0$

$<2.0$

$5.5 \mathrm{E}+01$

$3.6 \mathrm{E}+00$

$1.4 \mathrm{E}+00$

$1.1 E+00$
Mod Unit Flag Lab

$\mu g / L \quad 0 \quad$ GE

$\mu \mathrm{g} / \mathrm{L} \quad 0 \quad \mathrm{GE}$

$\mu g / L \quad 0 \quad G E$

$\mu g / L \quad 0 \quad G E$

$\mu g / L \quad 0 \quad$ GE

$\mu g / L \quad 0 \quad G E$

$\mu g / L \quad 0 \quad G E$

$\mu g / L \quad 0 \quad G E$

$\mu g / L \quad 0 \quad G E$

$\mu g / L \quad 0 \quad G E$

$\mu g / L \quad 0 \quad G E$

$\mu g / L \quad 0 \quad G E$

$\mu \mathrm{g} / \mathrm{L} \quad 0 \quad \mathrm{GE}$

$\mu \mathrm{g} / \mathrm{L} \quad 0 \quad \mathrm{GE}$

$\mu g / L \quad 0 \quad G E$

$\mu g / L \quad 0 \quad G E$

$\mu g / L \quad 0 \quad G E$

$\mu g / L \quad 0 \quad G E$

$\mu g / L \quad 0 \quad G E$

$\mu \mathrm{g} / \mathrm{L} \quad 0 \quad \mathrm{GE}$

$\mu g / L \quad 0 \quad G E$

$\mu g / L \quad 0 \quad G E$

$\mu g / L \quad O \quad G E$

$\mu g / L \quad 0 \quad G E$

$\mu \mathrm{g} / \mathrm{L} \quad 0 \quad \mathrm{GE}$

$\mu g / L \quad 0 \quad \mathrm{GE}$

$\mu \mathrm{g} / \mathrm{L} \quad 0 \quad \mathrm{GE}$

$\mu g / L \quad 0 \quad \mathrm{GE}$

$\mu g / \mathrm{L} \quad 0 \quad \mathrm{GE}$

$\mu \mathrm{g} / \mathrm{L} \quad 0 \quad \mathrm{GE}$

$\mu \mathrm{g} / \mathrm{L} \quad \mathrm{O} \quad \mathrm{GE}$

$\mu g / L \quad 0 \quad G E$

$\mu g / L \quad 0 \quad G E$

$\mu g / L \quad 0 \quad G E$

$\mu g / L \quad 0 \quad G E$

$\mu g / L \quad 0 \quad \mathrm{GE}$

JV2 $\mu g / L \quad 0 \quad G E$

$\mu g / L \quad 0 \quad G E$

$\mu \mathrm{g} / \mathrm{L} \quad 0 \quad \mathrm{GE}$

$\mu \mathrm{g} / \mathrm{L} \quad 0 \quad \mathrm{GE}$

$\mu g / L \quad O \quad G E$

$\mu g / L \quad 0 \quad$ GE

$\mu \mathrm{g} / \mathrm{L} \quad 0 \quad \mathrm{GE}$

$\mu \mathrm{g} / \mathrm{L} \quad 0 \quad \mathrm{GE}$

$\mu g / L \quad 0 \quad G E$

$\mu \mathrm{g} / \mathrm{L} \quad \mathrm{O} \quad \mathrm{GE}$

$\mu \mathrm{g} / \mathrm{L} \quad \mathrm{O} \quad \mathrm{GE}$

$\mu \mathrm{g} / \mathrm{L} \quad 0 \quad \mathrm{GE}$

JV2 $\mu g / L \quad 0 \quad$ GE

$\mu g / L \quad 0 \quad G E$

$\mu \mathrm{g} / \mathrm{L} \quad \mathrm{O} \quad \mathrm{GE}$

$\mu \mathrm{g} / \mathrm{L} \quad 0 \quad \mathrm{GE}$

pCi/L $\quad 0 \quad$ GP

pCi/L $0 \quad$ GP

$\mathrm{pCi} / \mathrm{L} \quad \mathrm{O} \quad \mathrm{GP}$

$\mathrm{pCi} / \mathrm{L} \quad 0 \quad \mathrm{GP}$

$\overline{- \text { = exceeded holding time. }} \mathbf{a}=$ exceeded screening level or final primary drinking water standard. 
WELL BGO 9D collected on 04/04/94, laboratory analyses (cont.)

\author{
브 ㅁ Analyte \\ Total activity \\ - Tritium \\ Uranium-233/234 \\ Uranium-235 \\ Uranium-238
}

\begin{tabular}{|c|c|c|c|c|}
\hline Result & $\underline{\mathrm{DF}}$ & Mod & Unit & Flag \\
\hline $\begin{array}{r}1.6 \mathrm{E}+05 \\
1.6 \mathrm{E}+02 \\
1.2 \mathrm{E}-02 \\
<0.0 \mathrm{E}+00 \\
1.0 \mathrm{E}-01\end{array}$ & $\begin{array}{l}1 \\
1 \\
1 \\
1 \\
1\end{array}$ & $\begin{array}{l}\text { UI } \\
\text { J }\end{array}$ & $\begin{array}{l}\mathrm{pCi} / \mathrm{L} \\
\mathrm{pCi} / \mathrm{mL} \\
\mathrm{pCi} / \mathrm{L} \\
\mathrm{pCi} / \mathrm{L} \\
\mathrm{pCi} / \mathrm{L}\end{array}$ & $\begin{array}{l}0 \\
2 \\
0 \\
0 \\
0\end{array}$ \\
\hline
\end{tabular}

WELL BGO 10AA

\begin{tabular}{|c|c|c|c|c|c|c|}
\hline SRS Coord. & Lat/Longitude & Screen Zone Elevation & Top of Casing & Casing & Pump & Formation \\
\hline $\begin{array}{l}\text { N76997.9 } \\
\text { E56990.5 }\end{array}$ & $\begin{array}{l}33.289057^{\circ} \mathrm{N} \\
81.666300^{\circ} \mathrm{W}\end{array}$ & $90.8-80.8 \mathrm{ft} \mathrm{msl}$ & $300.7 \mathrm{ft} \mathrm{msl}$ & $4^{\prime \prime}$ PVC & $\mathbf{s}$ & L. Congaree (IIA) \\
\hline
\end{tabular}

Sample date: 04/04/94

Depth to water: $142.98 \mathrm{ft}(43.58 \mathrm{~m})$ below TOC

Water elevation: $157.72 \mathrm{ft}(48.07 \mathrm{~m}) \mathrm{ms}$ )

Sp. conductance: $222 \mu \mathrm{S} / \mathrm{cm}$

Turbidity: 1.9 NTU

Water evacuated before sampling: $131 \mathrm{gal}$

\section{LABORATORY ANALYSES}

\section{H D Analyte}

$\mathrm{pH}$

Specific conductance

Turbidity

- Acetophenone

Aldrin

Aluminum, total recoverable

Aluminum, total recoverable

Antimony, total recoverable

Arsenic, total recoverable

Barium, total recoverable

Barium, total recoverable

Benzene

Bromodichloromethane

Bromoform

Bromomethane (Methyl bromide)

Cadmium, total recoverable

Cadmium, total recoverable

Calcium, total recoverable

Calcium, total recoverable

Carbon tetrachloride

Chloride

Chlorobenzene

Chloroethane

Chloroethene (Vinyl chloride)

2-Chloroethyl vinyl ether

Chloroform

Chloromethane (Methyl chloride)

Chromium, total recoverable

Chromium, total recoverable
Time: 15:50

pH: 7.6

Alkalinity: $74 \mathrm{mg} / \mathrm{L}$

Water temperature: $19.7^{\circ} \mathrm{C}$

Volumes purged: 2.6 well volumes

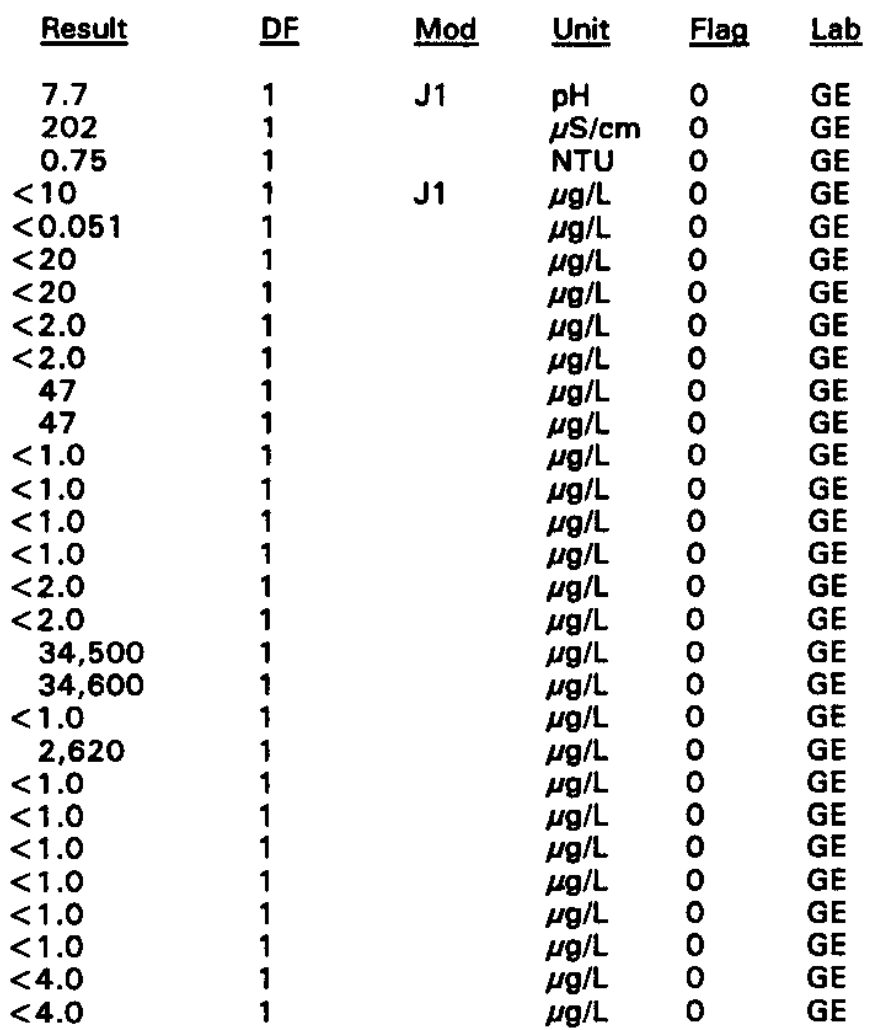

$\overline{- \text { = exceeded holding time. }} \mathbf{a}=$ exceeded screening level or final primary drinking water standard. 
WELL BGO 10AA collected on 04/04/94, laboratory analyses (cont.)

H D Analyte

Copper, total recoverable

Copper, total recoverable

Cyanide

p.p'-DDT

Dibromochloromethane

1,1-Dichloroethane

1,2-Dichloroethane

1,1-Dichloroethylene

trans-1,2-Dichloroethylene

Dichloromethane (Methylene chloride)

2,4-Dichlorophenoxyacetic acid

1,2-Dichloropropane

cis-1,3-Dichloropropene

cis-1,3-Dichloropropene

trans-1,3-Dichloropropene

Dieldrin

Endrin

Ethylbenzene

Fluoride

Fluoride

Heptachlor

Iron, total recoverable

Iron, total recoverable

Lead, total recoverable

Lindane

Magnesium, total recoverable

Magnesium, total recoverable

Manganese, total recoverable

Manganese, total recoverable

Mercury, total recoverable

Methoxychlor

Naphthalene

Nickel, total recoverable

Nickel, total recoverable

Nitrate-nitrite as nitrogen

Phenols

Potassium, total recoverable

Potassium, total recoverable

Selenium, total recoverable

Silica, total recoverable

Silica, total recoverable

Silver, total recoverable

Silver, total recoverable

Sodium, total recoverable

Sodium, total recoverable

Sulfate

1,1,2,2-Tetrachloroethane

Tetrachloroethylene

Tin, total recoverable

Tin, total recoverable

Toluene

Total dissolved solids

Total dissolved solids

Total organic carbon

Total organic carbon

Total organic halogens

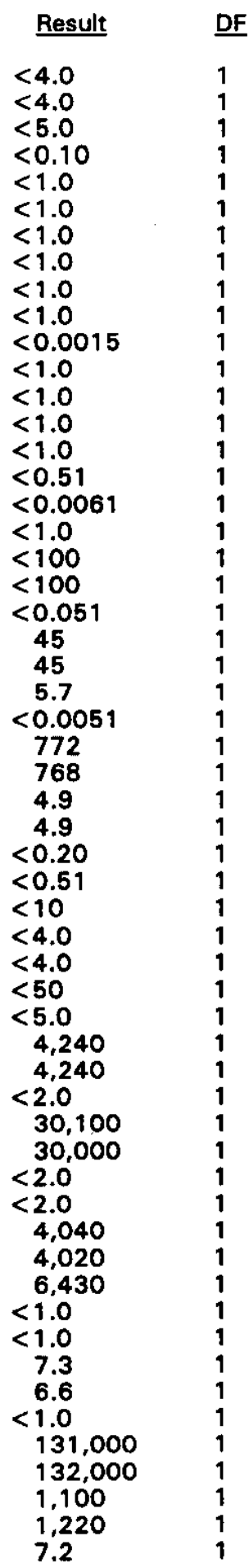

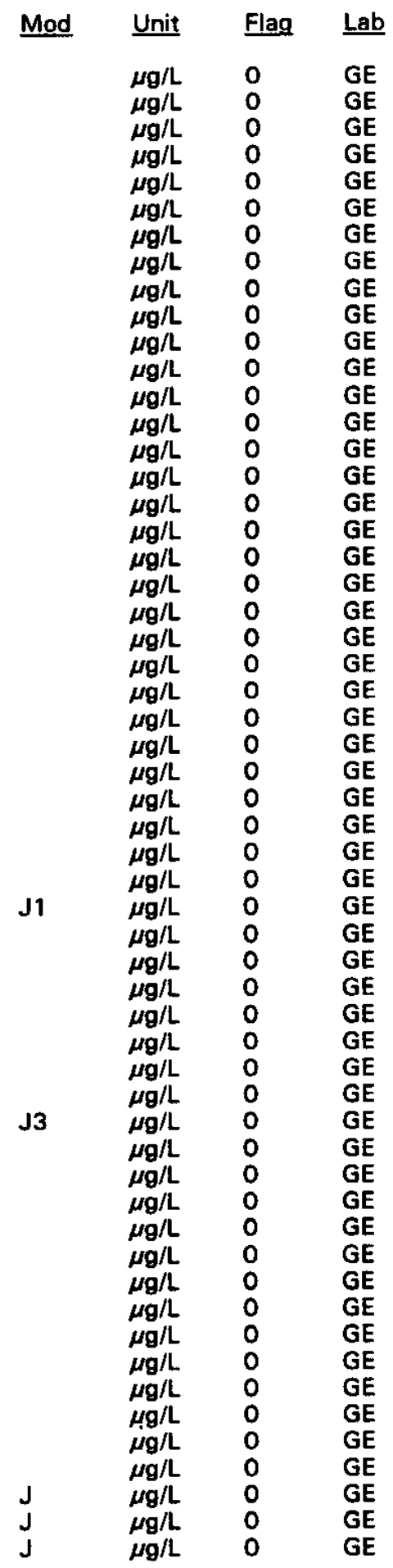

$\overline{- \text { = exceeded holding time. }}$ = exceeded screening level or final primary drinking water standard. 
WELL BGO 1OAA collected on 04/04/94, laboratory analyses (cont.)

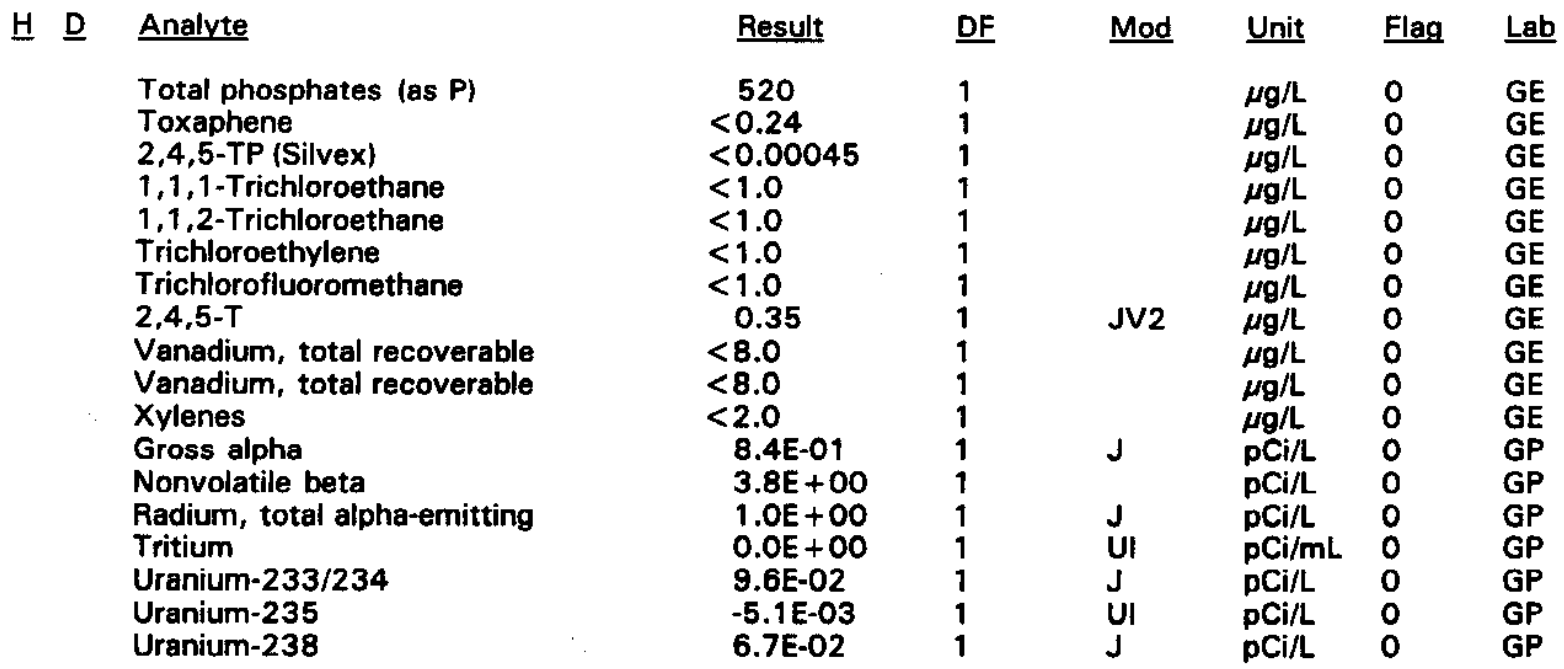

\section{WELL BGO 10AR}

\begin{tabular}{|c|c|c|c|c|c|c|}
\hline SRS Coord. & Lat/Longitude & Screen Zone Elevation & Top of Casing & Cosing & Pump & Formation \\
\hline $\begin{array}{l}\text { N76806.0 } \\
\text { E57063.8 }\end{array}$ & $\begin{array}{l}33.2887522^{\circ} \mathrm{N} \\
81.665734^{\circ} \mathrm{W}\end{array}$ & $106.5-96.5 \mathrm{ft} \mathrm{msl}$ & $300.5 \mathrm{ft} \mathrm{msl}$ & 4" PVC & $\mathbf{S}$ & U. Congaree (IIA) \\
\hline
\end{tabular}

\section{FIELD MEASUREMENTS}

Sample date: $04 / 05 / 94$

Depth to water: $142.24 \mathrm{ft}(43.36 \mathrm{~m})$ below TOC

Water elevation: $158.26 \mathrm{ft}(48.24 \mathrm{~m}) \mathrm{msl}$

Sp. conductance: $261 \mu \mathrm{S} / \mathrm{cm}$

Turbidity: 0.3 NTU

Water evacuated before sampling: $150 \mathrm{gal}$

\section{LABORATORY ANALYSES}

H D Analyte

$\mathrm{pH}$

Specific conductance

Turbidity

Acetophenone

Aldrin

Aluminum, total recoverable

Antimony, total recoverable

Arsenic, total recoverable

Barium, total recoverable

Benzene

Bromodichloromethane

Bromoform

Bromomethane (Methyl bromide)

Cadmium, total recoverable

Calcium, total recoverable

Carbon tetrachloride
Time: $11: 43$

pH: 7.8

Alkalinity: $99 \mathrm{mg} / \mathrm{L}$

Water temperature: $20.1^{\circ} \mathrm{C}$

Volumes purged: 3.7 well volumes

\begin{tabular}{|c|c|c|c|c|}
\hline Result & DF & Mod & Unit & Flag \\
\hline $\begin{aligned} & 8.0 \\
& 244 \\
& 0.26 \\
&< 10 \\
&< 0.051 \\
&< 20 \\
&< 2.0 \\
&< 2.0 \\
& 18 \\
&< 1.0 \\
&<1.0 \\
&<1.0 \\
&<1.0 \\
&<2.0 \\
&<6.000 \\
&<1.0\end{aligned}$ & $\begin{array}{l}1 \\
1 \\
1 \\
1 \\
1 \\
1 \\
1 \\
1 \\
1 \\
1 \\
1 \\
1 \\
1 \\
1 \\
1 \\
1\end{array}$ & J1 & $\begin{array}{l}\mathrm{pH} \\
\mu S / \mathrm{cm} \\
\mathrm{NTU} \\
\mu \mathrm{g} / \mathrm{L} \\
\mu g / \mathrm{L} \\
\mu \mathrm{g} / \mathrm{L} \\
\mu \mathrm{g} / \mathrm{L} \\
\mu \mathrm{g} / \mathrm{L} \\
\mu \mathrm{g} / \mathrm{L} \\
\mu \mathrm{g} / \mathrm{L} \\
\mu \mathrm{g} / \mathrm{L} \\
\mu \mathrm{g} / \mathrm{L} \\
\mu \mathrm{g} / \mathrm{L} \\
\mu \mathrm{g} / \mathrm{L} \\
\mu \mathrm{g} / \mathrm{L} \\
\mu \mathrm{g} / \mathrm{L}\end{array}$ & $\begin{array}{l}0 \\
0 \\
0 \\
0 \\
0 \\
0 \\
0 \\
0 \\
0 \\
0 \\
0 \\
0 \\
0 \\
0 \\
0 \\
0\end{array}$ \\
\hline
\end{tabular}

- =xceeded holding time. = exceeded screening level or final primary drinking water standard. 
WELL BGO 1OAR collected on 04/05/94, laboratory analyses (cont.)

브

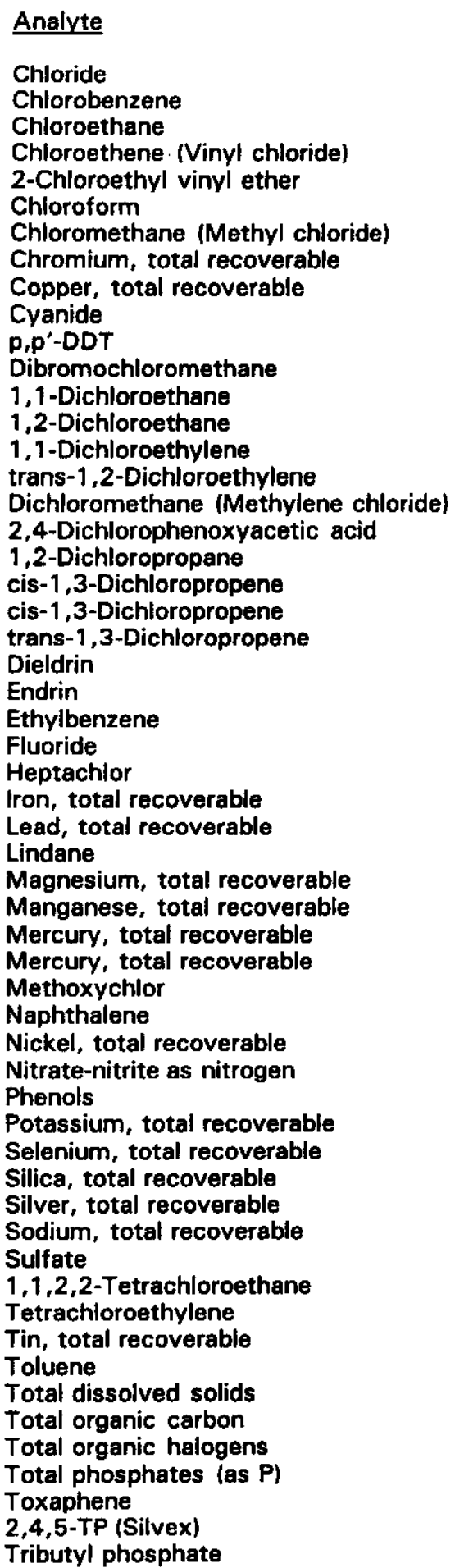

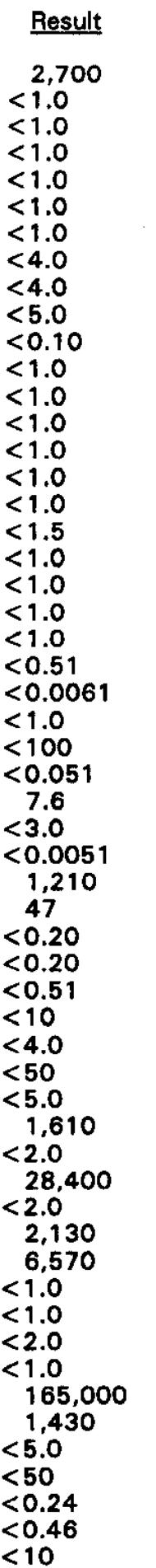

Mod Unit Flag Lab

$\mu \mathrm{g} / \mathrm{L} \quad 0 \quad \mathrm{GE}$

$\mu g / L \quad 0 \quad G E$

$\mu g / L \quad 0 \quad G E$

$\mu g / L \quad 0 \quad G E$

$\mu \mathrm{g} / \mathrm{L} \quad 0 \quad \mathrm{GE}$

$\mu \mathrm{g} / \mathrm{L} \quad 0 \quad \mathrm{GE}$

$\mu g / L \quad 0 \quad$ GE

$\mu \mathrm{g} / \mathrm{L} \quad \mathrm{O} \quad \mathrm{GE}$

$\mu g / L \quad 0 \quad$ GE

$\mu g / L \quad 0 \quad G E$

$\mu \mathrm{g} / \mathrm{L} \quad 0 \quad \mathrm{GE}$

$\mu g / L \quad 0 \quad$ GE

$\mu g / L \quad 0 \quad$ GE

$\mu g / L \quad 0 \quad$ GE

$\mu g / L \quad 0 \quad$ GE

$\mu g / L \quad 0 \quad$ GE

$\mu g / L \quad 0 \quad$ GE

$\mu g / L \quad 0 \quad$ GE

$\mu \mathrm{g} / \mathrm{L} \quad 0 \quad \mathrm{GE}$

$\mu \mathrm{g} / \mathrm{L} \quad 0 \quad \mathrm{GE}$

$\mu \mathrm{g} / \mathrm{L} \quad 0 \quad \mathrm{GE}$

$\mu g / L \quad 0 \quad G E$

$\mu g / L \quad 0 \quad$ GE

$\mu g / L \quad 0 \quad G E$

$\mu g / L \quad 0 \quad G E$

$\mu \mathrm{g} / \mathrm{L} \quad 0 \quad \mathrm{GE}$

$\mu g / L \quad 0 \quad$ GE

$\mu \mathrm{g} / \mathrm{L} \quad 0 \quad \mathrm{GE}$

$\mu g / L \quad 0 \quad G E$

$\mu g / L \quad 0 \quad$ GE

$\mu g / L \quad 0 \quad$ GE

$\mu \mathrm{g} / \mathrm{L} \quad 1 \quad \mathrm{GE}$

$\mu \mathrm{g} / \mathrm{L} \quad 0 \quad \mathrm{GE}$

$\mu g / L \quad O \quad$ GE

$\mu g / L \quad 0 \quad G E$

$\mu g / L \quad O \quad$ GE

$\mu g / L \quad 0 \quad G E$

$\mu \mathrm{g} / \mathrm{L} \quad \mathrm{G} \quad \mathrm{GE}$

$\mu g / L \quad 0 \quad G E$

$\mu \mathrm{g} / \mathrm{L} \quad \mathrm{O} \quad \mathrm{GE}$

$\mu g / L \quad O \quad$ GE

$\mu g / L \quad 0 \quad$ GE

$\mu g / L \quad 0 \quad G E$

$\mu g / L \quad 0 \quad$ GE

$\mu g / L \quad 0 \quad$ GE

$\mu \mathrm{g} / \mathrm{L} \quad 0 \quad \mathrm{GE}$

$\mu g / L \quad 0 \quad$ GE

$\mu g / L \quad 0 \quad G E$

$\mu g / L \quad 0 \quad$ GE

JV2 $\mu g / L \quad 0 \quad$ GE

J $\mu g / L \quad 0 \quad$ GE

$\mu g / L \quad 0 \quad$ GE

$\mu g / L \quad 0 \quad$ GE

$\mu \mathrm{g} / \mathrm{L} \quad 0 \quad \mathrm{GE}$

$\mu g / L \quad 0 \quad$ GE

- =exceeded holding time. $=$ exceeded screening level or final primary drinking water standard. 
WELL BGO 10AR collected on 04/05/94, laboratory analyses (cont.)

H $\underline{\text { Analyte }}$

1,1,1-Trichloroethane

1,1,2-Trichloroethane

Trichloroethylene

Trichlorofluoromethane

2,4,5-T

Vanadium, total recoverable

Xylenes

Zinc, total recoverable

Carbon-14

Gross alpha

Nonvolatile beta

Radium, total alpha-emitting

Tritium

Uranium-233/234

Uranium-235

Uranium-238

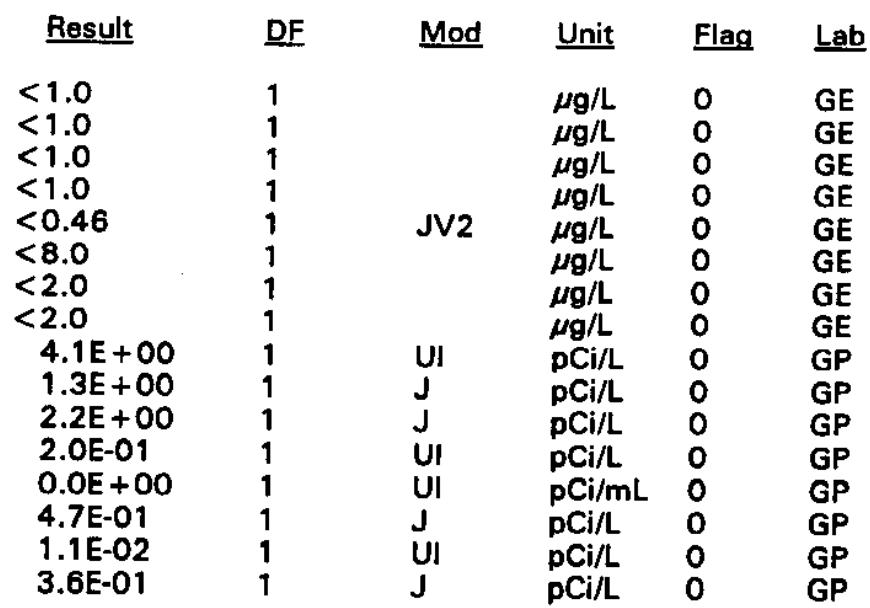

\section{WELL BGO 10B}

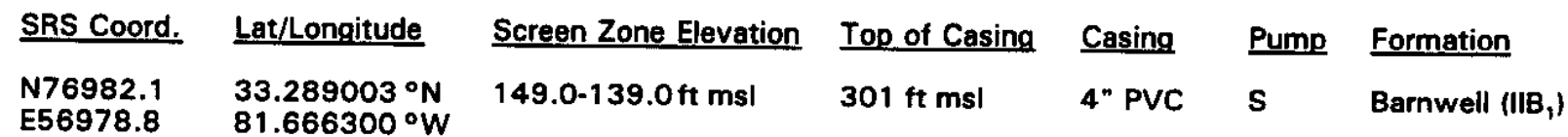

\section{FIELD MEASUREMENTS}

Sample date: 04/05/94

Depth to water: $81.05 \mathrm{ft}(24.70 \mathrm{~m})$ below TOC

Water elevation: $219.95 \mathrm{ft}(67.04 \mathrm{~m}) \mathrm{msl}$

Sp. conductance: $253 \mu \mathrm{S} / \mathrm{cm}$

Turbidity: 1.3 NTU

Water evacuated before sampling: $51 \mathrm{gal}$

The well went dry during purging.

\section{LABORATORY ANALYSES}

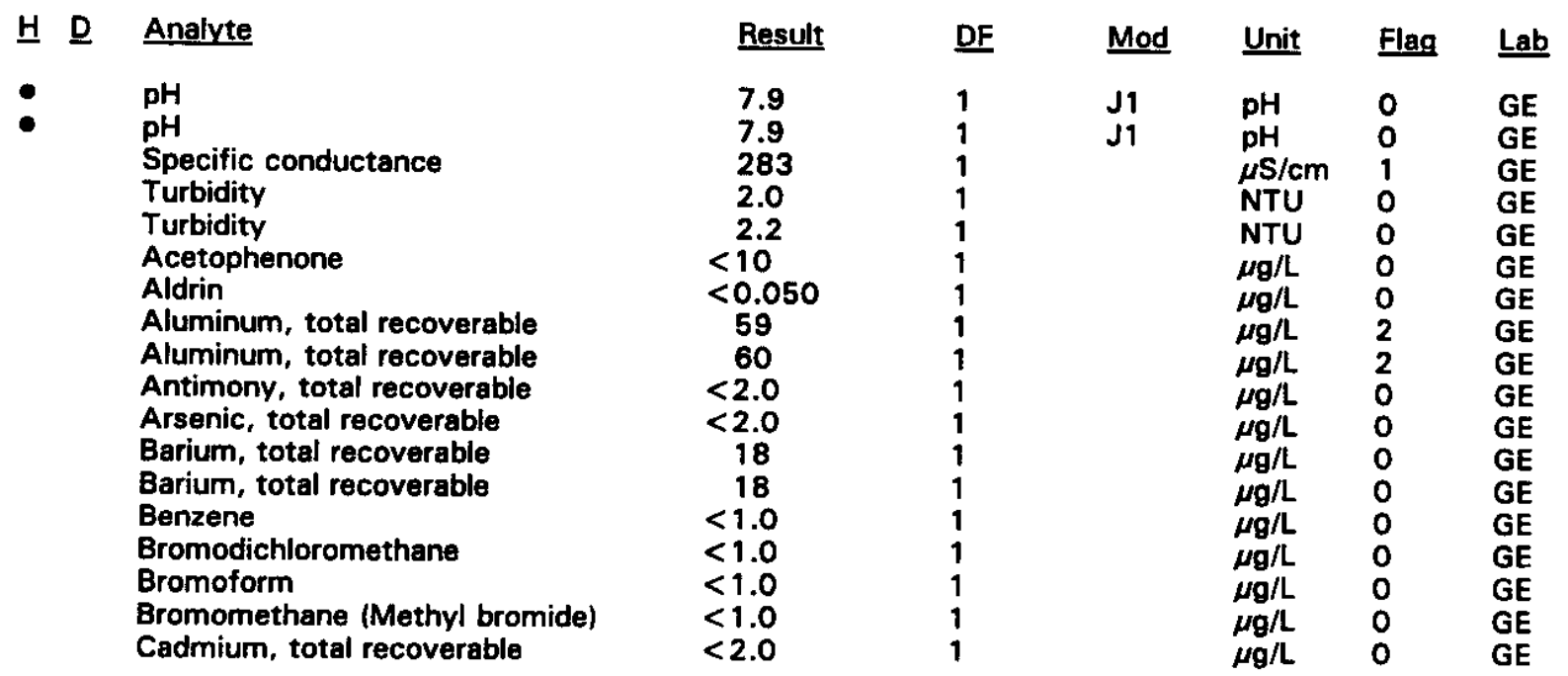

pH: 8.6

Alkalinity: $95 \mathrm{mg} / \mathrm{L}$

Water temperature: $18.4^{\circ} \mathrm{C}$

Volumes purged: 1.0 well volumes
Time: 9:51 
WELL BGO $10 B$ collected on $04 / 05 / 94$, laboratory analyses (cont.)

H $\underline{\text { Analyte }}$

Cadmium, total recoverable

Calcium, total recoverable

Calcium, total recoverable

Carbon tetrachloride

Chloride

Chlorobenzene

Chloroethane

Chloroethene (Vinyl chloride)

2-Chloroethyl vinyl ether

Chloroform

Chloromethane (Methyl chloride)

Chromium, total recoverable

Chromium, total recoverable

Copper, total recoverable

Copper, total recoverable

Cyanide

$p, p^{\prime}$-DDT

Dibromochloromethane

1,1-Dichloroethane

1,2-Dichloroethane

1,1-Dichloroethylene

trans-1,2-Dichloroethylene

Dichloromethane (Methylene chloride)

2,4-Dichlorophenoxyacetic acid

1,2-Dichloropropane

cis-1,3-Dichloropropene

cis-1,3-Dichloropropene

trans-1,3-Dichloropropene

Dieldrin

Endrin

Ethylbenzene

Fluoride

Fluoride

Heptachlor

Iron, total recoverable

Iron, total recoverable

Lead, total recoverable

Lindane

Magnesium, total recoverable

Magnesium, total recoverable

Manganese, total recoverable

Manganese, total recoverable

Mercury, total recoverable

Methoxychlor

Naphthalene

Nickel, total recoverable

Nickel, total recoverable

Nitrate-nitrite as nitrogen

Nitrate-nitrite as nitrogen

Nitrate-nitrite as nitrogen

Phenols

Potassium, total recoverable

Potassium, total recoverable

Selenium, total recoverable

Silica, total recoverable

Silica, total recoverable
Result $\quad \underline{D F}$

$<2.0$
46,300
46,200

$<1.0$

2,510

$<1.0$

$<1.0$

$<1.0$

$<1.0$

$<1.0$

$<1.0$

$<4.0$

$<4.0$

$<4.0$

$<4.0$

$<5.0$

$<0.10$

$<1.0$

$<1.0$

$<1.0$

$<1.0$

$<1.0$

$<1.0$

$<0.0015$

$<1.0$

$<1.0$

$<1.0$

$<1.0$

$<0.50$

$<0.0060$

$<1.0$

$<100$

$<100$

$<0.050$

105

106

$<3.0$

$<0.0050$

1,560

1,560

2.8

2.9

$<0.20$

$<0.50$

$<10$

5.4

5.5

$<50$

$<50$

$<50$

$<5.0$

3,840

3,780

$<2.0$

38,300

38,200
$\underline{\mathrm{DF}}$

Mod

$\underline{\text { Unit }}$

Flag

$\underline{\text { Lab }}$

$\mu \mathrm{g} / \mathrm{L} \quad 0 \quad \mathrm{GE}$

$\mu g / L \quad 0 \quad$ GE

$\mu \mathrm{g} / \mathrm{L} \quad \mathrm{O} \quad \mathrm{GE}$

$\mu g / L \quad 0 \quad G E$

$\mu \mathrm{g} / \mathrm{L} \quad 0 \quad \mathrm{GE}$

$\mu g / L \quad 0 \quad G E$

$\mu g / L \quad 0 \quad$ GE

$\mu \mathrm{g} / \mathrm{L} \quad 0 \quad \mathrm{GE}$

$\mu \mathrm{g} / \mathrm{L} \quad 0 \quad \mathrm{GE}$

$\mu g / L \quad 0 \quad$ GE

$\mu g / L \quad 0 \quad$ GE

$\mu g / L \quad 0 \quad$ GE

$\mu g / L \quad 0 \quad G E$

$\mu \mathrm{g} / \mathrm{L} \quad 0 \quad \mathrm{GE}$

$\mu \mathrm{g} / \mathrm{L} \quad 0 \quad \mathrm{GE}$

$\mu g / L \quad 0 \quad G E$

$\mu g / L \quad 0 \quad$ GE

$\mu \mathrm{g} / \mathrm{L} \quad 0 \quad \mathrm{GE}$

$\mu \mathrm{g} / \mathrm{L} \quad \mathrm{O} \quad \mathrm{GE}$

$\mu g / L \quad 0 \quad$ GE

$\mu \mathrm{g} / \mathrm{L} \quad 0 \quad \mathrm{GE}$

$\mu g / L \quad 0 \quad$ GE

$\mu g / \mathrm{L} \quad 0 \quad \mathrm{GE}$

$\mu g / L \quad 0 \quad$ GE

$\mu \mathrm{g} / \mathrm{L} \quad 0 \quad \mathrm{GE}$

$\mu \mathrm{g} / \mathrm{L} \quad 0 \quad \mathrm{GE}$

$\mu g / L \quad 0 \quad G E$

$\mu g / L \quad 0 \quad$ GE

$\mu \mathrm{g} / \mathrm{L} \quad 0 \quad \mathrm{GE}$

$\mu \mathrm{g} / \mathrm{L} \quad 0 \quad \mathrm{GE}$

$\mu g / L \quad 0 \quad$ GE

$\mu g / L \quad 0 \quad$ GE

$\mu \mathrm{g} / \mathrm{L} \quad 0 \quad \mathrm{GE}$

$\mu \mathrm{g} / \mathrm{L} \quad \mathrm{O} \quad \mathrm{GE}$

$\mu \mathrm{g} / \mathrm{L} \quad 0 \quad \mathrm{GE}$

$\mu \mathrm{g} / \mathrm{h} \quad \mathrm{O} \quad \mathrm{GE}$

$\mu \mathrm{g} / \mathrm{L} \quad 0 \quad \mathrm{GE}$

$\mu \mathrm{g} / \mathrm{L} \quad 0 \quad \mathrm{GE}$

$\mu \mathrm{g} / \mathrm{L} \quad \mathrm{O} \quad \mathrm{GE}$

$\mu g / L \quad O \quad$ GE

$\mu g / L \quad 0 \quad$ GE

$\mu g / L \quad 0 \quad$ GE

$\mu \mathrm{g} / \mathrm{L} \quad 0 \quad \mathrm{GE}$

$\mu g / L \quad 0 \quad$ GE

$\mu \mathrm{g} / \mathrm{L} \quad 0 \quad \mathrm{GE}$

$\mu g / L \quad 0 \quad$ GE

$\mu \mathrm{g} / \mathrm{L} \quad 0 \quad \mathrm{GE}$

$\mu g / L \quad 0 \quad$ GE

$\mu \mathrm{g} / \mathrm{L} \quad 0 \quad \mathrm{GE}$

$\mu \mathrm{g} / \mathrm{L} \quad 0 \quad \mathrm{GE}$

$\mu \mathrm{g} / \mathrm{L} \quad 0 \quad \mathrm{GE}$

$\mu g / L \quad 0 \quad$ GE

$\mu g / L \quad 0 \quad$ GE

$\mu g / L \quad 0 \quad$ GE

$\mu g / L \quad 0 \quad$ GE

$\mu g / L \quad 0 \quad G E$

$\overline{-}=$ exceeded holding time. $=$ exceeded screening level or final primary drinking water standard. 
WELL BGO $10 \mathrm{~B}$ collected on $04 / 05 / 94$, laboratory analyses (cont.)

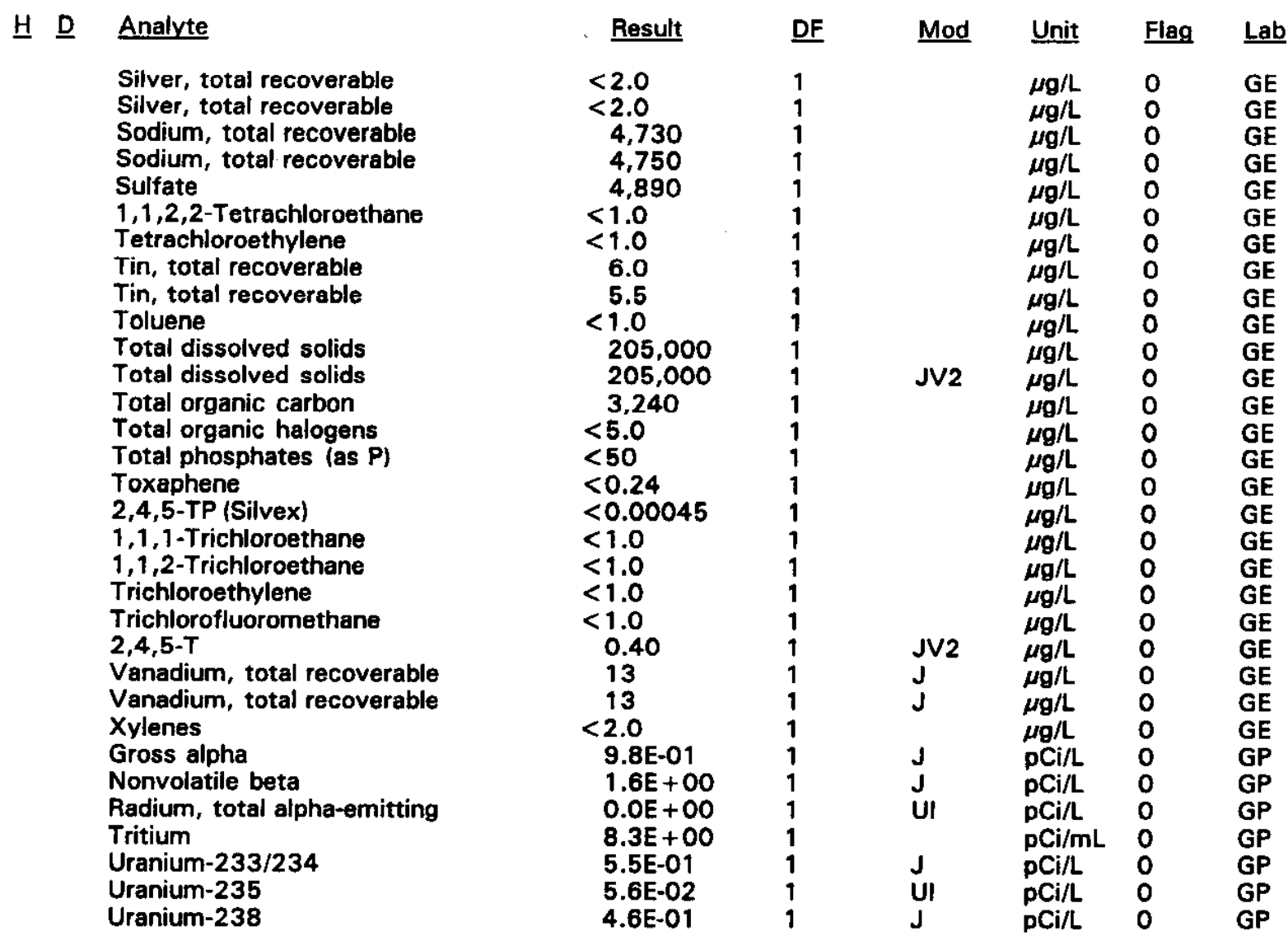

\section{WELL BGO 10C}

\begin{tabular}{|c|c|c|c|c|c|c|}
\hline SRS Coord. & Lat/Longitude & Screen Zone Elevation & Top of Casing & Casing & Pump & Formation \\
\hline $\begin{array}{l}\text { N76805.2 } \\
\text { E57041.1 }\end{array}$ & $\begin{array}{l}33.288713^{\circ} \mathrm{N} \\
81.665792^{\circ} \mathrm{W}\end{array}$ & $167.3-157.3 \mathrm{ft} \mathrm{msl}$ & $301.3 \mathrm{ft} \mathrm{msl}$ & 4" PVC & $\mathbf{s}$ & Barnwe \\
\hline
\end{tabular}

\section{FIELD MEASUREMENTS}

Sample date: 04/05/94

Depth to water: $80.63 \mathrm{ft}(24.58 \mathrm{~m})$ below TOC

Water elevation: $220.67 \mathrm{ft}(67.26 \mathrm{~m}) \mathrm{msl}$

Sp. conductance: $98 \mu \mathrm{S} / \mathrm{cm}$

Turbidity: 94.4 NTU

Water evacuated before sampling: $40 \mathrm{gal}$

The well went dry during purging.

\section{LABORÁTORY ANALYSES}

H D Analyte

$\bullet \quad \mathrm{pH}$
Result

7.9
Time: $11: 19$

pH: 9.3

Alkalinity: $40 \mathrm{mg} / \mathrm{L}$

Water temperature: $20.2^{\circ} \mathrm{C}$

Volumes purged: 1.0 well volumes

$\overline{-}=$ exceeded holding time. $=$ exceeded screening level or final primary drinking water standard. 
WELL BGO $10 \mathrm{C}$ collected on $04 / 05 / 94$, laboratory analyses (cont.)

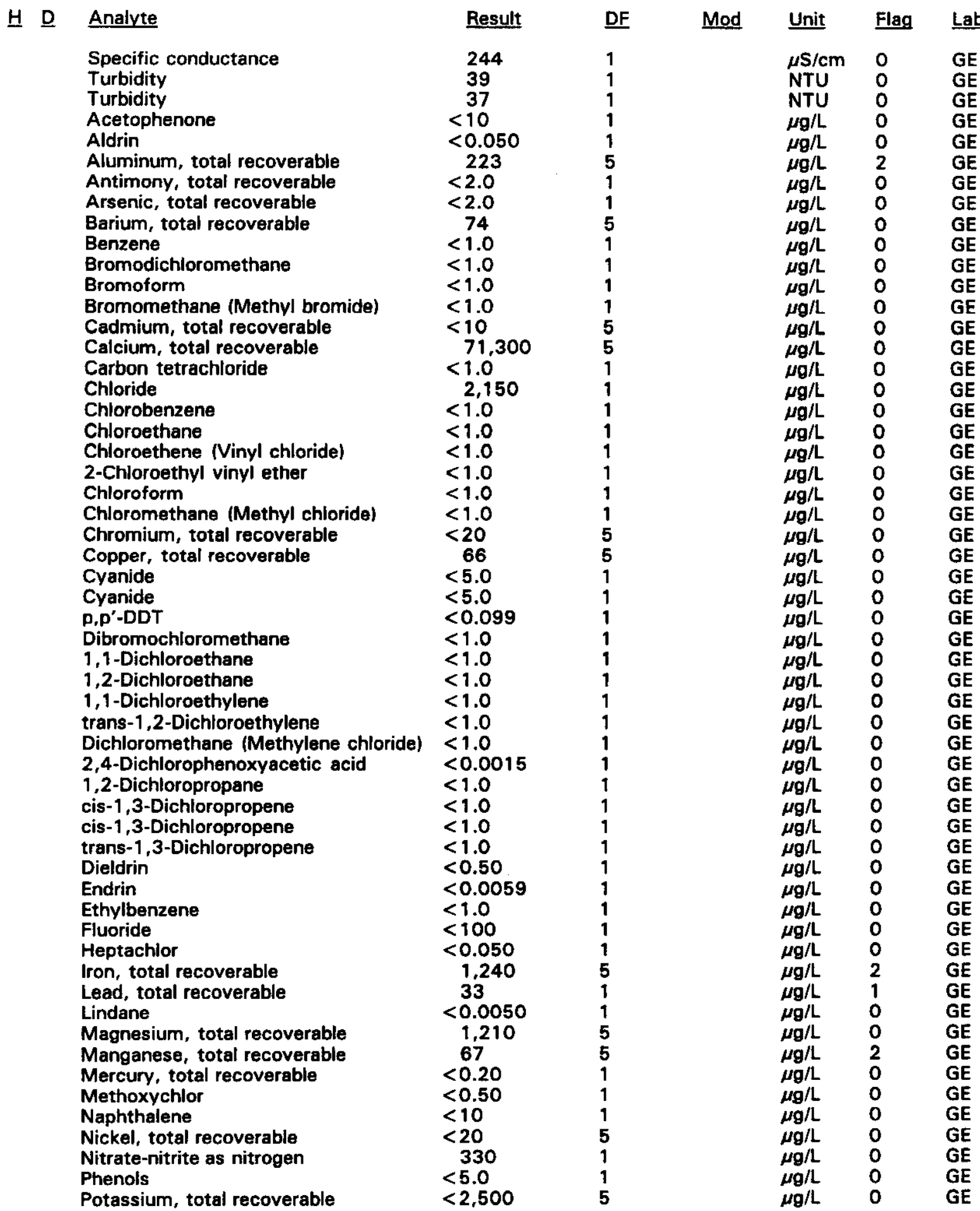

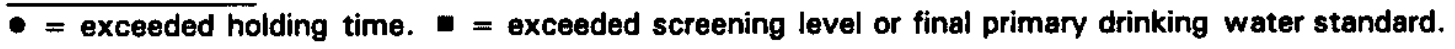


WELL BGO $10 \mathrm{C}$ collected on $04 / 05 / 94$, laboratory analyses (cont.)

\section{H ㅁ Analyte}

Selenium, total recoverable

Silica, total recoverable

Silver, total recoverable

Sodium, total recoverable

Sulfate

$1,1,2,2$-Tetrachloroethane

Tetrachloroethylene

Tin, total recoverable

Toluene

Total dissolved solids

Total dissolved solids

Total organic carbon

Total organic halogens

Total phosphates (as P)

Toxaphene

2,4,5-TP (Silvex)

1,1,1-Trichloroethane

1,1,2-Trichloroethane

Trichloroethylene

Trichlorofluoromethane

2,4,5-T

Vanadium, total recoverable

Xylenes

Zinc, total recoverable

Carbon-14

Gross alpha

Nonvolatile beta

Radium, total alpha-emitting

Tritium

Tritium

Uranium-233/234

Uranium-235

Uranium-238
Result DF

$<2.0$

23,900

$<10$

3,630

3,930

$<1.0$

$<1.0$

$<10$

$<1.0$

161,000

161,000

1,340

$<5.0$

190

$<0.24$

$<0.00044$

$<1.0$

$<1.0$

$<1.0$

$<1.0$

0.39

$<40$

$<2.0$

124

$4.8 \mathrm{E}+00$

8.7E-01

$1.0 E+00$

3.0E-01

$1.9 E+00$

$1.9 E+00$

6.9E-01

4.5E-02

5.2E-01
DF

Mod

Unit Flag Lab

$\mu g / L \quad 0 \quad G E$

$\mu g / L \quad O \quad G E$

$\mu g / L \quad 0 \quad G E$

$\mu g / L \quad 0 \quad G E$

$\mu g / L \quad 0 \quad G E$

$\mu g / L \quad 0 \quad G E$

$\mu g / L \quad 0 \quad \mathrm{GE}$

$\mu \mathrm{g} / \mathrm{L} \quad 0 \quad \mathrm{GE}$

$\mu g / L \quad 0 \quad$ GE

$\mu g / L \quad 0 \quad \mathrm{GE}$

JV2 $\mu g / L \quad 0 \quad G E$

$J \quad \mu g / L \quad O \quad G E$

$\mu g / L \quad 0 \quad G E$

$\mu g / L \quad O \quad G E$

$\mu g / L \quad O \quad G E$

$\mu g / L \quad 0 \quad G E$

$\mu g / L \quad 0 \quad$ GE

$\mu g / L \quad 0 \quad G E$

$\mu g / L \quad 0 \quad G E$

$\mu g / L \quad 0 \quad G E$

$\begin{array}{llll}J V 2 & \mu g / L & 0 & G E\end{array}$

$\mu g / L \quad 0 \quad G E$

$\mu \mathrm{g} / \mathrm{L} \quad \mathrm{O} \quad \mathrm{GE}$

$\mu g / L \quad 0 \quad \mathrm{GE}$

$\mathrm{pCi} / \mathrm{L} \quad 0 \quad \mathrm{GP}$

$\mathrm{pCi} / L \quad 0 \quad$ GP

$p C i / L \quad 0 \quad G P$

$\mathrm{pCi} / \mathrm{L} \quad 0 \quad \mathrm{GP}$

$\mathrm{pCi} / \mathrm{mL} \quad 0 \quad \mathrm{GP}$

$\mathrm{pCi} / \mathrm{mL} \quad 0 \quad \mathrm{GP}$

$\mathrm{pCi} / \mathrm{L} \quad \mathrm{GP}$

$\mathrm{pCi} / \mathrm{L} \quad 0 \quad \mathrm{GP}$

pCi/L 0 GP

- exceeded holding time. - =xceeded screening level or final primary drinking water standard. 
WELL BGO 1ODR collected on 04/05/94, laboratory analyses (cont.)

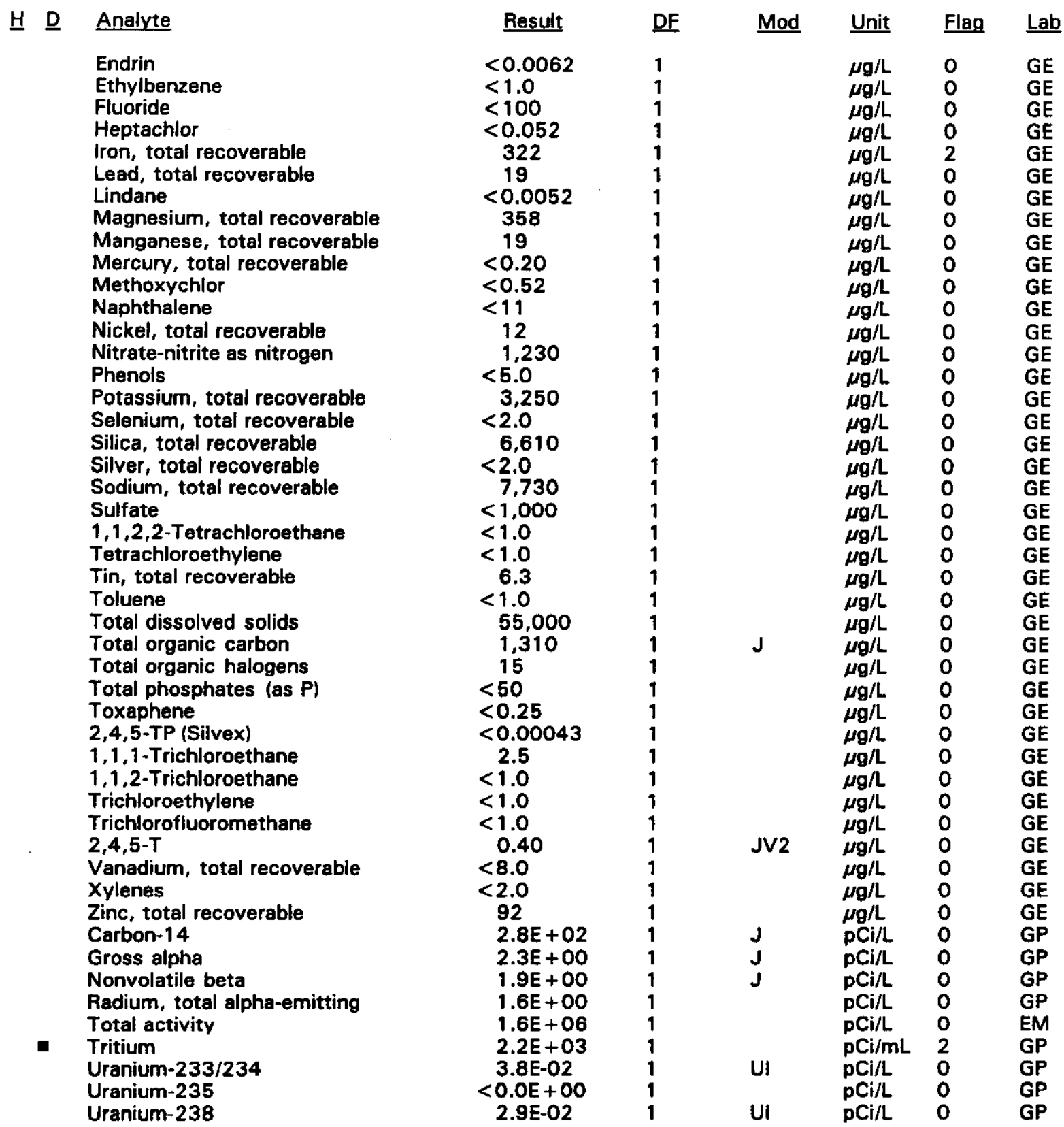

- = exceeded holding time. = exceeded screening level or final primary drinking water standard. 
WELL BGO 11D

\begin{tabular}{|c|c|c|c|c|c|c|}
\hline SRS Coord. & Lat/Longitude & Screen Zone Elevation & Top of Casing & Casing & Pump & Formation \\
\hline $\begin{array}{l}\text { N76805.1 } \\
\text { E56651.3 }\end{array}$ & $\begin{array}{l}33.288077^{\circ} \mathrm{N} \\
81.666819^{\circ} \mathrm{W}\end{array}$ & $236.3-216.3 \mathrm{ft} \mathrm{msl}$ & $305.3 \mathrm{ft} \mathrm{msl}$ & 4" PVC & $\mathbf{S}$ & Water Table $\left(\| \mathrm{IB}_{2}\right)$ \\
\hline
\end{tabular}

\section{FIELD MEASUREMENTS}

Sample date: 04/05/94

Depth to water: $73.47 \mathrm{ft}(22.39 \mathrm{~m})$ below TOC

Water elevation: $231.83 \mathrm{ft}(70.66 \mathrm{~m}) \mathrm{msl}$

Sp. conductance: $42 \mu \mathrm{S} / \mathrm{cm}$

Turbidity: 0.3 NTU

Water evacuated before sampling: $\mathbf{4 0}$ gal

Time: 12:41

$\mathrm{pH}: 4.8$

Alkalinity: $0 \mathrm{mg} / \mathrm{L}$

Water temperature: $21.2^{\circ} \mathrm{C}$

Volumes purged: 3.9 well volumes

\section{LABORATORY ANALYSES}

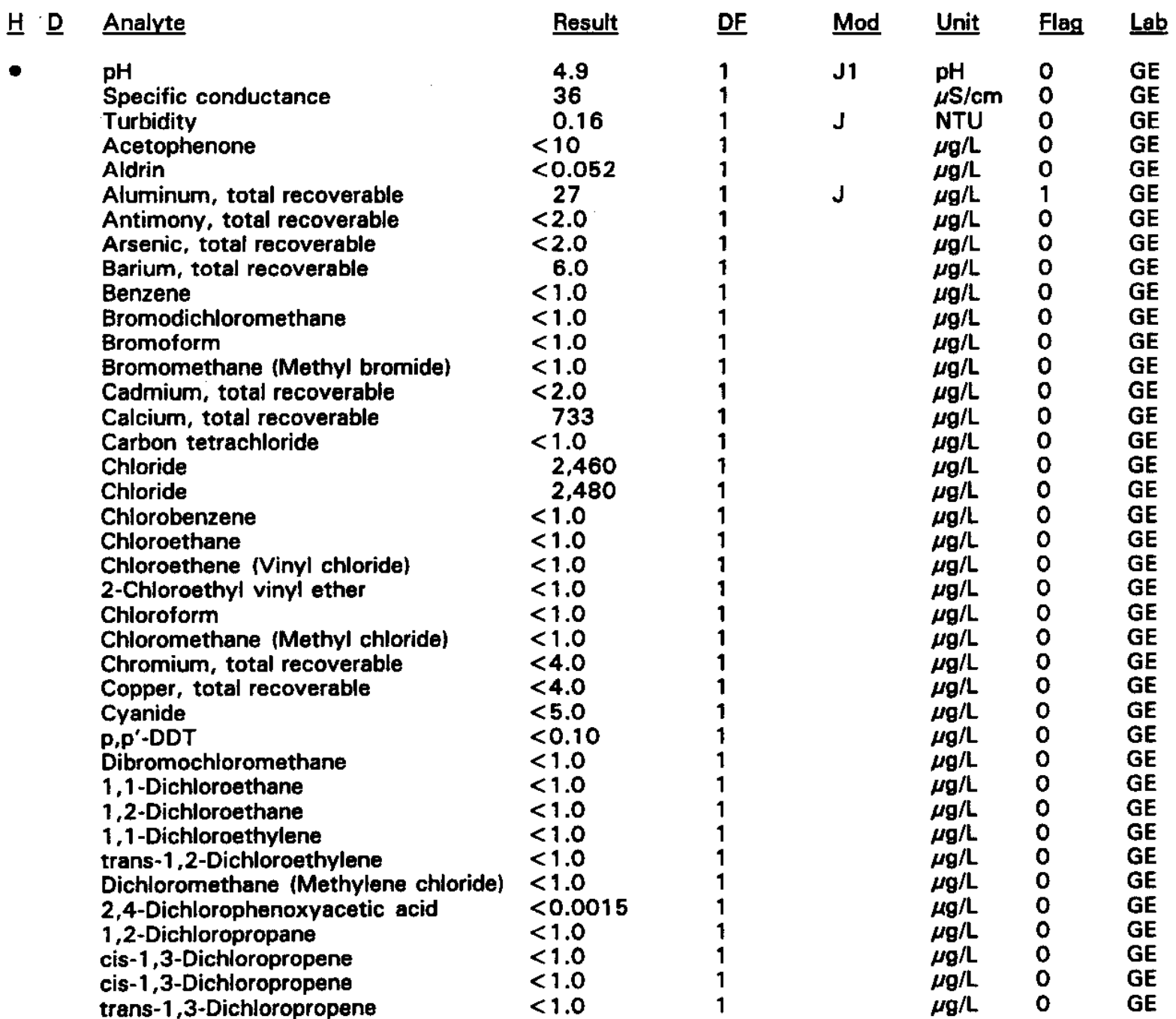

- = exceeded holding time. = exceeded screening level or final primary drinking water standard. 
WELL BGO $11 \mathrm{D}$ collected on 04/05/94, laboratory analyses (cont.)

H

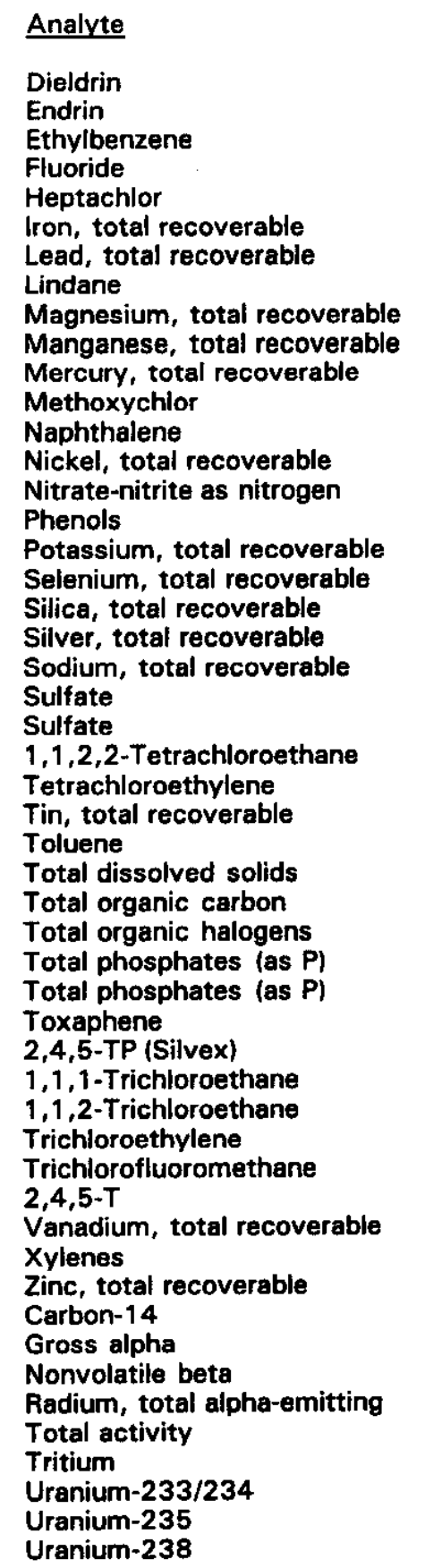

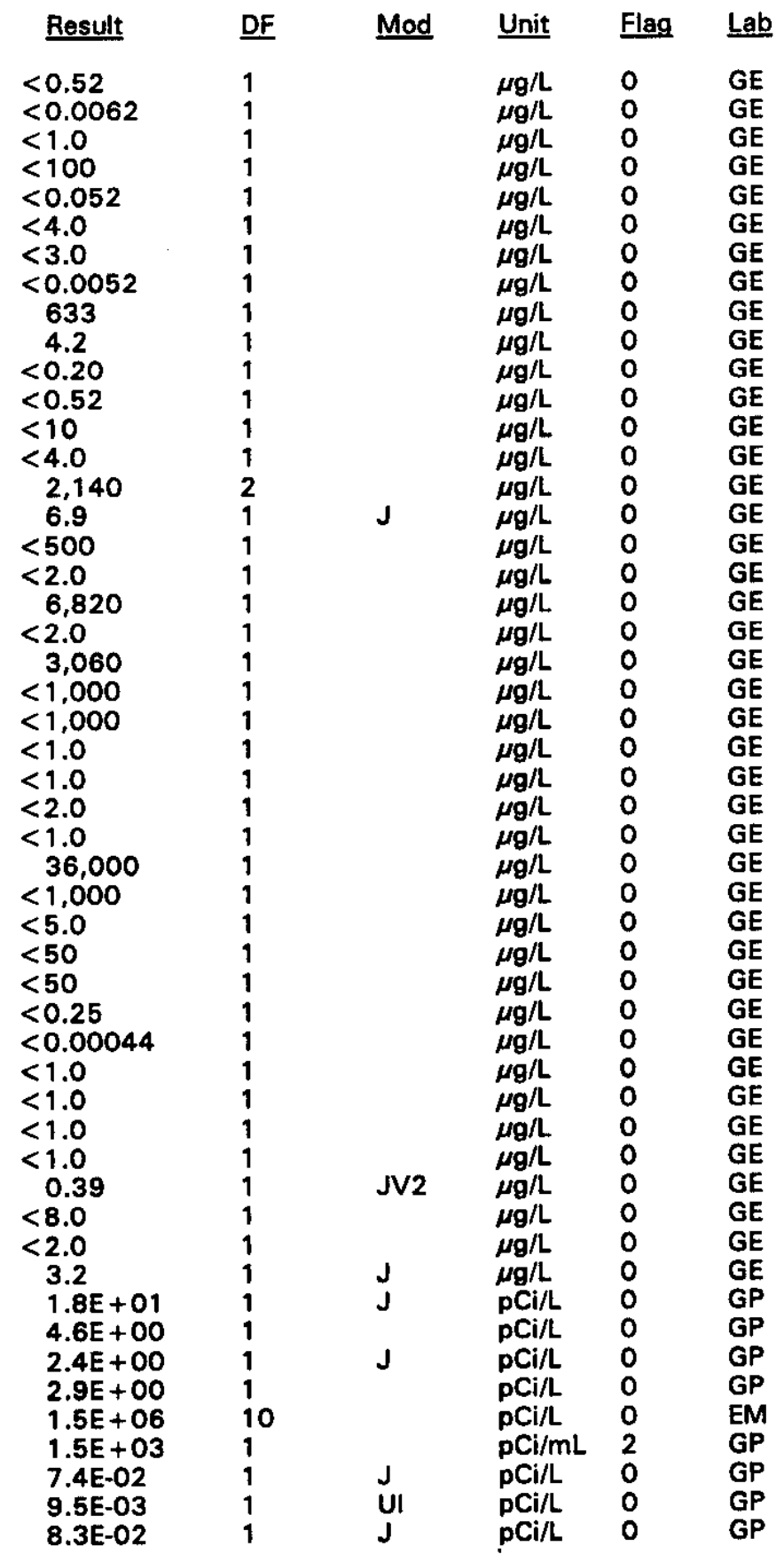

- = exceeded holding time. $\square=$ exceeded screening level or final primary drinking water standard. 


\section{WELL BGO 12AR}

\begin{tabular}{|c|c|c|c|c|c|c|}
\hline SRS Coord. & Lat/Longitude & Screen Zone Elevation & Top of Casing & Casing & Pump & Formation \\
\hline $\begin{array}{l}\text { N76803.8 } \\
\text { E56259.9 }\end{array}$ & $\begin{array}{l}33.287435^{\circ} \mathrm{N} \\
81.667847^{\circ} \mathrm{W}\end{array}$ & $109.3-99.3 \mathrm{ft} \mathrm{msl}$ & $313.4 \mathrm{ft} \mathrm{msl}$ & 4" PVC & $\mathbf{s}$ & U. Congaree (IIA) \\
\hline
\end{tabular}

\section{FIELD MEASUREMENTS}

Sample date: 04/05/94

Depth to water: $155.79 \mathrm{ft}(47.49 \mathrm{~m})$ below TOC

Water elevation: $157.61 \mathrm{ft}(48.04 \mathrm{~m}) \mathrm{msl}$

Sp. conductance: $191 \mu \mathrm{S} / \mathrm{cm}$

Turbidity: 0.4 NTU

Water evacuated before sampling: $168 \mathrm{gal}$

\section{LABORATORY ANALYSES}

H $\underline{\text { Analyte }}$

$\mathrm{pH}$

Specific conductance

Turbidity

Acetophenone

Aldrin

Aldrin

Aluminum, total recoverable

Aluminum, total recoverable

Antimony, total recoverable

Arsenic, total recoverable

Barium, total recoverable

Barium, total recoverable

Benzene

Benzene

Bromodichloromethane

Bromodichloromethane

Bromoform

Bromoform

Bromomethane (Methyl bromide)

Bromomethane (Methyl bromide)

Cadmium, total recoverable

Cadmium, total recoverable

Calcium, total recoverable

Calcium, total recoverable

Carbon tetrachloride

Carbon tetrachloride

Chloride

Chlorobenzene

Chlorobenzene

Chloroethane

Chloroethane

Chloroethene (Vinyl chloride)

Chloroethene (Vinyl chloride)

2-Chloroethyl vinyl ether

2-Chloroethyl vinyl ether

2-Chloroethyl vinyl ether

Chloroform

Chloroform

Chloromethane (Methyl chloride)

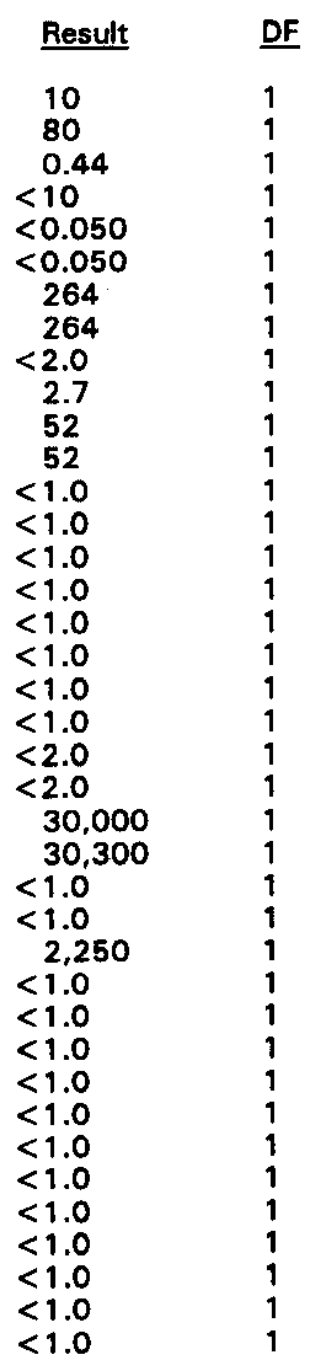

Time: 13:56

pH: 10.3

Alkalinity: $66 \mathrm{mg} / \mathrm{L}$

Water temperature: $20.3^{\circ} \mathrm{C}$

Volumes purged: 4.4 well volumes

\begin{tabular}{|c|c|c|c|c|}
\hline DF & Mod & $\underline{\text { Unit }}$ & Flag & Lak \\
\hline 1 & J1 & $\mathrm{pH}$ & 2 & GE \\
\hline 1 & & $\mu \mathrm{S} / \mathrm{cm}$ & 0 & GE \\
\hline 1 & & NTU & 0 & $\mathrm{GE}$ \\
\hline 1 & & $\mu g / L$ & 0 & GE \\
\hline 1 & & $\mu g / L$ & 0 & $\mathrm{GE}$ \\
\hline 1 & & $\mu \mathrm{g} / \mathrm{L}$ & 0 & $\mathrm{GE}$ \\
\hline 1 & & $\mu \mathrm{g} / \mathrm{L}$ & 2 & GE \\
\hline 1 & & $\mu \mathrm{g} / \mathrm{L}$ & 2 & GE \\
\hline 1 & & $\mu \mathrm{g} / \mathrm{L}$ & 0 & GE \\
\hline 1 & $\mathbf{J}$ & $\mu \mathrm{g} / \mathrm{L}$ & 0 & GE \\
\hline 1 & & $\mu g / L$ & 0 & GE \\
\hline 1 & & $\mu \mathrm{g} / \mathrm{L}$ & 0 & GE \\
\hline 1 & & $\mu g / L$ & 0 & GE \\
\hline 1 & & $\mu \mathrm{g} / \mathrm{L}$ & 0 & $\mathrm{GE}$ \\
\hline 1 & & $\mu \mathrm{g} / \mathrm{L}$ & 0 & GE \\
\hline 1 & & $\mu \mathrm{g} / \mathrm{L}$ & 0 & $\mathrm{GE}$ \\
\hline 1 & & $\mu \mathrm{g} / \mathrm{L}$ & 0 & GE \\
\hline 1 & & $\mu \mathrm{g} / \mathrm{L}$ & 0 & GE \\
\hline 1 & & $\mu \mathrm{g} / \mathrm{L}$ & 0 & GE \\
\hline 1 & & $\mu g / L$ & 0 & GE \\
\hline 1 & & $\mu g / L$ & 0 & GE \\
\hline 1 & & $\mu \mathrm{g} / \mathrm{L}$ & 0 & GE \\
\hline 1 & & $\mu g / L$ & 0 & GE \\
\hline 1 & & $\mu \mathrm{g} / \mathrm{L}$ & 0 & GE \\
\hline 1 & & $\mu \mathrm{g} / \mathrm{L}$ & 0 & $\mathrm{GE}$ \\
\hline 1 & & $\mu g / L$ & 0 & GE \\
\hline 1 & & $\mu g / L$ & 0 & GE \\
\hline 1 & & $\mu g / L$ & 0 & GE \\
\hline 1 & & $\mu g / L$ & 0 & GE \\
\hline 1 & & $\mu g / L$ & 0 & GE \\
\hline 1 & & $\mu g / L$ & 0 & GE \\
\hline 1 & & $\mu \mathrm{g} / \mathrm{L}$ & 0 & GE \\
\hline 1 & & $\mu g / L$ & 0 & GE \\
\hline 1 & & $\mu g / L$ & 0 & GE \\
\hline 1 & & $\mu g / L$ & 0 & GE \\
\hline 1 & & $\mu g / L$ & 0 & $\mathrm{GE}$ \\
\hline 1 & & $\mu \mathrm{g} / \mathrm{L}$ & 0 & $\mathrm{GE}$ \\
\hline 1 & & $\mu \mathrm{g} / \mathrm{L}$ & 0 & $\mathrm{GE}$ \\
\hline & & $\mu g / L$ & 0 & GE \\
\hline
\end{tabular}

- = exceeded holding time. - = exceeded screening level or final primary drinking water standard. 
WELL BGO 12AR collected on 04/05/94, laboratory analyses (cont.)

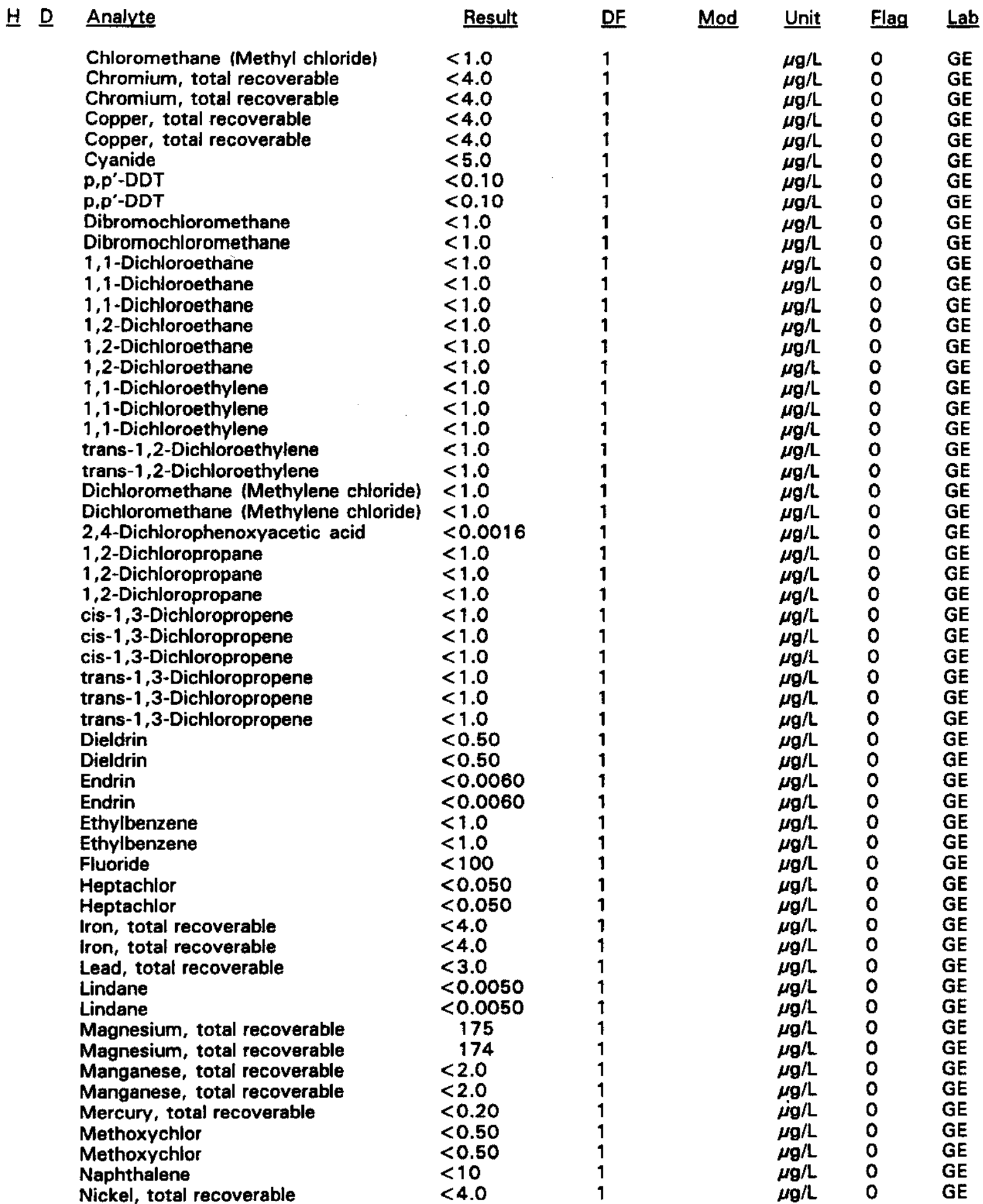

- =xceeded holding time. = exceeded screening level or final primary drinking water standard. 
WELL BGO $12 \mathrm{AR}$ collected on 04/05/94, laboratory analyses (cont.)

\section{$\underline{H} \underline{\text { D }}$ Analyte}

Nickel, total recoverable Nitrate-nitrite as nitrogen Phenols

Potassium, total recoverable

Potassium, total recoverable

Selenium, total recoverable

Silica, total recoverable

Silica, total recoverable

Silver, total recoverable

Silver, total recoverable

Sodium, total recoverable

Sodium, total recoverable

Sulfate

1,1,2,2-Tetrachloroethane

$1,1,2,2$-Tetrachloroethane

Tetrachloroethylene

Tetrachloroethylene

Tin, total recoverable

Tin, total recoverable

Toluene

Toluene

Total dissolved solids

Total organic carbon

Total organic halogens

Total phosphates (as P)

Toxaphene

Toxaphene

2,4,5-TP (Silvex)

Tributyl phosphate

1,1,1-Trichloroethane

1,1,1-Trichloroethane

1,1,1-Trichloroethane

1,1,2-Trichloroethane

1,1,2-Trichloroethane

1,1,2-Trichloroethane

Trichloroethylene

Trichloroethylene

Trichlorofluoromethane

Trichlorofluoromethane

2,4,5-T

Vanadium, total recoverable

Vanadium, total recoverable

Xylenes

Xylenes

Zinc, total recoverable

Zinc, total recoverable

Carbon-14

Gross alpha

Nonvolatile beta

Radium, total alpha-emitting

Tritium

Uranium-233/234

Uranium-233/234

Uranium-235

Uranium-235

Uranium-238

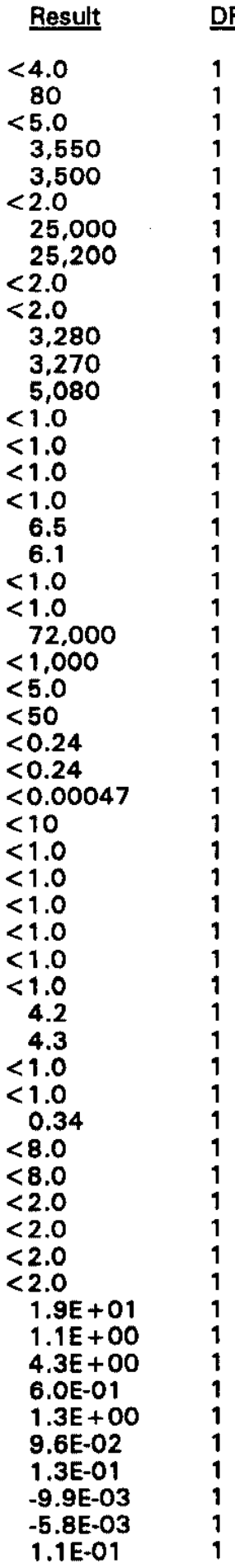

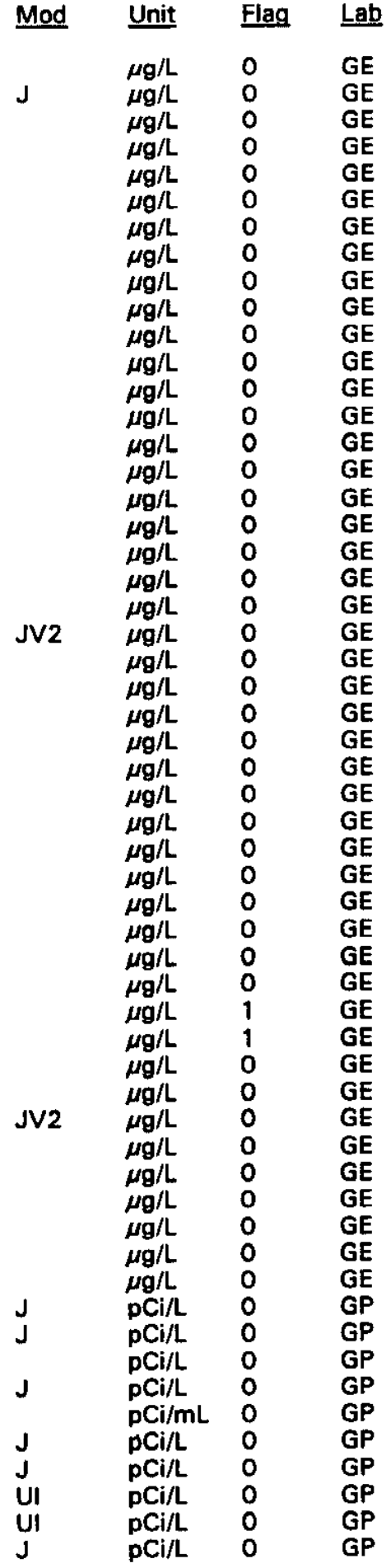

= exceeded holding time. = exceeded screening level or final primary drinking water standard. 
WELL BGO 12AR collected on 04/05/94, laboratory analyses (cont.)

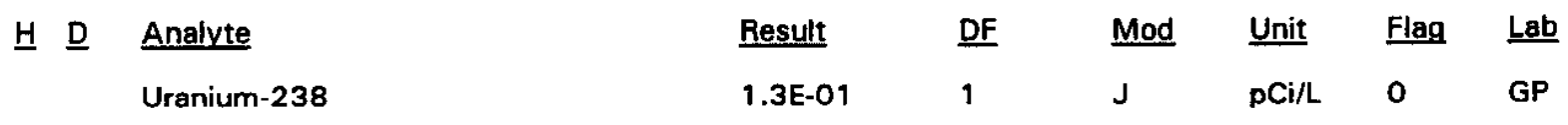

\section{WELL BGO 12CR}

\begin{tabular}{|c|c|c|c|c|c|c|}
\hline SRS Coord. & Lat/Longitude & Screen Zone Elevation & Top of Casing & Casing & Pump & Formation \\
\hline $\begin{array}{l}\text { N76806.0 } \\
\text { E56215.2 }\end{array}$ & $\begin{array}{l}33.287367^{\circ} \mathrm{N} \\
81.667969^{\circ} \mathrm{W}\end{array}$ & $154.0-144.0 \mathrm{ft} \mathrm{msl}$ & $314 \mathrm{ft} \mathrm{msl}$ & 4" PVC & $\mathbf{s}$ & Barnwell (IIB, ) \\
\hline
\end{tabular}

\section{FIELD MEASUREMENTS}

Sample date: $04 / 06 / 94$

Depth to water: $92.57 \mathrm{ft}(28.22 \mathrm{~m})$ below TOC

Water elevation: $221.43 \mathrm{ft}(67.49 \mathrm{~m}) \mathrm{msl}$

Sp. conductance: $127 \mu \mathrm{S} / \mathrm{cm}$

Turbidity: $1.0 \mathrm{NTU}$

Water evacuated before sampling: $35 \mathrm{gal}$

The well went dry during purging.

\section{LABORATORY ANALYSES}

H $\underline{\text { Analyte }}$

pH

Specific conductance

Turbidity

Turbidity

Acetophenone

Aldrin

Aluminum, total recoverable

Antimony, total recoverable

Arsenic, total recoverable

Barium, total recoverable

Benzene

Bromodichloromethane

Bromoform

Bromomethane (Methyl bromide)

Cadmium, total recoverable

Calcium, total recoverable

Carbon tetrachloride

Chloride

Chlorobenzene

Chloroethane

Chloroethene (Vinyl chloride)

2-Chloroethyl vinyl ether

Chloroform

Chloromethane (Methyl chloride)

Chromium, total recoverable

Copper, total recoverable

Cyanide

Cyanide

p.p'-DDT

Dibromochloromethane

1,1-Dichloroethane

1,1-Dichloroethane

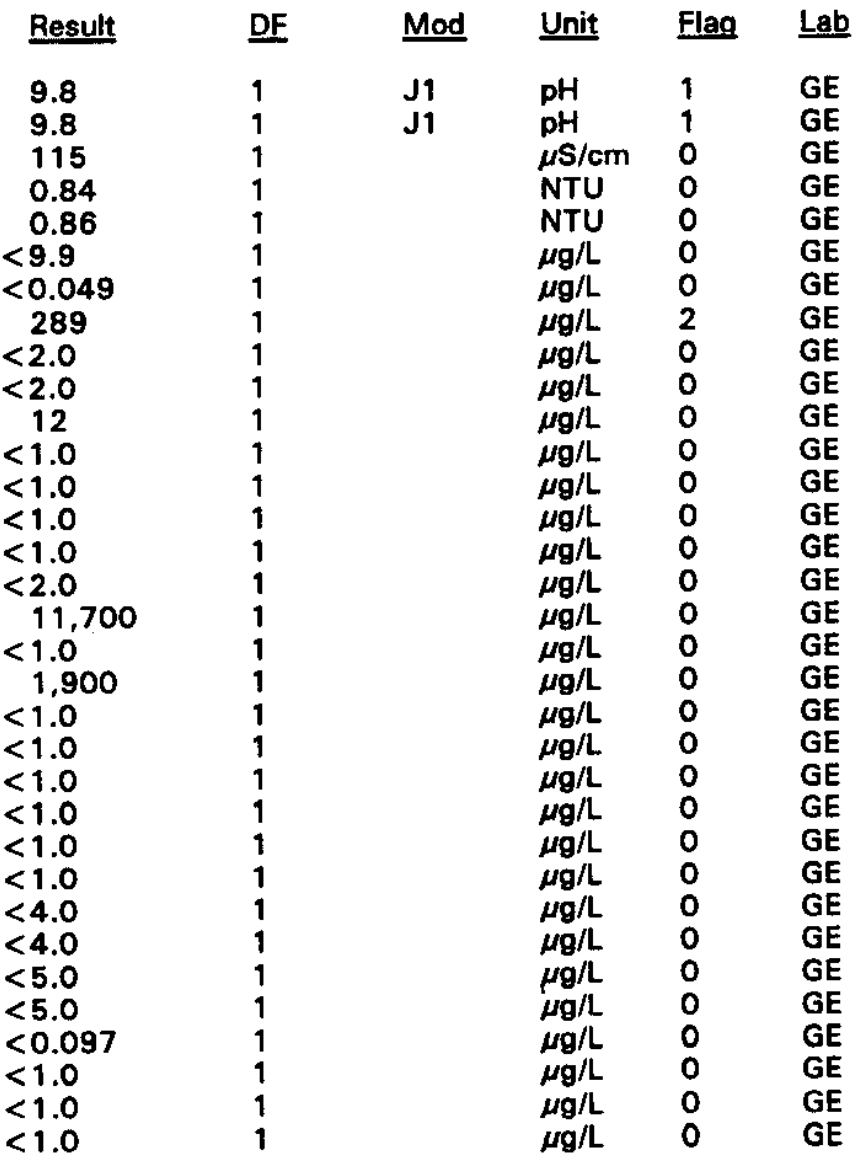

Time: 9:55

pH: 9.8

Alkalinity: $37 \mathrm{mg} / \mathrm{L}$

Water temperature: $19.0^{\circ} \mathrm{C}$

Volumes purged: 0.7 well volumes 
WELL BGO 12CR collected on 04/06/94, laboratory analyses (cont.)

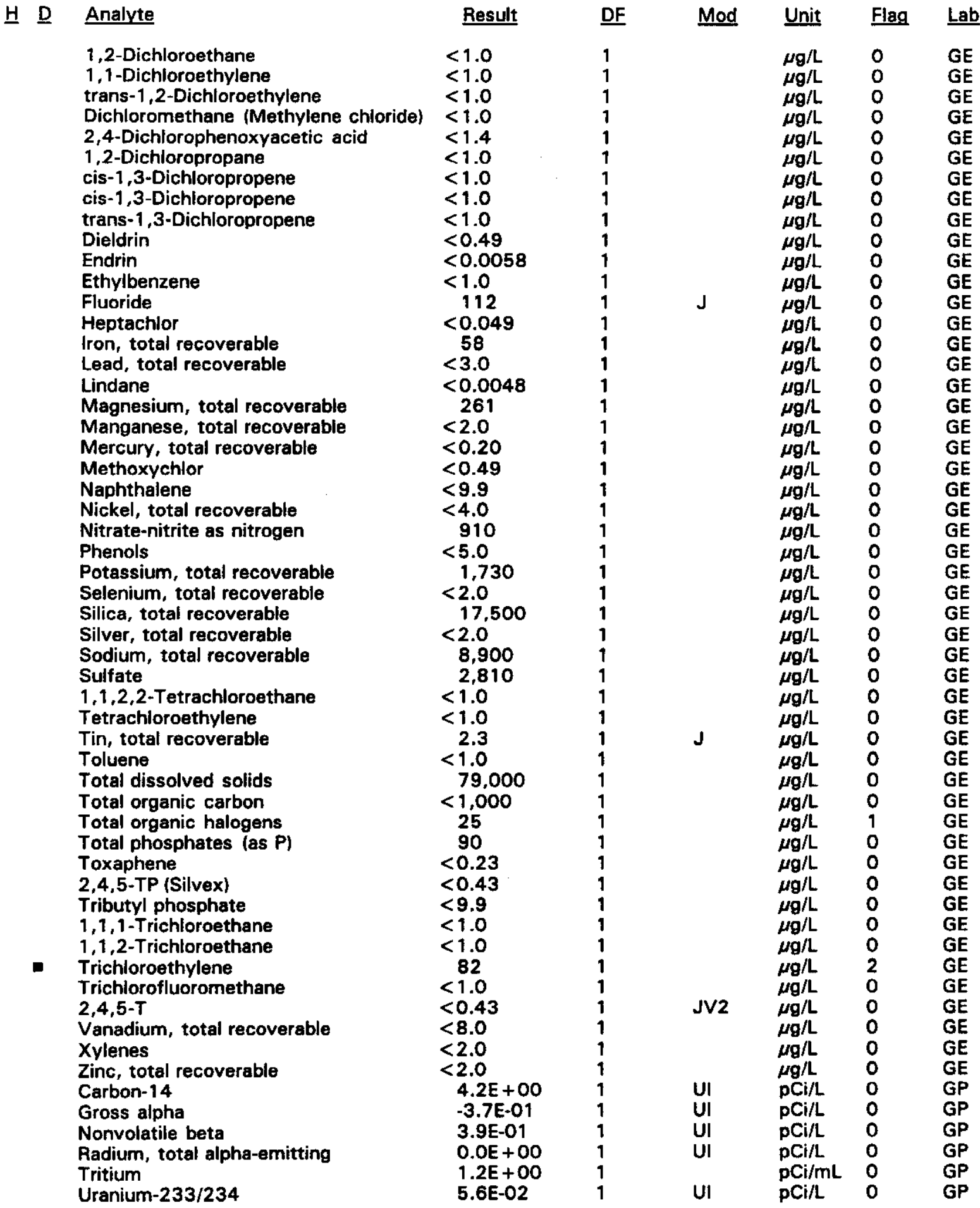

- = exceeded holding time. - exceeded screening level or final primary drinking water standard. 
WELL BGO 12CR collected on 04/06/94, laboratory analyses (cont.)

H $\underline{\text { Analyte }}$

Uranium-235

Uranium-238
Result

7.4E-02

7.4E-02
DF

1

1

Mod

Unit

Flag Lab

UI

UI

$\mathrm{pCi} / \mathrm{L}$

0

GP

GP

\section{WELL BGO 12D}

\begin{tabular}{|c|c|c|c|c|c|c|}
\hline SRS Coord. & Lat/Longitude & Screen Zone Elevation & Top of Casing & Casing & Pump & Formation \\
\hline $\begin{array}{l}\text { N76805.2 } \\
\text { E56231.1 }\end{array}$ & $\begin{array}{l}33.287391^{\circ} \mathrm{N} \\
81.667925^{\circ} \mathrm{W}\end{array}$ & $237.8-217.8 \mathrm{ft} \mathrm{msl}$ & $313.7 \mathrm{ft} \mathrm{msl}$ & 4" PVC & $\mathbf{s}$ & Water Table $\left(11 \mathrm{~B}_{2}\right)$ \\
\hline
\end{tabular}

\section{FIELD MEASUREMENTS}

Sample date: 04/06/94

Depth to water: $82.01 \mathrm{ft}(25.00 \mathrm{~m})$ below TOC

Water elevation: $231.69 \mathrm{ft}(70.62 \mathrm{~m}) \mathrm{ms} \mid$

Sp. conductance: $89 \mu \mathrm{S} / \mathrm{cm}$

Turbidity: 4.5 NTU

Water evacuated before sampling: $4 \mathrm{gal}$

The well went dry during purging.

\section{LABORATORY ANALYSES}

H

Analyte
pH
Specific conductance
Turbidity
Acetophenone
Aldrin
Aluminum, total recoverable
Antimony, total recoverable
Arsenic, total recoverable
Barium, total recoverable
Benzene
Bromodichloromethane
Bromoform
Bromomethane (Methyl bromide)
Cadmium, total recoverable
Calcium, total recoverable
Carbon tetrachloride
Chloride
Chlorobenzene
Chloroethane
Chloroethene (Vinyl chloride)
2-Chloroethyl vinyl ether
Chloroform
Chloromethane (Methyl chloride)
Chromium, total recoverable
Copper, total recoverable
Cyanide
p,p'-DDT
Dibromochloromethane
1,1 -Dichloroethane
$1,2-$ Dichloroethane
1,1-Dichloroethylene
trans-1,2-Dichloroethylene

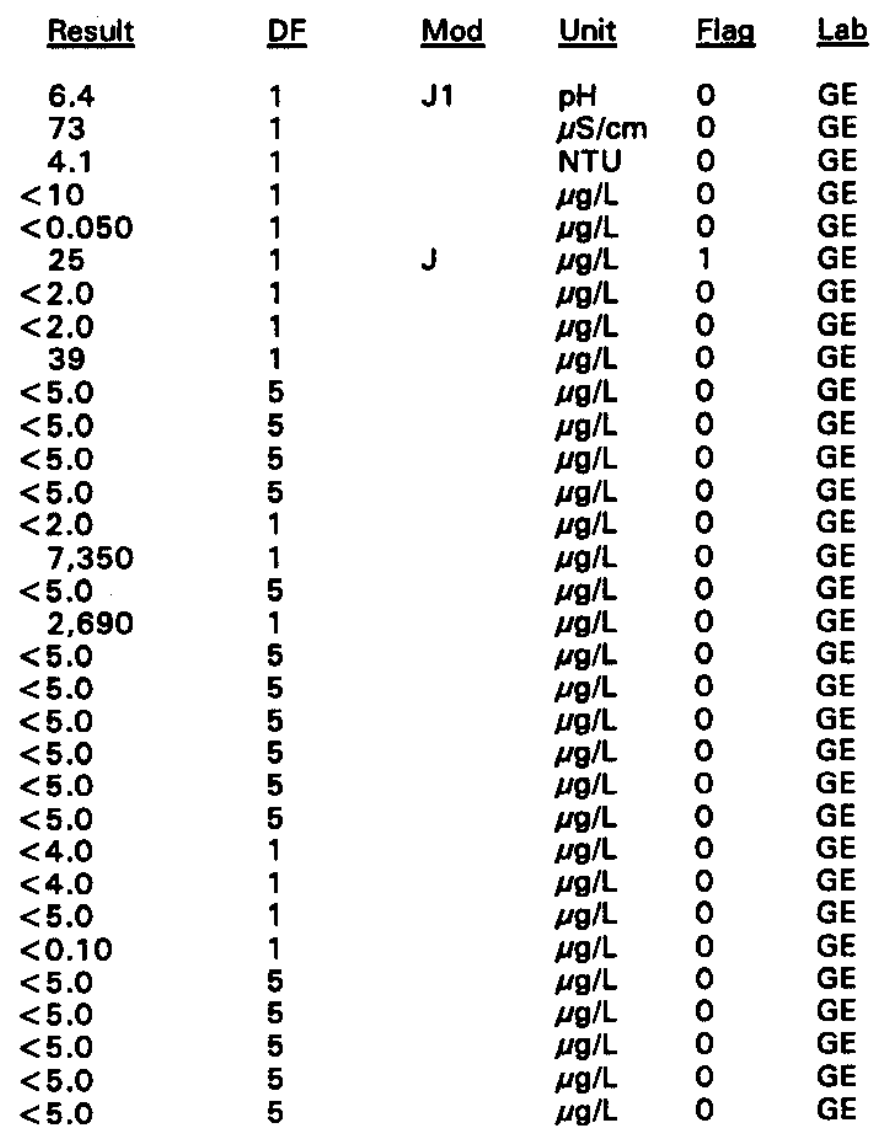

Time: $9: 40$

pH: 6.4

Alkalinity: $12 \mathrm{mg} / \mathrm{L}$

Water temperature: $18.5^{\circ} \mathrm{C}$

Volumes purged: 0.4 well volumes 
WELL BGO 12D collected on $04 / 06 / 94$, laboratory analyses (cont.)

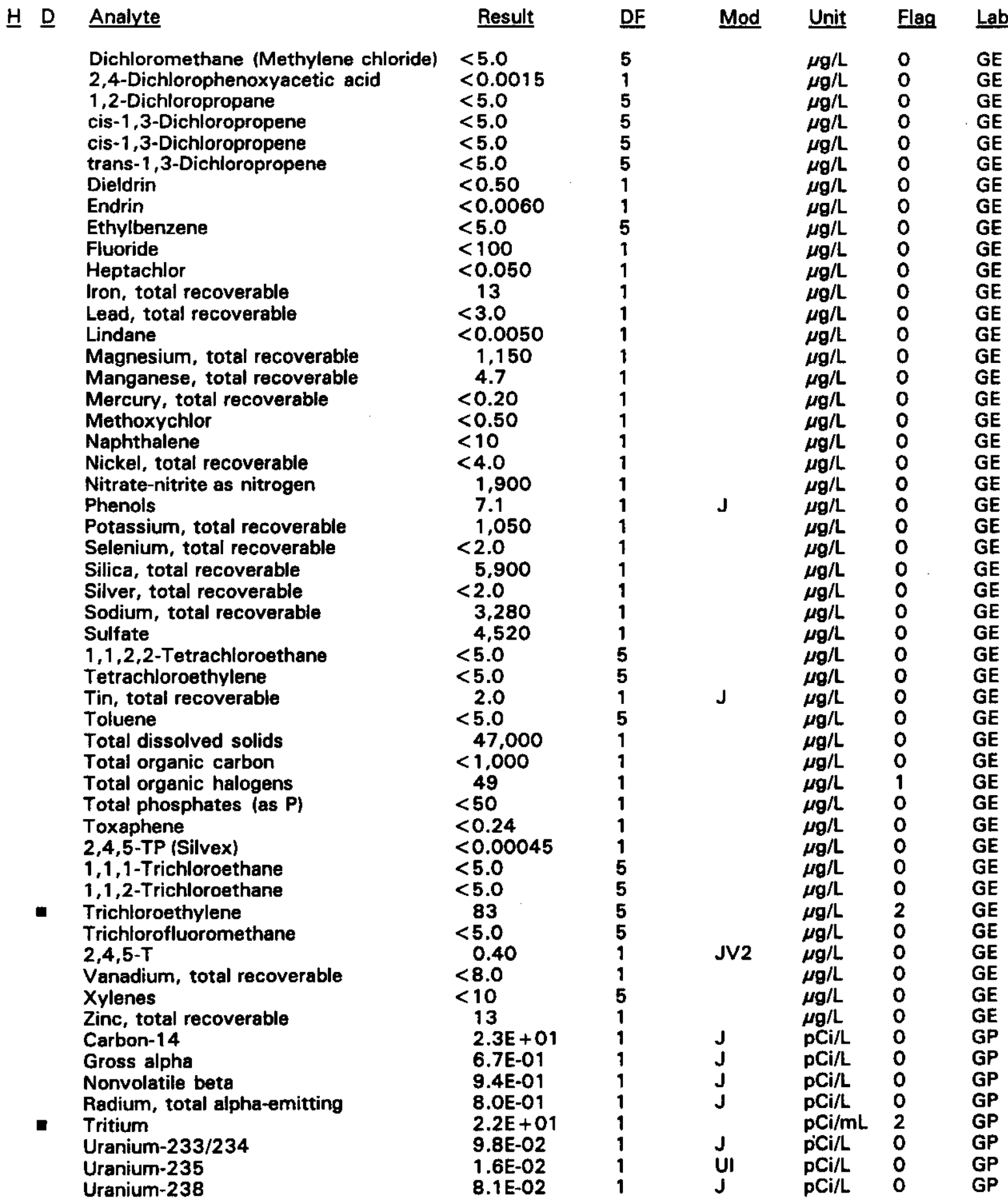

- = exceeded holding time. = exceeded screening level or final primary drinking water standard. 
WELL BGO 13DR

\begin{tabular}{|c|c|c|c|c|c|c|}
\hline SRS Coord. & Lat/Longitude & Screen Zone Elevation & Top of Casing & Casing & Pump & Formation \\
\hline $\begin{array}{l}N 76824.7 \\
E 55840.4\end{array}$ & $\begin{array}{l}33.286797^{\circ} \mathrm{N} \\
81.668992^{\circ} \mathrm{W}\end{array}$ & $220.3-210.3 \mathrm{ft} \mathrm{msl}$ & $319.3 \mathrm{ft} \mathrm{msl}$ & 4" PVC & $\mathbf{s}$ & Barnwell (IIB $)_{1}$ \\
\hline
\end{tabular}

\section{FIELD MEASUREMENTS}

Sample date: 04/19/94

Depth to water: $88.17 \mathrm{ft}(26.87 \mathrm{~m})$ below TOC

Water elevation: $231.13 \mathrm{ft}(70.45 \mathrm{~m}) \mathrm{msl}$

Sp. conductance: $82 \mu \mathrm{S} / \mathrm{cm}$

Turbidity: 11.8 NTU

Water evacuated before sampling: $8 \mathrm{gal}$

The well went dry during purging.

\section{LABORATORY ANALYSES}

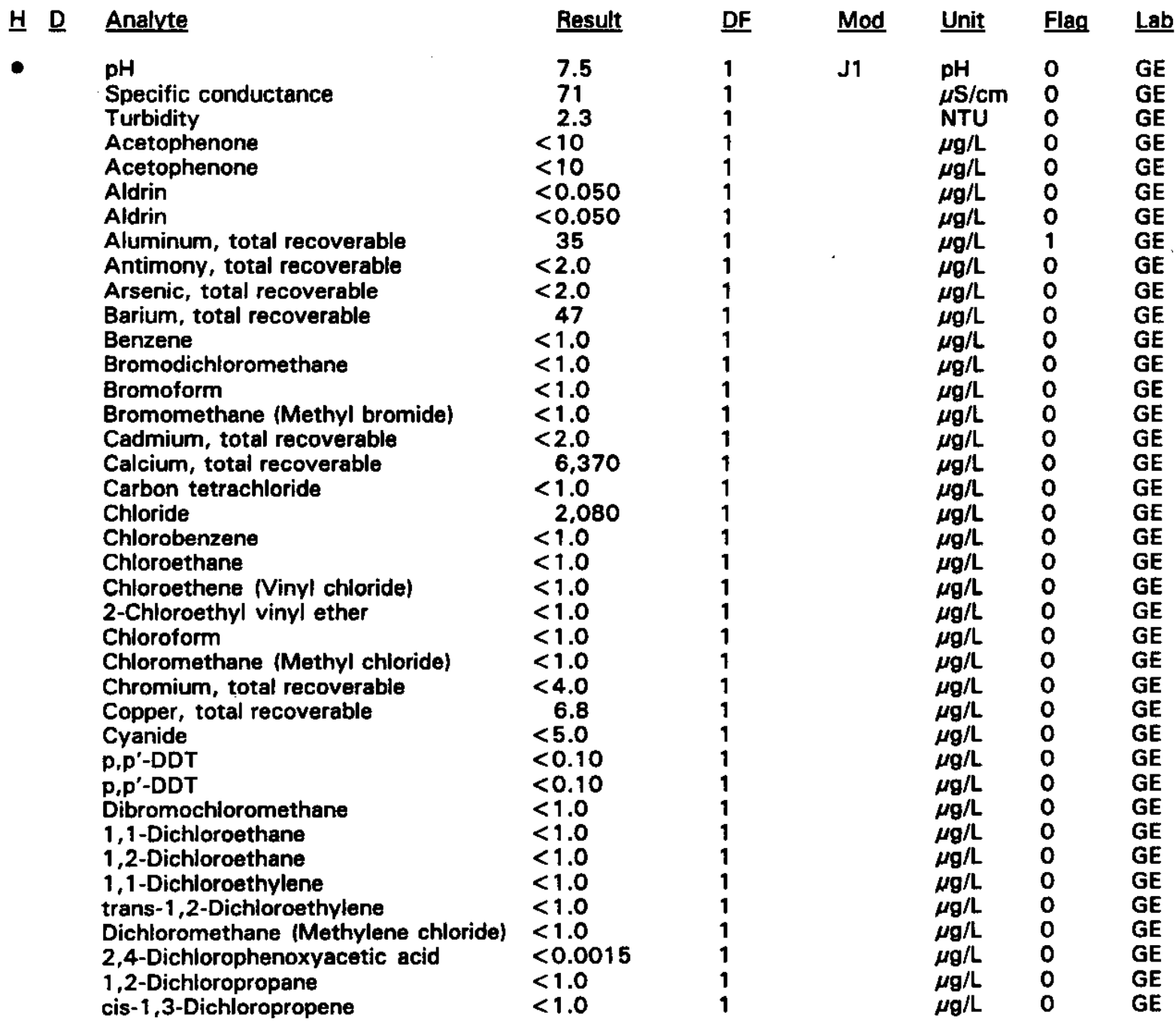

Time: 11:17

pH: 7.1

Alkalinity: $20 \mathrm{mg} / \mathrm{L}$

Water temperature: $20.1^{\circ} \mathrm{C}$

Volumes purged: 0.6 well volumes 
WELL. BGO 13DR collected on 04/19/94, laboratory analyses (cont.)

H D Analyte

trans-1,3-Dichloropropene

Dieldrin

Dieldrin

Endrin

Endrin

Ethylbenzene

Fluoride

Heptachior

Heptachlor

Iron, total recoverable

Lead, total recoverable

Lindane

Lindane

Magnesium, total recoverable

Manganese, total recoverable

Mercury, total recoverable

Methoxychlor

Methoxychlor

Naphthalene

Naphthalene

Nickel, total recoverable

Nitrate-nitrite as nitrogen

Phenols

Potassium, total recoverable

Selenium, total recoverable

Silica, total recoverable

Silver, total recoverable

Sodium, total recoverable

Sulfate

1,1,2,2-Tetrachloroethane

Tetrachloroethylene

Tin, total recoverable

Toluene

Total dissolved solids

Total dissolved solids

Total organic carbon

Total organic halogens

Total phosphates (as P)

Toxaphene

Toxaphene

2,4,5-TP (Silvex)

Tributyl phosphate

Tributyl phosphate

1,1,1-Trichloroethane

1,1,2-Trichloroethane

Trichloroethylene

Trichlorofluoromethane

2,4,5-T

Vanadium, total recoverable

Xylenes

Zinc, total recoverable

Carbon-14

Gross alpha

Nonvolatile beta

Radium, total alpha-emitting

Tritium
Result DF

$<1.0$

$<0.50$

$<0.50$

$<0.0060$

$<0.0060$

$<1.0$

$<100$

$<0.050$

$<0.050$

189

22

$<0.0050$

$<0.0050$

383

73

$<0.20$

$<0.50$

$<0.50$

$<10$

$<10$

5.8

830

$<5.0$

1,280

$<2.0$

6,970

$<2.0$

6,000

5,070

$<1.0$

$<1.0$

2.6

$<1.0$

43,000

46,000

$<1,000$

$<5.0$

$<50$

$<0.24$

$<0.24$

$<0.00045$

$<10$

$<10$

$<1.0$

$<1.0$

$<1.0$

$<1.0$

$<0.00045$

$<8.0$

$<2.0$

63

$2.3 E+00$

$1.8 E+00$

$2.5 E+00$

$-3.0 \mathrm{E}-01$

$1.8 \mathrm{E}+01$
Mod Unit Flag Lab

$\mu g / L \quad 0 \quad$ GE

$\mu g / L \quad 0 \quad$ GE

$\mu g / L \quad 0 \quad$ GE

$\mu g / L \quad 0 \quad$ GE

$\mu g / L \quad 0 \quad G E$

$\mu g / L \quad 0 \quad G E$

$\mu g / L \quad 0 \quad$ GE

$\mu g / L \quad 0 \quad G E$

$\mu g / L \quad 0 \quad$ GE

$\mu \mathrm{g} / \mathrm{L} \quad 1 \quad \mathrm{GE}$

$\mu \mathrm{g} / \mathrm{L} \quad \mathrm{O} \quad \mathrm{GE}$

$\mu g / L \quad 0 \quad G E$

$\mu \mathrm{g} / \mathrm{L} \quad 0 \quad \mathrm{GE}$

$\mu g / L \quad 0 \quad$ GE

$\mu \mathrm{g} / \mathrm{L} \quad 2 \quad \mathrm{GE}$

$\mu \mathrm{g} / \mathrm{L} \quad 0 \quad \mathrm{GE}$

$\mu g / L \quad 0 \quad$ GE

$\mu \mathrm{g} / \mathrm{L} \quad \mathrm{O} \quad \mathrm{GE}$

$\mu \mathrm{g} / \mathrm{L} \quad 0 \quad \mathrm{GE}$

$\mu g / L \quad 0 \quad G E$

$\mu g / L \quad O \quad G E$

$\mu g / L \quad 0 \quad G E$

$\mu g / L \quad 0 \quad$ GE

$\mu g / L \quad 0 \quad G E$

$\mu g / L \quad 0 \quad G E$

$\mu g / L \quad 0 \quad G E$

$\mu g / L \quad 0 \quad$ GE

$\mu \mathrm{g} / \mathrm{L} \quad 0 \quad \mathrm{GE}$

$\mu g / L \quad 0 \quad$ GE

$\mu g / L \quad 0 \quad$ GE

$\mu g / L \quad 0 \quad$ GE

J $\mu g / L \quad 0 \quad$ GE

$\mu g / L \quad 0 \quad$ GE

$\mu g / L \quad 0 \quad$ GE

$\mu \mathrm{g} / \mathrm{L} \quad 0$

$\mu g / L \quad 0 \quad$ GE

$\mu g / L \quad 0 \quad$ GE

$\mu g / L \quad 0 \quad$ GE

$\mu g / L \quad 0$

$\mu g / L \quad 0 \quad$ GE

$\mu g / L \quad 0 \quad$ GE

$\mu \mathrm{g} / \mathrm{L} \quad \mathrm{O} \quad \mathrm{GE}$

$\mu g / L \quad O \quad G E$

$\mu g / L \quad 0 \quad G E$

$\mu g / L \quad 0 \quad$ GE

$\mu g / L \quad 0 \quad$ GE

$\mu \mathrm{g} / \mathrm{L} \quad \mathrm{O} \quad \mathrm{GE}$

$\mu g / L \quad 0 \quad G E$

$\mu \mathrm{g} / \mathrm{L} \quad \mathrm{O} \quad \mathrm{GE}$

$\mu g / L \quad 0 \quad$ GE

$\mu g / L \quad 0 \quad G E$

$\mathrm{pCi} / \mathrm{L} \quad 0 \quad \mathrm{GP}$

$\mathrm{pCi} / \mathrm{L} \quad \mathrm{O} \quad \mathrm{GP}$

$\mathrm{pCi} / \mathrm{L} \quad 0 \quad \mathrm{GP}$

$\mathrm{pCi} / \mathrm{L} \quad \mathrm{O} \quad \mathrm{GP}$

$\mathrm{pCi} / \mathrm{mL} \quad 1 \quad \mathrm{GP}$

- = exceeded holding time. = exceeded screening level or final primary drinking water standard. 
WELL BGO 13DR collected on 04/19/94, laboratory analyses (cont.)

\begin{tabular}{|c|c|c|c|c|c|}
\hline H $\underline{D} \quad$ Analyte & Result & DF & Mod & Unit & Flag \\
\hline $\begin{array}{l}\text { Uranium-233/234 } \\
\text { Uranium-235 } \\
\text { Uranium-238 }\end{array}$ & $\begin{array}{l}1.5 E-01 \\
-6.5 E-03 \\
9.7 E-02\end{array}$ & $\begin{array}{l}1 \\
1 \\
1\end{array}$ & $\begin{array}{l}\text { J } \\
\text { UI } \\
\text { UI }\end{array}$ & $\begin{array}{l}\mathrm{pCi} / \mathrm{L} \\
\mathrm{pCi} / \mathrm{L} \\
\mathrm{pCi} / \mathrm{L}\end{array}$ & $\begin{array}{l}0 \\
0 \\
0\end{array}$ \\
\hline
\end{tabular}

WELL BGO 14AR

\begin{tabular}{|c|c|c|c|c|c|c|}
\hline SRS Coord. & Lat/Longitude & Screen Zone Elevation & Top of Casing & Casing & Pump & Formation \\
\hline $\begin{array}{l}\text { N76351.8 } \\
\text { E55788.9 }\end{array}$ & $\begin{array}{l}33.285667^{\circ} \mathrm{N} \\
81.668209^{\circ} \mathrm{W}\end{array}$ & $106.8-96.8 \mathrm{ft} \mathrm{msl}$ & $300.7 \mathrm{ft} \mathrm{msl}$ & 4" PVC & $\mathbf{S}$ & U. Congaree (IIA) \\
\hline
\end{tabular}

FIELD MEASUREMENTS

Sample date: 04/05/94

Depth to water: $140.99 \mathrm{ft}(42.97 \mathrm{~m})$ below TOC

Water elevation: $159.71 \mathrm{ft}(48.68 \mathrm{~m}) \mathrm{msl}$

Sp. conductance: $219 \mu \mathrm{S} / \mathrm{cm}$

Turbidity: 0.5 NTU

Water evacuated before sampling: $215 \mathrm{gal}$

LABORATORY ANALYSES

H D Analyte

pH

Specific conductance

Specific conductance

Turbidity

Acetophenone

Aldrin

Aluminum, total recoverable

Antimony, total recoverable

Arsenic, total recoverable

Barium, total recoverable

Benzene

Bromodichloromethane

Bromoform

Bromomethane (Methyl bromide)

Cadmium, total recoverable

Calcium, total recoverable

Carbon tetrachloride

Chloride

Chlorobenzene

Chloroethane

Chloroethene (Vinyl chloride)

2-Chloroethyl vinyl ether

Chloroform

Chloromethane (Methyl chloride)

Chromium, total recoverable

Copper, total recoverable

Cyanide

p, $\mathbf{p}^{\prime}-\mathrm{DDT}$

Dibromochloromethane

1,1-Dichloroethane

1,2-Dichloroethane
Time: 15:13

pH: 9.8

Alkalinity: $85 \mathrm{mg} / \mathrm{L}$

Water temperature: $20.2{ }^{\circ} \mathrm{C}$

Volumes purged: 5.2 well volumes

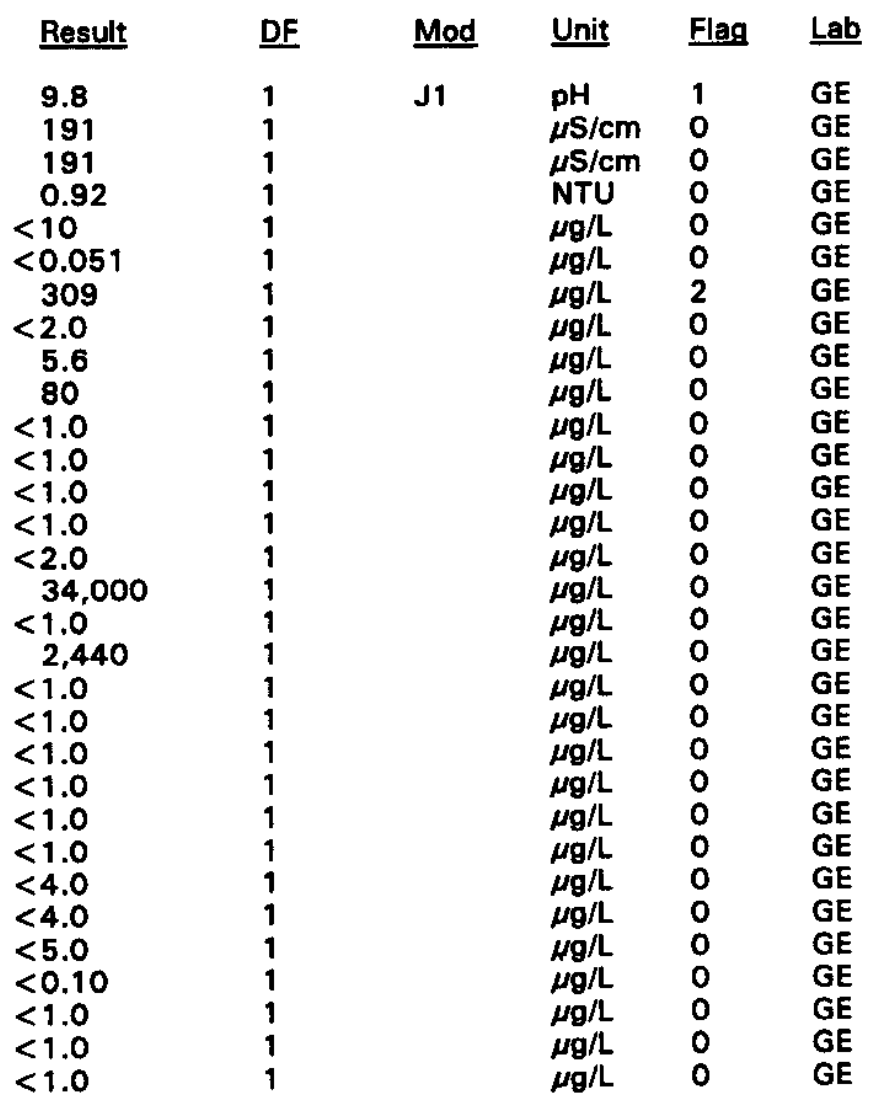

- = exceeded holding time. $\square=$ exceeded screening level or final primary drinking water standard. 
WELL BGO 14AR collected on 04/05/94, laboratory analyses (cont.)

H $\underline{\text { Analyte }}$

1,1-Dichloroethylene

trans-1,2-Dichloroethylene

Dichloromethane (Methylene chloride)

2,4-Dichlorophenoxyacetic acid

2,4-Dichlorophenoxyacetic acid

1,2-Dichloropropane

cis-1,3-Dichloropropene

cis-1,3-Dichloropropene

trans-1,3-Dichloropropene

Dieldrin

Endrin

Ethylbenzene

Fluoride

Heptachlor

Iron, total recoverable

Lead, total recoverable

Lindane

Magnesium, total recoverable

Manganese, total recoverable

Mercury, total recoverable

Methoxychlor

Naphthalene

Nickel, total recoverable

Nitrate-nitrite as nitrogen

Phenols

Potassium, total recoverable

Selenium, total recoverable

Silica, total recoverable

Silver, total recoverable

Sodium, total recoverable

Sulfate

1,1,2,2-Tetrachloroethane

Tetrachloroethylene

Tin, total recoverable

Toluene

Total dissolved solids

Total organic carbon

Total organic halogens

Total phosphates (as P)

Toxaphene

2,4,5-TP (Silvex)

2,4,5-TP (Silvex)

Tributyl phosphate

1,1,1-Trichloroethane

1,1,2-Trichloroethane

Trichloroethylene

Trichlorofluoromethane

2,4,5-T

2,4,5-T

Vanadium, total recoverable

Xylenes

Zinc, total recoverable

Carbon-14

Gross alpha

Nonvolatile beta

Radium, total alpha-emitting
Result DF

$<1.0$

$<1.0$

$<1.0$

$<0.0016$

$<0.0016$

$<1.0$

$<1.0$

$<1.0$

$<1.0$

$<0.51$

$<0.0061$

$<1.0$

105

$<0.051$

5.4

$<3.0$

$<0.0051$

533

11

$<0.20$

$<0.51$

$<10$

$<4.0$

220

$<5.0$

7,530

$<2.0$

24,700

$<2.0$

4,860

4,150

$<1.0$

$<1.0$

15

$<1.0$

143,000

1,200

$<5.0$

160

$<0.24$

$<0.00047$

$<0.00047$

$<10$

$<1.0$

$<1.0$

1.8

$<1.0$

0.39

0.36

$<8.0$

$<2.0$

$<2.0$

$7.0 E+00$

$2.2 E+0 O$

$5.6 E+00$

9.OE-01
Mod

Unit

Flag Lab

$\mu g / L \quad 0 \quad$ GE

$\mu g / L \quad 0 \quad G E$

$\mu g / L \quad 0 \quad$ GE

$\mu \mathrm{g} / \mathrm{L} \quad \mathrm{O} \quad \mathrm{GE}$

$\mu \mathrm{g} / \mathrm{L} \quad \mathrm{O} \quad \mathrm{GE}$

$\mu g / L \quad 0 \quad$ GE

$\mu \mathrm{g} / \mathrm{L} \quad \mathrm{O} \quad \mathrm{GE}$

$\mu g / L \quad 0 \quad \mathrm{GE}$

$\mu \mathrm{g} / \mathrm{L} \quad \mathrm{O} \quad \mathrm{GE}$

$\mu \mathrm{g} / \mathrm{L} \quad \mathrm{O} \quad \mathrm{GE}$

$\mu g / L \quad 0 \quad$ GE

$\mu \mathrm{g} / \mathrm{L} \quad 0 \quad \mathrm{GE}$

J $\mu \mathrm{g} / \mathrm{L} \quad 0 \quad \mathrm{GE}$

$\mu g / L \quad 0 \quad$ GE

J $\mu g / L \quad 0 \quad$ GE

$\mu g / L \quad 0 \quad$ GE

$\mu g / L \quad 0 \quad$ GE

$\mu \mathrm{g} / \mathrm{L} \quad 0 \quad \mathrm{GE}$

$\mu g / L \quad O \quad G E$

$\mu g / L \quad 0 \quad$ GE

$\mu g / L \quad 0 \quad$ GE

$\mu g / L \quad 0 \quad$ GE

$\mu \mathrm{g} / \mathrm{L} \quad \mathrm{O} \quad \mathrm{GE}$

$\mu g / L \quad 0 \quad G E$

$\mu \mathrm{g} / \mathrm{L} \quad \mathrm{O} \quad \mathrm{GE}$

$\mu g / L \quad 0 \quad G E$

$\mu g / L \quad 0 \quad G E$

$\mu g / L \quad O \quad G E$

$\mu \mathrm{g} / \mathrm{L} \quad 0 \quad \mathrm{GE}$

$\mu g / L \quad 0 \quad \mathrm{GE}$

$\mu g / L \quad 0 \quad$ GE

$\mu g / L \quad 0 \quad$ GE

$\mu \mathrm{g} / \mathrm{L} \quad 0 \quad \mathrm{GE}$

$\mu \mathrm{g} / \mathrm{L} \quad 1 \quad \mathrm{GE}$

$\mu g / L \quad 0 \quad G E$

JV2 $\mu g / L \quad 0 \quad$ GE

$J \quad \mu g / L \quad 0 \quad$ GE

$\mu \mathrm{g} / \mathrm{L} \quad 0 \quad \mathrm{GE}$

$\mu g / L \quad 0 \quad G E$

$\mu \mathrm{g} / \mathrm{L} \quad \mathrm{O} \quad \mathrm{GE}$

$\mu \mathrm{g} / \mathrm{L} \quad 0 \quad \mathrm{GE}$

$\mu \mathrm{g} / \mathrm{L} \quad 0 \quad \mathrm{GE}$

$\mu g / L \quad 0 \quad$ GE

$\mu g / L \quad 0 \quad$ GE

$\mu g / L \quad 0 \quad G E$

$\mu \mathrm{g} / \mathrm{L}$

$\mu g / L$

JV2 $\mu g / L$

JV2 $\mu g / L$

$\mu \mathrm{g} / \mathrm{L}$

$\mu g / L$

$\mu \boldsymbol{g} / \mathrm{L}$

pCi/L

pCi/L

$\mathrm{pCi} / \mathrm{L}$

pCi/L

- exceeded holding time. = exceeded screening level or final primary drinking water standard. 
WELL BGO 14AR collected on 04/05/94, laboratory analyses (cont.)

\begin{tabular}{|c|c|c|c|c|c|}
\hline Analyte & Result & $\underline{\mathrm{DF}}$ & Mod & Unit & Flag \\
\hline $\begin{array}{l}\text { Tritium } \\
\text { Uranium-233/234 } \\
\text { Uranium-235 } \\
\text { Uranium-238 }\end{array}$ & $\begin{array}{l}2.4 \mathrm{E}+00 \\
6.6 \mathrm{E}-02 \\
2.5 \mathrm{E}-01 \\
2.5 \mathrm{E}-01\end{array}$ & $\begin{array}{l}1 \\
1 \\
1 \\
1\end{array}$ & $\begin{array}{l}\mathrm{UI} \\
\mathrm{J}\end{array}$ & $\begin{array}{l}\mathrm{pCi} / \mathrm{mL} \\
\mathrm{pCi} / \mathrm{L} \\
\mathrm{pCi} / \mathrm{L} \\
\mathrm{pCi} / \mathrm{L}\end{array}$ & $\begin{array}{l}0 \\
0 \\
0 \\
0\end{array}$ \\
\hline
\end{tabular}

\section{WELL BGO 14CR}

\begin{tabular}{|c|c|c|c|c|c|c|}
\hline RS Coord. & Lat/Longitude & Screen Zone Elevation & Top of Casing & Casing & Pump & Formation \\
\hline & & $200.1-190.1 \mathrm{ft} \mathrm{msl}$ & $300.5 \mathrm{ft} \mathrm{msl}$ & 4" PVC & $\mathbf{S}$ & Barnw \\
\hline
\end{tabular}

\section{FIELD MEASUREMENTS}

Sample date: 04/06/94

Depth to water: $76.59 \mathrm{ft}(23.34 \mathrm{~m})$ below TOC

Water elevation: $223.91 \mathrm{ft}(68.25 \mathrm{~m}) \mathrm{msl}$

Sp. conductance: $117 \mu \mathrm{S} / \mathrm{cm}$

Turbidity: 5.3 NTU

Water evacuated before sampling: $18 \mathrm{gal}$

The well went dry during purging.

\section{LABORATORY ANALYSES}

H $\underline{\text { Analyte }}$

- $\quad$ pH

Specific conductance

Turbidity

Acetophenone

Acetophenone

Aldrin

Aluminum, total recoverable

Antimony, total recoverable

Arsenic, total recoverable

Barium, total recoverable

Benzene

Bromodichloromethane

Bromoform

Bromomethane (Methyl bromide)

Cadmium, total recoverable

Calcium, total recoverable

Carbon tetrachloride

Chloride

Chlorobenzene

Chloroethane

Chloroethene (Vinyl chloride)

2-Chloroethyl vinyl ether

Chloroform

Chloromethane (Methyl chloride)

Chromium, total recoverable

Copper, total recoverable

Cyanide

p,p'-DDT

Dibromochloromethane

1,1-Dichloroethane
Time: $9: 17$

pH: 6.7

Alkalinity: $24 \mathrm{mg} / \mathrm{L}$

Water temperature: $19.1^{\circ} \mathrm{C}$

Volumes purged: 0.8 well volumes

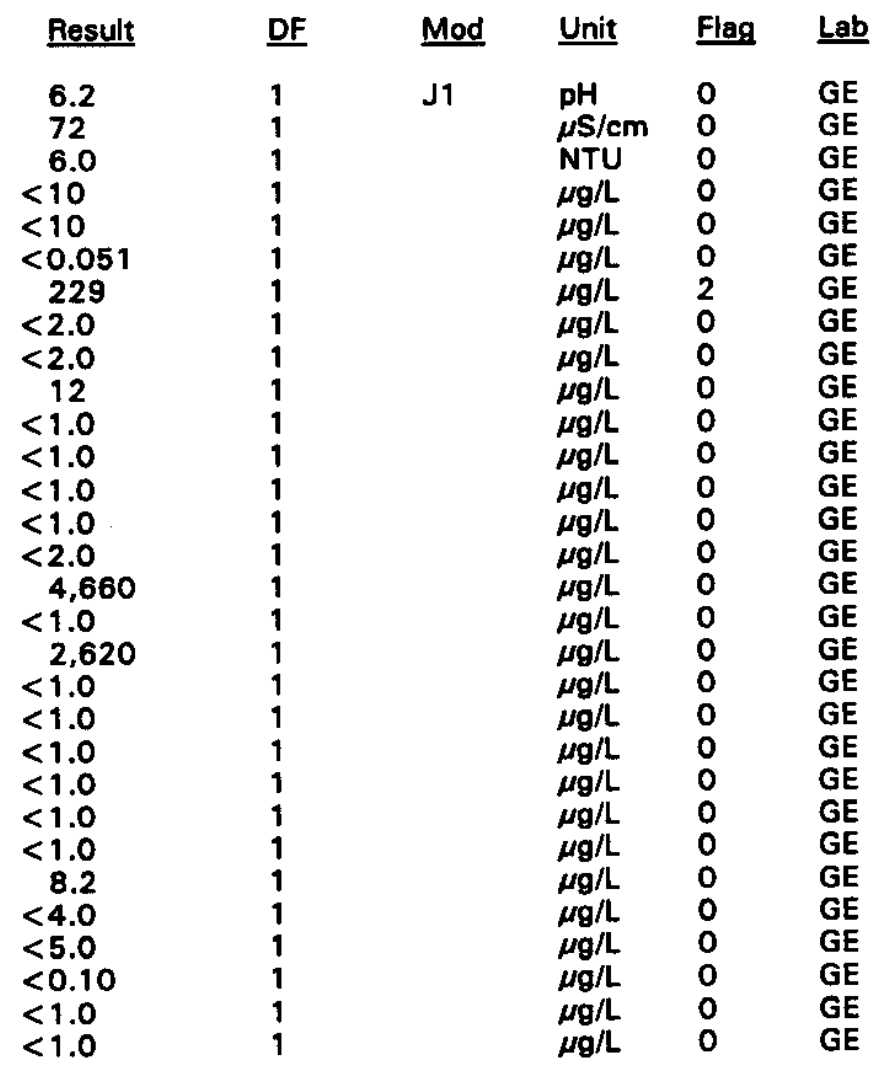

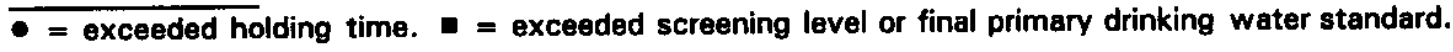


WELL BGO 14CR collected on 04/06/94, laboratory analyses (cont.)

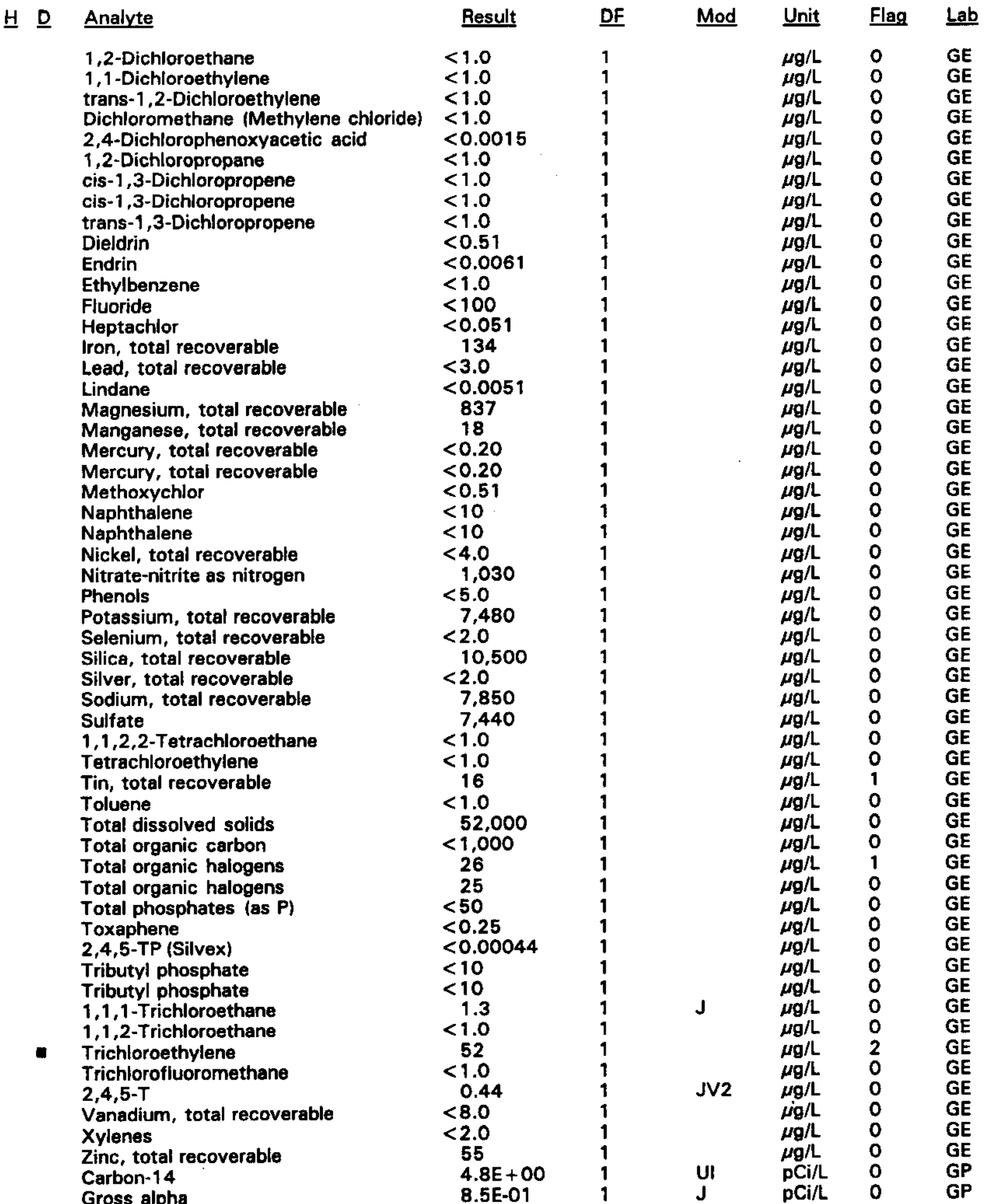

- exceeded holding time. $=$ =xceeded screening level or final primary drinking water standard. 
WELL BGO 14CR collected on 04/06/94, laboratory analyses (cont.)

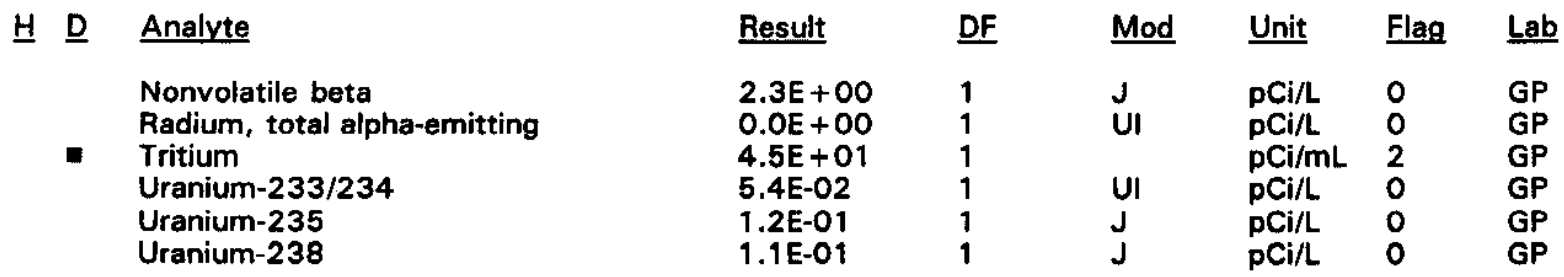

WELL BGO 14DR

\begin{tabular}{|c|c|c|c|c|c|c|}
\hline SRS Coord. & Lat/Longitude & Screen Zone Elevation & Top of Casing & Casing & Pump & Formation \\
\hline $\begin{array}{l}\text { N76322.1 } \\
\text { E55789.4 }\end{array}$ & $\begin{array}{l}33.285602^{\circ} \mathrm{N} \\
81.668150^{\circ} \mathrm{W}\end{array}$ & $238.1-218.1 \mathrm{ft} \mathrm{msl}$ & $300.3 \mathrm{ft} \mathrm{msl}$ & 4" PVC & s & Water Table $\left(I \mathrm{~B}_{2}\right)$ \\
\hline
\end{tabular}

\section{FIELD MEASUREMENTS}

Sample date: $04 / 05 / 94$

Depth to water: $69.34 \mathrm{ft}(21.14 \mathrm{~m})$ below TOC

Water elevation: $230.96 \mathrm{ft}(70.40 \mathrm{~m}) \mathrm{ms}$ l

Sp. conductance: $45 \mu \mathrm{S} / \mathrm{cm}$

Turbidity: 0.4 NTU

Water evacuated before sampling: 53 gal

\section{LABORATORY ANALYSES}

H D Analyte

- $\quad \mathrm{pH}$

pH
Specific conductance
Turbidity
Acetophenone
Aldrin
Aluminum, total recoverable
Antimony, total recoverable
Arsenic, total recoverable
Barium, total recoverable
Benzene
Bromodichloromethane
Bromoform
Bromomethane (Methyl bromide)
Cadmium, total recoverable
Calcium, total recoverable
Carbon tetrachloride
Chloride
Chlorobenzene
Chloroethane
Chloroethene (Vinyl chloride)
2-Chloroethyl vinyl ether
Chloroform
Chloromethane (Methyl chloride)
Chromium, total recoverable
Copper, total recoverable
Cyanide
p,p'-DDT
Dibromochloromethane

Time: 14:42

pH: 5.1

Alkalinity: $1 \mathrm{mg} / \mathrm{L}$

Water temperature: $20.4^{\circ} \mathrm{C}$

Volumes purged: 6.3 well volumes

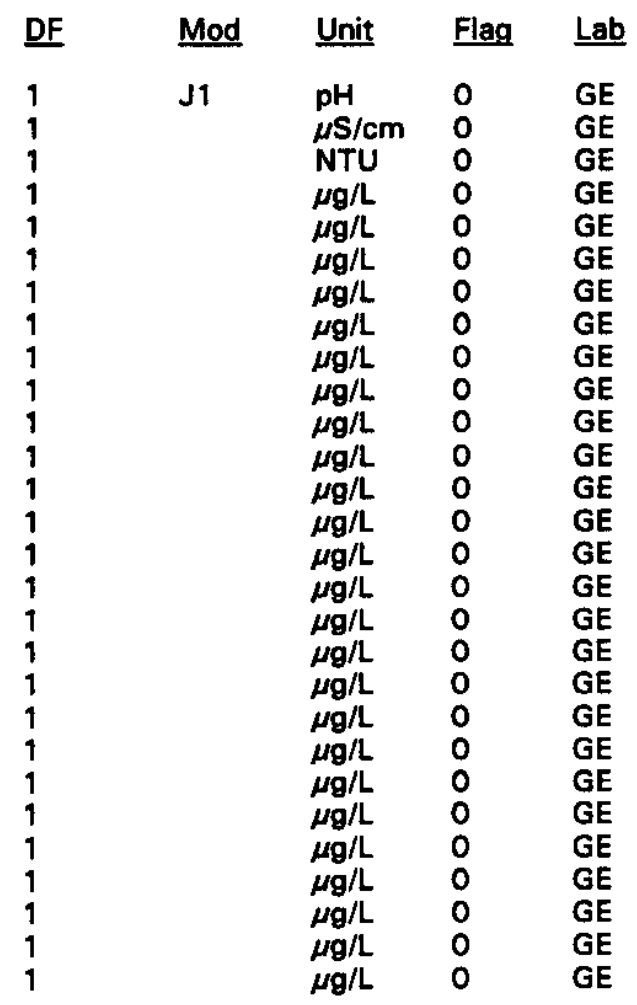

\footnotetext{
$\overline{0}=$ exceeded holding time. $\omega=$ exceeded screening level or final primary drinking water standard.
} 
WELL BGO 14DR collected on 04/05/94, laboratory analyses (cont.)

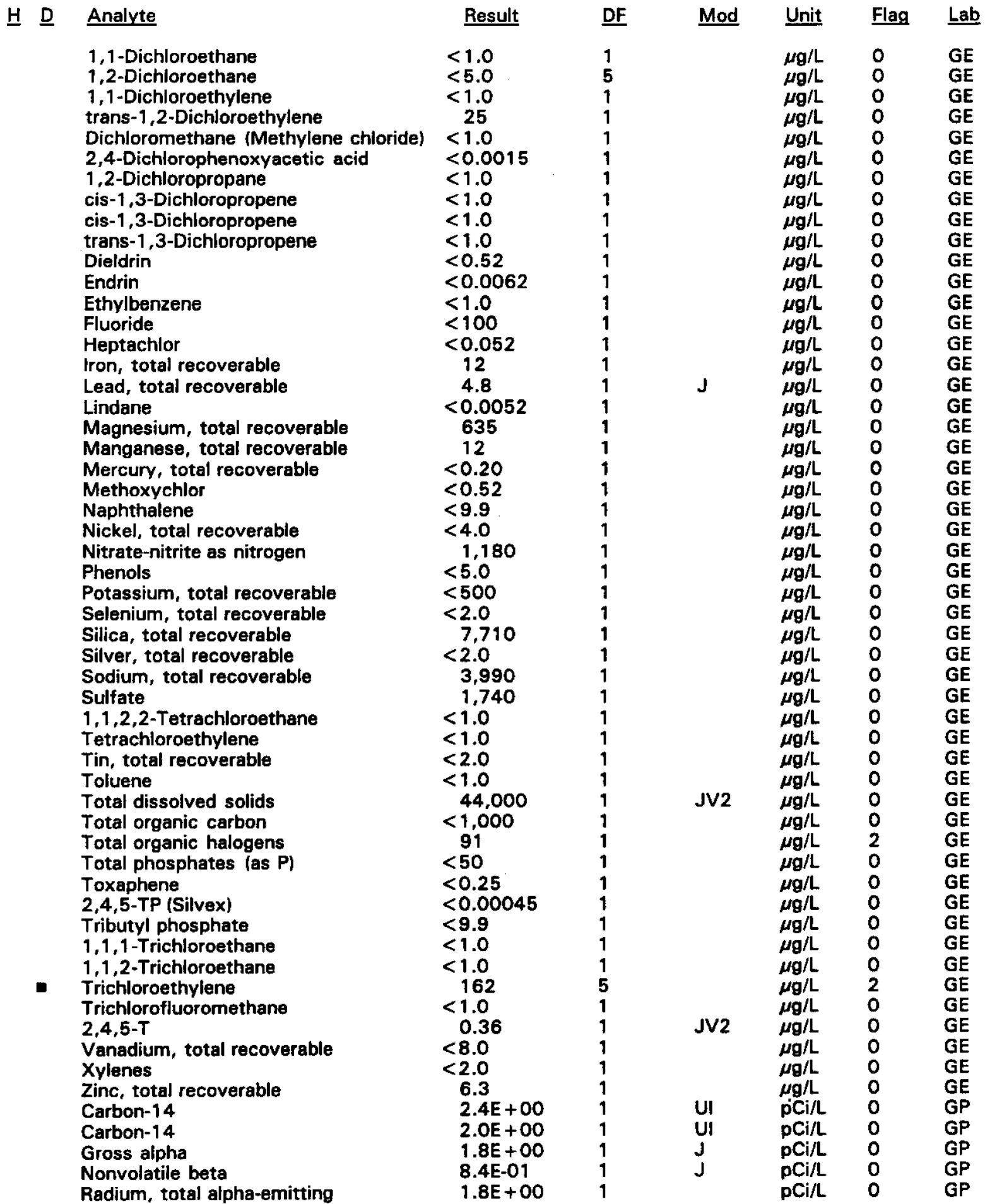

- = exceeded holding time. = exceeded screening level or final primary drinking water standard. 
WELL BGO 14DR collected on $04 / 05 / 94$, laboratory analyses (cont.)

\begin{tabular}{|c|c|c|c|c|c|}
\hline H $\underline{D}$ Analyte & Result & DF & Mod & Unit & Flag \\
\hline $\begin{array}{l}\text { Tritium } \\
\text { Uranium-233/234 } \\
\text { Uranium-235 } \\
\text { Uranium-238 }\end{array}$ & $\begin{array}{l}1.2 \mathrm{E}+01 \\
8.8 \mathrm{E}-02 \\
6.8 \mathrm{E}-02 \\
1.3 \mathrm{E}-01\end{array}$ & $\begin{array}{l}1 \\
1 \\
1 \\
1\end{array}$ & $\begin{array}{l}\mathbf{J} \\
\mathbf{J}\end{array}$ & $\begin{array}{l}\mathrm{pCi} / \mathrm{mL} \\
\mathrm{pCi} / \mathrm{L} \\
\mathrm{pCi} / \mathrm{L} \\
\mathrm{pCi} / \mathrm{L}\end{array}$ & $\begin{array}{l}1 \\
0 \\
0 \\
0\end{array}$ \\
\hline
\end{tabular}

\section{WELL BGO 15D}

$\begin{array}{lllllll}\text { SRS Coord, } & \text { Lat/Longitude } & \text { Screen Zone Elevation } & \text { Top of Casing } & \text { Casing } & \text { Pump } & \text { Formation } \\ \text { N75973.5 } & 33.284945^{\circ} \mathrm{N} & 238.7-218.7 \mathrm{ft} \mathrm{msl} & 298.7 \mathrm{ft} \mathrm{msl} & \text { 4" PVC } & \text { S } & \text { Water Table (IIB })_{2}\end{array}$

\section{FIELD MEASUREMENTS}

Sample date: 04/06/94

Depth to water: $68.06 \mathrm{ft}(20.74 \mathrm{~m})$ below TOC

Water elevation: $230.64 \mathrm{ft}(70.30 \mathrm{~m}) \mathrm{msl}$

Sp. conductance: $35 \mu \mathrm{S} / \mathrm{cm}$

Turbidity: 2.3 NTU

Water evacuated before sampling: $\mathbf{4 9}$ gal

\section{LABORATORY ANALYSES}

\section{H $\underline{\text { Analyte }}$}

pH

Specific conductance

Specific conductance

Turbidity

Acetophenone

Aldrin

Aluminum, total recoverable

Antimony, total recoverable

Arsenic, total recoverable

Barium, total recoverable

Benzene

Bromodichloromethane

Bromoform

Bromomethane (Methyl bromide)

Cadmium, total recoverable

Calcium, total recoverable

Carbon tetrachloride

Chloride

Chloride

Chlorobenzene

Chloroethane

Chloroethene (Vinyl chloride)

2-Chloroethyl vinyl ether

Chloroform

Chloromethane (Methyl chloride)

Chromium, total recoverable

Copper, total recoverable

Cyanide

p,p'-DDT

Dibromochloromethane

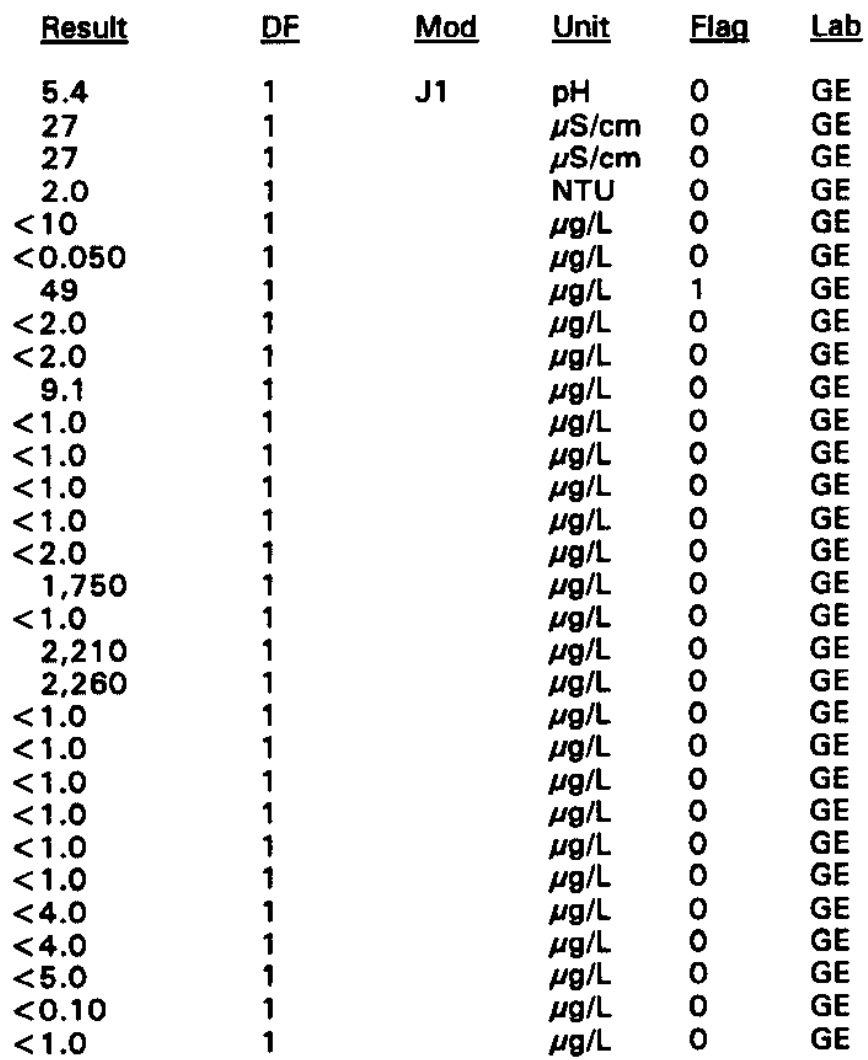

Time: $14: 43$

pH: 5.3

Alkalinity: $1 \mathrm{mg} / \mathrm{L}$

Water temperature: $21.1^{\circ} \mathrm{C}$

Volumes purged: 6.3 well volumes 
WELL BGO 15D collected on 04/06/94, laboratory analyses (cont.)

H D Analyte

1,1-Dichloroethane

1,2-Dichloroethane

1,1-Dichloroethylene

trans-1,2-Dichloroethylene

Dichloromethane (Methylene chloride)

2,4-Dichlorophenoxyacetic acid

1,2-Dichloropropane

cis-1,3-Dichloropropene

trans-1,3-Dichloropropene

Dieldrin

Endrin

Ethylbenzene

Fluoride

Heptachlor

Iron, total recoverable

Lead, total recoverable

Lindane

Magnesium, total recoverable

Manganese, total recoverable

Mercury, total recoverable

Methoxychior

Naphthalene

Nickel, total recoverable

Nitrate-nitrite as nitrogen

Phenols

Potassium, total recoverable

Selenium, total recoverable

Silica, total recoverable

Silver, total recoverable

Sodium, total recoverable

Sulfate

Sulfate

$1,1,2,2$-Tetrachloroethane

- Tetrachloroethylene

Tin, total recoverable

Toluene

Total dissolved solids

Total organic carbon

Total organic halogens

Total phosphates (as P)

Toxaphene

- 2,4,5-TP (Silvex)

1,1,1-Trichloroethane

1,1,2-Trichloroethane

Trichloroethylene

Trichlorofluoromethane

2,4,5-T

Vanadium, total recoverable

Xylenes

Zinc, total recoverable

Carbon-14

Gross alpha

Nonvolatile beta

Radium, total alpha-emitting

Total activity

- Tritium
Result DF

$<1.0$

$<1.0$

$<1.0$

$<1.0$

$<1.0$

$<0.0015$

$<1.0$

$<1.0$

$<1.0$

$<0.50$

$<0.0060$

$<1.0$

$<100$

$<0.050$

12

$<3.0$

$<0.0050$

427

7.8

$<0.20$

$<0.50$

$<10$

$<4.0$

1,140

$<5.0$

$<500$

$<2.0$

6,930

$<2.0$

2,160

$<1,000$

$<1,000$

$<1.0$

5.8

$<2.0$

$<1.0$

26,000

$<1,000$

53

$<50$

$<0.24$

$<0.00046$

$<1.0$

$<1.0$

69

1.4

$<0.00046$

$<8.0$

$<2.0$

$<2.0$

$1.9 \mathrm{E}+00$

$2.1 E+00$

$1.9 \mathrm{E}+00$

$1.2 \mathrm{E}+00$

2.7E + 05

$2.6 E+02$
Mod

Unit

Flag

Lab

$\mu \mathrm{g} / \mathrm{L}$

O GE

$\begin{array}{lll}\mu g / L & 0 & \mathrm{GE} \\ \mu \mathrm{g} / \mathrm{L} & 0 & \mathrm{GE}\end{array}$

$\mu \mathrm{g} / \mathrm{L} \quad 0 \quad \mathrm{GE}$

$\mu \mathrm{g} / \mathrm{L} \quad 0 \quad \mathrm{GE}$

J1

$\begin{array}{lll}\mu \mathrm{g} / \mathrm{L} & 0 & \mathrm{GE} \\ \mu \mathrm{g} / \mathrm{L} & 0 & \mathrm{GE}\end{array}$

$\mu g / L \quad 0 \quad G E$

$\mu g / L \quad O \quad$ GE

$\mu g / L \quad 0 \quad \mathrm{GE}$

$\mu \mathrm{g} / \mathrm{L} \quad 0 \quad \mathrm{GE}$

$\mu \mathrm{g} / \mathrm{L} \quad 0 \quad$ GE

$\mu \mathrm{g} / \mathrm{L} \quad \mathrm{O} \quad \mathrm{GE}$

$\mu \mathrm{g} / \mathrm{L} \quad \mathrm{O} \quad \mathrm{GE}$

$\mu \mathrm{g} / \mathrm{L} \quad \mathrm{O} \quad \mathrm{GE}$

$\mu g / L \quad 0 \quad G E$

$\mu g / L \quad 0 \quad G E$

$\mu g / L \quad 0 \quad$ GE

$\mu g / L \quad 0 \quad G E$

$\mu g / L \quad 0 \quad G E$

$\mu g / L \quad 0 \quad$ GE

$\mu g / L \quad 0 \quad$ GE

$\mu g / \mathrm{L} \quad 0 \quad \mathrm{GE}$

$\mu \mathrm{g} / \mathrm{L} \quad 0 \quad \mathrm{GE}$

$\mu g / L \quad 0 \quad$ GE

$\mu \mathrm{g} / \mathrm{L} \quad 0 \quad \mathrm{GE}$

$\mu \mathrm{g} / \mathrm{L} \quad \mathrm{O} \quad \mathrm{GE}$

$\mu \mathrm{g} / \mathrm{L} \quad \mathrm{O} \quad \mathrm{GE}$

$\mu \mathrm{g} / \mathrm{L} \quad 0 \quad \mathrm{GE}$

$\mu g / L \quad 0 \quad$ GE

$\mu \mathrm{g} / \mathrm{L} \quad 0 \quad \mathrm{GE}$

$\mu \mathrm{g} / \mathrm{L} \quad 0 \quad \mathrm{GE}$

$\mu \mathrm{g} / \mathrm{L} \quad 0 \quad \mathrm{GE}$

$\mu g / L \quad 2 \quad$ GE

$\mu g / L \quad 0 \quad$ GE

$\mu g / L \quad 0 \quad G E$

JV2

$\mu g / L$

$\mu \mathrm{g} / \mathrm{L}$

$\mu g / L$

$\mu g / L$

$\mu g / L$

J1 $\mu g / L$

$\mu \mathrm{g} / \mathrm{L}$

$\mu g / L$

$\mu g / L$

$\mu g / L$

$\mu g / L$

$\mu g / L$

$\mu g / L$

$\mu \mathrm{g} / \mathrm{L}$

pCi/L

$\mathrm{pCi} / \mathrm{L}$

pCi/L

$\mathrm{pCi} / \mathrm{L}$

pCi/L

$\mathrm{pCi} / \mathrm{mL}$

GE

GE

GE

GE

GE

GE

GE

GE

GE

GE

GE

GE

GE

GE

GP

GP

GP

EM

GP

- = exceeded holding time. = exceeded screening level or final primary drinking water standard. 
WELL BGO 15D collected on 04/06/94, laboratory analyses (cont.)

H D Analyte

Uranium-233/234
Uranium-233/234
Uranium-235
Uranium-235
Uranium-238
Uranium-238

Uranium-233/234

Uranium-235

Uranium-238

Uranium-238

Result
$1.8 \mathrm{E}-02$
$8.9 \mathrm{E}-02$
$9.0 \mathrm{E}-03$
$1.3 \mathrm{E}-02$
$6.5 \mathrm{E}-02$
$-2.3 \mathrm{E}-02$

DF

$\begin{array}{lllll}\text { Mod } & & \text { Unit } & \text { Flag } & \text { Lab } \\ \text { UI } & & \text { pCi/L } & 0 & \text { GP } \\ J & & \text { pCi/L } & 0 & \text { GP } \\ \text { UI } & \text { pCi/L } & 0 & \text { GP } \\ U I & \text { pCi/L } & 0 & \text { GP } \\ J & \text { pCi/L } & 0 & \text { GP } \\ \text { UI } & \text { pCi/L } & 0 & \text { GP }\end{array}$

\section{WELL BGO 16AR}

\begin{tabular}{|c|c|c|c|c|c|c|}
\hline SRS Coord. & Lat/Longitude & Screen Zone Elevation & Top of Casing & Casing & Pump & Formation \\
\hline $\begin{array}{l}\text { N75743.2 } \\
=56217.1\end{array}$ & $\begin{array}{l}33.285020^{\circ} \mathrm{N} \\
81.665899^{\circ} \mathrm{W}\end{array}$ & $113.7-103.7 \mathrm{ft} \mathrm{ms}$ & $303.7 \mathrm{ft} \mathrm{msl}$ & 4" PVC & $\mathbf{s}$ & \\
\hline
\end{tabular}

\section{FIELD MEASUREMENTS}

Sample date: 04/07/94

Depth to water: $142.75 \mathrm{ft}(\mathbf{4 3 . 5 1} \mathrm{m})$ below TOC

Water elevation: $160.95 \mathrm{ft}(49.06 \mathrm{~m}) \mathrm{msl}$

Sp. conductance: $200 \mu \mathrm{S} / \mathrm{cm}$

Turbidity: 0.1 NTU

Water evacuated before sampling: $103 \mathrm{gal}$

\section{LABORATORY ANALYSES}

\section{H $\underline{\text { Analyte }}$}

$\mathrm{pH}$

Specific conductance

Turbidity

Acetophenone

Aldrin

Aluminum, total recoverable

Aluminum, total recoverable

Antimony, total recoverable

Arsenic, total recoverable

Barium, total recoverable

Barium, total recoverable

Benzene

Bromodichloromethane

Bromoform

Bromomethane (Methyl bromide)

Cadmium, total recoverable

Cadmium, total recoverable

Calcium, total recoverable

Calcium, total recoverable

Carbon tetrachloride

Chioride

Chlorobenzene

Chioroethane

Chloroethene (Vinyl chloride)

2-Chloroethyl vinyl ether

Chloroform

Chloromethane (Methyl chloride)

Chromium, total recoverable

$\quad$ Result
7.6
180
$<0.10$
$<10$
$<0.050$
$<20$
$<20$
$<2.0$
$<2.0$
39
38
$<1.0$
$<1.0$
$<1.0$
$<1.0$
$<2.0$
$<2.0$
29.000
28,700
$<1.0$
2.030
$<1.0$
$<1.0$
$<1.0$
$<1.0$
$<1.0$
$<1.0$
$<4.0$

Time: 13:46

pH: 7.5

Alkalinity: $78 \mathrm{mg} / \mathrm{L}$

Water temperature: $19.8^{\circ} \mathrm{C}$

Volumes purged: 2.7 well volumes

\begin{tabular}{|c|c|c|c|c|}
\hline DF & Mod & Unit & Flag & Lat \\
\hline 1 & J1 & $\mathrm{pH}$ & $\begin{array}{l}0 \\
0\end{array}$ & $\begin{array}{l}\mathrm{GE} \\
\mathrm{GE}\end{array}$ \\
\hline $\begin{array}{l}1 \\
1\end{array}$ & & $\begin{array}{l}\mu \mathrm{s} / \mathrm{cm} \\
\text { NTU }\end{array}$ & 0 & $\mathrm{GE}$ \\
\hline 1 & & $\mu \mathrm{g} / \mathrm{L}$ & 0 & GE \\
\hline 1 & & $\mu g / L$ & 0 & GE \\
\hline 1 & & $\mu g / L$ & 0 & $\mathrm{GE}$ \\
\hline 1 & & $\mu g / L$ & 0 & GE \\
\hline 1 & & $\mu g / L$ & 0 & GE \\
\hline 1 & & $\mu \mathrm{g} / \mathrm{L}$ & 0 & $\mathrm{GE}$ \\
\hline 1 & & $\mu g / L$ & 0 & $\mathrm{GE}$ \\
\hline 1 & & $\mu g / L$ & 0 & GE \\
\hline 1 & & $\mu \mathrm{g} / \mathrm{L}$ & 0 & GE \\
\hline 1 & & $\mu g / L$ & 0 & GE \\
\hline 1 & & $\mu \mathrm{g} / \mathrm{L}$ & 0 & GE \\
\hline 1 & & $\mu \mathrm{g} / \mathrm{L}$ & 0 & SE \\
\hline 1 & & $\mu \boldsymbol{g} / \mathrm{L}$ & 0 & GE \\
\hline 1 & & $\mu g / L$ & 0 & \\
\hline 1 & & $\mu g / L$ & 0 & GE \\
\hline 1 & & $\mu \mathrm{g} / \mathrm{L}$ & 0 & GE \\
\hline 1 & & $\mu \mathrm{g} / \mathrm{L}$ & 0 & G \\
\hline 1 & & $\mu \mathrm{g} / \mathrm{L}$ & 0 & GE \\
\hline 1 & & $\mu \mathrm{g} / \mathrm{L}$ & 0 & GE \\
\hline 1 & & $\mu \mathrm{g} / \mathrm{L}$ & 0 & GE \\
\hline 1 & & $\mu \mathrm{g} / \mathrm{L}$ & 0 & $\mathrm{GE}$ \\
\hline 1 & & $\mu g / L$ & 0 & \\
\hline 1 & & $\mu g / L$ & 0 & GE \\
\hline 1 & & & 0 & \\
\hline & & & & \\
\hline
\end{tabular}

- = exceeded holding time. = exceeded screening level or final primary drinking water standard. 
WELL BGO 16AR collected on 04/07/94, laboratory analyses (cont.)

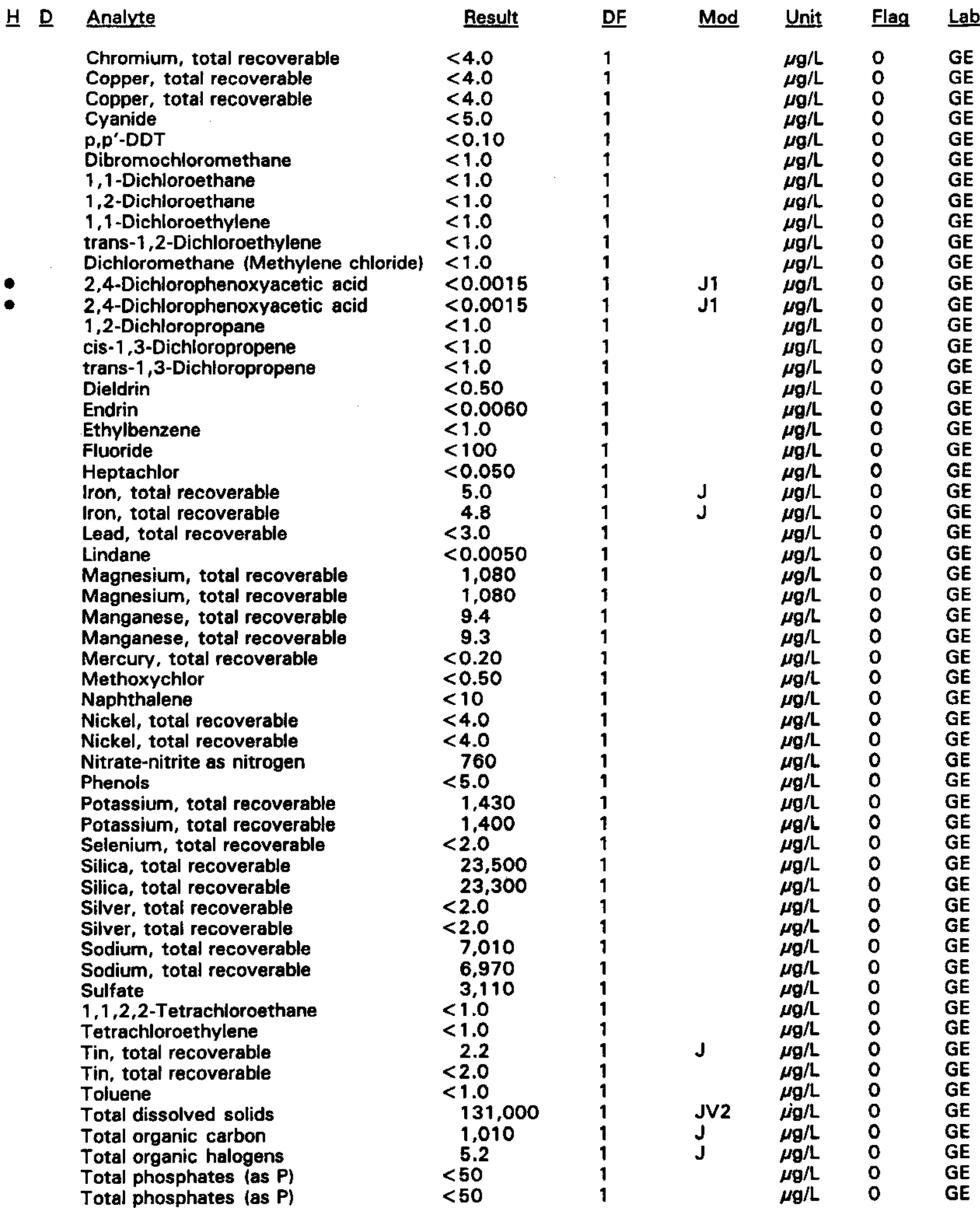

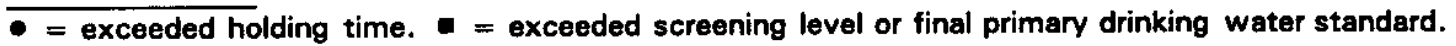


WELL BGO 16AR collected on 04/07/94, laboratory analyses (cont.)

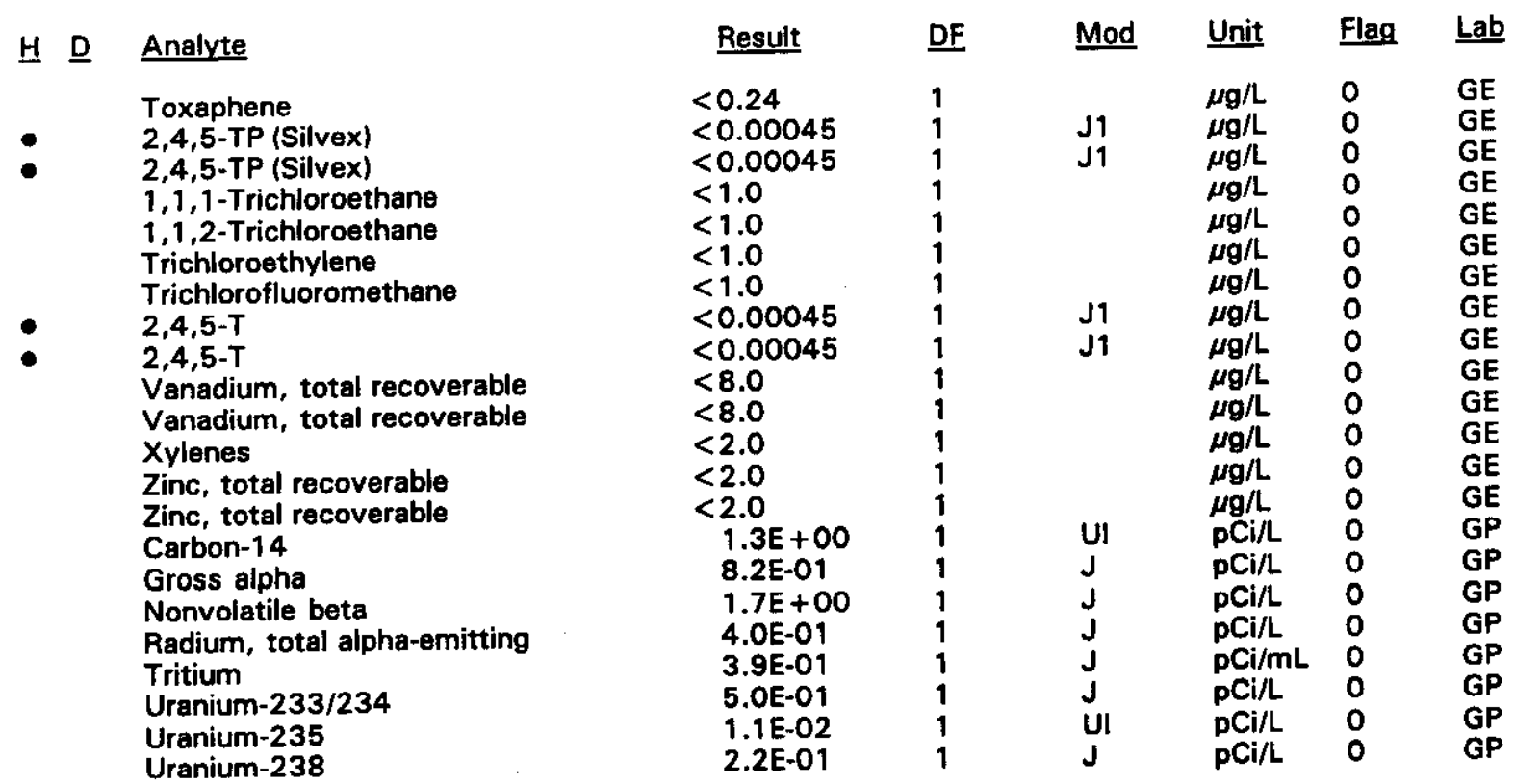

\section{WELL BGO 16B}

\begin{tabular}{|c|c|c|c|c|c|c|}
\hline SRS Coord. & Lat/Longitude & Screen Zone Elevation & Top of Casing & Casing & Pump & Formation \\
\hline $\begin{array}{l}175767.5 \\
56183.8\end{array}$ & $\begin{array}{l}33.285019^{\circ} \mathrm{N} \\
81.666034^{\circ} \mathrm{W}\end{array}$ & 146.0-136.0 ft msl & $305.1 \mathrm{ft} \mathrm{msl}$ & 4" PVC & $\mathbf{S}$ & McBean (IIB, \\
\hline
\end{tabular}

\section{FIELD MEASUREMENTS}

Sample date: 04/08/94

Depth to water: $86.47 \mathrm{ft}(26.36 \mathrm{~m})$ below TOC

Water elevation: $218.63 \mathrm{ft}(66.64 \mathrm{~m}) \mathrm{msl}$

Sp. conductance: $227 \mu \mathrm{S} / \mathrm{cm}$

Turbidity: 18.8 NTU

Water evacuated before sampling: $46 \mathrm{gal}$

The well went dry during purging.

\section{LABORATORY ANALYSES}

\section{므 Analyte}

- $\quad$ pH

Specific conductance

Specific conductance

- Turbidity

Acetophenone

Aldrin

Aluminum, total recoverable

Antimony, total recoverable

Arsenic, total recoverable

Barium, total recoverable

Benzene

Benzene
Time: 10:16

pH: 9.9

Alkalinity: 85 mg/L

Water temperature: $18.1^{\circ} \mathrm{C}$

Volumes purged: 0.8 well volumes

\begin{tabular}{|c|c|c|c|c|}
\hline Result & DF & Mod & Unit & Flag \\
\hline $\begin{aligned} & 8.0 \\
& 232 \\
& 231 \\
& 4.4 \\
&<9.7 \\
&<0.049 \\
& 82 \\
&<2.0 \\
&<2.0 \\
& 61 \\
&<1.0 \\
&<1.0\end{aligned}$ & \begin{tabular}{l|l}
1 & 1 \\
1 & \\
1 \\
1 \\
1 \\
1 \\
1 \\
1 \\
1 \\
1 \\
1 \\
1
\end{tabular} & $\begin{array}{l}\text { J1 } \\
\text { J1 }\end{array}$ & $\begin{array}{l}\mathrm{pH} \\
\mu \mathrm{S} / \mathrm{cm} \\
\mu \mathrm{S} / \mathrm{cm} \\
\mathrm{NTU} \\
\mu \mathrm{g} / \mathrm{L} \\
\mu \mathrm{g} / \mathrm{L} \\
\mu \mathrm{g} / \mathrm{L} \\
\mu \mathrm{g} / \mathrm{L} \\
\mu \mathrm{g} / \mathrm{L} \\
\mu \mathrm{g} / \mathrm{L} \\
\mu \mathrm{g} / \mathrm{L} \\
\mu \mathrm{g} / \mathrm{L}\end{array}$ & $\begin{array}{l}0 \\
0 \\
0 \\
0 \\
0 \\
0 \\
2 \\
0 \\
0 \\
0 \\
0 \\
0\end{array}$ \\
\hline
\end{tabular}

- = exceeded holding time. - = exceeded screening level or final primary drinking water standard. 
WELL BGO 16B collected on 04/08/94, laboratory analyses (cont.)

H ㅁ Analyte

Bromodichloromethane

Bromodichloromethane

Bromoform

Bromoform

Bromomethane (Methyl bromide)

Bromomethane (Methyl bromide)

Cadmium, total recoverable

Calcium, total recoverable

Carbon tetrachloride

Carbon tetrachloride

Chloride

Chlorobenzene

Chlorobenzene

Chloroethane

Chloroethane

Chloroethene (Vinyl chloride)

Chloroethene (Vinyl chloride)

2-Chloroethyl vinyl ether

2-Chloroethyl vinyl ether

Chloroform

Chloroform

Chloromethane (Methyl chloride)

Chloromethane (Methyl chloride)

Chromium, total recoverable

Copper, total recoverable

Cyanide

p,p'-DDT

Dibromochloromethane

Dibromochloromethane

1,1-Dichloroethane

1,1-Dichloroethane

1,2-Dichloroethane

1,2-Dichloroethane

1,1-Dichloroethylene

1,1-Dichloroethylene

trans-1,2-Dichloroethylene

trans-1,2-Dichloroethylene

Dichloromethane (Methylene chloride)

Dichloromethane (Methylene chloride)

2,4-Dichlorophenoxyacetic acid

1,2-Dichloropropane

1,2-Dichloropropane

cis-1,3-Dichloropropene

cis-1,3-Dichloropropene

cis-1,3-Dichloropropene

trans-1,3-Dichloropropene

trans-1,3-Dichloropropene

Dieldrin

Endrin

Ethylbenzene

Ethylbenzene

Fluoride

Heptachlor

Iron, total recoverable

Lead, total recoverable

Lindane

Result DF

$<1.0$

$<1.0$

$<1.0$

$<1.0$

$<1.0$

$<1.0$

$<2.0$

39,800

$<1.0$

$<1.0$

2,110

$<1.0$

$<1.0$

$<1.0$

$<1.0$

$<1.0$

$<1.0$

$<1.0$

$<1.0$

$<1.0$

$<1.0$

$<1.0$

$<1.0$

4.4

15

$<5.0$

$<0.098$

$<1.0$

$<1.0$

$<1.0$

$<1.0$

$<1.0$

$<1.0$

$<1.0$

$<1.0$

$<1.0$

$<1.0$

$<1.0$

$<1.0$

$<0.0015$

$<1.0$

$<1.0$

$<1.0$

$<1.0$

$<1.0$

$<1.0$

$<1.0$

$<0.49$

$<0.0059$

$<1.0$

$<1.0$

$<100$

$<0.049$

332

3.2

$<0.0049$

\section{DF}

Mod

Unit

Flag Lab

$\mu g / L$

$\mu g / L$

$\mu g / L$

$\mu \mathrm{g} / \mathrm{L}$

$\mu g / L$

$\mu \mathrm{g} / \mathrm{L}$

$\mu \mathrm{g} / \mathrm{L}$

$\mu \mathrm{g} / \mathrm{L}$

$\mu g / L$

$\mu \mathrm{g} / \mathrm{L}$

$\mu \mathrm{g} / \mathrm{L}$

$\mu \mathrm{g} / \mathrm{L}$

$\mu g / L$

$\mu \mathrm{g} / \mathrm{L}$

$\mu g / L$

$\mu g / L$

$\mu \mathrm{g} / \mathrm{L}$

$\mu \mathrm{g} / \mathrm{L}$

$\mu g / L$

$\mu g / L$

$\mu \mathrm{g} / \mathrm{L}$

$\mu g / L$

$\mu \mathrm{g} / \mathrm{L}$

$\mu g / L$

$\mu g / L$

$\mu g / L$

$\mu g / L$

$\mu g / L$

$\mu g / L$

$\mu g / L$

$\mu \mathrm{g} / \mathrm{L}$

$\mu g / L$

$\mu g / L$

$\mu g / L$

$\mu g / L$

$\mu g / L$

$\mu g / L$

$\mu \mathrm{g} / \mathrm{L}$

$\mu \mathrm{g} / \mathrm{L}$

$\mu g / L$

$\mu \mathrm{g} / \mathrm{L}$

$\mu \mathrm{g} / \mathrm{L}$

$\mu \mathrm{g} / \mathrm{L}$

$\mu g / L$.

$\mu g / L$

$\mu \mathrm{g} / \mathrm{L}$

$\mu \mathrm{g} / \mathrm{L}$

$\mu \mathrm{g} / \mathrm{L}$

$\mu \mathrm{g} / \mathrm{L}$.

$\mu \mathrm{g} / \mathrm{L}$

$\mu \mathrm{g} / \mathrm{L}$

$\mu \mathrm{g} / \mathrm{L}$

$\mu \mathrm{g} / \mathrm{L}$

$\mu g / L$

$\mu \mathrm{g} / \mathrm{L}$

$\mu g / L$ o GE

O GE

O GE

O GE

GE

GE

GE

O GE

O GE

O GE

O GE

O GE

O GE

o GE

O GE

GE

O GE

0 GE

O GE

O GE

O GE

o GE

o GE

0 GE

0 GE

O GE

0 GE

O GE

O GE

GE

GE

GE

GE

GE

GE

GE

GE

GE

GE

GE

GE

GE

GE

GE

GE

GE

GE

GE

GE

GE
O GE

O GE

- = exceeded holding time. = exceeded screening level or final primary drinking water standard. 
WELL BGO $16 \mathrm{~B}$ collected on 04/08/94, laboratory analyses (cont.)

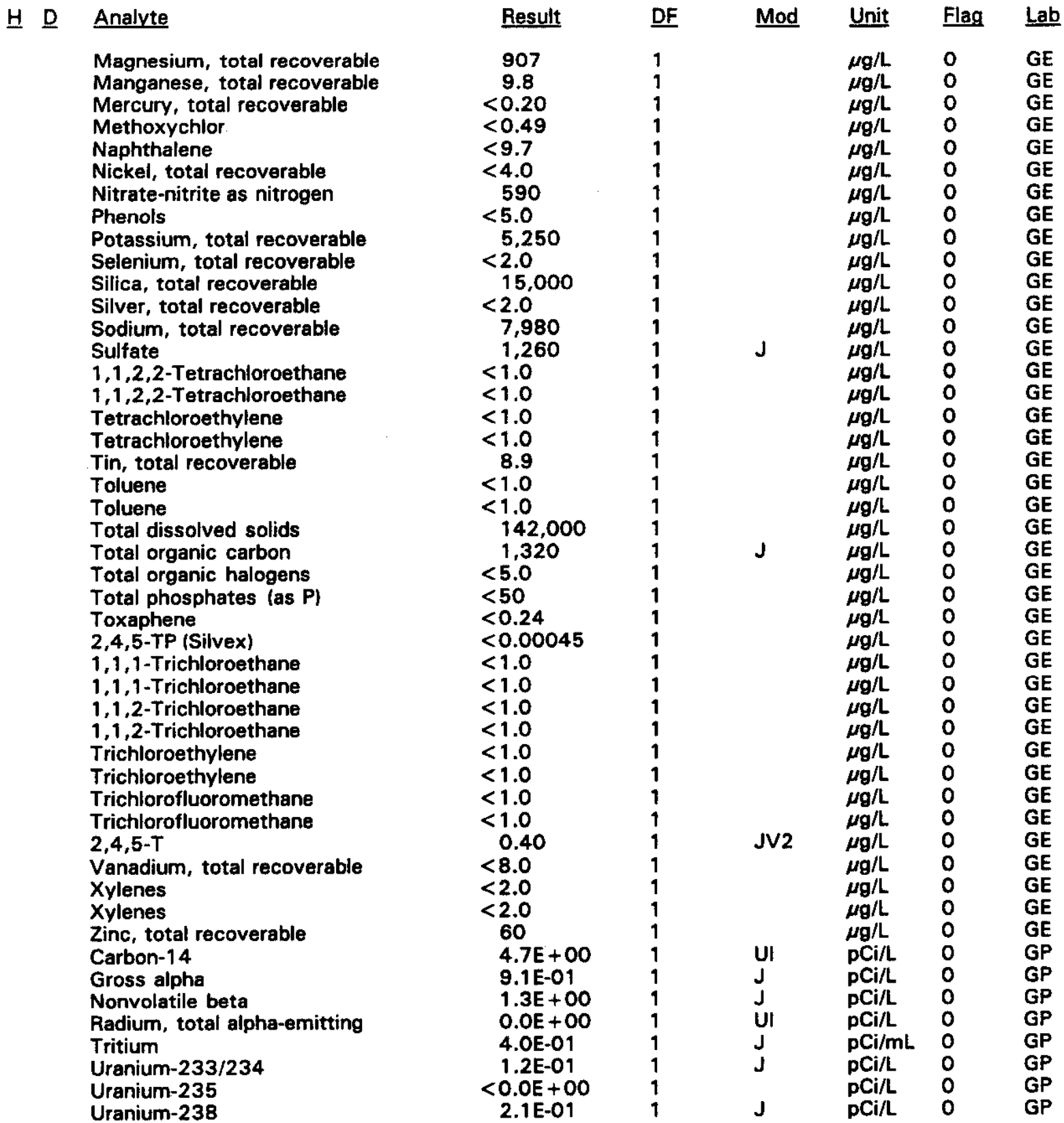

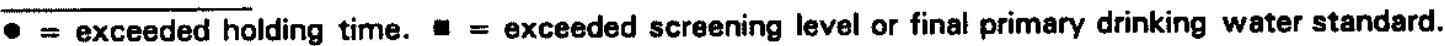




\section{WELL BGO 16D}

\begin{tabular}{|c|c|c|c|c|c|c|}
\hline SRS Coord. & Lat/Longitude & Screen Zone Elevation & Top of Casing & Casing & Pump & Formation \\
\hline $\begin{array}{l}N 75751.4 \\
\text { E56202.1 }\end{array}$ & $\begin{array}{l}33.285013^{\circ} \mathrm{N} \\
81.665954^{\circ} \mathrm{W}\end{array}$ & $237.3-217.3 \mathrm{ft} \mathrm{msl}$ & $304.6 \mathrm{ft} \mathrm{msl}$ & 4" PVC & s & ble $\left(\|_{2}\right)$ \\
\hline
\end{tabular}

FIELD MEASUREMENTS

Sample date: 04/08/94

Depth to water: $73.37 \mathrm{ft}(22.36 \mathrm{~m})$ below TOC

Water elevation: $231.23 \mathrm{ft}(70.48 \mathrm{~m}) \mathrm{ms}$

Sp. conductance: $198 \mu \mathrm{S} / \mathrm{cm}$

Turbidity: 2.8 NTU

Water evacuated before sampling: 3 gal

The well went dry during purging.

\section{LABORATORY ANALYSES}

$$
\text { : }
$$

-
Specific conductance

Turbidity

Turbidity

Acetophenone

Aldrin

Aluminum, total recoverable

Antimony, total recoverable

Arsenic, total recoverable

Barium, total recoverable

Benzene

Bromodichloromethane

Bromoform

Bromomethane (Methyl bromide)

Cadmium, total recoverable

Calcium, total recoverable

Carbon tetrachloride

Chloride

Chloride

Chlorobenzene

Chloroethane

Chloroethene (Vinyl chloride)

2-Chloroethyl vinyl ether

Chloroform

Chloromethane (Methyl chloride)

Chromium, total recoverable

Copper, total recoverable

Cyanide

p.p'-DDT

Dibromochloromethane

1,1-Dichloroethane

1,2-Dichloroethane

1,1-Dichloroethylene

trans-1,2-Dichloroethylene

Dichloromethane (Methylene chloride)

2,4-Dichlorophenoxyacetic acid

1,2-Dichloropropane

cis-1,3-Dichloropropene

trans-1,3-Dichloropropene

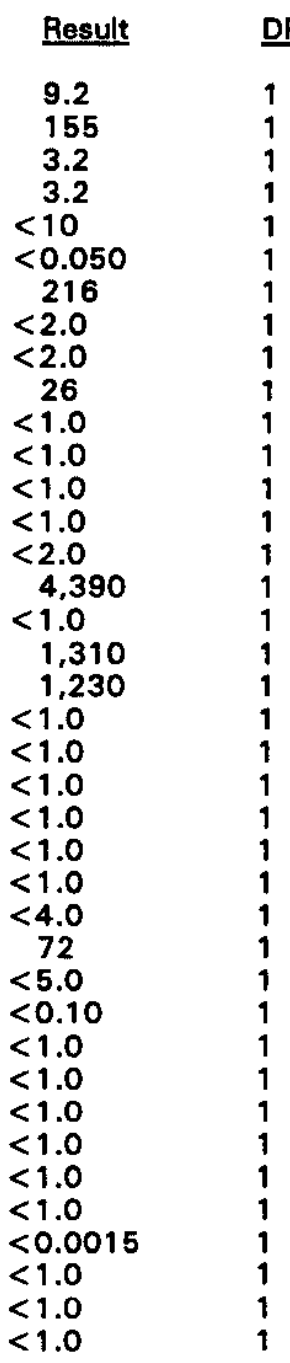

Time: 10:04

pH: 9.4

Alkalinity: $78 \mathrm{mg} / \mathrm{L}$

Water temperature: $17.8^{\circ} \mathrm{C}$

\begin{tabular}{|c|c|c|c|c|}
\hline DF & Mod & Unit & Flag & Lab \\
\hline 1 & J1 & $\mathrm{pH}$ & 1 & GE \\
\hline 1 & & $\mu \mathrm{S} / \mathrm{cm}$ & 0 & $\mathrm{GE}$ \\
\hline 1 & J1 & NTU & 0 & GE \\
\hline 1 & J1 & NTU & 0 & $\mathrm{GE}$ \\
\hline 1 & & $\mu g / L$ & 0 & GE \\
\hline 1 & & $\mu \mathrm{g} / \mathrm{L}$ & 0 & $\mathrm{GE}$ \\
\hline 1 & & $\mu g / L$ & 2 & GE \\
\hline 1 & & $\mu \mathrm{g} / \mathrm{L}$ & 0 & GE \\
\hline 1 & & $\mu g / L$ & 0 & GE \\
\hline 1 & & $\mu g / L$ & 0 & GE \\
\hline 1 & & $\mu g / L$ & 0 & GE \\
\hline 1 & & $\mu g / L$ & 0 & GE \\
\hline 1 & & $\mu g / L$ & 0 & GE \\
\hline 1 & & $\mu g / L$ & 0 & GE \\
\hline 1 & & $\mu g / L$ & 0 & GE \\
\hline 1 & & $\mu \mathrm{g} / \mathrm{L}$ & 0 & GE \\
\hline 1 & & $\mu g / L$ & 0 & GE \\
\hline 1 & & $\mu g / L$ & 0 & GE \\
\hline 1 & & $\mu g / L$ & 0 & GE \\
\hline 1 & & $\mu \mathrm{g} / \mathrm{L}$ & 0 & GE \\
\hline 1 & & $\boldsymbol{\mu g} / \mathrm{L}$ & 0 & GE \\
\hline 1 & & $\mu \mathrm{g} / \mathrm{L}$ & 0 & GE \\
\hline 1 & & $\mu g / L$ & 0 & GE \\
\hline $\begin{array}{l}1 \\
1\end{array}$ & & $\mu g / L$ & 0 & GE \\
\hline 1 & & $\mu g / L$ & $\begin{array}{l}0 \\
0\end{array}$ & \\
\hline 1 & & $\mu \mathrm{g} / \mathrm{L}$ & 0 & $\mathrm{GE}$ \\
\hline 1 & & $\mu g / L$ & 0 & \\
\hline 1 & & $\mu g / L$ & 0 & GE \\
\hline 1 & & $\mu \mathrm{g} / \mathrm{L}$ & 0 & GE \\
\hline 1 & & $\mu g / L$ & 0 & GE \\
\hline 1 & & $\mu g / L$ & 0 & GE \\
\hline 1 & & $\mu g / L$ & 0 & GE \\
\hline 1 & & $\mu g / L$ & 0 & GE \\
\hline 1 & & $\mu g / L$ & 0 & GE \\
\hline 1 & & $\mu g / L$ & 0 & \\
\hline 1 & & $\mu g / L$ & 0 & \\
\hline 1 & & $\mu \mathrm{g} / \mathrm{L}$ & 0 & GE \\
\hline 1 & & $\mu \mathrm{g} / \mathrm{L}$ & 0 & \\
\hline
\end{tabular}

Volumes purged: 0.3 well volumes

- = exceeded holding time. a = exceeded screening level or final primary drinking water standard. 
WELL BGO 16D collected on 04/08/94, laboratory analyses (cont.)

브몽

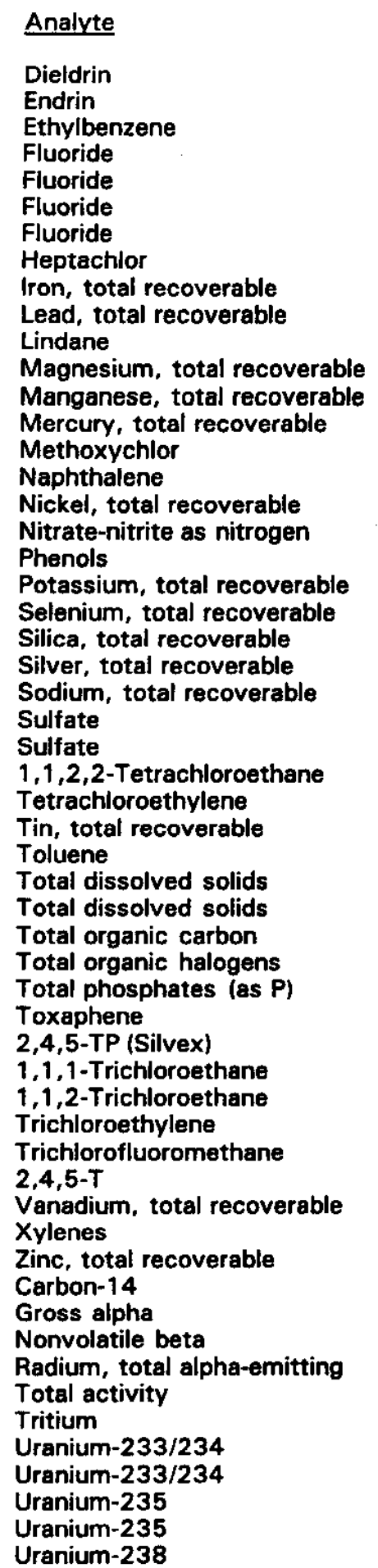

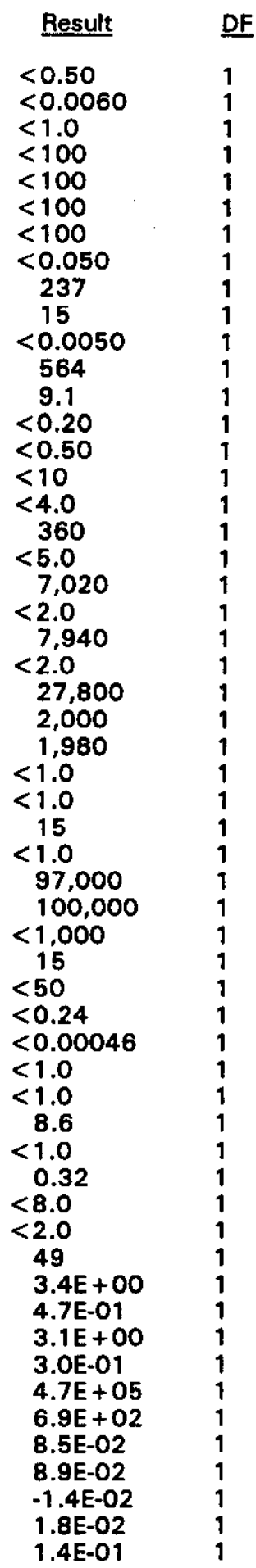

\begin{tabular}{|c|c|c|}
\hline Mod & Unit & Flag \\
\hline $\begin{array}{l}\text { UI } \\
\text { UI } \\
\text { UI } \\
\text { UI } \\
\text { UI }\end{array}$ & $\begin{array}{l}\mu g / L \\
\mu g / L \\
\mu g / L \\
\mu g / L \\
\mu g / L \\
\mu g / L \\
\mu g / L \\
\mu g / L \\
\mu g / L \\
\mu g / L \\
\mu g / L \\
\mu g / L \\
\mu g / L \\
\mu g / L \\
\mu g / L \\
\mu g / L \\
\mu g / L \\
\mu g / L \\
\mu g / L \\
\mu g / L\end{array}$ & $\begin{array}{l}0 \\
0 \\
0 \\
0 \\
0 \\
0 \\
0 \\
0 \\
1 \\
0 \\
0 \\
0 \\
0 \\
0 \\
0 \\
0 \\
0 \\
0 \\
0 \\
0 \\
0 \\
0 \\
0 \\
0 \\
0 \\
0 \\
0 \\
0 \\
1 \\
0 \\
0 \\
0 \\
0 \\
0 \\
0 \\
0 \\
0 \\
0 \\
0 \\
2 \\
0 \\
0 \\
0 \\
0 \\
0 \\
0 \\
0 \\
0 \\
0 \\
0 \\
2 \\
0 \\
0 \\
0 \\
0 \\
0 \\
0\end{array}$ \\
\hline
\end{tabular}

$\overline{-}=$ exceeded holding time. $\square=$ exceeded screening level or final primary drinking water standard. 
WELL BGO 16D collected on $04 / 08 / 94$, laboratory analyses (cont.)

H D $\begin{array}{lllllll}\text { Analyte } & \text { Result } & \underline{D F} & \underline{\text { Mod }} & \underline{\text { Unit }} & \text { Flag } & \text { Lab } \\ \text { Uranium-238 } & 5.8 \mathrm{E}-02 & 1 & \mathrm{UI} & \mathrm{pCi} / \mathrm{L} & 0 & \mathrm{GP}\end{array}$

\section{WELL BGO 17DR}

\begin{tabular}{|c|c|c|c|c|c|c|}
\hline SRS Coord. & Lat/Longitude & Screen Zone Elevation & Top of Casing & Casing & Pump & Formation \\
\hline 75 & $\begin{array}{l}33.285022^{\circ} \mathrm{N} \\
81.665128^{\circ} \mathrm{W}\end{array}$ & $236.9-216.9 \mathrm{ft} \mathrm{msl}$ & $299.2 \mathrm{ft} \mathrm{msl}$ & 4" PVC & $\mathbf{s}$ & Water Table $\left(\|_{2}\right)_{2}$ \\
\hline
\end{tabular}

\section{FIELD MEASUREMENTS}

Sample date: $04 / 08 / 94$

Depth to water: $65.89 \mathrm{ft}(20.08 \mathrm{~m})$ below TOC

Water elevation: $233.31 \mathrm{ft}(71.11 \mathrm{~m}) \mathrm{msl}$

Sp. conductance: $26 \mu \mathrm{S} / \mathrm{cm}$

Turbidity: 15.5 NTU

Water evacuated before sampling: 8 gal

The well went dry during purging.

\section{LABORATORY ANALYSES}

\section{H $\underline{\text { Analvte }}$}

$-\quad$ pH

pH
pH
Specific conductance
Turbidity
Acetophenone
Aldrin
Aluminum, total recoverable
Antimony, total recoverable
Arsenic, total recoverable
Barium, total recoverable
Benzene
Bromodichloromethane
Bromoform
Bromomethane (Methyl bromide)
Cadmium, total recoverable
Calcium, total recoverable
Carbon tetrachloride
Chloride
Chloride
Chlorobenzene
Chloroethane
Chloroethene (Vinyl chloride)
2-Chloroethyl vinyl ether
Chloroform
Chloromethane (Methyl chloride)
Chromium, total recoverable
Copper, total recoverable
Cyanide
p,p'-DDT
Dibromochloromethane
1,1-Dichloroethane
1,2-Dichloroethane
1,1-Dichloroethylene

Time: 9:49

pH: 5.6

Alkalinity: $2 \mathrm{mg} / \mathrm{L}$

Water temperature: $17.3^{\circ} \mathrm{C}$

Volumes purged: 0.7 well volumes

\begin{tabular}{|c|c|c|c|c|c|}
\hline Result & DF & Mod & $\underline{\text { Unit }}$ & Flag & Lab \\
\hline 5.8 & 1 & J1 & pH & 0 & GE \\
\hline 5.8 & $i$ & J1 & pH & 0 & $\mathrm{GE}$ \\
\hline 19 & 1 & & $\mu \mathrm{S} / \mathrm{cm}$ & 0 & GE \\
\hline 38 & $i$ & J1 & NTU & 0 & GE \\
\hline$<10$ & 1 & & $\mu \mathrm{g} / \mathrm{L}$ & 0 & \\
\hline$<0.052$ & 1 & & $\mu g / L$ & 0 & \\
\hline $\begin{array}{r}93 \\
<2.0\end{array}$ & 1 & & $\mu \mathrm{g} / \mathrm{L}$ & 2 & G \\
\hline $\begin{array}{l}<2.0 \\
<2.0\end{array}$ & 1 & & $\mu g / L$ & 0 & \\
\hline $\begin{array}{r}<2.0 \\
8.4\end{array}$ & 1 & & $\mu g / L$ & 0 & GE \\
\hline $\begin{array}{r}8.4 \\
<1.0\end{array}$ & 1 & & $\mu \mathrm{g} / \mathrm{L}$ & 0 & GE \\
\hline$<1.0$ & 1 & & $\mu \mathrm{g} / \mathrm{L}$ & 0 & GE \\
\hline$<1.0$ & 1 & & $\mu \mathrm{g} / \mathrm{L}$ & 0 & GE \\
\hline$<1.0$ & 1 & & $\mu g / L$ & 0 & GE \\
\hline$<1.0$ & 1 & & $\mu g / L$ & 0 & GE \\
\hline$<2.0$ & 1 & & $\mu \mathrm{g} / \mathrm{L}$ & 0 & GE \\
\hline 1,130 & 1 & & $\mu \boldsymbol{g} / \mathrm{L}$ & 0 & GE \\
\hline$<1.0$ & 1 & & $\mu g / L$ & 0 & \\
\hline $\begin{array}{r}2,470 \\
2,460\end{array}$ & 1 & & $\mu \mathrm{g} / \mathrm{L}$ & 0 & \\
\hline $\begin{array}{l}2,460 \\
<1.0\end{array}$ & 1 & & $\mu \mathrm{g} / \mathrm{L}$ & 0 & $\mathrm{GE}$ \\
\hline$<1.0$ & 1 & & $\mu g / L$ & 0 & \\
\hline$<1.0$ & 1 & & $\mu g / L$ & 0 & EE \\
\hline$<1.0$ & 1 & & $\mu g / L$ & 0 & GE \\
\hline$<1.0$ & 1 & & $\mu g / L$ & 0 & \\
\hline$<1.0$ & 1 & & $\mu g / L$ & 0 & GE \\
\hline$<1.0$ & 1 & & $\mu g / L$ & 0 & iE \\
\hline$<4.0$ & 1 & & $\mu g / L$ & 0 & \\
\hline$<4.0$ & 1 & & $\mu g / L$ & 0 & GE \\
\hline$<5.0$ & 1 & & $\mu \mathfrak{g} / \mathbf{L}$ & 0 & $\mathrm{SE}$ \\
\hline$<0.10$ & 1 & & $\mu \mathrm{g} / \mathrm{L}$ & 0 & \\
\hline$<1.0$ & 1 & & $\mu g / L$ & 0 & \\
\hline$<1.0$ & 1 & & $\mu g / L$ & 0 & \\
\hline$<1.0$ & 1 & & $\mu g / L$ & 0 & \\
\hline$<1.0$ & 1 & & $\mu \mathrm{g} / \mathrm{L}$ & 0 & \\
\hline
\end{tabular}

- = exceeded holding time. = exceeded screening level or final primary drinking water standard. 
WELL BGO 17DR collected on 04/08/94, laboratory analyses (cont.)

브

Analyte
trans-1,2-Dichloroethylene
Dichloromethane (Methylene chloride)
2,4-Dichlorophenoxyacetic acid
1,2-Dichloropropane
cis-1,3-Dichloropropene
cis-1,3-Dichloropropene
trans-1,3-Dichloropropene
Dieldrin
Endrin
Ethylbenzene
Fluoride
Fluoride
Heptachlor
Iron, total recoverable
Lead, total recoverable
Lindane
Magnesium, total recoverable
Manganese, total recoverable
Mercury, total recoverable
Mercury, total recoverable
Methoxychlor
Naphthalene
Nickel, total recoverable
Nitrate-nitrite as nitrogen
Phenols
Potassium, total recoverable
Selenium, total recoverable
Silica, total recoverable
Silver, total recoverable
Sodium, total recoverable
Sulfate
Sulfate
1,1,2,2-Tetrachloroethane
Tetrachloroethylene
Tin, total recoverable
Toluene
Total dissolved solids
Total organic carbon
Total organic halogens
Total phosphates (as P)
Toxaphene
2,4,5-TP (Silvex)
Tributyl phosphate
1,1,1-Trichloroethane
1,1,2-Trichloroethane
Trichloroethylene
Trichlorofluoromethane
2,4,5-T
Vanadium, total recoverable
Xylenes
Zinc, total recoverable
Carbon-14
Gross alpha
Nonvolatile beta
Radium, total alpha-emitting
Tritium

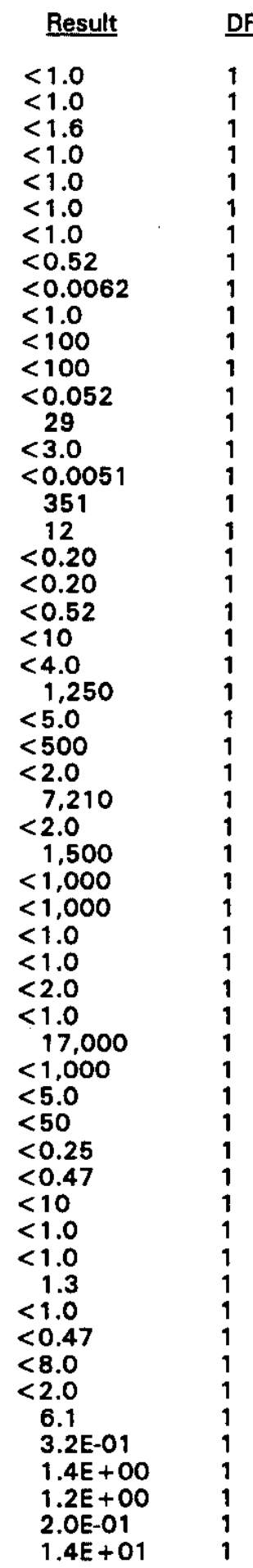

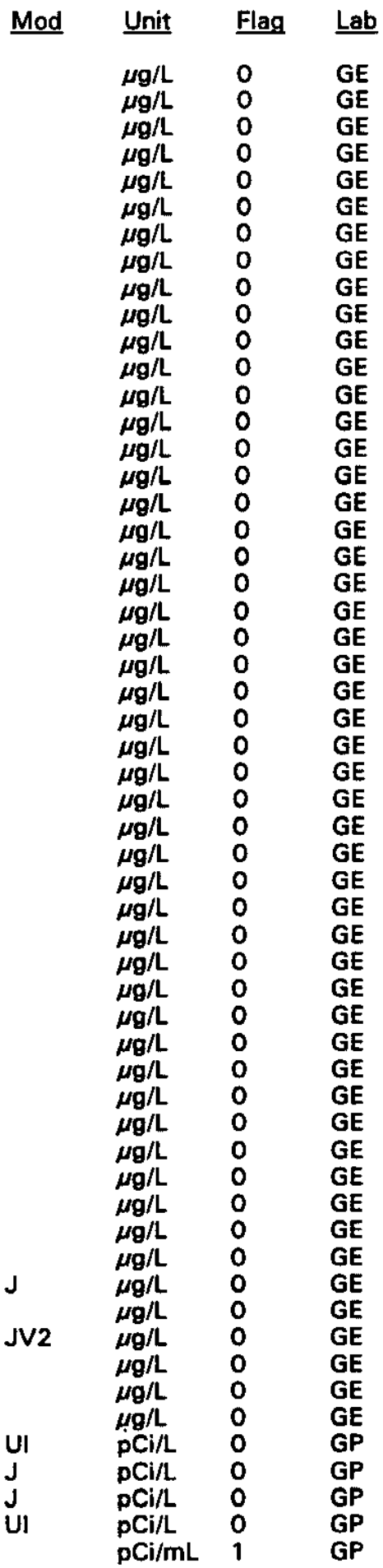

$\overline{0}$ = exceeded holding time. = =xceeded screening level or final primary drinking water standard. 
WELL BGO 17DR collected on 04/08/94, laboratory analyses (cont.)

H D Analyte

Uranium-233/234

Uranium-235

Uranium-238
Result DF

\subsection{E-01}

2.2E-02

2.1E-01
Mod

Unit

Flag L Lab

$J \quad \mathrm{pCi} / \mathrm{L}$

UI $\mathrm{pCi} / \mathrm{L}$

$\mathrm{J} \quad \mathrm{pCi} / \mathrm{L}$

$\begin{array}{ll}0 & \text { GP } \\ 0 & \text { GP } \\ 0 & \text { GP }\end{array}$

WELL BGO 18A

\begin{tabular}{|c|c|c|c|c|c|c|}
\hline SRS Coord. & Lat/Longitude & Screen Zone Elevation & Top of Casing & Casing & Pump & Formation \\
\hline $\begin{array}{l}\text { N75599.9 } \\
\text { E56699.7 }\end{array}$ & $\begin{array}{l}33.285490^{\circ} \mathrm{N} \\
81.664350^{\circ} \mathrm{W}\end{array}$ & $109.5-99.5 \mathrm{ft} \mathrm{msl}$ & $295.2 \mathrm{ft} \mathrm{msl}$ & 4" PVC & $\mathbf{s}$ & U. Congaree (IIA) \\
\hline
\end{tabular}

FIELD MEASUREMENTS

Sample date: 04/07/94

Depth to water: $133.91 \mathrm{ft}(40.82 \mathrm{~m})$ below TOC

Water elevation: $161.29 \mathrm{ft}(49.16 \mathrm{~m}) \mathrm{msl}$

Sp. conductance: $201 \mu \mathrm{S} / \mathrm{cm}$

Turbidity: 0.1 NTU

Water evacuated before sampling: $192 \mathrm{gal}$

\section{LABORATORY ANALYSES}

H D Analyte

$\mathrm{pH}$

Specific conductance

Turbidity

Acetophenone

Aldrin

Aluminum, total recoverable

Antimony, total recoverable

Arsenic, total recoverable

Barium, total recoverable

Benzene

Benzene

Bromodichloromethane

Bromodichloromethane

Bromoform

Bromoform

Bromomethane (Methyl bromide)

Bromomethane (Methyl bromide)

Cadmium, total recoverable

Calcium, total recoverable

Carbon tetrachloride

Carbon tetrachloride

Chloride

Chlorobenzene

Chlorobenzene

Chloroethane

Chloroethane

Chloroethene (Vinyl chloride)

Chloroethene (Vinyl chloride)

2-Chloroethyl vinyl ether

2-Chloroethyl vinyl ether

Chloroform
Time: 11:36

pH: 6.9

Alkalinity: $75 \mathrm{mg} / \mathrm{L}$

Water temperature: $19.4^{\circ} \mathrm{C}$

Volumes purged: 4.7 well volumes

\begin{tabular}{|c|c|c|c|c|c|}
\hline Result & DF & Mod & Unit & Flag & Lab \\
\hline $\begin{array}{l}7.0 \\
182\end{array}$ & 1 & J1 & $\mathrm{pH}$ & 0 & $\mathrm{GE}$ \\
\hline $\begin{array}{r}182 \\
<0.10\end{array}$ & 1 & & $\mu \mathrm{S} / \mathrm{cm}$ & 0 & GE \\
\hline$<10$ & 1 & & $\mu \mathrm{g} / \mathrm{L}$ & 0 & $\begin{array}{l}\text { GE } \\
\text { GE }\end{array}$ \\
\hline$<0.051$ & 1 & & $\mu \mathrm{g} / \mathrm{L}$ & 0 & GE \\
\hline$<20$ & 1 & & $\mu g / \mathrm{L}$ & 0 & GE \\
\hline$<2.0$ & 1 & & $\mu \mathrm{g} / \mathrm{L}$ & 0 & GE \\
\hline$<2.0$ & 1 & & $\mu g / L$ & 0 & GE \\
\hline 32 & 1 & & $\mu \mathrm{g} / \mathrm{L}$ & 0 & \\
\hline$<1.0$ & 1 & & $\mu \mathrm{g} / \mathrm{L}$ & 0 & GE \\
\hline$<1.0$ & $i$ & & $\mu g / \mathrm{L}$ & 0 & $\overrightarrow{G E}$ \\
\hline$<1.0$ & 1 & & $\mu g / L$ & 0 & GE \\
\hline$<1.0$ & 1 & & $\mu g / L$ & 0 & $\mathbf{E}$ \\
\hline$<1.0$ & 1 & & $\mu g / L$ & 0 & $\mathrm{GE}$ \\
\hline$<1.0$ & $i$ & & $\mu g / L$ & 0 & $\mathrm{EE}$ \\
\hline$<1.0$ & 1 & & $\mu g / L$ & 0 & GE \\
\hline$<1.0$ & 1 & & $\mu g / L$ & 0 & $\mathrm{GE}$ \\
\hline$<2.0$ & $i$ & & $\mu g / L$ & 0 & $\mathrm{GE}$ \\
\hline 33,100 & 1 & & $\mu \mathrm{g} / \mathrm{L}$ & 0 & G \\
\hline$<1.0$ & 1 & & $\mu \mathrm{g} / \mathrm{L}$ & 0 & \\
\hline$<1.0$ & 1 & & $\mu \mathrm{g} / \mathrm{L}$ & 0 & $\underline{E}$ \\
\hline 2,440 & 1 & & $\mu g / L$ & 0 & GE \\
\hline$<1.0$ & 1 & & $\mu \mathrm{g} / \mathrm{L}$ & 0 & E \\
\hline$<1.0$ & 1 & & $\mu \mathrm{g} / \mathrm{L}$ & 0 & \\
\hline$<1.0$ & 1 & & $\mu g / L$ & 0 & GE \\
\hline$<1.0$ & 1 & & $\mu g / L$ & 0 & \\
\hline$<1.0$ & 1 & & $\mu g / L$ & 0 & \\
\hline$<1.0$ & 1 & & $\mu g / L$ & 0 & $\mathrm{GE}$ \\
\hline$<1.0$ & $i$ & & $\mu g / L$ & 0 & \\
\hline$<1.0$ & 1 & & $\mu g / L$ & 0 & \\
\hline$<1.0$ & 1 & & $\mu \mathrm{g} / \mathrm{L}$ & 0 & $\mathrm{SE}$ \\
\hline
\end{tabular}

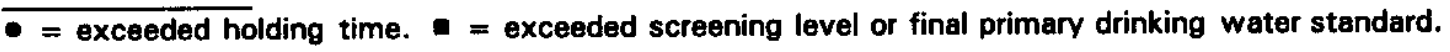


WELL BGO 18A collected on 04/07/94, laboratory analyses (cont.)

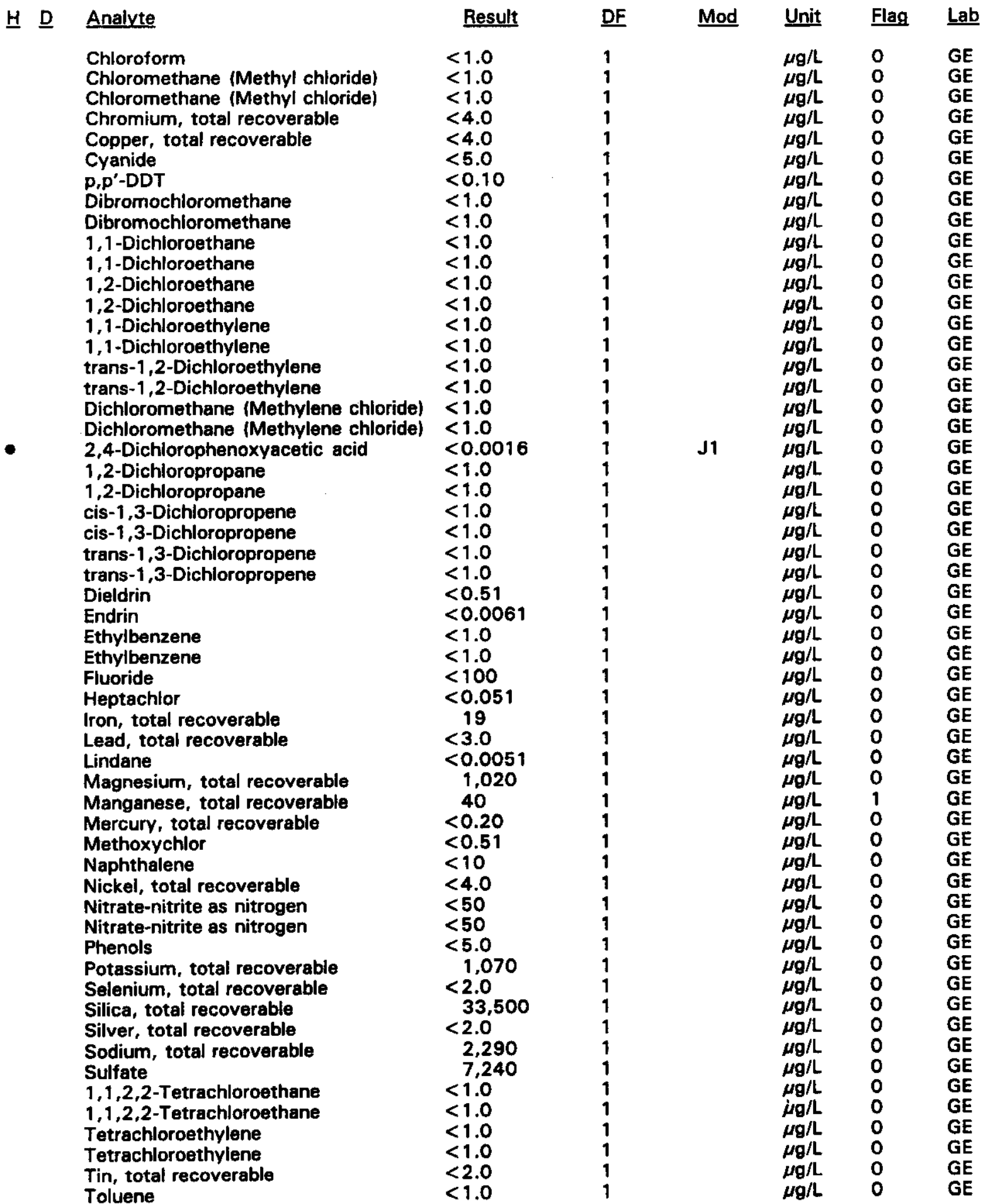

- exceeded holding time. = exceeded screening level or final primary drinking water standard. 
WELL BGO 18A collected on 04/07/94, laboratory analyses (cont.)

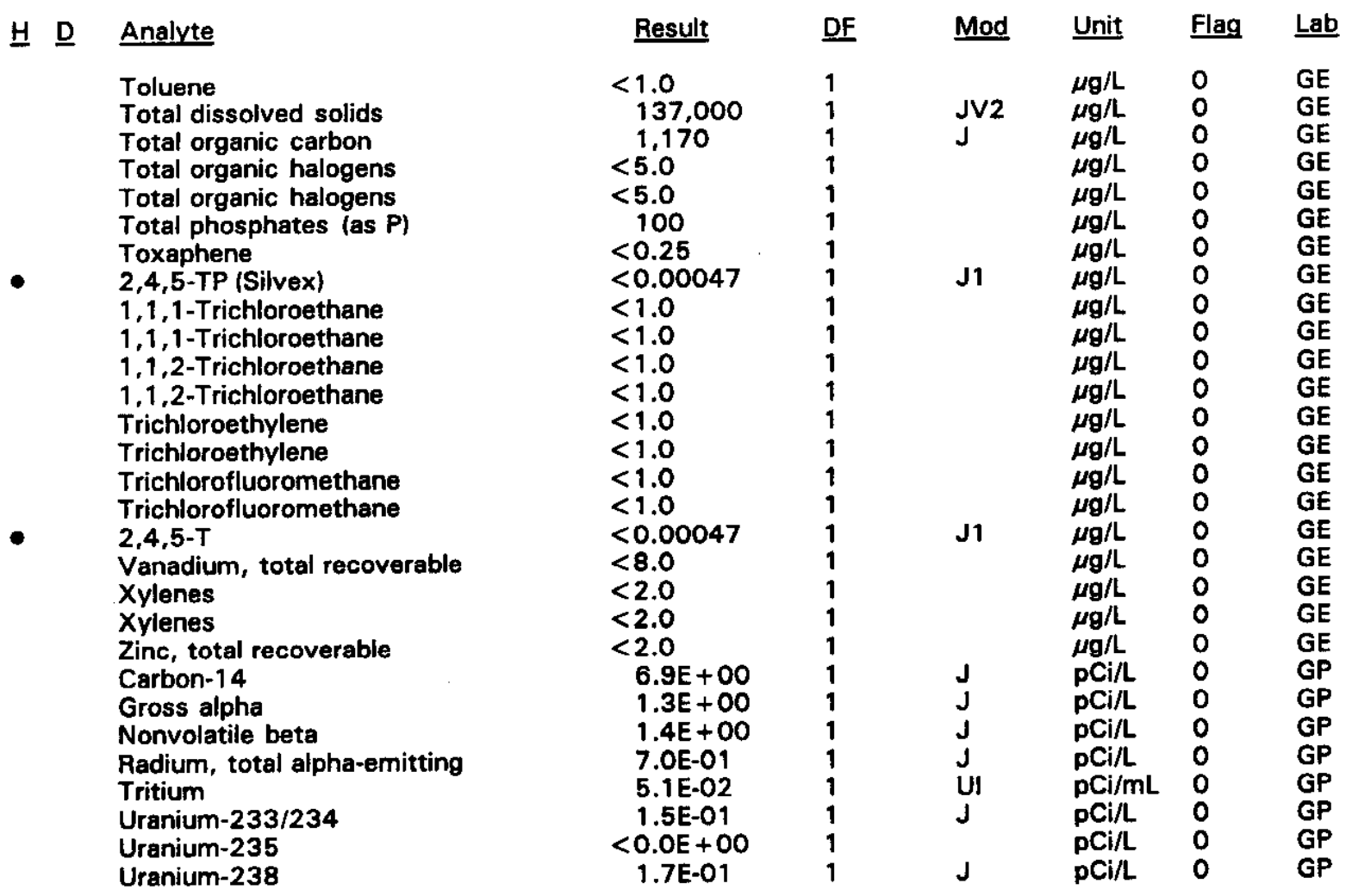

\section{WELL BGO 18D}

\begin{tabular}{|c|c|c|c|c|c|c|}
\hline SRS Coord. & Lat/Longitude & Screen Zone Elevation & Top of Casing & Casing & Pump & Formation \\
\hline $\begin{array}{l}N 75600.0 \\
E 56711.2\end{array}$ & $\begin{array}{l}33.285509^{\circ} \mathrm{N} \\
81.664320^{\circ} \mathrm{W}\end{array}$ & $239.6-219.6 \mathrm{ft} \mathrm{ms!}$ & $294.9 \mathrm{ft} \mathrm{msl}$ & 4" PVC & $\mathbf{S}$ & Water Table $\left(\mathrm{IIB}_{2}\right)$ \\
\hline
\end{tabular}

\section{FIELD MEASUREMENTS}

\section{Sample date: 04/07/94}

Depth to water: $62.71 \mathrm{ft}(19.11 \mathrm{~m})$ below TOC

Water elevation: $232.19 \mathrm{ft}(70.77 \mathrm{~m}) \mathrm{msl}$

Sp. conductance: $29 \mu \mathrm{S} / \mathrm{cm}$

Turbidity: 0.1 NTU

Water evacuated before sampling: $131 \mathrm{gal}$

\section{LABORATORY ANALYSES}

H D $\quad$ Analyte
pH
Specific conductance
Turbidity
Acetophenone
Aldrin

$\begin{array}{llllll}\text { Result } & \text { DF } & \text { Mod } & \text { Unit } & \text { Flag } & \text { Lab } \\ 5.3 & 1 & \text { J1 } & \text { pH } & 0 & \text { GE } \\ 24 & 1 & & \text { iS/cm } & 0 & \text { GE } \\ <0.10 & 1 & & \text { NTU } & 0 & \text { GE } \\ <9.7 & 1 & & \mu g / L & 0 & \text { GE } \\ <0.053 & 1 & & \mu g / L & 0 & \text { GE }\end{array}$

Time: $11: 25$

pH: 4.6

Alkalinity: $0 \mathrm{mg} / \mathrm{L}$

Volumes purged: 15.9 well volumes
Water temperature: $19.3^{\circ} \mathrm{C}$

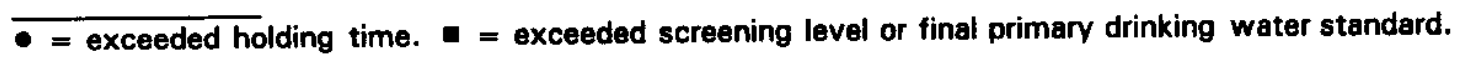


WELL BGO 18D collected on 04/07/94, laboratory analyses (cont.)

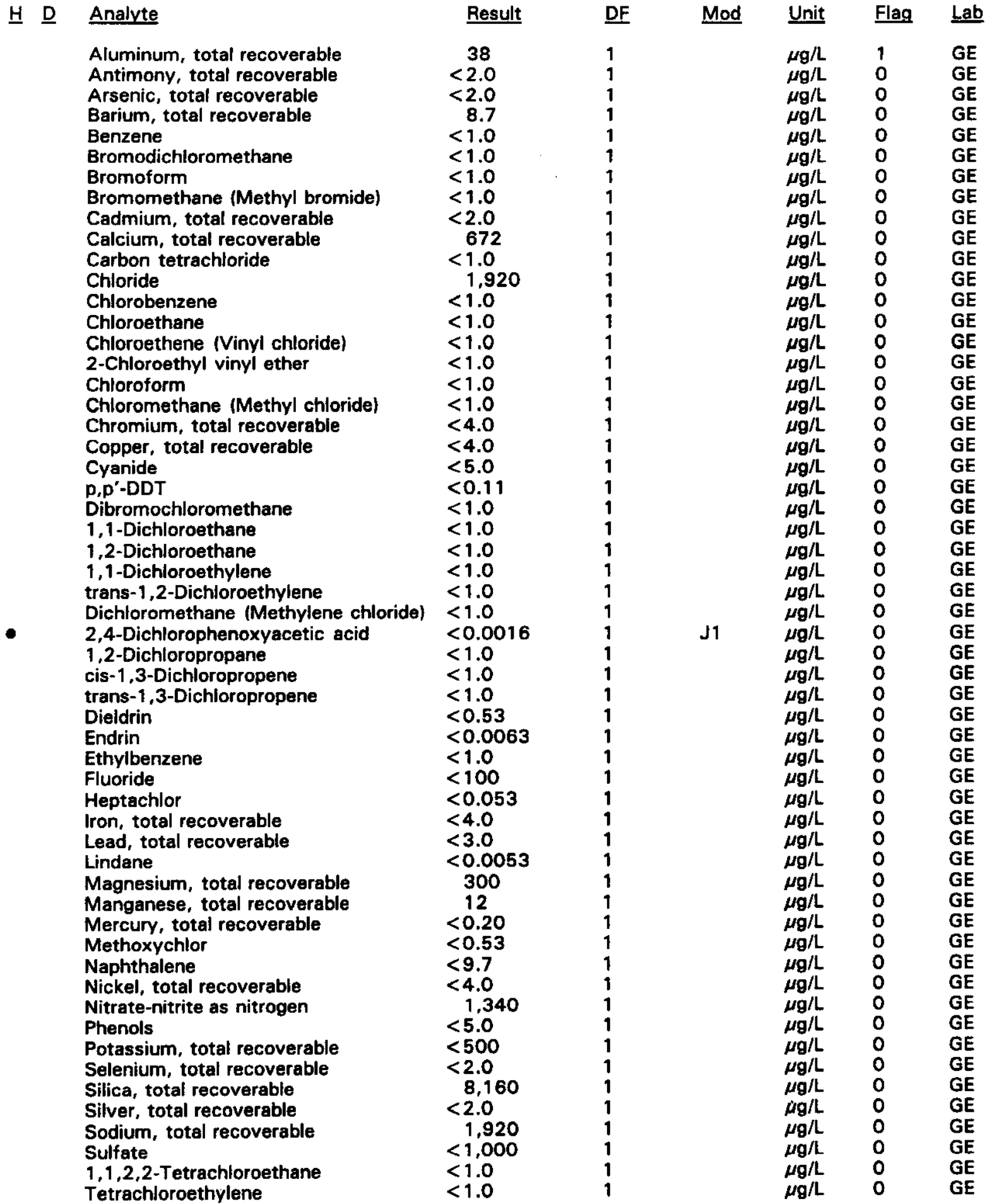

- = exceeded holding time. = exceeded screening level or final primary drinking water standard. 
WELL BGO $18 D$ collected on $04 / 07 / 94$, laboratory analyses (cont.)

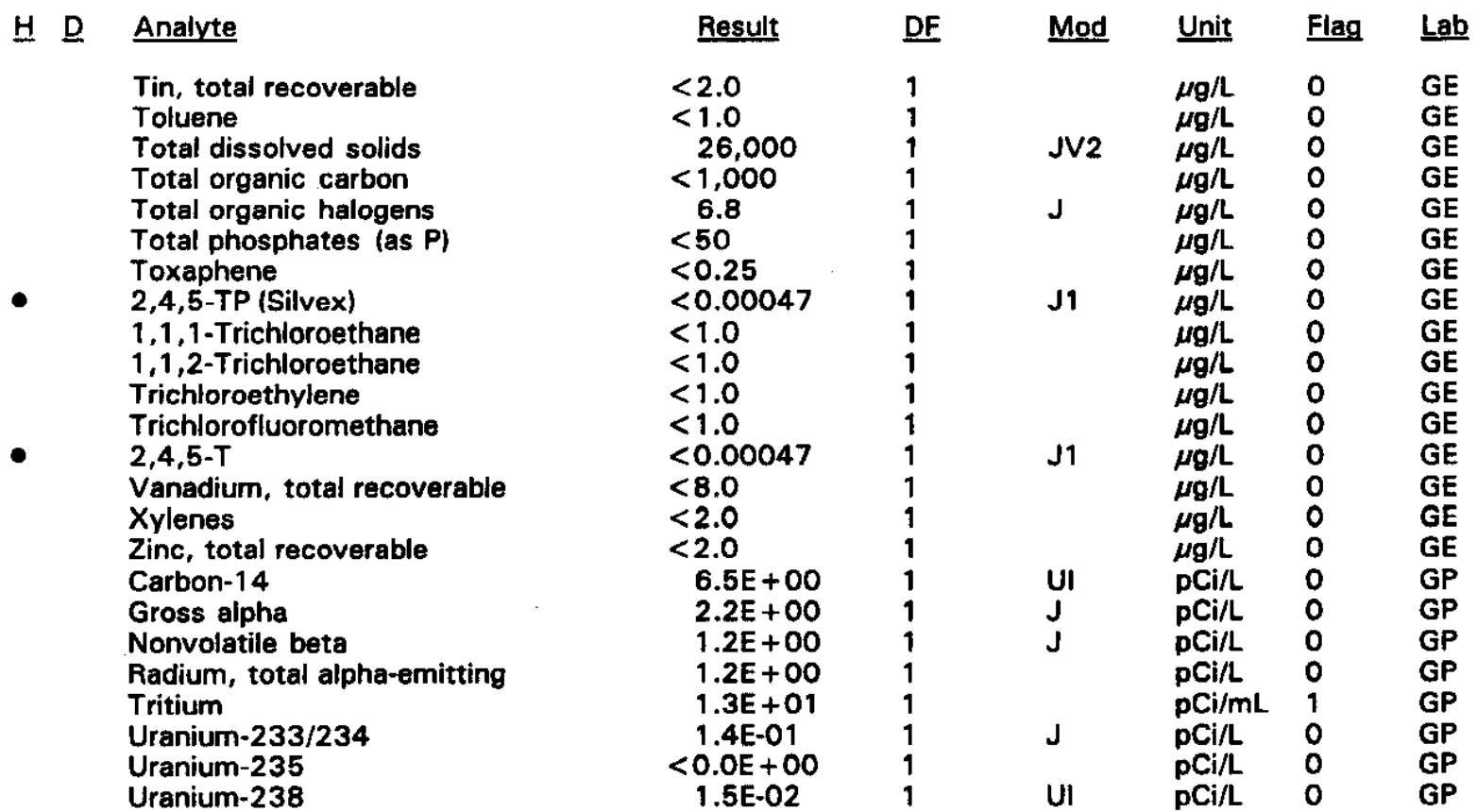

\section{WELL. BGO 19D}

\begin{tabular}{|c|c|c|c|c|c|c|}
\hline SRS Coord. & Lat/Longitude & Screen Zone Elevation & Top of Casing & Casing & Pump & Formation \\
\hline $\begin{array}{l}\text { N75350.0 } \\
\text { E56997.3 }\end{array}$ & $\begin{array}{l}33.285423^{\circ} \mathrm{N} \\
81.663081^{\circ} \mathrm{W}\end{array}$ & $213.9-193.9 \mathrm{ft} \mathrm{msl}$ & $287.2 \mathrm{ft} \mathrm{msl}$ & $4^{n}$ PVC & $\mathbf{S}$ & Water Table $\left(\mathrm{IIB}_{2}\right)$ \\
\hline
\end{tabular}

\section{FIELD MEASUREMENTS}

Sample date: 04/07/94

Depth to water: $57.29 \mathrm{ft}(17.46 \mathrm{~m})$ below TOC

Water elevation: $229.91 \mathrm{ft}(70.08 \mathrm{~m}) \mathrm{msl}$

Sp. conductance: $40 \mu \mathrm{s} / \mathrm{cm}$

Turbidity: 2.1 NTU

Water evacuated before sampling: $160 \mathrm{gal}$

\section{LABORATORY ANALYSES}

H $\underline{\text { Analyte }}$

- $\quad \mathrm{pH}$

Specific conductance

Turbidity

Acetophenone

Aldrin

Aluminum, total recoverable

Aluminum, total recoverable

Antimony, total recoverable

Arsenic, total recoverable

Barium, total recoverable
Time: 10:56

pH: 6.1

Alkalinity: $5 \mathrm{mg} / \mathrm{L}$

Water temperature: $19.3^{\circ} \mathrm{C}$

Volumes purged: 6.8 well volumes

\begin{tabular}{|c|c|c|c|c|}
\hline Result & DF & Mod & Unit & Flag \\
\hline $\begin{aligned} & 6.0 \\
& 31 \\
& 9.9 \\
&<10 \\
&<0.050 \\
& 38 \\
& 36 \\
&<2.0 \\
&<2.0 \\
& 21\end{aligned}$ & $\begin{array}{l}1 \\
1 \\
1 \\
1 \\
1 \\
1 \\
1 \\
1 \\
1 \\
1\end{array}$ & J1 & $\begin{array}{l}\mathrm{pH} \\
\mu \mathrm{S} / \mathrm{cm} \\
N T U \\
\mu g / L \\
\mu g / L \\
\mu g / L \\
\mu g / L \\
\mu g / L \\
\mu g / L \\
\mu g / L\end{array}$ & $\begin{array}{l}0 \\
0 \\
0 \\
0 \\
0 \\
1 \\
1 \\
0 \\
0 \\
0\end{array}$ \\
\hline
\end{tabular}

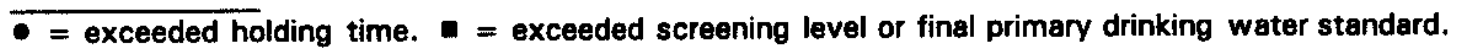


WELL BGO 190 collected on 04/07/94, laboratory analyses (cont.)

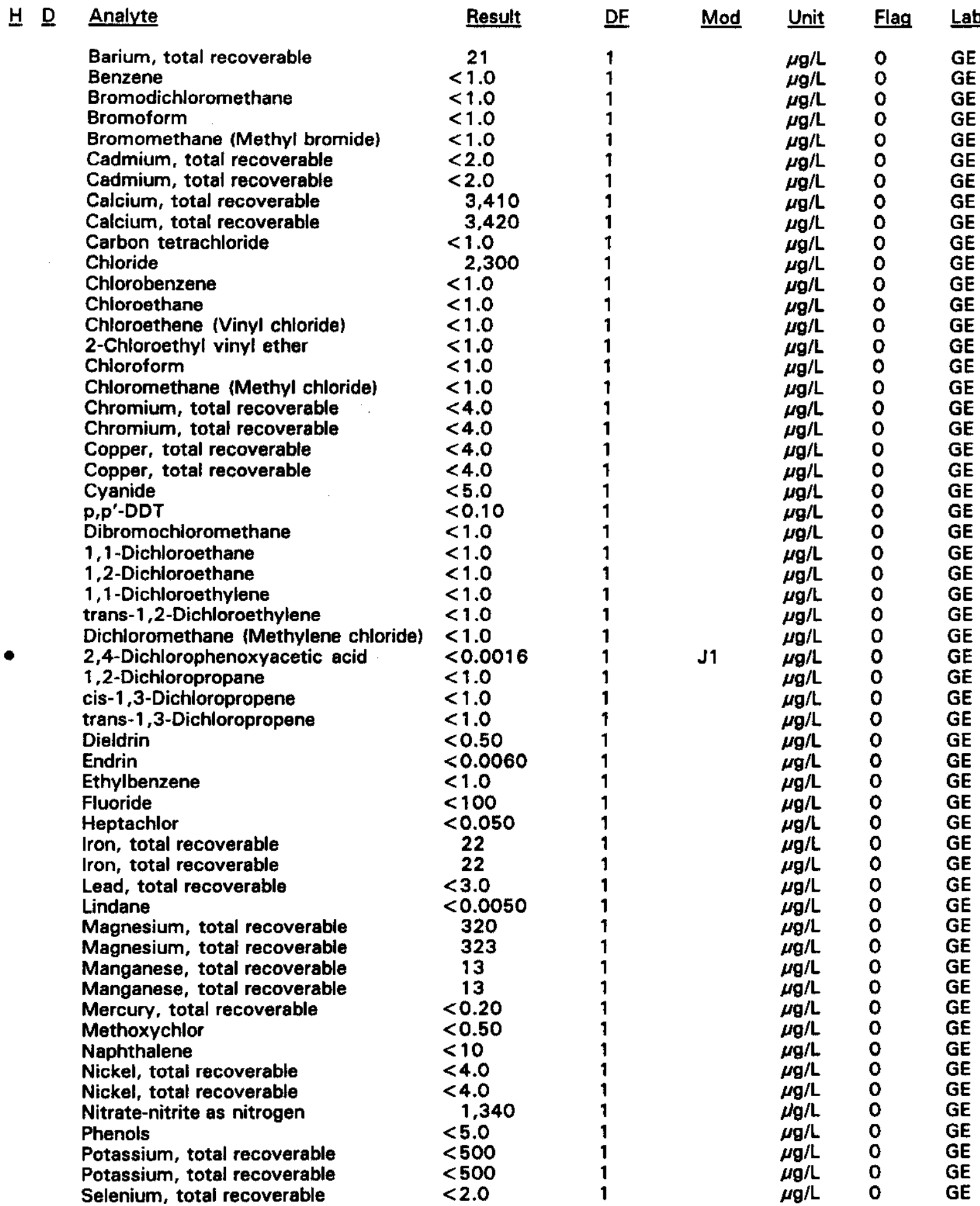

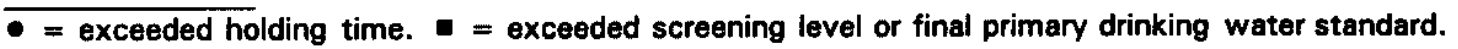


WELL BGO 19D collected on 04/07/94, laboratory analyses (cont.)

브 $\underline{\text { Analyte }}$

Silica, total recoverable

Silica, total recoverable

Silver, total recoverable

Silver, total recoverable

Sodium, total recoverable

Sodium, total recoverable

Sulfate

1,1,2,2-Tetrachloroethane

Tetrachloroethylene

Tin, total recoverable

Tin, total recoverable

Toluene

Total dissolved solids

Total organic carbon

Total organic halogens

Total phosphates (as P)

Toxaphene

- 2,4,5-TP (Silvex)

1,1,1-Trichloroethane

1,1,2-Trichloroethane

Trichloroethylene

Trichlorofluoromethane

- 2,4,5-T

Vanadium, total recoverable

Vanadium, total recoverable

Xylenes

Zinc, total recoverable

Zinc, total recoverable

Carbon-14

Gross alpha

Nonvolatile beta

Radium, total alpha-emitting

Tritium

Uranium-233/234

Uranium-235

Uranium-238
Result

8,940
8,980

$<2.0$

$<2.0$

2,890

2,920

$<1,000$

$<1.0$

$<1.0$

$<2.0$

$<2.0$

$<1.0$

39,000

$<1,000$

$<5.0$

$<50$

$<0.24$

$<0.00049$

$<1.0$

$<1.0$

$<1.0$

$<1.0$

$<0.00049$

$<8.0$

$<8.0$

$<2.0$

7.6

7.5

$2.6 E+00$

$1.1 E+00$

5.5E-01

4.0E-01

$7.5 \mathrm{E}+01$

2.3E-02

$-1.6 \mathrm{E}-03$

5.9E-02
DF

Mod

Unit

Flag Lab

$\mu \mathrm{g} / \mathrm{L} \quad 0 \quad \mathrm{GE}$

$\mu g / L \quad 0 \quad G E$

$\mu g / L \quad 0 \quad$ GE

$\mu g / L \quad 0 \quad$ GE

$\mu g / L \quad 0 \quad$ GE

$\mu \mathrm{g} / \mathrm{L} \quad 0 \quad \mathrm{GE}$

$\mu \mathrm{g} / \mathrm{L} \quad 0 \quad \mathrm{GE}$

$\mu g / L \quad 0 \quad$ GE

$\mu \mathrm{g} / \mathrm{L} \quad 0 \quad \mathrm{GE}$

$\mu g / L \quad 0 \quad$ GE

$\mu g / L \quad O \quad$ GE

$\mu \mathrm{g} / \mathrm{L} \quad 0 \quad \mathrm{GE}$

JV2 $\mu g / L \quad 0 \quad$ GE

$\mu g / L \quad 0 \quad$ GE

$\mu \mathrm{g} / \mathrm{L} \quad 0 \quad \mathrm{GE}$

$\mu \mathrm{g} / \mathrm{L} \quad 0 \quad \mathrm{GE}$

$\mu \mathrm{g} / \mathrm{L} \quad 0 \quad \mathrm{GE}$

$\mu \mathrm{g} / \mathrm{L} \quad 0 \quad \mathrm{GE}$

$\mu g / L \quad 0 \quad$ GE

$\mu g / L \quad 0 \quad$ GE

$\mu g / L \quad 0 \quad$ GE

$\mu g / L \quad 0 \quad$ GE

$\mu \mathrm{g} / \mathrm{L} \quad 0 \quad \mathrm{GE}$

$\mu g / L \quad 0 \quad$ GE

$\mu g / L \quad 0 \quad$ GE

$\mu \mathrm{g} / \mathrm{L} \quad 0 \quad \mathrm{GE}$

$\mu g / L \quad O \quad$ GE

$\mu g / L \quad 0 \quad$ GE

$\mathrm{pCi} / \mathrm{L} \quad 0 \quad \mathrm{GP}$

$\mathrm{pCi} / \mathrm{L} \quad 0 \quad \mathrm{GP}$

pCi/L $\quad 0 \quad$ GP

pCi/L 0 GP

$\mathrm{pCi} / \mathrm{mL} 2 \quad \mathrm{GP}$

$\mathrm{pCi} / \mathrm{L} \quad 0 \quad \mathrm{GP}$

pCi/L $\quad 0 \quad$ GP

$\mathrm{PCi} / \mathrm{L} \quad \mathrm{G} \quad \mathrm{GP}$

- = exceeded holding time. = exceeded screening level or final primary drinking water standard. 


\section{WELL BGO 20D}

\begin{tabular}{|c|c|c|c|c|c|c|}
\hline SRS Coord. & Lat/Longitude & Screen Zone Elevation & Top of Casing & Casing & Pump & Formation \\
\hline $\begin{array}{l}\text { N74962.2 } \\
\text { E57113.8 }\end{array}$ & $\begin{array}{l}33.284755^{\circ} \mathrm{N} \\
81.662021^{\circ} \mathrm{W}\end{array}$ & $236.3-216.3 \mathrm{ft} \mathrm{msl}$ & $283.7 \mathrm{ft} \mathrm{msl}$ & 4" PVC & $\mathbf{s}$ & Water Table $\left(11 \mathrm{~B}_{2}\right)$ \\
\hline
\end{tabular}

\section{FIELD MEASUREMENTS}

Sample date: 04/08/94

Depth to water: $49.54 \mathrm{ft}(15.10 \mathrm{~m})$ below TOC

Water elevation: $234.16 \mathrm{ft}(71.37 \mathrm{~m}) \mathrm{ms}$ !

Sp. conductance: $82 \mu \mathrm{S} / \mathrm{cm}$

Turbidity: 5.2 NTU

Water evacuated before sampling: 7 gal

The well went dry during purging.

Time: 10:32

pH: 5.5

Alkalinity: $3 \mathrm{mg} / \mathrm{L}$

Water temperature: $17.2{ }^{\circ} \mathrm{C}$

Volumes purged: 0.6 well volumes

\section{LABORATORY ANALYSES}

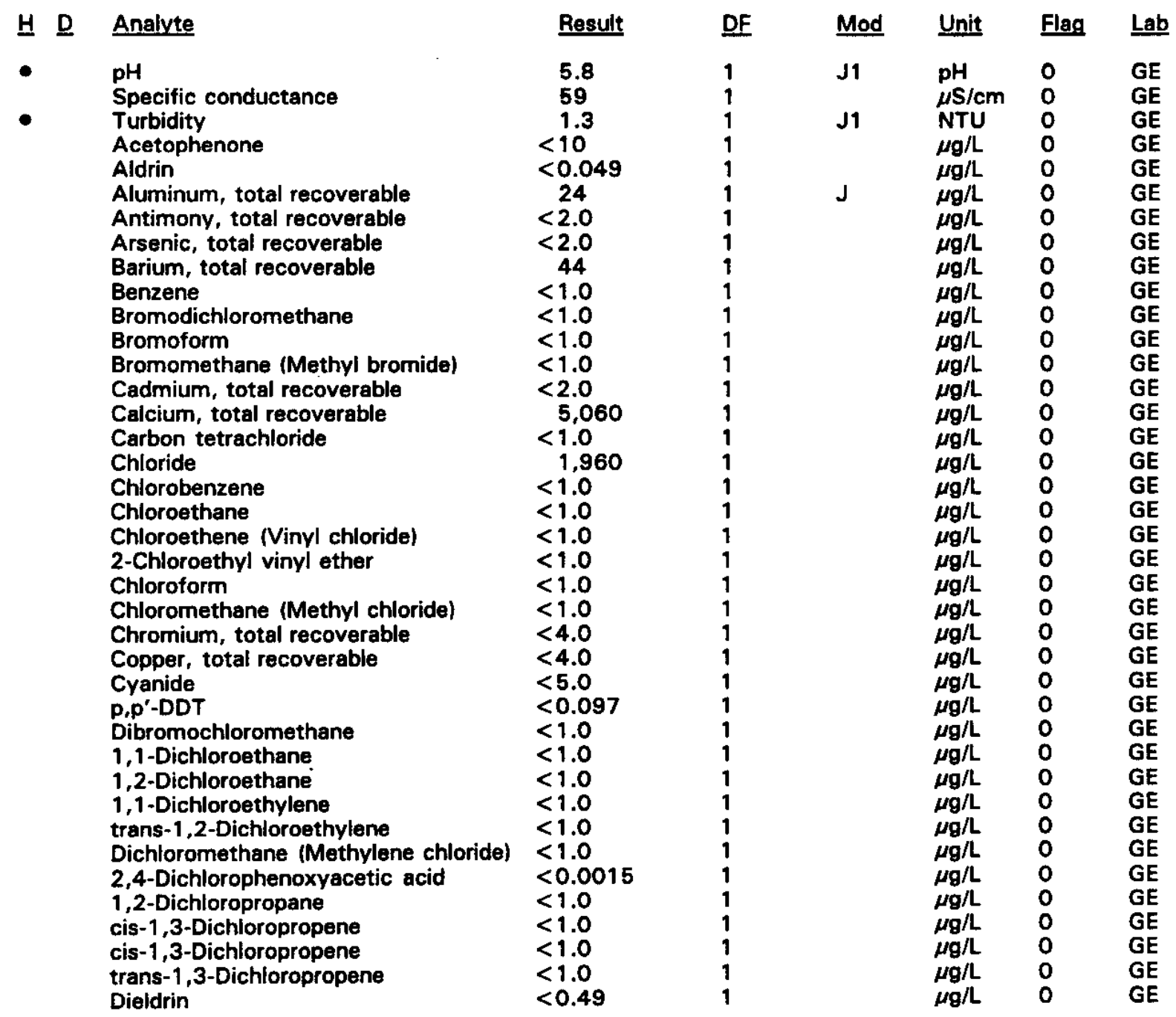

- = exceeded holding time. = exceeded screening tevel or final primary drinking water standard. 
WELL BGO 2OD collected on 04/08/94, laboratory analyses (cont.)

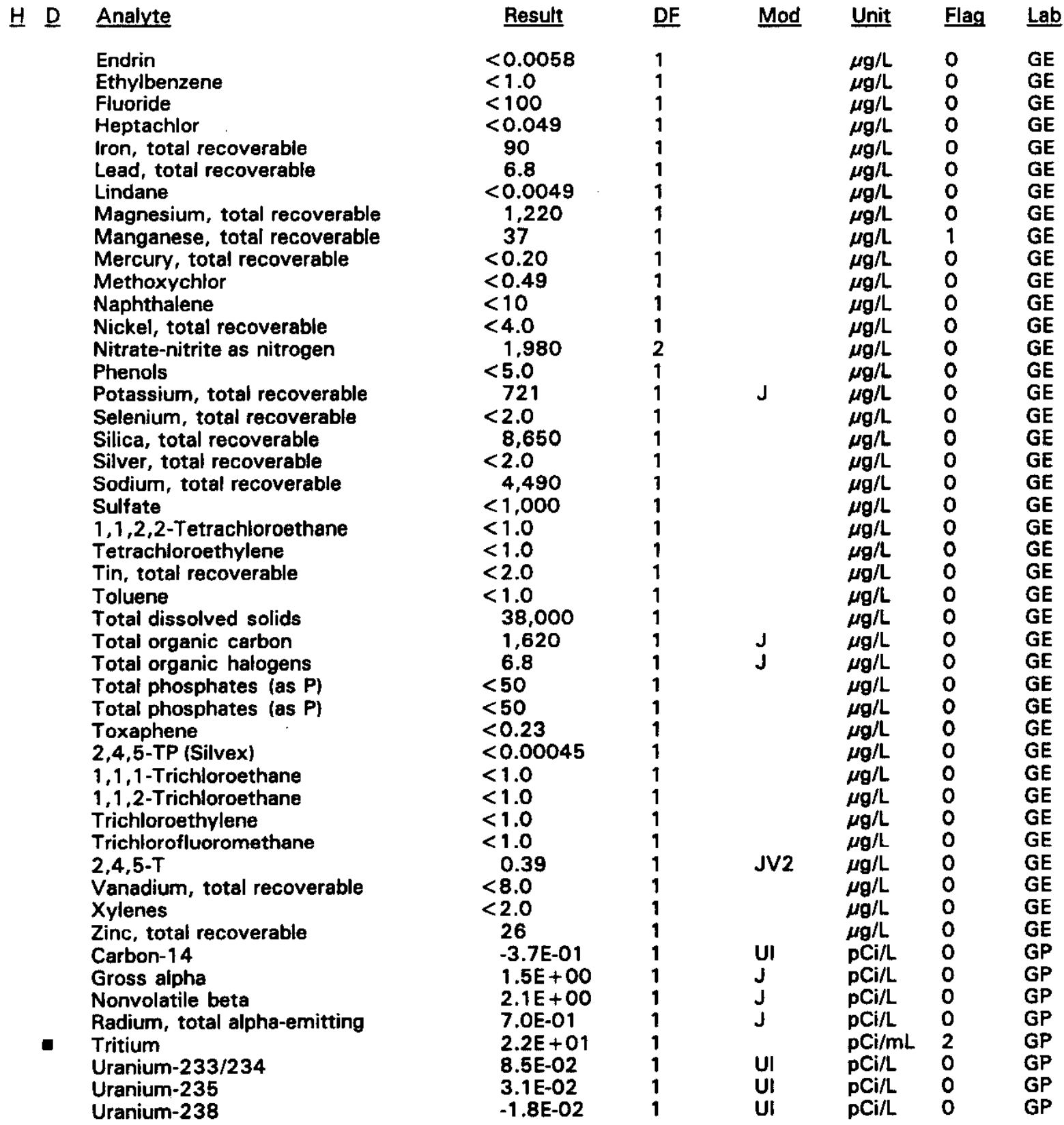

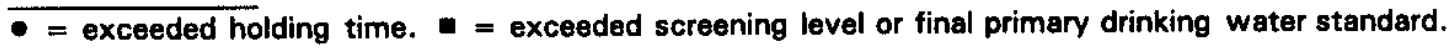


WELL BGO 21D

\begin{tabular}{|c|c|c|c|c|c|c|}
\hline SRS Coord. & Lat/Longitude & Screen Zone Elevation & Top of Casing & Casing & Pump & Formation \\
\hline $\begin{array}{l}\text { N74688.5 } \\
\text { E57470.7 }\end{array}$ & $\begin{array}{l}33.284732^{\circ} \mathrm{N} \\
81.660549^{\circ} \mathrm{W}\end{array}$ & $237.7-217.7 \mathrm{ft} \mathrm{msl}$ & $285.4 \mathrm{ft} \mathrm{msl}$ & 4" PVC & $\mathbf{s}$ & Water Table $\left(\mathrm{IIB}_{2}\right)$ \\
\hline
\end{tabular}

\section{FIELD MEASUREMENTS}

Sample date: 04/08/94

Depth to water: $50.47 \mathrm{ft}(15.38 \mathrm{~m})$ below TOC

Water elevation: $234.93 \mathrm{ft}(71.61 \mathrm{~m}) \mathrm{msl}$

Sp. conductance: $87 \mu \mathrm{S} / \mathrm{cm}$

Turbidity: 27.3 NTU

Water evacuated before sampling: $7 \mathrm{gal}$

The well went dry during purging.

\section{LABORATORY ANALYSES}

H. Analyte
pH
Specific conductance
Turbidity
Acetophenone
Aldrin
Aluminum, total recoverable
Antimony, total recoverable
Arsenic, total recoverable
Barium, total recoverable
Benzene
Bromodichloromethane
Bromoform
Bromomethane (Methyl bromide)
Cadmium, total recoverable
Calcium, total recoverable
Carbon tetrachloride
Chloride
Chlorobenzene
Chloroethane
Chloroethene (Vinyl chloride)
2-Chloroethyl vinyl ether
Chloroform
Chloromethane (Methyl chloride)
Chromium, total recoverable
Copper, total recoverable
Cyanide
p,p'-DDT
Dibromochloromethane
1,1-Dichloroethane
1,2-Dichloroethane
1,1-Dichloroethylene
trans-1,2-Dichloroethylene
Dichloromethane (Methylene chloride)
2,4-Dichlorophenoxyacetic acid
2,4-Dichlorophenoxyacetic acid
1,2-Dichloropropane
cis-1,3-Dichloropropene
cis-1,3-Dichloropropene
trans-1,3-Dichloropropene
(a)

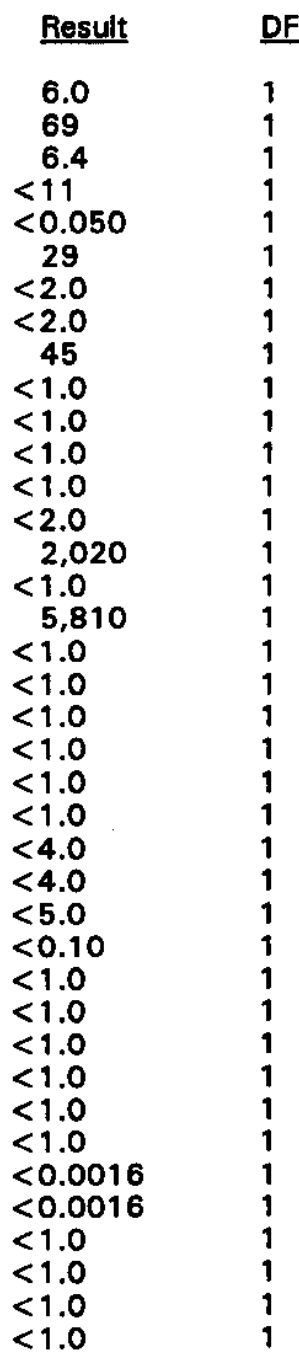

Time: $9: 31$

pH: $\mathbf{5 . 9}$

Alkalinity: $2 \mathrm{mg} / \mathrm{L}$

Water temperature: $15.7^{\circ} \mathrm{C}$

Volumes purged: 0.6 well volumes 
WELL BGO 21D collected on 04/08/94, laboratory analyses (cont.)

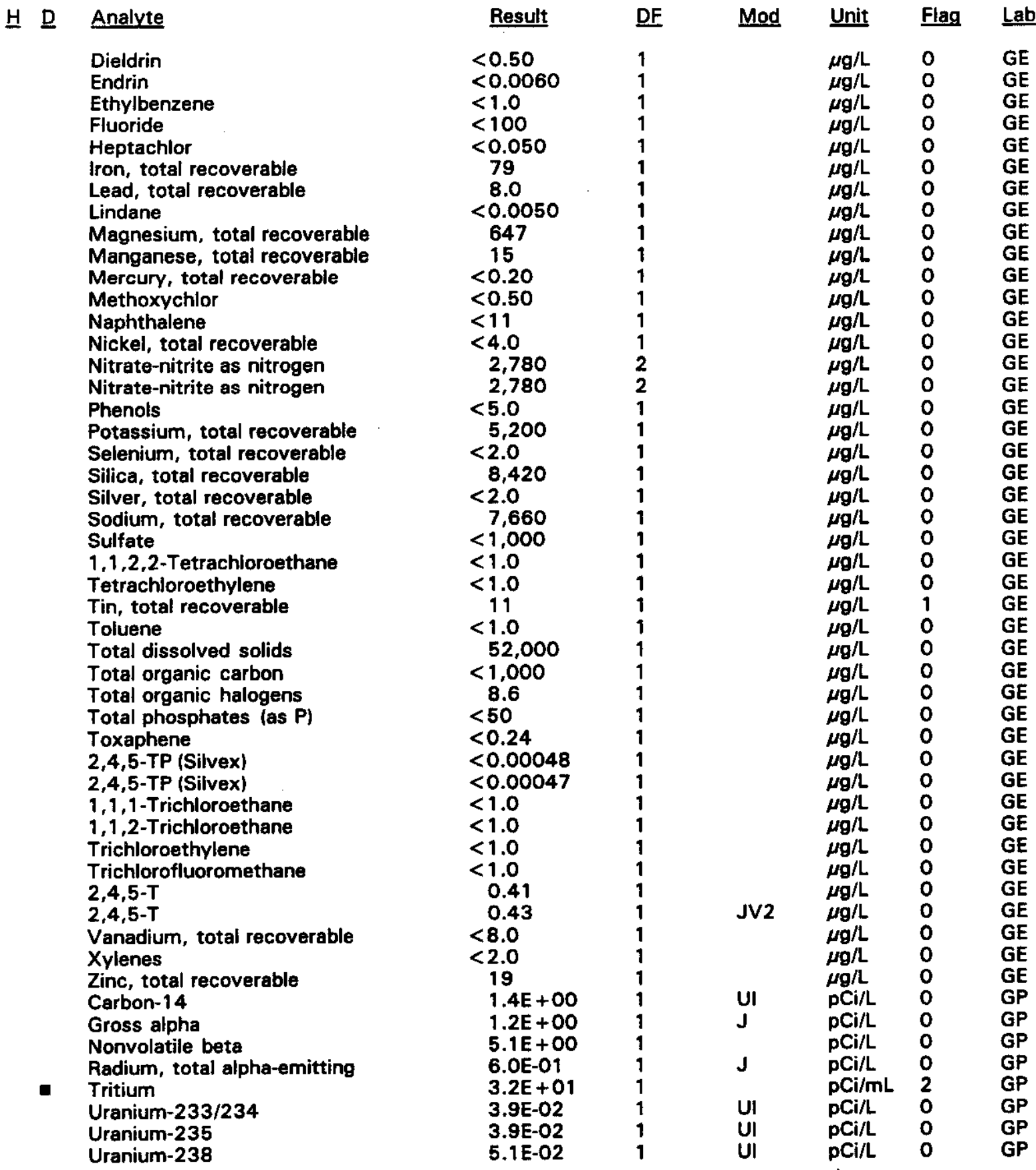

- = exceeded holding time. E = exceeded screening level or final primary drinking water standard. 
WELL BGO 22DR

\begin{tabular}{|c|c|c|c|c|c|c|}
\hline SRS Coord. & Lat/Longitude & Screen Zone Elevation & Top of Casing & Casing & Pump & Formation \\
\hline $\begin{array}{l}\text { N744711.5 } \\
\text { E57831.5 }\end{array}$ & $\begin{array}{l}33.284841^{\circ} \mathrm{N} \\
81.659178{ }^{\circ} \mathrm{W}\end{array}$ & 239.2-219.2ft msl & $286.1 \mathrm{ft} \mathrm{msl}$ & 4" PVC & $\mathbf{S}$ & Water $\mathrm{Ta}$ \\
\hline
\end{tabular}

\section{FIELD MEASUREMENTS}

Sample date: 04/08/94

Depth to water: $49.06 \mathrm{ft}(14.95 \mathrm{~m})$ below TOC

Water elevation: $237.04 \mathrm{ft}(72.25 \mathrm{~m}) \mathrm{ms}$

Sp. conductance: $26 \mu \mathrm{S} / \mathrm{cm}$

Turbidity: 219 NTU

Water evacuated before sampling: 14 gal

The well went dry during purging.

\section{LABORATORY ANALYSES}

H D Analyte

- $\quad$ pH

pH

- Turbidity

Acetophenone

Aldrin

Aluminum, total recoverable

Antimony, total recoverable

Arsenic, total recoverable

Barium, total recoverable

Benzene

Bromodichloromethane

Bromoform

Bromomethane (Methyl bromide)

Cadmium, total recoverable

Calcium, total recoverable

Carbon tetrachloride

Chloride

Chlorobenzene

Chloroethane

Chloroethene (Vinyl chloride)

2-Chloroethyl vinyl ether

Chloroform

Chloromethane (Methyl chloride)

Chromium, total recoverable

Copper, total recoverable

Cyanide

p,p'-DDT

Dibromochloromethane

1,1-Dichloroethane

1,2-Dichloroethane

1,1-Dichloroethylene

trans-1,2-Dichloroethylene

Dichloromethane (Methylene chloride)

2,4-Dichlorophenoxyacetic acid

1,2-Dichloropropane

cis-1,3-Dichloropropene

trans-1,3-Dichloropropene

Dieldrin
Time: $9: 16$

pH: 5.6

Alkalinity: $3 \mathrm{mg} / \mathrm{L}$

Water temperature: $17.9^{\circ} \mathrm{C}$

Volumes purged: 1.2 well volumes

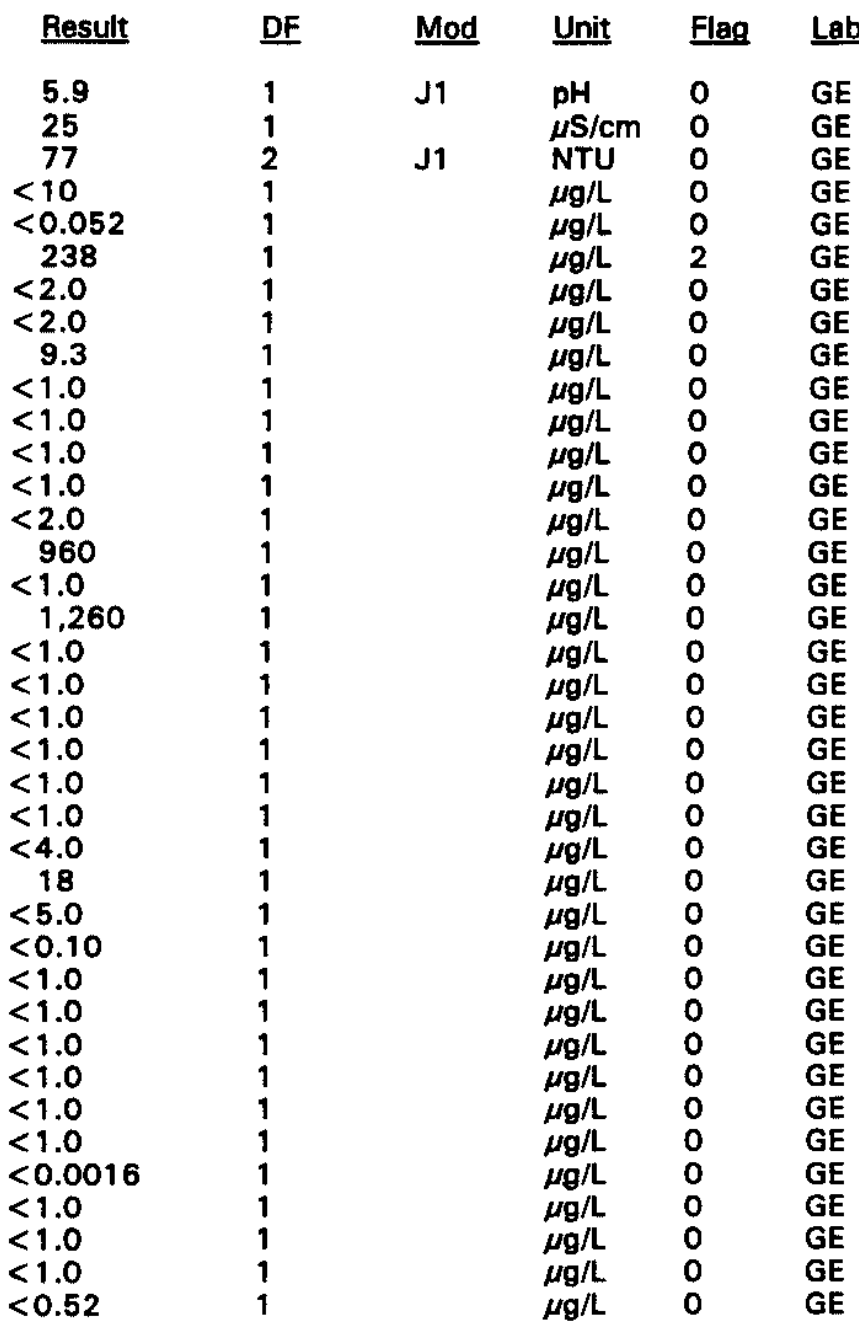

$\overline{-}=$ exceeded holding time. $=$ exceeded screening level or final primary drinking water standard. 
WELL BGO 22DR collected on 04/08/94, laboratory analyses (cont.)

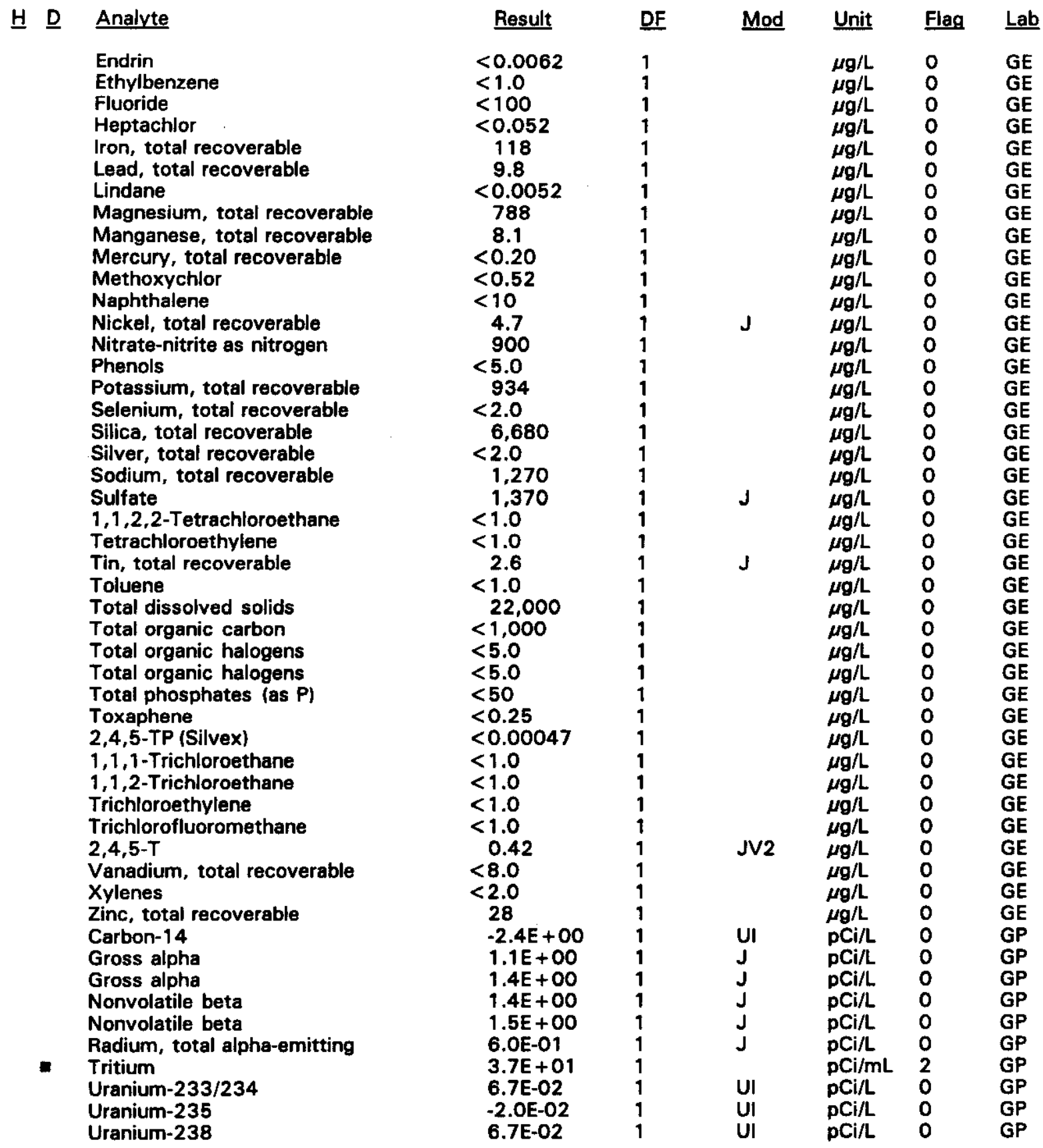

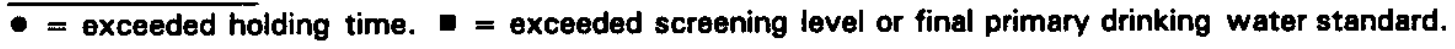


WELL BGO 23D

\begin{tabular}{|c|c|c|c|c|c|c|}
\hline SRS Coord. & Lat/Longitude & Screen Zone Elevation & Top of Casing & Casing & Pump & Formation \\
\hline $\begin{array}{l}N 74238.1 \\
\text { E58133.0 }\end{array}$ & $\begin{array}{l}33.284817^{\circ} \mathrm{N} \\
81.657930^{\circ} \mathrm{W}\end{array}$ & $242.0-222.0 \mathrm{ft} \mathrm{msl}$ & $289.2 \mathrm{ft} \mathrm{msl}$ & 4" PVC & $\mathbf{S}$ & le $\left(\| B_{2}\right)$ \\
\hline
\end{tabular}

\section{FIELD MEASUREMENTS}

Sample date: 04/07/94

Depth to water: $53.10 \mathrm{ft}(16.19 \mathrm{~m})$ below TOC

Water elevation: $236.10 \mathrm{ft}(71.96 \mathrm{~m}) \mathrm{msl}$

Sp. conductance: $40 \mu \mathrm{S} / \mathrm{cm}$

Turbidity: 0.3 NTU

Water evacuated before sampling: 34 gal

\section{LABORATORY ANALYSES}

H. Analyte
pH
Specific conductance
Turbidity
Acetophenone
Aldrin
Aluminum, total recoverable
Antimony, total recoverable
Arsenic, total recoverable
Barium, total recoverable
Benzene
Bromodichloromethane
Bromoform
Bromomethane (Methyl bromide)
Cadmium, total recoverable
Calcium, total recoverable
Carbon tetrachloride
Chloride
Chlorobenzene
Chloroethane
Chloroethene (Vinyl chloride)
2-Chloroethyl vinyl ether
Chloroform
Chloromethane (Methyl chloride)
Chromium, total recoverable
Copper, total recoverable
Cyanide
p,p'-DDT
Dibromochloromethane
1,1 -Dichloroethane
$1,2-$ Dichloroethane
1,1-Dichloroethylene
trans-1,2-Dichloroethylene
Dichloromethane (Methylene chloride)
2,4-Dichlorophenoxyacetic acid
$1,2-$ Dichloropropane
cis-1,3-Dichloropropene
trans-1,3-Dichloropropene
Dieldrin
Endrin

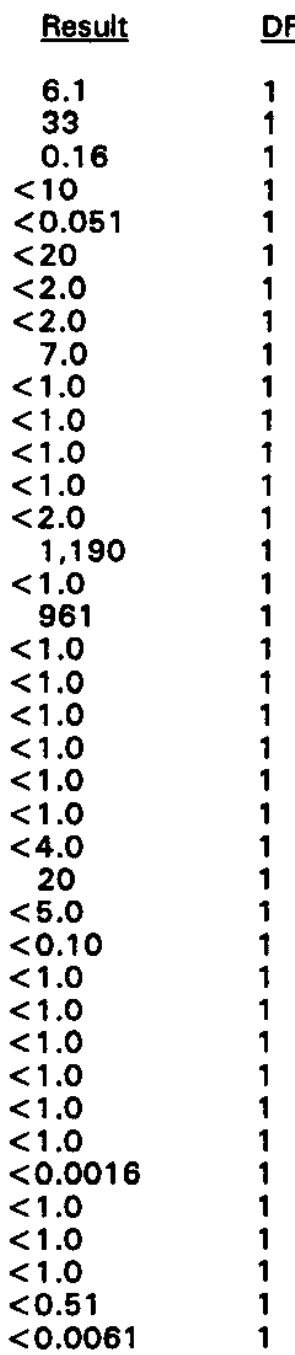

Time: 12:4

pH: 5.8

Alkalinity: $4 \mathrm{mg} / \mathrm{L}$

Water temperature: $20.8^{\circ} \mathrm{C}$

Volumes purged: $\mathbf{3 . 7}$ well volumes 
WELL BGO 23D collected on 04/07/94, laboratory analyses (cont.)

H D Analyte

Ethylbenzene

Fluoride

Heptachlor

Iron, total recoverable

Lead, total recoverable

Lindane

Magnesium, total recoverable

Manganese, total recoverable

Mercury, total recoverable

Methoxychlor

Naphthalene

Nickel, total recoverable

Nitrate-nitrite as nitrogen

Phenols

Potassium, total recoverable

Selenium, total recoverable

Silica, total recoverable

Silver, total recoverable

Sodium, total recoverable

Sulfate

$1,1,2,2$-Tetrachloroethane

Tetrachloroethylene

Tin, total recoverable

Toluene

Total dissolved solids

Total organic carbon

Total organic halogens

Total phosphates (as P)

Toxaphene

- 2,4,5-TP (Silvex)

$1,1,1$-Trichloroethane

1,1,2-Trichloroethane

Trichloroethylene

Trichlorofluoromethane

2,4,5-T

Vanadium, total recoverable

Xylenes

Zinc, total recoverable

Carbon-14

Gross alpha

Nonvolatile beta

Radium, total alpha-emitting

- Tritium

Uranium-233/234

Uranium-235

Uranium-238

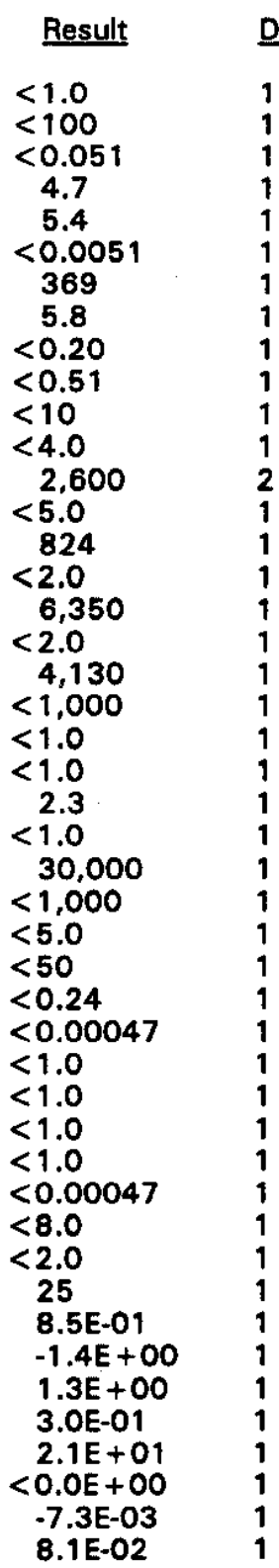

Mod Unit Flag Lab

$\mu g / L \quad O \quad$ GE

$\mu g / L \quad 0 \quad G E$

$\mu g / L \quad 0 \quad$ GE

$\mu g / L \quad 0 \quad G E$

$\mu g / L \quad O \quad G E$

$\mu \mathrm{g} / \mathrm{L} \quad 0 \quad \mathrm{GE}$

$\mu \mathrm{g} / \mathrm{L} \quad \mathrm{O} \quad \mathrm{GE}$

$\mu \mathrm{g} / \mathrm{L} \quad 0 \quad \mathrm{GE}$

$\mu g / L \quad 0 \quad$ GE

$\mu g / L \quad 0 \quad G E$

$\mu \mathrm{g} / \mathrm{L} \quad 0 \quad \mathrm{GE}$

$\mu g / L \quad 0 \quad G E$

$\mu \mathrm{g} / \mathrm{L} \quad \mathrm{O} \quad \mathrm{GE}$

$\mu \mathrm{g} / \mathrm{L} \quad 0 \quad \mathrm{GE}$

$\mu g / L \quad 0 \quad$ GE

$\mu \mathrm{g} / \mathrm{L} \quad \mathrm{O} \quad \mathrm{GE}$

$\mu g / L \quad 0 \quad$ GE

$\mu g / L \quad 0 \quad$ GE

$\mu g / L \quad 0 \quad$ GE

$\mu g / L \quad 0 \quad G E$

$\mu g / L \quad 0 \quad$ GE

$\mu g / L \quad 0 \quad$ GE

$\mu \mathrm{g} / \mathrm{L} \quad \mathrm{O} \quad \mathrm{GE}$

$\mu g / L \quad 0 \quad$ GE

$\mu g / L \quad 0 \quad$ GE

$\mu g / L \quad 0 \quad$ GE

$\mu \mathrm{g} / \mathrm{L} \quad 0 \quad \mathrm{GE}$

$\mu g / L \quad 0 \quad$ GE

$\mu g / L \quad 0 \quad G E$

$\mu g / L \quad 0 \quad$ GE

$\mu g / L \quad 0 \quad G E$

$\mu g / L \quad 0 \quad$ GE

$\mu \mathrm{g} / \mathrm{L} \quad \mathrm{O} \quad \mathrm{GE}$

$\mu g / L \quad 0 \quad$ GE

$\mu \mathrm{g} / \mathrm{L} \quad \mathrm{O} \quad \mathrm{GE}$

$\mu \mathrm{g} / \mathrm{L} \quad \mathrm{O} \quad \mathrm{GE}$

$\mu \mathrm{g} / \mathrm{L} \quad 0 \quad \mathrm{GE}$

$\mu \mathrm{g} / \mathrm{L} \quad 0 \quad \mathrm{GE}$

$\mathrm{pCi} / \mathrm{L} \quad 0 \quad \mathrm{GP}$

$\mathrm{pCi} \quad 0 \quad \mathrm{GP}$

pCi/L $\quad 0 \quad$ GP

$\mathrm{PCi} / \mathrm{L} \quad 0 \quad \mathrm{GP}$

$\mathrm{pCi} / \mathrm{mL} 2 \quad \mathrm{GP}$

PCi/L 0 GP

pCi/L 0 GP

pCi/L 0 GP

- = exceeded holding time. $\|=$ exceeded screening level or final primary drinking water standard. 
WELL BGO 24D

\begin{tabular}{|c|c|c|c|c|c|c|}
\hline SRS Coord. & Lat/Longitude & Screen Zone Elevation & Top of Casing & Casing & Pump & Formation \\
\hline $\begin{array}{l}\text { N74012.4 } \\
\text { E58438.8 }\end{array}$ & $\begin{array}{l}33.284816^{\circ} \mathrm{N} \\
81.656687^{\circ} \mathrm{W}\end{array}$ & $241.0-221.0 \mathrm{ft} \mathrm{msl}$ & $293.2 \mathrm{ft} \mathrm{msl}$ & 4" PVC & $\mathbf{S}$ & Water Table $\left(\mathrm{IB}_{2}\right)$ \\
\hline
\end{tabular}

FIELD MEASUREMENTS

Sample date: 04/08/94

Depth to water: $56.35 \mathrm{ft}(17.18 \mathrm{~m})$ below TOC

Water elevation: $236.85 \mathrm{ft}(72.19 \mathrm{~m}) \mathrm{ms}$

Sp. conductance: $80 \mu \mathrm{S} / \mathrm{cm}$

Turbidity: 18.8 NTU

Water evacuated before sampling: 7 gal

The well went dry during purging.

Time: $8: 57$

pH: 9.6

Alkalinity: $23 \mathrm{mg} / \mathrm{L}$

Water temperature: $17.1^{\circ} \mathrm{C}$

Volumes purged: 0.7 well volumes

\section{LABORATORY ANALYSES}

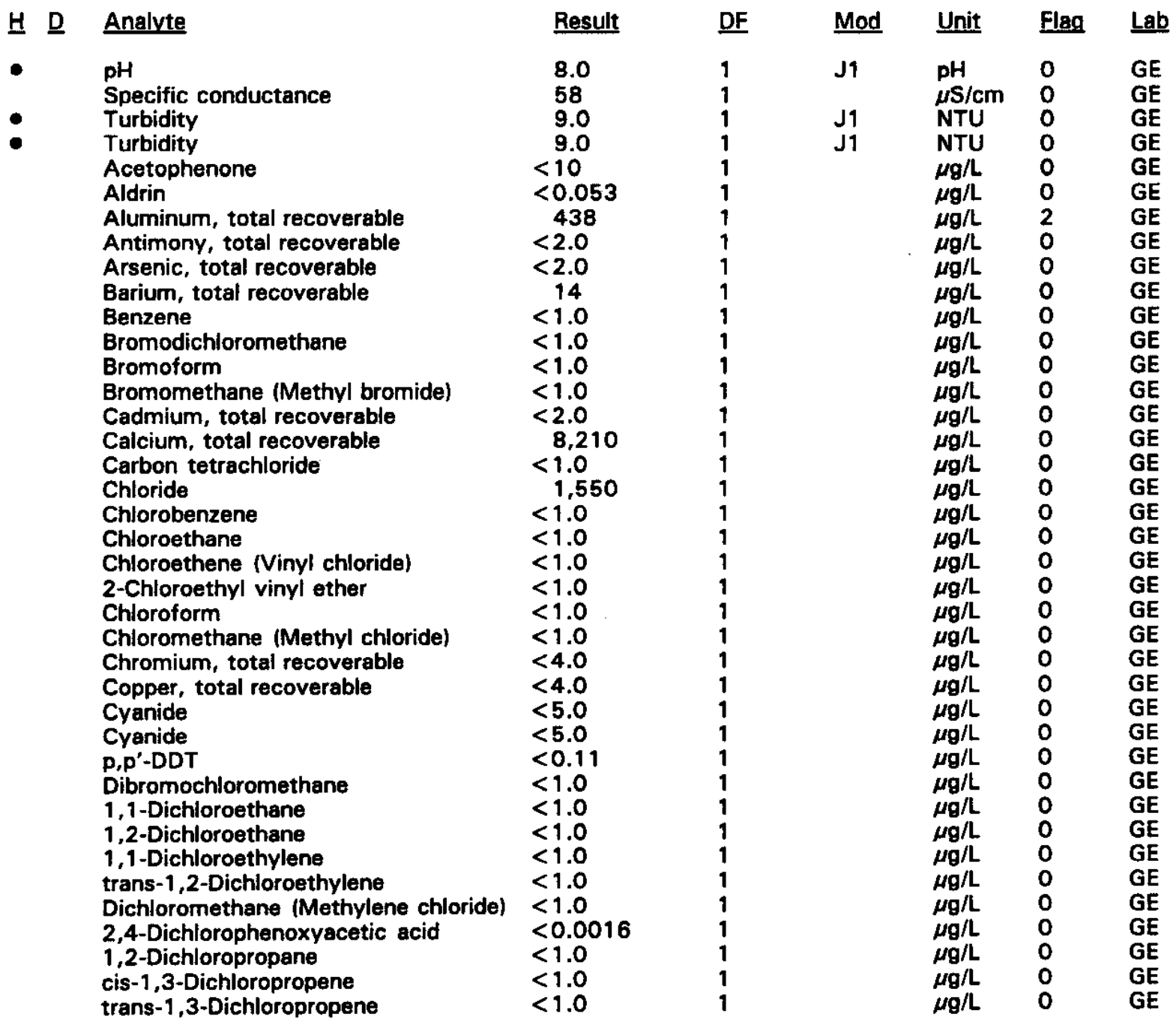

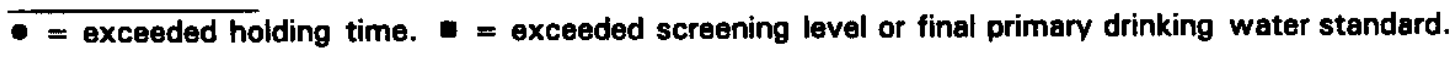


WELL BGO 24D collected on 04/08/94, laboratory analyses (cont.)

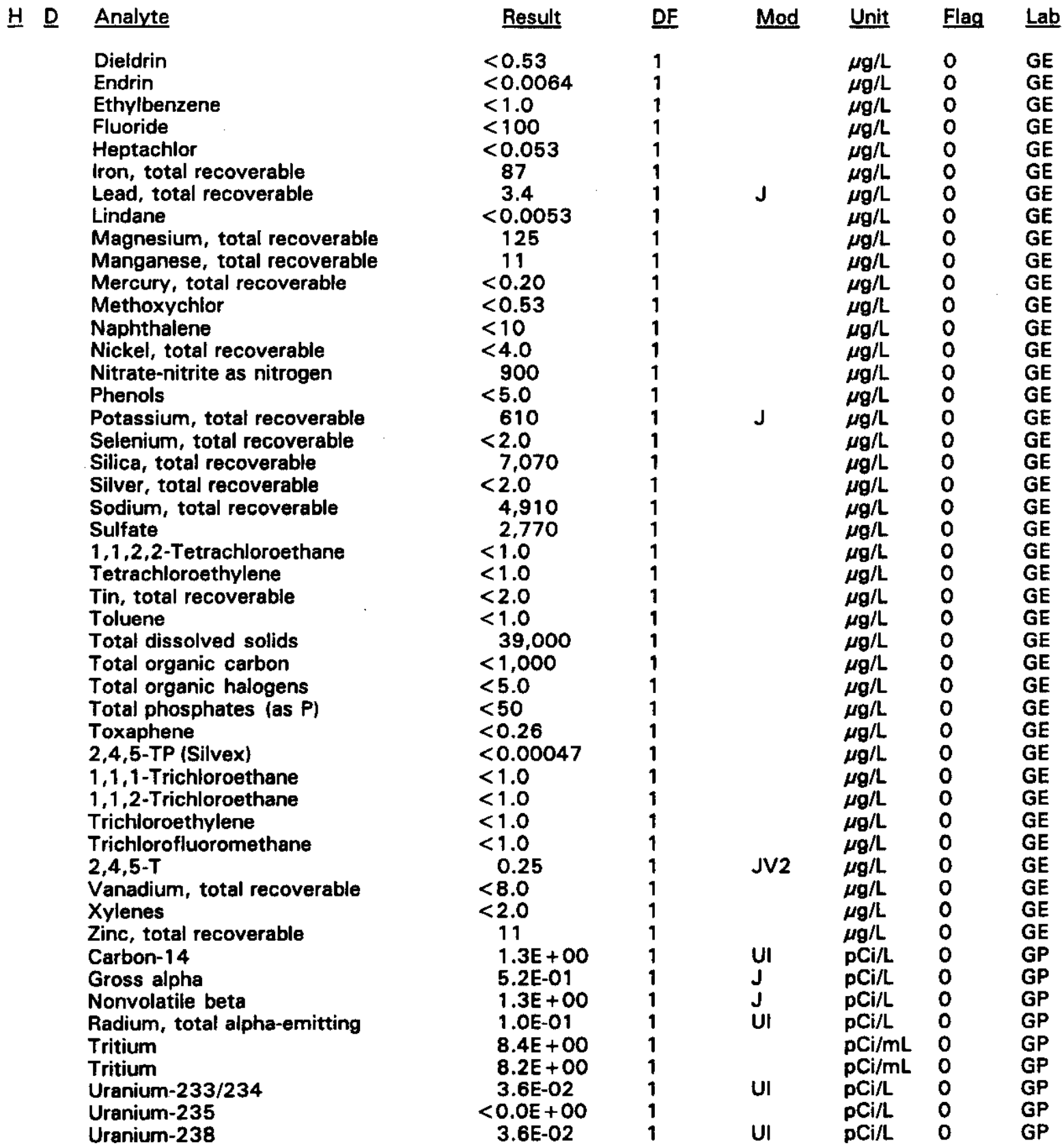

- = exceeded holding time. = exceeded screening level or final primary drinking water standard. 


\section{WELL BGO 25A}

\begin{tabular}{|c|c|c|c|c|c|c|}
\hline SRS Coord. & Lat/Longitude & Screen Zone Elevation & Top of Casing & Casing & Pump & Formation \\
\hline $\begin{array}{l}N 76158.5 \\
\text { E55668.1 }\end{array}$ & $\begin{array}{l}33.285042^{\circ} \mathrm{N} \\
81.668151^{\circ} \mathrm{W}\end{array}$ & $114.1-104.1 \mathrm{ft} \mathrm{msl}$ & $296.5 \mathrm{ft} \mathrm{msl}$ & 4" PVC & $\mathbf{S}$ & U. Congaree (IIA) \\
\hline
\end{tabular}

\section{FIELD MEASUREMENTS}

Sample date: 04/06/94

Depth to water: $135.64 \mathrm{ft}(41.34 \mathrm{~m})$ below TOC

Water elevation: $160.86 \mathrm{ft}(49.03 \mathrm{~m}) \mathrm{ms}$ l

Sp. conductance: $265 \mu \mathrm{S} / \mathrm{cm}$

Turbidity: 0.9 NTU

Water evacuated before sampling: 23 gal

The well went dry during purging.

\section{LABORATORY ANALYSES}

H D Analyte

- $\quad$ pH

pH
Specific conductance
Specific conductance
Turbidity
Acetophenone
Aldrin
Aluminum, total recoverable
Antimony, total recoverable
Arsenic, total recoverable
Barium, total recoverable
Benzene
Benzene
Bromodichloromethane
Bromodichloromethane
Bromoform
Bromoform
Bromomethane (Methyl bromide)
Bromomethane (Methyl bromide)
Cadmium, total recoverable
Calcium, total recoverable
Carbon tetrachloride
Carbon tetrachloride
Chloride
Chlorobenzene
Chlorobenzene
Chloroethane
Chloroethane
Chloroethene (Vinyl chloride)
Chloroethene (Vinyl chloride)
2-Chloroethyl vinyl ether
2-Chloroethyl vinyl ether
Chloroform
Chloroform
Chloromethane (Methyl chloride)
Chloromethane (Methyl chloride)
Chromium, total recoverable
Copper, total recoverable
Cyanide
p,p'-DDT

$\begin{aligned} & \text { Result } \\ & 7.7 \\ & 243 \\ & 245 \\ & 0.32 \\ &<10 \\ &<0.052 \\ &<20 \\ &<2.0 \\ &<2.0 \\ &<37 \\ &<1.0 \\ &<1.0 \\ &<1.0 \\ &<1.0 \\ &<1.0 \\ &<1.0 \\ &<1.0 \\ &<1.0 \\ &<2.0 \\ &<47.600 \\ &<1.0 \\ &<1.0 \\ &<2.240 \\ &<1.0 \\ &<1.0 \\ &<1.0 \\ &<1.0 \\ &<1.0 \\ &<1.0 \\ &<1.0 \\ &<1.0 \\ &<1.0 \\ &<1.0 \\ &<1.0 \\ &<1.0 \\ &<4.0 \\ &<5.7 \\ &<5.0 \\ &<0.10 \\ &< \\ &< \\ &<\end{aligned}$

Time: $\mathbf{8 : 3 3}$

pH: 7.6

Alkalinity: $101 \mathrm{mg} / \mathrm{L}$

Water temperature: $18.9^{\circ} \mathrm{C}$

Volumes purged: 0.6 well volumes

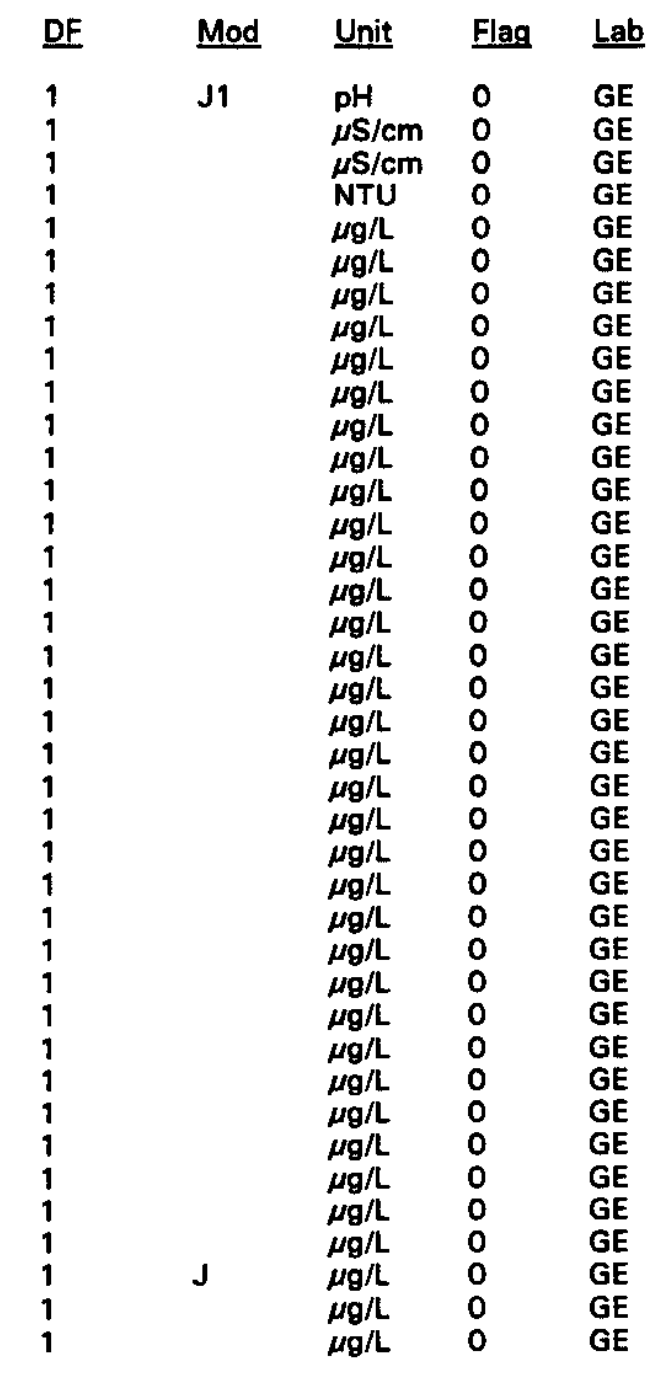

- = exceeded holding time. - = exceeded screening level or final primary drinking water standard. 
WELL BGO 25A collected on 04/06/94, laboratory analyses (cont.)

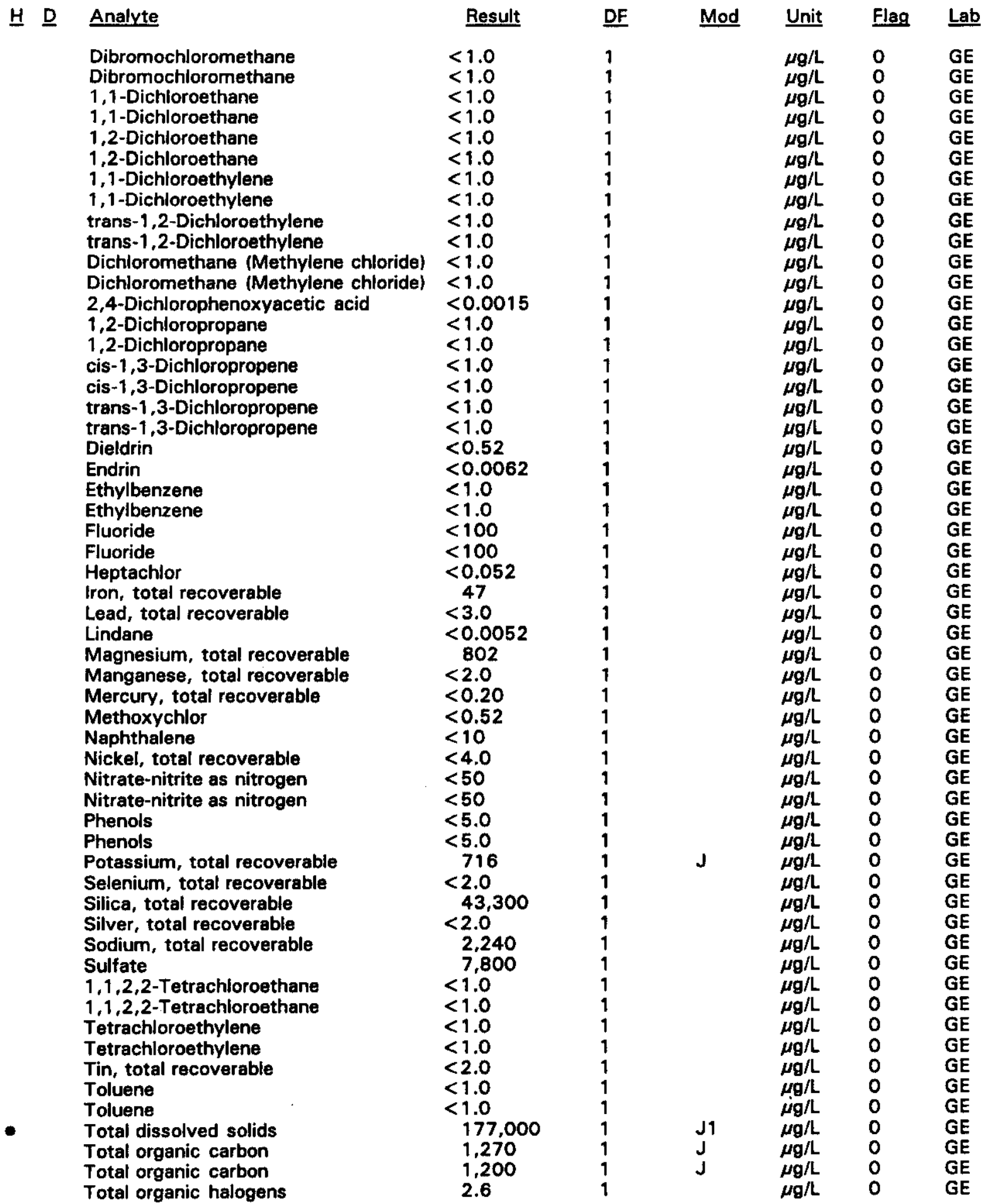

$\overline{-=\text { exceeded holding time. }}=$ = exceeded screening level or final primary drinking water standard. 
WELL BGO 25A collected on 04/06/94, laboratory analyses (cont.)

H D Analyte

Total phosphates (as P)

Toxaphene

2,4,5-TP (Silvex)

1,1,1-Trichloroethane

1,1,1-Trichloroethane

1,1,2-Trichloroethane

1,1,2-Trichloroethane

Trichloroethylene

Trichloroethylene

Trichlorofluoromethane

Trichlorofluoromethane

2,4,5-T

Vanadium, total recoverable

Xylenes

Xylenes

Gross alpha

Nonvolatile beta

Radium, total alpha-emitting

Tritium

Uranium-233/234

Uranium-235

Uranium-238

\begin{tabular}{lll}
\multicolumn{1}{l}{ Result } & DF \\
$<50$ & \\
$<0.25$ & 1 \\
$<0.00045$ & 1 \\
$<1.0$ & 1 \\
$<1.0$ & 1 \\
$<1.0$ & 1 \\
$<1.0$ & 1 \\
$<1.0$ & 1 \\
$<1.0$ & 1 \\
$<1.0$ & 1 \\
$<1.0$ & 1 \\
0.42 & 1 \\
$<8.0$ & 1 \\
$<2.0$ & 1 \\
$<2.0$ & 1 \\
$-7.6 E-02$ & 1 \\
$6.4 E-01$ & 1 \\
$2.0 E-01$ & 1 \\
$1.1 E-01$ & 1 \\
$7.3 E-02$ & 1 \\
$<0.0$ E +00 & 1 \\
$1.3 E-01$ & 1
\end{tabular}

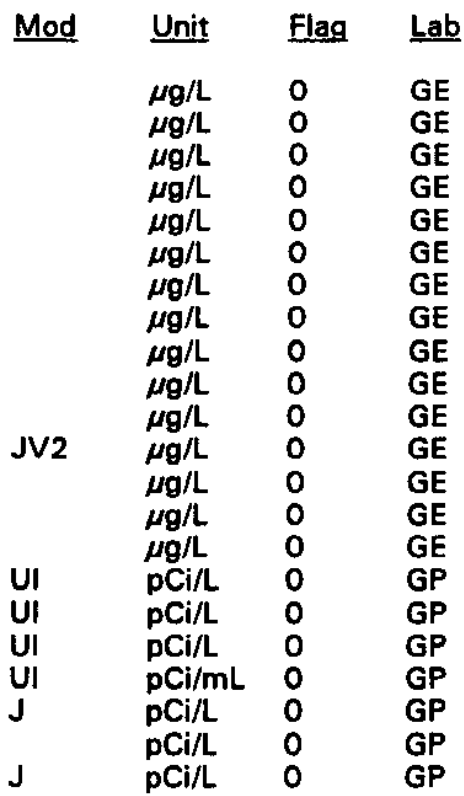

WELL BGO 26D

\begin{tabular}{|c|c|c|c|c|c|c|}
\hline SRS coord. & Lat/Longitude & Screen Zone Elevation & Top of Casing & Casing & Pump & Formation \\
\hline $\begin{array}{l}N 76128.0 \\
E 55015.2\end{array}$ & $\begin{array}{l}33.283910^{\circ} \mathrm{N} \\
81.669811^{\circ} \mathrm{W}\end{array}$ & $233.5-213.4 \mathrm{ft} \mathrm{msl}$ & $285.5 \mathrm{ft} \mathrm{msl}$ & 4" PVC & $\mathbf{s}$ & $\left(|1| B_{2}\right)$ \\
\hline
\end{tabular}

\section{FIELD MEASUREMENTS}

Sample date: $04 / 12 / 94$

Depth to water: $57.21 \mathrm{ft}(17.44 \mathrm{~m})$ below TOC

Water elevation: $228.29 \mathrm{ft}(69.58 \mathrm{~m}) \mathrm{msl}$

Sp. conductance: $30 \mu \mathrm{S} / \mathrm{cm}$

Turbidity: 7.7 NTU

Water evacuated before sampling: 9 gal

The well went dry during purging.

\section{LABORATORY ANALYSES}

H D

Analyte

pH

Specific conductance

Turbidity

Turbidity

Acetophenone

Acetophenone

Aldrin

Aluminum, total recoverable

Antimony, total recoverable

Arsenic, total recoverable

Barium, total recoverable

Benzene
Time: 9:56

pH: 4.9

Alkalinity: $0 \mathrm{mg} / \mathrm{L}$

Water temperature: $19.1^{\circ} \mathrm{C}$

Volumes purged: 0.9 well volumes

$\begin{array}{rlllll}\text { Result } & \text { DF } & \text { Mod } & \text { Unit } & \text { Flag } & \text { Lab } \\ 5.6 & 1 & \text { J1 } & \text { pH } & 0 & \text { GE } \\ 26 & 1 & & \mu S / c m & 0 & \text { GE } \\ 23 & 1 & & \text { NTU } & 0 & \text { GE } \\ 22 & 1 & & \text { NTU } & 0 & \text { GE } \\ <10 & 1 & & \mu g / L & 0 & \text { GE } \\ <10 & 1 & & \mu g / L & 0 & \text { GE } \\ <0.051 & 1 & & \mu g / L & 0 & \text { GE } \\ 223 & 1 & & \mu g / L & 2 & \text { GE } \\ <2.0 & 1 & & \mu g / L & 0 & \text { GE } \\ <2.0 & 1 & & \mu g / L & 0 & \text { GE } \\ 6.4 & 1 & & \mu g / L & 0 & \text { GE } \\ <1.0 & 1 & & \mu g / L & 0 & \text { GE }\end{array}$

- = exceeded holding time. $\square=$ exceeded screening level or final primary drinking water standard. 
WELL BGO 26D collected on 04/12/94, laboratory analyses (cont.)

H D Analyte

Bromodichloromethane

Bromoform

Bromomethane (Methyl bromide)

Cadmium, total recoverable

Calcium, total recoverable

Carbon tetrachloride

Chloride

Chlorobenzene

Chloroethane

Chloroethene (Vinyl chloride)

2-Chloroethyl vinyl ether

Chloroform

Chloromethane (Methyl chloride)

Chromium, total recoverable

Copper, total recoverable

Cyanide

p,p'-DDT

Dibromochloromethane

1,1-Dichloroethane

1,2-Dichloroethane

1,1-Dichloroethylene

trans-1,2-Dichloroethylene

Dichloromethane (Methylene chloride)

2,4-Dichlorophenoxyacetic acid

1,2-Dichloropropane

cis-1,3-Dichloropropene

trans-1,3-Dichloropropene

Dieldrin

Endrin

Ethylbenzene

Fluoride

Heptachlor

Iron, total recoverable

Lead, total recoverable

Lindane

Magnesium, total recoverable

Manganese, total recoverable

Mercury, total recoverable

Mercury, total recoverable

Methoxychlor

Naphthalene

Naphthalene

Nickel, total recoverable

Nitrate-nitrite as nitrogen

Phenols

Potassium, total recoverable

Selenium, total recoverable

Silica, total recoverable

Silver, total recoverable

Sodium, total recoverable

Sulfate

1,1,2,2-Tetrachloroethane

Tetrachloroethylene

Tin, total recoverable

Toluene

Total dissolved solids

Result DF

$<1.0$

$<1.0$

$<1.0$

$<2.0$

1,020

$<1.0$

2,460

$<1.0$

$<1.0$

$<1.0$

$<1.0$

$<1.0$

$<1.0$

$<4.0$

37

$<5.0$

$<0.10$

$<1.0$

$<1.0$

$<1.0$

$<1.0$

$<1.0$

$<1.0$

$<0.0016$

$<1.0$

$<1.0$

$<1.0$

$<0.51$

$<0.0061$

$<1.0$

$<100$

$<0.051$

151

9.7

$<0.0051$

430

12

$<0.20$

0.24

$<0.51$

$<10$

$<10$

$<4.0$

910

$<5.0$

$<500$

$<2.0$

7,840

$<2.0$

2,140

$<1,000$

$<1.0$

1.3

$<2.0$

$<1.0$

22,000
Mod Unit Flag Lab

$\mu g / L \quad O \quad G E$

$\mu g / L \quad 0 \quad G E$

$\mu g / L \quad 0 \quad \mathrm{GE}$

$\mu g / L \quad 0 \quad G E$

$\mu g / L \quad 0 \quad G E$

$\mu g / L \quad 0 \quad G E$

$\mu g / L \quad O \quad G E$

$\mu g / L \quad 0 \quad G E$

$\mu g / L \quad O \quad G E$

$\mu \mathrm{g} / \mathrm{L} \quad 0 \quad \mathrm{GE}$

$\mu g / L \quad 0 \quad G E$

$\mu \mathrm{g} / \mathrm{L} \quad 0 \quad \mathrm{GE}$

$\mu \mathrm{g} / \mathrm{L} \quad \mathrm{O} \quad \mathrm{GE}$

$\mu g / L \quad 0 \quad G E$

$\mu g / L \quad 0 \quad$ GE

$\mu g / L \quad 0 \quad G E$

$\mu g / L \quad 0 \quad G E$

$\mu \mathrm{g} / \mathrm{L} \quad 0 \quad \mathrm{GE}$

$\mu \mathrm{g} / \mathrm{L} \quad 0 \quad \mathrm{GE}$

$\mu \mathrm{g} / \mathrm{L} \quad 0 \quad \mathrm{GE}$

$\mu g / L \quad 0 \quad$ GE

$\mu g / L \quad 0 \quad G E$

$\mu \mathrm{g} / \mathrm{L} \quad 0 \quad \mathrm{GE}$

$\mu \mathrm{g} / \mathrm{L} \quad 0 \quad \mathrm{GE}$

$\mu g / L \quad 0 \quad G E$

$\mu \mathrm{g} / \mathrm{L} \quad \mathrm{O} \quad \mathrm{GE}$

$\mu g / L \quad 0 \quad G E$

$\mu \mathrm{g} / \mathrm{L} \quad 0 \quad \mathrm{GE}$

$\mu g / L \quad 0 \quad$ GE

$\mu \mathrm{g} / \mathrm{L} \quad 0 \quad \mathrm{GE}$

$\mu \mathrm{g} / \mathrm{L} \quad \mathrm{O} \quad \mathrm{GE}$

$\mu \mathrm{g} / \mathrm{L} \quad 0 \quad \mathrm{GE}$

$\mu \mathrm{g} / \mathrm{L} \quad 1 \quad \mathrm{GE}$

$\mu g / L \quad 0 \quad$ GE

$\mu \mathrm{g} / \mathrm{L} \quad \mathrm{O} \quad \mathrm{GE}$

$\mu \mathrm{g} / \mathrm{L} \quad 0 \quad \mathrm{GE}$

$\mu \mathrm{g} / \mathrm{L} \quad 0 \quad \mathrm{GE}$

$\mu \mathrm{g} / \mathrm{L} \quad 0 \quad \mathrm{GE}$

$\mu g / L \quad 0 \quad G E$

$\mu g / L \quad 0 \quad$ GE

$\mu g / L \quad 0 \quad G E$

$\mu g / L \quad 0 \quad$ GE

$\mu \mathrm{g} / \mathrm{L} \quad 0 \quad \mathrm{GE}$

$\mu g / L \quad 0 \quad$ GE

$\mu g / L \quad 0 \quad G E$

$\mu \mathrm{g} / \mathrm{L} \quad \mathrm{O} \quad \mathrm{GE}$

$\mu g / L \quad 0 \quad G E$

$\mu \mathrm{g} / \mathrm{L} \quad \mathrm{O} \quad \mathrm{GE}$

$\mu g / L \quad 0 \quad G E$

$\mu \mathrm{g} / \mathrm{L} \quad \mathrm{O} \quad \mathrm{GE}$

$\mu \mathrm{g} / \mathrm{L} \quad 0 \quad \mathrm{GE}$

$\mu \mathrm{g} / \mathrm{L} \quad 0 \quad \mathrm{GE}$

$\mu \mathrm{g} / \mathrm{h} \quad 0 \quad \mathrm{GE}$

$\mu g / L \quad 0 \quad G E$

$\mu \mathrm{g} / \mathrm{L} \quad \mathrm{O} \quad \mathrm{GE}$

$\mu g / L \quad 0 \quad$ GE

- = exceeded holding time. $\|$ = exceeded screening level or final primary drinking water standard. 
WELL BGO 260 collected on 04/12/94, laboratory analyses (cont.)

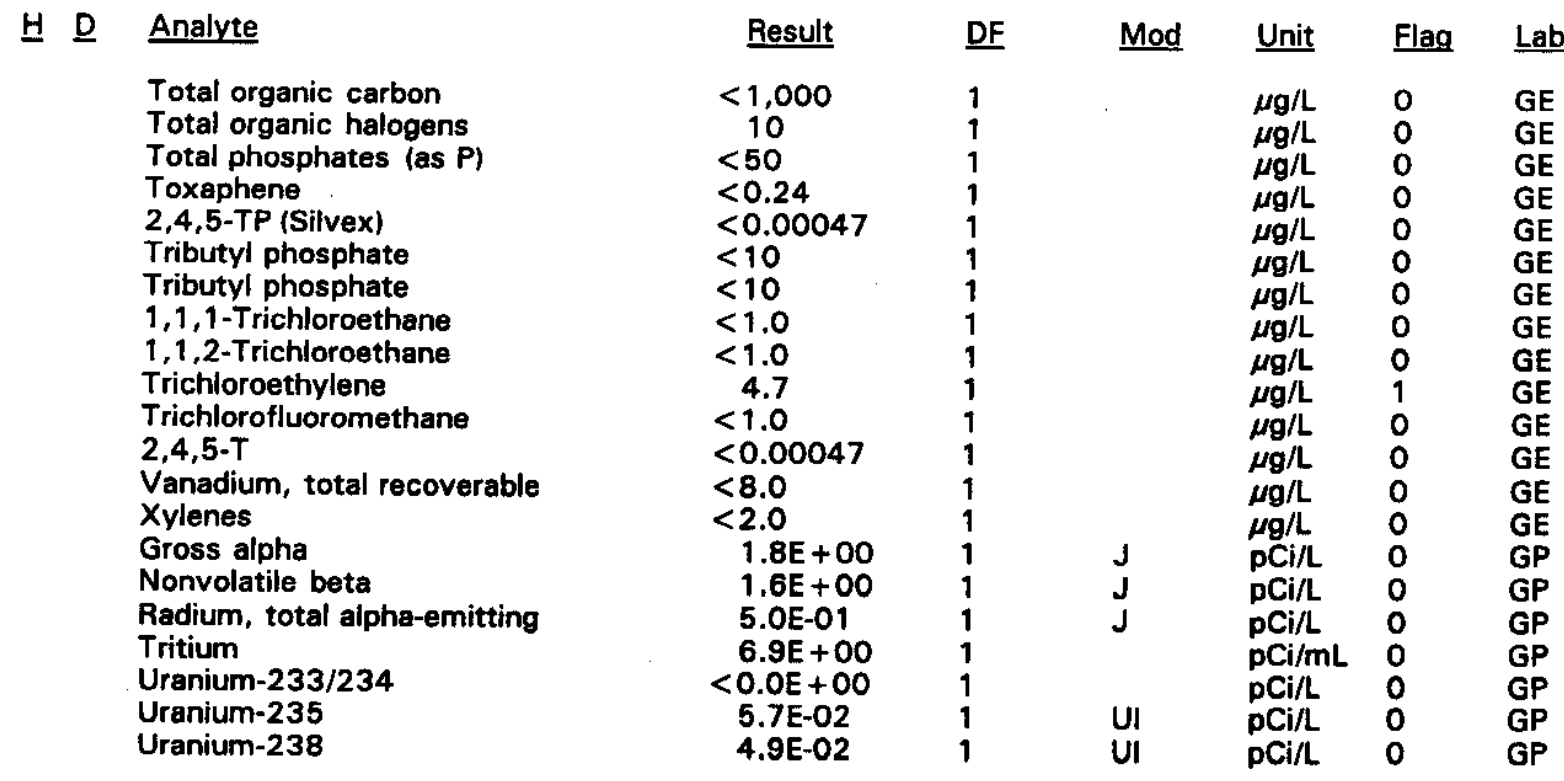

\section{WELL BGO 27C}

\author{
SRS Coord. \\ N75666.3 \\ E54671.4 \\ Lat/Longitude \\ $33.282328^{\circ} \mathrm{N}$ \\ $81.669820^{\circ} \mathrm{W}$ \\ FIELD MEASUREMENTS \\ Sample date: 04/11/94 \\ Depth to water: $54.79 \mathrm{ft}(16.70 \mathrm{~m})$ below TOC \\ Water elevation: $221.21 \mathrm{ft}(67.43 \mathrm{~m}) \mathrm{msl}$ \\ Sp. conductance: $118 \mu \mathrm{S} / \mathrm{cm}$ \\ Turbidity: 0.7 NTU \\ Water evacuated before sampling: $150 \mathrm{gal}$
}

Screen Zone Elevation

$163.9-154.9 \mathrm{ft} \mathrm{ms}$

$\begin{array}{llll}\text { Top of Casing } & \text { Casing } & \text { Pump } & \text { Formation } \\ 276 \mathrm{ft} \mathrm{msl} & 4^{\text {" PVC }} & \mathrm{s} & \text { McBean (IIB, }\end{array}$

Time: 14:08

pH: 7.1

Alkalinity: $35 \mathrm{mg} / \mathrm{L}$

Water temperature: $19.8^{\circ} \mathrm{C}$

Volumes purged: $\mathbf{3 . 4}$ well volumes

\section{LABORATORY ANALYSES}

H D $\quad$ Analvte
- pH
pH
Specific conductance
Turbidity
Acetophenone
Aldrin
Aldrin
Aluminum, total recoverable
Antimony, total recoverable
Arsenic, total recoverable
Barium, total recoverable
Benzene
Benzene

$\overline{-}=$ exceeded holding time. $=$ exceeded screening level or final primary drinking water standard.

\begin{tabular}{|c|c|c|c|c|}
\hline Result & DF & Mod & Unit & Flag \\
\hline $\begin{array}{l}7.3 \\
7.3 \\
106 \\
0.29 \\
<9.7 \\
<0.050 \\
<0.050 \\
39 \\
<2.0 \\
<2.0 \\
3.3 \\
<1.0 \\
<1.0\end{array}$ & $\begin{array}{l}1 \\
1 \\
1 \\
1 \\
1 \\
1 \\
1 \\
1 \\
1 \\
1 \\
1 \\
1 \\
1\end{array}$ & $\begin{array}{l}\text { J1 } \\
\text { J1 }\end{array}$ & $\begin{array}{l}\mathrm{pH} \\
\mathrm{pH} \\
\mu \mathrm{S} / \mathrm{cm} \\
\mathrm{NTU} \\
\mu \mathrm{g} / \mathrm{L} \\
\mu \mathrm{g} / \mathrm{L} \\
\mu \mathrm{g} / \mathrm{L} \\
\mu \mathrm{g} / \mathrm{L} \\
\mu \mathrm{g} / \mathrm{L} \\
\mu \mathrm{g} / \mathrm{L} \\
\mu \mathrm{g} / \mathrm{L} \\
\mu \mathrm{g} / \mathrm{L} \\
\mu \mathrm{g} / \mathrm{L}\end{array}$ & $\begin{array}{l}0 \\
0 \\
0 \\
0 \\
0 \\
0 \\
0 \\
1 \\
0 \\
0 \\
0 \\
0 \\
0\end{array}$ \\
\hline
\end{tabular}


WELL BGO 27C collected on 04/11/94, laboratory analyses (cont.)

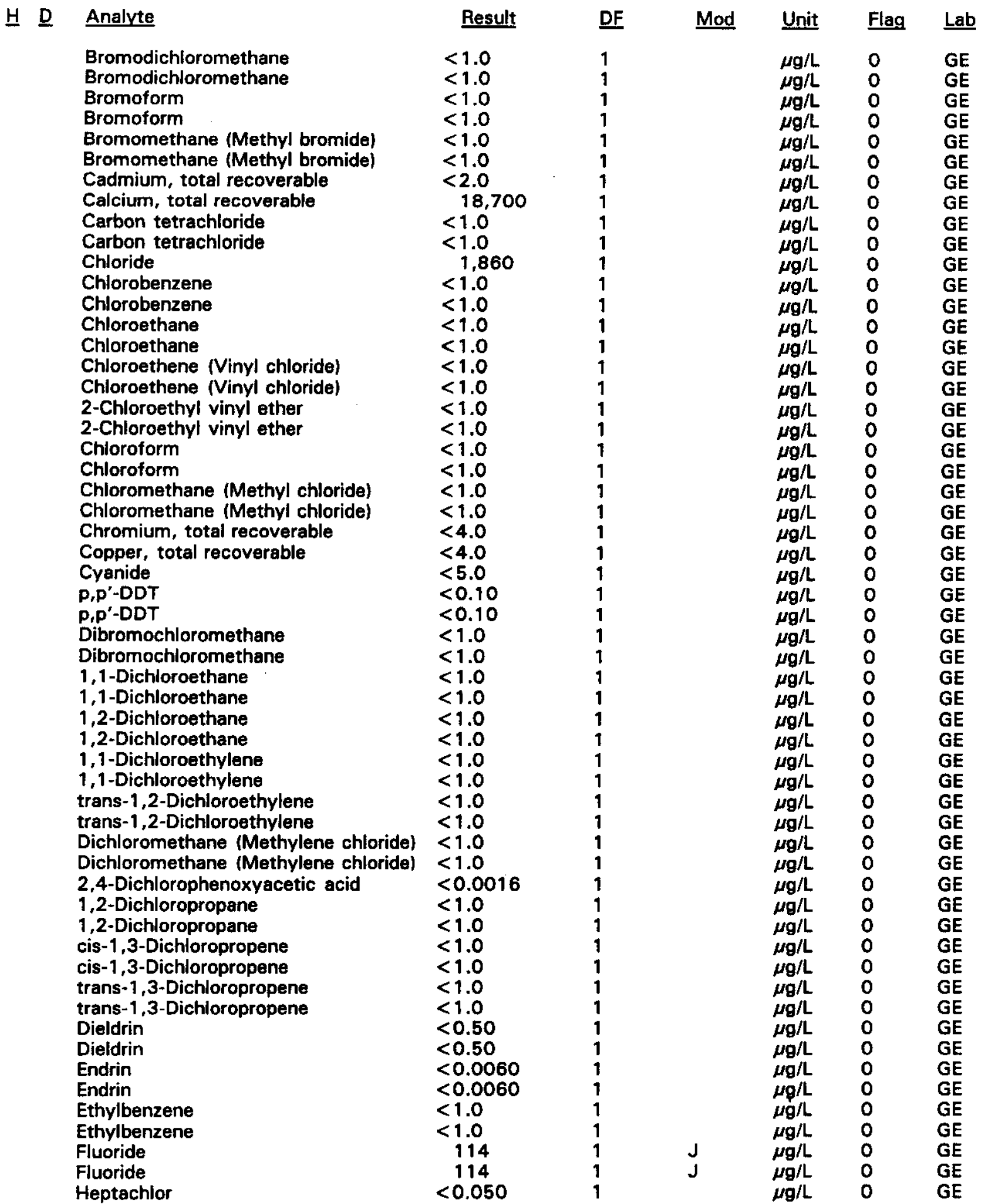

- exceeded holding time. $=$ exceeded screening level or final primary drinking water standard. 
WELL BGO $27 \mathrm{C}$ collected on $04 / 11 / 94$, laboratory analyses (cont.)

H $\underline{\text { Analyte }}$

Heptachlor

Iron, total recoverable

Lead, total recoverable

Lindane

Lindane

Magnesium, total recoverable

Manganese, total recoverable

Mercury, total recoverable

Methoxychlor

Methoxychlor

Naphthalene

Nickel, total recoverable

Nitrate-nitrite as nitrogen

Phenols

Potassium, total recoverable

Selenium, total recoverable

Silica, total recoverable

Silver, total recoverable

Sodium, total recoverable

Sulfate

1,1,2,2-Tetrachloroethane

1,1,2,2-Tetrachloroethane

Tetrachloroethylene

Tetrachloroethylene

Tin, total recoverable

Toluene

Toluene

Total dissolved solids

Total organic carbon

Total organic halogens

Total organic halogens

Total phosphates (as P)

Toxaphene

Toxaphene

2,4,5-TP (Silvex)

Tributyl phosphate

1,1,1-Trichloroethane

1,1,1-Trichloroethane

1,1,2-Trichloroethane

1,1,2-Trichloroethane

- Trichloroethylene

- Trichloroethylene

Trichlorofluoromethane

Trichlorofluoromethane

2,4,5-T

Vanadium, total recoverable

Xylenes

Xylenes

Gross alpha

Nonvolatile beta

Radium, total alpha-emitting

- Tritium

Uranium-233/234

Uranium-235

Uranium-238
$<0.050$

9.9

$<3.0$

$<0.0050$

$<0.0050$

297

$<2.0$

$<0.20$

$<0.50$

$<0.50$

$<9.7$

$<4.0$

1.280

$<5.0$

$<500$

$<2.0$

9,210

$<2.0$

1,630

$<1,000$

$<1.0$

$<1.0$

$<1.0$

$<1.0$

$<2.0$

$<1.0$

$<1.0$

68,000

$<1,000$

14

16

280

$<0.24$

$<0.24$

$<0.00046$

$<9.7$

$<1.0$

$<1.0$

$<1.0$

$<1.0$

26

26

$<1.0$

$<1.0$

0.40

$<8.0$

$<2.0$

$<2.0$

4.1E-01

$1.1 \mathrm{E}+00$

1. OE-O1

7.4E + 01

5.1E-02

$<0.0 \mathrm{E}+00$

5.5E-02

\section{DF}

1

1

1

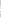

1

1

1

1

$\begin{array}{ll}\text { DF } & \text { Mod } \\ 1 \\ 1 \\ 1 \\ 1 \\ 1 \\ 1 \\ 1 \\ 1 \\ 1 \\ 1 \\ 1 \\ 1 \\ 1 \\ 1 \\ 1 \\ 1 \\ 1 \\ 1 \\ 1 \\ 1 \\ 1 \\ 1 \\ 1 \\ 1 \\ 1 \\ 1 \\ 1 \\ 1 \\ 1 \\ 1 \\ 1 \\ 1 \\ 1 \\ 1 \\ 1 \\ 1 \\ 1 \\ 1 \\ 1\end{array}$

$\begin{array}{ll}\text { DF } & \text { Mod } \\ 1 \\ 1 \\ 1 \\ 1 \\ 1 \\ 1 \\ 1 \\ 1 \\ 1 \\ 1 \\ 1 \\ 1 \\ 1 \\ 1 \\ 1 \\ 1 \\ 1 \\ 1 \\ 1 \\ 1 \\ 1 \\ 1 \\ 1 \\ 1 \\ 1 \\ 1 \\ 1 \\ 1 \\ 1 \\ 1 \\ 1 \\ 1 \\ 1 \\ 1 \\ 1 \\ 1 \\ 1 \\ 1 \\ 1\end{array}$

1

$\begin{array}{ll}\text { DF } & \text { Mod } \\ 1 \\ 1 \\ 1 \\ 1 \\ 1 \\ 1 \\ 1 \\ 1 \\ 1 \\ 1 \\ 1 \\ 1 \\ 1 \\ 1 \\ 1 \\ 1 \\ 1 \\ 1 \\ 1 \\ 1 \\ 1 \\ 1 \\ 1 \\ 1 \\ 1 \\ 1 \\ 1 \\ 1 \\ 1 \\ 1 \\ 1 \\ 1 \\ 1 \\ 1 \\ 1 \\ 1 \\ 1 \\ 1 \\ 1\end{array}$

$\begin{array}{ll}\text { DF } & \text { Mod } \\ 1 \\ 1 \\ 1 \\ 1 \\ 1 \\ 1 \\ 1 \\ 1 \\ 1 \\ 1 \\ 1 \\ 1 \\ 1 \\ 1 \\ 1 \\ 1 \\ 1 \\ 1 \\ 1 \\ 1 \\ 1 \\ 1 \\ 1 \\ 1 \\ 1 \\ 1 \\ 1 \\ 1 \\ 1 \\ 1 \\ 1 \\ 1 \\ 1 \\ 1 \\ 1 \\ 1 \\ 1 \\ 1 \\ 1 \\ 1 \\ 1 \\ 1 \\ 1 \\ 1 \\ \\ 1 \\ 1\end{array}$

$\begin{array}{ll}\text { DF } & \text { Mod } \\ 1 \\ 1 \\ 1 \\ 1 \\ 1 \\ 1 \\ 1 \\ 1 \\ 1 \\ 1 \\ 1 \\ 1 \\ 1 \\ 1 \\ 1 \\ 1 \\ 1 \\ 1 \\ 1 \\ 1 \\ 1 \\ 1 \\ 1 \\ 1 \\ 1 \\ 1 \\ 1 \\ 1 \\ 1 \\ 1 \\ 1 \\ 1 \\ 1 \\ 1 \\ 1 \\ 1 \\ 1 \\ 1 \\ 1 \\ 1 \\ 1 \\ 1 \\ 1 \\ 1 \\ 1 \\ 1 \\ 1 \\ 1\end{array}$

$\begin{array}{ll}\text { DF } & \text { Mod } \\ 1 \\ 1 \\ 1 \\ 1 \\ 1 \\ 1 \\ 1 \\ 1 \\ 1 \\ 1 \\ 1 \\ 1 \\ 1 \\ 1 \\ 1 \\ 1 \\ 1 \\ 1 \\ 1 \\ 1 \\ 1 \\ 1 \\ 1 \\ 1 \\ 1 \\ 1 \\ 1 \\ 1 \\ 1 \\ 1 \\ 1 \\ 1 \\ 1 \\ 1 \\ 1 \\ 1 \\ 1 \\ 1 \\ 1 \\ 1 \\ 1 \\ 1 \\ 1 \\ 1 \\ \\ 1 \\ 1\end{array}$

$\begin{array}{ll}\text { DF } & \text { Mod } \\ 1 \\ 1 \\ 1 \\ 1 \\ 1 \\ 1 \\ 1 \\ 1 \\ 1 \\ 1 \\ 1 \\ 1 \\ 1 \\ 1 \\ 1 \\ 1 \\ 1 \\ 1 \\ 1 \\ 1 \\ 1 \\ 1 \\ 1 \\ 1 \\ 1 \\ 1 \\ 1 \\ 1 \\ 1 \\ 1 \\ 1 \\ 1 \\ 1 \\ 1 \\ 1 \\ 1 \\ 1 \\ 1 \\ 1 \\ 1 \\ 1\end{array}$

$\begin{array}{ll}\text { DF } & \text { Mod } \\ 1 \\ 1 \\ 1 \\ 1 \\ 1 \\ 1 \\ 1 \\ 1 \\ 1 \\ 1 \\ 1 \\ 1 \\ 1 \\ 1 \\ 1 \\ 1 \\ 1 \\ 1 \\ 1 \\ 1 \\ 1 \\ 1 \\ 1 \\ 1 \\ 1 \\ 1 \\ 1 \\ 1 \\ 1 \\ 1 \\ 1 \\ 1 \\ 1 \\ 1 \\ 1 \\ 1 \\ 1 \\ 1 \\ 1 \\ 1 \\ 1 \\ 1 \\ 1 \\ 1 \\ 1 \\ 1 \\ 1 \\ 1\end{array}$

DF
1
1
1
1
1
1
1
1
1
1
1
1
1
1
1
1
1
1
1
1
1
1
1
1
1
1
1
1
1
1
1
1
1
1
1
1
1
1
1
1
1
1
1

1
1

1
1

DF
1
1
1
1
1
1
1
1
1
1
1
1
1
1
1
1
1
1
1
1
1
1
1
1
1
1
1
1
1
1
1
1
1
1
1
1
1
1
1
1
1
1
1

1
1

1
1

DF
1
1
1
1
1
1
1
1
1
1
1
1
1
1
1
1
1
1
1
1
1
1
1
1
1
1
1
1
1
1
1
1
1
1
1
1
1
1
1
1
1
1
1

1
1

1
1

DF
1
1
1
1
1
1
1
1
1
1
1
1
1
1
1
1
1
1
1
1
1
1
1
1
1
1
1
1
1
1
1
1
1
1
1
1
1
1
1
1
1
1
1

1
1

1
1

$\begin{array}{ll}\text { DF } & \text { Mod } \\ 1 \\ 1 \\ 1 \\ 1 \\ 1 \\ 1 \\ 1 \\ 1 \\ 1 \\ 1 \\ 1 \\ 1 \\ 1 \\ 1 \\ 1 \\ 1 \\ 1 \\ 1 \\ 1 \\ 1 \\ 1 \\ 1 \\ 1 \\ 1 \\ 1 \\ 1 \\ 1 \\ 1 \\ 1 \\ 1 \\ 1 \\ 1 \\ 1 \\ 1 \\ 1 \\ 1 \\ 1 \\ 1 \\ 1 \\ 1 \\ 1 \\ 1 \\ 1 \\ 1 \\ 1 \\ 1 \\ 1 \\ 1\end{array}$

$\begin{array}{ll}\text { DF } & \text { Mod } \\ 1 \\ 1 \\ 1 \\ 1 \\ 1 \\ 1 \\ 1 \\ 1 \\ 1 \\ 1 \\ 1 \\ 1 \\ 1 \\ 1 \\ 1 \\ 1 \\ 1 \\ 1 \\ 1 \\ 1 \\ 1 \\ 1 \\ 1 \\ 1 \\ 1 \\ 1 \\ 1 \\ 1 \\ 1 \\ 1 \\ 1 \\ 1 \\ 1 \\ 1 \\ 1 \\ 1 \\ 1 \\ 1 \\ 1 \\ 1 \\ 1 \\ 1 \\ 1 \\ 1 \\ 1 \\ 1 \\ 1 \\ 1\end{array}$

$\begin{array}{ll}\text { DF } & \text { Mod } \\ 1 \\ 1 \\ 1 \\ 1 \\ 1 \\ 1 \\ 1 \\ 1 \\ 1 \\ 1 \\ 1 \\ 1 \\ 1 \\ 1 \\ 1 \\ 1 \\ 1 \\ 1 \\ 1 \\ 1 \\ 1 \\ 1 \\ 1 \\ 1 \\ 1 \\ 1 \\ 1 \\ 1 \\ 1 \\ 1 \\ 1 \\ 1 \\ 1 \\ 1 \\ 1 \\ 1 \\ 1 \\ 1 \\ 1 \\ 1 \\ 1 \\ 1 \\ 1 \\ 1 \\ 1 \\ 1 \\ 1 \\ 1\end{array}$

DF
1
1
1
1
1
1
1
1
1
1
1
1
1
1
1
1
1
1
1
1
1
1
1
1
1
1
1
1
1
1
1
1
1
1
1
1
1
1
1
1
1
1
1

1
1

1
1

$\begin{array}{ll}\text { DF } & \text { Mod } \\ 1 \\ 1 \\ 1 \\ 1 \\ 1 \\ 1 \\ 1 \\ 1 \\ 1 \\ 1 \\ 1 \\ 1 \\ 1 \\ 1 \\ 1 \\ 1 \\ 1 \\ 1 \\ 1 \\ 1 \\ 1 \\ 1 \\ 1 \\ 1 \\ 1 \\ 1 \\ 1 \\ 1 \\ 1 \\ 1 \\ 1 \\ 1 \\ 1 \\ 1 \\ 1 \\ 1 \\ 1 \\ 1 \\ 1 \\ 1 \\ 1 \\ 1 \\ 1 \\ 1 \\ 1 \\ 1 \\ 1 \\ 1\end{array}$

$\begin{array}{ll}\text { DF } & \text { Mod } \\ 1 \\ 1 \\ 1 \\ 1 \\ 1 \\ 1 \\ 1 \\ 1 \\ 1 \\ 1 \\ 1 \\ 1 \\ 1 \\ 1 \\ 1 \\ 1 \\ 1 \\ 1 \\ 1 \\ 1 \\ 1 \\ 1 \\ 1 \\ 1 \\ 1 \\ 1 \\ 1 \\ 1 \\ 1 \\ 1 \\ 1 \\ 1 \\ 1 \\ 1 \\ 1 \\ 1 \\ 1 \\ 1 \\ 1 \\ 1 \\ 1 \\ 1 \\ 1 \\ 1 \\ 1 \\ 1 \\ 1 \\ 1\end{array}$

DF
1
1
1
1
1
1
1
1
1
1
1
1
1
1
1
1
1
1
1
1
1
1
1
1
1
1
1
1
1
1
1
1
1
1
1
1
1
1
1
1
1
1
1
1
1
1
1
1
1
1

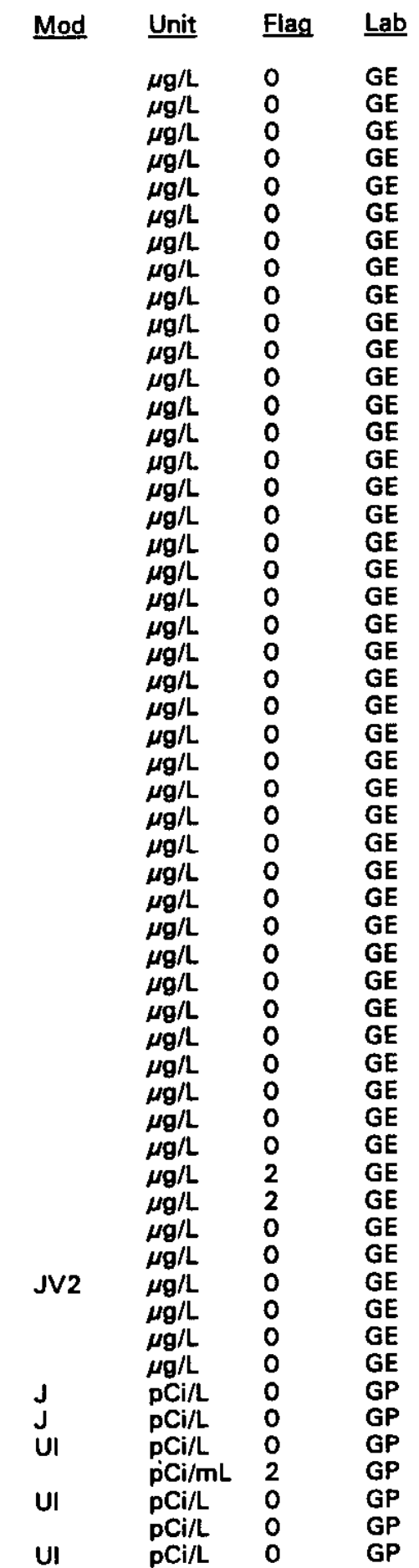

UI 


\section{WELL BGO 27D}

$\begin{array}{lllllll}\text { SRS Coord. } & \text { Lat/Longitude } & \text { Screen Zone Elevation } & \text { Top of Casing } & \text { Casing } & \text { Pump } & \text { Formation } \\ \text { N75677.3 } & 33.282366^{\circ} \mathrm{N} & 229.3-209.3 \mathrm{ft} \mathrm{msl} & 276.3 \mathrm{ft} \mathrm{msl} & \text { 4" PVC } & \text { S } & \text { Water Table (IIB }{ }_{2} \text { ) } \\ \text { E54680.2 } & 81.669818^{\circ} \mathrm{W} & & & & & \end{array}$

\section{FIELD MEASUREMENTS}

Sample date: 04/12/94

Depth to water: $48.31 \mathrm{ft}(14.73 \mathrm{~m})$ below TOC

Water elevation: $227.99 \mathrm{ft}(69.49 \mathrm{~m}) \mathrm{ms}$ l

Sp. conductance: $37 \mu \mathrm{S} / \mathrm{cm}$

Turbidity: 35.2 NTU

Water evacuated before sampling: $13 \mathrm{gal}$

The well went dry during purging.

\section{LABORATORY ANALYSES}

H D Analyte

- $\quad \mathrm{pH}$

Specific conductance
Turbidity
Acetophenone
Aldrin
Aluminum, total recoverable
Antimony, total recoverable
Arsenic, total recoverable
Barium, total recoverable
Benzene
Bromodichloromethane
Bromoform
Bromomethane (Methyl bromide)
Cadmium, total recoverable
Calcium, total recoverable
Carbon tetrachloride
Chloride
Chlorobenzene
Chloroethane
Chloroethene (Vinyl chloride)
2-Chloroethyl vinyl ether
Chloroform
Chloromethane (Methyl chloride)
Chromium, total recoverable
Copper, total recoverable
Cyanide
p,p'-DDT
Dibromochloromethane
1,1-Dichloroethane
1,2-Dichloroethane
1,1-Dichloroethylene
trans-1,2-Dichloroethylene
Dichloromethane (Methylene chloride)
2,4-Dichlorophenoxyacetic acid
1,2-Dichloropropane
cis-1,3-Dichloropropene
trans-1,3-Dichloropropene
Dieldrin
Endrin

\begin{tabular}{|c|c|c|c|c|}
\hline Result & DF & Mod & Unit & Flag \\
\hline $\begin{aligned} & 5.5 \\
& 29 \\
& 24 \\
&<10 \\
&<0.050 \\
& 4.000 \\
&<2.0 \\
&<2.0 \\
& 18 \\
&<1.0 \\
&<1.0 \\
&<1.0 \\
&<1.0 \\
&<2.0 \\
& 1.030 \\
&<1.0 \\
& 1.630 \\
&<1.0 \\
&<1.0 \\
&<1.0 \\
&<1.0 \\
&<1.0 \\
&<1.0 \\
& 6.6 \\
& 112 \\
&<5.0 \\
&<0.10 \\
&<1.0 \\
&<1.0 \\
&<1.0 \\
&<1.0 \\
&<1.0 \\
&<1.0 \\
&<0.0015 \\
&<1.0 \\
&<1.0 \\
&<1.0 \\
&<0.50 \\
&<0.0060\end{aligned}$ & $\begin{array}{l}1 \\
1 \\
1 \\
1 \\
1 \\
1 \\
1 \\
1 \\
1 \\
1 \\
1 \\
1 \\
1 \\
1 \\
1 \\
1 \\
1 \\
1 \\
1 \\
1 \\
1 \\
1 \\
1 \\
1 \\
1 \\
1 \\
1 \\
1 \\
1 \\
1 \\
1 \\
1 \\
1 \\
1 \\
1 \\
1 \\
1 \\
1 \\
1\end{array}$ & $J 1$ & 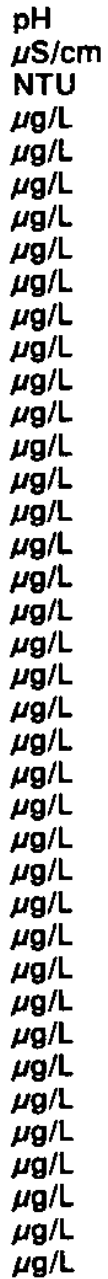 & $\begin{array}{l}0 \\
0 \\
0 \\
0 \\
0 \\
2 \\
0 \\
0 \\
0 \\
0 \\
0 \\
0 \\
0 \\
0 \\
0 \\
0 \\
0 \\
0 \\
0 \\
0 \\
0 \\
0 \\
0 \\
0 \\
0 \\
0 \\
0 \\
0 \\
0 \\
0 \\
0 \\
0 \\
0 \\
0 \\
0 \\
0 \\
0 \\
0 \\
0\end{array}$ \\
\hline
\end{tabular}

Time: 10:21

pH: 4.9

Alkalinity: $0 \mathrm{mg} / \mathrm{L}$

Water temperature: $19.4{ }^{\circ} \mathrm{C}$

Volumes purged: 1.1 well volumes 
WELL BGO 27D collected on 04/12/94, laboratory analyses (cont.)

H $\underline{\text { Analyte }}$

Ethylbenzene

Fluoride

Heptachlor

Iron, total recoverable

Lead, total recoverable

Lindane

Magnesium, total recoverable

Manganese, total recoverable

Mercury, total recoverable

Methoxychlor

Naphthalene

Nickel, total recoverable

Nitrate-nitrite as nitrogen

Phenols

Potassium, total recoverable

Selenium, total recoverable

Silica, total recoverable

Silver, total recoverable

Sodium, total recoverable

Sulfate

$1,1,2,2$-Tetrachloroethane

Tetrachloroethylene

Tin, total recoverable

Toluene

Total dissolved solids

Total organic carbon

Total organic halogens

Total organic halogens

Total phosphates (as P)

Toxaphene

2,4,5-TP (Silvex)

Tributyl phosphate

1,1,1-Trichloroethane

1,1,2-Trichloroethane

Trichloroethylene

Trichlorofluoromethane

2,4,5-T

Vanadium, total recoverable

Xylenes

Gross alpha

Nonvolatile beta

Radium, total alpha-emitting

- Tritium

Uranium-233/234

Uranium-235

Uranium-238
Result DF

$<1.0$

$<100$

$<0.050$

1,810

34

$<0.0050$

1,220

29

$<0.20$

$<0.50$

$<10$

4.4

1,840

$<5.0$

990

$<2.0$

47,000

$<2.0$

1,380

$<1,000$

$<1.0$

$<1.0$

$<2.0$

$<1.0$

23,000

$<1,000$

15

16

$<50$

$<0.24$

$<0.00046$

$<10$

$<1.0$

$<1.0$

$<1.0$

$<1.0$

$<0.00046$

$<8.0$

$<2.0$

2.9E + 00

$3.2 \mathrm{E}+00$

$1.0 \mathrm{E}+00$

$3.1 \mathrm{E}+01$

8.3E-02

$<0.0 \mathrm{E}+00$

1.OE-01
DF

Mod Unit Flag Lab

$\mu \mathrm{g} / \mathrm{L} \quad 0 \quad \mathrm{GE}$

$\mu g / L \quad 0 \quad G E$

$\mu \mathrm{g} / \mathrm{L} \quad 0 \quad \mathrm{GE}$

$\mu \mathrm{g} / \mathrm{L} \quad 2 \quad \mathrm{GE}$

$\mu g / L \quad 1 \quad G E$

$\mu g / L \quad 0 \quad G E$

$\mu g / L \quad 0 \quad G E$

$\mu g / L \quad 1 \quad G E$

$\mu g / L \quad 0 \quad G E$

$\mu g / L \quad 0 \quad$ GE

$\mu g / L \quad 0 \quad G E$

J $\mu g / L \quad 0 \quad$ GE

$\mu g / L \quad 0 \quad G E$

$\mu g / L \quad 0 \quad G E$

$\mu \mathrm{g} / \mathrm{L} \quad 0 \quad \mathrm{GE}$

$\mu g / L \quad 0 \quad G E$

$\mu \mathrm{g} / \mathrm{L} \quad 0 \quad \mathrm{GE}$

$\mu \mathrm{g} / \mathrm{L} \quad 0 \quad \mathrm{GE}$

$\mu g / L \quad O \quad G E$

$\mu g / L \quad 0 \quad G E$

$\mu \mathrm{g} / \mathrm{L} \quad 0 \quad \mathrm{GE}$

$\mu g / L \quad 0 \quad G E$

$\mu \mathrm{g} / \mathrm{L} \quad 0 \quad \mathrm{GE}$

$\mu g / L \quad O \quad G E$

$\mu g / L \quad O \quad G E$

$\mu g / L \quad 0 \quad G E$

$\mu g / L \quad 0 \quad G E$

$\mu \mathrm{g} / \mathrm{L} \quad 0 \quad \mathrm{GE}$

$\mu g / L \quad 0 \quad G E$

$\mu g / L \quad 0 \quad$ GE

$\mu g / L \quad 0 \quad G E$

$\mu g / L \quad 0 \quad \mathrm{GE}$

$\mu g / L \quad 0 \quad G E$

$\mu g / \mathrm{L} \quad 0 \quad \mathrm{GE}$

$\mu g / L \quad 0 \quad G E$

$\mu \mathrm{g} / \mathrm{L} \quad 0 \quad \mathrm{GE}$

$\mu g / \mathrm{L} \quad 0 \quad \mathrm{GE}$

$\mu g / L \quad 0 \quad$ GE

$\mu \mathrm{g} / \mathrm{L} \quad 0$

$\mathrm{pCi} / \mathrm{L} \quad 0 \quad \mathrm{GP}$

$\begin{array}{llll}J & \mathrm{pCi} / \mathrm{L} & 0 & \mathrm{GP}\end{array}$

UI $\quad \mathrm{pCi} / \mathrm{L} \quad 0 \quad \mathrm{GP}$

UI $\quad \begin{array}{lll}\mathrm{pCl} / \mathrm{L} & 0 & \mathrm{G}\end{array}$

- = exceeded holding time. = exceeded screening level or final primary drinking water standard. 
WELL BGO 28D

\begin{tabular}{|c|c|c|c|c|c|c|}
\hline SAS Coord. & Lat/Longitude & Screen Zone Elevation & Top of Casing & Casing & Pump & Formation \\
\hline $\begin{array}{l}N 75348.3 \\
\text { E54457.9 }\end{array}$ & $\begin{array}{l}33.281276^{\circ} \mathrm{N} \\
81.669764^{\circ} \mathrm{W}\end{array}$ & $230.1-210.1 \mathrm{ft} \mathrm{msl}$ & $277.4 \mathrm{ft} \mathrm{msl}$ & 4" PVC & $\mathbf{S}$ & able $\left(I I B_{2}\right)$ \\
\hline
\end{tabular}

\section{FIELD MEASUREMENTS}

Sample date: 04/12/94

Depth to water: $50.72 \mathrm{ft}(15.46 \mathrm{~m})$ below TOC

Water elevation: $226.68 \mathrm{ft}(69.09 \mathrm{~m}) \mathrm{msl}$

Sp. conductance: $108 \mu \mathrm{S} / \mathrm{cm}$

Turbidity: 51.7 NTU

Water evacuated before sampling: $10 \mathrm{gal}$

The well went dry during purging.

\section{LABORATORY ANALYSES}

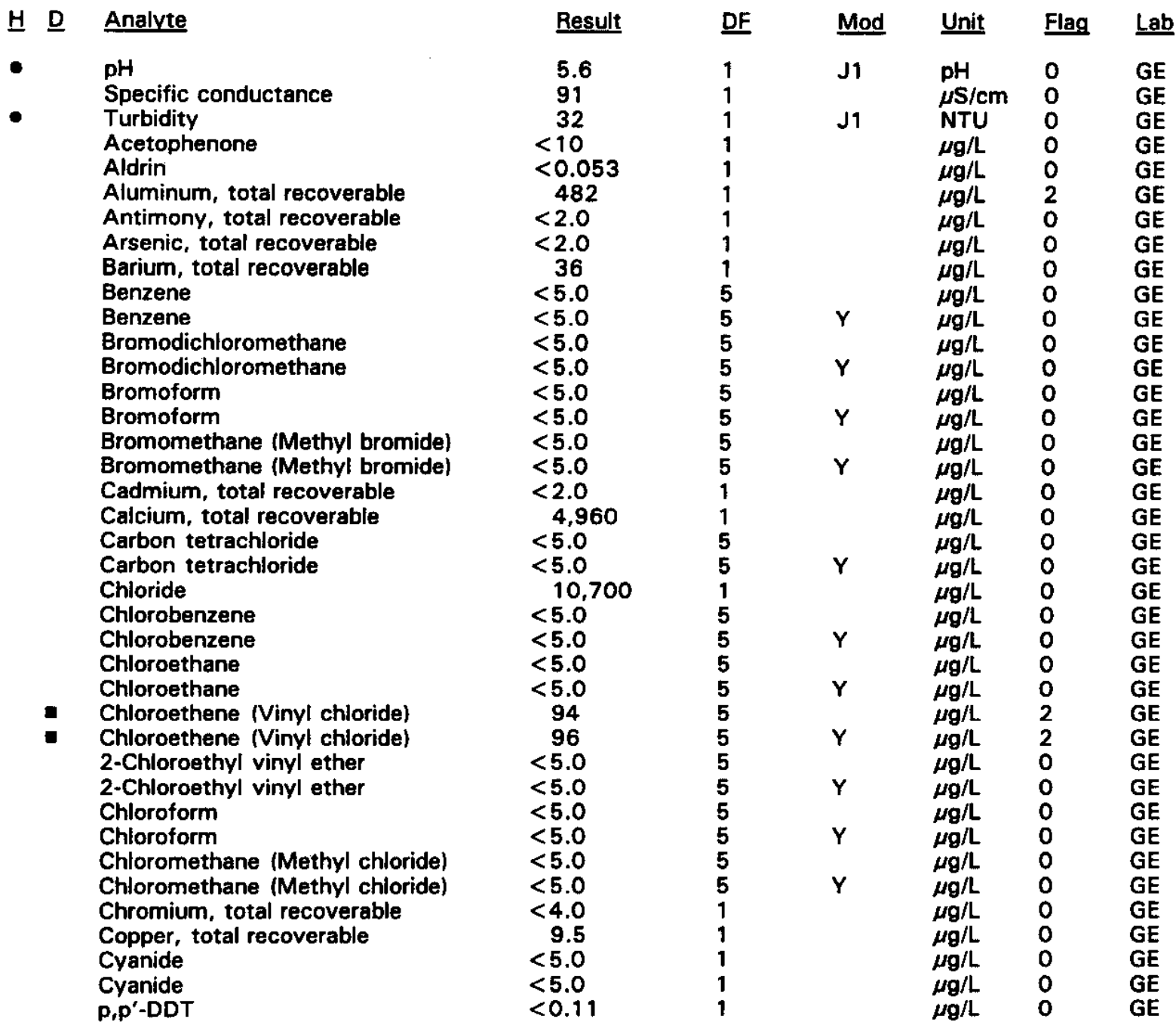

- = exceeded holding time. $\|=$ exceeded screening level or final primary drinking water standard.
pH: 5.4

Alkalinity: $7 \mathrm{mg} / \mathrm{L}$

Water temperature: $20.6^{\circ} \mathrm{C}$

Volumes purged: 0.9 well volumes 
WELL BGO 28D collected on 04/12/94, laboratory analyses (cont.)

H D Analyte

Dibromochloromethane

Dibromochloromethane

1,1-Dichloroethane

1,1-Dichloroethane

1,2-Dichloroethane

1,2-Dichloroethane

1,1-Dichloroethylene

1,1-Dichloroethylene

trans-1,2-Dichloroethylene

trans-1,2-Dichloroethylene

Dichloromethane (Methylene chloride)

Dichloromethane (Methylene chloride)

2,4-Dichlorophenoxyacetic acid

1,2-Dichloropropane

1,2-Dichloropropane

cis-1,3-Dichloropropene

cis-1,3-Dichloropropene

trans-1,3-Dichloropropene

trans-1,3-Dichloropropene

Dieldrin

Endrin

Ethylbenzene

Ethylbenzene

Fluoride

Fluoride

Heptachlor

Iron, total recoverable

Lead, total recoverable

Lindane

Magnesium, total recoverable

Manganese, total recoverable

Mercury, total recoverable

Methoxychlor

Naphthalene

Nickel, total recoverable

Nitrate-nitrite as nitrogen

Phenols

Potassium, total recoverable

Selenium, total recoverable

Silica, total recoverable

Silver, total recoverable

Sodium, total recoverable

Sulfate

1,1,2,2-Tetrachloroethane

$1,1,2,2$-Tetrachloroethane

Tetrachloroethylene

Tetrachloroethylene

Tin, total recoverable

Toluene

Toluene

Total dissolved solids

Total organic carbon

Total organic halogens

Total phosphates (as P)

Toxaphene

2,4,5-TP (Silvex)

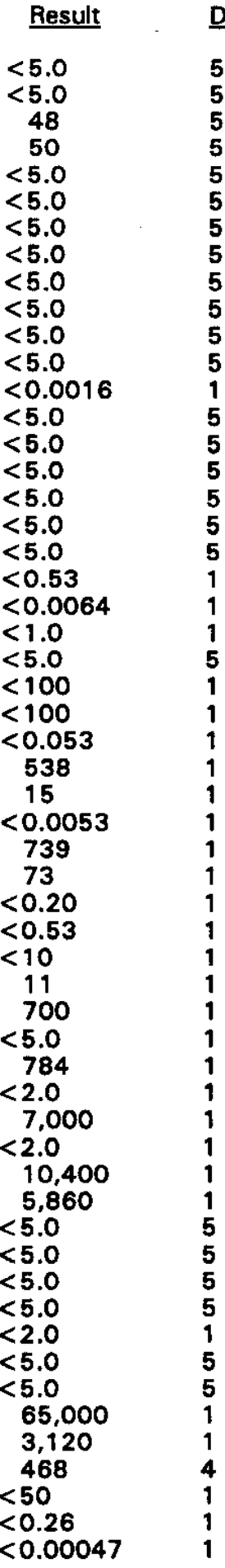

= exceeded holding time. $\quad$ = exceeded screening level or final primary drinking water standard. 
WELL BGO 28 D collected on $04 / 12 / 94$, laboratory analyses (cont.)

$\begin{array}{ll}\text { H } & \text { Analyte } \\ \text { Tributyl phosphate } \\ 1,1,1-T \text { Trichloroethane } \\ 1,1,1-\text { Trichloroethane } \\ 1,1,2-\text { Trichloroethane } \\ 1,1,2-\text { Trichloroethane } \\ \text { Trichloroethylene } \\ \text { Trichloroethylene } \\ \text { Trichlorofluoromethane } \\ \text { Trichlorofluoromethane } \\ \text { 2,4,5-T } \\ \text { Vanadium, total recoverable } \\ \text { Xylenes } \\ \text { Xylenes } \\ \text { Gross alpha } \\ \text { Nonvolatile beta } \\ \text { Radium, total alpha-emitting } \\ \text { Total activity } \\ \text { Tritium } \\ \text { Uranium-233/234 } \\ \text { Uranium-235 } \\ \text { Uranium-238 }\end{array}$

\begin{tabular}{lll}
\multicolumn{1}{c}{ Result } & & DF \\
15 & 1 \\
$<5.0$ & 5 \\
$<5.0$ & 5 \\
$<5.0$ & 5 \\
$<5.0$ & 5 \\
208 & 5 \\
204 & 5 \\
$<5.0$ & 5 \\
$<5.0$ & 5 \\
$<0.00047$ & 1 \\
$<8.0$ & 1 \\
$<10$ & 5 \\
$<10$ & 5 \\
$2.2 \mathrm{E}+00$ & 1 \\
$2.8 \mathrm{E}+00$ & 1 \\
$1.0 \mathrm{E}+00$ & 1 \\
$2.2 \mathrm{E}+08$ & 100 \\
$1.6 \mathrm{E}+05$ & 1 \\
$7.7 \mathrm{E}-02$ & 1 \\
$<0.0 \mathrm{E}+00$ & 1 \\
$1.6 \mathrm{E}-02$ & 1
\end{tabular}

\begin{tabular}{|c|c|c|}
\hline Mod & Unit & Flag \\
\hline$J$ & $\begin{array}{l}\mu \mathrm{g} / \mathrm{L} \\
\mu \mathrm{g} / \mathrm{L}\end{array}$ & $\begin{array}{l}0 \\
0\end{array}$ \\
\hline Y & $\begin{array}{l}\mu g / L \\
\mu g / L\end{array}$ & $\begin{array}{l}0 \\
0\end{array}$ \\
\hline $\mathbf{Y}$ & $\begin{array}{l}\mu \mathrm{g} / \mathrm{L} \\
\mu \mathrm{g} / \mathrm{L}\end{array}$ & $\begin{array}{l}0 \\
2\end{array}$ \\
\hline$Y$ & $\begin{array}{l}\mu g / L \\
\mu g / L\end{array}$ & $\begin{array}{l}2 \\
0\end{array}$ \\
\hline Y & $\begin{array}{l}\mu g / L \\
\mu g / L \\
\mu g / L \\
\mu g / L\end{array}$ & $\begin{array}{l}0 \\
0 \\
0 \\
0\end{array}$ \\
\hline Y & $\mu \mathrm{g} / \mathrm{L}$ & 0 \\
\hline $\mathbf{J}$ & $p \bar{C} i / L$ & 0 \\
\hline $\begin{array}{l}\mathbf{J} \\
\mathbf{J}\end{array}$ & $\begin{array}{l}\mathrm{pCi} / \mathrm{L} \\
\mathrm{DCi} / \mathrm{L}\end{array}$ & $\begin{array}{l}0 \\
0\end{array}$ \\
\hline & $\begin{array}{l}\mathrm{pCi} / \mathrm{L} \\
\mathrm{pCi} / \mathrm{mL}\end{array}$ & $\begin{array}{l}0 \\
2\end{array}$ \\
\hline Ul & $\begin{array}{l}\mathrm{pCi} / \mathrm{L} \\
\mathrm{pCi} / \mathrm{L}\end{array}$ & $\begin{array}{l}0 \\
0\end{array}$ \\
\hline UI & $\mathrm{pCi} / \mathrm{L}$ & 0 \\
\hline
\end{tabular}

\section{WELL BGO 29A}

\begin{tabular}{|c|c|c|c|c|c|c|}
\hline SRS Coord. & Lat/Longitude & Screen Zone Elevation & Top of Casing & Casing & Pump & Formation \\
\hline $\begin{array}{l}\text { N75560.0 } \\
\text { E54103.5 }\end{array}$ & $\begin{array}{l}33.281166^{\circ} \mathrm{N} \\
81.671108^{\circ} \mathrm{W}\end{array}$ & $112.5-102.5 \mathrm{ft} \mathrm{msl}$ & $264.2 \mathrm{ft} \mathrm{msl}$ & 4" PVC & $\mathbf{S}$ & U. Congaree (IIA) \\
\hline
\end{tabular}

\section{FIELD MEASUREMENTS}

Sample date: 04/12/94

Depth to water: $104.47 \mathrm{ft}(31.84 \mathrm{~m})$ below TOC

Water elevation: $159.73 \mathrm{ft}(48.69 \mathrm{~m}) \mathrm{msl}$

Sp. conductance: $117 \mu \mathrm{S} / \mathrm{cm}$

Turbidity: 14.9 NTU

Water evacuated before sampling: $38 \mathrm{gal}$

The well went dry during purging.

\section{LABORATORY ANALYSES}

\section{H D Analyte}

pH

Specific conductance

Turbidity

Acetophenone

Aldrin

Aluminum, total recoverable

Antimony, total recoverable

Antimony, total recoverable

Arsenic, total recoverable

Arsenic, total recoverable

Barium, total recoverable

Benzene

Bromodichloromethane
Time: 9:05

pH: 7.5

Alkalinity: $37 \mathrm{mg} / \mathrm{L}$

Water temperature: $19.0^{\circ} \mathrm{C}$

Volumes purged: 1.0 well volumes

\begin{tabular}{|c|c|c|c|c|}
\hline Result & DF & Mod & Unit & Flag \\
\hline $\begin{aligned} & 7.9 \\
& 115 \\
& 20 \\
&<10 \\
&<0.052 \\
& 643 \\
&<2.0 \\
&<2.0 \\
&<2.0 \\
&<2.0 \\
& 21 \\
&<1.0 \\
&<1.0\end{aligned}$ & $\begin{array}{l}1 \\
1 \\
1 \\
1 \\
1 \\
1 \\
1 \\
1 \\
1 \\
1 \\
1 \\
1 \\
1\end{array}$ & J1 & $\begin{array}{l}\mathrm{pH} \\
\mu \mathrm{S} / \mathrm{cm} \\
\mathrm{NTU} \\
\mu \mathrm{g} / \mathrm{L} \\
\mu \mathrm{g} / \mathrm{L} \\
\mu \mathrm{g} / \mathrm{L} \\
\mu \mathrm{g} / \mathrm{L} \\
\mu \mathrm{g} / \mathrm{L} \\
\mu \mathrm{g} / \mathrm{L} \\
\mu \mathrm{g} / \mathrm{L} \\
\mu \mathrm{g} / \mathrm{L} \\
\mu \mathrm{g} / \mathrm{L} \\
\mu \mathrm{g} / \mathrm{L}\end{array}$ & $\begin{array}{l}0 \\
0 \\
0 \\
0 \\
0 \\
2 \\
0 \\
0 \\
0 \\
0 \\
0 \\
0 \\
0\end{array}$ \\
\hline
\end{tabular}

- = exceeded holding time. = = exceeded screening level or final primary drinking water standard. 
WELL BGO 29A collected on 04/12/94, laboratory analyses (cont.)

H D Analyte

Bromoform

Bromomethane (Methyl bromide)

Cadmium, total recoverable

Calcium, total recoverable

Carbon tetrachloride

Chloride

Chlorobenzene

Chloroethane

Chloroethene (Vinyl chloride)

2-Chloroethyl vinyl ether

Chloroform

Chloromethane (Methyl chloride)

Chromium, total recoverable

Copper, total recoverable

Cyanide

p,p'-DDT

Dibromochloromethane

1,1-Dichloroethane

1,2-Dichloroethane

1,1-Dichloroethylene

trans-1,2-Dichloroethylene

Dichloromethane (Methylene chloride)

2,4-Dichlorophenoxyacetic acid

1,2-Dichloropropane

cis-1,3-Dichloropropene

trans-1,3-Dichloropropene

Dieldrin

Endrin

Ethylbenzene

Fluoride

Heptachlor

Iron, total recoverable

Lead, total recoverable

Lead, total recoverable

Lindane

Magnesium, total recoverable

Manganese, total recoverable

Mercury, total recoverable

Methoxychlor

Naphthalene

Nickel, total recoverable

Nitrate-nitrite as nitrogen

Phenols

Potassium, total recoverable

Selenium, total recoverable

Selenium, total recoverable

Silica, total recoverable

Silver, total recoverable

Sodium, total recoverable

Sulfate

1,1,2,2-Tetrachloroethane

Tetrachloroethylene

Tin, total recoverable

Toluene

Total dissolved solids

Total organic carbon

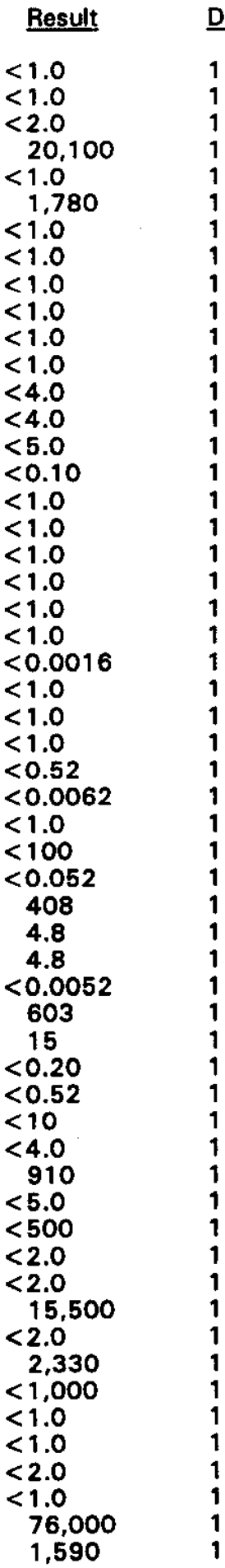

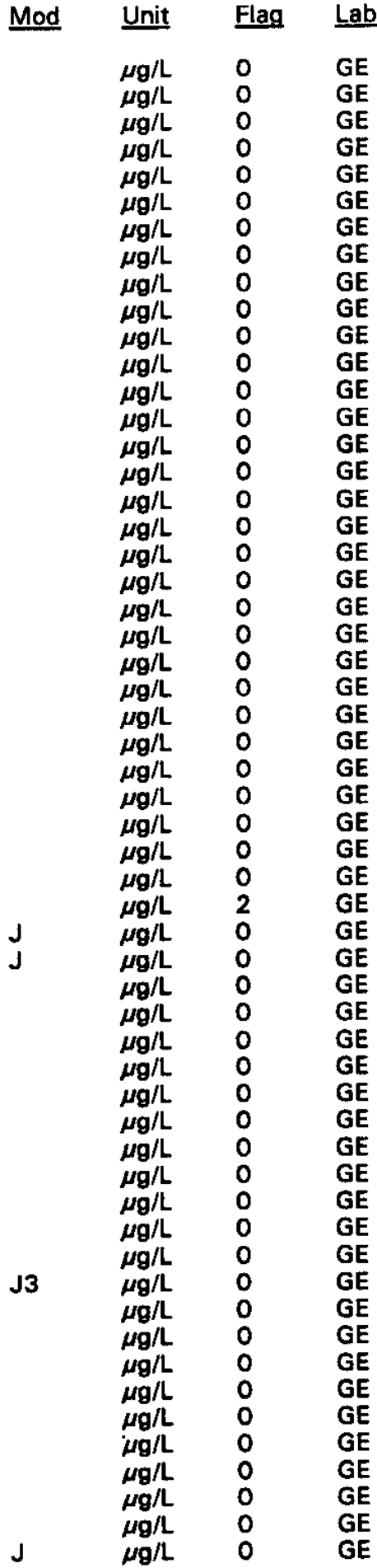

- = exceeded holding time. = exceeded screening level or final primary drinking water standard. 
WELL. BGO 29A collected on 04/12/94, laboratory analyses (cont.)

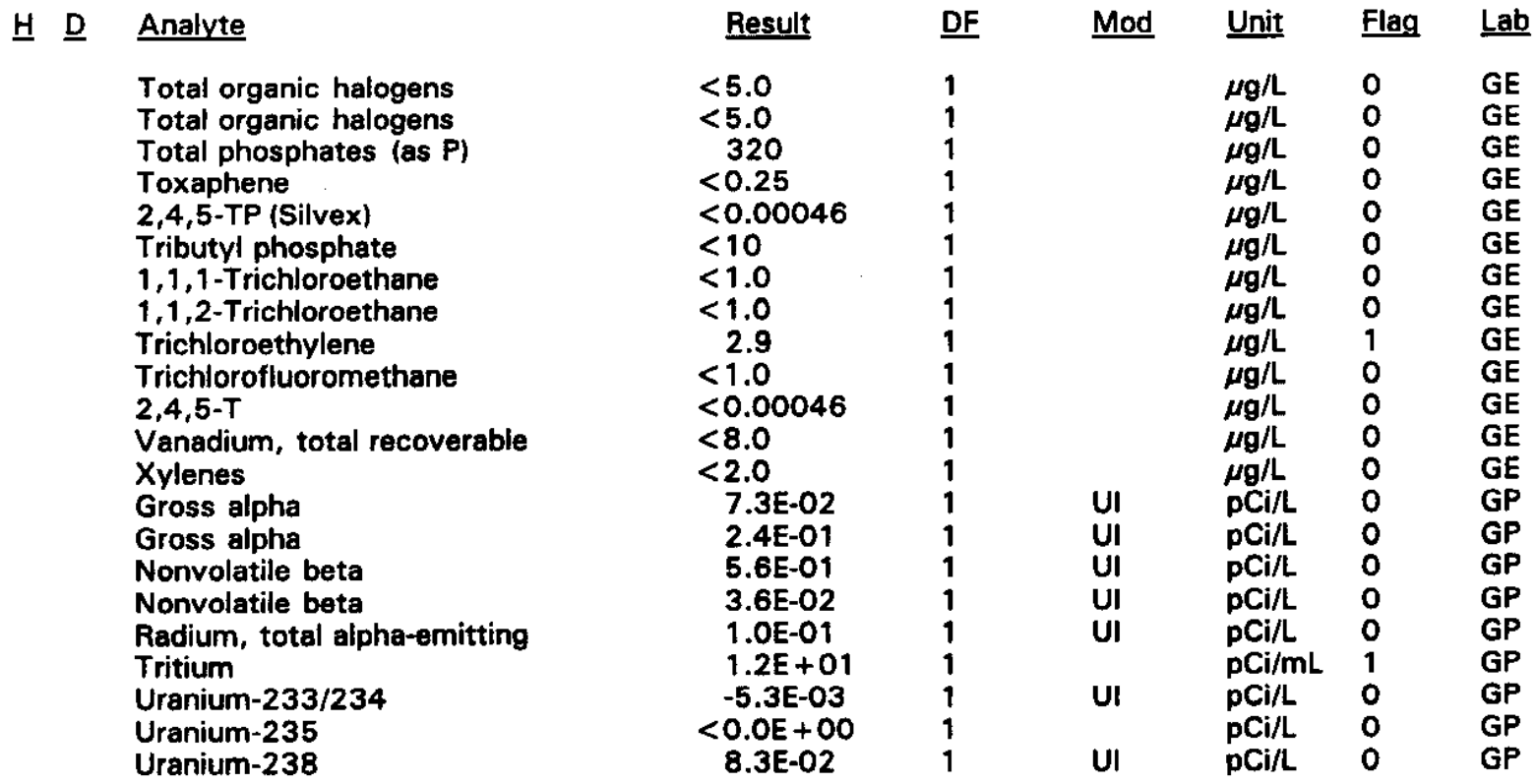

\section{WELL BGO 29C}

$\begin{array}{lllllll}\text { SRS Coord. } & \text { Lat/Longitude } & \text { Screen Zone Elevation } & \text { Top of Casing } & \text { Casing } & \text { Pump } & \text { Formation } \\ \text { N75577.8 } & 33.281198^{\circ} \mathrm{N} & 186.8-176.8 \mathrm{ft} \mathrm{msl} & 264.8 \mathrm{ft} \mathrm{msl} & 4^{\prime \prime} \text { PVC } & \text { S } & \text { Barnwell (IIB,) }\end{array}$

E54099.1 $81.671154^{\circ} \mathrm{W}$

\section{FIELD MEASUREMENTS}

Sample date: $04 / 12 / 94$

Depth to water: $41.59 \mathrm{ft}(12.68 \mathrm{~m})$ below TOC

Water elevation: $223.21 \mathrm{ft}(68.04 \mathrm{~m}) \mathrm{msl}$

Sp. conductance: $50 \mu \mathrm{S} / \mathrm{cm}$

Turbidity: $1.3 \mathrm{NTU}$

Water evacuated before sampling: 26 gal

The well went dry during purging.

\section{LABORATORY ANALYSES}

H $\underline{\text { Analivte }}$

pH

Specific conductance

Turbidity

Acetophenone

Aldrin

Aluminum, total recoverable

Antimony, total recoverable

Arsenic, total recoverable

Barium, total recoverable

Benzene

Bromodichloromethane

Bromoform
Time: $8: 47$

pH: 5.4

Alkalinity: $1 \mathrm{mg} / \mathrm{L}$

Water temperature: $18.9^{\circ} \mathrm{C}$

Volumes purged: 0.9 well volumes

- = exceeded holding time. $=$ exceeded screening level or final primary drinking water standard. 
WELL BGO $29 \mathrm{C}$ collected on $04 / 12 / 94$, laboratory analyses (cont.)

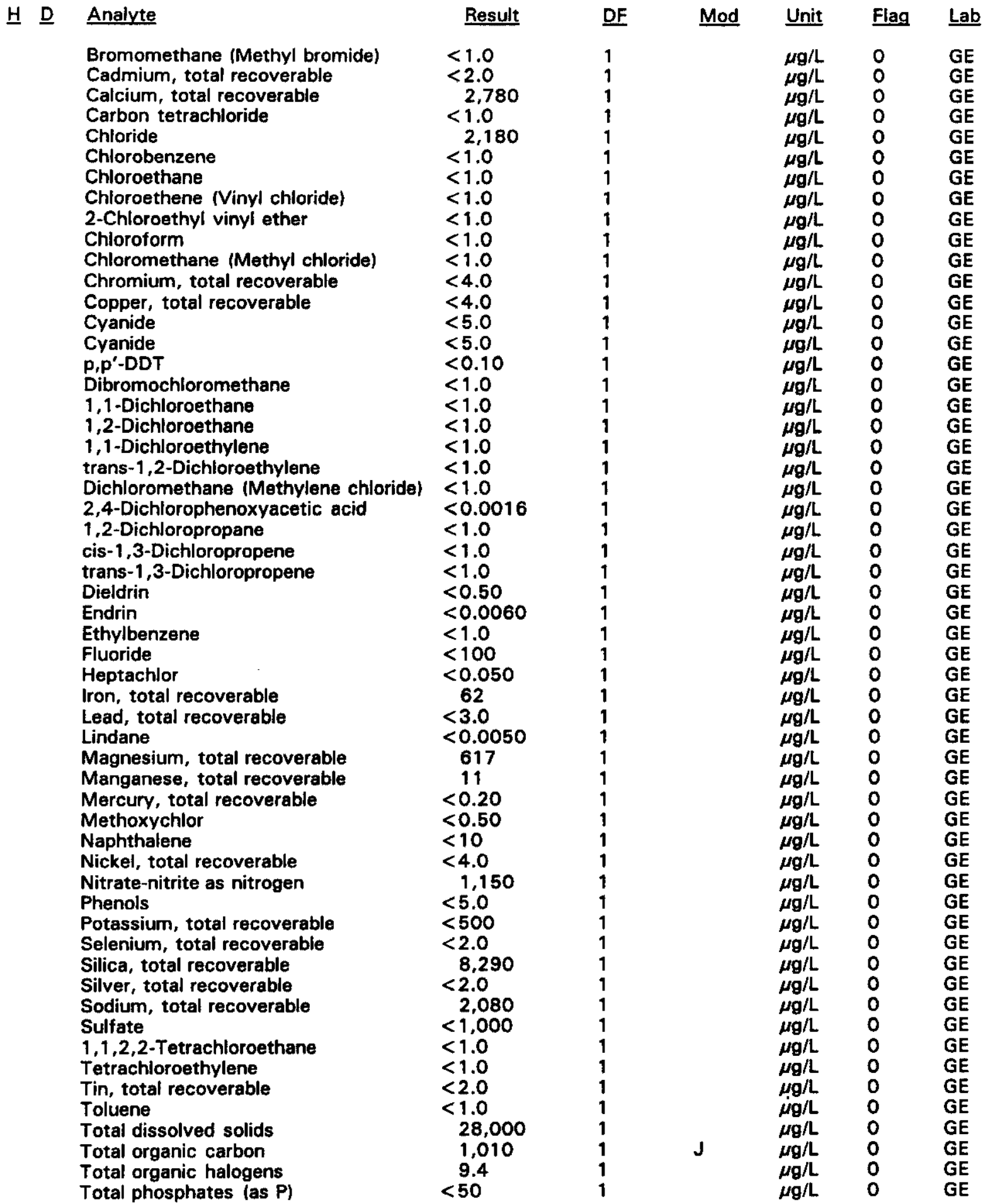

- = exceeded holding time. - =xceeded screening level or final primary drinking water standard. 
WELL BGO $29 \mathrm{C}$ collected on $04 / 12 / 94$, laboratory analyses (cont.)

$\underline{H} \underline{D}$

Analyte
Toxaphene
$2,4,5-T P$ (Silvex)
Tributyl phosphate
$1,1,1-T$ richloroethane
$1,1,2-T$ richloroethane
Trichloroethylene
Trichlorofluoromethane
$2,4,5-T$
Vanadium, total recoverable
Xylenes
Gross alpha
Nonvolatile beta
Radium, total alpha-emitting
Tritium
Uranium-233/234
Uranium-235
Uranium-238

\begin{tabular}{|c|}
\hline Result \\
\hline $\begin{aligned} &< 0.24 \\
&< 0.00046 \\
&< 10 \\
&< 1.0 \\
&< 1.0 \\
& 1.2 \\
&< 1.0 \\
&< 0.00046 \\
&< 8.0 \\
&< 2.0 \\
& 1.0 E+00 \\
& 1.8 E+00 \\
& 4.0 E-01 \\
& 1.1 E+01 \\
& 5.7 E-02 \\
&< 0.0 E+00 \\
&<0.0 E+00\end{aligned}$ \\
\hline
\end{tabular}

$\begin{array}{llll}\text { Mod } & \text { Unit } & \text { Flag } & \text { Lab } \\ & & & \\ & \mu g / L & 0 & \text { GE } \\ & \mu g / L & 0 & \text { GE } \\ & \mu g / L & 0 & \text { GE } \\ & \mu g / L & 0 & \text { GE } \\ & \mu g / L & 0 & \text { GE } \\ & \mu g / L & 0 & \text { GE } \\ & \mu g / L & 0 & \text { GE } \\ & \mu g / L & 0 & \text { GE } \\ & \mu g / L & 0 & \text { GE } \\ & \mu g / L & 0 & \text { GE } \\ & \mathrm{pCi} / L & 0 & \text { GP } \\ & \mathrm{pCi} / L & 0 & \text { GP } \\ & \mathrm{pCi} / L & 0 & \text { GP } \\ J & \mathrm{pCi} / \mathrm{mL} & 1 & \text { GP } \\ \mathrm{J} & \mathrm{pCi} / \mathrm{L} & 0 & \mathrm{GP} \\ \mathrm{J} & \mathrm{pCi} / \mathrm{L} & 0 & \mathrm{GP} \\ & \mathrm{pCi} / \mathrm{L} & 0 & \mathrm{GP}\end{array}$

\section{WELL BGO 29D}

\begin{tabular}{|c|c|c|c|c|c|c|}
\hline SRS Coord. & Lat/Longitude & Screen Zone Elevation & Top of Casing & Casing & Pump & Formation \\
\hline $\begin{array}{l}\text { N75592.5 } \\
\text { E54099.4 }\end{array}$ & $\begin{array}{l}33.281231^{\circ} \mathrm{N} \\
81.671182{ }^{\circ} \mathrm{W}\end{array}$ & $228.5-208.5 \mathrm{ft} \mathrm{msl}$ & $265.5 \mathrm{ft} \mathrm{msl}$ & 4" PVC & $\mathbf{S}$ & able $\left(I I B_{2}\right)$ \\
\hline
\end{tabular}

\section{FIELD MEASUREMENTS}

Sample date: $04 / 12 / 94$

Depth to water: $38.88 \mathrm{ft}(11.85 \mathrm{~m})$ below TOC

Water elevation: $226.62 \mathrm{ft}(69.07 \mathrm{~m}) \mathrm{msl}$

Sp. conductance: $72 \mu \mathrm{S} / \mathrm{cm}$

Turbidity: 15.7 NTU

Water evacuated before sampling: $11 \mathrm{gal}$

The well went dry during purging.

\section{LABORATORY ANALYSES}

\section{브 Analvte}

pH

Specific conductance

Turbidity

Acetophenone

Aldrin

Aluminum, total recoverable

Antimony, total recoverable

Arsenic, total recoverable

Barium, total recoverable

Benzene

Bromodichloromethane

Bromoform

Bromomethane (Methyl bromide)

Cadmium, total recoverable

Calcium, total recoverable

Carbon tetrachloride

Chloride
Time: 8:31

pH: 4.9

Alkalinity: $0 \mathrm{mg} / \mathrm{L}$

Water temperature: $18.6^{\circ} \mathrm{C}$

Volumes purged: 0.9 well volumes

\begin{tabular}{|c|c|c|c|c|}
\hline Result & DF & Mod & Unit & Flag \\
\hline $\begin{aligned} & 5.3 \\
& 48 \\
& 35 \\
&<10 \\
&<0.050 \\
& 1.820 \\
&<2.0 \\
&<2.0 \\
& 35 \\
&<1.0 \\
&<1.0 \\
&<1.0 \\
&<1.0 \\
&<2.0 \\
& 3.570 \\
&<1.0 \\
& 2.310\end{aligned}$ & $\begin{array}{l}1 \\
1 \\
1 \\
1 \\
1 \\
1 \\
1 \\
1 \\
1 \\
1 \\
1 \\
1 \\
1 \\
1 \\
1 \\
1 \\
1\end{array}$ & J1 & $\begin{array}{l}\text { pH } \\
\mu S / c m \\
N T U \\
\mu g / L \\
\mu g / L \\
\mu g / L \\
\mu g / L \\
\mu g / L \\
\mu g / L \\
\mu g / L \\
\mu g / L \\
\mu g / L \\
\mu g / L \\
\mu g / L \\
\mu g / L \\
\mu g / L \\
\mu g / L\end{array}$ & $\begin{array}{l}0 \\
0 \\
0 \\
0 \\
0 \\
2 \\
0 \\
0 \\
0 \\
0 \\
0 \\
0 \\
0 \\
0 \\
0 \\
0 \\
0\end{array}$ \\
\hline
\end{tabular}

- = exceeded holding time. $\quad=$ exceeded screening level or final primary drinking water standard. 
WELL BGO 29D collected on 04/12/94, laboratory analyses (cont.)

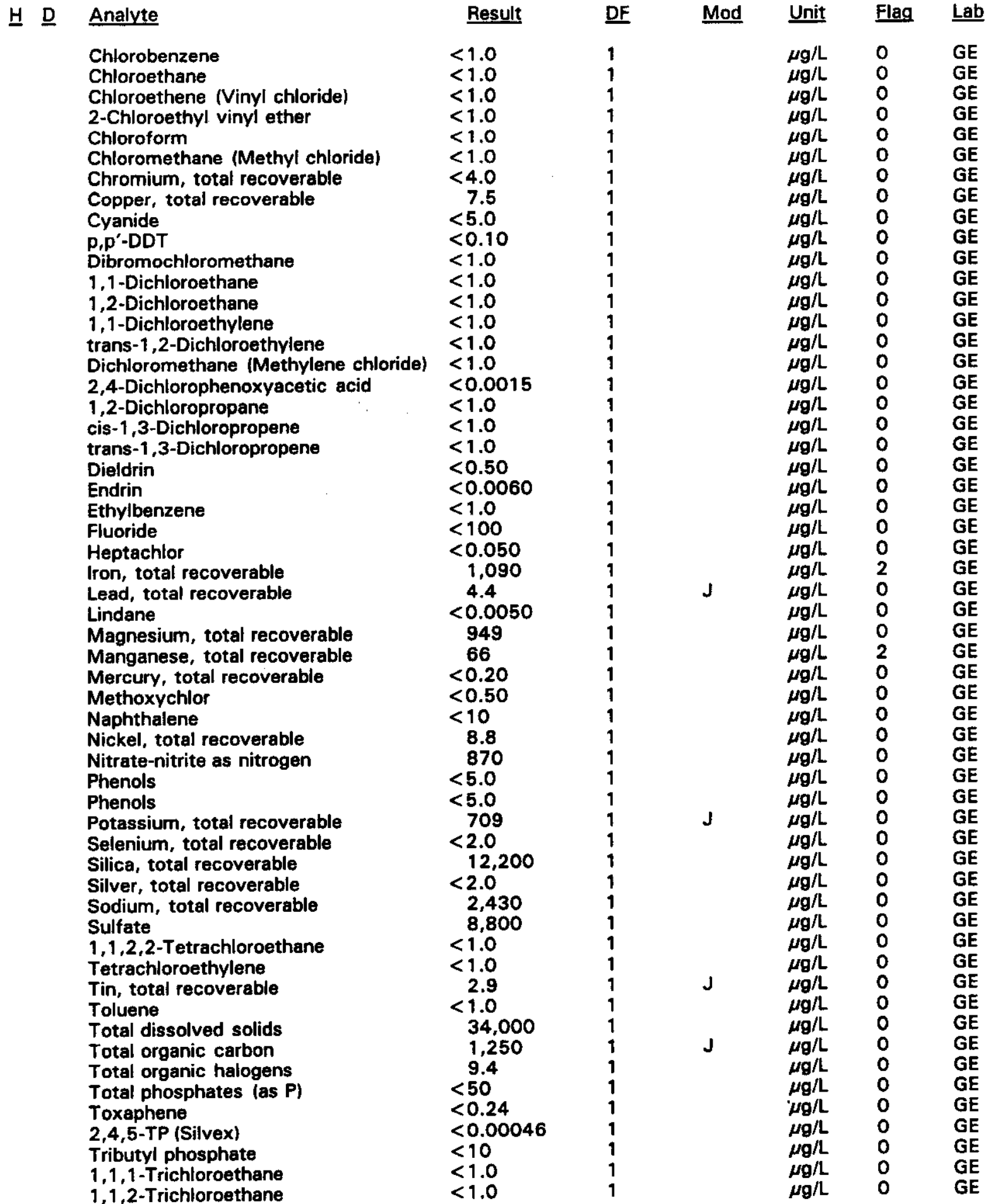

- = exceeded holding time. $n=$ exceeded screening level or final primary drinking water standard. 
WELL BGO 290 collected on 04/12/94, laboratory analyses (cont.)

\begin{tabular}{|c|c|c|c|c|c|}
\hline Analyte & Result & DF & Mod & Unit & Flag \\
\hline $\begin{array}{l}\text { Trichloroethylene } \\
\text { Trichlorofluoromethane } \\
2,4,5-T \\
\text { Vanadium, total recoverable } \\
\text { Xylenes } \\
\text { Gross alpha } \\
\text { Nonvolatile beta } \\
\text { Radium, total alpha-emitting } \\
\text { Tritium } \\
\text { Uranium-233/234 } \\
\text { Uranium-235 } \\
\text { Uranium-238 }\end{array}$ & $\begin{aligned} &< 1.0 \\
&<1.0< \\
&<0.00046<8.0 \\
&< 2.0 \\
& 3.1 E+00 \\
& 3.8 E+00 \\
& 2.6 E+00 \\
& 4.2 E+01 \\
&-5.8 E-03 \\
&< 0.0 E+00 \\
& 9.1 E-02\end{aligned}$ & $\begin{array}{l}1 \\
1 \\
1 \\
1 \\
1 \\
1 \\
1 \\
1 \\
1 \\
1 \\
1 \\
1\end{array}$ & $\begin{array}{l}\text { UI } \\
\text { UI }\end{array}$ & $\begin{array}{l}\mu \mathrm{g} / \mathrm{L} \\
\mu \mathrm{g} / \mathrm{L} \\
\mu \mathrm{g} / \mathrm{L} \\
\mu \mathrm{g} / \mathrm{L} \\
\mu \mathrm{g} / \mathrm{L} \\
\mathrm{pCi} / \mathrm{L} \\
\mathrm{pCi} / \mathrm{L} \\
\mathrm{pCi} / \mathrm{L} \\
\mathrm{pCi} / \mathrm{mL} \\
\mathrm{pCi} / \mathrm{L} \\
\mathrm{pCi} / \mathrm{L} \\
\mathrm{pCi} / \mathrm{L}\end{array}$ & $\begin{array}{l}0 \\
0 \\
0 \\
0 \\
0 \\
0 \\
0 \\
0 \\
2 \\
0 \\
0 \\
0\end{array}$ \\
\hline
\end{tabular}

WELL BGO 30C

$\begin{array}{lllllll}\text { SRS Coord. } & \text { Lat/Longitude } & \text { Screen Zone Elevation } & \text { Top of Casing } & \text { Casing } & \text { Pump } & \text { Formation } \\ \text { N75181.0 } & 33.280995^{\circ} \mathrm{N} & 188.4-178.4 \mathrm{ft} \mathrm{msl} & 274.5 \mathrm{ft} \mathrm{msl} & 4^{\text {" PVC }} & \text { S } & \text { McBean (IIB,) } \\ \text { E54512.3 } & 81.669296^{\circ} \mathrm{W} & & & \end{array}$

\section{FIELD MEASUREMENTS}

Sample date: 04/12/94

Depth to water: $54.81 \mathrm{ft}(16.71 \mathrm{~m})$ below TOC

Water elevation: $219.69 \mathrm{ft}(66.96 \mathrm{~m}) \mathrm{msl}$

Sp. conductance: $44 \mu \mathrm{S} / \mathrm{cm}$

Turbidity: 26.5 NTU

Water evacuated before sampling: 25 gal

The well went dry during purging.

\section{LABORATORY ANALYSES}

Time: $11: 00$

pH: 6.1

Alkalinity: $8 \mathrm{mg} / \mathrm{L}$

Water temperature: $21.2{ }^{\circ} \mathrm{C}$

Volumes purged: 0.9 well volumes

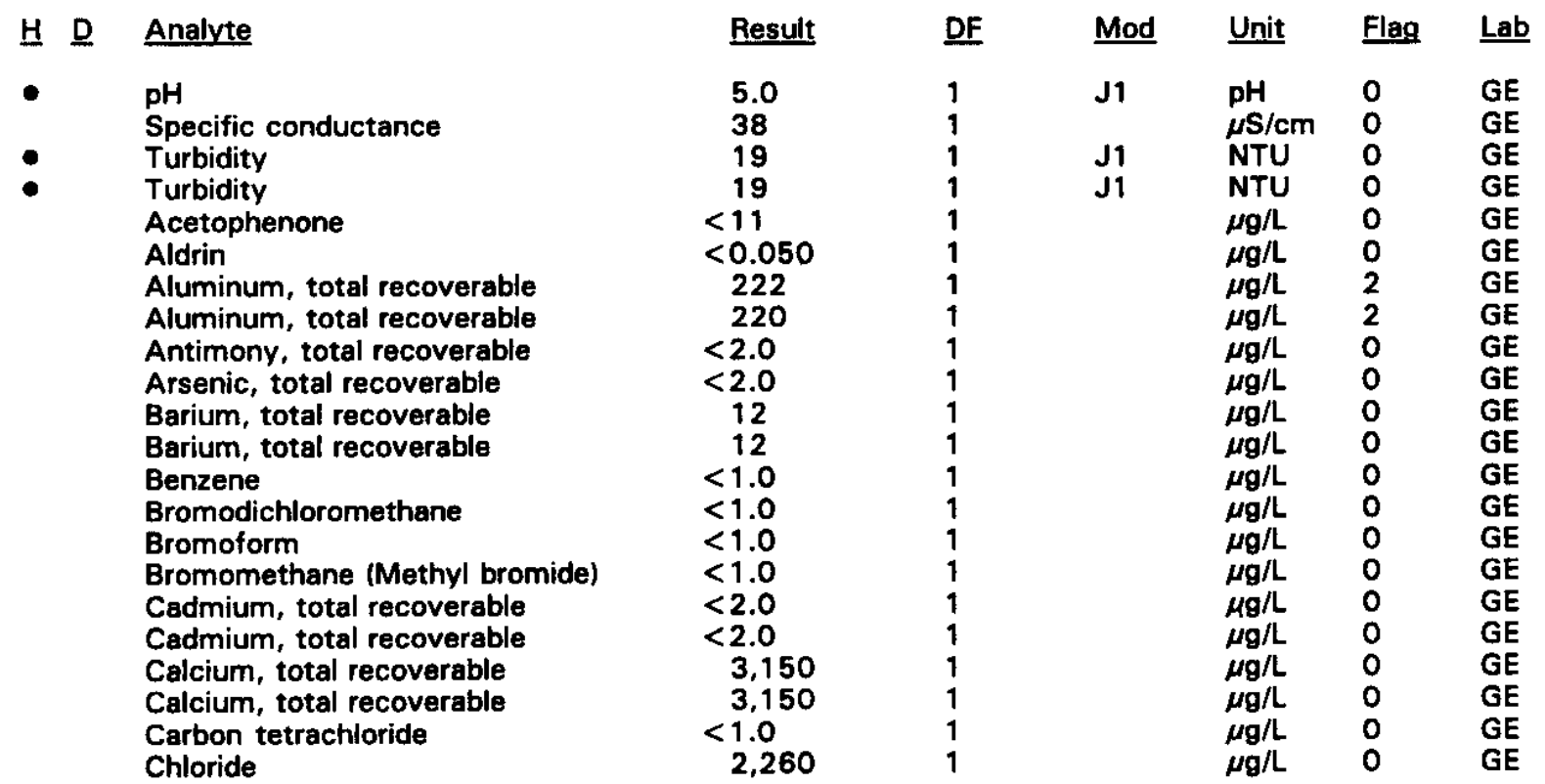

- = exceeded holding time. = exceeded screening level or final primary drinking water standard. 
WELL. BGO $30 \mathrm{C}$ collected on $04 / 12 / 94$, laboratory analyses (cont.)

H $\underline{\text { Analyte }}$

Chlorobenzene

Chloroethane

Chloroethene (Vinyl chloride)

2-Chloroethyl vinyl ether

Chloroform

Chloromethane (Methyl chloride)

Chromium, total recoverable

Chromium, total recoverable

Copper, total recoverable

Copper, total recoverable

Cyanide

p,p'-DDT

Dibromochloromethane

1,1-Dichloroethane

1,2-Dichloroethane

1,1-Dichloroethylene

trans-1,2-Dichloroethylene

Dichloromethane (Methylene chloride)

2,4-Dichlorophenoxyacetic acid

1,2-Dichloropropane

cis-1,3-Dichloropropene

trans-1,3-Dichloropropene

Dieldrin

Endrin

Ethylbenzene

Fluoride

Fluoride

Heptachlor

Iron, total recoverable

iron, total recoverable

Lead, total recoverable

Lindane

Magnesium, total recoverable

Magnesium, total recoverable

Manganese, total recoverable

Manganese, total recoverable

Mercury, total recoverable

Mercury, total recoverable

Methoxychlor

Naphthalene

Nickel, total recoverable

Nickel, total recoverable

Nitrate-nitrite as nitrogen

Phenols

Potassium, total recoverable

Potassium, total recoverable

Selenium, total recoverable

Silica, total recoverable

Silica, total recoverable

Silver, total recoverable

Silver, total recoverable

Sodium, total recoverable

Sodium, total recoverable

Sulfate

1,1,2,2-Tetrachloroethane

Tetrachloroethylene

\begin{tabular}{|c|c|c|c|c|c|}
\hline Result & DF & Mod & Unit & Flag & Lat \\
\hline$<1.0$ & 1 & & $\mu \mathrm{g} / \mathrm{L}$ & 0 & $\mathrm{GE}$ \\
\hline$<1.0$ & 1 & & $\mu g / L$ & 0 & $\mathrm{GE}$ \\
\hline $\begin{array}{l}<1.0 \\
<1.0\end{array}$ & 1 & & $\mu \mathrm{g} / \mathrm{L}$ & 0 & GE \\
\hline $\begin{array}{l}<1.0 \\
<1.0\end{array}$ & 1 & & $\mu g / L$ & 0 & GE \\
\hline $\begin{array}{l}<1.0 \\
<1.0\end{array}$ & 1 & & $\mu g / L$ & 0 & $\mathrm{GE}$ \\
\hline $\begin{array}{l}<1.0 \\
<4.0\end{array}$ & 1 & & $\mu g / L$ & 0 & GE \\
\hline $\begin{array}{l}<4.0 \\
<4.0\end{array}$ & 1 & & $\mu g / L$ & 0 & GE \\
\hline $\begin{array}{r}<4.0 \\
4.0\end{array}$ & 1 & & $\mu g / \mathrm{L}$ & 0 & $\mathrm{GE}$ \\
\hline $\begin{array}{r}4.0 \\
<4.0\end{array}$ & 1 & $J$ & $\mu \mathrm{g} / \mathrm{L}$ & 0 & GE \\
\hline $\begin{array}{l}<4.0 \\
<5.0\end{array}$ & 1 & & $\mu g / L$ & 0 & GE \\
\hline $\begin{array}{l}<5.0 \\
<0.10\end{array}$ & 1 & & $\mu g / L$ & 0 & GE \\
\hline $\begin{array}{l}<0.10 \\
<1.0\end{array}$ & 1 & & $\mu \mathrm{g} / \mathrm{L}$ & 0 & GE \\
\hline$<1.0$ & 1 & & $\mu g / L$ & 0 & GE \\
\hline$<1.0$ & 1 & & $\mu g / L$ & 0 & GE \\
\hline$<1.0$ & 1 & & $\mu g / L$ & 0 & $G E$ \\
\hline$<1.0$ & 1 & & $\mu \mathrm{g} / \mathrm{L}$ & 0 & $\mathrm{GE}$ \\
\hline$<1.0$ & 1 & & $\mu g / L$ & 0 & GE \\
\hline$<1.0$ & 1 & & $\mu \mathrm{g} / \mathrm{L}$ & 0 & GE \\
\hline$<0.0015$ & 1 & & $\mu g / L$ & 0 & GE \\
\hline $\begin{array}{l}<1.0 \\
<1.0\end{array}$ & $\begin{array}{l}1 \\
1\end{array}$ & & $\mu g / L$ & 0 & GE \\
\hline$<1.0$ & $i$ & & $\underset{\mu \mathrm{g} / \mathrm{L}}{\mu \mathrm{g} / \mathrm{L}}$ & $\begin{array}{l}0 \\
0\end{array}$ & $\begin{array}{l}\mathrm{GE} \\
\mathrm{GE}\end{array}$ \\
\hline$<0.50$ & 1 & & $\mu g / \mathrm{L}$ & 0 & $\mathrm{GE}$ \\
\hline$<0.0060$ & 1 & & $\mu \mathrm{g} / \mathrm{L}$ & 0 & GE \\
\hline $\begin{array}{l}<1.0 \\
<100\end{array}$ & 1 & & $\mu \mathrm{g} / \mathrm{L}$ & 0 & \\
\hline $\begin{array}{l}<100 \\
<100\end{array}$ & $\begin{array}{l}1 \\
1\end{array}$ & & $\begin{array}{l}\mu g / L \\
\mu g / L\end{array}$ & $\begin{array}{l}0 \\
0\end{array}$ & GE \\
\hline$<0.050$ & 1 & & $\mu g / L$ & 0 & $\begin{array}{l}\text { GE } \\
\text { GE }\end{array}$ \\
\hline 171 & 1 & & $\mu g / L$ & 1 & GE \\
\hline 171 & 1 & & $\mu g / L$ & 1 & $\mathrm{EE}$ \\
\hline 4.1 & 1 & $J$ & $\mu g / L$ & 0 & GE \\
\hline$<0.0050$ & 1 & & $\mu g / L$ & 0 & GE \\
\hline 260 & 1 & & $\mu \mathrm{g} / \mathrm{L}$ & 0 & GE \\
\hline 259 & 1 & & $\mu g / L$ & 0 & GE \\
\hline 18 & 1 & & $\mu g / L$ & 0 & GE \\
\hline 18 & 1 & & $\mu g / L$ & 0 & GE \\
\hline $\begin{array}{l}<0.20 \\
<0.20\end{array}$ & 1 & & $\mu \mathrm{g} / \mathrm{L}$ & 0 & GE \\
\hline $\begin{array}{l}<0.20 \\
<0.50\end{array}$ & 1 & & $\mu g / L$ & 0 & GE \\
\hline $\begin{array}{l}<0.50 \\
<11\end{array}$ & 1 & & $\mu g / L$ & 0 & GE \\
\hline $\begin{array}{l}<11 \\
<4.0\end{array}$ & 1 & & $\mu \mathrm{g} / \mathrm{L}$ & 0 & GE \\
\hline $\begin{array}{l}<4.0 \\
<4.0\end{array}$ & 1 & & $\mu g / L$ & 0 & GE \\
\hline $\begin{array}{r}<4.0 \\
430\end{array}$ & 1 & & $\mu g / L$ & 0 & GE \\
\hline $\begin{array}{r}430 \\
<5.0\end{array}$ & $\begin{array}{l}1 \\
1\end{array}$ & & $\mu g / L$ & 0 & $\mathbf{G}$ \\
\hline $\begin{array}{r}<5.0 \\
946\end{array}$ & $i$ & & $\begin{array}{l}\mu g / \mathrm{L} \\
\mu \mathrm{g} / \mathrm{L}\end{array}$ & $\begin{array}{l}0 \\
0\end{array}$ & $\begin{array}{l}\text { GE } \\
\text { GE }\end{array}$ \\
\hline $\begin{array}{r}949 \\
<20\end{array}$ & 1 & & $\mu \mathrm{g} / \mathrm{L}$ & 0 & $\mathrm{GE}$ \\
\hline $\begin{array}{l}<2.0 \\
9.280\end{array}$ & $\begin{array}{l}1 \\
1\end{array}$ & & $\mu g / L$ & 0 & GE \\
\hline $\begin{array}{l}9,280 \\
9,250\end{array}$ & 1 & & $\mu g / L$ & $\begin{array}{l}0 \\
0\end{array}$ & $\begin{array}{l}\text { GE } \\
\text { GE }\end{array}$ \\
\hline $\begin{array}{l}\mathbf{9 , 2 5 0} \\
<2.0\end{array}$ & 1 & & $\begin{array}{l}\mu g / L \\
\mu g / L\end{array}$ & 0 & GE \\
\hline$<2.0$ & 1 & & $\mu \mathrm{g} / \mathrm{L}$ & 0 & \\
\hline 3,110 & 1 & & $\mu g / L$ & 0 & GE \\
\hline 3,090 & 1 & & $\mu g / L$ & 0 & GE \\
\hline$<1,000$ & 1 & & $\mu g / L$ & 0 & \\
\hline$<1.0$ & 1 & & $\mu \mathrm{g} / \mathrm{L}$ & 0 & $\mathrm{GE}$ \\
\hline$<1.0$ & 1 & & $\mu \mathrm{g} / \mathrm{L}$ & 0 & \\
\hline
\end{tabular}

- = exceeded holding time. $a=$ exceeded screening level or final primary drinking water standard. 
WELL BGO 3OC collected on 04/12/94, laboratory analyses (cont.)

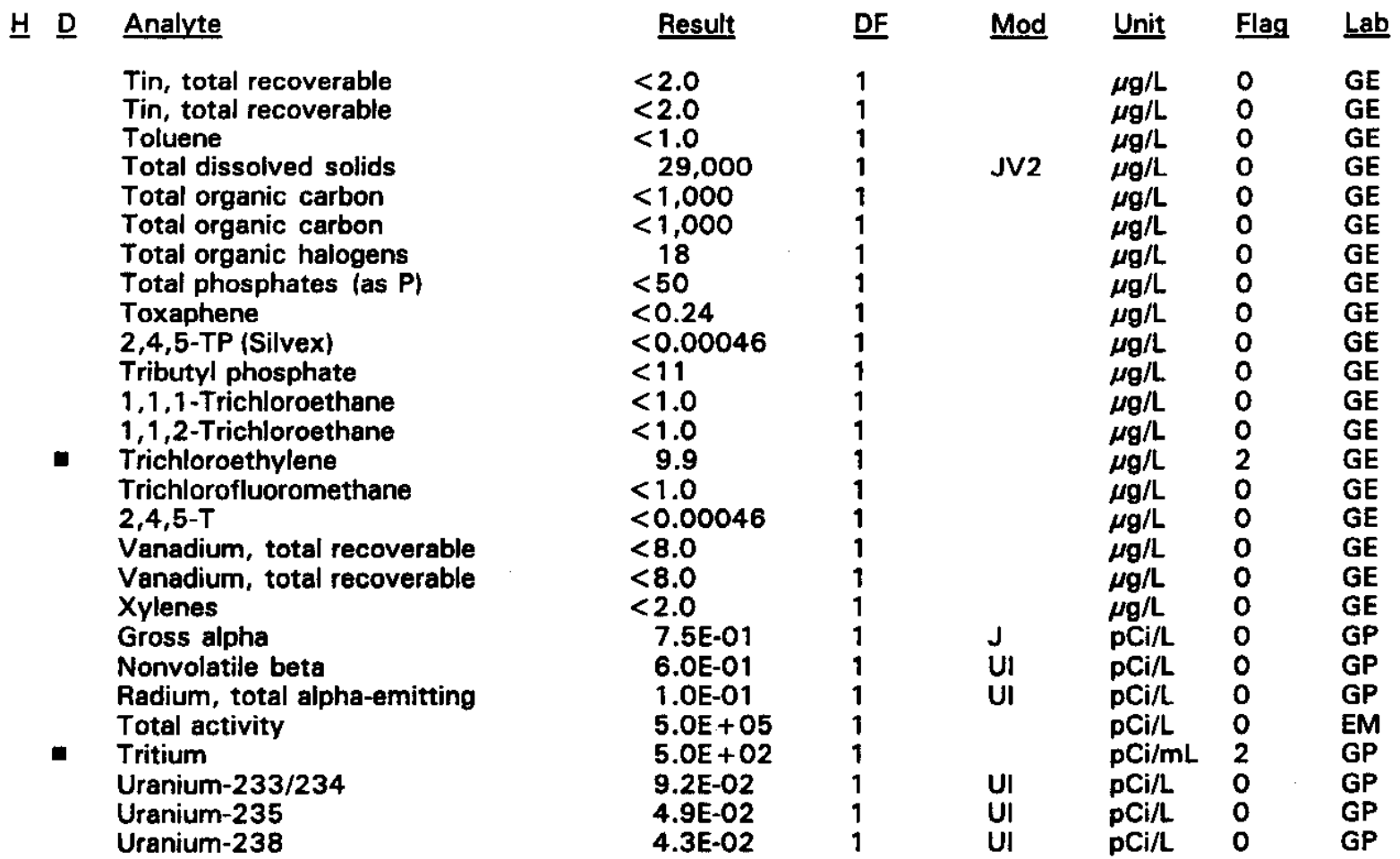

\section{WELL BGO 30D}

\begin{tabular}{|c|c|c|c|c|c|c|}
\hline SRS Coord. & Lat/Longitude & Screen Zone Elevation & Top of Casing & $\underline{\text { Casing }}$ & Pump & Formation \\
\hline $\begin{array}{l}\text { N75187.7 } \\
\text { E54499.2 }\end{array}$ & $\begin{array}{l}33.280988^{\circ} \mathrm{N} \\
81.669343^{\circ} \mathrm{W}\end{array}$ & $227.8-207.8 \mathrm{ft} \mathrm{msl}$ & $274.8 \mathrm{ft} \mathrm{msl}$ & 4" PVC & $\mathbf{S}$ & Water Table $\left(\mathrm{IIB}_{2}\right)$ \\
\hline
\end{tabular}

\section{FIELD MEASUREMENTS}

Sample date: 04/12/94

Depth to water: $48.57 \mathrm{ft}(14.80 \mathrm{~m})$ below TOC

Water elevation: $226.23 \mathrm{ft}(68.96 \mathrm{~m}) \mathrm{ms}$ !

Sp. conductance: $156 \mu \mathrm{S} / \mathrm{cm}$

Turbidity: 7.9 NTU

Water evacuated before sampling: $14 \mathrm{gal}$

The well went dry during purging.

\section{LABORATORY ANALYSES}

H D Analyte

- $\quad \mathrm{pH}$

Specific conducte
Turbidity
Acetophenone

Acetophe
Aldrin

Aluminum, total recoverable

Antimony, total recoverable

$\begin{array}{lllllll}\text { Result } & \text { DF } & \text { Mod } & & \text { Unit } & \text { Flag } & \text { Lab } \\ 5.9 & & & & & & \\ 133 & 1 & J 1 & \text { pH } & 0 & \text { GE } \\ 2.6 & 1 & & \mu S / c m & 0 & \text { GE } \\ <10 & 1 & J 1 & \text { NTU } & 0 & \text { GE } \\ <0.052 & 1 & 1 & & \mu g / L & 0 & \text { GE } \\ 249 & 1 & & \mu g / L & 0 & \text { GE } \\ <2.0 & 1 & & \mu g / L & 2 & \text { GE } \\ & & & \mu g / L & 0 & \text { GE }\end{array}$

Time: $11: 16$

pH: 5.7

Alkalinity: $17 \mathrm{mg} / \mathrm{L}$

Water temperature: $21.8^{\circ} \mathrm{C}$

Volumes purged: 1.2 well volumes

- = exceeded holding time. = exceeded screening level or final primary drinking water standard. 
WELL BGO $30 D$ collected on $04 / 12 / 94$, laboratory analyses (cont.)

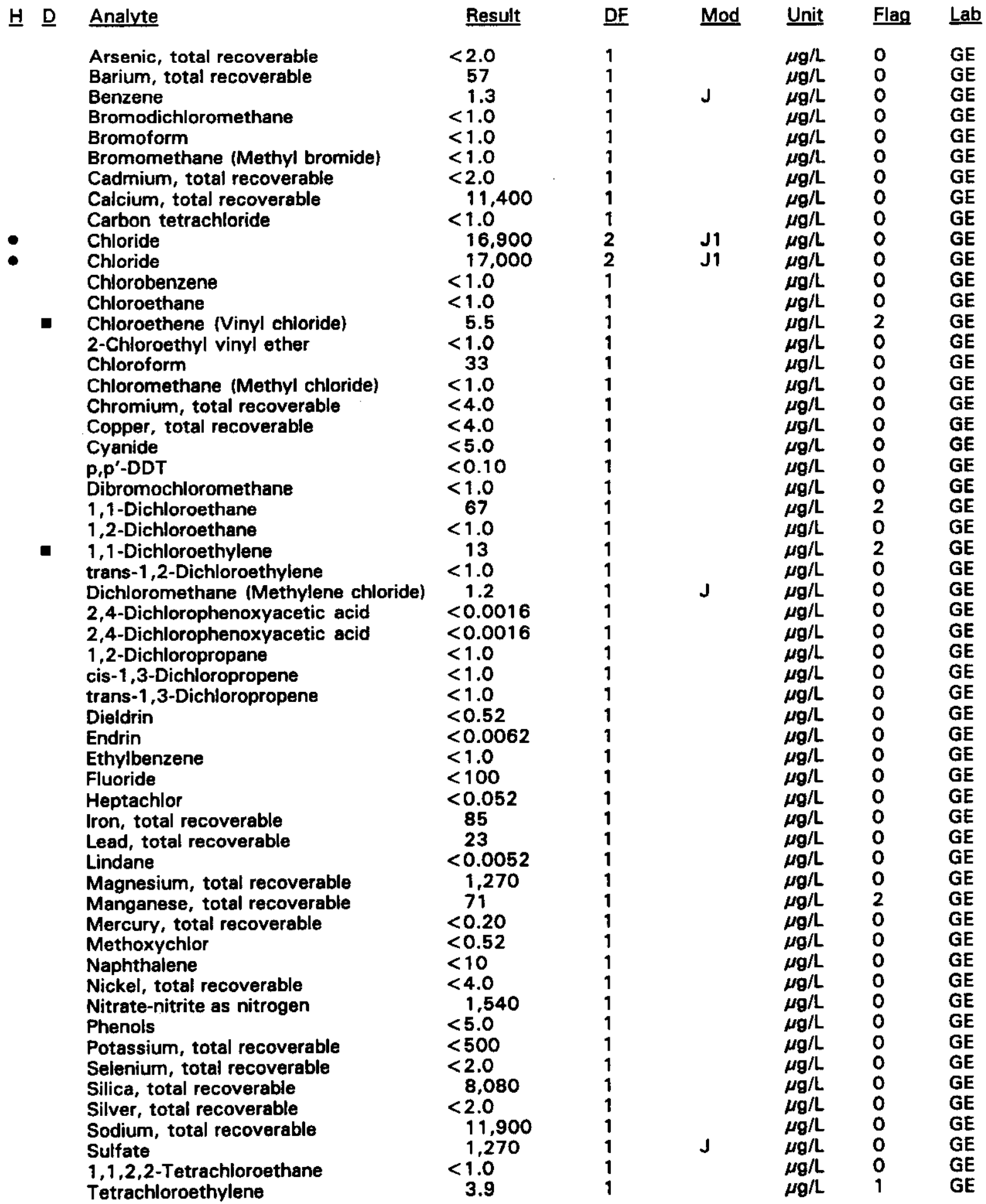

- =xceeded holding time. = exceeded screening level or final primary drinking water standard. 
WELL BGO 3OD collected on 04/12/94, laboratory analyses (cont.)

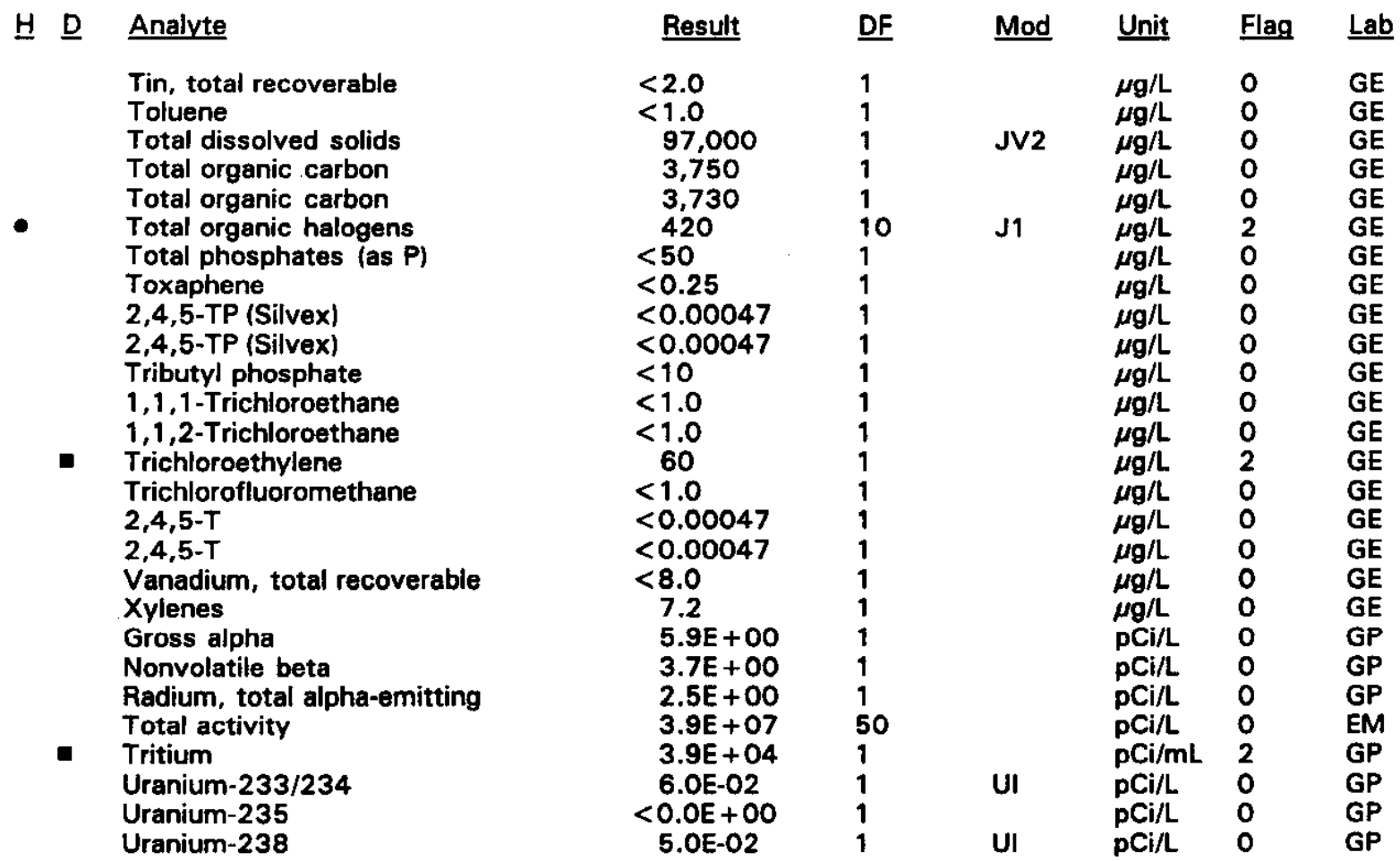

\section{WELL BGO 31C}

\begin{tabular}{|c|c|c|c|c|c|c|}
\hline SRS Coord. & Lat/Longitude & Screen Zone Elevation & Top of Casing & Casing & Pump & Formation \\
\hline $\begin{array}{l}\text { N74978.0 } \\
\text { E54816.2 }\end{array}$ & $\begin{array}{l}33.281042^{\circ} \mathrm{N} \\
81.668101^{\circ} \mathrm{W}\end{array}$ & $186.4-176.4 \mathrm{ft} \mathrm{msl}$ & $273.1 \mathrm{ft} \mathrm{msl}$ & 4" PVC & $\mathbf{s}$ & McBean $\left(I \mid B_{1}\right)$ \\
\hline
\end{tabular}

\section{FIELD MEASUREMENTS}

Sample date: $04 / 12 / 94$

Depth to water: $47.10 \mathrm{ft}(14.36 \mathrm{~m})$ below TOC

Water elevation: $226.00 \mathrm{ft}(68.89 \mathrm{~m}) \mathrm{ms}$ l

Sp. conductance: $31 \mu \mathrm{S} / \mathrm{cm}$

Turbidity: 4.1 NTU

Water evacuated before sampling: 27 gal

The well went dry during purging.

\section{LABORATORY ANALYSES}

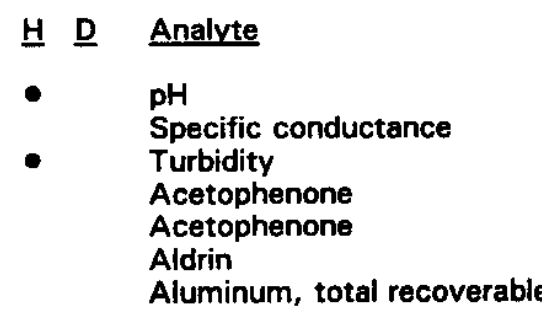

Time: $11: 44$

pH: 5.1

Alkalinity: $0 \mathrm{mg} / \mathrm{L}$

Water temperature: $22.0^{\circ} \mathrm{C}$

Volumes purged: 0.8 well volumes

= exceeded holding time. = exceeded screening level or final primary drinking water standard. 
WELL BGO $31 \mathrm{C}$ collected on 04/12/94, laboratory analyses (cont.)

H D Analyte

Antimony, total recoverable

Arsenic, total recoverable

Barium, total recoverable

Benzene

Bromodichloromethane

Bromoform

Bromomethane (Methyl bromide)

Cadmium, total recoverable

Calcium, total recoverable

Carbon tetrachloride

Chloride

Chlorobenzene

Chloroethane

Chloroethene (Vinyl chloride)

2-Chloroethyl vinyl ether

Chloroform

Chloromethane (Methyl chloride)

Chromium, total recoverable

Copper, total recoverable

Cyanide

p,p'-DDT

Dibromochloromethane

1,1-Dichloroethane

1,2-Dichloroethane

1,1-Dichloroethylene

trans-1,2-Dichloroethylene

Dichloromethane (Methylene chloride)

2,4-Dichlorophenoxyacetic acid

1,2-Dichloropropane

cis-1,3-Dichloropropene

trans-1,3-Dichloropropene

Dieldrin

Endrin

Ethylbenzene

Fluoride

Heptachlor

Iron, total recoverable

Lead, total recoverable

Lindane

Magnesium, total recoverable

Manganese, total recoverable

Mercury, total recoverable

Methoxychlor

Naphthalene

Naphthalene

Nickel, total recoverable

Nitrate-nitrite as nitrogen

Phenols

Potassium, total recoverable

Selenium, total recoverable

Silica, total recoverable

Silver, total recoverable

Sodium, total recoverable

Sulfate

1,1,2,2-Tetrachloroethane

Tetrachloroethylene
Result DF

$<2.0$

$<2.0$

7.4

$<1.0$

$<1.0$

$<1.0$

$<1.0$

$<2.0$

1,530

$<1.0$

1,990

$<1.0$

$<1.0$

$<1.0$

$<1.0$

$<1.0$

$<1.0$

$<4.0$

4.6

$<5.0$

$<0.10$

$<1.0$

$<1.0$

$<1.0$

$<1.0$

$<1.0$

$<1.0$

$<0.0016$

$<1.0$

$<1.0$

$<1.0$

$<0.50$

$<0.0060$

$<1.0$

$<100$

$<0.050$

14

$<3.0$

$<0.0050$

511

8.2

$<0.20$

$<0.50$

$<11$

$<10$

$<4.0$

1,100

$<5.0$

$<500$

$<2.0$

8,320

$<2.0$

1,680

$<1,000$

$<1.0$

2.4
Mod Unit Flag Lab

$\mu g / L \quad 0 \quad$ GE

$\mu g / L \quad 0 \quad G E$

$\mu \mathrm{g} / \mathrm{L} \quad \mathrm{O} \quad \mathrm{GE}$

$\mu g / L \quad 0 \quad G E$

$\mu g / L \quad 0 \quad$ GE

$\mu \mathrm{g} / \mathrm{L} \quad 0 \quad \mathrm{GE}$

$\mu \mathrm{g} / \mathrm{L} \quad 0 \quad \mathrm{GE}$

$\mu \mathrm{g} / \mathrm{L} \quad \mathrm{O} \quad \mathrm{GE}$

$\mu g / L \quad 0 \quad$ GE

$\mu g / L \quad 0 \quad$ GE

$\mu g / L \quad 0 \quad$ GE

$\mu g / L \quad 0 \quad$ GE

$\mu \mathrm{g} / \mathrm{L} \quad 0 \quad \mathrm{GE}$

$\mu g / L \quad 0 \quad G E$

$\mu \mathrm{g} / \mathrm{L} \quad 0 \quad \mathrm{GE}$

$\mu g / L \quad 0 \quad G E$

$\mu g / L \quad 0 \quad$ GE

$\mu g / L \quad 0 \quad G E$

$\mu g / L \quad 0 \quad$ GE

$\mu g / L \quad 0 \quad G E$

$\mu g / L \quad 0 \quad$ GE

$\mu g / L \quad 0 \quad$ GE

$\mu \mathrm{g} / \mathrm{L} \quad 0 \quad \mathrm{GE}$

$\mu \mathrm{g} / \mathrm{L} \quad \mathrm{O} \quad \mathrm{GE}$

$\mu \mathrm{g} / \mathrm{L} \quad 0 \quad \mathrm{GE}$

$\mu g / L \quad 0 \quad$ GE

$\mu \mathrm{g} / \mathrm{L} \quad \mathrm{O} \quad \mathrm{GE}$

$\mu g / L \quad 0 \quad$ GE

$\mu g / L \quad 0 \quad G E$

$\mu g / L \quad 0 \quad$ GE

$\mu \mathrm{g} / \mathrm{L} \quad \mathrm{O} \quad \mathrm{GE}$

J3 $\mu g / L \quad 0 \quad$ GE

J3 $\mu \mathrm{g} / \mathrm{L} \quad 0 \quad \mathrm{GE}$

$\mu g / L \quad O \quad$ GE

$\mu g / L \quad 0 \quad G E$

J3 $\mu \mathrm{g} / \mathrm{L} \quad 0 \quad \mathrm{GE}$

$\mu \mathrm{g} / \mathrm{L} \quad 0 \quad \mathrm{GE}$

$\mu g / L \quad 0 \quad$ GE

$\mu \mathrm{g} / \mathrm{L} \quad \mathrm{O} \quad \mathrm{GE}$

$\mu g / L \quad 0 \quad$ GE

$\mu g / L \quad 0 \quad G E$

$\mu g / L \quad 0 \quad G E$

$\mu g / L \quad 0 \quad G E$

$\mu \mathrm{g} / \mathrm{L} \quad \mathrm{O} \quad \mathrm{GE}$

$\mu \mathrm{g} / \mathrm{L} \quad 0 \quad \mathrm{GE}$

$\mu g / L \quad 0 \quad G E$

$\mu g / L \quad 0 \quad G E$

$\mu \mathrm{g} / \mathrm{L} \quad 0 \quad \mathrm{GE}$

$\mu \mathrm{g} / \mathrm{L} \quad \mathrm{O} \quad \mathrm{GE}$

$\mu g / L \quad O \quad G E$

$\mu \mathrm{g} / \mathrm{L} \quad 0 \quad \mathrm{GE}$

$\dot{\mu g} / \mathrm{L} \quad \mathrm{O} \quad \mathrm{GE}$

$\mu \mathrm{g} / \mathrm{L} \quad 0 \quad \mathrm{GE}$

$\mu g / L \quad O \quad G E$

$\begin{array}{lll}\mu g / L & 0 & \mathrm{GE} \\ \mu \mathrm{g} / \mathrm{L} & 0 & \mathrm{GE}\end{array}$

- = exceeded holding time. $=$ exceeded screening level or final primary drinking water standard. 
WELL BGO $31 \mathrm{C}$ collected on 04/12/94, laboratory analyses (cont.)

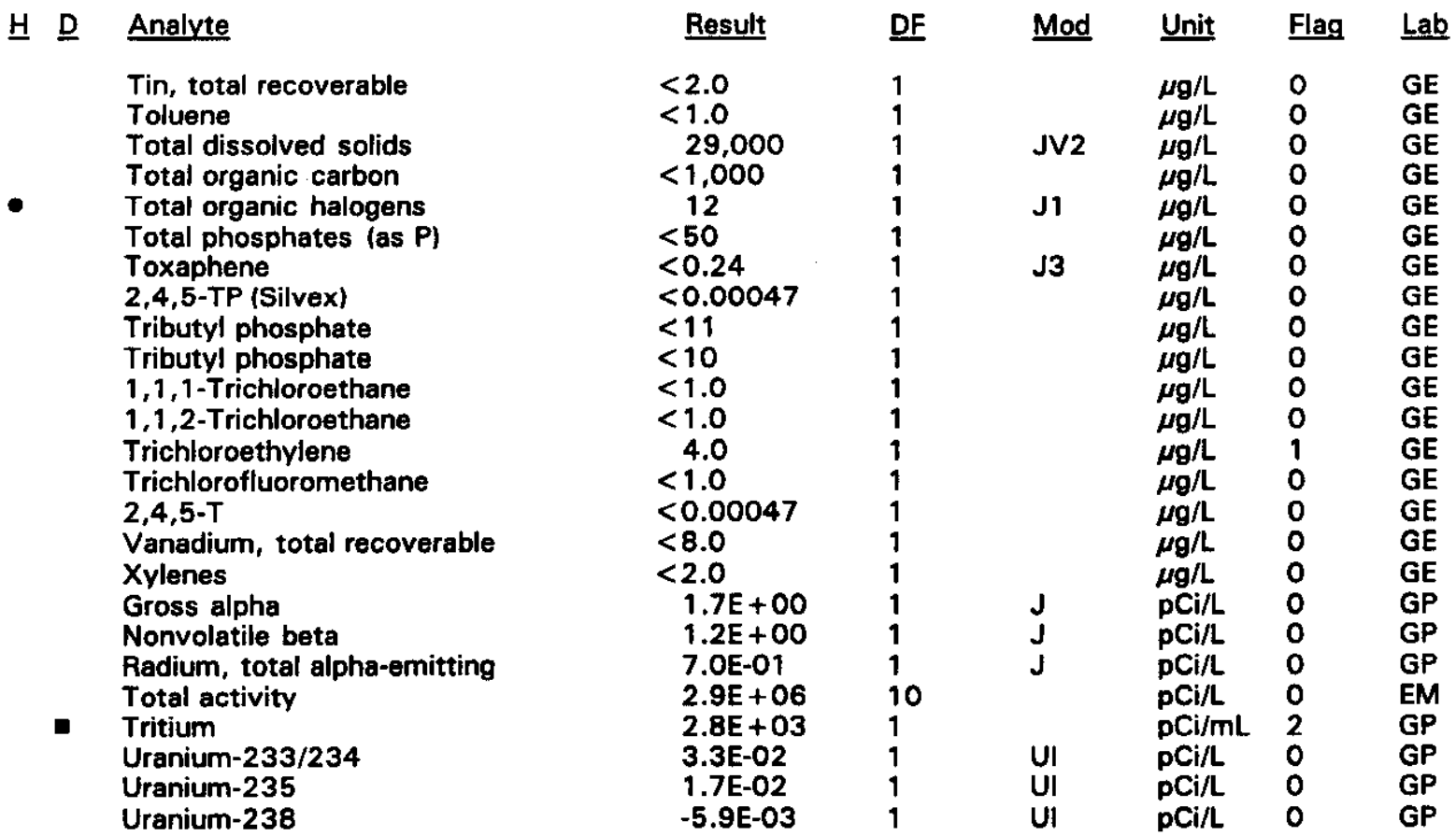

\section{WELL BGO 31D}

\begin{tabular}{|c|c|c|c|c|c|c|}
\hline SRS Coord. & Lat/Longitude & Scroen Zone Elevation & Top of Casing & Casing & Pump & Formation \\
\hline $\begin{array}{l}\text { N74985.3 } \\
\text { E54841.7 }\end{array}$ & $\begin{array}{l}33.281099^{\circ} \mathrm{N} \\
81.668048^{\circ} \mathrm{W}\end{array}$ & $231.1-211.1 \mathrm{ft} \mathrm{msl}$ & $273.7 \mathrm{ft} \mathrm{msl}$ & 4" PVC & $\mathbf{S}$ & Water Table $\left(\mathrm{IIB}_{2}\right)$ \\
\hline
\end{tabular}

FIELD MEASUREMENTS

Sample date: 04/12/94

Depth to water: $46.46 \mathrm{ft}(14.16 \mathrm{~m})$ below TOC

Water elevation: $227.24 \mathrm{ft}(69.26 \mathrm{~m}) \mathrm{msl}$

Sp. conductance: $37 \mu \mathrm{S} / \mathrm{cm}$

Turbidity: 28.1 NTU

Water evacuated before sampling: $6 \mathrm{gal}$

The well went dry during purging.

\section{LABORATORY ANALYSES}

\section{H $\underline{\text { D }}$ Analyte}

pH

Specific conductance

Turbidity

Acetophenone

Aldrin

Aldrin

Aluminum, total recoverable

Antimony, total recoverable

Arsenic, total recoverable
Time: $11: 59$

pH: 4.8

Alkalinity: $0 \mathrm{mg} / \mathrm{L}$

Water temperature: $21.3^{\circ} \mathrm{C}$

Volumes purged: 0.6 well volumes

- exceeded holding time. = exceeded screening level or final primary drinking water standard. 
WELL BGO 31D collected on 04/12/94, laboratory analyses (cont.)

\section{H $\underline{D}$}

Analyte

Barium, total recoverable

Benzene

Bromodichloromethane

Bromoform

Bromomethane (Methyl bromide)

Cadmium, total recoverable

Calcium, total recoverable

Carbon tetrachloride

Chloride

Chlorobenzene

Chloroethane

Chloroethene (Vinyl chloride)

2-Chloroethyl vinyl ether

Chioroform

Chloromethane (Methyl chloride)

Chromium, total recoverable

Copper, total recoverable

Cyanide

p.p'-DDT

p,p'-DDT

Dibromochloromethane

1,1-Dichloroethane

1,2-Dichloroethane

1,1-Dichloroethylene

trans-1,2-Dichloroethylene

Dichloromethane (Methylene chloride)

2,4-Dichlorophenoxyacetic acid

1,2-Dichloropropane

cis-1,3-Dichloropropene

trans-1,3-Dichloropropene

Dieldrin

Dieldrin

Endrin

Endrin

Ethylbenzene

Fluoride

Heptachlor

Heptachlor

Iron, total recoverable

Lead, total recoverable

Lindane

Lindane

Magnesium, total recoverable

Manganese, total recoverable

Mercury, total recoverable

Methoxychior

Methoxychior

Naphthalene

Nickel, total recoverable

Nitrate-nitrite as nitrogen

Phenols

Potassium, total recoverable

Selenium, total recoverable

Silica, total recoverable

Silver, total recoverable

Sodium, total recoverable
Result

$$
7.7
$$

$<1.0$

$<1.0$

$<1.0$

$<1.0$

$<2.0$

512

$<1.0$

3,270

$<1.0$

$<1.0$

$<1.0$

$<1.0$

$<1.0$

$<1.0$

$<4.0$

15

$<5.0$

$<0.10$

$<0.10$

$<1.0$

$<1.0$

$<1.0$

$<1.0$

$<1.0$

$<1.0$

$<0.0016$

$<1.0$

$<1.0$

$<1.0$

$<0.50$

$<0.50$

$<0.0060$

$<0.0060$

$<1.0$

$<100$

$<0.050$

$<0.050$

685

8.3

$<0.0050$

$<0.0050$

1,480

8.3

0.37

$<0.50$

$<0.50$

$<10$

$<4.0$

1,120

$<5.0$

$<500$

$<2.0$

9,430

$<2.0$

1,260
Mod

Unit Flag Lab

$\mu \mathrm{g} / \mathrm{L} \quad \mathrm{O} \quad \mathrm{GE}$

$\mu \mathrm{g} / \mathrm{L} \quad 0 \quad \mathrm{GE}$

$\mu \mathrm{g} / \mathrm{L} \quad \mathrm{O} \quad \mathrm{GE}$

$\mu g / L \quad 0 \quad$ GE

$\mu \mathrm{g} / \mathrm{L} \quad 0 \quad \mathrm{GE}$

$\mu \mathrm{g} / \mathrm{L} \quad 0 \quad \mathrm{GE}$

$\mu g / \mathrm{L} \quad 0 \quad \mathrm{GE}$

$\mu g / L \quad 0 \quad G E$

$\mu g / L \quad 0 \quad$ GE

$\mu g / L \quad 0 \quad G E$

$\mu g / L \quad 0 \quad G E$

$\mu g / L \quad 0 \quad G E$

$\mu \mathrm{g} / \mathrm{L} \quad 0 \quad \mathrm{GE}$

$\mu g / L \quad 0 \quad$ GE

$\mu g / L \quad 0 \quad G E$

$\mu \mathrm{g} / \mathrm{L} \quad 0 \quad \mathrm{GE}$

$\mu g / L \quad 0 \quad G E$

$\mu g / L \quad 0 \quad$ GE

$\mu g / L \quad 0 \quad G E$

$\mu g / L \quad 0 \quad G E$

$\mu \mathrm{g} / \mathrm{L} \quad 0 \quad \mathrm{GE}$

$\mu g / L \quad 0 \quad G E$

$\mu \mathrm{g} / \mathrm{L} \quad 0$

$\mu g / L \quad 0 \quad G E$

$\mu \mathrm{g} / \mathrm{L} \quad 0 \quad \mathrm{GE}$

$\mu \mathrm{g} / \mathrm{L} \quad \mathrm{O} \quad \mathrm{GE}$

$\mu \mathrm{g} / \mathrm{L} \quad 0 \quad \mathrm{GE}$

$\mu \mathrm{g} / \mathrm{L} \quad \mathrm{O} \quad \mathrm{GE}$

$\mu g / L \quad 0 \quad G E$

$\mu g / L \quad 0 \quad$ GE

$\mu g / L \quad 0 \quad G E$

$\mu \mathrm{g} / \mathrm{L} \quad 0 \quad \mathrm{GE}$

$\mu g / L \quad 0 \quad$ GE

$\mu g / L \quad 0 \quad G E$

$\mu g / L \quad 0 \quad$ GE

$\mu g / L \quad 0 \quad G E$

$\mu g / L \quad 0 \quad$ GE

$\mu g / \mathrm{L} \quad 0 \quad \mathrm{GE}$

$\mu g / L \quad 2 \quad G E$

$\mu g / L \quad 0 \quad \mathrm{GE}$

$\mu g / \mathrm{L} \quad 0 \quad \mathrm{GE}$

$\mu \mathrm{g} / \mathrm{L} \quad 0 \quad \mathrm{GE}$

$\mu g / L \quad 0 \quad$ GE

$\mu \mathrm{g} / \mathrm{L} \quad 0 \quad \mathrm{GE}$

$\mu g / \mathrm{L} \quad 0 \quad \mathrm{GE}$

$\mu \mathrm{g} / \mathrm{L} \quad 0 \quad \mathrm{GE}$

$\mu g / L \quad 0 \quad$ GE

$\mu \mathrm{g} / \mathrm{L} \quad 0 \quad \mathrm{GE}$

$\mu g / L \quad 0 \quad G E$

$\mu \mathrm{g} / \mathrm{L} \quad 0 \quad \mathrm{GE}$

$\mu g / L \quad 0 \quad$ GE

$\mu g / L \quad 0 \quad$ GE

$\mu g / L \quad 0 \quad G E$

$\mu \mathrm{g} / \mathrm{L} \quad 0 \quad \mathrm{GE}$

$\mu g / L \quad 0 \quad G E$

$\mu g / L \quad 0 \quad$ GE

- exceeded holding time. = exceeded screening level or final primary drinking water standard. 
WELL BGO 310 collected on 04/12/94, laboratory analyses (cont.)

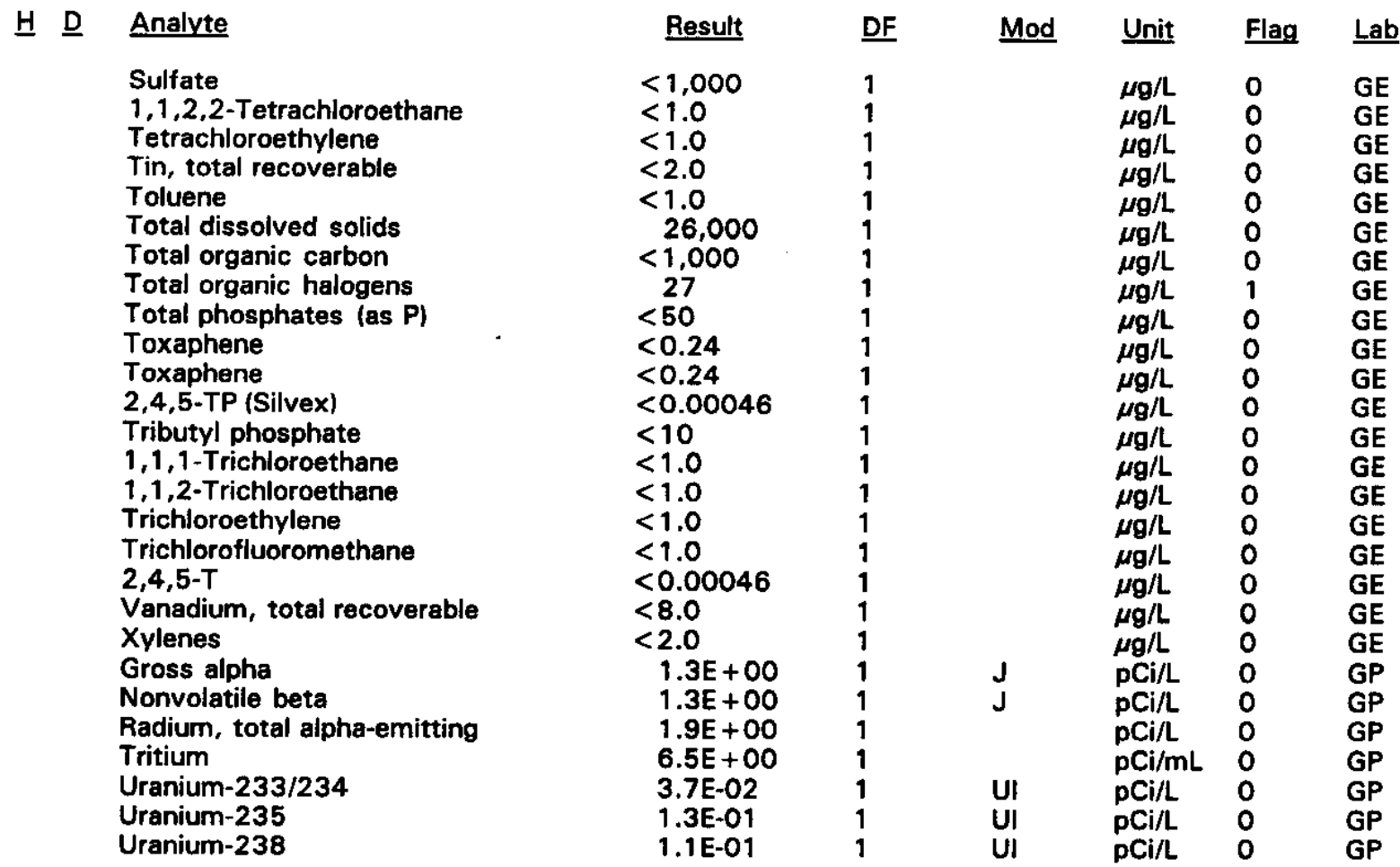

\section{WELL BGO 32D}

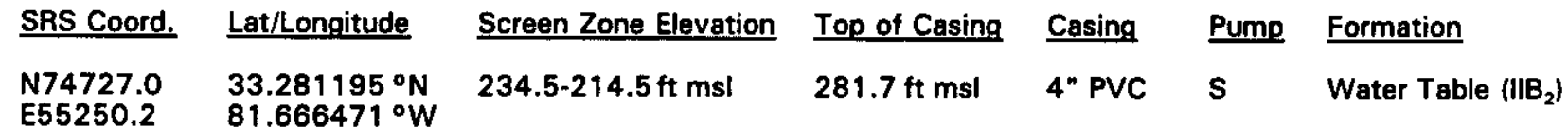

\section{FIELD MEASUREMENTS}

Sample date: 04/15/94

Depth to water: $53.67 \mathrm{ft}(16.36 \mathrm{~m})$ below TOC

Water elevation: $228.03 \mathrm{ft}(69.50 \mathrm{~m}) \mathrm{msl}$

Sp. conductance: $86 \mu \mathrm{S} / \mathrm{cm}$

Turbidity: 65.1 NTU

Water evacuated before sampling: $7 \mathrm{gal}$

The well went dry during purging.

\section{LABORATORY ANALYSES}

H D Analyte

- $\mathrm{pH}$

Specific conductance

Specific conductance

- Turbidity

Acetophenone

Aldrin

Aluminum, total recoverable

\begin{tabular}{|c|c|c|c|c|}
\hline Result & DF & Mod & Unit & Flag \\
\hline $\begin{array}{c}5.0 \\
73 \\
73 \\
79 \\
<10 \\
<0.051 \\
855\end{array}$ & $\begin{array}{l}1 \\
1 \\
1 \\
8 \\
1 \\
1 \\
1\end{array}$ & $\begin{array}{l}\mathbf{J 1} \\
\mathrm{J} 1\end{array}$ & $\begin{array}{l}\mathrm{pH} \\
\mu \mathrm{S} / \mathrm{cm} \\
\mu S / \mathrm{cm} \\
\mathrm{NTU} \\
\mu \mathrm{g} / \mathrm{L} \\
\mu \mathrm{g} / \mathrm{L} \\
\mu \mathrm{g} / \mathrm{L}\end{array}$ & $\begin{array}{l}0 \\
0 \\
0 \\
0 \\
0 \\
0 \\
2\end{array}$ \\
\hline
\end{tabular}

Time: $8: 41$

pH: 4.8

Alkalinity: $0 \mathrm{mg} / \mathrm{L}$

Water temperature: $20.3^{\circ} \mathrm{C}$

Volumes purged: 0.8 well volumes

- exceeded holding time. $=$ exceeded screening level or final primary drinking water standard. 
WELL BGO 32D collected on 04/15/94, laboratory analyses (cont.)

H $\underline{\text { Analyte }}$

Antimony, total recoverable

Arsenic, total recoverable

Barium, total recoverable

Benzene

Benzene

Bromodichloromethane

Bromoform

Bromomethane (Methyl bromide)

Cadmium, total recoverable

Calcium, total recoverable

Carbon tetrachloride

Chloride

Chlorobenzene

Chlorobenzene

Chloroethane

Chloroethene (Vinyl chloride)

2-Chloroethyl vinyl ether

Chioroform

Chloromethane (Methyl chloride)

Chromium, total recoverable

Copper, total recoverable

Cyanide

p.p'-DDT

Dibromochloromethane

1,1-Dichloroethane

1,2-Dichloroethane

1,1-Dichloroethylene

1,1-Dichloroethylene

trans-1,2-Dichloroethylene

Dichloromethane (Methylene chloride)

2,4-Dichlorophenoxyacetic acid

1,2-Dichloropropane

cis-1,3-Dichloropropene

trans-1,3-Dichloropropene

Dieldrin

Endrin

Ethylbenzene

Fluoride

Heptachlor

Iron, total recoverable

Lead, total recoverable

Lindane

Magnesium, total recoverable

Manganese, total recoverable

Mercury, total recoverable

Methoxychlor

Naphthalene

Nickel, total recoverable

Nitrate-nitrite as nitrogen

Phenols

Potassium, total recoverable

Selenium, total recoverable

Silica, total recoverable

Silver, total recoverable

Sodium, total recoverable

Sulfate
Result D

$<2.0$

$<2.0$

90

$<1.0$

$<1.0$

$<1.0$

$<1.0$

$<1.0$

$<2.0$

794

$<1.0$

6,730

$<1.0$

$<1.0$

$<1.0$

$<1.0$

$<1.0$

$<1.0$

$<1.0$

$<4.0$

308

$<5.0$

$<0.10$

$<1.0$

$<1.0$

$<1.0$

$<1.0$

$<1.0$

$<1.0$

$<1.0$

$<0.0016$

$<1.0$

$<1.0$

$<1.0$

$<0.51$

$<0.0061$

$<1.0$

$<100$

$<0.051$

393

31

$<0.0051$

1,430

18

$<0.20$

$<0.51$

$<10$

5.5

4,100

$<5.0$

1,050

$<2.0$

7,430

$<2.0$

7,430

$<1,000$
DF

Mod

$\underline{\text { Unit }}$ Flag Lab

$\mu \mathrm{g} / \mathrm{L} \quad 0 \quad \mathrm{GE}$

$\mu \mathrm{g} / \mathrm{L} \quad 0 \quad \mathrm{GE}$

$\mu \mathrm{g} / \mathrm{L} \quad 0 \quad \mathrm{GE}$

$\mu g / L \quad 0 \quad G E$

$\mu \mathrm{g} / \mathrm{L} \quad 0 \quad \mathrm{GE}$

$\mu \mathrm{g} / \mathrm{L} \quad 0 \quad \mathrm{GE}$

$\mu g / L \quad 0 \quad G E$

$\mu g / L \quad 0 \quad G E$

$\mu g / L \quad 0 \quad G E$

$\mu \mathrm{g} / \mathrm{L} \quad \mathrm{O} \quad \mathrm{GE}$

$\mu g / L \quad 0 \quad G E$

$\mu \mathrm{g} / \mathrm{L} \quad 0 \quad \mathrm{GE}$

$\mu g / L \quad 0 \quad$ GE

$\mu g / L \quad 0 \quad G E$

$\mu g / L \quad 0 \quad$ GE

$\mu g / L \quad 0 \quad G E$

$\mu g / L \quad 0 \quad$ GE

$\mu g / L \quad 0 \quad G E$

$\mu g / L \quad O \quad G E$

$\mu g / L \quad 0 \quad G E$

$\mu g / L \quad 0 \quad G E$

$\mu \mathrm{g} / \mathrm{L} \quad 0 \quad \mathrm{GE}$

$\mu \mathrm{g} / \mathrm{L} \quad 0 \quad \mathrm{GE}$

$\mu g / L \quad 0 \quad G E$

$\mu g / L \quad 0 \quad G E$

$\mu g / L \quad 0 \quad G E$

$\mu g / L \quad 0 \quad G E$

$\mu g / L \quad 0 \quad G E$

$\mu g / L \quad 0 \quad G E$

$\mu g / L \quad 0 \quad G E$

$\mu g / L \quad 0 \quad G E$

$\mu g / L \quad 0 \quad G E$

$\mu \mathrm{g} / \mathrm{L} \quad 0 \quad \mathrm{GE}$

$\mu g / L \quad 0 \quad G E$

$\mu g / L \quad 0 \quad$ GE

$\mu g / L \quad 0 \quad G E$

$\mu g / L \quad 0 \quad$ GE

$\mu g / L \quad 0 \quad G E$

$\mu g / L \quad 0 \quad G E$

$\mu g / L \quad 2 \quad G E$

$\mu g / L \quad 1 \quad$ GE

$\mu g / L \quad 0 \quad$ GE

$\mu g / L \quad 0 \quad G E$

$\mu g / L \quad 0 \quad G E$

$\mu g / L \quad 0 \quad \mathrm{GE}$

$\mu g / L \quad 0 \quad G E$

$\mu g / L \quad 0 \quad G E$

$\mu g / L \quad 0 \quad G E$

$\mu g / L \quad 0 \quad$ GE

$\mu g / L \quad 0 \quad G E$

$\mu g / L \quad 0 \quad G E$

$\mu \mathrm{jg} / \mathrm{L} \quad \mathrm{O} \quad \mathrm{GE}$

$\mu \mathrm{g} / \mathrm{L} \quad \mathrm{O} \quad \mathrm{GE}$

$\mu \mathrm{g} / \mathrm{L} \quad \mathrm{O} \quad \mathrm{GE}$

$\mu g / L \quad 0 \quad G E$

- = exceeded holding time. - = exceeded screening level or final primary drinking water standard. 
WELL BGO 32D collected on 04/15/94, laboratory analyses (cont.)

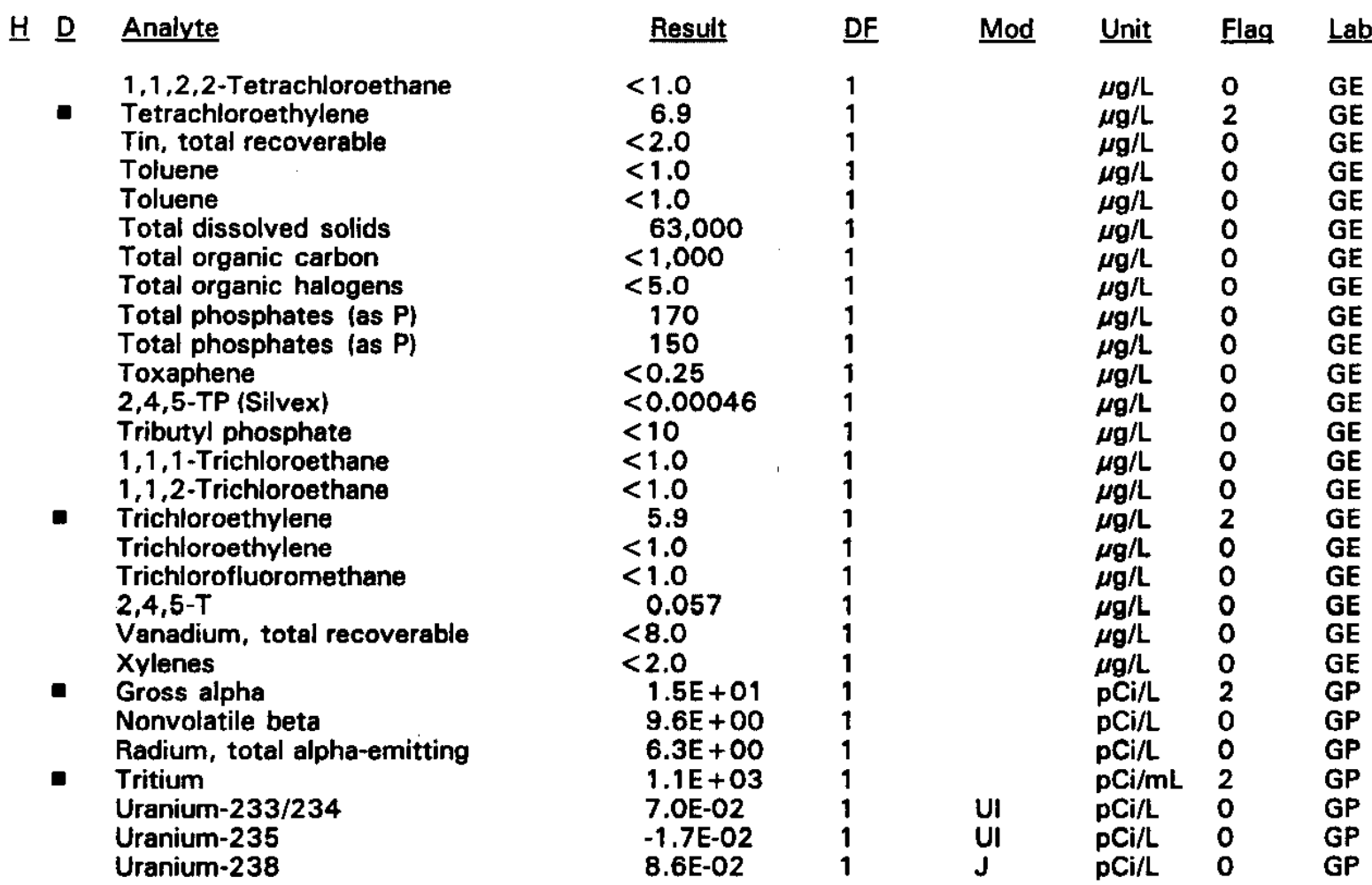

\section{WELL BGO 33C}

\begin{tabular}{|c|c|c|c|c|c|c|}
\hline SRS Coord. & Lat/Longitude & Screen Zone Elevation & Top of Casing & Casing & Pump & Formation \\
\hline $\begin{array}{l}\text { N74479.7 } \\
\text { E55681.4 }\end{array}$ & $\begin{array}{l}33.281351^{\circ} \mathrm{N} \\
81.664855^{\circ} \mathrm{W}\end{array}$ & $187.8-177.8 \mathrm{ft} \mathrm{msl}$ & $279.4 \mathrm{ft} \mathrm{msl}$ & 4" PVC & $\mathbf{S}$ & McBean (IIB, \\
\hline
\end{tabular}

\section{FIELD MEASUREMENTS}

Sample date: $04 / 11 / 94$

Depth to water: $53.88 \mathrm{ft}(16.42 \mathrm{~m})$ below TOC

Water elevation: $225.52 \mathrm{ft}(68.74 \mathrm{~m}) \mathrm{ms}$ |

Sp. conductance: $59 \mu \mathrm{S} / \mathrm{cm}$

Turbidity: 0.6 NTU

Water evacuated before sampling: $\mathbf{2 2 0}$ gal

\section{LABORATORY ANALYSES}

Time: 10:33

pH: 5.7

Alkalinity: $4 \mathrm{mg} / \mathrm{L}$

Water temperature: $19.9^{\circ} \mathrm{C}$

Volumes purged: 7.0 well volumes

\begin{tabular}{|c|c|c|c|c|c|c|}
\hline$\underline{\text { H }} \underline{\mathrm{D}}$ & Analyte & Result & DF & Mod & Unit & Flag \\
\hline - & $\begin{array}{l}\text { pH } \\
\text { Specific conductance } \\
\text { Specific conductance } \\
\text { Turbidity } \\
\text { Acetophenone } \\
\text { Aldrin }\end{array}$ & $\begin{array}{l}5.9 \\
50 \\
50 \\
0.29 \\
<10 \\
<0.050\end{array}$ & $\begin{array}{l}1 \\
1 \\
1 \\
1 \\
1 \\
1\end{array}$ & $\begin{array}{l}\text { J1 } \\
\text { J1 }\end{array}$ & $\begin{array}{l}\mathrm{pH} \\
\mu \mathrm{S} / \mathrm{cm} \\
\mu S / \mathrm{cm} \\
\mathrm{NTU} \\
\mu \mathrm{g} / \mathrm{L} \\
\mu \mathrm{g} / \mathrm{L}\end{array}$ & $\begin{array}{l}0 \\
0 \\
0 \\
0 \\
0 \\
0\end{array}$ \\
\hline
\end{tabular}

\footnotetext{
- = exceeded holding time. - = exceeded screening level or final primary drinking water standard.
} 
WELL. BGO $33 \mathrm{C}$ collected on $04 / 11 / 94$, laboratory analyses (cont.)

H $\underline{\text { Analyte }}$

Aluminum, total recoverable

Antimony, total recoverable

Arsenic, total recoverable

Barium, total recoverable

Benzene

Bromodichloromethane

Bromoform

Bromomethane (Methyl bromide)

Cadmium, total recoverable

Calcium, total recoverable

Carbon tetrachloride

Chloride

Chloride

Chlorobenzene

Chloroethane

Chloroethene (Vinyl chloride)

2-Chloroethyl vinyl ether

Chloroform

Chloromethane (Methyl chloride)

Chromium, total recoverable

Copper, total recoverable

Cyanide

p,p'-DDT

Dibromochloromethane

1,1-Dichloroethane

1,2-Dichloroethane

1,1-Dichloroethylene

trans-1,2-Dichloroethylene

Dichloromethane (Methylene chloride)

2,4-Dichlorophenoxyacetic acid

1,2-Dichloropropane

cis-1,3-Dichloropropene

trans-1,3-Dichloropropene

Dieldrin

Endrin

Ethylbenzene

Fluoride

Heptachlor

Iron, total recoverable

Lead, total recoverable

Lindane

Magnesium, total recoverable

Manganese, total recoverable

Mercury, total recoverable

Mercury, total recoverable

Methoxychlor

Naphthalene

Nickel, total recoverable

Nitrate-nitrite as nitrogen

Phenols

Phenols

Potassium, total recoverable

Selenium, total recoverable

Silica, total recoverable

Silver, total recoverable

Sodium, total recoverable
Result DF

38

$<2.0$

$<2.0$

11

$<1.0$

$<1.0$

$<1.0$

$<1.0$

$<2.0$

4,370

$<1.0$

5,970

5,990

$<1.0$

$<1.0$

$<1.0$

$<1.0$

14

$<1.0$

$<4.0$

$<4.0$

$<5.0$

$<0.10$

$<1.0$

$<1.0$

$<1.0$

$<1.0$

$<1.0$

$<1.0$

$<0.0015$

$<1.0$

$<1.0$

$<1.0$

$<0.50$

$<0.0060$

$<1.0$

$<100$

$<0.050$

6.4

$<3.0$

$<0.0050$

671

30

1.4

1.3

$<0.50$

$<10$

$<4.0$

990

$<5.0$

$<5.0$

$<500$

$<2.0$

10,600

$<2.0$

3,280
Mod Unit Flag Lab

$\mu \mathrm{g} / \mathrm{L} \quad 1 \quad \mathrm{GE}$

$\mu \mathrm{g} / \mathrm{L} \quad 0 \quad \mathrm{GE}$

$\mu g / L \quad 0 \quad G E$

$\mu \mathrm{g} / \mathrm{L} \quad 0 \quad \mathrm{GE}$

$\mu \mathrm{g} / \mathrm{L} \quad 0 \quad \mathrm{GE}$

$\mu \mathrm{g} / \mathrm{L} \quad 0 \quad \mathrm{GE}$

$\mu g / L \quad 0 \quad G E$

$\mu \mathrm{g} / \mathrm{L} \quad 0 \quad \mathrm{GE}$

$\mu g / L \quad 0 \quad G E$

$\mu g / L \quad 0 \quad G E$

$\mu g / L \quad 0 \quad G E$

$\mu \mathrm{g} / \mathrm{L} \quad 0 \quad \mathrm{GE}$

$\mu \mathrm{g} / \mathrm{L} \quad 0 \quad \mathrm{GE}$

$\mu g / L \quad 0 \quad G E$

$\mu g / L \quad 0 \quad$ GE

$\mu \mathrm{g} / \mathrm{L} \quad 0 \quad \mathrm{GE}$

$\mu g / L \quad 0 \quad$ GE

$\mu \mathrm{g} / \mathrm{L} \quad 0 \quad \mathrm{GE}$

$\mu g / L \quad 0 \quad$ GE

$\mu g / L \quad 0 \quad G E$

$\mu \mathrm{g} / \mathrm{L} \quad 0 \quad \mathrm{GE}$

$\mu g / L \quad 0 \quad G E$

$\mu \mathrm{g} / \mathrm{L} \quad 0$

$\mu g / L \quad 0 \quad G E$

$\mu g / L \quad 0$

$\mu g / L \quad 0 \quad G E$

$\mu g / L \quad 0$

$\mu g / L \quad 0 \quad G E$

$\mu g / L \quad 0 \quad G E$

$\mu g / L \quad 0 \quad G E$

$\mu g / L \quad 0 \quad G E$

$\mu g / L \quad 0 \quad$ GE

$\mu \mathrm{g} / \mathrm{L} \quad 0 \quad \mathrm{GE}$

$\mu g / L \quad 0 \quad G E$

$\mu g / L \quad 0 \quad G E$

$\mu \mathrm{g} / \mathrm{L} \quad 0 \quad \mathrm{GE}$

$\mu \mathrm{g} / \mathrm{L} \quad 0 \quad \mathrm{GE}$

$\mu g / L \quad 0 \quad G E$

$\mu g / L \quad 0 \quad G E$

$\mu g / L \quad 0 \quad G E$

$\mu g / L \quad 0 \quad$ GE

$\mu g / L \quad 0 \quad G E$

$\mu g / L \quad 1 \quad G E$

$\mu \mathrm{g} / \mathrm{L} \quad 1 \quad \mathrm{GE}$

$\mu \mathrm{g} / \mathrm{L} \quad 1 \quad \mathrm{GE}$

$\mu g / L \quad 0 \quad G E$

$\mu g / L \quad 0 \quad G E$

$\mu g / L \quad 0 \quad$ GE

$\mu g / L \quad 0 \quad G E$

$\mu g / L \quad 0 \quad \mathrm{GE}$

$\mu g / L \quad 0 \quad G E$

$\mu g / L \quad 0 \quad$ GE

$\mu g / L \quad 0 \quad G E$

$\mu g / L \quad 0 \quad$ GE

$\mu g / L \quad 0 \quad G E$

$\mu g / L \quad 0 \quad$ GE

- = exceeded holding time. = exceeded screening level or final primary drinking water standard. 
WELL BGO 33C collected on 04/11/94, laboratory analyses (cont.)

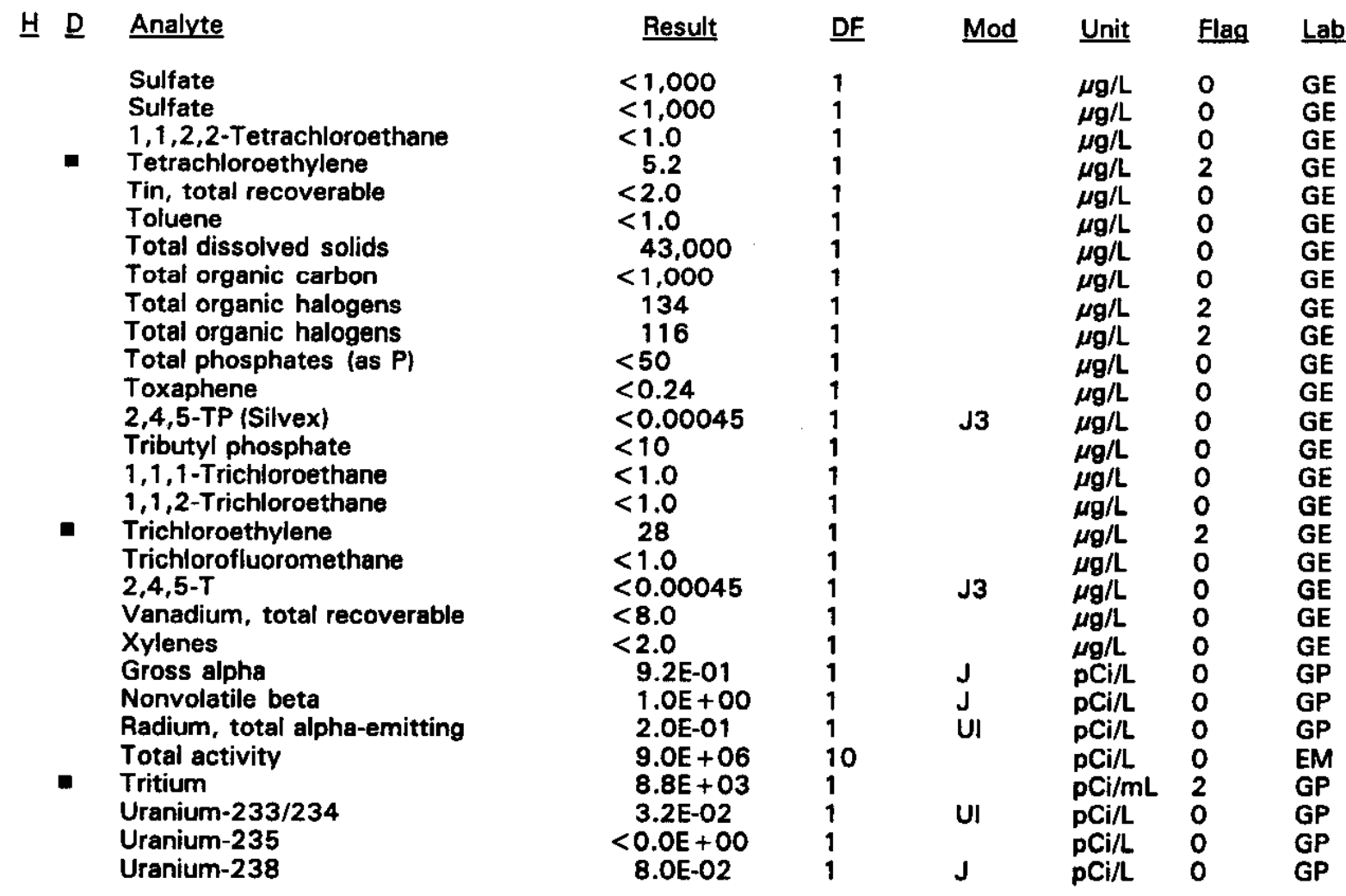

\section{WELL BGO 33D}

\begin{tabular}{|c|c|c|c|c|c|c|}
\hline SRS Coord. & Lat/Longitude & Screen Zone Elevation & Top of Casing & Casing & Pump & Formation \\
\hline $\begin{array}{l}\text { N74468.7 } \\
\text { E55695.4 }\end{array}$ & $\begin{array}{l}33.281350^{\circ} \mathrm{N} \\
81.664797^{\circ} \mathrm{W}\end{array}$ & $233.1-213.1 \mathrm{ft} \mathrm{msl}$ & $280.3 \mathrm{ft} \mathrm{msl}$ & 4" PVC & $\mathbf{S}$ & Water Ta \\
\hline
\end{tabular}

\section{FIELD MEASUREMENTS}

Sample date: 04/15/94

Depth to water: $49.45 \mathrm{ft}(15.07 \mathrm{~m})$ below TOC

Water elevation: $230.85 \mathrm{ft}(70.36 \mathrm{~m}) \mathrm{msl}$

Sp. conductance: $75 \mu \mathrm{S} / \mathrm{cm}$

Turbidity: 29.6 NTU

Water evacuated before sampling: $8 \mathrm{gal}$

The well went dry during purging.

\section{LABORATORY ANALYSES}

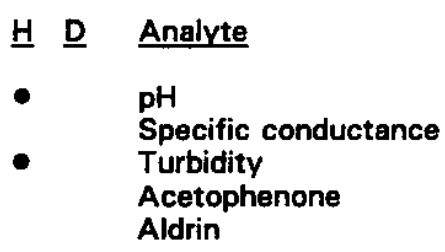

- exceeded holding time.

$\begin{aligned} & \text { Result } \\ & 5.5 \\ & 59 \\ & 28 \\ &<10 \\ &<0.050\end{aligned}$

Time: $9: 10$

pH: 5.0

Alkalinity: $0 \mathrm{mg} / \mathrm{L}$

Water temperature: $19.9^{\circ} \mathrm{C}$

Volumes purged: 0.7 well volumes 
WELL BGO 330 collected on $04 / 15 / 94$, laboratory analyses (cont.)

H $\underline{\text { Analyte }}$

Aluminum, total recoverable

Aluminum, total recoverable

Antimony, total recoverable

Antimony, total recoverable

Arsenic, total recoverable

Arsenic, total recoverable

Barium, total recoverable

Barium, total recoverable

Benzene

Bromodichloromethane

Bromoform

Bromomethane (Methyl bromide)

Cadmium, total recoverable

Cadmium, total recoverable

Calcium, total recoverable

Calcium, total recoverable

Carbon tetrachloride

Chloride

Chloride

Chlorobenzene

Chloroethane

Chloroethene (Vinyl chloride)

2-Chloroethyl vinyl ether

Chloroform

Chloromethane (Methyl chloride)

Chromium, total recoverable

Chromium, total recoverable

Copper, total recoverable

Copper, total recoverable

Cyanide

p,p'-DDT

Dibromochloromethane

1,1-Dichloroethane

1,2-Dichloroethane

1,1-Dichloroethylene

trans-1,2-Dichloroethylene

Dichloromethane (Methylene chloride)

2,4-Dichlorophenoxyacetic acid

1,2-Dichloropropane

cis-1,3-Dichloropropene

trans-1,3-Dichloropropene

Dieldrin

Endrin

Ethylbenzene

Fluoride

Heptachlor

Iron, total recoverable

Iron, total recoverable

Lead, total recoverable

Lead, total recoverable

Lindane

Magnesium, total recoverable

Magnes im, total recoverable

Mangar ise, total recoverable

Mangariese, total recoverable

Mercury, total recoverable

\section{Result}

332

329

$<2.0$

$<2.0$

$<2.0$

$<2.0$

16

16

$<1.0$

$<1.0$

$<1.0$

$<1.0$

$<2.0$

$<2.0$

1,380

1,390

$<1.0$

8,630

8,640

$<1.0$

$<1.0$

$<1.0$

$<1.0$

$<1.0$

$<1.0$

$<4.0$

$<4.0$

66

67

$<5.0$

$<0.10$

$<1.0$

$<1.0$

$<1.0$

$<1.0$

$<1.0$

$<1.0$

$<0.0016$

$<1.0$

$<1.0$

$<1.0$

$<0.50$

$<0.0060$

$<1.0$

$<100$

$<0.050$

260

259

15

15

$<0.0050$

1,580

1,610

6.0

6.0

0.21

\section{DF}

Mod

$\mu \mathrm{g} / \mathrm{L}$

$\mu g / L$

$\mu g / L$

$\sqrt{3}$

$\mu g /$

$\mu \mathrm{g} / \mathrm{L}$

$\mu g / L$

$\mu g / L$

$\mu g / L$

$\mu g / L$

$\mu g / L$

$\mu g / L$

$\mu g / L$

$\mu g / L$

$\mu \mathrm{g} / \mathrm{L}$

$\mu g / L$

$\mu g / L$

$\mu g / L$

$\mu \mathrm{g} / \mathrm{L}$

$\mu g / L$

$\mu g / L$

$\mu g / L$

$\mu g / L$

$\mu g / L$

$\mu g / L$

$\mu \mathrm{g} / \mathrm{L}$

$\mu g / L$

$\mu \mathrm{g} / \mathrm{L}$

$\mu g / L$

$\mu g / L$

$\mu g / L$

$\mu \mathrm{g} / \mathrm{L}$

$\mu \mathrm{g} / \mathrm{L}$

$\mu g / L$

$\mu \mathrm{g} / \mathrm{L}$

$\mu g / L$

$\mu g / L$

$\mu g / L$

$\mu g / L$

$\mu g / L$

$\mu g / L$

$\mu g / L$

$\mu g / L$

$\mu g / L$

$\mu g / L$

$\mu g / L$

$\mu g / L$

$\mu \mathrm{g} / \mathrm{L}$

$\mu g / L$

$\mu g / L$

$\mu g / L$

$\mu g / L$

$\mu g / L$

$\mu g / L$

$\mu g / L$

$\mu \mathrm{g} / \mathrm{L}$
Flag Lab

2 GE

2 GE

o GE

O GE

O GE

GE

O GE

O GE

O GE

O GE

O GE

O GE

O GE

GE

O GE

O GE

GE

O GE

O GE

GE

O GE

O GE

O GE

$\begin{array}{ll}0 & \text { GE } \\ 0 & \text { GE }\end{array}$

O GE

O GE

O GE

O GE

O GE

O GE

O GE

O GE

O GE

O GE

O GE

1 GE

1 GE

0 GE

O GE

o GE

O GE

O GE

$0 \quad$ GE

- exceeded holding time. = exceeded screening level or final primary drinking water standard. 
WELL BGO 33D collected on $04 / 15 / 94$, laboratory analyses (cont.)

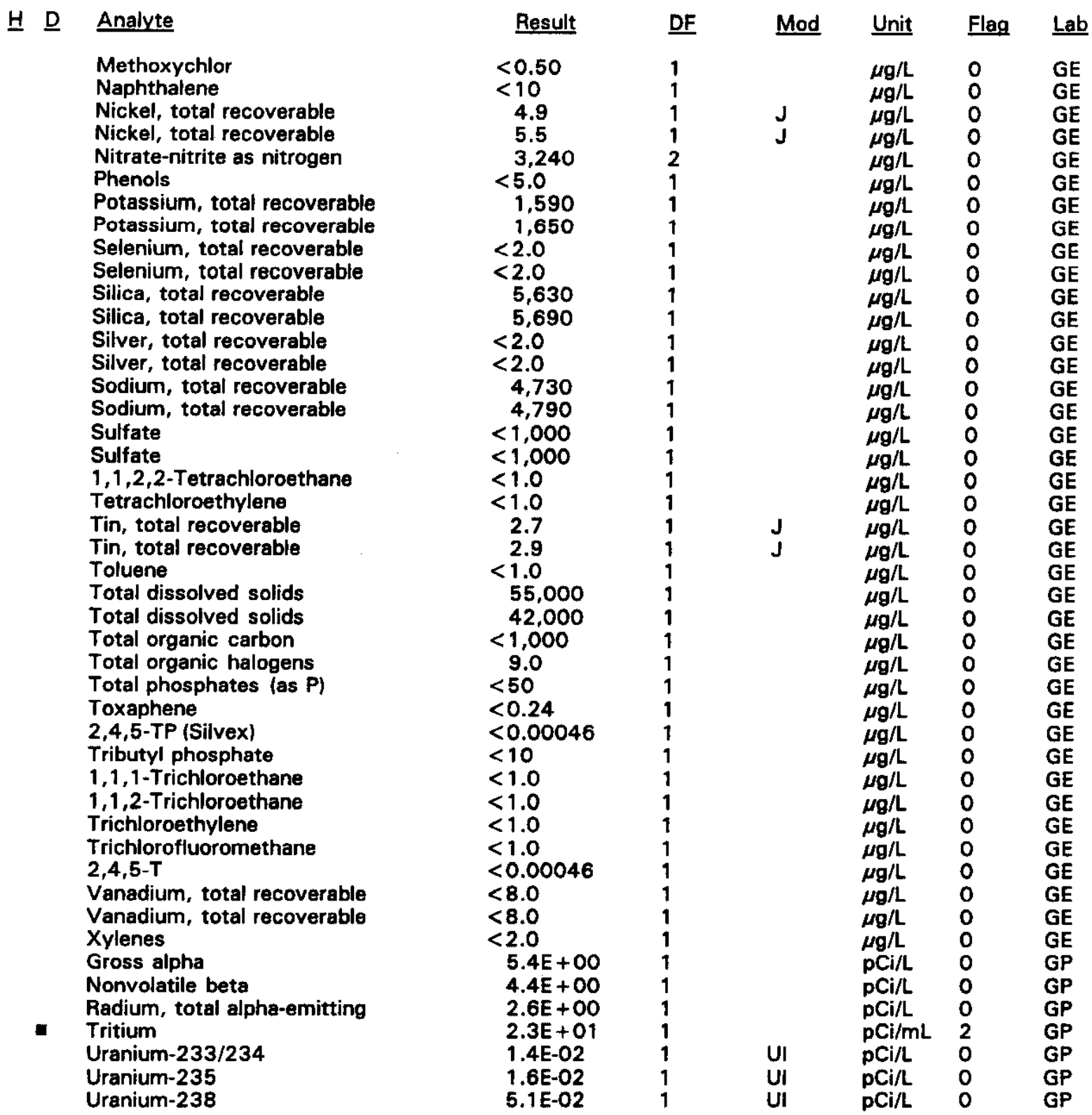

- = exceeded holding time. $=$ =xceeded screening level or final primary drinking water standerd. 


\section{WELL BGO 34D}

\begin{tabular}{|c|c|c|c|c|c|c|}
\hline SRS Coord. & Lat/Longitude & Screen Zone Elevation & Top of Casing & Casing & Pump & Formation \\
\hline & $\begin{array}{l}33.281451^{\circ} \mathrm{N} \\
81.663311^{\circ} \mathrm{W}\end{array}$ & $232.7-212.7 \mathrm{ft} \mathrm{msl}$ & $274.9 \mathrm{ft} \mathrm{msl}$ & 4" PVC & s & \\
\hline
\end{tabular}

\section{FIELD MEASUREMENTS}

Sample date: 04/15/94

Depth to water: $41.25 \mathrm{ft}(12.57 \mathrm{~m})$ below TOC

Water elevation: $233.65 \mathrm{ft}(71.22 \mathrm{~m}) \mathrm{ms}$ I

Sp. conductance: $100 \mu \mathrm{S} / \mathrm{cm}$

Turbidity: 5.1 NTU

Water evacuated before sampling: 13 gal

The well went dry during purging.

\section{LABORATORY ANALYSES}

H $\underline{\text { Analyte }}$

- $\quad$ pH

Specific conductance

Turbidity

Acetophenone

Aldrin

Aluminum, total recoverable

Antimony, total recoverable

Arsenic, total recoverable

Barium, total recoverable

Benzene

Bromodichloromethane

Bromoform

Bromomethane (Methyl bromide)

Cadmium, total recoverable

Calcium, total recoverable

Carbon tetrachloride

Chloride

Chlorobenzene

Chioroethane

Chloroethene (Vinyl chloride)

2-Chloroethyl vinyl ether

Chloroform

Chloromethane (Methyl chloride)

Chromium, total recoverable

Copper, total recoverable

Cyanide

p,p'-DDT

Dibromochloromethane

1,1-Dichloroethane

1,2-Dichloroethane

1,1-Dichloroethylene

trans-1,2-Dichloroethylene

Dichloromethane (Methylene chloride)

2,4-Dichlorophenoxyacetic acid

1,2-Dichloropropane

cis-1,3-Dichloropropene

trans-1,3-Dichloropropene

Dieldrin

Endrin

\begin{tabular}{|c|c|c|c|c|}
\hline Result & DF & Mod & Unit & Flag \\
\hline $\begin{array}{rl} & 7.8 \\
92 & 1.5 \\
< & 10 \\
< & 0.050 \\
& 146 \\
< & 2.0 \\
< & 2.0 \\
& 49 \\
< & 1.0 \\
<1.0 \\
<1.0 \\
<1.0 \\
<2.0 \\
14.200 \\
<1.0 \\
3.630 \\
<1.0 \\
<1.0 \\
<1.0 \\
<1.0 \\
<1.0 \\
<1.0 \\
<4.0 \\
<4.0 \\
<5.0 \\
<0.10 \\
<1.0 \\
<1.0 \\
<1.0 \\
<1.0 \\
<1.0 \\
<1.0 \\
<0.0016 \\
<1.0 \\
<1.0 \\
<1.0 \\
<0.50 \\
<0.0060\end{array}$ & $\begin{array}{l}1 \\
1 \\
1 \\
1 \\
1 \\
1 \\
1 \\
1 \\
1 \\
1 \\
1 \\
1 \\
1 \\
1 \\
1 \\
1 \\
1 \\
1 \\
1 \\
1 \\
1 \\
1 \\
1 \\
1 \\
1 \\
1 \\
1 \\
1 \\
1 \\
1 \\
1 \\
1 \\
1 \\
1 \\
1 \\
1 \\
1 \\
1 \\
1\end{array}$ & $\begin{array}{l}\text { J1 } \\
\text { J1 }\end{array}$ & 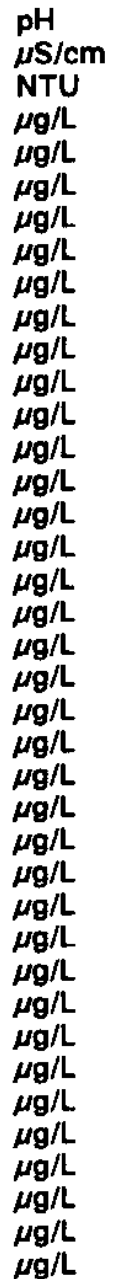 & $\begin{array}{l}0 \\
0 \\
0 \\
0 \\
0 \\
2 \\
0 \\
0 \\
0 \\
0 \\
0 \\
0 \\
0 \\
0 \\
0 \\
0 \\
0 \\
0 \\
0 \\
0 \\
0 \\
0 \\
0 \\
0 \\
0 \\
0 \\
0 \\
0 \\
0 \\
0 \\
0 \\
0 \\
0 \\
0 \\
0 \\
0 \\
0 \\
0 \\
0\end{array}$ \\
\hline
\end{tabular}

Time: 9:37

$\mathrm{pH}: \mathbf{7 . 8}$

Alkalinity: $27 \mathrm{mg} / \mathrm{L}$

Water temperature: $19.9^{\circ} \mathrm{C}$

Volumes purged: 0.9 well volumes 
WELL BGO 34D collected on $04 / 15 / 94$, laboratory analyses (cont.)

H $\underline{\text { D Analyte }}$

Ethylbenzene

Fluoride

Heptachlor

Iron, total recoverable

Lead, total recoverable

Lindane

Magnesium, total recoverable

Manganese, total recoverable

Mercury, total recoverable

Methoxychlor

Naphthalene

Nickel, total recoverable

Nitrate-nitrite as nitrogen

Phenols

Potassium, total recoverable

Selenium, total recoverable

Silica, total recoverable

Silver, total recoverable

Sodium, total recoverable

Sulfate

1,1,2,2-Tetrachloroethane

Tetrachloroethylene

Tin, total recoverable

Toluene

Total dissolved solids

Total organic carbon

Total organic halogens

Total phosphates (as P)

Toxaphene

2,4,5-TP (Silvex)

Tributyl phosphate

1,1,1-Trichloroethane

1,1,2-Trichloroethane

Trichloroethylene

Trichlorofluoromethane

2,4,5-T

Vanadium, total recoverable

Xylenes

Gross aipha

Gross alpha

Nonvolatile beta

Nonvolatile beta

Radium, total alpha-emitting

- Tritium

Uranium-233/234

Uranium-235

Uranium-238

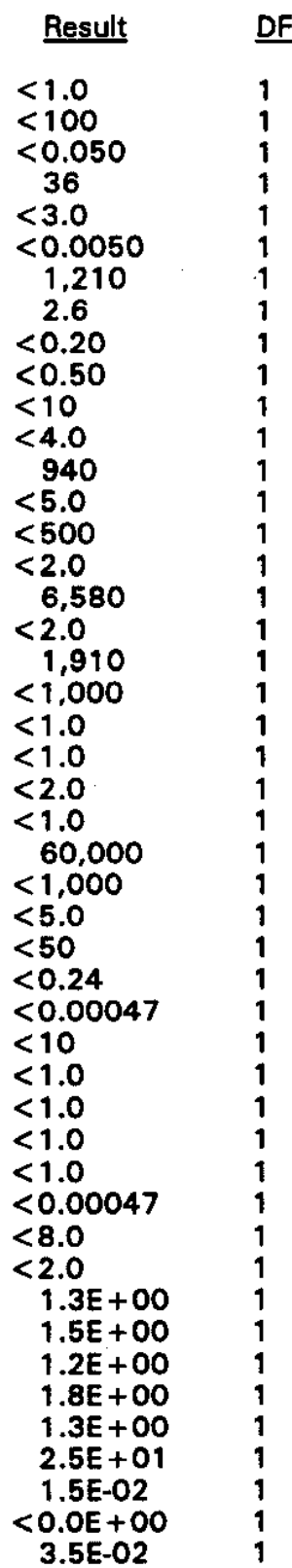

Mod Unit Flag Lab

$\mu g / L \quad 0 \quad$ GE

$\mu \mathrm{g} / \mathrm{L} \quad 0 \quad \mathrm{GE}$

$\mu \mathrm{g} / \mathrm{L} \quad 0 \quad \mathrm{GE}$

$\mu \mathrm{g} / \mathrm{L} \quad \mathrm{O} \quad \mathrm{GE}$

$\mu \mathrm{g} / \mathrm{L} \quad \mathrm{O} \quad \mathrm{GE}$

$\mu g / L \quad 0 \quad$ GE

$\mu g / L \quad 0 \quad \mathrm{GE}$

$\mu g / L \quad 0 \quad$ GE

$\mu g / L \quad 0 \quad G E$

$\mu g / L \quad 0 \quad \mathrm{GE}$

$\mu \mathrm{g} / \mathrm{L} \quad 0 \quad \mathrm{GE}$

$\mu g / L \quad 0 \quad G E$

$\mu \mathrm{g} / \mathrm{L} \quad 0 \quad \mathrm{GE}$

$\mu \mathrm{g} / \mathrm{L} \quad \mathrm{O} \quad \mathrm{GE}$

$\mu \mathrm{g} / \mathrm{L} \quad 0 \quad \mathrm{GE}$

$\mu g / L \quad 0 \quad G E$

$\mu g / L \quad 0 \quad$ GE

$\mu g / L \quad 0 \quad$ GE

$\mu g / L \quad 0 \quad$ GE

$\mu \mathrm{g} / \mathrm{L} \quad 0 \quad \mathrm{GE}$

$\mu \mathrm{g} / \mathrm{L} \quad \mathrm{O} \quad \mathrm{GE}$

$\mu \mathrm{g} / \mathrm{L} \quad \mathrm{O} \quad \mathrm{GE}$

$\mu \mathrm{g} / \mathrm{L} \quad \mathrm{O} \quad \mathrm{GE}$

$\mu g / L \quad 0 \quad G E$

$\mu g / L \quad 0 \quad$ GE

$\mu g / L \quad 0 \quad G E$

$\mu g / L \quad 0 \quad$ GE

$\mu g / L \quad 0 \quad$ GE

$\mu \mathrm{g} / \mathrm{L} \quad \mathrm{O} \quad \mathrm{GE}$

$\mu \mathrm{g} / \mathrm{L} \quad \mathrm{O} \quad \mathrm{GE}$

$\mu \mathrm{g} / \mathrm{L} \quad 0 \quad \mathrm{GE}$

$\mu \mathrm{g} / \mathrm{L} \quad 0 \quad \mathrm{GE}$

$\mu g / L \quad 0 \quad$ GE

$\mu g / L \quad O \quad G E$

$\mu g / L \quad 0 \quad$ GE

$\mu g / L \quad 0 \quad$ GE

$\mu g / L \quad 0 \quad$ GE

$\mu g / L \quad 0 \quad$ GE

$\begin{array}{llll}J & p C i / L & 0 & G P \\ J & p C i / L & 0 & G P\end{array}$

UI $\quad \mathrm{PCi} / \mathrm{L} \quad 0 \quad \mathrm{GP}$

J $\quad \mathrm{Ci} / \mathrm{L} \quad 0 \quad \mathrm{GP}$

$\mathrm{pCi} / \mathrm{L} \quad 0 \quad \mathrm{GP}$

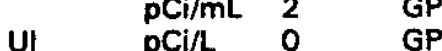

$\begin{array}{lll}\mathrm{PCi} / \mathrm{L} & 0 & \mathrm{GP} \\ \mathrm{pCi} & 0 & \mathrm{GP}\end{array}$

- = exceeded holding time. $=$ exceeded screening level or final primary drinking water standard. 
WELL BGO 35C

$\begin{array}{lllllll}\text { SRS Coord. } & \text { Lat/Longitude } & \text { Screen Zone Elevation } & \text { Top of Casing } & \text { Casing } & \text { Pump } & \text { Formation } \\ \text { N73953.9 } & 33.281598^{\circ} \mathrm{N} & 171.9-161.9 \mathrm{ft} \mathrm{msl} & 273.4 \mathrm{ft} \mathrm{msl} & \text { 4" PVC } & \text { S } & \text { McBean (IIB, }) \\ \text { E56545.7 } & 81.661558^{\circ} \mathrm{W} & & & & \end{array}$

FIELD MEASUREMENTS

Sample date: 04/11/94

Depth to water: $44.73 \mathrm{ft}(13.63 \mathrm{~m})$ below TOC

Water elevation: $228.67 \mathrm{ft}(69.70 \mathrm{~m}) \mathrm{msl}$

Sp. conductance: $50 \mu \mathrm{S} / \mathrm{cm}$

Turbidity: 0.3 NTU

Water evacuated before sampling: $207 \mathrm{gal}$

\section{LABORATORY ANALYSES}

H D Analyte

- $\quad$ pH

- $\quad \mathrm{pH}$

- $\quad \mathrm{pH}$

- $\quad$ pH

Specific conductance

Specific conductance

Specific conductance

Specific conductance

Specific conductance

Turbidity

Turbidity

- Turbidity

Turbidity

Acetophenone

Acetophenone

Acetophenone

Acetophenone

Acetophenone

Aldrin

Aldrin

Aluminum, total recoverable

Aluminum, total recoverable

Aluminum, total recoverable

Aluminum, total recoverable

Aluminum, total recoverable

Antimony, total recoverable

Antimony, total recoverable

Antimony, total recoverable

Antimony, total recoverable

Antimony, total recoverable

Arsenic, total recoverable

Arsenic, total recoverable

Arsenic, total recoverable

Arsenic, total recoverable

Arsenic, total recoverable

Barium, total recoverable

Barium, total recoverable

Barium, total recoverable

Barium, total recoverable
Time: $11: 11$

pH: 6.4

Alkalinity: $9 \mathrm{mg} / \mathrm{L}$

Water temperature: $20.1^{\circ} \mathrm{C}$

Volumes purged: $\mathbf{4 . 7}$ well volumes

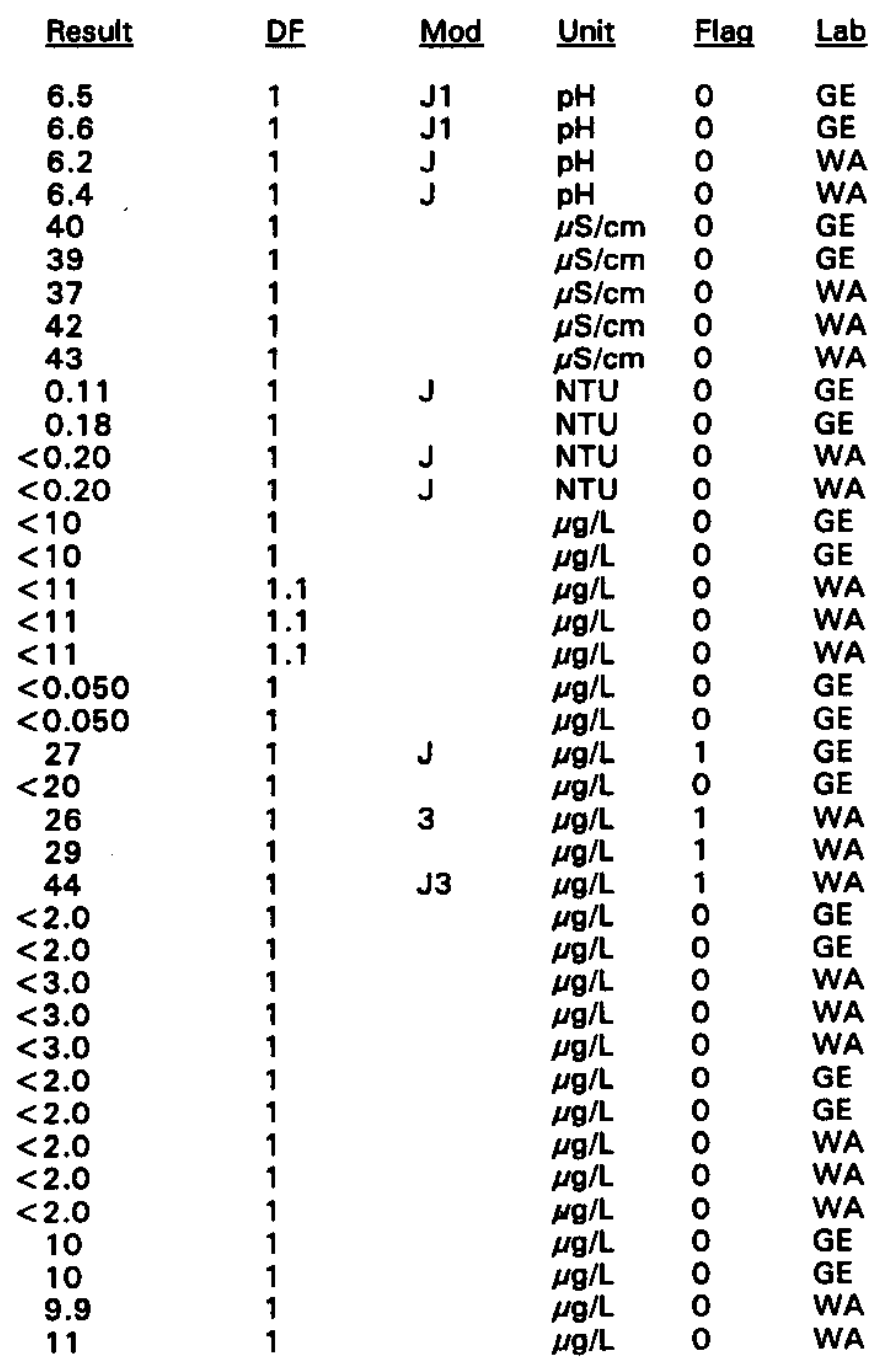

- = exceeded holding time. - =xceeded screening level or final primary drinking water standard. 
WELL BGO 35C collected on 04/11/94, laboratory analyses (cont.)

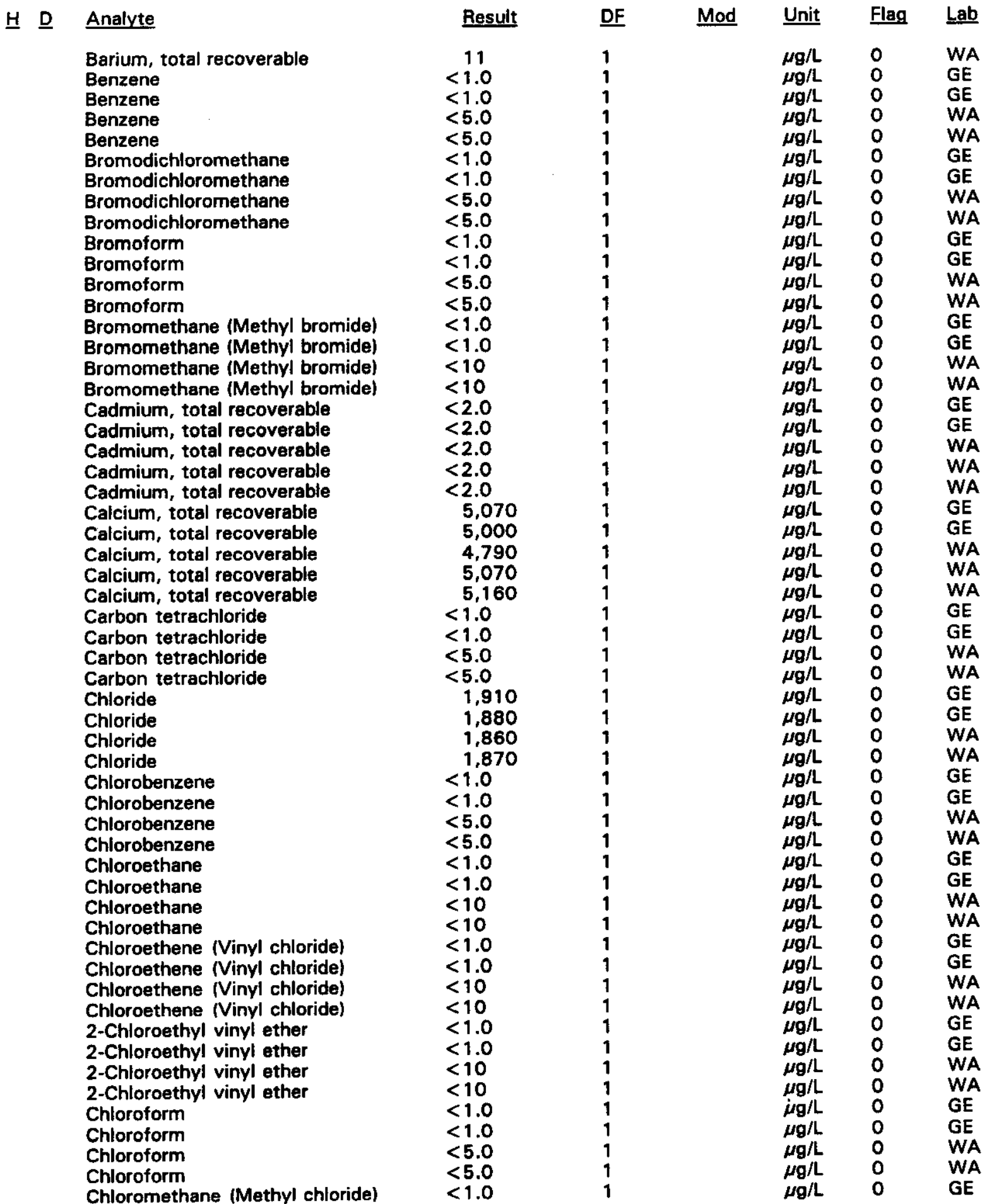

\footnotetext{
- = exceeded holding time. = exceeded screening level or final primary drinking water standard.
} 
WELL BGO $35 \mathrm{C}$ collected on $04 / 11 / 94$, laboratory analyses (cont.)

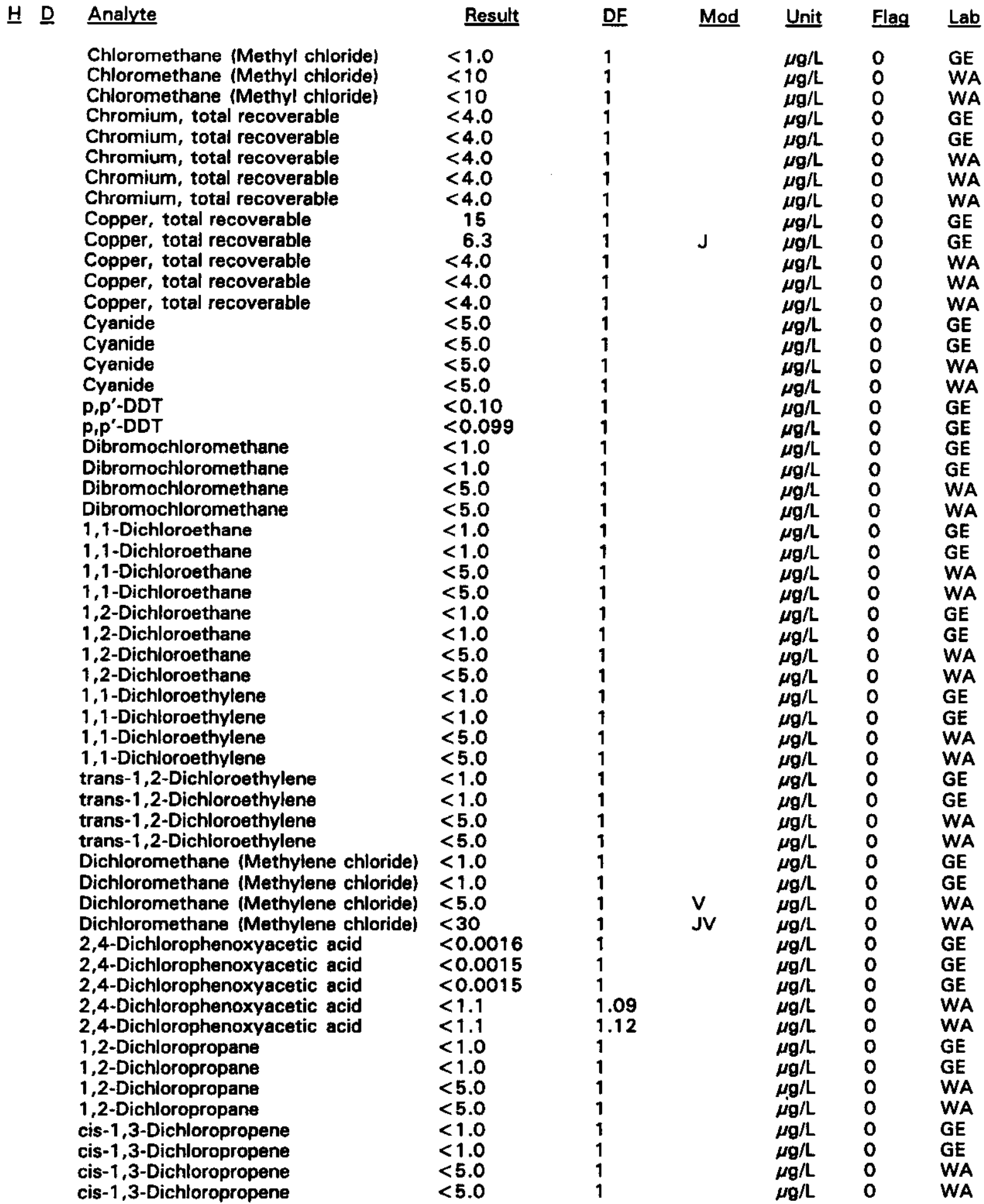

- = exceeded holding time. $\|$ = exceeded screening level or final primary drinking water standard. 
WELL BGO 35C collected on 04/11/94, laboratory analyses (cont.)

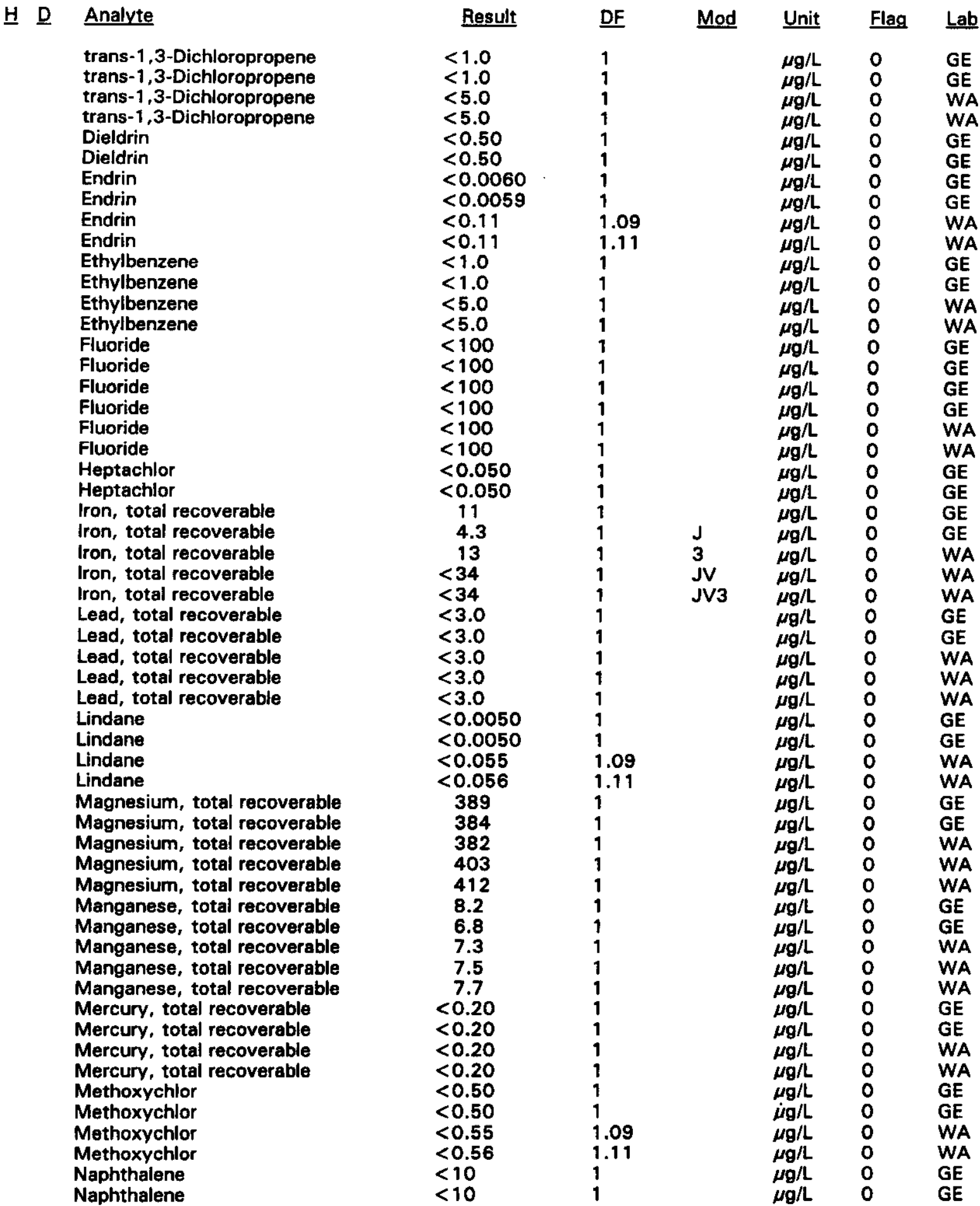

- exceeded holding time. = exceeded screening level or final primary drinking water standard. 
WELL BGO 35C collected on 04/11/94, laboratory analyses (cont.)

\section{H $\underline{\text { Analyte }}$}

Nickel, total recoverable Nickel, total recoverable

Nickel, total recoverable

Nickel, total recoverable

Nickel, total recoverable

Nitrate as nitrogen

Nitrate as nitrogen

Nitrate-nitrite as nitrogen

Nitrate-nitrite as nitrogen

Phenols

Phenols

Phenols

Phenols

Phenols

Potassium, total recoverable

Potassium, total recoverable

Potassium, total recoverable

Potassium, total recoverable

Potassium, total recoverable

Selenium, total recoverable

Selenium, total recoverable

Selenium, total recoverable

Selenium, total recoverable

Selenium, total recoverable

Silica, total recoverable

Silica, total recoverable

Silica, total recoverable

Silica, total recoverable

Silica, total recoverable

Silver, total recoverable

Silver, total recoverable

Silver, total recoverable

Silver, total recoverable

Silver, total recoverable

Sodium, total recoverable

Sodium, total recoverable

Sodium, total recoverable

Sodium, total recoverable

Sodium, total recoverable

Sulfate

Sulfate

Sulfate

Sulfate

1,1,2,2-Tetrachloroethane

1,1,2,2-Tetrachloroethane

1,1,2,2-Tetrachloroethane

1,1,2,2-Tetrachloroethane

Tetrachloroethylene

Tetrachloroethylene

Tetrachloroethylene

Tetrachloroethylene

Tin, total recoverable

Tin, total recoverable

Tin, total recoverable

Tin, total recoverable

Tin, total recoverable

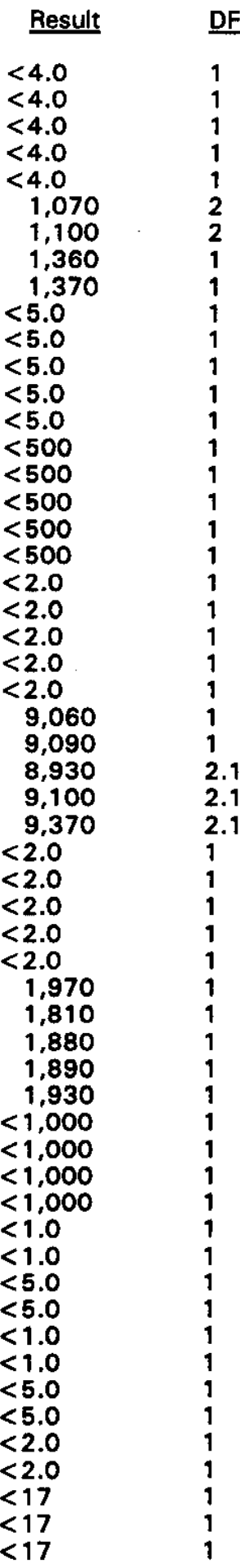

\begin{tabular}{|c|c|c|}
\hline Mod & Unit & Flag \\
\hline $\begin{array}{l}\text { J3 } \\
\text { J3 }\end{array}$ & $\begin{array}{l}\mu g / L \\
\mu g / L \\
\mu g / L \\
\mu g / L \\
\mu g / L \\
\mu g / L \\
\mu g / L \\
\mu g / L \\
\mu g / L \\
\mu g / L \\
\mu g / L \\
\mu g / L \\
\mu g / L \\
\mu g / L \\
\mu g / L \\
\mu g / L \\
\mu g / L \\
\mu g / L \\
\mu g / L \\
\mu g / L \\
\mu g / L \\
\mu g / L \\
\mu g / L \\
\mu g / L \\
\mu g / L \\
\mu g / L \\
\mu g / L\end{array}$ & $\begin{array}{l}0 \\
0 \\
0 \\
0 \\
0 \\
0 \\
0 \\
0 \\
0\end{array}$ \\
\hline
\end{tabular}

- = exceeded holding time. = exceeded screening level or final primary drinking water standard. 
WELL BGO $35 \mathrm{C}$ collected on $04 / 11 / 94$, laboratory analyses (cont.)

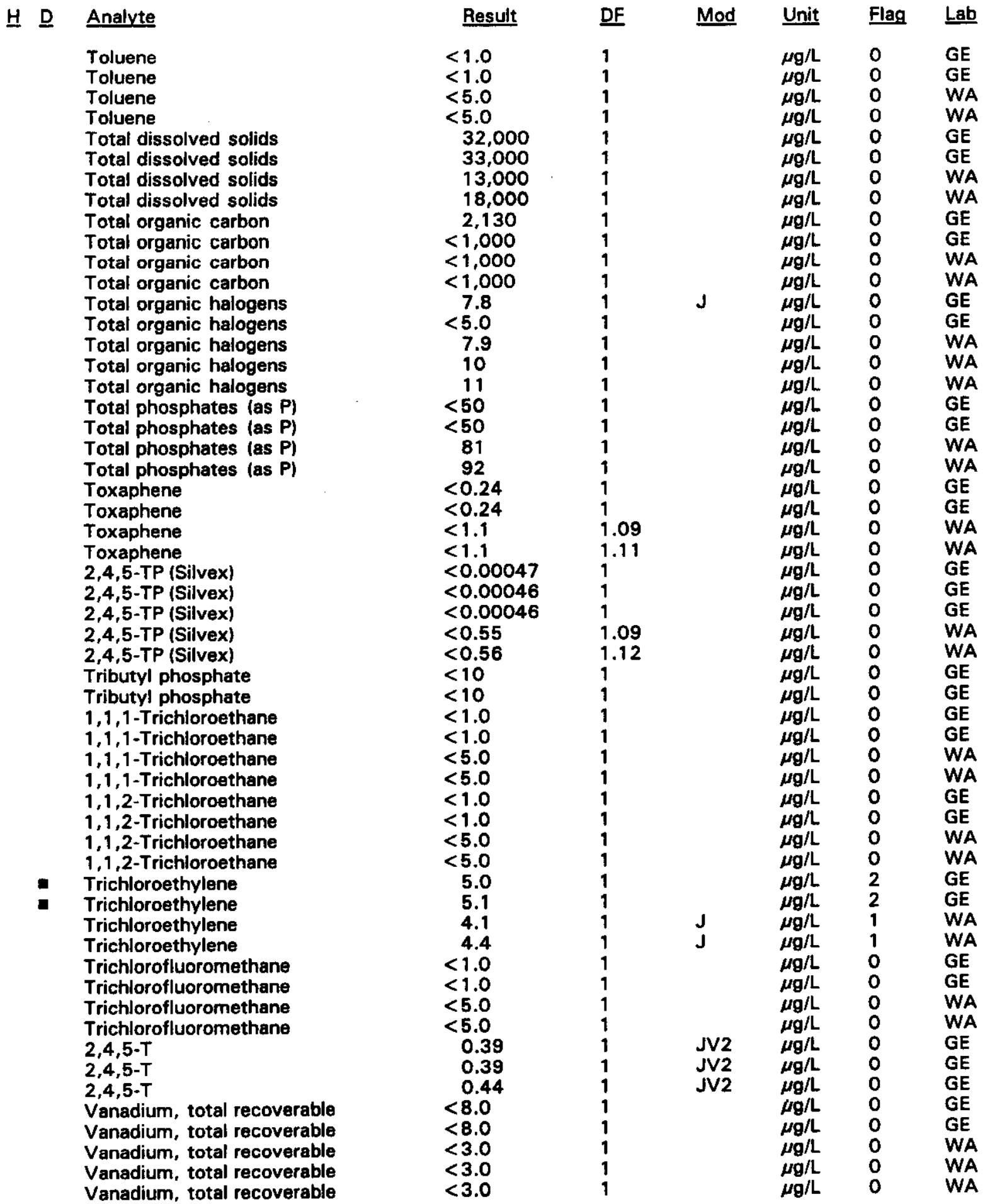

$\overline{-~=~ e x c e e d e d ~ h o l d i n g ~ t i m e . ~}-$ = exceeded screening level or final primary drinking water standard. 
WELL BGO 35C collected on 04/11/94, laboratory analyses (cont.)

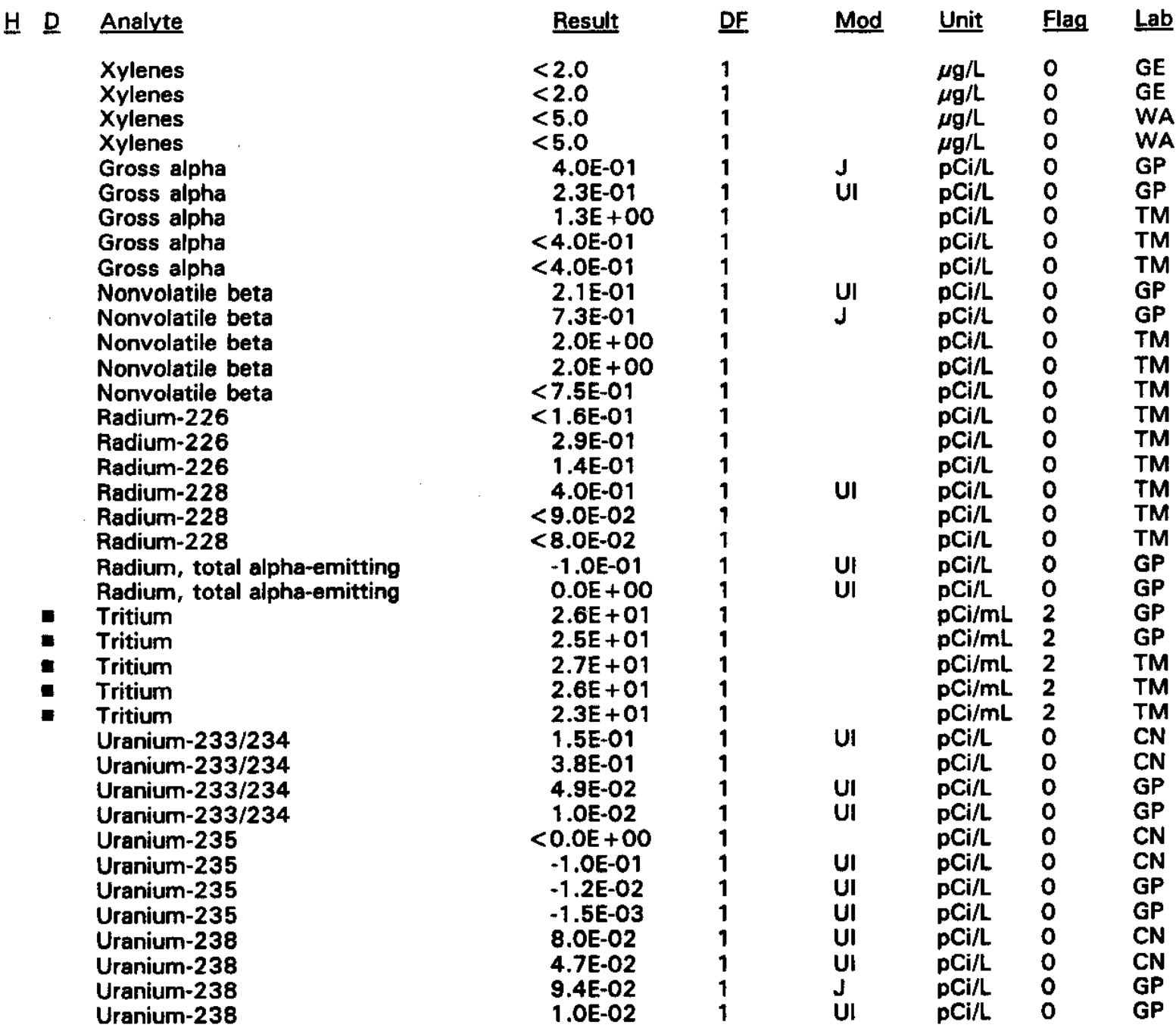

- = exceeded holding time. $\quad$ = exceeded screening level or final primary drinking water standard. 


\section{WELL BGO 35D}

\begin{tabular}{|c|c|c|c|c|c|c|}
\hline SRS Coord. & Lat/Longitude & Screen Zone Elevation & Top of Casing & Casing & Pump & Formation \\
\hline $\begin{array}{l}\text { N73946.0 } \\
\text { E56556.5 }\end{array}$ & $\begin{array}{l}33.281599^{\circ} \mathrm{N} \\
81.661514^{\circ} \mathrm{W}\end{array}$ & $239.4-219.4 \mathrm{ft} \mathrm{msl}$ & $273.5 \mathrm{ft} \mathrm{msl}$ & 4" PVC & $\mathbf{S}$ & Water Table $\left(\| B_{2}\right)$ \\
\hline
\end{tabular}

\section{FIELD MEASUREMENTS}

Sample date: 04/15/94

Depth to water: $38.38 \mathrm{ft}(11.70 \mathrm{~m})$ below TOC

Water elevation: $235.12 \mathrm{ft}(71.67 \mathrm{~m}) \mathrm{msl}$

Sp. conductance: $45 \mu \mathrm{S} / \mathrm{cm}$

Turbidity: 37.5 NTU

Water evacuated before sampling: $11 \mathrm{gal}$

The well went dry during purging.

\section{LABORATORY ANALYSES}

- Analyte
pH
Specific conductance
Turbidity
Acetophenone
Aldrin
Aluminum, total recoverable
Antimony, total recoverable
Arsenic, total recoverable
Barium, total recoverable
Benzene
Bromodichloromethane
Bromoform
Bromomethane (Methyl bromide)
Cadmium, total recoverable
Calcium, total recoverable
Carbon tetrachloride
Chloride
Chlorobenzene
Chloroethane
Chloroethene (Vinyl chloride)
2-Chloroethyl vinyl ether
Chloroform
Chloromethane (Methyl chloride)
Chromium, total recoverable
Copper, total recoverable
Cyanide
p,p'-DDT
Dibromochioromethane
1,1-Dichloroethane
1,2-Dichloroethane
1,1-Dichloroethylene
trans-1,2-Dichloroethylene
Dichloromethane (Methylene chloride)
2,4-Dichlorophenoxyacetic acid
2,4-Dichlorophenoxyacetic acid
1,2-Dichloropropane
cis-1,3-Dichloropropene
trans-1,3-Dichloropropene
Dieldrin

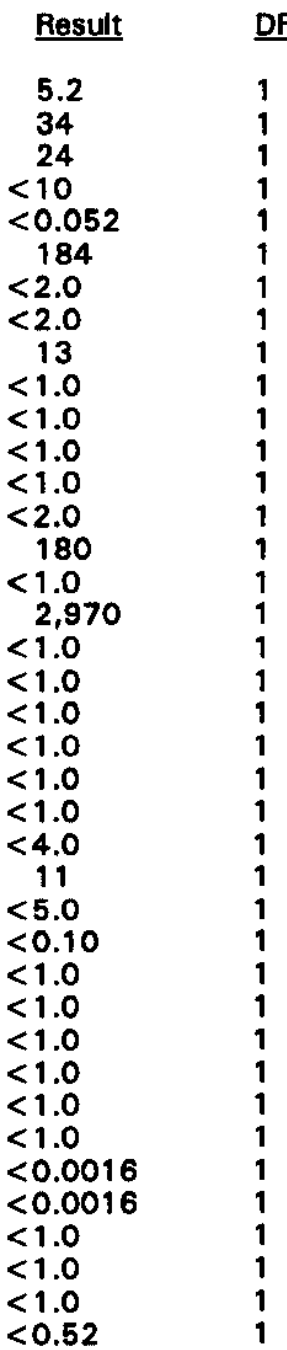

Time: 10:06

pH: 4.8

Alkalinity: $0 \mathrm{mg} / \mathrm{L}$

Water temperature: $20.5^{\circ} \mathrm{C}$

Volumes purged: 1.1 well volumes 
WELL BGO 35D collected on $04 / 15 / 94$, laboratory analyses (cont.)

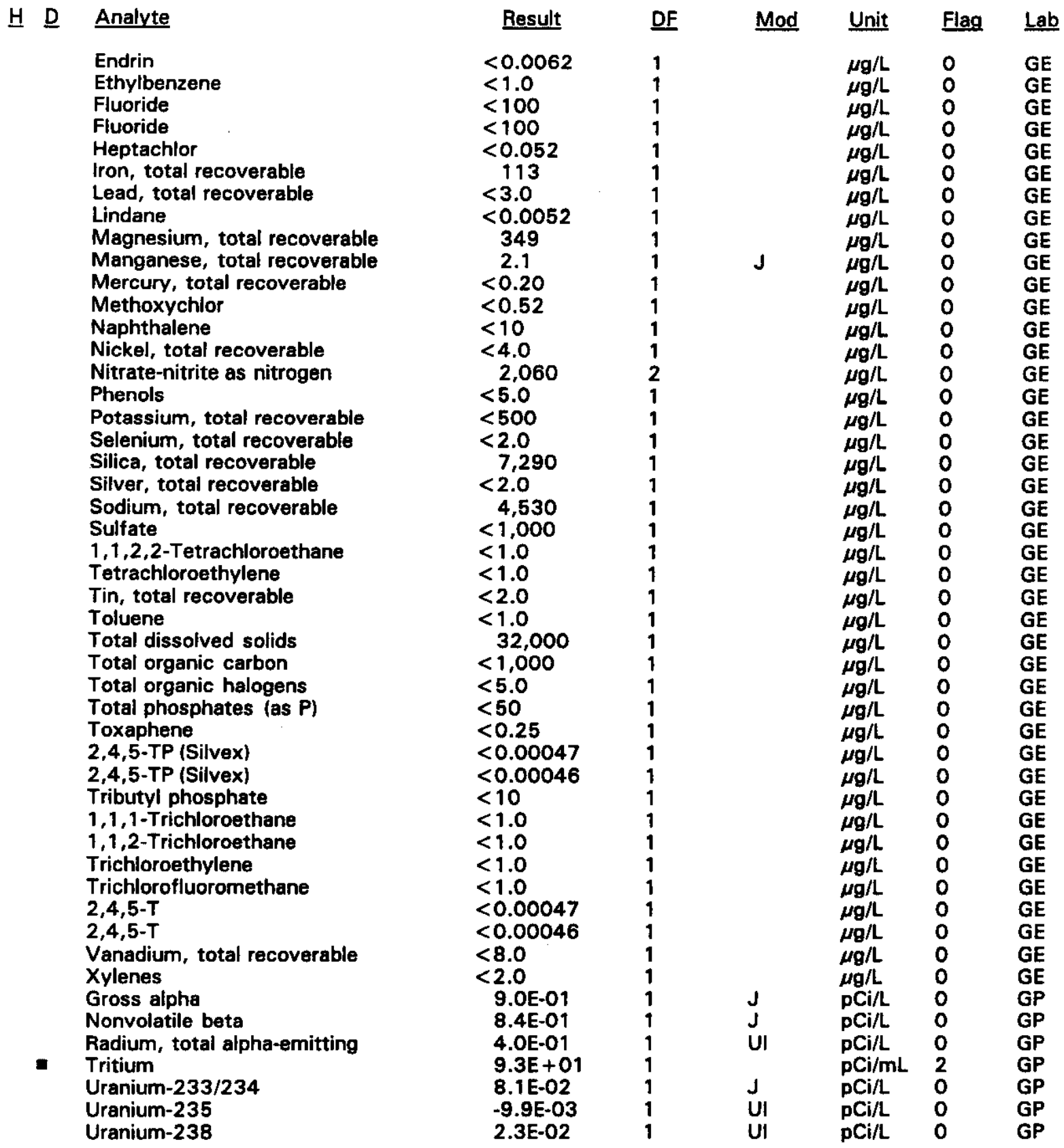

- = exceeded holding time. - = exceeded screening level or final primary drinking water standard. 
WELL BGO 36D

\begin{tabular}{|c|c|c|c|c|c|c|}
\hline SRS Coord. & Lat/Longitude & Screen Zone Elevation & Top of Casing & Casing & Pump & Formation \\
\hline $\begin{array}{l}N 73743.8 \\
E 56888.1\end{array}$ & $\begin{array}{l}33.281692^{\circ} \mathrm{N} \\
81.660248^{\circ} \mathrm{W}\end{array}$ & $243.3-223.3 \mathrm{ft} \mathrm{msl}$ & $275.4 \mathrm{ft} \mathrm{msl}$ & 4" PVC & $\mathbf{S}$ & Water Table $\left(\mathrm{IIB}_{2}\right.$ ) \\
\hline
\end{tabular}

\section{FIELD MEASUREMENTS}

Sample date: 04/15/94

Depth to water: $37.68 \mathrm{ft}(11.49 \mathrm{~m})$ below TOC

Water elevation: $237.72 \mathrm{ft}(72.46 \mathrm{~m}) \mathrm{ms}$ l

Sp. conductance: $35 \mu \mathrm{S} / \mathrm{cm}$

Turbidity: 161 NTU

Water evacuated before sampling: $7 \mathrm{gal}$

The well went dry during purging.

\section{LABORATORY ANALYSES}

H $\underline{D}$

-
Analyte

$\mathrm{pH}$

Specific conductance

Turbidity

Acetophenone

Aldrin

Aluminum, total recoverable

Antimony, total recoverable

Arsenic, total recoverable

Barium, total recoverable

Benzene

Bromodichloromethane

Bromoform

Bromomethane (Methyl bromide)

Cadmium, total recoverable

Calcium, total recoverable

Carbon tetrachloride

Chloride

Chlorobenzene

Chloroethane

Chloroethene (Vinyl chloride)

2-Chloroethyl vinyl ether

Chloroform

Chloromethane (Methyl chloride)

Chromium, total recoverable

Copper, total recoverable

Cyanide

p,p'-DDT

Dibromochloromethane

1,1-Dichloroethane

1,2-Dichloroethane

1,1-Dichloroethylene

trans-1,2-Dichloroethylene

Dichloromethane (Methylene chloride)

2,4-Dichlorophenoxyacetic acid

1,2-Dichloropropane

cis-1,3-Dichloropropene

trans-1,3-Dichloropropene

Dieldrin
Time: 10:31

pH: 4.8

Alkalinity: $0 \mathrm{mg} / \mathrm{L}$

Water temperature: $20.6^{\circ} \mathrm{C}$

Volumes purged: 0.7 well volumes

\begin{tabular}{|c|c|c|c|c|c|}
\hline Result & DF & Mod & Unit & Flag & Lat \\
\hline 5.3 & 1 & J1 & pH & 0 & GE \\
\hline 30 & 1 & & $\mu \mathrm{S} / \mathrm{cm}$ & 0 & GE \\
\hline 47 & 2 & J1 & & & GE \\
\hline$<10$ & 1 & & $\mu g / L$ & 0 & GE \\
\hline$<0.049$ & 1 & & $\mu \mathrm{g} / \mathrm{L}$ & 0 & $\mathrm{GE}$ \\
\hline 1,050 & 1 & & $\mu g / L$ & 2 & GE \\
\hline$<2.0$ & 1 & & $\mu g / L$ & 0 & GE \\
\hline$<2.0$ & 1 & & $\mu g / L$ & 0 & $\mathrm{GE}$ \\
\hline 17 & 1 & & $\mu \mathrm{g} / \mathrm{L}$ & 0 & GE \\
\hline$<1.0$ & 1 & & $\mu g / L$ & 0 & GE \\
\hline$<1.0$ & 1 & & $\mu g / L$ & 0 & GE \\
\hline$<1.0$ & 1 & & $\mu g / L$ & 0 & GE \\
\hline$<1.0$ & 1 & & $\mu g / L$ & 0 & GE \\
\hline$<2.0$ & 1 & & $\mu \mathrm{g} / \mathrm{L}$ & 0 & GE \\
\hline 82 & 1 & & $\mu g / L$ & 0 & $\mathrm{GE}$ \\
\hline$<1.0$ & 1 & & $\mu g / L$ & 0 & iE \\
\hline 3,650 & 1 & & $\mu g / L$ & 0 & GE \\
\hline$<1.0$ & 1 & & $\mu g / L$ & 0 & $\mathrm{GE}$ \\
\hline$<1.0$ & 1 & & $\mu g / L$ & 0 & GE \\
\hline$<1.0$ & 1 & & $\mu g / L$ & 0 & GE \\
\hline$<1.0$ & 1 & & $\mu g / L$ & 0 & GE \\
\hline$<1.0$ & 1 & & $\mu g / L$ & 0 & GE \\
\hline$<1.0$ & 1 & & $\mu g / L$ & 0 & GE \\
\hline$<4.0$ & 1 & & $\mu \mathrm{g} / \mathrm{L}$ & 0 & GE \\
\hline 192 & 1 & & $\mu g / L$ & 0 & E \\
\hline$<5.0$ & 1 & & $\mu \mathrm{g} / \mathrm{L}$ & 0 & \\
\hline$<0.097$ & 1 & & $\mu g / L$ & 0 & GE \\
\hline$<1.0$ & 1 & & $\mu g / L$ & 0 & \\
\hline$<1.0$ & 1 & & $\mu g / L$ & 0 & \\
\hline$<1.0$ & 1 & & $\mu g / L$ & 0 & GE \\
\hline$<1.0$ & 1 & & $\mu \mathrm{g} / \mathrm{L}$ & 0 & GE \\
\hline$<1.0$ & 1 & & $\mu g / L$ & 0 & \\
\hline$<1.0$ & 1 & & $\mu \mathrm{g} / \mathrm{L}$ & 0 & GE \\
\hline$<0.0015$ & 1 & & $\mu g / L$ & 0 & GE \\
\hline$<1.0$ & 1 & & $\mu \mathrm{g} / \mathrm{L}$ & 0 & \\
\hline$<1.0$ & 1 & & $\mu g / L$ & 0 & $\mathrm{GE}$ \\
\hline$<1.0$ & 1 & & $\mu \mathrm{g} / \mathrm{L}$ & 0 & GE \\
\hline$<0.49$ & 1 & & $\mu \mathrm{g} / \mathrm{L}$ & 0 & \\
\hline
\end{tabular}

- = exceeded holding time. = exceeded screening level or final primary drinking water standard. 
WELL BGO 36D collected on 04/15/94, laboratory analyses (cont.)

H

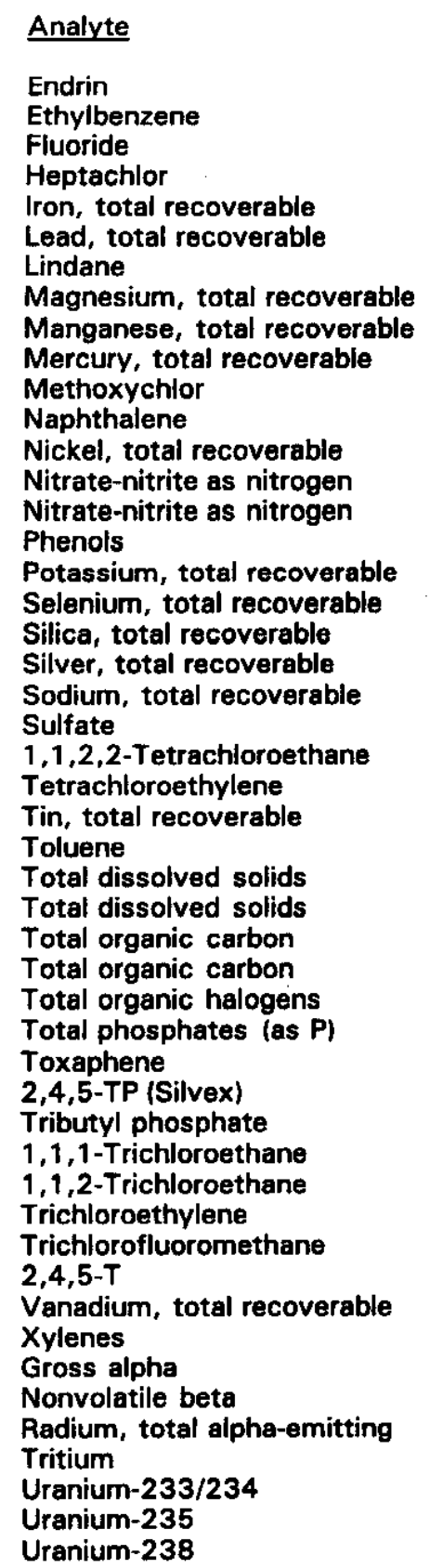

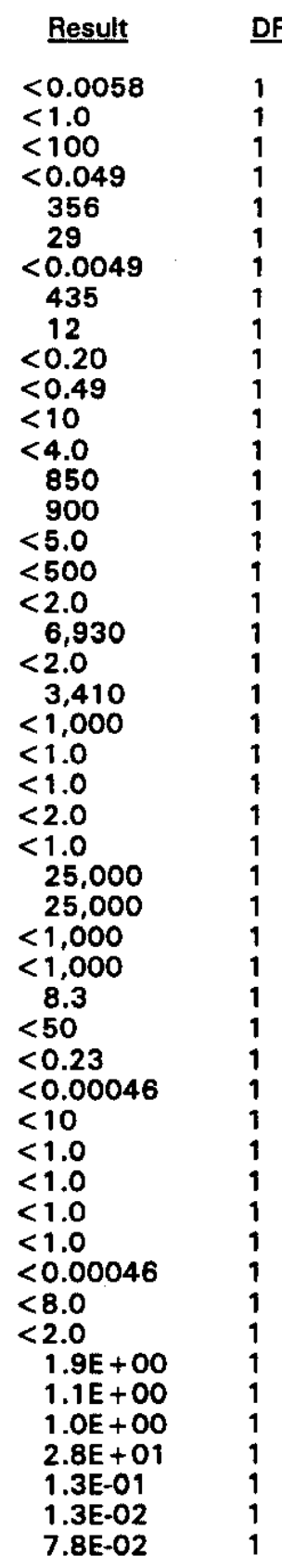

\begin{tabular}{|c|c|c|}
\hline Mod & Unit & Flag \\
\hline $\begin{array}{l}J \\
J \\
J \\
J \\
\mathbf{U} \\
J\end{array}$ & $\begin{array}{l}\mu g / L \\
\mu g / L \\
\mu g / L \\
\mu g / L \\
\mu g / L \\
\mu g / L \\
\mu g / L \\
\mu g / L \\
\mu g / L \\
\mu g / L \\
\mu g / L \\
\mu g / L \\
\mu g / L \\
\mu g / L \\
\mu g / L \\
\mu g / L \\
\mu g / L \\
\mu g / L \\
\mu g / L \\
\mu g / L \\
\mu g / L \\
\mu g / L\end{array}$ & $\begin{array}{l}0 \\
0 \\
0 \\
0 \\
2 \\
1 \\
0 \\
0 \\
0 \\
0 \\
0 \\
0 \\
0 \\
0 \\
0 \\
0 \\
0 \\
0 \\
0 \\
0 \\
0 \\
0 \\
0 \\
0 \\
0 \\
0 \\
0 \\
0 \\
0 \\
0 \\
0 \\
0 \\
0 \\
0 \\
0 \\
0 \\
0 \\
0 \\
0 \\
0 \\
0 \\
0 \\
0 \\
0 \\
0 \\
2 \\
0 \\
0 \\
0\end{array}$ \\
\hline
\end{tabular}

- = exceeded holding time. = exceeded screening level or final primary drinking water standard. 


\section{WELL BGO 37D}

\begin{tabular}{|c|c|c|c|c|c|c|}
\hline SRS Coord. & Lat/Longitude & Screen Zone Elevation & Top of Casing & Casing & Pump & Formation \\
\hline $\begin{array}{l}\text { N73490.8 } \\
\text { E57292.9 }\end{array}$ & $\begin{array}{l}33.281793^{\circ} \mathrm{N} \\
81.658691^{\circ} \mathrm{W}\end{array}$ & $246.1-226.1 \mathrm{ft} \mathrm{msl}$ & $287.3 \mathrm{ft} \mathrm{msl}$ & 4" PVC & $\mathbf{S}$ & Water Table $\left(I \mathrm{BB}_{2}\right)$ \\
\hline
\end{tabular}

\section{FIELD MEASUREMENTS}

Sample date: 04/15/94

Depth to water: $49.80 \mathrm{ft}(15.18 \mathrm{~m})$ below TOC

Water elevation: $237.50 \mathrm{ft}(72.39 \mathrm{~m}) \mathrm{msl}$

Sp. conductance: $39 \mu \mathrm{S} / \mathrm{cm}$

Turbidity: 13.8 NTU

Water evacuated before sampling: $5 \mathrm{gal}$

The well went dry during purging.

\section{LABORATORY ANALYSES}

므 Analyte

- $\mathrm{pH}$

Specific conductance

Turbidity

Acetophenone

Aldrin

Aluminum, total recoverable

Antimony, total recoverable

Arsenic, total recoverable

Barium, total recoverable

Benzene

Bromodichloromethane

Bromoform

Bromomethane (Methyl bromide)

Cadmium, total recoverable

Calcium, total recoverable

Carbon tetrachloride

Chloride

Chlorobenzene

Chioroethane

Chloroethene (Vinyl chloride)

2-Chloroethyl vinyl ether

Chloroform

Chloromethane (Methyl chloride)

Chromium, total recoverable

Copper, total recoverable

Cyanide

p, $p^{\prime}$-DDT

Dibromochloromethane

1,1-Dichloroethane

1,2-Dichioroethane

1,1-Dichloroethylene

trans-1,2-Dichloroethylene

Dichloromethane (Methylene chloride)

2,4-Dichlorophenoxyacetic acid

1,2-Dichloropropane

cis-1,3-Dichloropropene

trans-1,3-Dichloropropene

Dieldrin

Endrin

\begin{tabular}{ll}
$\quad$ Result & DF \\
\hline 5.8 & 1 \\
30 & 1 \\
30 & 1 \\
$<10$ & 1 \\
$<0.051$ & 1 \\
179 & 1 \\
$<2.0$ & 1 \\
$<2.0$ & 1 \\
8.9 & 1 \\
$<1.0$ & 1 \\
$<1.0$ & 1 \\
$<1.0$ & 1 \\
$<1.0$ & 1 \\
$<2.0$ & 1 \\
285 & 1 \\
$<1.0$ & 1 \\
1.740 & 1 \\
$<1.0$ & 1 \\
$<1.0$ & 1 \\
$<1.0$ & 1 \\
$<1.0$ & 1 \\
$<1.0$ & 1 \\
$<1.0$ & 1 \\
$<1.0$ & 1 \\
$<4$ & 13
\end{tabular}

Time: 10:59

pH: 5.3

Alkalinity: $1 \mathrm{mg} / \mathrm{L}$

Water temperature: $19.5^{\circ} \mathrm{C}$

Volumes purged: 0.7 well volumes

\begin{tabular}{|c|c|c|c|c|}
\hline DF & Mod & Unit & Fiag & $\underline{\mathrm{L}}$ \\
\hline 1 & J1 & pH & 0 & G \\
\hline 1 & & $\mu \mathrm{S} / \mathrm{cm}$ & 0 & $\mathrm{G}$ \\
\hline 1 & J1 & NTU & 0 & $\mathbf{G}$ \\
\hline 1 & & $\mu \mathrm{g} / \mathrm{L}$ & 0 & G \\
\hline 1 & & $\mu \mathrm{g} / \mathrm{L}$ & 0 & G \\
\hline 1 & & $\mu \mathrm{g} / \mathrm{L}$ & 2 & G \\
\hline 1 & & $\mu \mathrm{g} / \mathrm{L}$ & 0 & GI \\
\hline 1 & & $\mu g / L$ & 0 & \\
\hline 1 & & $\mu \mathrm{g} / \mathrm{L}$ & 0 & \\
\hline 1 & & $\mu g / L$ & 0 & \\
\hline 1 & & $\mu g / L$ & 0 & \\
\hline 1 & & $\mu g / L$ & 0 & GI \\
\hline 1 & & $\mu g / L$ & 0 & \\
\hline 1 & & $\mu g / L$ & 0 & \\
\hline 1 & & $\mu g / L$ & 0 & \\
\hline 1 & & $\mu \mathrm{g} / \mathrm{L}$ & 0 & G \\
\hline 1 & & $\mu g / L$ & 0 & G \\
\hline 1 & & $\mu g / L$ & 0 & \\
\hline 1 & & $\mu g / L$ & 0 & \\
\hline 1 & & $\mu g / L$ & 0 & \\
\hline 1 & & $\mu g / L$ & 0 & \\
\hline 1 & & $\mu g / L$ & 0 & \\
\hline 1 & & $\mu g / L$ & 0 & \\
\hline 1 & & $\mu \mathrm{g} / \mathrm{L}$ & 0 & \\
\hline 1 & & $\mu g / L$ & 0 & \\
\hline 1 & & $\mu \mathrm{g} / \mathrm{L}$ & 0 & \\
\hline 1 & & $\mu g / L$ & 0 & \\
\hline 1 & & $\mu \mathrm{g} / \mathrm{L}$ & 0 & \\
\hline 1 & & $\mu \mathrm{g} / \mathrm{L}$ & 0 & \\
\hline 1 & & $\mu \mathrm{g} / \mathrm{L}$ & 0 & \\
\hline 1 & & $\mu g / L$ & 0 & \\
\hline 1 & & $\mu g / L$ & 0 & \\
\hline 1 & & $\mu g / L$ & 0 & \\
\hline 1 & & $\mu \mathrm{g} / \mathrm{L}$ & 0 & \\
\hline 1 & & $\mu g / L$ & 0 & \\
\hline 1 & & $\mu g / L$ & 0 & \\
\hline 1 & & $\mu g / L$ & 0 & \\
\hline 1 & & $\mu g / L$ & 0 & \\
\hline 1 & & $\mu g / L$ & 0 & \\
\hline
\end{tabular}

- = exceeded holding time. = exceeded screening level or final primary drinking water standard. 
WELL BGO 37D collected on 04/15/94, laboratory analyses (cont.)

\section{H $\underline{D} \quad$ Analyte}

Ethylbenzene

Fluoride

Heptachlor

Iron, total recoverable

Lead, total recoverable

Lindane

Magnesium, total recoverable

Manganese, total recoverable

Mercury, total recoverable

Methoxychlor

Naphthalene

Nickel, total recoverable

Nitrate-nitrite as nitrogen

Phenols

Potassium, total recoverable

Selenium, total recoverable

Silica, total recoverable

Silver, total recoverable

Sodium, total recoverable

Sulfate

1,1,2,2-Tetrachloroethane

Tetrachloroethylene

Tin, total recoverable

Toluene

Total dissolved solids

Total organic carbon

Total organic halogens

Total phosphates (as P)

Toxaphene

2,4,5-TP (Silvex)

Tributyl phosphate

1,1,1-Trichloroethane

1,1,2-Trichloroethane

Trichloroethylene

Trichlorofluoromethane

2,4,5-T

Vanadium, total recoverable

Xylenes

Gross alpha

Nonvolatile beta

Radium, total alpha-emitting

- Tritium

Uranium-233/234

Uranium-235

Uranium-238

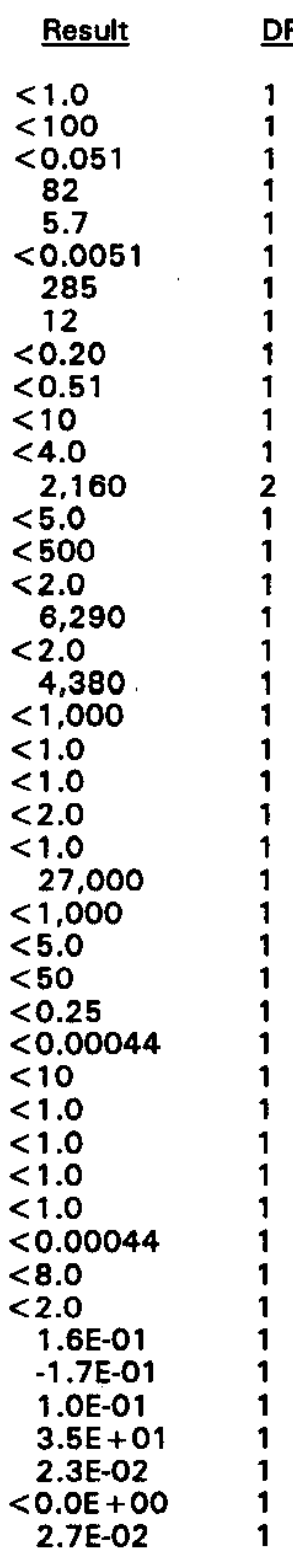

- = exceeded holding time. $\quad$ = exceeded screening level or final primary drinking water standard. 


\section{WELL BGO 38D}

\begin{tabular}{|c|c|c|c|c|c|c|}
\hline SRS Coord. & Lat/Longitude & Screen Zone Elevation & Top of Casing & Casing & Pump & Formation \\
\hline $\begin{array}{l}\text { N73329.3 } \\
\text { E57557.5 }\end{array}$ & $\begin{array}{l}33.281868^{\circ} \mathrm{N} \\
81.657681^{\circ} \mathrm{W}\end{array}$ & $242.3-222.3 \mathrm{ft} \mathrm{msl}$ & $291.6 \mathrm{ft} \mathrm{msl}$ & 4" PVC & $\mathbf{S}$ & Water Table $\left(\mid \mathrm{IB}_{2}\right)$ \\
\hline
\end{tabular}

\section{FIELD MEASUREMENTS}

Sample date: 04/15/94

Depth to water: $56.60 \mathrm{ft}(17.25 \mathrm{~m})$ below TOC

Water elevation: $235.00 \mathrm{ft}(71.63 \mathrm{~m}) \mathrm{msl}$

Sp. conductance: $42 \mu \mathrm{S} / \mathrm{cm}$

Turbidity: 20.5 NTU

Water evacuated before sampling: $7 \mathrm{gal}$

The well went dry during purging.

\section{LABORATORY ANALYSES}

H D Analyte

- $\quad$ H

Specific conductance

- Turbidity

Acetophenone

Aldrin

Aluminum, total recoverable

Antimony, total recoverable

Arsenic, total recoverable

Barium, total recoverable

Benzene

Bromodichloromethane

Bromoform

Bromomethane (Methyl bromide)

Cadmium, total recoverable

Calcium, total recoverable

Carbon tetrachloride

Chloride

Chlorobenzene

Chloroethane

Chloroethene (Vinyl chloride)

2-Chloroethyl vinyl ether

Chloroform

Chloromethane (Methyl chloride)

Chromium, total recoverable

Copper, total recoverable

Cyanide

p, p'-DDT

Dibromochloromethane

1,1-Dichloroethane

1,2-Dichloroethane

1,1-Dichloroethylene

trans-1,2-Dichloroethylene

Dichloromethane (Methylene chloride)

2,4-Dichlorophenoxyacetic acid

1,2-Dichloropropane

cis-1,3-Dichloropropene

trans-1,3-Dichloropropene

Dieldrin

Endrin

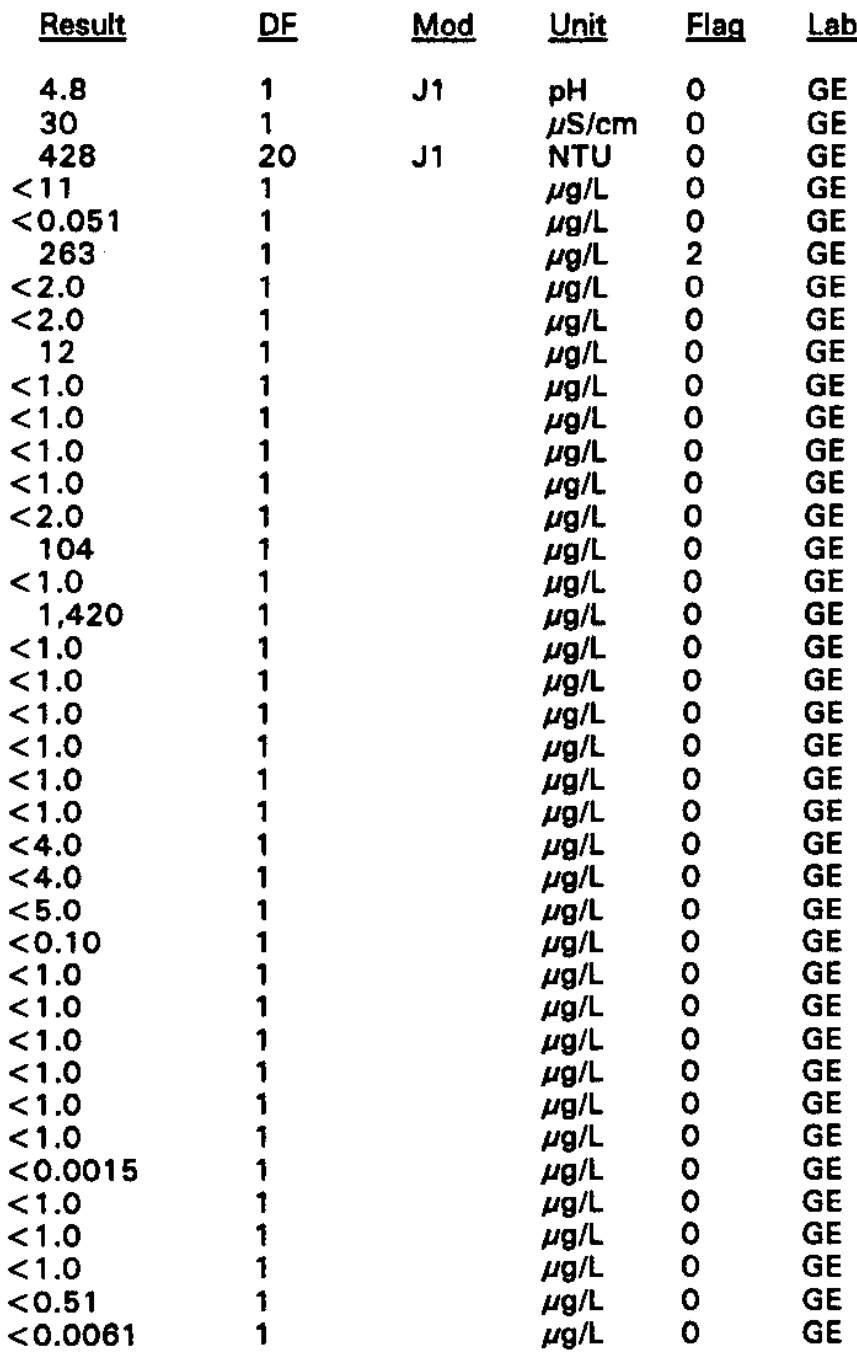

Time: $11: 28$

pH: 4.4

Alkalinity: $0 \mathrm{mg} / \mathrm{L}$

Water temperature: $20.3^{\circ} \mathrm{C}$

Volumes purged: 0.8 well volumes 
WELL BGO 38D collected on $04 / 15 / 94$, laboratory analyses (cont.)

\begin{tabular}{|c|c|c|c|c|c|c|}
\hline$\underline{H} \underline{D}$ & Analyte & Result & DF & Mod & Unit & Flag \\
\hline 口 & 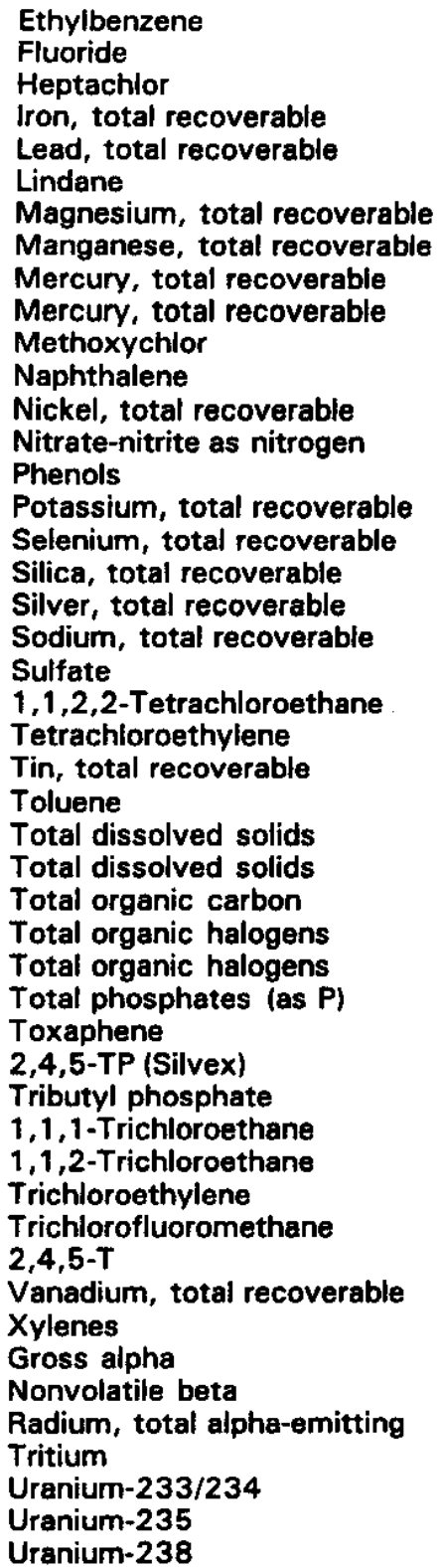 & 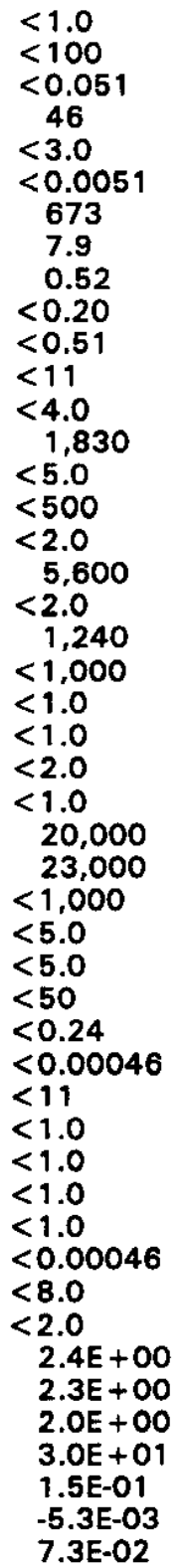 & $\begin{array}{l}1 \\
1 \\
1 \\
1 \\
1 \\
1 \\
1 \\
1 \\
1 \\
1 \\
1 \\
1 \\
1 \\
1 \\
1 \\
1 \\
1 \\
1 \\
1 \\
1 \\
1 \\
1 \\
1 \\
1 \\
1 \\
1 \\
1 \\
1 \\
1 \\
1 \\
1 \\
1 \\
1 \\
1 \\
1 \\
1 \\
1 \\
1 \\
1 \\
1 \\
1 \\
1 \\
1 \\
1 \\
1 \\
1 \\
1 \\
1\end{array}$ & $\begin{array}{l}\mathbf{J} \\
\mathrm{U} \mathbf{I}\end{array}$ & $\begin{array}{l}\mu g / L \\
\mu g / L \\
\mu g / L \\
\mu g / L \\
\mu g / L \\
\mu g / L \\
\mu g / L \\
\mu g / L \\
\mu g / L \\
\mu g / L \\
\mu g / L \\
\mu g / L \\
\mu g / L \\
\mu g / L \\
\mu g / L \\
\mu g / L \\
\mu g / L \\
\mu g / L \\
\mu g / L \\
\mu g / L \\
\mu g / L \\
\mu g / L \\
\mu g / L \\
\mu g / L \\
\mu g / L\end{array}$ & $\begin{array}{l}0 \\
0 \\
0 \\
0 \\
0 \\
0 \\
0 \\
0 \\
0 \\
0 \\
0 \\
0 \\
0 \\
0 \\
0 \\
0 \\
0 \\
0 \\
0 \\
0 \\
0 \\
0 \\
0 \\
0 \\
0 \\
0 \\
0 \\
0 \\
0 \\
0 \\
0 \\
0 \\
0 \\
0 \\
0 \\
0 \\
0 \\
0 \\
0 \\
0 \\
0 \\
0 \\
0 \\
0 \\
2 \\
0 \\
0 \\
0\end{array}$ \\
\hline
\end{tabular}

- exceeded holding time. = exceeded screening level or final primary drinking water standard. 


\section{WELL BGO 39D}

\begin{tabular}{|c|c|c|c|c|c|c|}
\hline SRS Coord. & Lat/Longitude & Screen Zone Elevation & Top of Casing & Casing & Pump & Formation \\
\hline $\begin{array}{l}N 73583.5 \\
\text { E57831.0 }\end{array}$ & $\begin{array}{l}33.282876^{\circ} \mathrm{N} \\
81.657454^{\circ} \mathrm{W}\end{array}$ & $244.7-224.7 \mathrm{ft} \mathrm{msl}$ & $295.7 \mathrm{ft} \mathrm{msl}$ & 4" PVC & $\mathbf{s}$ & Water Table $\left(\mathrm{IIB}_{2}\right)$ \\
\hline
\end{tabular}

\section{FIELD MEASUREMENTS}

Sample date: 04/15/94

Depth to water: $60.81 \mathrm{ft}(18.54 \mathrm{~m})$ below TOC

Water elevation: $234.89 \mathrm{ft}(71.60 \mathrm{~m}) \mathrm{ms}$ l

Sp. conductance: $38 \mu \mathrm{S} / \mathrm{cm}$

Turbidity: 20.4 NTU

Water evacuated before sampling: $5 \mathrm{gal}$

The well went dry during purging.

\section{LABORATORY ANALYSES}

\section{H $\underline{\text { Analyte }}$}

pH

Specific conductance

Turbidity

Acetophenone

Aldrin

Aluminum, total recoverable

Antimony, total recoverable

Arsenic, total recoverable

Barium, total recoverable

Benzene

Benzene

Benzene

Benzene

Bromodichloromethane

Bromodichloromethane

Bromoform

Bromoform

Bromomethane (Methyl bromide)

Bromomethane (Methyl bromide)

Cadmium, total recoverable

Calcium, total recoverable

Carbon tetrachloride

Carbon tetrachloride

Chloride

Chlorobenzene

Chlorobenzene

Chlorobenzene

Chlorobenzene

Chloroethane

Chloroethane

Chloroethene (Vinyl chloride)

Chloroethene (Vinyl chloride)

2-Chloroethyl vinyl ether

2-Chloroethyl vinyl ether

Chioroform

Chloroform

Chloromethane (Methyl chloride)

Chloromethane (Methyl chloride)

Chromium, total recoverable

\begin{tabular}{|c|c|c|c|c|c|}
\hline Result & DF & Mod & Unit & Flag & Lat \\
\hline 5.0 & 1 & J1 & pH & 0 & GE \\
\hline 28 & 1 & & $\mu \mathrm{S} / \mathrm{cm}$ & 0 & $\mathrm{GE}$ \\
\hline 19 & 1 & J1 & NTU & 0 & GE \\
\hline$<10$ & 1 & & $\mu \mathrm{g} / \mathrm{L}$ & 0 & GE \\
\hline$<0.050$ & 1 & & $\mu g / L$ & 0 & GE \\
\hline 108 & 1 & & $\mu \mathrm{g} / \mathrm{L}$ & 2 & GE \\
\hline$<2.0$ & 1 & & $\mu g / L$ & 0 & GE \\
\hline$<2.0$ & 1 & & $\mu \mathrm{g} / \mathrm{L}$ & 0 & GE \\
\hline 5.5 & 1 & & $\mu \mathrm{g} / \mathrm{L}$ & 0 & $\mathrm{GE}$ \\
\hline$<1.0$ & 1 & & $\mu \mathrm{g} / \mathrm{L}$ & 0 & GE \\
\hline$<1.0$ & 1 & & $\mu \mathrm{g} / \mathrm{L}$ & 0 & GE \\
\hline$<1.0$ & 1 & & $\mu g / L$ & 0 & GE \\
\hline$<1.0$ & 1 & & $\mu \mathrm{g} / \mathrm{L}$ & 0 & $\mathbf{E}$ \\
\hline$<1.0$ & 1 & & $\mu g / L$ & 0 & $\mathbf{E}$ \\
\hline$<1.0$ & 1 & & $\mu \mathrm{g} / \mathrm{L}$ & 0 & $\mathbf{E}$ \\
\hline$<1.0$ & 1 & & $\mu \mathrm{g} / \mathrm{L}$ & 0 & $\mathbf{E}$ \\
\hline$<1.0$ & 1 & & $\mu \mathrm{g} / \mathrm{L}$ & 0 & \\
\hline$<1.0$ & $i$ & & $\mu \mathrm{g} / \mathrm{L}$ & 0 & GE \\
\hline$<1.0$ & 1 & & $\mu \mathrm{g} / \mathrm{L}$ & 0 & GE \\
\hline$<2.0$ & 1 & & $\mu \mathrm{g} / \mathrm{L}$ & 0 & $\mathrm{GE}$ \\
\hline 35 & $i$ & & $\mu \mathrm{g} / \mathrm{L}$ & 0 & GE \\
\hline$<1.0$ & 1 & & $\mu \mathrm{g} / \mathrm{L}$ & 0 & GE \\
\hline$<1.0$ & $i$ & & $\mu \mathrm{g} / \mathrm{L}$ & 0 & $\overline{G E}$ \\
\hline 3,130 & 1 & & $\mu g / L$ & 0 & \\
\hline$<1.0$ & 1 & & $\mu \mathrm{g} / \mathrm{L}$ & 0 & \\
\hline$<1.0$ & $i$ & & $\mu \mathrm{g} / \mathrm{L}$ & 0 & $\mathbf{E}$ \\
\hline$<1.0$ & 1 & & $\mu \mathrm{g} / \mathrm{L}$ & 0 & \\
\hline$<1.0$ & 1 & & $\mu g / L$ & 0 & \\
\hline$<1.0$ & $i$ & & $\mu g / L$ & 0 & $\mathrm{E}$ \\
\hline$<1.0$ & 1 & & $\mu \mathrm{g} / \mathrm{L}$ & 0 & \\
\hline$<1.0$ & 1 & & $\mu \mathrm{g} / \mathrm{L}$ & 0 & \\
\hline$<1.0$ & $i$ & & $\mu \mathrm{g} / \mathrm{L}$ & 0 & jE \\
\hline$<1.0$ & 1 & & $\mu g / L$ & 0 & \\
\hline$<1.0$ & 1 & & $\mu \mathrm{g} / \mathrm{L}$ & 0 & GE \\
\hline$<1.0$ & $i$ & & $\mu g / L$ & 0 & \\
\hline$<1.0$ & 1 & & $\mu g / L$ & 0 & \\
\hline$<1.0$ & 1 & & $\mu \mathrm{g} / \mathrm{L}$ & 0 & \\
\hline$<1.0$ & 1 & & $\mu g / L$ & 0 & \\
\hline$<4.0$ & 1 & & $\mu \mathrm{g} / \mathrm{L}$ & 0 & \\
\hline
\end{tabular}

Time: 12:01

pH: 4.6

Alkalinity: $0 \mathrm{mg} / \mathrm{L}$

Water temperature: $20.1^{\circ} \mathrm{C}$

Volumes purged: 0.7 well volumes 
WELL. BGO 390 collected on 04/15/94, laboratory analyses (cont.)

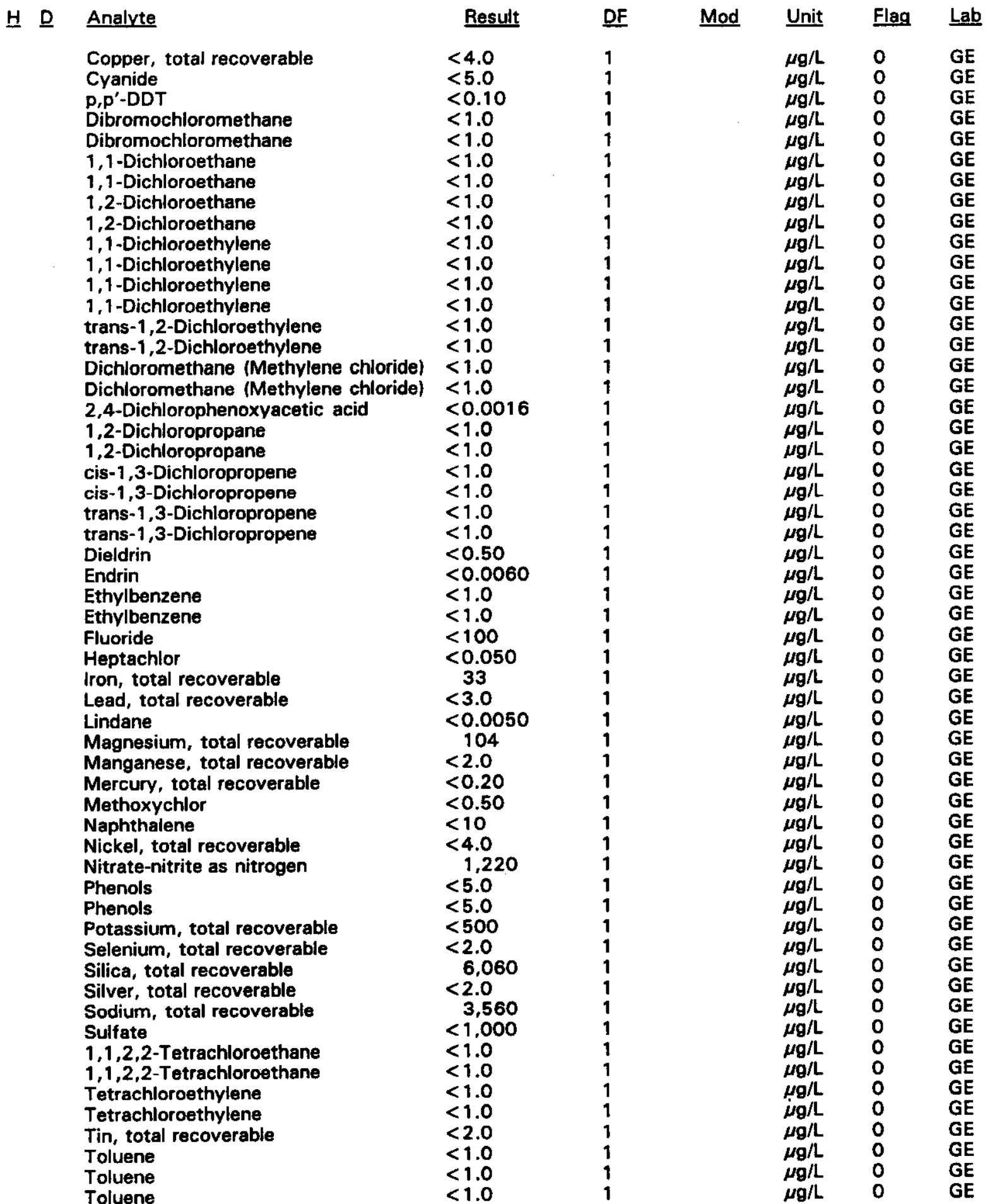

- = exceeded holding time. = exceeded screening level or final primary drinking water standard. 
WELL BGO 390 collected on $04 / 15 / 94$, laboratory analyses (cont.)

브

Analyte
Toluene
Total dissolved solids
Total organic carbon
Total organic halogens
Total phosphates (as P)
Toxaphene
2,4,5-TP (Silvex)
Tributyl phosphate
$1,1,1-T r i c h l o r o e t h a n e$
$1,1,1-T r i c h l o r o e t h a n e$
$1,1,2-T r i c h l o r o e t h a n e$
$1,1,2-T r i c h l o r o e t h a n e$
Trichloroethylene
Trichloroethylene
Trichloroethylene
Trichloroethylene
Trichlorofluoromethane
Trichlorofluoromethane
$2,4,5-T$
Vanadium, total recoverable
Xylenes
Xylenes
Xylenes
Gross alpha
Nonvolatile beta
Radium, total alpha-emitting
Tritium
Uranium-233/234
Uranium-235
Uranium-238

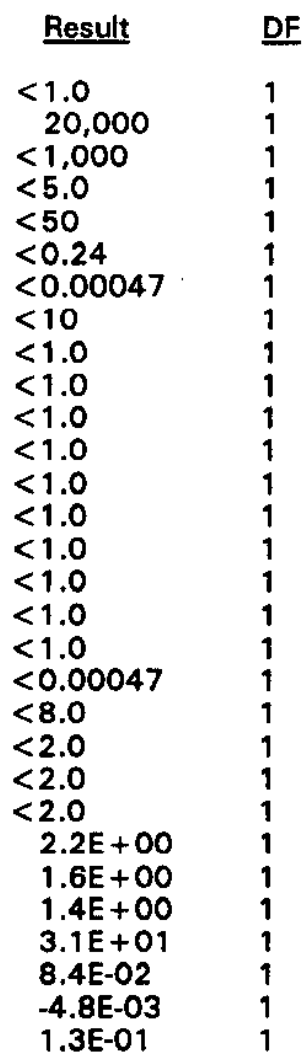

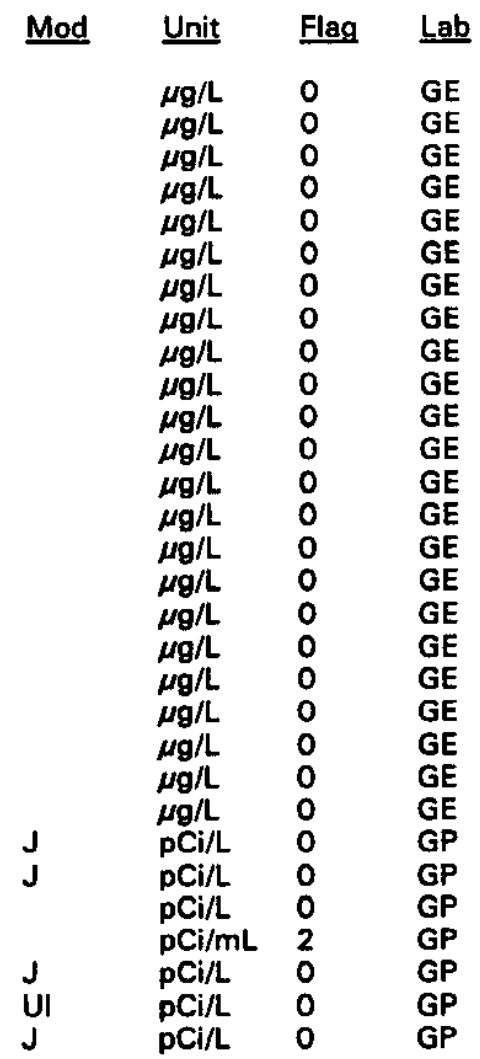

WELL BGO 40D

\begin{tabular}{|c|c|c|c|c|c|c|}
\hline SRS Coord. & Lat/Longitude & Screen Zone Elevation & Top of Casing & Casing & Pump & Formation \\
\hline $\begin{array}{l}N 76125.8 \\
\text { E54638.6 }\end{array}$ & $\begin{array}{l}33.283290^{\circ} \mathrm{N} \\
81.670799^{\circ} \mathrm{W}\end{array}$ & $226.5-216.6 \mathrm{ft} \mathrm{msl}$ & $288.4 \mathrm{ft} \mathrm{msl}$ & 4" PVC & $\mathbf{s}$ & Water Table $\left(\mathrm{IIB}_{2}\right)$ \\
\hline
\end{tabular}

\section{FIELD MEASUREMENTS}

Sample date: 04/14/94

Depth to water: $65.61 \mathrm{ft}(20.00 \mathrm{~m})$ below TOC

Water elevation: $222.79 \mathrm{ft}(67.91 \mathrm{~m}) \mathrm{msl}$

Sp. conductance: $348 \mu \mathrm{S} / \mathrm{cm}$

Turbidity: 9.6 NTU

Water evacuated before sampling: 2 gal

The well went dry during purging.

\section{LABORATORY ANALYSES}

H $\underline{\text { Analyte }}$

$\bullet$

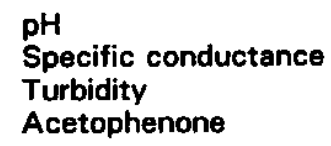

Time: 12:36

pH: 7.0

Alkalinity: $132 \mathrm{mg} / \mathrm{L}$

Water temperature: $21.5^{\circ} \mathrm{C}$

Volumes purged: 0.5 well volumes

- exceeded holding time. = exceeded screening level or final primary drinking water standard. 
WELL BGO 4OD collected on $04 / 14 / 94$, laboratory analyses (cont.)

브 $\underline{\text { Analyte }}$

Aldrin

Aluminum, total recoverable

Aluminum, total recoverable

Antimony, total recoverable

Antimony, total recoverable

Arsenic, total recoverable

Arsenic, total recoverable

Barium, total recoverable

Barium, total recoverable

Benzene

Bromodichloromethane

Bromoform

Bromomethane (Methyl bromide)

Cadmium, total recoverable

Cadmium, total recoverable

Calcium, total recoverable

Calcium, total recoverable

Carbon tetrachloride

Chloride

Chlorobenzene

Chloroethane

Chloroethene (Vinyl chloride)

2-Chloroethyl vinyl ether

Chloroform

Chloromethane (Methyl chloride)

Chromium, total recoverable

Chromium, total recoverable

Copper, total recoverable

Copper, total recoverable

Cyanide

p,p'-DDT

Dibromochloromethane

1,1-Dichioroethane

1,2-Dichioroethane

1,1-Dichloroethylene

trans-1,2-Dichloroethylene

Dichloromethane (Methylene chloride)

2,4-Dichlorophenoxyacetic acid

2,4-Dichlorophenoxyacetic acid

1,2-Dichloropropane

cis-1,3-Dichloropropene

trans-1,3-Dichloropropene

Dieldrin

Endrin

Ethylbenzene

Fluoride

Fluoride

Heptachlor

Iron, total recoverable

Iron, total recoverable

Lead, total recoverable

Lead, total recoverable

Lindane

Magnesium, total recoverable

Magnesium, total recoverable

Manganese, total recoverable
$<0.050$

560

619

$<2.0$

$<2.0$

$<2.0$

$<2.0$

103

104

$<1.0$

$<1.0$

$<1.0$

$<1.0$

$<2.0$

$<2.0$

9,680

9,780

$<1.0$

3,150

$<1.0$

$<1.0$

$<1.0$

$<1.0$

1.4

$<1.0$

$<4.0$

$<4.0$

9.3

9.2

$<5.0$

$<0.10$

$<1.0$

$<1.0$

$<1.0$

$<1.0$

$<1.0$

$<1.0$

$<0.0016$

$<0.0016$

$<1.0$

$<1.0$

$<1.0$

$<0.50$

$<0.0060$

$<1.0$

$<100$

$<100$

$<0.050$

470

497

32

31

$<0.0050$

1,820

1,840

46

\section{DF}

Mod Unit Flag Lab

J3

J3

$\mu \mathrm{g} / \mathrm{L} \quad 0 \quad \mathrm{GE}$

$\mu g / L \quad 2 \quad G E$

$\mu g / L \quad 2 \quad G E$

$\mu g / L \quad 0 \quad \mathrm{GE}$

$\mu \mathrm{g} / \mathrm{L} \quad 0 \quad \mathrm{GE}$

$\mu g / L \quad 0 \quad$ GE

$\mu \mathrm{g} / \mathrm{L} \quad 0 \quad \mathrm{GE}$

$\mu g / L \quad 0 \quad G E$

$\mu g / L \quad 0 \quad \mathrm{GE}$

$\mu g / L \quad 0 \quad G E$

$\mu g / L \quad 0 \quad \mathrm{GE}$

$\mu g / L \quad 0 \quad G E$

$\mu g / L \quad 0 \quad$ GE

$\mu g / L \quad 0 \quad \mathrm{GE}$

$\mu g / \mathrm{L} \quad 0 \quad \mathrm{GE}$

$\mu \mathrm{g} / \mathrm{L} \quad 0 \quad \mathrm{GE}$

$\mu g / L \quad 0 \quad$ GE

$\mu \mathrm{g} / \mathrm{L} \quad \mathrm{O} \quad \mathrm{GE}$

$\mu g / L \quad 0 \quad G E$

$\mu \mathrm{g} / \mathrm{L} \quad \mathrm{O} \quad \mathrm{GE}$

$\mu g / L \quad 0 \quad G E$

$\mu \mathrm{g} / \mathrm{L} \quad 0 \quad \mathrm{GE}$

$\mu g / L \quad 0 \quad G E$

J $\mu g / L \quad 0 \quad G E$

$\mu \mathrm{g} / \mathrm{L} \quad 0 \quad \mathrm{GE}$

$\mu g / L \quad 0 \quad G E$

$\mu \mathrm{g} / \mathrm{L} \quad 0 \quad \mathrm{GE}$

$\mu g / L \quad 0 \quad G E$

$\mu g / L \quad 0 \quad G E$

$\mu \mathrm{g} / \mathrm{L} \quad 0 \quad \mathrm{GE}$

J3 $\mu g / L \quad O \quad G E$

$\mu g / L \quad 0 \quad$ GE

$\mu g / L \quad 0 \quad G E$

$\mu g / L \quad O \quad G E$

$\mu g / L \quad 0 \quad G E$

$\mu g / L \quad O \quad G E$

$\mu g / L \quad 0 \quad G E$

$\mu g / L \quad O \quad G E$

$\mu g / L \quad 0 \quad G E$

$\mu g / L \quad 0 \quad G E$

$\mu g / L \quad 0 \quad G E$

$\begin{array}{lll}\mu g / L & 0 & \text { GE } \\ \text { J3 } & \mu g / L & 0\end{array}$

J3 $\mu \mathrm{g} / \mathrm{L} \quad \mathrm{O} \quad \mathrm{GE}$

$\begin{array}{lll}\mu g / L & 0 & \mathrm{GE} \\ \mu g / L & 0 & \mathrm{GE}\end{array}$

J3 $\mu g / L \quad 0 \quad$ GE

$\begin{array}{lll}\mu g / L & 2 & \mathrm{GE}\end{array}$

J3 $\mu \mathrm{g} / \mathrm{L} \quad 2 \quad \mathrm{GE}$

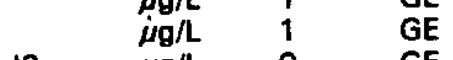

J3 $\mu g / L \quad 0 \quad$ GE

$\mu g / L \quad 0 \quad G E$

$\mu g / L \quad 0 \quad G E$

- = exceeded holding time. = exceeded screening level or final primary drinking water standard. 
WELL BGO 4OD collected on 04/14/94, laboratory analyses (cont.)

\section{H D Analyte}

Manganese, total recoverable

Mercury, total recoverable

Methoxychlor

Naphthalene

Nickel, total recoverable

Nickel, total recoverable

Nitrate-nitrite as nitrogen

Phenols

Potassium, total recoverable

Potassium, total recoverable

Selenium, total recoverable

Selenium, total recoverable

Silica, total recoverable

Silica, total recoverable

Silver, total recoverable

Silver, total recoverable

Sodium, total recoverable

Sodium, total recoverable

Sulfate

1,1,2,2-Tetrachloroethane

Tetrachloroethylene

Tin, total recoverable

Tin, total recoverable

Toluene

Total dissolved solids

Total organic carbon

Total organic halogens

Total phosphates (as P)

Toxaphene

2,4,5-TP (Silvex)

2,4,5-TP (Silvex)

Tributyl phosphate

1,1,1-Trichloroethane

1,1,2-Trichloroethane

Trichloroethylene

Trichlorofluoromethane

2,4,5-T

2,4,5-T

Vanadium, total recoverable

Vanadium, total recoverable

Xylenes

Gross alpha

Nonvolatile beta

Radium, total alpha-emitting

Tritium

Uranium-233/234

Uranium-235

Uranium-238
Result DF

47

$<0.20$

$<0.50$

$<10$

7.5

7.4

1,480

$<5.0$

33,600

33,900

$<2.0$

$<2.0$

7,060

7,350

$<2.0$

$<2.0$

37,900

38,300

4,050

$<1.0$

$<1.0$

74

74

$<1.0$

208,000

1,720

$<5.0$

$<50$

$<0.24$

$<0.00048$

$<0.00047$

$<10$

$<1.0$

$<1.0$

1.9

$<1.0$

$<0.00048$

$<0.00047$

$<8.0$

$<8.0$

$<2.0$

4.5E-01

$1.6 E+01$

1.1E + 00

$7.4 \mathrm{E}+00$

$<0.0 \mathrm{OE}+00$

$-1.7 E-02$

2.3E-01
DF

Mod

J3

Unit Flag Lab

$\mu g / h \quad 1 \quad$ GE

$\mu g / L \quad 0 \quad$ GE

O GE

$\mu \mathrm{g} / \mathrm{L} \quad \mathrm{O} \quad \mathrm{GE}$

$\mu \mathrm{g} / \mathrm{L} \quad 0 \quad \mathrm{GE}$

$\mu \mathrm{g} / \mathrm{L} \quad 0 \quad \mathrm{GE}$

$\mu \mathrm{g} / \mathrm{L} \quad \mathrm{O} \quad \mathrm{GE}$

$\mu g / L \quad 0 \quad$ GE

$\mu g / L \quad 0 \quad$ GE

$\mu \mathrm{g} / \mathrm{L} \quad \mathrm{O} \quad \mathrm{GE}$

$\mu g / L \quad 0 \quad G E$

$\mu g / L \quad 0 \quad$ GE

$\mu g / L \quad 0 \quad$ GE

$\mu g / L \quad 0 \quad G E$

$\mu g / L \quad 0 \quad$ GE

$\mu g / L \quad 0 \quad$ GE

$\mu g / L \quad 0 \quad$ GE

$\mu \mathrm{g} / \mathrm{L} \quad \mathrm{O} \quad \mathrm{GE}$

$\mu g / L \quad 0 \quad$ GE

$\mu \mathrm{g} / \mathrm{L} \quad \mathrm{O} \quad \mathrm{GE}$

$\mu g / L \quad 2 \quad$ GE

$\mu \mathrm{g} / \mathrm{L} \quad 2 \quad \mathrm{GE}$

$\mu g / L \quad 0 \quad$ GE

JV2 $\mu \mathrm{g} / \mathrm{L} \quad 0 \quad \mathrm{GE}$

$\mu \mathrm{g} / \mathrm{L} \quad \mathrm{O} \quad \mathrm{GE}$

$\mu g / L \quad 0 \quad$ GE

$\mu g / L \quad 0 \quad$ GE

J3 $\mu g / L \quad 0 \quad$ GE

$\mu g / L \quad O \quad G E$

$\mu g / L \quad 0 \quad$ GE

$\mu g / L \quad 0 \quad$ GE

$\mu g / L \quad 0 \quad$ GE

$\mu g / L \quad O \quad$ GE

$\mu g / L \quad 0 \quad$ GE

$\mu g / L \quad 0 \quad$ GE

$\mu g / L \quad 0 \quad$ GE

$\mu g / L \quad 0 \quad$ GE

$\mu \mathrm{g} / \mathrm{L} \quad 0 \quad \mathrm{GE}$

$\mu g / L \quad 0 \quad G E$

$\mu g / L \quad 0 \quad$ GE

UI $\quad \mathrm{PCi} / \mathrm{L} \quad \mathrm{P} \quad \mathrm{GP}$

pCi/L $\quad 0 \quad$ GP

$\mathrm{PCi} / \mathrm{L} \quad 0 \quad \mathrm{GP}$

$\mathrm{pCi} / \mathrm{mL} \quad 0 \quad \mathrm{GP}$

$\mathrm{pCi} / \mathrm{L} \quad \mathrm{O} \quad \mathrm{GP}$

$\begin{array}{llll}\text { UI } & \mathrm{PCi} / \mathrm{L} & 0 & \mathrm{GP} \\ \mathrm{J} & \mathrm{pCi} / \mathrm{L} & 0 & \mathrm{GP}\end{array}$

- = exceeded holding time. - = exceeded screening level or final primary drinking water standard. 
WELL BGO 42C

$\begin{array}{lllllll}\text { SRS Coord, } & \text { Lat/Longitude } & \text { Screen Zone Elevation } & \text { Top of Casing } & \text { Casing } & \text { Pump } & \text { Formation } \\ \text { N76404.7 } & 33.285349^{\circ} \mathrm{N} & 195.9-185.9 \mathrm{ft} \mathrm{msl} & 297.9 \mathrm{ft} \mathrm{msl} & \text { 4" PVC } & \text { S } & \text { Barnwell (IIB, } \\ \text { E55522.3 } & 81.669014^{\circ} \mathrm{W} & & & \end{array}$

\section{FIELD MEASUREMENTS}

Sample date: 04/06/94

Depth to water: $74.31 \mathrm{ft}(22.65 \mathrm{~m})$ below TOC

Water elevation: $223.59 \mathrm{ft}(68.15 \mathrm{~m}) \mathrm{ms}$ l

Sp. conductance: $56 \mu \mathrm{S} / \mathrm{cm}$

Turbidity: 21.0 NTU

Water evacuated before sampling: $18 \mathrm{gal}$

The well went dry during purging.

\section{LABORATORY ANALYSES}

\section{H $\underline{\text { Analyte }}$}

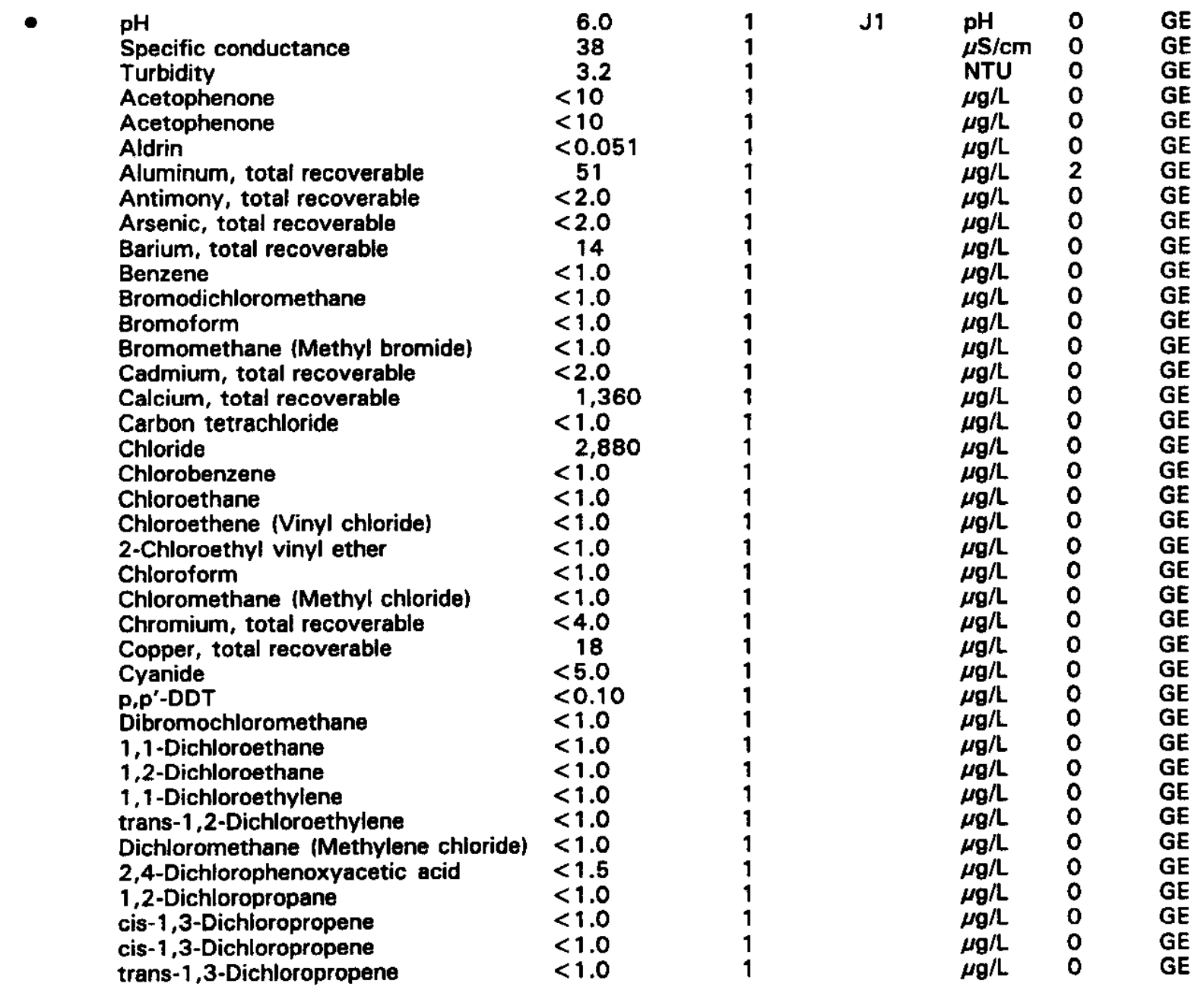

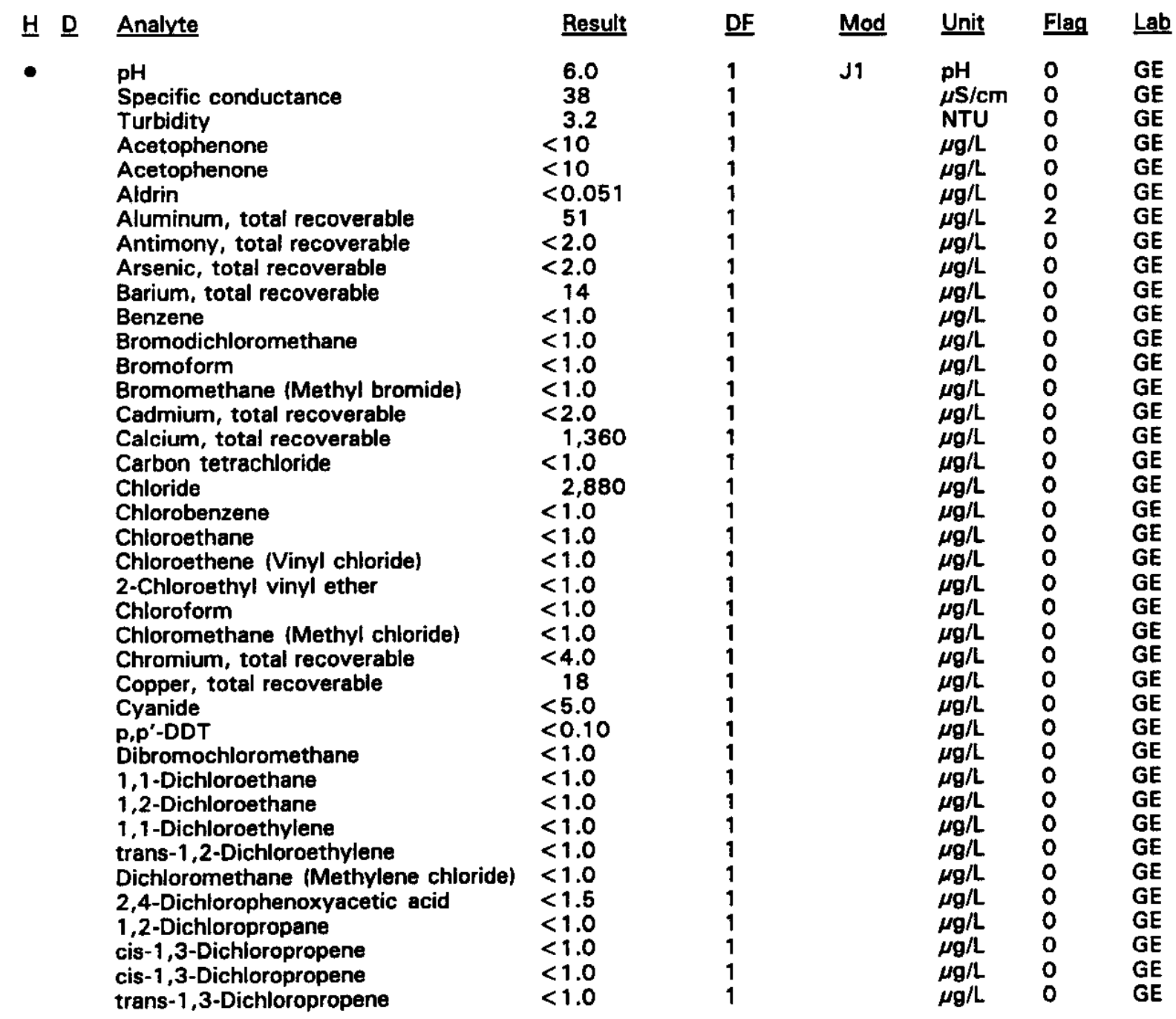

Time: 8:55

$\mathrm{pH}: 6.1$

Alkalinity: $10 \mathrm{mg} / \mathrm{L}$

Water temperature: $18.3^{\circ} \mathrm{C}$

Volumes purged: 0.7 well volumes

- = exceeded holding time. $\square=$ exceeded screening level or final primary drinking water standard. 
WELL BGO $42 \mathrm{C}$ collected on $04 / 06 / 94$, laboratory analyses (cont.)

H D Analyte

Dieldrin

Endrin

Ethylbenzene

Fluoride

Heptachlor

Iron, total recoverable

Lead, total recoverable

Lindane

Magnesium, total recoverable

Manganese, total recoverable

Mercury, total recoverable

Methoxychlor

Naphthalene

Naphthalene

Nickel, total recoverable

Nitrate-nitrite as nitrogen

Phenols

Potassium, total recoverable

Selenium, total recoverable

Silica, total recoverable

Silver, total recoverable

Sodium, total recoverable

Sulfate

1,1,2,2-Tetrachloroethane

Tetrachloroethylene

Tin, total recoverable

Toluene

Total dissolved solids

Total dissolved solids

Total organic carbon

Total organic halogens

Total phosphates (as P)

Toxaphene

2,4,5-TP (Silvex)

1,1,1-Trichloroethane

1,1,2-Trichloroethane

Trichloroethylene

Trichlorofluoromethane

2,4,5-T

Vanadium, total recoverable

Xylenes

Gross alpha

Nonvolatile beta

Radium, total alpha-emitting

Tritium

Uranium-233/234

Uranium-235

Uranium-238

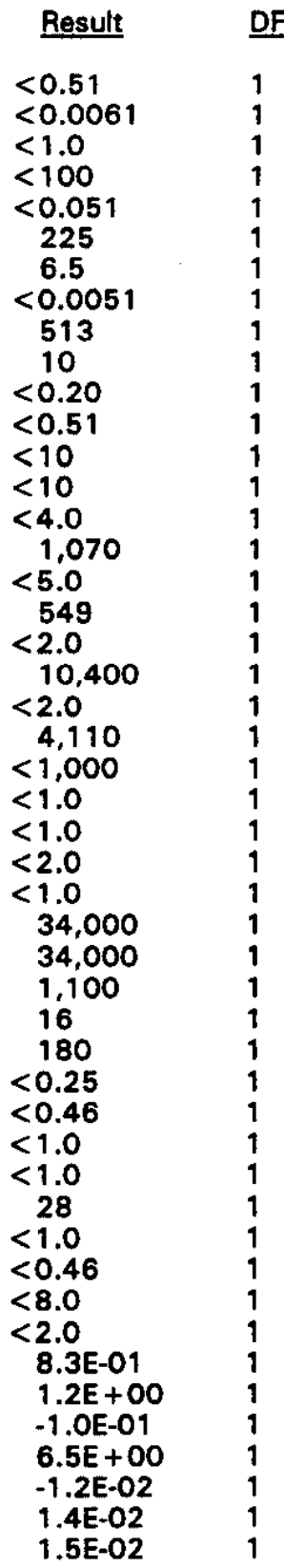

Mod

Unit Flag Lab

$\mu g / L \quad 0 \quad$ GE

$\mu \mathrm{g} / \mathrm{L} \quad \mathrm{O} \quad \mathrm{GE}$

$\mu \mathrm{g} / \mathrm{L} \quad \mathrm{O} \quad \mathrm{GE}$

$\mu g / L \quad 0 \quad G E$

$\mu g / L \quad O \quad G E$

$\mu g / L \quad 1 \quad$ GE

$\mu \mathrm{g} / \mathrm{L} \quad 0 \quad \mathrm{GE}$

$\mu \mathrm{g} / \mathrm{L} \quad \mathrm{O} \quad \mathrm{GE}$

$\mu g / L \quad 0 \quad G E$

$\mu g / L \quad 0 \quad G E$

$\mu g / L \quad 0 \quad \mathrm{GE}$

$\mu \mathrm{g} / \mathrm{L} \quad 0 \quad \mathrm{GE}$

$\mu g / L \quad 0 \quad G E$

$\mu g / \mathrm{L} \quad 0 \quad \mathrm{GE}$

$\mu g / L \quad 0 \quad$ GE

$\mu g / L \quad O \quad G E$

$\mu g / L \quad 0 \quad G E$

J $\mu g / L \quad 0 \quad$ GE

$\mu g / L \quad 0 \quad$ GE

$\mu g / L \quad 0 \quad G E$

$\mu g / L \quad 0 \quad$ GE

$\mu \mathrm{g} / \mathrm{L} \quad \mathrm{O} \quad \mathrm{GE}$

$\mu g / L \quad 0 \quad G E$

$\mu g / L \quad 0 \quad G E$

$\mu \mathrm{g} / \mathrm{L} \quad \mathrm{O} \quad \mathrm{GE}$

$\mu \mathrm{g} / \mathrm{L} \quad 0 \quad \mathrm{GE}$

$\mu \mathrm{g} / \mathrm{L} \quad 0 \quad \mathrm{GE}$

$\mu \mathrm{g} / \mathrm{L} \quad 0 \quad \mathrm{GE}$

$\mu g / L \quad 0 \quad G E$

$\mu g / L \quad 0 \quad \mathrm{GE}$

$\mu \mathrm{g} / \mathrm{L} \quad 0 \quad \mathrm{GE}$

$\mu \mathrm{g} / \mathrm{L} \quad 0 \quad \mathrm{GE}$

$\mu \mathrm{g} / \mathrm{h} \quad \mathrm{O} \quad \mathrm{GE}$

$\mu \mathrm{g} / \mathrm{L} \quad 0 \quad \mathrm{GE}$

$\mu \mathrm{g} / \mathrm{L} \quad 0 \quad \mathrm{GE}$

$\mu \mathrm{g} / \mathrm{L} \quad 0 \quad \mathrm{GE}$

$\mu \mathrm{g} / \mathrm{L} \quad 2 \quad \mathrm{GE}$

$\mu \mathrm{g} / \mathrm{L} \quad 0 \quad \mathrm{GE}$

JV2 $\mu g / L \quad 0 \quad$ GE

$\mu g / L \quad 0 \quad$ GE

$\mu \mathrm{g} / \mathrm{L} \quad 0 \quad \mathrm{GE}$

$\mathrm{pCi} / \mathrm{L} \quad \mathrm{O} \quad \mathrm{GP}$

$\mathrm{pCi} / \mathrm{L} \quad 0 \quad \mathrm{GP}$

pCi/L $\quad 0 \quad$ GP

$\mathrm{pCi} / \mathrm{mL} \quad 0 \quad \mathrm{GP}$

$\mathrm{pCi} / \mathrm{L} \quad 0 \quad \mathrm{GP}$

$\mathrm{pCi} / \mathrm{L} \quad 0 \quad \mathrm{GP}$

$\mathrm{pCi} / \mathrm{L} \quad 0 \quad \mathrm{GP}$

- = exceeded holding time. = exceeded screening level or final primary drinking water standard. 


\section{WELL BGO 43AA}

\begin{tabular}{|c|c|c|c|c|c|c|}
\hline SRS Coord. & Lat/Longitude & Screen Zone Elevation & Top of Casing & Casing & Pump & Formation \\
\hline $\begin{array}{l}N 77066.0 \\
E 56268.6\end{array}$ & $\begin{array}{l}33.288029^{\circ} \mathrm{N} \\
81.668333^{\circ} \mathrm{W}\end{array}$ & $72.2-62.2 \mathrm{ft} \mathrm{msl}$ & $314.3 \mathrm{ft} \mathrm{msl}$ & 4" PVC & $\mathbf{S}$ & L. Congaree (IIA) \\
\hline
\end{tabular}

\section{FIELD MEASUREMENTS}

Sample date: 04/06/94

Depth to water: $157.87 \mathrm{ft}(\mathbf{4 8 . 1 2} \mathrm{m})$ below TOC

Water elevation: $156.43 \mathrm{ft}(47.68 \mathrm{~m}) \mathrm{ms}$ |

Sp. conductance: $430 \mu \mathrm{S} / \mathrm{cm}$

Turbidity: 1.0 NTU

Water evacuated before sampling: $161 \mathrm{gal}$

\section{LABORATORY ANALYSES}

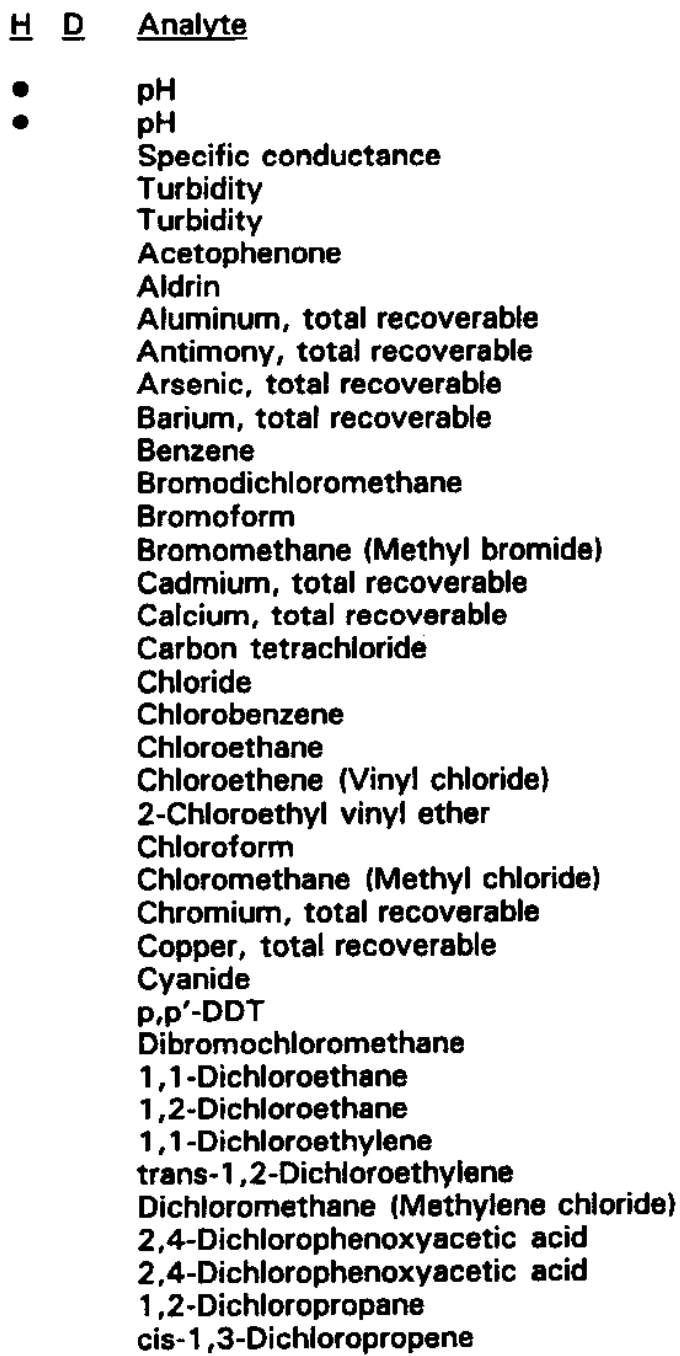

\begin{tabular}{|c|c|c|c|c|}
\hline Result & DF & Mod & Unit & Flag \\
\hline 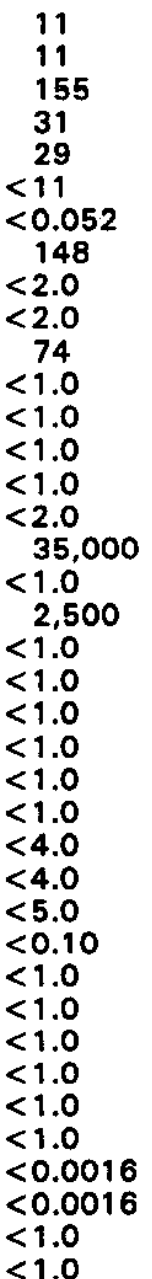 & $\begin{array}{l}1 \\
1 \\
1 \\
1 \\
1 \\
1 \\
1 \\
1 \\
1 \\
1 \\
1 \\
1 \\
1 \\
1 \\
1 \\
1 \\
1 \\
1 \\
1 \\
1 \\
1 \\
1 \\
1 \\
1 \\
1 \\
1 \\
1 \\
1 \\
1 \\
1 \\
1 \\
1 \\
1 \\
1 \\
1 \\
1 \\
1 \\
1 \\
1\end{array}$ & $\begin{array}{l}\text { J1 } \\
\text { J1 }\end{array}$ & 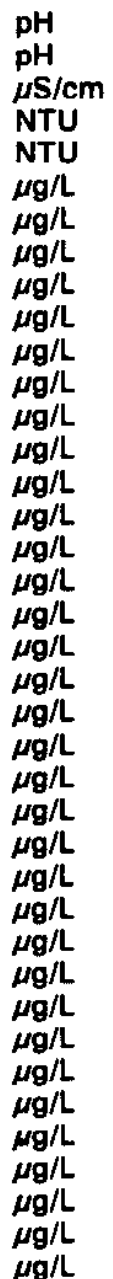 & $\begin{array}{l}2 \\
2 \\
0 \\
0 \\
0 \\
0 \\
0 \\
2 \\
0 \\
0 \\
0 \\
0 \\
0 \\
0 \\
0 \\
0 \\
0 \\
0 \\
0 \\
0 \\
0 \\
0 \\
0 \\
0 \\
0 \\
0 \\
0 \\
0 \\
0 \\
0 \\
0 \\
0 \\
0 \\
0 \\
0 \\
0 \\
0 \\
0 \\
0\end{array}$ \\
\hline
\end{tabular}

Time: $11: 20$

pH: 11.1

Alkalinity: $122 \mathrm{mg} / \mathrm{L}$

Water temperature: $20.0^{\circ} \mathrm{C}$

Volumes purged: 2.6 well volumes

\footnotetext{
- = exceeded holding time. $\square=$ exceeded screening level or final primary drinking water standard.
} 
WELL BGO 43AA collected on 04/06/94, laboratory analyses (cont.)

H $\underline{\text { Analyte }}$

cis-1,3-Dichloropropene

trans-1,3-Dichloropropene

Dieldrin

Endrin

Ethylbenzene

Fluoride

Heptachlor

Iron, total recoverable

Lead, total recoverable

Lindane

Magnesium, total recoverable

Manganese, total recoverable

Mercury, total recoverable

Methoxychlor

Naphthalene

Nickel, total recoverable

Nitrate-nitrite as nitrogen

Phenols

Potassium, total recoverable

Selenium, total recoverable

Silica, total recoverable

Silver, total recoverable

Sodium, total recoverable

Sulfate

1,1,2,2-Tetrachloroethane

Tetrachloroethylene

Tin, total recoverable

Toluene

Total dissolved solids

Total organic carbon

Total organic halogens

Total phosphates (as P)

Toxaphene

2,4,5-TP (Silvex)

2,4,5-TP (Silvex)

1,1,1-Trichloroethane

1,1,2-Trichloroethane

Trichloroethylene

Trichlorofluoromethane

2,4,5-T

2,4,5-T

Vanadium, total recoverable

Xylenes

Gross alpha

Nonvolatile beta

Radium, total alpha-emitting

Tritium

Uranium-233/234

Uranium-235

Uranium-238
Result DF

$<1.0$

$<1.0$

$<0.52$

$<0.0062$

$<1.0$

$<100$

$<0.052$

4.9

3.2

$<0.0052$

488

$<2.0$

$<0.20$

$<0.52$

$<11$

$<4.0$

$<50$

$<5.0$

10,400

$<2.0$

24,500

$<2.0$

8,060

6,420

$<1.0$

$<1.0$

20

$<1.0$

91,000

$<1,000$

$<5.0$

$<50$

$<0.25$

$<0.00047$

$<0.00046$

$<1.0$

$<1.0$

$<1.0$

$<1.0$

0.40

0.45

$<8.0$

$<2.0$

$1.0 E+00$

$5.5 \mathrm{E}+00$

4.OE-01

3.3E-01

2.1E-01

2.1E-02

2.3E-01
Mod Unit Flag Lab

$\mu \mathrm{g} / \mathrm{L} \quad 0 \quad \mathrm{GE}$

$\mu g / L \quad 0 \quad G E$

$\mu g / L \quad 0 \quad$ GE

$\mu \mathrm{g} / \mathrm{L} \quad \mathrm{O} \quad \mathrm{GE}$

$\mu g / L \quad 0 \quad$ GE

$\mu \mathrm{g} / \mathrm{L} \quad \mathrm{O} \quad \mathrm{GE}$

$\mu \mathrm{g} / \mathrm{L} \quad 0 \quad \mathrm{GE}$

$\mu \mathrm{g} / \mathrm{L} \quad \mathrm{O} \quad \mathrm{GE}$

$\mu g / L \quad 0 \quad$ GE

$\mu \mathrm{g} / \mathrm{L} \quad 0 \quad \mathrm{GE}$

$\mu g / L \quad 0 \quad$ GE

$\mu g / L \quad 0 \quad G E$

$\mu g / L \quad 0 \quad$ GE

$\mu g / L \quad 0 \quad$ GE

$\mu g / L \quad 0 \quad$ GE

$\mu g / L \quad 0 \quad$ GE

$\mu g / L \quad 0 \quad$ GE

$\mu \mathrm{g} / \mathrm{L} \quad 0 \quad \mathrm{GE}$

$\mu g / L \quad 0 \quad$ GE

$\mu g / L \quad 0 \quad$ GE

$\mu g / L . \quad 0 \quad$ GE

$\mu g / L \quad 0 \quad$ GE

$\mu \mathrm{g} / \mathrm{L} \quad 0 \quad \mathrm{GE}$

$\mu \mathrm{g} / \mathrm{L} \quad 0 \quad \mathrm{GE}$

$\mu \mathrm{g} / \mathrm{L} \quad 0 \quad \mathrm{GE}$

$\mu \mathrm{g} / \mathrm{L} \quad 0 \quad \mathrm{GE}$

$\mu g / L \quad 2 \quad$ GE

$\mu g / L \quad 0 \quad$ GE

$\mu g / L \quad 0 \quad$ GE

$\mu g / L \quad 0 \quad$ GE

$\mu \mathrm{g} / \mathrm{L} \quad 0 \quad \mathrm{GE}$

$\mu \mathrm{g} / \mathrm{L} \quad \mathrm{O} \quad \mathrm{GE}$

$\mu g / L \quad O \quad G E$

$\mu \mathrm{g} / \mathrm{L} \quad \mathrm{O} \quad \mathrm{GE}$

$\mu g / L \quad 0 \quad$ GE

$\mu g / L \quad 0 \quad$ GE

$\mu g / L \quad 0 \quad$ GE

$\mu g / L \quad 0 \quad$ GE

$\mu g / L \quad 0 \quad$ GE

$\mu g / L \quad 0 \quad$ GE

JV2 $\mu \mathrm{g} / \mathrm{L} \quad 0 \quad \mathrm{GE}$

$\mu g / L \quad 0 \quad$ GE

$\mu g / L \quad$ G $\quad$ GE

$\mathrm{pCi} / \mathrm{L} \quad \mathrm{O} \quad \mathrm{GP}$

$\mathrm{pCi} / \mathrm{L} \quad \mathrm{O} \quad \mathrm{GP}$

$\mathrm{pCi} / \mathrm{L} \quad \mathrm{O} \quad \mathrm{GP}$

$\mathrm{pCi} / \mathrm{mL} \quad 0 \quad \mathrm{GP}$

$\mathrm{pCi} / \mathrm{L} \quad \mathrm{O} \quad \mathrm{GP}$

$\mathrm{pCi} / \mathrm{L} \quad 0 \quad \mathrm{GP}$

$\mathrm{pCi} / \mathrm{L} \quad \mathrm{GP}$

= exceeded holding time. = exceeded screening level or final primary drinking water standard. 
WELL BGO 43CR

$\begin{array}{lllllll}\text { SRS Coord. } & \text { Lat/Longitude } & \text { Screen Zone Elevation } & \text { Top of Casing } & \text { Casing } & \text { Pump Formation } \\ \text { N77035.2 } & 33.287910^{\circ} \mathrm{N} & 188.4-178.4 \mathrm{ft} \mathrm{msl} & 315.3 \mathrm{ft} \mathrm{msl} & \text { 4" PVC } & \text { S } & \text { Barnwell (IIB, }) \\ \text { E56237.2 } & 81.668356^{\circ} \mathrm{W} & & & & \end{array}$

\section{FIELD MEASUREMENTS}

Sample date: 04/06/94

Depth to water: $89.78 \mathrm{ft}(27.37 \mathrm{~m})$ below TOC

Water elevation: $225.52 \mathrm{ft}(68.74 \mathrm{~m}) \mathrm{msl}$

Sp. conductance: $153 \mu \mathrm{S} / \mathrm{cm}$

Turbidity: 4.2 NTU

Water evacuated before sampling: $23 \mathrm{gal}$

The well went dry during purging.

\section{LABORATORY ANALYSES}

H $\underline{\text { Analyte }}$

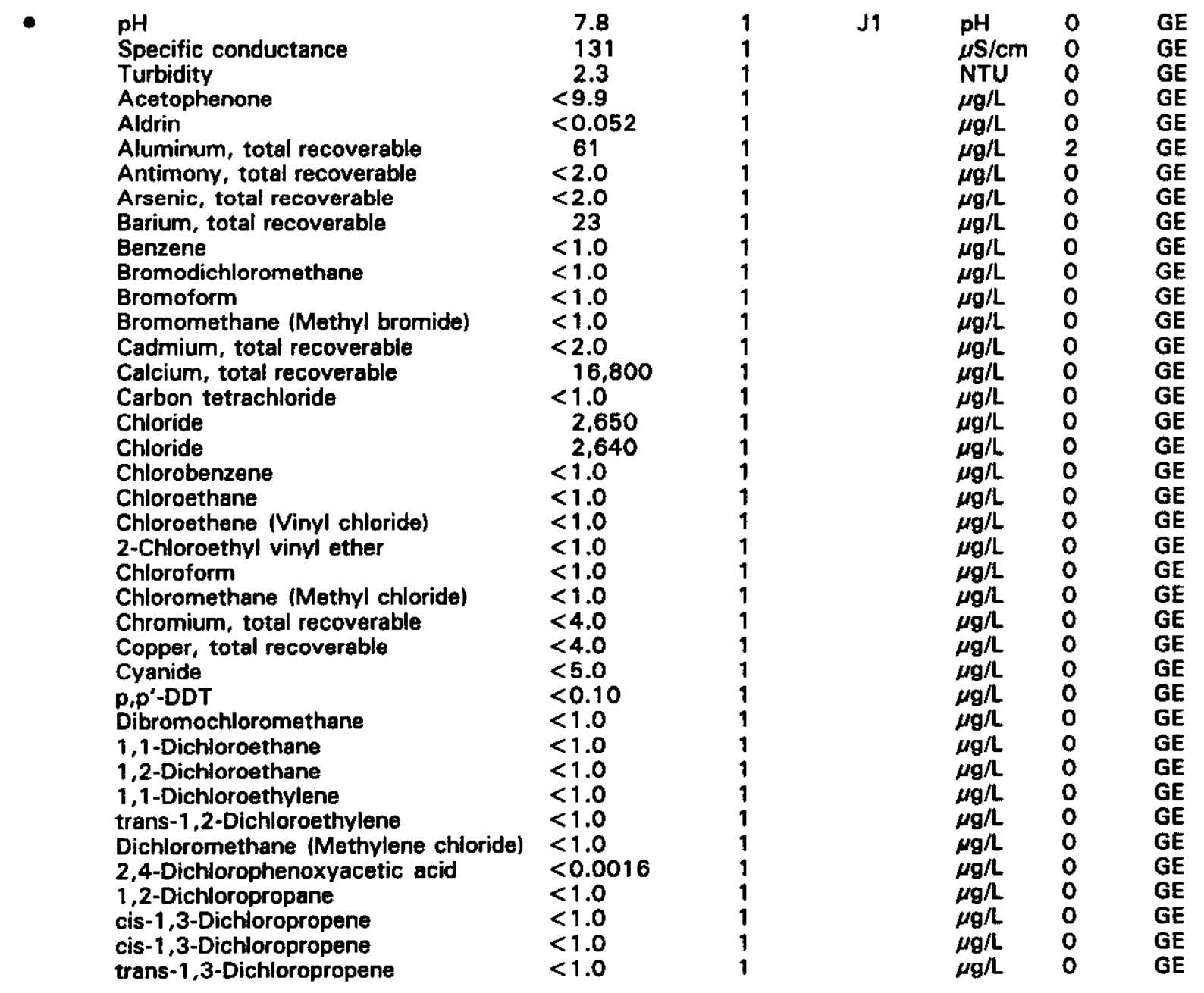

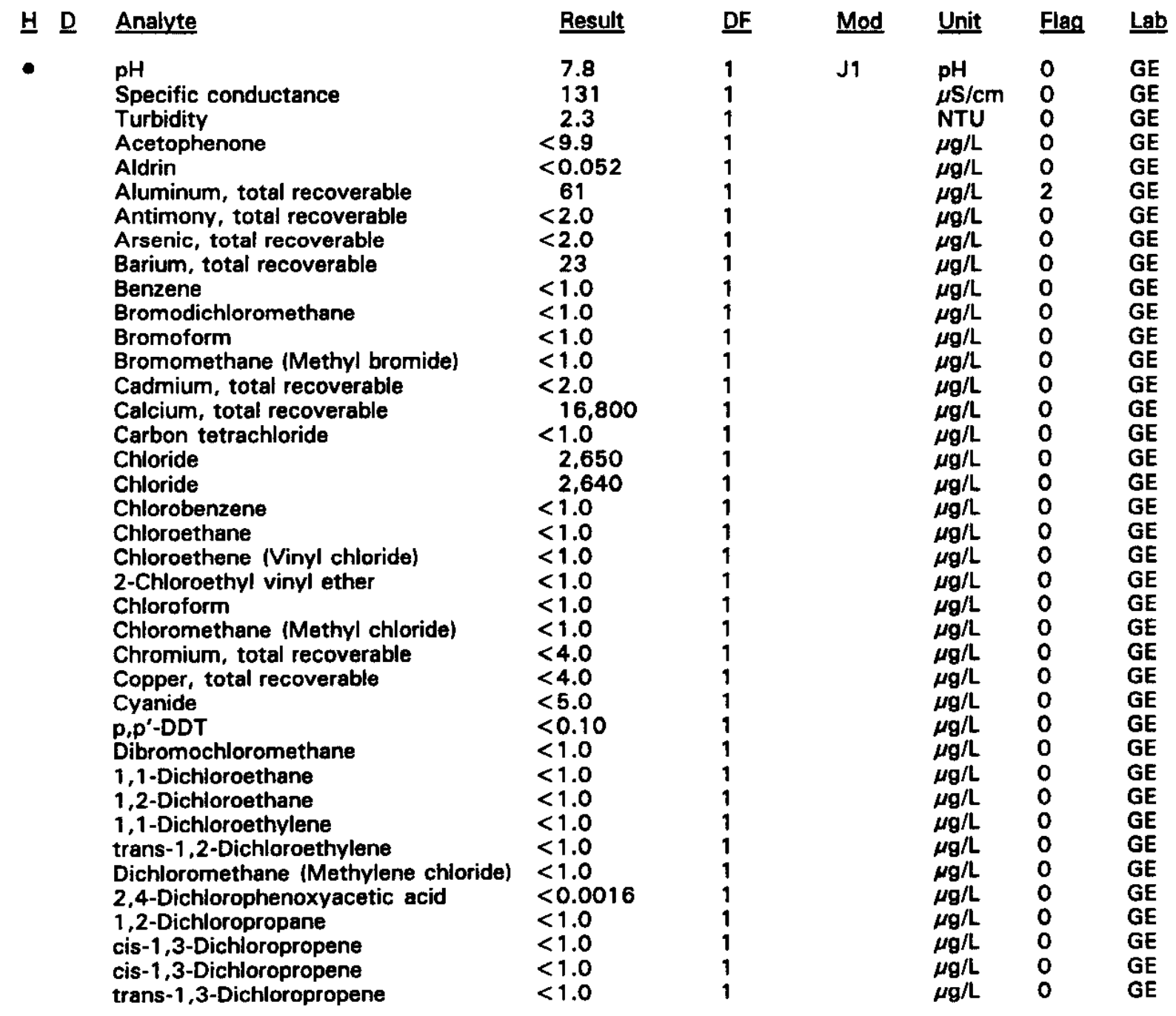

Time: 10:30

pH: 7.1

Alkalinity: $36 \mathrm{mg} / \mathrm{L}$

Water temperature: $18.8{ }^{\circ} \mathrm{C}$

Volumes purged: 0.7 well volumes

- = exceeded holding time. $\quad$ = exceeded screening level or final primary drinking water standard. 
WELL BGO 43CR collected on 04/06/94, laboratory analyses (cont.)

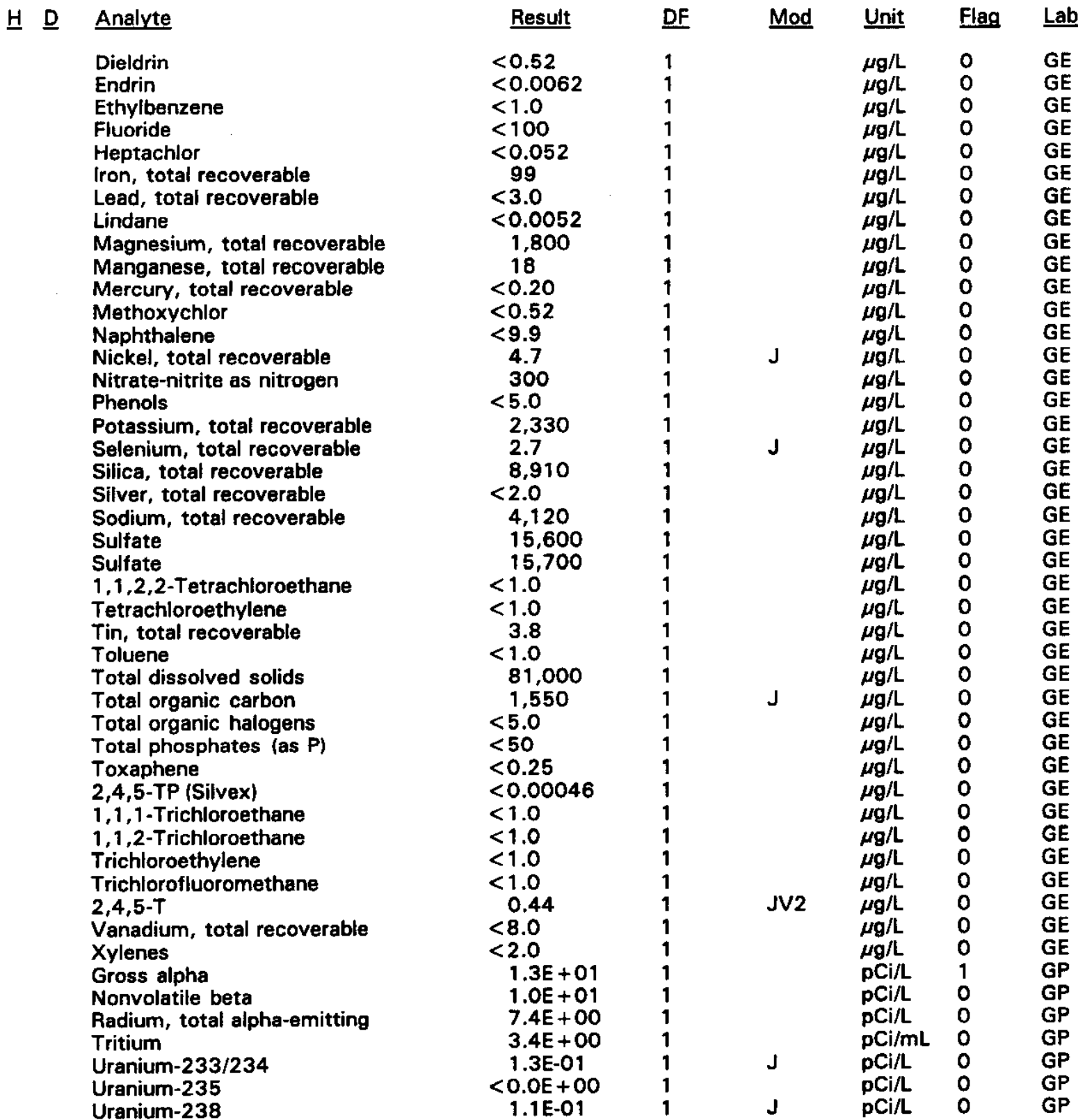

- = exceeded holding time. $\|$ = exceeded screening level or final primary drinking water standard. 
WELL BGO 43D

\begin{tabular}{|c|c|c|c|c|c|c|}
\hline SRS Coord. & Lat/Longitude & Screen Zone Elevation & Top of Casing & Casing & Pump & Formation \\
\hline $\begin{array}{l}N 77056.7 \\
E 56238.8\end{array}$ & $\begin{array}{l}33.287960^{\circ} \mathrm{N} \\
81.668394^{\circ} \mathrm{W}\end{array}$ & $208.2-198.2 \mathrm{ft} \mathrm{msl}$ & $315.3 \mathrm{ft} \mathrm{msl}$ & 4" PVC & $\mathbf{s}$ & Barnwell (IIB ${ }_{1}$ ) \\
\hline
\end{tabular}

\section{FIELD MEASUREMENTS}

Sample date: 04/06/94

Depth to water: $83.75 \mathrm{ft}(25.53 \mathrm{~m})$ below TOC

Water elevation: $231.55 \mathrm{ft}(70.58 \mathrm{~m}) \mathrm{msl}$

Sp. conductance: $80 \mu \mathrm{S} / \mathrm{cm}$

Turbidity: 0.6 NTU

Water evacuated before sampling: $103 \mathrm{gal}$

\section{LABORATORY ANALYSES}

H D Analyte

pH

Specific conductance

Turbidity

Acetophenone

Aldrin

Aluminum, total recoverable

Antimony, total recoverable

Arsenic, total recoverable

Barium, total recoverable

Benzene

Bromodichloromethane

Bromoform

Bromomethane (Methyl bromide)

Cadmium, total recoverable

Calcium, total recoverable

Carbon tetrachloride

Chloride

Chlorobenzene

Chloroethane

Chloroethene (Vinyl chloride)

2-Chloroethyl vinyl ether

Chloroform

Chloromethane (Methyl chloride)

Chromium, total recoverable

Copper, total recoverable

Cyanide

p, p'-DDT

Dibromochloromethane

1,1-Dichloroethane

1,2-Dichloroethane

1,1-Dichloroethylene

trans-1,2-Dichloroethylene

Dichloromethane (Methylene chloride)

- 2,4-Dichlorophenoxyacetic acid

1,2-Dichloropropane

cis-1,3-Dichloropropene

trans-1,3-Dichloropropene

Dieldrin

Endrin

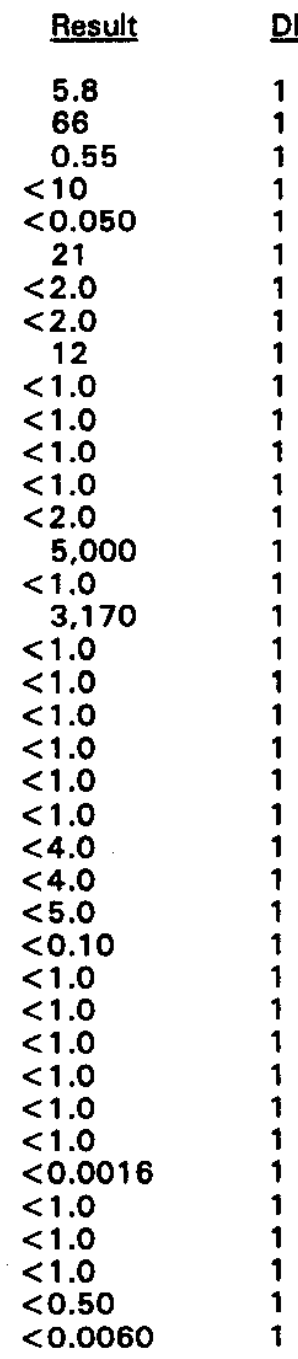

Time: $10: 57$

pH: $\mathbf{5 . 9}$

Alkalinity: $4 \mathrm{mg} / \mathrm{L}$

Water temperature: $19.1^{\circ} \mathrm{C}$

Volumes purged: 4.7 well volumes

\begin{tabular}{|c|c|c|c|c|}
\hline DF & Mod & Unit & Flag & Lak \\
\hline 1 & J1 & pH & 0 & GE \\
\hline 1 & & $\mu \mathrm{S} / \mathrm{cm}$ & 0 & $\mathrm{GE}$ \\
\hline 1 & & NTU & 0 & GE \\
\hline 1 & & $\mu g / L$ & 0 & GE \\
\hline 1 & & $\mu g / L$ & 0 & GE \\
\hline 1 & $J$ & $\mu g / L$ & 0 & GE \\
\hline$i$ & & $\mu \mathrm{g} / \mathrm{L}$ & 0 & GE \\
\hline 1 & & $\mu \mathrm{g} / \mathrm{L}$ & 0 & GE \\
\hline 1 & & $\mu g / L$ & 0 & \\
\hline 1 & & $\mu \mathrm{g} / \mathrm{L}$ & 0 & GE \\
\hline 1 & & $\mu g / L$ & 0 & GE \\
\hline 1 & & $\mu \mathrm{g} / \mathrm{L}$ & 0 & GE \\
\hline 1 & & $\mu g / L$ & 0 & GE \\
\hline 1 & & $\mu g / L$ & 0 & $\mathrm{GE}$ \\
\hline 1 & & $\mu g / L$ & 0 & GE \\
\hline 1 & & $\mu g / L$ & 0 & GE \\
\hline 1 & & $\mu \mathrm{g} / \mathrm{L}$ & 0 & GE \\
\hline 1 & & $\mu \mathrm{g} / \mathrm{L}$ & 0 & GE \\
\hline 1 & & $\mu \mathrm{g} / \mathrm{L}$ & 0 & $\mathrm{GE}$ \\
\hline 1 & & $\mu g / L$ & 0 & GE \\
\hline 1 & & $\mu g / L$ & 0 & \\
\hline 1 & & $\mu \mathrm{g} / \mathrm{L}$ & 0 & GE \\
\hline 1 & & $\mu g / L$ & 0 & GE \\
\hline 1 & & $\mu g / L$ & 0 & \\
\hline 1 & & $\mu g / L$ & 0 & GE \\
\hline 1 & & $\mu g / L$ & 0 & \\
\hline 1 & & $\mu g / L$ & 0 & \\
\hline 1 & & $\mu g / L$ & 0 & GE \\
\hline 1 & & $\mu g / L$ & 0 & \\
\hline 1 & & $\mu \mathrm{g} / \mathrm{L}$ & 0 & \\
\hline 1 & & $\mu g / L$ & 0 & $\mathrm{SE}$ \\
\hline 1 & & $\mu \mathrm{g} / \mathrm{L}$ & 0 & \\
\hline 1 & J1 & $\mu g / L$ & 0 & \\
\hline 1 & & $\mu g / L$ & O & \\
\hline $\begin{array}{l}1 \\
1\end{array}$ & & $\mu g / L$ & $\begin{array}{l}0 \\
0\end{array}$ & \\
\hline 1 & & $\mu g / L$ & 0 & GE \\
\hline 1 & & $\mu \mathrm{g} / \mathrm{L}$ & 0 & \\
\hline
\end{tabular}

$\overline{- \text { = exceeded holding time. }}$ = exceeded screening level or final primary drinking water standard. 
WELL BGO 430 collected on $04 / 06 / 94$, laboratory analyses (cont.)

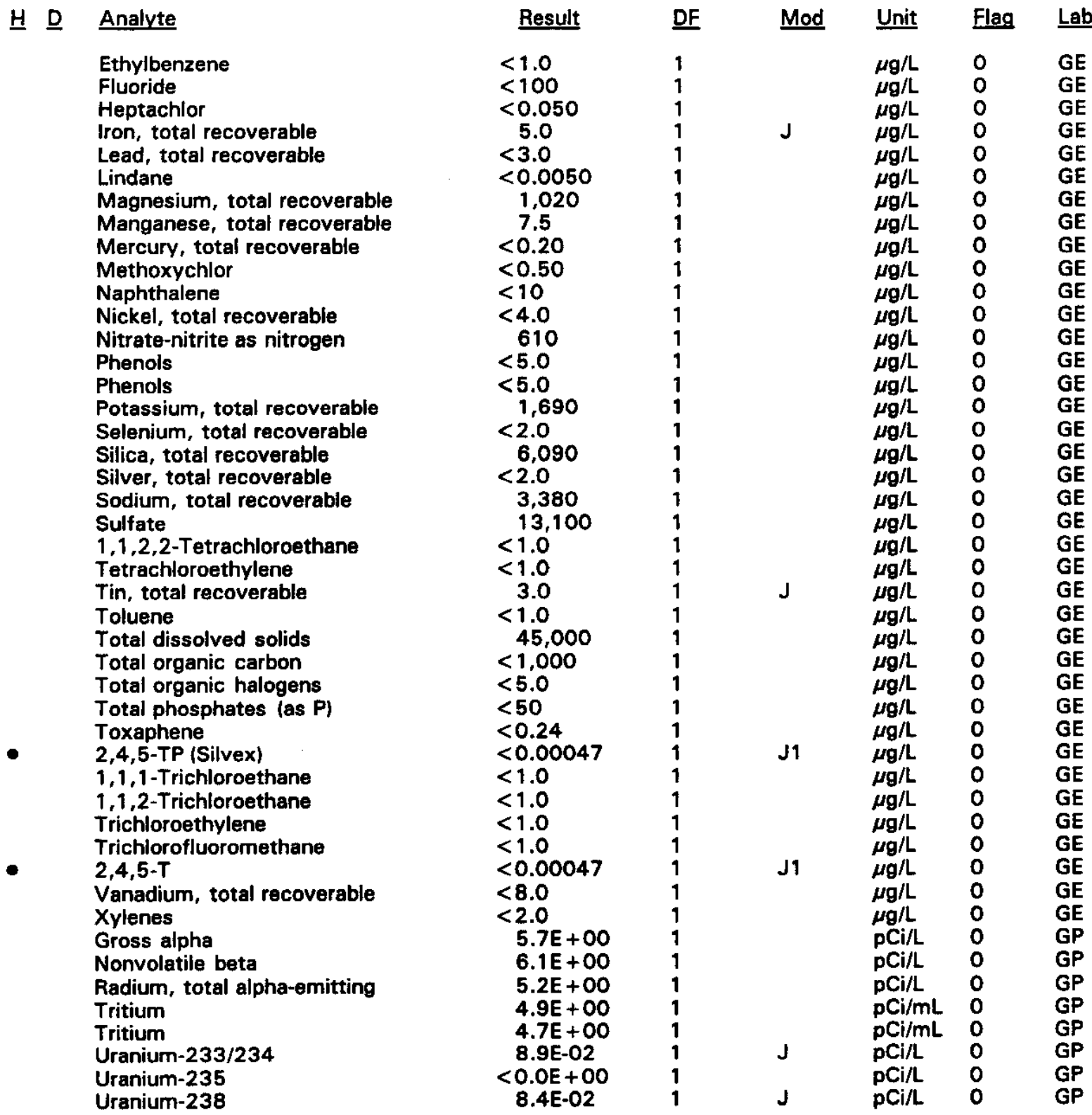

- = exceeded holding time. $\square=$ exceeded screening level or final primary drinking water standard. 
WELL BGO 44A

$\begin{array}{lllllll}\text { SRS Coord. } & \text { Lat/Longitude } & \text { Screen Zone Elevation } & \text { Top of Casing } & \text { Casing } & \text { Pump } & \text { Formation } \\ \text { N76755.2 } & 33.289924^{\circ} \mathrm{N} & 108.0-98.0 \mathrm{ft} \mathrm{msl} & 285.3 \mathrm{ft} \mathrm{msl} & 4^{\prime \prime} \text { PVC } & \text { S } & \text { U. Congaree (IIA) } \\ \text { E57851.2 } & 81.663562^{\circ} \mathrm{W} & & & & \end{array}$

\section{FIELD MEASUREMENTS}

Sample date: 04/06/94

Depth to water: $126.89 \mathrm{ft}(38.68 \mathrm{~m})$ below TOC

Water elevation: $158.41 \mathrm{ft}(48.28 \mathrm{~m}) \mathrm{msl}$

Sp. conductance: $212 \mu \mathrm{S} / \mathrm{cm}$

Turbidity: 0.2 NTU

Water evacuated before sampling: $289 \mathrm{gal}$

\section{LABORATORY ANALYSES}

H Analyte
pH
Specific conductance
Turbidity
Acetophenone
Aldrin
Aluminum, total recoverable
Antimony, total recoverable
Antimony, total recoverable
Arsenic, total recoverable
Arsenic, total recoverable
Barium, total recoverable
Benzene
Bromodichloromethane
Bromoform
Bromomethane (Methyl bromide)
Cadmium, total recoverable
Calcium, total recoverable
Carbon tetrachloride
Chloride
Chlorobenzene
Chloroethane
Chloroethene (Vinyl chloride)
2-Chloroethyl vinyl ether
Chloroform
Chloromethane (Methyl chloride)
Chromium, total recoverable
Copper, total recoverable
Cyanide
p,p'-DDT
Dibromochloromethane
1,1-Dichloroethane
1,2-Dichloroethane
1,1-Dichloroethylene
trans-1,2-Dichloroethylene
Dichloromethane (Methylene chloride)
2,4-Dichlorophenoxyacetic acid
1,2-Dichloropropane
cis-1,3-Dichloropropene
trans-1,3-Dichloropropene

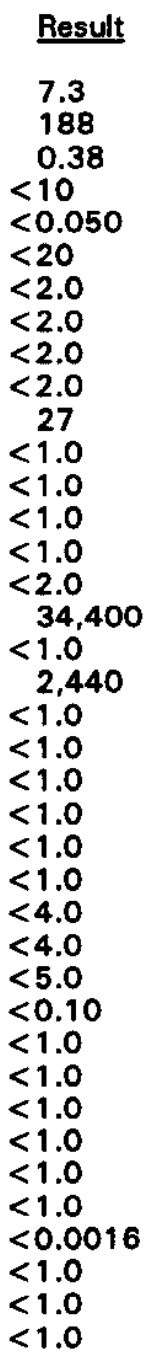

Time: 13:37

pH: 7.3

Alkalinity: $77 \mathrm{mg} / \mathrm{L}$

Water temperature: $19.8^{\circ} \mathrm{C}$

Volumes purged: 7.3 well volumes 
WELL. BGO 44A collected on 04/06/94, laboratory analyses (cont.)

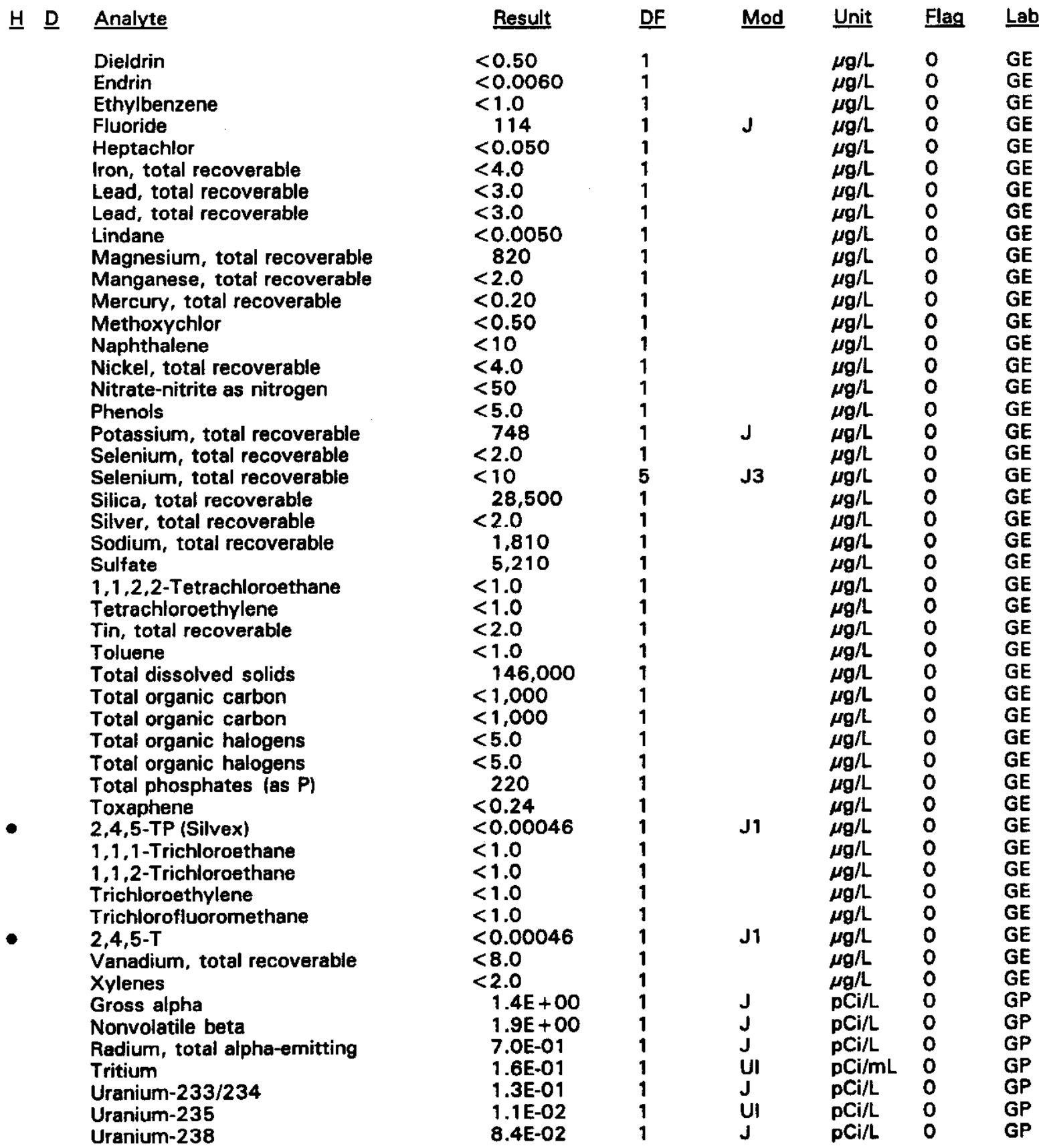

- = exceeded holding time. $\square=$ exceeded screening level or final primary drinking water standard. 


\section{WELL BGO 44AA}

\begin{tabular}{|c|c|c|c|c|c|c|}
\hline SRS Coord. & Lat/Longitude & Screen Zone Elevation & Top of Casing & Casing & Pump & Formation \\
\hline $\begin{array}{l}\text { N76757.0 } \\
\text { E57880.5 }\end{array}$ & $\begin{array}{l}33.289976^{\circ} \mathrm{N} \\
81.663488^{\circ} \mathrm{W}\end{array}$ & $71.3-61.2 \mathrm{ft} \mathrm{msl}$ & $285.3 \mathrm{ft} \mathrm{msl}$ & 4" PVC & $\mathbf{S}$ & L. Congaree (IIA) \\
\hline
\end{tabular}

\section{FIELD MEASUREMENTS}

Sample date: 04/06/94

Depth to water: $126.74 \mathrm{ft}(38.63 \mathrm{~m})$ below TOC

Water elevation: $158.56 \mathrm{ft}(48.33 \mathrm{~m}) \mathrm{ms}$ l

Sp. conductance: $196 \mu \mathrm{S} / \mathrm{cm}$

Turbidity: 0.4 NTU

Water evacuated before sampling: $\mathbf{3 2 4}$ gal

\section{LABORATORY ANALYSES}

\section{H $\underline{D} \quad$ Analyte}

pH

Specific conductance

Turbidity

Acetophenone

Aldrin

Aluminum, total recoverable

Aluminum, total recoverable

Antimony, total recoverable

Arsenic, total recoverable

Barium, total recoverable

Barium, total recoverable

Benzene

Bromodichloromethane

Bromoform

Bromomethane (Methyl bromide)

Cadmium, total recoverable

Cadmium, total recoverable

Calcium, total recoverable

Calcium, total recoverable

Carbon tetrachloride

Chloride

Chlorobenzene

Chloroethane

Chloroethene (Vinyl chloride)

2-Chloroethyl vinyl ether

Chloroform

Chloromethane (Methyl chioride)

Chromium, total recoverable

Chromium, total recoverable

Copper, total recoverable

Copper, total recoverable

Cyanide

Cyanide

p,p'-DDT

Dibromochloromethane

1,1-Dichloroethane

1,2-Dichloroethane

1,1-Dichloroethylene

trans-1,2-Dichloroethylene
Time: 13:16

pH: 9.7

Alkalinity: $75 \mathrm{mg} / \mathrm{L}$

Water temperature: $20.0^{\circ} \mathrm{C}$

Volumes purged: 5.1 well volumes

\begin{tabular}{|c|c|c|c|c|c|}
\hline Result & DF & Mod & Unit & Flag & Lat \\
\hline 9.4 & 1 & J1 & pH & 1 & GE \\
\hline $\begin{array}{l}163 \\
0.23\end{array}$ & 1 & & $\mu \mathrm{S} / \mathrm{cm}$ & 0 & \\
\hline $\begin{array}{l}0.23 \\
<9.7\end{array}$ & 1 & & NTU & 0 & GE \\
\hline$<0.051$ & 1 & & $\begin{array}{l}\mu g / \mathrm{L} \\
\mu \mathrm{g} / \mathrm{L}\end{array}$ & 0 & $\mathrm{GE}$ \\
\hline 124 & 1 & & $\mu g / L$ & 2 & $\mathbf{G E}$ \\
\hline 125 & 1 & & $\mu g / L$ & 2 & GE \\
\hline$<2.0$ & 1 & & $\mu g / L$ & 0 & GE \\
\hline$<2.0$ & 1 & & $\mu g / L$ & 0 & GE \\
\hline 66 & 1 & & $\mu g / L$ & 0 & GE \\
\hline 66 & 1 & & $\mu g / L$ & 0 & GE \\
\hline$<1.0$ & 1 & & $\mu g / L$ & 0 & GE \\
\hline$<1.0$ & 1 & & $\mu g / L$ & 0 & GE \\
\hline$<1.0$ & 1 & & $\mu g / L$ & 0 & GE \\
\hline$<1.0$ & 1 & & $\mu \mathrm{g} / \mathrm{L}$ & 0 & GE \\
\hline$<2.0$ & 1 & & $\mu g / L$ & 0 & GE \\
\hline$<2.0$ & 1 & & $\mu \mathrm{g} / \mathrm{L}$ & 0 & GE \\
\hline 27,700 & 1 & & $\mu g / L$ & 0 & GE \\
\hline $\begin{aligned} & 27,800 \\
< & 1.0\end{aligned}$ & $\begin{array}{l}1 \\
1\end{array}$ & & $\begin{array}{l}\mu g / L \\
\mu g / L\end{array}$ & $\begin{array}{l}0 \\
0\end{array}$ & $\begin{array}{l}\mathrm{GE} \\
\mathrm{GE}\end{array}$ \\
\hline 2,120 & $i$ & & $\mu g / L$ & 0 & GE \\
\hline$<1.0$ & 1 & & $\mu g / L$ & 0 & GE \\
\hline$<1.0$ & 1 & & $\mu g / L$ & 0 & GE \\
\hline$<1.0$ & 1 & & $\mu g / L$ & 0 & GE \\
\hline$<1.0$ & 1 & & $\mu g / L$ & 0 & GE \\
\hline$<1.0$ & 1 & & $\mu g / L$ & 0 & GE \\
\hline$<1.0$ & 1 & & $\mu g / L$ & 0 & GE \\
\hline$<4.0$ & 1 & & $\mu g / L$ & 0 & GE \\
\hline$<4.0$ & 1 & & $\mu g / L$ & 0 & GE \\
\hline$<4.0$ & 1 & & $\mu \mathrm{g} / \mathrm{L}$ & 0 & GE \\
\hline$<4.0$ & 1 & & $\mu \mathrm{g} / \mathrm{L}$ & 0 & GE \\
\hline$<5.0$ & 1 & & $\mu g / L$ & 0 & GE \\
\hline$<5.0$ & 1 & & $\mu g / L$ & 0 & GE \\
\hline$<0.10$ & 1 & & $\mu g / L$ & 0 & GE \\
\hline$<1.0$ & 1 & & $\mu \mathrm{g} / \mathrm{L}$ & 0 & GE \\
\hline$<1.0$ & 1 & & $\mu g / L$ & 0 & GE \\
\hline$<1.0$ & 1 & & $\mu g / L$ & 0 & GE \\
\hline$<1.0$ & 1 & & $\mu g / L$ & 0 & GE \\
\hline$<1.0$ & 1 & & $\mu \mathrm{g} / \mathrm{L}$ & 0 & \\
\hline
\end{tabular}

- =xceeded holding time. $\quad$ = exceeded screening level or final primary drinking water standard. 
WELL BGO 44AA collected on 04/06/94, laboratory analyses (cont.)

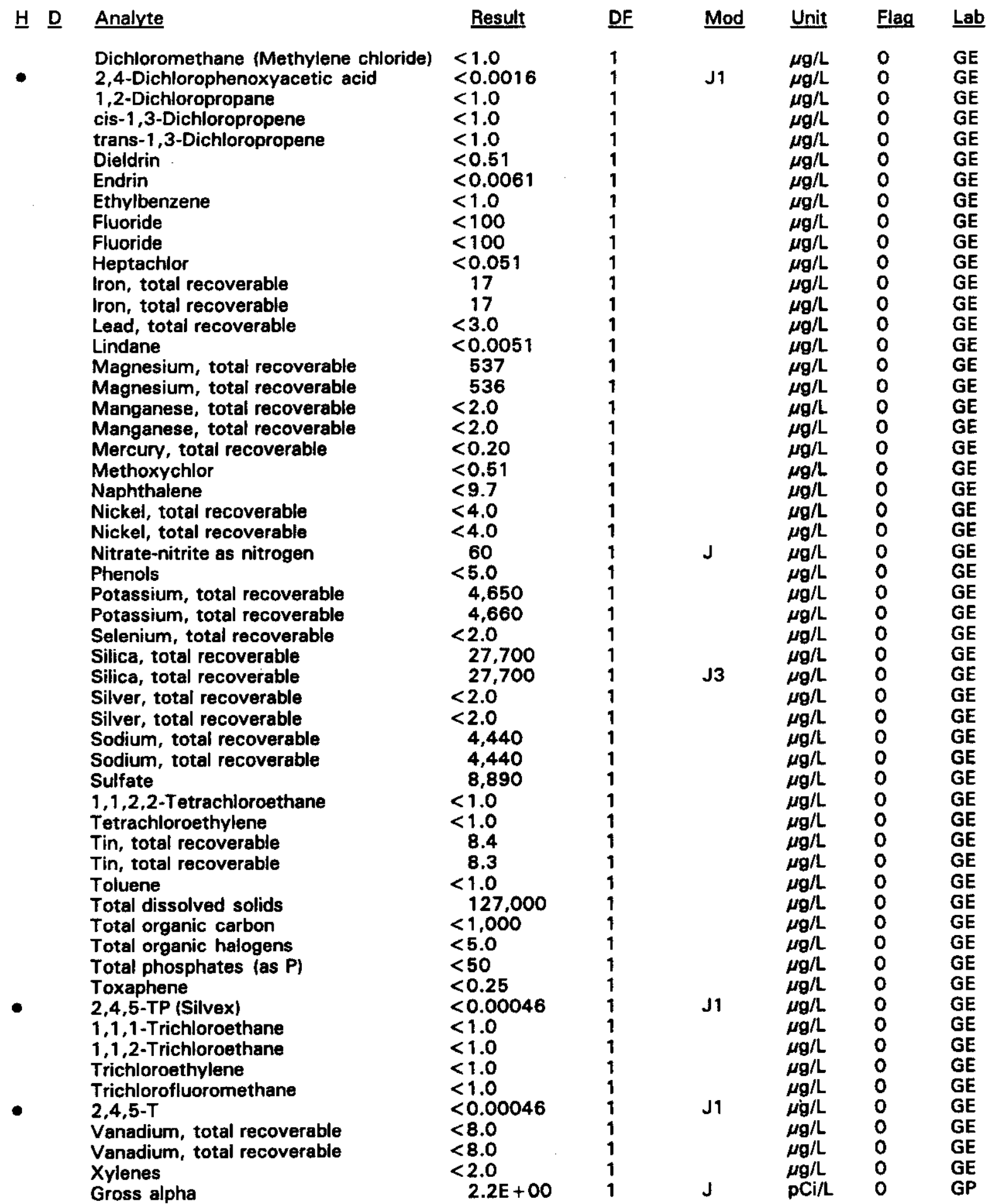

$\overline{- \text { = exceeded holding time. }}$ = exceeded screening level or final primary drinking water standard. 
WELL BGO 44AA collected on 04/06/94, laboratory analyses (cont.)

Hㅁ Analyte

Nonvolatile beta

Radium, total alpha-emitting

Tritium

Uranium-233/234

Uranium-235

Uranium-238
Result

$1.8 \mathrm{E}+00$
$1.0 \mathrm{E}+00$
$2.2 \mathrm{E}+00$
$1.9 \mathrm{E}-01$
$5.4 \mathrm{E}-02$
$3.1 \mathrm{E}-01$

DF

$\begin{array}{lllll}\text { Mod } & & \text { Unit } & \text { Flag } & \text { Lab } \\ \mathrm{J} & & \mathrm{pCi} / \mathrm{L} & 0 & \mathrm{GP} \\ & \mathrm{pCi} / \mathrm{L} & 0 & \mathrm{GP} \\ & \mathrm{pCi} / \mathrm{mL} & 0 & \mathrm{GP} \\ \mathrm{J} & \mathrm{pCi} / \mathrm{L} & 0 & \mathrm{GP} \\ \mathrm{UI} & \mathrm{pCi} / \mathrm{L} & 0 & \mathrm{GP} \\ \mathrm{J} & \mathrm{pCi} / \mathrm{L} & 0 & \text { GP }\end{array}$

\section{WELL BGO 44B}

\begin{tabular}{|c|c|c|c|c|c|c|}
\hline SRS Coord. & Lat/Longitude & Screen Zone Elevation & Top of Casing & Casing & Pump & Formation \\
\hline $\begin{array}{l}\text { N76756.0 } \\
\text { E57865.8 }\end{array}$ & $\begin{array}{l}33.289950^{\circ} \mathrm{N} \\
81.663525^{\circ} \mathrm{W}\end{array}$ & $158.1-148.1 \mathrm{ft} \mathrm{msl}$ & $285.2 \mathrm{ft} \mathrm{msl}$ & 4" PVC & $\mathbf{s}$ & McBean (IIB $\left.{ }_{1}\right)$ \\
\hline
\end{tabular}

\section{FIELD MEASUREMENTS}

Sample date: $04 / 06 / 94$

Depth to water: $64.04 \mathrm{ft}(19.52 \mathrm{~m})$ below TOC

Water elevation: $221.16 \mathrm{ft}(67.41 \mathrm{~m}) \mathrm{msl}$

Sp. conductance: $244 \mu \mathrm{S} / \mathrm{cm}$

Turbidity: 211 NTU

Water evacuated before sampling: $\mathbf{4 0} \mathrm{gal}$

The well went dry during purging.

\section{LABORATORY ANALYSES}

H D Analyte

pH

Specific conductance

Turbidity

Acetophenone

Aldrin

Aluminum, total recoverable

Antimony, total recoverable

Arsenic, total recoverable

Barium, total recoverable

Benzene

Benzene

Bromodichloromethane

Bromodichloromethane

Bromoform

Bromoform

Bromomethane (Methyl bromide)

Bromomethane (Methyl bromide)

Cadmium, total recoverable

Calcium, total recoverable

Carbon tetrachloride

Carbon tetrachloride

Chloride

Chlorobenzene

Chlorobenzene

Chloroethane

Chloroethane

Chloroethene (Vinyl chloride)
Time: 12:28

pH: 8.4

Alkalinity: $121 \mathrm{mg} / \mathrm{h}$

Water temperature: $19.5^{\circ} \mathrm{C}$

Volumes purged: 0.8 well volumes

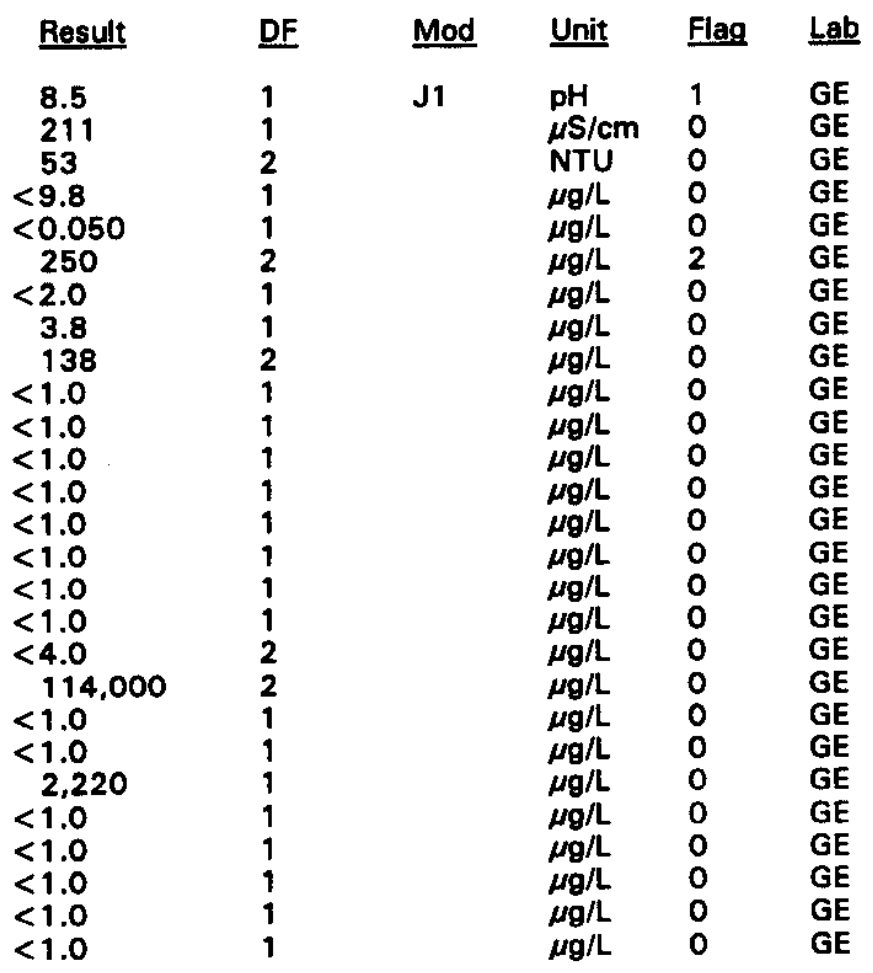

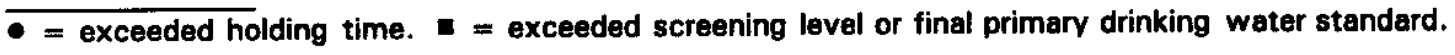


WELL BGO 44B collected on 04/06/94, laboratory analyses (cont.)

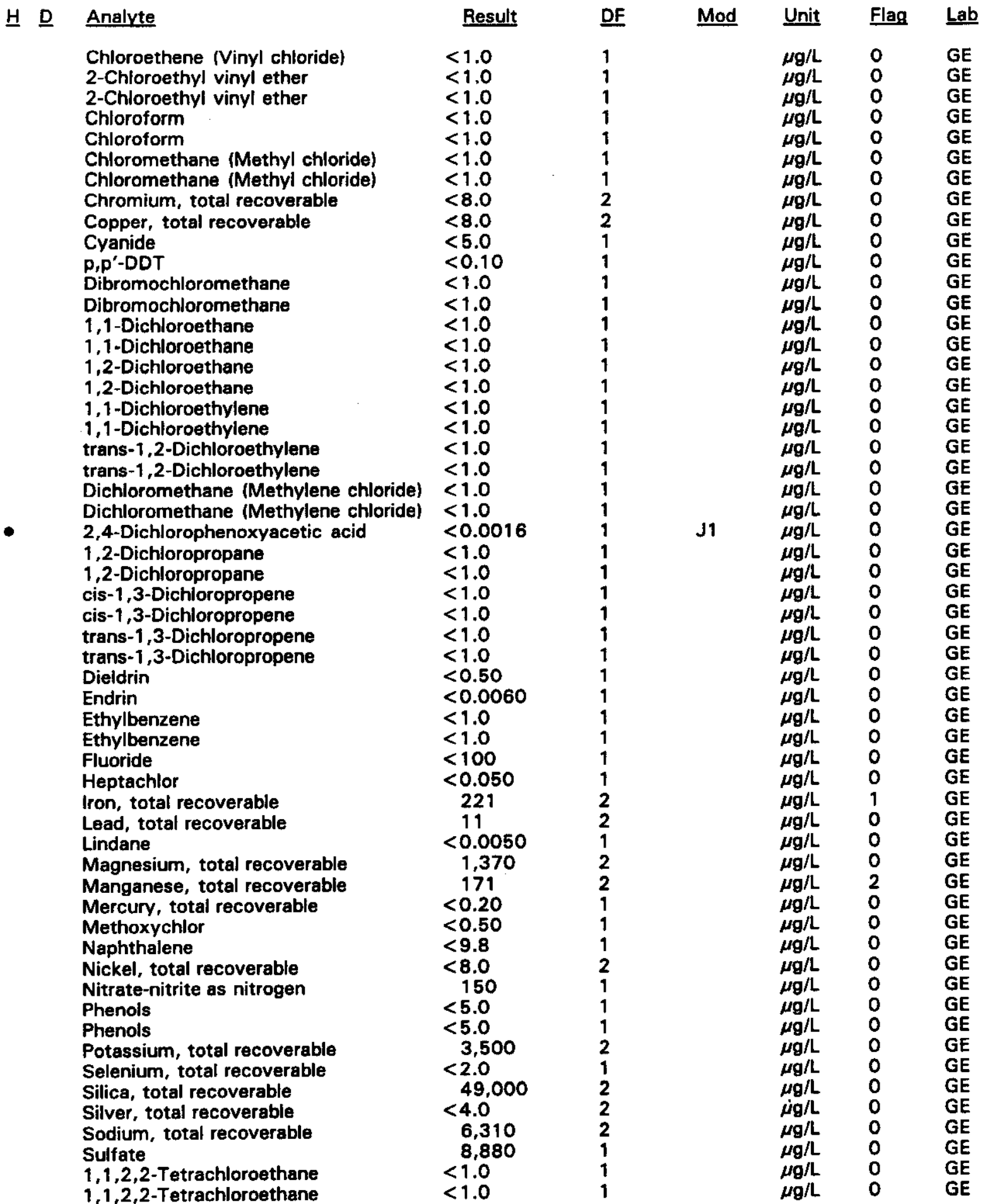

$\overline{0}$ = exceeded holding time. $-=$ exceeded screening level or final primary drinking water standard. 
WELL BGO 44B collected on 04/06/94, laboratory analyses (cont.)

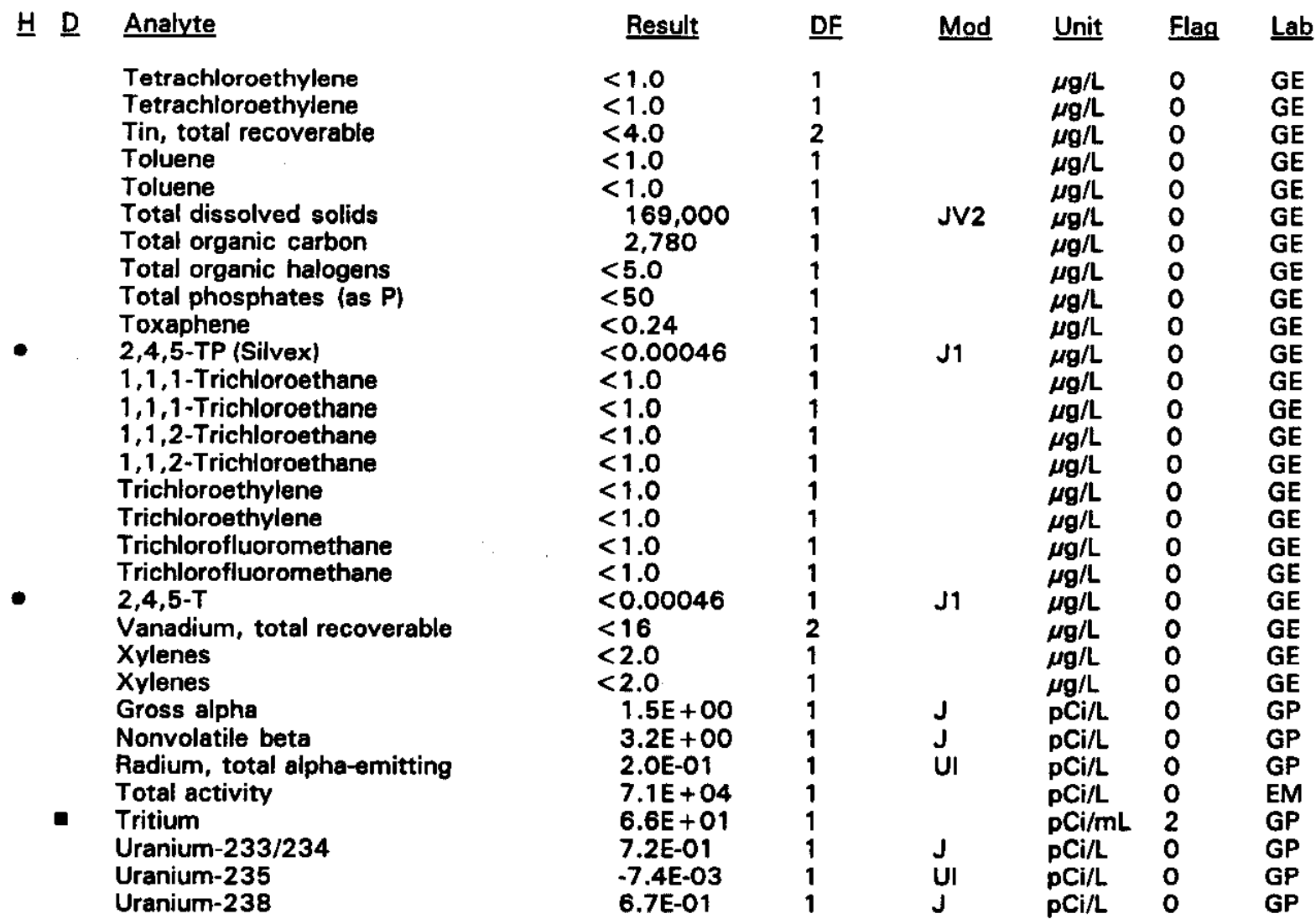

WELL BGO 44C

\begin{tabular}{|c|c|c|c|c|c|c|}
\hline SRS Coord. & Lat/Longitude & Screen Zone Elevation & Top of Casing & Casing & Pump & Formation \\
\hline $\begin{array}{l}N 76757.8 \\
E 57894.9\end{array}$ & $\begin{array}{l}33.290001^{\circ} \mathrm{N} \\
81.663452^{\circ} \mathrm{W}\end{array}$ & $200.6-190.6 \mathrm{ft} \mathrm{msl}$ & $285.6 \mathrm{ft} \mathrm{msl}$ & 4" PVC & $\mathbf{S}$ & Barnwell (IIB, ) \\
\hline
\end{tabular}

FIELD MEASUREMENTS

Sample date: 04/06/94

Depth to water: $64.58 \mathrm{ft}(19.68 \mathrm{~m})$ below TOC

Water elevation: $221.02 \mathrm{ft}(67.37 \mathrm{~m}) \mathrm{msl}$

Sp. conductance: $165 \mu \mathrm{S} / \mathrm{cm}$

Turbidity: 11.6 NTU

Water evacuated before sampling: $20 \mathrm{gal}$

The well went dry during purging.

\section{LABORATORY ANALYSES}

$\begin{array}{ll}\text { ․ㅡ } & \text { Analyte } \\ & \text { pH } \\ & \begin{array}{l}\text { Specific conductance } \\ \text { Turbidity }\end{array}\end{array}$

- exceeded holding time.

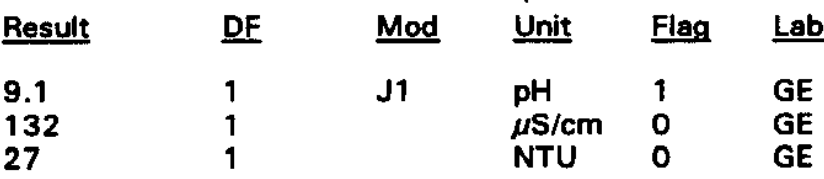

Time: 12:45

pH: 7.7

Alkalinity: $61 \mathrm{mg} / \mathrm{L}$

Water temperature: $19.5^{\circ} \mathrm{C}$

Volumes purged: 1.0 well volumes 
WELL BGO 44C collected on $04 / 06 / 94$, laboratory analyses (cont.)

H D Analyte

Acetophenone

Aldrin

Aluminum, total recoverable

Antimony, total recoverable

Antimony, total recoverable

Arsenic, total recoverable

Arsenic, total recoverable

Barium, total recoverable

Benzene

Bromodichloromethane

Bromoform

Bromomethane (Methyl bromide)

Cadmium, total recoverable

Calcium, total recoverable

Carbon tetrachloride

Chloride

Chlorobenzene

Chloroethane

Chloroethene (Vinyl chloride)

2-Chloroethyl vinyl ether

Chloroform

Chloromethane (Methyl chloride)

Chromium, total recoverable

Copper, total recoverable

Cyanide

p.p'-DDT

Dibromochloromethane

1,1-Dichloroethane

1,2-Dichloroethane

1,1-Dichloroethylene

trans-1,2-Dichloroethylene

Dichloromethane (Methylene chloride)

2,4-Dichlorophenoxyacetic acid

1,2-Dichloropropane

cis-1,3-Dichloropropene

cis-1,3-Dichloropropene

trans-1,3-Dichloropropene

Dieldrin

Endrin

Ethylbenzene

Fluoride

Heptachlor

Iron, total recoverable

Lead, total recoverable

Lead, total recoverable

Lindane

Magnesium, total recoverable

Manganese, total recoverable

Mercury, total recoverable

Methoxychlor

Naphthalene

Nickel, total recoverable

Nitrate-nitrite as nitrogen

Phenols

Phenols

Potassium, total recoverable
Result DF

$<9.9$

$<0.051$

391

$<2.0$

$<2.0$

$<2.0$

$<2.0$

74

$<1.0$

$<1.0$

$<1.0$

$<1.0$

$<2.0$

15,100

$<1.0$

2,120

$<1.0$

$<1.0$

$<1.0$

$<1.0$

$<1.0$

$<1.0$

$<4.0$

$<4.0$

$<5.0$

$<0.10$

$<1.0$

$<1.0$

$<1.0$

$<1.0$

$<1.0$

$<1.0$

$<1.5$

$<1.0$

$<1.0$

$<1.0$

$<1.0$

$<0.51$

$<0.0061$

$<1.0$

$<100$

$<0.051$

257

9.2

10

$<0.0051$

965

338

$<0.20$

$<0.51$

$<9.9$

$<4.0$

800

$<5.0$

$<5.0$

5,360
Mod Unit Flag Lab

$\mu \mathrm{g} / \mathrm{L} \quad 0 \quad \mathrm{GE}$

$\mu \mathrm{g} / \mathrm{L} \quad \mathrm{O} \quad \mathrm{GE}$

$\mu \mathrm{g} / \mathrm{L} \quad 2 \quad \mathrm{GE}$

$\mu \mathrm{g} / \mathrm{L} \quad 0 \quad \mathrm{GE}$

$\mu g / L \quad 0 \quad$ GE

$\mu \mathrm{g} / \mathrm{L} \quad 0 \quad \mathrm{GE}$

$\mu g / L \quad 0 \quad G E$

$\mu \mathrm{g} / \mathrm{L} \quad \mathrm{O} \quad \mathrm{GE}$

$\mu \mathrm{g} / \mathrm{L} \quad \mathrm{O} \quad \mathrm{GE}$

$\mu \mathrm{g} / \mathrm{L} \quad \mathrm{O} \quad \mathrm{GE}$

$\mu g / L \quad 0 \quad$ GE

$\mu \mathrm{g} / \mathrm{L} \quad 0 \quad \mathrm{GE}$

$\mu g / L \quad 0 \quad$ GE

$\mu g / L \quad 0 \quad$ GE

$\mu g / L \quad 0 \quad$ GE

$\mu g / L \quad 0 \quad$ GE

$\mu g / L \quad 0 \quad G E$

$\mu g / L \quad 0 \quad$ GE

$\mu \mathrm{g} / \mathrm{L} \quad 0 \quad \mathrm{GE}$

$\mu g / L \quad 0 \quad$ GE

$\mu g / L \quad 0 \quad$ GE

$\mu g / L \quad 0 \quad G E$

$\mu \mathrm{g} / \mathrm{L} \quad \mathrm{O} \quad \mathrm{GE}$

$\mu g / L \quad 0 \quad$ GE

$\mu \mathrm{g} / \mathrm{L} \quad 0 \quad \mathrm{GE}$

$\mu g / L \quad 0 \quad G E$

$\mu g / L \quad 0 \quad$ GE

$\mu g / L \quad 0 \quad G E$

$\mu \mathrm{g} / \mathrm{L} \quad 0 \quad \mathrm{GE}$

$\mu \mathrm{g} / \mathrm{L} \quad 0 \quad \mathrm{GE}$

$\mu \mathrm{g} / \mathrm{L} \quad 0 \quad \mathrm{GE}$

$\mu \mathrm{g} / \mathrm{L} \quad 0 \quad \mathrm{GE}$

$\mu \mathrm{g} / \mathrm{L} \quad 0 \quad \mathrm{GE}$

$\mu g / L \quad 0 \quad$ GE

$\mu \mathrm{g} / \mathrm{L} \quad 0 \quad \mathrm{GE}$

$\mu g / L \quad 0 \quad$ GE

$\mu \mathrm{g} / \mathrm{L} \quad 0 \quad \mathrm{GE}$

$\mu \mathrm{g} / \mathrm{L} \quad \mathrm{O} \quad \mathrm{GE}$

$\mu g / L \quad 0 \quad$ GE

$\mu \mathrm{g} / \mathrm{L} \quad 0 \quad \mathrm{GE}$

$\mu \mathrm{g} / \mathrm{L} \quad \mathrm{O} \quad \mathrm{GE}$

$\mu \mathrm{g} / \mathrm{L} \quad 0 \quad \mathrm{GE}$

$\mu g / L \quad 1 \quad G E$

$\mu g / L \quad 0 \quad G E$

$\mu \mathrm{g} / \mathrm{L} \quad \mathrm{O} \quad \mathrm{GE}$

$\mu g / L \quad 0 \quad$ GE

$\mu g / \mathrm{L} \quad 0 \quad \mathrm{GE}$

$\mu g / L \quad 2 \quad$ GE

$\mu g / L \quad 0 \quad$ GE

$\mu \mathrm{g} / \mathrm{L} \quad 0 \quad \mathrm{GE}$

$\mu \mathrm{g} / \mathrm{L} \quad 0 \quad \mathrm{GE}$

$\mu \mathrm{g} / \mathrm{L} \quad \mathrm{O} \quad \mathrm{GE}$

$\mu \mathrm{g} / \mathrm{L} \quad 0 \quad \mathrm{GE}$

$\mu g / L \quad 0 \quad G E$

$\mu g / L \quad 0 \quad G E$

$\mu g / L \quad$ GE

- exceeded holding time. = exceeded screening level or final primary drinking water standard. 
WELL BGO $44 \mathrm{C}$ collected on $04 / 06 / 94$, laboratory analyses (cont.)

\section{H D Analyte}

Selenium, total recoverable

Selenium, total recoverable

Silica, total recoverable

Silver, total recoverable

Sodium, total recoverable

Sulfate

1,1,2,2-Tetrachloroethane

Tetrachloroethylene

Tin, total recoverable

Toluene

Total dissolved solids

Total organic carbon

Total organic halogens

Total phosphates (as P)

Toxaphene

2,4,5-TP (Silvex)

1,1,1-Trichloroethane

1,1,2-Trichloroethane

Trichloroethylene

Trichlorofluoromethane

2,4,5-T

Vanadium, total recoverable

Xylenes

Gross alpha

Nonvolatile beta

Radium, total alpha-emitting

Total activity

- Tritium

Uranium-233/234

Uranium-235

Uranium-238

Result
$<2.0$
$<2.0$
8.050
$<2.0$
9.970
1,830
$<1.0$
$<1.0$
11
$<1.0$
89,000
2,140
$<5.0$
90
$<0.24$
$<0.46$
$<1.0$
$<1.0$
2.1
$<1.0$
$<0.46$
$<8.0$
$<2.0$
$6.9 \mathrm{E}-01$
$3.7 \mathrm{E}+00$
$6.0 \mathrm{E}-01$
$4.0 \mathrm{E}+05$
$3.2 \mathrm{E}+02$
$2.6 \mathrm{E}-01$
$2.0 \mathrm{E}-02$
$3.2 \mathrm{E}-01$

$\underline{\mathrm{DF}}$

Mod Unit Flag Lab

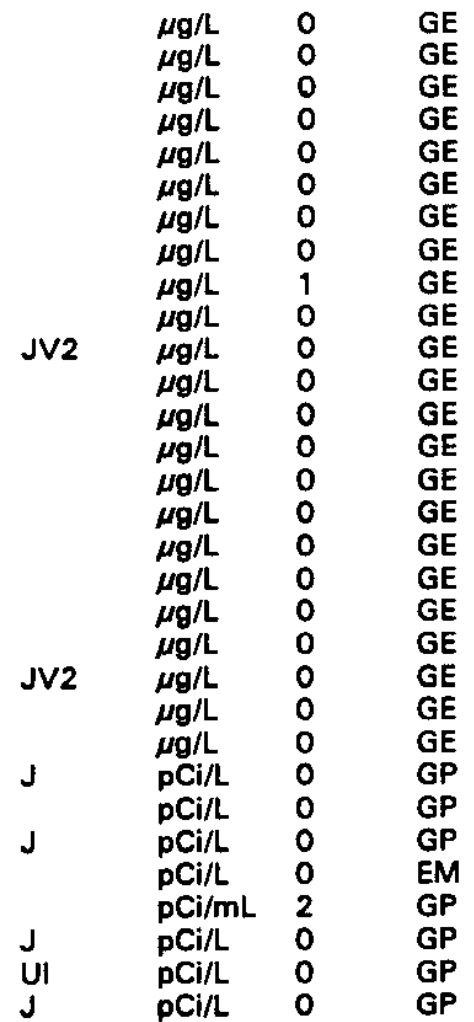

\section{WELL BGO 44D}

\begin{tabular}{|c|c|c|c|c|c|c|}
\hline SRS Coord. & Lat/Longitude & Screen Zone Elevation & Top of Casing & Casing & Pump & Formation \\
\hline $\begin{array}{l}N 76759.5 \\
\text { E57910.0 }\end{array}$ & $\begin{array}{l}33.290030^{\circ} \mathrm{N} \\
81.663415^{\circ} \mathrm{W}\end{array}$ & $233.4-223.4 \mathrm{ft} \mathrm{msl}$ & $285.4 \mathrm{ft} \mathrm{msl}$ & 4" PVC & $\mathbf{s}$ & Water Table $\left(I I B_{2}\right)$ \\
\hline
\end{tabular}

\section{FIELD MEASUREMENTS}

Sample date: 04/06/94

Depth to water: $52.85 \mathrm{ft}(16.11 \mathrm{~m})$ below TOC

Water elevation: $232.55 \mathrm{ft}(70.88 \mathrm{~m}) \mathrm{msl}$

Sp. conductance: $34 \mu \mathrm{S} / \mathrm{cm}$

Turbidity: 0.6 NTU

Water evacuated before sampling: $96 \mathrm{gal}$

\section{LABORATORY ANALYSES}

\begin{tabular}{|c|c|c|c|c|c|}
\hline H D Analvte & Result & $\underline{\mathrm{DF}}$ & Mod & Unit & Flag \\
\hline $\begin{array}{l}\text { pH } \\
\text { pH } \\
\text { Specific conductance }\end{array}$ & $\begin{array}{l}5.2 \\
5.2 \\
24\end{array}$ & $\begin{array}{l}1 \\
1 \\
1\end{array}$ & $\begin{array}{l}\text { J1 } \\
\text { J1 }\end{array}$ & $\begin{array}{l}\mathrm{pH} \\
\mathrm{pH} \\
\mu \mathrm{S} / \mathrm{cm}\end{array}$ & $\begin{array}{l}0 \\
0 \\
0\end{array}$ \\
\hline
\end{tabular}

Time: 14:01

pH: 4.8

Alkalinity: $0 \mathrm{mg} / \mathrm{L}$

Water temperature: $20.0^{\circ} \mathrm{C}$

Volumes purged: 16.0 well volumes

- = exceeded holding time. = exceeded screening level or final primary drinking water standard. 
WELL BGO 44D collected on 04/06/94, laboratory analyses (cont.)

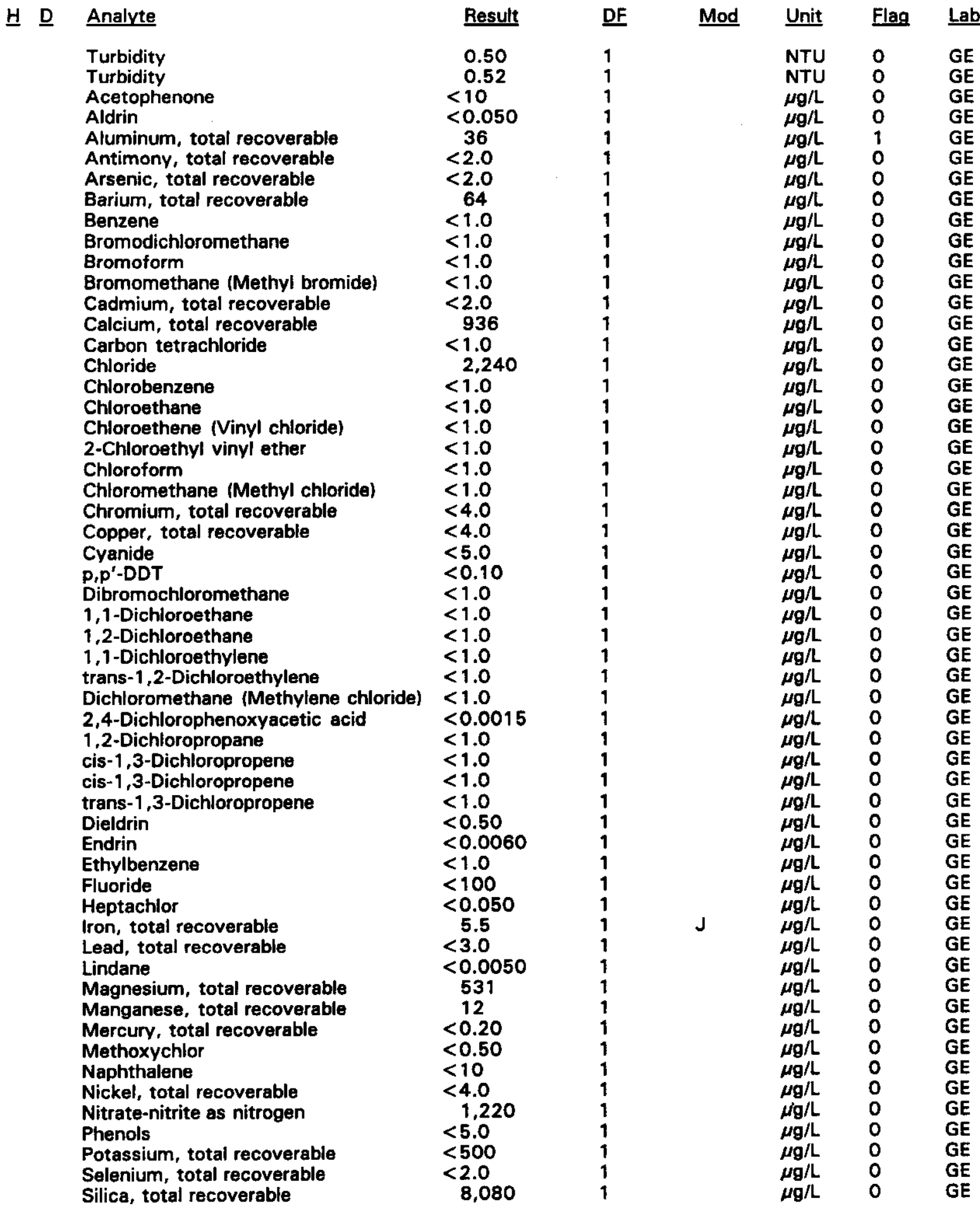

- = exceeded holding time. $\square=$ exceeded screening level or final primary drinking water standard. 
WELL BGO 44D collected on 04/06/94, laboratory analyses (cont.)

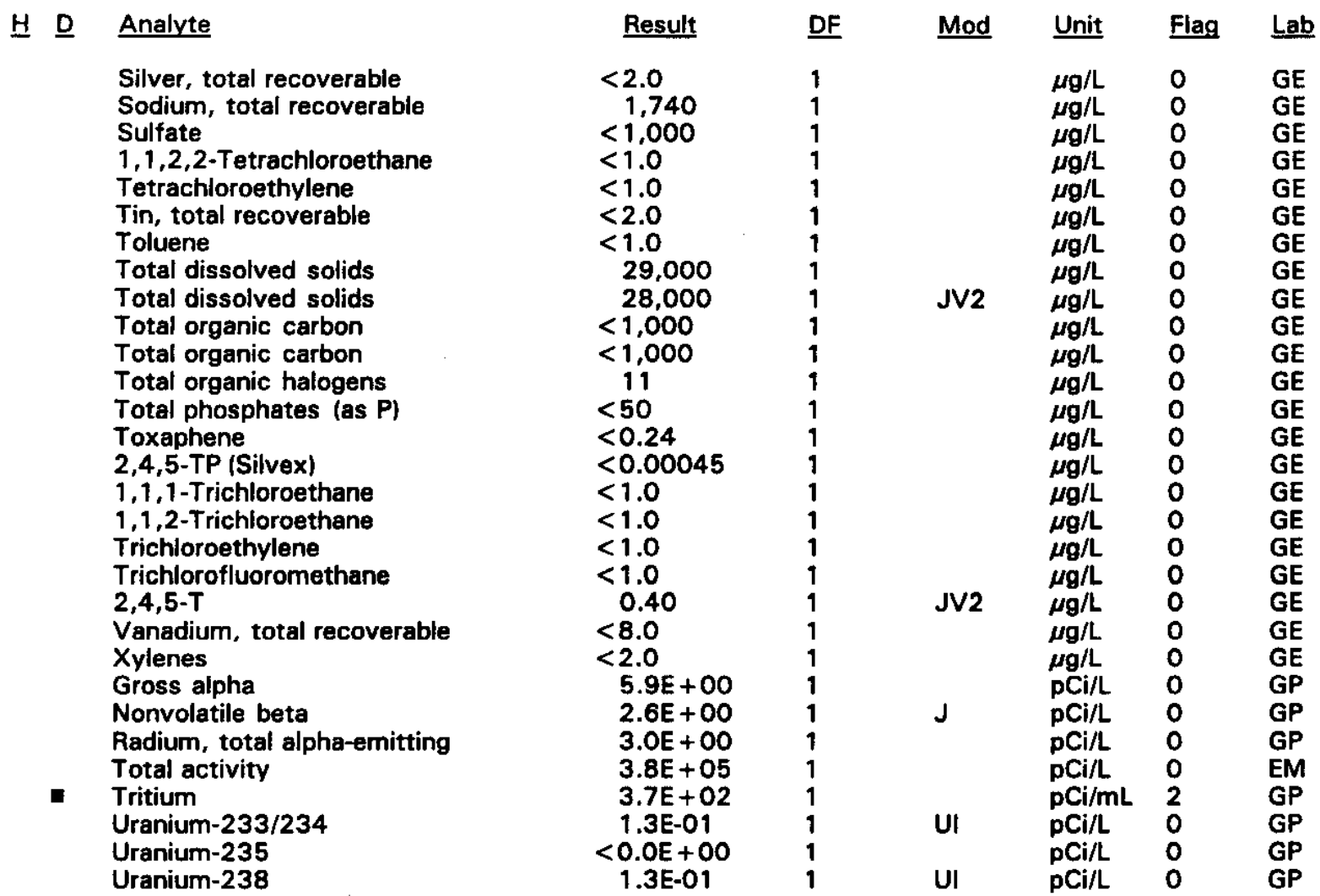

\section{WELL BGO 45A}

\begin{tabular}{|c|c|c|c|c|c|c|}
\hline SRS Coord. & Lat/Longitude & Screen Zone Elevation & Top of Casing & Casing & Pump & Formation \\
\hline $\begin{array}{l}\text { N75830.0 } \\
\text { E54550.1 }\end{array}$ & $\begin{array}{l}33.282492^{\circ} \mathrm{N} \\
81.670457^{\circ} \mathrm{W}\end{array}$ & $126.9-116.9 \mathrm{ft} \mathrm{msl}$ & $278.9 \mathrm{ft} \mathrm{msl}$ & 4" PVC & $\mathbf{S}$ & U. Congaree (IIA) \\
\hline
\end{tabular}

\section{FIELD MEASUREMENTS}

Sample date: 04/06/94

Depth to water: $118.16 \mathrm{ft}(36.02 \mathrm{~m})$ below TOC

Water elevation: $160.74 \mathrm{ft}(48.99 \mathrm{~m}) \mathrm{msl}$

Sp. conductance: $203 \mu \mathrm{S} / \mathrm{cm}$

Turbidity: 0.1 NTU

Water evacuated before sampling: $103 \mathrm{gal}$
Time: 15:48

pH: 7.7

Alkalinity: $72 \mathrm{mg} / \mathrm{L}$

Water temperature: $19.6^{\circ} \mathrm{C}$

Volumes purged: 3.6 well volumes

\section{LABORATORY ANALYSES}

\begin{tabular}{|c|c|c|c|c|c|c|}
\hline$\underline{H} \underline{D}$ & Analyte & Result & DF & Mod & Unit & Flag \\
\hline - & $\begin{array}{l}\text { pH } \\
\text { Specific conductance } \\
\text { Turbidity } \\
\text { Acetophenone }\end{array}$ & $\begin{array}{l}8.0 \\
177 \\
0.93 \\
<9.8\end{array}$ & $\begin{array}{l}1 \\
1 \\
1 \\
1\end{array}$ & J1 & $\begin{array}{l}\mathrm{pH} \\
\mu \mathrm{S} / \mathrm{cm} \\
\mathrm{NTU} \\
\mu \mathrm{g} / \mathrm{L}\end{array}$ & $\begin{array}{l}0 \\
0 \\
0 \\
0\end{array}$ \\
\hline
\end{tabular}

\footnotetext{
- exceeded holding time. $\square=$ exceeded screening level or final primary drinking water standard.
} 
WELL BGO $45 \mathrm{~A}$ collected on 04/06/94, laboratory analyses (cont.)

H $\underline{\text { Analyte }}$

Aldrin

Aluminum, total recoverable

Antimony, total recoverable

Arsenic, total recoverable

Barium, total recoverable

Benzene

Bromodichloromethane

Bromoform

Bromomethane (Methyl bromide)

Cadmium, total recoverable

Calcium, total recoverable

Carbon tetrachloride

Chloride

Chlorobenzene

Chloroethane

Chloroethene (Vinyl chloride)

2-Chloroethyl vinyl ether

Chloroform

Chloromethane (Methyl chloride)

Chromium, total recoverable

Copper, total recoverable

Cyanide

p.p'-DDT

Dibromochloromethane

1,1-Dichloroethane

1,2-Dichloroethane

1,1-Dichloroethylene

trans-1,2-Dichloroethylene

Dichloromethane (Methylene chloride)

2,4-Dichlorophenoxyacetic acid

1,2-Dichloropropane

cis-1,3-Dichloropropene

trans-1,3-Dichloropropene

Dieldrin

Endrin

Ethylbenzene

Fluoride

Heptachlor

Iron, total recoverable

Lead, total recoverable

Lindane

Magnesium, total recoverable

Manganese, total recoverable

Mercury, total recoverable

Methoxychlor

Naphthalene

Nickel, total recoverable

Nitrate-nitrite as nitrogen

Phenols

Phenols

Potassium, total recoverable

Selenium, total recoverable

Silica, total recoverable

Silver, total recoverable

Sodium, total recoverable

Sulfate

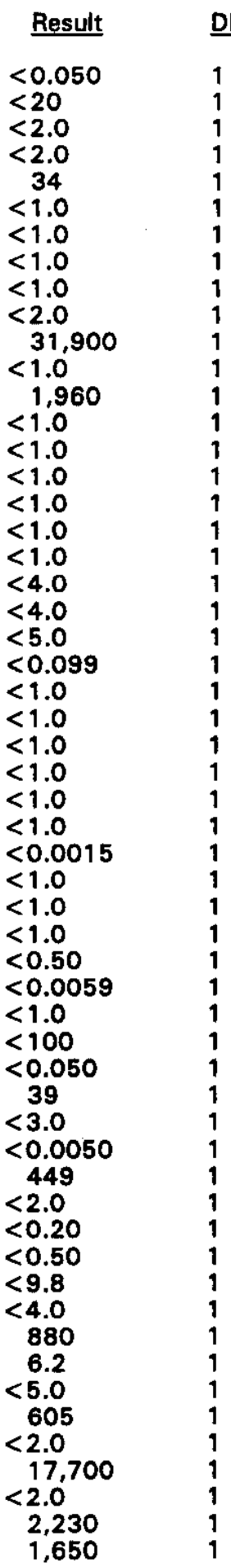

Mod Unit Flag Lab

$\mu g / L \quad 0 \quad G E$

$\mu \mathrm{g} / \mathrm{L} \quad \mathrm{O} \quad \mathrm{GE}$

$\mu g / L \quad O \quad G E$

$\mu \mathrm{g} / \mathrm{L} \quad 0 \quad \mathrm{GE}$

$\mu g / L \quad 0 \quad$ GE

$\mu g / L \quad 0 \quad G E$

$\mu g / L \quad 0 \quad G E$

$\mu \mathrm{g} / \mathrm{L} \quad 0 \quad \mathrm{GE}$

$\mu g / L \quad 0 \quad$ GE

$\mu \mathrm{g} / \mathrm{L} \quad 0 \quad \mathrm{GE}$

$\mu \mathrm{g} / \mathrm{L} \quad 0 \quad \mathrm{GE}$

$\mu g / L \quad 0 \quad G E$

$\mu g / L \quad 0 \quad$ GE

$\mu \mathrm{g} / \mathrm{L} \quad 0 \quad \mathrm{GE}$

$\mu \mathrm{g} / \mathrm{L} \quad 0 \quad \mathrm{GE}$

$\mu g / L \quad 0 \quad G E$

$\mu g / L \quad 0 \quad$ GE

$\mu \mathrm{g} / \mathrm{L} \quad \mathrm{O} \quad \mathrm{GE}$

$\mu \mathrm{g} / \mathrm{L} \quad 0 \quad \mathrm{GE}$

$\mu \mathrm{g} / \mathrm{L} \quad 0 \quad \mathrm{GE}$

$\mu g / L \quad 0 \quad$ GE

$\mu g / L \quad 0 \quad$ GE

$\mu \mathrm{g} / \mathrm{L} \quad 0 \quad \mathrm{GE}$

$\mu g / L \quad 0 \quad$ GE

$\mu \mathrm{g} / \mathrm{L} \quad 0 \quad \mathrm{GE}$

$\mu g / L \quad 0 \quad$ GE

$\mu \mathrm{g} / \mathrm{L} \quad 0 \quad \mathrm{GE}$

$\mu g / L \quad 0 \quad G E$

$\mu g / L \quad 0 \quad G E$

$\mu g / L \quad 0 \quad$ GE

$\mu g / L \quad 0 \quad G E$

$\mu g / L \quad 0 \quad G E$

$\mu g / L \quad 0 \quad G E$

$\mu g / L \quad 0 \quad G E$

$\mu \mathrm{g} / \mathrm{L} \quad \mathrm{O} \quad \mathrm{GE}$

$\mu g / L \quad 0 \quad$ GE

$\mu g / L \quad 0 \quad$ GE

$\mu g / L \quad O \quad$ GE

$\mu \mathrm{g} / \mathrm{L} \quad \mathrm{O} \quad \mathrm{GE}$

$\mu g / L \quad 0 \quad$ GE

$\mu g / L \quad 0 \quad G E$

$\mu g / L \quad 0 \quad$ GE

$\mu g / L \quad 0 \quad$ GE

$\mu g / L \quad 0 \quad$ GE

$\mu \mathrm{g} / \mathrm{L} \quad \mathrm{O} \quad \mathrm{GE}$

$\mu \mathrm{g} / \mathrm{L} \quad \mathrm{O} \quad \mathrm{GE}$

$\mu g / L \quad O \quad$ GE

$\mu g / L \quad 0 \quad$ GE

J $\mu g / L \quad 0 \quad$ GE

$\mu g / L$ o $\quad$ GE

J $\mu g / L \quad 0 \quad$ GE

$\dot{\mu g} / \mathrm{L} \quad 0 \quad$ GE

$\mu g / L \quad 0 \quad G E$

$\mu g / L \quad 0 \quad$ GE

$\mu g / L \quad O \quad G E$

$\mu g / L \quad O \quad G E$

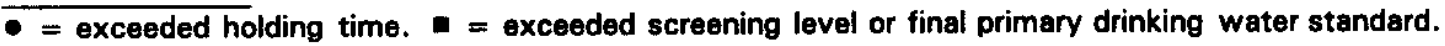


WELL BGO 45A collected on 04/06/94, laboratory analyses (cont.)

H D Analyte

$1,1,2,2-$ Tetrachloroethane

Tetrachloroethylene

Tin, total recoverable

Toluene

- Total dissolved solids

Total organic carbon

Total organic halogens

Total phosphates (as P)

Toxaphene

2,4,5-TP (Silvex)

Tributyl phosphate

1,1,1-Trichloroethane

1,1,2-Trichloroethane

Trichloroethylene

Trichlorofluoromethane

2,4,5-T

Vanadium, total recoverable

Xylenes

Gross alpha

Nonvolatile beta

Radium, total alpha-emitting

Tritium

Uranium-233/234

Uranium-235

Uranium-238

\begin{tabular}{ll}
\multicolumn{1}{l}{ Result } & D \\
$<1.0$ & 1 \\
$<1.0$ & 1 \\
$<2.0$ & 1 \\
$<1.0$ & 1 \\
117,000 & 1 \\
1,170 & 1 \\
$<5.0$ & 1 \\
$<50$ & 1 \\
$<0.24$ & 1 \\
$<0.00044$ & 1 \\
$<9.8$ & 1 \\
$<1.0$ & 1 \\
$<1.0$ & 1 \\
$<1.0$ & 1 \\
$<1.0$ & 1 \\
0.42 & 1 \\
$<8.0$ & 1 \\
$<2.0$ & 1 \\
$3.8 E-02$ & 1 \\
$7.2 E+00$ & 1 \\
$9.0 E-01$ & 1 \\
$3.8 E+00$ & 1 \\
$1.7 E-01$ & 1 \\
$7.3 E-02$ & 1 \\
$1.8 E-01$ & 1
\end{tabular}

\begin{tabular}{|c|c|c|}
\hline Mod & Unit & Flag \\
\hline & $\mu g / L$ & 0 \\
\hline & $\mu g / L$ & 0 \\
\hline & $\mu g / L$ & 0 \\
\hline & $\mu g / L$ & 0 \\
\hline J1 & $\mu g / L$ & 0 \\
\hline$J$ & $\mu g / L$ & 0 \\
\hline & $\mu g / L$ & 0 \\
\hline & $\begin{array}{l}\mu g / L \\
\mu g / L\end{array}$ & 0 \\
\hline & $\mu g / L$ & 0 \\
\hline & $\mu g / L$ & 0 \\
\hline & $\begin{array}{l}\mu g / L \\
\mu g / L\end{array}$ & $\begin{array}{l}0 \\
0\end{array}$ \\
\hline & $\mu g / L$ & 0 \\
\hline & $\mu g / L$ & 0 \\
\hline JV2 & $\mu g / L$ & 0 \\
\hline & $\mu g / L$ & 0 \\
\hline UI & $\mathrm{pCi} / \mathrm{L}$ & 0 \\
\hline & $\mathrm{pCi} / \mathrm{L}$ & 0 \\
\hline$J$ & pCi/L & 0 \\
\hline & $\mathrm{pCi} / \mathrm{mL}$ & 0 \\
\hline J & $\mathrm{pCi} / \mathrm{L}$ & 0 \\
\hline J & $\mathrm{pCi} / \mathrm{L}$ & 0 \\
\hline & pCi/L & 0 \\
\hline
\end{tabular}

WELL BGO 45B

$\begin{array}{lllllll}\text { SRS Coord. } & \text { Lat/Longitude } & \text { Screen Zone Elevation } & \text { Top of Casing } & \text { Casing } & \text { Pump } & \text { Formation } \\ \text { N75840.3 } & 33.282536^{\circ} \mathrm{N} & 147.0-137.0 \mathrm{ft} \text { msl } & 278.6 \mathrm{ft} \mathrm{msl} & 4^{\text {" PVC }} & \text { S } & \text { McBean (IIB, }) \\ \text { E54563.6 } & 81.670441^{\circ} \mathrm{W} & & & & \end{array}$

\section{FIELD MEASUREMENTS}

Sample date: 04/14/94

Depth to water: $59.06 \mathrm{ft}(18.00 \mathrm{~m})$ below TOC

Water elevation: $219.54 \mathrm{ft}(66.92 \mathrm{~m}) \mathrm{msl}$

Sp. conductance: $156 \mu \mathrm{S} / \mathrm{cm}$

Turbidity: 1.1 NTU

Water evacuated before sampling: $\mathbf{4 4} \mathrm{gal}$

The well went dry during purging.

\section{LABORATORY ANALYSES}

H D Analyte

$\mathrm{pH}$

Specific conductance

Turbidity

Acetophenone

Aldrin

Aldrin

Aluminum, total recoverable

Antimony, total recoverable

Arsenic, total recoverable
Time: 13:27

pH: 10.3

Alkalinity: $49 \mathrm{mg} / \mathrm{L}$

Water temperature: $20.4^{\circ} \mathrm{C}$

Volumes purged: 0.8 well volumes

- = exceeded holding time. $\square=$ exceeded screening level or final primary drinking water standard. 
WELL BGO 45B collected on $04 / 14 / 94$, laboratory analyses (cont.)

H $\underline{\text { Analyte }}$

Barium, total recoverable

Benzene

Bromodichloromethane

Bromoform

Bromomethane (Methyl bromide)

Cadmium, total recoverable

Calcium, total recoverable

Carbon tetrachloride

Chloride

Chlorobenzene

Chloroethane

Chloroethene (Vinyl chloride)

2-Chloroethyl vinyl ether

Chloroform

Chloromethane (Methyl chloride)

Chromium, total recoverable

Copper, total recoverable

Cyanide

$p, p^{\prime}$-DDT

p.p'-DDT

Dibromochloromethane

1,1-Dichloroethane

1,2-Dichloroethane

1,1-Dichloroethylene

trans-1,2-Dichloroethylene

Dichloromethane (Methylene chloride)

2,4-Dichlorophenoxyacetic acid

1,2-Dichloropropane

cis-1,3-Dichloropropene

trans-1,3-Dichloropropene

Dieldrin

Dieldrin

Endrin

Endrin

Ethylbenzene

Fluoride

Fluoride

Fluoride

Fluoride

Heptachlor

Heptachlor

Iron, total recoverable

Lead, total recoverable

Lindane

Lindane

Magnesium, total recoverable

Manganese, total recoverable

Mercury, total recoverable

Methoxychlor

Methoxychlor

Naphthalene

Nickel, total recoverable

Nitrate-nitrite as nitrogen

Phenols

Phenols

Potassium, total recoverable
Result DF

44
$<1.0$

$<1.0$

$<1.0$

$<1.0$

$<2.0$

14,600

$<1.0$

2,070

$<1.0$

$<1.0$

$<1.0$

$<1.0$

$<1.0$

$<1.0$

4.5

$<4.0$

$<5.0$

$<0.10$

$<0.10$

$<1.0$

$<1.0$

$<1.0$

$<1.0$

$<1.0$

$<1.0$

$<0.0015$

$<1.0$

$<1.0$

$<1.0$

$<0.50$

$<0.50$

$<0.0060$

$<0.0060$

$<1.0$

$<100$

$<100$

$<100$

$<100$

$<0.050$

$<0.050$

7.4

$<3.0$

$<0.0050$

$<0.0050$

262

$<2.0$

$<0.20$

$<0.50$

$<0.50$

$<10$

$<4.0$

830

$<5.0$

$<5.0$

5,380 $\underline{\text { Mod }}$

Flag Lab

$\mu \mathrm{g} / \mathrm{L} \quad 0 \quad \mathrm{GE}$

$\mu \mathrm{g} / \mathrm{L} \quad 0 \quad \mathrm{GE}$

$\mu \mathrm{g} / \mathrm{L} \quad 0 \quad \mathrm{GE}$

$\mu g / L \quad 0 \quad$ GE

$\mu g / L \quad 0 \quad$ GE

$\mu g / L \quad 0 \quad G E$

$\mu g / L \quad 0 \quad G E$

$\mu \mathrm{g} / \mathrm{L} \quad 0 \quad \mathrm{GE}$

$\mu g / L \quad 0 \quad G E$

$\mu g / L \quad 0 \quad$ GE

$\mu g / L \quad 0 \quad$ GE

$\mu g / L \quad 0 \quad$ GE

$\mu \mathrm{g} / \mathrm{L} \quad 0 \quad \mathrm{GE}$

$\mu g / L \quad 0 \quad$ GE

$\mu \mathrm{g} / \mathrm{L} \quad 0 \quad \mathrm{GE}$

$\mu g / L \quad 0 \quad$ GE

$\mu g / L \quad 0 \quad$ GE

$\mu g / L \quad 0 \quad G E$

$\mu g / L \quad 0 \quad G E$

$\mu \mathrm{g} / \mathrm{L} \quad 0 \quad \mathrm{GE}$

$\mu g / L \quad 0 \quad$ GE

$\mu g / L \quad 0 \quad G E$

$\mu \mathrm{g} / \mathrm{L} \quad 0 \quad \mathrm{GE}$

$\mu g / L \quad 0 \quad$ GE

$\mu \mathrm{g} / \mathrm{L} \quad \mathrm{O} \quad \mathrm{GE}$

$\mu g / L \quad 0 \quad$ GE

$\mu g / L \quad 0 \quad$ GE

$\mu \mathrm{g} / \mathrm{L} \quad \mathrm{O} \quad \mathrm{GE}$

$\mu \mathrm{g} / \mathrm{L} \quad 0 \quad \mathrm{GE}$

$\mu \mathrm{g} / \mathrm{L} \quad 0 \quad \mathrm{GE}$

$\mu \mathrm{g} / \mathrm{L} \quad 0 \quad \mathrm{GE}$

$\mu g / L \quad 0 \quad \mathrm{GE}$

$\mu \mathrm{g} / \mathrm{L} \quad 0 \quad \mathrm{GE}$

$\mu g / L \quad 0 \quad$ GE

$\mu \mathrm{g} / \mathrm{L} \quad 0 \quad \mathrm{GE}$

$\mu \mathrm{g} / \mathrm{L} \quad \mathrm{O}$ GE

$\mu \mathrm{g} / \mathrm{h} \quad 0 \quad \mathrm{GE}$

$\mu g / L \quad 0 \quad$ GE

$\mu \mathrm{g} / \mathrm{L} \quad 0 \quad \mathrm{GE}$

$\mu g / L \quad 0 \quad$ GE

$\mu g / L \quad 0 \quad$ GE

$\mu g / L \quad 0 \quad$ GE

$\mu g / L \quad 0 \quad$ GE

$\mu \mathrm{g} / \mathrm{L} \quad \mathrm{O}$ GE

$\mu \mathrm{g} / \mathrm{L} \quad 0 \quad \mathrm{GE}$

$\mu g / L \quad 0 \quad \mathrm{GE}$

$\mu \mathrm{g} / \mathrm{L} \quad 0 \quad \mathrm{GE}$

$\mu \mathrm{g} / \mathrm{L} \quad 0 \quad \mathrm{GE}$

$\mu \mathrm{g} / \mathrm{L} \quad 0 \quad \mathrm{GE}$

$\mu g / L \quad 0 \quad$ GE

$\mu g / L \quad 0 \quad$ GE

$\mu g / L \quad 0 \quad$ GE

$\mu \mathrm{g} / \mathrm{L} \quad 0 \quad \mathrm{GE}$

$\mu g / L \quad 0 \quad$ GE

$\mu \mathrm{g} / \mathrm{L} \quad 0 \quad \mathrm{GE}$

$\mu g / L \quad 0 \quad$ GE

- = exceeded holding time. = = exceeded screening level or final primary drinking water standard. 
WELL BGO 45B collected on 04/14/94, laboratory analyses (cont.)

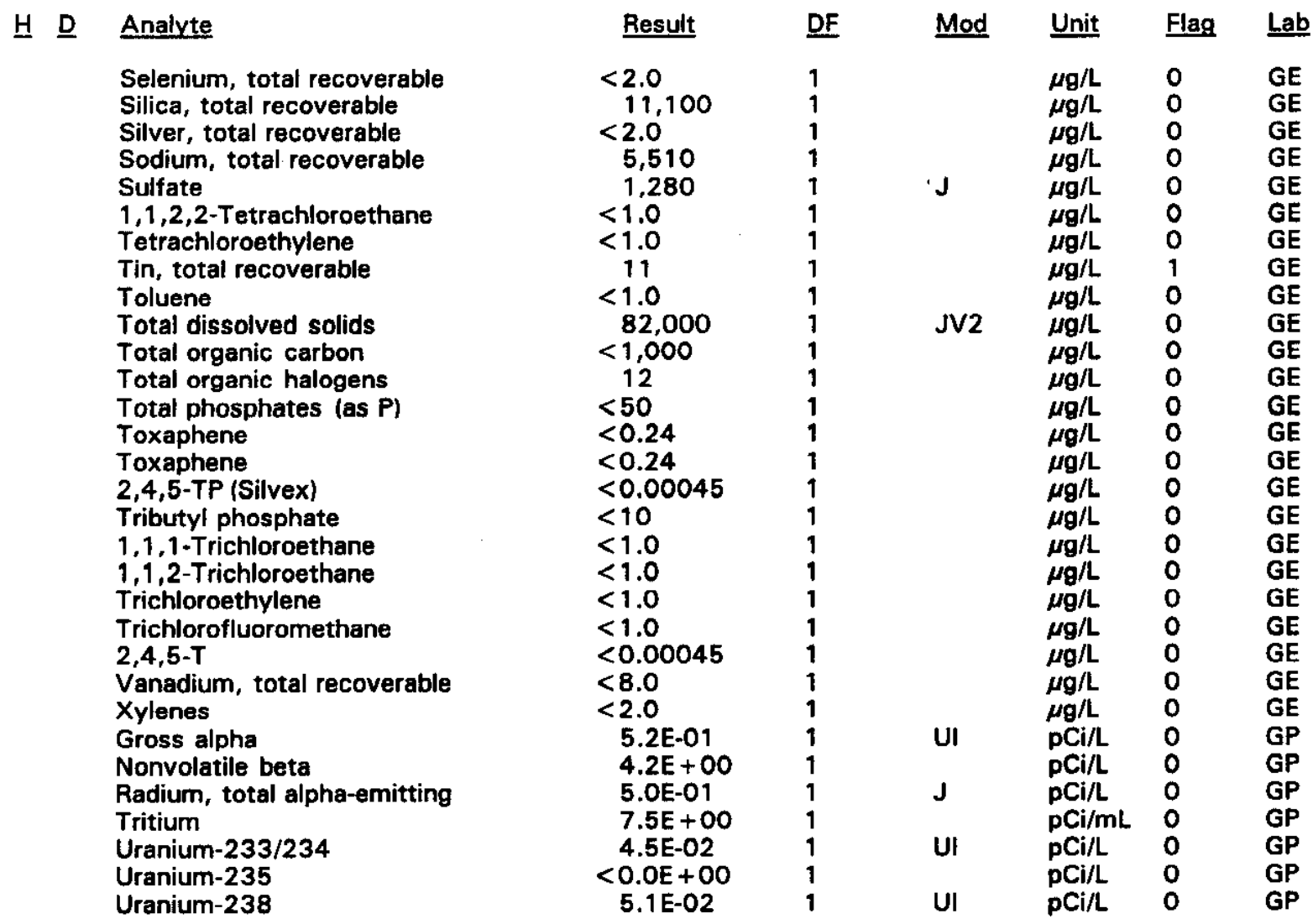

\section{WELL BGO 45C}

\begin{tabular}{|c|c|c|c|c|c|c|}
\hline SRS Coord. & Lat/Longitude & Screen Zone Elevation & Top of Casing & Casing & Pump & Formation \\
\hline $\begin{array}{l}\text { N75835.0 } \\
\text { E54577.4 }\end{array}$ & $\begin{array}{l}33.282547^{\circ} \mathrm{N} \\
81.670395^{\circ} \mathrm{W}\end{array}$ & $200.5-190.5 \mathrm{ft} \mathrm{msl}$ & $278.6 \mathrm{ft} \mathrm{msl}$ & 4" PVC & $\mathbf{S}$ & Barnwell (IIB ${ }_{1}$ ) \\
\hline
\end{tabular}

\section{FIELD MEASUREMENTS}

Sample date: 04/14/94

Depth to water: $55.23 \mathrm{ft}(16.83 \mathrm{~m})$ below TOC

Water elevation: $223.37 \mathrm{ft}(68.08 \mathrm{~m}) \mathrm{msl}$

Sp. conductance: $46 \mu \mathrm{S} / \mathrm{cm}$

Turbidity: 4.4 NTU

Water evacuated before sampling: $21 \mathrm{gal}$

The well went dry during purging.

\section{LABORATORY ANALYSES}

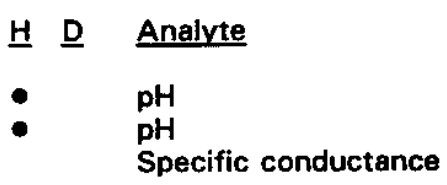

Time: 13:10

pH: 5.4

Alkalinity: $4 \mathrm{mg} / \mathrm{L}$

Water temperature: $20.7^{\circ} \mathrm{C}$

Volumes purged: 1.0 well volumes

- = exceeded holding time. - = exceeded screening level or final primary drinking water standard. 
WELL BGO $45 \mathrm{C}$ collected on $04 / 14 / 94$, laboratory analyses (cont.)

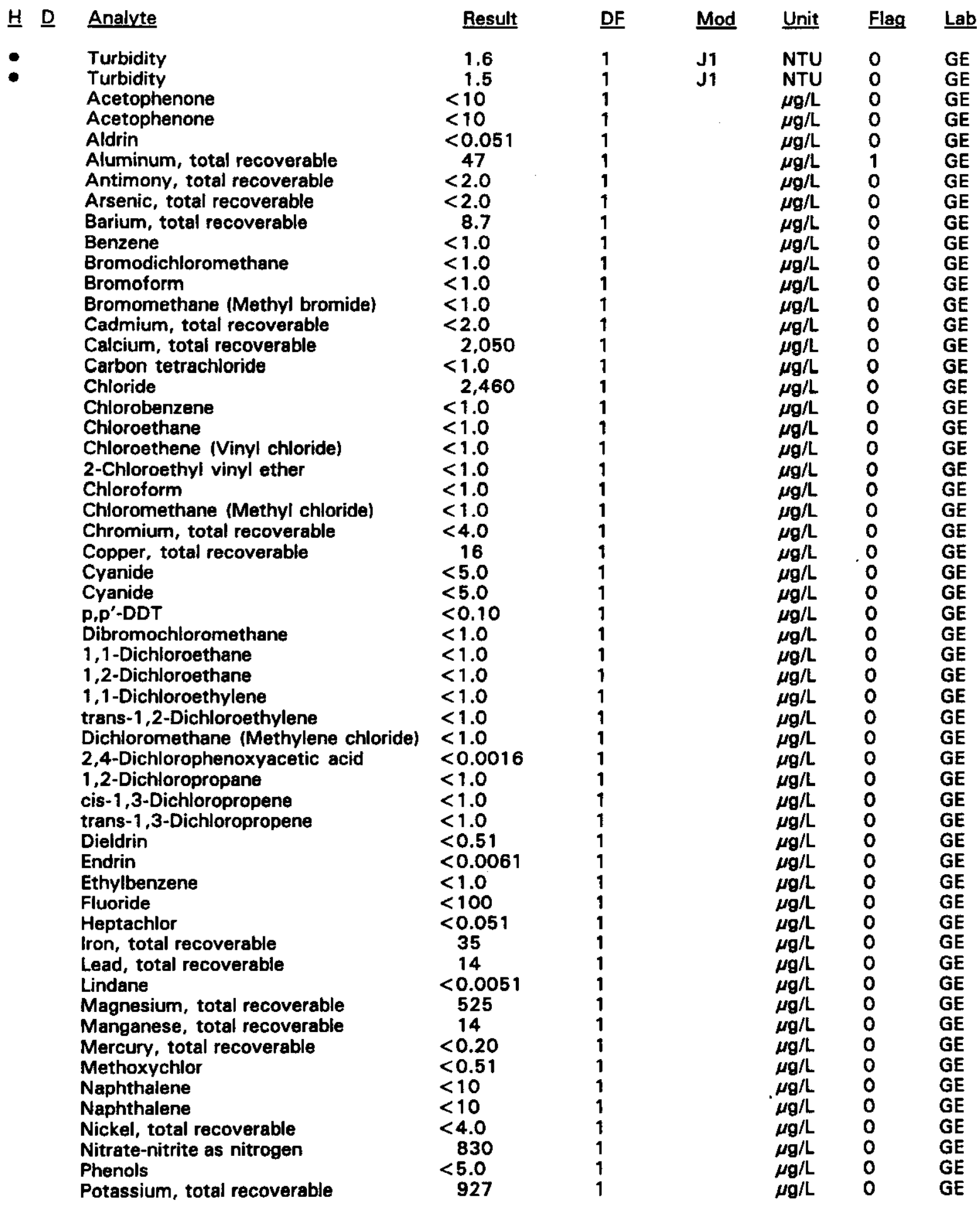

- = exceeded holding time. $\square=$ exceeded screening level or final primary drinking water standard. 
WELL BGO 45C coliected on $04 / 14 / 94$, laboratory analyses (cont.)

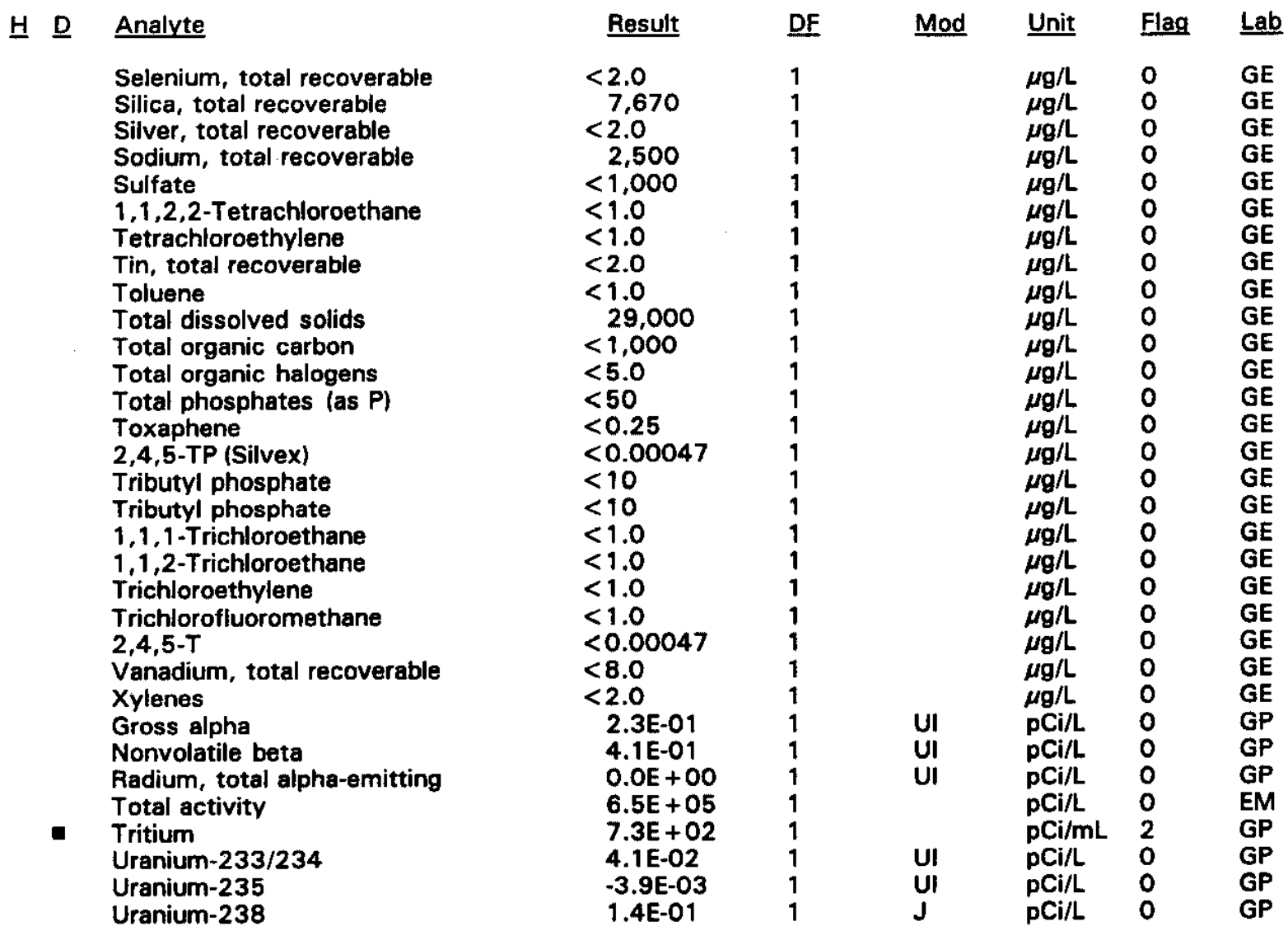

\section{WELL BGO 45D}

\begin{tabular}{|c|c|c|c|c|c|c|}
\hline SRS Coord. & Lat/Longitude & Screen Zone Elevation & Top of Casing & Casing & Pump & Formation \\
\hline $\begin{array}{l}\text { N75854.3 } \\
\text { E54585.6 }\end{array}$ & $\begin{array}{l}33.282603^{\circ} \mathrm{N} \\
81.670411^{\circ} \mathrm{W}\end{array}$ & $229.6-209.6 \mathrm{ft} \mathrm{msl}$ & $278.6 \mathrm{ft} \mathrm{msl}$ & 4" PVC & $\mathbf{S}$ & Water $\mathrm{Ta}$ \\
\hline
\end{tabular}

\section{FIELD MEASUREMENTS}

Sample date: 04/06/94

Depth to water: $50.53 \mathrm{ft}(15.40 \mathrm{~m})$ below TOC

Water elevation: $228.07 \mathrm{ft}(69.52 \mathrm{~m}) \mathrm{ms}$ l

Sp. conductance: $35 \mu \mathrm{S} / \mathrm{cm}$

Turbidity: 1.3 NTU

Water evacuated before sampling: 33 gal

LABORATORY ANALYSES

$\begin{array}{ll}\text { H } & \text { Analyte } \\ & \text { pH } \\ & \text { Specific conductance }\end{array}$
Time: 15:28

pH: 5.3

Alkalinity: $1 \mathrm{mg} / \mathrm{L}$

Water temperature: $20.1^{\circ} \mathrm{C}$

Volumes purged: 2.7 well volumes

- = exceeded holding time. $\square=$ exceeded screening level or final primary drinking water standard. 
WELL BGO 45D collected on 04/06/94, laboratory analyses (cont.)

\section{H D Analyte}

Turbidity

Acetophenone

Aldrin

Aluminum, total recoverable

Antimony, total recoverable

Antimony, total recoverable

Arsenic, total recoverable

Arsenic, total recoverable

Barium, total recoverable

Benzene

Bromodichloromethane

Bromoform

Bromomethane (Methyl bromide)

Cadmium, total recoverable

Calcium, total recoverable

Carbon tetrachloride

Chloride

Chlorobenzene

Chloroethane

Chloroethene (Vinyl chloride)

2-Chloroethyl vinyl ether

Chloroform

Chloromethane (Methyl chloride)

Chromium, total recoverable

Copper, total recoverable

Cyanide

p,p'-DDT

Dibromochloromethane

1,1-Dichloroethane

1,2-Dichioroethane

1,1-Dichloroethylene

trans-1,2-Dichloroethylene

Dichloromethane (Methylene chloride)

2,4-Dichlorophenoxyacetic acid

1,2-Dichloropropane

cis-1,3-Dichloropropene

cis-1,3-Dichloropropene

trans-1,3-Dichloropropene

Dieldrin

Endrin

Ethylbenzene

Fluoride

Heptachlor

Iron, total recoverable

Lead, total recoverable

Lead, total recoverable

Lindane

Magnesium, total recoverable

Manganese, total recoverable

Mercury, total recoverable

Methoxychlor

Naphthalene

Nickel, total recoverable

Nitrate-nitrite as nitrogen

Phenols

Potassium, total recoverable

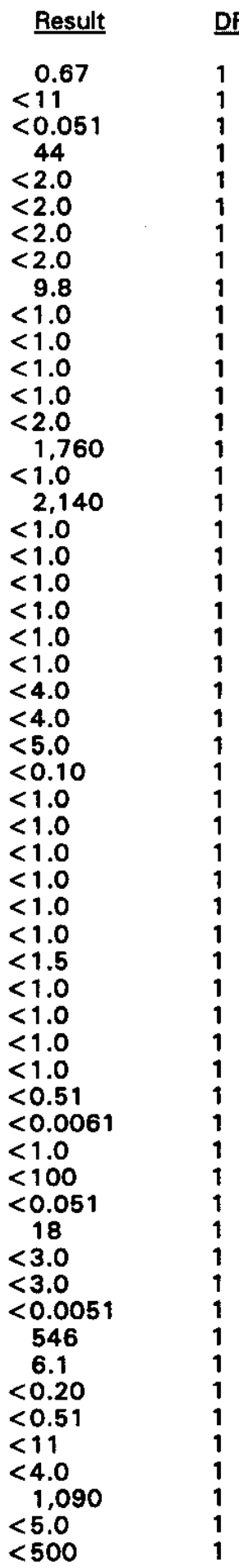

Mod Unit Flag Lab

$\begin{array}{lll}N T U & 0 & G E\end{array}$

$\mu \mathrm{g} / \mathrm{L} / \mathrm{L} \quad \mathrm{O} \quad \mathrm{GE}$

$\mu g / L \quad 1 \quad G E$

$\mu g / L \quad 0 \quad \mathrm{GE}$

$\mu \mathrm{g} / \mathrm{L} \quad 0 \quad \mathrm{GE}$

J3

$\mu \mathrm{g} / \mathrm{L}$

$\mu g / L$

$\mu g / L$

$\mu g / L$

$\mu g / L$

$\mu g / L$

$\mu g / L$

$\mu g / L$

$\mu g / L$

$\mu g / L$

$\mu g / L$

$\mu g / L$

$\mu g / L$

$\mu g / L$

$\mu g / L$

$\mu g / L$

$\mu \mathrm{g} / \mathrm{L}$

$\mu g / L$

$\mu g / L$

$\mu \mathrm{g} / \mathrm{L}$

$\mu \mathrm{g} / \mathrm{L}$

$\mu g / L$

$\mu g / L$

$\mu g / L$

$\mu g / L$

$\mu g / L$

$\mu \mathrm{g} / \mathrm{L}$

$\mu \mathrm{g} / \mathrm{L}$

$\mu g / L$

$\mu \mathrm{g} / \mathrm{L}$

$\mu g / L$

$\mu \mathrm{g} / \mathrm{L}$

$\mu g / L$

$\mu g / L$

$\mu g / L$

$\mu g / L$

$\mu g / L$

$\mu \mathrm{g} / \mathrm{L}$

$\mu g / L$

$\mu \mathrm{g} / \mathrm{L}$

$\mu g / L$

$\mu \mathrm{g} / \mathrm{L}$

$\mu g / L$

$\mu g / L$

$\mu g / L$

$\mu \mathrm{g} / \mathrm{L}$

$\mu g / L$

$\mu g / L$

GE
$\mathrm{GE}$
$\mathrm{GE}$
$\mathrm{GE}$
$\mathrm{GE}$
$\mathrm{GE}$
$\mathrm{GE}$
$\mathrm{GE}$
$\mathrm{GE}$
$\mathrm{GE}$
$\mathrm{GE}$
$\mathrm{GE}$
$\mathrm{GE}$
$\mathrm{GE}$
$\mathrm{GE}$
$\mathrm{GE}$
$\mathrm{GE}$
$\mathrm{GE}$
$\mathrm{GE}$
$\mathrm{GE}$
$\mathrm{GE}$
$\mathrm{GE}$
$\mathrm{GE}$
$\mathrm{GE}$
$\mathrm{GE}$
$\mathrm{GE}$
$\mathrm{GE}$
$\mathrm{GE}$
$\mathrm{GE}$
$\mathrm{GE}$

- = exceeded holding time. = exceeded screening level or final primary drinking water standard. 
WELL BGO 45D collected on 04/06/94, laboratory analyses (cont.)

H

Analyte
Selenium, total recoverable
Selenium, total recoverable
Silica, total recoverable
Silver, total recoverable
Sodium, total recoverable
Sulfate
1,1,2,2-Tetrachloroethane
Tetrachloroethylene
Tin, total recoverable
Toluene
Total dissolved solids
Total organic carbon
Total organic halogens
Total phosphates (as P)
Toxaphene
2,4,5-TP (Silvex)
Tributyl phosphate
1,1,1-Trichloroethane
1,1,2-Trichloroethane
1,1,2-Trichloroethane
Trichloroethylene
Trichlorofluoromethane
$2,4,5-T$
Vanadium, total recoverable
Xylenes
Gross alpha
Nonvolatile beta
Radium, total alpha-emitting
Tritium
Uranium-233/234
Uranium-235
Uranium-238

\begin{tabular}{ll}
\multicolumn{1}{r}{ Result } & DF \\
$<2.0$ & 1 \\
$<2.0$ & 1 \\
7,190 & 1 \\
$<2.0$ & 1 \\
2.050 & 1 \\
$<1,000$ & 1 \\
$<1.0$ & 1 \\
$<1.0$ & 1 \\
$<2.0$ & 1 \\
$<1.0$ & 1 \\
37,000 & 1 \\
$<1.000$ & 1 \\
6.4 & 1 \\
$<50$ & 1 \\
$<0.25$ & 1 \\
$<0.46$ & 1 \\
$<11$ & 1 \\
$<1.0$ & 1 \\
$<1.0$ & 1 \\
$<1.0$ & 1 \\
$<1.0$ & 1 \\
$<1.0$ & 1 \\
$<0.46$ & 1 \\
$<8.0$ & 1 \\
$<2.0$ & 1 \\
$8.8 E-01$ & 1 \\
$3.0 E+00$ & 1 \\
$7.0 E-01$ & 1 \\
$5.0 E+02$ & 1 \\
$1.6 E-01$ & 1 \\
$5.9 E-02$ & 1 \\
$8.1 E-02$ & 1 \\
&
\end{tabular}

DF

Mod

Unit Flag Lab

$\mu g / L \quad 0 \quad$ GE

$\mu g / L \quad 0 \quad$ GE

$\mu \mathrm{g} / \mathrm{L} \quad \mathrm{O} \quad \mathrm{GE}$

$\mu g / L \quad 0 \quad$ GE

$\mu \mathrm{g} / \mathrm{L} \quad 0 \quad \mathrm{GE}$

$\mu g / L \quad 0 \quad G E$

$\mu g / L \quad 0 \quad$ GE

$\mu \mathrm{g} / \mathrm{L} \quad \mathrm{O} \quad \mathrm{GE}$

$\mu g / L \quad 0 \quad$ GE

$\mu \mathrm{g} / \mathrm{L} \quad \mathrm{O} \quad \mathrm{GE}$

$\mu \mathrm{g} / \mathrm{L} \quad \mathrm{O} \quad \mathrm{GE}$

$\mu \mathrm{g} / \mathrm{L} \quad \mathrm{O} \quad \mathrm{GE}$

J

$\mu \mathrm{g} / \mathrm{L} \quad 0 \quad \mathrm{GE}$

$\mu g / L \quad 0 \quad G E$

$\mu g / L \quad 0 \quad$ GE

$\mu g / L \quad 0 \quad$ GE

$\mu g / L \quad 0 \quad$ GE

$\mu g / L \quad 0 \quad G E$

$\mu \mathrm{g} / \mathrm{L} \quad 0 \quad \mathrm{GE}$

$\mu \mathrm{g} / \mathrm{L} \quad \mathrm{O} \quad \mathrm{GE}$

$\mu \mathrm{g} / \mathrm{L} \quad 0 \quad \mathrm{GE}$

$\mu \mathrm{g} / \mathrm{L} \quad 0 \quad \mathrm{GE}$

JV2 $\mu g / L \quad 0 \quad$ GE

$\mu g / L \quad 0 \quad$ GE

$\mu g / L \quad 0 \quad$ GE

pCi/L $0 \quad$ GP

PCi/L $\quad 0 \quad$ GP

pCi/L $0 \quad$ GP

pCi/mL 2 GP

pCi/L 0 GP

$\mathrm{pCi} / \mathrm{L} \quad 0 \quad \mathrm{GP}$

pCi/L 0 GP

WELL BGO 46B

\begin{tabular}{|c|c|c|c|c|c|c|}
\hline SRS Coord. & Lat/Longitude & Screen Zone Elevation & Top of Casing & Casing & Pump & Formation \\
\hline 75 & $\begin{array}{l}33.280511^{\circ} \mathrm{N} \\
81.669146^{\circ} \mathrm{W}\end{array}$ & $150.4-140.4 \mathrm{ft} \mathrm{msl}$ & $265.4 \mathrm{ft} \mathrm{msl}$ & 4" PVC & $\mathbf{s}$ & \\
\hline
\end{tabular}

\section{FIELD MEASUREMENTS}

Sample date: 04/12/94

Depth to water: $47.39 \mathrm{ft}(14.44 \mathrm{~m})$ below TOC

Water elevation: $218.01 \mathrm{ft}(66.45 \mathrm{~m}) \mathrm{msl}$

Sp. conductance: $64 \mu \mathrm{S} / \mathrm{cm}$

Turbidity: 0.3 NTU

Water evacuated before sampling: 335 gal
Time: 15:10

pH: 6.3

Alkalinity: $16 \mathrm{mg} / \mathrm{L}$

Water temperature: $20.1^{\circ} \mathrm{C}$

Volumes purged: 6.6 well volumes

\section{LABORATORY ANALYSES}

\begin{tabular}{|c|c|c|c|c|c|c|}
\hline$\underline{H} \underline{\mathrm{D}}$ & Analyte & Result & DF & Mod & Unit & Flag \\
\hline & $\begin{array}{l}\mathrm{pH} \\
\mathrm{pH}\end{array}$ & $\begin{array}{l}6.6 \\
6.6\end{array}$ & $\begin{array}{l}1 \\
1\end{array}$ & $\begin{array}{l}\mathrm{J} 1 \\
\mathrm{~J} 1\end{array}$ & $\begin{array}{l}\mathrm{pH} \\
\mathrm{pH}\end{array}$ & $\begin{array}{l}0 \\
0\end{array}$ \\
\hline
\end{tabular}

- = exceeded holding time. = exceeded screening level or final primary drinking water standard. 
WELL BGO $46 B$ collected on $04 / 12 / 94$, laboratory analyses (cont.)

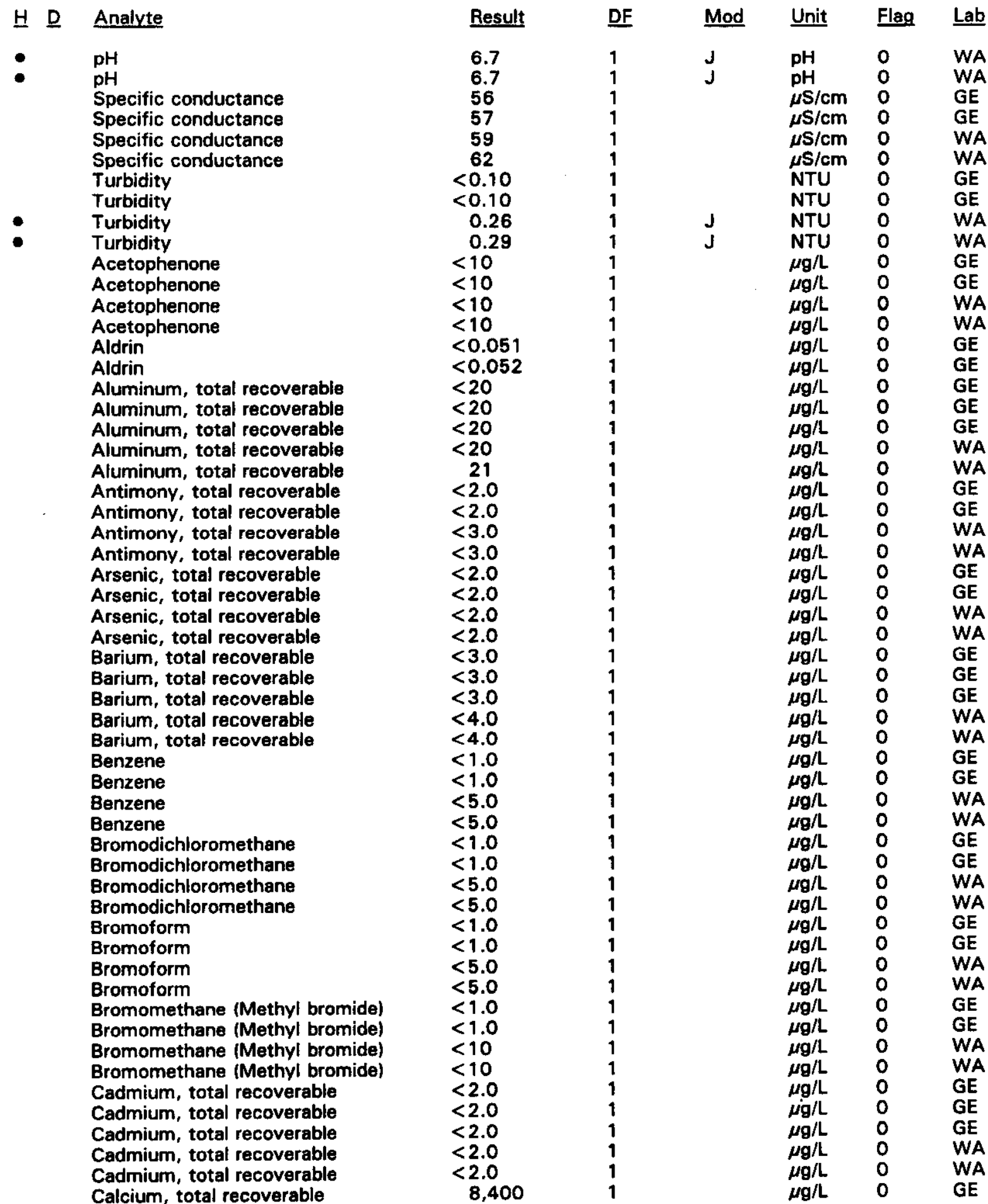

- = exceeded holding time. $=$ exceeded screening level or final primary drinking water standard. 
WELL BGO $46 B$ collected on $04 / 12 / 94$, laboratory analyses (cont.)

H $\underline{\text { D }}$ Analyte

Calcium, total recoverable

Calcium, total recoverable

Calcium, total recoverable

Calcium, total recoverable

Carbon tetrachloride

Carbon tetrachloride

Carbon tetrachloride

Carbon tetrachloride

Chloride

Chiloride

Chloride

Chloride

Chlorobenzene

Chlorobenzene

Chlorobenzene

Chlorobenzene

Chloroethane

Chloroethane

Chloroethane

Chloroethane

Chloroethene (Vinyl chloride)

Chloroethene (Vinyl chloride)

Chloroethene (Vinyl chloride)

Chloroethene (Vinyl chloride)

2-Chloroethyl vinyl ether

2-Chloroethyl vinyl ether

2-Chloroethyl vinyl ether

2-Chloroethyl vinyl ether

Chloroform

Chloroform

Chloroform

Chloroform

Chloromethane (Methyl chloride)

Chloromethane (Methyl chloride)

Chloromethane (Methyl chloride)

Chloromethane (Methyl chloride)

Chromium, total recoverable

Chromium, total recoverable

Chromium, total recoverable

Chromium, total recoverable

Chromium, total recoverable

Copper, total recoverable

Copper, total recoverable

Copper, total recoverable

Copper, total recoverable

Copper, total recoverable

Cyanide

Cyanide

Cyanide

Cyanide

p,p'-DDT

p,p'-DDT

Dibromochloromethane

Dibromochloromethane

Dibromochloromethane

Dibromochloromethane

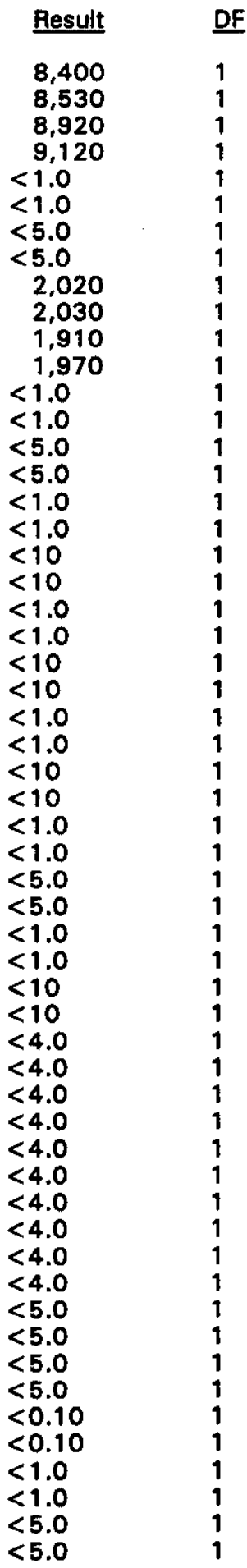

Mod Unit Flag Lab

$\mu \mathrm{g} / \mathrm{L}$

$\mu \mathrm{g} / \mathrm{L}$

$\mu g / L$

$\mu \mathrm{g} / \mathrm{L}$

$\mu \mathrm{g} / \mathrm{L}$

$\mu \mathrm{g} / \mathrm{L}$

$\mu g / L$

$\mu \mathrm{g} / \mathrm{L}$

$\mu \mathrm{g} / \mathrm{L}$

$\mu g / L$

$\mu \mathrm{g} / \mathrm{L}$

$\mu g / L$

$\mu g / L$

$\mu g / L$

$\mu \mathrm{g} / \mathrm{L}$

$\mu g / L$

$\mu g / L$

$\mu \mathrm{g} / \mathrm{L}$

$\mu g / L$

$\mu g / L$

$\mu g / L$

$\mu g / L$

$\mu \mathrm{g} / \mathrm{L}$

$\mu \mathrm{g} / \mathrm{L}$

$\mu g / L$

$\mu \mathrm{g} / \mathrm{L}$

$\mu g / L$

$\mu g / L$

$\mu g / L$

$\mu g / L$

$\mu \mathrm{g} / \mathrm{L}$

$\mu \mathrm{g} / \mathrm{L}$

$\mu g / L$

$\mu \mathrm{g} / \mathrm{L}$

$\mu \mathrm{g} / \mathrm{h}$

$\mu g / L$

$\mu g / L$

$\mu \mathrm{g} / \mathrm{L}$

$\mu g / L$

$\mu \mathrm{g} / \mathrm{L}$

$\mu g / L$

$\mu g / L$

$\mu g / L$

$\mu \mathrm{g} / \mathrm{L}$

$\mu g / L$

$\mu g / L$

$\mu g / L$

$\mu g / L$

$\mu g / L$

$\mu g / L$

$\mu g / L$

$\mu \mathrm{g} / \mathrm{L}$

$\mu \mathrm{g} / \mathrm{L}$

$\mu \mathrm{g} / \mathrm{L}$

$\mu \mathrm{g} / \mathrm{L}$

$\mu \mathrm{g} / \mathrm{L}$
GE

GE

WA

WA

GE

GE

WA

WA

GE

GE

WA

WA

GE

GE

WA

WA

GE

GE

WA

WA

GE

GE

WA

WA

GE

GE

WA

WA

GE

GE

WA

WA

GE

GE

WA

WA

GE

GE

GE

WA

WA

GE

GE

GE

WA

WA

GE

GE

WA

WA

GE

GE

GE

GE

WA

WA

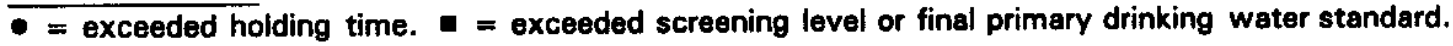


WELL BGO $46 \mathrm{~B}$ collected on 04/12/94, laboratory analyses (cont.)

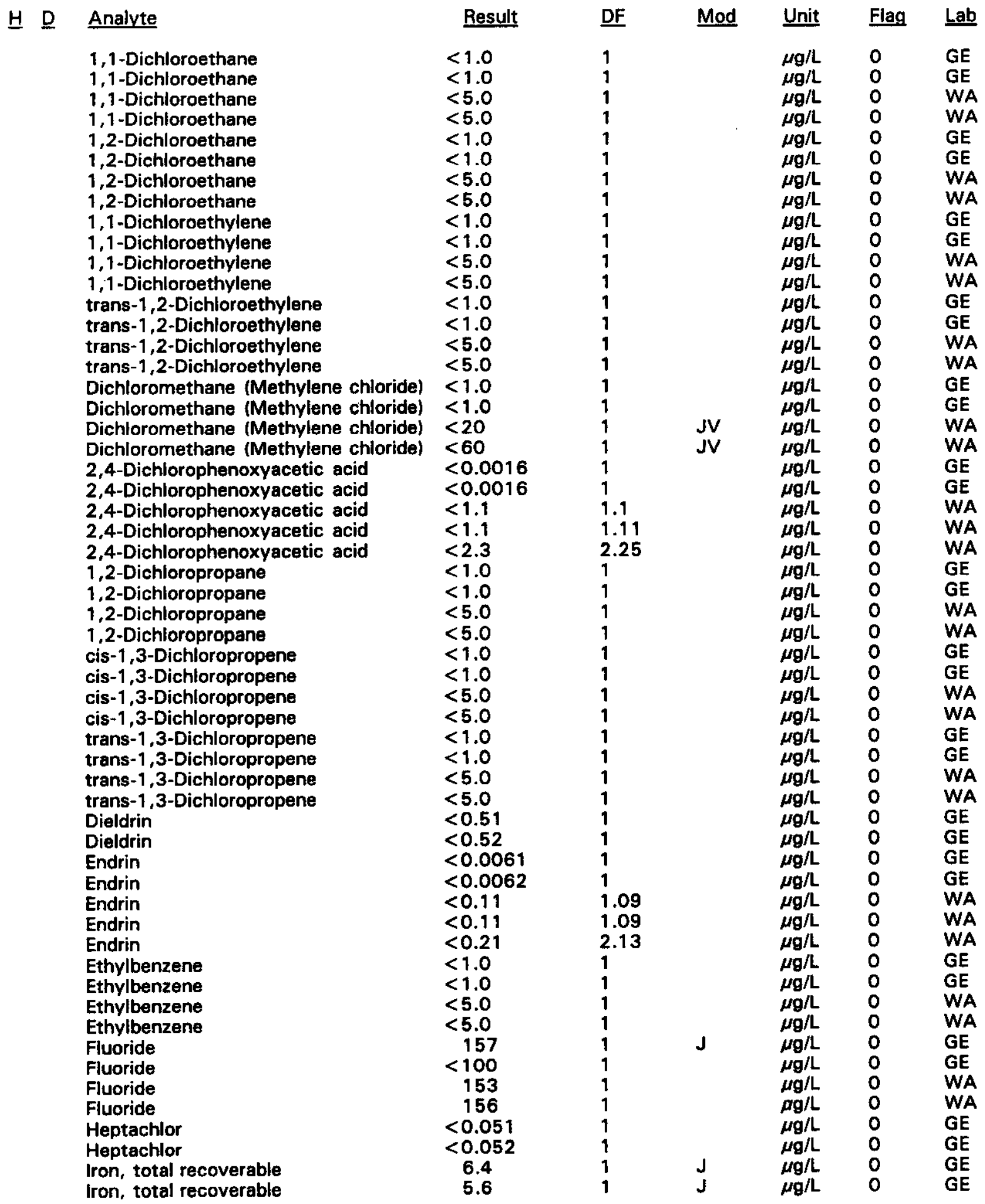

$\overline{-}=$ exceeded holding time. $=$ exceeded screening level or final primary drinking water standard. 
WELL. BGO 46B collected on 04/12/94, laboratory analyses (cont.)

\section{H D Analyte}

Iron, total recoverable

Iron, total recoverable

Iron, total recoverable

Lead, total recoverable

Lead, total recoverable

Lead, total recoverable

Lead, total recoverable

Lindane

Lindane

Lindane

Lindane

Lindane

Magnesium, total recoverable

Magnesium, total recoverable

Magnesium, total recoverable

Magnesium, total recoverable

Magnesium, total recoverable

Manganese, total recoverable

Manganese, total recoverable

Manganese, total recoverable

Manganese, total recoverable

Manganese, total recoverable

Mercury, total recoverable

Mercury, total recoverable

Mercury, total recoverable

Mercury, total recoverable

Mercury, total recoverable

Methoxychlor

Methoxychlor

Methoxychior

Methoxychlor

Methoxychlor

Methoxychior

Naphthalene

Naphthalene

Nickel, total recoverable

Nickel, total recoverable

Nickel, total recoverable

Nickel, total recoverable

Nickel, total recoverable

Nitrate as nitrogen

Nitrate as nitrogen

Nitrate-nitrite as nitrogen

Nitrate-nitrite as nitrogen

Phenols

Phenols

Phenols

Phenois

Potassium, total recoverable

Potassium, total recoverable

Potassium, total recoverable

Potassium, total recoverable

Potassium, total recoverable

Selenium, total recoverable

Selenium, total recoverable

Selenium, total recoverable

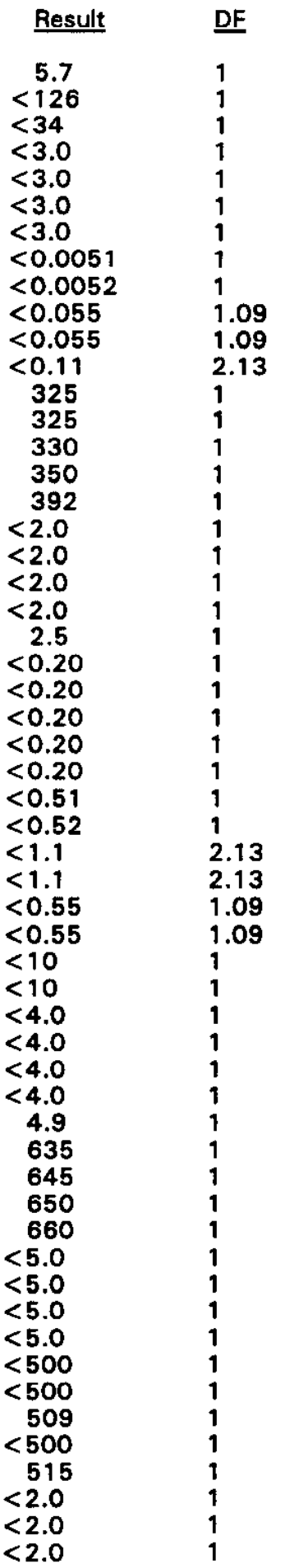

Mod Unit Flag Lab

$$
J
$$

JV

$\mu g / L$

$\mu g / L$

$\mu g / L$

$\mu g / L$

$\mu g / L$

$\mu \mathrm{g} / \mathrm{L}$

$\mu g / L \quad 0 \quad$ GE

$\mu \mathrm{g} / \mathrm{L} \quad 0$ WA

$\mu g / L \quad 0 \quad$ WA

$\mu g / L \quad 0 \quad$ WA

$\mu g / L \quad 0 \quad$ GE

$\mu g / L \quad 0 \quad G E$

$\mu g / L \quad 0 \quad$ GE

$\begin{array}{lll}\mu g / L & 0 & \text { WA } \\ \mu g / L & 0 & \text { WA }\end{array}$

$\mu g / L \quad 0 \quad$ GE

$\mu \mathrm{g} / \mathrm{L} \quad 0 \quad \mathrm{GE}$

$\mu g / L \quad 0 \quad$ GE

$\mu g / L \quad 0 \quad$ WA

$\mu g / L \quad 0$ WA

$\mu g / L \quad 0 \quad$ GE

$\mu \mathrm{g} / \mathrm{L} \quad \mathrm{O} \quad \mathrm{GE}$

$\mu g / L \quad 0 \quad$ GE

$\mu \mathrm{g} / \mathrm{L} \quad 0 \quad$ WA

$\mu g / L \quad 0 \quad$ WA

$\mu g / L \quad 0 \quad$ GE

$\mu g / L \quad O \quad$ GE

$\mu g / L \quad 0$ WA

$\mu g / L \quad 0 \quad$ WA

$\mu g / L \quad 0$ WA

$\mu g / L \quad 0 \quad$ WA

$\mu g / L \quad 0 \quad$ GE

$\mu g / L \quad 0 \quad$ GE

$\mu \mathrm{g} / \mathrm{L} \quad 0 \quad \mathrm{GE}$

$\mu g / L \quad 0 \quad$ GE

$\mu \mathrm{g} / \mathrm{L} \quad 0 \quad \mathrm{GE}$

$\mu \mathrm{g} / \mathrm{L} \quad 0 \quad$ WA

$\mu g / L \quad 0 \quad$ WA

$\mu g / L \quad 0 \quad$ WA

$\mu g / L \quad 0$ WA

$\mu g / L \quad 0 \quad$ GE

$\mu g / L \quad 0 \quad$ GE

$\mu \mathrm{g} / \mathrm{L} \quad 0 \quad \mathrm{GE}$

$\mu \mathrm{g} / \mathrm{L} \quad 0 \quad \mathrm{GE}$

$\mu g / L \quad 0 \quad$ WA

$\mu g / L \quad 0 \quad$ WA

$\mu g / L \quad 0 \quad$ GE

$\mu g / L \quad 0 \quad$ GE

$\mu g / L \quad 0 \quad$ GE

$\mu \mathrm{g} / \mathrm{L} \quad 0 \quad$ WA

$\mu \mathrm{g} / \mathrm{L} \quad 0 \quad$ WA

$\mu \mathrm{g} / \mathrm{L} \quad 0 \quad \mathrm{GE}$

$\mu \mathrm{g} / \mathrm{L} \quad \mathrm{O} \quad \mathrm{GE}$

$\mu \mathrm{g} / \mathrm{L} \quad 0 \quad$ WA

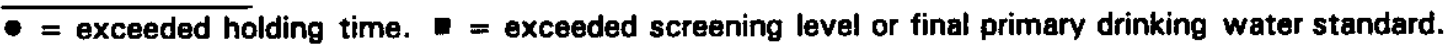


WELL BGO 468 collected on $04 / 12 / 94$, laboratory analyses (cont.)

H D Analyte

Selenium, total recoverable

Silica, total recoverable

Silica, total recoverable

Silica, total recoverable

Silica, total recoverable

Silica, total recoverable

Silver, total recoverable

Silver, total recoverable

Silver, total recoverable

Silver, total recoverable

Silver, total recoverable

Sodium, total recoverable

Sodium, total recoverable

Sodium, total recoverable

Sodium, total recoverable

Sodium, total recoverable

Sulfate

Sulfate

Sulfate

Sulfate

1,1,2,2-Tetrachloroethane

1,1,2,2-Tetrachloroethane

1,1,2,2-Tetrachloroethane

1,1,2,2-Tetrachloroethane

Tetrachloroethylene

Tetrachloroethylene

Tetrachloroethylene

Tetrachloroethylene

Tin, total recoverable

Tin, total recoverable

Tin, total recoverable

Tin, total recoverable

Tin, total recoverable

Toluene

Toluene

Toluene

Toluene

Total dissolved solids

Total dissolved solids

Total dissolved solids

Total dissolved solids

Total organic carbon

Total organic carbon

Total organic carbon

Total organic carbon

Total organic carbon

Total organic halogens

Total organic halogens

Total organic halogens

Total organic halogens

Total organic halogens

Total phosphates (as P)

Total phosphates (as P)

Total phosphates (as P)

Total phosphates (as P)

Toxaphene

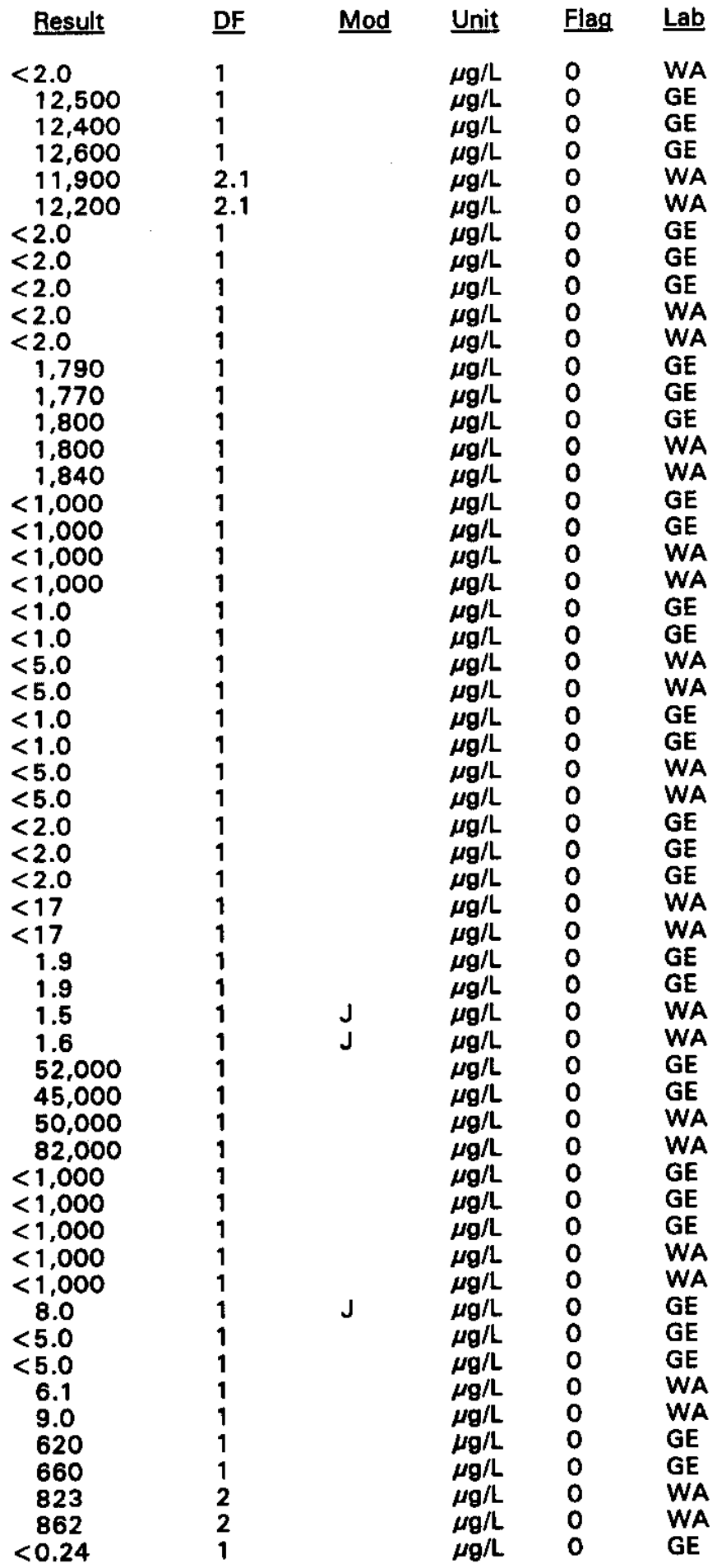

$\overline{- \text { = exceeded holding time. }}=$ exceeded screening level or final primary drinking water standard. 
WELL BGO $46 \mathrm{~B}$ collected on 04/1 2/94, laboratory analyses (cont.)

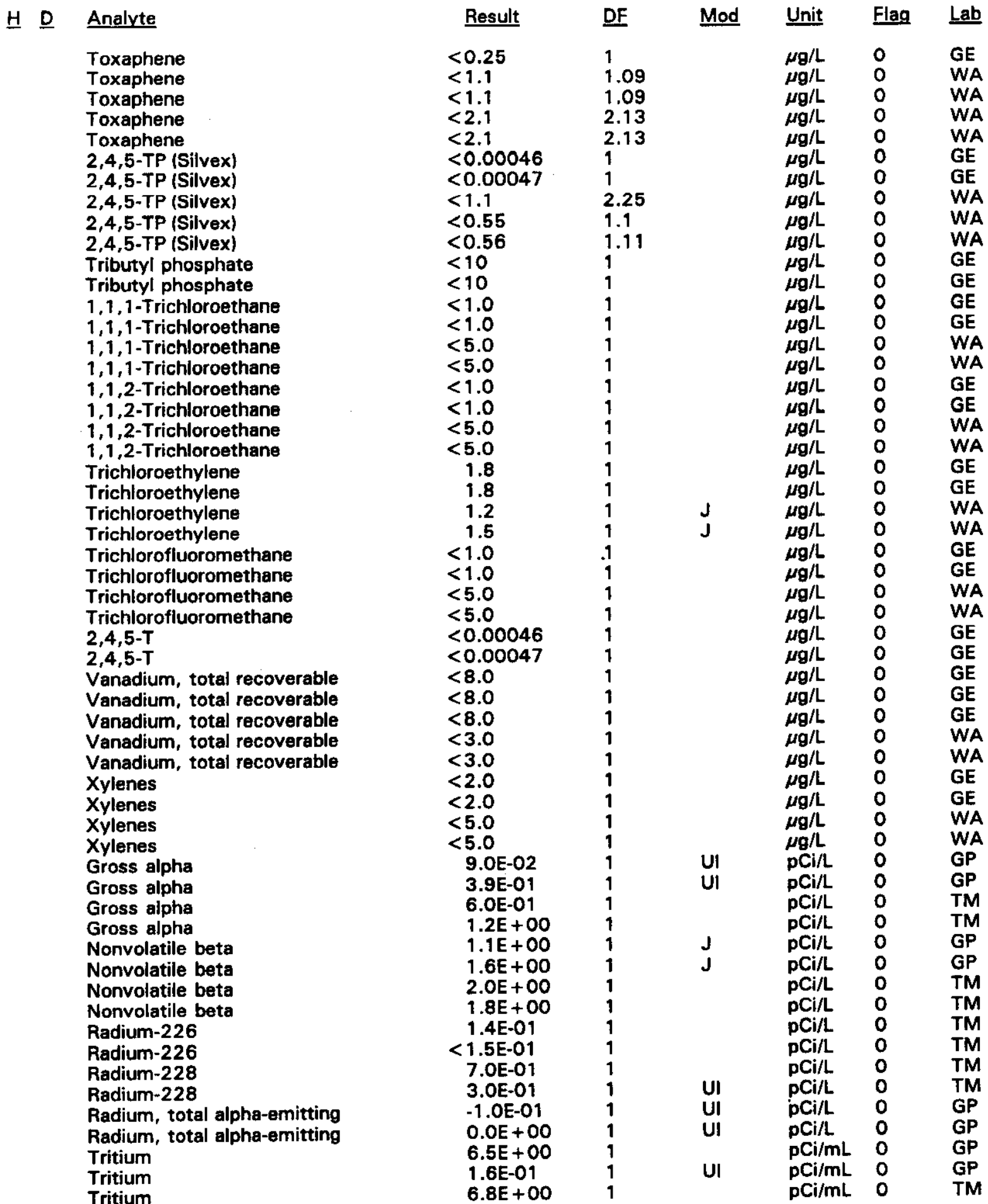

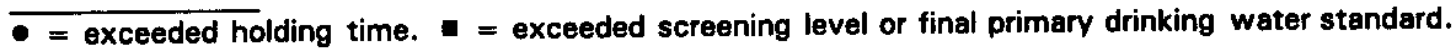


WELL BGO 46B collected on 04/12/94, laboratory analyses (cont.)

H D $\quad$ Analyte
Tritium
Uranium-233/234
Uranium-233/234
Uranium-233/234
Uranium-233/234
Uranium-235
Uranium-235
Uranium-235
Uranium-235
Uranium-238
Uranium-238
Uranium-238
Uranium-238

$\begin{array}{cc}\text { Result } & \text { DF } \\ \text { 7.6E }+00 & 1 \\ -1.3 E-02 & 1 \\ 1.2 E-01 & 1 \\ <0.0 E+00 & 1 \\ 1.0 E-01 & 1 \\ <0.0 E+00 & 1 \\ 1.2 E-01 & 1 \\ -1.3 E-02 & 1 \\ 1.0 E-01 & 1 \\ 9.9 E-02 & 1 \\ 9.1 E-02 & 1 \\ <0.0 E+00 & 1 \\ 1.0 E-01 & 1\end{array}$

\begin{tabular}{|c|c|c|}
\hline Mod & Unit & Flag \\
\hline $\begin{array}{l}\text { UI } \\
\text { UI }\end{array}$ & $\begin{array}{l}\mathrm{pCi} / \mathrm{mL} \\
\mathrm{pCi} / \mathrm{L} \\
\mathrm{pCi} / \mathrm{L} \\
\mathrm{pCi} / \mathrm{L}\end{array}$ & $\begin{array}{l}0 \\
0 \\
0 \\
0\end{array}$ \\
\hline UI & $\begin{array}{l}\mathrm{pCi} / \mathrm{L} \\
\mathrm{pCi} / \mathrm{L} \\
\mathrm{pCi} / \mathrm{L}\end{array}$ & $\begin{array}{l}0 \\
0 \\
0\end{array}$ \\
\hline UI & $\mathrm{pCi} / \mathrm{L}$ & 0 \\
\hline UI & $p C i / L$ & 0 \\
\hline U1 & $\mathrm{pCi} / \mathrm{L}$ & 0 \\
\hline UI & $\mathrm{pCi} / \mathrm{L}$ & 0 \\
\hline UI & pCi/L & 0 \\
\hline
\end{tabular}

\section{WELL BGO 46C}

\begin{tabular}{|c|c|c|c|c|c|c|}
\hline SRS Coord. & Lat/Longitude & Screen Zone Elevation & Top of Casing & Casing & Pump & Formation \\
\hline $\begin{array}{l}\text { N75022.2 } \\
\text { E54433.9 }\end{array}$ & $\begin{array}{l}33.280515^{\circ} \mathrm{N} \\
81.669194^{\circ} \mathrm{W}\end{array}$ & 188.0-178.0ft ms & $265.1 \mathrm{ft} \mathrm{msl}$ & 4" PVC & $\mathbf{S}$ & Barnw \\
\hline
\end{tabular}

\section{FIELD MEASUREMENTS}

Sample date: $04 / 14 / 94$

Depth to water: $45.43 \mathrm{ft}(13.85 \mathrm{~m})$ below TOC Water elevation: $219.67 \mathrm{ft}(66.96 \mathrm{~m}) \mathrm{ms}$ l Sp. conductance: $56 \mu \mathrm{S} / \mathrm{cm}$ Turbidity: 12.7 NTU

Water evacuated before sampling: 30 gal The well went dry during purging.

\section{LABORATORY ANALYSES}

H D Analyte

- $\quad \mathrm{pH}$

Specific conductance
Turbidity
Acetophenone
Aldrin
Aluminum, total recoverable
Antimony, total recoverable
Arsenic, total recoverable
Barium, total recoverable
Benzene
Benzene
Bromodichloromethane
Bromodichloromethane
Bromoform
Bromoform
Bromomethane (Methyl bromide)
Bromomethane (Methyl bromide)
Cadmium, total recoverable
Calcium, total recoverable
Carbon tetrachloride
Carbon tetrachloride

$\begin{array}{lll}\quad \text { Result } & & \text { DF } \\ 7.0 & & 1 \\ 46 & & 1 \\ 13 & & 1 \\ <10 & 1 \\ <0.052 & 1 \\ 46 & 1 \\ <2.0 & 1 \\ <2.0 & 1 \\ 6.8 & 1 \\ <1.0 & 1 \\ <1.0 & 1 \\ <1.0 & 1 \\ <1.0 & 1 \\ <1.0 & 1 \\ <1.0 & 1 \\ <1.0 & 1 \\ <1.0 & 1 \\ <2.0 & 1 \\ 1.440 & 1 \\ <1.0 & 1 \\ <1.0 & 1 \\ <1.0 & 1\end{array}$

Time: $8: 26$

pH: 6.3

Alkalinity: $11 \mathrm{mg} / \mathrm{L}$

Water temperature: $18.3^{\circ} \mathrm{C}$

Volumes purged: 1.1 well volumes 
WELL BGO $46 C$ collected on $04 / 14 / 94$, laboratory analyses (cont.)

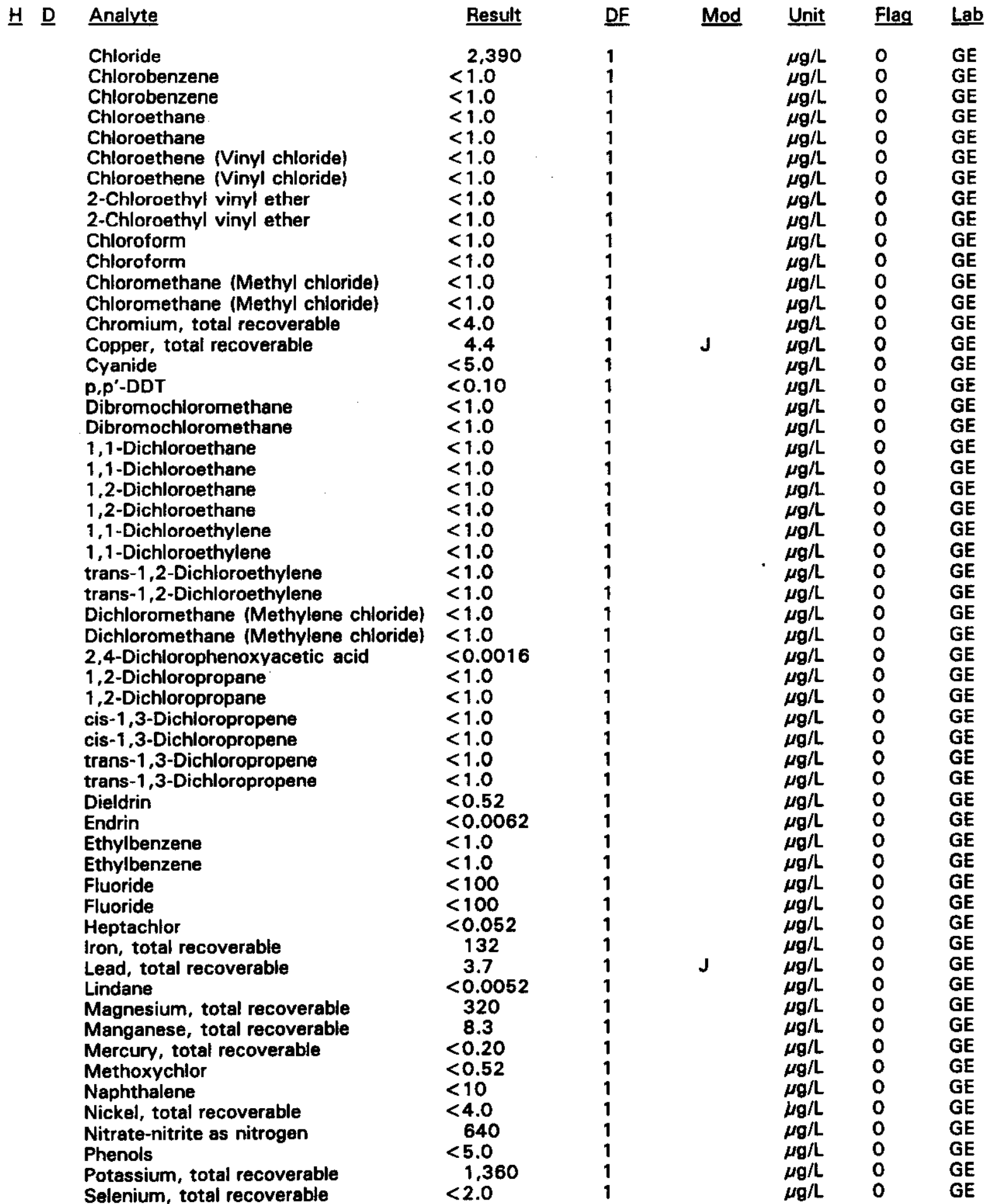

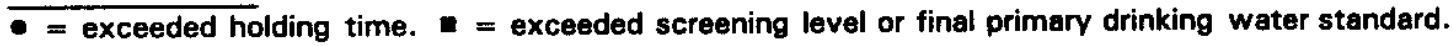


WELL BGO $46 \mathrm{C}$ collected on $04 / 14 / 94$, laboratory analyses (cont.)

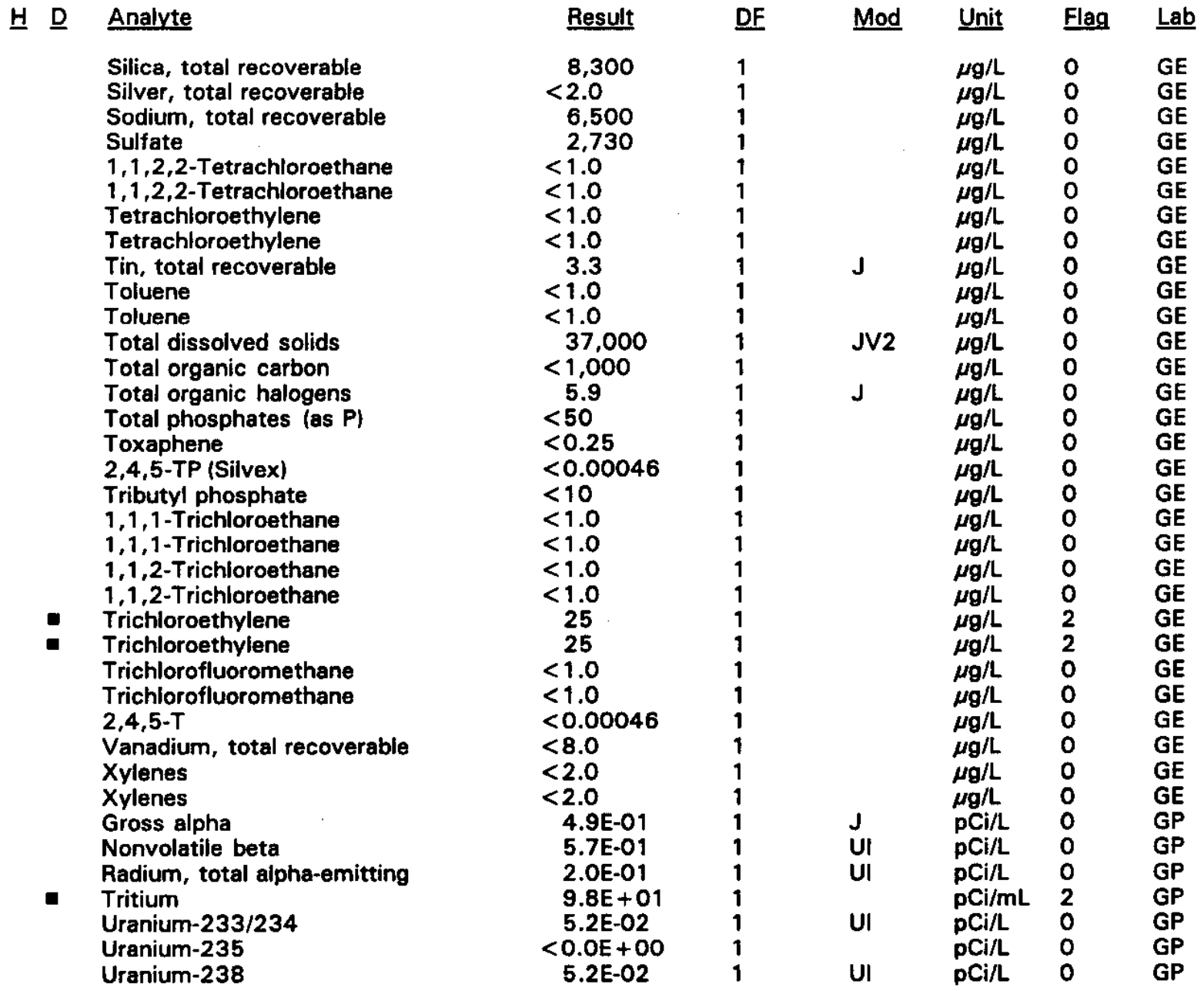

- = exceeded holding time. a = exceeded screening level or final primary drinking water standard. 
WELL BGO 46D

\begin{tabular}{|c|c|c|c|c|c|c|}
\hline SRS Coord. & Lat/Longitude & Screen Zone Elevation & Top of Casing & Casing & Pump & Formation \\
\hline $\begin{array}{l}N 75033.8 \\
\text { E54420.0 }\end{array}$ & $\begin{array}{l}33.280518^{\circ} \mathrm{N} \\
81.669253^{\circ} \mathrm{W}\end{array}$ & $212.1-202.1 \mathrm{ft} \mathrm{msl}$ & $265.1 \mathrm{ft} \mathrm{msl}$ & 4" PVC & $\mathbf{S}$ & Water Table $\left(\mathrm{IIB}_{2}\right)$ \\
\hline
\end{tabular}

\section{FIELD MEASUREMENTS}

Sample date: 04/12/94

Depth to water: $39.56 \mathrm{ft}(12.06 \mathrm{~m})$ below TOC

Water elevation: $225.54 \mathrm{ft}(68.75 \mathrm{~m}) \mathrm{msl}$

Sp. conductance: $49 \mu \mathrm{S} / \mathrm{cm}$

Turbidity: 0.1 NTU

Water evacuated before sampling: $118 \mathrm{gal}$

\section{LABORATORY ANALYSES}

\section{H D Analyte}

- $\quad \mathrm{pH}$

Specific conductance

Turbidity

Acetophenone

Aldrin

Aldrin

Aluminum, total recoverable

Antimony, total recoverable

Arsenic, total recoverable

Barium, total recoverable

Benzene

Benzene

Bromodichloromethane

Bromodichloromethane

Bromoform

Bromoform

Bromomethane (Methyl bromide)

Bromomethane (Methyl bromide)

Cadmium, total recoverable

Calcium, total recoverable

Carbon tetrachloride

Carbon tetrachloride

Chloride

Chloride

Chlorobenzene

Chlorobenzene

Chloroethane

Chloroethane

- Chloroethene (Vinyl chloride)

- Chloroethene (Vinyl chloride)

2-Chloroethyl vinyl ether

2-Chloroethyl vinyl ether

Chloroform

Chloroform

Chloromethane (Methyl chloride)

Chloromethane (Methyl chloride)

Chromium, total recoverable

Copper, total recoverable

Cyanide
Time: 14:26

pH: 4.6

Alkalinity: $0 \mathrm{mg} / \mathrm{L}$

Water temperature: $20.6^{\circ} \mathrm{C}$

Volumes purged: 7.7 well volumes

\begin{tabular}{|c|c|c|c|c|c|}
\hline Result & DF & Mod & Unit & Flag & Lab \\
\hline 5.1 & 1 & J1 & & 0 & GE \\
\hline 43 & 1 & & $\mu \mathrm{S} / \mathrm{cm}$ & 0 & $\mathrm{GE}$ \\
\hline$<0.10$ & 1 & J1 & NTU & 0 & GE \\
\hline$<10$ & 1 & & $\mu g / L$ & 0 & $\mathrm{GE}$ \\
\hline$<0.050$ & 1 & & $\mu \mathrm{g} / \mathrm{L}$ & 0 & GE \\
\hline$<0.050$ & $i$ & & $\mu \mathrm{g} / \mathrm{L}$ & 0 & $\overline{G E}$ \\
\hline 23 & $i$ & J & $\mu \mathrm{g} / \mathrm{L}$ & 0 & GE \\
\hline$<2.0$ & 1 & & $\mu \mathrm{g} / \mathrm{L}$ & 0 & GE \\
\hline$<2.0$ & $i$ & & $\mu g / L$ & 0 & GE \\
\hline 6.7 & 1 & & $\mu \mathrm{g} / \mathrm{L}$ & 0 & GE \\
\hline$<1.0$ & 1 & & $\mu g / L$ & 0 & $\mathrm{GE}$ \\
\hline$<1.0$ & 1 & & $\mu \mathrm{g} / \mathrm{L}$ & 0 & $\mathrm{GE}$ \\
\hline$<1.0$ & 1 & & $\mu \mathrm{g} / \mathrm{L}$ & 0 & $\mathrm{GE}$ \\
\hline$<1.0$ & 1 & & $\mu g / L$ & 0 & GE \\
\hline$<1.0$ & 1 & & $\mu g / L$ & 0 & GE \\
\hline$<1.0$ & 1 & & $\mu \mathrm{g} / \mathrm{L}$ & 0 & GE \\
\hline$<1.0$ & $j$ & & $\mu g / L$ & 0 & GE \\
\hline$<1.0$ & 1 & & $\mu g / L$ & 0 & GE \\
\hline$<2.0$ & 1 & & $\mu \mathrm{g} / \mathrm{L}$ & 0 & GE \\
\hline 584 & 1 & & $\mu g / L$ & 0 & GE \\
\hline$<1.0$ & 1 & & $\mu \mathrm{g} / \mathrm{L}$ & 0 & \\
\hline$<1.0$ & 1 & & $\mu \mathrm{g} / \mathrm{L}$ & 0 & GE \\
\hline 6,930 & 1 & & $\mu \mathrm{g} / \mathrm{L}$ & 0 & GE \\
\hline 7,080 & 1 & & $\mu \mathrm{g} / \mathrm{L}$ & 0 & GE \\
\hline$<1.0$ & 1 & & $\mu g / L$ & 0 & GE \\
\hline$<1.0$ & 1 & & $\mu \mathrm{g} / \mathrm{L}$ & 0 & GE \\
\hline$<1.0$ & 1 & & $\mu g / L$ & 0 & GE \\
\hline$<1.0$ & 1 & & $\mu \mathrm{g} / \mathrm{L}$ & 0 & \\
\hline 3.6 & 1 & & $\mu g / L$ & 2 & \\
\hline 2.7 & 1 & & $\mu \mathrm{g} / \mathrm{L}$ & 2 & \\
\hline$<1.0$ & 1 & & $\mu g / L$ & 0 & \\
\hline$<1.0$ & 1 & & $\mu g / L$ & 0 & GE \\
\hline 3.5 & 1 & & $\mu g / L$ & 0 & \\
\hline 2.7 & 1 & & $\mu g / L$ & 0 & \\
\hline$<1.0$ & 1 & & $\mu g / L$ & 0 & GE \\
\hline$<1.0$ & 1 & & $\mu \mathrm{g} / \mathrm{L}$ & 0 & \\
\hline$<4.0$ & 1 & & $\mu \mathrm{g} / \mathrm{L}$ & 0 & \\
\hline$<4.0$ & 1 & & $\mu \mathrm{g} / \mathrm{L}$ & 0 & $\mathrm{GE}$ \\
\hline$<5.0$ & $i$ & & $\mu g / L$ & 0 & \\
\hline
\end{tabular}

- = exceeded holding time. - = exceeded screening level or final primary drinking water standard. 
WELL BGO 46D collected on $04 / 12 / 94$, laboratory analyses (cont.)

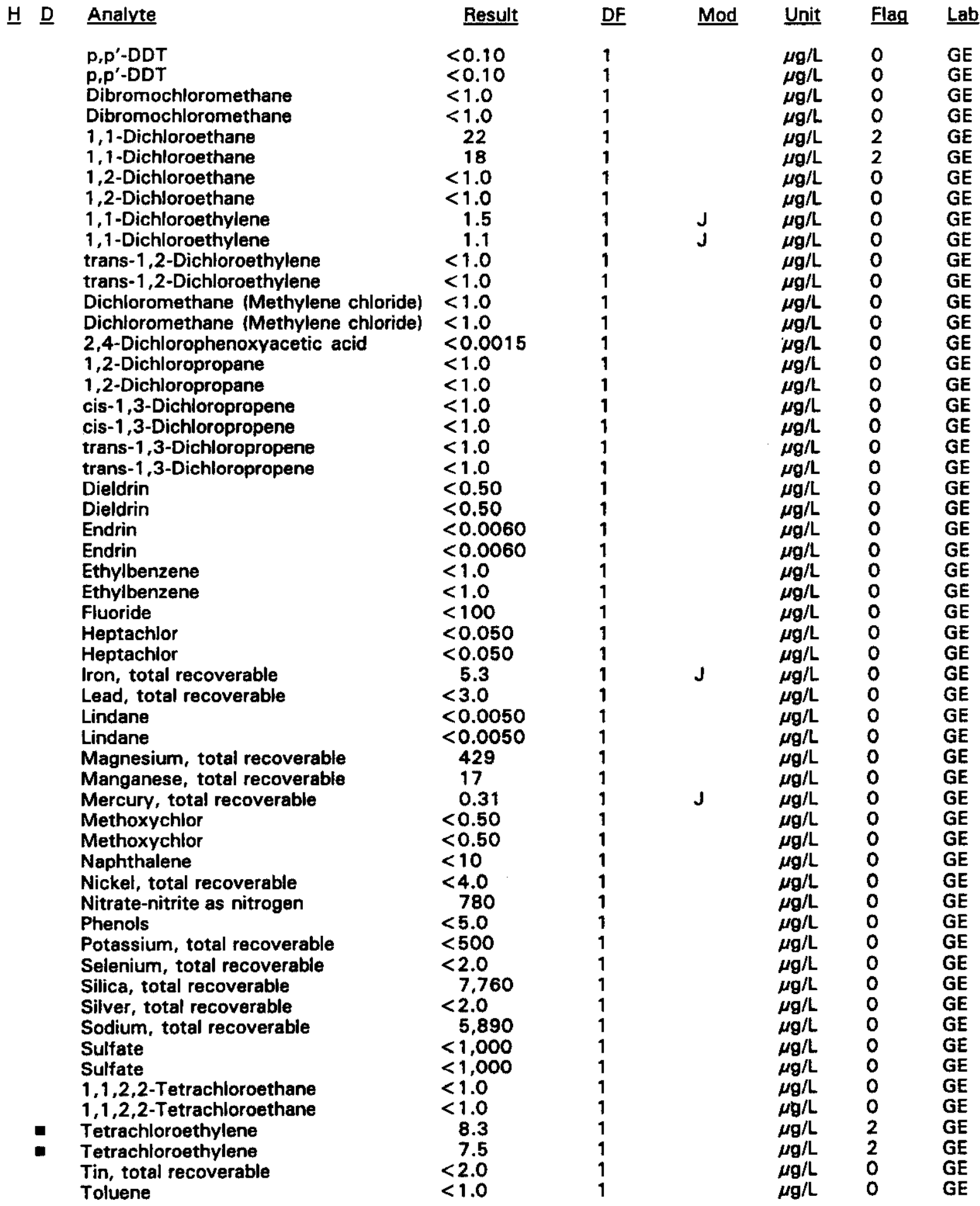

- exceeded holding time. = exceeded screening level or final primary drinking water standard. 
WELL BGO 46D collected on 04/12/94, laboratory analyses (cont.)

$\begin{array}{ll}\text { H } & \text { Analyte } \\ \text { Toluene } \\ \text { Total dissolved solids } \\ \text { Total organic carbon } \\ \text { Total organic halogens } \\ \text { Total phosphates (as P) } \\ \text { Toxaphene } \\ \text { Toxaphene } \\ 2,4,5-\text { TP (Silvex) } \\ \text { Tributyl phosphate } \\ 1,1,1-T r i c h l o r o e t h a n e \\ 1,1,1-\text { Trichloroethane } \\ 1,1,2-T \text { Trichloroethane } \\ 1,1,2-\text { Trichloroethane } \\ \text { Trichloroethylene } \\ \text { Trichloroethylene } \\ \text { Trichlorofluoromethane } \\ \text { Trichlorofluoromethane } \\ 2,4,5-T \\ \text { Vanadium, total recoverable } \\ \text { Xylenes } \\ \text { Xylenes } \\ \text { Gross alpha } \\ \text { Nonvolatile beta } \\ \text { Radium, total alpha-emitting } \\ \text { Total activity } \\ \text { Tritium } \\ \text { Uranium-233/234 } \\ \text { Uranium-235 } \\ \text { Uranium-238 }\end{array}$

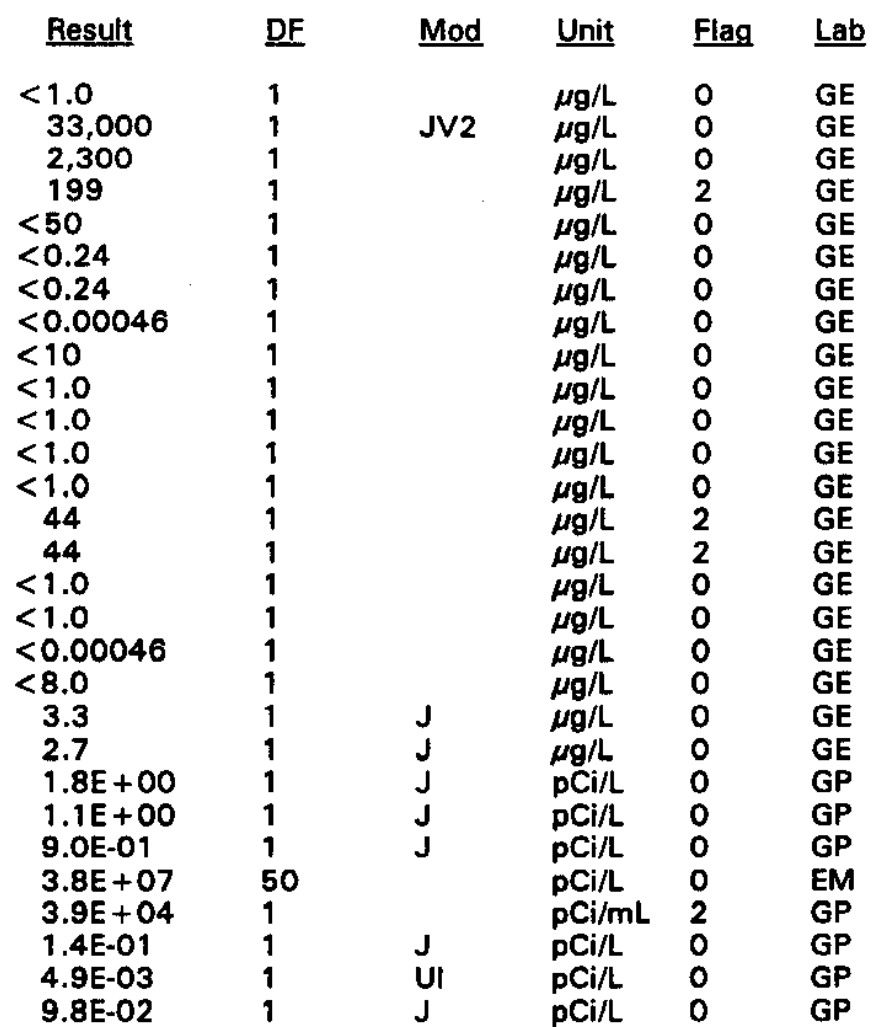

\section{WELL BGO 47A}

\begin{tabular}{|c|c|c|c|c|c|c|}
\hline SRS Coord. & Lat/Longitude & Screen Zone Elevation & Top of Casing & Casing & Pump & Formation \\
\hline & $80650^{\circ} \mathrm{N}$ & $96.8-86.8 \mathrm{ft} \mathrm{msl}$ & $266.9 \mathrm{ft} \mathrm{msl}$ & 4" PVC & $\mathbf{S}$ & U. Co \\
\hline
\end{tabular}

\section{FIELD MEASUREMENTS}

Sample date: $04 / 11 / 94$

Depth to water: $104.60 \mathrm{ft}(31.88 \mathrm{~m})$ below TOC Water elevation: $162.30 \mathrm{ft}(49.47 \mathrm{~m}) \mathrm{msl}$

Sp. conductance: $161 \mu \mathrm{S} / \mathrm{cm}$

Turbidity: 0.7 NTU

Water evacuated before sampling: $262 \mathrm{gal}$
Time: 15:58

pH: 7.2

Alkalinity: $49 \mathrm{mg} / \mathrm{L}$

Water temperature: $20.1{ }^{\circ} \mathrm{C}$

Volumes purged: $\mathbf{5 . 3}$ well volumes

\section{LABORATORY ANALYSES}

H D Analyte

$\bullet$

pH
Specific conductance

Turbidity

Acetophenone

Aldrin

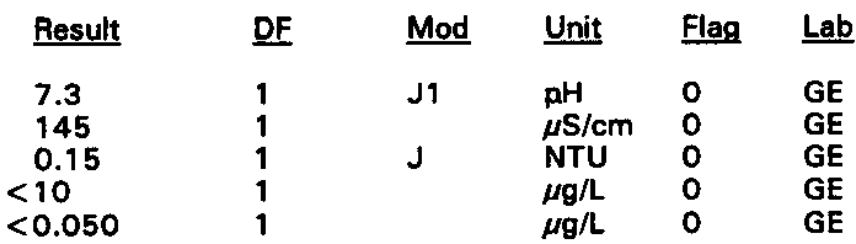

- = exceeded holding time. $=$ exceeded screening level or final primary drinking water standard. 
WELL BGO 47A collected on 04/11/94, laboratory analyses (cont.)

\section{H D Analyte}

Aluminum, total recoverable

Antimony, total recoverable

Antimony, total recoverable

Arsenic, total recoverable

Arsenic, total recoverable

Barium, total recoverable

Benzene

Bromodichloromethane

Bromoform

Bromomethane (Methyl bromide)

Cadmium, total recoverable

Calcium, total recoverable

Carbon tetrachloride

Chloride

Chlorobenzene

Chloroethane

Chloroethene (Vinyl chloride)

2-Chloroethyl vinyl ether

Chloroform

Chloromethane (Methyl chloride)

Chromium, total recoverable

Copper, total recoverable

Cyanide

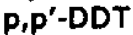

Dibromochloromethane

1,1-Dichloroethane

1,2-Dichloroethane

1,1-Dichloroethylene

trans-1,2-Dichloroethylene

Dichloromethane (Methylene chloride)

2,4-Dichlorophenoxyacetic acid

1,2-Dichloropropane

cis-1,3-Dichloropropene

trans-1,3-Dichloropropene

Dieldrin

Endrin

Ethylbenzene

Fluoride

Fluoride

Heptachlor

Iron, total recoverable

Lead, total recoverable

Lead, total recoverable

Lindane

Magnesium, total recoverable

Manganese, total recoverable

Mercury, total recoverable

Methoxychlor

Naphthalene

Nickel, total recoverable

Nitrate-nitrite as nitrogen

Phenols

Potassium, total recoverable

Selenium, total recoverable

Selenium, total recoverable

Silica, total recoverable

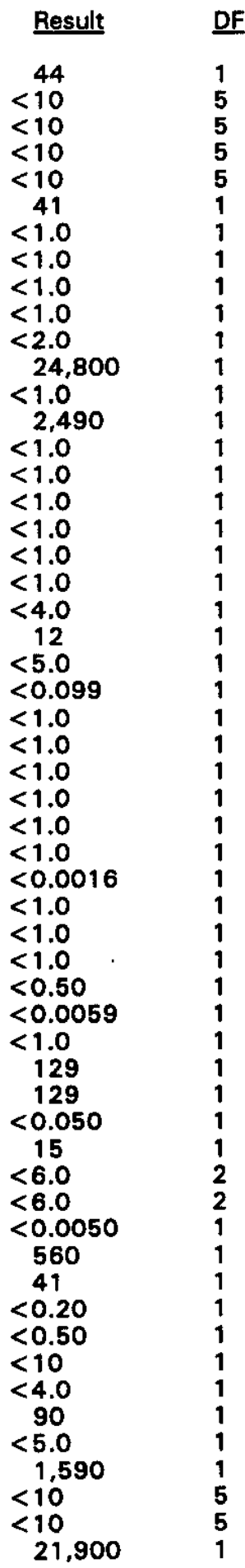

\begin{tabular}{|c|c|c|}
\hline Mod & Unit & Flag \\
\hline $\begin{array}{l}J \\
J\end{array}$ & $\begin{array}{l}\mu \mathrm{g} / \mathrm{L} \\
\mu \mathrm{g} / \mathrm{L} \\
\mu \mathrm{g} / \mathrm{L} \\
\mu \mathrm{g} / \mathrm{L} \\
\mu \mathrm{g} / \mathrm{L} \\
\mu \mathrm{g} / \mathrm{L} \\
\mu \mathrm{g} / \mathrm{L} \\
\mu \mathrm{g} / \mathrm{L} \\
\mu \mathrm{g} / \mathrm{L} \\
\mu \mathrm{g} / \mathrm{L} \\
\mu \mathrm{g} / \mathrm{L} \\
\mu \mathrm{g} / \mathrm{L} \\
\mu \mathrm{g} / \mathrm{L} \\
\mu \mathrm{g} / \mathrm{L} \\
\mu \mathrm{g} / \mathrm{L} \\
\mu \mathrm{g} / \mathrm{L} \\
\mu \mathrm{g} / \mathrm{L} \\
\mu \mathrm{g} / \mathrm{L} \\
\mu \mathrm{g} / \mathrm{L} \\
\mu \mathrm{g} / \mathrm{L} \\
\mu \mathrm{g} / \mathrm{L} \\
\mu \mathrm{g} / \mathrm{L} \\
\mu \mathrm{g} / \mathrm{L} \\
\mu \mathrm{g} / \mathrm{L} \\
\mu \mathrm{g} / \mathrm{L} \\
\mu \mathrm{g} / \mathrm{L} \\
\mu \mathrm{g} / \mathrm{L} \\
\mu \mathrm{g} / \mathrm{L} \\
\mu \mathrm{g} / \mathrm{L} \\
\mu \mathrm{g} / \mathrm{L} \\
\mu \mathrm{g} / \mathrm{L} \\
\mu \mathrm{g} / \mathrm{L} \\
\mu \mathrm{g} / \mathrm{L} \\
\mu g / \mathrm{L} \\
\mu g / \mathrm{L} \\
\mu \mathrm{g} / \mathrm{L} \\
\mu \mathrm{g} / \mathrm{L} \\
\mu \mathrm{g} / \mathrm{L} \\
\mu \mathrm{g} / \mathrm{L} \\
\mu \mathrm{g} / \mathrm{L} \\
\mu \mathrm{g} / \mathrm{L} \\
\mu \mathrm{g} / \mathrm{L} \\
\mu \mathrm{g} / \mathrm{L} \\
\mu \mathrm{g} / \mathrm{L} \\
\mu \mathrm{g} / \mathrm{L} \\
\mu \mathrm{g} / \mathrm{L} \\
\mu \mathrm{g} / \mathrm{L} \\
\mu \mathrm{g} / \mathrm{L} \\
\mu \mathrm{g} / \mathrm{L} \\
\mu \mathrm{g} / \mathrm{L} \\
\mu \mathrm{g} / \mathrm{L} \\
\mu \mathrm{g} / \mathrm{L} \\
\mu \mathrm{g} / \mathrm{L} \\
\mu \mathrm{g} / \mathrm{L} \\
\mu \mathrm{g} / \mathrm{L} \\
\mu \mathrm{g} / \mathrm{L}\end{array}$ & $\begin{array}{l}1 \\
0 \\
0 \\
0 \\
0 \\
0 \\
0 \\
0 \\
0 \\
0 \\
0 \\
0 \\
0 \\
0 \\
0 \\
0 \\
0 \\
0 \\
0 \\
0 \\
0 \\
0 \\
0 \\
0 \\
0 \\
0\end{array}$ \\
\hline
\end{tabular}

- = exceeded holding time. = exceeded screening level or final primary drinking water standard. 
WELL BGO 47A collected on 04/11/94, laboratory analyses (cont.)

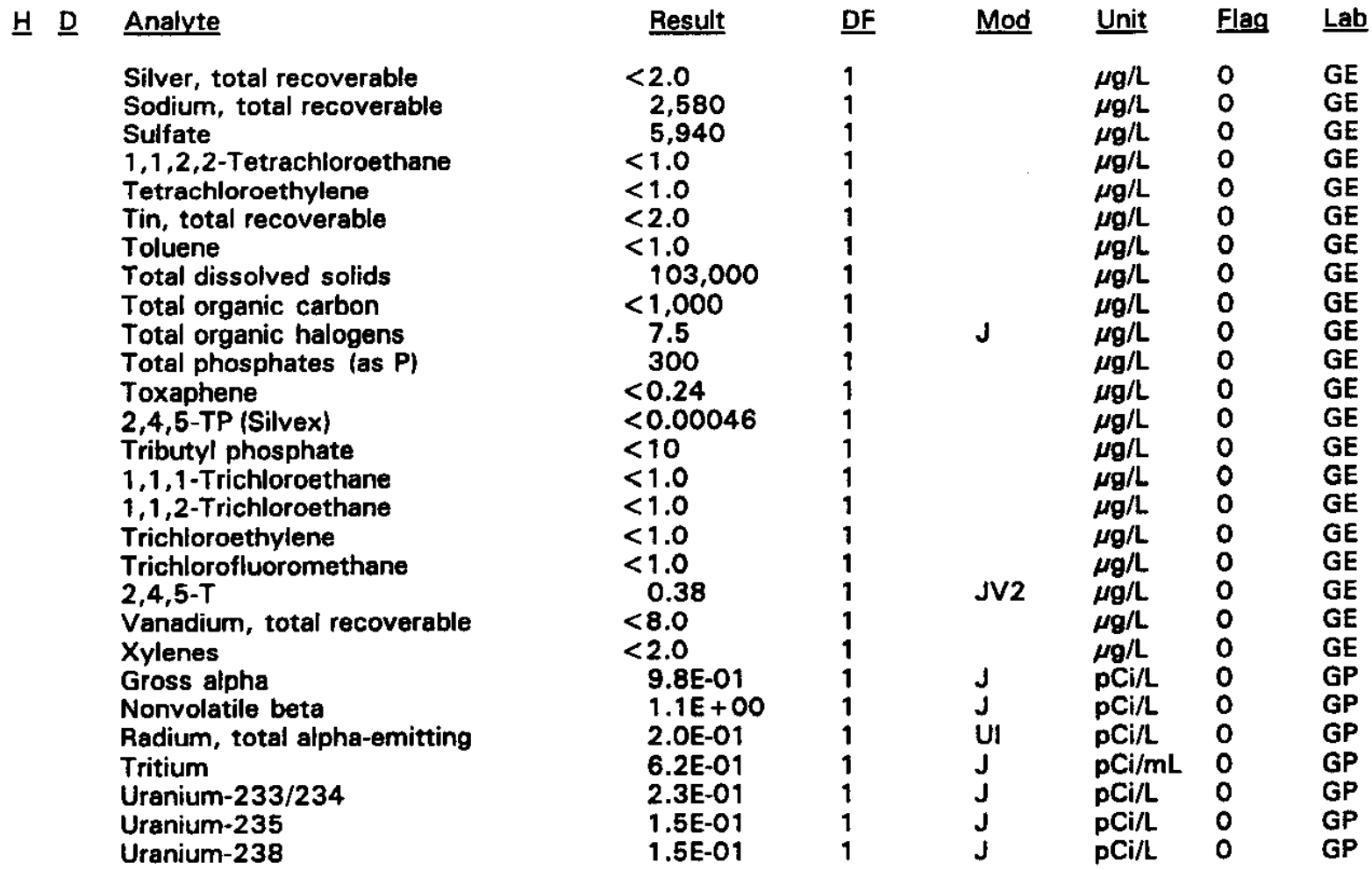

\section{WELL BGO 47C}

\begin{tabular}{|c|c|c|c|c|c|c|}
\hline SAS Coord. & Lat/Longitude & Screen Zone Elevation & Top of Casing & Casing & Pump & Formation \\
\hline $\begin{array}{l}\text { N74752.0 } \\
\text { E54933.4 }\end{array}$ & $\begin{array}{l}33.280733^{\circ} \mathrm{N} \\
81.667354^{\circ} \mathrm{W}\end{array}$ & $188.6-178.6 \mathrm{ft} \mathrm{msl}$ & $267.6 \mathrm{ft} \mathrm{msl}$ & 4" PVC & $\mathbf{s}$ & Barnwell (IIB, \\
\hline
\end{tabular}

\section{FIELD MEASUREMENTS}

Sample date: $04 / 11 / 94$

Time: 15:23

Depth to water: $44.82 \mathrm{ft}(13.66 \mathrm{~m})$ below TOC

Water elevation: $222.78 \mathrm{ft}(67.90 \mathrm{~m}) \mathrm{msl}$

No water evacuated before sampling.

Inaccessibility or pump failure prevented sample collection.

$\overline{-}=$ exceeded holding time. $\quad$ = exceeded screening level or final primary drinking water standard. 
WELL BGO 47D

\begin{tabular}{|c|c|c|c|c|c|c|}
\hline SRS Coord. & Lat/Longitude & Screen Zone Elevation & Top of Casing & Casing & Pump & Formation \\
\hline $\begin{array}{l}N 74739.7 \\
\text { E54922.9 }\end{array}$ & $\begin{array}{l}33.280689^{\circ} \mathrm{N} \\
81.667357^{\circ} \mathrm{W}\end{array}$ & $213.4-203.4 \mathrm{ft} \mathrm{msl}$ & $267.4 \mathrm{ft} \mathrm{msl}$ & 4" PVC & $\mathbf{S}$ & able $\left(\mathrm{IIB}_{2}\right)$ \\
\hline
\end{tabular}

Sample date: 04/11/94

Depth to water: $40.94 \mathrm{ft}(12.48 \mathrm{~m})$ below TOC

Water elevation: $226.46 \mathrm{ft}(69.03 \mathrm{~m}) \mathrm{ms}$

Sp. conductance: $47 \mu \mathrm{S} / \mathrm{cm}$

Turbidity: 0.3 NTU

Water evacuated before sampling: $192 \mathrm{gal}$

\section{LABORATORY ANALYSES}

H D Analyte

\section{- $\quad \mathrm{pH}$}

Specific conductance

Turbidity

Acetophenone

Aldrin

Aluminum, total recoverable

Antimony, total recoverable

Arsenic, total recoverable

Barium, total recoverable

Benzene

Bromodichloromethane

Bromoform

Bromomethane (Methyl bromide)

Cadmium, total recoverable

Calcium, total recoverable

Carbon tetrachloride

Chloride

Chlorobenzene

Chloroethane

Chloroethene (Vinyl chloride)

2-Chloroethyl vinyl ether

Chloroform

Chloromethane (Methyl chloride)

Chromium, total recoverable

Copper, total recoverable

Cyanide

p, $p^{\prime}$-DDT

Dibromochloromethane

1,1-Dichloroethane

1,2-Dichloroethane

1,1-Dichloroethylene

trans-1,2-Dichloroethylene

Dichloromethane (Methylene chloride)

2,4-Dichlorophenoxyacetic acid

1,2-Dichloropropane

cis-1,3-Dichloropropene

trans-1,3-Dichloropropene

Dieldrin

Endrin
Time: 15:40

pH: 5.2

Alkalinity: $2 \mathrm{mg} / \mathrm{L}$

Water temperature: $20.6^{\circ} \mathrm{C}$

Volumes purged: 12.7 well volumes

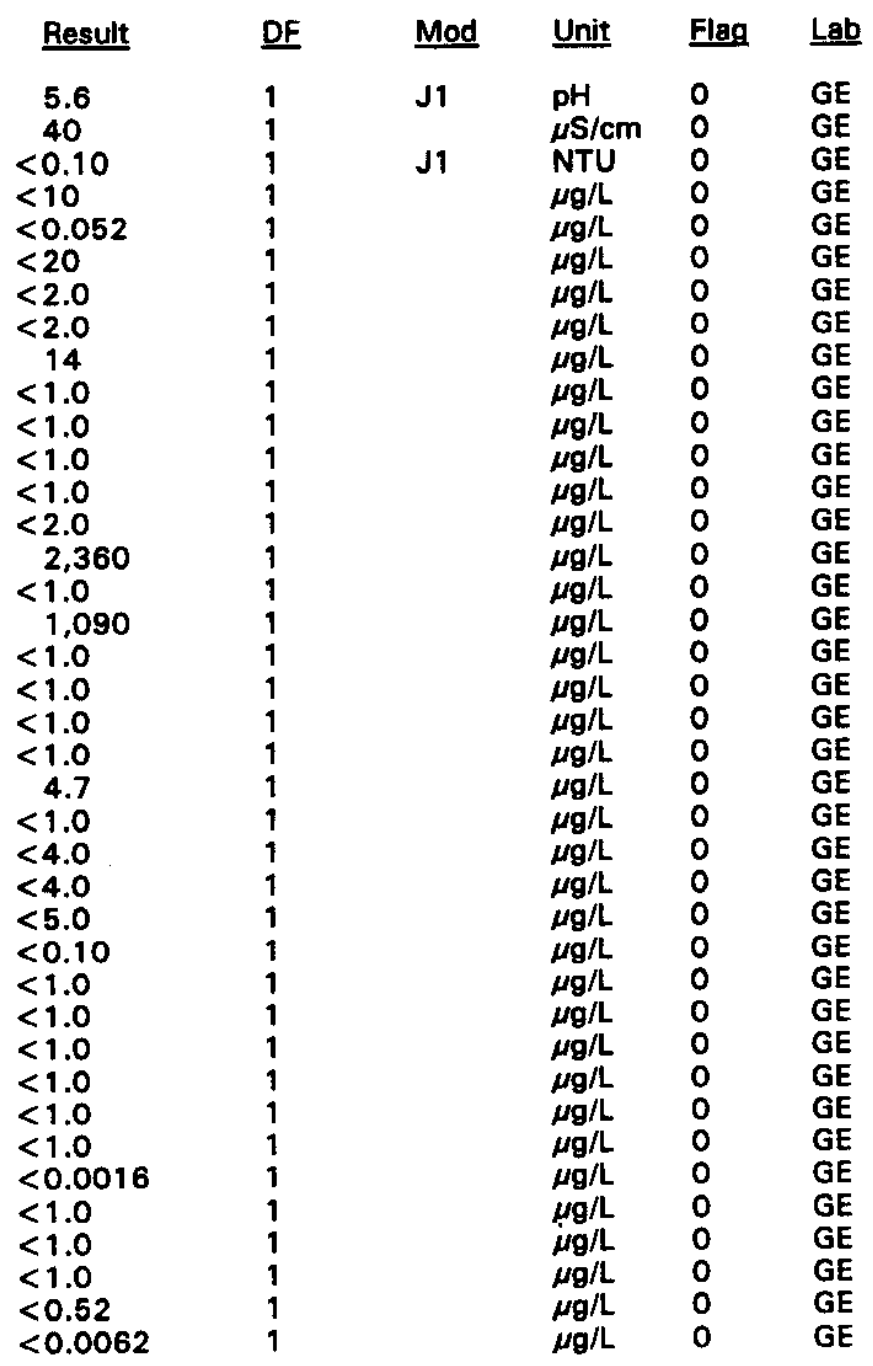

- = exceeded holding time. = exceeded screening level or final primary drinking water standard. 
WELL BGO 47D collected on 04/11/94, laboratory analyses (cont.)

H D Analyte

Ethylbenzene

- Fluoride

Heptachlor

Iron, total recoverable

Lead, total recoverable

Lindane

Magnesium, total recoverable

Manganese, total recoverable

Mercury, total recoverable

Methoxychior

Naphthalene

Nickel, total recoverable

Nitrate-nitrite as nitrogen

Phenols

Potassium, total recoverable

Selenium, total recoverable

Silica, total recoverable

Silver, total recoverable

Sodium, total recoverable

Sulfate

1,1,2,2-Tetrachloroethane

Tetrachloroethylene

Tin, total recoverable

Toluene

Total dissolved solids

Total organic carbon

Total organic halogens

Total organic halogens

Total phosphates (as P)

Toxaphene

2,4,5-TP (Silvex)

Tributyl phosphate

1,1,1-Trichloroethane

1,1,2-Trichloroethane

- Trichloroethylene

Trichlorofluoromethane

2,4,5-T

Vanadium, total recoverable

Xylenes

Gross alpha

Nonvolatile beta

Radium, total alpha-emitting

Total activity

- Tritium

Uranium-233/234

Uranium-235

Uranium-238

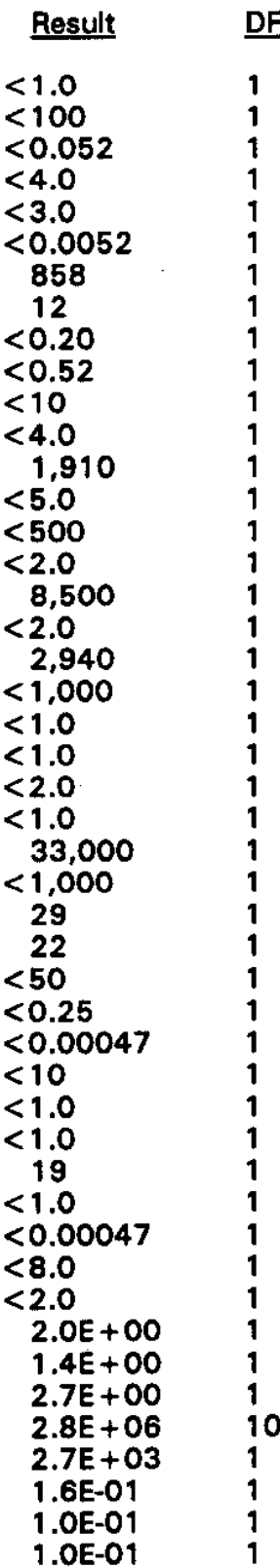

Mod

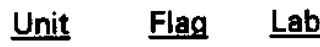

J1

$\mu \mathrm{g} / \mathrm{L}$

$\mu g / L$

$\mu g / L$

$\mu g / L$

$\mu g / L$

$\mu g / L$

$\mu \mathrm{g} / \mathrm{L}$

$\mu g / L$

$\mu g / L$

$\mu g / L$

$\mu g / L$

$\mu g / L$

$\mu g / L$

$\mu g / L$

$\mu g / L$

$\mu g / L$

$\mu g / L$

$\mu g / L$

$\mu \mathrm{g} / \mathrm{L}$

$\mu \mathrm{g} / \mathrm{L}$

$\mu g / L$

$\mu \mathrm{g} / \mathrm{L}$

$\mu g / L$

$\mu g / L$

$\mu g / L$

$\mu g / L$

$\mu g / L$

$\mu g / L$

$\mu \mathrm{g} / \mathrm{L}$

$\mu g / L$

$\mu \mathrm{g} / \mathrm{L}$

$\mu g / L$

$\mu \mathrm{g} / \mathrm{L}$

$\mu g / L$

$\mu g / L$

$\mu g / L$

$\mu \mathrm{g} / \mathrm{L}$

$\mu g / L$

pCi/L

pCi/L

pCi/L

pCi/L

$\mathrm{pCi} / \mathrm{mL}$

pCi/L

pCi/L

pCi/L
GE

GE

GE

GE

GE

GE

GE

GE

GE

GE

GE

GE

GE

GE

GE

GE

GE

GE

GE

GE

GE

GE

GE

GE

GE

GE

GE

GE

$\mathrm{GE}$

GE

GE

GE

GE

GE

GE

GE

GE

GE

GE

GP

GP

GP

EM

GP

GP

GP

- =xceeded holding time. = exceeded screening level or final primary drinking water standard. 
WELL BGO 48C

\begin{tabular}{|c|c|c|c|c|c|c|}
\hline SRS Coord. & Lat/Longitude & Screen Zone Elevation & Top of Casing & Casing & Pump & Formation \\
\hline $\begin{array}{l}N 74599.6 \\
E 55124.4\end{array}$ & $\begin{array}{l}33.280708^{\circ} \mathrm{N} \\
81.666555^{\circ} \mathrm{W}\end{array}$ & $186.7-176.7 \mathrm{ft} \mathrm{msl}$ & $276.6 \mathrm{ft} \mathrm{msl}$ & 4" PVC & $\mathbf{s}$ & Barnwell (IIB ${ }_{1}$ ) \\
\hline
\end{tabular}

\section{FIELD MEASUREMENTS}

Sample date: 04/11/94

Depth to water: $53.11 \mathrm{ft}(16.19 \mathrm{~m})$ below TOC

Water elevation: $223.49 \mathrm{ft}(68.12 \mathrm{~m}) \mathrm{msl}$

Sp. conductance: $39 \mu \mathrm{S} / \mathrm{cm}$

Turbidity: 0.5 NTU

Water evacuated before sampling: $160 \mathrm{gal}$

\section{LABORATORY ANALYSES}

\section{H $\underline{\text { Analyte }}$}

- pH

Specific conductance

Turbidity

Acetophenone

Acetophenone

Aldrin

Aluminum, total recoverable

Antimony, total recoverable

Arsenic, total recoverable

Barium, total recoverable

Benzene

Bromodichloromethane

Bromoform

Bromomethane (Methyl bromide)

Cadmium, total recoverable

Calcium, total recoverable

Carbon tetrachloride

Chloride

Chlorobenzene

Chloroethane

Chloroethene (Vinyl chloride)

2-Chloroethyl vinyl ether

Chloroform

Chloromethane (Methyl chloride)

Chromium, total recoverable

Copper, total recoverable

Cyanide

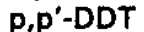

Dibromochloromethane

1,1-Dichloroethane

1,2-Dichloroethane

1,1-Dichloroethylene

trans-1,2-Dichloroethylene

Dichloromethane (Methylene chloride)

2,4-Dichlorophenoxyacetic acid

1,2-Dichloropropane

cis-1,3-Dichloropropene

trans-1,3-Dichloropropene

Dieldrin
Time: 12:34

pH: 5.0

Alkalinity: $1 \mathrm{mg} / \mathrm{L}$

Water temperature: $20.6^{\circ} \mathrm{C}$

Volumes purged: $\mathbf{5 . 2}$ well volumes

\begin{tabular}{|c|c|c|c|c|}
\hline Result & DF & Mod & Unit & Flag \\
\hline $\begin{aligned} & 5.5 \\
& 33 \\
& 0.26 \\
&< 10 \\
&<10 \\
&<0.052 \\
&< 20 \\
&<2.0 \\
&<2.0 \\
&<11 \\
&<1.0 \\
&<1.0 \\
&<1.0 \\
&<1.0 \\
&<2.0 \\
&<1.680 \\
&<1.0 \\
& 2,280 \\
&<1.0 \\
&<1.0 \\
&<1.0 \\
&<1.0 \\
&<1.3 \\
&<1.0 \\
&<4.0 \\
&<4.0 \\
&<5.0 \\
&<0.10 \\
&<1.0 \\
&<1.0 \\
&<1.0 \\
&<1.0 \\
&<1.0 \\
&<1.0 \\
&<0.0016 \\
&<1.0 \\
&<1.0 \\
&<1.0 \\
&<0.52 \\
&<1\end{aligned}$ & $\begin{array}{l}1 \\
1 \\
1 \\
1 \\
1 \\
1 \\
1 \\
1 \\
1 \\
1 \\
1 \\
1 \\
1 \\
1 \\
1 \\
1 \\
1 \\
1 \\
1 \\
1 \\
1 \\
1 \\
1 \\
1 \\
1 \\
1 \\
1 \\
1 \\
1 \\
1 \\
1 \\
1 \\
1 \\
1 \\
1 \\
1 \\
1 \\
1 \\
1\end{array}$ & $\begin{array}{l}\text { J1 } \\
\text { J1 }\end{array}$ & 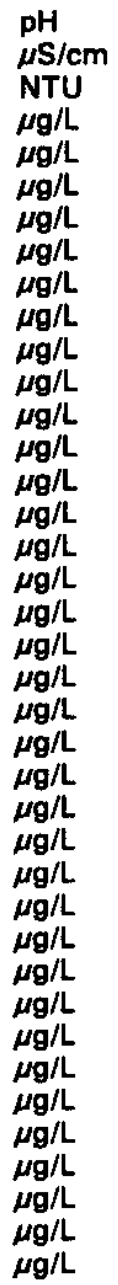 & $\begin{array}{l}0 \\
0 \\
0 \\
0 \\
0 \\
0 \\
0 \\
0 \\
0 \\
0 \\
0 \\
0 \\
0 \\
0 \\
0 \\
0 \\
0 \\
0 \\
0 \\
0 \\
0 \\
0 \\
0 \\
0 \\
0 \\
0 \\
0 \\
0 \\
0 \\
0 \\
0 \\
0 \\
0 \\
0 \\
0 \\
0 \\
0 \\
0 \\
0\end{array}$ \\
\hline
\end{tabular}

\footnotetext{
- = exceeded holding time. = exceeded screening level or final primary drinking water standard.
} 
WELL BGO $48 \mathrm{C}$ collected on $04 / 11 / 94$, laboratory analyses (cont.)

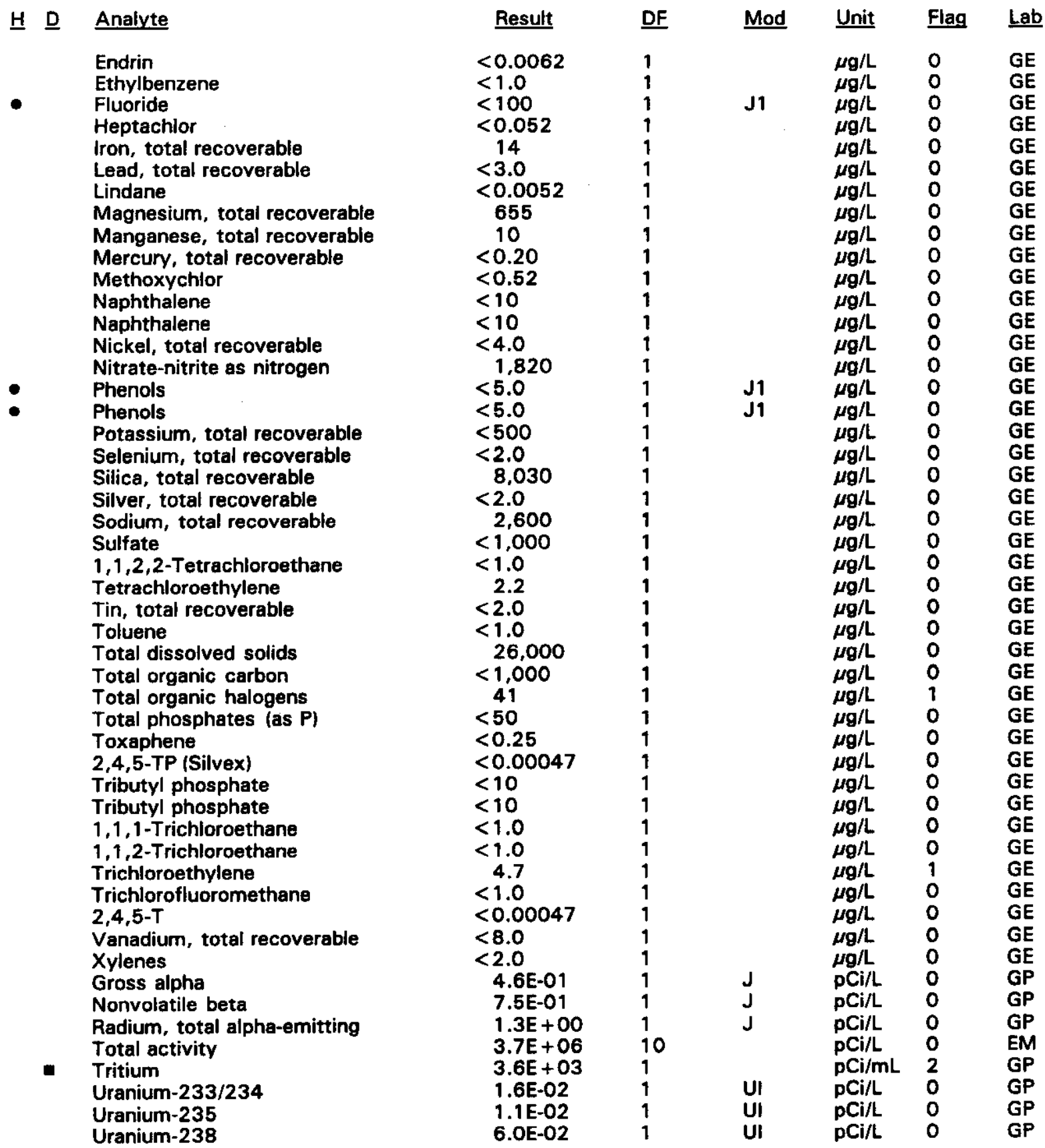

- = exceeded holding time. $=$ exceeded screening level or final primary drinking water standard. 


\section{WELL BGO 48D}

$\begin{array}{lllllll}\text { SRS Coord. } & \text { Lat/Longitude } & \text { Screen Zone Elevation } & \text { Top of Casing } & \text { Casing } & \text { Pump } & \text { Formation } \\ \text { N74586.4 } & 33.280673^{\circ} \mathrm{N} & 212.0-202.0 \mathrm{ft} \mathrm{msl} & 276.9 \mathrm{ft} \mathrm{msl} & 4^{\text {" PVC }} & \text { S } & \text { Water Table (IIB })_{2} \\ \text { E55121.0 } & 81.666538^{\circ} \mathrm{W} & & & \end{array}$

\section{FIELD MEASUREMENTS}

Sample date: $04 / 11 / 94$

Depth to water: $50.11 \mathrm{ft}(15.27 \mathrm{~m})$ below TOC

Water elevation: $226.79 \mathrm{ft}(69.13 \mathrm{~m}) \mathrm{msl}$

Sp. conductance: $65 \mu \mathrm{S} / \mathrm{cm}$

Turbidity: 1.4 NTU

Water evacuated before sampling: $236 \mathrm{gal}$

\section{LABORATORY ANALYSES}

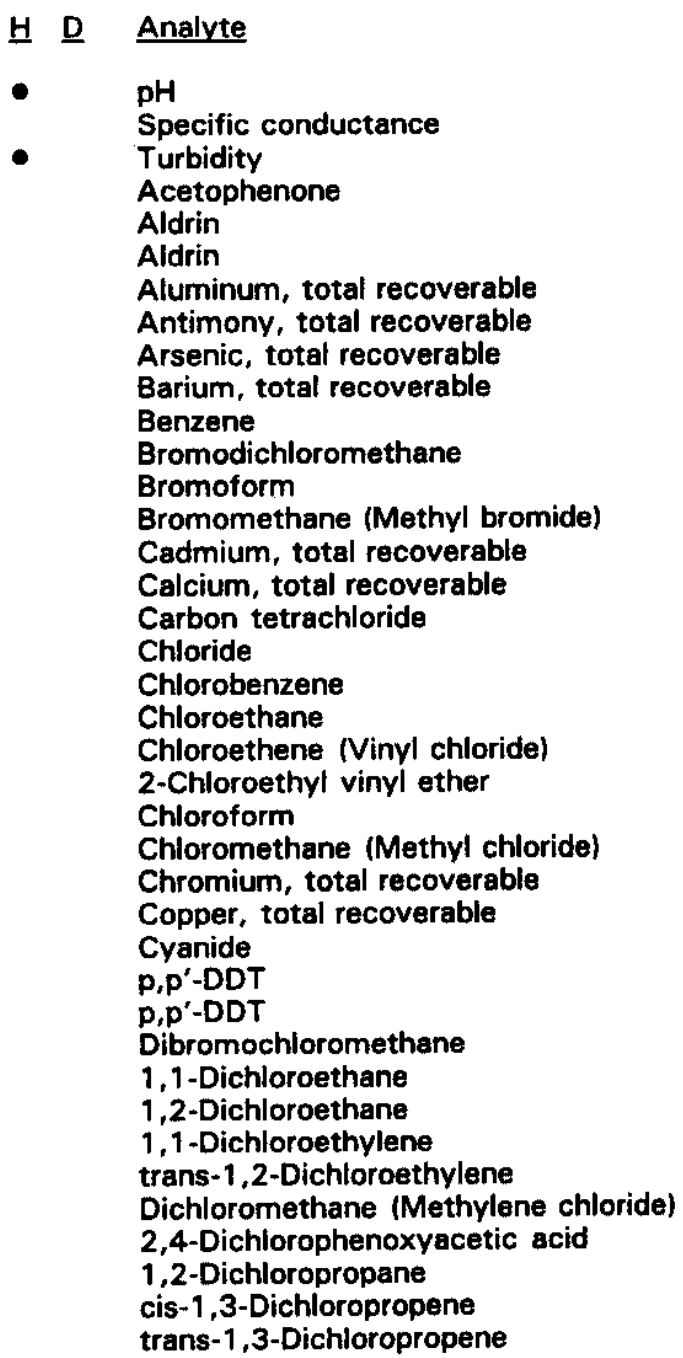

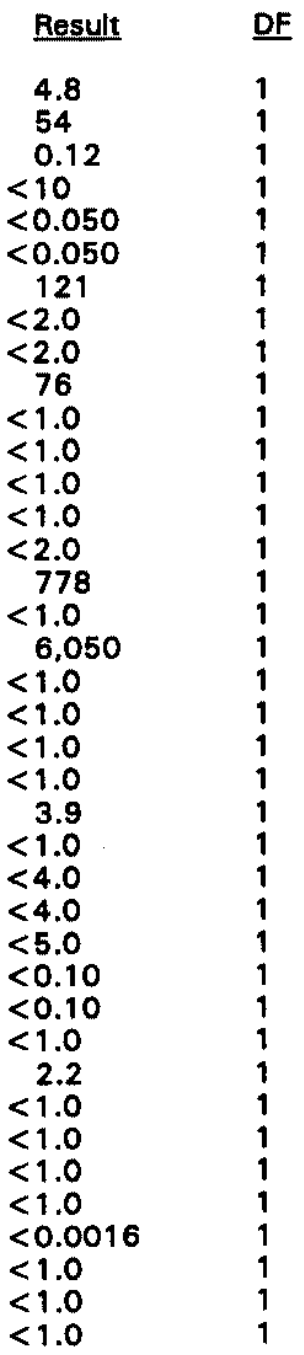

Time: $12: 49$

$\mathrm{pH}: 4.4$

Alkalinity: $0 \mathrm{mg} / \mathrm{L}$

Water temperature: $21.0^{\circ} \mathrm{C}$

Volumes purged: 14.5 well volumes 
WELL. BGO 480 collected on $04 / 11 / 94$, laboratory analyses (cont.)

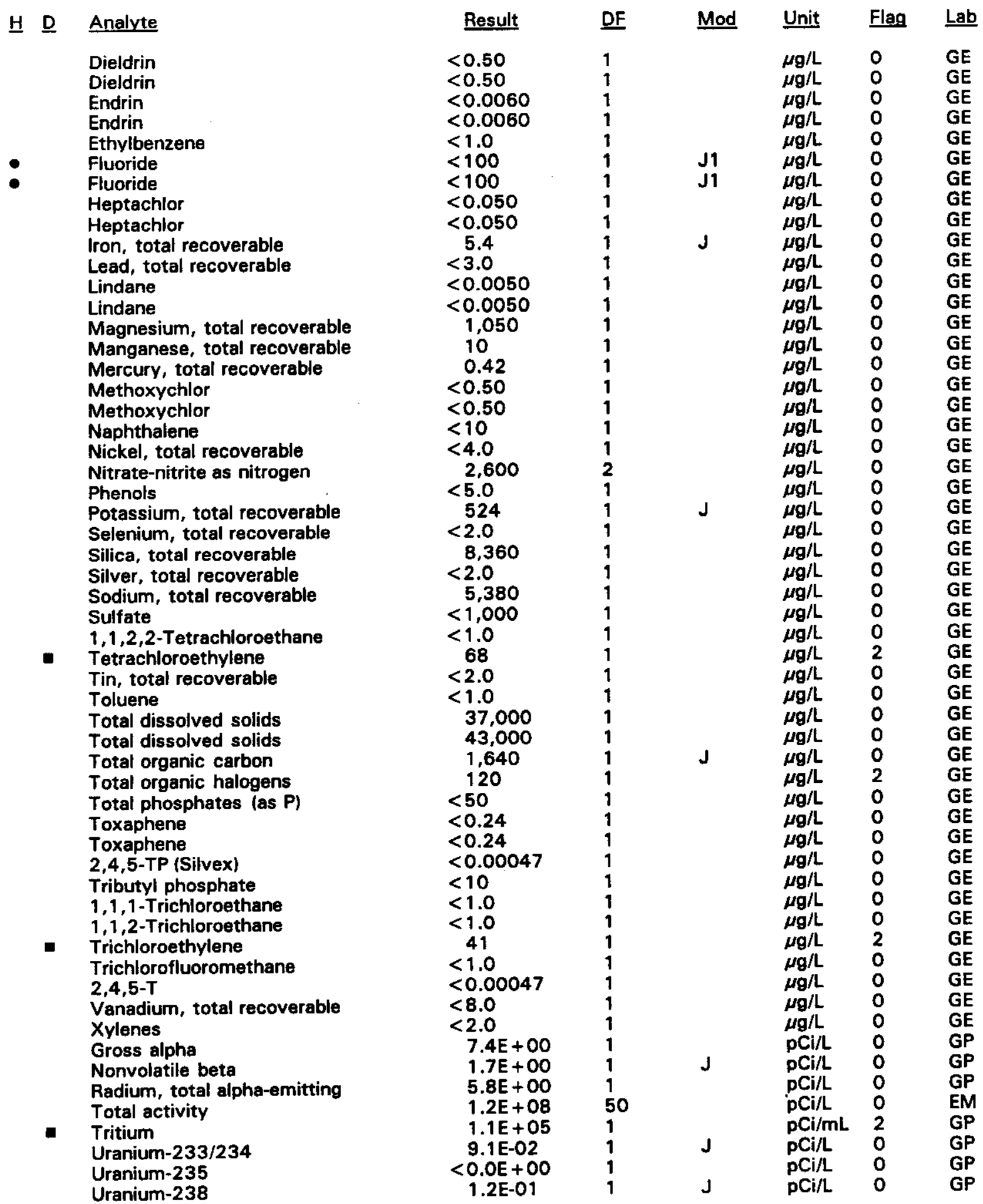

\footnotetext{
- = exceeded holding time. - = exceeded screening level or final primary drinking water standard.
} 


\section{WELL BGO 49A}

$\begin{array}{lllllll}\text { SRS Coord. } & \text { Lat/Longitude } & \text { Screen Zone Elevation } & \text { Top of Casing } & \text { Casing } & \text { Pump } & \text { Formation } \\ \text { N73902.8 } & 33.280930^{\circ} \mathrm{N} & 85.1-75.1 \mathrm{ft} \mathrm{msl} & 271.2 \mathrm{ft} \mathrm{msl} & \text { 4" PVC } & \text { S } & \text { U. Congaree (IIA) } \\ \text { E56205.1 } & 81.662356^{\circ} \mathrm{W} & & & & \end{array}$

\section{FIELD MEASUREMENTS}

Sample date: 04/13/94

Depth to water: Not available

Water elevation: Not available

Sp. conductance: $276 \mu \mathrm{S} / \mathrm{cm}$

Turbidity: 0.3 NTU

Water evacuated before sampling: $\mathbf{2 3 0}$ gal

\section{LABORATORY ANALYSES}

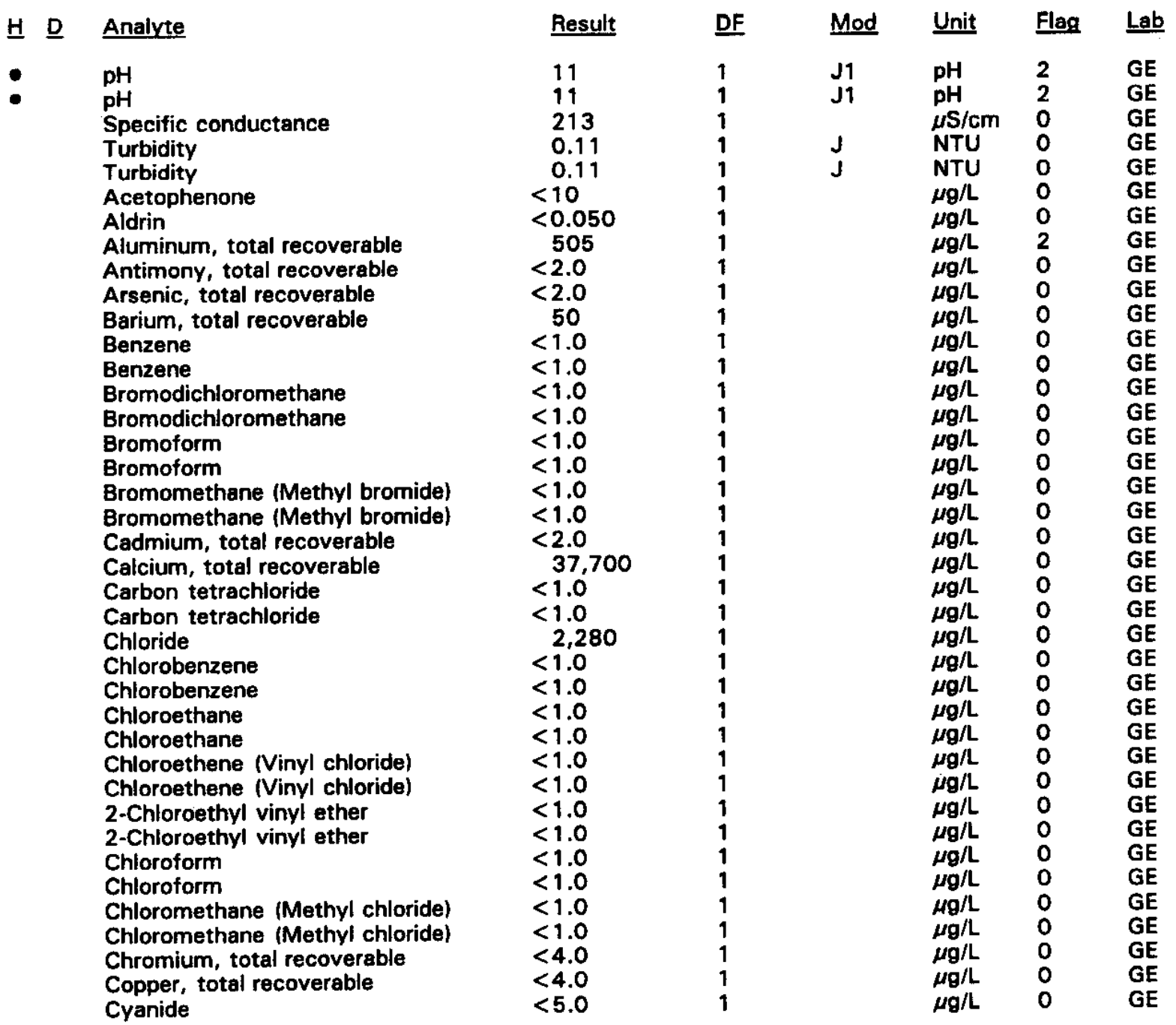

Time: $11: 58$

$\mathrm{pH}: 10.8$

Alkalinity: $97 \mathrm{mg} / \mathrm{L}$

Water temperature: $19.9^{\circ} \mathrm{C}$ 
WELL. BGO $49 \mathrm{~A}$ collected on $04 / 13 / 94$, laboratory analyses (cont.)

HE D Analyte

$p, p^{\prime}-D D T$

Dibromochloromethane

Dibromochloromethane

1,1-Dichloroethane

1,1-Dichloroethane

1,2-Dichloroethane

1,2-Dichloroethane

1,1-Dichloroethylene

1,1-Dichloroethylene

trans-1,2-Dichloroethylene

trans-1,2-Dichloroethylene

Dichloromethane (Methylene chloride)

Dichloromethane (Methylene chloride)

2,4-Dichlorophenoxyacetic acid

1,2-Dichloropropane

1,2-Dichloropropane

cis-1,3-Dichloropropene

cis-1,3-Dichloropropene

cis-1,3-Dichloropropene

trans-1,3-Dichloropropene

trans-1,3-Dichloropropene

Dieldrin

Endrin

Ethylbenzene

Ethylbenzene

Fluoride

Heptachlor

Iron, total recoverable

Lead, total recoverable

Lindane

Magnesium, total recoverable

Manganese, total recoverable

Mercury, total recoverable

Methoxychlor

Naphthalene

Nickel, total recoverable

Nitrate-nitrite as nitrogen

Nitrate-nitrite as nitrogen

Phenols

Potassium, total recoverable

Selenium, total recoverable

Silica, total recoverable

Silver, total recoverable

Sodium, total recoverable

Sulfate

1,1,2,2-Tetrachloroethane

1,1,2,2-Tetrachloroethane

Tetrachloroethylene

Tetrachloroethylene

Tin, total recoverable

Toluene

Toluene

Total dissolved solids

Total organic carbon

Total organic halogens

Total phosphates (as P)
Result DF

$<0.10$

$<1.0$

$<1.0$

$<1.0$

$<1.0$

$<1.0$

$<1.0$

$<1.0$

$<1.0$

$<1.0$

$<1.0$

$<1.0$

$<1.0$

$<1.5$

$<1.0$

$<1.0$

$<1.0$

$<1.0$

$<1.0$

$<1.0$

$<1.0$

$<0.50$

$<0.0060$

$<1.0$

$<1.0$

$<100$

$<0.050$

4.9

$<3.0$

$<0.0050$

449

$<2.0$

$<0.20$

$<0.50$

$<10$

$<4.0$

$<50$

$<50$

$<5.0$

6,330

$<2.0$

6,340

$<2.0$

4,000

2,450

$<1.0$

$<1.0$

$<1.0$

$<1.0$

14

$<1.0$

$<1.0$

116,000

$<1,000$

$<5.0$

90
Mod Unit Flag Lab

$\mu g / L \quad 0 \quad$ GE

$\mu g / L \quad 0 \quad G E$

$\mu g / L \quad 0 \quad$ GE

$\mu g / L \quad 0 \quad G E$

$\mu g / L \quad 0 \quad \mathrm{GE}$

$\mu g / L \quad 0 \quad$ GE

$\mu \mathrm{g} / \mathrm{L} \quad \mathrm{O} \quad \mathrm{GE}$

$\mu g / L \quad 0 \quad$ GE

$\mu \mathrm{g} / \mathrm{L} \quad 0 \quad \mathrm{GE}$

$\mu g / L \quad 0 \quad \mathrm{GE}$

$\mu g / L \quad O \quad$ GE

$\mu \mathrm{g} / \mathrm{L} \quad 0 \quad \mathrm{GE}$

$\mu g / L \quad 0 \quad$ GE

$\mu g / L \quad 0 \quad$ GE

$\mu \mathrm{g} / \mathrm{L} \quad 0 \quad \mathrm{GE}$

$\mu g / L \quad 0 \quad$ GE

$\mu g / L \quad 0 \quad$ GE

$\mu g / L \quad 0 \quad \mathrm{GE}$

$\mu g / L \quad 0 \quad$ GE

$\mu g / L \quad 0 \quad$ GE

$\mu g / L \quad 0 \quad$ GE

$\mu g / L \quad 0 \quad$ GE

$\mu \mathrm{g} / \mathrm{L} \quad 0 \quad \mathrm{GE}$

$\mu \mathrm{g} / \mathrm{L} \quad 0 \quad \mathrm{GE}$

$\mu g / L \quad 0 \quad$ GE

$\mu \mathrm{g} / \mathrm{L} \quad 0 \quad \mathrm{GE}$

$\mu g / L \quad 0 \quad$ GE

$\mu g / L \quad 0 \quad$ GE

$\mu g / L \quad 0 \quad$ GE

$\mu g / L \quad 0 \quad$ GE

$\mu g / L \quad 0 \quad$ GE

$\mu g / L \quad 0 \quad$ GE

$\mu \mathrm{g} / \mathrm{L} \quad 0 \quad \mathrm{GE}$

$\mu g / L \quad 0 \quad$ GE

$\mu \mathrm{g} / \mathrm{L} \quad 0 \quad \mathrm{GE}$

$\mu g / L \quad 0 \quad$ GE

$\mu g / L \quad 0 \quad$ GE

$\mu g / L \quad O \quad$ GE

$\mu g / L \quad 0 \quad$ GE

$\mu \mathrm{g} / \mathrm{L} \quad 0 \quad \mathrm{GE}$

$\mu \mathrm{g} / \mathrm{L} \quad 0 \quad \mathrm{GE}$

$\mu \mathrm{g} / \mathrm{L} \quad 0 \quad \mathrm{GE}$

$\mu g / L \quad 0 \quad G E$

$\mu g / L \quad 0 \quad$ GE

$\mu g / L \quad 0 \quad G E$

$\mu g / L \quad 0 \quad$ GE

$\mu g / L \quad 0 \quad G E$

$\mu g / L \quad O \quad$ GE

$\mu g / L \quad 0 \quad$ GE

$\mu \mathrm{g} / \mathrm{L} \quad 1 \quad \mathrm{GE}$

$\mu \mathrm{g} / \mathrm{L} \quad 0 \quad \mathrm{GE}$

$\mu g / L \quad 0 \quad$ GE

$\mu \mathrm{g} / \mathrm{L} \quad 0 \quad \mathrm{GE}$

$\mu g / L \quad 0 \quad$ GE

$\mu g / L \quad 0 \quad$ GE

$\mu \mathrm{g} / \mathrm{L} \quad 0 \quad \mathrm{GE}$

- = exceeded holding time. $=$ = exceeded screening level or final primary drinking water standard. 
WELL BGO 49A collected on $04 / 13 / 94$, laboratory analyses (cont.)

H. $\mathrm{D} \quad$ Analyte
Toxaphene
$2,4,5-T P$ (Silvex)
Tributyl phosphate
$1,1,1-$ Trichloroethane
$1,1,1-$ Trichloroethane
$1,1,2$-Trichloroethane
$1,1,2-T$ richloroethane
Trichloroethylene
Trichloroethylene
Trichlorofluoromethane
Trichlorofluoromethane
2,4,5-T
Vanadium, total recoverable
Xylenes
Xylenes
Gross alpha
Nonvolatile beta
Radium, total alpha-emitting
Tritium
Uranium-233/234
Uranium-235
Uranium-238

\begin{tabular}{lll}
\multicolumn{1}{l}{ Result } & & DF \\
$<0.24$ & & 1 \\
$<0.46$ & & 1 \\
$<10$ & 1 \\
$<1.0$ & 1 \\
$<1.0$ & 1 \\
$<1.0$ & 1 \\
$<1.0$ & 1 \\
$<1.0$ & 1 \\
$<1.0$ & 1 \\
$<1.0$ & 1 \\
$<1.0$ & 1 \\
$<0.46$ & 1 \\
$<8.0$ & 1 \\
$<2.0$ & 1 \\
$<2.0$ & 1 \\
$1.0 E+00$ & 1 \\
$5.0 E+00$ & 1 \\
$7.0 E-01$ & 1 \\
$6.9 E-01$ & 1 \\
$-1.3 E-02$ & 1 \\
$-6.3 E-03$ & 1 \\
$5.2 E-02$ & 1
\end{tabular}

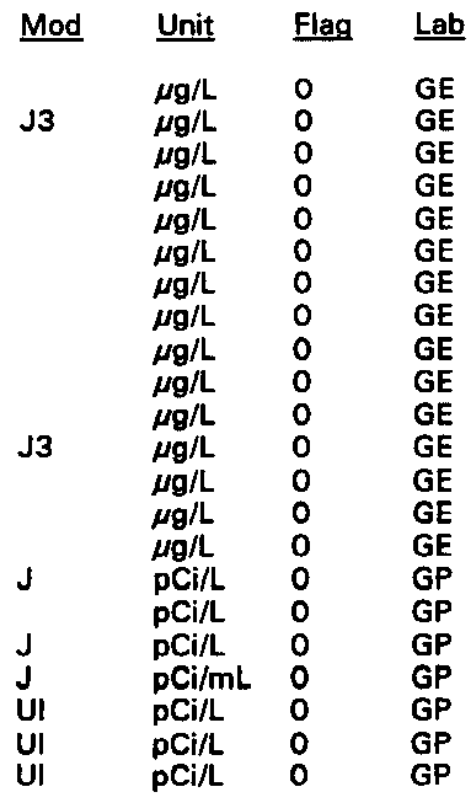

\section{WELL BGO $49 C$}

$\begin{array}{lllllll}\text { SRS Coord. } & \text { Lat/Longitude } & \text { Screen Zone Elevation } & \text { Top of Casing } & \text { Casing } & \text { Pump Formation } \\ \text { N73917.2 } & 33.280957^{\circ} \mathrm{N} & 176.0-166.0 \mathrm{ft} \mathrm{msl} & 271.1 \mathrm{ft} \mathrm{msl} & 4^{\text {" PVC }} & \text { S } & \text { Barnwell (IIB,) } \\ \text { E56202.2 } & 81.662391^{\circ} \mathrm{W} & & & & \end{array}$

\section{FIELD MEASUREMENTS}

Sample date: $04 / 13 / 94$

Depth to water: $42.89 \mathrm{ft}(13.07 \mathrm{~m})$ below TOC

Water elevation: $228.21 \mathrm{ft}(69.56 \mathrm{~m}) \mathrm{msl}$

Sp. conductance: $80 \mu \mathrm{S} / \mathrm{cm}$

Turbidity: 0.2 NTU

Water evacuated before sampling: $154 \mathrm{gal}$

\section{LABORATORY ANALYSES}

\section{H $\underline{\text { Analyte }}$}

$\mathrm{pH}$

Specific conductance

Turbidity

Acetophenone

Aldrin

Aldrin

Aluminum, total recoverable

Antimony, total recoverable

Arsenic, total recoverable

Barium, total recoverable

Benzene

Bromodichloromethane
Time: $11: 34$

pH: 7.3

Alkalinity: $20 \mathrm{mg} / \mathrm{L}$

Water temperature: $19.2^{\circ} \mathrm{C}$

Volumes purged: 3.8 well volumes

\begin{tabular}{|c|c|c|c|c|}
\hline Result & DF & Mod & Unit & Flag \\
\hline $\begin{aligned} & 7.5 \\
& 69 \\
& 0.11 \\
&<10 \\
&<0.050 \\
&<0.050 \\
& 21 \\
&<2.0 \\
&<2.0 \\
& 34 \\
&<1.0 \\
&<1.0\end{aligned}$ & $\begin{array}{l}1 \\
1 \\
1 \\
1 \\
1 \\
1 \\
1 \\
1 \\
1 \\
1 \\
1 \\
1\end{array}$ & $\begin{array}{l}\text { J1 } \\
\text { J }\end{array}$ & $\begin{array}{l}\text { pH } \\
\mu S / \mathrm{cm} \\
\mathrm{NTU} \\
\mu \mathrm{g} / \mathrm{L} \\
\mu \mathrm{g} / \mathrm{L} \\
\mu \mathrm{g} / \mathrm{L} \\
\mu \mathrm{g} / \mathrm{L} \\
\mu \mathrm{g} / \mathrm{L} \\
\mu \mathrm{g} / \mathrm{L} \\
\mu \mathrm{g} / \mathrm{L} \\
\mu \mathrm{g} / \mathrm{L} \\
\mu \mathrm{g} / \mathrm{L}\end{array}$ & $\begin{array}{l}0 \\
0 \\
0 \\
0 \\
0 \\
0 \\
0 \\
0 \\
0 \\
0 \\
0 \\
0\end{array}$ \\
\hline
\end{tabular}

- exceeded holding time. $\quad=$ exceeded screening level or final primary drinking water standard. 
WELL BGO $49 \mathrm{C}$ coliected on $04 / 13 / 94$, laboratory analyses (cont.)

H

Analyte
Bromoform
Bromomethane (Methyl bromide)
Cadmium, total recoverable
Calcium, total recoverable
Carbon tetrachloride
Chloride
Chloride
Chlorobenzene
Chloroethane
Chloroethene (Vinyl chloride)
2-Chloroethyl vinyl ether
Chloroform
Chloromethane (Methyl chloride)
Chromium, total recoverable
Copper, total recoverable
Cyanide
p,p'-DDT
p,p'-DDT
Dibromochloromethane
1,1-Dichloroethane
1,2-Dichloroethane
1,1-Dichloroethylene
trans-1,2-Dichloroethylene
Dichloromethane (Methylene chloride)
2,4-Dichlorophenoxyacetic acid
1,2-Dichloropropane
cis-1,3-Dichloropropene
trans-1,3-Dichloropropene
Dieldrin
Dieldrin
Endrin
Endrin
Ethylbenzene
Fluoride
Heptachlor
Heptachlor
lron, total recoverable
Lead, total recoverable
Lindane
Lindane
Magnesium, total recoverable
Manganese, total recoverable
Mercury, total recoverable
Methoxychlor
Methoxychlor
Naphthalene
Nickel, total recoverable
Nitrate-nitrite as nitrogen
Phenols
Potassium, total recoverable
Selenium, total recoverable
Silica, total recoverable
Silver, total recoverable
Sodium, total recoverable
Sulfate

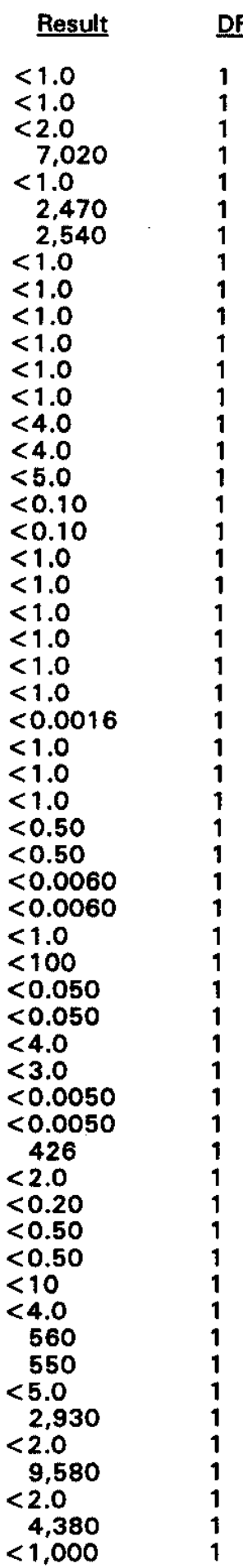

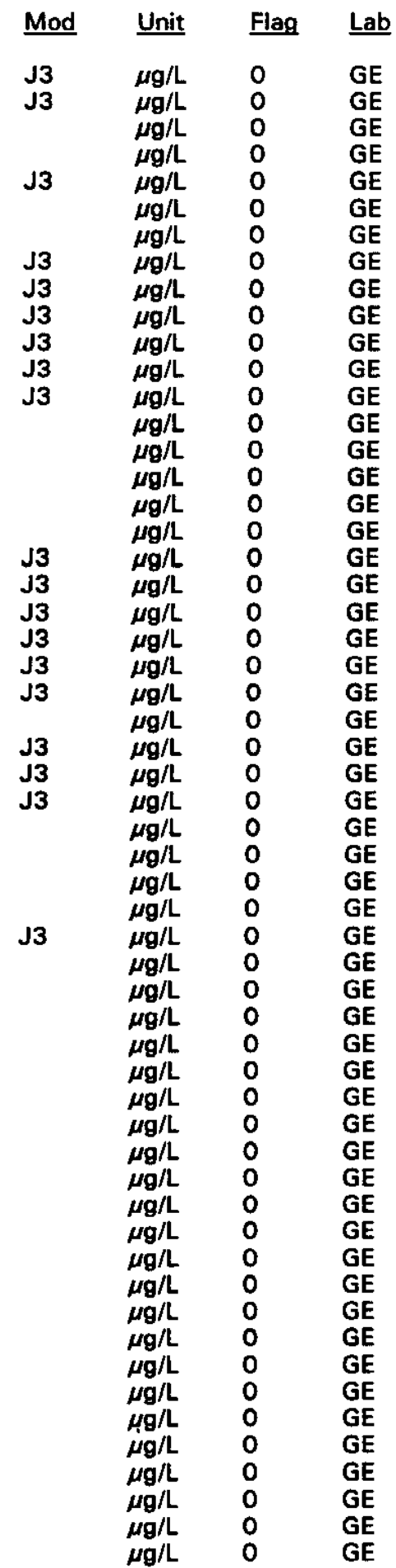

$\overline{-}=$ exceeded holding time. $\square=$ exceeded screening level or final primary drinking water standard. 
WELL BGO $49 \mathrm{C}$ collected on $04 / 13 / 94$, laboratory analyses (cont.)

H.$\quad$ Analyte
Sulfate
$1,1,2,2-T e t r a c h l o r o e t h a n e$
Tetrachloroethylene
Tin, total recoverable
Toluene
Total dissolved solids
Total organic carbon
Total organic halogens
Total phosphates las P)
Toxaphene
Toxaphene
$2,4,5-T P$ (Silvex)
Tributyl phosphate
1,1,1-Trichloroethane
1,1,2-Trichloroethane
Trichloroethylene
Trichlorofluoromethane
$2,4,5-T$
Vanadium, total recoverable
Xylenes
Gross alpha
Nonvolatile beta
Radium, total alpha-emitting
Tritium
Uranium-233/234
Uranium-235
Uranium-238

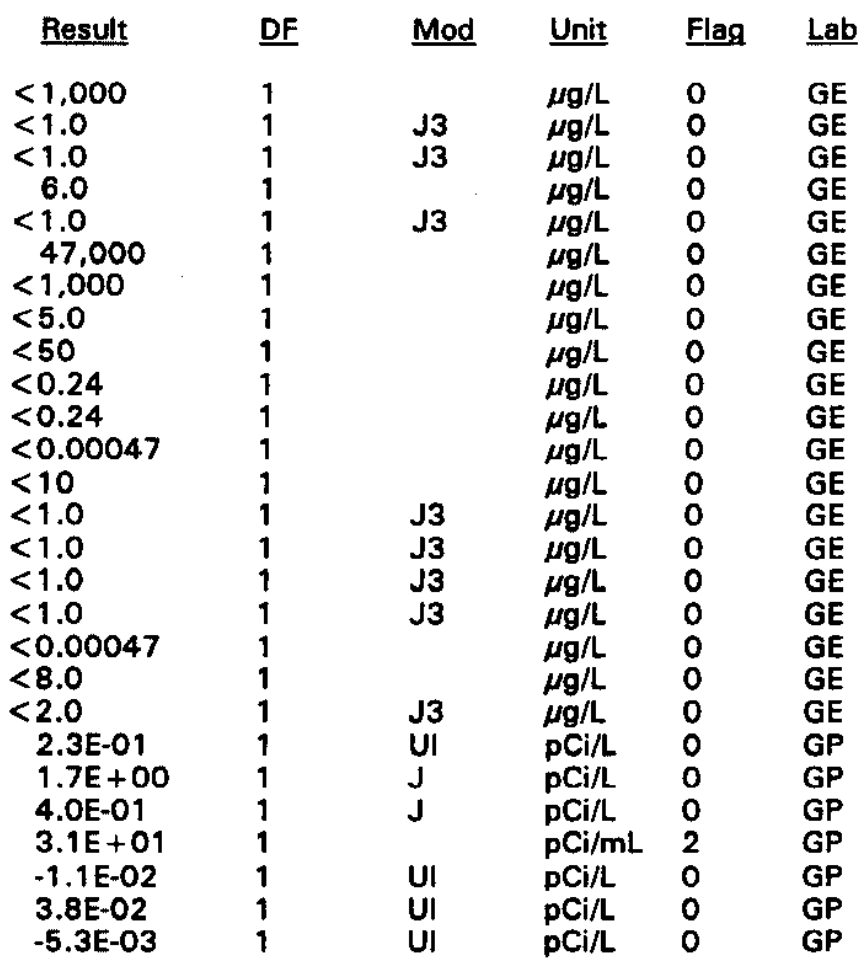

\section{WELL BGO 49D}

\begin{tabular}{|c|c|c|c|c|c|c|}
\hline SRS Coord. & Lat/Longitude & $\underline{\text { Screen Zone Elevation }}$ & Top of Casing & Casing & Pump & Formation \\
\hline $\begin{array}{l}N 73931.5 \\
\text { E56198.8 }\end{array}$ & $\begin{array}{l}33.280983^{\circ} \mathrm{N} \\
81.662428^{\circ} \mathrm{W}\end{array}$ & $238.5-218.5 \mathrm{ft} \mathrm{msl}$ & $271.5 \mathrm{ft} \mathrm{msl}$ & 4" PVC & $\mathbf{s}$ & $\left(1 \mathrm{IB}_{2}\right)$ \\
\hline
\end{tabular}

\section{FIELD MEASUREMENTS}

\section{Sample date: 04/14/94}

Depth to water: $36.91 \mathrm{ft}(11.25 \mathrm{~m})$ below TOC Water elevation: $234.59 \mathrm{ft}(71.50 \mathrm{~m}) \mathrm{msl}$

Sp. conductance: $39 \mu \mathrm{S} / \mathrm{cm}$

Turbidity: 9.9 NTU

Water evacuated before sampling: 9 gal The well went dry during purging.

\section{LABORATORY ANALYSES}

HE D Analyte

- $\quad$ pH

Specific conductance

Turbidity

Acetophenone

Aldrin

Aluminum, total recoverable

Antimony, total recoverable
Time: 9:34

pH: 5.4

Alkalinity: $1 \mathrm{mg} / \mathrm{L}$

Water temperature: $17.7^{\circ} \mathrm{C}$

Volumes purged: 0.9 well volumes 
WELL BGO $49 D$ collected on $04 / 14 / 94$, laboratory analyses (cont.)

H $\underline{\text { Analyte }}$

Arsenic, total recoverable

Barium, total recoverable

Benzene

Bromodichloromethane

Bromoform

Bromomethane (Methyl bromide)

Cadmium, total recoverable

Calcium, total recoverable

Carbon tetrachloride

Chloride

Chlorobenzene

Chloroethane

Chloroethene (Vinyl chloride)

2-Chloroethyl vinyl ether

Chloroform

Chloromethane (Methyl chloride)

Chromium, total recoverable

Copper, total recoverable

Cyanide

p,p'-DDT

Dibromochloromethane

1,1-Dichloroethane

1,2-Dichloroethane

1,1-Dichloroethylene

trans-1,2-Dichloroethylene

Dichloromethane (Methylene chloride)

2,4-Dichlorophenoxyacetic acid

1,2-Dichloropropane

cis-1,3-Dichloropropene

trans-1,3-Dichloropropene

Dieldrin

Endrin

Ethylbenzene

Fluoride

Fluoride

Heptachlor

Iron, total recoverable

Lead, total recoverable

Lindane

Magnesium, total recoverable

Manganese, total recoverable

Mercury, total recoverable

Methoxychlor

Naphthalene

Nickel, total recoverable

Nitrate-nitrite as nitrogen

Phenols

Potassium, total recoverable

Selenium, total recoverable

Silica, total recoverable

Silver, total recoverable

Sodium, total recoverable

Sulfate

1,1,2,2-Tetrachloroethane

Tetrachloroethylene

Tin, total recoverable
Result DF

$<2.0$

7.8

$<1.0$

$<1.0$

$<1.0$

$<1.0$

$<2.0$

417

$<1.0$

1,620

$<1.0$

$<1.0$

$<1.0$

$<1.0$

$<1.0$

$<1.0$

$<4.0$

$<4.0$

$<5.0$

$<0.10$

$<1.0$

$<1.0$

$<1.0$

$<1.0$

$<1.0$

$<1.0$

$<0.0016$

$<1.0$

$<1.0$

$<1.0$

$<0.50$

$<0.0060$

$<1.0$

$<100$

$<100$

$<0.050$

113

4.1

$<0.0050$

354

2.7

$<0.20$

$<0.50$

$<11$

$<4.0$

2,000

$<5.0$

$<500$

$<2.0$

6,120

$<2.0$

3,550

$<1,000$

$<1.0$

$<1.0$

$<2.0$
Mod Unit Flag Lab

$\mu \mathrm{g} / \mathrm{L} \quad \mathrm{O} \quad \mathrm{GE}$

$\mu g / L \quad 0 \quad \mathrm{GE}$

$\mu g / L \quad 0 \quad G E$

$\mu g / L \quad 0 \quad$ GE

$\mu g / L \quad O \quad G E$

$\mu g / L \quad 0 \quad$ GE

$\mu \mathrm{g} / \mathrm{L} \quad \mathrm{O} \quad \mathrm{GE}$

$\mu g / L \quad 0 \quad G E$

$\mu g / L \quad 0 \quad G E$

$\mu \mathrm{g} / \mathrm{L} \quad 0 \quad \mathrm{GE}$

$\mu \mathrm{g} / \mathrm{L} \quad \mathrm{O} \quad \mathrm{GE}$

$\mu g / L \quad 0 \quad$ GE

$\mu g / L \quad 0 \quad$ GE

$\mu g / L \quad 0 \quad$ GE

$\mu g / L \quad 0 \quad$ GE

$\mu \mathrm{g} / \mathrm{L} \quad 0 \quad \mathrm{GE}$

$\mu g / L \quad O \quad G E$

$\mu g / L \quad 0 \quad$ GE

$\mu \mathrm{g} / \mathrm{L} \quad 0 \quad \mathrm{GE}$

J3 $\mu g / L \quad 0 \quad$ GE

$\mu \mathrm{g} / \mathrm{L} \quad 0 \quad \mathrm{GE}$

$\mu g / L \quad 0 \quad$ GE

$\mu g / L \quad 0 \quad$ GE

$\mu \mathrm{g} / \mathrm{L} \quad 0 \quad \mathrm{GE}$

$\mu g / L \quad 0 \quad G E$

$\mu g / L \quad 0 \quad$ GE

$\mu g / L \quad 0 \quad G E$

$\mu \mathrm{g} / \mathrm{L} \quad 0 \quad \mathrm{GE}$

$\mu \mathrm{g} / \mathrm{L} \quad 0 \quad \mathrm{GE}$

$\mu g / L \quad 0 \quad$ GE

J3 $\mu g / L \quad O \quad$ GE

J3 $\mu g / L \quad 0 \quad$ GE

$\mu g / L \quad 0 \quad G E$

$\mu g / L \quad 0 \quad G E$

$\mu g / L \quad 0 \quad G E$

J3 $\mu \mathrm{g} / \mathrm{L} \quad \mathrm{O} \quad \mathrm{GE}$

$\begin{array}{llll} & \mu g / L & 0 & G E \\ J & \mu g / L & 0 & G E\end{array}$

J3 $\mu g / L \quad 0 \quad$ GE

$\mu g / L \quad 0 \quad$ GE

J $\mu g / L \quad 0 \quad$ GE

$\mu \mathrm{g} / \mathrm{L} \quad 0 \quad \mathrm{GE}$

$\mu \mathrm{g} / \mathrm{L} \quad 0 \quad \mathrm{GE}$

$\mu \mathrm{g} / \mathrm{L} \quad 0 \quad \mathrm{GE}$

$\mu \mathrm{g} / \mathrm{L} \quad 0 \quad \mathrm{GE}$

$\mu \mathrm{g} / \mathrm{L} \quad \mathrm{O} \quad \mathrm{GE}$

$\mu \mathrm{g} / \mathrm{L} \quad \mathrm{O} \quad \mathrm{GE}$

$\mu g / L \quad 0 \quad$ GE

$\mu g / L \quad 0 \quad$ GE

$\mu g / L \quad 0 \quad$ GE

$\mu \mathrm{g} / \mathrm{L} \quad 0 \quad \mathrm{GE}$

$\mu g / L \quad 0 \quad$ GE

$\mu g / L \quad 0 \quad$ GE

$\mu g / L \quad 0 \quad$ GE

$\begin{array}{lll}\mu g / L & 0 & \text { GE } \\ \mu \mathrm{g} / \mathrm{L} & 0 & \mathrm{GE}\end{array}$

- exceeded holding time. = exceeded screening level or final primary drinking water standard. 
WELL BGO 49D collected on 04/14/94, laboratory analyses (cont.)

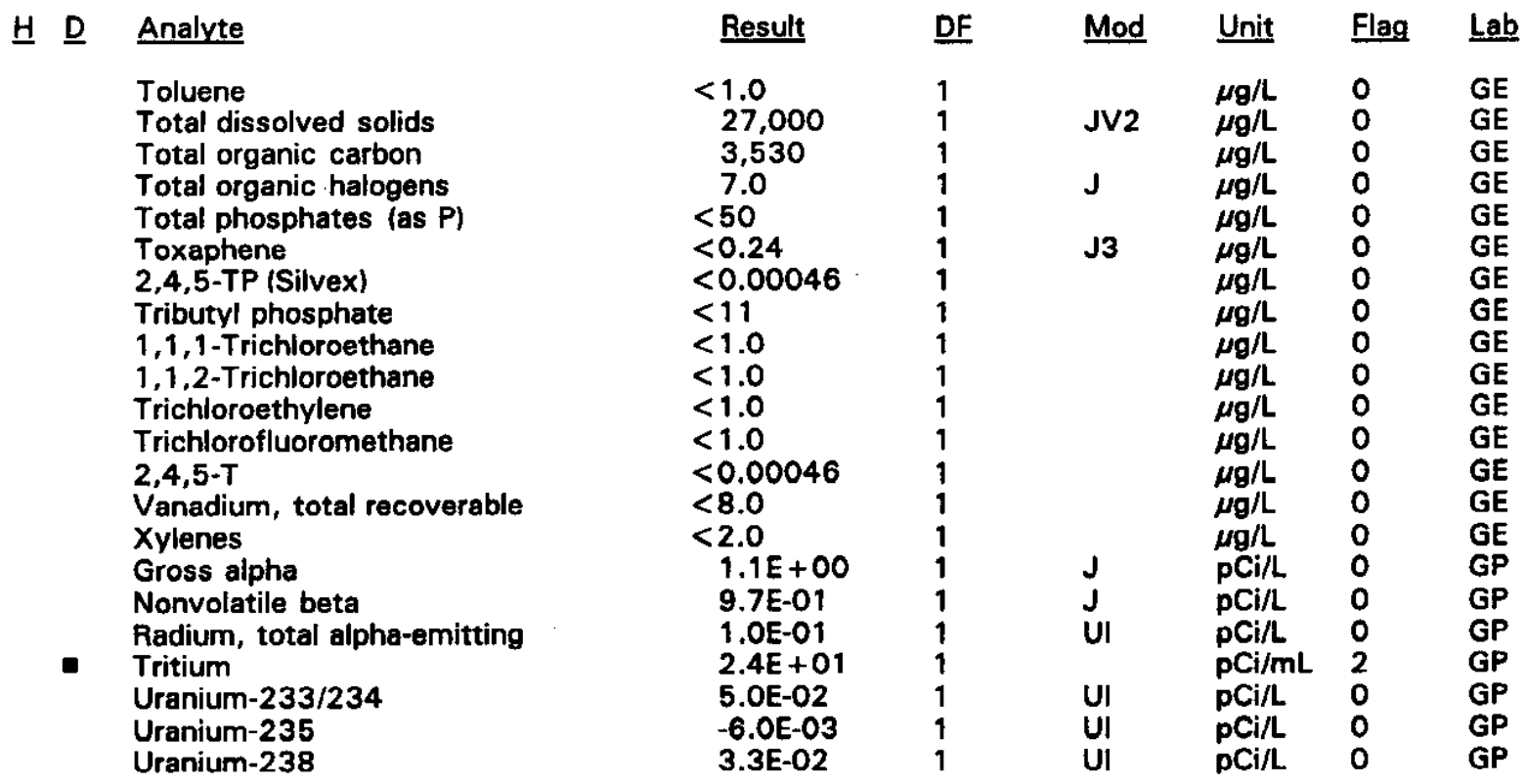

\section{WELL BGO 50A}

\begin{tabular}{|c|c|c|c|c|c|c|}
\hline SRS Coord. & Lat/Longitude & Screen Zone Elevation & Top of Casing & Casing & Pump & Formation \\
\hline $\begin{array}{l}\text { N75201.2 } \\
\text { E54179.8 }\end{array}$ & $\begin{array}{l}33.280497^{\circ} \mathrm{N} \\
81.670210^{\circ} \mathrm{W}\end{array}$ & $100.5-90.5 \mathrm{ft} \mathrm{msl}$ & $255.4 \mathrm{ft} \mathrm{msl}$ & 4" PVC & $\mathbf{S}$ & U. Congaree (IIA) \\
\hline
\end{tabular}

\section{FIELD MEASUREMENTS}

Sample date: 04/14/94

Depth to water: $95.37 \mathrm{ft}(29.07 \mathrm{~m})$ below TOC

Water elevation: $160.03 \mathrm{ft}(48.78 \mathrm{~m}) \mathrm{ms}$

Sp. conductance: $591 \mu \mathrm{S} / \mathrm{cm}$

Turbidity: 2.6 NTU

Water evacuated before sampling: $\mathbf{4 0}$ gal

The well went dry during purging.

\section{LABORATORY ANALYSES}

H $\underline{\text { Analyte }}$

pH

Specific conductance

Turbidity

Acetophenone

Aldrin

Aluminum, total recoverable

Antimony, total recoverable

Arsenic, total recoverable

Barium, total recoverable

Benzene

Bromodichloromethane

Bromoform
Time: 9:07

pH: 11.6

Alkalinity: $132 \mathrm{mg} / \mathrm{L}$

Water temperature: $18.6^{\circ} \mathrm{C}$

Volumes purged: 0.9 well volumes

- = exceeded holding time. = exceeded screening level or final primary drinking water standard. 
WELL BGO 5OA collected on 04/14/94, laboratory analyses (cont.)

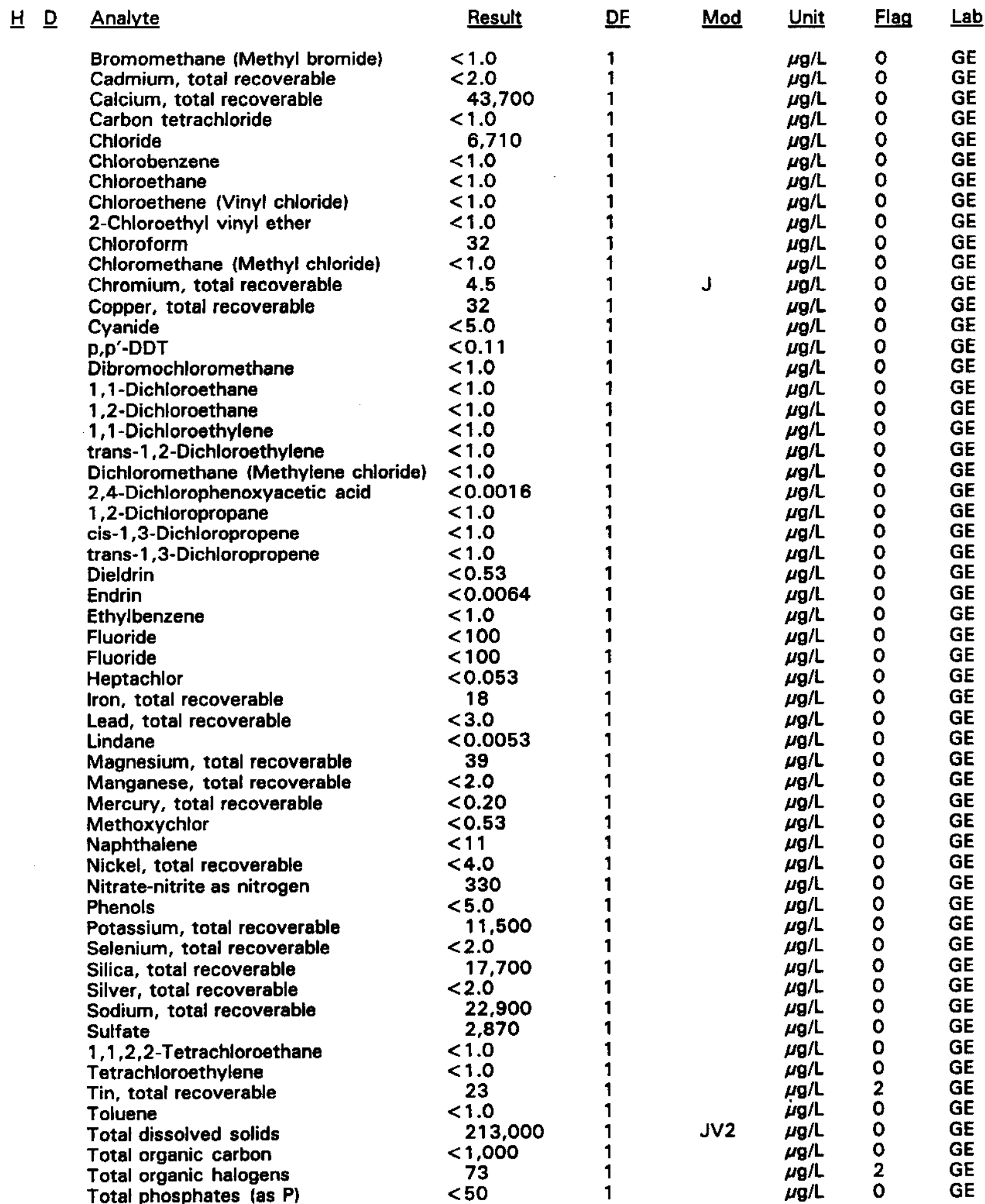

\footnotetext{
- = exceeded holding time. $=$ exceeded screening level or final primary drinking water standard.
} 
WELL. BGO 5OA collected on 04/14/94, laboratory analyses (cont.)

H $\underline{\text { Analyte }}$
Toxaphene
$2,4,5-T P$ (Silvex)
Tributyl phosphate
$1,1,1-$ Trichloroethane
1,1,2-Trichloroethane
Trichloroethylene
Trichlorofluoromethane
2,4,5-T
Vanadium, total recoverable
Xylenes
Gross alpha
Nonvolatile beta
Radium, total alpha-emitting
Tritium
Uranium-233/234
Uranium-235
Uranium-238

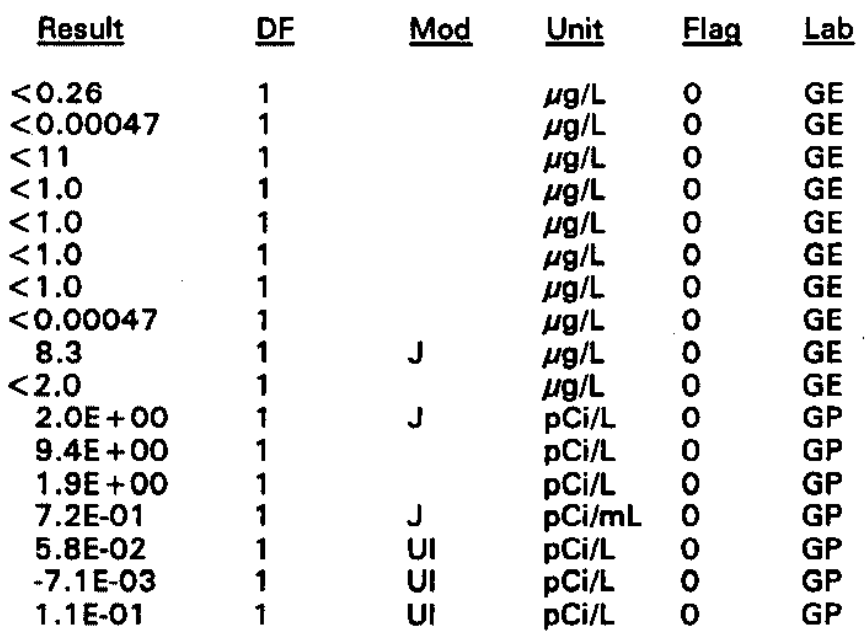

\section{WELL BGO 50C}

\begin{tabular}{|c|c|c|c|c|c|c|}
\hline SRS Coord. & Lat/Longitude & Screen Zone Elevation & Top of Casing & Casing & Pump & Formation \\
\hline $\begin{array}{l}\text { N75190.4 } \\
\text { E54197.0 }\end{array}$ & $\begin{array}{l}33.280501^{\circ} \mathrm{N} \\
81.670144^{\circ} \mathrm{W}\end{array}$ & $172.5-162.5 \mathrm{ft} \mathrm{msl}$ & $255.5 \mathrm{ft} \mathrm{msl}$ & 4" PVC & $\mathbf{S}$ & Barnwell (IIB $)$ \\
\hline
\end{tabular}

\section{FIELD MEASUREMENTS}

\section{Sample date: 04/14/94}

Depth to water: $47.54 \mathrm{ft}(14.49 \mathrm{~m})$ below TOC

Water elevation: $207.96 \mathrm{ft}(63.39 \mathrm{~m}) \mathrm{msl}$

Sp. conductance: $33 \mu \mathrm{S} / \mathrm{cm}$

Turbidity: 10.7 NTU

Water evacuated before sampling: $\mathbf{3 8} \mathrm{gal}$

The well went dry during purging.

\section{LABORATORY ANALYSES}

\section{H D Analyte}

- $\quad \mathrm{pH}$

Specific conductance

Turbidity

Acetophenone

Aldrin

Aluminum, total recoverable

Antimony, total recoverable

Arsenic, total recoverable

Barium, total recoverable

Benzene

Bromodichloromethane

Bromoform

Bromomethane (Methyl bromide)

Cadmium, total recoverable

Calcium, total recoverable

Carbon tetrachloride

Chloride
Time: $8: 50$

pH: 5.4

Alkalinity: $1 \mathrm{mg} / \mathrm{L}$

Water temperature: $11.7^{\circ} \mathrm{C}$

Volumes purged: 1.3 well volumes

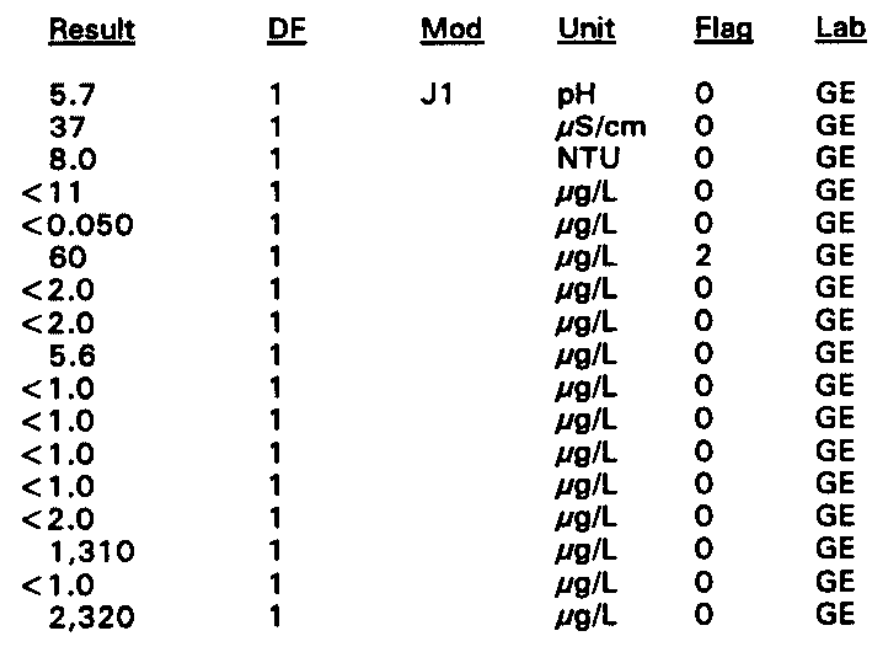

$\overline{-=}$ exceeded holding time. $=$ exceeded screening level or final primary drinking water standard. 
WELL BGO 50C collected on 04/14/94, laboratory analyses (cont.)

H

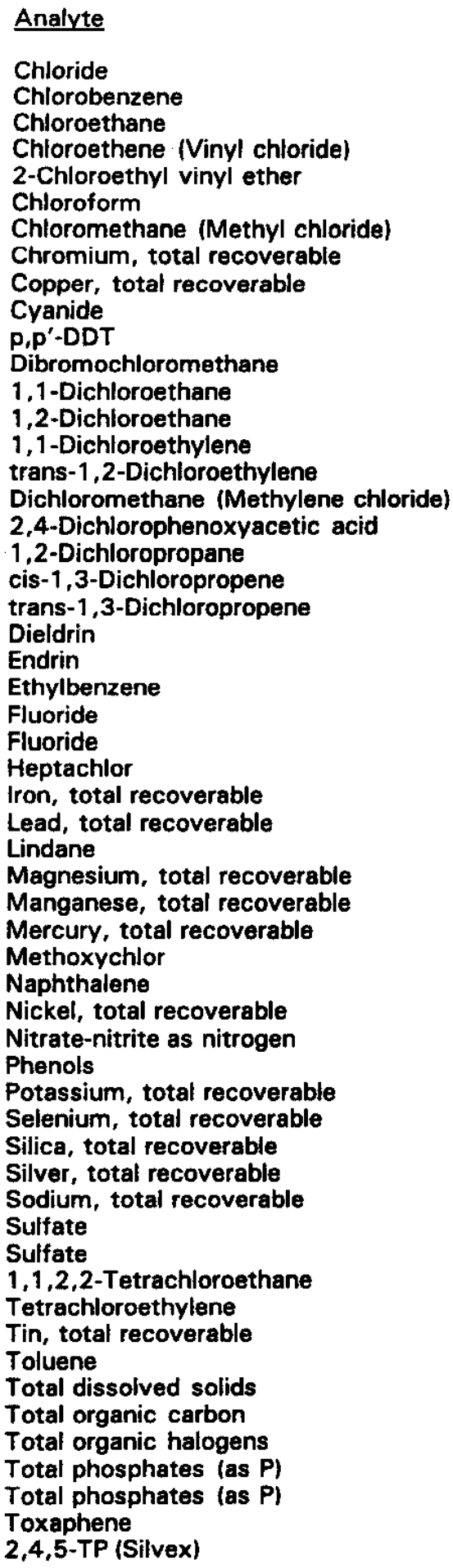

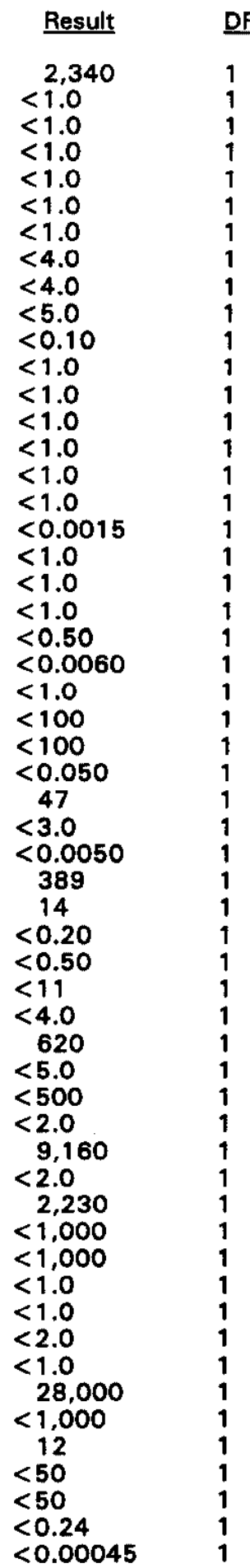

\begin{tabular}{|c|c|c|}
\hline Mod & Unit & Flag \\
\hline JV2 & $\begin{array}{l}\mu \mathrm{g} / \mathrm{L} \\
\mu \mathrm{g} / \mathrm{L} \\
\mu \mathrm{g} / \mathrm{L} \\
\mu \mathrm{g} / \mathrm{L} \\
\mu \mathrm{g} / \mathrm{L} \\
\mu \mathrm{g} / \mathrm{L} \\
\mu \mathrm{g} / \mathrm{L} \\
\mu \mathrm{g} / \mathrm{L} \\
\mu \mathrm{g} / \mathrm{L} \\
\mu \mathrm{g} / \mathrm{L} \\
\mu \mathrm{g} / \mathrm{L} \\
\mu \mathrm{g} / \mathrm{L} \\
\mu \mathrm{g} / \mathrm{L} \\
\mu \mathrm{g} / \mathrm{L} \\
\mu \mathrm{g} / \mathrm{L} \\
\mu \mathrm{g} / \mathrm{L} \\
\mu \mathrm{g} / \mathrm{L} \\
\mu \mathrm{g} / \mathrm{L} \\
\mu \mathrm{g} / \mathrm{L} \\
\mu \mathrm{g} / \mathrm{L} \\
\mu \mathrm{g} / \mathrm{L} \\
\mu \mathrm{g} / \mathrm{L} \\
\mu \mathrm{g} / \mathrm{L} \\
\mu \mathrm{g} / \mathrm{L} \\
\mu \mathrm{g} / \mathrm{L} \\
\mu \mathrm{g} / \mathrm{L} \\
\mu \mathrm{g} / \mathrm{L} \\
\mu \mathrm{g} / \mathrm{L} \\
\mu \mathrm{g} / \mathrm{L} \\
\mu \mathrm{g} / \mathrm{L} \\
\mu \mathrm{g} / \mathrm{L} \\
\mu \mathrm{g} / \mathrm{L} \\
\mu \mathrm{g} / \mathrm{L} \\
\mu \mathrm{g} / \mathrm{L} \\
\mu \mathrm{g} / \mathrm{L} \\
\mu \mathrm{g} / \mathrm{L} \\
\mu \mathrm{g} / \mathrm{L} \\
\mu \mathrm{g} / \mathrm{L} \\
\mu \mathrm{g} / \mathrm{L} \\
\mu \mathrm{g} / \mathrm{L} \\
\mu \mathrm{g} / \mathrm{L} \\
\mu \mathrm{g} / \mathrm{L} \\
\mu \mathrm{g} / \mathrm{L} \\
\mu \mathrm{g} / \mathrm{L} \\
\mu \mathrm{g} / \mathrm{L} \\
\mu \mathrm{g} / \mathrm{L} \\
\mu \mathrm{g} / \mathrm{L} \\
\mu \mathrm{g} / \mathrm{L} \\
\mu \mathrm{g} / \mathrm{L} \\
\mu \mathrm{g} / \mathrm{L} \\
\mu \mathrm{g} / \mathrm{L} \\
\mu \mathrm{g} / \mathrm{L} \\
\mu \mathrm{g} / \mathrm{L} \\
\mu \mathrm{g} / \mathrm{L} \\
\mu \mathrm{g} / \mathrm{L} \\
\mu \mathrm{g} / \mathrm{L}\end{array}$ & $\begin{array}{l}0 \\
0 \\
0 \\
0 \\
0 \\
0 \\
0 \\
0 \\
0 \\
0 \\
0 \\
0 \\
0\end{array}$ \\
\hline
\end{tabular}

- = exceeded holding time. $\omega=$ exceeded screening level or final primary drinking water standard. 
WELL BGO $50 C$ collected on 04/14/94, laboratory analyses (cont.)

브 D Analyte

Tributyl phosphate

$1,1,1$-Trichloroethane

1,1,2-Trichloroethane

- Trichloroethylene

Trichiorofluoromethane

2,4,5-T

Vanadium, total recoverable

Xylenes

Gross alpha

Nonvolatile beta

Radium, total alpha-emitting

- Tritium

Uranium-233/234

Uranium-235

Uranium-238

\begin{tabular}{ll}
\multicolumn{1}{l}{ Result } & \\
\hline$<11$ & \\
$<1.0$ & 1 \\
$<1.0$ & 1 \\
11 & 1 \\
$<1.0$ & 1 \\
$<0.00045$ & 1 \\
$<8.0$ & 1 \\
$<2.0$ & 1 \\
$2.3 E-01$ & 1 \\
$6.2 E-01$ & 1 \\
$0.0 E+00$ & 1 \\
$1.0 E+02$ & 1 \\
$1.4 E-01$ & 1 \\
$<0.0 E+00$ & 1 \\
$4.7 E-02$ & 1
\end{tabular}

$\begin{array}{clll}\text { Mod } & \text { Unit } & \text { Flag } & \text { Lab } \\ & & & \\ & \mu \mathrm{g} / \mathrm{L} & 0 & \mathrm{GE} \\ & \mu \mathrm{g} / \mathrm{L} & 0 & \mathrm{GE} \\ & \mu \mathrm{g} / \mathrm{L} & 0 & \mathrm{GE} \\ & \mu \mathrm{g} / \mathrm{L} & 2 & \mathrm{GE} \\ & \mu \mathrm{g} / \mathrm{L} & 0 & \mathrm{GE} \\ & \mu \mathrm{g} / \mathrm{L} & 0 & \mathrm{GE} \\ & \mu \mathrm{g} / \mathrm{L} & 0 & \mathrm{GE} \\ & \mu \mathrm{g} / \mathrm{L} & 0 & \mathrm{GE} \\ & \mathrm{pCi} / \mathrm{L} & 0 & \mathrm{GP} \\ \text { UI } & \mathrm{pCi} / \mathrm{L} & 0 & \mathrm{GP} \\ \text { UI } & \mathrm{pCi} / \mathrm{L} & 0 & \mathrm{GP} \\ \text { UI } & \mathrm{pCi} / \mathrm{mL} & 2 & \mathrm{GP} \\ & \mathrm{pCi} / \mathrm{L} & 0 & \mathrm{GP} \\ \text { UI } & \mathrm{pCi} / \mathrm{L} & 0 & \mathrm{GP} \\ & \mathrm{pCi} / \mathrm{L} & 0 & \mathrm{GP}\end{array}$

\section{WELL BGO 50D}

$\begin{array}{lllllll}\text { SRS Coord } & \text { Lat/Longitude } & \text { Screen Zone Elevation } & \text { Top of Casing } & \text { Casing } & \text { Pump } & \text { Formation } \\ \text { N75181.3 } & 33.280501^{\circ} \mathrm{N} & 228.0-208.0 \mathrm{ft} \mathrm{msl} & 256 \mathrm{ft} \mathrm{msl} & 4^{\text {" PVC }} & \text { S } & \text { Water Table (IIB }) \\ \text { E54209.1 } & \mathbf{8 1 . 6 7 0 0 9 5 ^ { \circ } \mathrm { W }} & & & \end{array}$

\section{FIELD MEASUREMENTS}

Sample date: $04 / 13 / 94$

Depth to water: $30.57 \mathrm{ft}(9.32 \mathrm{~m})$ below TOC

Water elevation: $225.43 \mathrm{ft}(68.71 \mathrm{~m}) \mathrm{msl}$

Sp. conductance: $74 \mu \mathrm{S} / \mathrm{cm}$

Turbidity: $1.0 \mathrm{NTU}$

Water evacuated before sampling: $\mathbf{4 9} \mathrm{gal}$

\section{LABORATORY ANALYSES}

$\begin{array}{ll}\text { H } & \text { Analyte } \\ \text { - } & \text { pH } \\ \text { SH } & \\ \text { Turbific conductance } \\ \text { Acetophenone } \\ \text { Aldrin } \\ \text { Aluminum, total recoverable } \\ \text { Antimony, total recoverable } \\ \text { Arsenic, total recoverable } \\ \text { Barium, total recoverable } \\ \text { Benzene } \\ \text { Bromodichloromethane } \\ \text { Bromoform } \\ \text { Bromomethane (Methyl bromide) } \\ \text { Cadmium, total recoverable } \\ \text { Calcium, total recoverable } \\ \text { Carbon tetrachloride } \\ \text { Chloride } \\ \text { Chlorobenzene }\end{array}$

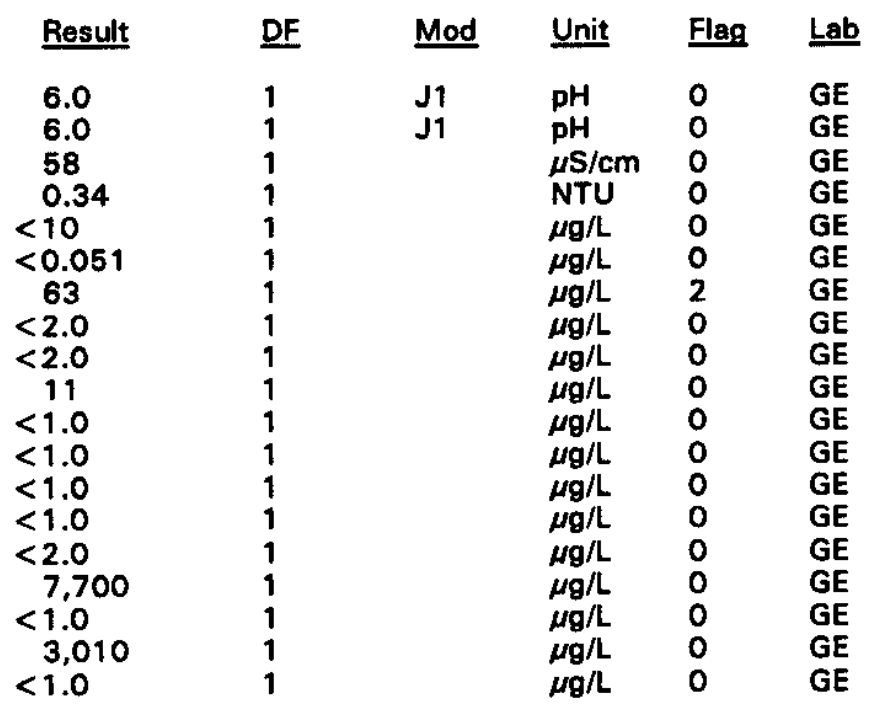

Time: 9:57

pH: 6.0

Alkalinity: $14 \mathrm{mg} / \mathrm{L}$

Water temperature: $20.0^{\circ} \mathrm{C}$

Volumes purged: 4.3 well volumes 
WELL BGO 5OD collected on 04/13/94, laboratory analyses (cont.)

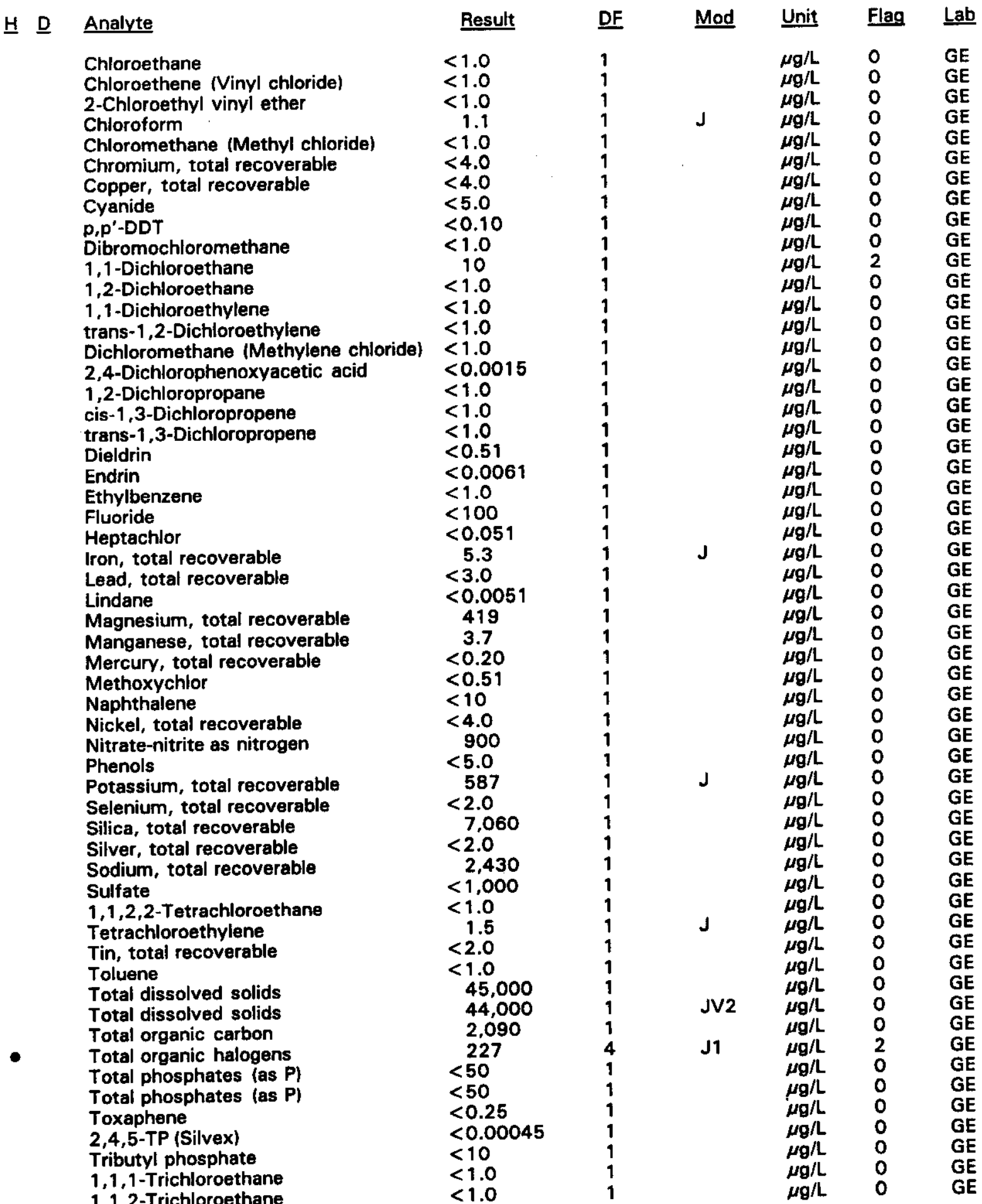

\footnotetext{
- =xceeded holding time. = exceeded screening level or final primary drinking water standard.
} 
WELL BGO 500 collected on $04 / 13 / 94$, laboratory analyses (cont.)

H

- Trichloroethylene

Trichlorofluoromethane

2,4,5-T

Vanadium, total recoverable

Xylenes

Gross alpha

Nonvolatile beta

Radium, total alpha-emitting

Radium, total alpha-emitting

Total activity

- Tritium

Uranium-233/234

Uranium-235

Uranium-238

Result
19
$<1.0$
$<0.00045$
$<8.0$
$<2.0$
$2.4 E+00$
$1.6 E+00$
$1.1 E+00$
$1.7 E+00$
$6.3 E+06$
$6.1 E+03$
$5.1 E-02$
$1.6 E-02$
$4.1 E-02$

DF
1
1
1
1
1
1
1
1
1
1
1
1
1

$\begin{array}{llll}\text { Mod } & \text { Unit } & \text { Flag } & \text { Lab } \\ & & & \\ & \mu g / L & 2 & \text { GE } \\ & \mu g / L & 0 & \text { GE } \\ & \mu g / L & 0 & \text { GE } \\ & \mu g / L & 0 & \text { GE } \\ & \mu g / L & 0 & \text { GE } \\ & \mathrm{pCi} / L & 0 & \text { GP } \\ J & \mathrm{pCi} / L & 0 & \text { GP } \\ J & \mathrm{pCi} / \mathrm{L} & 0 & \text { GP } \\ & \mathrm{pCi} / \mathrm{L} & 0 & \mathrm{GP} \\ & \mathrm{pCi} / \mathrm{L} & 0 & \mathrm{EM} \\ & \mathrm{pCi} / \mathrm{mL} & 2 & \mathrm{GP} \\ & \mathrm{pCi} / \mathrm{L} & 0 & \mathrm{GP} \\ \text { UI } & \mathrm{pCi} / \mathrm{L} & 0 & \mathrm{GP} \\ \mathrm{UI} & \mathrm{pCi} / \mathrm{L} & 0 & \mathrm{GP}\end{array}$

WELL BGX 1C

\begin{tabular}{|c|c|c|c|c|c|c|}
\hline SRS Coord. & Lat/Longitude & Screen Zone Elevation & Top of Casing & Casing & Pump & Formation \\
\hline $\begin{array}{l}\text { N76820.0 } \\
\text { E58599.8 }\end{array}$ & $\begin{array}{l}33.291289^{\circ} \mathrm{N} \\
81.661716^{\circ} \mathrm{W}\end{array}$ & $186.0-176.0 \mathrm{ft} \mathrm{msl}$ & $291.3 \mathrm{ft} \mathrm{msl}$ & 4" PVC & $\mathbf{s}$ & \\
\hline
\end{tabular}

\section{FIELD MEASUREMENTS}

Sample date: 04/14/94

Depth to water: $75.54 \mathrm{ft}(23.02 \mathrm{~m})$ below TOC

Water elevation: $215.76 \mathrm{ft}(65.76 \mathrm{~m}) \mathrm{msl}$

Sp. conductance: $156 \mu \mathrm{S} / \mathrm{cm}$

Turbidity: 3.9 NTU

Water evacuated before sampling: $16 \mathrm{gal}$

The well went dry during purging.

\section{LABORATORY ANALYSES}

H D Analyte

- $\mathrm{pH}$

Specific conductance

Turbidity

Acetophenone

Aldrin

Aluminum, total recoverable

Antimony, total recoverable

Antimony, total recoverable

Arsenic, total recoverable

Arsenic, total recoverable

Barium, total recoverable

Benzene

Bromodichloromethane

Bromoform

Bromomethane (Methyl bromide)

Cadmium, total recoverable

Calcium, total recoverable

Carbon tetrachloride

Chloride

Chlorobenzene
Time: 11:45

pH: 11.9

Alkalinity: $358 \mathrm{mg} / \mathrm{L}$

Water temperature: $19.6^{\circ} \mathrm{C}$

Volumes purged: 0.6 well volumes

\begin{tabular}{|c|c|c|c|c|c|}
\hline Result & DF & Mod & Unit & Flag & $\underline{\text { Lat }}$ \\
\hline 12 & 1 & J1 & $\mathrm{pH}$ & 2 & GE \\
\hline 1.740 & 1 & & $\mu \mathrm{S} / \mathrm{cm}$ & 2 & GE \\
\hline $\begin{array}{r}3.0 \\
<10\end{array}$ & 1 & J1 & NTU & 0 & GE \\
\hline $\begin{array}{l}<10 \\
<0.051\end{array}$ & $\begin{array}{l}1 \\
1\end{array}$ & & $\begin{array}{l}\mu g / L \\
\mu g / L\end{array}$ & $\begin{array}{l}0 \\
0\end{array}$ & $\begin{array}{l}\mathrm{GE} \\
\mathrm{GE}\end{array}$ \\
\hline 665 & 2 & & $\mu g / L$ & 2 & GE \\
\hline$<2.0$ & 1 & & $\mu g / L$ & 0 & \\
\hline$<2.0$ & 1 & & $\mu g / L$ & 0 & \\
\hline$<2.0$ & 1 & & $\mu g / L$ & 0 & \\
\hline$<2.0$ & 1 & & $\mu g / L$ & 0 & $\mathrm{GE}$ \\
\hline 199 & 2 & & $\mu g / L$ & 0 & \\
\hline$<1.0$ & 1 & & $\mu \mathrm{g} / \mathrm{L}$ & 0 & \\
\hline$<1.0$ & 1 & & $\mu g / L$ & 0 & GE \\
\hline$<1.0$ & 1 & & $\mu \mathrm{g} / \mathrm{L}$ & 0 & \\
\hline$<1.0$ & 1 & & $\mu g / L$ & 0 & \\
\hline$<4.0$ & 2 & & $\mu g / L$ & 0 & GE \\
\hline$<1.0$ & 1 & & $\mu \mathrm{g} / \mathrm{L}$ & 0 & \\
\hline 935 & 1 & & $\mu g / L$ & 0 & \\
\hline$<1.0$ & 1 & & $\mu \mathrm{g} / \mathrm{L}$ & 0 & \\
\hline
\end{tabular}

- = exceeded holding time. $=$ exceeded screening level or final primary drinking water standard. 
WELL BGX IC collected on 04/14/94, laboratory analyses (cont.)

H $\underline{\text { Analyte }}$

Chloroethane

Chloroethene (Vinyl chloride)

2-Chloroethyl vinyl ether

Chloroform

Chloromethane (Methyl chloride)

Chromium, total recoverable

Copper, total recoverable

Cyanide

$p, p^{\prime}-D D T$

Dibromochloromethane

1,1-Dichloroethane

1,2-Dichloroethane

1,1-Dichloroethylene

trans-1,2-Dichloroethylene

Dichloromethane (Methylene chloride)

2,4-Dichlorophenoxyacetic acid

1,2-Dichloropropane

cis-1,3-Dichloropropene

trans-1,3-Dichloropropene

Dieldrin

Endrin

Ethylbenzene

Fluoride

Heptachlor

Iron, total recoverable

Lead, total recoverable

Lead, total recoverable

Lindane

Magnesium, total recoverable

Manganese, total recoverable

Mercury, total recoverable

Methoxychlor

Naphthalene

Nickel, total recoverable

Nitrate-nitrite as nitrogen

Phenols

Potassium, total recoverable

Selenium, total recoverable

Selenium, total recoverable

Silica, total recoverable

Silver, total recoverable

Sodium, total recoverable

Sulfate

1,1,2,2-Tetrachloroethane

Tetrachloroethylene

Tin, total recoverable

Toluene

Total dissolved solids

Total organic carbon

Total organic halogens

Total phosphates (as P)

Toxaphene

2,4,5-TP (Silvex)

1,1,1-Trichloroethane

1,1,2-Trichloroethane

Trichloroethylene
Result DF

$<1.0$

$<1.0$

$<1.0$

$<1.0$

$<1.0$

$<8.0$

$<8.0$

$<5.0$

$<0.10$

$<1.0$

$<1.0$

$<1.0$

$<1.0$

$<1.0$

$<1.0$

$<0.0016$

$<1.0$

$<1.0$

$<1.0$

$<0.51$

$<0.0061$

$<1.0$

$<100$

$<0.051$

11

8.1

8.8

$<0.0051$

170

$<4.0$

$<0.20$

$<0.51$

$<10$

$<8.0$

380

$<5.0$

5,710

$<2.0$

$<2.0$

6,600

$<4.0$

5,220

$<1,000$

$<1.0$

$<1.0$

4.7

$<1.0$

412,000

$<1,000$

$<5.0$

60

$<0.24$

$<0.00046$

$<1.0$

$<1.0$

$<1.0$
Mod Unit Flag Lab

$\mu g / L \quad 0 \quad$ GE

$\mu g / L \quad 0 \quad$ GE

$\mu \mathrm{g} / \mathrm{L} \quad \mathrm{O} \quad \mathrm{GE}$

$\mu \mathrm{g} / \mathrm{L} \quad 0 \quad \mathrm{GE}$

$\mu \mathrm{g} / \mathrm{L} \quad 0 \quad \mathrm{GE}$

$\mu \mathrm{g} / \mathrm{L} \quad 0 \quad \mathrm{GE}$

$\mu g / L \quad 0 \quad$ GE

$\mu g / L \quad 0 \quad G E$

$\mu g / L \quad 0 \quad$ GE

$\mu g / L \quad 0 \quad$ GE

$\mu g / L \quad 0 \quad$ GE

$\mu \mathrm{g} / \mathrm{L} \quad \mathrm{O} \quad \mathrm{GE}$

$\mu \mathrm{g} / \mathrm{L} \quad 0 \quad \mathrm{GE}$

$\mu g / L \quad 0 \quad$ GE

$\mu \mathrm{g} / \mathrm{L} \quad 0 \quad \mathrm{GE}$

$\mu g / L \quad 0 \quad$ GE

$\mu \mathrm{g} / \mathrm{L} \quad 0 \quad \mathrm{GE}$

$\mu g / L \quad 0 \quad$ GE

$\mu g / L \quad 0 \quad$ GE

$\mu \mathrm{g} / \mathrm{L} \quad 0 \quad \mathrm{GE}$

$\mu g / L \quad 0 \quad$ GE

$\mu \mathrm{g} / \mathrm{L} \quad 0 \quad \mathrm{GE}$

$\mu \mathrm{g} / \mathrm{L} \quad 0$

$\mu \mathrm{g} / \mathrm{L} \quad 0 \quad \mathrm{GE}$

$\mu \mathrm{g} / \mathrm{L} \quad 0 \quad \mathrm{GE}$

$\mu \mathrm{g} / \mathrm{L} \quad 0 \quad \mathrm{GE}$

$\mu g / L \quad 0 \quad$ GE

$\mu \mathrm{g} / \mathrm{L} \quad 0 \quad \mathrm{GE}$

$\mu \mathrm{g} / \mathrm{L} \quad 0 \quad \mathrm{GE}$

$\mu \mathrm{g} / \mathrm{L} \quad \mathrm{O} \quad \mathrm{GE}$

$\mu \mathrm{g} / \mathrm{L} \quad 0 \quad \mathrm{GE}$

$\mu g / L \quad 0 \quad$ GE

$\mu g / L \quad 0 \quad$ GE

$\mu g / L \quad 0 \quad$ GE

$\mu \mathrm{g} / \mathrm{L} \quad 0 \quad \mathrm{GE}$

$\mu g / L \quad 0 \quad$ GE

$\mu \mathrm{g} / \mathrm{L} \quad 0 \quad \mathrm{GE}$

$\mu \mathrm{g} / \mathrm{L} \quad 0 \quad \mathrm{GE}$

$\mu \mathrm{g} / \mathrm{L} \quad 0 \quad \mathrm{GE}$

$\mu \mathrm{g} / \mathrm{L} \quad \mathrm{O} \quad \mathrm{GE}$

$\mu \mathrm{g} / \mathrm{L} \quad 0 \quad \mathrm{GE}$

$\mu \mathrm{g} / \mathrm{L} \quad 0 \quad \mathrm{GE}$

$\mu \mathrm{g} / \mathrm{L} \quad 0 \quad \mathrm{GE}$

$\mu g / L \quad 0 \quad$ GE

$\mu g / L \quad 0 \quad$ GE

$\mu g / L \quad 0 \quad$ GE

$\mu \mathrm{g} / \mathrm{L}$

$\mu \mathrm{g} / \mathrm{L}$

$\mu \mathrm{g} / \mathrm{L}$

$\mu \mathrm{g} / \mathrm{L}$

$\mu g / L$

$\mu g / L$

$\mu \mathrm{g} / \mathrm{L}$

$\mu g / L$

$\mu g / L$

$\mu \mathrm{g} / \mathrm{L}$

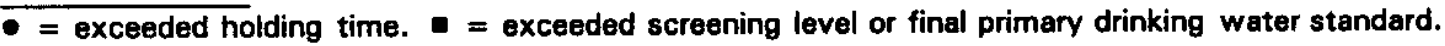


WELL BGX $1 \mathrm{C}$ collected on $04 / 14 / 94$, laboratory analyses (cont.)
H $\underline{\text { Analyte }}$
Trichlorofluoromethane
2,4,5-T
Vanadium, total recoverable
Xylenes
Gross alpha
Nonvolatile beta
Radium, total alpha-emitting
Total activity
- Tritium

\begin{tabular}{ll}
\multicolumn{1}{l}{ Result } & DF \\
$<1.0$ & \\
$<0.00046$ & 1 \\
$<16$ & 2 \\
$<2.0$ & 1 \\
$1.8 E+00$ & 1 \\
$3.5 E+00$ & 1 \\
$1.5 E+00$ & 1 \\
$1.5 E+06$ & 10 \\
$1.6 E+03$ & 1
\end{tabular}

$\begin{array}{llll}\text { Mod } & \underline{\text { Unit }} & \text { Flag } & \text { Lab } \\ & & & \\ & \mu g / L & 0 & \text { GE } \\ & \mu g / L & 0 & \text { GE } \\ & \mu g / L & 0 & \text { GE } \\ & \mu \mathrm{g} / \mathrm{L} & 0 & \mathrm{GE} \\ \mathrm{pCi} / \mathrm{L} & 0 & \mathrm{GP} \\ \mathrm{J} & \mathrm{pCi} / \mathrm{L} & 0 & \mathrm{GP} \\ & \mathrm{pCi} / \mathrm{L} & 0 & \mathrm{GP} \\ & \mathrm{pCi} / \mathrm{L} & 0 & \mathrm{EM} \\ & \mathrm{pCi} / \mathrm{mL} & 2 & \mathrm{GP}\end{array}$

WELL BGX 1D

\begin{tabular}{|c|c|c|c|c|c|c|}
\hline SRS Coord. & Lat/Longitude & Screen Zone Elevation & Top of Casing & Casing & Pump & Formation \\
\hline $\begin{array}{l}\text { N768 } \\
5586 C\end{array}$ & $\begin{array}{l}33.291280^{\circ} \mathrm{N} \\
81.661673^{\circ} \mathrm{W}\end{array}$ & $234.7-214.7 \mathrm{ft} \mathrm{msl}$ & $291.3 \mathrm{ft} \mathrm{msl}$ & 4" PVC & $\mathbf{s}$ & Water Table $\left(\mathrm{IIB}_{2}\right)$ \\
\hline
\end{tabular}

\section{FIELD MEASUREMENTS}

Sample date: $04 / 14 / 94$

Depth to water: $61.49 \mathrm{ft}(18.74 \mathrm{~m})$ below TOC

Water elevation: $229.81 \mathrm{ft}(70.05 \mathrm{~m}) \mathrm{msl}$

Sp. conductance: $109 \mu \mathrm{S} / \mathrm{cm}$

Turbidity: 45.0 NTU

Water evacuated before sampling: $4 \mathrm{gal}$

The well went dry during purging.

\section{LABORATORY ANALYSES}

H $\underline{\text { Analyte }}$

$\mathrm{pH}$

Specific conductance

Specific conductance

Turbidity

Acetophenone

Aldrin

Aluminum, total recoverable

Antimony, total recoverable

Arsenic, total recoverable

Barium, total recoverable

Benzene

Bromodichloromethane

Bromoform

Bromomethane (Methyl bromide)

Cadmium, total recoverable

Calcium, total recoverable

Carbon tetrachloride

Chloride

Chlorobenzene

Chloroethane

Chloroethene (Vinyl chloride)

2-Chloroethyl vinyl ether

Chloroform

Chloromethane (Methyl chloride)

Chromium, total recoverable
Time: 12:01

pH: 5.4

Alkalinity: $4 \mathrm{mg} / \mathrm{L}$

Water temperature: $19.8^{\circ} \mathrm{C}$

Volumes purged: 0.4 well volumes

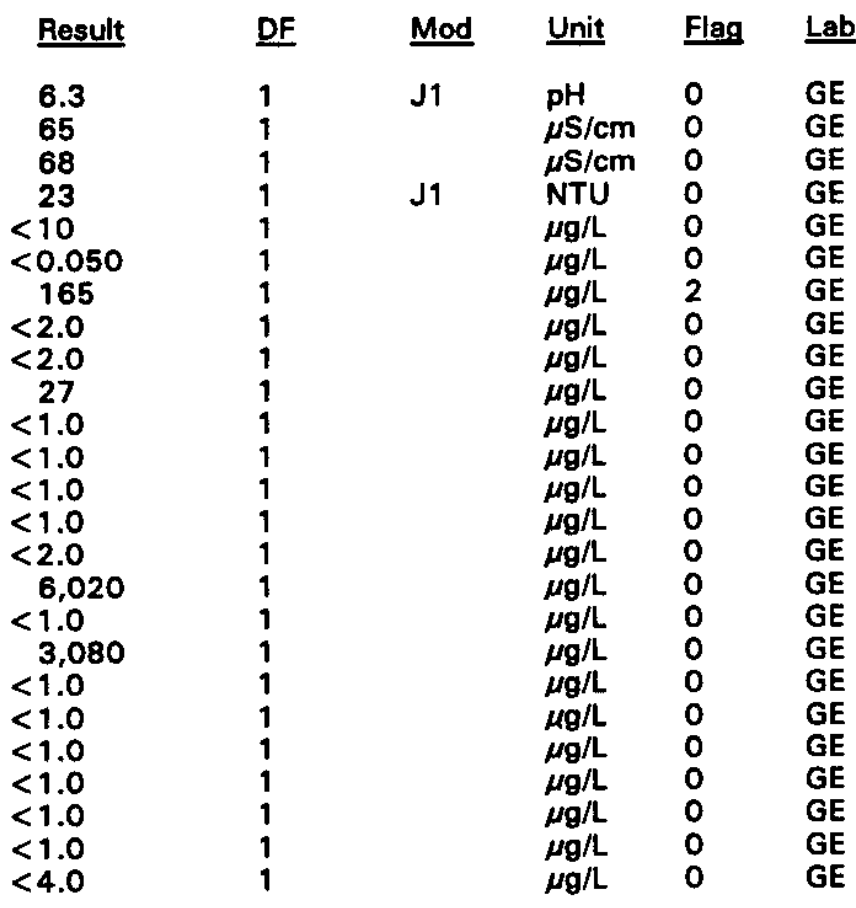

- = exceeded holding time. $=$ exceeded screening level or final primary drinking water standard. 
WELL BGX 1D collected on 04/14/94, laboratory analyses (cont.)

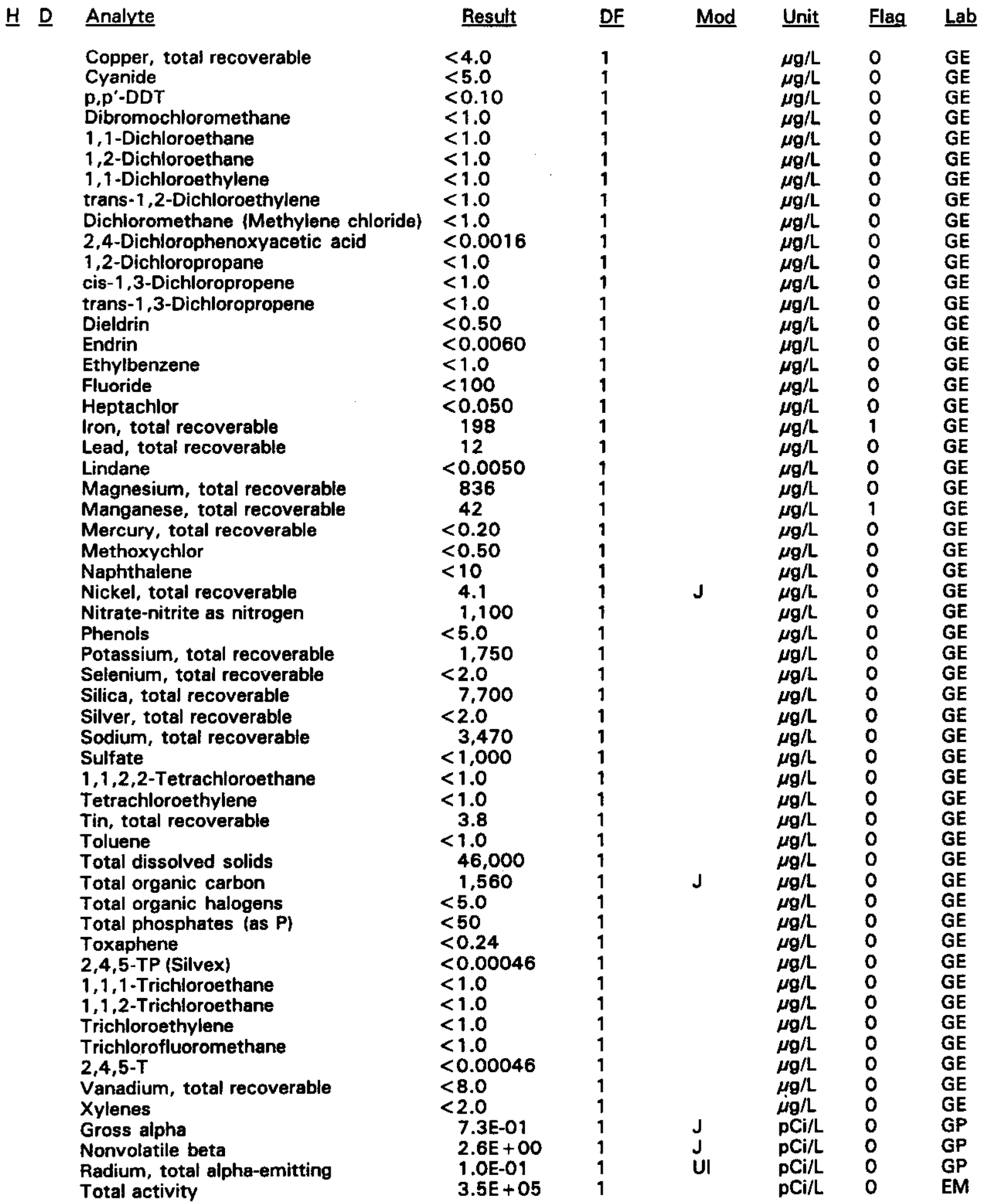

- = exceeded holding time. = exceeded screening level or final primary drinking water standard. 
WSRC-TR-94-0345

Unclassified

WELL BGX 1D collected on 04/14/94, laboratory analyses (cont.)
H D Analyte
Result
$\underline{\text { DF }}$
Mod
Unit Flag $\underline{\mathrm{Lab}}$
- Tritium
$3.5 E+02$
$\mathrm{pCi} / \mathrm{mL} \quad 2$
GP

WELL BGX 2B

$\begin{array}{llllllll}\text { SRS Coord. } & \text { Lat/Longitude } & \text { Screen Zone Elevation } & \text { Top of Casing } & \text { Casing } & \text { Pump } & \text { Formation } \\ \text { N77203.4 } & 33.291577^{\circ} \mathrm{N} & 147.2-137.2 \mathrm{ft} \mathrm{msl} & 291.3 \mathrm{ft} \mathrm{msl} & 4^{\text {" PVC }} & \text { S } & \text { McBean (IIB,) } \\ \text { E58256.5 } & 81.663365^{\circ} \mathrm{W} & & & & \end{array}$

\section{FIELD MEASUREMENTS}

Sample date: $04 / 14 / 94$

Depth to water: $78.92 \mathrm{ft}(24.06 \mathrm{~m})$ below TOC

Water elevation: $212.38 \mathrm{ft}(64.73 \mathrm{~m}) \mathrm{msl}$

Sp. conductance: $261 \mu \mathrm{S} / \mathrm{cm}$

Turbidity: 17.3 NTU

Water evacuated before sampling: $\mathbf{5 0}$ gal

The well went dry during purging.

\section{LABORATORY ANALYSES}

H $\underline{\text { Analyte }}$

-

$\mathrm{pH}$
Specific conductance

Specific conductance

Turbidity

Acetophenone

Aldrin

Aluminum, total recoverable

Antimony, total recoverable

Arsenic, total recoverable

Barium, total recoverable

Benzene

Bromodichloromethane

Bromoform

Bromomethane (Methyl bromide)

Cadmium, total recoverable

Calcium, total recoverable

Carbon tetrachloride

Chloride

Chlorobenzene

Chloroethane

Chloroethene (Vinyl chloride)

2-Chloroethyl vinyl ether

Chloroform

Chloromethane (Methyl chloride)

Chromium, total recoverable

Copper, total recoverable

Cyanide

p,p'-DDT

Dibromochloromethane

1,1-Dichloroethane

1,2-Dichloroethane

1,1-Dichloroethylene

trans-1,2-Dichloroethylene
Time: 10:56

pH: 7.6

Alkalinity: $99 \mathrm{mg} / \mathrm{L}$

Water temperature: $19.1^{\circ} \mathrm{C}$

Volumes purged: 1.0 well volumes

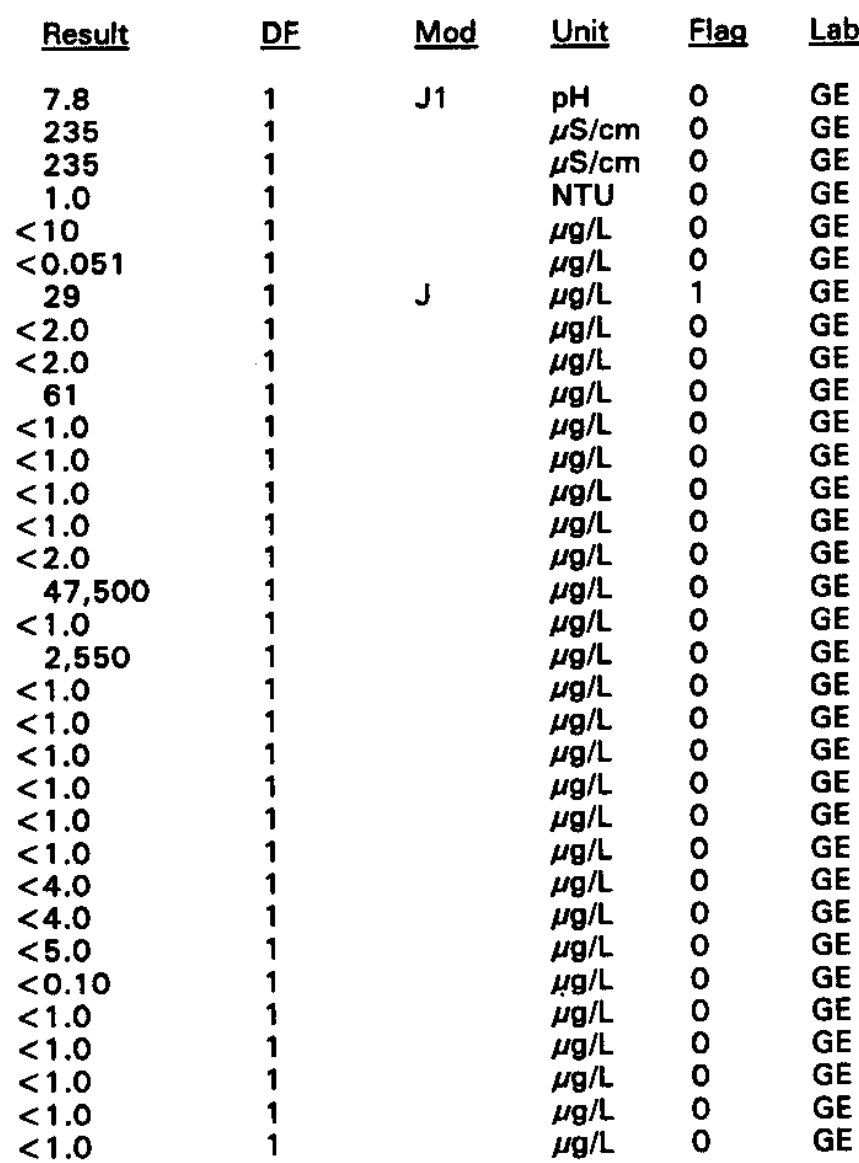

- = exceeded holding time. - = exceeded screening level or final primary drinking water standard. 
WELL BGX 2B collected on 04/14/94, laboratory analyses (cont.)

Analyte
Dichloromethane (Methylene chloride)
2,4-Dichlorophenoxyacetic acid
1,2-Dichloropropane
cis-1,3-Dichloropropene
trans-1,3-Dichloropropene
Dieldrin
Endrin
Ethylbenzene
Fluoride
Fluoride
Heptachlor
Iron, total recoverable
Lead, total recoverable
Lindane
Magnesium, total recoverable
Manganese, total recoverable
Mercury, total recoverable
Methoxychlor
Naphthalene
Nickel, total recoverable
Nitrate-nitrite as nitrogen
Phenols
Potassium, total recoverable
Selenium, total recoverable
Silica, total recoverable
Silver, total recoverable
Sodium, total recoverable
Sulfate
1,1,2,2-Tetrachloroethane
Tetrachloroethylene
Tin, total recoverable
Toluene
Total dissolved solids
Total organic carbon
Total organic halogens
Total organic halogens
Total phosphates (as P)
Toxaphene
$2,4,5-T P$ (Silvex)
1,1,1-Trichloroethane
1,1,2-Trichloroethane
Trichloroethylene
Trichlorofluoromethane
2,4,5-T
Vanadium, total recoverable
Xylenes
Gross alpha
Nonvolatile beta
Radium, total alpha-emitting
Tritium

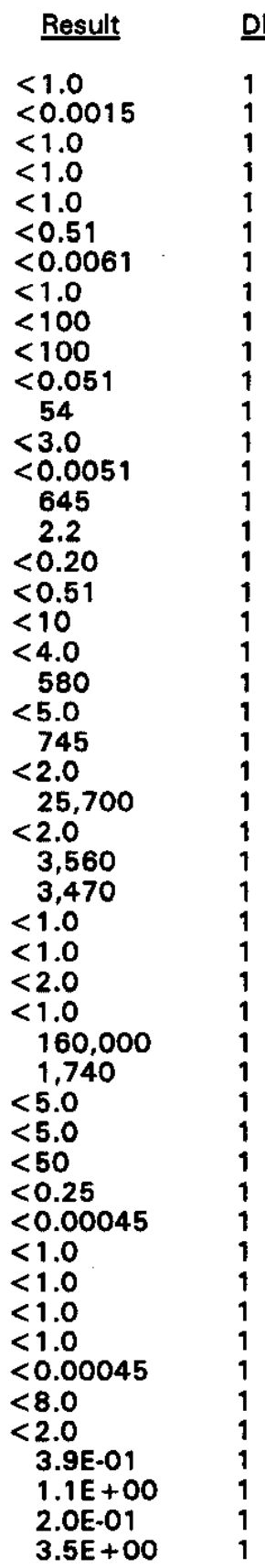

Mod

Unit Flag Lab

$\mu \mathrm{g} / \mathrm{L} \quad 0 \quad \mathrm{GE}$

$\mu \mathrm{g} / \mathrm{L}$

$\mu g / L$

$\mu g / L$

$\mu g / L$

$\mu g / L$

$\mu g / L$

$\mu \mathrm{g} / \mathrm{L}$

$\mu g / L$

$\mu g / L$

$\mu g / L$

$\mu g / L$

$\mu g / L$

$\mu g / L$

$\mu g / L$

$\mu g / L$

$\mu g / L$

$\mu \mathrm{g} / \mathrm{L}$

$\mu g / L$

$\mu \mathrm{g} / \mathrm{L}$

$\mu \mathrm{g} / \mathrm{L}$

$\mu \mathrm{g} / \mathrm{L}$

$\mu \mathrm{g} / \mathrm{L}$

$\mu g / L$

$\mu \mathrm{g} / \mathrm{L}$

$\mu g / L$

$\mu g / L$

$\mu g / L$

$\mu g / L$

$\mu \mathrm{g} / \mathrm{L}$

$\mu \mathrm{g} / \mathrm{L}$

$\mu g / L$

JV2

$\mu \mathrm{g} / \mathrm{L}$

$\mu g / L$

$\mu g / L$

$\mu g / L$

$\mu g / L$

$\mu g / L$

$\mu g / L$

$\mu g / L$

$\mu g / L$

$\mu \mathrm{g} / \mathrm{L}$

$\mu g / L$

$\mu g / L$

$\mu g / L$

$\mu g / L$

UI $\quad \mathrm{pCi} / \mathrm{L}$

J

pCi/L

pCi/L

pCi/mL

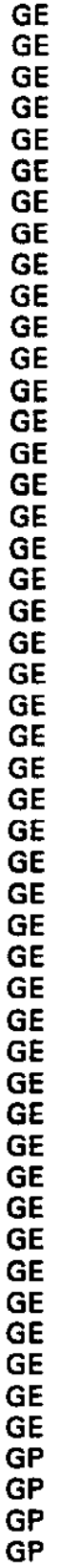

- = exceeded holding time. - = exceeded screening level or final primary drinking water standard. 
WELL BGX 20

$\begin{array}{lllllll}\text { SRS Coord. } & \text { Lat/Longitude } & \text { Screen Zone Elevation } & \text { Top of Casing } & \text { Casing } & \text { Pump Formation } & \text { Fun } \\ \text { N77192.4 } & 33.291567^{\circ} \mathrm{N} & 191.1-181.1 \mathrm{ft} \mathrm{msl} & 291.1 \mathrm{ft} \mathrm{msl} & \text { 4" PVC } & \text { S } & \text { Barnwell (IIB,) } \\ \text { E58265.6 } & 81.663320^{\circ} \mathrm{W} & & & & \end{array}$

\section{FIELD MEASUREMENTS}

Sample date: $04 / 14 / 94$

Depth to water: $75.88 \mathrm{ft}(23.13 \mathrm{~m})$ below TOC

Water elevation: $215.22 \mathrm{ft}(65.60 \mathrm{~m}) \mathrm{ms}$

Sp. conductance: $75 \mu \mathrm{S} / \mathrm{cm}$

Turbidity: 2.3 NTU

Water evacuated before sampling: $20 \mathrm{gal}$

The well went dry during purging.

\section{LABORATORY ANALYSES}

H $\underline{\text { Analyte }}$

\section{pH}

pH

Specific conductance

Turbidity

Acetophenone

Aldrin

Aluminum, total recoverable

Antimony, total recoverable

Arsenic, total recoverable

Barium, total recoverable

Benzene

Bromodichloromethane

Bromoform

Bromomethane (Methyl bromide)

Cadmium, total recoverable

Calcium, total recoverable

Carbon tetrachloride

Chloride

Chlorobenzene

Chloroethane

Chloroethene (Vinyl chloride)

2-Chloroethyl vinyl ether

Chloroform

Chloromethane (Methyl chloride)

Chromium, total recoverable

Copper, total recoverable

Cyanide

p,p'-DDT

Dibromochloromethane

1,1-Dichloroethane

1,2-Dichloroethane

1,1-Dichloroethylene

trans-1,2-Dichloroethylene

Dichloromethane (Methylene chloride)

2,4-Dichlorophenoxyacetic acid

1,2-Dichloropropane

cis-1,3-Dichloropropene

trans-1,3-Dichloropropene

Dieldrin
Time: $11: 12$

pH: 5.4

Alkalinity: $3 \mathrm{mg} / \mathrm{L}$

Water temperature: $19.2^{\circ} \mathrm{C}$

Volumes purged: 0.9 well volumes

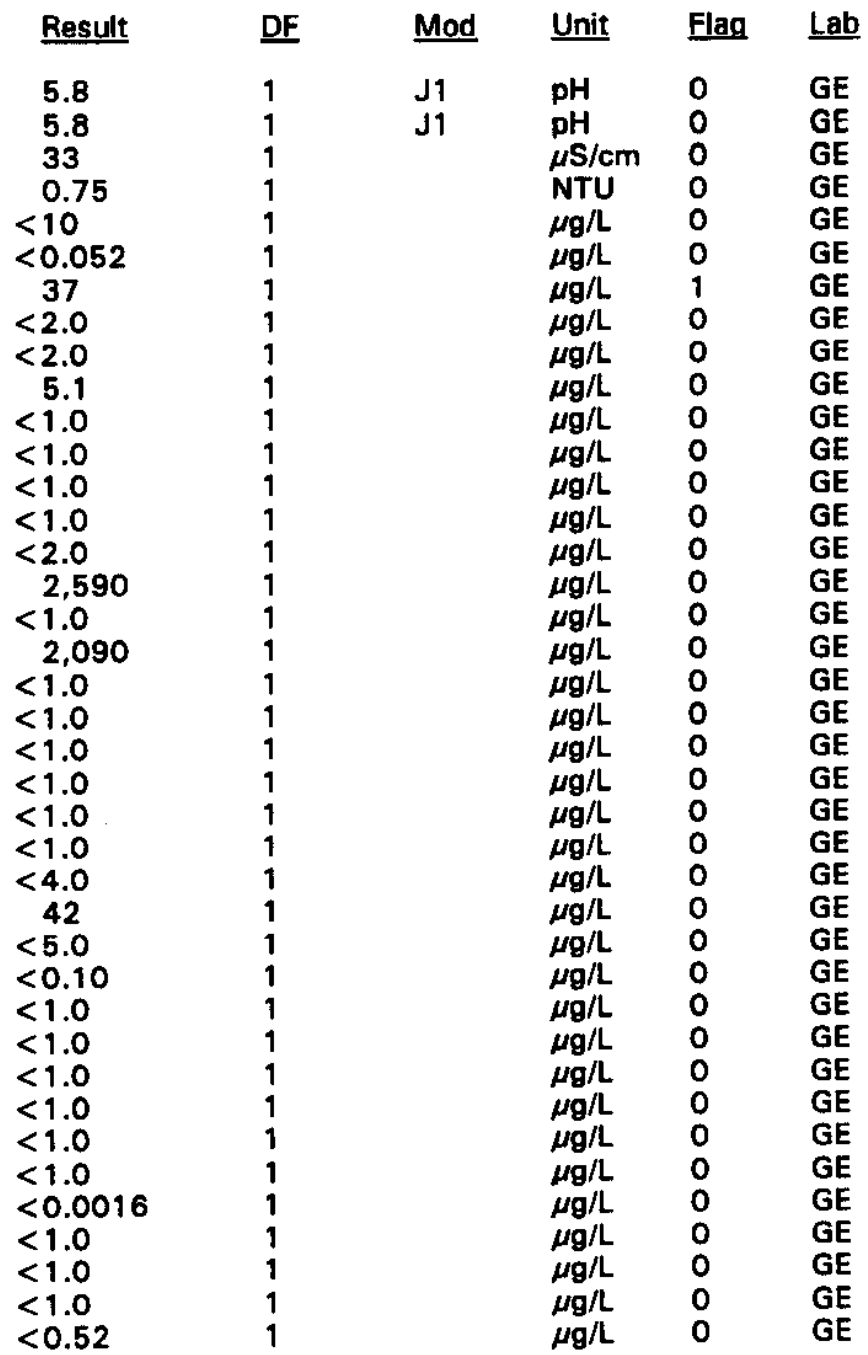

- = exceeded holding time. $=$ = exceeded screening level or final primary drinking water standard. 
WELL BGX 2D collected on $04 / 14 / 94$, laboratory analyses (cont.)

H $\underline{\text { D }}$ Analyte

Endrin

Ethylbenzene

Fluoride

Fluoride

Fluoride

Fluoride

Heptachlor

Iron, total recoverable

Lead, total recoverable

Lindane

Magnesium, total recoverable

Manganese, total recoverable

Mercury, total recoverable

Methoxychlor

Naphthalene

Nickel, total recoverable

Nitrate-nitrite as nitrogen

Nitrate-nitrite as nitrogen

Phenols

Potassium, total recoverable

Selenium, total recoverable

Silica, total recoverable

Silver, total recoverable

Sodium, total recoverable

Sulfate

1,1,2,2-Tetrachloroethane

Tetrachloroethylene

Tin, total recoverable

Toluene

Total dissolved solids

Total dissolved solids

Total organic carbon

Total organic halogens

Total phosphates (as P)

Toxaphene

2,4,5-TP (Silvex)

1,1,1-Trichloroethane

1,1,2-Trichloroethane

- Trichloroethylene

Trichlorofluoromethane

2,4,5-T

Vanadium, total recoverable

Xylenes

Gross alpha

Nonvolatile beta

Radium, total alpha-emitting

- Tritium

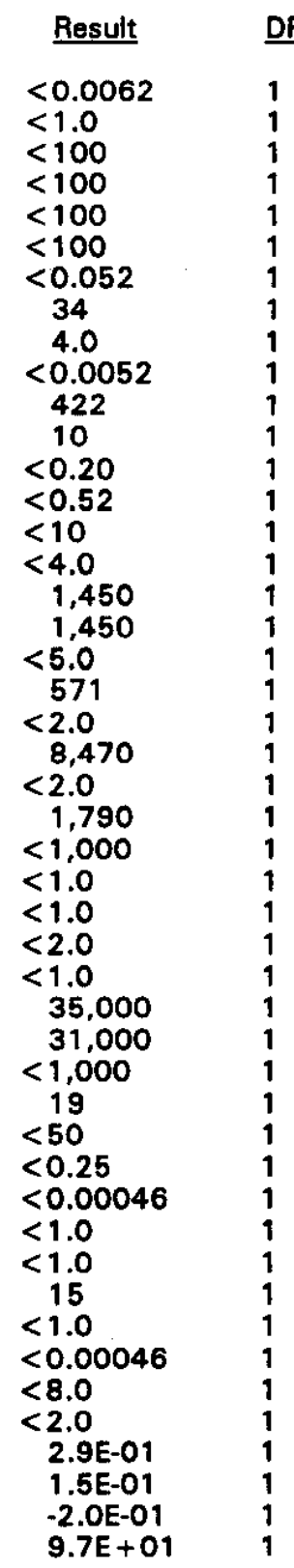

Mod Unit Flag Lab

$\mu \mathrm{g} / \mathrm{L} \quad 0 \quad \mathrm{GE}$

$\mu \mathrm{g} / \mathrm{L} \quad \mathrm{O} \quad \mathrm{GE}$

$\mu g / L \quad 0 \quad G E$

$\mu \mathrm{g} / \mathrm{L} \quad 0 \quad \mathrm{GE}$

$\mu g / L \quad 0 \quad$ GE

$\mu \mathrm{g} / \mathrm{L} \quad \mathrm{O} \quad \mathrm{GE}$

$\mu g / L \quad 0 \quad$ GE

$\mu \mathrm{g} / \mathrm{L} \quad 0 \quad \mathrm{GE}$

$\mu \mathrm{g} / \mathrm{L} \quad 0 \quad \mathrm{GE}$

$\mu \mathrm{g} / \mathrm{L} \quad 0 \quad \mathrm{GE}$

$\mu \mathrm{g} / \mathrm{L} \quad 0 \quad \mathrm{GE}$

$\mu g / L \quad 0 \quad$ GE

$\mu g / L \quad 0 \quad$ GE

$\mu g / L \quad 0 \quad G E$

$\mu g / L \quad 0 \quad$ GE

$\mu \mathrm{g} / \mathrm{L} \quad \mathrm{O} \quad \mathrm{GE}$

$\mu g / L \quad 0 \quad G E$

$\mu g / L \quad 0 \quad$ GE

$\mu g / L \quad 0 \quad G E$

$\mu g / L \quad 0 \quad G E$

$\mu g / L \quad O \quad G E$

$\mu g / L \quad 0 \quad$ GE

$\mu \mathrm{g} / \mathrm{L} \quad \mathrm{O} \quad \mathrm{GE}$

$\mu \mathrm{g} / \mathrm{L} \quad 0 \quad \mathrm{GE}$

$\mu g / L \quad 0 \quad$ GE

$\mu \mathrm{g} / \mathrm{L} \quad \mathrm{O} \quad \mathrm{GE}$

$\mu g / L \quad 0 \quad G E$

$\mu \mathrm{g} / \mathrm{L} \quad 0 \quad \mathrm{GE}$

$\mu g / L \quad 0 \quad G E$

JV2 $\mu g / L \quad 0 \quad$ GE

JV2 $\mu \mathrm{g} / \mathrm{L} \quad 0 \quad \mathrm{GE}$

$\mu \mathrm{g} / \mathrm{L} \quad \mathrm{O} \quad \mathrm{GE}$

$\mu g / L \quad 0 \quad G E$

$\mu g / L \quad 0 \quad G E$

$\mu g / L \quad 0 \quad G E$

$\mu g / L \quad 0 \quad$ GE

$\mu g / L \quad 0 \quad G E$

$\mu g / L \quad 0 \quad G E$

$\mu g / L \quad 2 \quad G E$

$\mu g / L \quad 0 \quad$ GE

$\mu g / L \quad 0 \quad$ GE

$\mu \mathrm{g} / \mathrm{L} \quad 0 \quad \mathrm{GE}$

$\mu \mathrm{g} / \mathrm{L} \quad \mathrm{O} \quad \mathrm{GE}$

PCi/L 0 GP

$\mathrm{pCi} / \mathrm{L} \quad 0 \quad \mathrm{GP}$

PCi/L 0 GP

$\mathrm{pCi} / \mathrm{mL} 2$ GP

- = exceeded holding time. = exceeded screening level or final primary drinking water standard. 
WELL BGX 3D

\begin{tabular}{|c|c|c|c|c|c|c|}
\hline SRS Coord. & Lat/Longitude & Screen Zone Elevation & Top of Casing & Casing & Pump & Formation \\
\hline $\begin{array}{l}\text { N77577.0 } \\
\text { E57780.1 }\end{array}$ & $\begin{array}{l}33.291626^{\circ} \mathrm{N} \\
81.665346^{\circ} \mathrm{W}\end{array}$ & $221.6-201.6 \mathrm{ft} \mathrm{msI}$ & $291.2 \mathrm{ft} \mathrm{msl}$ & 4" PVC & $\mathbf{s}$ & Barnwell (IIB, \\
\hline
\end{tabular}

\section{FIELD MEASUREMENTS}

Sample date: 04/13/94

Depth to water: $76.18 \mathrm{ft}(23.22 \mathrm{~m})$ below TOC

Water elevation: $215.02 \mathrm{ft}(65.54 \mathrm{~m}) \mathrm{msl}$

Sp. conductance: $36 \mu \mathrm{S} / \mathrm{cm}$

Turbidity: 0.6 NTU

Water evacuated before sampling: $46 \mathrm{gal}$

Time: $15: 16$

pH: 5.6

Alkalinity: $4 \mathrm{mg} / \mathrm{L}$

Water temperature: $19.7^{\circ} \mathrm{C}$

Volumes purged: 5.2 well volumes

\section{LABORATORY ANALYSES}

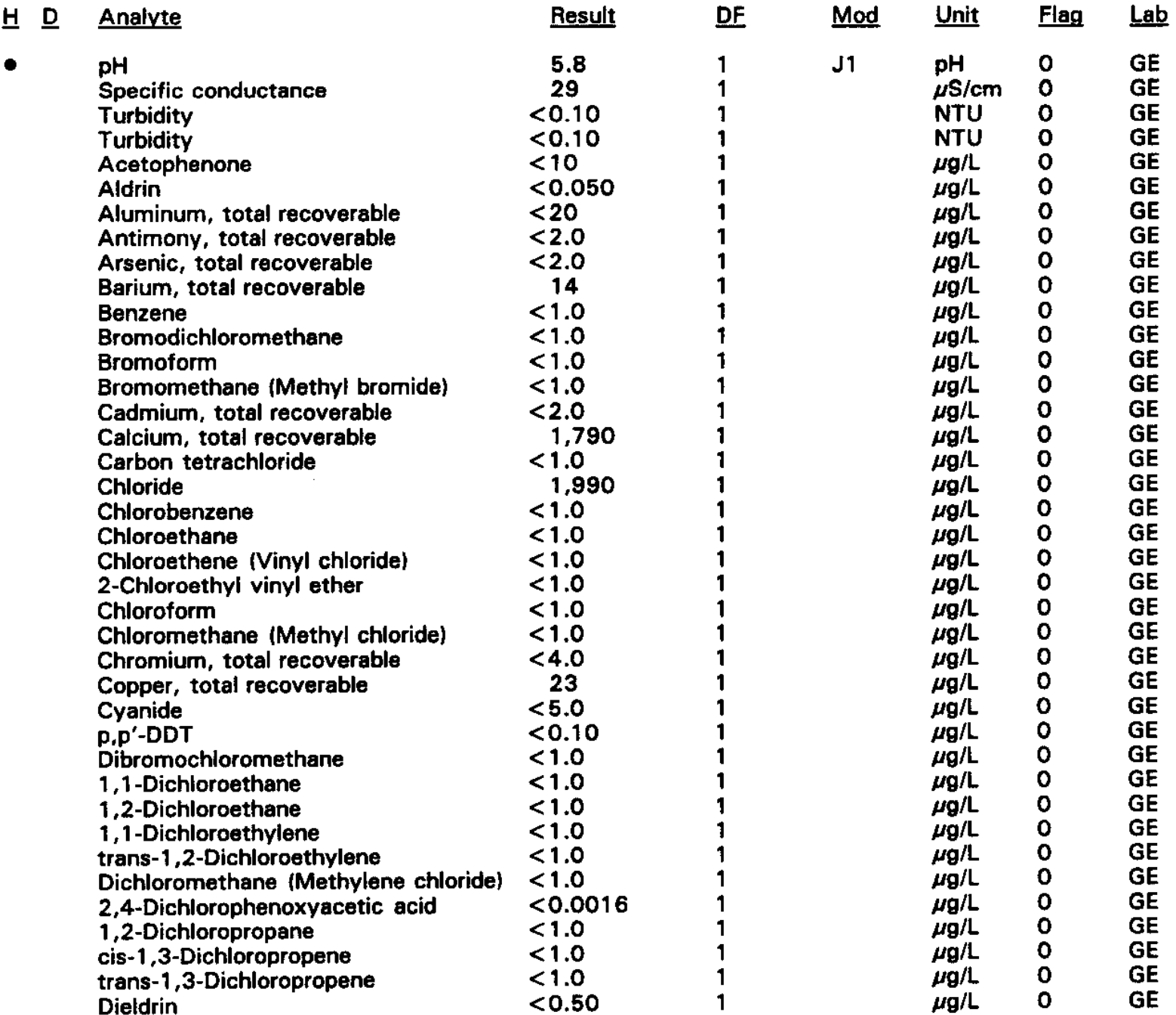

$\overline{-}=$ exceeded holding time. $\square=$ exceeded screening level or final primary drinking water standard. 
WELL BGX 3D collected on 04/13/94, laboratory analyses (cont.)

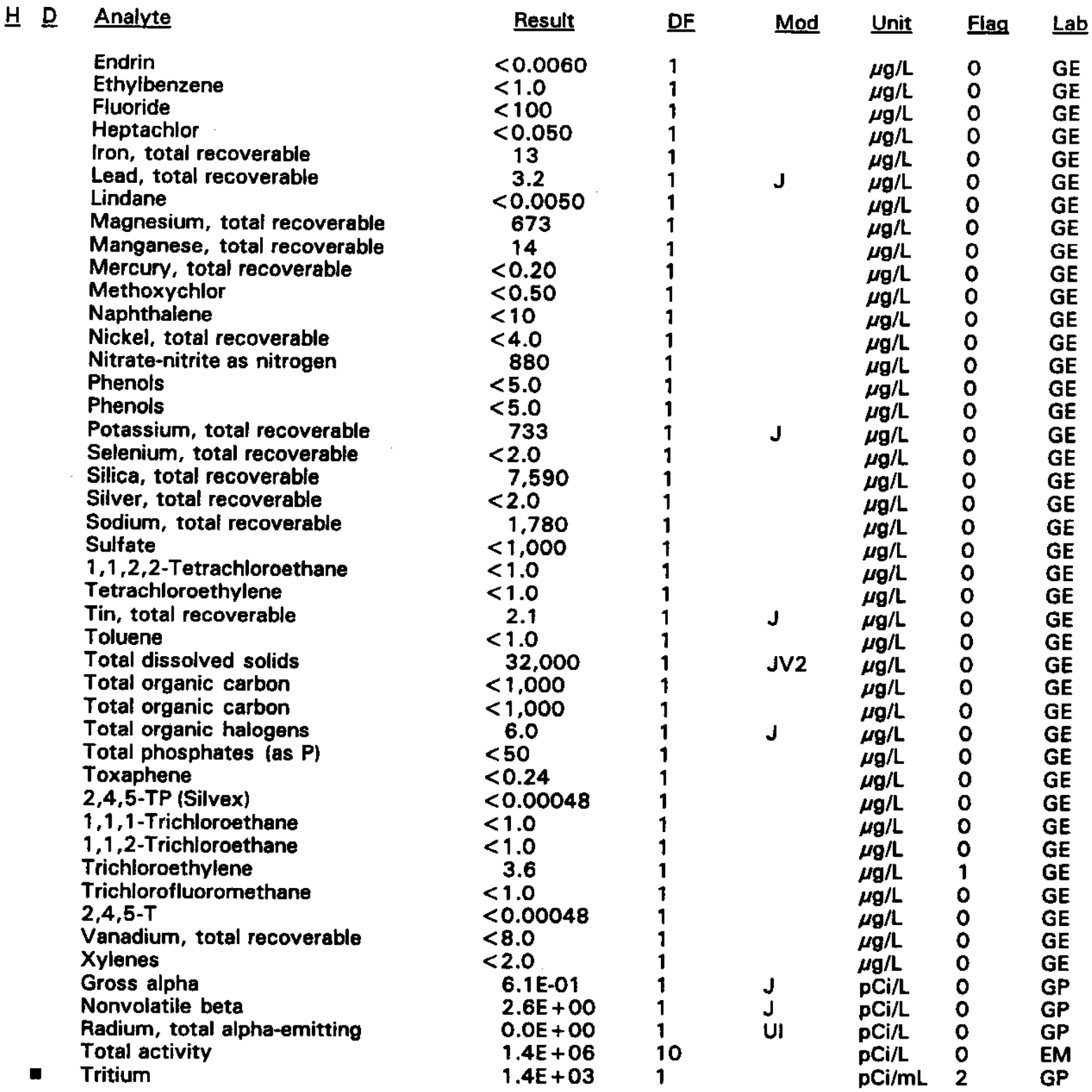

- exceeded holding time. = exceeded screening level or final primary drinking water standard. 


\section{WELL BGX 4A}

$\begin{array}{lllllll}\text { SRS Coord. } & \text { Lat/Longitude } & \text { Screen Zone Elevation } & \text { Top of Casing } & \text { Casing } & \text { Pump } & \text { Formation } \\ \text { N77879.2 } & 33.291373^{\circ} \mathrm{N} & 116.8-106.8 \mathrm{ft} \mathrm{msl} & 290.9 \mathrm{ft} \mathrm{msl} & \text { 4" PVC } & \text { S } & \text { U. Congaree (IIA) } \\ \text { E57215.6 } & 81.667419^{\circ} \mathrm{W} & \mathbf{8 1 . 6 7 4} & & \end{array}$

\section{FIELD MEASUREMENTS}

Sample date: 04/14/94

Depth to water: $135.81 \mathrm{ft}(41.40 \mathrm{~m})$ below TOC

Water elevation: $155.09 \mathrm{ft}(47.27 \mathrm{~m}) \mathrm{msl}$

Sp. conductance: $303 \mu \mathrm{S} / \mathrm{cm}$

Turbidity: 0.7 NTU

Water evacuated before sampling: $108 \mathrm{gal}$

\section{LABORATORY ANALYSES}

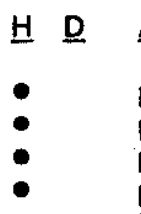

Analyte
pH
pH
pH
pH
Specific conductance
Specific conductance
Specific conductance
Specific conductance
Turbidity
Turbidity
Turbidity
Turbidity
Acetophenone
Acetophenone
Acetophenone
Acetophenone
Acetophenone
Aldrin

Aldrin

Aluminum, total recoverable

Aluminum, total recoverable

Aluminum, total recoverable

Aluminum, total recoverable

Aluminum, total recoverable

Antimony, total recoverable

Antimony, total recoverable

Antimony, total recoverable

Antimony, total recoverable

Antimony, total recoverable

Antimony, total recoverable

Arsenic, total recoverable

Arsenic, total recoverable

Arsenic, total recoverable

Arsenic, total recoverable

Arsenic, total recoverable

Arsenic, total recoverable

Barium, total recoverable

Barium, total recoverable

Barium, total recoverable
Time: 15:32

pH: 7.7

Alkalinity: $119 \mathrm{mg} / \mathrm{L}$

Water temperature: $20.0^{\circ} \mathrm{C}$

Volumes purged: 3.4 well volumes

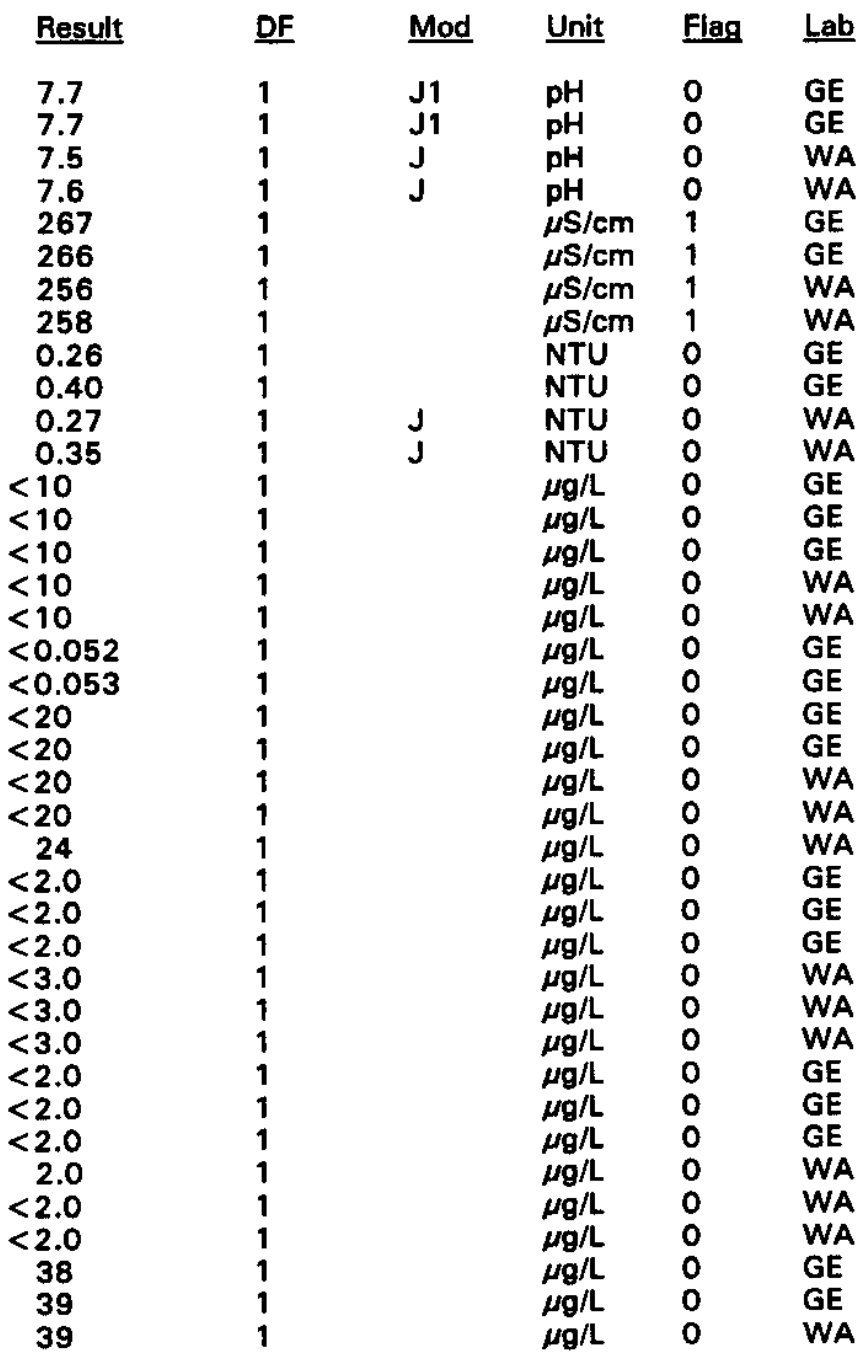

- = exceeded holding time. $\square=$ exceeded screening level or final primary drinking water standard. 
WELL BGX 4A collected on 04/14/94, laboratory analyses (cont.)

H $\underline{\text { Analyte }}$

Barium, total recoverable

Barium, total recoverable

Benzene

Benzene

Benzene

Benzene

Bromodichloromethane

Bromodichloromethane

Bromodichloromethane

Bromodichloromethane

Bromoform

Bromoform

Bromoform

Bromoform

Bromomethane (Methyl bromide)

Bromomethane (Methyl bromide)

Bromomethane (Methyl bromide)

Bromomethane (Methyl bromide)

Cadmium, total recoverable

Cadmium, total recoverable

Cadmium, total recoverable

Cadmium, total recoverable

Cadmium, total recoverable

Calcium, total recoverable

Calcium, total recoverable

Calcium, total recoverable

Calcium, total recoverable

Calcium, total recoverable

Carbon tetrachloride

Carbon tetrachloride

Carbon tetrachloride

Carbon tetrachloride

Chloride

Chloride

Chloride

Chloride

Chloride

Chlorobenzene

Chlorobenzene

Chlorobenzene

Chlorobenzene

Chloroethane

Chloroethane

Chloroethane

Chloroethane

Chloroethene (Vinyl chloride)

Chloroethene (Vinyl chloride)

Chloroethene (Vinyl chloride)

Chloroethene (Vinyl chloride)

2-Chloroethyl vinyl ether

2-Chloroethyl vinyl ether

2-Chloroethyl vinyl ether

2-Chloroethyl vinyl ether

Chloroform

Chloroform

Chloroform
Result DF

$$
40
$$$$
41
$$

$<1.0$

$<1.0$

$<5.0$

$<5.0$

$<1.0$

$<1.0$

$<5.0$

$<5.0$

$<1.0$

$<1.0$

$<5.0$

$<5.0$

$<1.0$

$<1.0$

$<10$

$<10$

$<2.0$

$<2.0$

$<2.0$

$<2.0$

$<2.0$

52,900

52,700

54,300

54,800

56,000

$<1.0$

$<1.0$

$<5.0$

$<5.0$

2,950

2,940

2,640

2,640

2,700

$<1.0$

$<1.0$

$<5.0$

$<5.0$

$<1.0$

$<1.0$

$<10$

$<10$

$<1.0$

$<1.0$

$<10$

$<10$

$<1.0$

$<1.0$

$<10$

$<10$

$<1.0$

$<1.0$

$<5.0$
Mod Unit $\underline{\text { Flag }}$

$\mu \mathrm{g} / \mathrm{L} \quad \mathrm{O}$ WA

$\mu g / L \quad 0 \quad$ WA

$\mu \mathrm{g} / \mathrm{L} \quad 0 \quad \mathrm{GE}$

$\mu g / L \quad$ WA

$\mu \mathrm{g} / \mathrm{L} \quad 0 \quad$ WA

$\mu g / L \quad 0 \quad$ GE

$\mu g / L \quad 0 \quad$ GE

$\mu g / L \quad 0 \quad$ WA

$\mu \mathrm{g} / \mathrm{L} \quad 0 \quad$ WA

$\mu g / L \quad 0 \quad$ GE

$\mu g / L \quad O \quad G E$

$\mu g / L \quad 0 \quad$ WA

$\mu g / L \quad 0 \quad$ WA

$\mu g / L \quad 0 \quad$ GE

$\mu g / L \quad 0 \quad$ GE

$\mu g / L \quad 0$ WA

$\mu g / L \quad 0 \quad$ WA

$\mu g / L \quad 0 \quad$ GE

$\mu g / L \quad 0 \quad$ GE

$\mu g / L \quad 0 \quad$ WA

$\mu g / L \quad 0 \quad$ WA

$\mu \mathrm{g} / \mathrm{L} \quad 0 \quad$ WA

$\mu \mathrm{g} / \mathrm{L} \quad \mathrm{O} \quad \mathrm{GE}$

$\mu g / L \quad 0 \quad$ GE

J3

$\mu g / L$

$\mu g / L$

$\mu g / L$

$\mu g / L$

$\mu g / L$

$\mu g / L$

$\mu g / L$

$\mu g / L$

$\mu g / L$

$\mu g / L$

$\mu g / L$

$\mu g / L$

$\mu \mathrm{g} / \mathrm{L}$

$\mu \mathrm{g} / \mathrm{L}$

$\mu g / L$

$\mu g / L$

$\mu g / L$

$\mu g / L$

$\mu g / L$

$\mu g / L$

$\mu g / L$

$\mu g / L$

$\mu g / L$

$\mu \mathrm{g} / \mathrm{L}$

$\mu g / L$

$\mu g / L$

$\mu g / L$

$\mu \mathrm{g} / \mathrm{L}$

$\mu g / L$

$\mu g / L$

$\mu g / L$

- = exceeded holding time. $\square=$ exceeded screening level or final primary drinking water standard. 
WELL BGX 4 A collected on $04 / 14 / 94$, laboratory analyses (cont.)

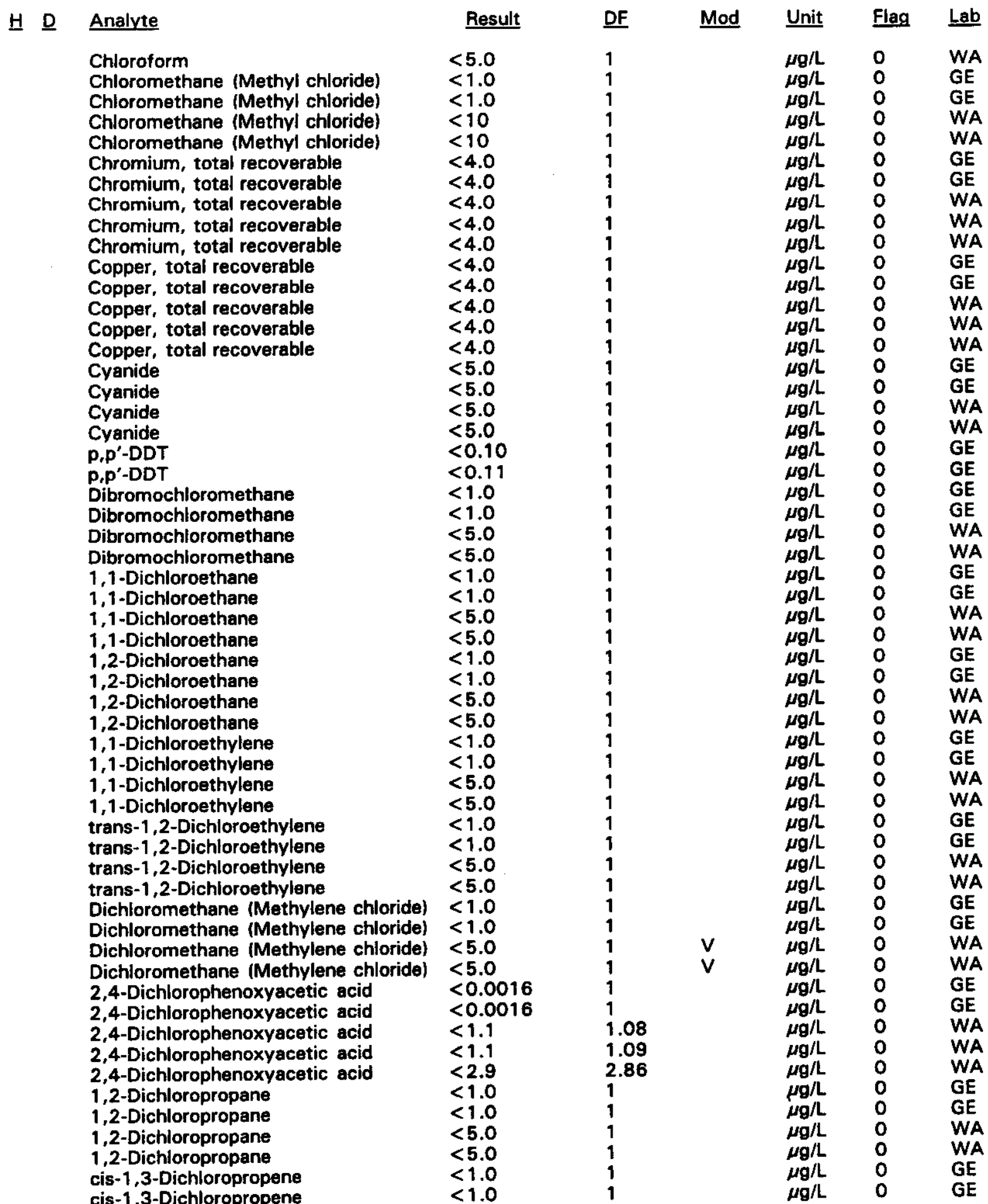

- exceeded holding time. $\square=$ exceeded screening level or final primary drinking water standard. 
WELL BGX 4A collected on 04/14/94, laboratory analyses (cont.)

H D Analyte

cis-1,3-Dichloropropene

cis-1,3-Dichloropropene

trans-1,3-Dichloropropene

trans-1,3-Dichloropropene

trans-1,3-Dichloropropene

trans-1,3-Dichloropropene

Dieldrin

Dieldrin

Endrin

Endrin

Endrin

Endrin

Endrin

Ethylbenzene

Ethylbenzene

Ethylbenzene

Ethylbenzene

Fluoride

Fluoride

Fluoride

Fluoride

Heptachlor

Heptachlor

Iron, total recoverable

Iron, total recoverable

Iron, total recoverable

Iron, total recoverable

Iron, total recoverable

Lead, total recoverable

Lead, total recoverable

Lead, total recoverable

Lead, total recoverable

Lead, total recoverable

Lead, total recoverable

Lindane

Lindane

Lindane

Lindane

Lindane

Magnesium, total recoverable

Magnesium, total recoverable

Magnesium, total recoverable

Magnesium, total recoverable

Magnesium, total recoverable

Manganese, total recoverable

Manganese, total recoverable

Manganese, total recoverable

Manganese, total recoverable

Manganese, total recoverable

Mercury, total recoverable

Mercury, total recoverable

Mercury, total recoverable

Mercury, total recoverable

Mercury, total recoverable

Methoxychlor

Methoxychlor
Result DF

$\begin{array}{ll}<5.0 & 1 \\ <5.0 & 1 \\ <1.0 & 1 \\ <1.0 & 1 \\ <5.0 & 1 \\ <5.0 & 1 \\ <0.52 & 1 \\ <0.53 & 1 \\ <0.0062 & 1 \\ <0.0064 & 1 \\ <0.11 & 1.09 \\ <0.11 & 1.09 \\ <0.22 & 2.17\end{array}$

$<1.0$

$<1.0$

$<5.0$

$<5.0$

$<100$

$<100$

$<100$

$<100$

$<0.052$

$<0.053$

16

16

18

18

21

$<3.0$

$<3.0$

$<3.0$

$<3.0$

$<3.0$

$<3.0$

$<0.0052$

$<0.0053$

$<0.055$

$<0.055$

$<0.11$

1,260

1,250

1,260

1,270

1,310

10.0

9.6

11

12

12

$<0.20$

$<0.20$

$<0.20$

$<0.20$

$<0.20$

$<0.52$

$<0.53$
Mod

$\underline{\text { Unit }}$ Flag Lab

$\mu \mathrm{g} / \mathrm{L} \quad 0 \quad$ WA

$\mu g / L \quad 0 \quad$ WA

$\mu g / L \quad 0 \quad G E$

$\begin{array}{lll}\mu g / L & 0 & \mathrm{GE} \\ \mu \mathrm{g} / \mathrm{L} & 0 & \mathrm{WA}\end{array}$

$\mu g / L \quad 0 \quad$ WA

$\mu g / L \quad 0 \quad$ GE

$\mu g / L \quad 0 \quad G E$

$\mu g / L \quad 0 \quad \mathrm{GE}$

$\mu g / L \quad 0 \quad G E$

$\mu g / L \quad 0 \quad W A$

$\mu g / L \quad 0 \quad$ WA

$\mu g / L \quad 0 \quad$ WA

$\mu g / L \quad 0 \quad G E$

$\mu \mathrm{g} / \mathrm{L} \quad 0 \quad \mathrm{GE}$

$\mu \mathrm{g} / \mathrm{L} \quad 0 \quad$ WA

$\mu g / L \quad 0 \quad$ WA

$\mu \mathrm{g} / \mathrm{L} \quad 0 \quad \mathrm{GE}$

$\mu \mathrm{g} / \mathrm{L} \quad \mathrm{O} \quad \mathrm{GE}$

$\mu \mathrm{g} / \mathrm{L} \quad 0 \quad$ WA

$\mu \mathrm{g} / \mathrm{L} \quad 0 \quad$ WA

$\mu \mathrm{g} / \mathrm{L} \quad 0 \quad \mathrm{GE}$

$\mu \mathrm{g} / \mathrm{L} \quad \mathrm{O} \quad \mathrm{GE}$

$\mu \mathrm{g} / \mathrm{L} \quad 0 \quad \mathrm{GE}$

$\mu \mathrm{g} / \mathrm{L} \quad \mathrm{O} \quad \mathrm{GE}$

$\mu g / L \quad 0 \quad$ WA

$\mu \mathrm{g} / \mathrm{L} \quad 0 \quad$ WA

$\mu \mathrm{g} / \mathrm{L} \quad \mathrm{O}$ WA

$\mu g / L \quad 0 \quad$ GE

$\mu \mathrm{g} / \mathrm{L} \quad 0 \quad \mathrm{GE}$

$\mu g / L \quad 0 \quad$ GE

$\mu g / L \quad 0 \quad W A$

$\mu \mathrm{g} / \mathrm{L} \quad 0 \quad$ WA

$\mu g / L \quad 0 \quad$ WA

$\mu g / L \quad 0 \quad$ GE

$\mu g / L \quad 0 \quad$ GE

$\mu g / L \quad 0 \quad$ WA

$\mu g / L \quad 0 \quad$ WA

$\mu g / L \quad 0 \quad$ WA

$\mu g / L \quad 0 \quad$ GE

$\mu g / L \quad 0 \quad \mathrm{GE}$

$\mu g / L \quad 0 \quad W A$

$\mu g / L \quad 0 \quad$ WA

$\mu g / L \quad 0 \quad W A$

$\mu \mathrm{g} / \mathrm{L} \quad 0 \quad \mathrm{GE}$

$\mu g / L \quad 0 \quad$ GE

$\mu \mathrm{g} / \mathrm{L} \quad 0 \quad$ WA

$\mu g / L \quad 0 \quad$ WA

$\mu g / L \quad 0 \quad$ WA

$\mu g / L \quad 0 \quad$ GE

$\mu g / L \quad 0 \quad G E$

$\mu \mathrm{g} / \mathrm{L} \quad \mathrm{O}$ GE

$\mu g / \mathrm{L} \quad 0 \quad$ WA

$\mu g / L \quad 0 \quad W A$

$\mu g / L \quad 0 \quad G E$

- = exceeded holding time. = exceeded screening level or final primary drinking water standard. 
WELL BGX 4A collected on 04/14/94, laboratory analyses (cont.)

\section{H $\underline{\text { Analvte }}$}

Methoxychlor

Methoxychlor

Methoxychlor

Methoxychior

Naphthalene

Naphthalene

Naphthalene

Nickel, total recoverable

Nickel, total recoverable

Nickel, total recoverable

Nickel, total recoverable

Nickel, total recoverable

Nitrate as nitrogen

Nitrate as nitrogen

Nitrate-nitrite as nitrogen

Nitrate-nitrite as nitrogen

Phenols

Phenols

Phenols

Phenols

Potassium, total recoverable

Potassium, total recoverable

Potassium, total recoverable

Potassium, total recoverable

Potassium, total recoverable

Selenium, total recoverable

Selenium, total recoverable

Selenium, total recoverable

Selenium, total recoverable

Selenium, total recoverable

Selenium, total recoverable

Silica, total recoverable

Silica, total recoverable

Silica, total recoverable

Silica, total recoverable

Silica, total recoverable

Silver, total recoverable

Silver, total recoverable

Silver, total recoverable

Silver, total recoverable

Silver, total recoverable

Sodium, total recoverable

Sodium, total recoverable

Sodium, total recoverable

Sodium, total recoverable

Sodium, total recoverable

Sulfate

Sulfate

Sulfate

Sulfate

$1,1,2,2$-Tetrachloroethane

1,1,2,2-Tetrachloroethane

$1,1,2,2$-Tetrachloroethane

$1,1,2,2$-Tetrachloroethane

Tetrachloroethylene

Tetrachloroethylene
Result

$<1.1$

$<1.1$

$<0.55$

$<0.55$

$<10$

$<10$

$<10$

$<4.0$

$<4.0$

$<4.0$

$<4.0$

$<4.0$

$<20$

$<20$

$<50$

$<50$

$<5.0$

$<5.0$

$<5.0$

$<5.0$

1,430

1,500

1,270

1,550

1,590

$<2.0$

$<2.0$

$<2.0$

$<2.0$

$<2.0$

$<2.0$

35,000

34,900

32,600

33,100

33,600

$<2.0$

$<2.0$

$<2.0$

$<2.0$

$<2.0$

2,420

2,420

2,510

2,540

2,550

8,160

8,110

$\mathbf{9 , 9 4 0}$

10,200

$<1.0$

$<1.0$

$<5.0$

$<5.0$

$<1.0$

$<1.0$

\section{DE}

2.17

2.17

1.09

1.09

1

1

1

1

1

1

1

1

1

1

1

1
1

1

1

1

1

1

1

1

1

1

1

1

1

1

2.1

2.1
2.1

1

1

1

1

1

1

1

1

1

1

4
4
1

1

1
1
1

1

1
Mod Unit Flag Lab

$\mu g / L \quad 0 \quad W A$

$\mu g / L \quad O \quad$ WA

$\mu g / L$ WA

$\mu g / L \quad O \quad G E$

$\mu g / L \quad O \quad G E$

$\mu g / h \quad 0 \quad G E$

$\mu g / L \quad O \quad G E$

$\mu g / L \quad O \quad G E$

$\mu g / L$ WA

$\mu g / L$ WA

$\mu g / L \quad 0 \quad W A$

$\mu g / L \quad 0 \quad W A$

$\mu g / L$ WA

$\mu g / L \quad 0 \quad G E$

$\mu g / L \quad O \quad G E$

$\mu g / L \quad O \quad G E$

$\mu g / L \quad O \quad G E$

$\mu g / L$ WA

$\mu g / L$ WA

$\mu g / L \quad 0 \quad$ GE

$\mu g / L \quad O \quad G E$

$\mu g / L \quad 0 \quad W A$

$\mu g / L$ WA

$\mu g / L$ WA

J3 $\mu g / L \quad \circ \quad$ GE

J3 $\mu g / L$ L $\quad$ GE

$\mu g / L$ WA

$\mu g / L$ WA

$\mu g / L$ WA

$\mu g / L \quad 0 \quad$ GE

$\mu g / L \quad 0 \quad G E$

$\mu \mathrm{g} / \mathrm{L} \quad \mathrm{O}$ WA

J3 $\quad \underset{\mu g / L}{\mu} \quad 0 \quad$ WA

J3 $\mu \mathrm{g} / \mathrm{L} \quad \mathrm{O}$ WA

$\mu g / L \quad O \quad G E$

$\mu g / L \quad O \quad G E$

$\mu g / L$ WA

$\mu g / L$ WA

$\mu g / L$ WA

$\mu g / L \quad 0 \quad$ GE

$\mu g / L \quad O \quad G E$

$\mu g / L$ WA

$\mu g / L$ WA

$\mu g / L$ WA

$\mu g / L \quad 0 \quad G E$

$\mu g / L \quad O \quad G E$

$\mu g / L$ WA

$\mu g / \mathrm{L}$ O WA

$\mu g / L \quad 0 \quad \mathrm{GE}$

$\boldsymbol{\mu} \boldsymbol{g / L} \quad \mathrm{O}$ GE

$\mu \mathrm{g} / \mathrm{L}$

$\mu g / L$ WA

$\mu g / L \quad 0 \quad G E$

$\mu g / L \quad 0 \quad$ GE

- = exceeded holding time. = exceeded screening level or final primary drinking water standard. 
WELL BGX 4A collected on 04/14/94, laboratory analyses (cont.)

브

Result

Tetrachloroethylene

Tetrachloroethylene

Tin, total recoverable

Tin, total recoverable

Tin, total recoverable

Tin, total recoverable

Tin, total recoverable

Toluene

Toluene

Toluene

Toluene

Total dissolved solids

Total dissolved solids

Total dissolved solids

Total dissolved solids

Total organic carbon

Total organic carbon

Total organic carbon

Total organic carbon

Total organic carbon

Total organic halogens

Total organic halogens

Total organic halogens

Total organic halogens

Total phosphates (as P)

Total phosphates (as P)

Total phosphates (as P)

Total phosphates (as P)

Total phosphates (as P)

Toxaphene

Toxaphene

Toxaphene

Toxaphene

Toxaphene

Toxaphene

2,4,5-TP (Silvex)

2,4,5-TP (Silvex)

2,4,5-TP (Silvex)

2,4,5-TP (Silvex)

2,4,5-TP (Silvex)

$1,1,1$-Trichloroethane

$1,1,1$-Trichloroethane

$1,1,1$-Trichloroethane

1,1,1-Trichloroethane

1,1,2-Trichloroethane

1,1,2-Trichloroethane

1,1,2-Trichloroethane

1,1,2-Trichloroethane

Trichloroethylene

Trichloroethylene

Trichloroethylene

Trichloroethylene

Trichlorofluoromethane

Trichlorofluoromethane

Trichlorofluoromethane

Trichiorofluoromethane
$<5.0$

$<5.0$

$<2.0$

$<2.0$

$<17$

$<17$

$<17$

$<1.0$

$<1.0$

$<5.0$

$<5.0$

188,000

211,000

179,000

186,000

1,010

1,260

$<1,000$

$<1,000$

$<1,000$

$<5.0$

$<5.0$

$<5.0$

13

$<50$

$<50$

$<50$

$<50$

$<50$

$<0.25$

$<0.26$

$<1.1$

$<1.1$

$<2.2$

$<2.2$

$<0.00046$

$<0.00046$

$<1.4$

$<0.54$

$<0.55$

$<1.0$

$<1.0$

$<5.0$

$<5.0$

$<1.0$

$<1.0$

$<5.0$

$<5.0$

$<1.0$

$<1.0$

$<5.0$

$<5.0$

$<1.0$

$<1.0$

$<5.0$

$<5.0$
DF

Mod

$\underline{\mu n i t}$

$\mu \mathrm{g} / \mathrm{L}$

$\mu g / L$

$\mu g / L$

$\mu \mathrm{g} / \mathrm{L}$

$\mu \mathrm{g} / \mathrm{L}$

$\mu g / L$

$\mu g / L$

$\mu g / L$

$\mu g / L$

JV2

$\mu g / L$

$\mu g / L$

$\mu \mathrm{g} / \mathrm{L}$

$\mu g / L$

$\mu g / L$

$\mu \mathrm{g} / \mathrm{L}$

$\mu \mathrm{g} / \mathrm{L}$

$\mu \mathrm{g} / \mathrm{L}$

$\mu \mathrm{g} / \mathrm{L}$

$\mu g / L$

$\mu \mathrm{g} / \mathrm{L}$

$\mu g / L$

$\mu \mathrm{g} / \mathrm{L}$

$\mu g / L$

$\mu g / L$

$\mu \mathrm{g} / \mathrm{L}$

$\mu g / L$

$\mu g / L$

$\mu g / L$

$\mu g / L$

$\mu \mathrm{g} / \mathrm{L}$

$\mu \mathrm{g} / \mathrm{L}$

$\mu g / L$

$\mu \mathrm{g} / \mathrm{L}$

$\mu g / L$

$\mu \mathrm{g} / \mathrm{L}$

$\mu g / L$

$\mu \mathrm{g} / \mathrm{L}$

$\mu g / L$

$\mu g / L$

$\mu \mathrm{g} / \mathrm{L}$

$\mu \mathrm{g} / \mathrm{L}$

$\mu g / L$

$\mu g / L$

$\mu g / L$

$\mu g / L$

$\mu g / L$

$\mu g / L$

$\mu g / L$

$\mu \mathrm{g} / \mathrm{L}$

$\mu g / L$

$\mu g / L$

$\mu g / L$

$\mu \mathrm{g} / \mathrm{L}$
Flag Lab

$\begin{array}{ll}0 & \text { WA } \\ 0 & \text { WA } \\ 0 & \text { GE } \\ 0 & \text { GE } \\ 0 & \text { WA } \\ 0 & \text { WA } \\ 0 & \text { WA } \\ 0 & \text { GE } \\ 0 & \text { GE }\end{array}$

WA

WA

GE

GE

WA

WA

GE

GE

WA

WA

WA

GE

GE

WA

WA

GE

GE

WA

WA

WA

GE

GE

WA

WA

WA

WA

GE

GE

WA

WA

WA

GE

GE

WA

WA

GE

GE

WA

WA

GE

GE

WA

WA

GE

GE

WA

WA

$\overline{-~=~ e x c e e d e d ~ h o l d i n g ~ t i m e . ~} \quad$ = exceeded screening level or final primary drinking water standard. 
WELL BGX $4 A$ collected on $04 / 14 / 94$, laboratory analyses (cont.)

H $\underline{\text { Analyte }}$

$2,4,5-\mathrm{T}$

2,4,5-T

Vanadium, total recoverable

Vanadium, total recoverable

Vanadium, total recoverable

Vanadium, total recoverable

Vanadium, total recoverable

Xylenes

Xylenes

Xylenes

Xylenes

Gross alpha

Gross alpha

Gross alpha

Gross alpha

Nonvolatile beta

Nonvolatile beta

Nonvolatile beta

Nonvolatile beta

Radium-226

Radium-226

Radium-226

Radium-228

Radium-228

Radium-228

Radium, total alpha-emitting

Radium, total alpha-emitting

Tritium

Tritium

Tritium

Tritium

Tritium
Result DF

$<0.00046$

$<0.00046$

$<8.0$

$<8.0$

$<3.0$

$<3.0$

$<3.0$

$<2.0$

$<2.0$

$<5.0$

$<5.0$

4.8E-01

5.8E-01

$<4.9 \mathrm{E}-01$

$<3.5 \mathrm{E}-01$

$1.4 \mathrm{E}+00$

7.1E-01

5.1E + 00

3.1E+O0

2.1E-01

2.0E-01

1.8E-01

$1.0 E+00$

$1.5 E+00$

5.OE-01

$-1.0 E-01$

$0.0 E+00$

2.8E-01

9.7E-02

2.8E-01

2.3E-01

2.8E-01

Mod

Unit Flag Lab

$\mu \mathrm{g} / \mathrm{L} \quad \mathrm{O} \quad \mathrm{GE}$

$\mu g / L \quad 0 \quad$ GE

$\mu g / L \quad 0 \quad \mathrm{GE}$

$\mu \mathrm{g} / \mathrm{L} \quad \mathrm{O} \quad \mathrm{GE}$

$\mu g / L \quad 0 \quad$ WA

$\mu g / L \quad 0 \quad$ WA

$\mu \mathrm{g} / \mathrm{L} \quad \mathrm{O}$ WA

$\mu g / L \quad 0 \quad$ GE

$\mu \mathrm{g} / \mathrm{L} \quad \mathrm{O} \quad \mathrm{GE}$

$\mu g / L \quad 0 \quad$ WA

$\mu g / L \quad 0 \quad$ WA

$\begin{array}{llll}\text { UI } & \mathrm{pCi} / \mathrm{L} & 0 & \mathrm{GP} \\ \text { UI } & \mathrm{pCi} / \mathrm{L} & 0 & \mathrm{GP}\end{array}$

pCi/L $\quad 0 \quad$ TM

$\mathrm{pCi} / \mathrm{L} \quad 0 \quad \mathrm{TM}$

J $\quad \mathrm{pCi} / \mathrm{L} \quad 0 \quad \mathrm{GP}$

UI $\mathrm{PCi} / \mathrm{L} \quad 0 \quad \mathrm{GP}$

pCi/L $\quad 0 \quad T M$

pCi/L 00 TM

$\mathrm{pCi} / \mathrm{L} \quad 0 \quad \mathrm{TM}$

pCi/L 0 TM

PCi/L $\quad 0 \quad T M$

$\mathrm{pCi} / \mathrm{L} \quad 0 \quad \mathrm{TM}$

pCi/L 0 TM

UI $\quad \mathrm{pCi} / \mathrm{L} \quad 0 \quad \mathrm{TM}$

UI $\mathrm{pCi} / \mathrm{L} \quad 0 \quad \mathrm{GP}$

UI $\quad \mathrm{pCi} / \mathrm{L} \quad 0 \quad \mathrm{GP}$

UI $\quad \mathrm{pCi} / \mathrm{mL} \quad 0 \quad \mathrm{GP}$

UI $\quad \mathrm{pCi} / \mathrm{mL} \quad 0 \quad G P$

$\mathrm{pCi} / \mathrm{mL} \quad 0 \quad \mathrm{TM}$

UI $\quad \mathrm{pCi} / \mathrm{mL} \quad 0 \quad T M$

$\mathrm{pCi} / \mathrm{mL} \quad 0 \quad T M$

\section{WELL BGX 4C}

\begin{tabular}{|c|c|c|c|c|c|c|}
\hline SRS Coord. & Lat/Longitude & Screen Zone Elevation & Top of Casing & Casing & Pump & Formation \\
\hline $\begin{array}{l}\text { N77886.2 } \\
\text { E57202.2 }\end{array}$ & $\begin{array}{l}33.291367^{\circ} \mathrm{N} \\
81.667468^{\circ} \mathrm{W}\end{array}$ & $180.7-170.7 \mathrm{ft} \mathrm{msl}$ & $290.8 \mathrm{ft} \mathrm{msl}$ & ve & $\mathbf{s}$ & \\
\hline
\end{tabular}

\section{FIELD MEASUREMENTS}

Sample date: 04/13/94

Depth to water: $76.14 \mathrm{ft}(23.21 \mathrm{~m})$ below TOC

Water elevation: $214.66 \mathrm{ft}(65.43 \mathrm{~m}) \mathrm{ms}$ !

Sp. conductance: $84 \mu \mathrm{S} / \mathrm{cm}$

Turbidity: 0.7 NTU

Water evacuated before sampling: $191 \mathrm{gal}$

\section{LABORATORY ANALYSES}

H D Analyte

- $\quad \mathrm{pH}$

Specific conductance
Time: 14:33

pH: 6.3

Alkalinity: $24 \mathrm{mg} / \mathrm{L}$

Water temperature: $19.4^{\circ} \mathrm{C}$

Volumes purged: 6.6 well volumes

A


WELL BGX 4C collected on $04 / 13 / 94$, laboratory analyses (cont.)

H D Analyte

Turbidity

Acetophenone

Aldrin

Aluminum, total recoverable

Antimony, total recoverable

Arsenic, total recoverable

Barium, total recoverable

Benzene

Bromodichloromethane

Bromoform

Bromomethane (Methyl bromide)

Cadmium, total recoverable

Calcium, total recoverable

Carbon tetrachloride

Chloride

Chlorobenzene

Chioroethane

Chloroethene (Vinyl chloride)

2-Chloroethyl vinyl ether

Chloroform

Chloromethane (Methyl chloride)

Chromium, total recoverable

Copper, total recoverable

Cyanide

Cyanide

p,p'-DDT

Dibromochloromethane

1,1-Dichloroethane

1,2-Dichloroethane

1,1-Dichloroethylene

trans-1,2-Dichloroethylene

Dichloromethane (Methylene chloride)

2,4-Dichlorophenoxyacetic acid

1,2-Dichloropropane

cis-1,3-Dichloropropene

trans-1,3-Dichloropropene

Dieldrin

Endrin

Ethylbenzene

Fluoride

Heptachlor

Iron, total recoverable

Lead, total recoverable

Lindane

Magnesium, total recoverable

Manganese, total recoverable

Mercury, total recoverable

Methoxychlor

Naphthalene

Nickel, total recoverable

Nitrate-nitrite as nitrogen

Phenols

Potassium, total recoverable

Selenium, total recoverable

Silica, total recoverable

Silver, total recoverable

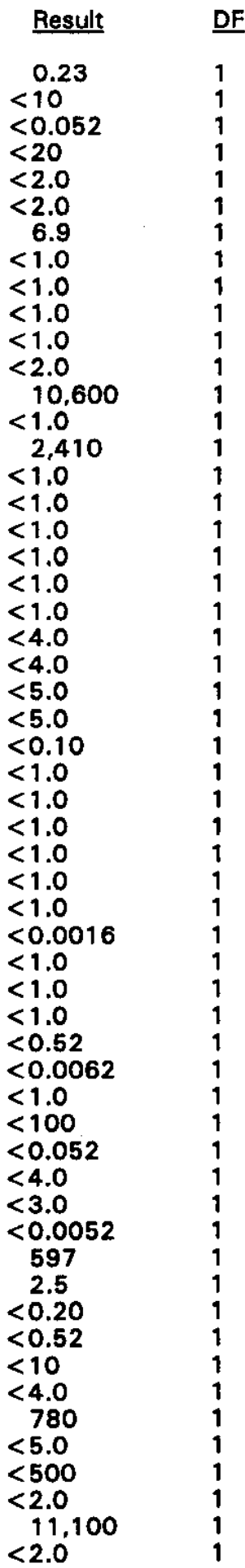

Mod

Unit Flag Lab

NTU $0 \quad$ GE

$\mu g / L \quad 0 \quad G E$

$\mu g / L \quad 0 \quad G E$

$\mu g / L \quad 0 \quad G E$

$\mu g / L \quad 0 \quad$ GE

$\mu \mathrm{g} / \mathrm{L} \quad 0 \quad \mathrm{GE}$

$\mu \mathrm{g} / \mathrm{L} \quad \mathrm{O} \quad \mathrm{GE}$

$\mu g / L \quad 0 \quad$ GE

$\mu g / L \quad 0 \quad$ GE

$\mu g / L \quad 0 \quad$ GE

$\mu g / L \quad 0 \quad$ GE

$\mu g / L \quad 0 \quad$ GE

$\mu g / L \quad 0 \quad G E$

$\mu g / L \quad 0 \quad$ GE

$\mu g / L \quad 0 \quad$ GE

$\mu g / L \quad 0 \quad$ GE

$\mu \mathrm{g} / \mathrm{L} \quad \mathrm{O} \quad \mathrm{GE}$

$\mu g / L \quad 0 \quad$ GE

$\mu g / L \quad 0 \quad$ GE

$\mu g / L \quad 0 \quad$ GE

$\mu \mathrm{g} / \mathrm{L} \quad 0 \quad \mathrm{GE}$

$\mu g / L \quad 0 \quad$ GE

$\mu g / L \quad O \quad G E$

$\mu g / L \quad 0 \quad G E$

$\mu g / L \quad 0 \quad G E$

$\mu g / L \quad 0 \quad G E$

$\mu \mathrm{g} / \mathrm{L} \quad \mathrm{O} \quad \mathrm{GE}$

$\mu \mathrm{g} / \mathrm{L} \quad 0 \quad \mathrm{GE}$

$\mu g / L \quad 0 \quad G E$

$\mu \mathrm{g} / \mathrm{L} \quad \mathrm{O} \quad \mathrm{GE}$

$\mu \mathrm{g} / \mathrm{L} \quad \mathrm{O} \quad \mathrm{GE}$

$\mu g / L \quad 0 \quad$ GE

$\mu g / L \quad 0 \quad$ GE

$\mu g / L \quad 0 \quad G E$

$\mu g / L \quad 0 \quad$ GE

$\mu g / L \quad 0 \quad$ GE

$\mu g / L \quad 0 \quad$ GE

$\mu g / L \quad 0 \quad \mathrm{GE}$

$\mu g / L \quad 0 \quad$ GE

$\mu g / L \quad 0 \quad$ GE

$\mu g / L \quad 0 \quad G E$

$\mu \mathrm{g} / \mathrm{L} \quad \mathrm{O} \quad \mathrm{GE}$

$\mu \mathrm{g} / \mathrm{L} \quad \mathrm{O} \quad \mathrm{GE}$

$\mu g / L \quad 0 \quad$ GE

$\mu g / L \quad 0 \quad$ GE

$\mu g / L \quad 0 \quad$ GE

$\mu \mathrm{g} / \mathrm{L} \quad 0 \quad \mathrm{GE}$

$\mu g / L \quad 0 \quad$ GE

$\mu g / L \quad 0 \quad G E$

$\mu \mathrm{g} / \mathrm{L} \quad \mathrm{O} \quad \mathrm{GE}$

$\mu g / L \quad 0 \quad G E$

$\mu \mathrm{g} / \mathrm{L} \quad \mathrm{O} \quad \mathrm{GE}$

$\mu g / L \quad 0 \quad G E$

$\mu g / L \quad 0 \quad$ GE

$\mu \mathrm{g} / \mathrm{L} \quad \mathrm{O} \quad \mathrm{GE}$

$\mu \mathrm{g} / \mathrm{L} \quad 0 \quad \mathrm{GE}$

- = exceeded holding time. = exceeded screening level or final primary drinking water standard. 
WELL BGX 4C collected on 04/13/94, laboratory analyses (cont.)

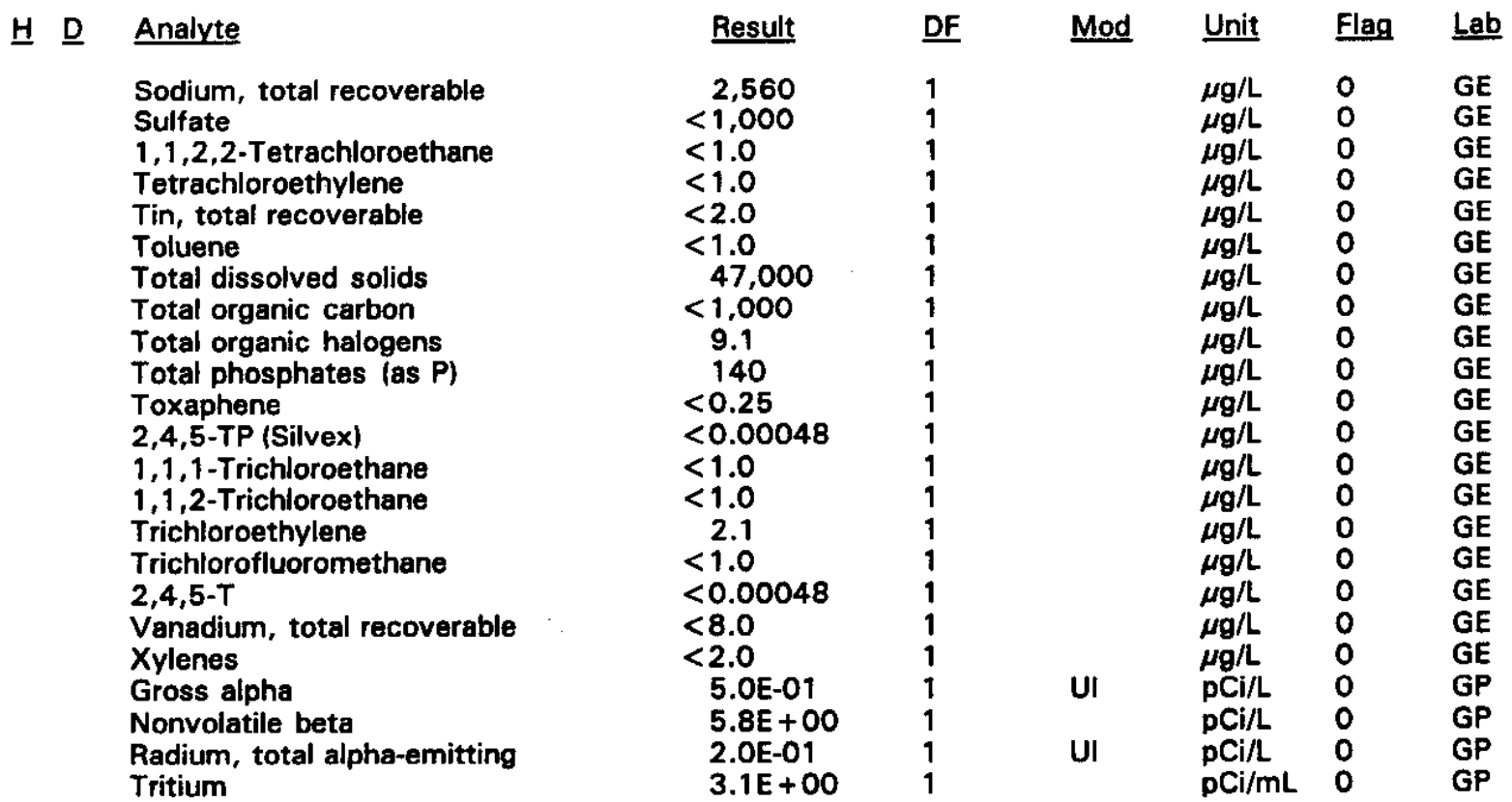

\section{WELL BGX 4D}

\begin{tabular}{|c|c|c|c|c|c|c|}
\hline SRS Coord. & Lat/Longitude & Screen Zone Elevation & Top of Casing & Casing & Pump & Formation \\
\hline $\begin{array}{l}\text { N77893.9 } \\
\text { E57186.2 }\end{array}$ & $\begin{array}{l}33.291358^{\circ} \mathrm{N} \\
81.667525^{\circ} \mathrm{W}\end{array}$ & $223.8-203.8 \mathrm{ft} \mathrm{msl}$ & $290.9 \mathrm{ft} \mathrm{msl}$ & 4" PVC & $\mathbf{S}$ & Barnwell (IIB $\left.B_{1}\right)$ \\
\hline
\end{tabular}

\section{FIELD MEASUREMENTS}

Sample date: 04/13/94

Depth to water: $75.00 \mathrm{ft}(22.86 \mathrm{~m})$ below TOC

Water elevation: $215.90 \mathrm{ft}(65.81 \mathrm{~m}) \mathrm{ms}$ !

Sp. conductance: $45 \mu \mathrm{S} / \mathrm{cm}$

Turbidity: 1.2 NTU

Water evacuated before sampling: $32 \mathrm{gal}$
Time: $14: 13$

pH: 5.7

Alkalinity: $5 \mathrm{mg} / \mathrm{L}$

Water temperature: $19.8^{\circ} \mathrm{C}$

Volumes purged: 4.0 well volumes

\section{LABORATORY ANALYSES}

H D Analyte

$\mathrm{pH}$

Specific conductance

Specific conductance

Turbidity

Acetophenone

Aldrin

Aluminum, total recoverable

Antimony, total recoverable

Antimony, total recoverable

Arsenic, total recoverable

Arsenic, total recoverable

\begin{tabular}{l}
$\quad$ Result \\
\hline 6.4 \\
55 \\
55 \\
0.32 \\
$<10$ \\
$<0.052$ \\
23 \\
$<2.0$ \\
$<2.0$ \\
$<2.0$ \\
$<2.0$
\end{tabular}

\begin{tabular}{llll} 
Mod & Unit & Flag & Lab \\
\cline { 3 - 4 } $\mathrm{J1}$ & & & \\
& $\mathrm{pH}$ & 0 & $\mathrm{GE}$ \\
& $\mu \mathrm{S} / \mathrm{cm}$ & 0 & $\mathrm{GE}$ \\
& $\mu \mathrm{S} / \mathrm{cm}$ & 0 & $\mathrm{GE}$ \\
$\mathrm{NTU}$ & 0 & $\mathrm{GE}$ \\
& $\mu \mathrm{g} / \mathrm{L}$ & 0 & $\mathrm{GE}$ \\
& $\mu \mathrm{g} / \mathrm{L}$ & 0 & $\mathrm{GE}$ \\
& $\mu \mathrm{g} / \mathrm{L}$ & 0 & $\mathrm{GE}$ \\
$\mathrm{J}$ & $\mu \mathrm{g} / \mathrm{L}$ & 0 & $\mathrm{GE}$ \\
& $\mu \mathrm{g} / \mathrm{L}$ & 0 & $\mathrm{GE}$ \\
& $\mu \mathrm{g} / \mathrm{L}$ & 0 & $\mathrm{GE}$ \\
& $\mu \mathrm{g} / \mathrm{L}$ & 0 & $\mathrm{GE}$
\end{tabular}

- exceeded holding time. = exceeded screening level or final primary drinking water standard. 
WELL BGX 4D collected on $04 / 13 / 94$, laboratory analyses (cont.)

H

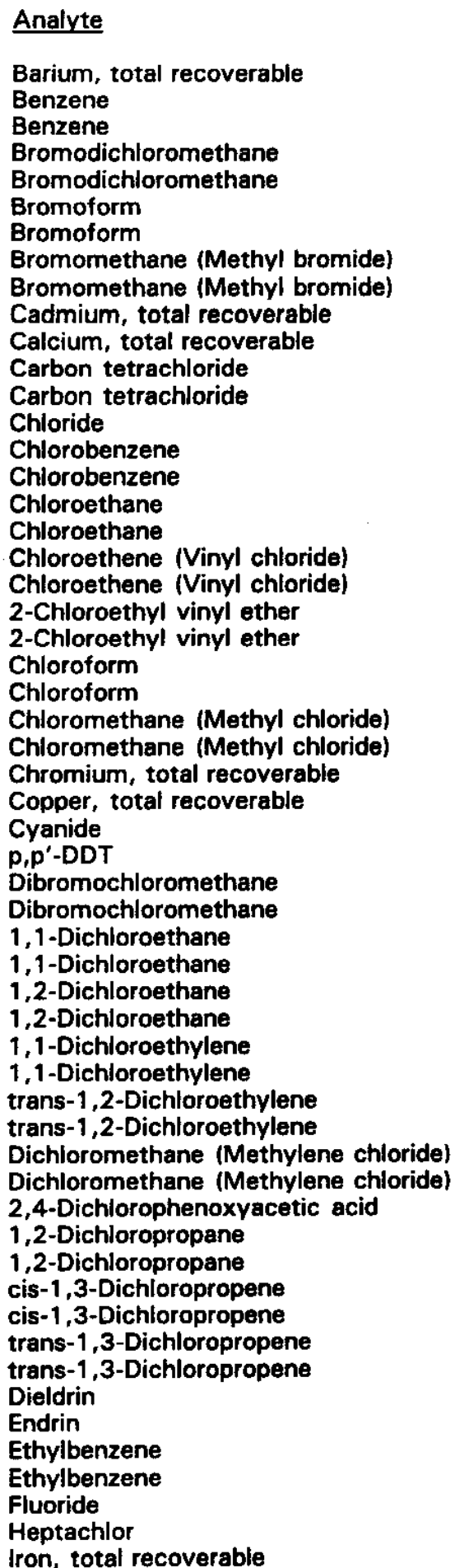

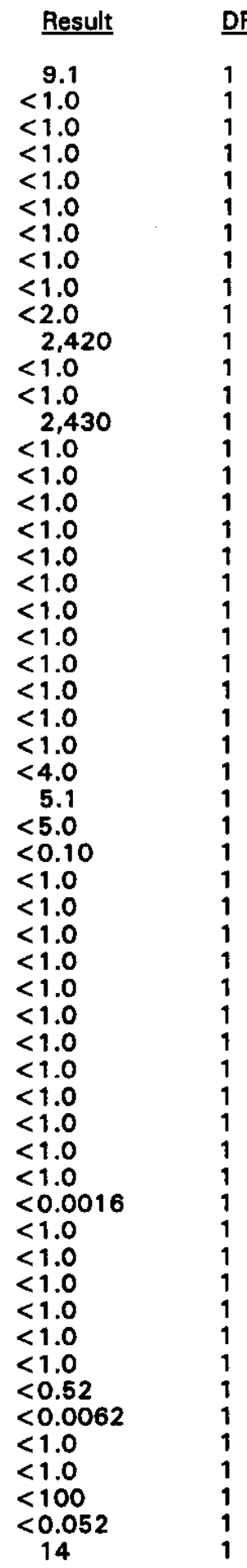

Mod Unit Flag Lab

$\mu g / L \quad 0 \quad$ GE

$\mu \mathrm{g} / \mathrm{L} \quad 0 \quad \mathrm{GE}$

$\mu g / L \quad 0 \quad$ GE

$\mu \mathrm{g} / \mathrm{L} \quad 0 \quad \mathrm{GE}$

$\mu g / L \quad 0 \quad$ GE

$\mu \mathrm{g} / \mathrm{L} \quad 0 \quad \mathrm{GE}$

$\mu \mathrm{g} / \mathrm{L} \quad \mathrm{O} \quad \mathrm{GE}$

$\mu \mathrm{g} / \mathrm{L} \quad 0 \quad \mathrm{GE}$

$\mu \mathrm{g} / \mathrm{L} \quad \mathrm{O} \quad \mathrm{GE}$

$\mu g / L \quad 0 \quad$ GE

$\mu \mathrm{g} / \mathrm{L} \quad 0 \quad \mathrm{GE}$

$\mu g / L \quad 0 \quad G E$

$\mu g / L \quad 0 \quad \mathrm{GE}$

$\mu g / L \quad 0 \quad G E$

$\mu g / L \quad 0 \quad$ GE

$\mu \mathrm{g} / \mathrm{L} \quad \mathrm{O} \quad \mathrm{GE}$

$\mu g / L \quad 0 \quad$ GE

$\mu g / L \quad 0 \quad$ GE

$\mu g / L \quad 0 \quad$ GE

$\mu \mathrm{g} / \mathrm{L} \quad 0 \quad \mathrm{GE}$

$\mu \mathrm{g} / \mathrm{L} \quad 0 \quad \mathrm{GE}$

$\mu g / L \quad 0 \quad$ GE

$\mu g / L \quad 0 \quad$ GE

$\mu g / L \quad 0 \quad G E$

$\mu g / L \quad 0 \quad$ GE

$\mu g / L \quad O \quad G E$

$\mu \mathrm{g} / \mathrm{L} \quad 0 \quad \mathrm{GE}$

$\mu \mathrm{g} / \mathrm{L} \quad \mathrm{O} \quad \mathrm{GE}$

$\mu g / L \quad 0 \quad$ GE

$\mu \mathrm{g} / \mathrm{L} \quad \mathrm{O} \quad \mathrm{GE}$

$\mu g / L \quad 0 \quad$ GE

$\mu g / L \quad O \quad G E$

$\mu g / L \quad 0 \quad G E$

$\mu g / L \quad O \quad G E$

$\mu g / L \quad 0 \quad G E$

$\mu g / L \quad 0 \quad G E$

$\mu g / L \quad 0 \quad G E$

$\mu \mathrm{g} / \mathrm{L} \quad 0 \quad \mathrm{GE}$

$\mu \mathrm{g} / \mathrm{L} \quad 0 \quad \mathrm{GE}$

$\mu g / L \quad 0 \quad$ GE

$\mu g / L \quad 0 \quad$ GE

$\mu \mathrm{g} / \mathrm{L} \quad 0 \quad \mathrm{GE}$

$\mu \mathrm{g} / \mathrm{L} \quad 0 \quad \mathrm{GE}$

$\mu \mathrm{g} / \mathrm{L} \quad 0 \quad \mathrm{GE}$

$\mu g / L \quad 0 \quad G E$

$\mu g / L \quad 0 \quad$ GE

$\mu g / L \quad 0 \quad$ GE

$\mu g / L \quad 0 \quad$ GE

$\mu \mathrm{g} / \mathrm{L} \quad 0 \quad \mathrm{GE}$

$\mu g / L \quad 0 \quad$ GE

$\mu g / L \quad 0 \quad G E$

$\mu \mathrm{g} / \mathrm{L} \quad 0 \quad \mathrm{GE}$

$\mu \mathrm{g} / \mathrm{L} \quad 0 \quad \mathrm{GE}$

$\mu \mathrm{g} / \mathrm{L} \quad 0 \quad \mathrm{GE}$

$\mu \mathrm{g} / \mathrm{L} \quad 0 \quad \mathrm{GE}$

$\mu \mathrm{g} / \mathrm{L} \quad \mathrm{O} \quad \mathrm{GE}$

- = exceeded holding time. = exceeded screening level or final primary drinking water standard. 
WELL BGX 4D collected on 04/13/94, laboratory analyses (cont.)

\section{H D Analyte}

Lead, total recoverable

Lead, total recoverable

Lindane

Magnesium, total recoverable

Manganese, total recoverable

Mercury, total recoverable

Methoxychlor

Naphthalene

Nickel, total recoverable

Nitrate-nitrite as nitrogen

Phenols

Potassium, total recoverable

Selenium, total recoverable

Selenium, total recoverable

Silica, total recoverable

Silver, total recoverable

Sodium, total recoverable

Sulfate

1,1,2,2-Tetrachloroethane

$1,1,2,2$-Tetrachloroethane

Tetrachloroethylene

Tetrachloroethylene

Tin, total recoverable

Toluene

Toluene

Total dissolved solids

Total organic carbon

Total organic halogens

Total phosphates (as P)

Toxaphene

2,4,5-TP (Silvex)

1,1,1-Trichloroethane

1,1,1-Trichloroethane

1,1,2-Trichloroethane

1,1,2-Trichioroethane

Trichloroethylene

Trichloroethylene

Trichlorofluoromethane

Trichlorofluoromethane

2,4,5-T

Vanadium, total recoverable

Xylenes

Xylenes

Gross alpha

Nonvolatile beta

Radium, total alpha-emitting

Tritium

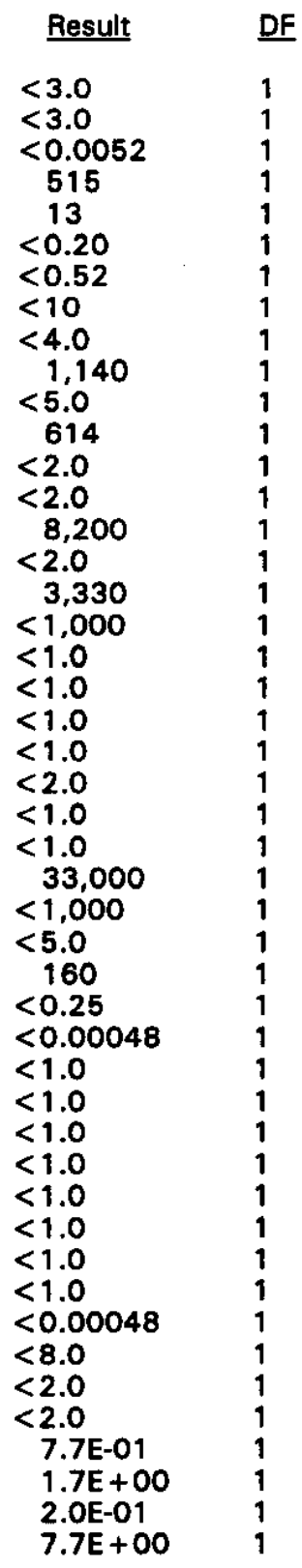

Mod Unit Flag Lab

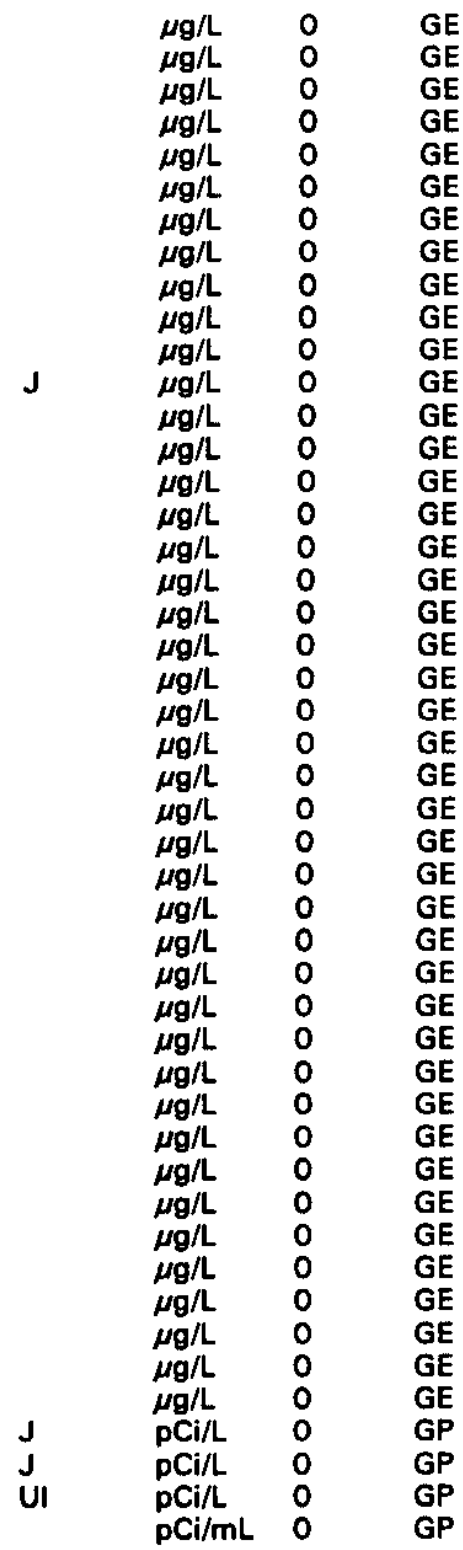

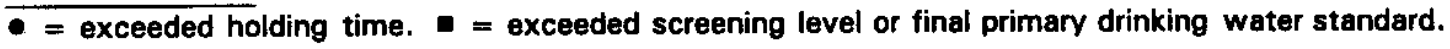




\section{WELL BGX 5D}

\begin{tabular}{|c|c|c|c|c|c|c|}
\hline SRS Coord. & Lat/Longitude & Screen Zone Elevation & Top of Casing & Casing & Pump & Formation \\
\hline $\begin{array}{l}N 78402.0 \\
E 57308.6\end{array}$ & $\begin{array}{l}33.292681^{\circ} \mathrm{N} \\
81.668190^{\circ} \mathrm{W}\end{array}$ & $215.0-195.0 \mathrm{ft} \mathrm{msl}$ & $285 \mathrm{ft} \mathrm{msl}$ & 4" PVC & $\mathbf{S}$ & Barnwell (IIB, \\
\hline
\end{tabular}

\section{FIELD MEASUREMENTS}

Sample date: 04/13/94

Depth to water: $76.19 \mathrm{ft}(23.22 \mathrm{~m})$ below TOC

Water elevation: $208.81 \mathrm{ft}(63.65 \mathrm{~m}) \mathrm{ms}$

Sp. conductance: $65 \mu \mathrm{S} / \mathrm{cm}$

Turbidity: 1.1 NTU

Water evacuated before sampling: $37 \mathrm{gal}$

\section{LABORATORY ANALYSES}

HE $\underline{\text { Analvte }}$

pH

Specific conductance

Turbidity

Acetophenone

Acetophenone

Aldrin

Aluminum, total recoverable

Antimony, total recoverable

Arsenic, total recoverable

Barium, total recoverable

Benzene

Bromodichloromethane

Bromoform

Bromomethane (Methyl bromide)

Cadmium, total recoverable

Calcium, total recoverable

Carbon tetrachloride

Chloride

Chlorobenzene

Chloroethane

Chloroethene (Vinyl chloride)

2-Chloroethyl vinyl ether

Chloroform

Chloromethane (Methyl chloride)

Chromium, total recoverable

Copper, total recoverable

Cyanide

p, $p^{\prime}$-DDT

Dibromochloromethane

1,1-Dichloroethane

1,2-Dichloroethane

1,1-Dichloroethylene

trans-1,2-Dichloroethylene

Dichloromethane (Methylene chloride)

2,4-Dichlorophenoxyacetic acid

1,2-Dichloropropane

cis-1,3-Dichloropropene

trans-1,3-Dichloropropene

Dieldrin
Time: 13:44

$\mathrm{pH}: \mathbf{5 . 3}$

Alkalinity: $1 \mathrm{mg} / \mathrm{L}$

Water temperature: $19.7^{\circ} \mathrm{C}$

Volumes purged: 4.1 well volumes

\begin{tabular}{|c|c|c|c|c|c|}
\hline Result & $\underline{D F}$ & Mod & Unit & Flag & Lat \\
\hline $\begin{array}{l}5.6 \\
55\end{array}$ & 1 & J1 & pH & 0 & GE \\
\hline $\begin{array}{l}55 \\
0.58\end{array}$ & 1 & & $\mu \mathrm{S} / \mathrm{cm}$ & 0 & \\
\hline $\begin{array}{l}0.58 \\
<10\end{array}$ & 1 & & NTU & 0 & GE \\
\hline$<10$ & $\begin{array}{l}1 \\
1\end{array}$ & & $\mu g / L$ & 0 & $\begin{array}{l}\text { GE } \\
\text { GE }\end{array}$ \\
\hline$<0.051$ & 1 & & $\mu \mathrm{g} / \mathrm{L}$ & 0 & $\mathrm{GE}$ \\
\hline 97 & 1 & & $\mu g / L$ & 2 & GE \\
\hline$<2.0$ & 1 & & $\mu g / L$ & 0 & GE \\
\hline$<2.0$ & 1 & & $\mu g / L$ & 0 & GE \\
\hline 24 & 1 & & $\mu g / L$ & 0 & GE \\
\hline $\begin{array}{l}<1.0 \\
<1.0\end{array}$ & $\begin{array}{l}1 \\
1\end{array}$ & & $\begin{array}{c}\mu \mathrm{g} / \mathrm{L} \\
\mu \mathrm{g} / \mathrm{L}\end{array}$ & $\begin{array}{l}0 \\
0\end{array}$ & $\begin{array}{l}\text { GE } \\
\text { GE }\end{array}$ \\
\hline$<1.0$ & 1 & & $\mu \mathrm{g} / \mathrm{L}$ & 0 & GE \\
\hline$<1.0$ & 1 & & $\mu \mathrm{g} / \mathrm{L}$ & 0 & GE \\
\hline$<2.0$ & 1 & & $\mu g / L$ & 0 & GE \\
\hline 2,270 & 1 & & $\mu g / L$ & 0 & \\
\hline$<1.0$ & 1 & & $\mu g / L$ & 0 & \\
\hline 4,040 & 1 & & $\mu g / L$ & 0 & \\
\hline $\begin{array}{l}<1.0 \\
<1.0\end{array}$ & 1 & & $\begin{array}{l}\mu g / L \\
\mu g / L\end{array}$ & $\begin{array}{l}0 \\
0\end{array}$ & $\begin{array}{l}\mathrm{GE} \\
\mathrm{GE}\end{array}$ \\
\hline$<1.0$ & 1 & & $\mu g / L$ & 0 & GE \\
\hline$<1.0$ & 1 & & $\mu g / L$ & 0 & \\
\hline$<1.0$ & 1 & & $\mu g / L$ & 0 & GE \\
\hline$<1.0$ & 1 & & $\mu g / L$ & 0 & \\
\hline$<4.0$ & 1 & & $\mu g / L$ & 0 & GE \\
\hline$<4.0$ & 1 & & $\mu g / L$ & 0 & \\
\hline$<5.0$ & 1 & & $\mu g / L$ & 0 & GE \\
\hline$<0.10$ & 1 & & $\mu \mathrm{g} / \mathrm{L}$ & 0 & \\
\hline$<1.0$ & 1 & & $\mu g / L$ & 0 & \\
\hline$<1.0$ & 1 & & $\mu g / L$ & 0 & GE \\
\hline$<1.0$ & 1 & & $\mu g / L$ & 0 & \\
\hline$<1.0$ & 1 & & $\mu g / L$ & 0 & \\
\hline$<1.0$ & 1 & & $\mu g / L$ & 0 & \\
\hline$<1.0$ & 1 & & $\mu g / L$ & 0 & \\
\hline$<0.0016$ & 1 & & $\mu g / L$ & 0 & \\
\hline$<1.0$ & 1 & & $\mu g / L$ & 0 & \\
\hline$<1.0$ & 1 & & $\mu g / L$ & 0 & \\
\hline$<1.0$ & 1 & & $\mu \mathrm{g} / \mathrm{L}$ & 0 & \\
\hline$<0.51$ & 1 & & $\mu g / L$ & 0 & \\
\hline
\end{tabular}

- = exceeded holding time. = exceeded screening level or final primary drinking water standard. 
WELL BGX 5D collected on 04/13/94, laboratory analyses (cont.)

H $\underline{\text { Analyte }}$

Endrin

Ethylbenzene

Fluoride

Heptachlor

Iron, total recoverable

Lead, total recoverable

Lindane

Magnesium, total recoverable

Manganese, total recoverable

Mercury, total recoverable

Methoxychlor

Naphthalene

Naphthalene

Nickel, total recoverable

Nitrate-nitrite as nitrogen

Phenols

Potassium, total recoverable

Selenium, total recoverable

Silica, total recoverable

Silver, total recoverable

Sodium, total recoverable

Sulfate

$1,1,2,2$-Tetrachloroethane

Tetrachloroethylene

Tin, total recoverable

Toluene

Total dissolved solids

Total organic carbon

Total organic halogens

Total phosphates (as P)

Toxaphene

2,4,5-TP (Silvex)

1,1,1-Trichloroethane

1,1,2-Trichloroethane

Trichloroethylene

Trichlorofluoromethane

2,4,5-T

Vanadium, total recoverable

Xylenes

Gross alpha

Gross alpha

Nonvolatile beta

Nonvolatile beta

Radium, total alpha-emitting

- Tritium
Result DF

$<0.0061$

$<1.0$

$<100$

$<0.051$

12

$<3.0$

$<0.0051$

1,080

335

$<0.20$

$<0.51$

$<10$

$<10$

5.8

1,270

$<5.0$

1,390

$<2.0$

7,090

$<2.0$

3,860

6,840

$<1.0$

$<1.0$

3.4

$<1.0$

44,000

$<1,000$

9.3

100

$<0.25$

$<0.00048$

$<1.0$

$<1.0$

$<1.0$

2.6

$<0.00048$

$<8.0$

$<2.0$

$2.9 E+00$

$3.6 \mathrm{E}+00$

$2.9 E+00$

$2.9 E+00$

$2.6 \mathrm{E}+00$

7.7E+01

Mod Unit Flag Lab

$\mu \mathrm{g} / \mathrm{L} \quad \mathrm{O} \quad \mathrm{GE}$

$\mu g / L \quad 0 \quad G E$

$\mu g / L \quad 0 \quad G E$

$\mu g / L \quad 0 \quad G E$

$\mu g / L \quad 0 \quad$ GE

$\mu g / L \quad 0 \quad G E$

$\mu \mathrm{g} / \mathrm{L} \quad \mathrm{O} \quad \mathrm{GE}$

$\mu g / L \quad 0 \quad G E$

$\mu g / L \quad 2 \quad G E$

$\mu g / L \quad 0 \quad G E$

$\mu g / L \quad 0 \quad$ GE

$\mu g / L \quad 0 \quad G E$

$\mu g / L \quad 0 \quad G E$

$J \quad \mu g / L \quad O \quad G E$

$\mu \mathrm{g} / \mathrm{L} \quad \mathrm{O} \quad \mathrm{GE}$

$\mu \mathrm{g} / \mathrm{L} \quad \mathrm{O} \quad \mathrm{GE}$

$\mu g / L \quad 0 \quad G E$

$\mu \mathrm{g} / \mathrm{L} \quad \mathrm{O} \quad \mathrm{GE}$

$\mu \mathrm{g} / \mathrm{L} \quad 0 \quad \mathrm{GE}$

$\mu g / L \quad 0 \quad G E$

$\mu \mathrm{g} / \mathrm{L} \quad \mathrm{O} \quad \mathrm{GE}$

$\mu g / L \quad 0 \quad G E$

$\mu \mathrm{g} / \mathrm{L} \quad \mathrm{O} \quad \mathrm{GE}$

$\mu \mathrm{g} / \mathrm{L} \quad 0 \quad \mathrm{GE}$

$\mu \mathrm{g} / \mathrm{L} \quad \mathrm{O} \quad \mathrm{GE}$

$\mu \mathrm{g} / \mathrm{L} \quad 0 \quad \mathrm{GE}$

$\mu g / L \quad 0 \quad G E$

$\mu \mathrm{g} / \mathrm{L} \quad \mathrm{O} \quad \mathrm{GE}$

$\mu g / L \quad O \quad G E$

$\mu g / L \quad 0 \quad$ GE

$\mu g / L \quad 0 \quad G E$

$\mu g / L \quad 0 \quad$ GE

$\mu \mathrm{g} / \mathrm{L} \quad 0 \quad \mathrm{GE}$

$\mu g / L \quad 0 \quad G E$

$\mu g / L \quad 0 \quad G E$

$\mu g / L \quad 0 \quad$ GE

$\mu g / L \quad 0 \quad G E$

$\mu g / L \quad 0 \quad G E$

$\mu g / L \quad 0 \quad G E$

pCi/L 0 GP

pCi/L $\quad 0 \quad$ GP

pCi/L $0 \quad$ GP

$\mathrm{pCi} / \mathrm{L} \quad 0$

$\mathrm{pCi} / \mathrm{L} \quad 0 \quad \mathrm{GP}$

$\mathrm{pCi} / \mathrm{mL} \quad 2 \quad \mathrm{GP}$

- = exceeded holding time. = exceeded screening level or final primary drinking water standard. 


$\begin{array}{lllllll}\text { SRS Coord. } & \text { Lat/Longitude } & \text { Screen Zone Elovation } & \text { Top of Casing } & \text { Casing } & \text { Pump } & \text { Formation } \\ \begin{array}{llllll}\text { N78740.1 } \\ \text { E57524.9 }\end{array} & \begin{array}{llll}33.293782^{\circ} \mathrm{N} \\ 81.668277^{\circ} \mathrm{W}\end{array} & 211.0-191.0 \mathrm{ft} \mathrm{ms} & 277 \mathrm{ft} \mathrm{msl} & \text { 4" PVC } & \text { S } & \text { Barnwell (IIB, })\end{array}$

\section{FIELD MEASUREMENTS}

Sample date: 04/18/94

Depth to water: $71.76 \mathrm{ft}(21.87 \mathrm{~m})$ below TOC

Water elevation: $205.24 \mathrm{ft}(62.56 \mathrm{~m}) \mathrm{msl}$

Sp. conductance: $74 \mu \mathrm{S} / \mathrm{cm}$

Turbidity: 1.4 NTU

Water evacuated before sampling: $\mathbf{4 1}$ gal

\section{LABORATORY ANALYSES}

\section{H D Analyte}

- $\mathrm{pH}$

Specific conductance

Turbidity

Acetophenone

Aldrin

Aluminum, total recoverable

Antimony, total recoverable

Arsenic, total recoverable

Barium, total recoverable

Benzene

Bromodichloromethane

Bromoform

Bromomethane (Methyl bromide)

Cadmium, total recoverable

Calcium, total recoverable

Carbon tetrachloride

Chloride

Chlorobenzene

Chloroethane

Chloroethene (Vinyl chloride)

2-Chloroethyl vinyl ether

Chloroform

Chloromethane (Methyl chloride)

Chromium, total recoverable

Copper, total recoverable

Cyanide

p.p'-DDT

Dibromochloromethane

1,1-Dichloroethane

1,2-Dichloroethane

1,1-Dichloroethylene

trans-1,2-Dichloroethylene

Dichloromethane (Methylene chloride)

2,4-Dichlorophenoxyacetic acid

1,2-Dichloropropane

cis-1,3-Dichloropropene

trans-1,3-Dichloropropene

Dieldrin

Endrin
Time: 11:03

pH: 6.5

Alkalinity: $14 \mathrm{mg} / \mathrm{L}$

Water temperature: $19.1^{\circ} \mathrm{C}$

Volumes purged: 4.4 well volumes

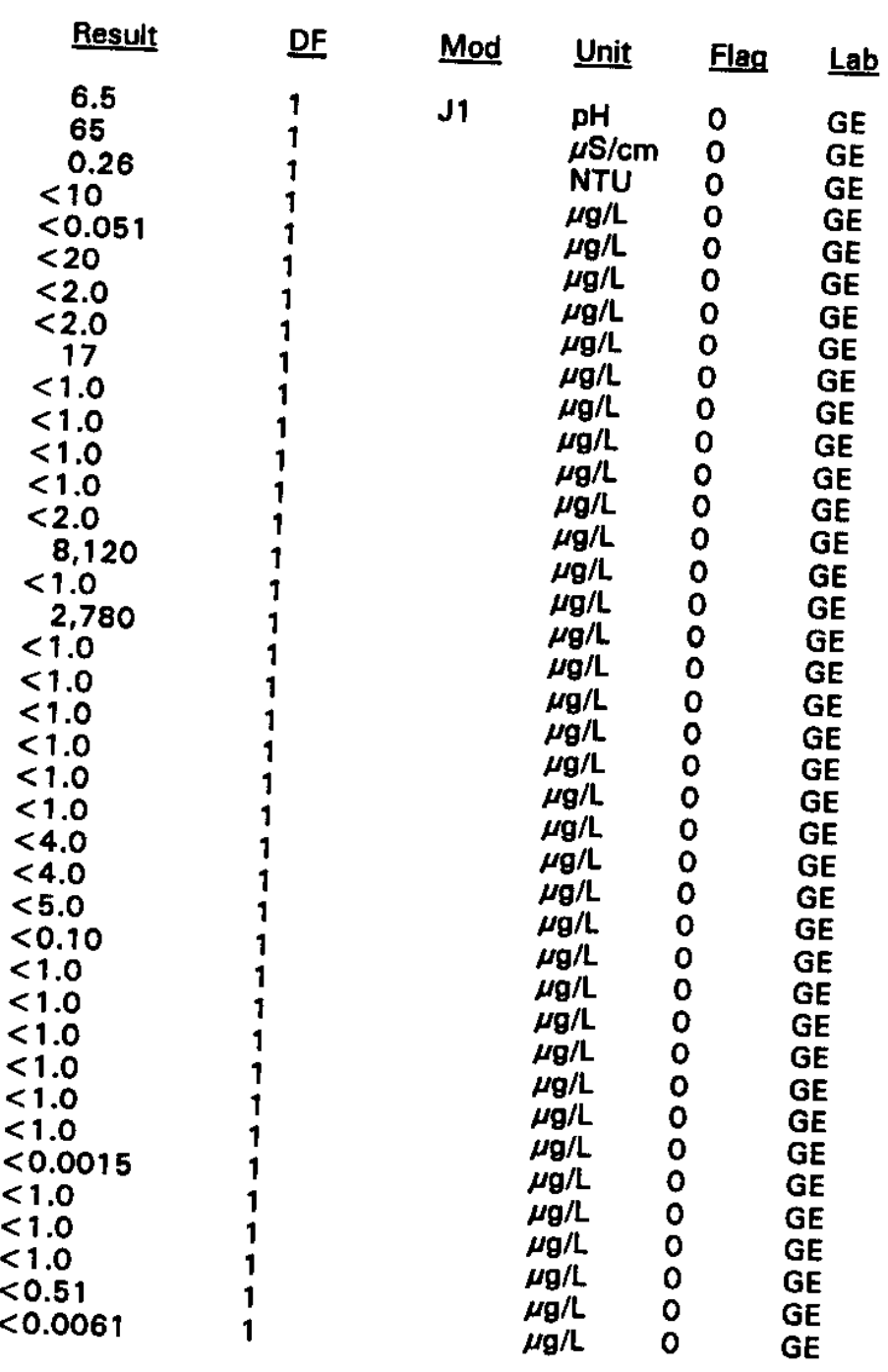

- exceeded holding time. = exceeded screening level or final primary drinking water standard. 
WELL BGX $6 D$ collected on $04 / 18 / 94$, laboratory analyses (cont.)

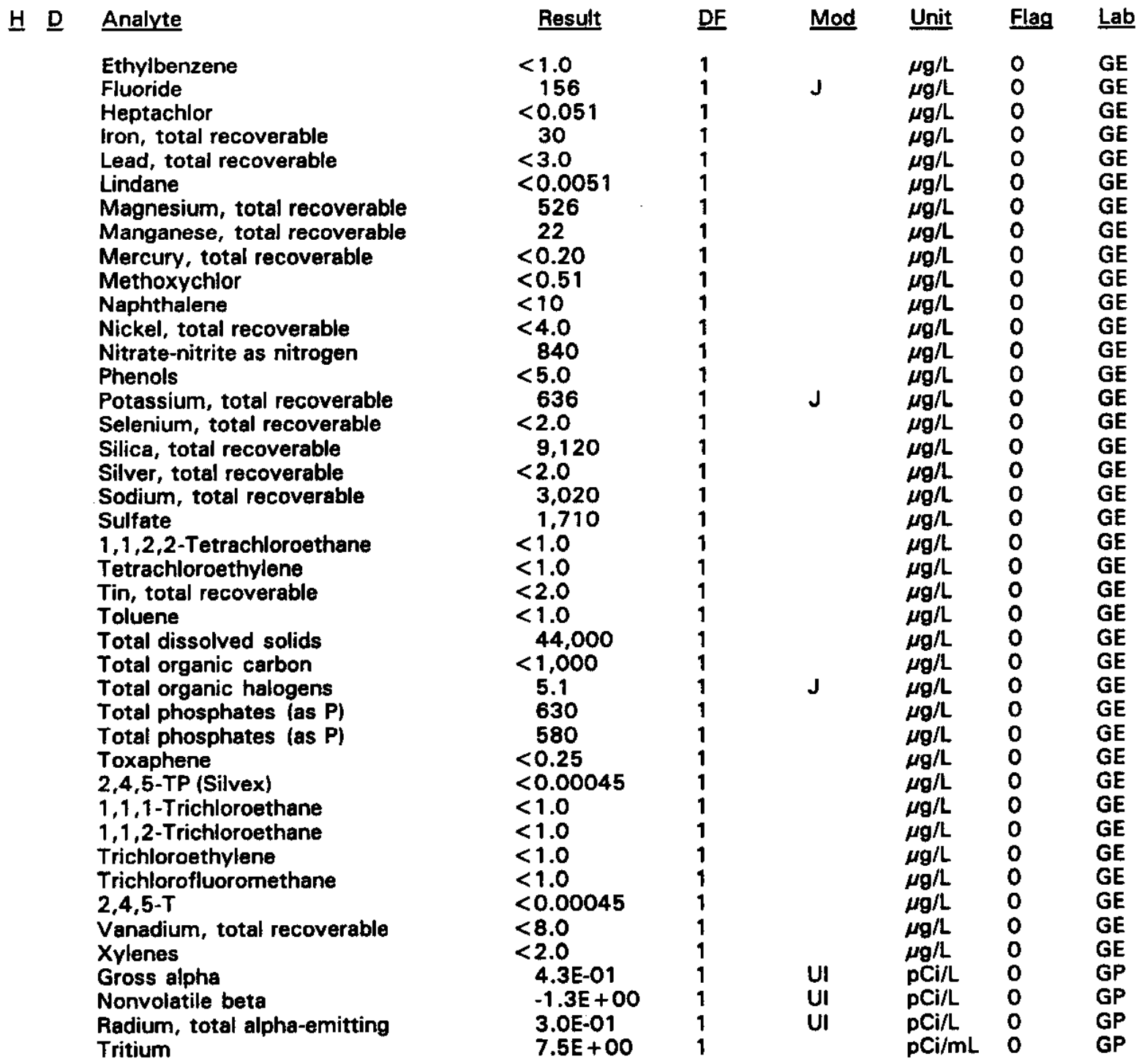

- = exceeded holding time. $\square=$ exceeded screening level or final primary drinking water standard. 
WELL BGX 7D

\begin{tabular}{|c|c|c|c|c|c|c|}
\hline SRS Coord. & Lat/Longitude & Screen Zone Elevation & Top of Casing & Casing & Pump & Formation \\
\hline $\begin{array}{l}178349.3 \\
58312.8\end{array}$ & $\begin{array}{l}33.294203^{\circ} \mathrm{N} \\
81.665443^{\circ} \mathrm{W}\end{array}$ & $214.1-194.1 \mathrm{ft} \mathrm{msl}$ & $279.2 \mathrm{ft} \mathrm{msl}$ & 4" PVC & $\mathbf{s}$ & Barn \\
\hline
\end{tabular}

\section{FIELD MEASUREMENTS}

Sample date: $04 / 18 / 94$

Depth to water: $74.02 \mathrm{ft}(22.56 \mathrm{~m})$ below TOC

Water elevation: $205.18 \mathrm{ft}(62.54 \mathrm{~m}) \mathrm{msl}$

Sp. conductance: $24 \mu \mathrm{S} / \mathrm{cm}$

Turbidity: 0.4 NTU

Water evacuated before sampling: $51 \mathrm{gal}$

Time: $11: 49$

pH: 5.3

Alkalinity: $0 \mathrm{mg} / \mathrm{L}$

Water temperature: $19.3^{\circ} \mathrm{C}$

Volumes purged: 7.0 well volumes

\section{LABORATORY ANALYSES}

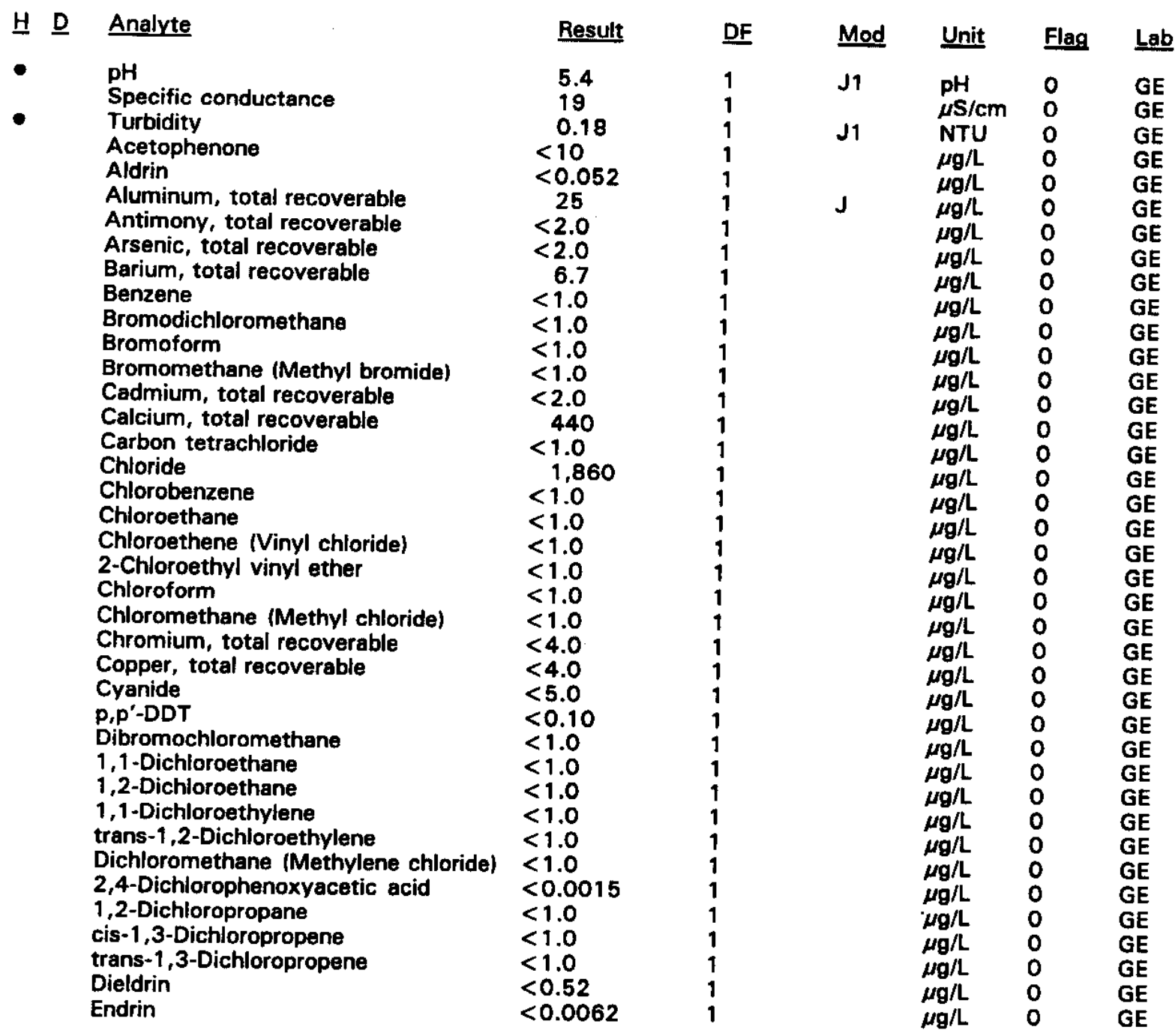

- = exceeded holding time. $\square=$ exceeded screening level or final primary drinking water standard. 
WELL BGX 7D collected on $04 / 18 / 94$, laboratory analyses (cont.)

H D Analyte

Ethylbenzene

Fluoride

Heptachlor

Iron, total recoverable

Lead, total recoverable

Lindane

Magnesium, total recoverable

Manganese, total recoverable

Mercury, total recoverable

Methoxychlor

Naphthalene

Nickel, total recoverable

Nitrate-nitrite as nitrogen

Phenols

Potassium, total recoverable

Selenium, total recoverable

Silica, total recoverable

Silver, total recoverable

Sodium, total recoverable

Sulfate

1,1,2,2-Tetrachloroethane

Tetrachloroethylene

Tin, total recoverable

Toluene

Total dissolved solids

Total organic carbon

Total organic halogens

Total phosphates (as P)

Toxaphene

2,4,5-TP (Silvex)

1,1,1-Trichloroethane

1,1,2-Trichloroethane

Trichloroethylene

Trichlorofluoromethane

2,4,5-T

Vanadium, total recoverable

Xylenes

Gross alpha

Nonvolatile beta

Radium, total alpha-emitting

Total activity

- Tritium

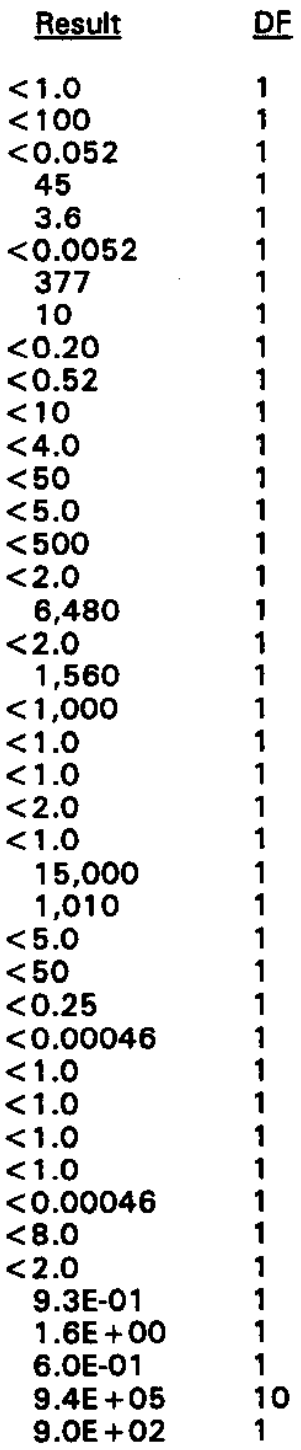

Mod Unit Flag Lab

$\mu g / L \quad 0 \quad G E$

$\mu g / L \quad 0 \quad G E$

$\mu \mathrm{g} / \mathrm{L} \quad 0 \quad \mathrm{GE}$

$\mu g / L \quad 0 \quad G E$

J $\mu g / L \quad O \quad G E$

$\mu \mathrm{g} / \mathrm{L} \quad 0 \quad \mathrm{GE}$

$\mu \mathrm{g} / \mathrm{L} \quad \mathrm{O} \quad \mathrm{GE}$

$\mu g / L \quad 0 \quad G E$

$\mu g / L \quad 0 \quad G E$

$\mu g / L \quad 0 \quad G E$

$\mu g / L \quad 0 \quad G E$

$\mu g / L \quad 0 \quad G E$

$\mu g / L \quad 0 \quad G E$

$\mu g / L \quad 0 \quad$ GE

$\mu g / L \quad 0 \quad G E$

$\mu g / L \quad 0 \quad G E$

$\mu g / L \quad 0 \quad G E$

$\mu g / L \quad 0 \quad G E$

$\mu g / L \quad 0 \quad G E$

$\mu g / L \quad 0 \quad G E$

$\mu g / L \quad 0 \quad G E$

$\mu g / L \quad 0 \quad G E$

$\mu g / L \quad 0 \quad G E$

$\mu g / L \quad 0 \quad G E$

$\mu g / \mathrm{L} \quad 0 \quad \mathrm{GE}$

$\mu g / L \quad O \quad G E$

$\mu g / L \quad O \quad G E$

$\mu g / \mathrm{L} \quad 0 \quad \mathrm{GE}$

$\mu g / L \quad 0 \quad$ GE

$\mu \mathrm{g} / \mathrm{L} \quad \mathrm{O} \quad \mathrm{GE}$

$\mu g / L \quad 0 \quad G E$

$\mu g / L \quad 0 \quad G E$

$\mu \mathrm{g} / \mathrm{L} \quad \mathrm{O} \quad \mathrm{GE}$

$\mu g / L \quad 0 \quad G E$

$\mu g / L \quad 0 \quad G E$

$\mu g / L \quad 0 \quad G E$

$\mu \mathrm{g} / \mathrm{L} \quad \mathrm{O} \quad \mathrm{GE}$

$\mathrm{pCi} / \mathrm{L} \quad 0 \quad \mathrm{GP}$

$\mathrm{pCi} / \mathrm{L} \quad 0 \quad \mathrm{GP}$

$\mathrm{pCi} / \mathrm{L} \quad \mathrm{G}$ GP

$\mathrm{pCi} / \mathrm{L}$ O EM

$\mathrm{pCi} / \mathrm{mL} 2$ GP

- exceeded holding time. $\omega=$ exceeded screening level or final primary drinking water standard. 


\section{WELL BGX 8DR}

\begin{tabular}{|c|c|c|c|c|c|c|}
\hline SRS Coord. & Lat/Longitude & Screen Zone Elevation & Top of Casing & Casing & Pump & Formation \\
\hline $\begin{array}{l}\text { N77589.6 } \\
\text { E58942.5 }\end{array}$ & $\begin{array}{l}33.293550^{\circ} \mathrm{N} \\
81.662309^{\circ} \mathrm{W}\end{array}$ & $203.1-183.1 \mathrm{ft} \mathrm{msl}$ & $278.2 \mathrm{ft} \mathrm{msl}$ & 4" PVC & $\mathbf{s}$ & Barnwell (IIB, \\
\hline
\end{tabular}

\section{FIELD MEASUREMENTS}

Sample date: 04/19/94

Depth to water: $73.03 \mathrm{ft}(22.26 \mathrm{~m})$ below TOC

Water elevation: $205.17 \mathrm{ft}(62.54 \mathrm{~m}) \mathrm{msl}$

Sp. conductance: $54 \mu \mathrm{S} / \mathrm{cm}$

Turbidity: 2.4 NTU

Water evacuated before sampling: $49 \mathrm{gal}$

\section{LABORATORY ANALYSES}

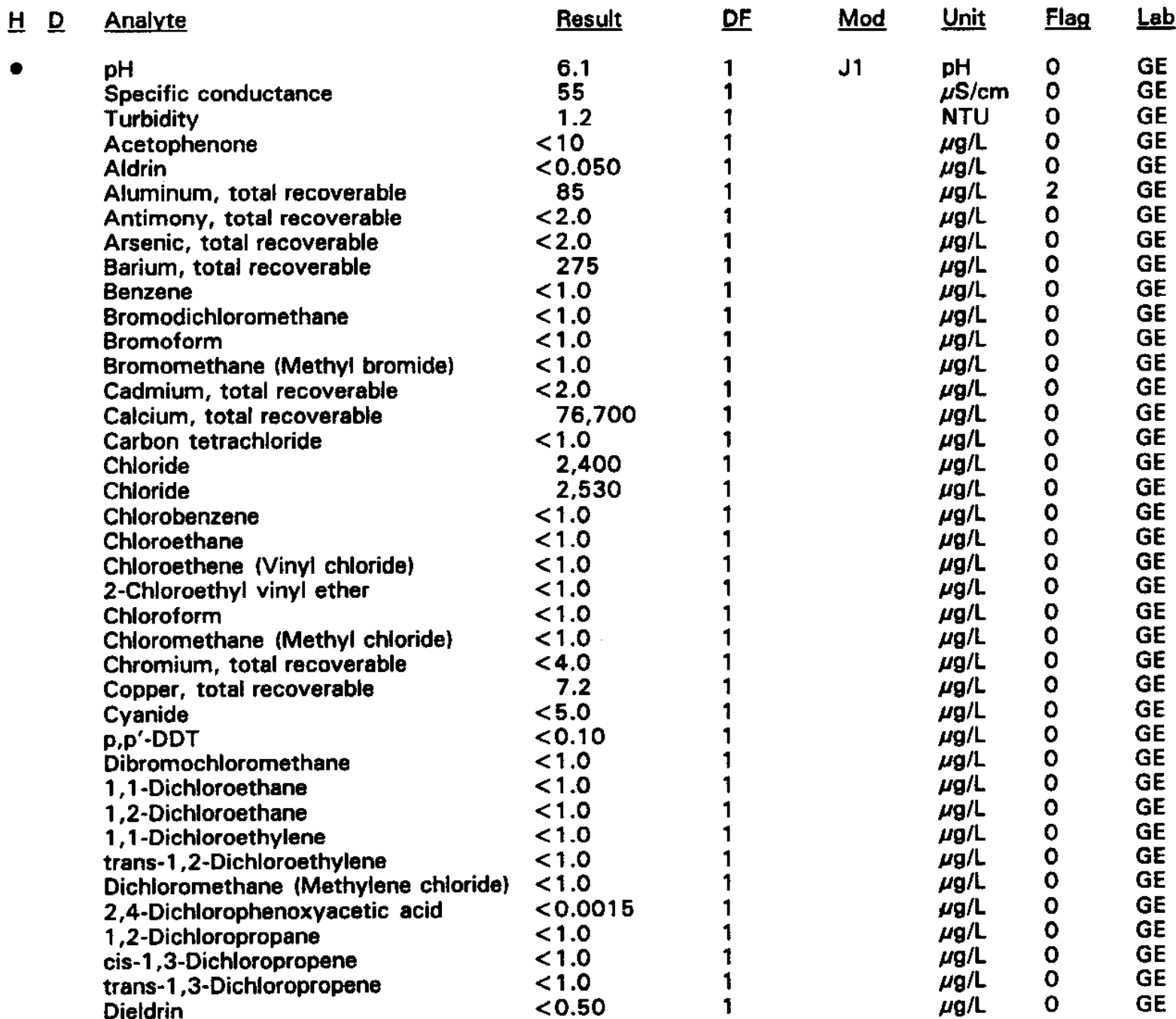

Time: $12: 50$

pH: $\mathbf{5 . 9}$

Alkalinity: $10 \mathrm{mg} / \mathrm{L}$

Water temperature: $20.0^{\circ} \mathrm{C}$

Volumes purged: $\mathbf{3 . 4}$ well volumes 
WELL BGX 8DR collected on 04/19/94, laboratory analyses (cont.)

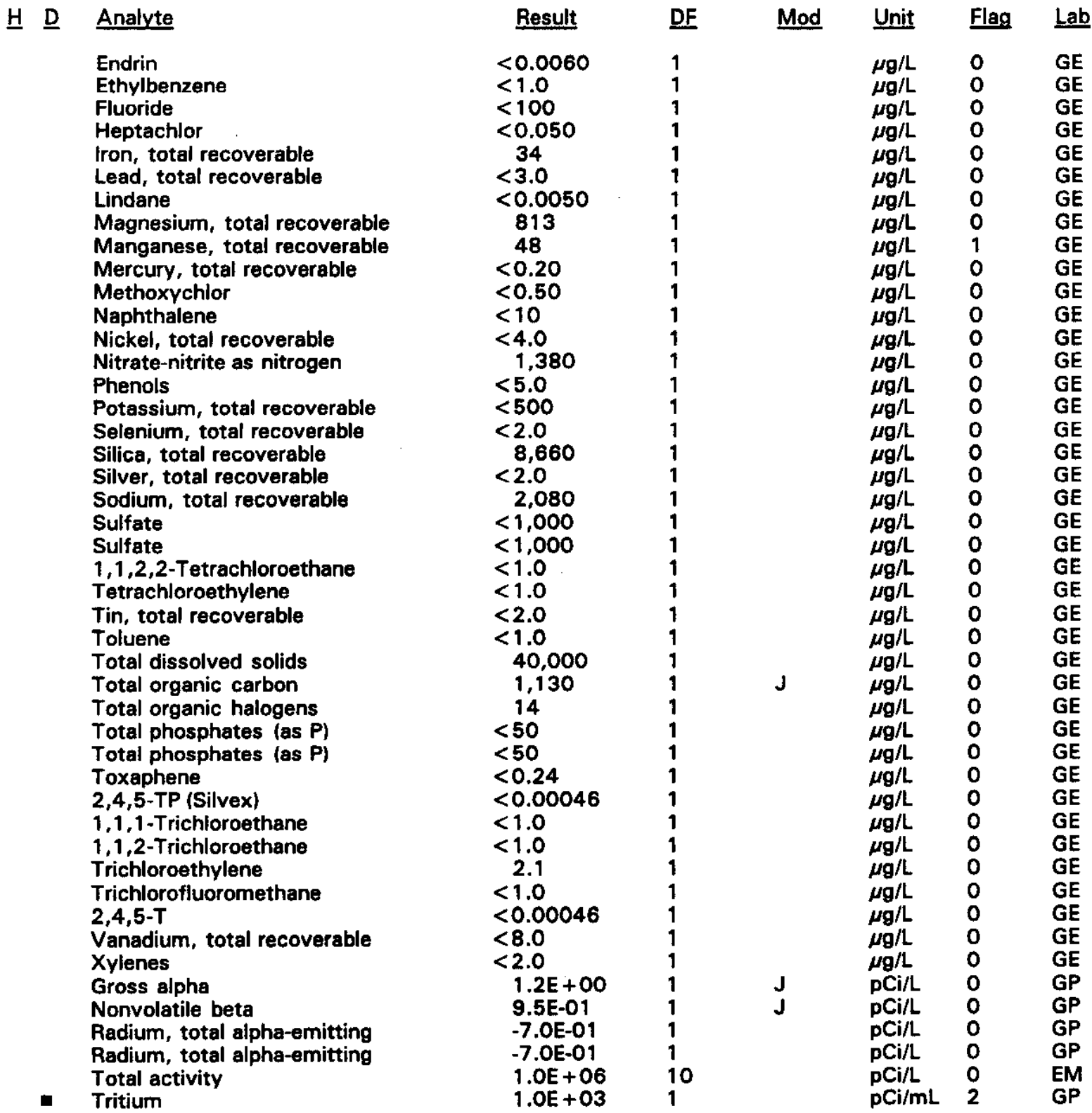

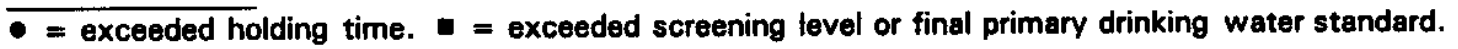




\section{WELL BGX 9D}

\begin{tabular}{|c|c|c|c|c|c|c|}
\hline SRS Coord. & Lat/Longitude & Screen Zone Elevation & Top of Casing & Casing & Pump & Formation \\
\hline $\begin{array}{l}\text { N76936.0 } \\
\text { E59522.1 }\end{array}$ & $\begin{array}{l}33.293050^{\circ} \mathrm{N} \\
81.659513^{\circ} \mathrm{W}\end{array}$ & $232.4-212.4 \mathrm{ft} \mathrm{msl}$ & $279.4 \mathrm{ft} \mathrm{msl}$ & 4" PVC & s & Water Table $\left(I \mathrm{~B}_{2}\right)$ \\
\hline
\end{tabular}

\section{FIELD MEASUREMENTS}

Sample date: 04/19/94

Depth to water: $52.87 \mathrm{ft}(16.11 \mathrm{~m})$ below TOC

Water elevation: $226.53 \mathrm{ft}(69.05 \mathrm{~m}) \mathrm{msl}$

Sp. conductance: $30 \mu \mathrm{S} / \mathrm{cm}$

Turbidity: 0.1 NTU

Water evacuated before sampling: $87 \mathrm{gal}$

\section{LABORATORY ANALYSES}

\section{H D Analyte}

- $\quad \mathrm{pH}$

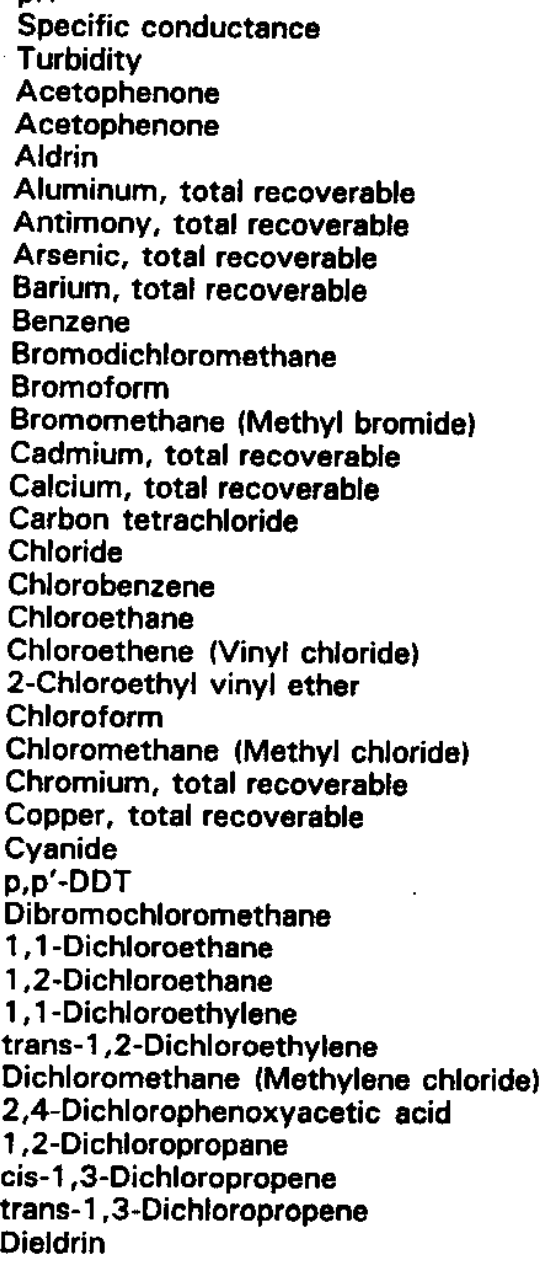

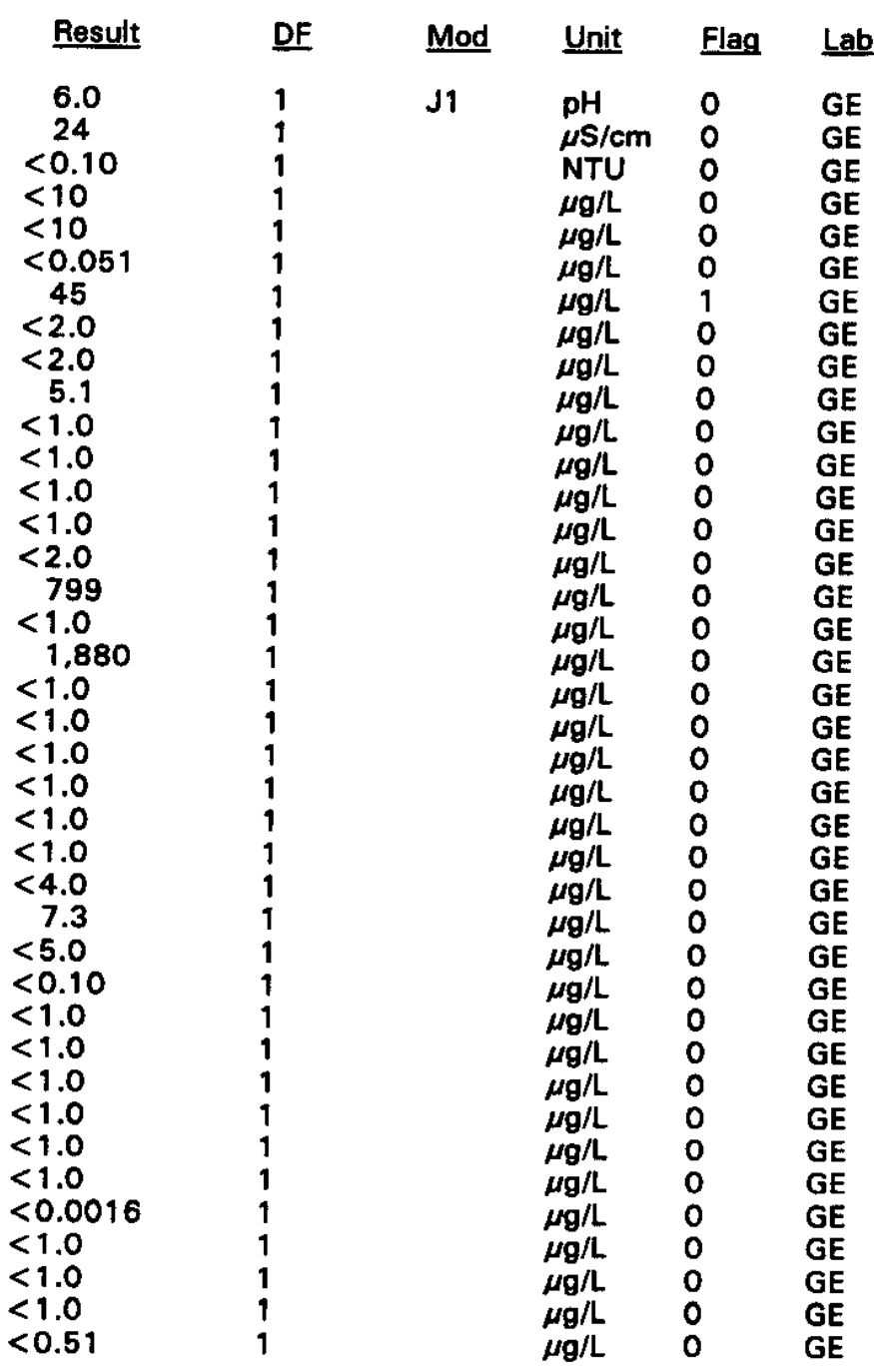

Time: 12:01

pH: 4.9

Alkalinity: $0 \mathrm{mg} / \mathrm{L}$

Water temperature: $19.1^{\circ} \mathrm{C}$

Volumes purged: 9.4 well volumes

\section{ES}


WELL BGX 9D collected on 04/19/94, laboratory analyses (cont.)

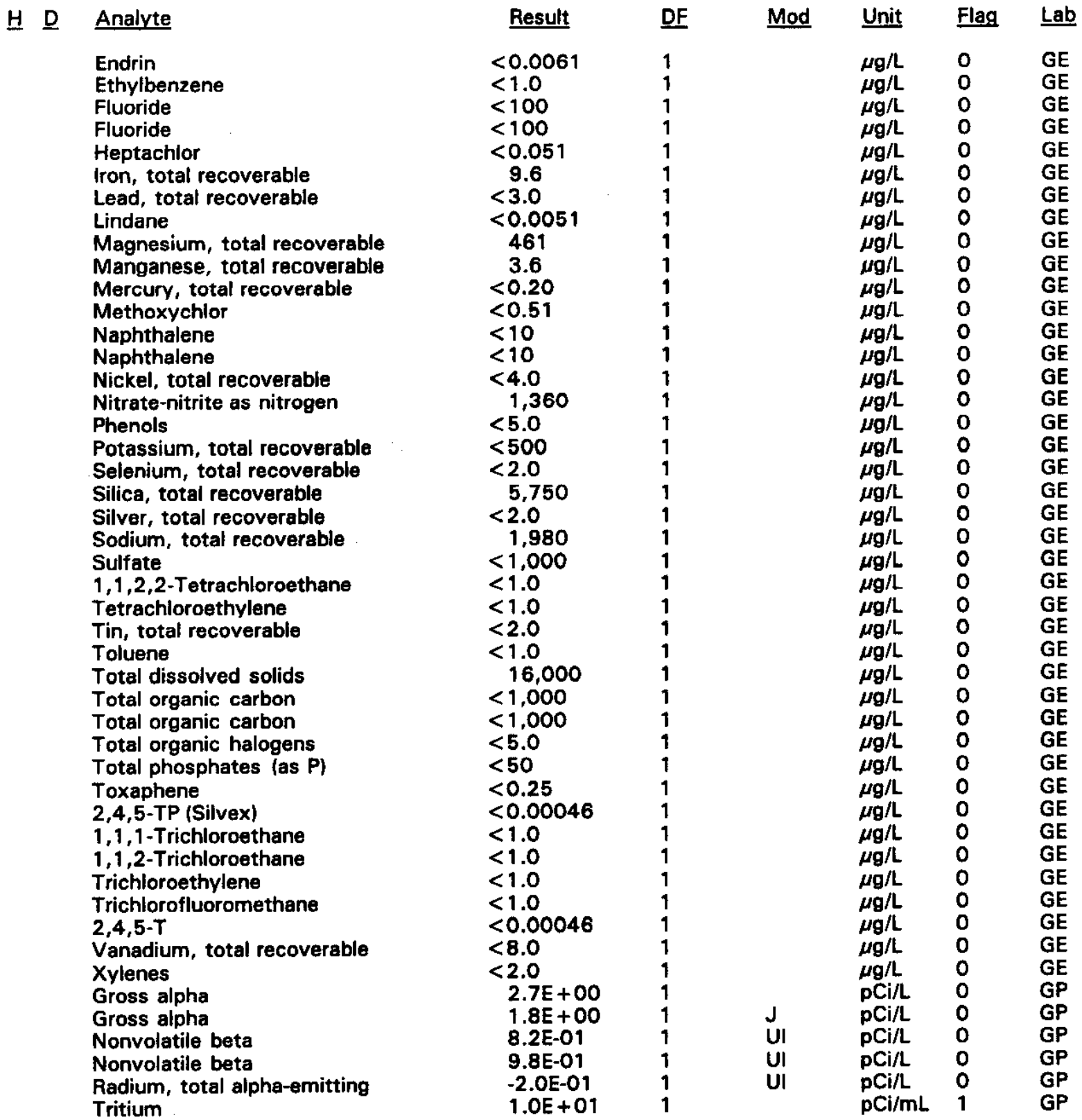

- = exceeded holding time. = exceeded screening level or final primary drinking water standard. 


\section{WELL BGX 10D}

\begin{tabular}{|c|c|c|c|c|c|c|}
\hline SRS Coord. & Lat/Longitude & Screen Zone Elevation & Top of Casing & Casing & Pump & Formation \\
\hline $\begin{array}{l}N 76183.3 \\
\text { E59765.5 }\end{array}$ & $\begin{array}{l}33.291783^{\circ} \mathrm{N} \\
81.657410^{\circ} \mathrm{W}\end{array}$ & $236.2-216.2 \mathrm{ft} \mathrm{msl}$ & $276.9 \mathrm{ft} \mathrm{msl}$ & 4" PVC & $\mathbf{S}$ & Water Ta \\
\hline
\end{tabular}

\section{FIELD MEASUREMENTS}

Sample date: 04/19/94

Depth to water: $51.57 \mathrm{ft}(15.72 \mathrm{~m})$ below TOC

Water elevation: $225.33 \mathrm{ft}(68.68 \mathrm{~m}) \mathrm{ms}$ l

Sp. conductance: $39 \mu \mathrm{S} / \mathrm{cm}$

Turbidity: 13.6 NTU

Water evacuated before sampling: $3 \mathrm{gal}$

The well went dry during purging.

Time: 10:30

pH: 6.1

Alkalinity: $4 \mathrm{mg} / \mathrm{L}$

Water temperature: $18.8^{\circ} \mathrm{C}$

Volumes purged: 0.5 well volumes

\section{LABORATORY ANALYSES}

H D Analyte

?

pH
Specific conductance

Turbidity

Acetophenone

Aldrin

Aluminum, total recoverable

Antimony, total recoverable

Arsenic, total recoverable

Barium, total recoverable

Benzene

Benzene

Bromodichloromethane

Bromodichloromethane

Bromoform

Bromoform

Bromomethane (Methyl bromide)

Bromomethane (Methyl bromide)

Cadmium, total recoverable

Calcium, total recoverable

Carbon tetrachloride

Carbon tetrachloride

Chloride

Chlorobenzene

Chlorobenzene

Chloroethane

Chloroethane

Chloroethene (Vinyl chloride)

Chloroethene (Vinyl chloride)

2-Chloroethyl vinyl ether

2-Chloroethyl vinyl ether

Chloroform

Chloroform

Chloromethane (Methyl chloride)

Chloromethane (Methyl chloride)

Chromium, total recoverable

Copper, total recoverable

Cyanide

p.p'-DDT

Dibromochloromethane
Result

6.6

38

19

$<10$

$<0.053$

159

$<2.0$

$<2.0$

11

$<1.0$

$<1.0$

$<1.0$

$<1.0$

$<1.0$

$<1.0$

$<1.0$

$<1.0$

$<2.0$

918

$<1.0$

$<1.0$

1,730

$<1.0$

$<1.0$

$<1.0$

$<1.0$

$<1.0$

$<1.0$

$<1.0$

$<1.0$

$<1.0$

$<1.0$

$<1.0$

$<1.0$

$<4.0$

38

$<5.0$

$<0.11$

$<1.0$

DF
1
1
1
1
1
1
1
1
1
1
1
1
1
1
1
1
1
1
1
1
1
1
1
1
1
1
1
1
1
1
1
1
1
1

\begin{tabular}{|c|c|c|}
\hline Mod & Unit & Flag \\
\hline J1 & 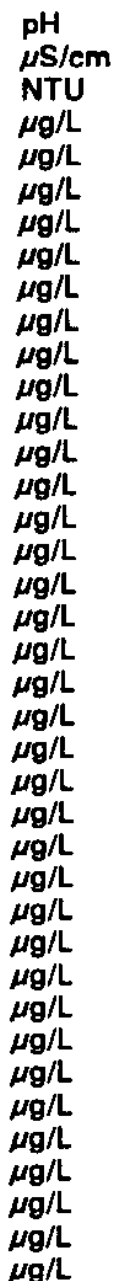 & $\begin{array}{l}0 \\
0 \\
0 \\
0 \\
0 \\
2 \\
0 \\
0 \\
0 \\
0 \\
0 \\
0 \\
0 \\
0 \\
0 \\
0 \\
0 \\
0 \\
0 \\
0 \\
0 \\
0 \\
0 \\
0 \\
0 \\
0 \\
0 \\
0 \\
0 \\
0 \\
0 \\
0 \\
0 \\
0 \\
0 \\
0 \\
0 \\
0 \\
0\end{array}$ \\
\hline
\end{tabular}

- = exceeded holding time. = exceeded screening level or final primary drinking water standard. 
WELL BGX 100 collected on 04/19/94, laboratory analyses (cont.)

H D Analyte

Dibromochloromethane

1,1-Dichloroethane

1,1-Dichloroethane

1,2-Dichloroethane

1,2-Dichloroethane

1,1-Dichloroethylene

1,1-Dichloroethylene

trans-1,2-Dichloroethylene

trans-1,2-Dichloroethylene

Dichloromethane (Methylene chloride)

Dichloromethane (Methylene chloride)

2,4-Dichlorophenoxyacetic acid

1,2-Dichloropropane

1,2-Dichloropropane

cis-1,3-Dichloropropene

cis-1,3-Dichloropropene

trans-1,3-Dichloropropene

trans-1,3-Dichloropropene

Dieldrin

Endrin

Ethylbenzene

Ethylbenzene

Fluoride

Heptachlor

Iron, total recoverable

Lead, total recoverable

Lindane

Magnesium, total recoverable

Manganese, total recoverable

Mercury, total recoverable

Methoxychlor

Naphthalene

Nickel, total recoverable

Nitrate-nitrite as nitrogen

Nitrate-nitrite as nitrogen

Phenols

Potassium, total recoverable

Selenium, total recoverable

Silica, total recoverable

Silver, total recoverable

Sodium, total recoverable

Sulfate

1,1,2,2-Tetrachloroethane

1,1,2,2-Tetrachloroethane

Tetrachloroethylene

Tetrachloroethylene

Tin, total recoverable

Toluene

Toluene

Total dissolved solids

Total organic carbon

Total organic halogens

Total phosphates (as P)

Toxaphene

2,4,5-TP (Silvex)

1,1,1-Trichloroethane

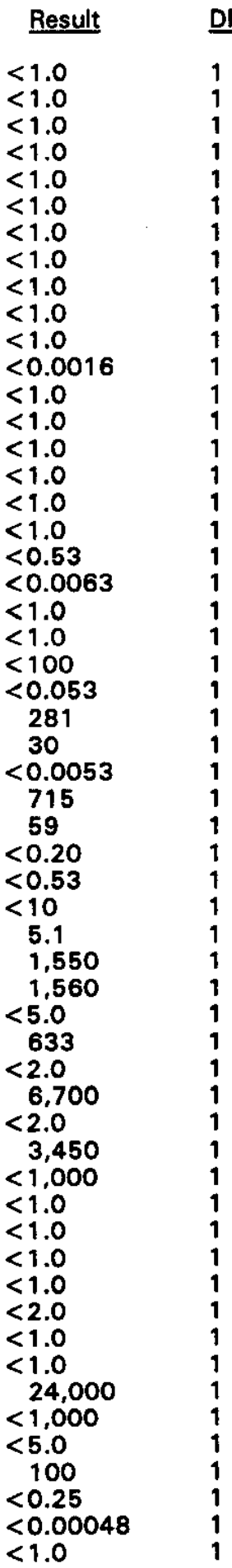

Mod Unit Flag Lab

$\mu \mathrm{g} / \mathrm{L} \quad 0 \quad \mathrm{GE}$

$\mu g / L \quad 0 \quad$ GE

$\mu g / L \quad 0 \quad G E$

$\mu g / L \quad 0 \quad G E$

$\mu g / L \quad 0 \quad G E$

$\mu g / L \quad 0 \quad$ GE

$\mu \mathrm{g} / \mathrm{L} \quad 0 \quad \mathrm{GE}$

$\mu g / L \quad 0 \quad$ GE

$\mu \mathrm{g} / \mathrm{L} \quad 0 \quad \mathrm{GE}$

$\mu g / L \quad 0 \quad G E$

$\mu g / L \quad 0 \quad$ GE

$\mu g / L \quad 0 \quad$ GE

$\mu g / L \quad 0 \quad$ GE

$\mu g / L \quad 0 \quad$ GE

$\mu \mathrm{g} / \mathrm{L} \quad 0 \quad \mathrm{GE}$

$\mu \mathrm{g} / \mathrm{L} \quad 0 \quad \mathrm{GE}$

$\mu \mathrm{g} / \mathrm{L} \quad 0 \quad \mathrm{GE}$

$\mu g / L \quad 0 \quad$ GE

$\mu g / L \quad 0 \quad$ GE

$\mu g / L \quad 0 \quad$ GE

$\mu g / L \quad 0 \quad$ GE

$\mu g / L \quad 0 \quad$ GE

$\mu g / L \quad 0 \quad$ GE

$\mu g / L \quad 0 \quad$ GE

$\mu g / L \quad 1 \quad$ GE

$\mu \mathrm{g} / \mathrm{L} \quad 1 \quad \mathrm{GE}$

$\mu \mathrm{g} / \mathrm{L} \quad 0 \quad \mathrm{GE}$

$\mu g / L \quad 0 \quad$ GE

$\mu g / L \quad 2 \quad$ GE

$\mu \mathrm{g} / \mathrm{L} \quad 0 \quad \mathrm{GE}$

$\mu \mathrm{g} / \mathrm{L} \quad \mathrm{O} \quad \mathrm{GE}$

$\mu g / L \quad 0 \quad$ GE

$\mu g / L \quad 0 \quad$ GE

$\mu g / L \quad 0 \quad$ GE

$\mu g / L \quad O \quad$ GE

$\mu g / L \quad 0 \quad$ GE

$\mu g / L \quad 0 \quad$ GE

$\mu g / L \quad 0 \quad$ GE

$\mu g / L \quad 0 \quad G E$

$\mu g / L \quad 0 \quad G E$

$\mu g / L \quad 0 \quad$ GE

$\mu g / L \quad 0 \quad$ GE

$\mu g / L \quad 0 \quad G E$

$\mu g / L \quad 0 \quad G E$

$\mu g / L \quad 0 \quad$ GE

$\mu g / L \quad 0 \quad$ GE

$\mu g / L \quad 0 \quad$ GE

$\mu g / L \quad 0 \quad G E$

$\mu \mathrm{g} / \mathrm{L} \quad 0 \quad \mathrm{GE}$

$\mu \mathrm{g} / \mathrm{L} \quad \mathrm{O} \quad \mathrm{GE}$

$\mu \mathrm{g} / \mathrm{L} \quad 0 \quad \mathrm{GE}$

$\dot{\mu} g / L \quad 0 \quad$ GE

$\mu g / L \quad 0 \quad$ GE

$\mu g / L \quad 0 \quad$ GE

$\mu g / L \quad 0 \quad G E$

- exceeded holding time. = exceeded screening level or final primary drinking water standard. 
WELL BGX $10 D$ collected on $04 / 19 / 94$, laboratory analyses (cont.)

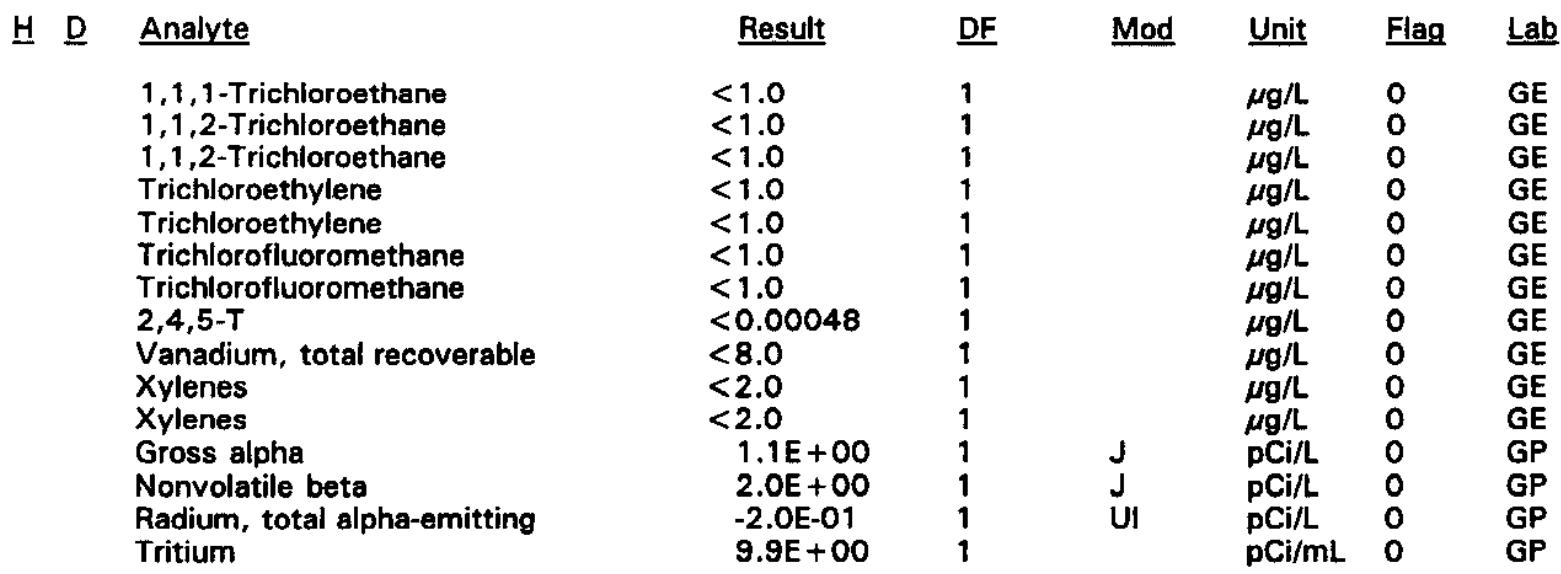

\section{WELL BGX 11D}

\begin{tabular}{|c|c|c|c|c|c|c|}
\hline SRS Coord. & Lat/Longitude & Screen Zone Elevation & Top of Casing & Casing & Pump & Formation \\
\hline $\begin{array}{l}\text { N75300.7 } \\
\text { E59581.4 }\end{array}$ & $\begin{array}{l}33.289530^{\circ} \mathrm{N} \\
81.656180^{\circ} \mathrm{W}\end{array}$ & $236.7-216.7 \mathrm{ft} \mathrm{msl}$ & $276.3 \mathrm{ft} \mathrm{msl}$ & 4" PVC & $\mathbf{s}$ & Water Table $\left(\|_{2} B_{2}\right)$ \\
\hline
\end{tabular}

\section{FIELD MEASUREMENTS}

Sample date: 04/19/94

Depth to water: $41.03 \mathrm{ft}(12.51 \mathrm{~m})$ below TOC

Water elevation: $235.27 \mathrm{ft}(71.71 \mathrm{~m}) \mathrm{ms}$ l

Sp. conductance: $44 \mu \mathrm{S} / \mathrm{cm}$

Turbidity: 69.4 NTU

Water evacuated before sampling: 4 gal

The well went dry during purging.

\section{LABORATORY ANALYSES}

H D Analyte

- $\quad$ pH

Specific conductance
Turbidity
Acetophenone
Aldrin
Aluminum, total recoverable
Aluminum, total recoverable
Antimony, total recoverable
Arsenic, total recoverable
Barium, total recoverable
Barium, total recoverable
Benzene
Bromodichloromethane
Bromoform
Bromomethane (Methyl bromide)
Cadmium, total recoverable
Cadmium, total recoverable
Calcium, total recoverable
Calcium, total recoverable

$\begin{array}{ccc}\text { Result } & & \text { DF } \\ 5.6 & & 1 \\ 37 & & 1 \\ 205 & & 10 \\ <9.9 & 1 \\ <0.050 & 1 \\ 1.130 & 1 \\ 1,460 & 1 \\ <2.0 & 1 \\ <2.0 & 1 \\ 19 & 1 \\ 22 & 1 \\ <1.0 & 1 \\ <1.0 & 1 \\ <1.0 & 1 \\ <1.0 & 1 \\ <2.0 & 1 \\ <2.0 & 1 \\ 2,590 & 1 \\ 2,590 & 1\end{array}$

Time: 10:05

pH: 5.1

Alkalinity: $0 \mathrm{mg} / \mathrm{L}$

Water temperature: $18.4^{\circ} \mathrm{C}$

Volumes purged: 0.3 well volumes

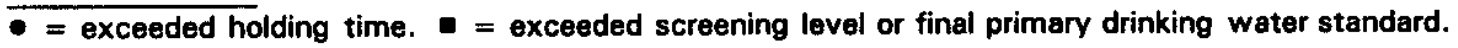


WELL BGX $11 D$ collected on 04/19/94, laboratory analyses (cont.)

H D Analyte

Carbon tetrachloride

Chloride

Chlorobenzene

Chloroethane

Chioroethene (Vinyl chloride)

2-Chloroethyl vinyl ether

Chloroform

Chloromethane (Methyl chloride)

Chromium, total recoverable

Chromium, total recoverable

Copper, total recoverable

Copper, total recoverable

Cyanide

Cyanide

p.p'-DDT

Dibromochloromethane

1,1-Dichloroethane

1,2-Dichloroethane

1,1-Dichloroethylene

trans-1,2-Dichloroethylene

Dichloromethane (Methylene chloride)

2,4-Dichlorophenoxyacetic acid

1,2-Dichloropropane

cis-1,3-Dichloropropene

trans-1,3-Dichloropropene

Dieldrin

Endrin

Ethylbenzene

Fluoride

Heptachlor

Iron, total recoverable

Iron, total recoverable

Lead, total recoverable

Lindane

Magnesium, total recoverable

Magnesium, total recoverable

Manganese, total recoverable

Manganese, total recoverable

Mercury, total recoverable

Mercury, total recoverable

Methoxychlor

Naphthalene

Nickel, total recoverable

Nickel, total recoverable

Nitrate-nitrite as nitrogen

Phenols

Potassium, total recoverable

Potassium, total recoverable

Selenium, total recoverable

Silica, total recoverable

Silica, total recoverable

Silver, total recoverable

Silver, total recoverable

Sodium, total recoverable

Sodium, total recoverable

Sulfate

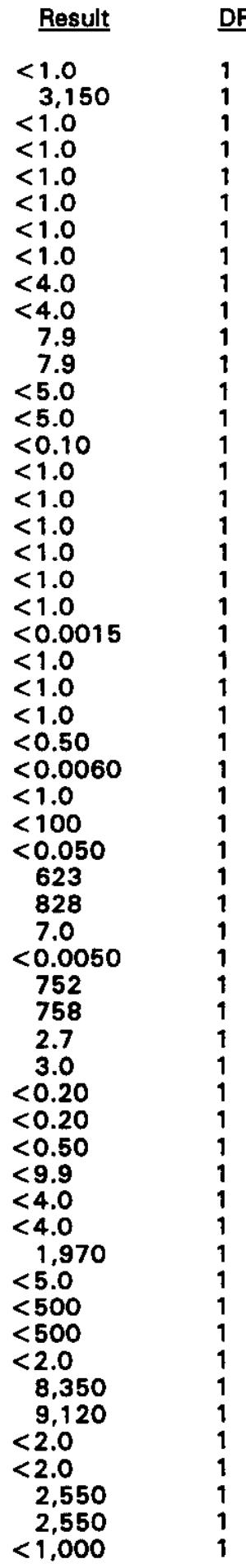

- = exceeded holding time. $\square=$ exceeded screening level or final primary drinking water standard. 
WELL BGX 11D collected on 04/19/94, laboratory analyses (cont.)

$\begin{array}{ll}\text { H. } & \text { Analyte } \\ \text { 1,1,2,2-Tetrachloroethane } \\ \text { Tetrachloroethylene } \\ \text { Tin, total recoverable } \\ \text { Tin, total recoverable } \\ \text { Toluene } \\ \text { Total dissolved solids } \\ \text { Total organic carbon } \\ \text { Total organic halogens } \\ \text { Total phosphates las P) } \\ \text { Toxaphene } \\ 2,4,5-T P \text { (Silvex) } \\ \text { 1,1,1-Trichloroethane } \\ \text { 1,1,2-Trichloroethane } \\ \text { Trichloroethylene } \\ \text { Trichlorofluoromethane } \\ 2,4,5-T \\ \text { Vanadium, total recoverable } \\ \text { Vanadium, total recoverable } \\ \text { Xylenes } \\ \text { Gross alpha } \\ \text { Nonvolatile beta } \\ \text { Radium, total alpha-emitting } \\ \text { Tritium }\end{array}$

\begin{tabular}{ll}
\multicolumn{1}{r}{ Result } & D \\
\hline$<1.0$ & 1 \\
$<1.0$ & 1 \\
$<2.0$ & 1 \\
$<2.0$ & 1 \\
$<1.0$ & 1 \\
30,000 & 1 \\
$<1.000$ & 1 \\
$<5.0$ & 1 \\
360 & 1 \\
$<0.24$ & 1 \\
$<0.00046$ & 1 \\
$<1.0$ & 1 \\
$<1.0$ & 1 \\
$<1.0$ & 1 \\
$<1.0$ & 1 \\
$<0.00046$ & 1 \\
$<8.0$ & 1 \\
$<8.0$ & 1 \\
$<2.0$ & 1 \\
$4.4 E+00$ & 1 \\
$8.6 E+00$ & 1 \\
$0.0 E+00$ & 1 \\
$2.1 E+01$ & 1
\end{tabular}

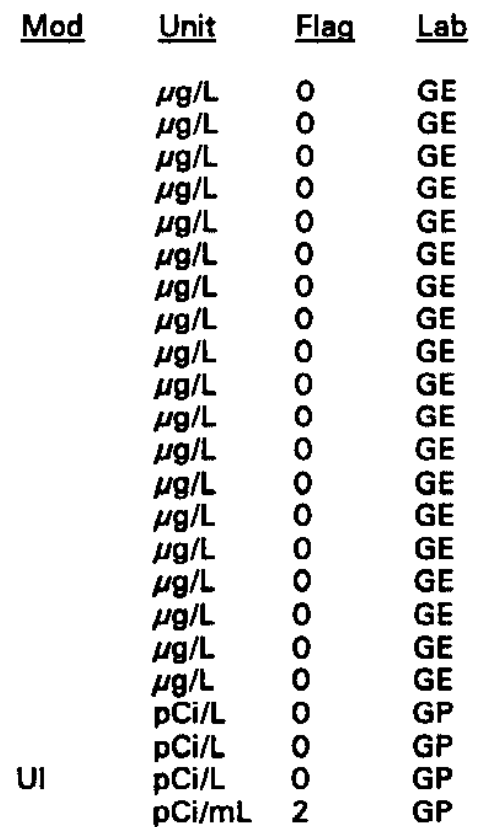

\section{WELL BGX $12 \mathrm{C}$}

\begin{tabular}{|c|c|c|c|c|c|c|}
\hline SRS Coord. & Lat/Longitude & Screen Zone Elevation & Top of Casing & Casing & Pump & Formation \\
\hline $\begin{array}{l}\text { N74427.9 } \\
\text { E59675.3 }\end{array}$ & $\begin{array}{l}33.287753^{\circ} \mathrm{N} \\
81.654237^{\circ} \mathrm{W}\end{array}$ & 184.1-174.1 ft mst & $275.1 \mathrm{ft} \mathrm{msl}$ & 4" PVC & $\mathbf{s}$ & Barnwell \\
\hline
\end{tabular}

\section{FIELD MEASUREMENTS}

Sample date: 04/13/94

Depth to water: $40.48 \mathrm{ft}(12.34 \mathrm{~m})$ below TOC

Water elevation: $234.62 \mathrm{ft}(71.51 \mathrm{~m}) \mathrm{ms}$ !

Sp. conductance: $39 \mu \mathrm{S} / \mathrm{cm}$

Turbidity: 0.9 NTU

Water evacuated before sampling: $107 \mathrm{gal}$
Time: 13:03

pH: 5.7

Alkalinity: $7 \mathrm{mg} / \mathrm{L}$

Water temperature: $19.5^{\circ} \mathrm{C}$

Volumes purged: 2.7 well volumes

\section{LABORATORY ANALYSES}

H D Analyte

$\bullet$

$\mathrm{pH}$
Specific conductance

Turbidity

Acetophenone

Aldrin

Aluminum, total recoverable

Antimony, total recoverable

Arsenic, total recoverable

Barium, total recoverable

Benzene

Bromodichloromethane

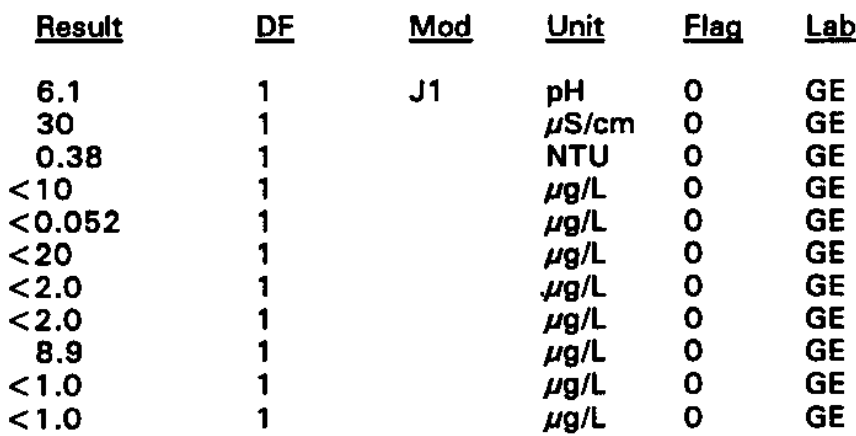

\footnotetext{
- = exceeded holding time. a = exceeded screening level or final primary drinking water standard.
} 
WELL BGX $12 \mathrm{C}$ collected on $04 / 13 / 94$, laboratory analyses (cont.)

\section{브}

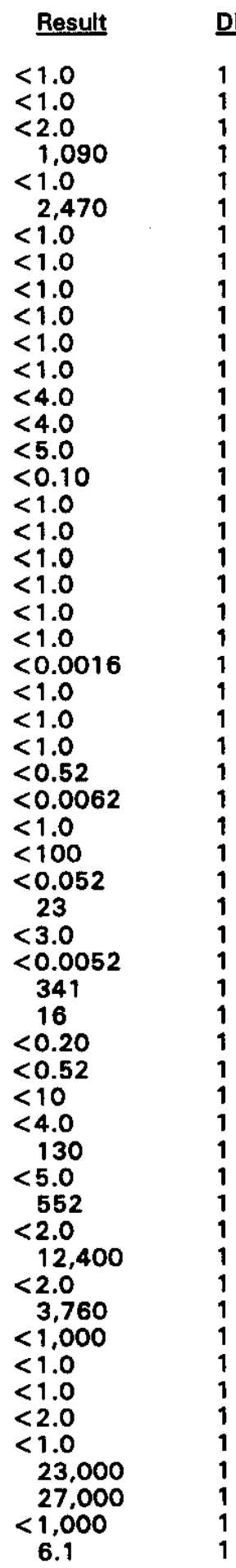

DF

$\underline{\text { Mod }}$

Unit Flag Lab

Bromoform

Bromomethane (Methyl bromide)

Cadmium, total recoverable

Calcium, total recoverable

Carbon tetrachloride

Chloride

Chlorobenzene

Chloroethane

Chloroethene (Vinyl chloride)

2-Chloroethyl vinyl ether

Chioroform

Chloromethane (Methyl chloride)

Chromium, total recoverable

Copper, total recoverable

Cyanide

p.p'-DDT

Dibromochloromethane

1,1-Dichloroethane

1,2-Dichloroethane

1,1-Dichloroethylene

trans-1,2-Dichloroethylene

Dichloromethane (Methylene chloride)

2,4-Dichlorophenoxyacetic acid

1,2-Dichloropropane

cis-1,3-Dichloropropene

trans-1,3-Dichloropropene

Dieldrin

Endrin

Ethylbenzene

Fluoride

Heptachlor

Iron, total recoverable

Lead, total recoverable

Lindane

Magnesium, total recoverable

Manganese, total recoverable

Mercury, total recoverable

Methoxychlor

Naphthalene

Nickel, total recoverable

Nitrate-nitrite as nitrogen

Phenols

Potassium, total recoverable

Selenium, total recoverable

Silica, total recoverable

Silver, total recoverable

Sodium, total recoverable

Sulfate

1,1,2,2-Tetrachloroethane

Tetrachloroethylene

Tin, total recoverable

Toluene

Total dissolved solids

Total dissolved solids

Total organic carbon

Total organic halogens

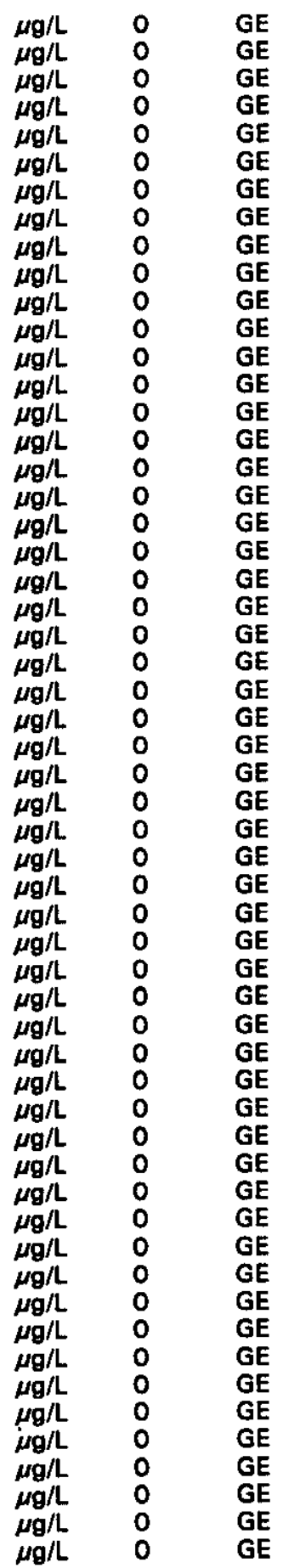

- = exceeded holding time. = exceeded screening level or final primary drinking water standard. 
WELL BGX 12C collected on 04/13/94, laboratory analyses (cont.)

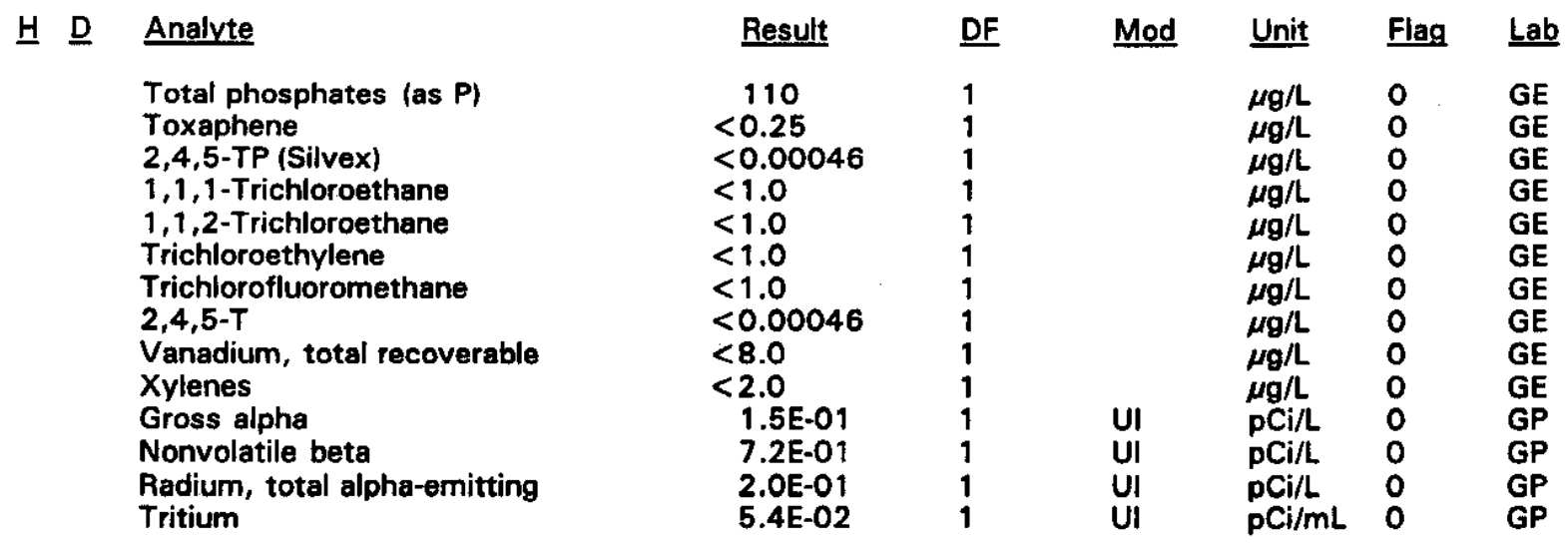

\section{WELL BGX 12D}

\begin{tabular}{|c|c|c|c|c|c|c|}
\hline SRS Coord. & Lat/Longitude & Screen Zone Elevation & Top of Casing & Casing & Pump & Formation \\
\hline $\begin{array}{l}\text { N74410.9 } \\
\text { E59674.3 }\end{array}$ & $\begin{array}{l}33.287714^{\circ} \mathrm{N} \\
81.654207^{\circ} \mathrm{W}\end{array}$ & $243.7-223.7 \mathrm{ft} \mathrm{msl}$ & $275.2 \mathrm{ft} \mathrm{msl}$ & 4" PVC & $\mathbf{S}$ & Water Table $\left(\mathrm{IB}_{2}\right)$ \\
\hline
\end{tabular}

\section{FIELD MEASUREMENTS}

Sample date: 04/14/94

Depth to water: $36.18 \mathrm{ft}(11.03 \mathrm{~m})$ below TOC

Water elevation: $239.02 \mathrm{ft}(72.85 \mathrm{~m}) \mathrm{msl}$

Sp. conductance: $24 \mu \mathrm{S} / \mathrm{cm}$

Turbidity: 17.4 NTU

Water evacuated before sampling: $9 \mathrm{gal}$

The well went dry during purging.

\section{LABORATORY ANALYSES}

H D Analyte

pH

Specific conductance

Turbidity

Acetophenone

Aldrin

Aluminum, total recoverable

Antimony, total recoverable

Arsenic, total recoverable

Barium, total recoverable

Benzene

Benzene

Bromodichloromethane

Bromodichloromethane

Bromoform

Bromoform

Bromomethane (Methyl bromide)

Bromomethane (Methyl bromide)

Cadmium, total recoverable

Calcium, total recoverable

Carbon tetrachloride
Time: 10:31

pH: 5.2

Alkalinity: $0 \mathrm{mg} / \mathrm{L}$

Water temperature: $19.2^{\circ} \mathrm{C}$

Volumes purged: 0.9 well volumes

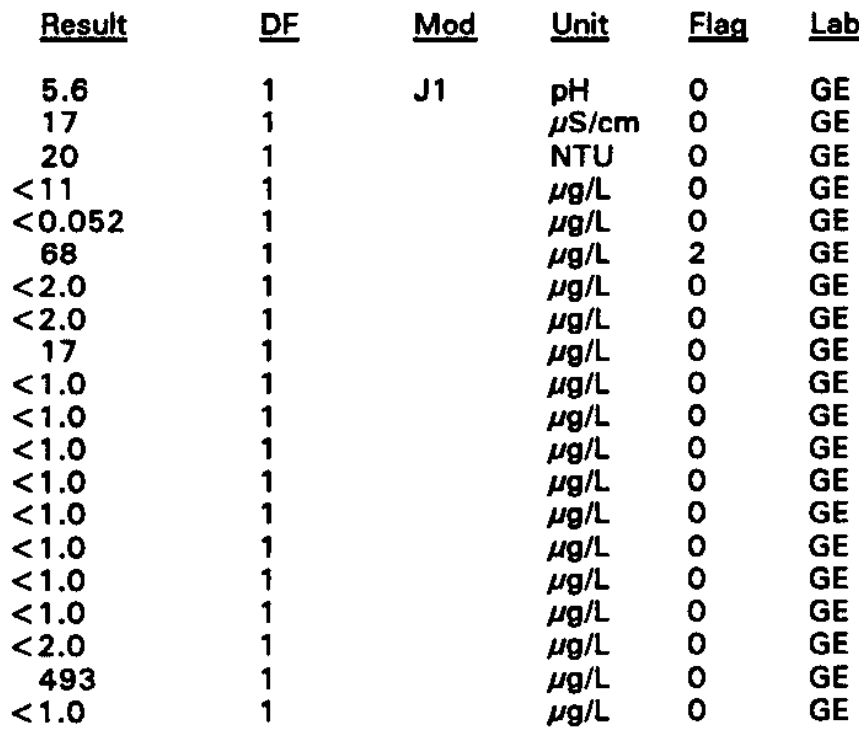

- = exceeded holding time. - = exceeded screening level or final primary drinking water standard. 
WELL BGX 120 collected on $04 / 14 / 94$, laboratory analyses (cont.)

H

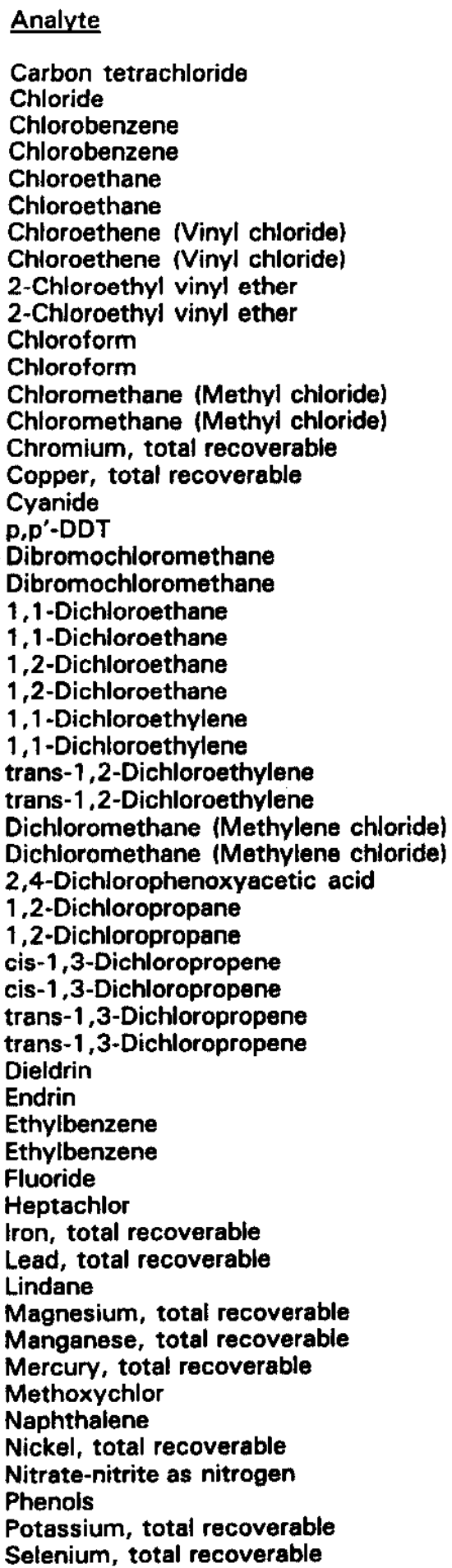

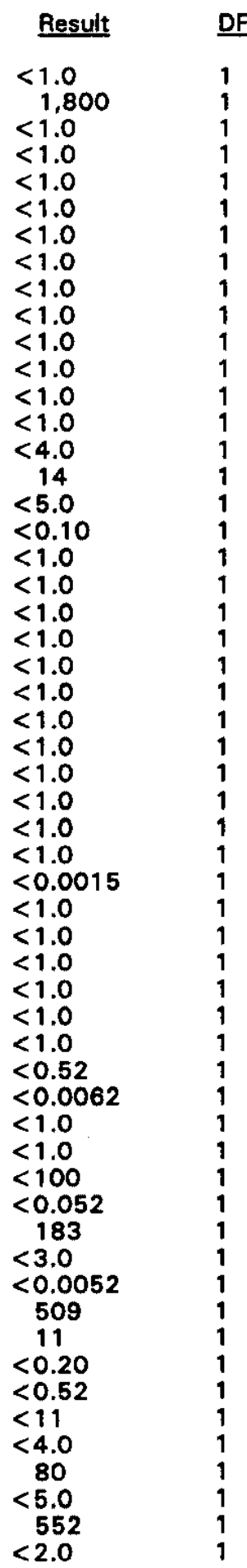

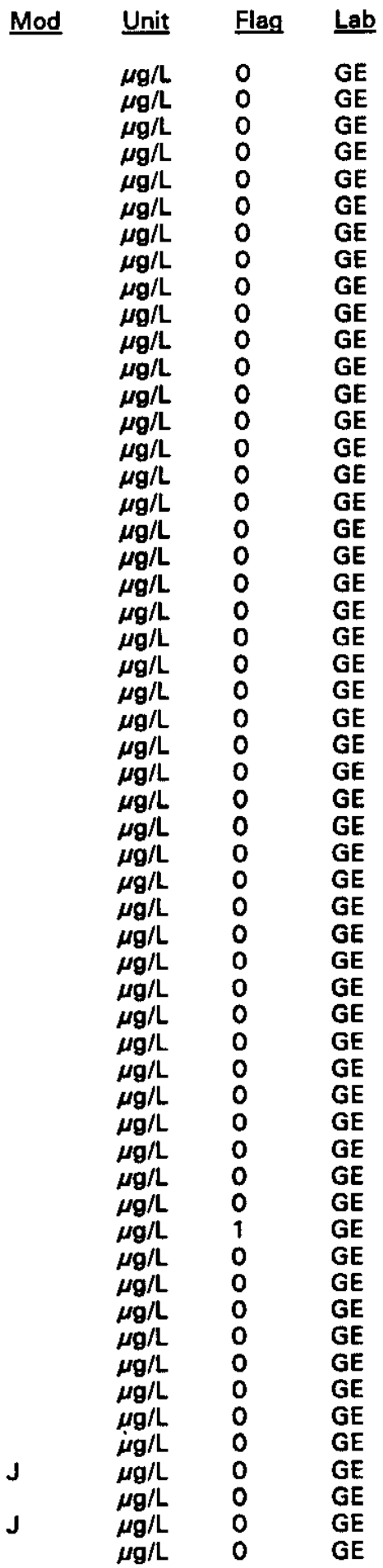

- = exceeded holding time. = exceeded screening level or final primary drinking water standerd. 
WELL BGX $12 D$ collected on $04 / 14 / 94$, laboratory analyses (cont.)

\section{$\underline{H} \underline{D}$ Analyte}

Silica, total recoverable

Silver, total recoverable

Sodium, total recoverable

Sulfate

1,1,2,2-Tetrachloroethane

$1,1,2,2$-Tetrachloroethane

Tetrachloroethylene

Tetrachloroethylene

Tin, total recoverable

Toluene

Toluene

Total dissolved solids

Total organic carbon

Total organic halogens

Total phosphates (as P)

Toxaphene

2,4,5-TP (Silvex)

1,1,1-Trichloroethane

$1,1,1$-Trichloroethane

1,1,2-Trichloroethane

1,1,2-Trichloroethane

Trichloroethylene

Trichloroethylene

Trichlorofluoromethane

Trichlorofluoromethane

2,4,5-T

Vanadium, total recoverable

Xylenes

Xylenes

Gross alpha

Nonvolatile beta

Radium, total alpha-emitting

- Tritium
Result DF

5,220

$<2.0$

1,210

$<1,000$

$<1.0$

$<1.0$

$<1.0$

$<1.0$

$<2.0$

$<1.0$

$<1.0$

20,000

$<1,000$

$<5.0$

$<50$

$<0.25$

$<0.00045$

$<1.0$

$<1.0$

$<1.0$

$<1.0$

$<1.0$

$<1.0$

$<1.0$

$<1.0$

$<0.00045$

$<8.0$

$<2.0$

$<2.0$

7.1E-01

$1.4 E+00$

4.0E-01

2.0E + 01
DF

Mod Unit Flag Lab

$\mu g / L \quad O \quad G E$

$\begin{array}{lll}\mu g / L & 0 & G E \\ \mu g / L & 0 & G E\end{array}$

$\mu g / L \quad 0 \quad G E$

$\mu \mathrm{g} / \mathrm{L} \quad 0 \quad \mathrm{GE}$

$\mu g / L \quad 0 \quad G E$

$\mu g / L \quad 0 \quad \mathrm{GE}$

$\mu \mathrm{g} / \mathrm{L} \quad \mathrm{O} \quad \mathrm{GE}$

$\mu \mathrm{g} / \mathrm{L} \quad \mathrm{O} \quad \mathrm{GE}$

$\mu \mathrm{g} / \mathrm{L} \quad 0 \quad \mathrm{GE}$

$\mu g / L \quad 0 \quad G E$

JV2 $\mu g / L \quad 0 \quad$ GE

$\mu \mathrm{g} / \mathrm{L} \quad 0 \quad \mathrm{GE}$

$\mu g / L \quad 0 \quad$ GE

$\mu g / L \quad 0 \quad \mathrm{GE}$

$\mu g / L \quad 0 \quad \mathrm{GE}$

$\mu g / L \quad 0 \quad G E$

$\mu g / L \quad 0 \quad \mathrm{GE}$

$\mu g / L \quad 0 \quad G E$

$\mu \mathrm{g} / \mathrm{L} \quad 0 \quad \mathrm{GE}$

$\mu g / L \quad 0 \quad G E$

$\mu g / L \quad O \quad G E$

$\mu g / L \quad O \quad G E$

$\mu \mathrm{g} / \mathrm{L} \quad \mathrm{O} \quad \mathrm{GE}$

$\mu g / L \quad 0 . \quad \mathrm{GE}$

$\mu \mathrm{g} / \mathrm{L} \quad \mathrm{O} \quad \mathrm{GE}$

$\mu \mathrm{g} / \mathrm{L} \quad \mathrm{O} \quad \mathrm{GE}$

$\mu g / L \quad 0 \quad$ GE

$\mu g / L \quad 0 \quad G E$

pCi/L 0 GP

$\mathrm{pCi} / \mathrm{L} \quad \mathrm{G} \quad \mathrm{GP}$

$\mathrm{pCi} / \mathrm{L} \quad 0 \quad \mathrm{GP}$

$\mathrm{pCi} / \mathrm{mL} 2 \mathrm{GP}$

- exceeded holding time. = exceeded screening level or final primary drinking water standard. 
WELL FSS 1D

$\begin{array}{lllllll}\text { SRS Coord. } & \text { Lat/Longitude } & \text { Screen Zone Elevation } & \text { Top of Casing } & \text { Casing } & \text { Pump } & \text { Formation } \\ \text { N75257.6 } & 33.280161^{\circ} \mathrm{N} & 229.9-209.9 \mathrm{ft} \mathrm{msl} & 266 \mathrm{ft} \mathrm{msl} & \text { 4" PVC } & \text { S } & \text { Water Table } \\ \text { E53897.6 } & 81.671063^{\circ} \mathrm{W} & & \mathbf{2 0} & & \end{array}$

\section{FIELD MEASUREMENTS}

Sample date: 04/27/94

Depth to water: $41.89 \mathrm{ft}(12.77 \mathrm{~m})$ below TOC

Water elevation: $224.11 \mathrm{ft}(68.31 \mathrm{~m}) \mathrm{msl}$

Sp. conductance: $81 \mu \mathrm{S} / \mathrm{cm}$

Turbidity: 27.9 NTU

Water evacuated before sampling: 5 gal

The well went dry during purging.

\section{LABORATORY ANALYSES}

\section{H D Analyte}

pH

Specific conductance

Specific conductance

Turbidity

Acetophenone

Aluminum, total recoverable

Antimony, total recoverable

Arsenic, total recoverable

Barium, total recoverable

Benzene

Bromodichloromethane

Bromoform

Bromomethane (Methyl bromide)

Cadmium, total recoverable

Calcium, total recoverable

Carbon tetrachloride

Chloride

Chlorobenzene

Chloroethane

Chloroethene (Vinyl chloride)

2-Chloroethyl vinyl ether

Chloroform

Chloromethane (Methyl chloride)

Chromium, total recoverable

Copper, total recoverable

Cyanide

Dibromochloromethane

1,1-Dichloroethane

1,2-Dichloroethane

1,1-Dichloroethylene

trans-1,2-Dichloroethylene

Dichloromethane (Methylene chloride)

2,4-Dichlorophenoxyacetic acid

2,4-Dichlorophenoxyacetic acid

1,2-Dichloropropane

cis-1,3-Dichloropropene

trans-1,3-Dichloropropene

Endrin

Ethylbenzene
Time: 10:05

$\mathrm{pH}: \mathbf{5 . 8}$

Alkalinity: $23 \mathrm{mg} / \mathrm{L}$

Water temperature: $18.9^{\circ} \mathrm{C}$

Volumes purged: 0.5 well volumes

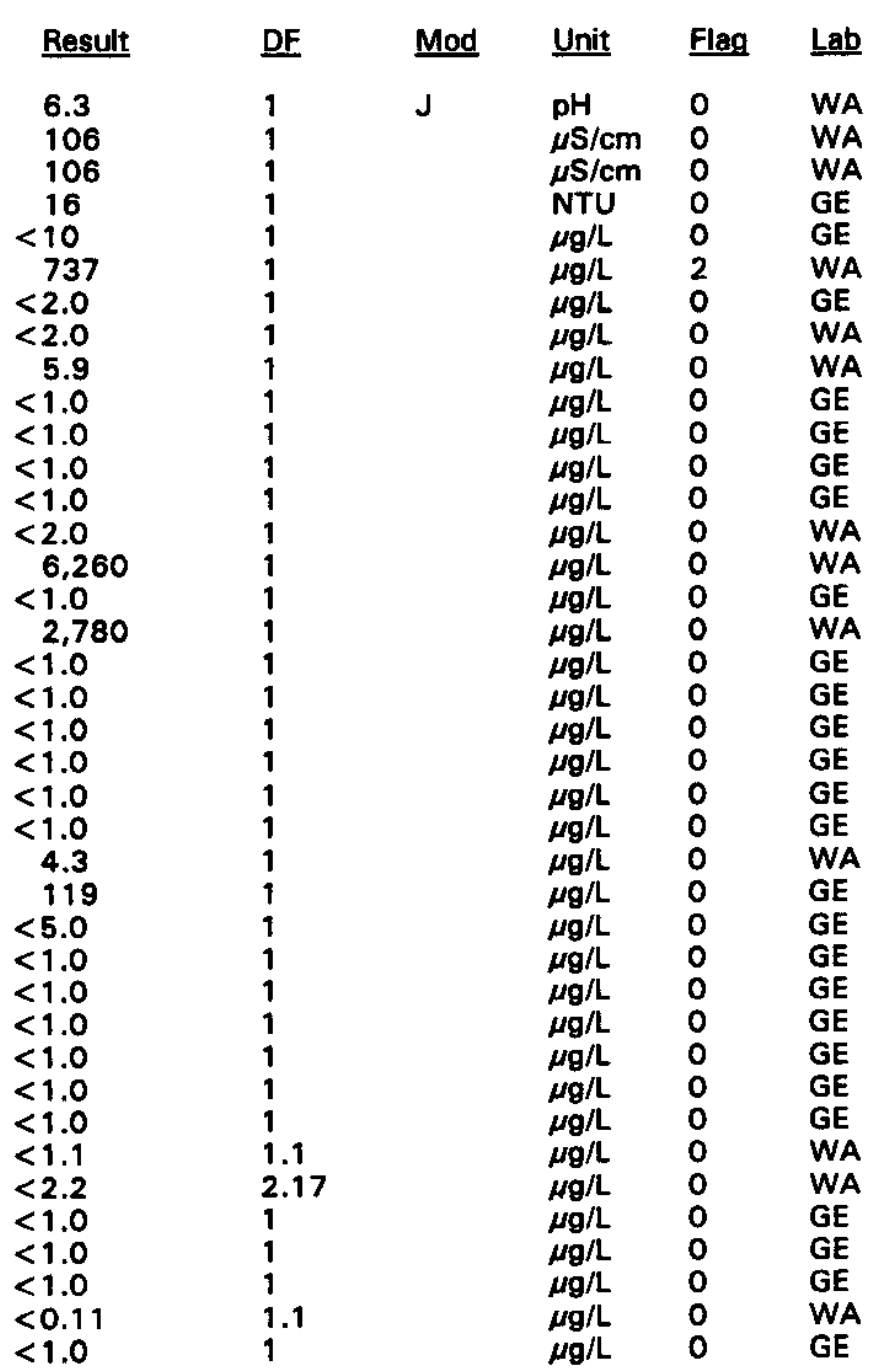

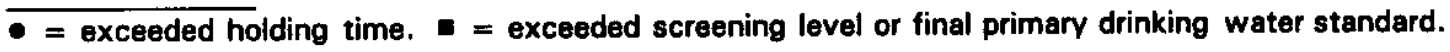


WELL FSS 1D collected on 04/27/94, laboratory analyses (cont.)

브 Analvte

Fluoride

Iron, total recoverable

Lead, total recoverable

Lindane

Magnesium, total recoverable

Manganese, total recoverable

Mercury, total recoverable

Methoxychlor

Naphthalene

Nickel, total recoverable

Nitrate as nitrogen

Phenols

Potassium, total recoverable

Selenium, total recoverable

Silica, total recoverable

Silver, total recoverable

Sodium, total recoverable

Sulfate

Sulfate

1,1,2,2-Tetrachloroethane

Tetrachloroethylene

Tin, total recoverable

Toluene

Total dissolved solids

Total organic carbon

Total organic halogens

Total phosphates (as P)

Toxaphene

2,4,5-TP (Silvex)

2,4,5-TP (Silvex)

Tributyl phosphate

1,1,1-Trichloroethane

1,1,2-Trichloroethane

Trichloroethylene

Trichlorofluoromethane

Vanadium, total recoverable

Xylenes

Gross alpha

Gross alpha

Nonvolatile beta

Nonvolatile beta

Radium-226

Radium-226

Radium-228

Radium-228

Tritium

Tritium

Uranium-233/234

Uranium-235

Uranium-238

Result DF

$<100$

845

16

$<0.055$

326

17

$<0.20$

$<0.55$

$<10$

$<4.0$

312

$<5.0$

$<500$

$<2.0$

4,270

$<2.0$

1,380

1,720

1,720

$<1.0$

$<1.0$

$<2.0$

$<1.0$

63,000

$<1,000$

$<5.0$

55

$<1.1$

$<1.1$

$<0.55$

$<10$

$<1.0$

$<1.0$

$<1.0$

$<1.0$

$<8.0$

$<2.0$

$1.6 \mathrm{E}+00$

2.4E + 00

$2.8 E+00$

$2.3 E+00$

4.4E-01

2.5E-01

8.0E-01

3.0E-01

9.9E + 00

1. $O E+01$

3.7E-02

3.3E-02

5. OE-02

1

1

1
1
1

1

1
1
1

1

1
1
1

1
1

1

1

1
1
1
1
1

1
1
1
1

DF

1

1

1.1

1

1.1

1

2.1

1

1

1

1

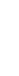

1

1

1

1

1

1

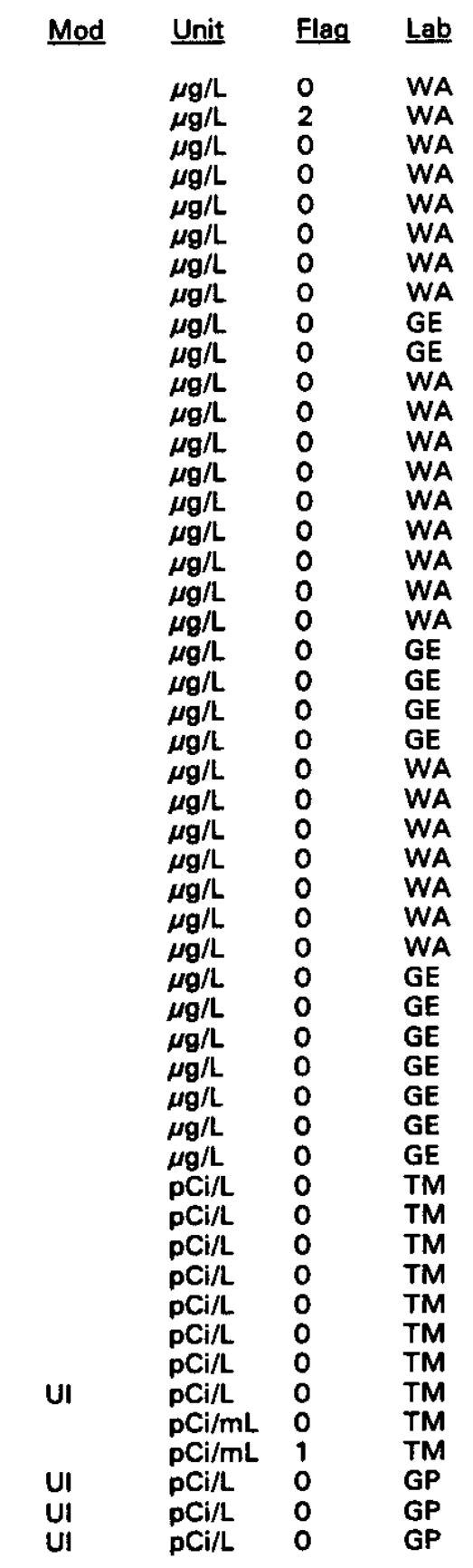

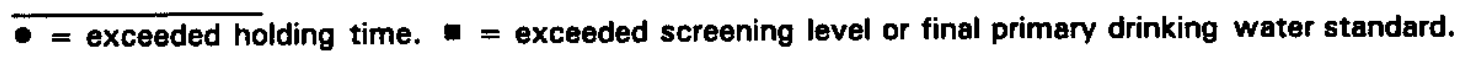


WELL FSS 2D

\begin{tabular}{|c|c|c|c|c|c|c|}
\hline SRS Coord. & Lat/Longitude & Screen Zone Elevation & Top of Casing & Casing & Pump & Formation \\
\hline $\begin{array}{l}\text { N75103.5 } \\
\text { E53918.9 }\end{array}$ & $\begin{array}{l}33.279855^{\circ} \mathrm{N} \\
81.670708^{\circ} \mathrm{W}\end{array}$ & $224.4-204.4 \mathrm{ft} \mathrm{ms}$ & $261.6 \mathrm{ft} \mathrm{msl}$ & 4" PVC & $\mathbf{S}$ & Water Table \\
\hline
\end{tabular}

\section{FIELD MEASUREMENTS}

Sample date: 04/27/94

Depth to water: $38.07 \mathrm{ft}(11.60 \mathrm{~m})$ below TOC

Water elevation: $223.53 \mathrm{ft}(68.13 \mathrm{~m}) \mathrm{msl}$

Sp. conductance: $146 \mu \mathrm{S} / \mathrm{cm}$

Turbidity: 48.8 NTU

Water evacuated before sampling: 12 gal

The well went dry during purging.

\section{LABORATORY ANALYSES}

H D Analyte

- $\quad \mathrm{pH}$

Specific conductance

Turbidity

Acetophenone

Aluminum, total recoverable

Antimony, total recoverable

Arsenic, total recoverable

Barium, total recoverable

Benzene

Bromodichloromethane

Bromoform

Bromomethane (Methyl bromide)

Cadmium, total recoverable

Calcium, total recoverable

Carbon tetrachloride

Chloride

Chlorobenzene

Chloroethane

Chloroethene (Vinyl chloride)

2-Chloroethyl vinyl ether

Chloroform

Chloromethane (Methyl chloride)

Chromium, total recoverable

Copper, total recoverable

Cyanide

Dibromochloromethane

1,1-Dichloroethane

1,2-Dichloroethane

1,1-Dichloroethylene

trans-1,2-Dichloroethylene

Dichloromethane (Methylene chloride)

2,4-Dichlorophenoxyacetic acid

1,2-Dichloropropane

cis-1,3-Dichloropropene

trans-1,3-Dichloropropene

Endrin

Ethylbenzene

Fluoride

Iron, total recoverable

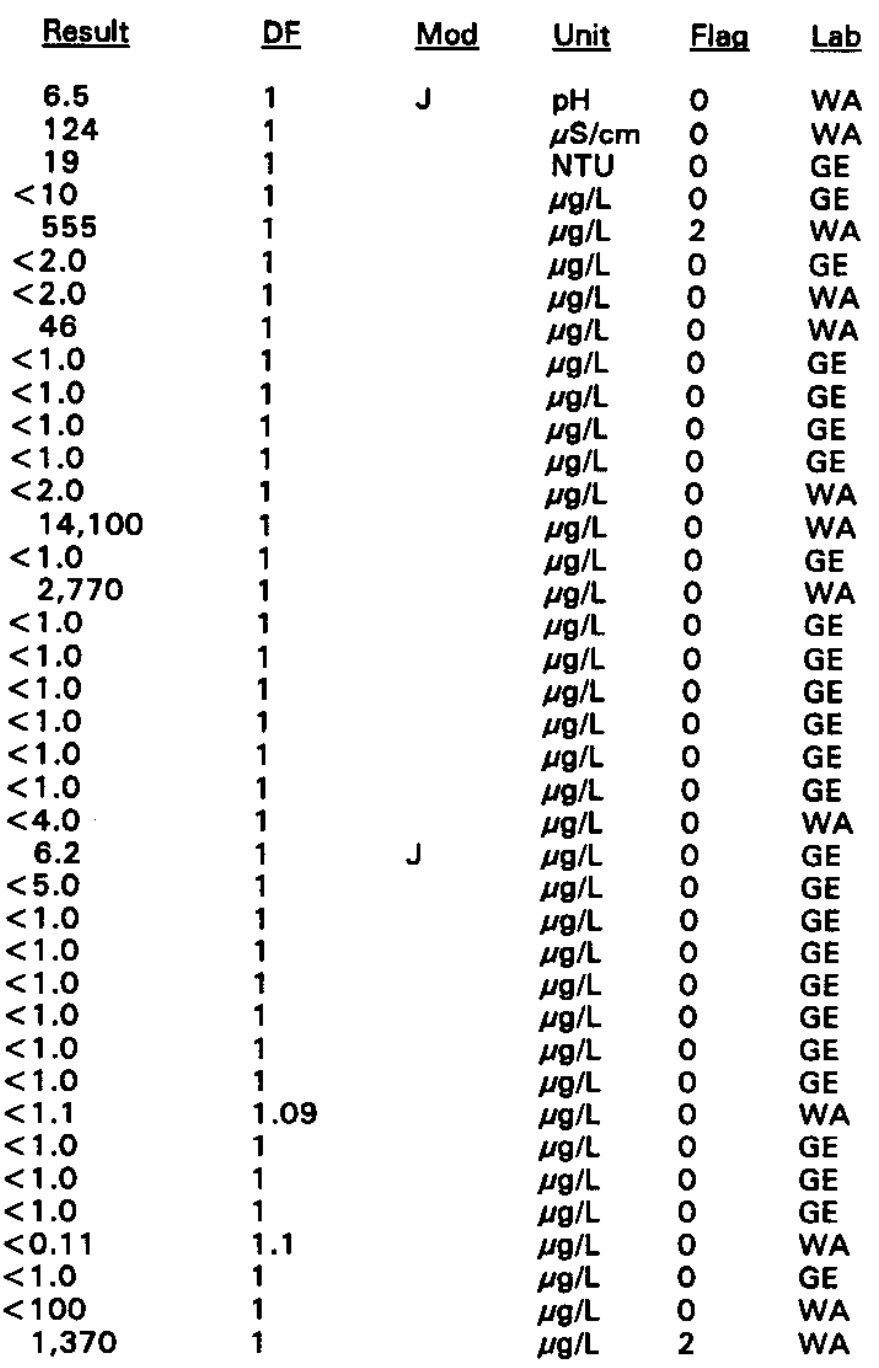

Time: 9:31

pH: 6.5

Alkalinity: $38 \mathrm{mg} / \mathrm{L}$

Water temperature: $20.3^{\circ} \mathrm{C}$

Volumes purged: 1.0 well volumes 
WELL FSS 20 coliected on $04 / 27 / 94$, laboratory analyses (cont.)

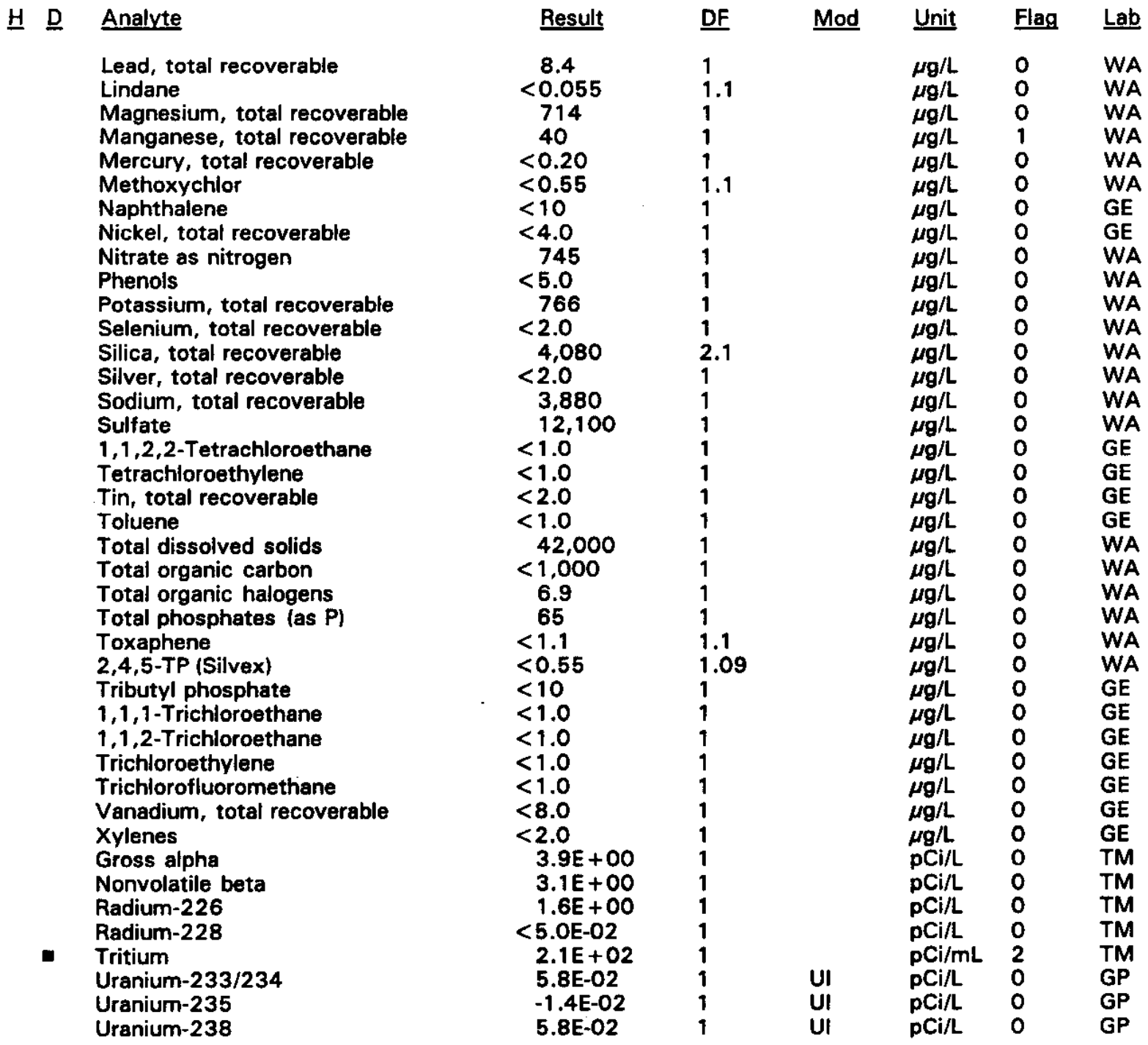

- = exceeded holding time. $=$ exceeded screening level or final primary drinking water standard. 


\section{WELL FSS 3D}

\begin{tabular}{|c|c|c|c|c|c|c|}
\hline SRS Coord. & Lat/Longitude & Screen Zone Elevation & Top of Casing & Casing & Pump & Formation \\
\hline $\begin{array}{l}\text { N74960.5 } \\
\text { E53548.0 }\end{array}$ & $\begin{array}{l}33.278933^{\circ} \mathrm{N} \\
81.671406^{\circ} \mathrm{W}\end{array}$ & $225.8-205.8 \mathrm{ft} \mathrm{msl}$ & $258.2 \mathrm{ft} \mathrm{msl}$ & 4" PVC & $\mathbf{S}$ & \\
\hline
\end{tabular}

\section{FIELD MEASUREMENTS}

Sample date: 04/27/94

Depth to water: $36.92 \mathrm{ft}(11.25 \mathrm{~m})$ below TOC

Water elevation: $221.28 \mathrm{ft}(67.45 \mathrm{~m}) \mathrm{ms} /$

Sp. conductance: $66 \mu \mathrm{S} / \mathrm{cm}$

Turbidity: 26.0 NTU

Water evacuated before sampling: 8 gal

The well went dry during purging.

Time: 8:57

pH: 5.2

Alkalinity: $2 \mathrm{mg} / \mathrm{L}$

Water temperature: $19.2{ }^{\circ} \mathrm{C}$

Volumes purged: 0.8 well volumes

\section{LABORATORY ANALYSES}

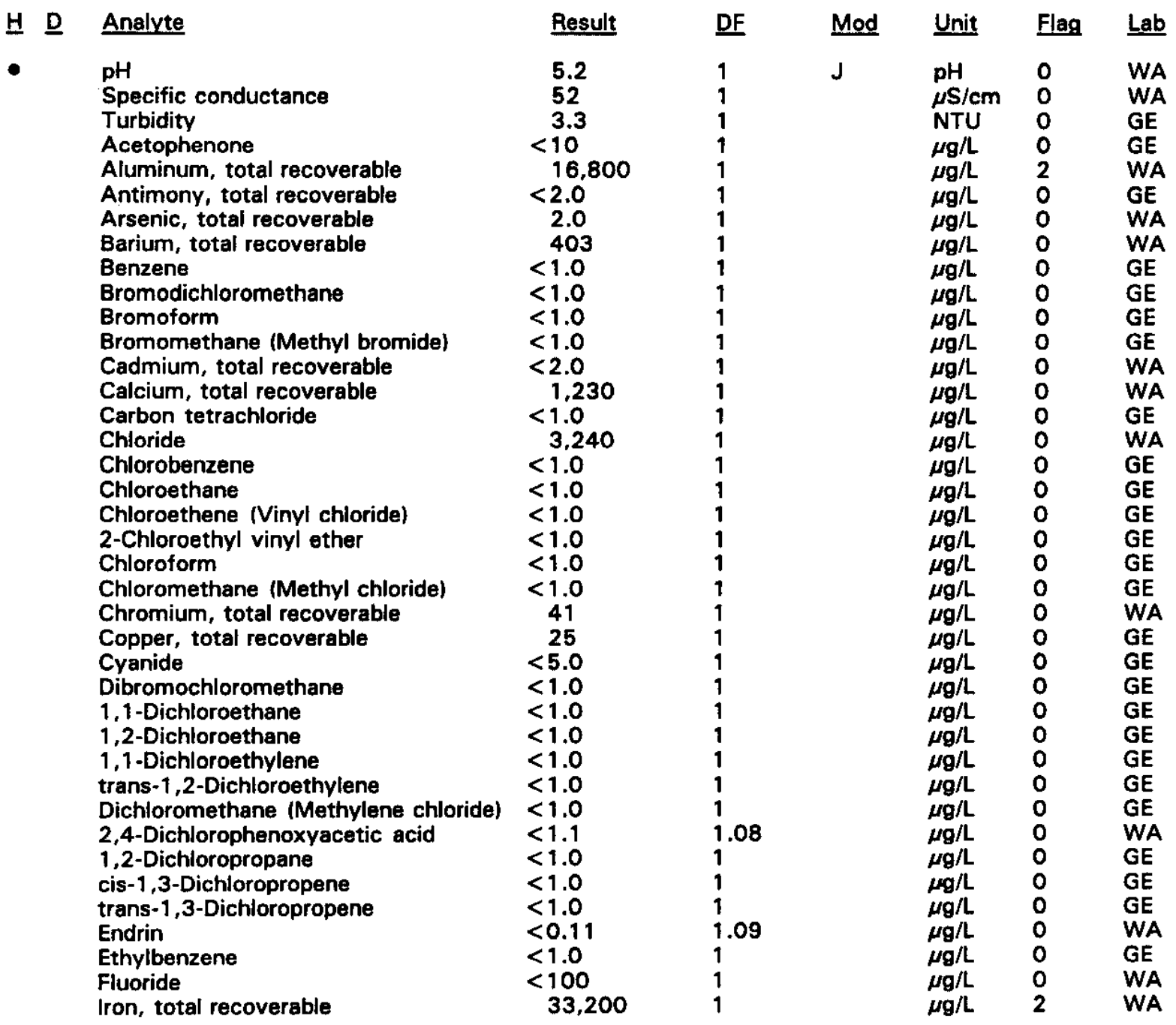

$\overline{- \text { = exceeded holding time. }}=$ exceeded screening level or final primary drinking water standard. 
WELL. FSS 3D collected on $04 / 27 / 94$, laboratory analyses (cont.)

H

Analyte
Lead, total recoverable
Lindane
Magnesium, total recoverable
Manganese, total recoverable
Mercury, total recoverable
Methoxychlor
Naphthalene
Nickel, total recoverable
Nitrate as nitrogen
Phenols
Potassium, total recoverable
Selenium, total recoverable
Silica, total recoverable
Silver, total recoverable
Sodium, total recoverable
Sulfate
1,1,2,2-Tetrachloroethane
Tetrachloroethylene
Tin, total recoverable
Toluene
Total dissolved solids
Total dissolved solids
Total organic carbon
Total organic halogens
Total phosphates (as P)
Toxaphene
$2,4,5-T P$ (Silvex)
Tributyl phosphate
1,1,1-Trichloroethane
1,1,2-Trichloroethane
Trichloroethylene
Trichlorofluoromethane
Vanadium, total recoverable
Xylenes
Gross alpha
Nonvolatile beta
Radium-226
Radium-228
Tritium
Uranium-233/234
Uranium-235
Uranium-238
Trum

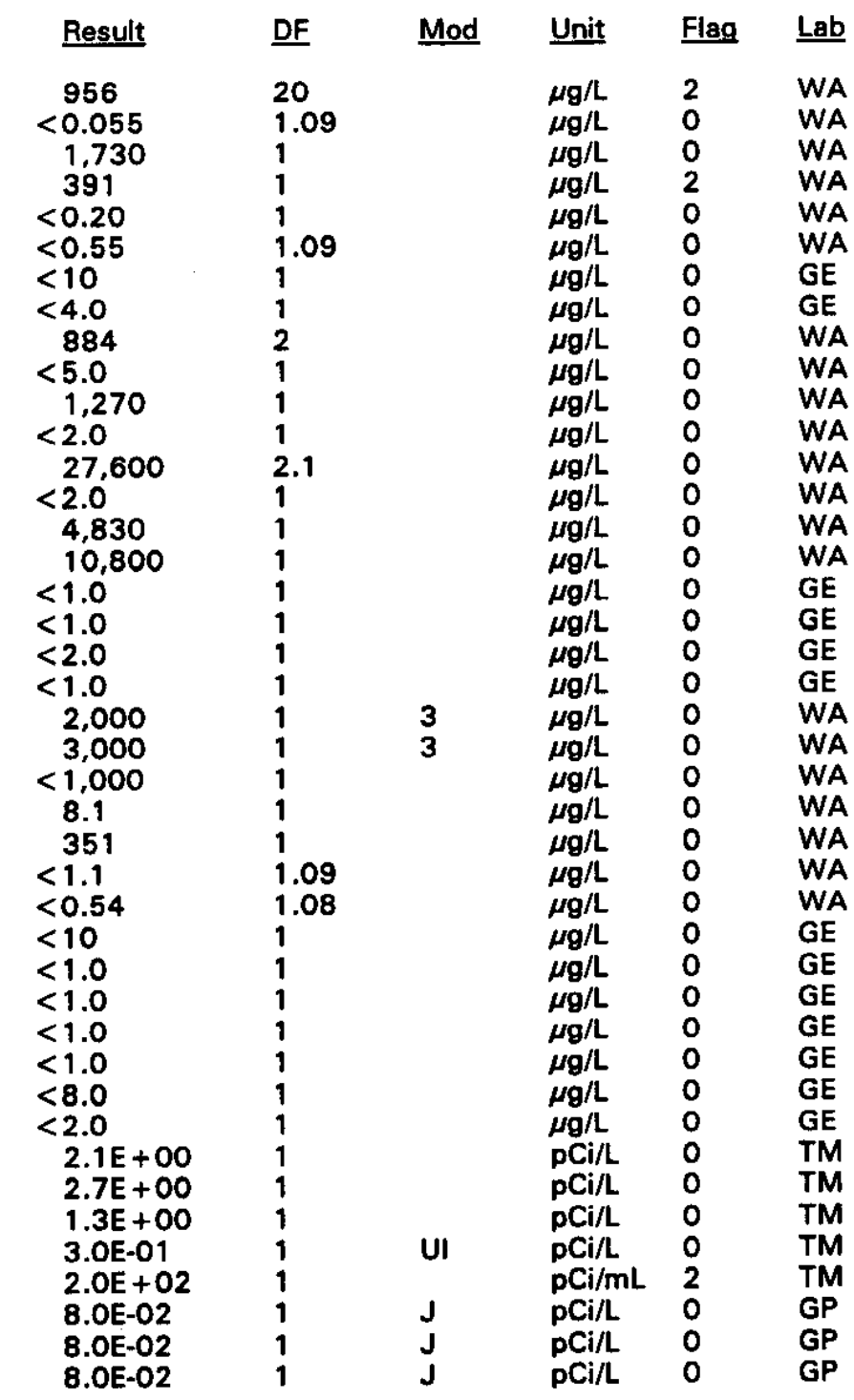

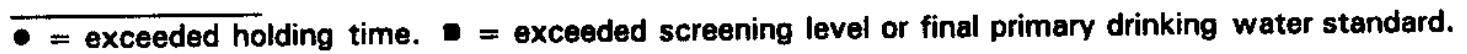


WELL FSS 4D

\begin{tabular}{|c|c|c|c|c|c|c|}
\hline SRS Coord. & Lat/Longitude & Screen Zone Elevation & Top of Casing & Casing & Pump & Formation \\
\hline $\begin{array}{l}N 75537.8 \\
E 52876.1\end{array}$ & $\begin{array}{l}33.279114^{\circ} \mathrm{N} \\
81.674297^{\circ} \mathrm{W}\end{array}$ & $222.6-202.6 \mathrm{ft} \mathrm{msl}$ & $291.8 \mathrm{ft} \mathrm{msl}$ & 4" PVC & s & Water Ta \\
\hline
\end{tabular}

\section{FIELD MEASUREMENTS}

Sample date: 04/27/94

Depth to water: $71.83 \mathrm{ft}(21.89 \mathrm{~m})$ below TOC

Water elevation: $219.97 \mathrm{ft}(67.05 \mathrm{~m}) \mathrm{msl}$

Sp. conductance: $54 \mu \mathrm{S} / \mathrm{cm}$

Turbidity: $31.0 \mathrm{NTU}$

Water evacuated before sampling: $13 \mathrm{gal}$

The well went dry during purging.

\section{LABORATORY ANALYSES}

\section{H D Analyte}

- $\quad \mathrm{pH}$

Specific conductance
Turbidity
Turbidity
Acetophenone
Aluminum, total recoverable
Antimony, total recoverable
Arsenic, total recoverable
Barium, total recoverable
Benzene
Bromodichloromethane
Bromoform
Bromomethane (Methyl bromide)
Cadmium, total recoverable
Calcium, total recoverable
Carbon tetrachloride
Chloride
Chlorobenzene
Chloroethane
Chloroethene (Vinyl chloride)
2-Chloroethyl vinyl ether
Chloroform
Chloromethane (Methyl chloride)
Chromium, total recoverable
Copper, total recoverable
Cyanide
Dibromochloromethane
1,1-Dichloroethane
1,2-Dichloroethane
1,1-Dichloroethylene
trans-1,2-Dichloroethylene
Dichloromethane (Methylene chloride)
2,4-Dichlorophenoxyacetic acid
1,2-Dichloropropane
cis-1,3-Dichloropropene
trans-1,3-Dichloropropene
Endrin
Endrin
Ethylbenzene

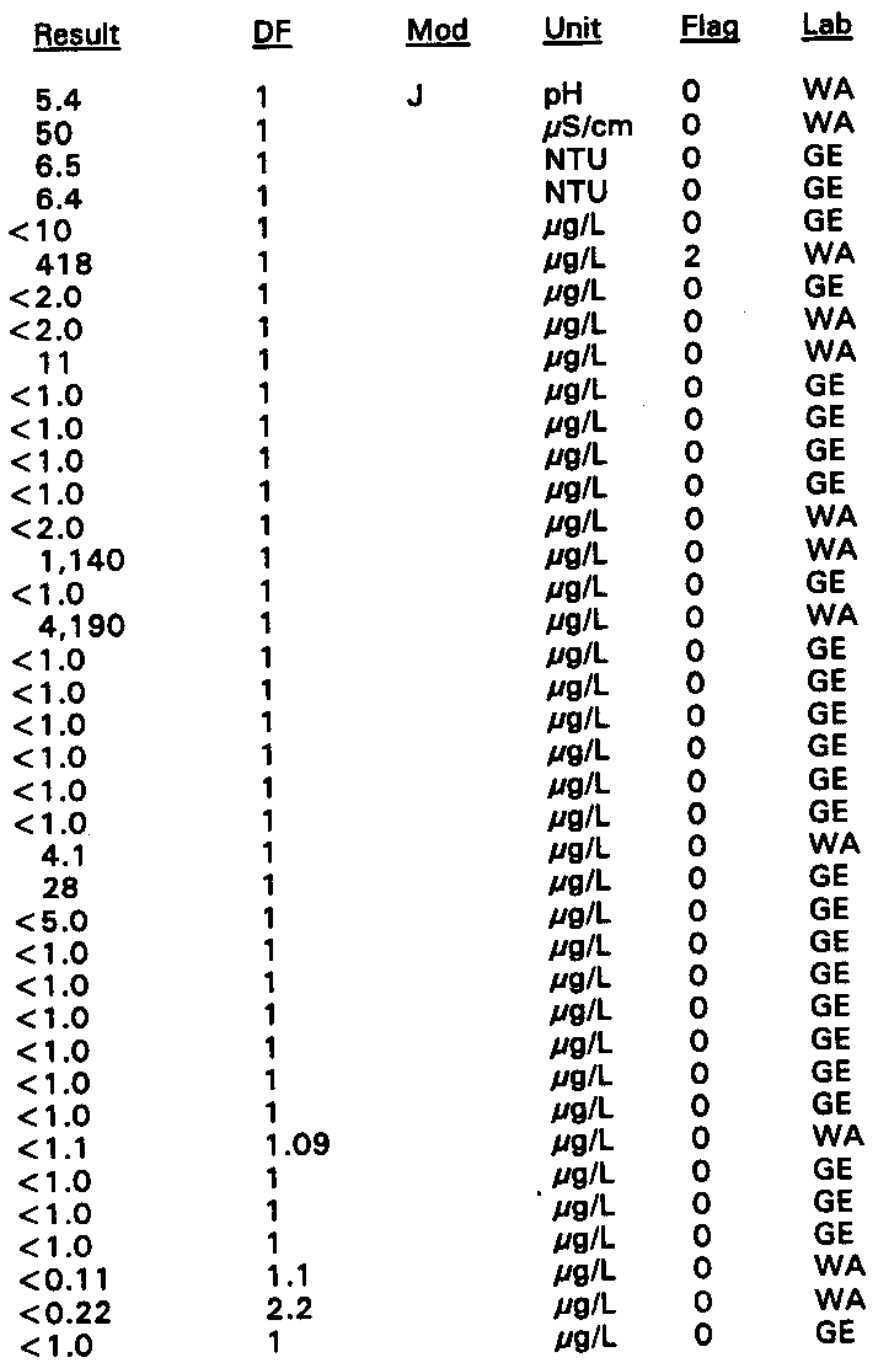

Time: 10:40

pH: 5.0

Alkalinity: $0 \mathrm{mg} / \mathrm{L}$

Water temperature: $20.5^{\circ} \mathrm{C}$

Volumes purged: 1.1 well volumes 
WELL FSS $4 D$ collected on 04/27/94, laboratory analyses (cont.)

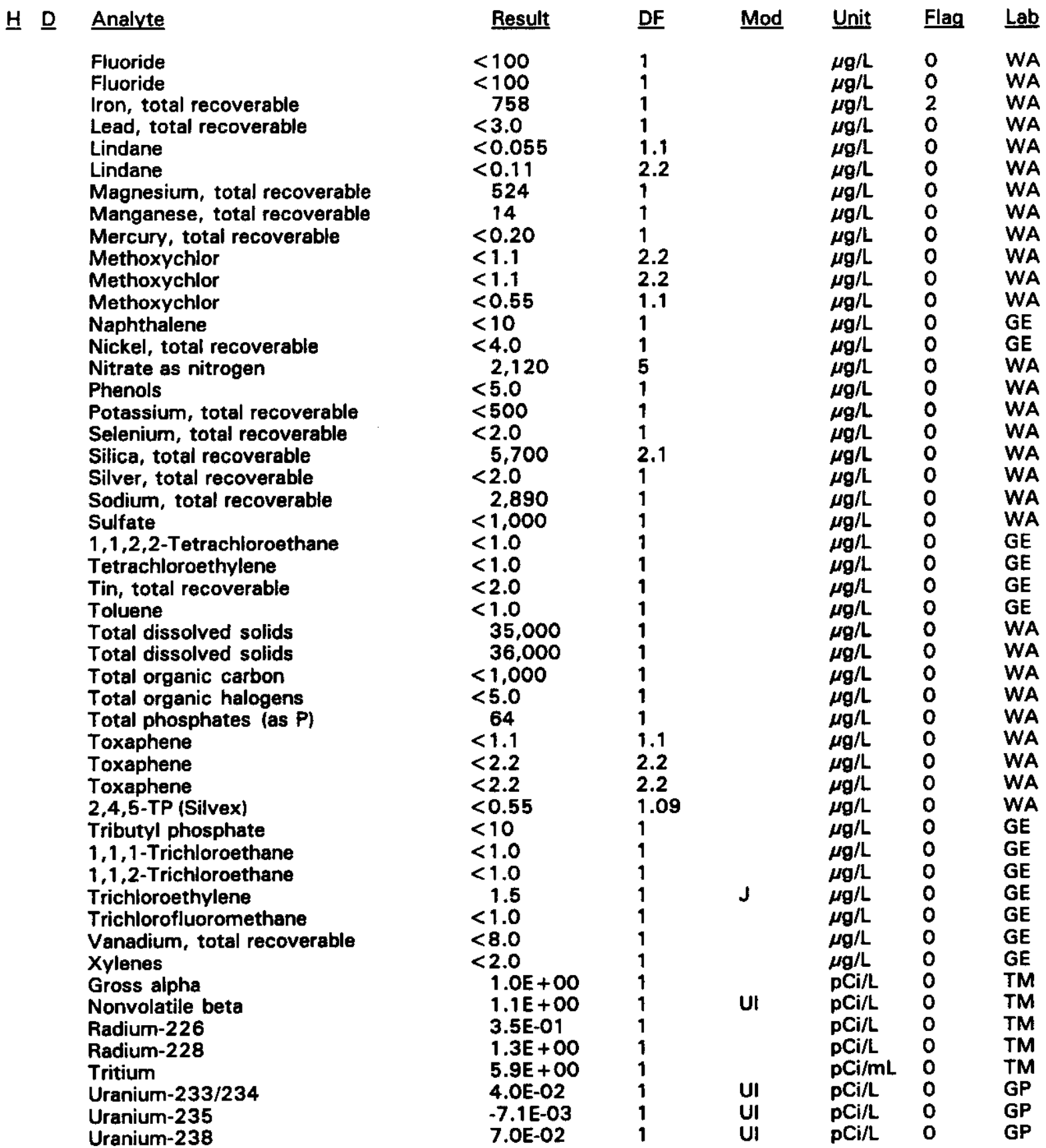

- = exceeded holding time. = exceeded screening level or final primary drinking water standard. 


\section{WELL HMD 1D}

\begin{tabular}{|c|c|c|}
\hline SRS Coord. & Lat/Longitude & Screen Zone E \\
\hline $\begin{array}{l}v 78731.7 \\
=56973.3\end{array}$ & $\begin{array}{l}33.292863^{\circ} \mathrm{N} \\
81.669714^{\circ} \mathrm{W}\end{array}$ & 219 \\
\hline \multicolumn{3}{|c|}{ FIELD MEASUREMENTS } \\
\hline \multicolumn{3}{|c|}{$\begin{array}{l}\text { Sample date: } 04 / 20 / 94 \\
\text { Depth to water: } 55.28 \mathrm{ft}(16.85 \mathrm{~m}) \text { below TOC } \\
\text { Water elevation: } 209.22 \mathrm{ft}(63.77 \mathrm{~m}) \mathrm{msl} \\
\text { Sp. conductance: } 95 \mu \mathrm{S} / \mathrm{cm} \\
\text { Turbidity: } 14.7 \mathrm{NTU} \\
\text { Water evacuated before sampling: } 4 \text { gal } \\
\text { The well went dry during purging. }\end{array}$} \\
\hline
\end{tabular}

Time: $9: 32$

pH: 6.2

Alkalinity: $28 \mathrm{mg} / \mathrm{L}$

Water temperature: $16.9^{\circ} \mathrm{C}$

Volumes purged: 0.6 well volumes

\section{LABORATORY ANALYSES}

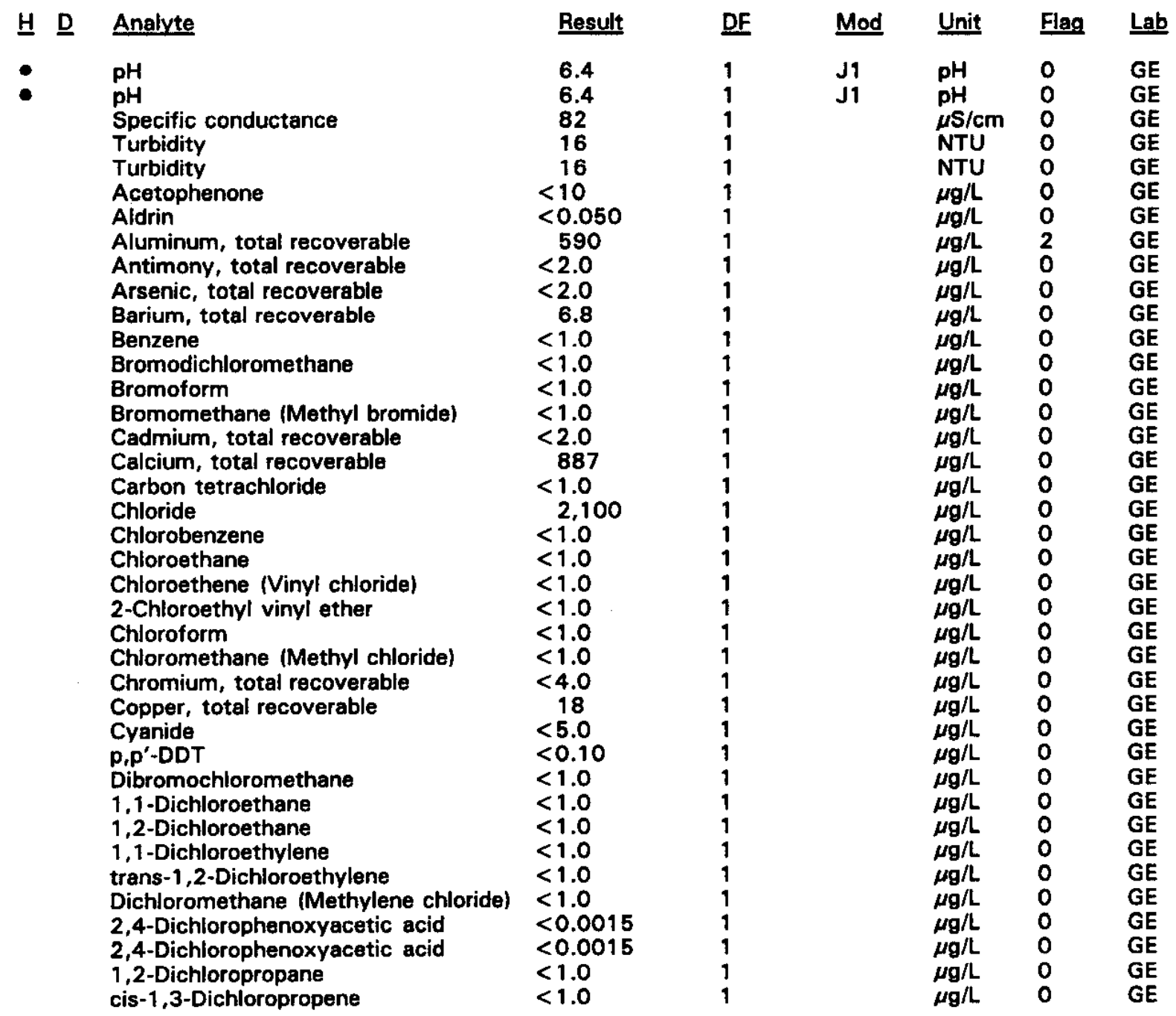

$\overline{-~=~ e x c e e d e d ~ h o l d i n g ~ t i m e . ~}-$ = exceeded screening level or final primary drinking water standard. 
WELL HMD $1 D$ collected on $04 / 20 / 94$, laboratory analyses (cont.)

H $\underline{\text { Analyte }}$

trans-1,3-Dichloropropene

Dieldrin

Endrin

Ethylbenzene

Fluoride

Heptachlor

Iron, total recoverable

Lead, total recoverable

Lindane

Magnesium, total recoverable

Manganese, total recoverable

Mercury, total recoverable

Methoxychlor

Naphthalene

Nickel, total recoverable

Nitrate-nitrite as nitrogen

Phenols

Potassium, total recoverable

Selenium, total recoverable

Silica, total recoverable

Silver, total recoverable

Sodium, total recoverable

Sulfate

1,1,2,2-Tetrachloroethane

Tetrachloroethylene

Tin, total recoverable

Toluene

Total dissolved solids

Total organic carbon

Total organic halogens

Total phosphates (as P)

Toxaphene

2,4,5-TP (Silvex)

2,4,5-TP (Silvex)

1,1,1-Trichloroethane

1,1,2-Trichloroethane

Trichloroethylene

Trichlorofluoromethane

2,4,5-T

2,4,5-T

Vanadium, total recoverable

Xylenes

Gross alpha

Nonvolatile beta

Radium, total alpha-emitting

- Tritium

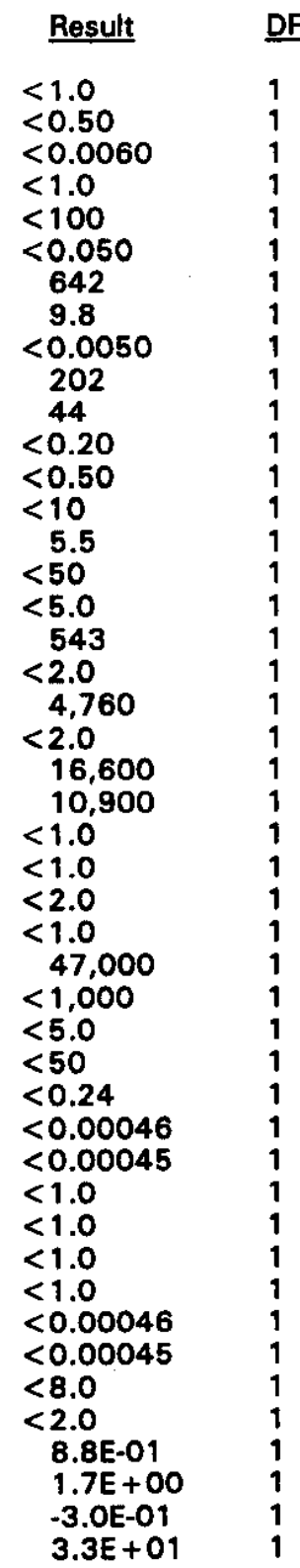

Mod Unit Flag Lab

$\mu g / L \quad O \quad G E$

$\mu \mathrm{g} / \mathrm{L} \quad 0 \quad \mathrm{GE}$

$\mu g / L \quad 0 \quad$ GE

$\mu g / L \quad 0 \quad$ GE

$\mu g / L \quad 0 \quad G E$

$\mu g / L \quad 0 \quad$ GE

$\mu g / L \quad 2 \quad G E$

$\mu g / L \quad 0 \quad G E$

$\mu g / L \quad 0 \quad$ GE

$\mu \mathrm{g} / \mathrm{L} \quad 0 \quad \mathrm{GE}$

$\mu g / L \quad 1 \quad G E$

$\mu \mathrm{g} / \mathrm{L} \quad 0 \quad \mathrm{GE}$

$\mu g / L \quad 0 \quad G E$

$\mu g / L \quad 0 \quad$ GE

$\mu \mathrm{g} / \mathrm{L} \quad 0 \quad \mathrm{GE}$

$\mu g / L \quad 0 \quad$ GE

$\mu g / L \quad 0 \quad G E$

$\mu \mathrm{g} / \mathrm{L} \quad 0 \quad \mathrm{GE}$

$\mu \mathrm{g} / \mathrm{L} \quad \mathrm{O} \quad \mathrm{GE}$

$\mu \mathrm{g} / \mathrm{L} \quad \mathrm{O} \quad \mathrm{GE}$

$\mu g / L \quad 0 \quad$ GE

$\mu \mathrm{g} / \mathrm{L} \quad \mathrm{O} \quad \mathrm{GE}$

$\mu \mathrm{g} / \mathrm{L} \quad \mathrm{O} \quad \mathrm{GE}$

$\mu g / L \quad O \quad G E$

$\mu \mathrm{g} / \mathrm{L} \quad 0 \quad \mathrm{GE}$

$\mu g / L \quad 0 \quad$ GE

$\mu g / L \quad 0 \quad$ GE

$\mu g / L \quad 0 \quad$ GE

$\mu g / L \quad 0 \quad$ GE

$\mu g / L \quad 0 \quad$ GE

$\mu g / L \quad 0 \quad$ GE

$\mu g / L \quad 0 \quad$ GE

$\mu \mathrm{g} / \mathrm{L} \quad 0, \mathrm{GE}$

$\mu g / L \quad 0 \quad$ GE

$\mu g / L \quad O \quad$ GE

$\mu g / L \quad 0 \quad$ GE

$\mu g / L \quad 0 \quad$ GE

$\mu g / L \quad 0 \quad$ GE

$\mu \mathrm{g} / \mathrm{L} \quad \mathrm{O} \quad \mathrm{GE}$

$\mu g / L \quad 0 \quad$ GE

$\mu \mathrm{g} / \mathrm{L} \quad \mathrm{O} \quad \mathrm{GE}$

$\mu \mathrm{g} / \mathrm{L} \quad \mathrm{O} \quad \mathrm{GE}$

$\mathrm{pCi} / \mathrm{L} \quad 0 \quad \mathrm{GP}$

$\mathrm{PCi} / \mathrm{L} \quad 0 \quad \mathrm{GP}$

pCi/L 0 GP

$\mathrm{pCi} / \mathrm{mL} 2 \mathrm{GP}$

- = exceeded holding time. $\square=$ exceeded screening level or final primary drinking water standard. 
WELL HMD 2D

$\begin{array}{lllllll}\text { SRS Coord, } & \text { Lat/Longitude } & \text { Screen Zone Elevation } & \text { Top of Casing } & \text { Casing } & \text { Pump Formation } & \text { Furnwell (IIB, }) \\ \text { N79665.8 } & 33.295413^{\circ} \mathrm{N} & 210.8-190.8 \mathrm{ft} \mathrm{msl} & 261.1 \mathrm{ft} \mathrm{msl} & \text { 4" PVC } & \text { S } & \text { Barnwell } \\ \text { E57269.7 } & 81.670748^{\circ} \mathrm{W} & & & & & \end{array}$

\section{FIELD MEASUREMENTS}

\section{Sample date: 04/15/94}

Depth to water: $61.29 \mathrm{ft}(18.68 \mathrm{~m})$ below TOC

Water elevation: $199.81 \mathrm{ft}(60.90 \mathrm{~m}) \mathrm{msl}$

Sp. conductance: $72 \mu \mathrm{S} / \mathrm{cm}$

Turbidity: 0.8 NTU

Water evacuated before sampling: $72 \mathrm{gal}$

\section{LABORATORY ANALYSES}

H $\underline{\text { Analyte }}$

\begin{tabular}{|c|}
\hline 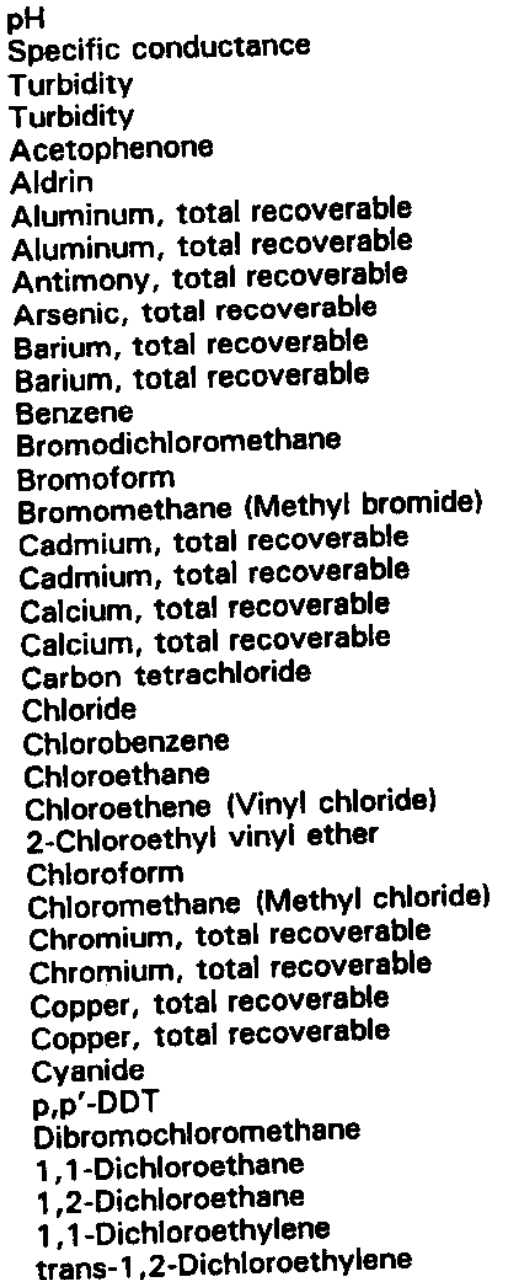 \\
\hline
\end{tabular}

\section{(n)}

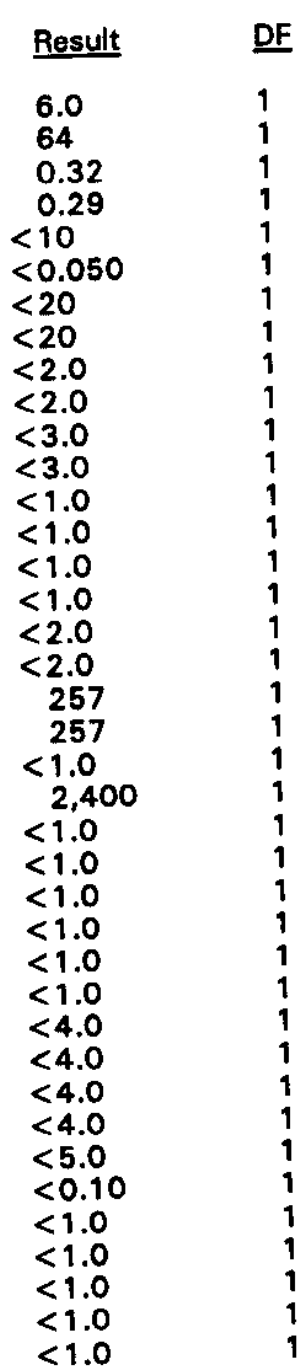

Time: 13:30

pH: 5.8

Alkalinity: $9 \mathrm{mg} / \mathrm{L}$

Water temperature: $19.8^{\circ} \mathrm{C}$

Volumes purged: 12.2 well volumes 
WELL HMD 2D collected on 04/15/94, laboratory analyses (cont.)

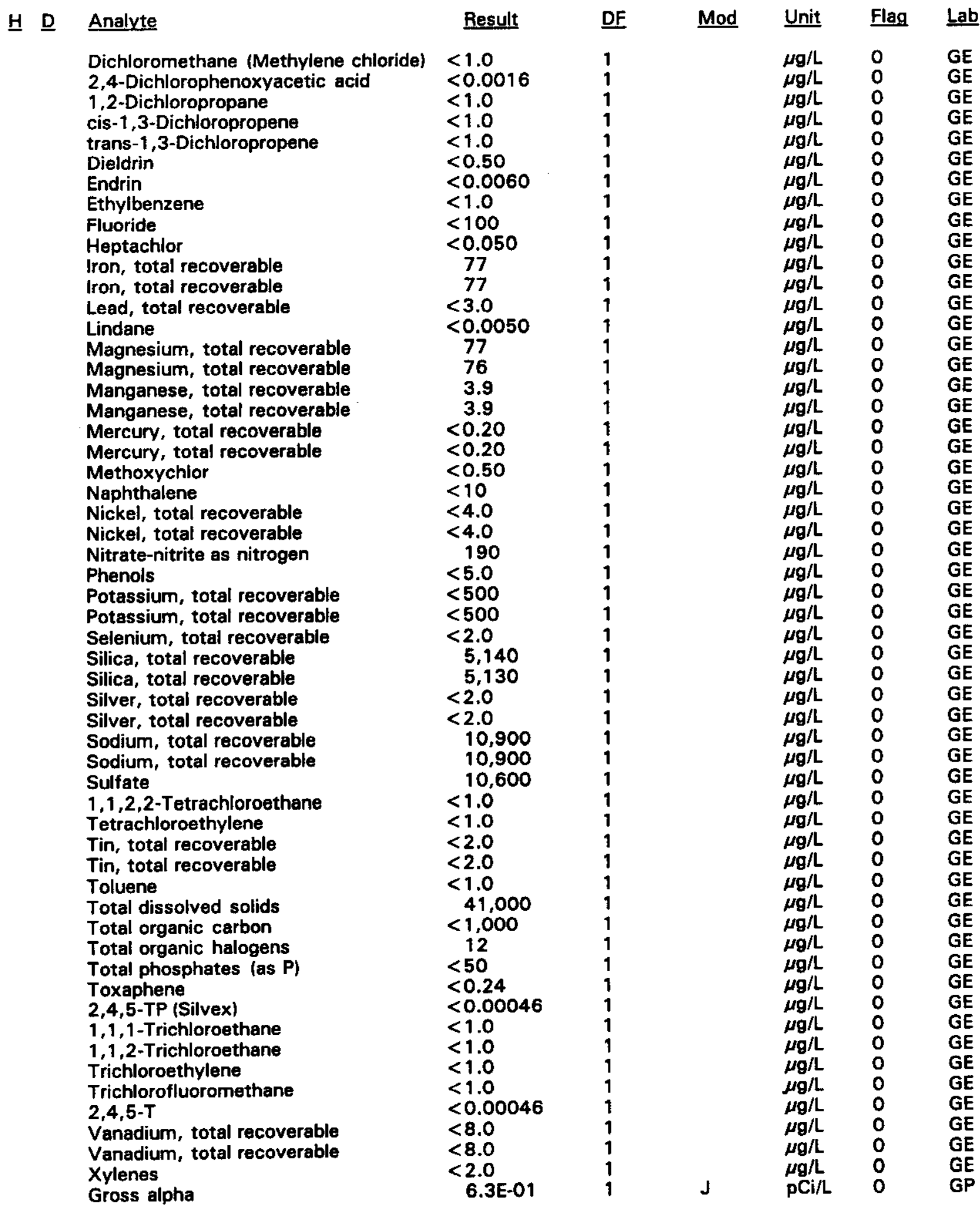

\footnotetext{
$\overline{-}=$ exceeded holding time. $=$ = exceeded screening level or final primary drinking water standard.
} 
WELL HMD 2D collected on 04/15/94, laboratory analyses (cont.)

\begin{tabular}{|c|c|c|c|c|c|c|}
\hline \multirow[t]{2}{*}{$\underline{H} \underline{D}$} & Analyte & Result & DF & Mod & Unit & Flag \\
\hline & $\begin{array}{l}\text { Nonvolatile beta } \\
\text { Radium, total alpha-emitting } \\
\text { Tritium }\end{array}$ & $\begin{array}{l}3.6 \mathrm{E}-01 \\
0.0 \mathrm{E}+00 \\
1.5 \mathrm{E}+01\end{array}$ & $\begin{array}{l}1 \\
1 \\
1\end{array}$ & $\begin{array}{l}\text { UI } \\
\text { UI }\end{array}$ & $\begin{array}{l}\mathrm{pCi} / \mathrm{L} \\
\mathrm{pCi} / \mathrm{L} \\
\mathrm{pCi} / \mathrm{mL}\end{array}$ & $\begin{array}{l}0 \\
0 \\
1\end{array}$ \\
\hline
\end{tabular}

\section{WELL HMD 3D}

$\begin{array}{lllllll}\text { SRS Coord. } & \text { Lat/Longitude } & \text { Screen Zone Elevation } & \text { Top of Casing } & \text { Casing } & \text { Pump } & \text { Formation } \\ \text { N79578.7 } & 33.295996^{\circ} \mathrm{N} & 207.7-187.7 \mathrm{ft} \mathrm{msl} & 259.5 \mathrm{ft} \mathrm{msl} & 4^{\text {" PVC }} & \text { S } & \text { Barnwell (IIB,) } \\ \text { E57745.2 } & 81.669327^{\circ} \mathrm{W} & & & & \end{array}$

\section{FIELD MEASUREMENTS}

Sample date: 04/15/94

Depth to water: $60.33 \mathrm{ft}(18.39 \mathrm{~m})$ below TOC

Water elevation: $199.17 \mathrm{ft}(60.71 \mathrm{~m}) \mathrm{msl}$

Sp. conductance: $67 \mu \mathrm{S} / \mathrm{cm}$

Turbidity: 1.5 NTU

Water evacuated before sampling: $80 \mathrm{gal}$

\section{LABORATORY ANALYSES}

브 ㅁ Analyte

- $\quad$ pH

Specific conductance

Specific conductance

- Turbidity

Acetophenone

Aldrin

Aluminum, total recoverable

Aluminum, total recoverable

Antimony, total recoverable

Arsenic, total recoverable

Barium, total recoverable

Barium, total recoverable

Benzene

Bromodichloromethane

Bromoform

Bromomethane (Methyl bromide)

Cadmium, total recoverable

Cadmium, total recoverable

Calcium, total recoverable

Calcium, total recoverable

Carbon tetrachloride

Chloride

Chlorobenzene

Chloroethane

Chloroethene (Vinyl chloride)

2-Chloroethyl vinyl ether

Chloroform

Chloromethane (Methyl chloride)

Chromium, total recoverable

Chromium, total recoverable

Copper, total recoverable
Time: 12:53

pH: 5.0

Alkalinity: $0 \mathrm{mg} / \mathrm{L}$

Water temperature: $14.0^{\circ} \mathrm{C}$

Volumes purged: 10.6 well volumes

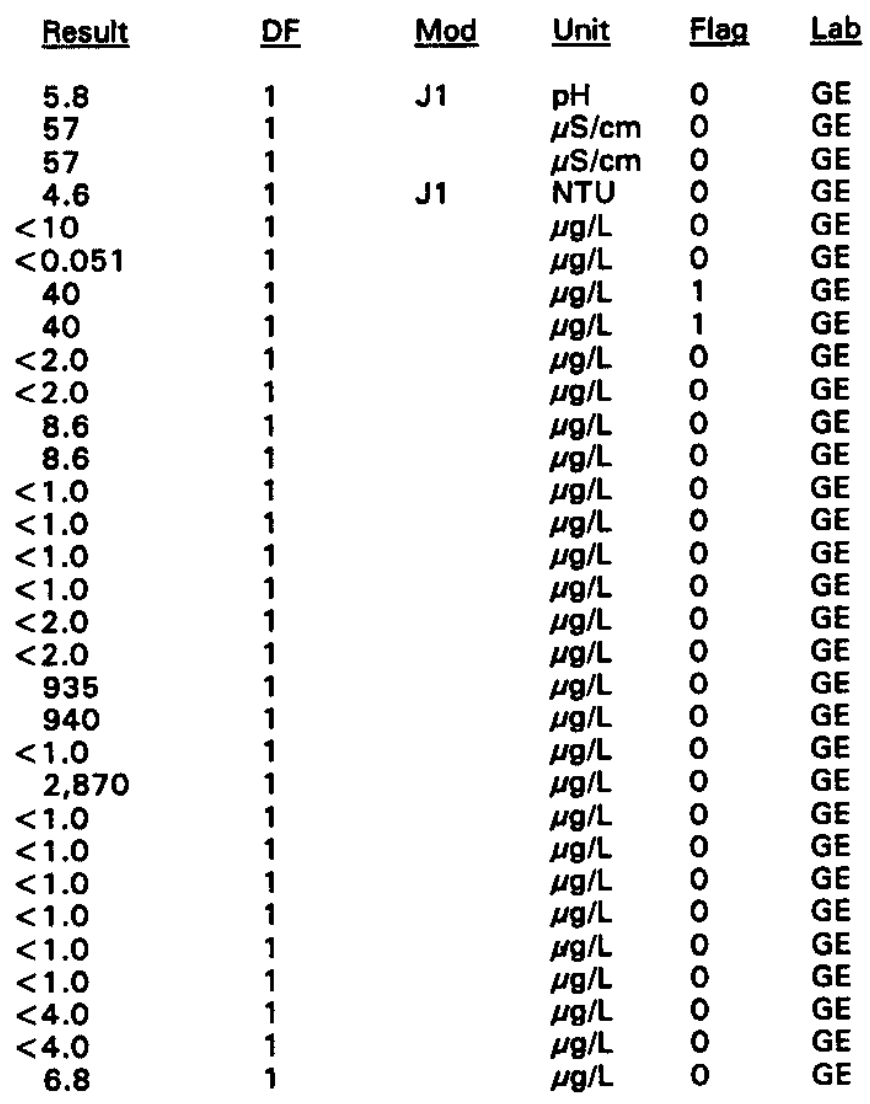

$\overline{-}=$ exceeded holding time. - = exceeded screening level of final primary drinking water standard. 
WELL HMD 3D collected on 04/15/94, laboratory analyses (cont.)

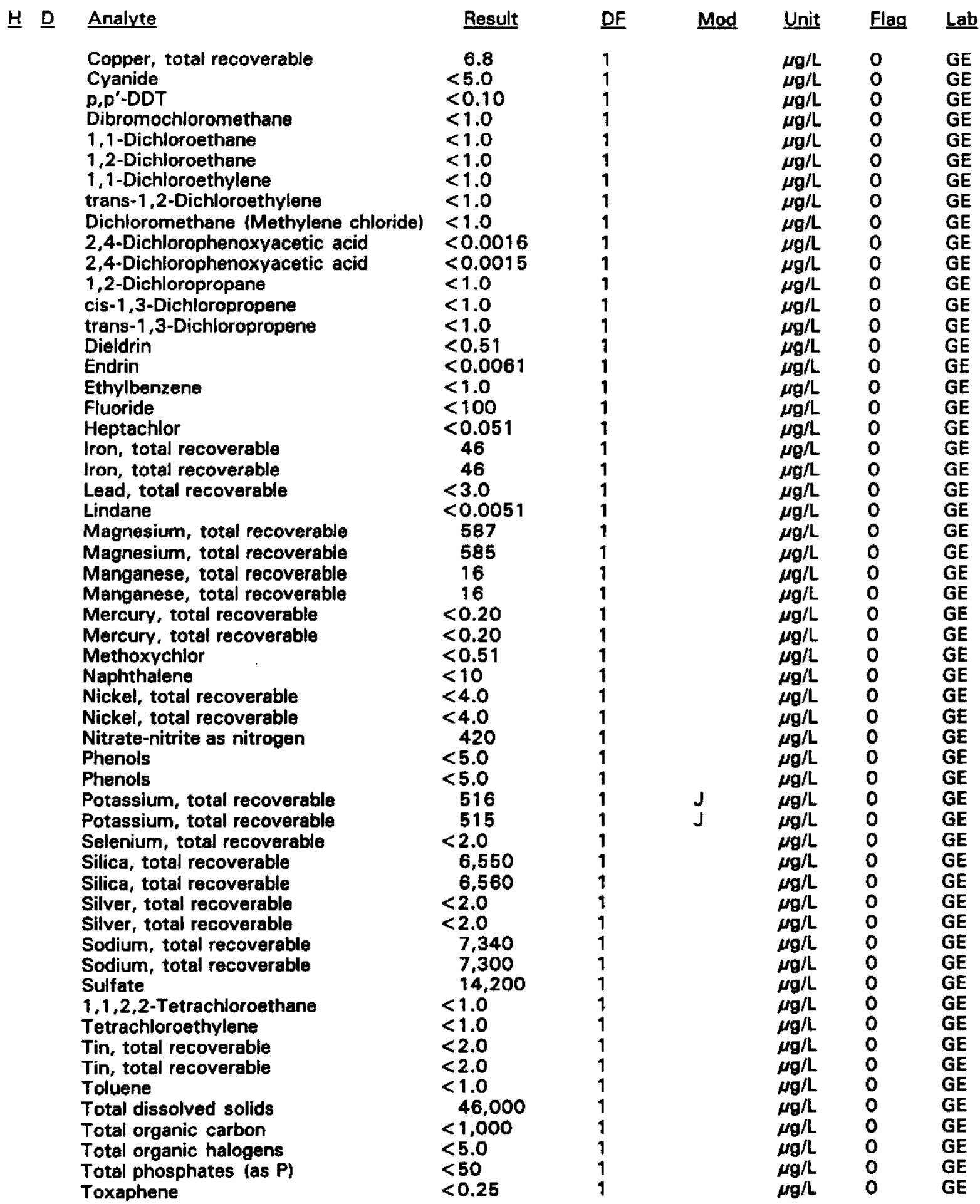

\footnotetext{
- = exceeded holding time. = exceeded screening level or final primary drinking water standard.
} 
WELL HMD 30 collected on $04 / 15 / 94$, laboratory analyses (cont.)

H $\underline{\text { Analyte }}$

2,4,5-TP (Silvex)

2,4,5-TP (Silvex)

1,1,1-Trichloroethane

1,1,2-Trichloroethane

Trichloroethylene

Trichlorofluoromethane

2,4,5-T

2,4,5-T

Vanadium, total recoverable

Vanadium, total recoverable

Xylenes

Gross alpha

Nonvolatile beta

Radium, total alpha-emitting

Tritium

\begin{tabular}{ll}
\multicolumn{1}{l}{ Result } & D \\
$<0.00046$ & 1 \\
$<0.00046$ & 1 \\
$<1.0$ & 1 \\
$<1.0$ & 1 \\
$<1.0$ & 1 \\
3.3 & 1 \\
$<0.00046$ & 1 \\
$<0.00046$ & 1 \\
$<8.0$ & 1 \\
$<8.0$ & 1 \\
$<2.0$ & 1 \\
$1.6 E+00$ & 1 \\
$1.0 E+00$ & 1 \\
$8.0 E-01$ & 1 \\
$1.2 E+01$ & 1
\end{tabular}

Mod Unit Flag Lab

$\mu \mathrm{g} / \mathrm{L} \quad 0 \quad \mathrm{GE}$

$\mu \mathrm{g} / \mathrm{L} \quad 0 \quad \mathrm{GE}$

$\mu g / L \quad 0 \quad$ GE

$\mu g / L \quad 0 \quad$ GE

$\mu g / L \quad 0 \quad$ GE

$\mu \mathrm{g} / \mathrm{L} \quad \mathrm{O} \quad \mathrm{GE}$

$\mu g / L \quad 0 \quad$ GE

$\mu g / L \quad 0 \quad$ GE

$\mu g / L \quad 0 \quad$ GE

$\mu \mathrm{g} / \mathrm{L} \quad \mathrm{O} \quad \mathrm{GE}$

$\mu g / L \quad 0 \quad$ GE

pCi/L 0 GP

PCi/L 00 GP

$\mathrm{PCi} / \mathrm{L} \quad 0 \quad \mathrm{GP}$

$\mathrm{pCi} / \mathrm{mL} \quad 1 \quad \mathrm{GP}$

WELL HMD 4D

\begin{tabular}{|c|c|c|c|c|c|c|}
\hline SRS Coord. & Lat/Longitude & Screen Zone Elevation & Top of Casing & $\underline{\text { Casing }}$ & Pump & Formation \\
\hline $\begin{array}{l}79160.4 \\
58188.5\end{array}$ & $\begin{array}{l}33.295794^{\circ} \mathrm{N} \\
81.667346^{\circ} \mathrm{W}\end{array}$ & $208.9-188.9 \mathrm{ft} \mathrm{msl}$ & $250.9 \mathrm{ft} \mathrm{msl}$ & 4" PVC & $\mathbf{s}$ & \\
\hline
\end{tabular}

\section{FIELD MEASUREMENTS}

Sample date: 04/20/94

Depth to water: $51.07 \mathrm{ft}(15.57 \mathrm{~m})$ below TOC

Water elevation: $199.83 \mathrm{ft}(60.91 \mathrm{~m}) \mathrm{msl}$

Sp. conductance: $28 \mu \mathrm{S} / \mathrm{cm}$

Turbidity: 19.9 NTU

Water evacuated before sampling: $6 \mathrm{gal}$

The well went dry during purging.

\section{LABORATORY ANALYSES}

H D Analyte

- $\quad \mathrm{pH}$

Specific conductance

Turbidity

Acetophenone

Aldrin

Aluminum, total recoverable

Antimony, total recoverable

Antimony, total recoverable

Arsenic, total recoverable

Arsenic, total recoverable

Barium, total recoverable

Benzene

Benzene

Bromodichloromethane

Bromodichloromethane

Bromoform

Bromoform

Bromomethane (Methyl bromide)

Bromomethane (Methyl bromide)
Time: 9:05

pH: 5.7

Alkalinity: $4 \mathrm{mg} / \mathrm{L}$

Water temperature: $18.1^{\circ} \mathrm{C}$

Volumes purged: 0.8 well volumes

\begin{tabular}{|c|c|c|c|c|}
\hline Result & DF & Mod & Unit & Flag \\
\hline 6.3 & 1 & J1 & pH & 0 \\
\hline $\begin{array}{l}29 \\
256\end{array}$ & 1 & & $\mu \mathrm{s} / \mathrm{cm}$ & 0 \\
\hline$<10$ & 1 & & $\mu g / L$ & 0 \\
\hline$<0.052$ & $i$ & & $\mu \mathrm{g} / \mathrm{L}$ & 0 \\
\hline 152 & 1 & & $\mu g / L$ & 2 \\
\hline$<2.0$ & 1 & & $\mu g / L$ & 0 \\
\hline$<2.0$ & 1 & J3 & $\mu g / L$ & 0 \\
\hline$<2.0$ & 1 & & $\mu g / L$ & 0 \\
\hline$<2.0$ & 1 & & $\mu \mathrm{g} / \mathrm{L}$ & 0 \\
\hline 20 & 1 & & $\mu g / L$ & 0 \\
\hline$<1.0$ & 1 & & $\mu \mathrm{g} / \mathrm{L}$ & 0 \\
\hline$<1.0$ & 1 & & $\mu g / L$ & 0 \\
\hline$<1.0$ & 1 & & $\mu g / L$ & 0 \\
\hline$<1.0$ & 1 & & $\mu \mathrm{g} / \mathrm{L}$ & 0 \\
\hline$<1.0$ & 1 & & $\mu g / L$ & 0 \\
\hline$<1.0$ & 1 & & $\mu g / L$ & 0 \\
\hline$<1.0$ & 1 & & $\mu g / L$ & 0 \\
\hline$<1.0$ & 1 & & $\mu g / L$ & 0 \\
\hline
\end{tabular}

- exceeded holding time. - = exceeded screening level or final primary drinking water standard. 
WELL HMD 4D collected on 04/20/94, laboratory analyses (cont.)

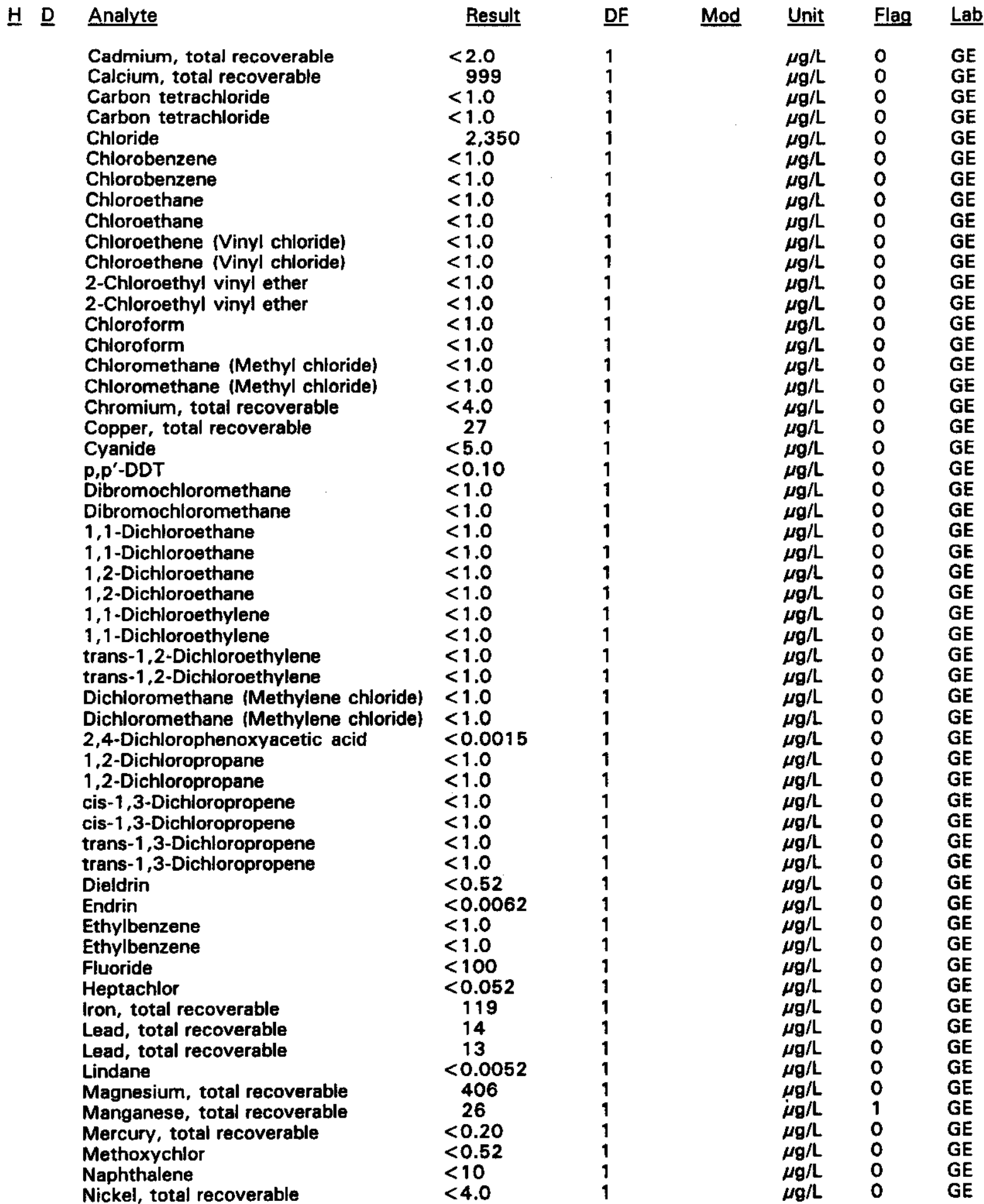

- = exceeded holding time. $\quad=$ exceeded screening level or final primary drinking water standard. 
WELL HMD 4D collected on 04/20/94, laboratory analyses (cont.)

H $\underline{\text { Analyte }}$

Nitrate-nitrite as nitrogen

Phenols

Potassium, total recoverable

Selenium, total recoverable

Selenium, total recoverable

Silica, total recoverable

Silver, total recoverable

Sodium, total recoverable

Sulfate

1,1,2,2-Tetrachloroethane

$1,1,2,2-$ Tetrachloroethane

Tetrachloroethylene

Tetrachloroethylene

Tin, total recoverable

Toluene

Toluene

Total dissolved solids

Total organic carbon

Total organic halogens

Total phosphates (as P)

Toxaphene

2,4,5-TP (Silvex)

1,1,1-Trichloroethane

1,1,1-Trichloroethane

1,1,2-Trichloroethane

1,1,2-Trichloroethane

Trichloroethylene

Trichloroethylene

Trichlorofluoromethane

Trichlorofluoromethane

2,4,5-T

Vanadium, total recoverable

Xylenes

Xylenes

Gross alpha

Nonvolatile beta

Radium, total alpha-emitting

Tritium

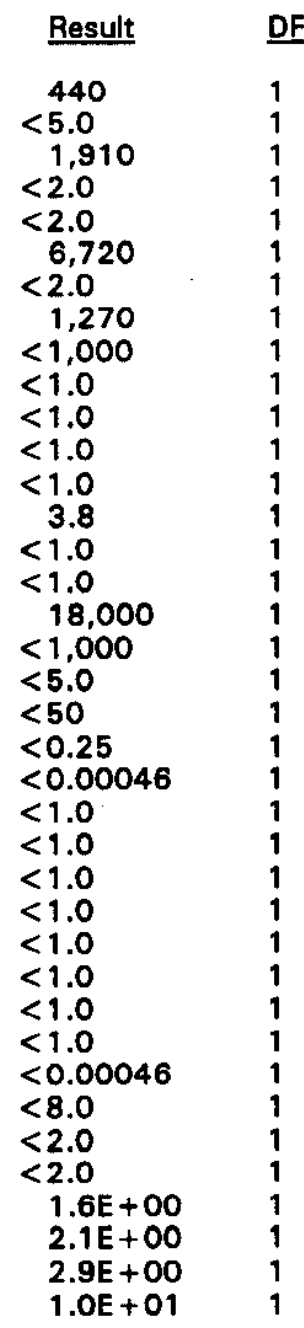

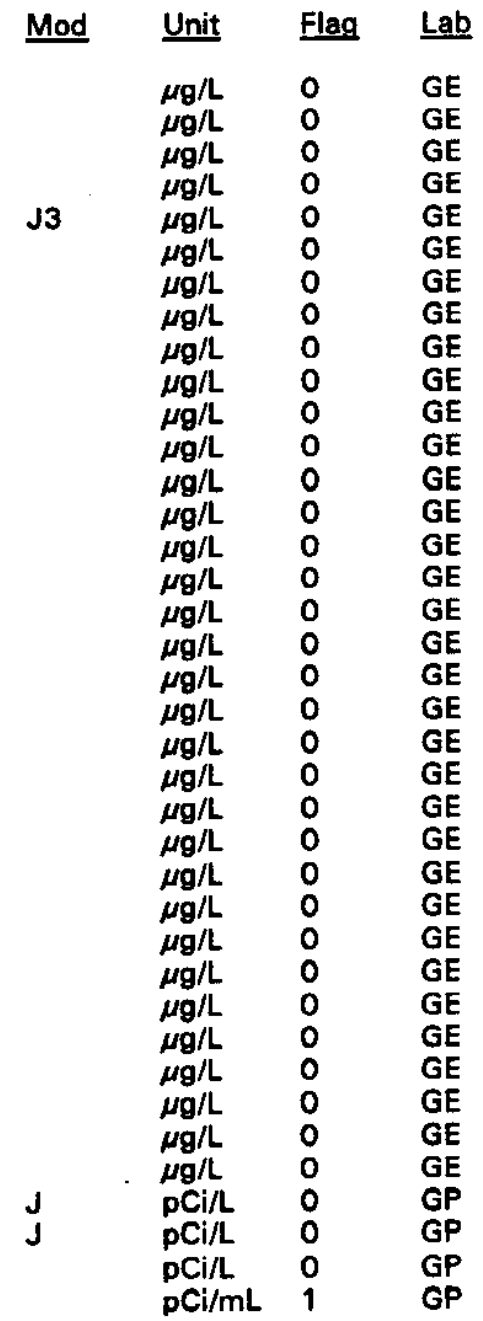

- = exceeded holding time. $\square=$ exceeded screening level or final primary drinking water standard. 


\section{WELL HSB 85A}

\begin{tabular}{|c|c|c|c|c|c|c|}
\hline SRS Coord. & Lat/Longitude & Screen Zone Elevation & Top of Casing & Casing & Pump & Formation \\
\hline $\begin{array}{l}\text { N73791.9 } \\
\text { E58943.4 }\end{array}$ & $\begin{array}{l}33.285152^{\circ} \mathrm{N} \\
81.654930^{\circ} \mathrm{W}\end{array}$ & $71.1-61.1 \mathrm{ft} \mathrm{msl}$ & $294.4 \mathrm{ft} \mathrm{msl}$ & 4" PVC & $\mathbf{s}$ & U. Congaree (IIA) \\
\hline
\end{tabular}

\section{FIELD MEASUREMENTS}

Sample date: 05/04/94

Depth to water: $125.81 \mathrm{ft}(38.35 \mathrm{~m})$ below TOC

Water elevation: $168.59 \mathrm{ft}(51.39 \mathrm{~m}) \mathrm{ms}$ l

Sp. conductance: $188 \mu \mathrm{S} / \mathrm{cm}$

Turbidity: 0.4 NTU

Water evacuated before sampling: $233 \mathrm{gal}$

Time: 13:20

pH: 7.0

Alkalinity: $68 \mathrm{mg} / \mathrm{L}$

Water temperature: $19.7^{\circ} \mathrm{C}$

Volumes purged: $\mathbf{3 . 3}$ well volumes

\section{LABORATORY ANALYSES}

\begin{tabular}{|c|c|c|c|c|c|}
\hline Analyte & Result & DF & Mod & Unit & Flag \\
\hline $\begin{array}{l}\text { pH } \\
\text { Specific conductance } \\
\text { Mercury, total recoverable } \\
\text { Nitrate as nitrogen } \\
\text { Gross alpha } \\
\text { Nonvolatile beta } \\
\text { Tritium }\end{array}$ & $\begin{array}{l}7.0 \\
177 \\
<0.20 \\
<50 \\
2.3 E-01 \\
1.3 E+00 \\
1.4 E-01\end{array}$ & $\begin{array}{l}1 \\
1 \\
1 \\
1 \\
1 \\
1 \\
1\end{array}$ & $\begin{array}{l}\text { UI } \\
\text { J } \\
\text { UI }\end{array}$ & $\begin{array}{l}\mathrm{pH} \\
\mu \mathrm{S} / \mathrm{cm} \\
\mu \mathrm{g} / \mathrm{L} \\
\mu \mathrm{g} / \mathrm{L} \\
\mathrm{pCi} / \mathrm{L} \\
\mathrm{pCi} / \mathrm{L} \\
\mathrm{pCi} / \mathrm{mL}\end{array}$ & $\begin{array}{l}0 \\
0 \\
0 \\
0 \\
0 \\
0 \\
0\end{array}$ \\
\hline
\end{tabular}

\section{WELL HSB 85B}

$\begin{array}{lllllll}\text { SRS Coord. Lat/Longitude } & \text { Screen Zone Elevation } & \text { Top of Casing } & \text { Casing } & \text { Pump } & \text { Formation } \\ \text { N73789.3 } & 33.285162^{\circ} \mathrm{N} & 143.2-133.2 \mathrm{ft} \mathrm{msl} & 294.5 \mathrm{ft} \mathrm{msl} & 4^{\prime \prime} \text { PVC } & \text { S } & \text { McBean (IIB, }) \\ \text { E58953.3 } & 81.654898^{\circ} \mathrm{W} & & & \end{array}$

FIELD MEASUREMENTS

Sample date: 04/29/94

Depth to water: $61.18 \mathrm{ft}(18.65 \mathrm{~m})$ below TOC

Water elevation: $233.32 \mathrm{ft}(71.12 \mathrm{~m}) \mathrm{ms}$

Sp. conductance: $183 \mu \mathrm{S} / \mathrm{cm}$

Turbidity: 1.4 NTU

Water evacuated before sampling: $51 \mathrm{gal}$

The well went dry during purging.
Time: $8: 14$

pH: 9.8

Alkalinity: $73 \mathrm{mg} / \mathrm{L}$

Water temperature: $19.0^{\circ} \mathrm{C}$

Volumes purged: 0.8 well volumes

\section{LABORATORY ANALYSES}

H D Analyte

pH

Specific conductance

Mercury, total recoverable

Nitrate-nitrite as nitrogen

Gross alpha

Nonvolatile beta

Tritium

\begin{tabular}{|c|c|c|c|c|}
\hline Result & DF & Mod & Unit & Flag \\
\hline $\begin{array}{l}7.9 \\
203 \\
<0.20 \\
<50 \\
4.7 E-01 \\
1.4 E+00 \\
3.4 E-01\end{array}$ & $\begin{array}{l}1 \\
1 \\
1 \\
1 \\
1 \\
1 \\
1\end{array}$ & ${ }_{\mathrm{J}}^{\mathrm{U}}$ & $\begin{array}{l}\mathrm{pH} \\
\mu \mathrm{S} / \mathrm{cm} \\
\mu \mathrm{g} / \mathrm{L} \\
\mu \mathrm{g} / \mathrm{L} \\
\mathrm{pCi} / \mathrm{L} \\
\mathrm{pCi} / \mathrm{L} \\
\mathrm{pCi} / \mathrm{mL}\end{array}$ & $\begin{array}{l}0 \\
0 \\
0 \\
0 \\
0 \\
0 \\
0\end{array}$ \\
\hline
\end{tabular}

- = exceeded holding time. $\square=$ exceeded screening level or final primary drinking water standard. 


\section{WELL HSB 85C}

\begin{tabular}{|c|c|c|c|c|c|c|}
\hline SRS Coord. & Lat/Longitude & Screen Zone Elevation & Top of Casing & Casing & Pump & Formation \\
\hline $\begin{array}{l}N 73802.3 \\
\text { E58947.4 }\end{array}$ & $\begin{array}{l}33.285182^{\circ} \mathrm{N} \\
81.654939^{\circ} \mathrm{W}\end{array}$ & $224.2 \cdot 214.2 \mathrm{ft} \mathrm{msl}$ & $294.1 \mathrm{ft} \mathrm{msl}$ & 4" PVC & $\mathbf{s}$ & Water Table $\left(\mathrm{IB}_{2}\right)$ \\
\hline
\end{tabular}

FIELD MEASUREMENTS

Sample date: 05/04/94

Depth to water: $55.46 \mathrm{ft}(16.90 \mathrm{~m})$ below TOC

Water elevation: $238.64 \mathrm{ft}(72.74 \mathrm{~m}) \mathrm{ms}$ I

Sp. conductance: $38 \mu \mathrm{S} / \mathrm{cm}$

Turbidity: 0.2 NTU

Water evacuated before sampling: 99 gal

\section{LABORATORY ANALYSES}

H $\underline{D}$ Analyte

- $\quad$ pH

Specific conductance

Mercury, total recoverable

Nitrate as nitrogen

Gross alpha

Nonvolatile beta

Tritium
Time: $12: 48$

pH: 4.7

Alkalinity: $0 \mathrm{mg} / \mathrm{L}$

Water temperature: $20.0^{\circ} \mathrm{C}$

Volumes purged: 6.2 well volumes

$\begin{array}{llllll}\text { Result } & \text { DF } & \text { Mod } & \text { Unit } & \text { Flag } & \text { Lab } \\ 5.1 & 1 & \mathrm{J1} & \mathrm{pH} & 0 & \mathrm{GE} \\ 32 & 1 & & \mu \mathrm{S} / \mathrm{cm} & 0 & \mathrm{GE} \\ <0.20 & 1 & & \mu \mathrm{g} / \mathrm{L} & 0 & \mathrm{GE} \\ 2,060 & 2 & & \mu \mathrm{g} / \mathrm{L} & 0 & \mathrm{GE} \\ 2.5 \mathrm{E}+00 & 1 & \mathrm{~J} & \mathrm{pCi} / \mathrm{L} & 0 & \mathrm{GP} \\ 1.1 \mathrm{E}+00 & 1 & \mathrm{~J} & \mathrm{pCi} / \mathrm{L} & 0 & \mathrm{GP} \\ 6.7 \mathrm{E}+00 & 1 & & \mathrm{pCi} / \mathrm{mL} & 0 & \mathrm{GP}\end{array}$

- = exceeded holding time. = exceeded screening level or final primary drinking water standard. 
WSRC-TR-94-0345

Unclassified

THIS PAGE LEFT BLANK INTENTIONALLY. 
WSRC-TR-94-0345

Unclassified

\section{Appendix E}

Data Quality/Useability Assessment 
WSRC-TR-94-0345

Unclassified

THIS PAGE LEFT BLANK INTENTIONALLY. 


\section{Data Quality/Useability Assessment}

Quality assurance/quality control (QAVC) procedures relating to accuracy and precision of analyses performed on groundwater samples are followed in the field and laboratory and are reviewed prior to publication of results. The review by the Environmental Protection Department/ Environmental Monitoring Section (EPD/EMS) of the volume of analytical data acquired each quarter and presented in various reports is an ongoing process; its review of the QAQC data cannot be completed in time to meet the deadlines for the reports required by the Resource Conservation and Recovery Act and associated regulations. Other site and regulatory personnel can obtain further information on the data quality and useability in a variety of ways, including those described below.

\section{Data Qualification}

The contract laboratories continually assess their own accuracy and precision according to U.S. Environmental Protection Agency (EPA) guidelines. They submit sample- or batch-specific QAVC information either at the same time as analytical results or in quarterly summaries. Properly defined and used result modifiers (also referred to as qualifiers) can be a key component in assessing data useability. Result modifiers designed by EPD/EMS and used by the primary laboratories are presented in Appendix D.

\section{Assessment of Accuracy of the Data}

Accuracy, or the nearness of the reported result to the true concentration of a constituent in a sample, can be assessed in several ways.

A laboratory's general accuracy can be judged by analysis of results obtained from known samples. The non-radionuclide contract laboratories analyze commercial reference samples every quarter at EPD/EMS' request. The results of these analyses are presented in the EPD/ EMS groundwater monitoring quarterly reports. The primary laboratories also seek or maintain state certification by participating periodically in performance studies; reference samples and analysis of results are provided by EPA. Results of these studies also are published in the EPD/EMS quarterly reports.

Analysis of blanks provides a tool for assessing the accuracy of both sampling and laboratory analysis. Results for all field blanks for the quarter can be found in the EPD/EMS quarterly reports. Any field or laboratory blanks that exceed established minimums are identified in the same reports, in tables associating them with groundwater samples analyzed in the same batches.

Surrogates, organic compounds similar in chemical behavior to the compounds of interest but not normally found in environmental samples, are used to monitor the effect of the matrix on the accuracy of analyses for organic parameters. For example, for analyses of volatile organics by EPA Method 8240, three surrogate compounds are added to all samples and blanks in each anaIytical batch. In analyses of semivolatile organics, three acid compounds and three base/neutral compounds are used. Two surrogates are used in organochlorine pesticides analyses. Percent recoveries for surrogate analyses are calculated by laboratory personnel, reported to EPD/EMS, reviewed, and entered into the database, but they are not published. If recoveries are not within specified limits, the laboratory is expected to reanalyze the samples or attach qualifiers to the data identifying the anomalous results. 
Sample-specific accuracy for both organic and inorganic parameters can be assessed by examination of matrix spike/matrix spike duplicate results. A sample is analyzed unspiked to determine a baseline set of values. A second portion of the sample is spiked with known concentrations of compounds appropriate to the analyses being performed, typically five volatile organic compounds for volatile organics analyses, eleven semivolatile compounds for semivolatiles, six pesticide compounds for pesticides, all metals for metals analyses by SW-846 methods (EPA, 1986), and a known quantity of cyanide for cyanide analysis. The percentage of the spike compound that is recovered (i.e., measured in excess of the value obtained for the unspiked sample) is a direct measure of analytical accuracy. EPA requires matrix spike/matrix spike duplicates to be run at least once per 20 samples of similar matrix.

Matrix spike/matrix spike duplicate results are reported to EPD/EMS but are not published. For organic compounds, according to EPA guidelines, no action is taken on the basis of matrix spike/matrix spike duplicate data alone (i.e., no result modifiers are assigned solely on the basis of matrix spike results); however, the results can indicate if a laboratory is having a systematic problem in the analysis of one or more analytes.

In the case of inorganic compounds, such as metals, the matrix spike sample analysis provides information about the effect of each sample matrix on the digestion and measurement methodology. Data qualifiers assigned by the laboratories on the basis of the percentage of spike recovery are reported in the published results tables.

\section{Assessment of Precision}

Precision of the analyses, or agreement of a set of replicate results among themselves, is assessed through the use of duplicates initiated by the laboratory and blind replicates (provided by EPD/EMS). The results of duplicate and replicate analyses are presented in the results tables of these reports for the first, second, and third quarters of each year. Duplicate and replicate results are not presented in fourth-quarter reports; the results tables present instead only the highest result for each analyte for each quarter of the year.

The laboratories assess precision by calculating the relative percent difference, or RPD, for each pair of laboratory-initiated duplicate results. One of the contract laboratories uses a data qualifier (J3) to modify metals analyses when the RPD for laboratory duplicates is greater than $20 \%$.

Additional statistical comparisons of laboratory duplicate and blind replicate results, both intraand interlaboratory, are presented in the EPD/EMS quarterly reports. The calculation used for these reports is the MRD, or mean relative difference, which is similar to EPA's RPD except that the MRD provides a single value for all of the analyses of a particular compound, either intra- or interlaboratory, during one quarter. Because detection limits may vary among samples, the MRD requires calculation of a reference detection limit, which is the detection limit at the 90th percentile of the array of limits in the population of all duplicate and replicate analyses for a given analyte during a particular quarter. The MRD is not method-specific.

\section{Method-Specific Accuracy and Precision}

The contract laboratories' EPA-approved laboratory procedures include QAVC requirements as an integral part of the methods. Thus, knowledge of the method used in obtaining data is an important component of determining data useability. EPA has conducted extensive research and development on the methods approved for the analysis of water and waste water; information on the accuracy and precision of a method is available from EPA publications, as is full information on required QAVC procedures. A listing of the methods used by the primary laboratories during 
fourth quarter 1993 is given below along with the source for the method description. Many, if not all, of these sources include presentations of representative accuracy and precision results.

Methods Used by the Contract Laboratories

Method

EPA120.1

EPA150.1

EPA160.1

EPA160.2

EPA180.1

EPA200.7

EPA204.2

EPA206.2

EPA239.2

EPA245.1

EPA270.2

EPA279.2

EPA300.0

EPA310.1

EPA325.2

EPA335.3

EPA340.2

EPA353.1

EPA353.2

EPA365.1

EPA365.2

EPA376.2

EPA413.1

EPA415.1

EPA418.1

EPA420.2

EPA900.0

EPA900.1

EPA906.0

EPA6010

EPA7041

EPA7060

EPA7421

EPA7470

EPA7740

EPA7841

EPA8010

EPA8080

EPAB150

EPA8240

EPA8270

EPA8280

EPA9012

EPA9020

EPA9020A

EPA9030

EPA9060
Used to Analyze

Specific conductance

$\mathrm{pH}$

Total dissolved solids

Total dissolved solids, total suspended solids

Turbidity

Metals

Antimony

Arsenic

Lead

Mercury

Selenium

Thallium

Chloride, nitrite, sulfate

Alkalinity

Chloride

Cyanide

Fluoride

Nitrogen, nitrate-nitrite

Nitrogen, nitrate, nitrite, or combined

Phosphorus, all forms (reported as total phosphates)

Phosphorus, all forms (reported as total phosphates)

Sulfide

Oil \& grease

Dissolved organic carbon, total inorganic carbon, total organic carbon

Total petroleum hydrocartons

Phenols

Gross alpha, nonvolatile beta

Total alpha-emitting radium

Tritium

Metals

Antimony

Arsenic

Lead

Mercury

Selenium

Thalium

Chlorinated volatile organics

Organochlorine pesticides and PCBs

Chlorinated herbicides

GCMS volatiles

GCMS semivolatiles

Dioxins and furans

Cyanide

Total organic halogens

Total organic halogens

Sulfide

Dissolved organic carbon, total inorganic carbon, total

organic carbon
Source

EPA EMSL 1983

EPA EMSL 1983

EPA EMSL 1983

EPA EMSL 1983

EPA EMSL 1983

EPA EMSL 1983

EPA EMSL 1983

EPA EMSL 1983

EPA EMSL 1983

EPA EMSL 1983

EPA EMSL 1983

EPA EMSL 1983

EPA EMSL 1991

EPA EMSL 1983

EPA EMSL 1983

EPA EMSL 1983

EPA EMSL 1983

EPA EMSL 1983

EPA EMSL 1983

EPA EMSL 1983

EPA EMSL 1983

EPA EMSL 1983

EPA EMSL 1983

EPA EMSL 1983

EPA EMSL 1983

EPA EMSL 1983

EPA EMSL 1980

EPA EMSL 1980

EPA EMSL 1980

EPA 1986

EPA 1986

EPA 1986

EPA 1986

EPA 1986

EPA 1986

EPA 1986

EPA 1986

EPA 1986

EPA 1986

EPA 1986

EPA 1986

EPA 1986

EPA 1986

EPA 1986

EPA 1986

EPA 1986

EPA 1986 
An example of available method-specific QAVQC information is that for the analysis of metals by EPA Method 6010/200.7 (EPA, 1986/EPA EMSL, 1983). The primary laboratories, General Engineering Laboratories (GE) and Roy F. Weston, Inc. (Weston), use this inductively coupled plasma (ICP) atomic emission spectrometric method.

The following precision and accuracy data are based on the experience of seven laboratories that applied the ICP technique to acid-distilled water matrices that had been spiked with various metal concentrates. (Note: Not all seven laboratories analyzed all 14 elements.) The references give results for samples having three concentration ranges; the results here are for samples having the lowest values, similar to actual groundwater results for SRS.

ICP Precision and Accuracy Data

$\begin{array}{llll}\text { Element } & \text { True value (wa/) } & \begin{array}{l}\text { Mean reported } \\ \text { value }(\mu g h)\end{array} & \begin{array}{l}\text { Mea } \\ \text { RSD }\end{array} \\ \text { Aluminum } & 60 & & 33 \\ \text { Arsenic } & 22 & 62 & 23 \\ \text { Beryllium } & 20 & 19 & 9.8 \\ \text { Cadmium } & 2.5 & 20 & 16 \\ \text { Chromium } & 10 & 2.9 & 18 \\ \text { Cobalt } & 20 & 10 & 4.1 \\ \text { Copper } & 11 & 20 & 40 \\ \text { Iron } & 20 & 11 & 15 \\ \text { Lead } & 24 & 19 & 32 \\ \text { Manganese } & 15 & 30 & 6.7 \\ \text { Nickel } & 30 & 15 & 11 \\ \text { Selenium } & 6 & 28 & 42 \\ \text { Vanadium } & 70 & 8.5 & 2.9 \\ \text { Zinc } & 16 & 69 & 45\end{array}$

a Relative standard deviation. In EPA (1986), the column heading is Mean Standard Deviation (\%).

As another example, EPA Method 601/8010 (EPA, 1991/EPA, 1986) is used by both GE and Weston for analyses of halogenated volatile organics. In the presentation of the method in both references, the following table gives method-specific accuracy and precision as functions of concentration. Contract laboratories are expected to achieve or at least approach these limits.

\section{Accuracy and Precision as Functions of Concentration for EPA Method 601/8010}

\section{Parameter}

Bromodichloromethane

Bromoform

Bromomethane

Carbon tetrachloride

Chlorobenzene

Chloroethane

2-Chloroethyl vinyl ether ${ }^{f}$

Chloroform

Chloromethane

Dibromochloromethane

1,2-Dichlorobenzene

1,3-Dichlorobenzene

1,4-Dichlorobenzene
Accuracy as

recovery, $X^{\prime a}$ (egh)

$1.12 C-1.02^{d}$

$0.96 C-2.05$

$0.76 C-1.27$

$0.98 C-1.04$

$1.00 \mathrm{C}-1.23$

$0.99 C-1.53$

$1.00 \mathrm{C}$

$0.93 C-0.39$

$0.77 C+0.18$

$0.94 C+2.72$

$0.93 C+1.70$

$0.95 C+0.43$

$0.93 C-0.09$
Single analyst

precision $(\mu \mathrm{g} h)^{b}$

$0.11 \bar{X}+0.04^{\circ}$

$0.12 \bar{X}+0.58$

$0.28 \bar{X}+0.27$

$0.15 \bar{X}+0.38$

$0.15 \bar{X}-0.02$

$0.14 \bar{X}-0.13$

$0.20 \bar{X}$

$0.13 \bar{X}+0.15$

$0.28 \bar{X}-0.31$

$0.11 \bar{X}+1.10$

$0.20 \bar{X}+0.97$

$0.14 \bar{X}+2.33$

$0.15 \bar{X}+0.29$
Overall precision $(\mu \mathrm{g} \Omega)^{\mathrm{c}}$

$0.20 \bar{X}+1.00$

$0.21 \bar{X}+2.41$

$0.36 \bar{X}+0.94$

$0.20 \bar{X}+0.39$

$0.18 \bar{X}+1.21$

$0.17 \bar{X}+0.63$

$0.35 \bar{X}$

$0.19 \bar{X}-0.02$

$0.52 \bar{X}+1.31$

$0.24 \bar{X}+1.68$

$0.13 \bar{X}+6.13$

$0.26 \bar{X}+2.34$

$0.20 \bar{X}+0.41$ 
Parameter

1.1-Dichloroethane

1,2-Dichloroethane

1,1-Dichloroethene

trans-1,2-Dichloroethene

Dichloromethane

(Methylene chloride)

1,2-Dichloropropane

cis-1,3-Dichloropropene ${ }^{f}$

trans-1,3-Dichloropropene $f$

1,1,2,2-Tetrachloroethane

Tetrachloroethylene

1,1,1-Trichloroethane

1,1,2-Trichloroethane

Trichloroethylene

Trichlorofluoromethane

Vinyl chloride
Accuracy as recovery $X^{\prime}$ ( $\left.\mu g / L\right)$

$0.95 \mathrm{C}-1.08$

$1.04 C-1.06$

$0.98 C-0.87$

$0.97 C-0.16$

$0.91 C-0.93$

$1.00 \mathrm{C}$

$1.00 \mathrm{C}$

$1.00 \mathrm{C}$

$0.95 C+0.19$

$0.94 C+0.06$

$0.90 C-0.16$

$0.86 C+0.30$

$0.87 C+0.48$

$0.89 C-0.07$

$0.97 C-0.36$
Single analyst

precision $(\mathrm{eg} / \mathrm{L})$

$0.09 \bar{X}+0.17$

$0.11 \bar{X}+0.70$

$0.21 \bar{X}-0.23$

$0.11 \bar{X}+1.46$

$0.11 \bar{X}+0.33$

$0.13 \bar{X}$

$0.18 \bar{X}$

$0.18 \bar{X}$

$0.14 \bar{X}+2.41$

$0.14 \bar{X}+0.38$

$0.15 \bar{X}+0.04$

$0.13 \bar{X}-0.14$

$0.13 \bar{X}-0.03$

$0.15 \bar{X}+0.67$

$0.13 \bar{X}+0.65$
Overalt

precision $(\mu \mathrm{g} / \mathrm{L})$

$0.14 \bar{X}+0.94$

$0.15 \bar{X}+0.94$

$0.29 \bar{X}-0.40$

$0.17 \bar{X}+1.46$

$0.21 \bar{X}+1.43$

$0.23 \bar{X}$

$0.32 \bar{X}$

$0.32 \bar{X}$

$0.23 \bar{X}+2.79$

$0.18 \bar{X}+2.21$

$0.20 \bar{X}+0.37$

$0.19 \bar{X}+0.67$

$0.23 \bar{X}+0.30$

$0.26 \bar{X}+0.91$

$0.27 \bar{X}+0.40$

a $X^{\prime}=$ expected recovery for one or more measurements of a sample containing a concentration of $C$, in $\mu g / L$.

b Expected single analyst standard deviation of measurements.

c Expected interlaboratory standard deviation of measurements.

d $C=$ true value for the concentration, in $\mu g / h$.

e $\bar{X}=$ average recovery found for measurements of samples containing a concentration of $C$, in $\mu g / L$.

Estimates based on performance of a single laboratory.

\section{References}

EPA (U.S. Environmental Protection Agency), 1986. Test Methods for Evaluating Solid Waste (SW-846), Volumes IA-IC. Washington, DC.

EPA (U.S. Environmental Protection Agency), 1991. Guidelines Establishing Test Procedures for the Analysis of Pollutants, Code of Federal Regulations, Title 40, Part 136, Appendix A. Washington, DC.

EPA EMSL (U.S. Environmental Protection Agency, Environmental Monitoring and Systems Laboratory), 1980. Prescribed Procedures for Measurement of Radioactivity in Drinking Water, EPA-600/4-80-032. Cincinnati, $\mathrm{OH}$.

EPA EMSL (U.S. Environmental Protection Agency, Environmental Monitoring and Systems Laboratory), 1983. Methods for Chemical Analysis of Water and Wastes. Cincinnati, OH.

EPA EMSL (U.S. Environmental Protection Agency, Environmental Monitoring and Systems Laboratory), 1991. Test Method, The Determination of Inorganic Anions in Water by lon Chromatography-Method 300.0. Cincinnati, $\mathrm{OH}$. 
WSRC-TR-94-0345

Unclassified

THIS PAGE LEFT BLANK INTENTIONALLY. 
WSRC-TR-94-0345

Unclassified

\section{Appendix F}

\section{Time Series Plots}


WSRC-TR-94-0345

Unclassified

THIS PAGE LEFT BLANK INTENTIONALLY. 
主

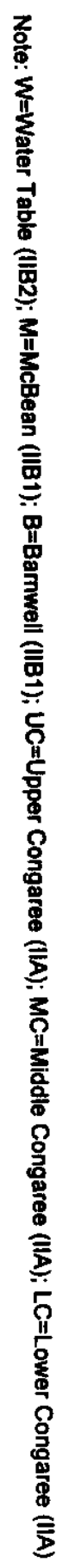

pH (pH units)

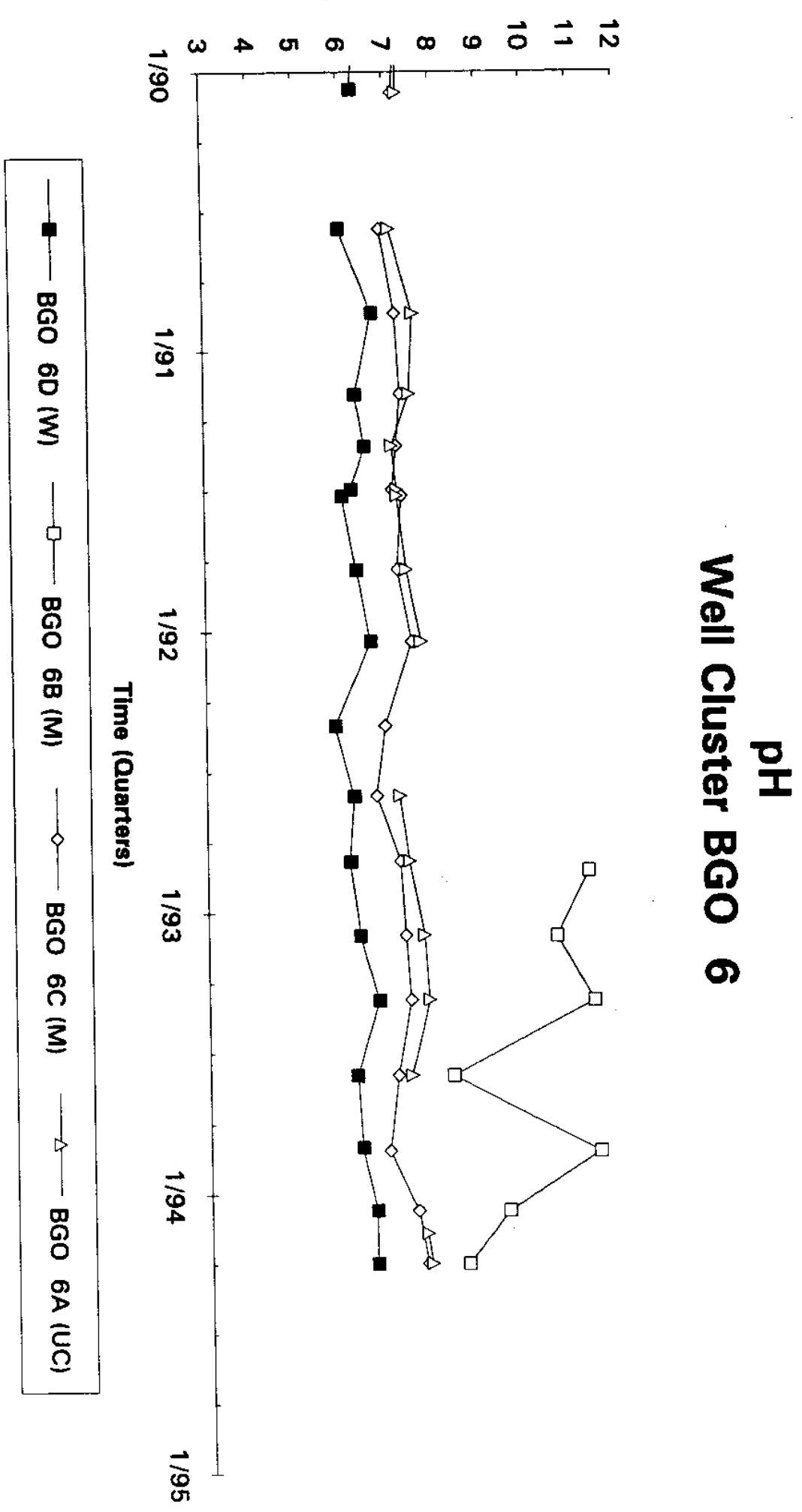


pH (pH units)

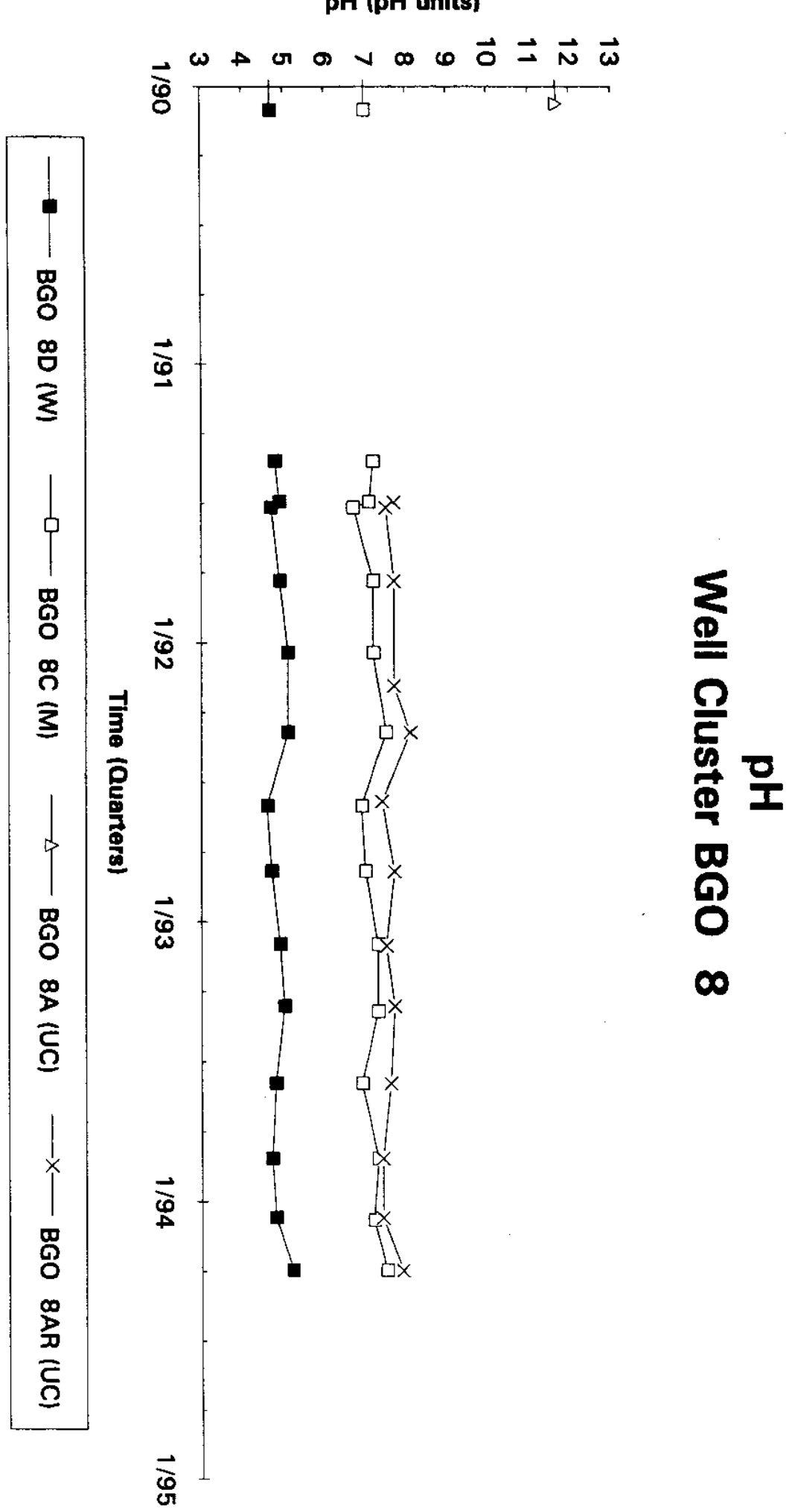




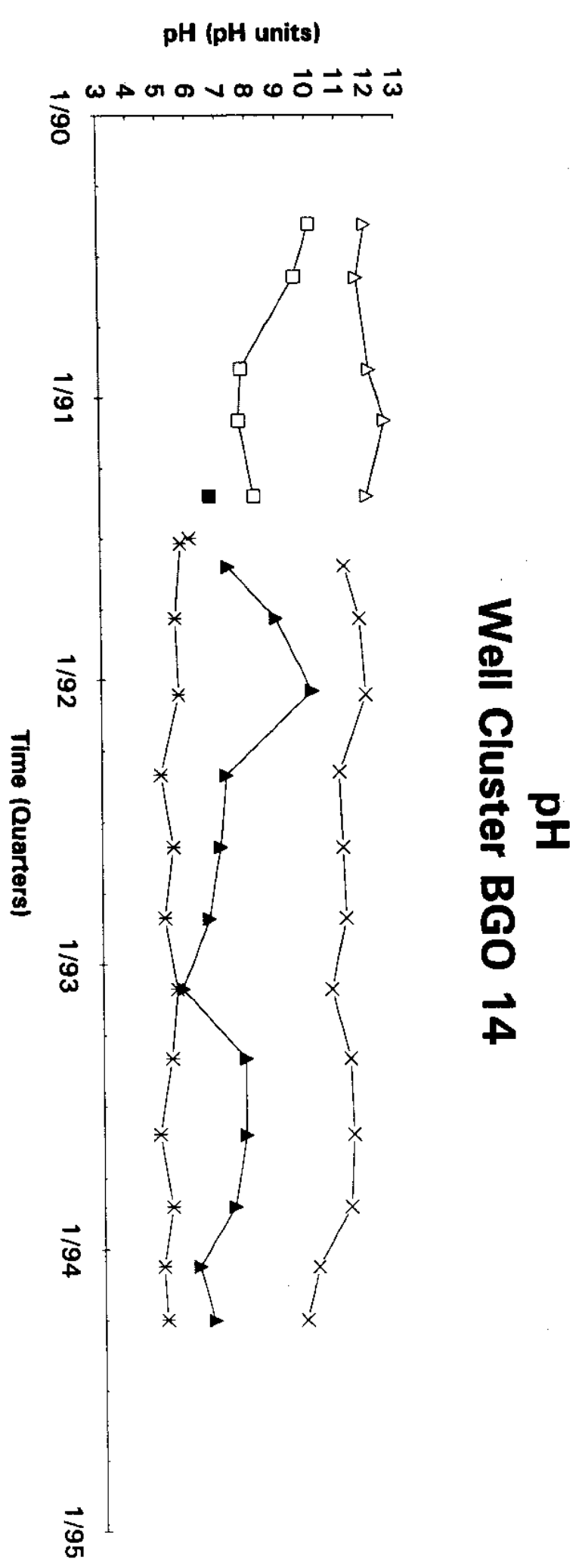

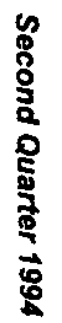

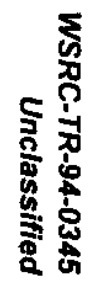


흥



产

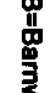

鿷

言

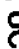

돔

8

:

言

ํㅗㅇ

출

8

\%

言

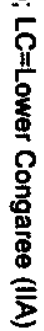

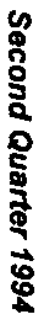

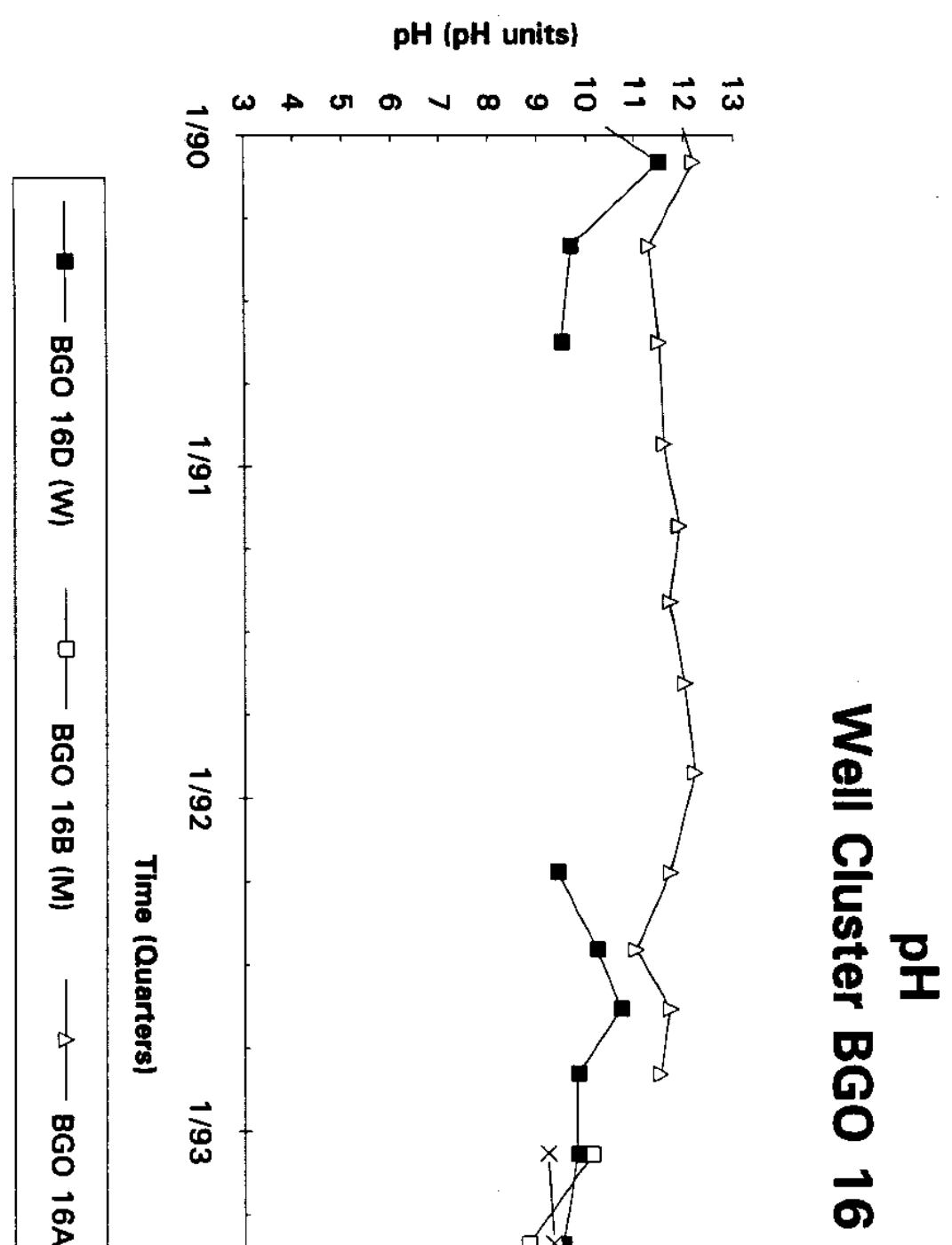




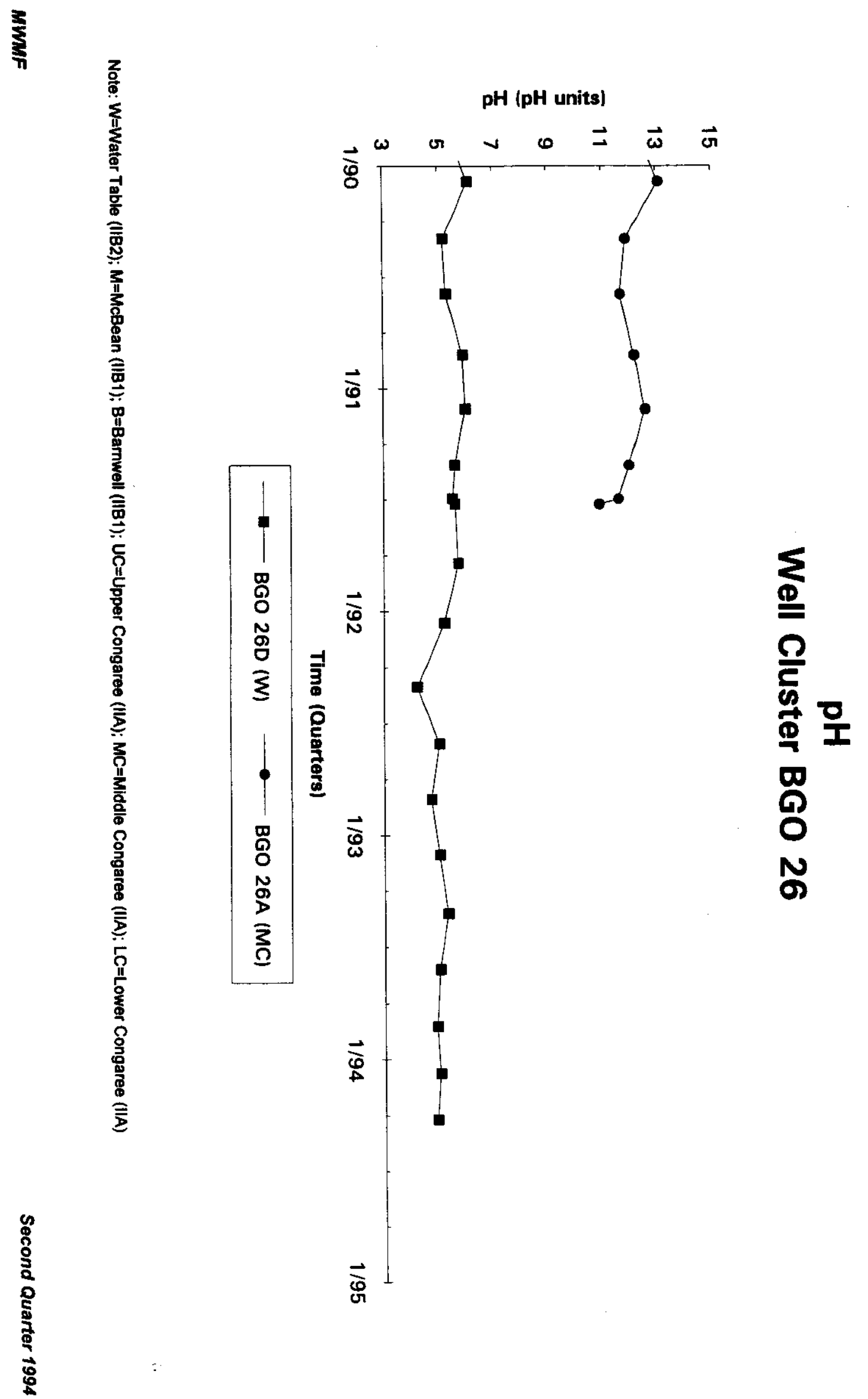

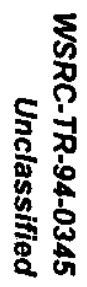




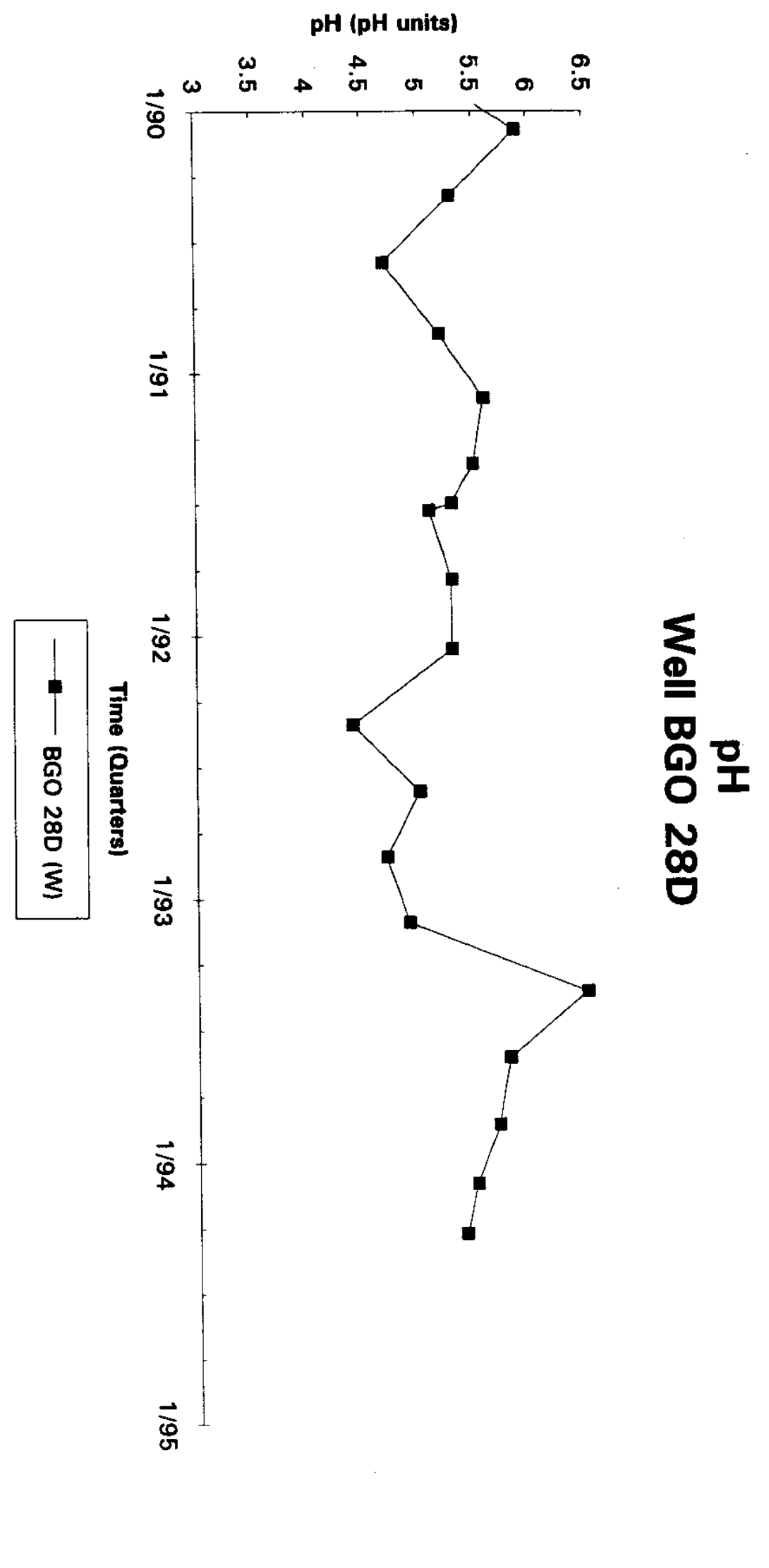

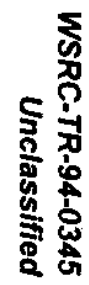




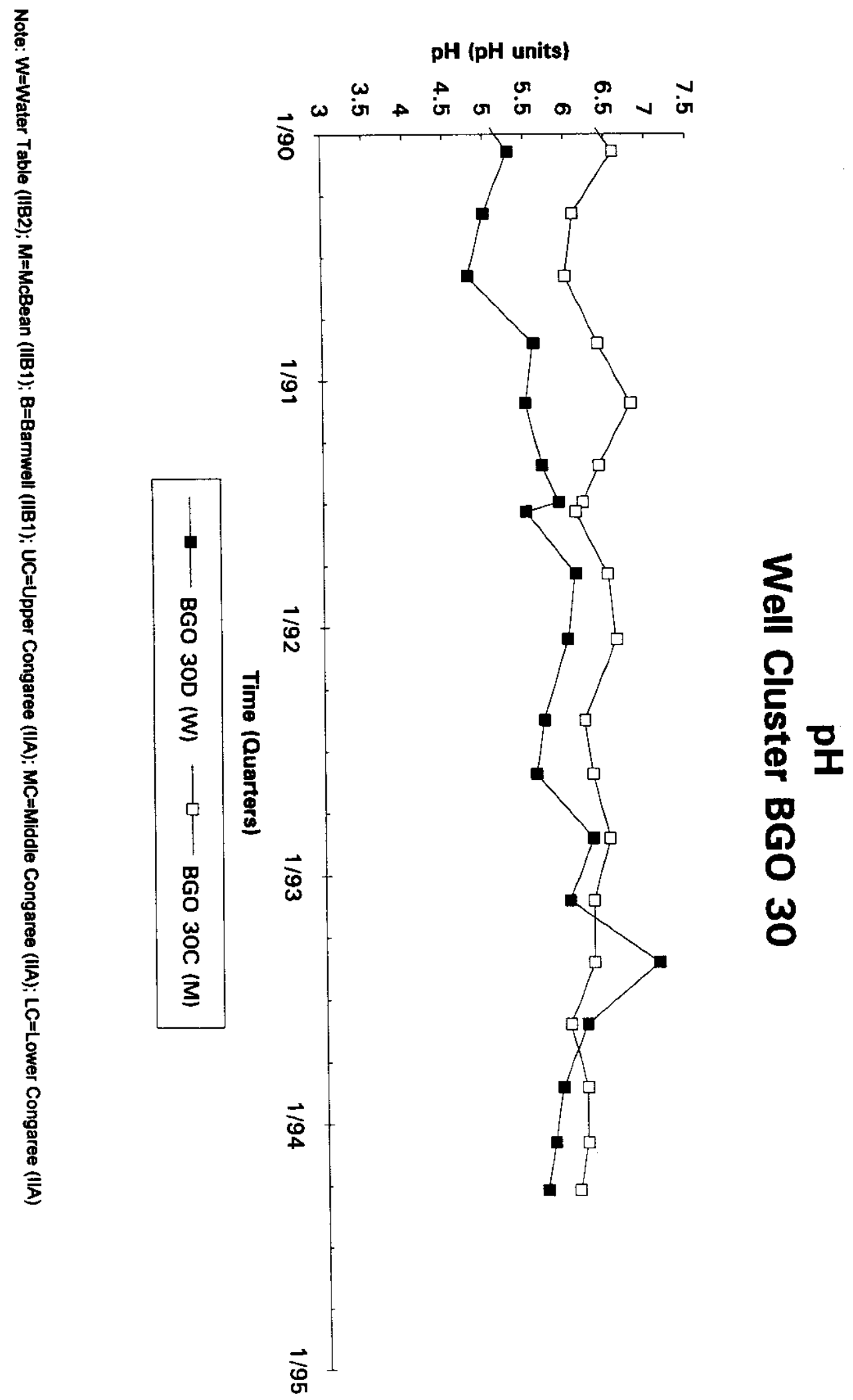




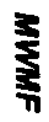

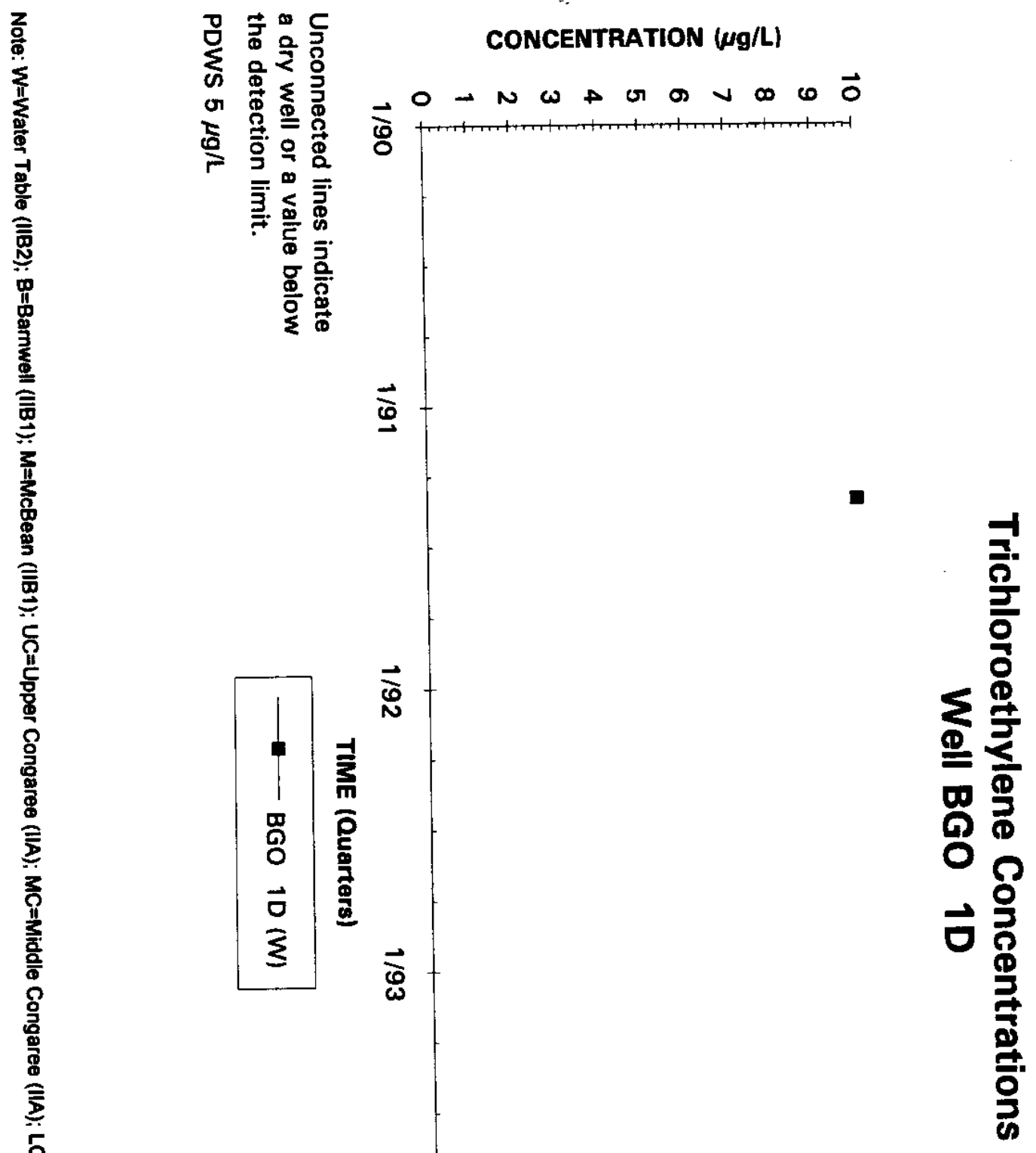


翟

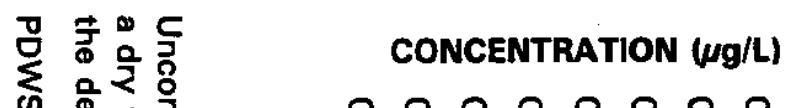

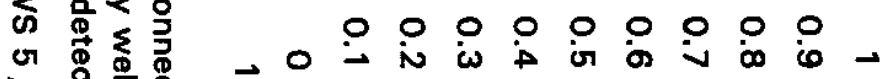

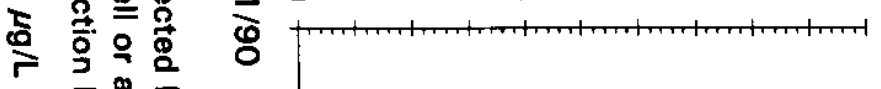

丞离

它

뭉

要

言

章

言

С

윰

$\stackrel{8}{3}$ 
$-\frac{5}{1}$

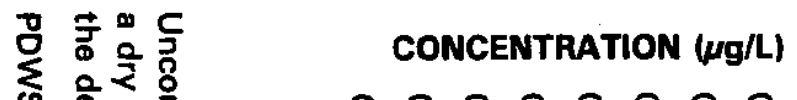

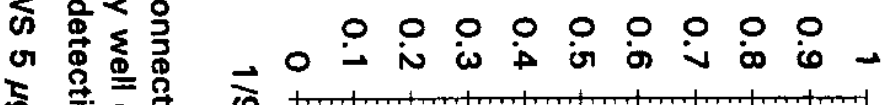

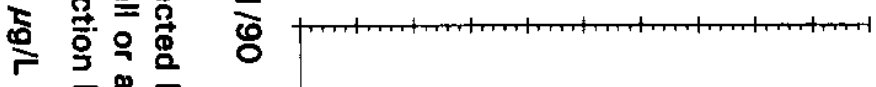

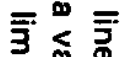

$\stackrel{\%}{\rightleftarrows}$

言

蕒

崖

言

ت્

竞

言

с

뭉

웅

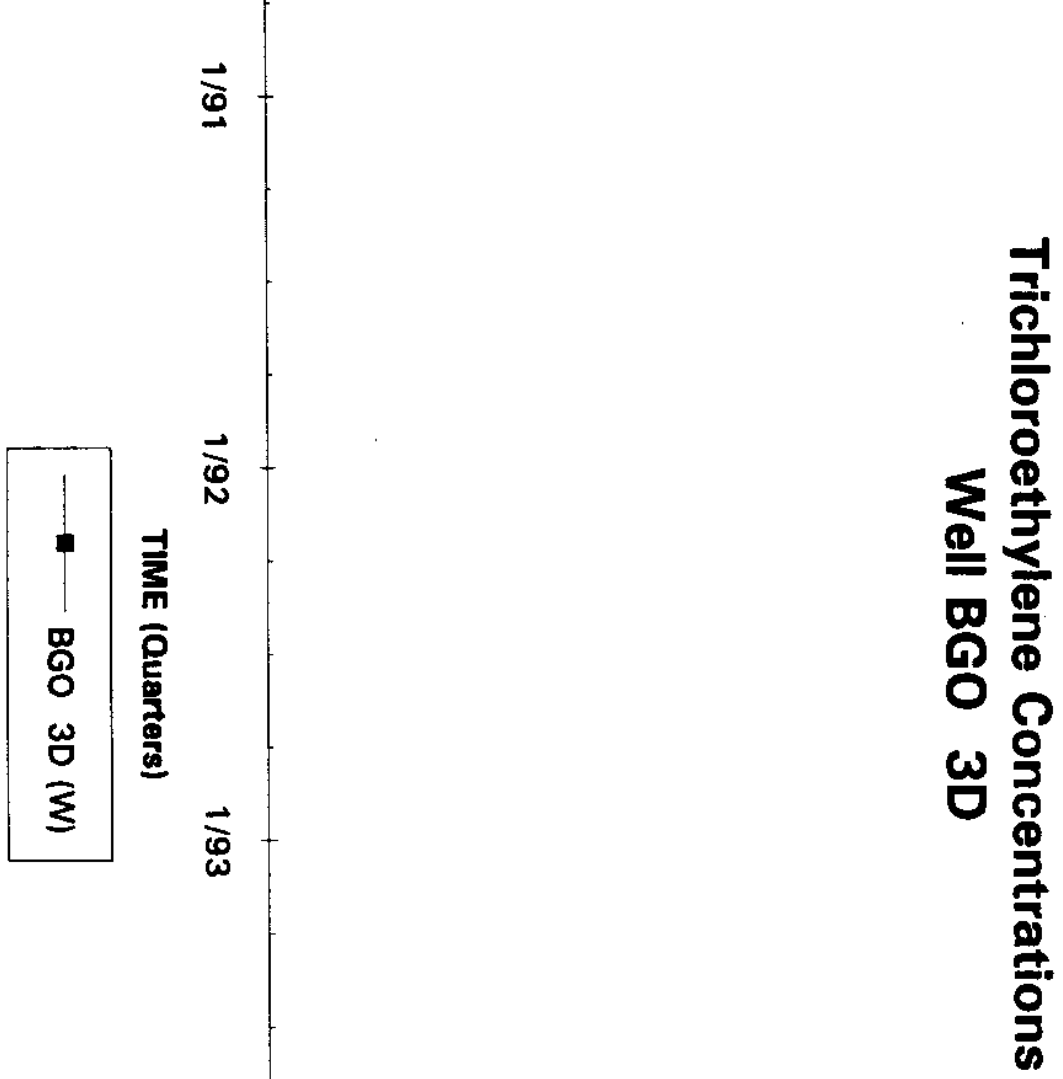


3

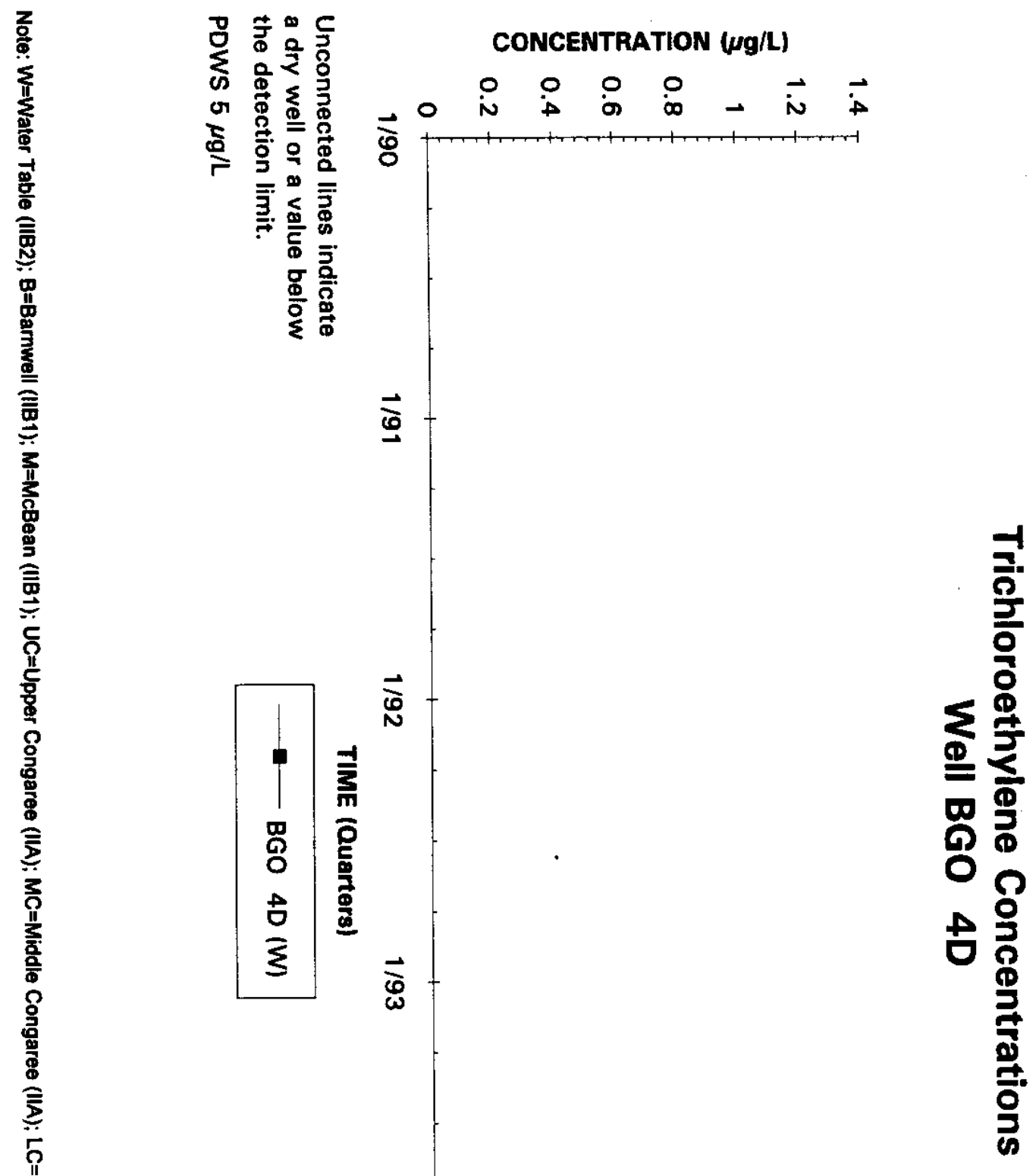




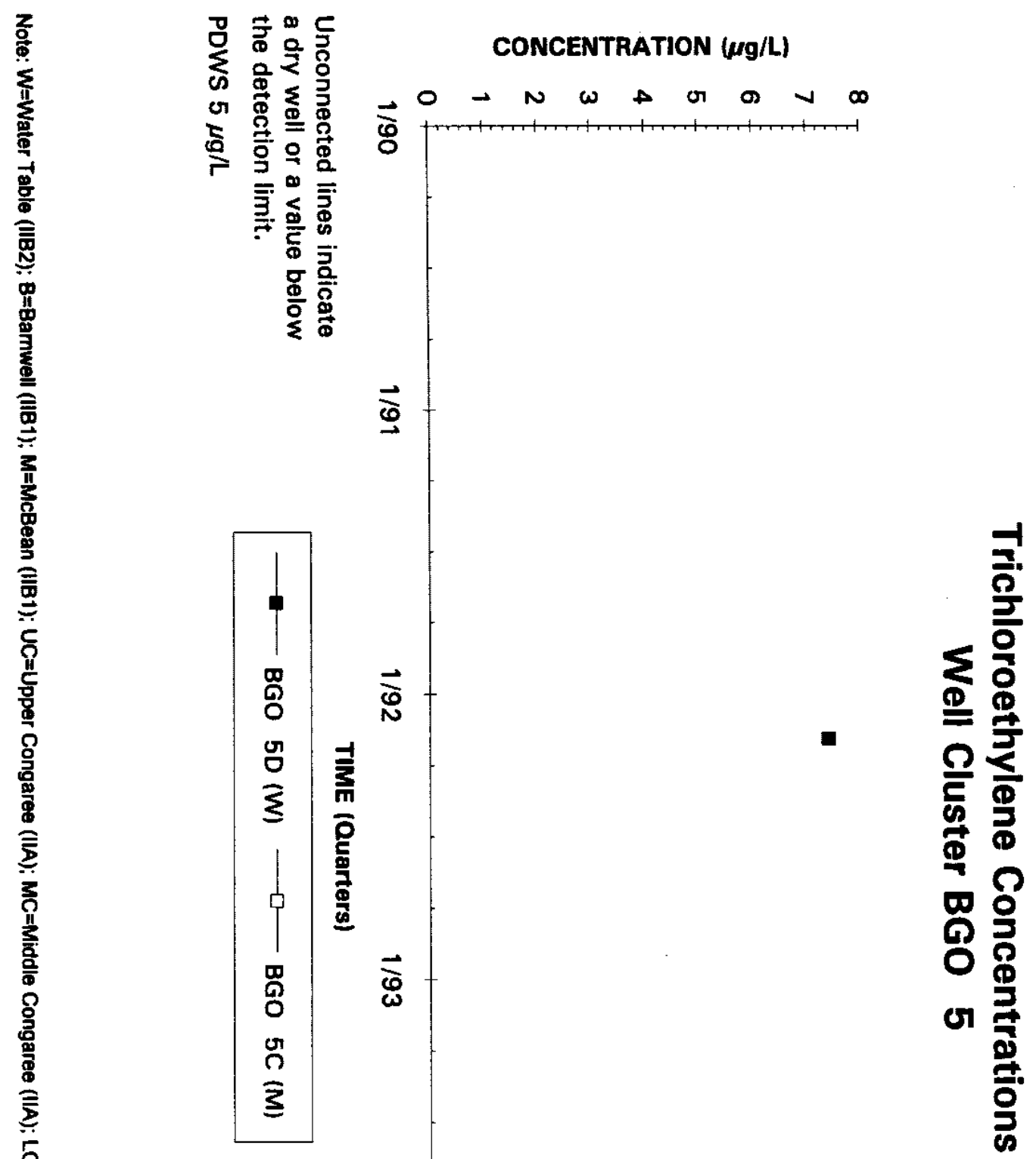



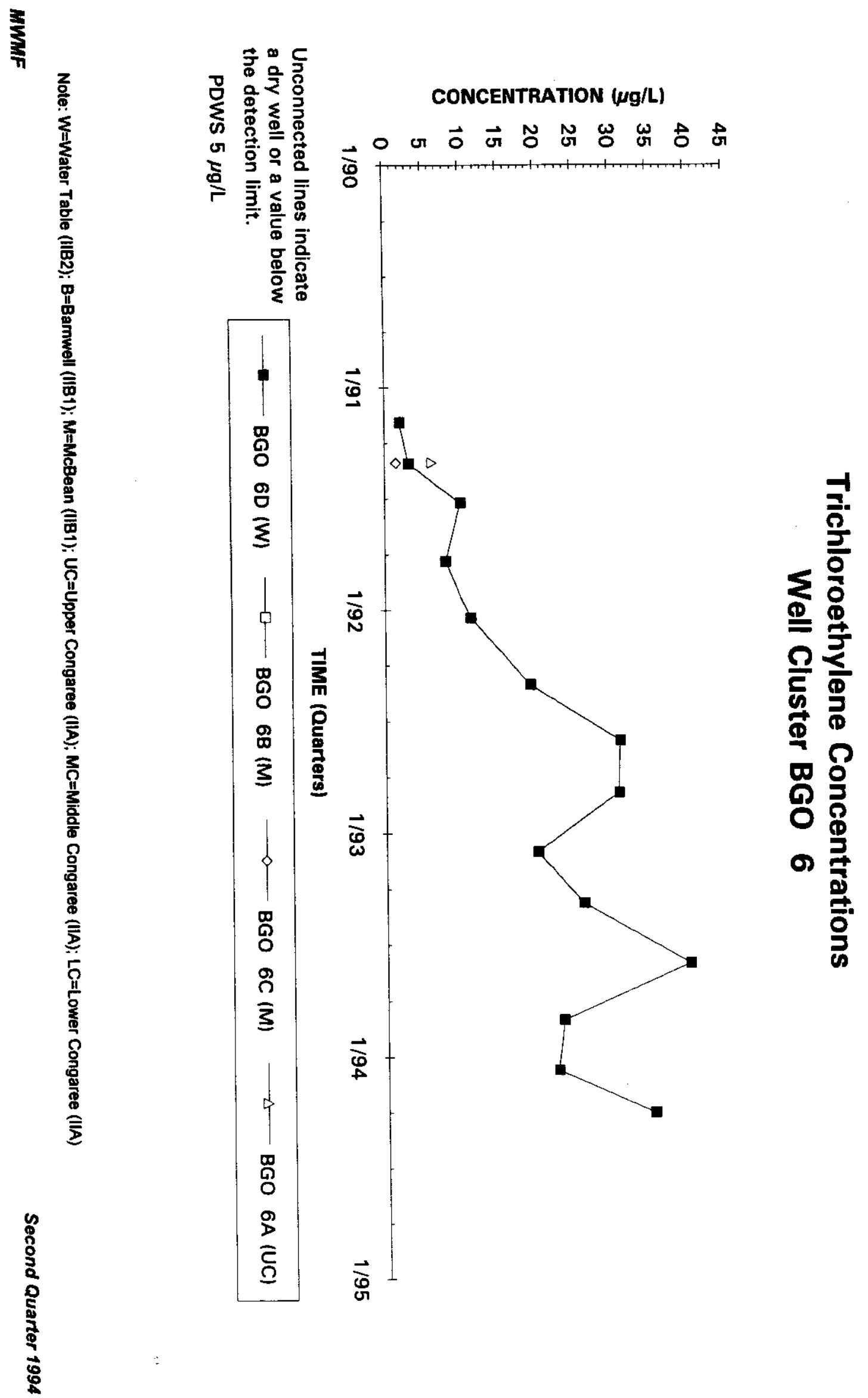


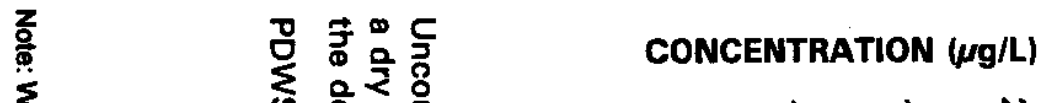

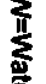

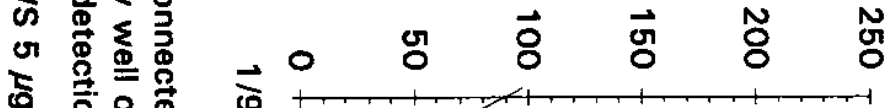

$\frac{\overrightarrow{\mathrm{m}}}{\mathrm{\sigma}}$

言

罚

尊

言

夏
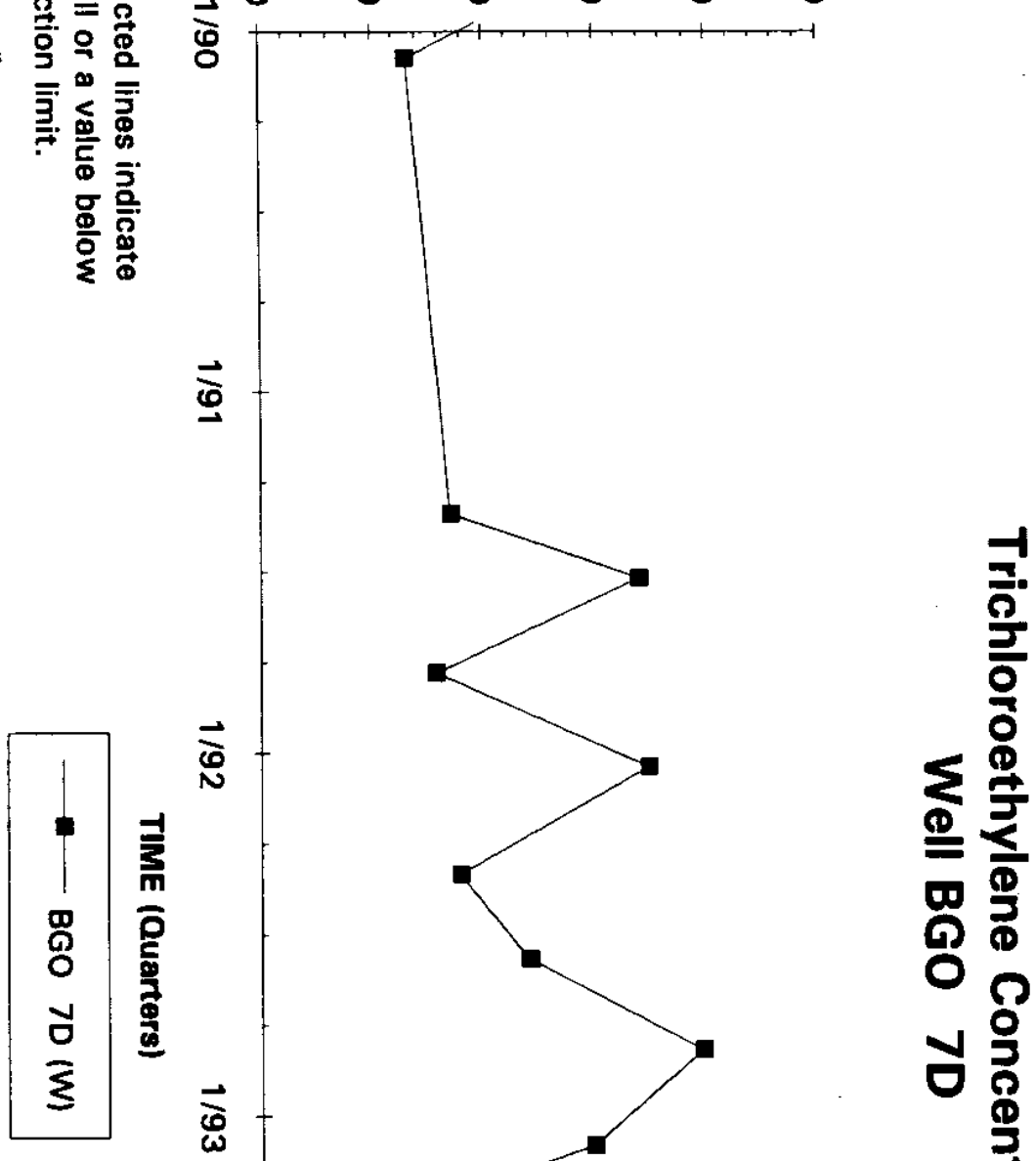

0

옹

言

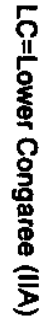


翠

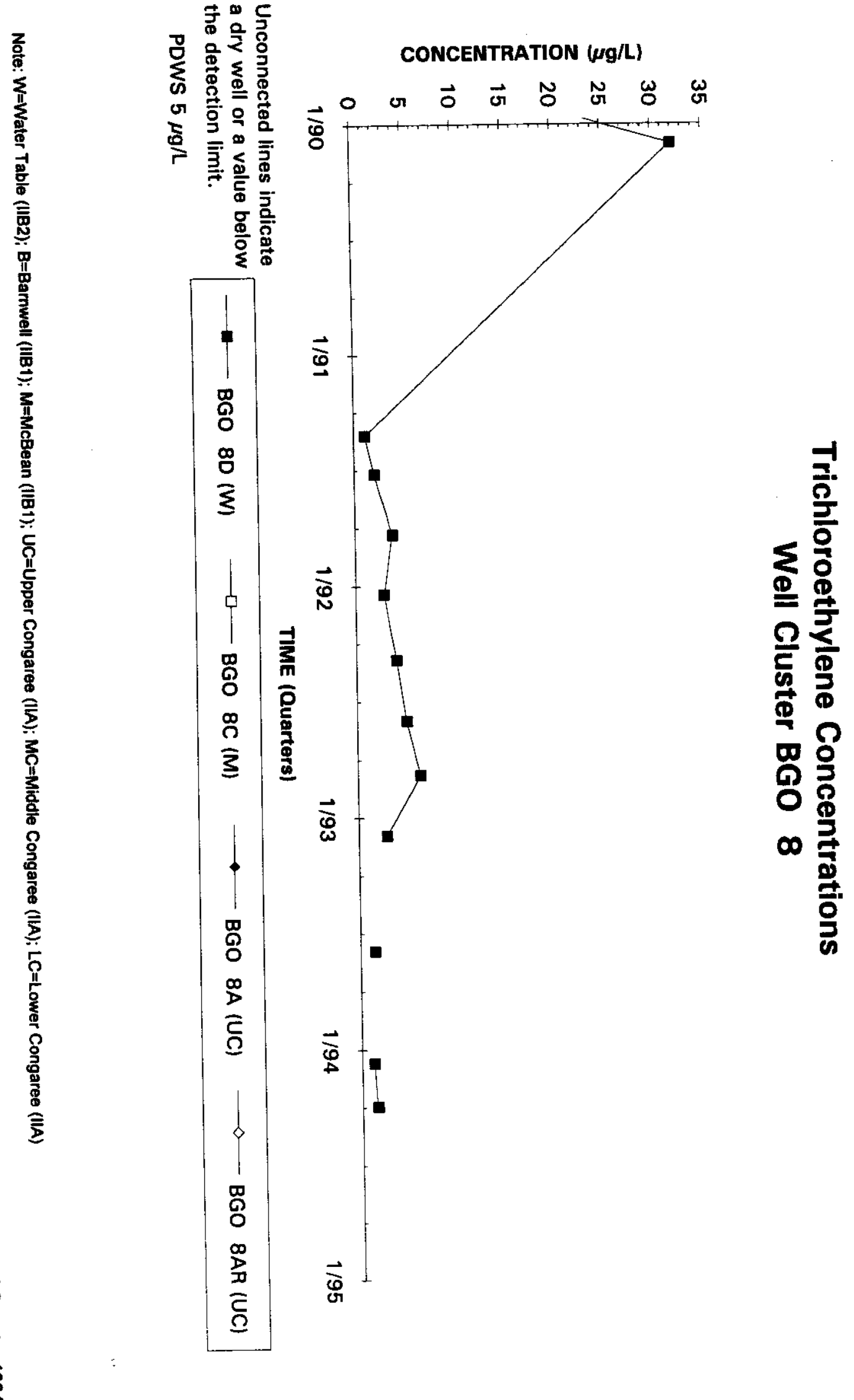

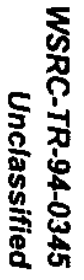




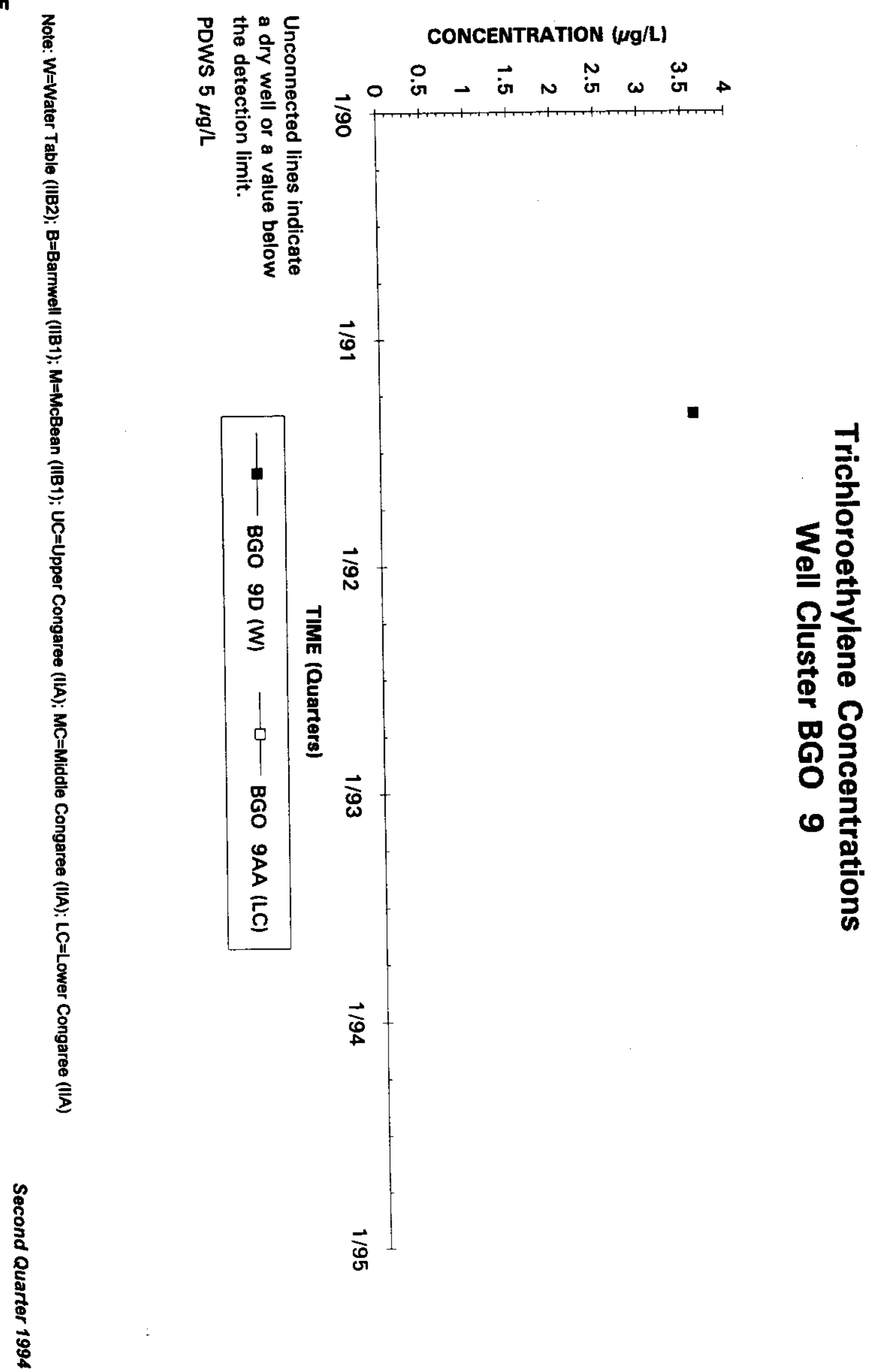


䓌员

응

西

궁요 훙

产 咅空
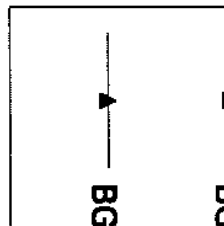

器

$\overrightarrow{\mathrm{s}}$

产

ㄷำ

8 용

हू
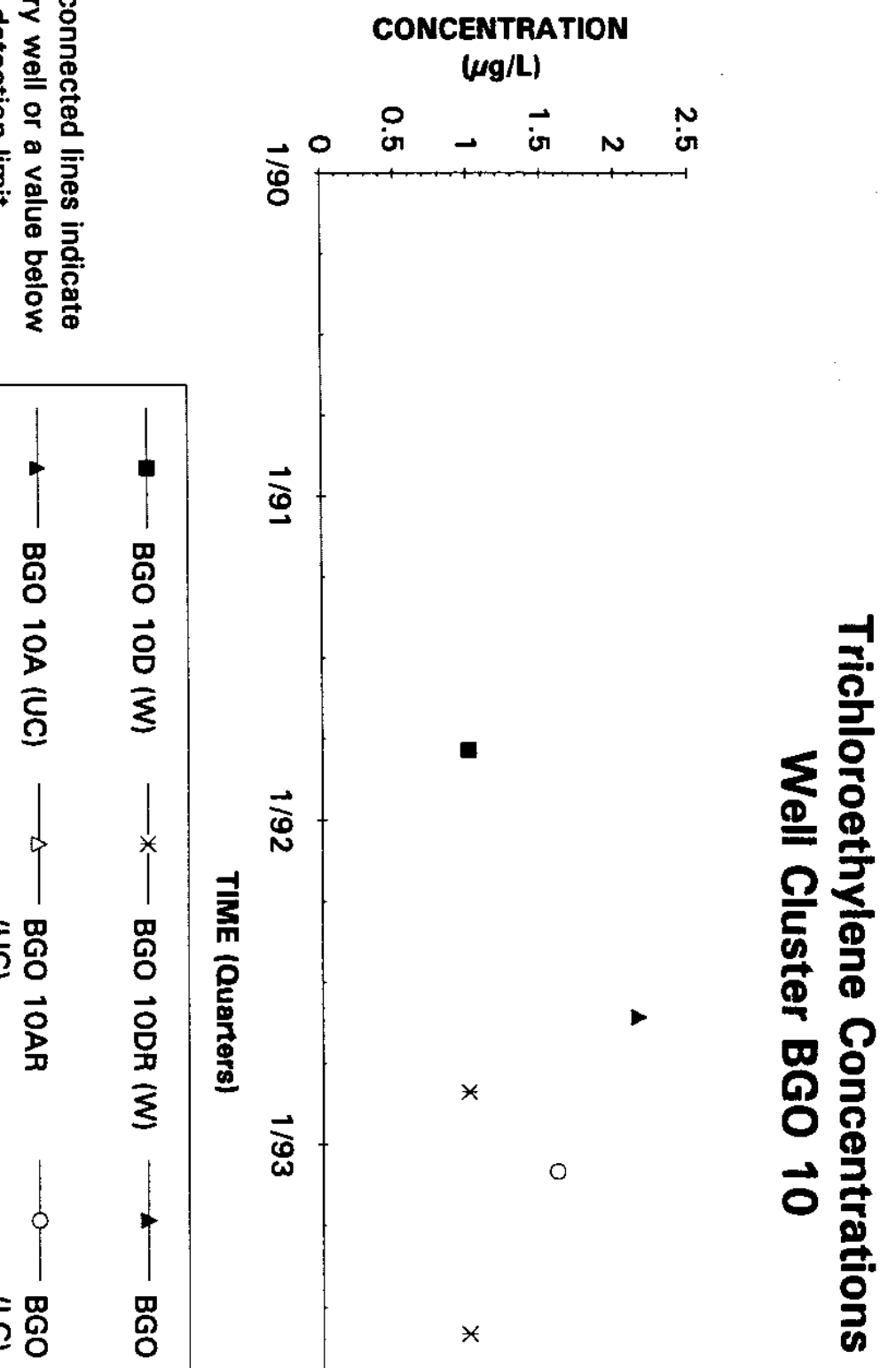

ธิธ

ธิธ

옹

$\Sigma$ 


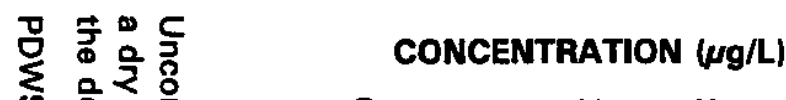

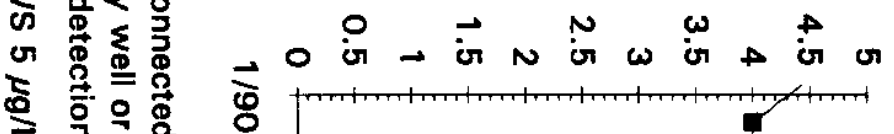

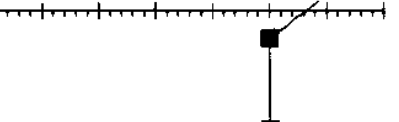

홍

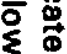

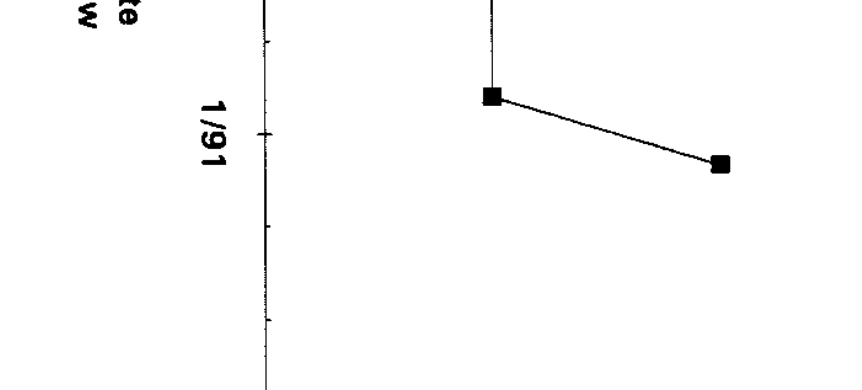

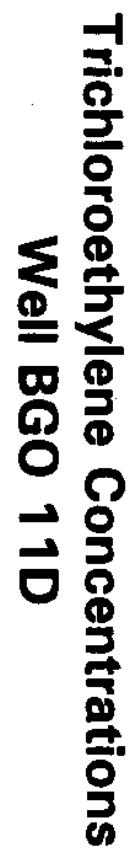




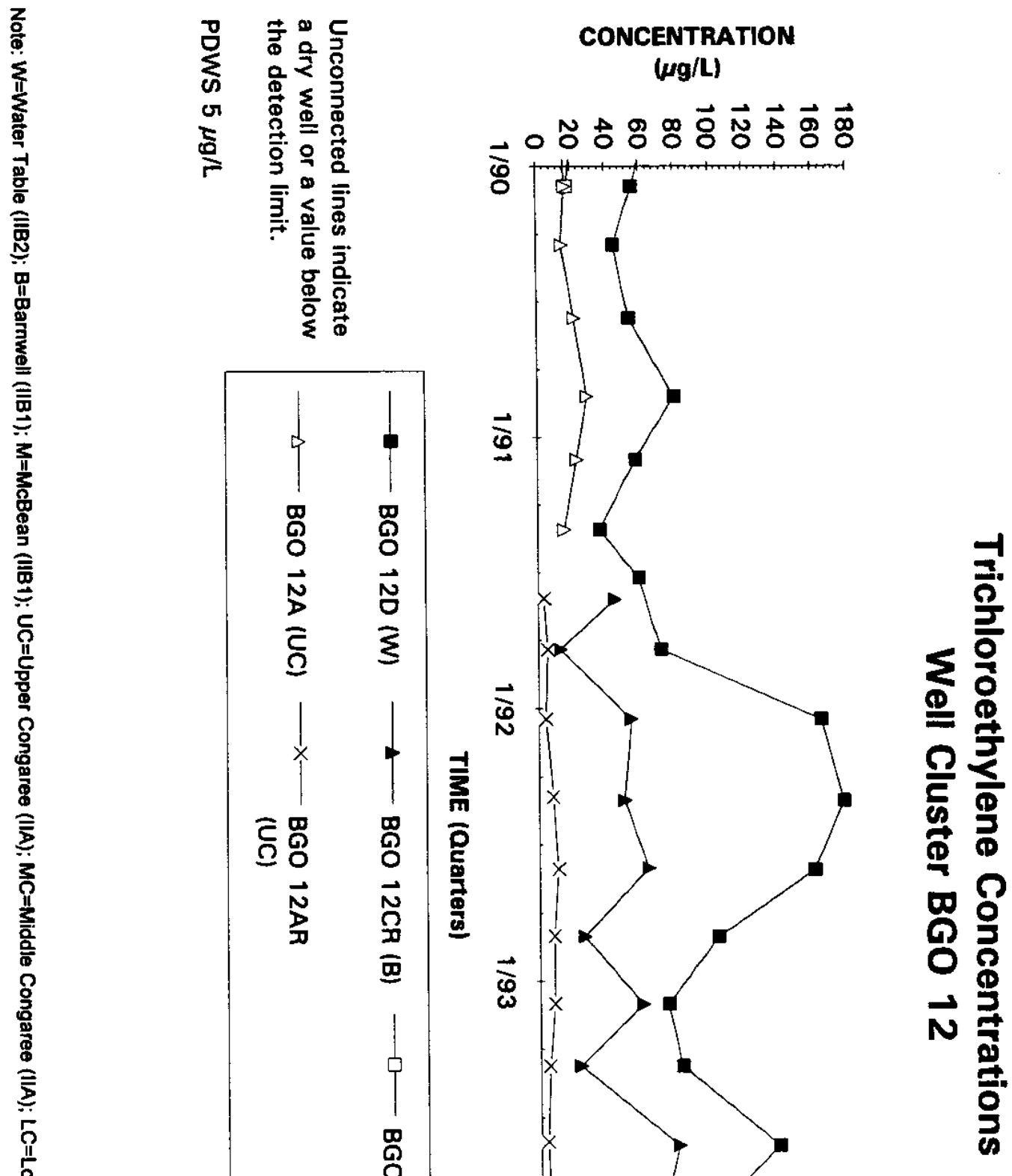




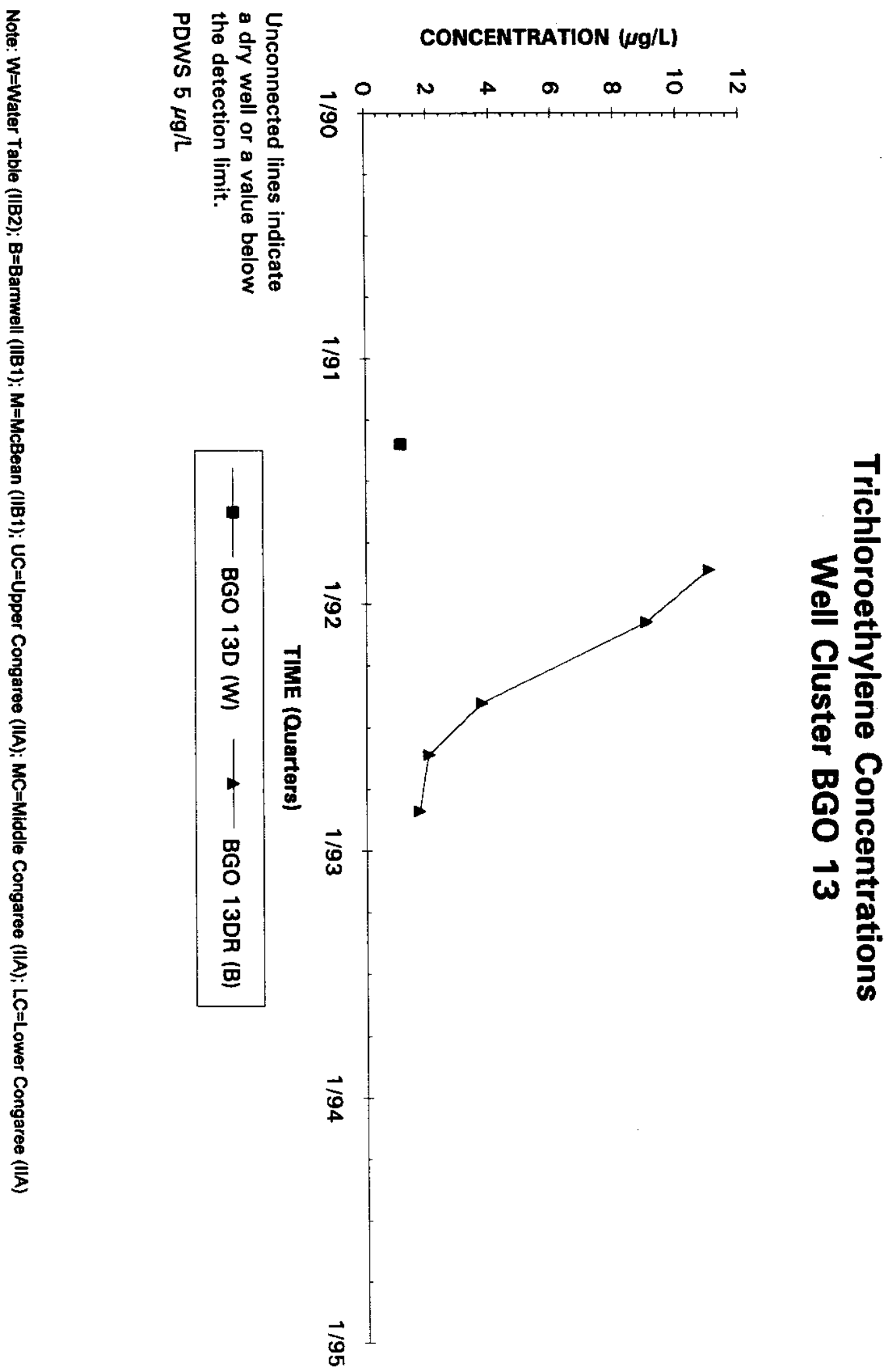



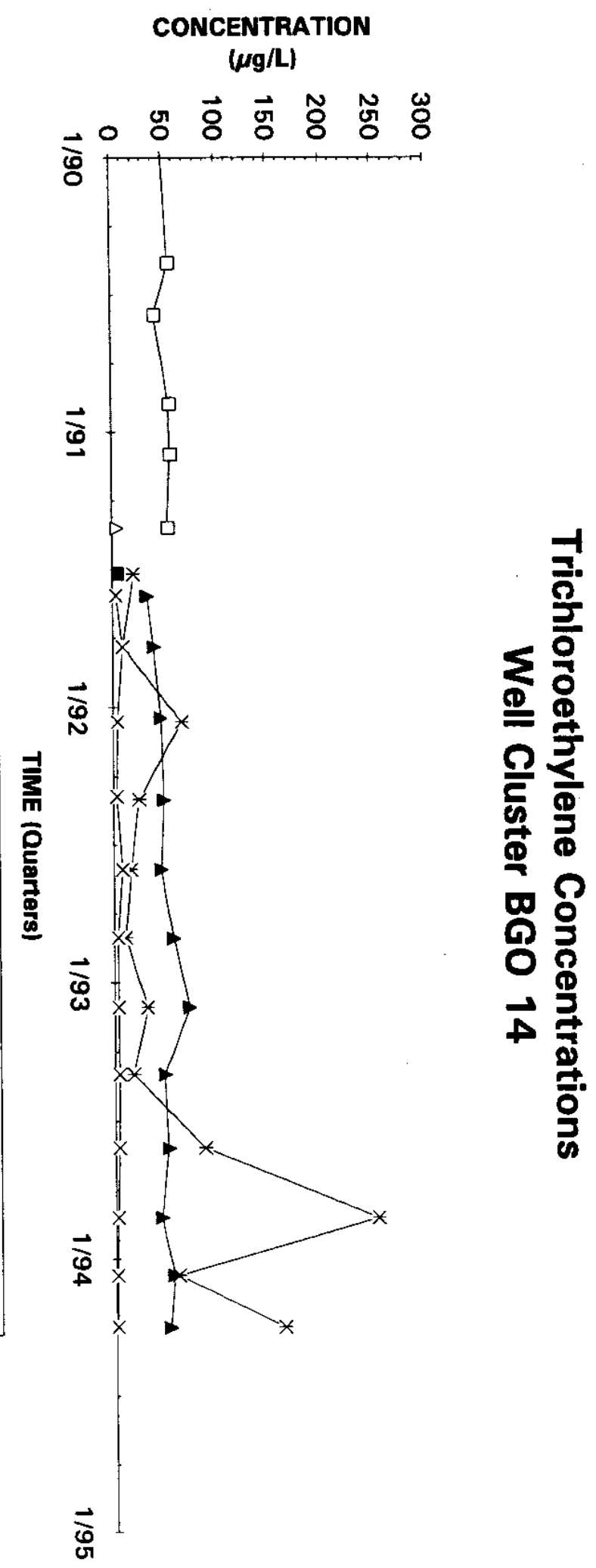


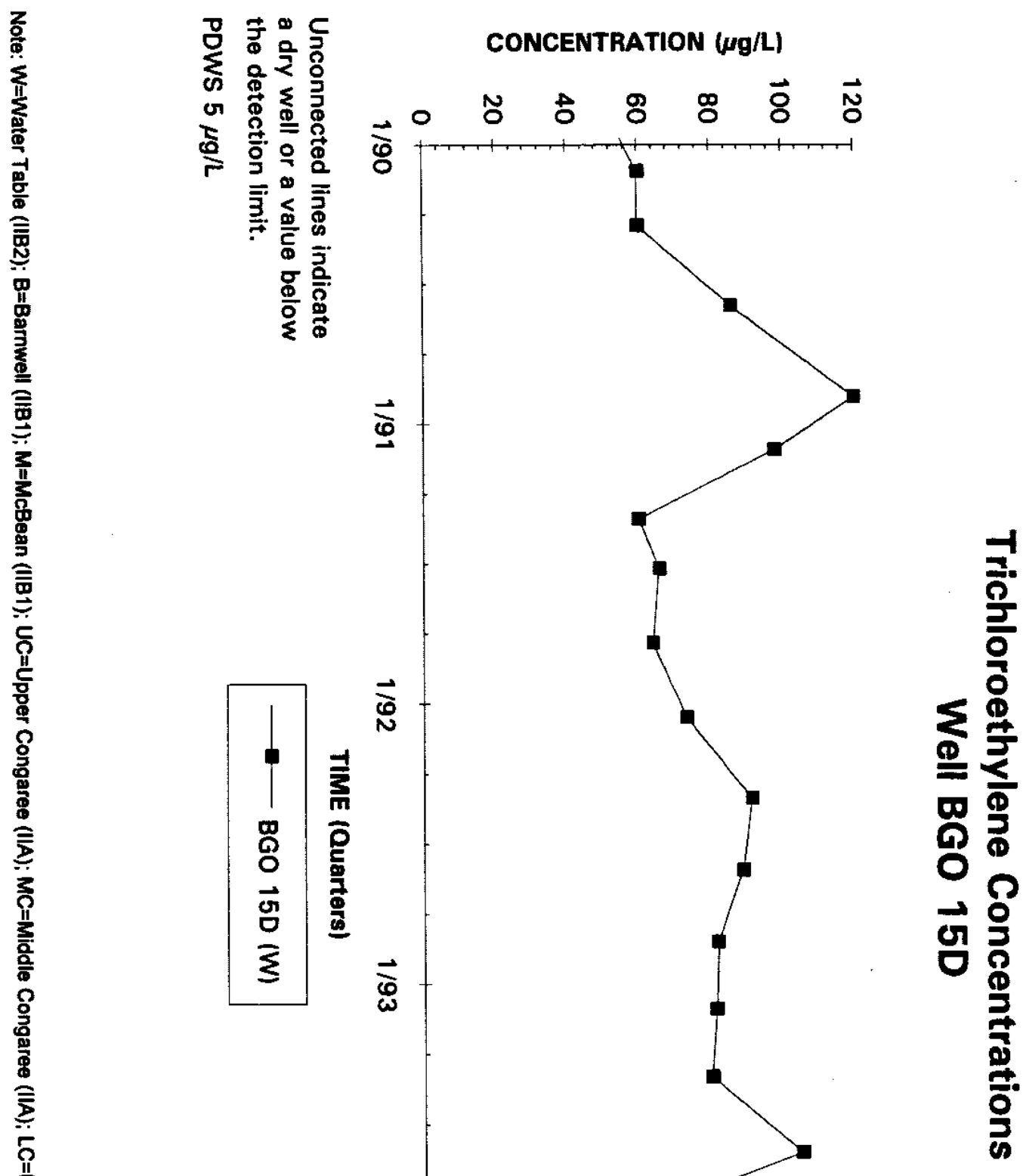




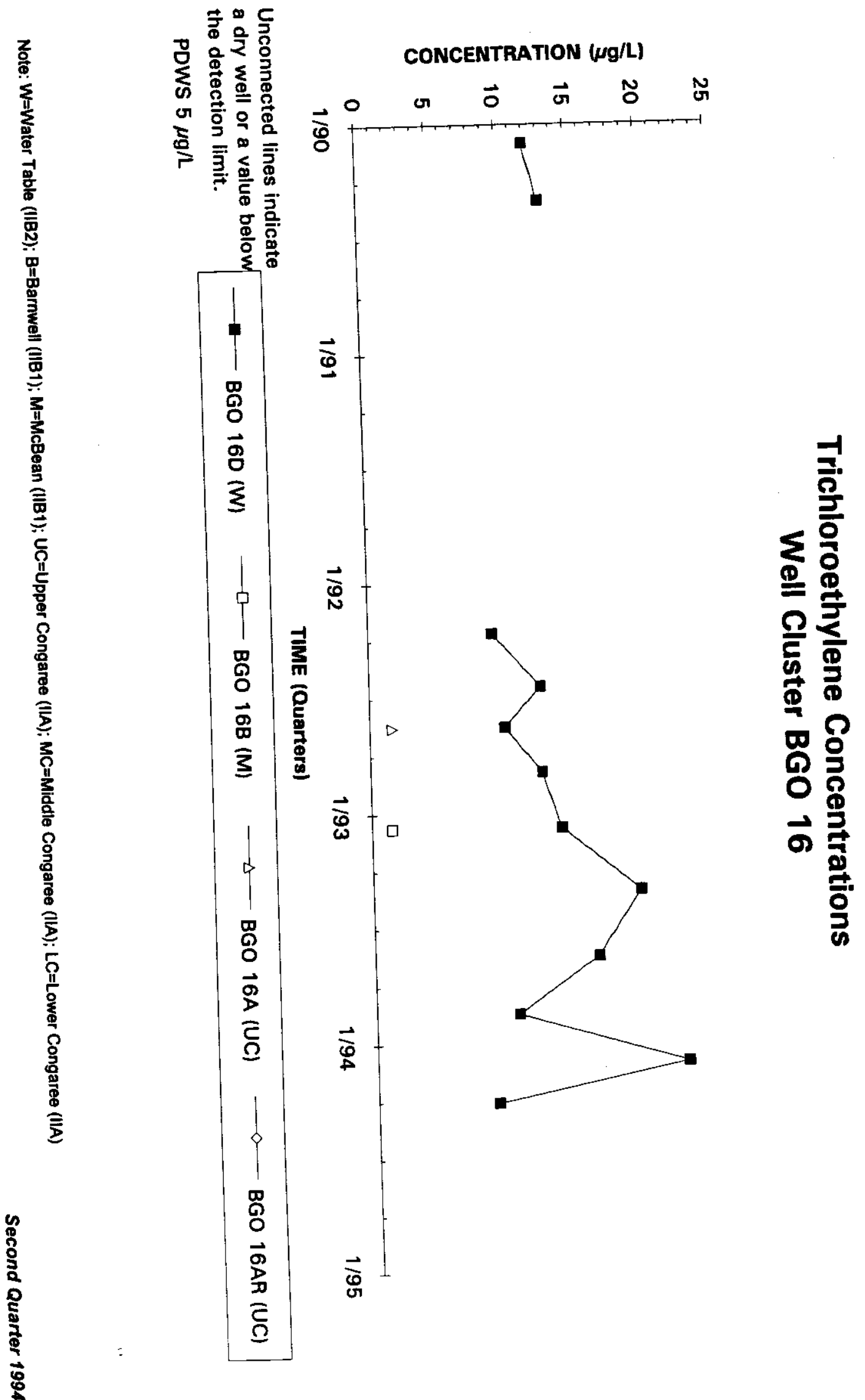




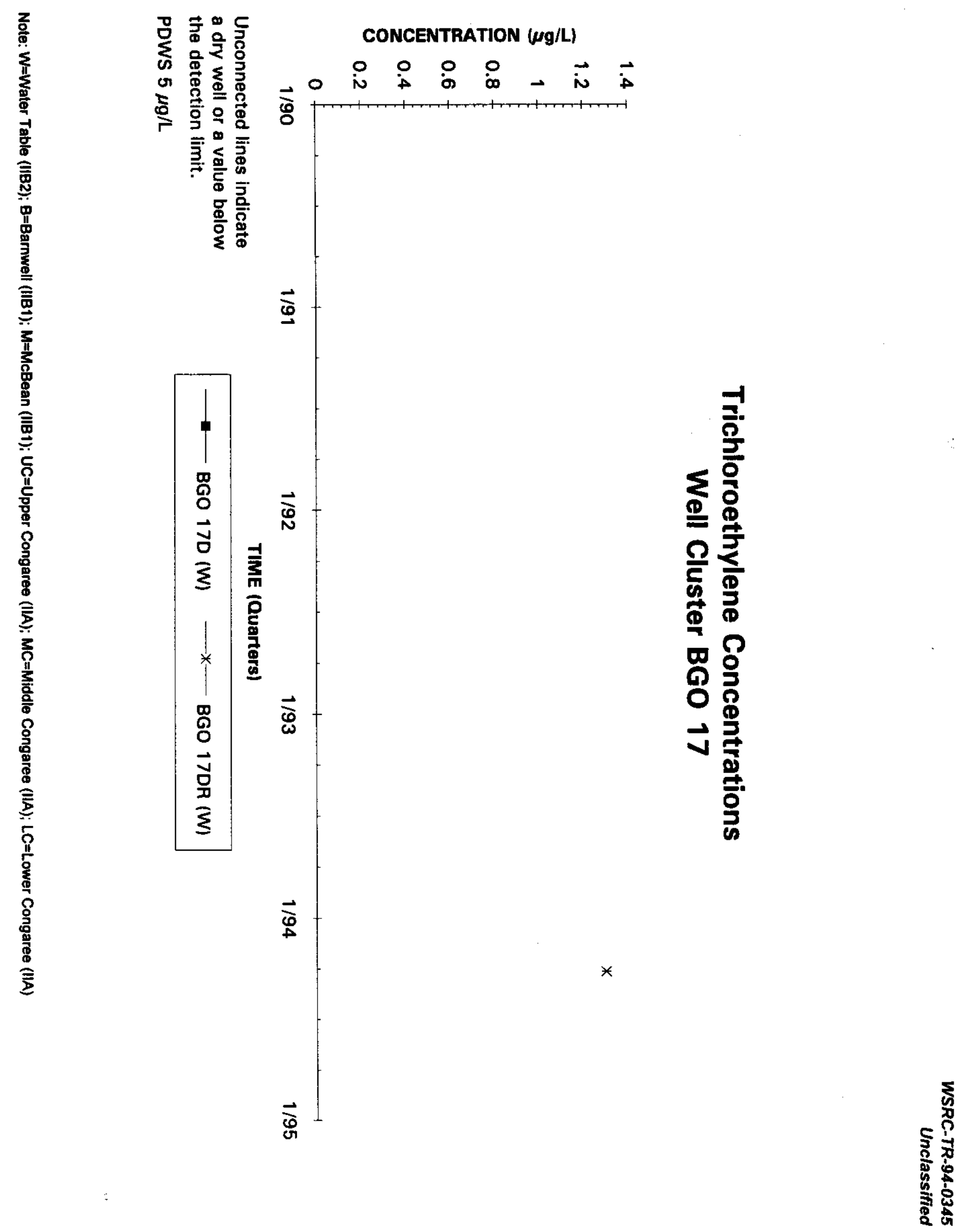




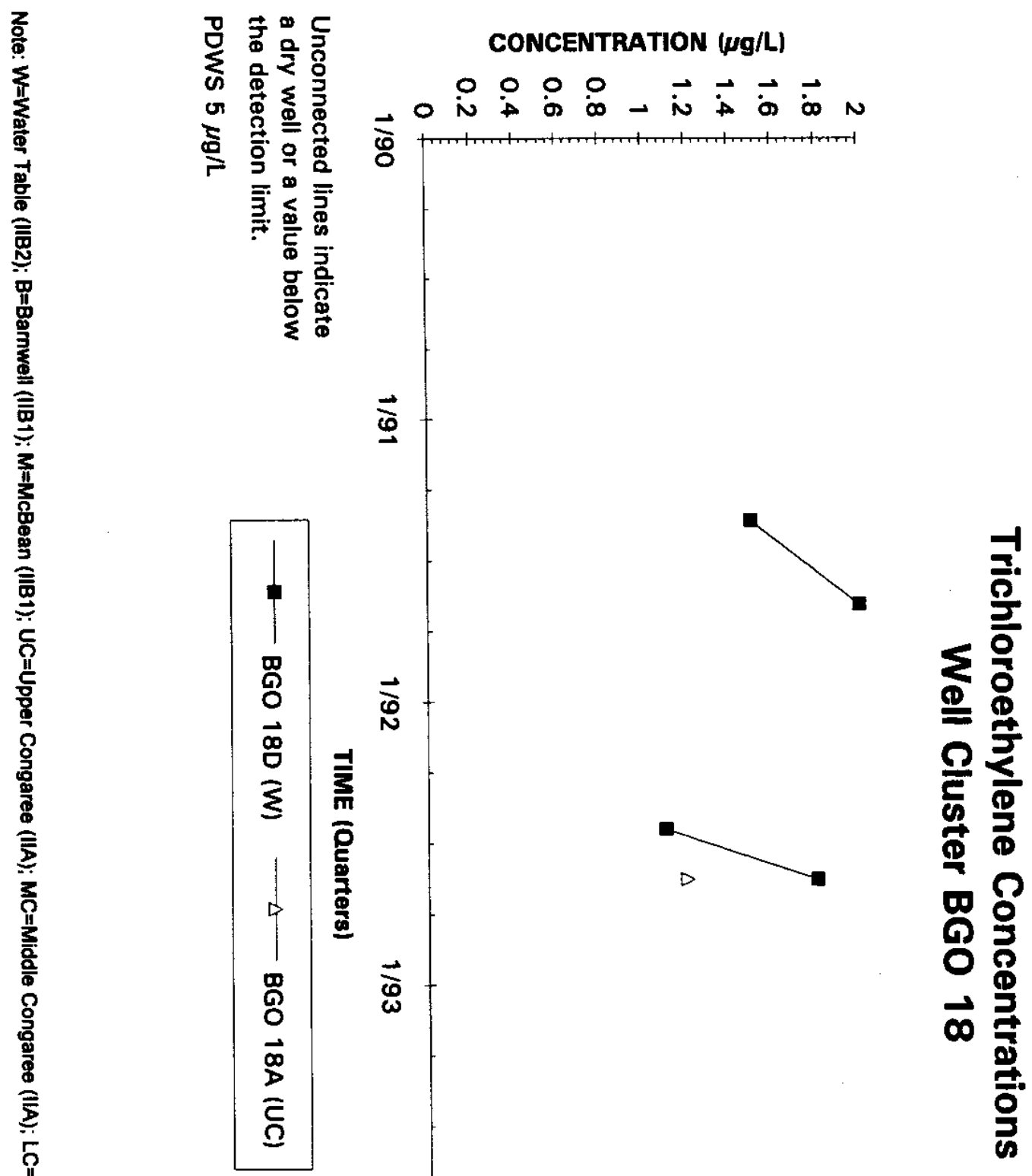




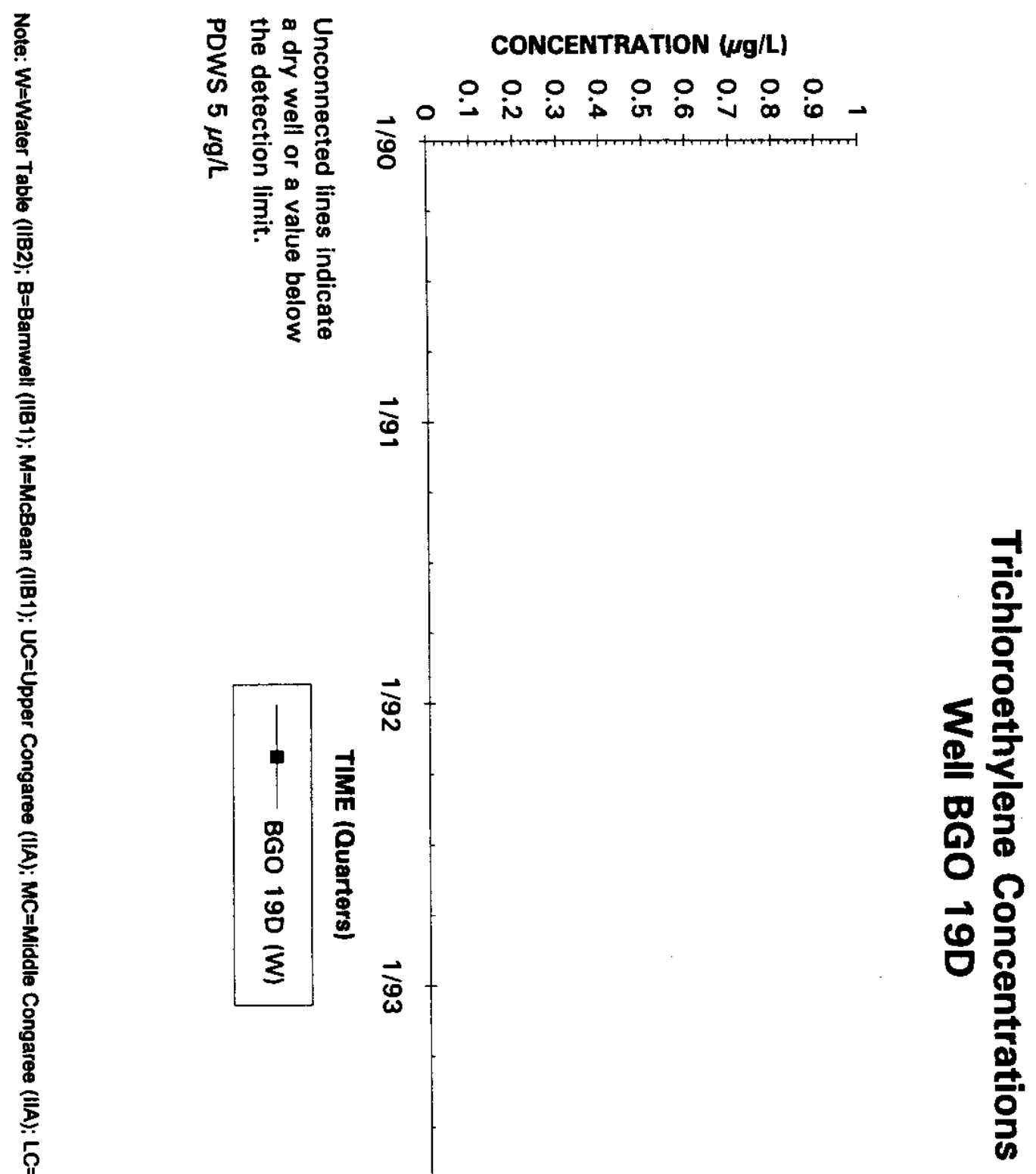


登

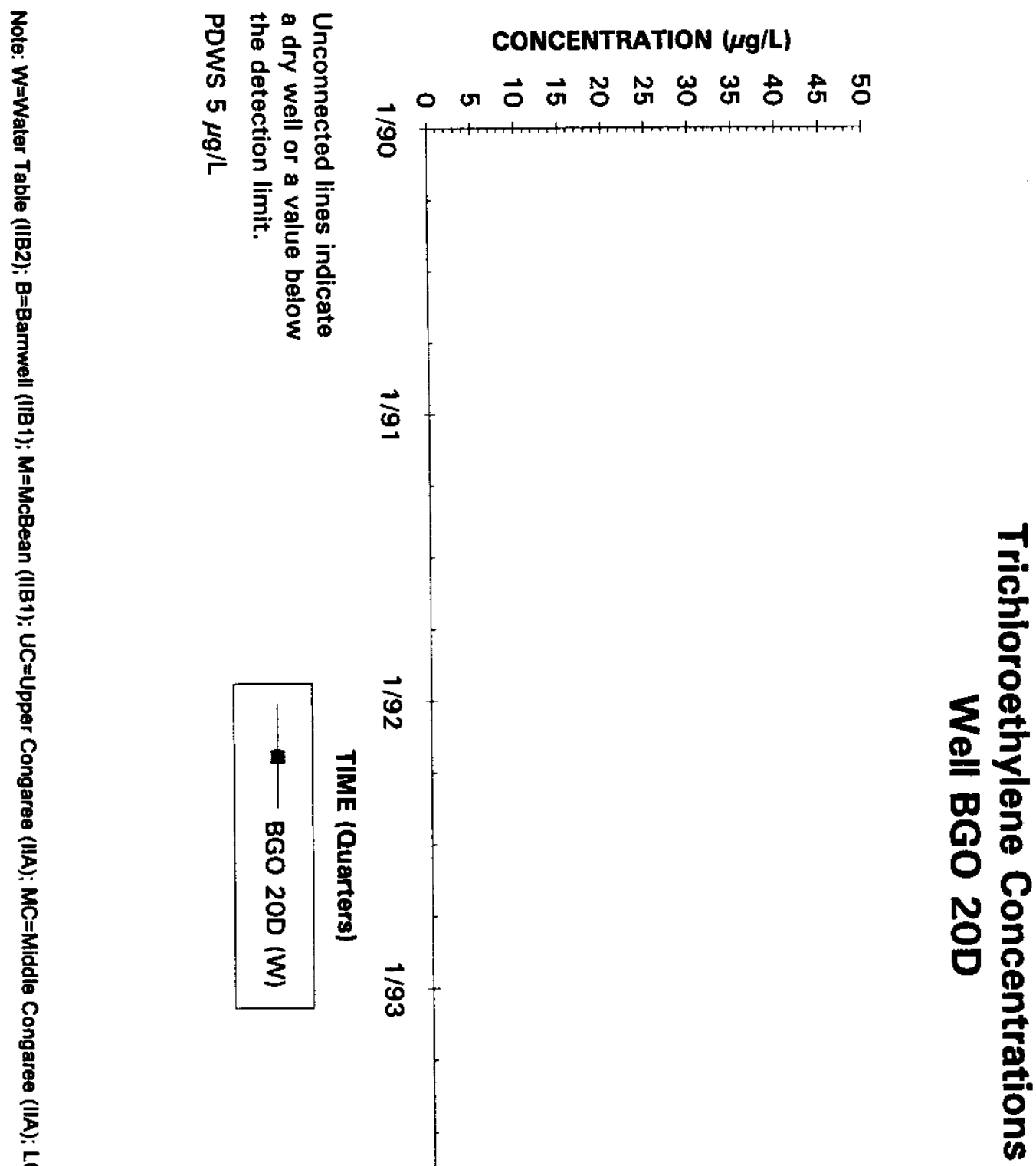



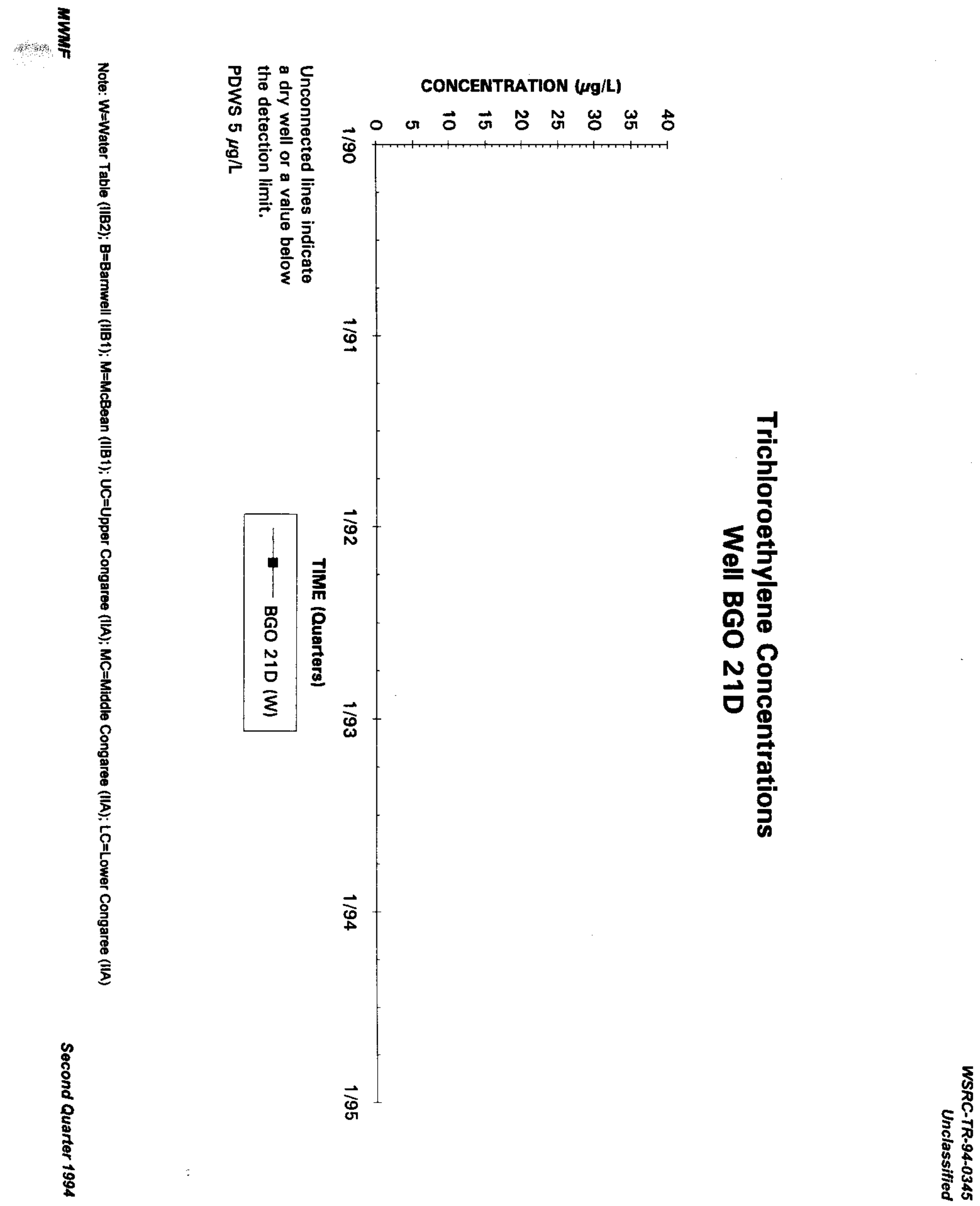
3

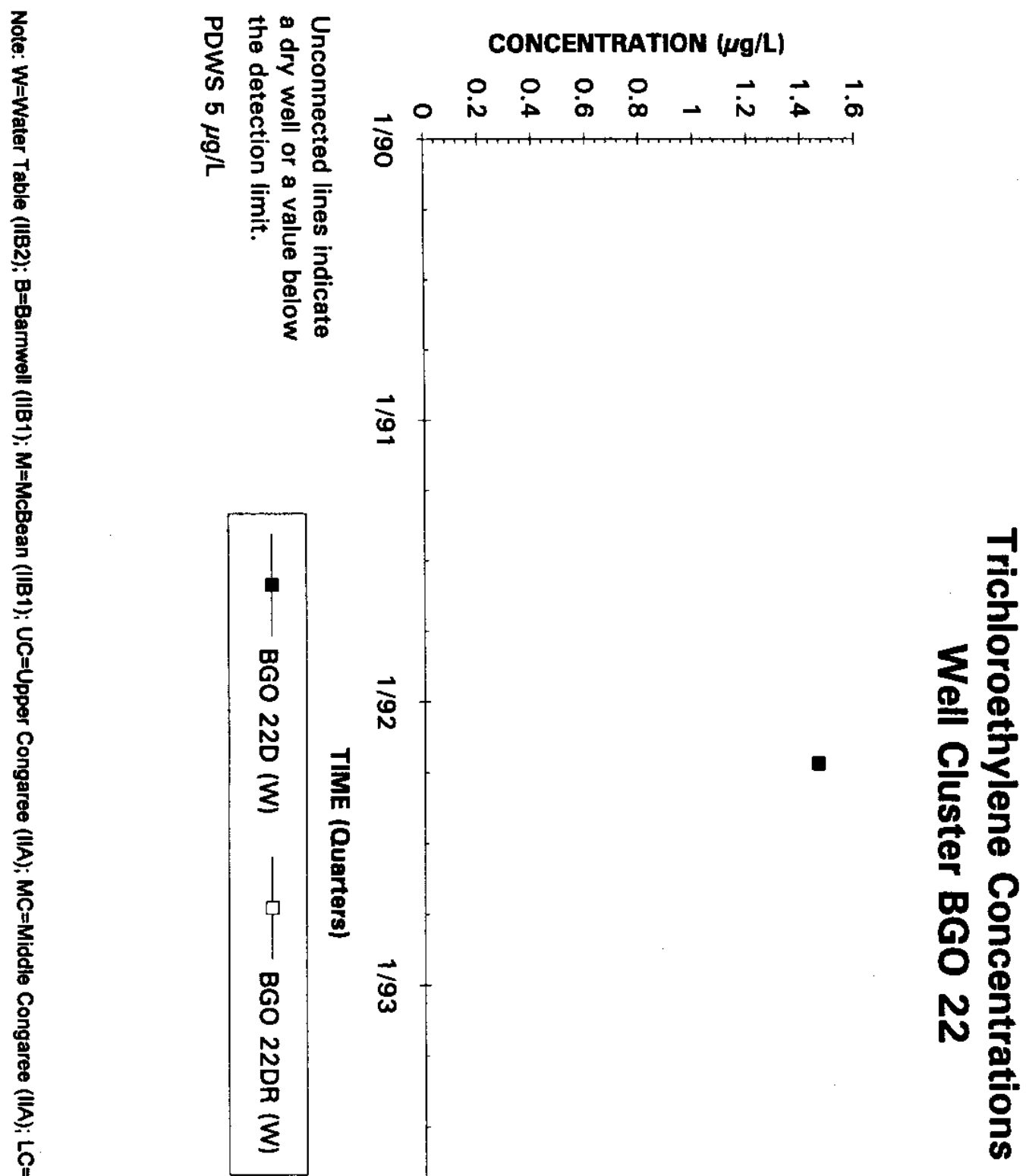




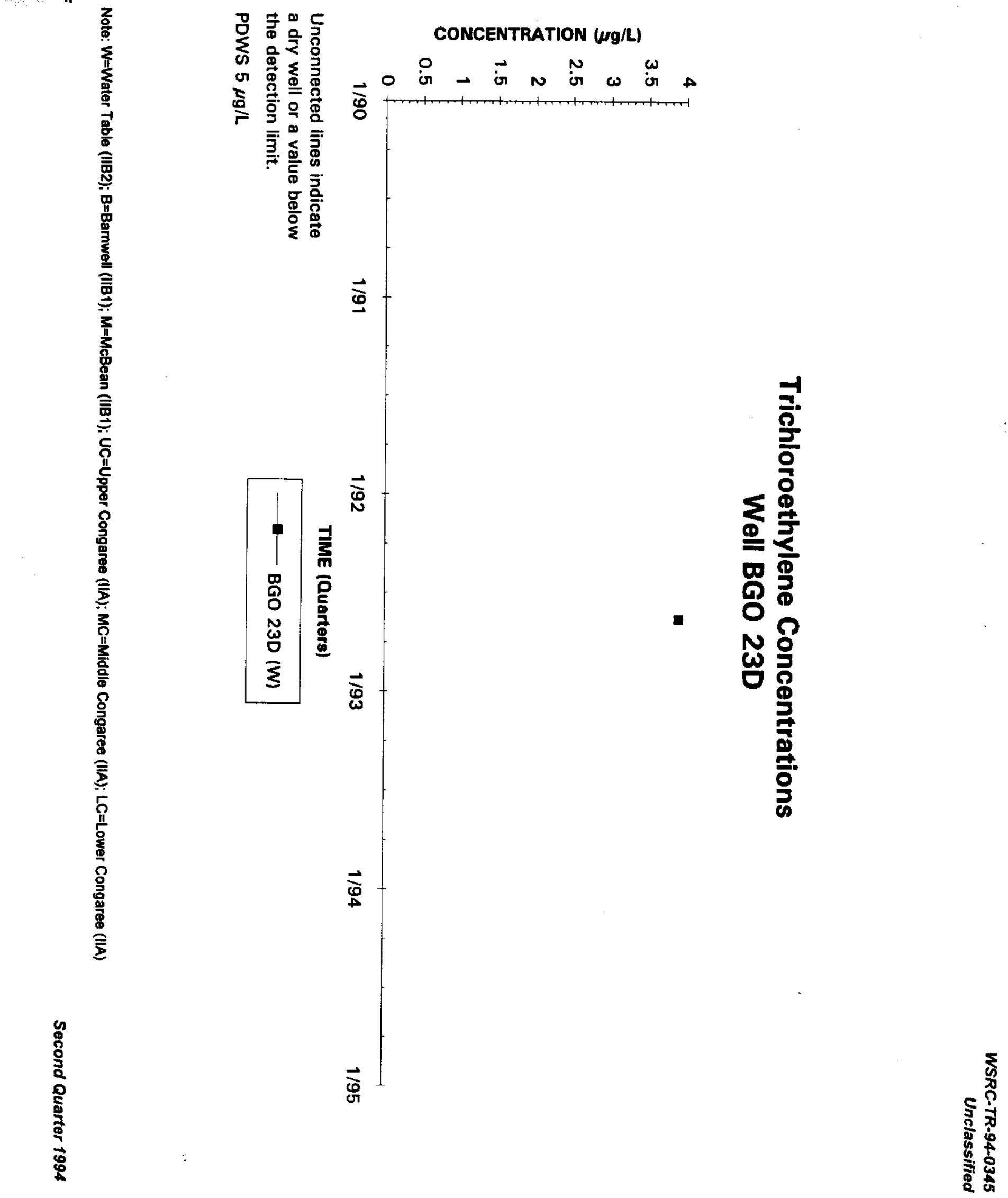


3

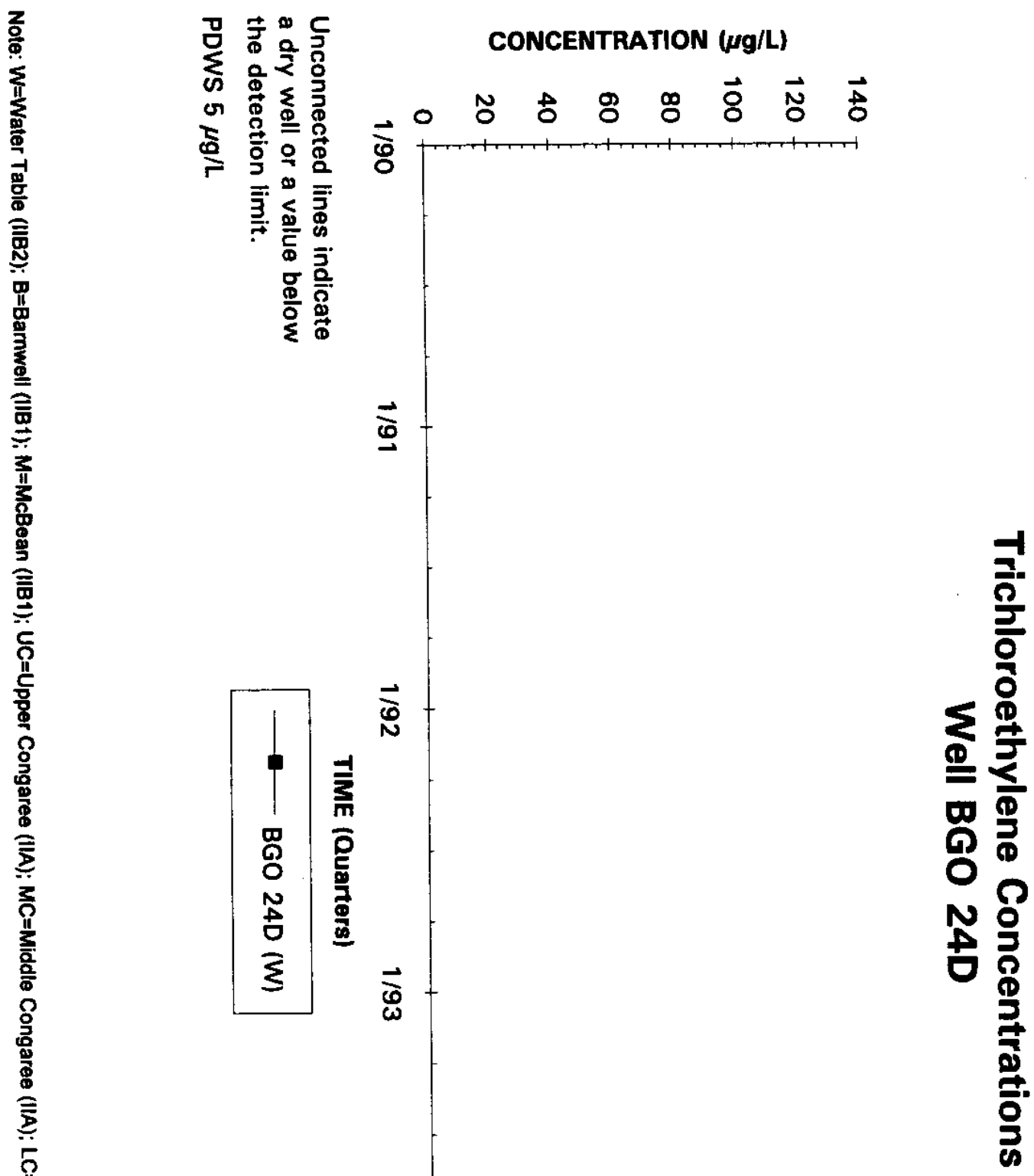

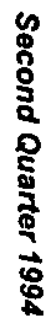

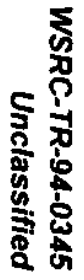




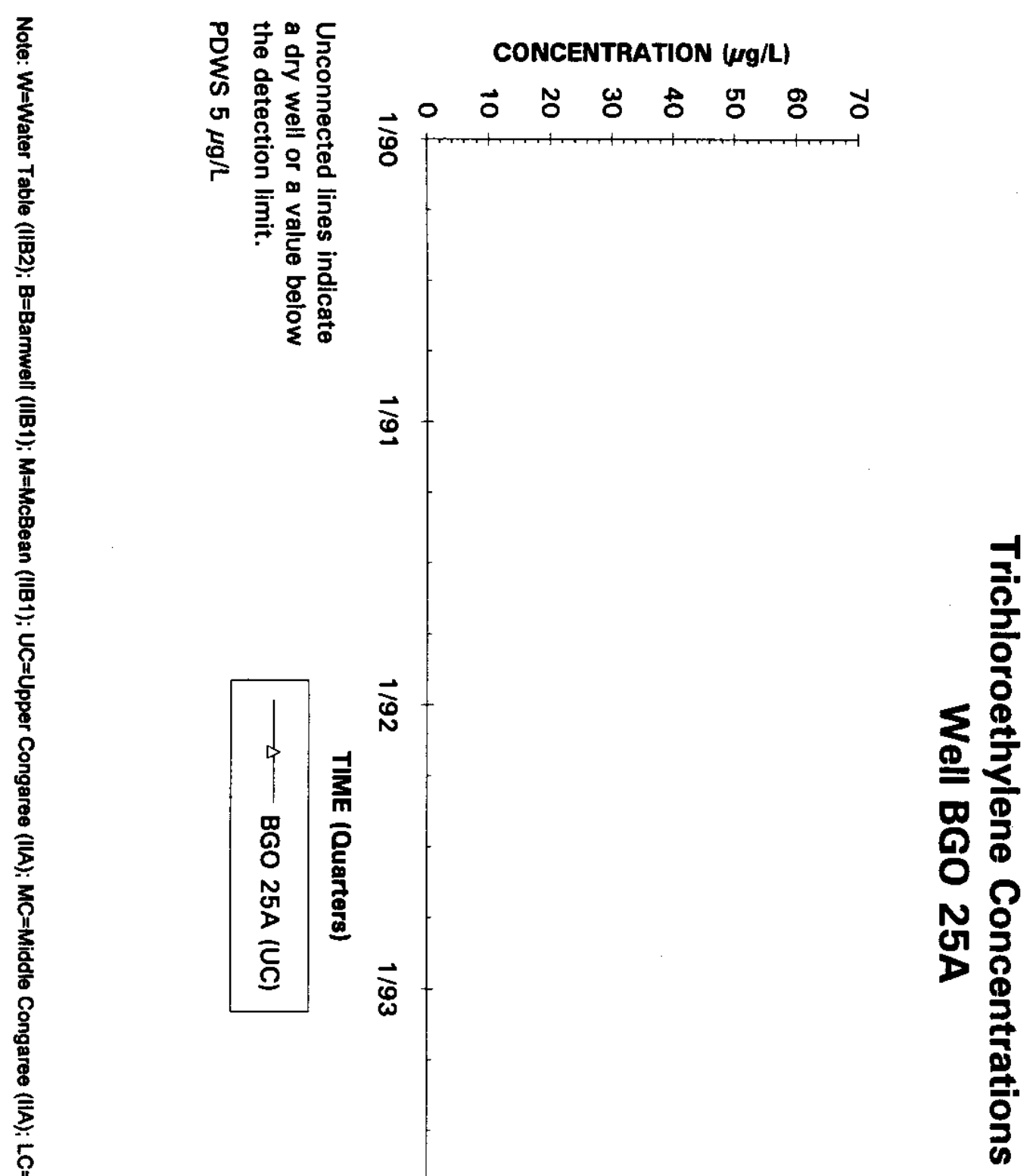




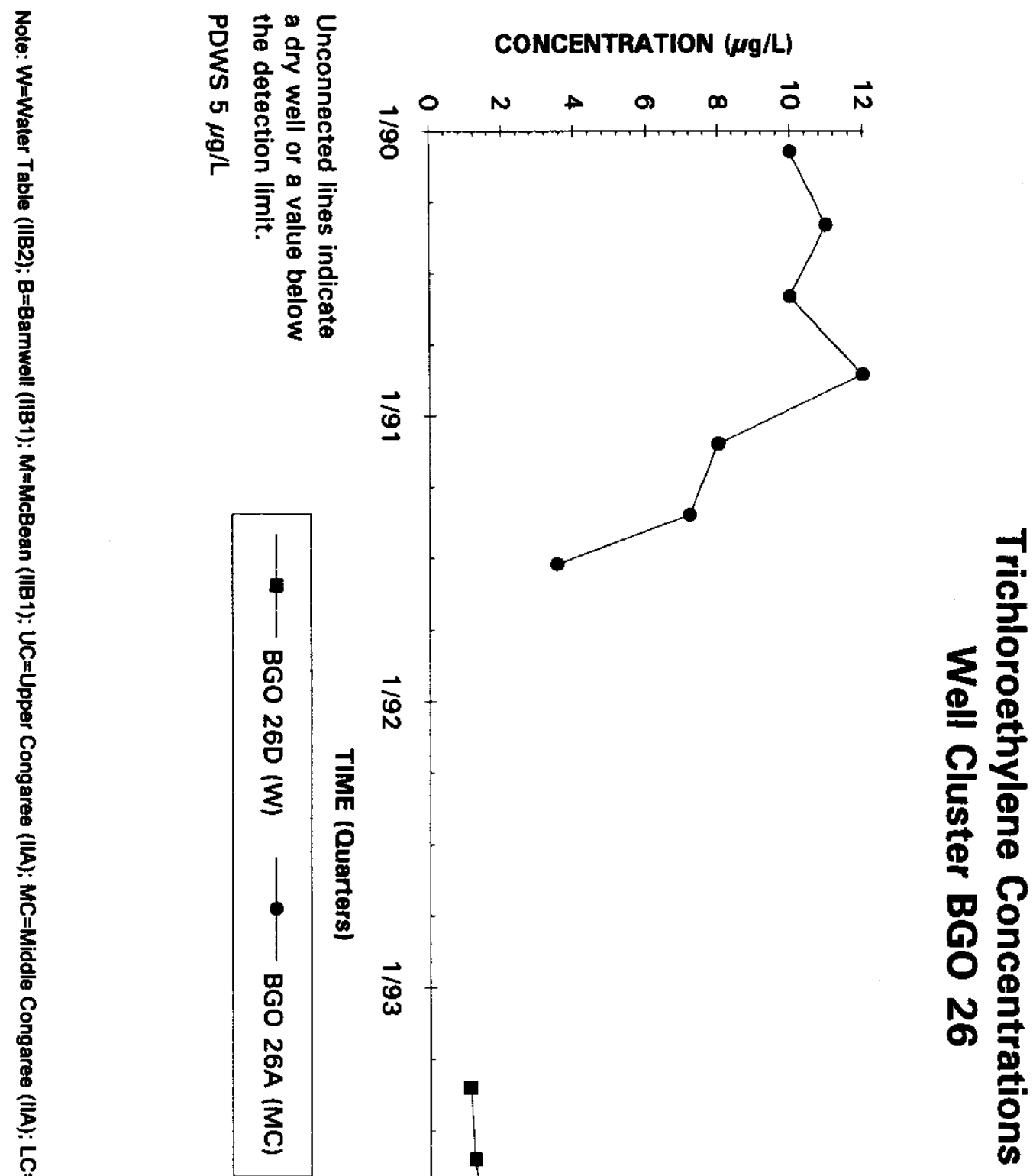




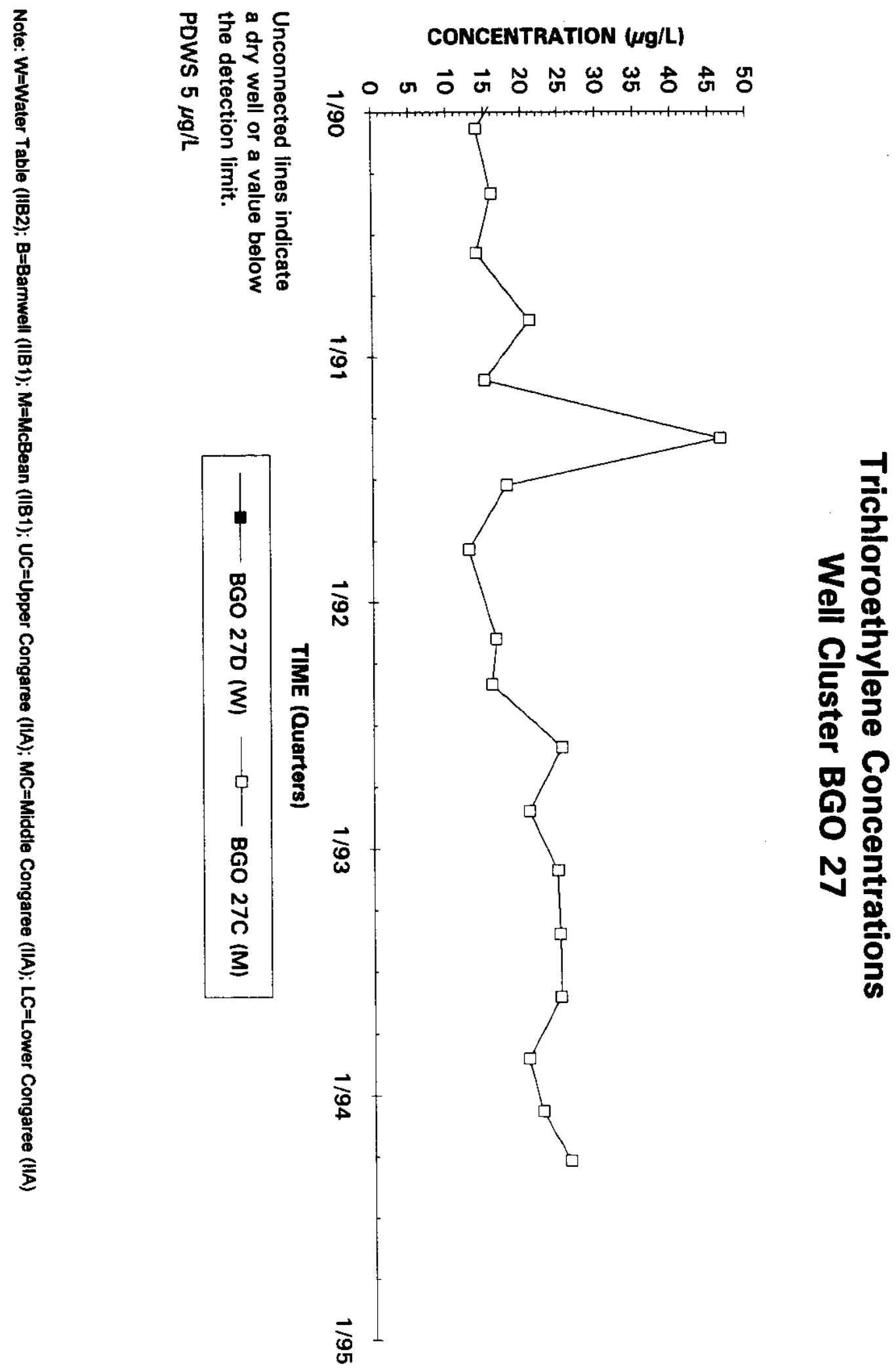


3

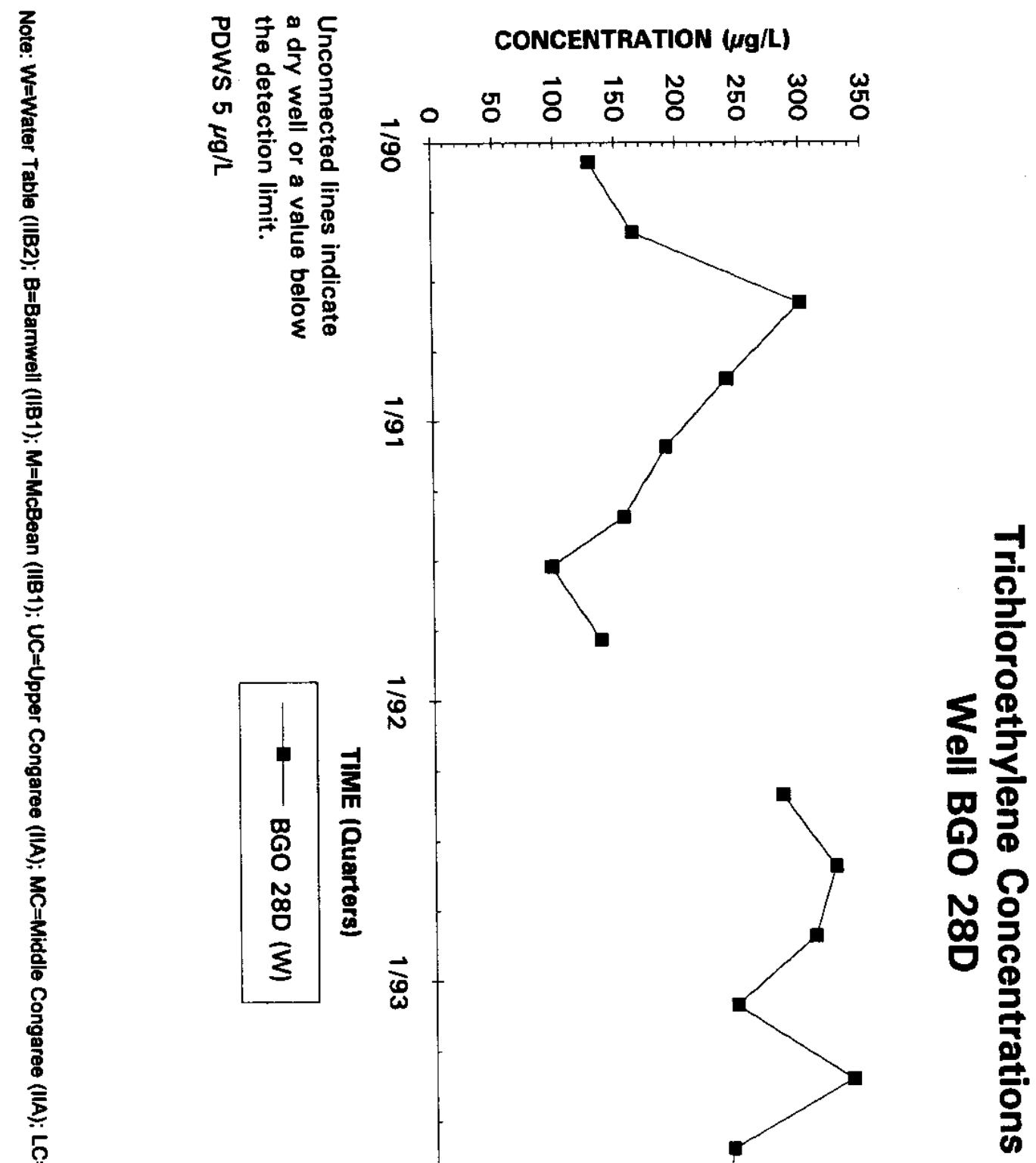

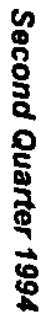

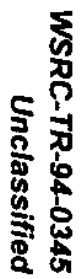


2

훟

言

思

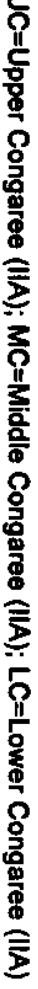

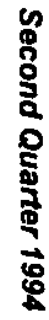
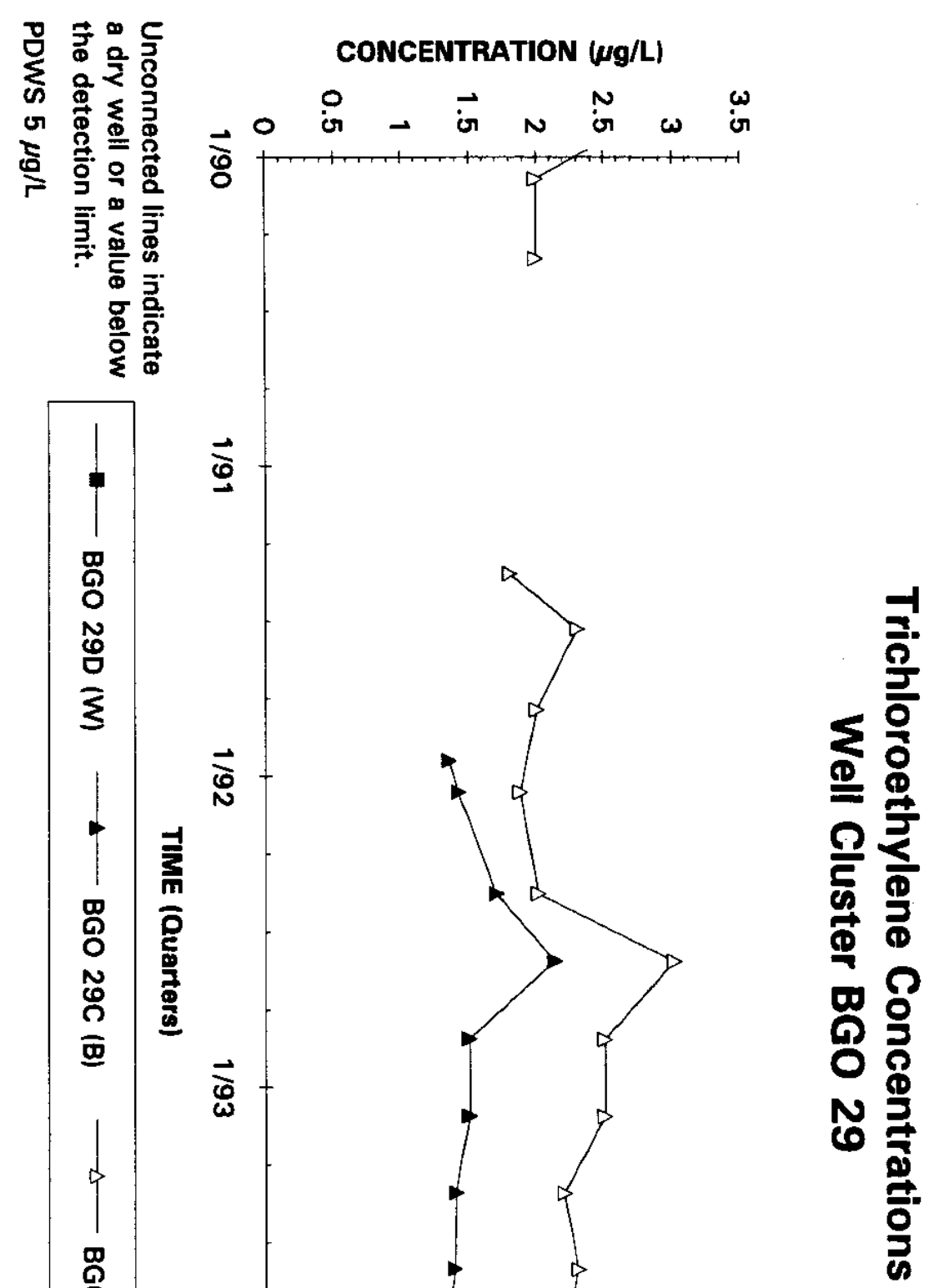
疍

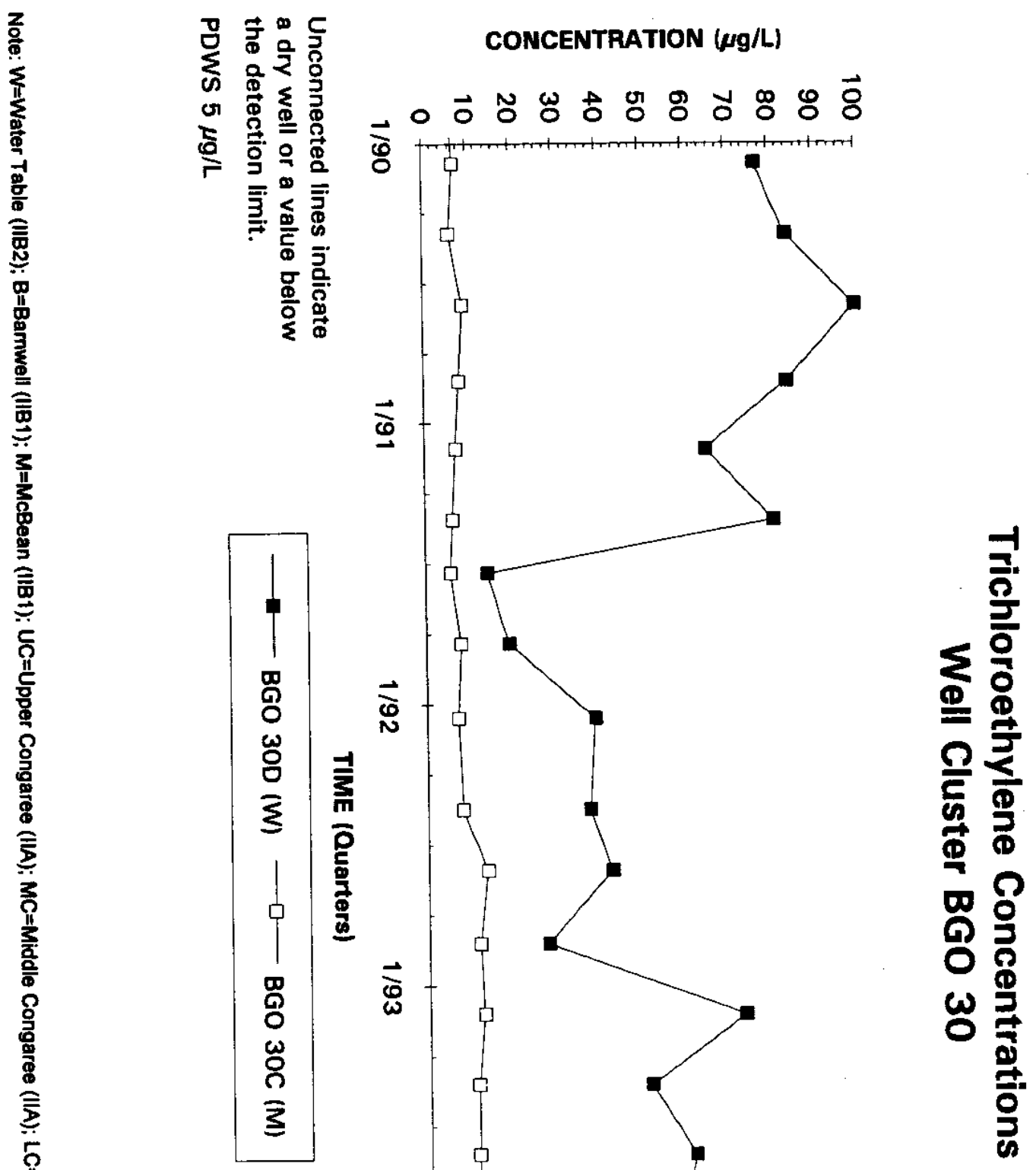




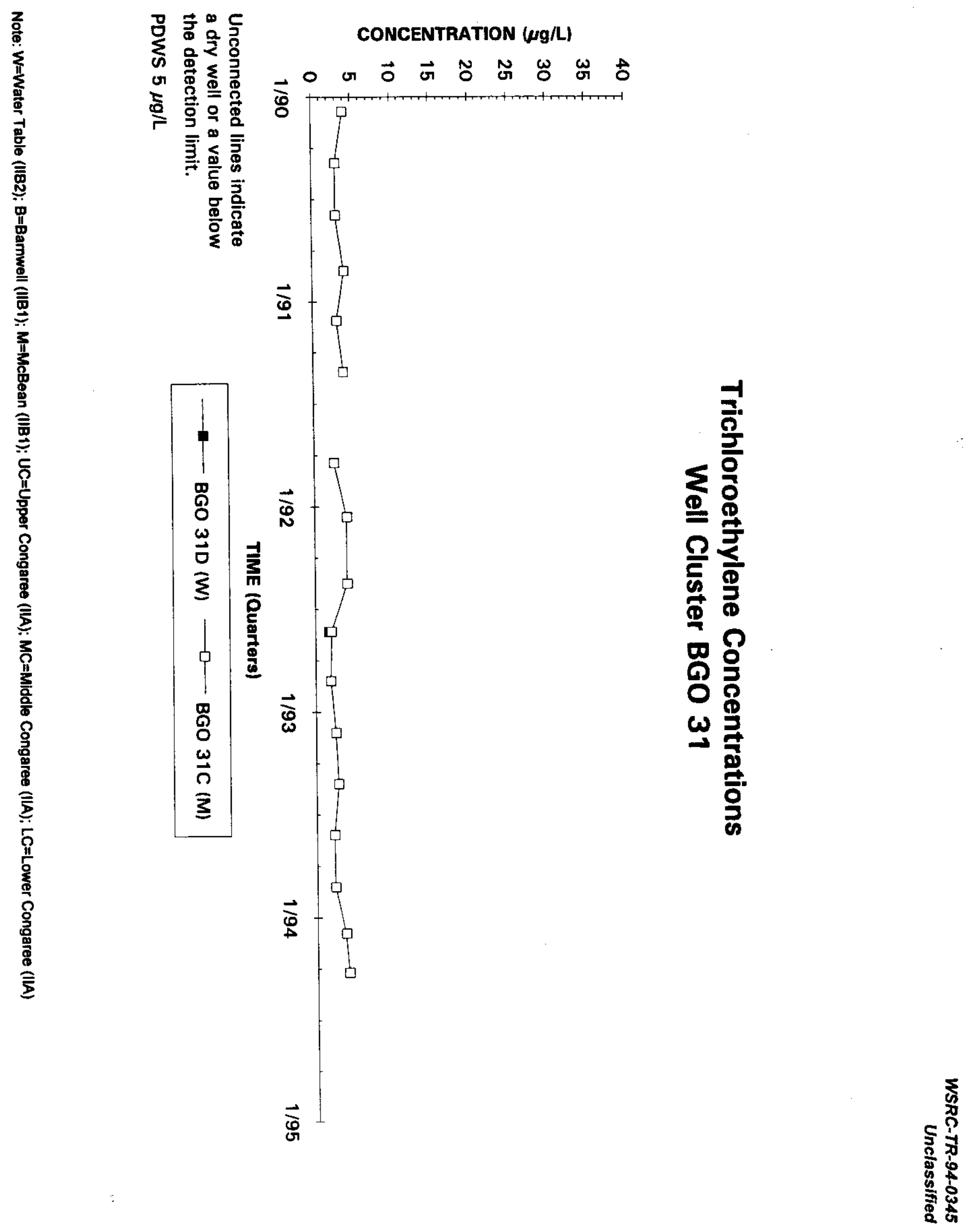




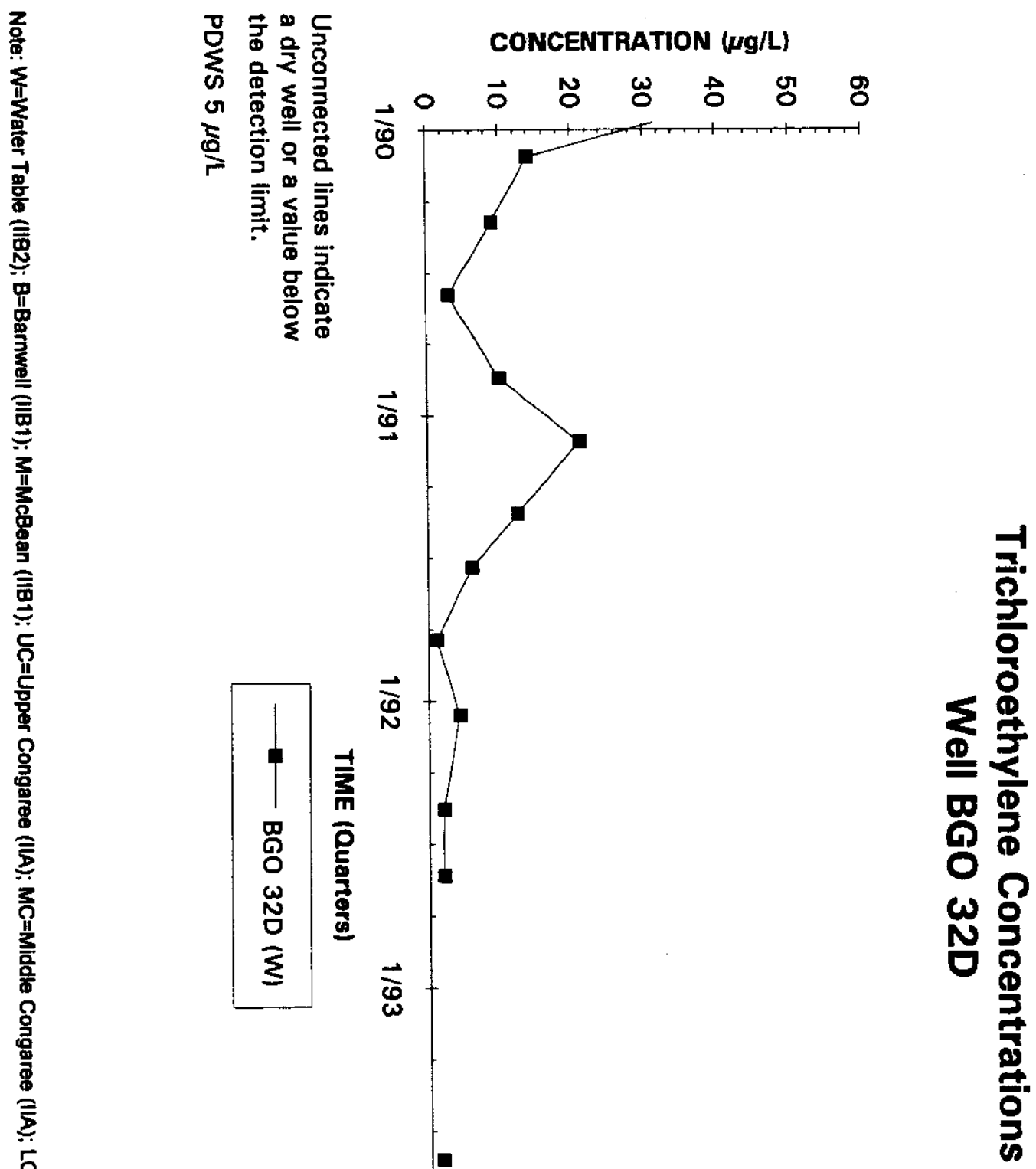



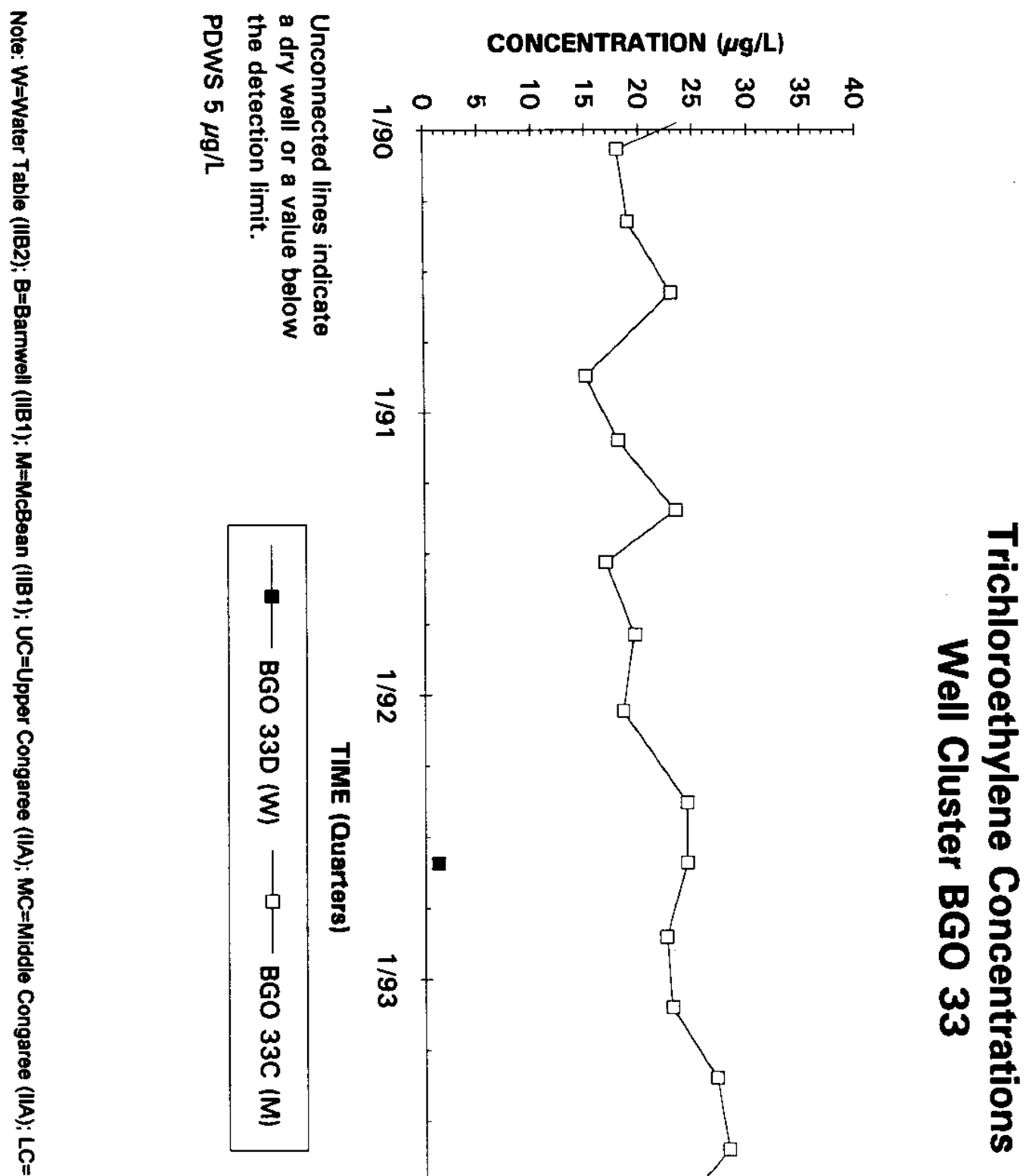

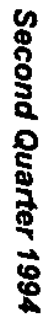

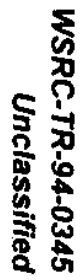




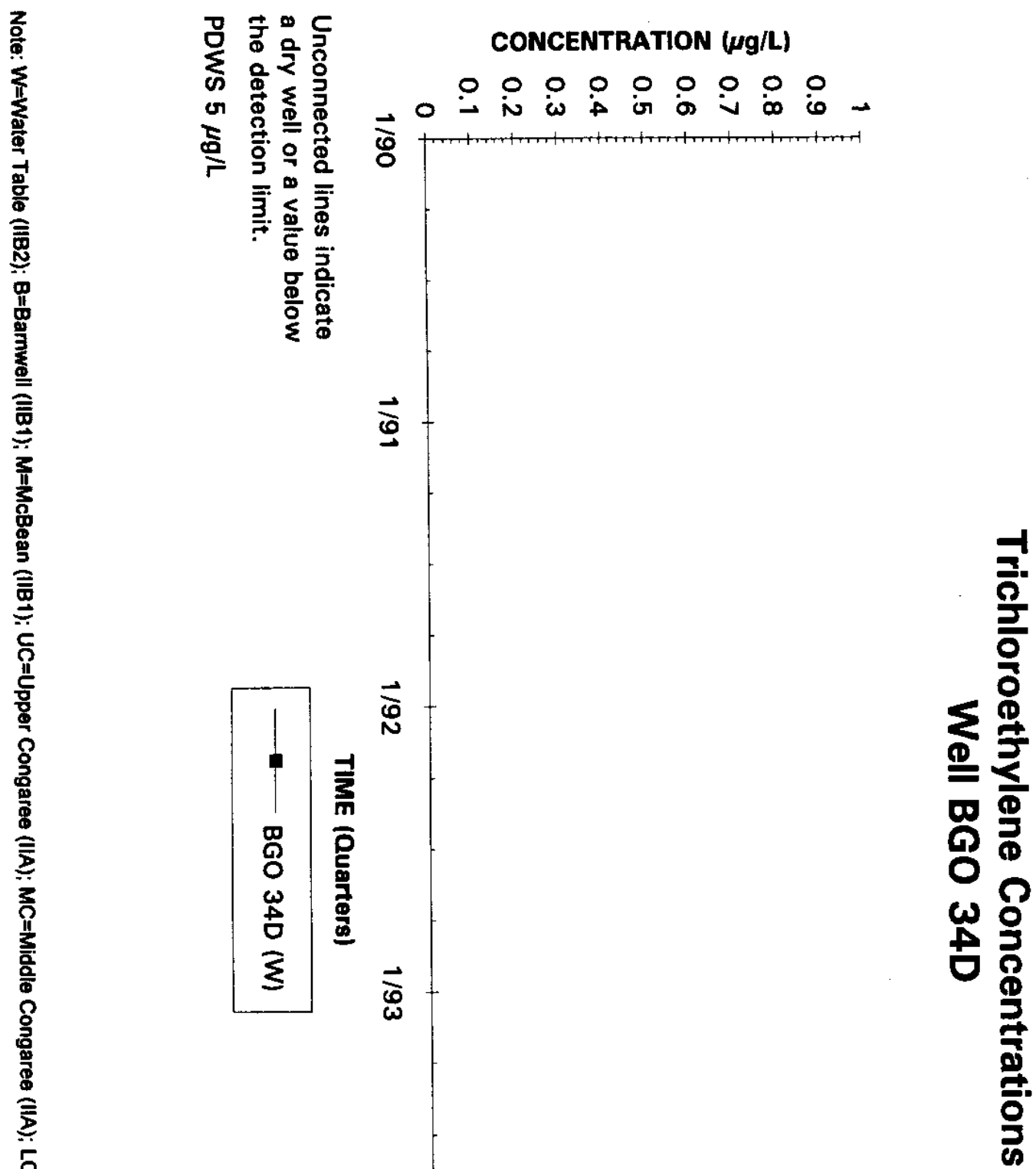




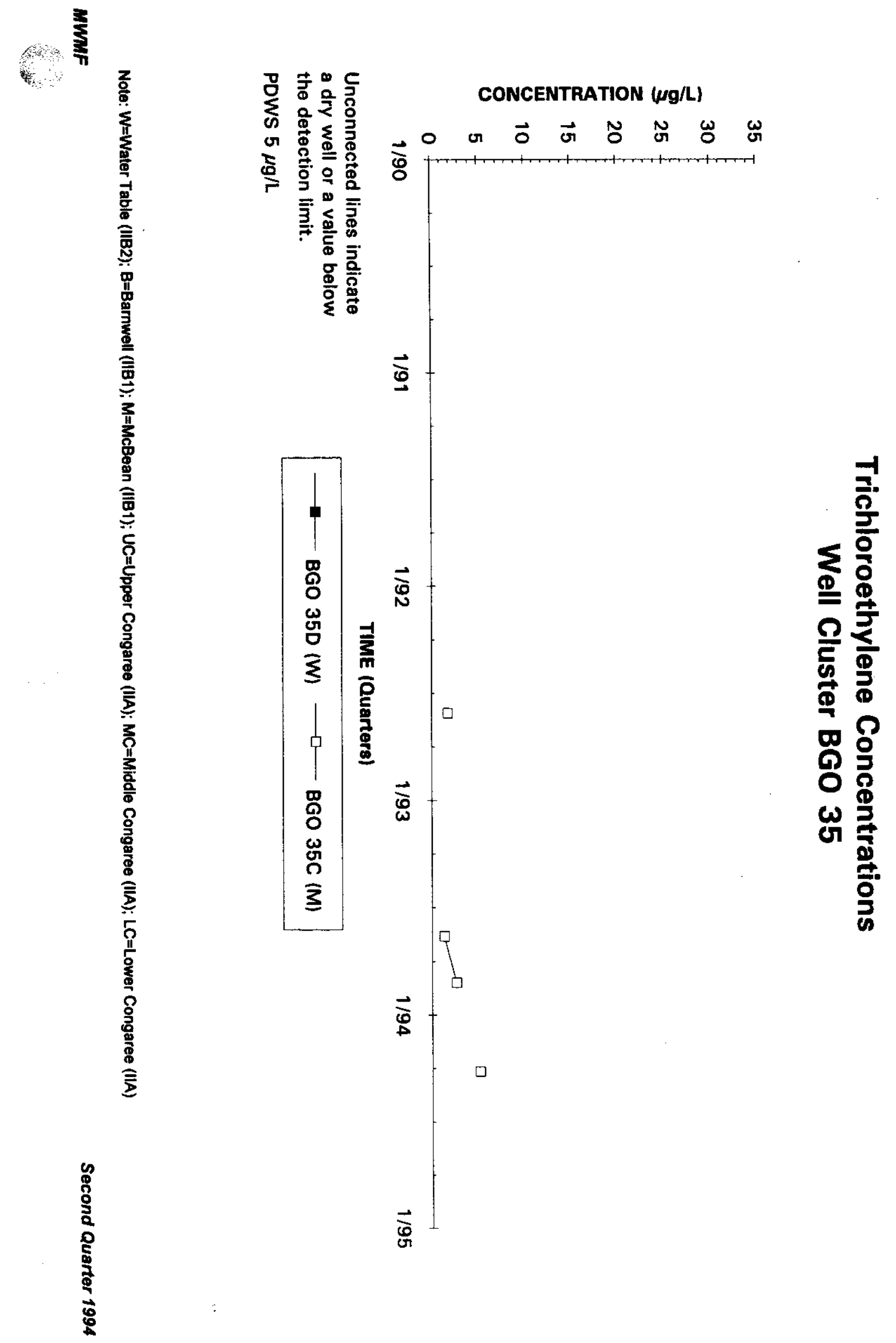

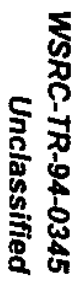




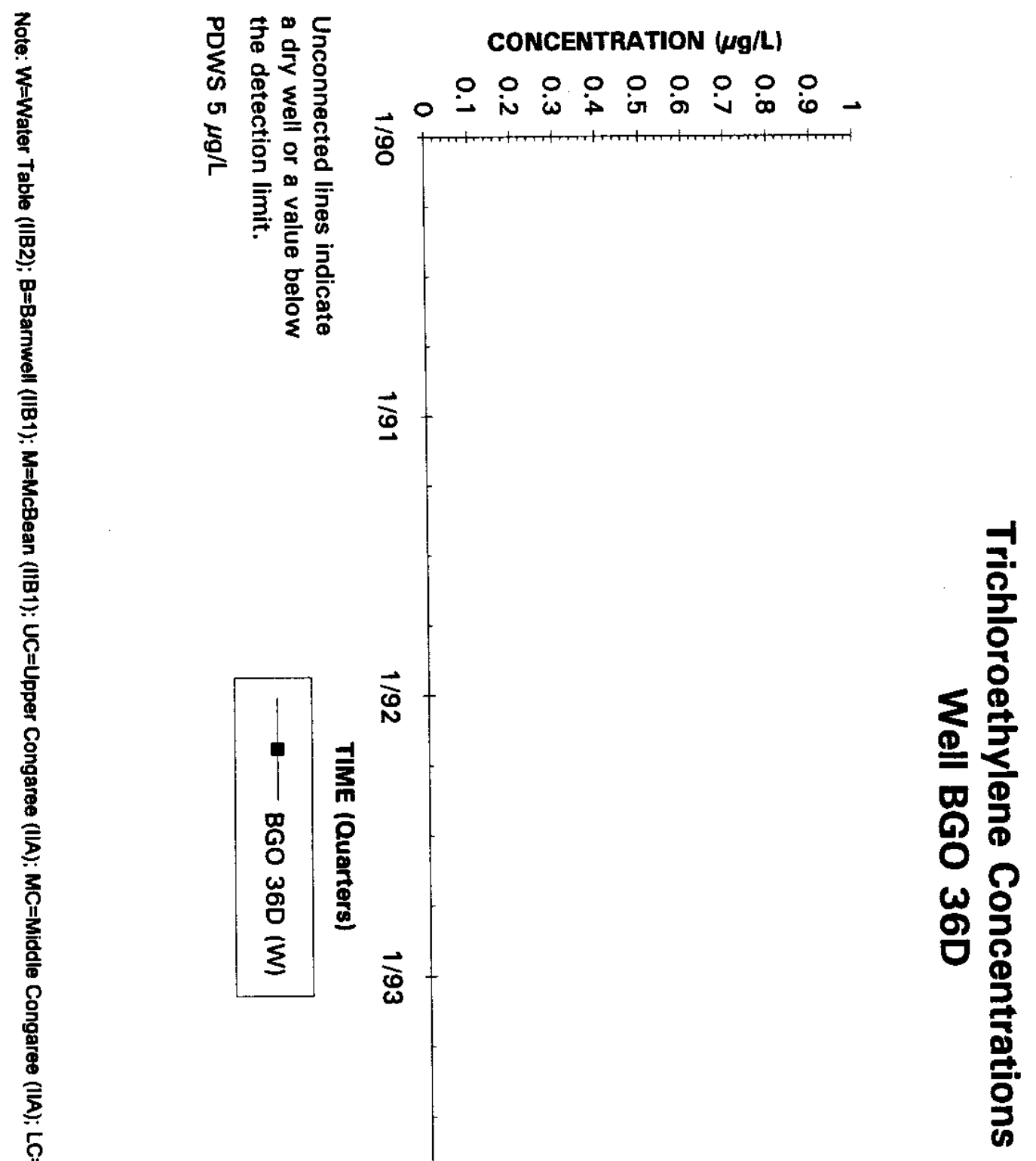




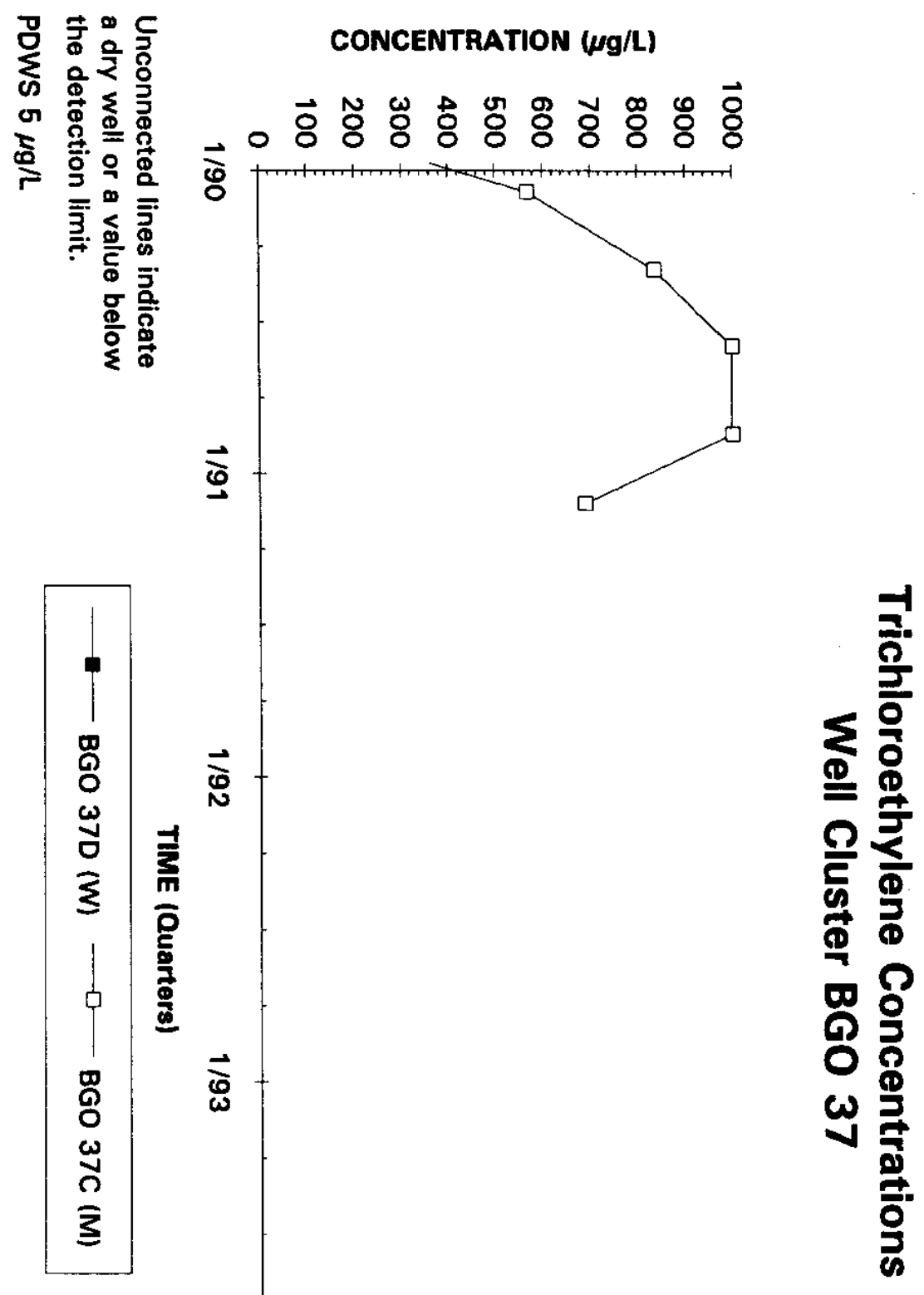

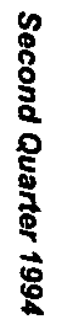

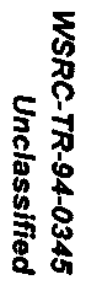




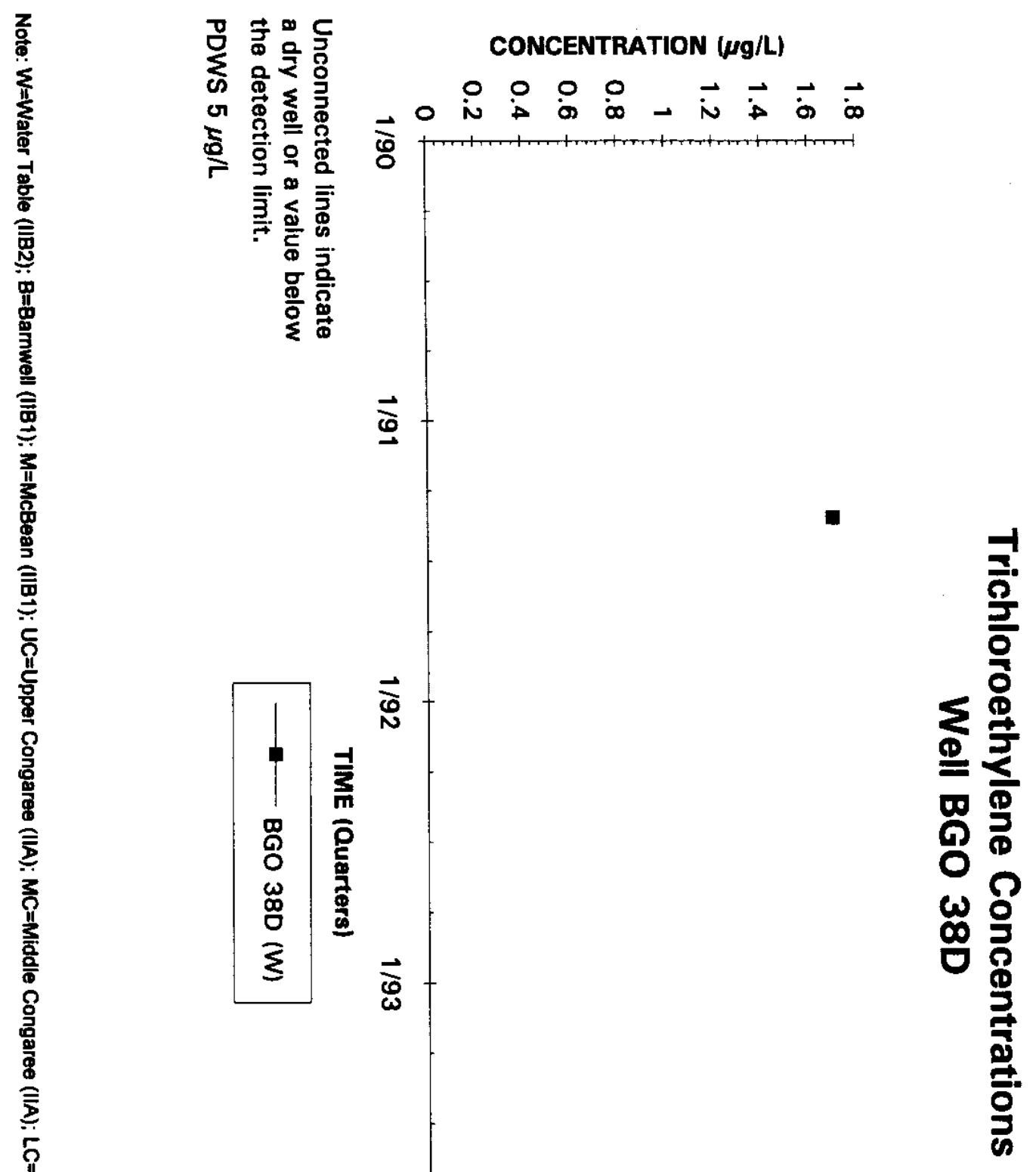



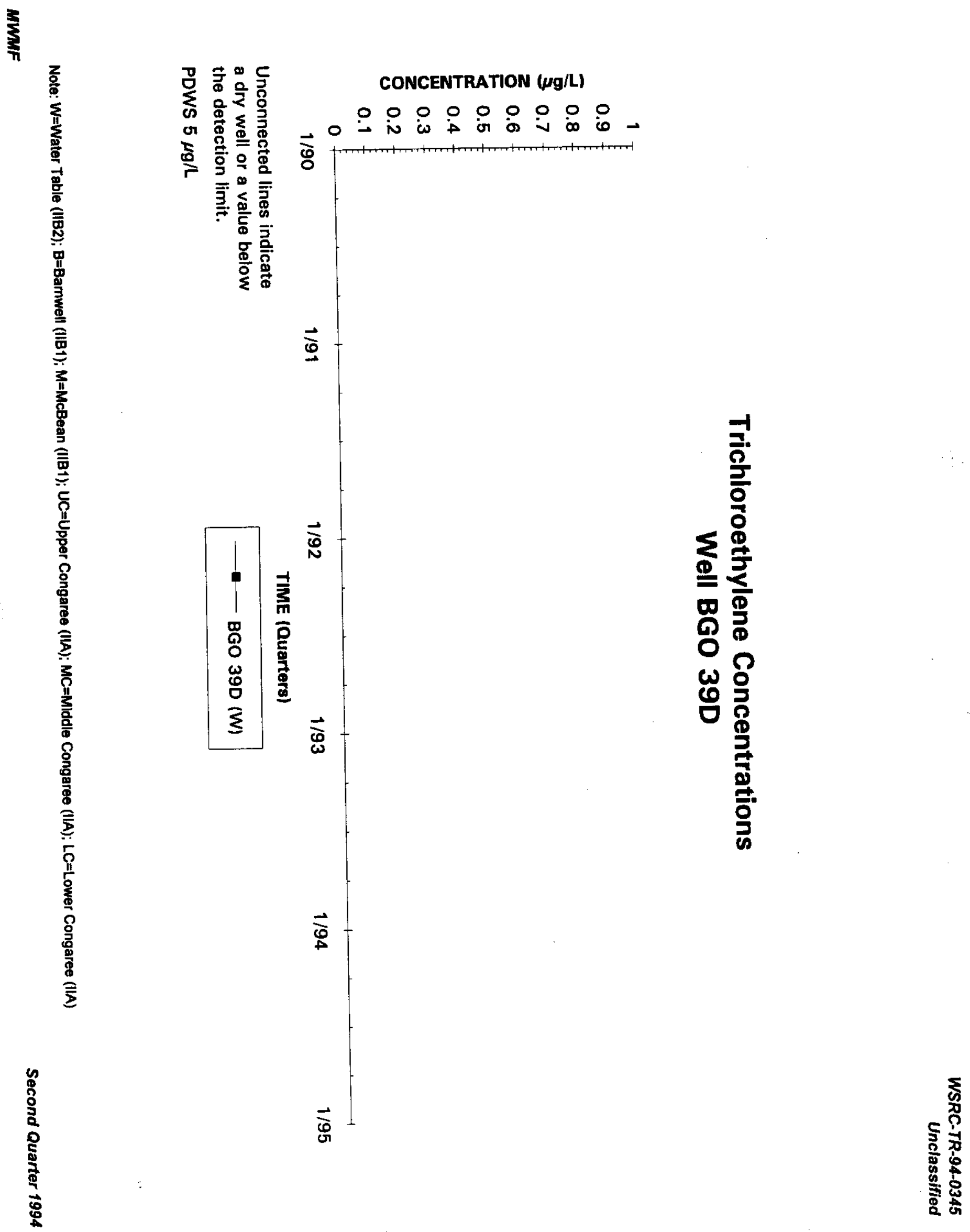


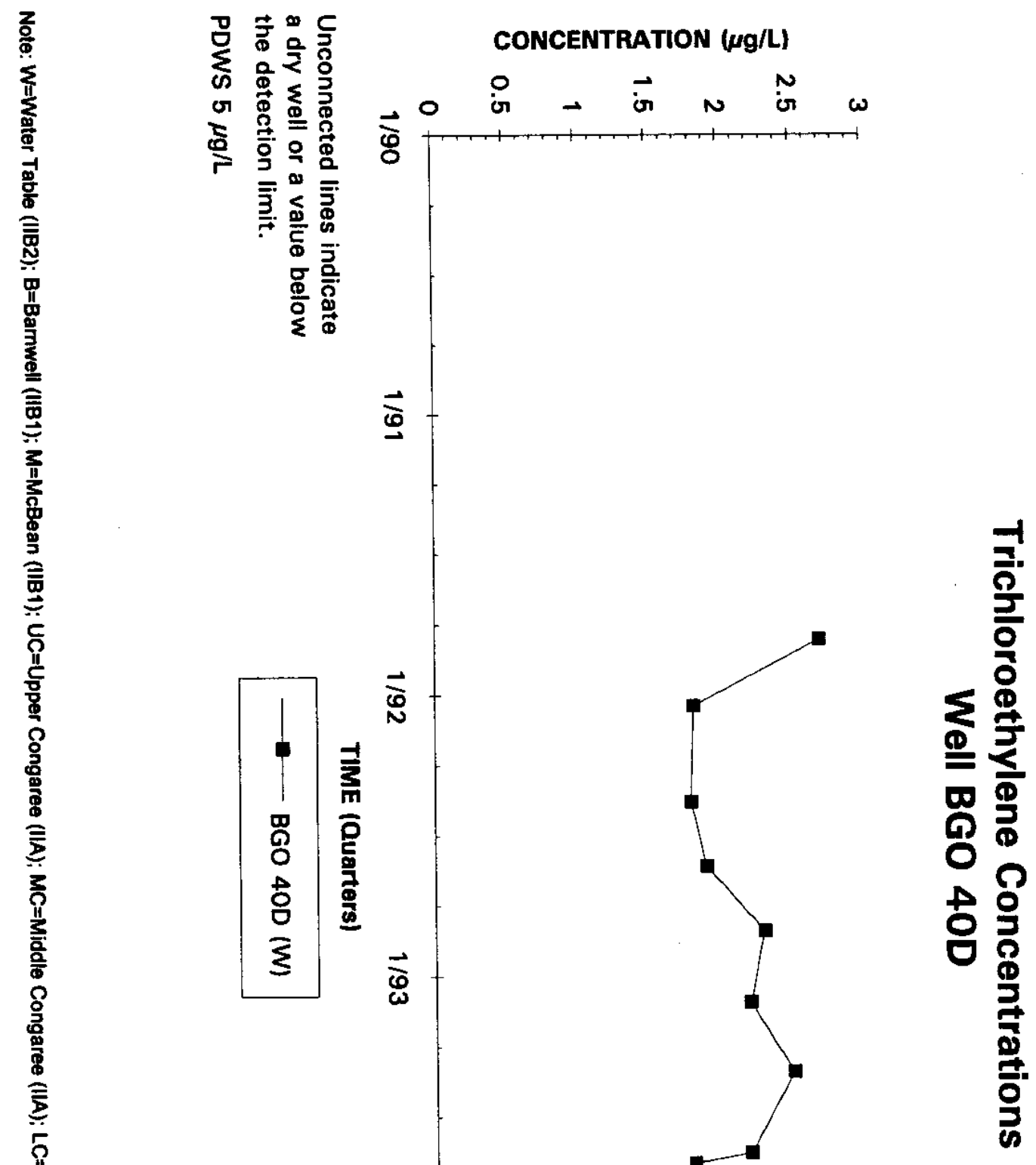




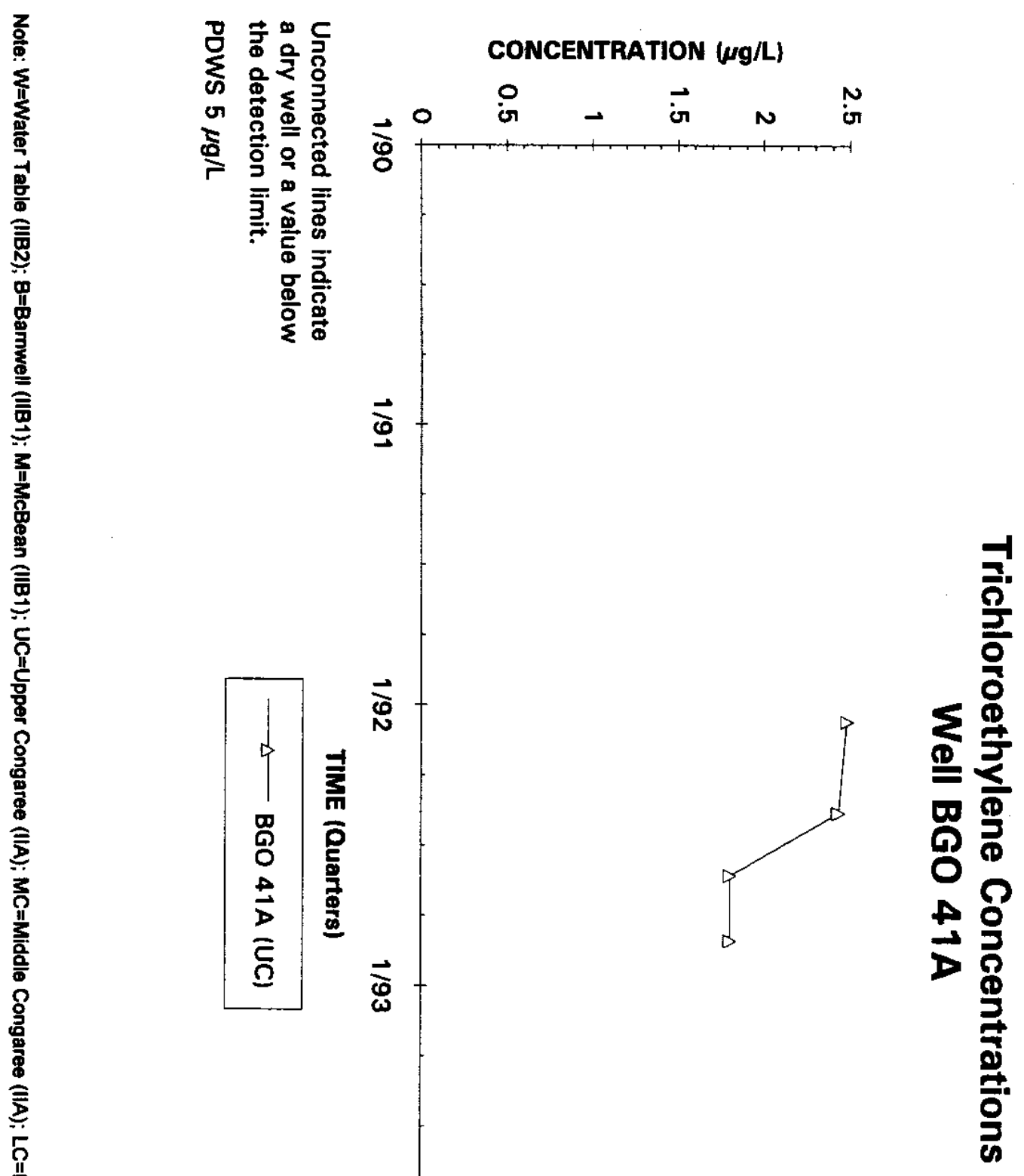

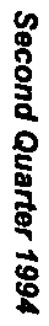




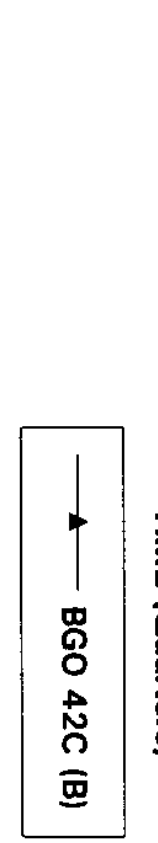

$\overrightarrow{\underline{\omega}}$

$\overrightarrow{0}$
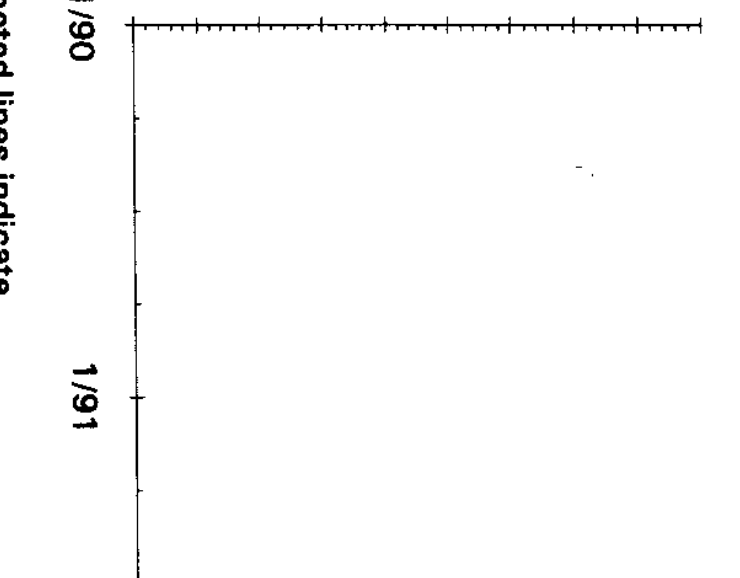
3

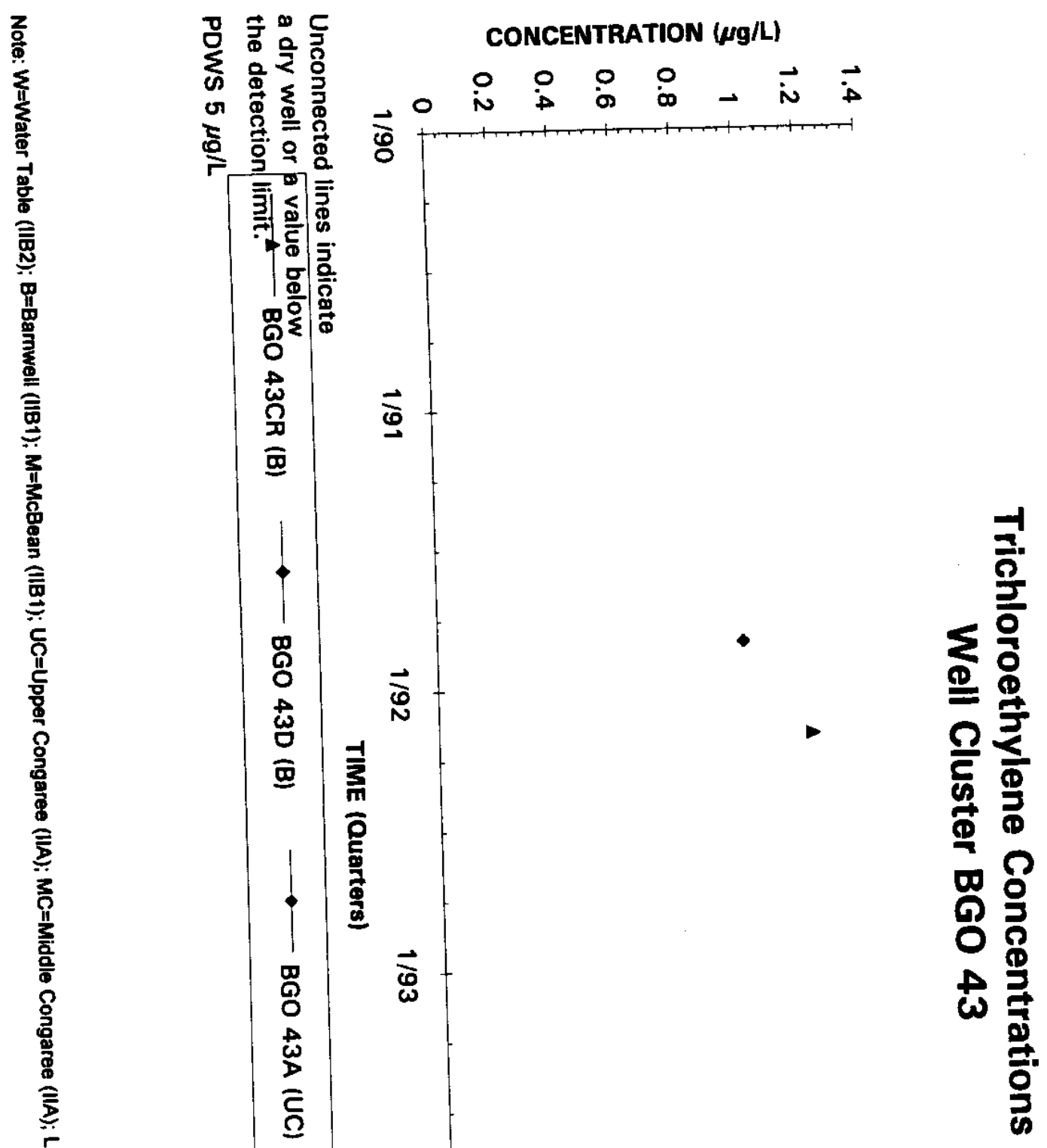


䍂
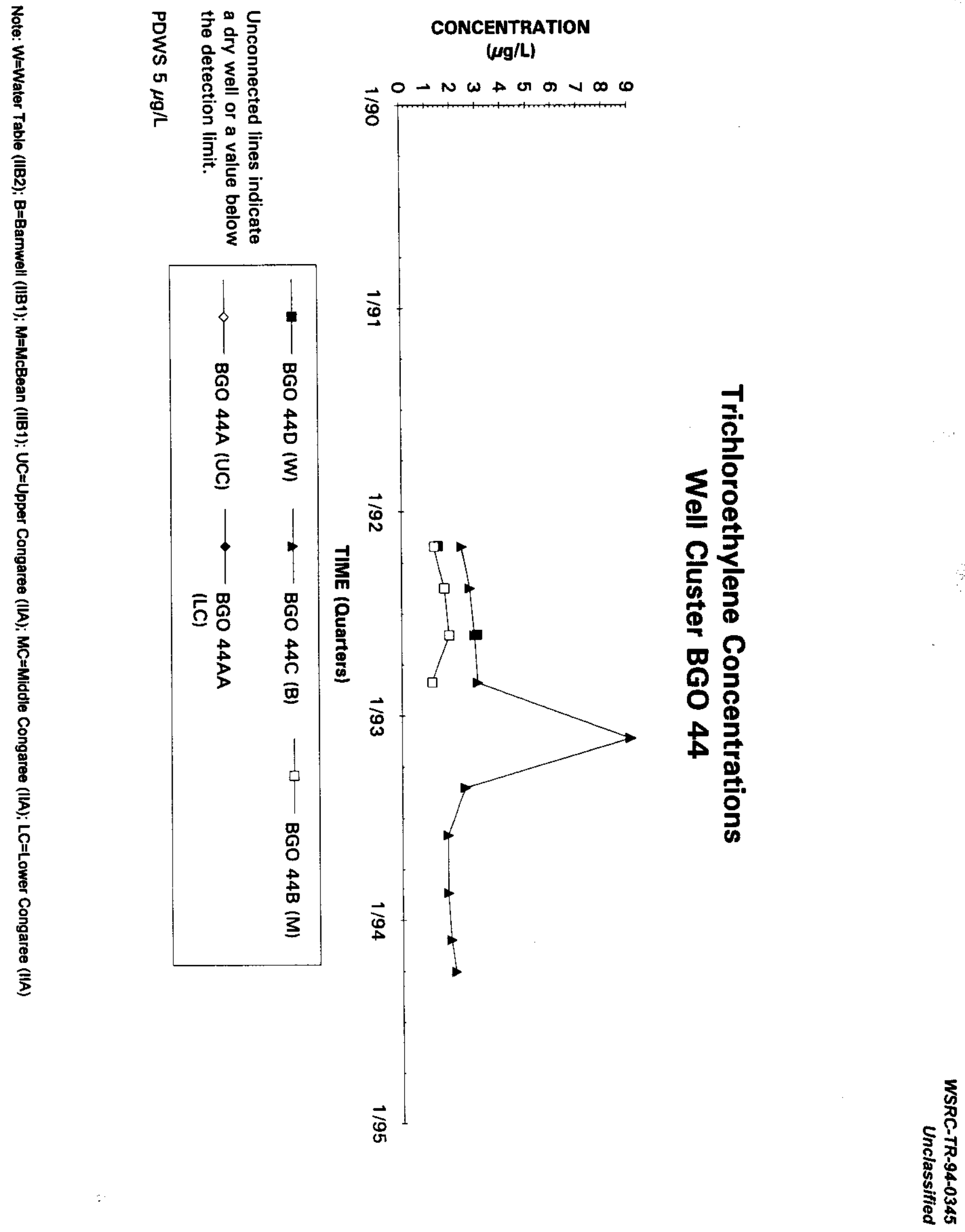


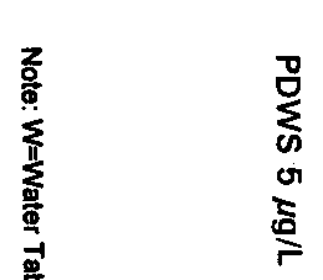

후을

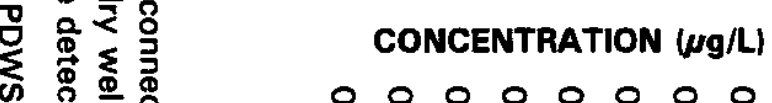

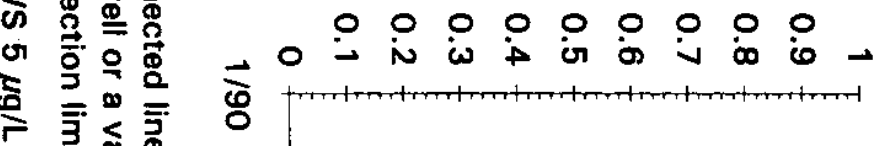

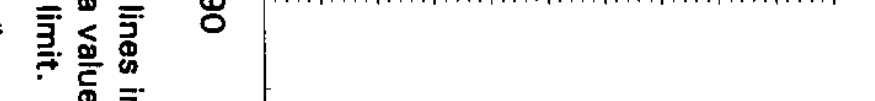

产

要

응

웅

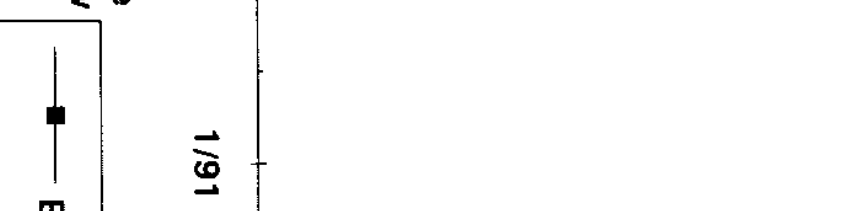

랄

吕

남 
萐

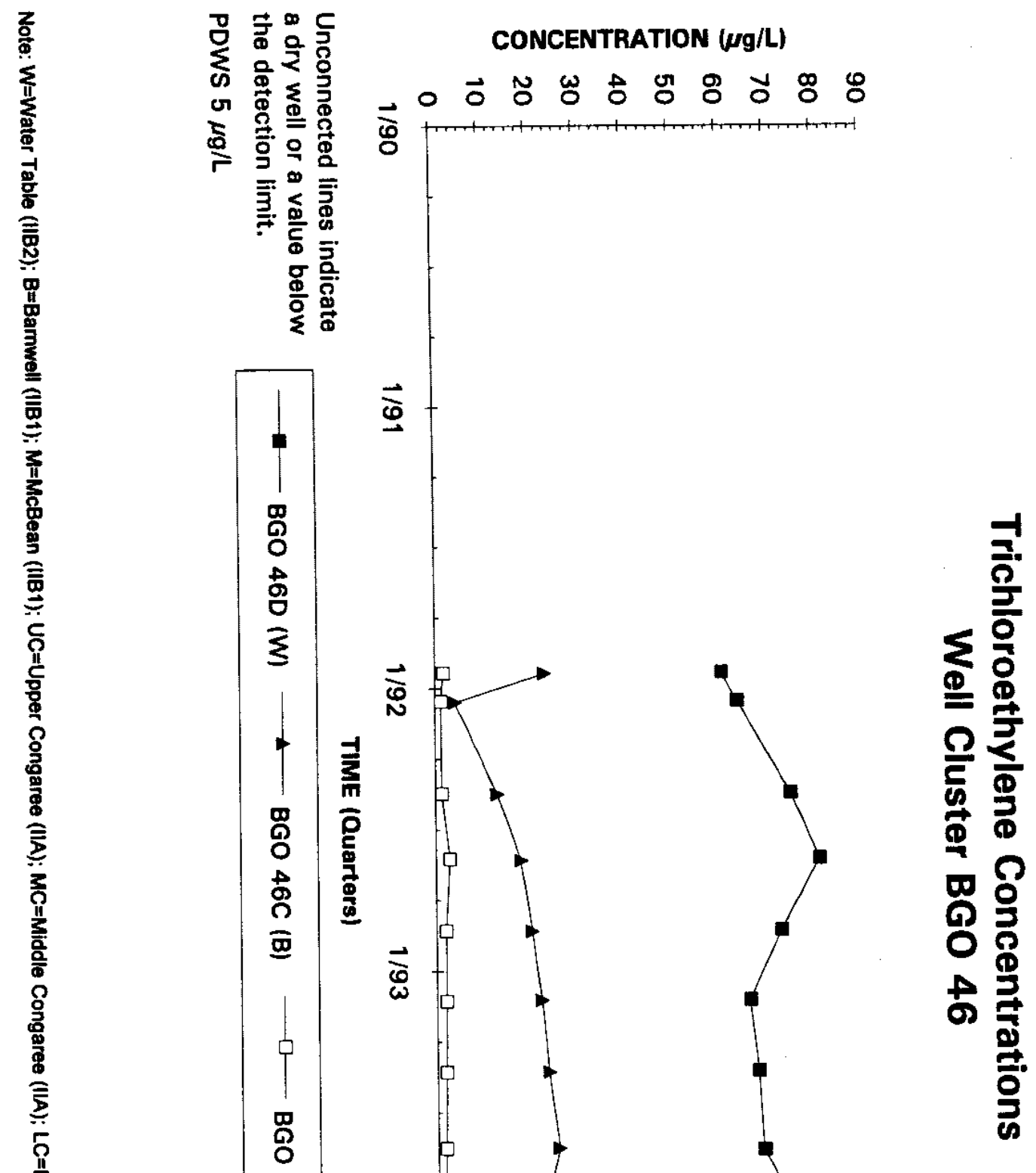




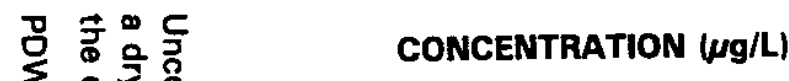

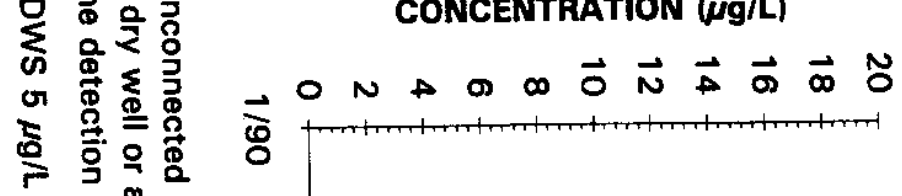

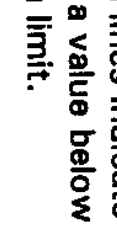

西

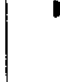

1

호

N

ล

畧
ثี
产

产

0

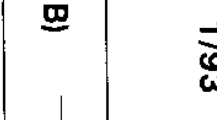

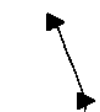
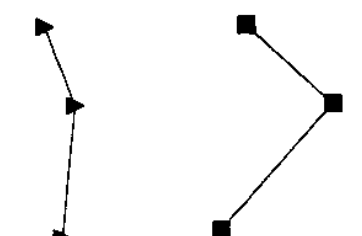

$\sum$

D

을 $\frac{5}{9}$

()

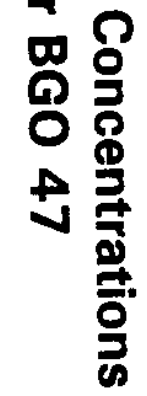




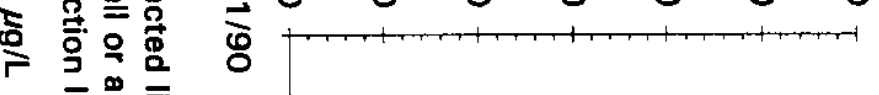

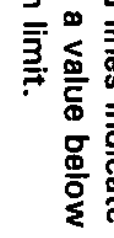




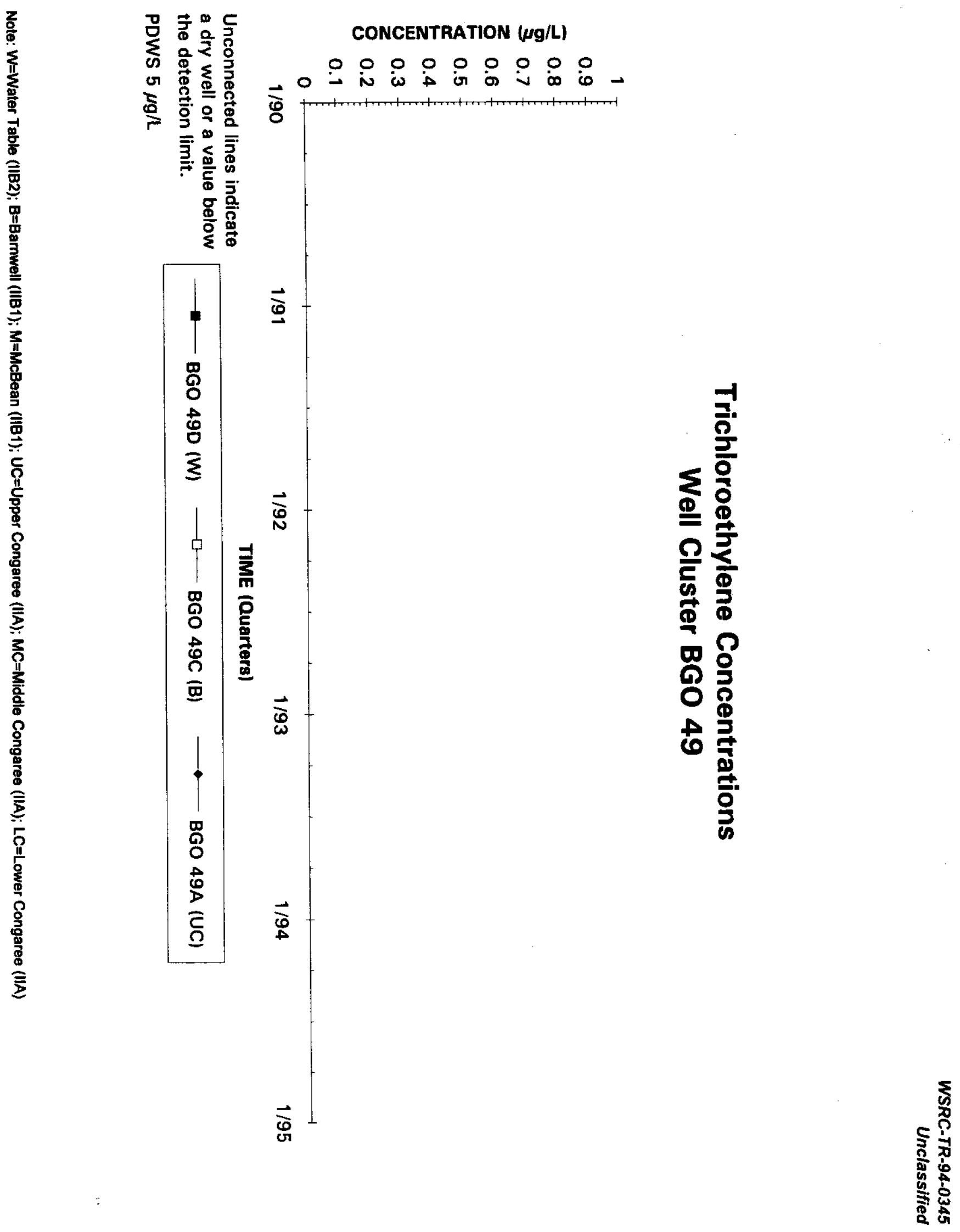




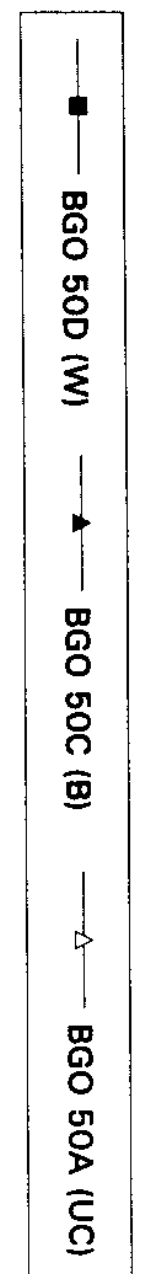



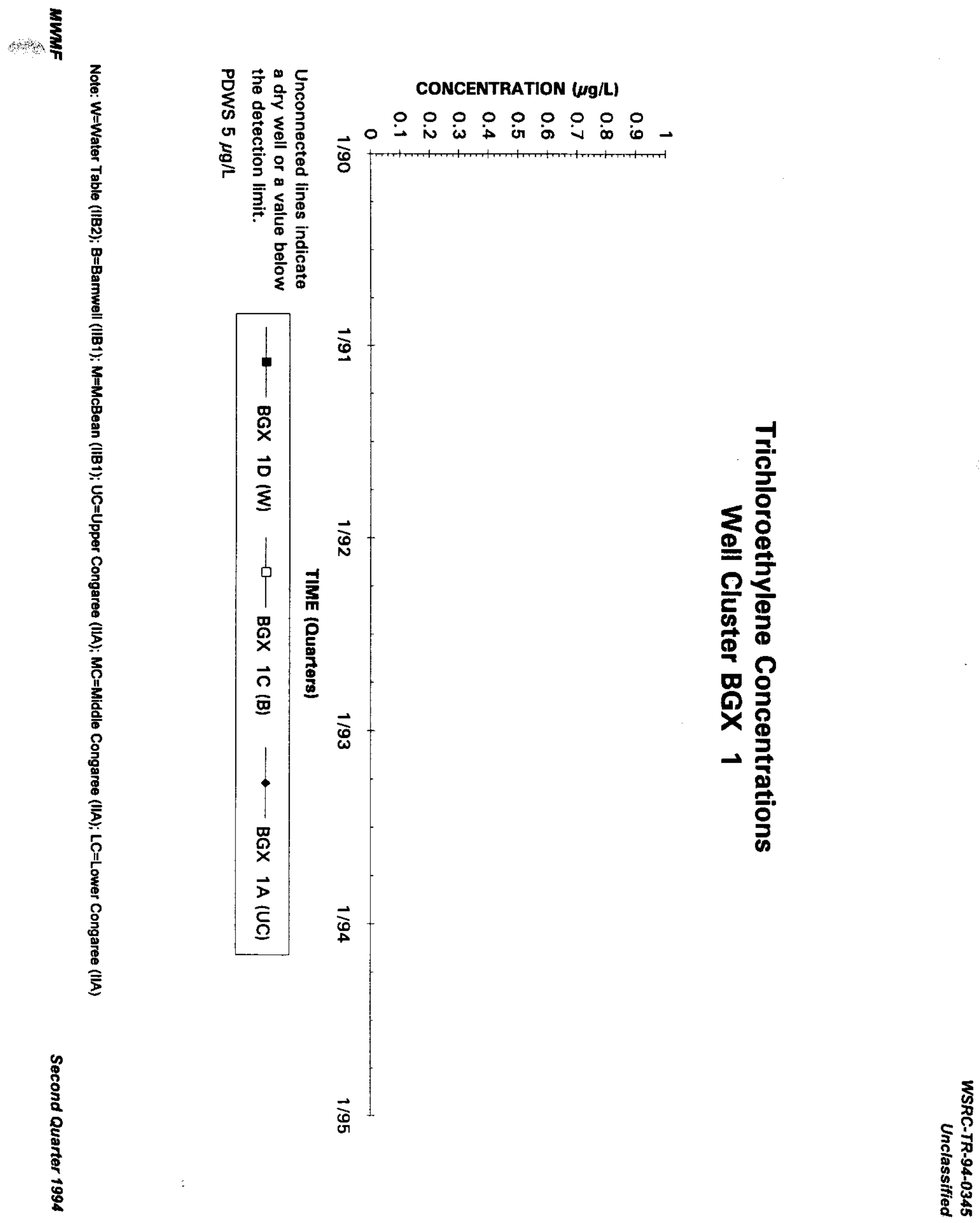

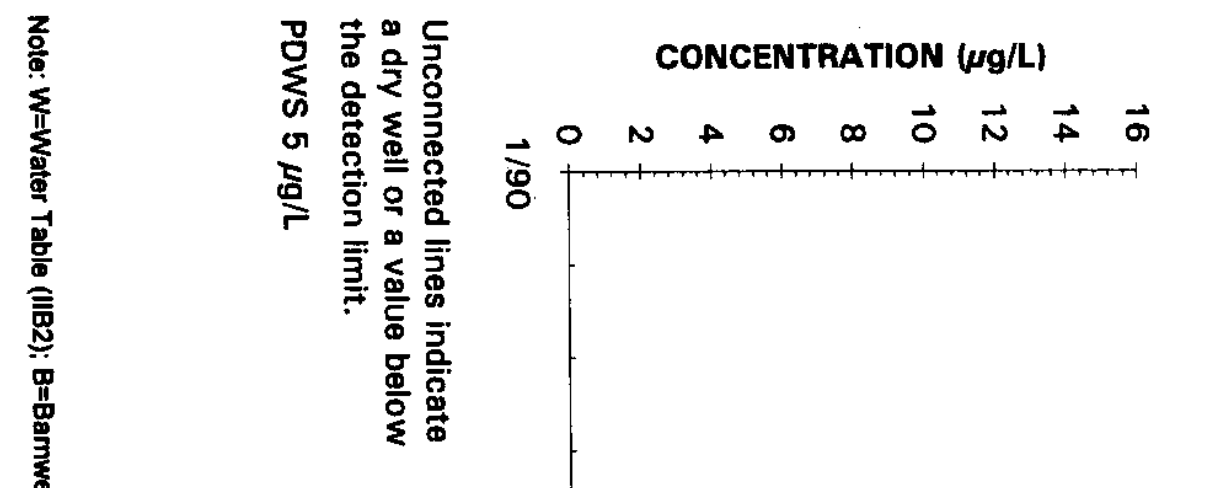


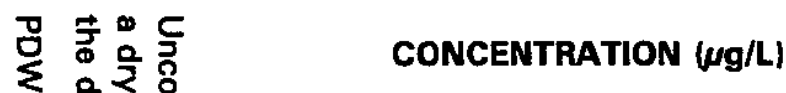

ज

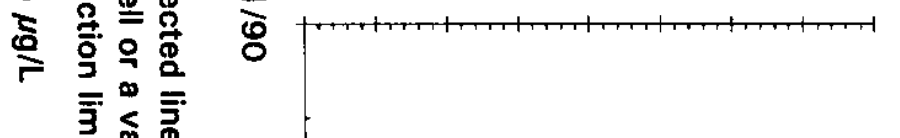

罍

言

置 灵

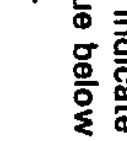

言

$\stackrel{乛}{3}$

$\frac{\overline{1}}{2}$

言

§ิ

듬

옹
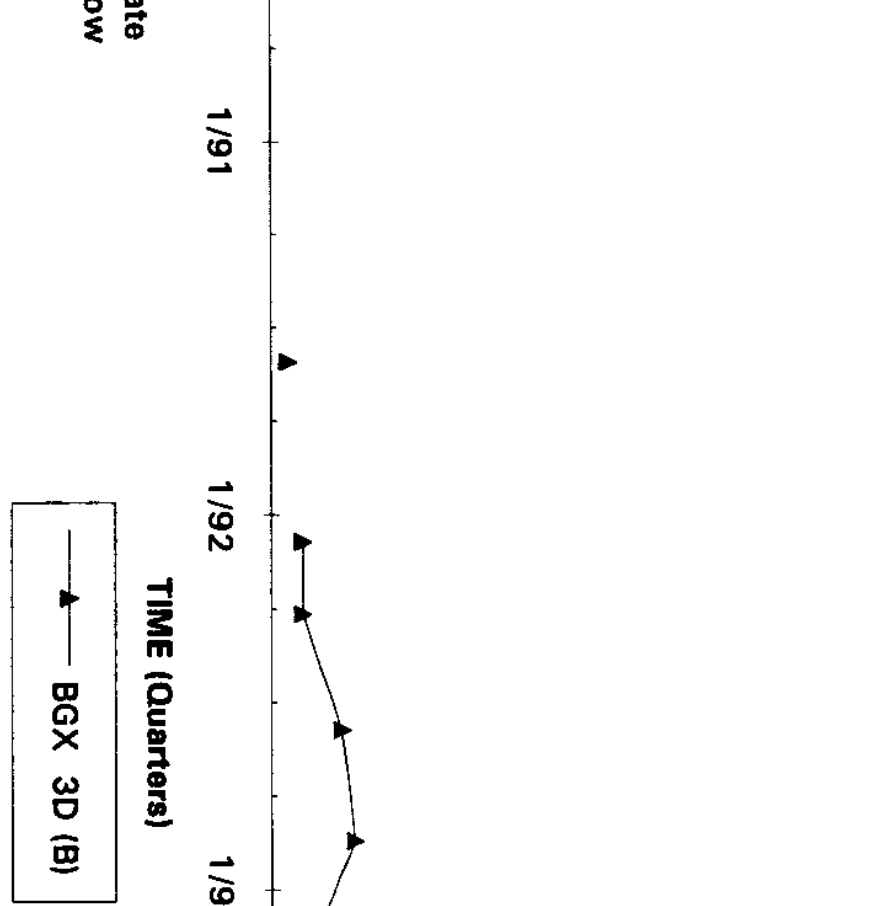

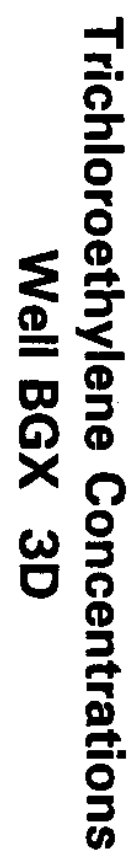

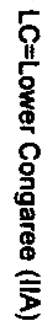

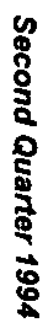

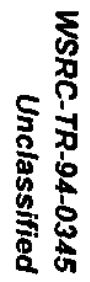


1

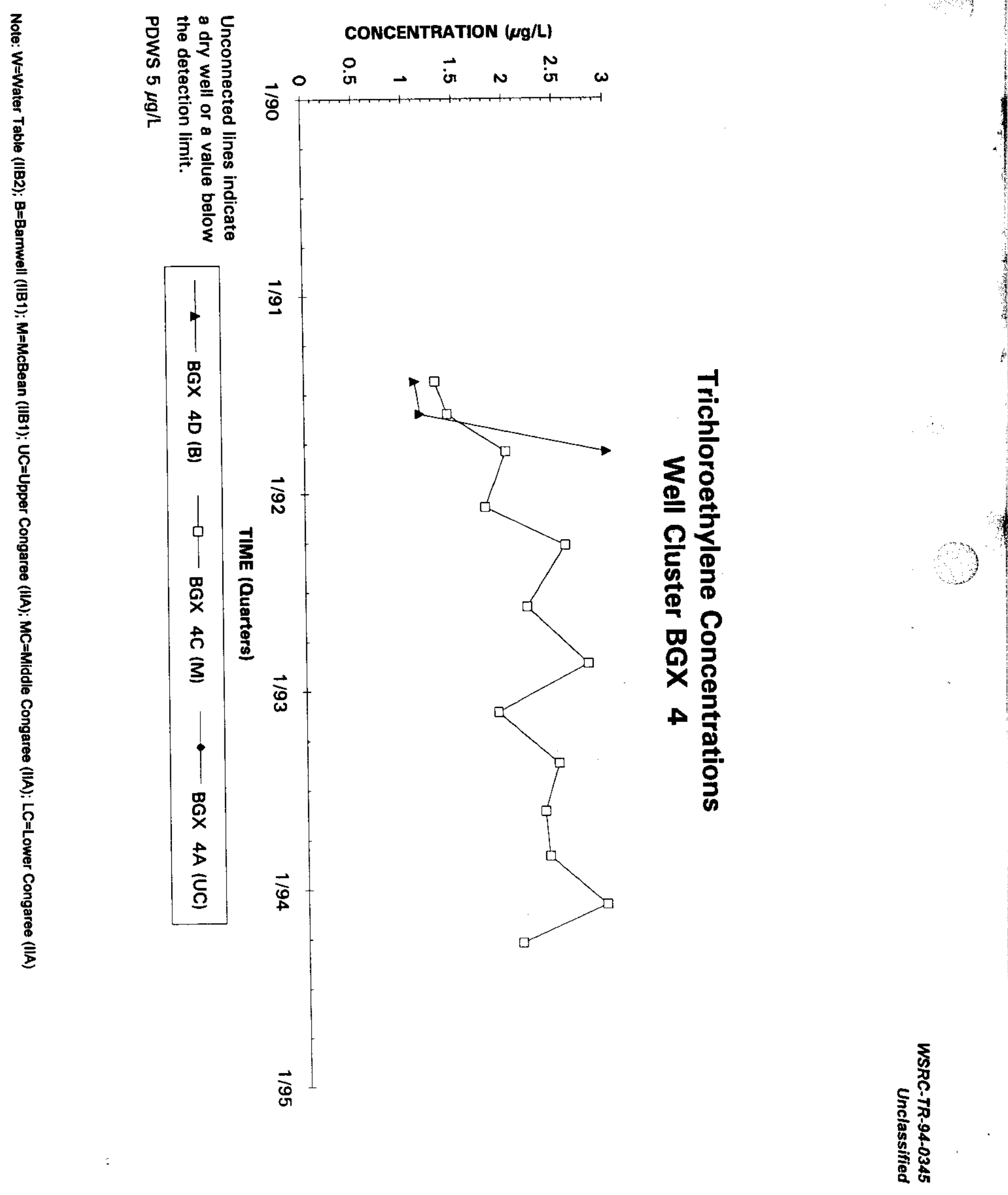




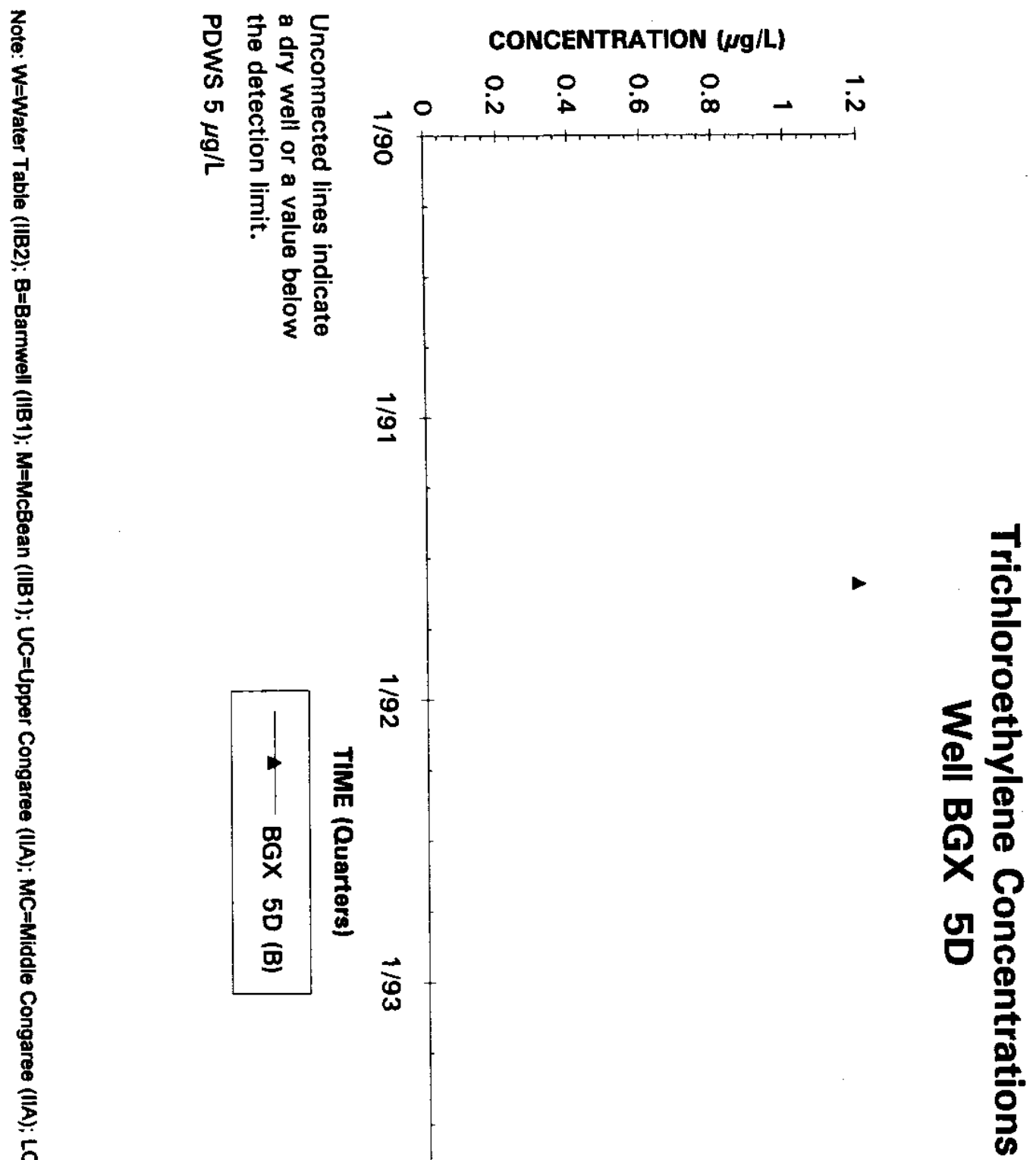


率

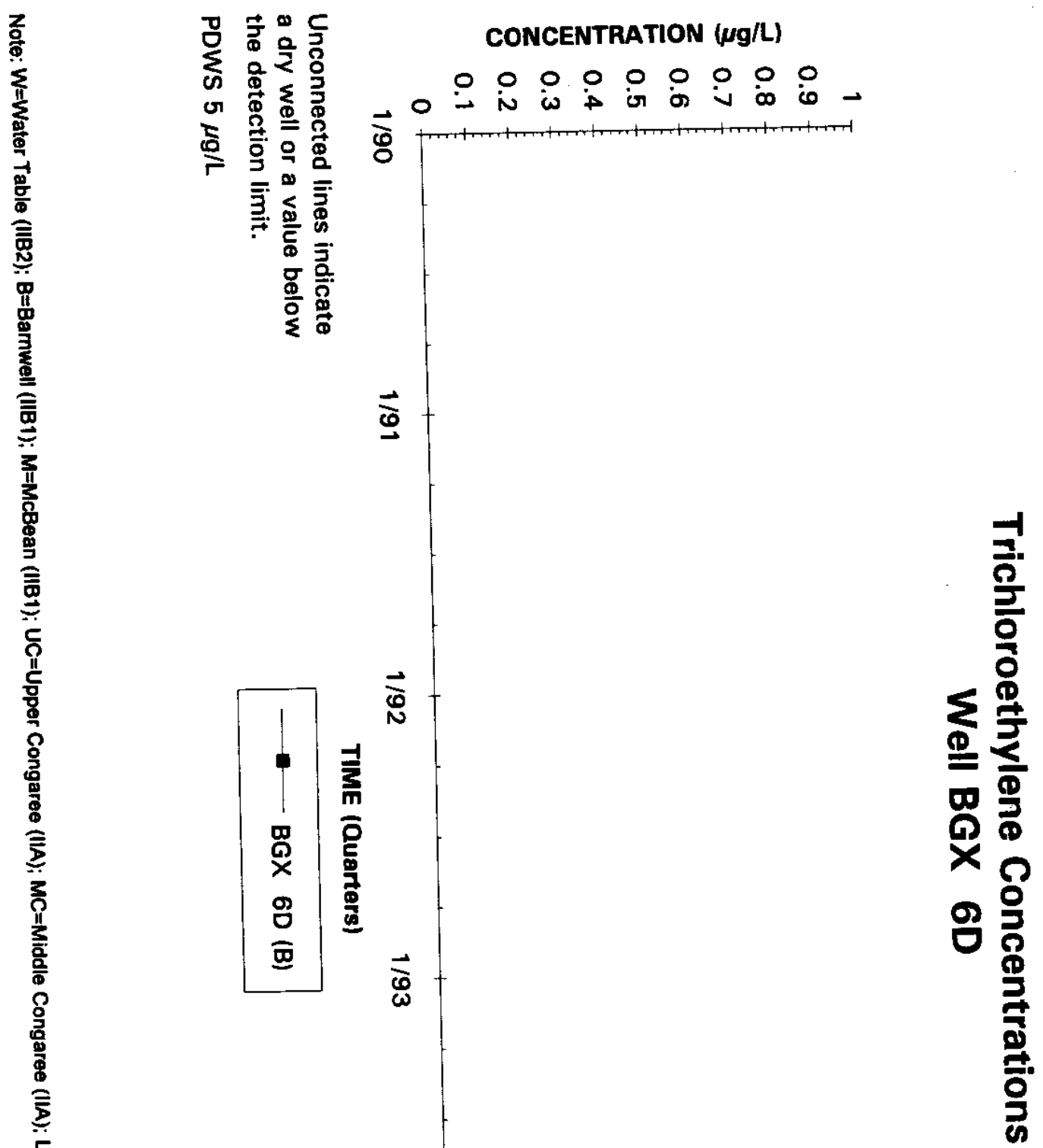




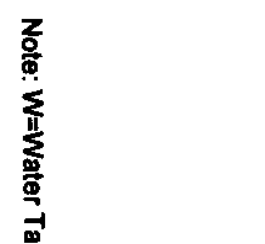

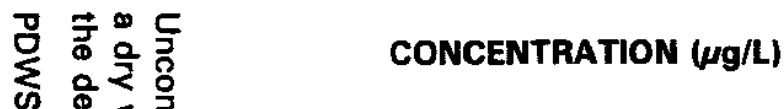

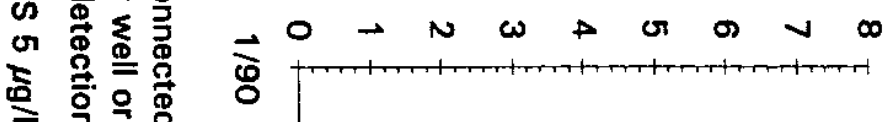

蛋空

它

음

这

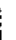

Ф્

줍

量

ิ-

당

동

$\S$

要

产

종

$\frac{\bar{t}}{6}$

$\S$

๑

స్

II

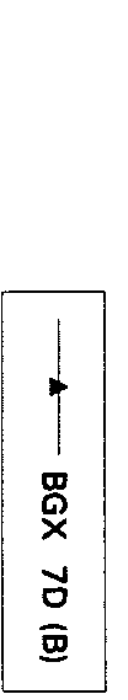

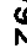

$\vec{\Xi}$

궁

ثे

-

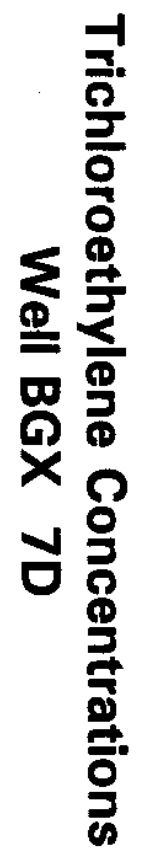



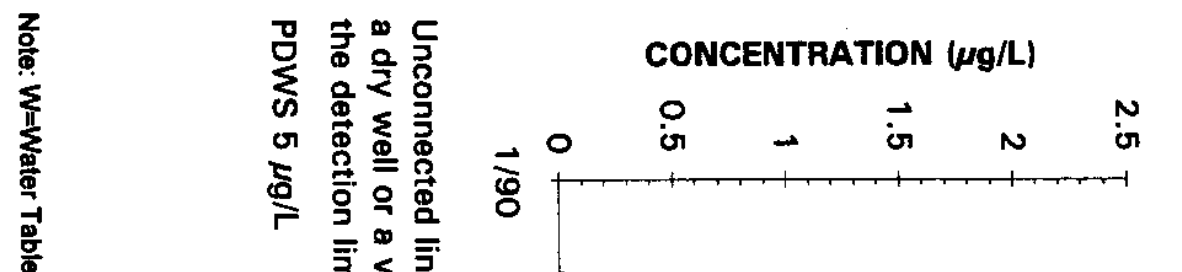

읗 方尔

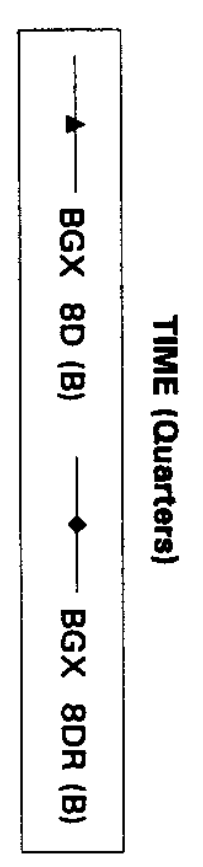

$\vec{\omega}$ 审 응
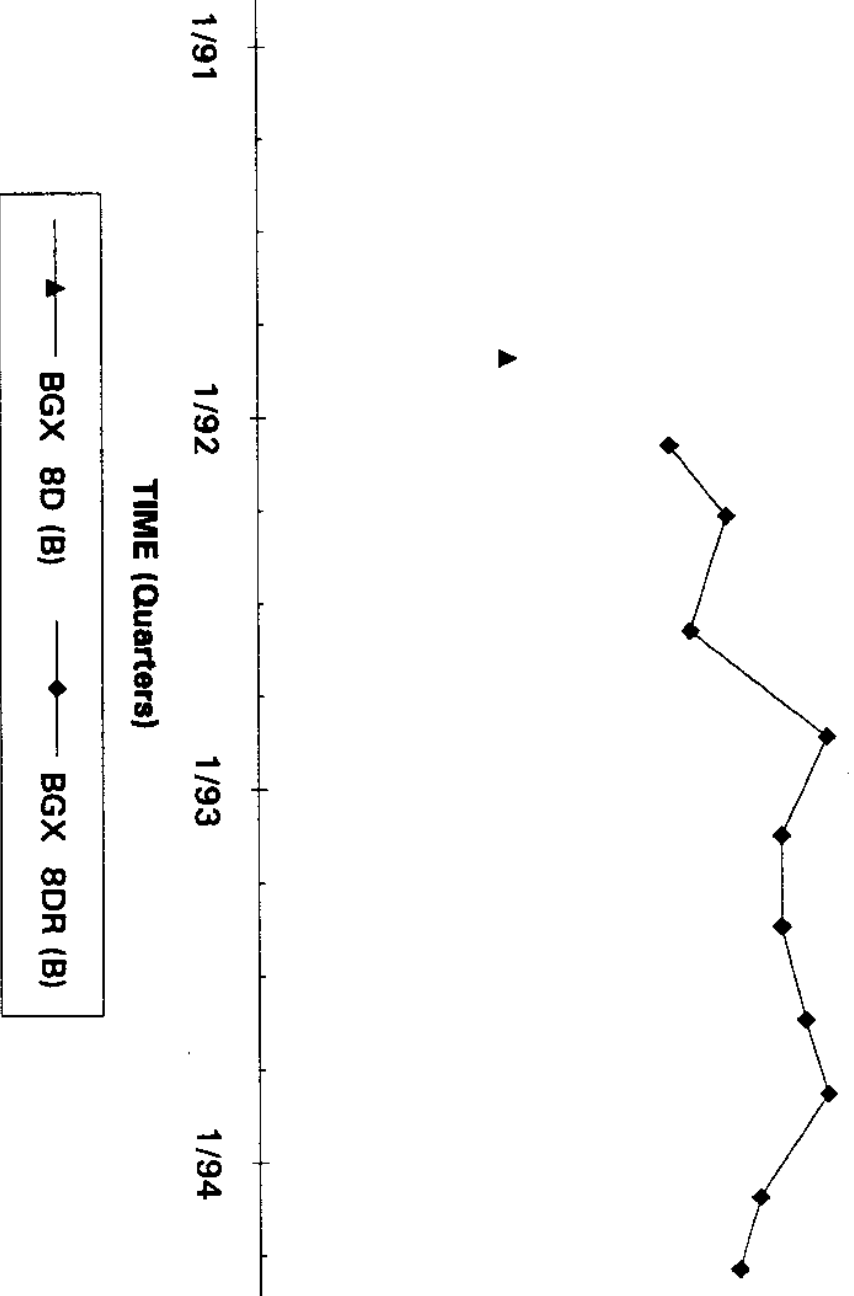

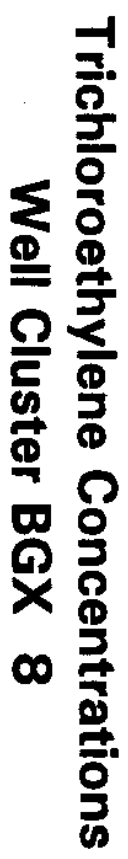




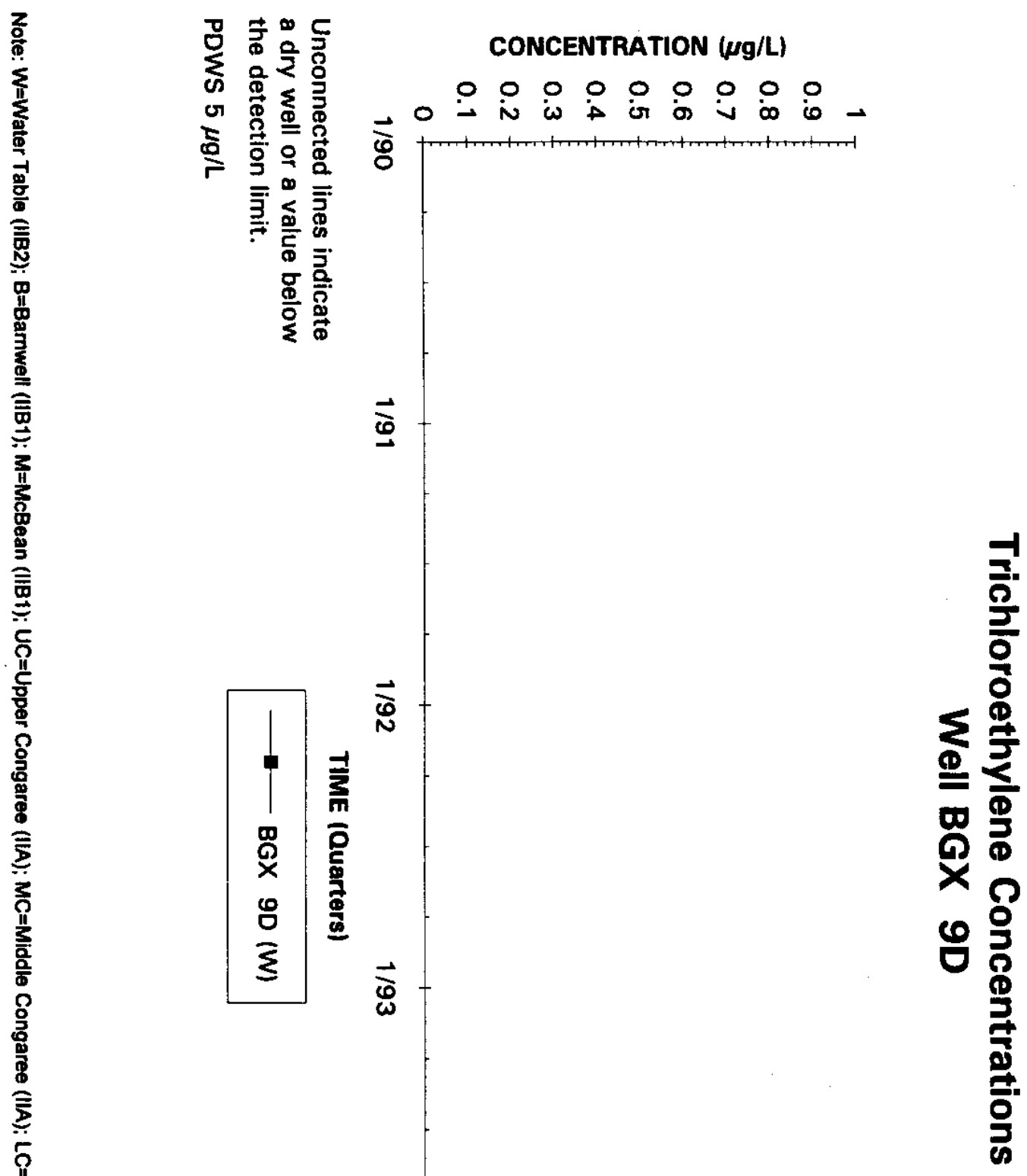


1

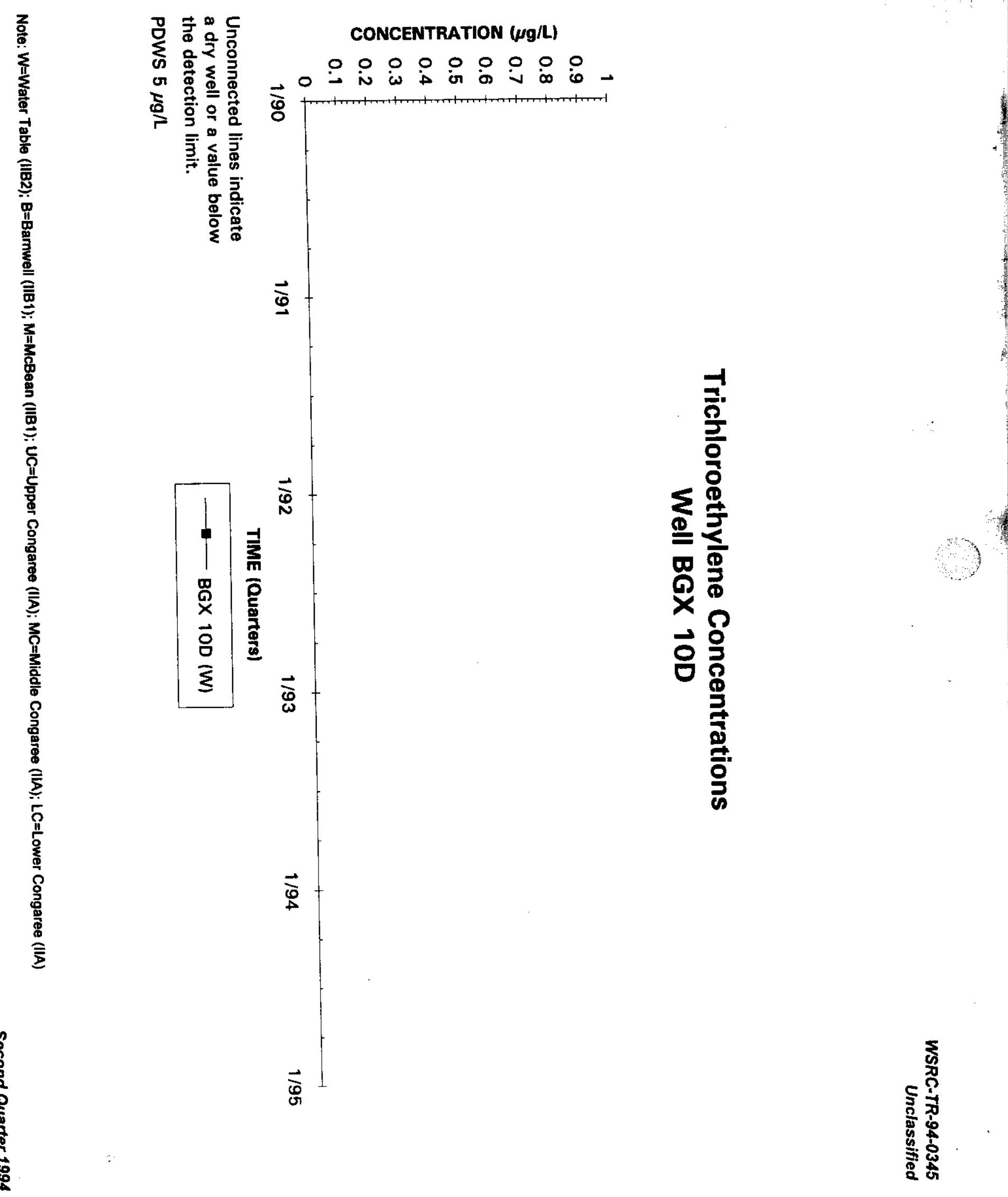




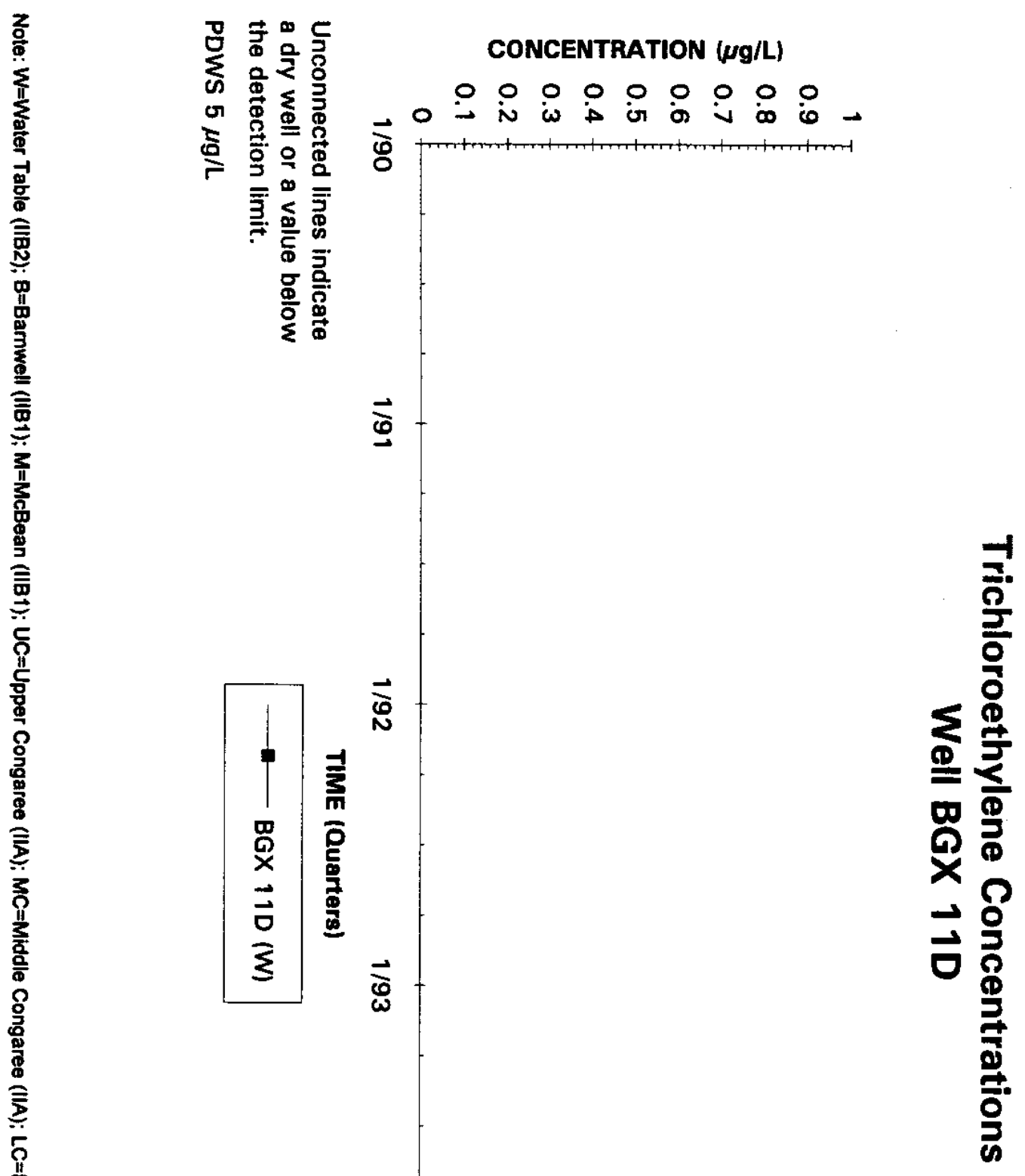



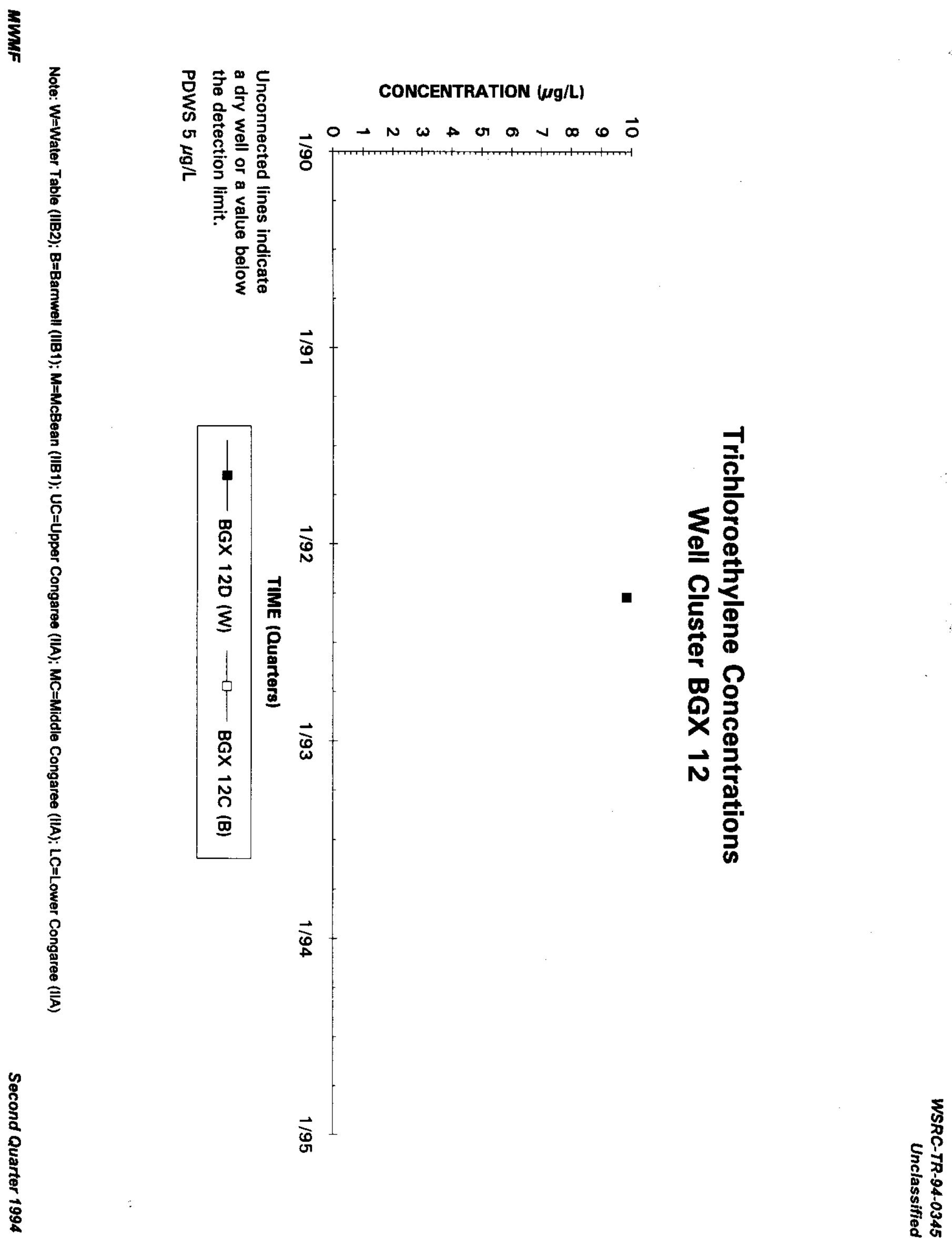


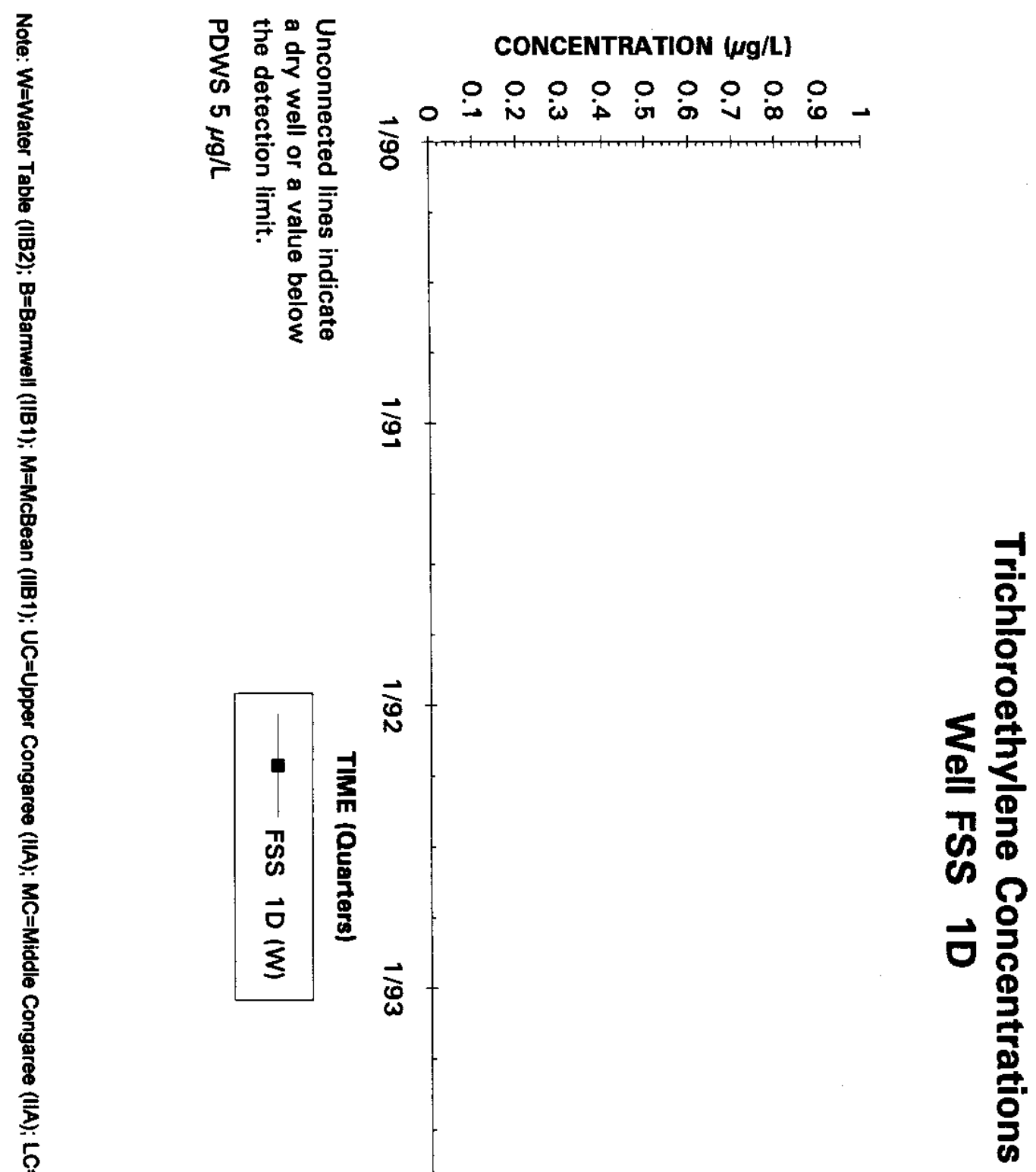




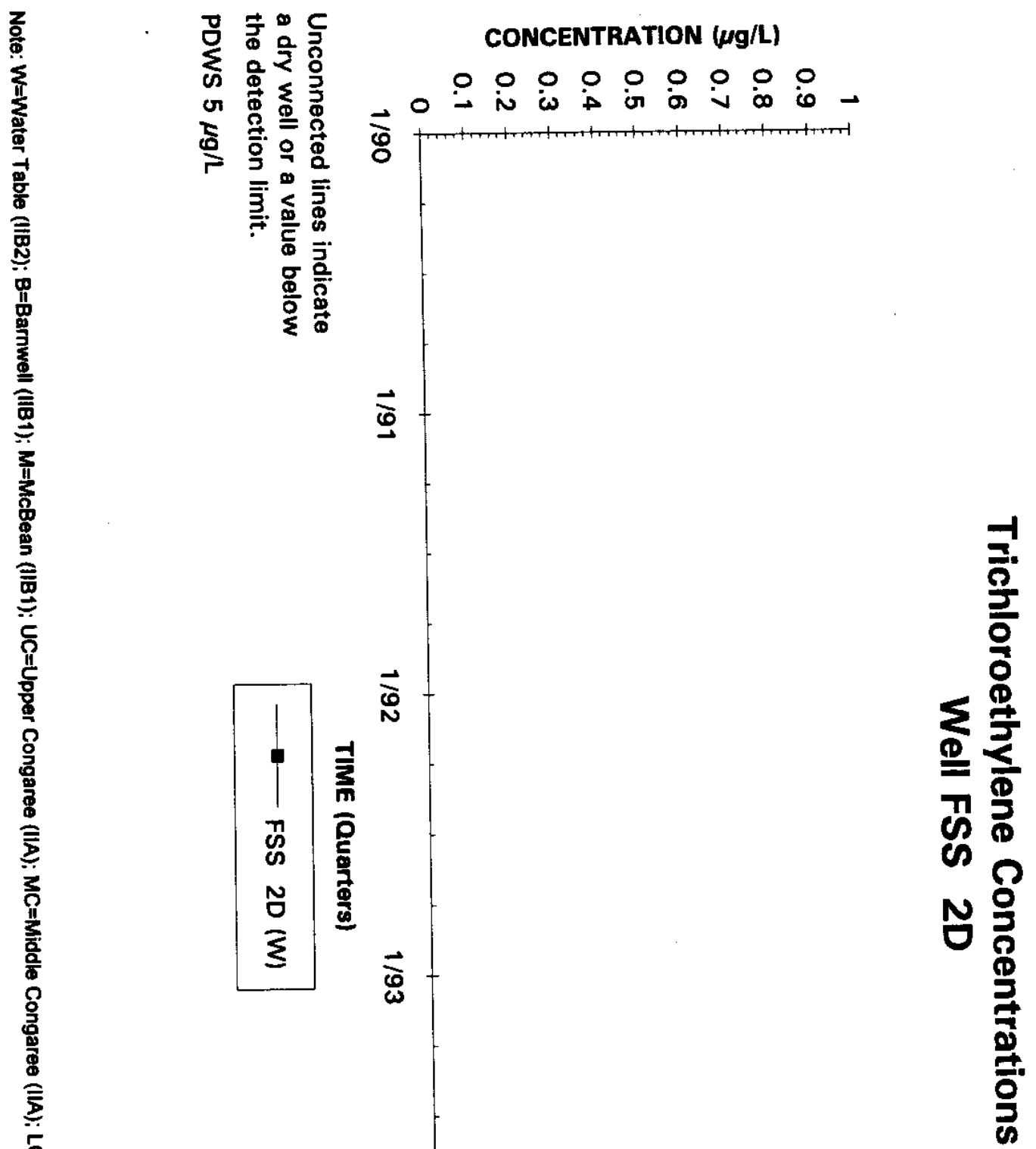




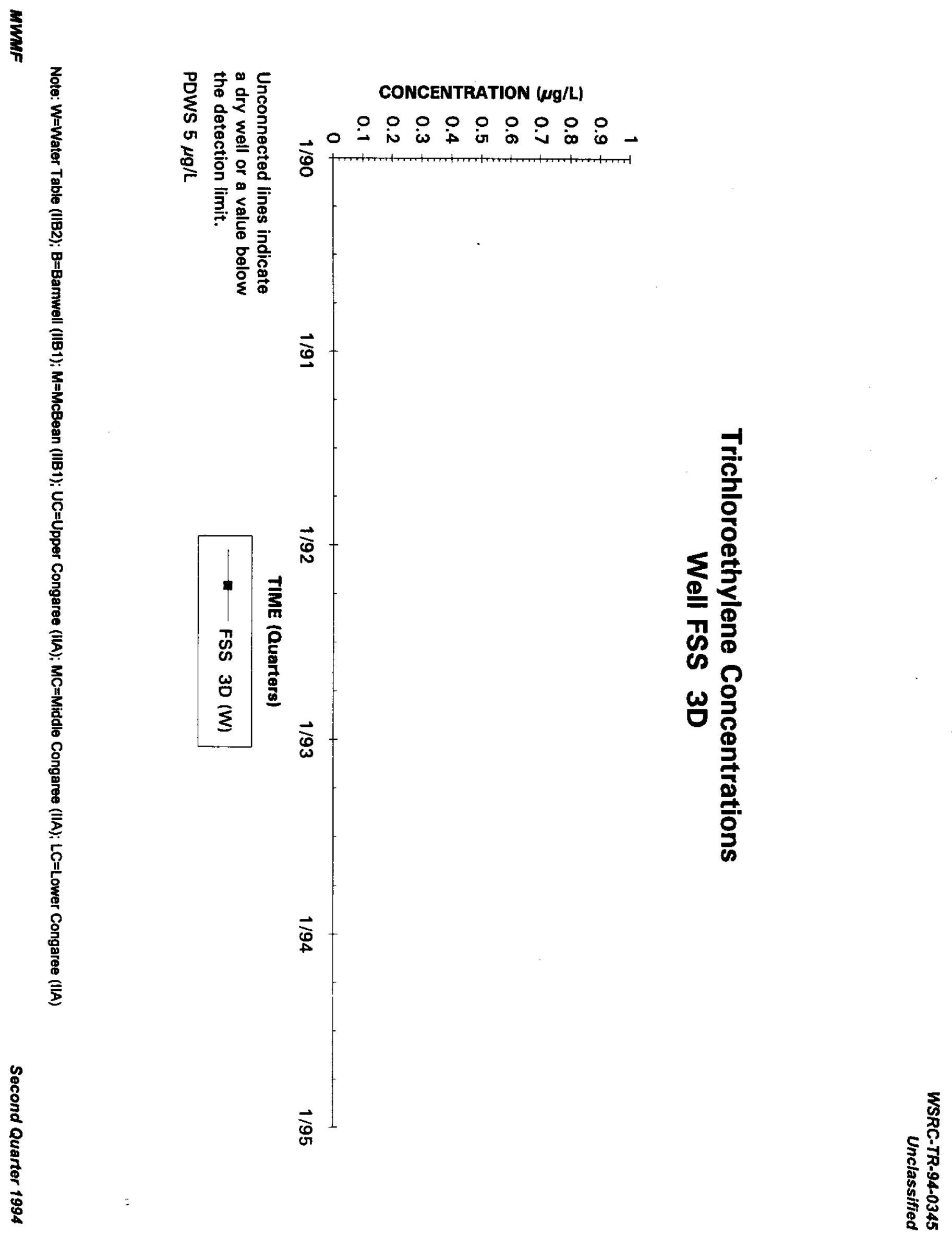




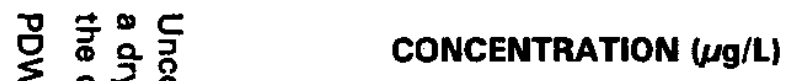

के 웅

जा 局

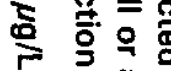

$=\infty$

类梀

के $0 \quad \begin{aligned} & 0 \\ & 0\end{aligned}$

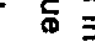

응

응

产

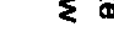

产

产

ริ

ถ

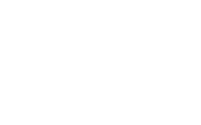

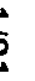
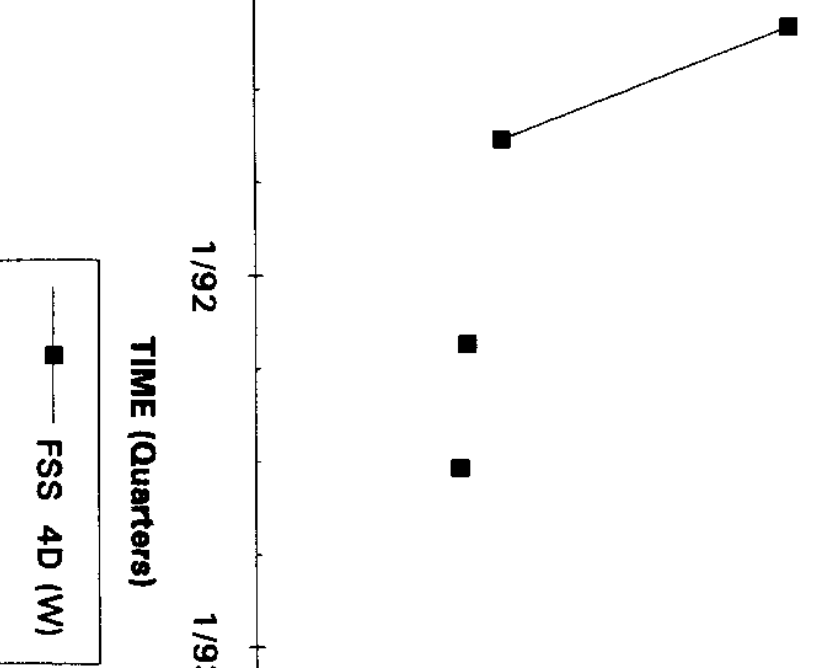

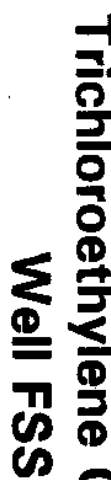

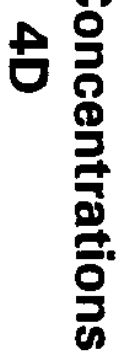



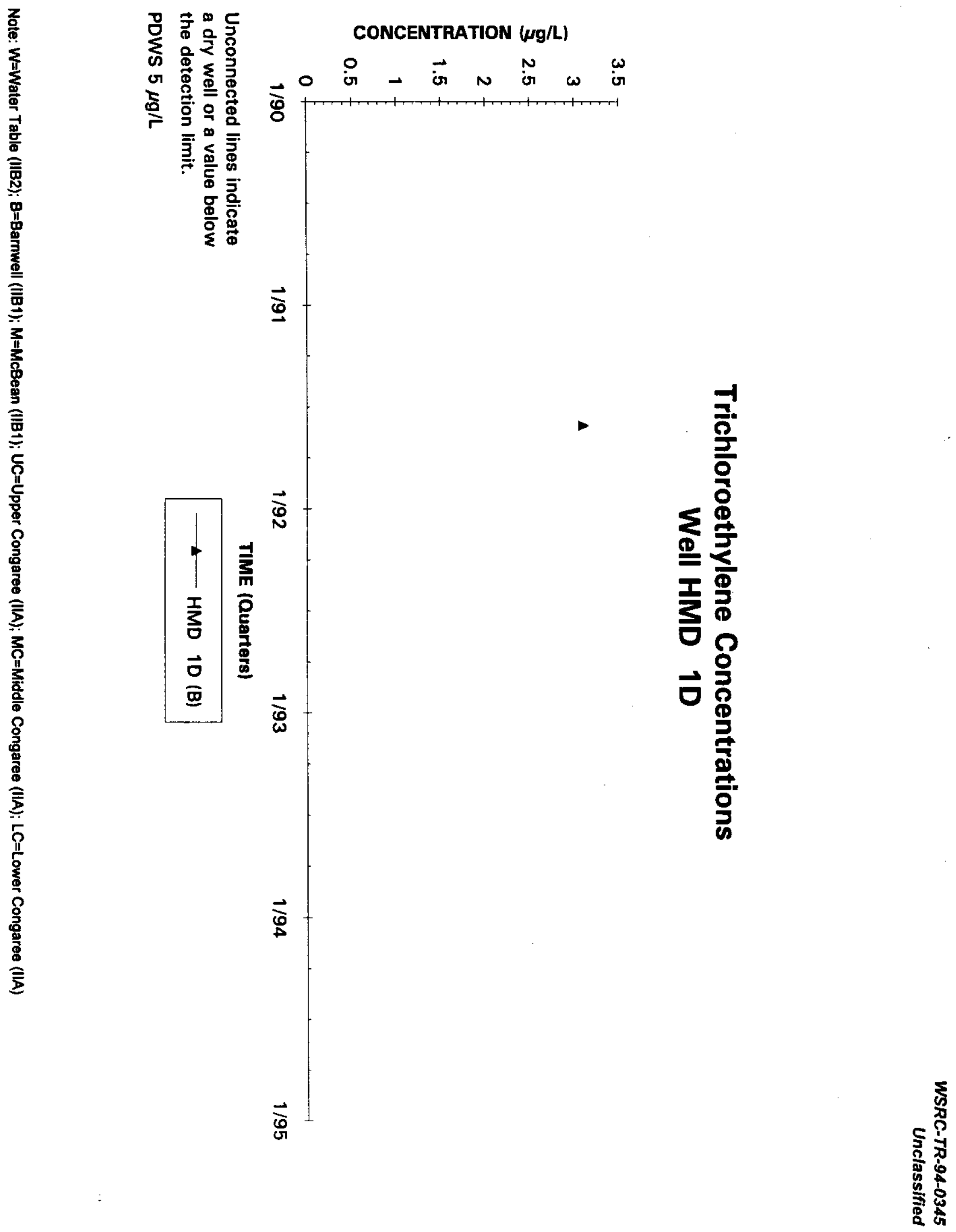
录

马ु
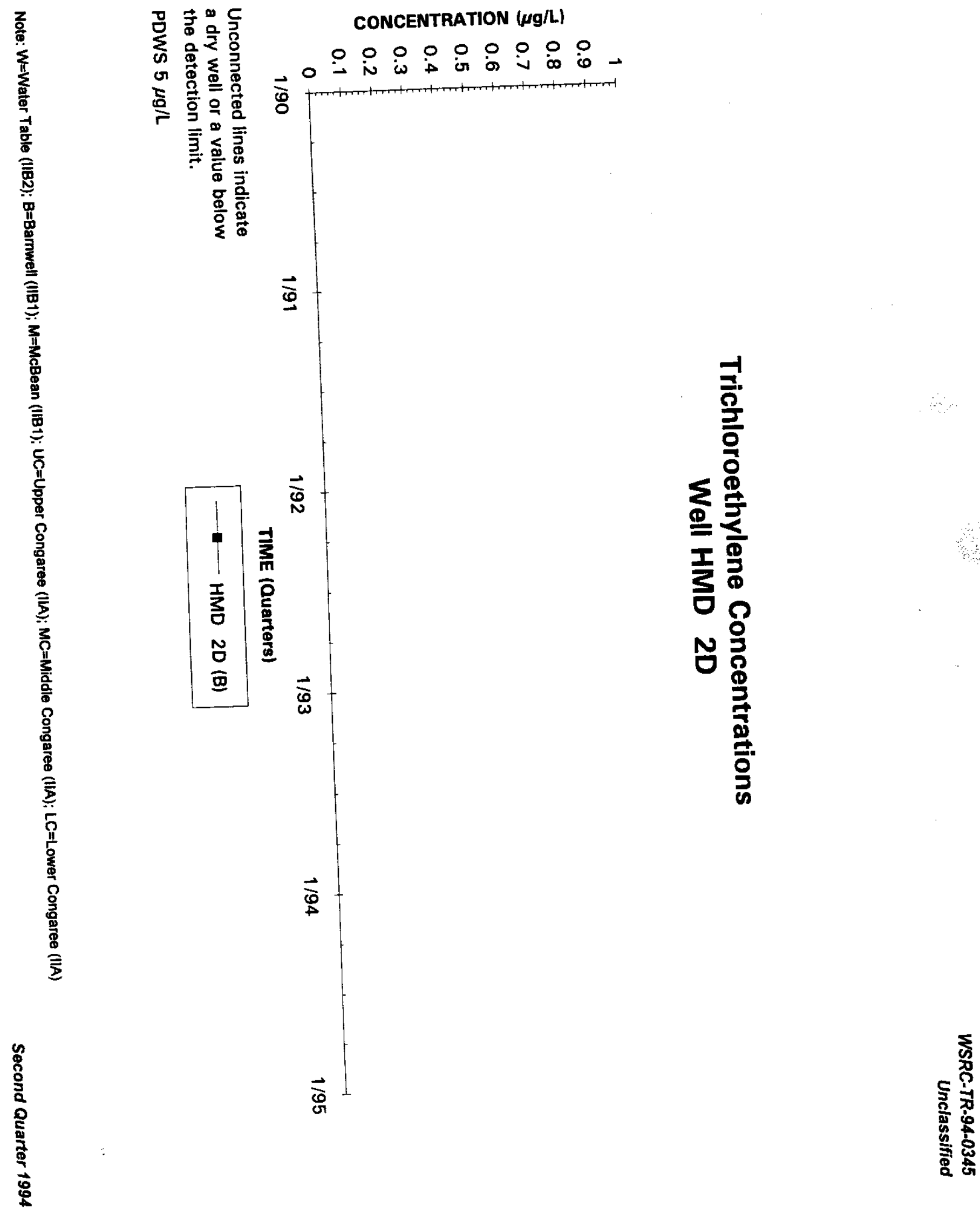


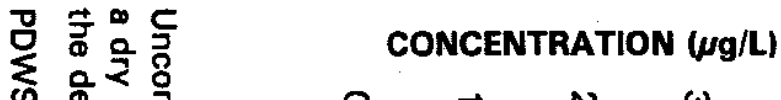

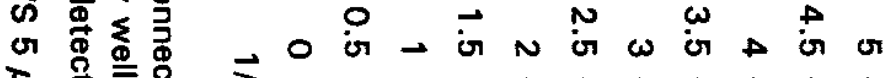

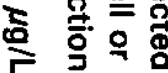

F

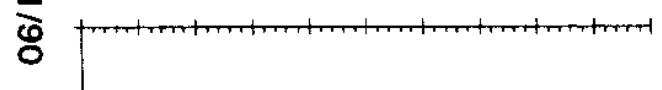

产

.

家

Ф

₹

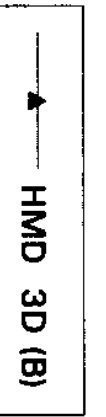

굴

产

$\stackrel{0}{0}$

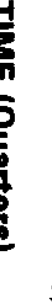

$\Sigma$

$\$$

エ

言

ํํำ

言

8

Ф़

产

iI

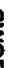

今

产

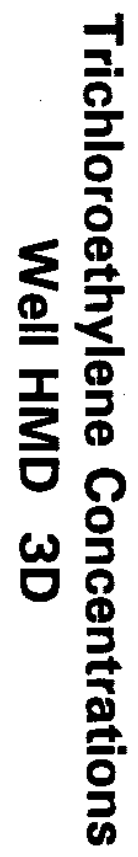




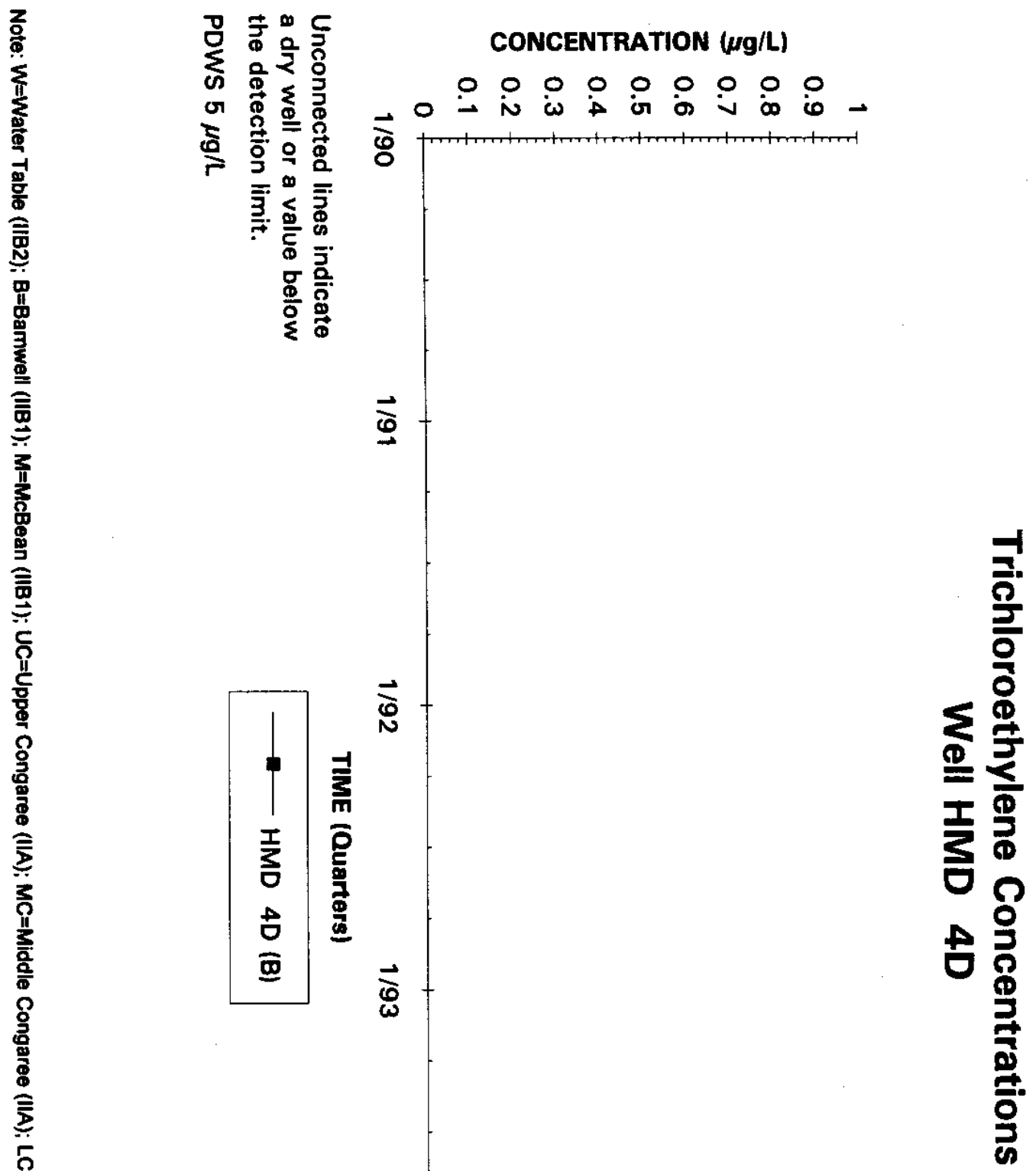


步

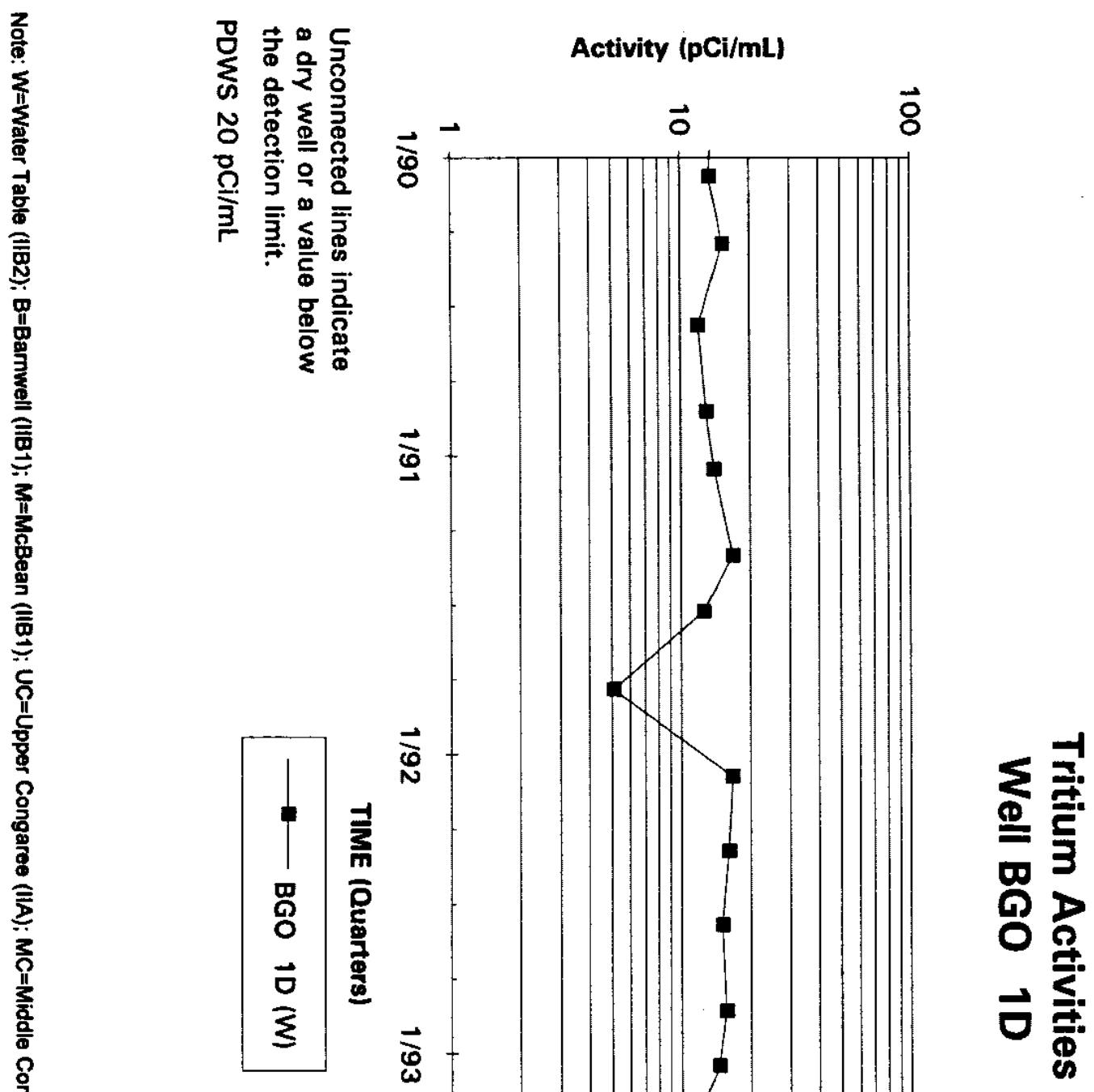


3
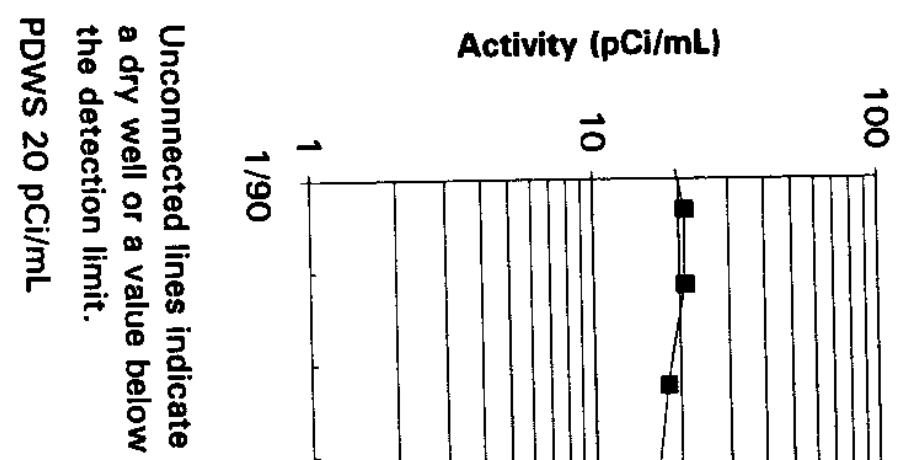

言

동

晏

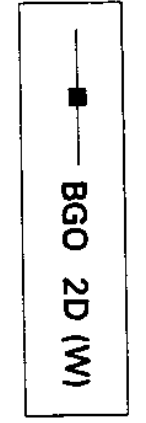

$\frac{1}{3}$

$\overrightarrow{0}$

ثิ

言

조

춯

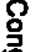

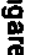

言

II

害

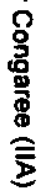

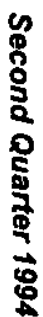

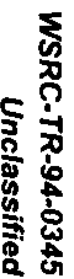




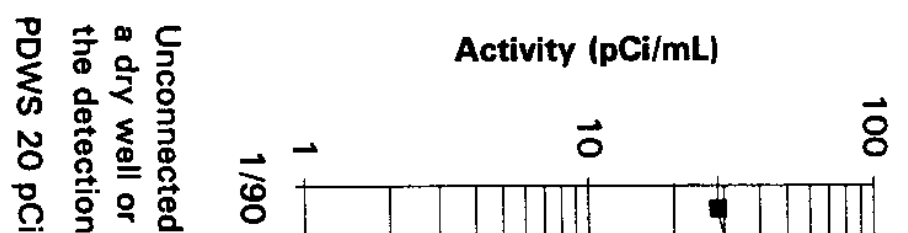

ํㅜㅇㅎㅎ응

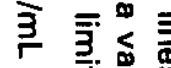

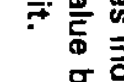

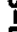

몽

.

言

.

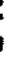

言

§

竞

ํํำ

言

క

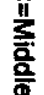

8

芦

言

5

$\frac{11}{5}$

今ั

言 
I

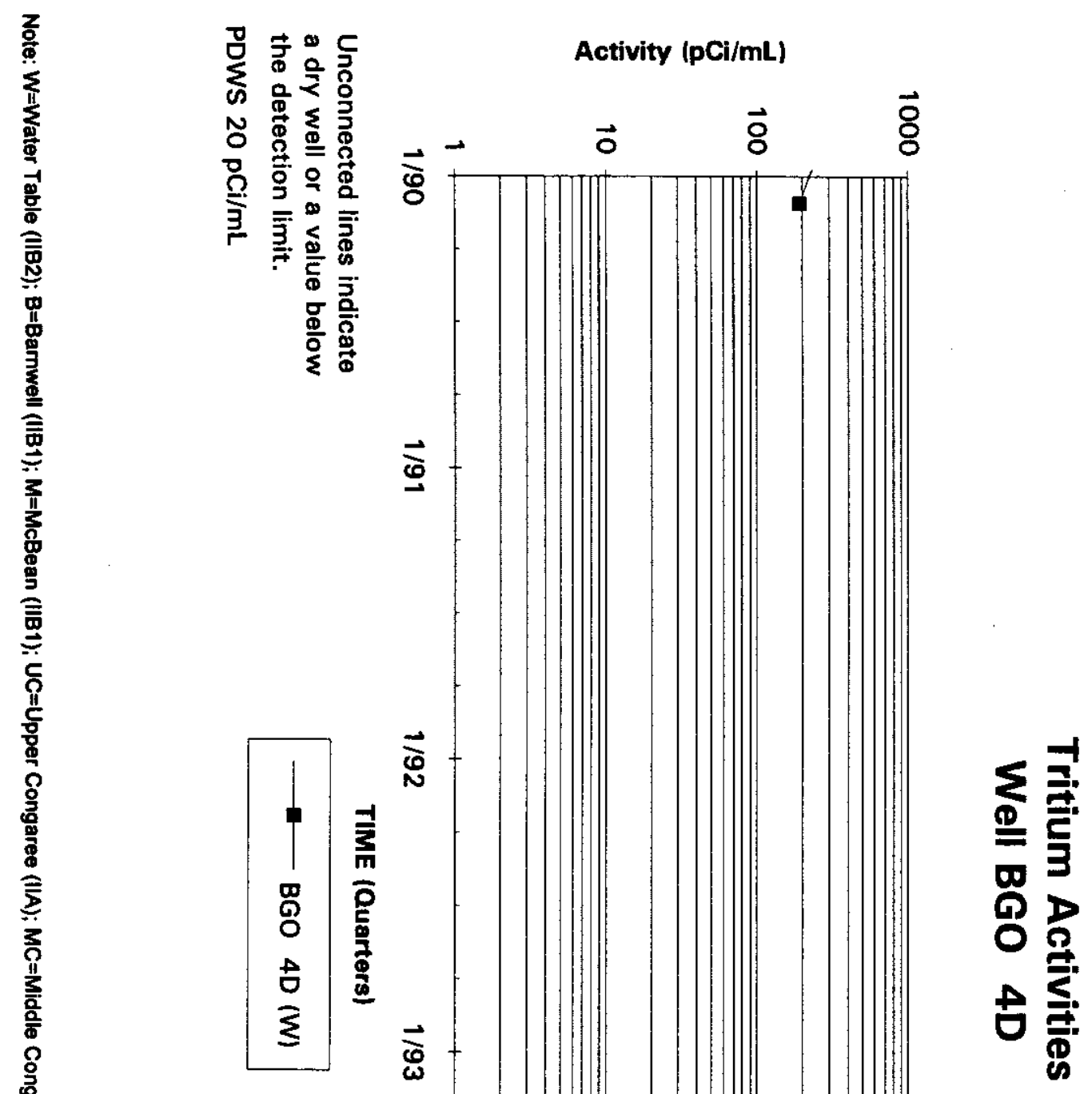

6
8
8
5
0
0
5
0
0
0 
3

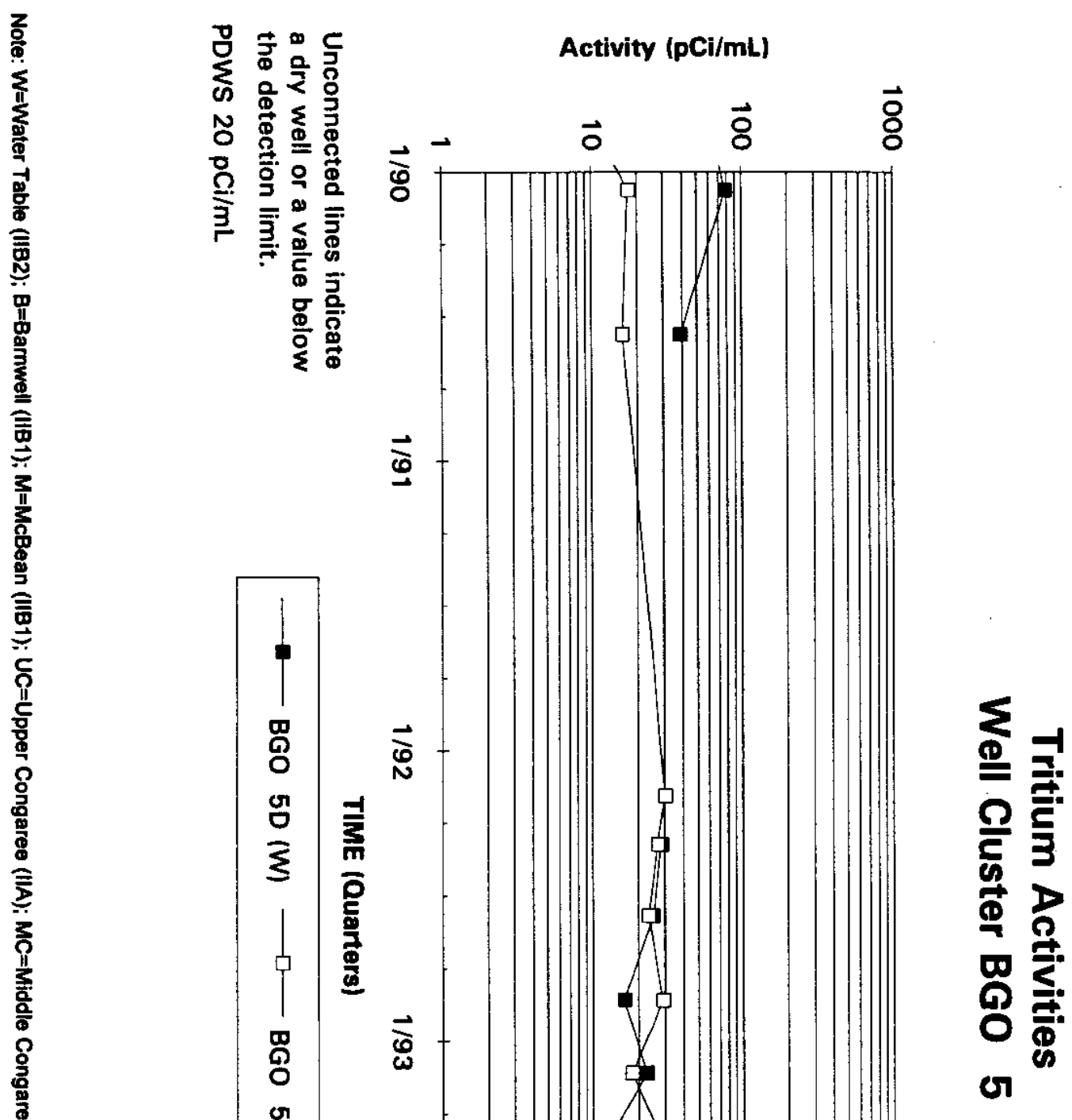


I

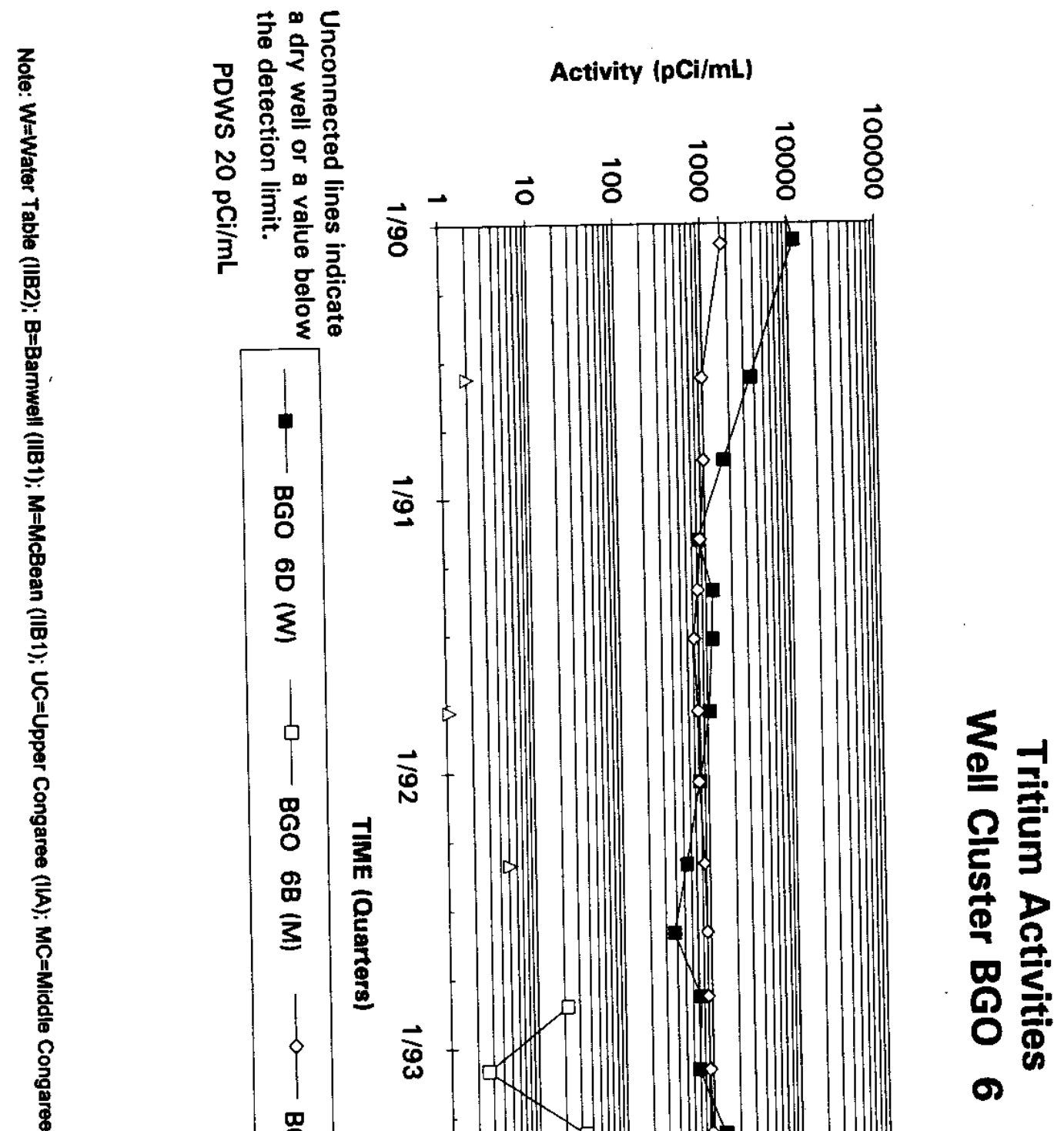

8
8
8
0
0
5
3
0
0
0

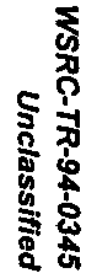


1

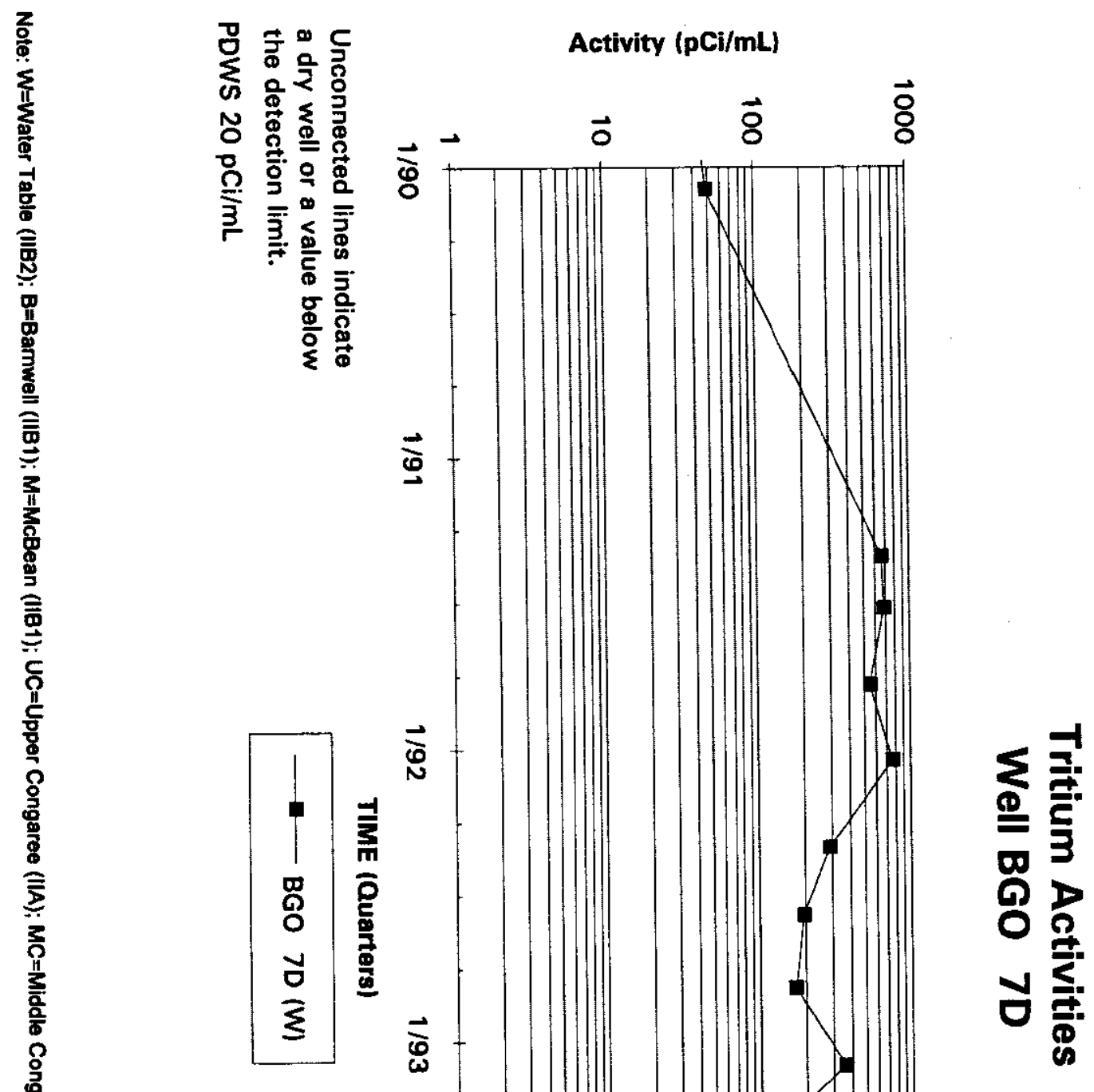

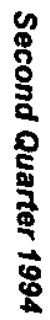

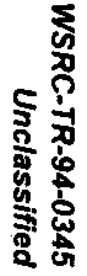


1

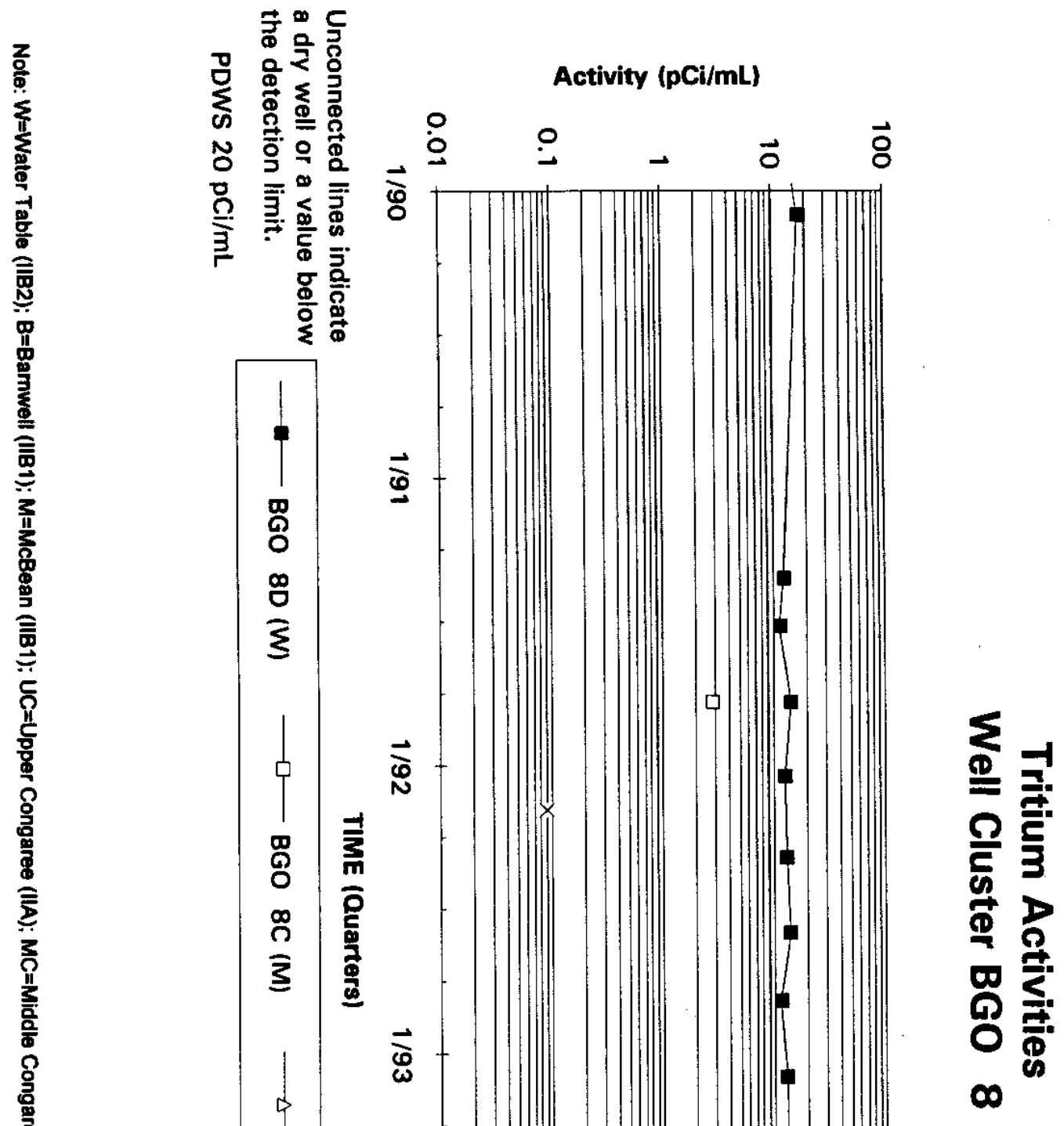

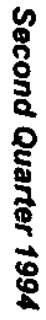

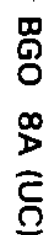

|

产

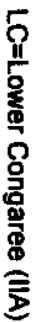

总

罚

$\overrightarrow{0}$

$\vec{\circ}$

ఏ్

S尔 


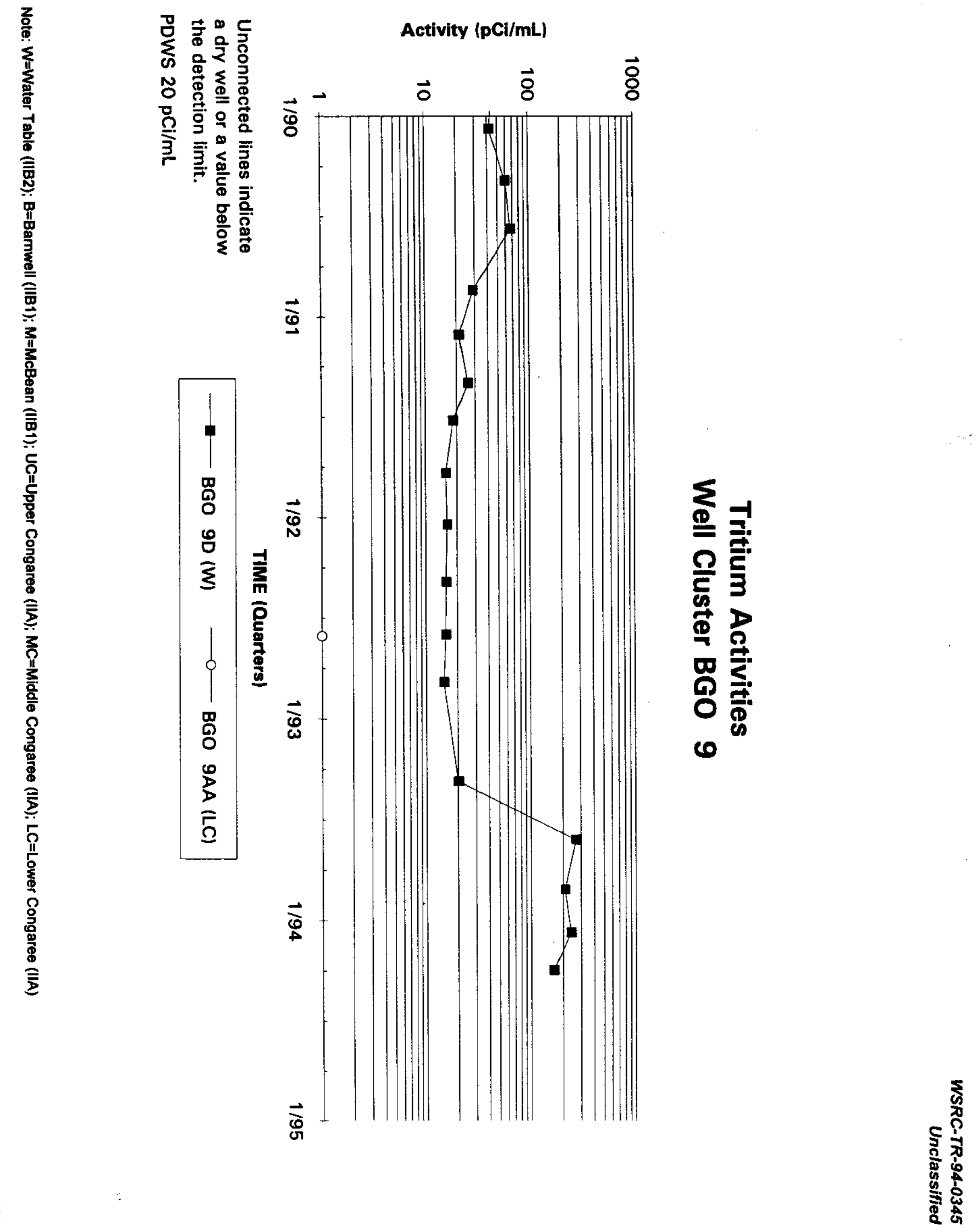


产

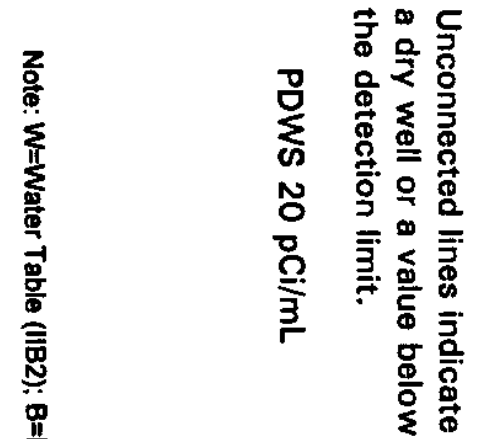

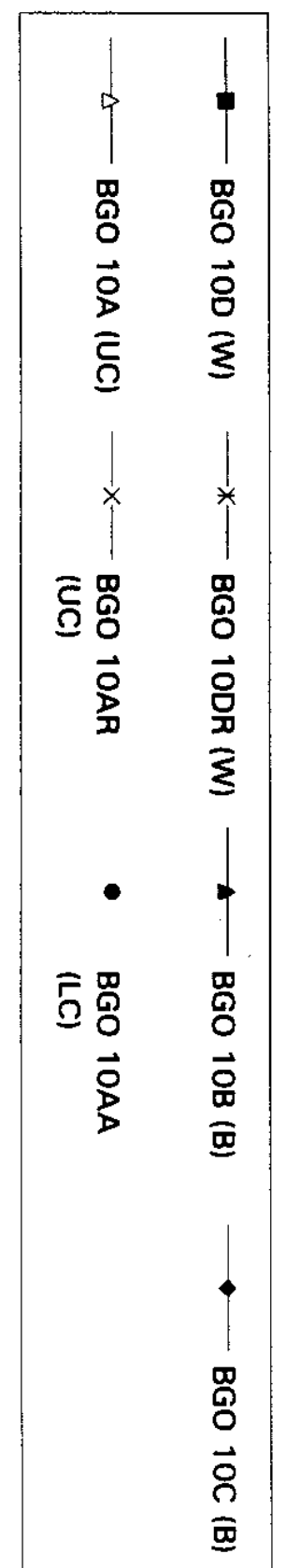

Activity (pCi/mL) 
I

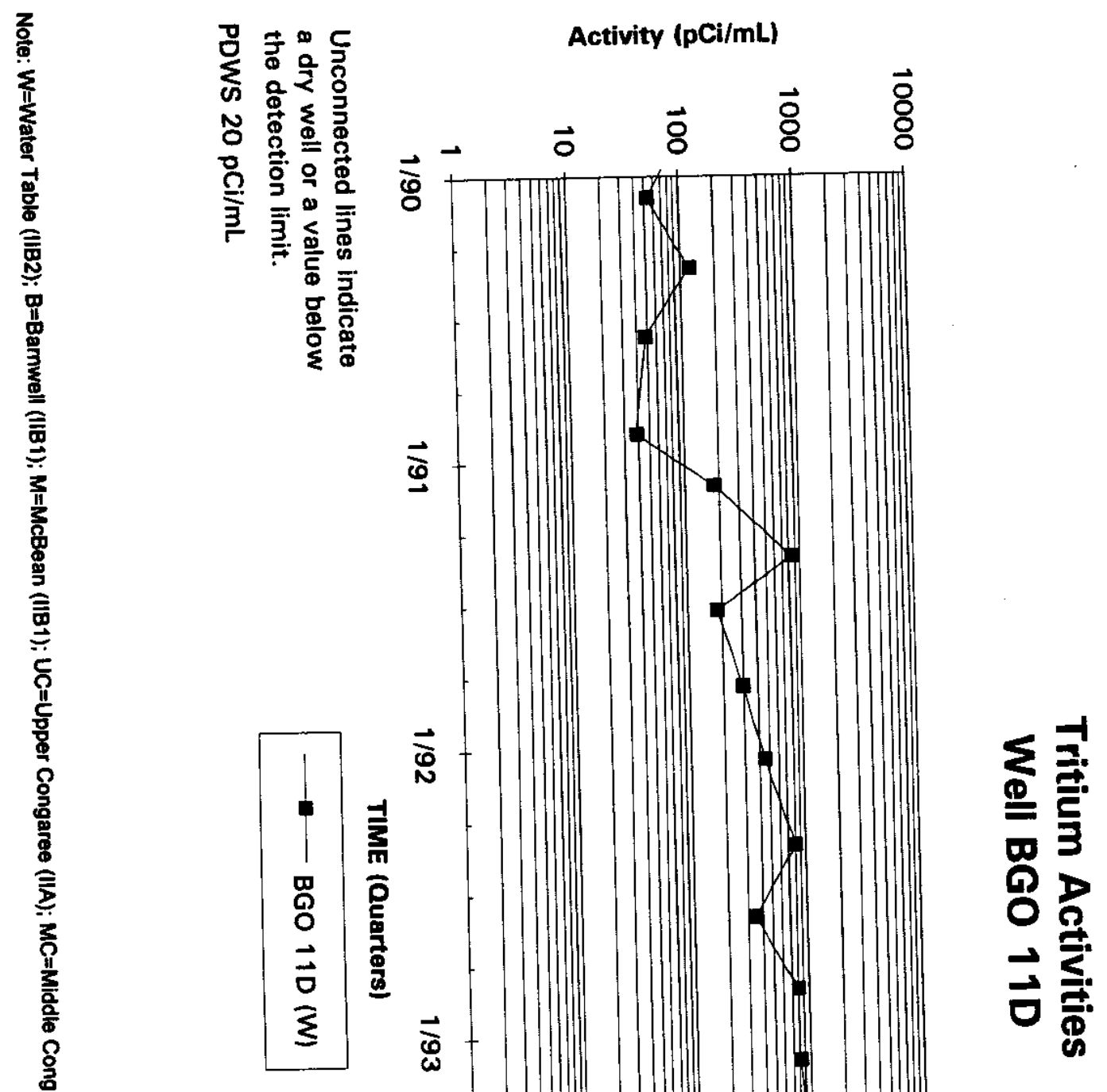


I

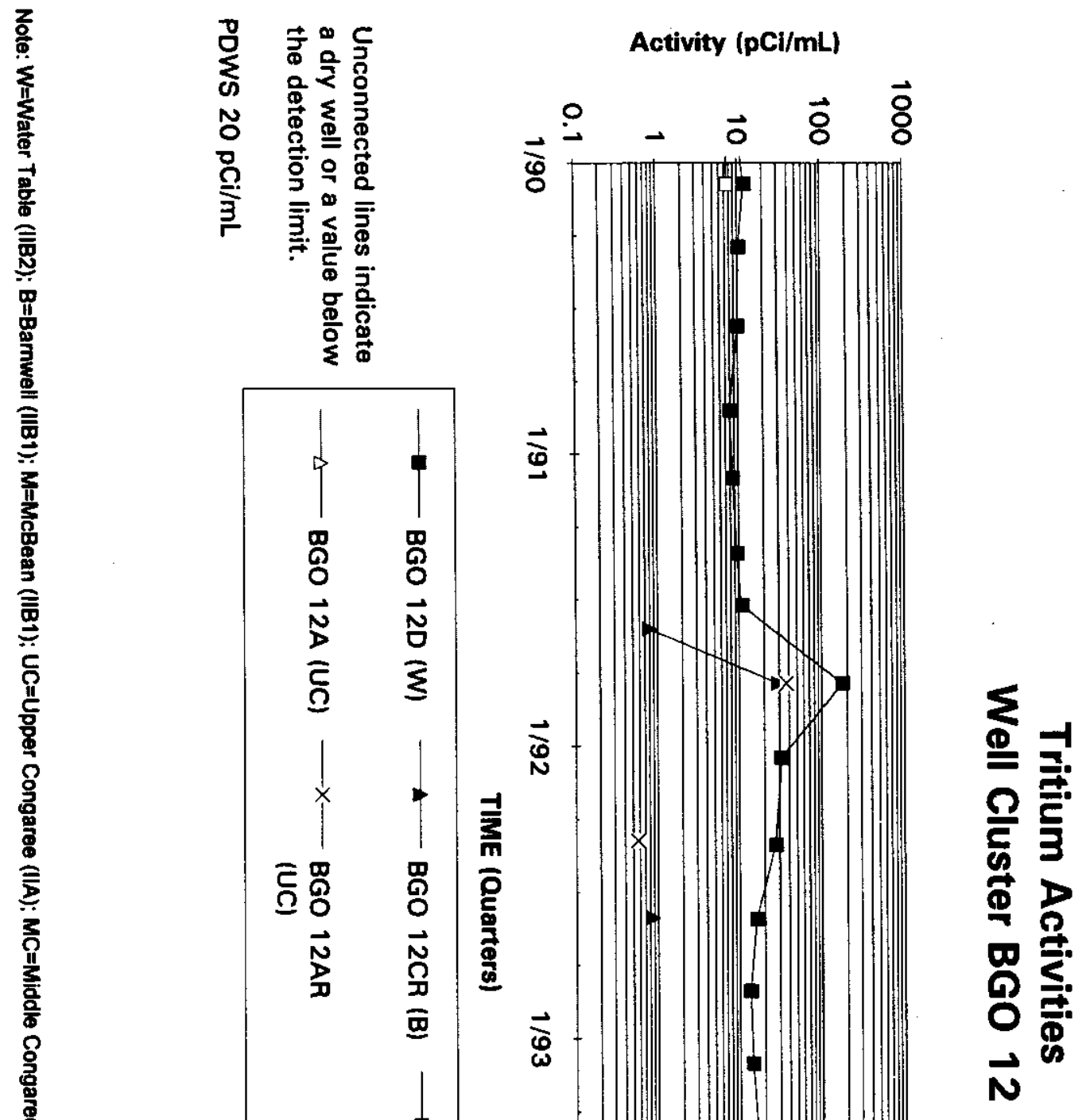

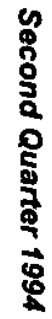

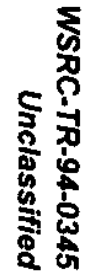




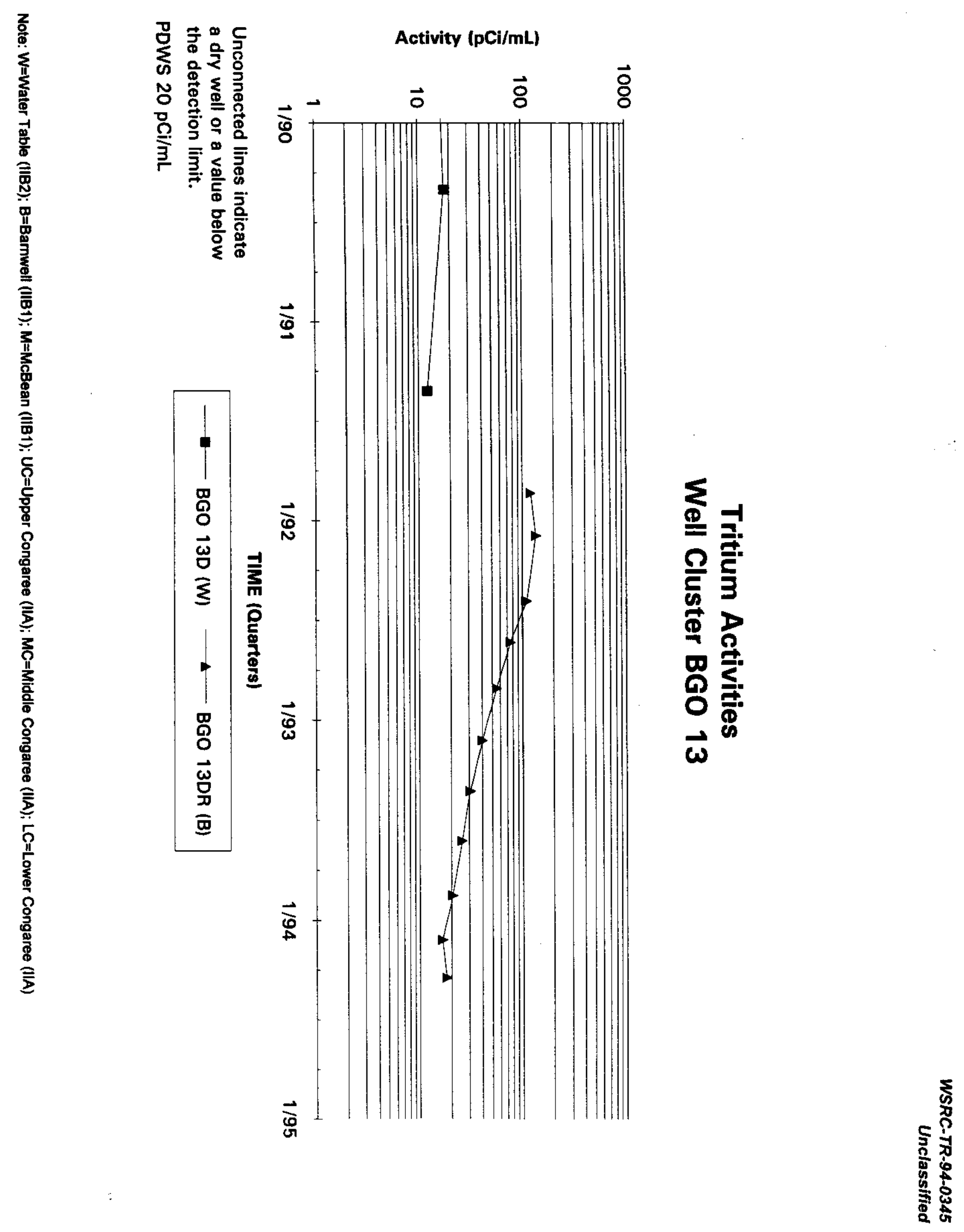


翠

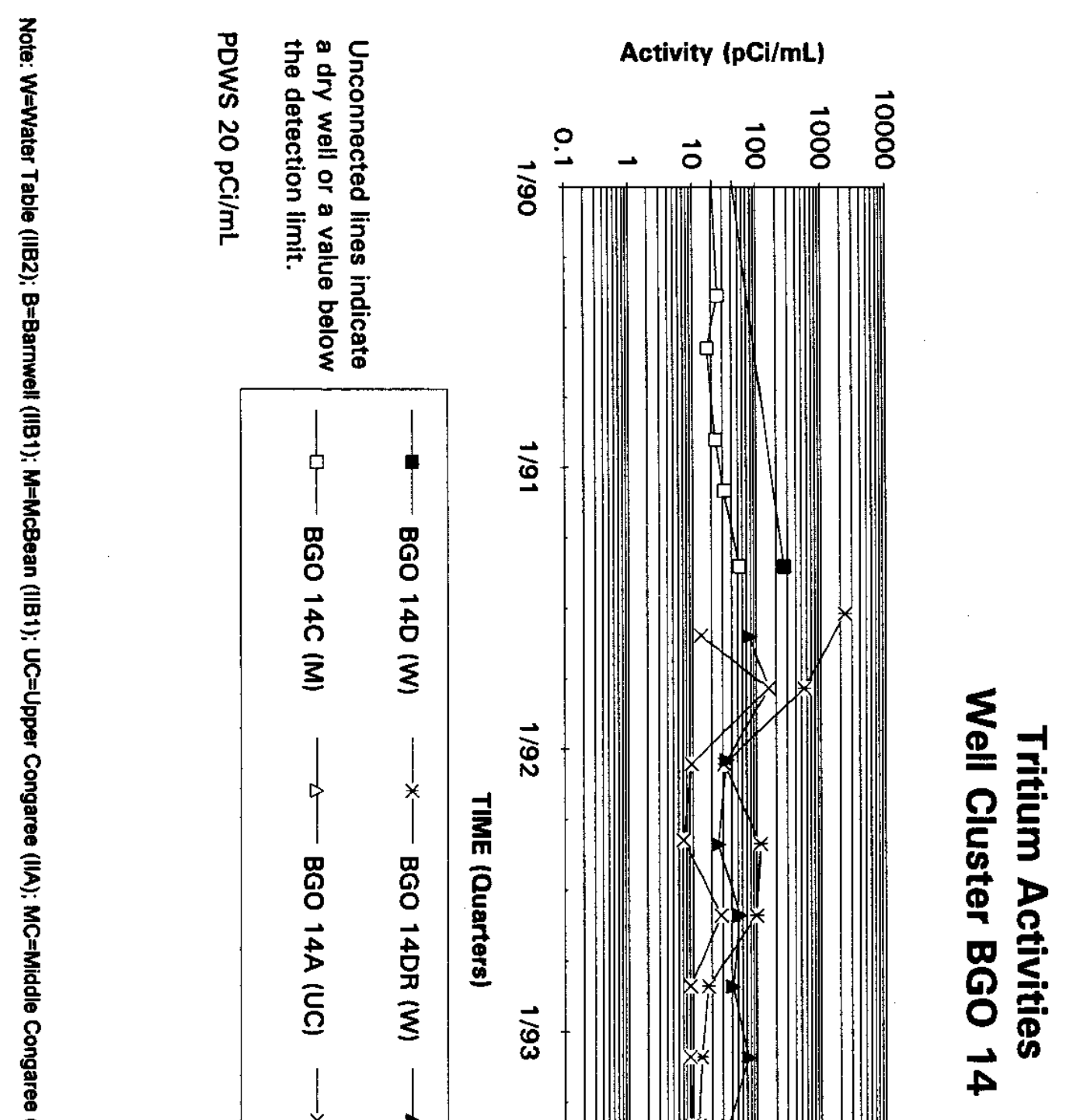

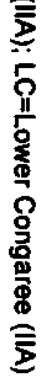

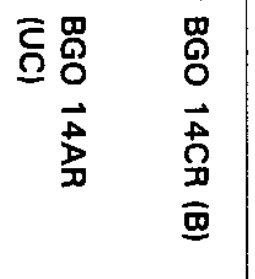

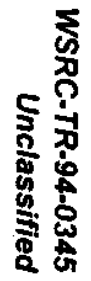


3

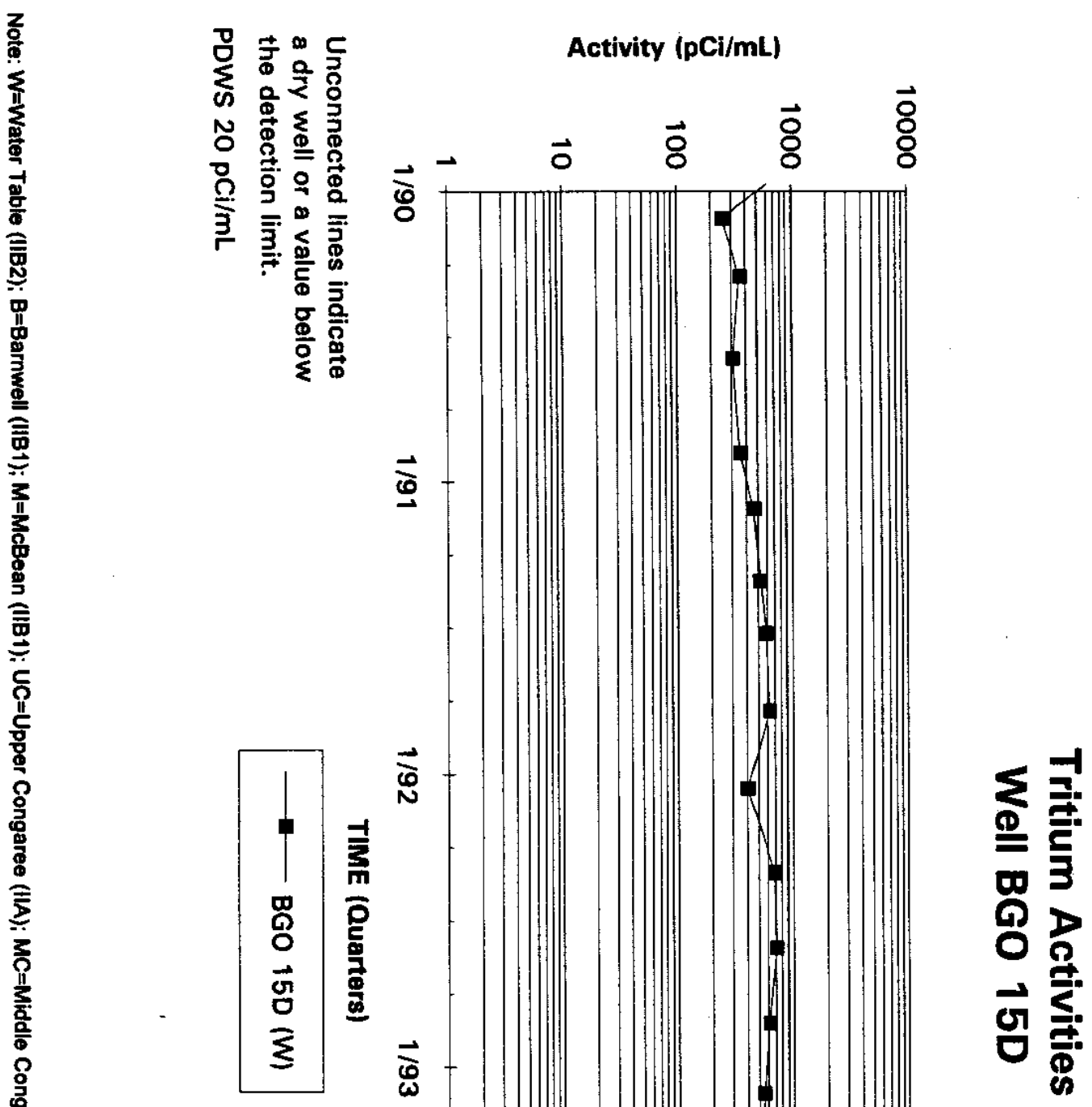


1

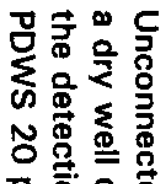

Activity (pCi/mL)

응응

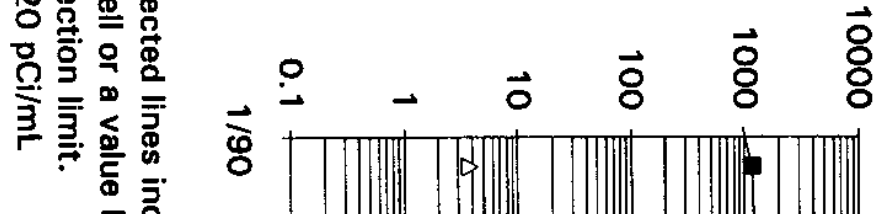

兽

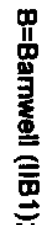

II

응 음

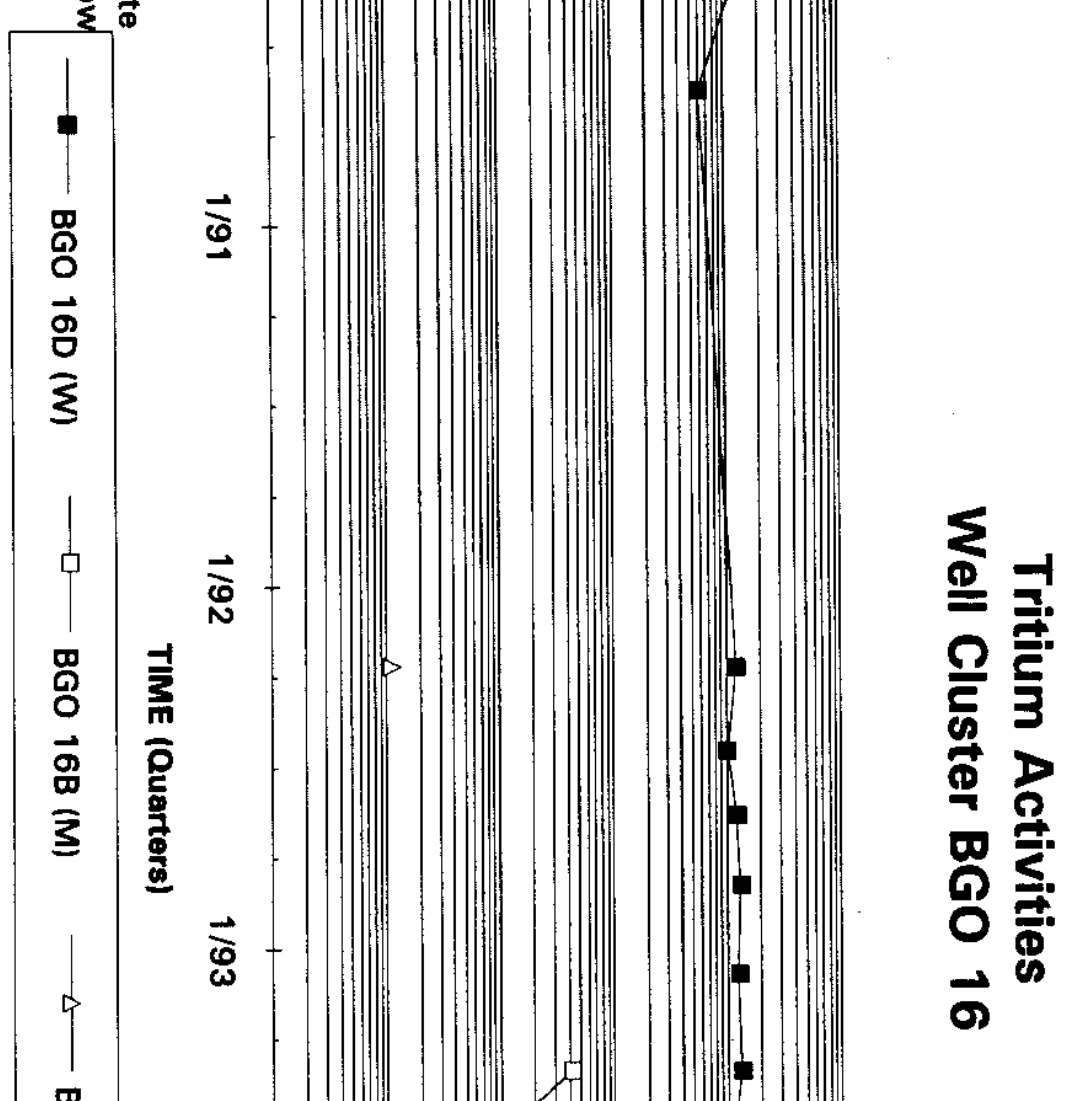

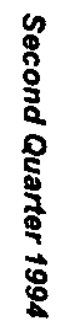

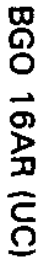

$\vec{G}$

$\vec{\Phi}$

言

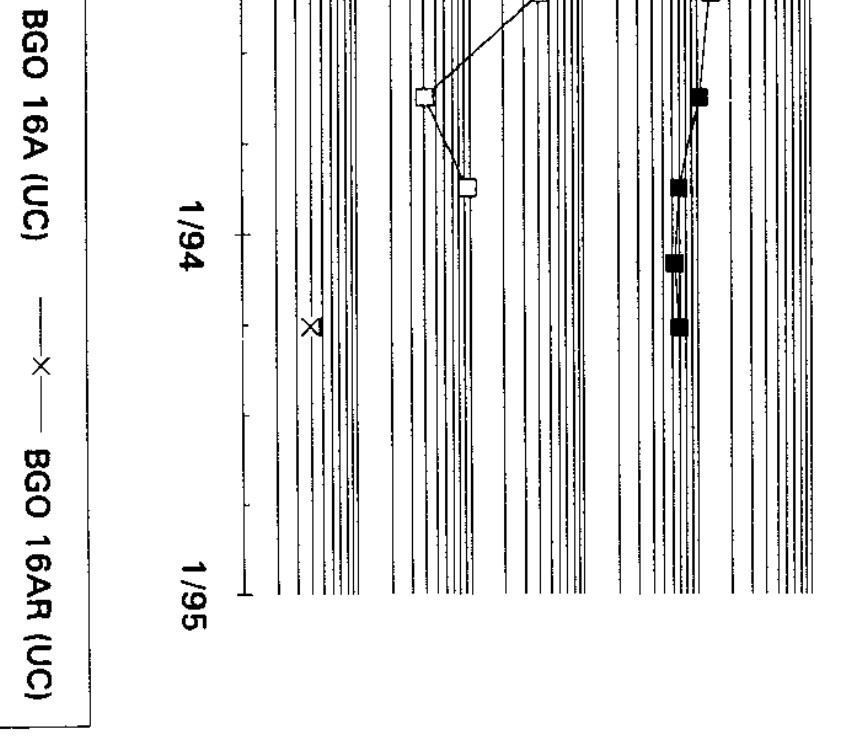

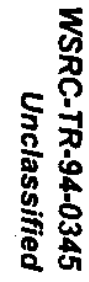




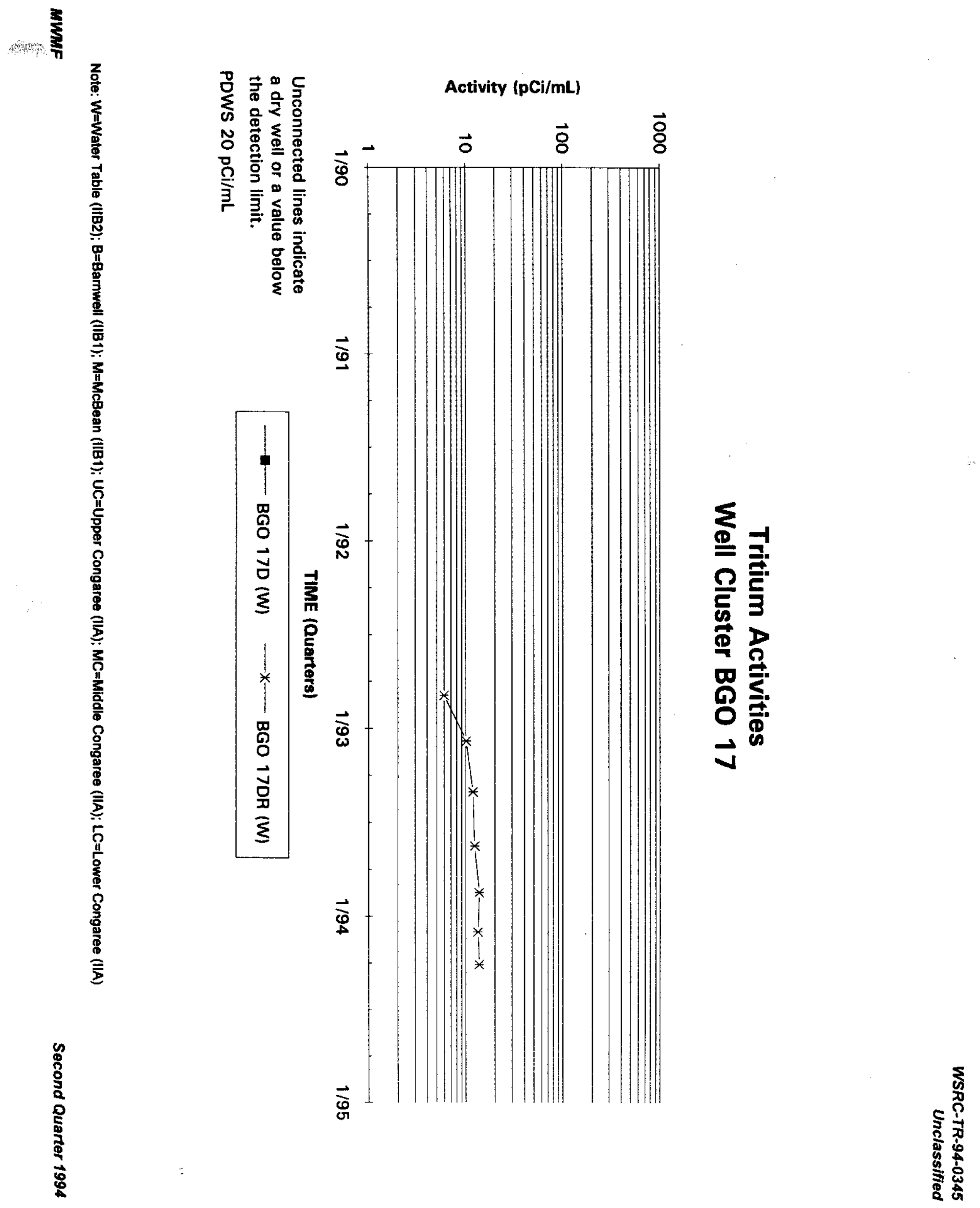


3

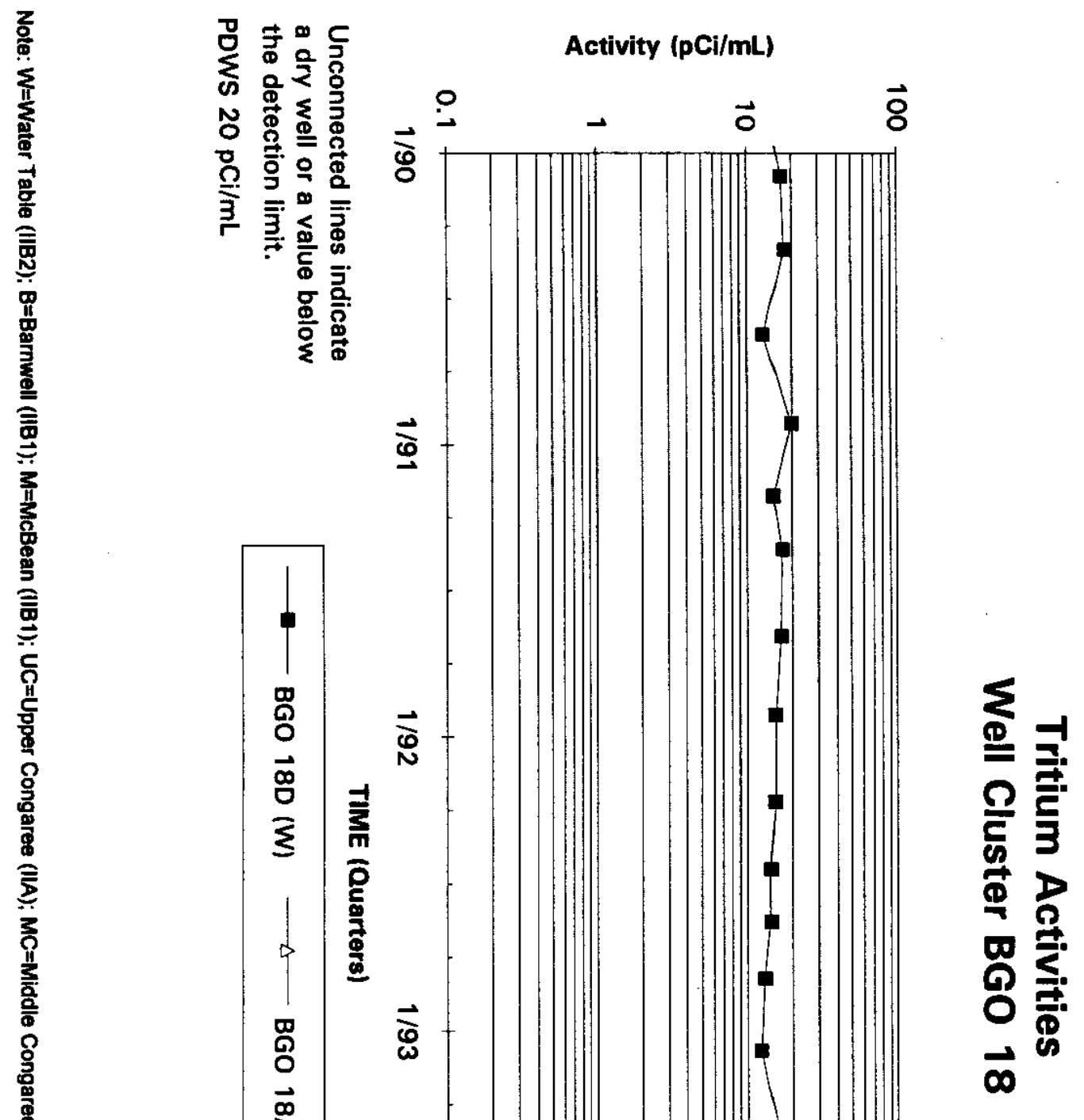

0
8
8
0
0
0
5
5
9
8
8

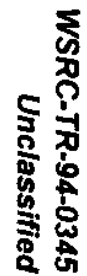


3

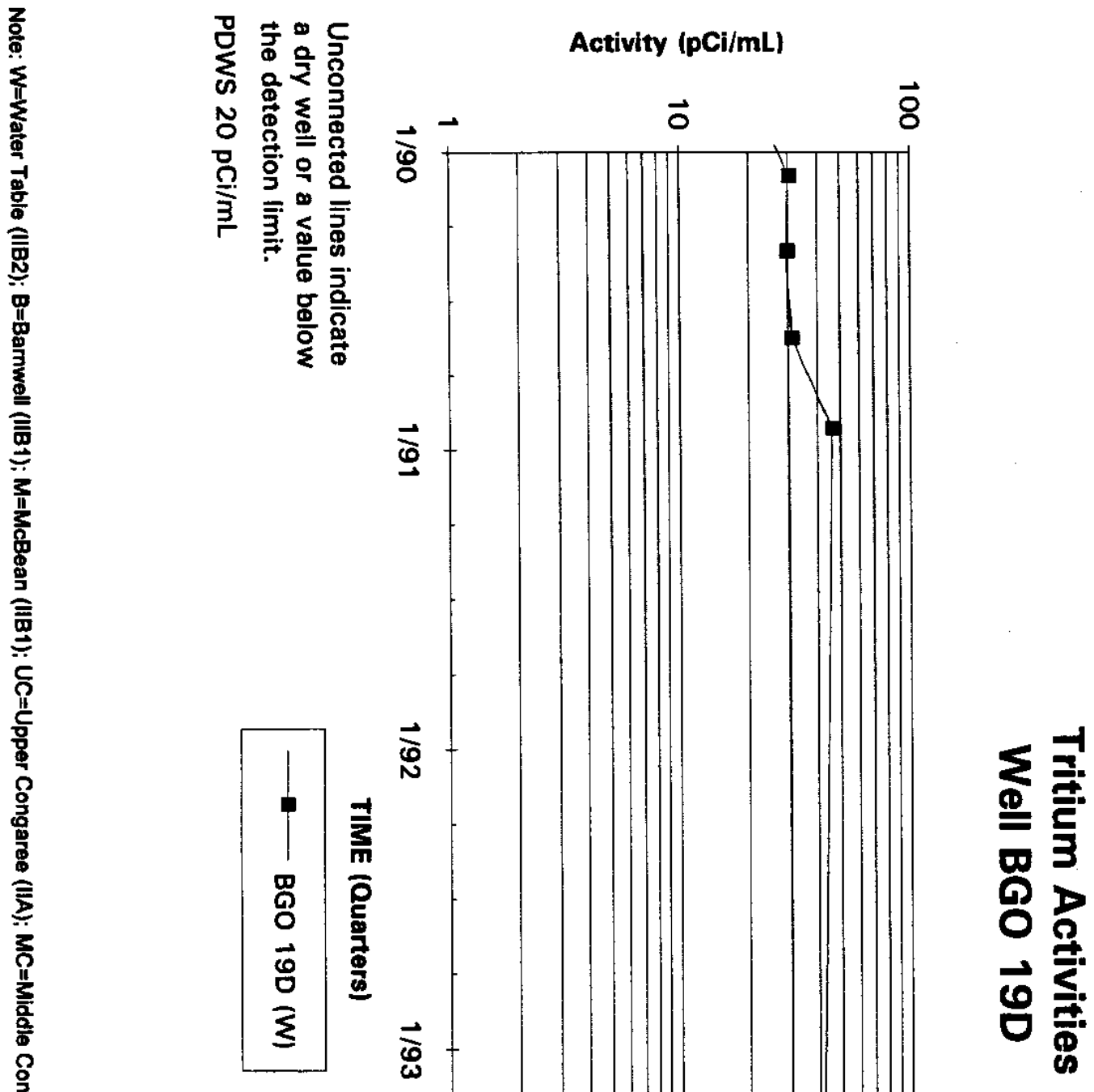

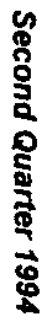

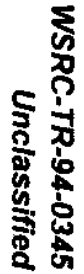


3

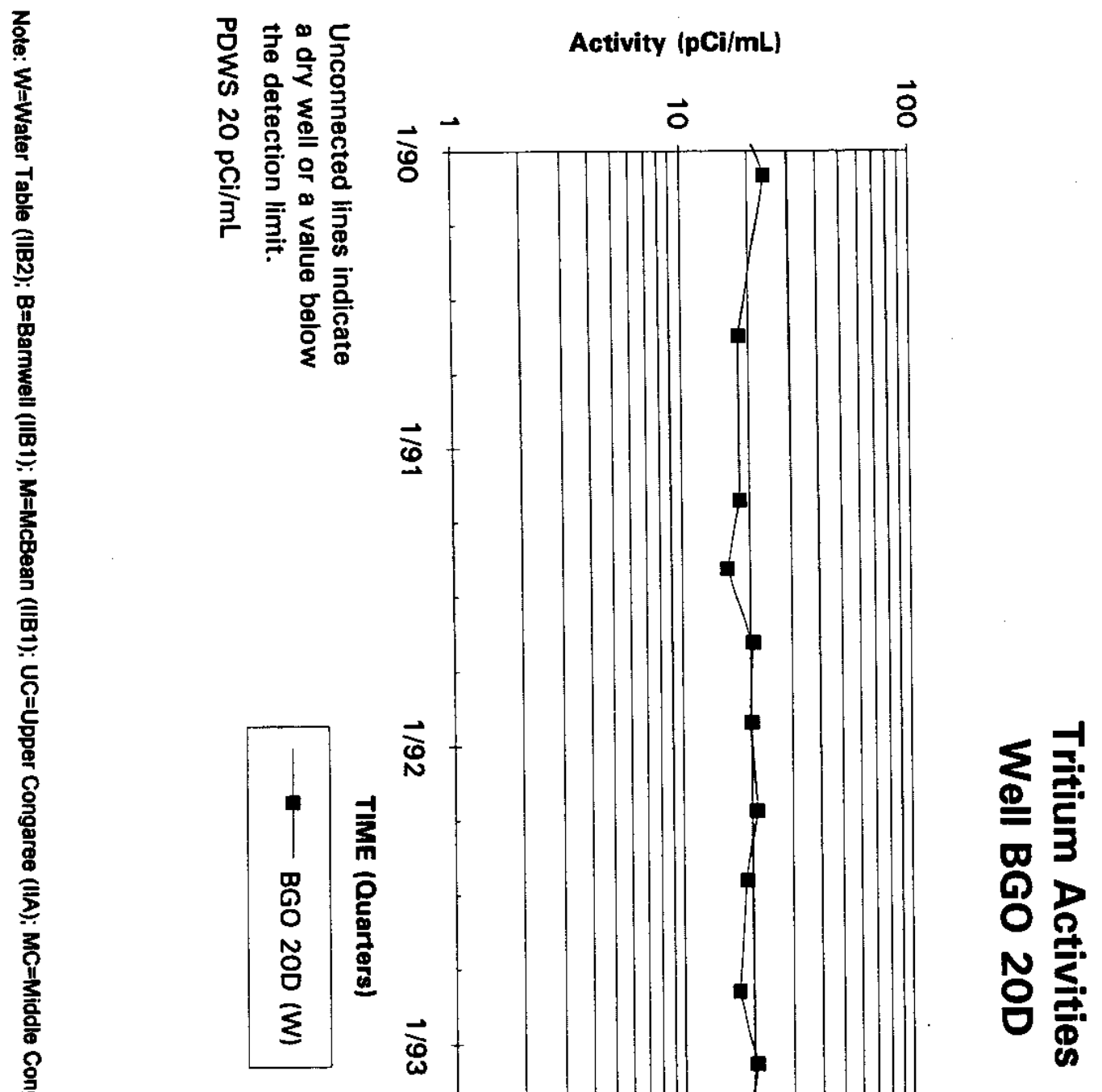


3

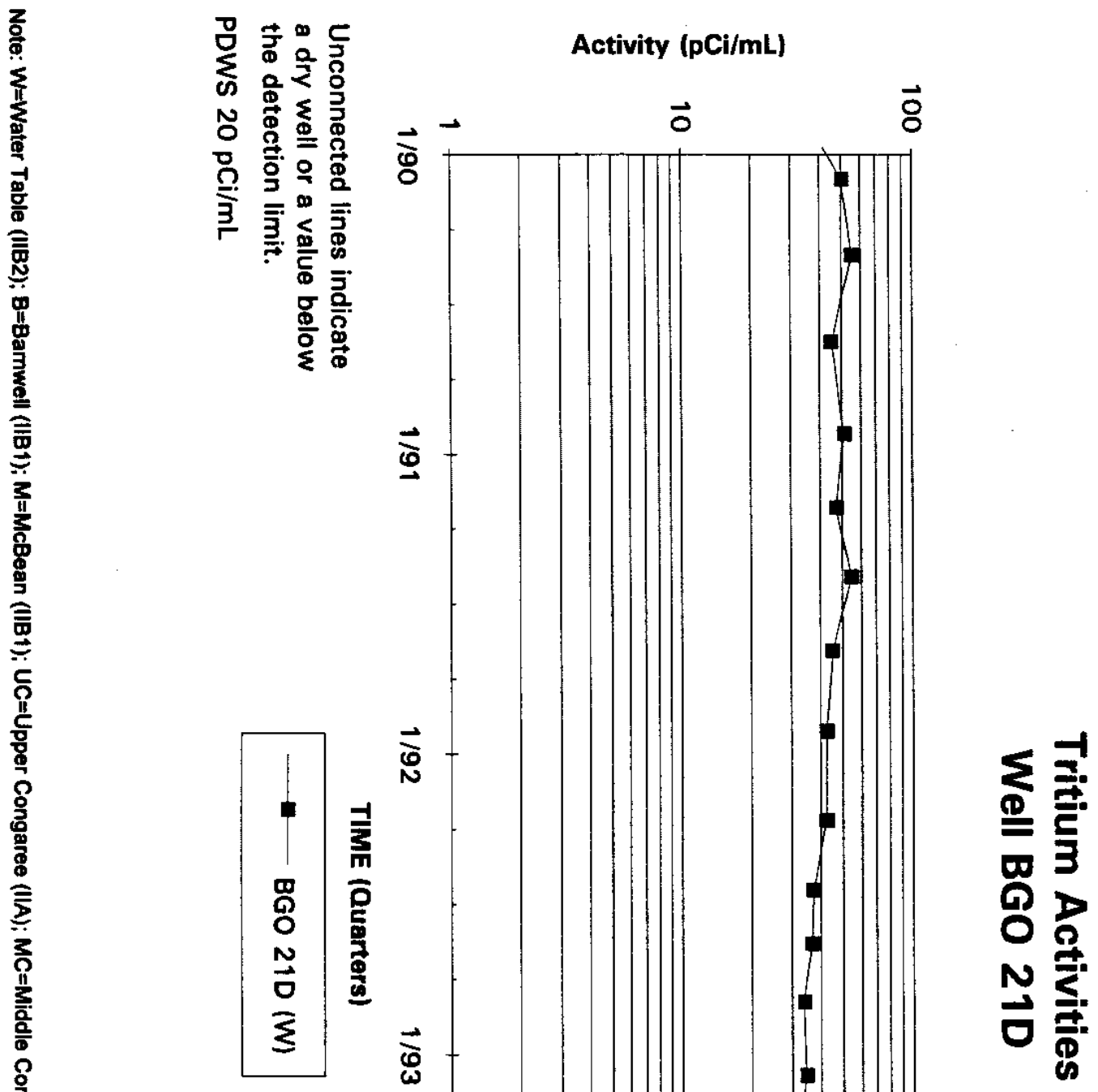


㛚

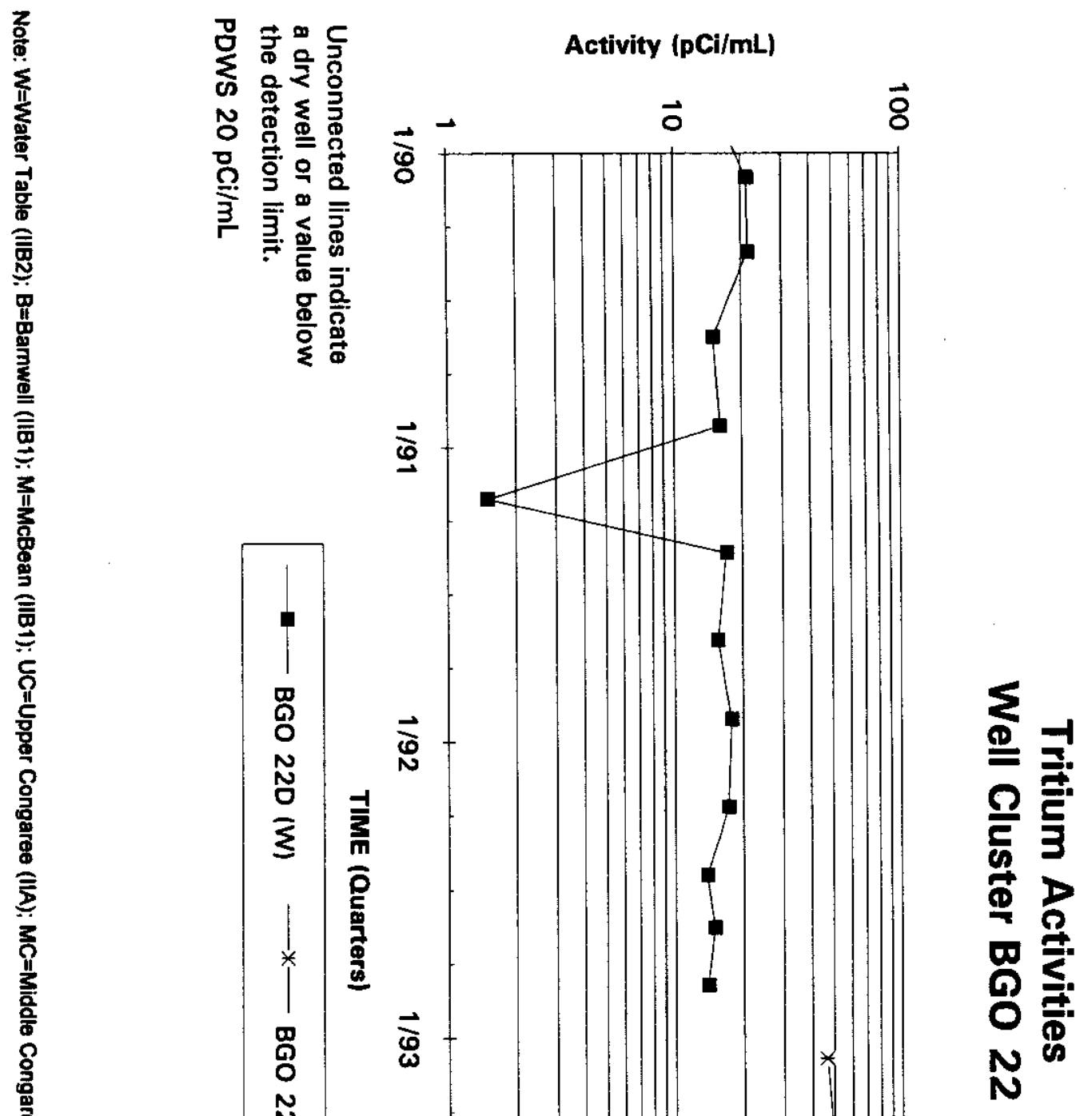

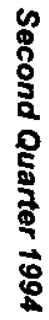

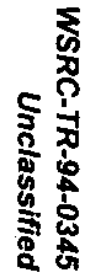




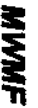

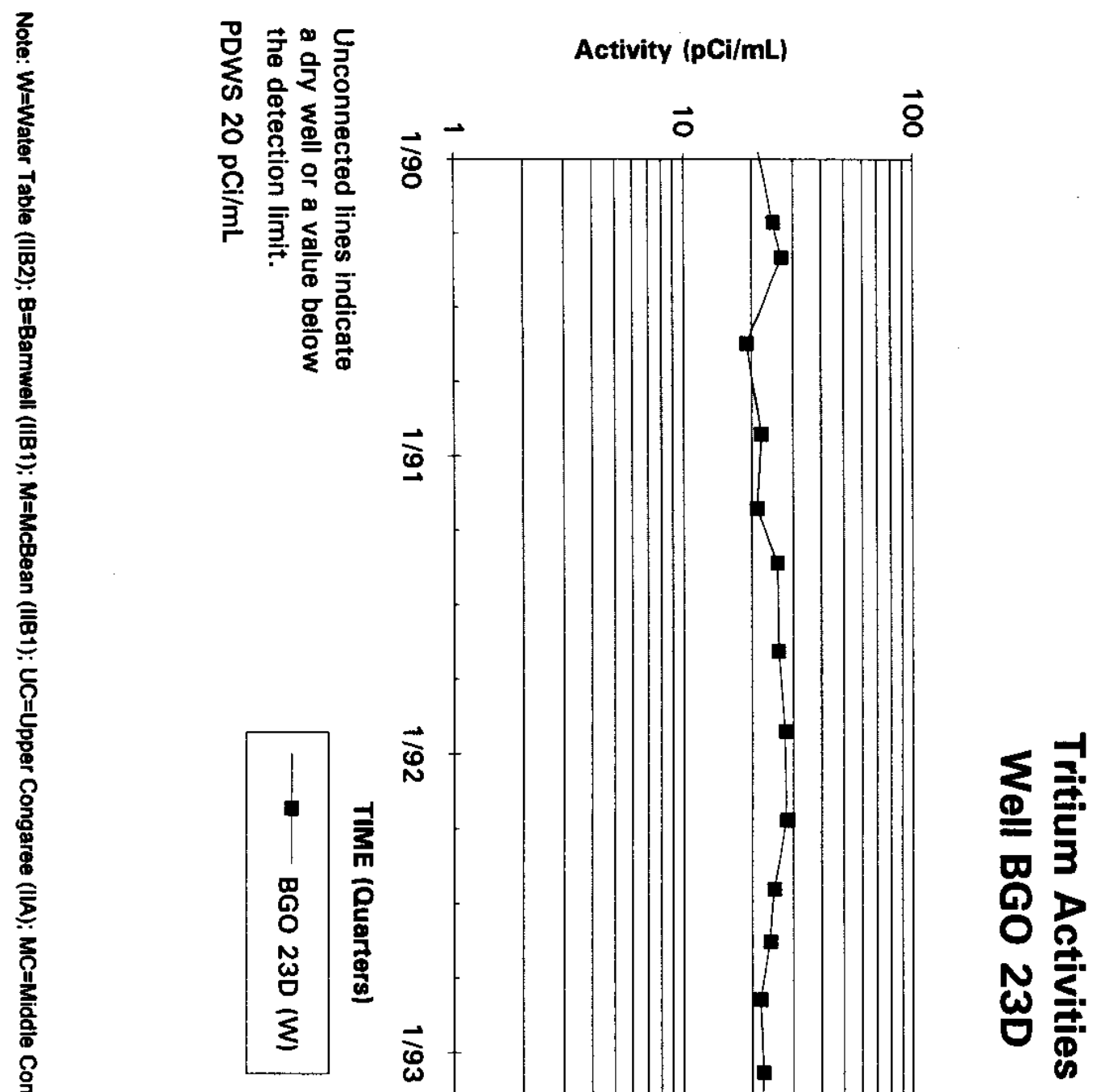

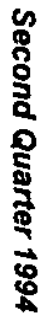

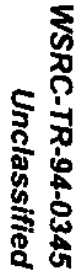




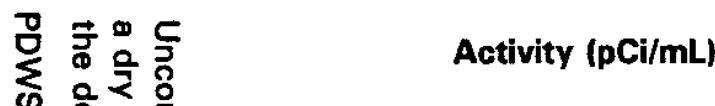

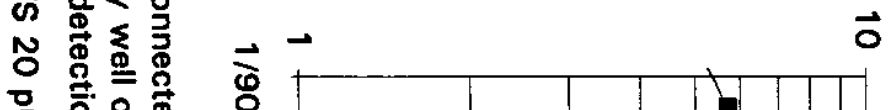

产

훙융

实雪文

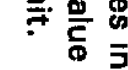

罾

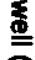

言

$\frac{3}{11}$

高

言

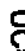

듬

产

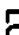

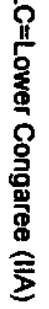
동 응
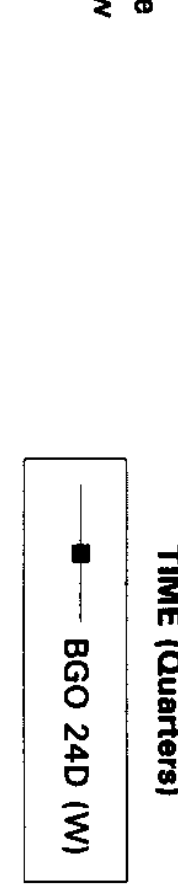

ฮे
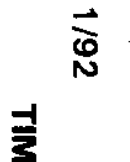

章

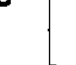

픔

产

$\vec{\Phi}$ 


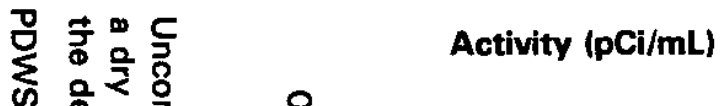

N

承 를.

产水

章

四
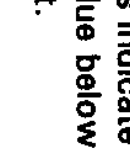

言

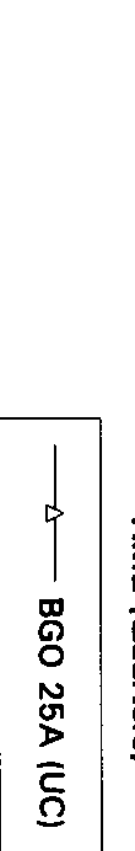

$\vec{\Phi}$

-

高

言

(2)

듬

8

$\stackrel{\$}{\overline{\bar{~}}}$

3

츨

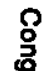

\%

言

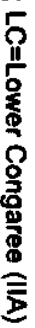

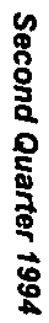

空 
雺

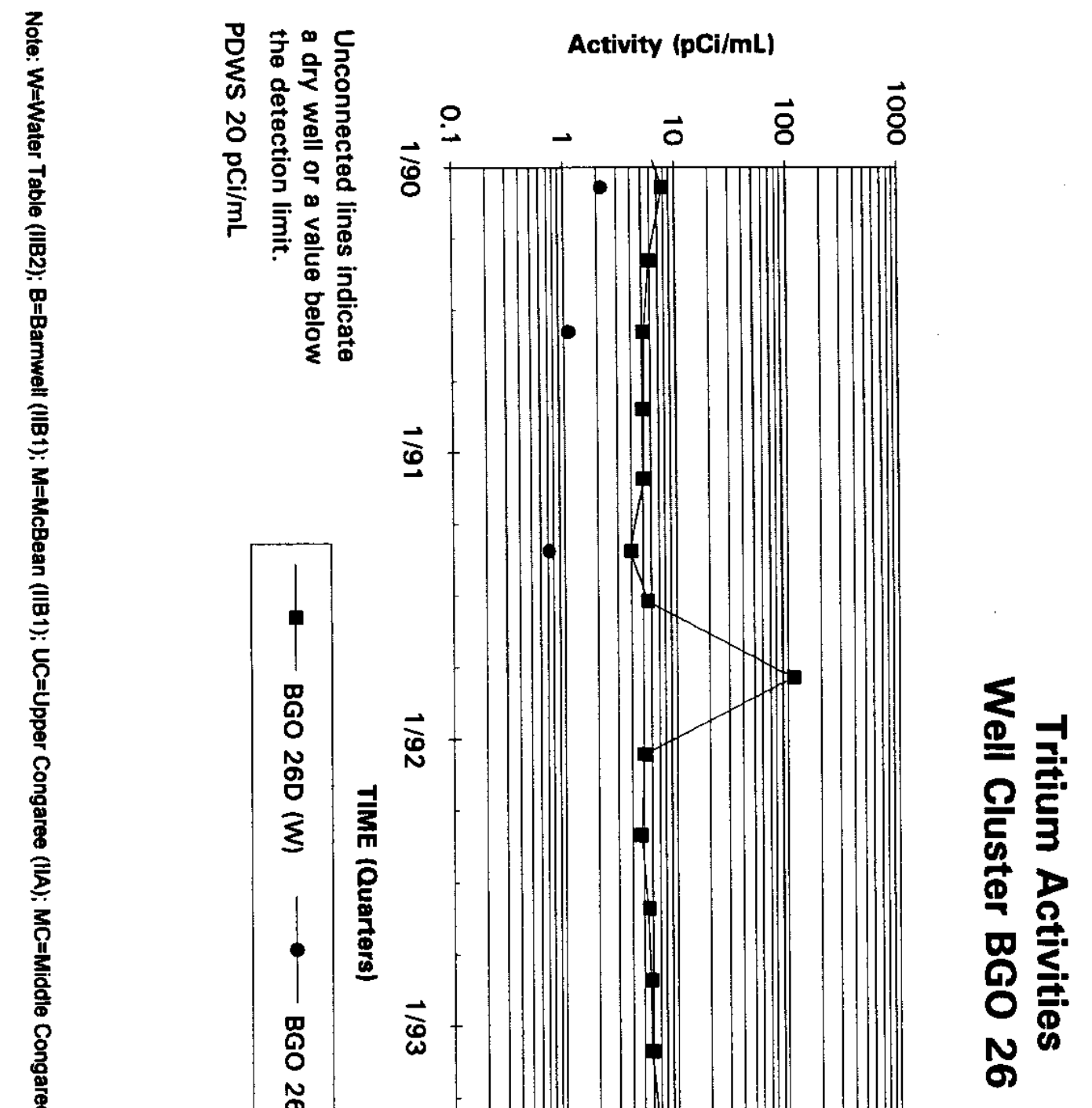

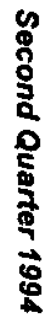

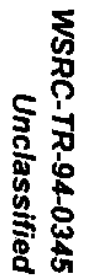




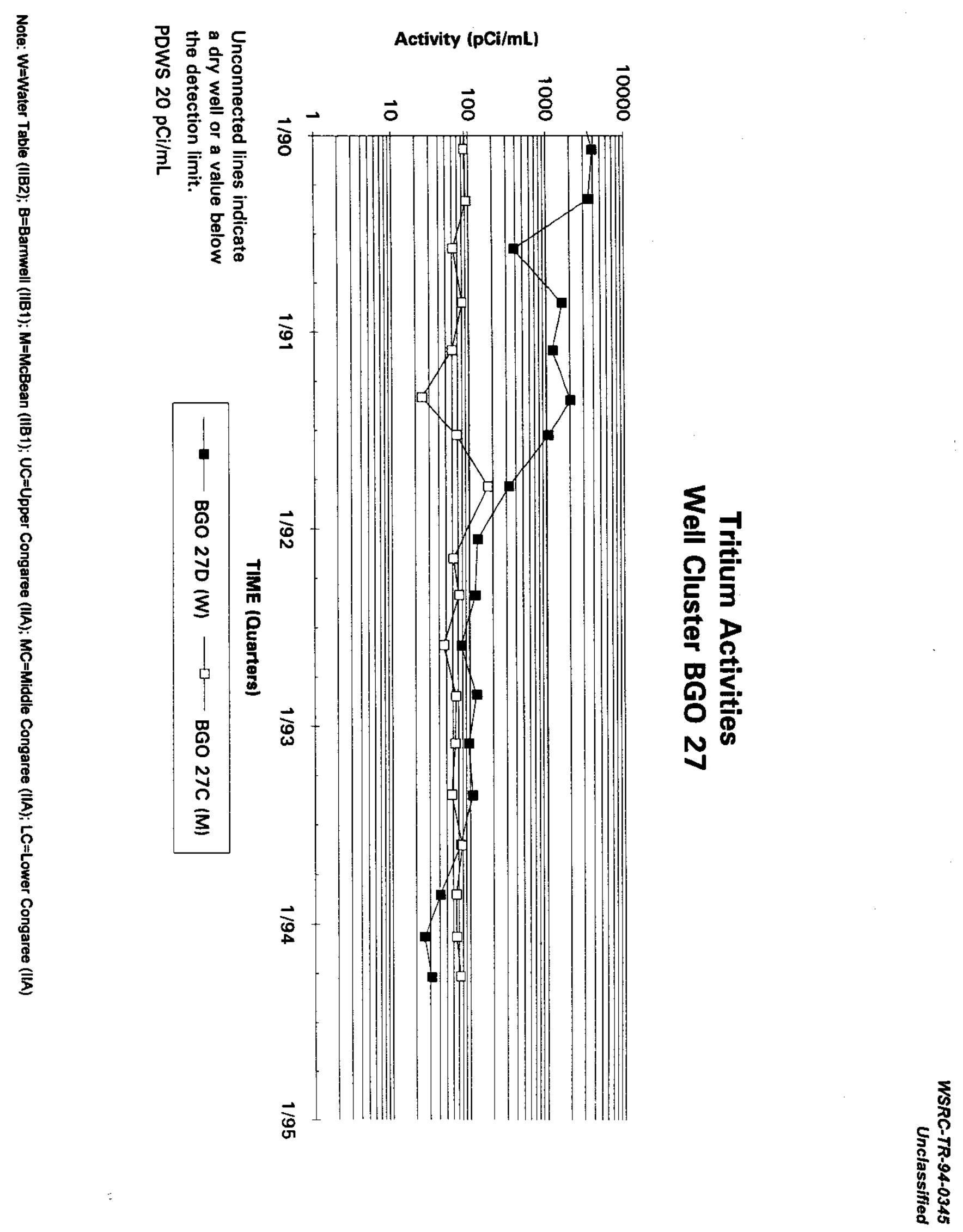


$\sum_{3}^{3}$

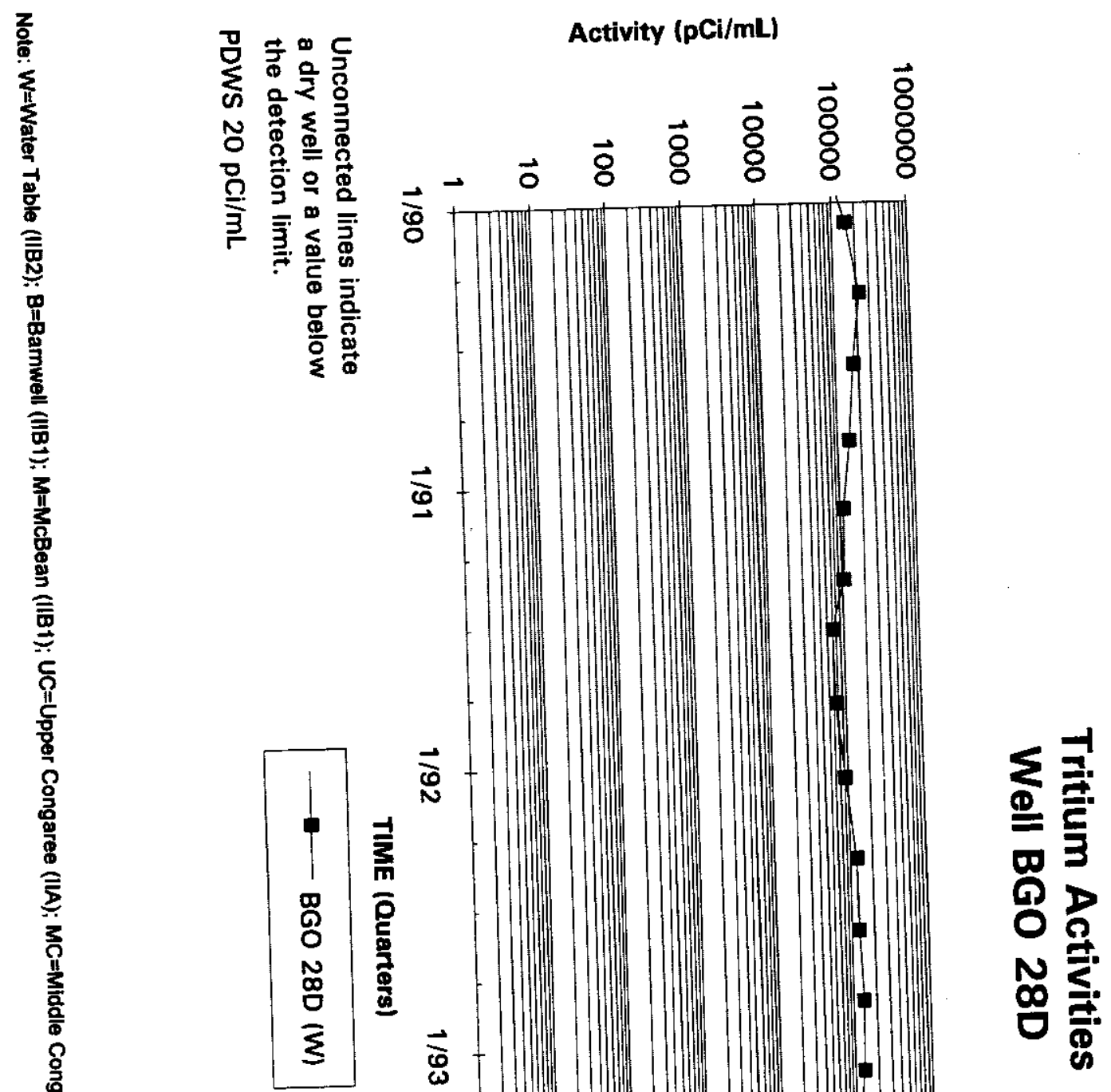

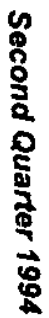

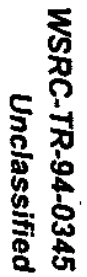




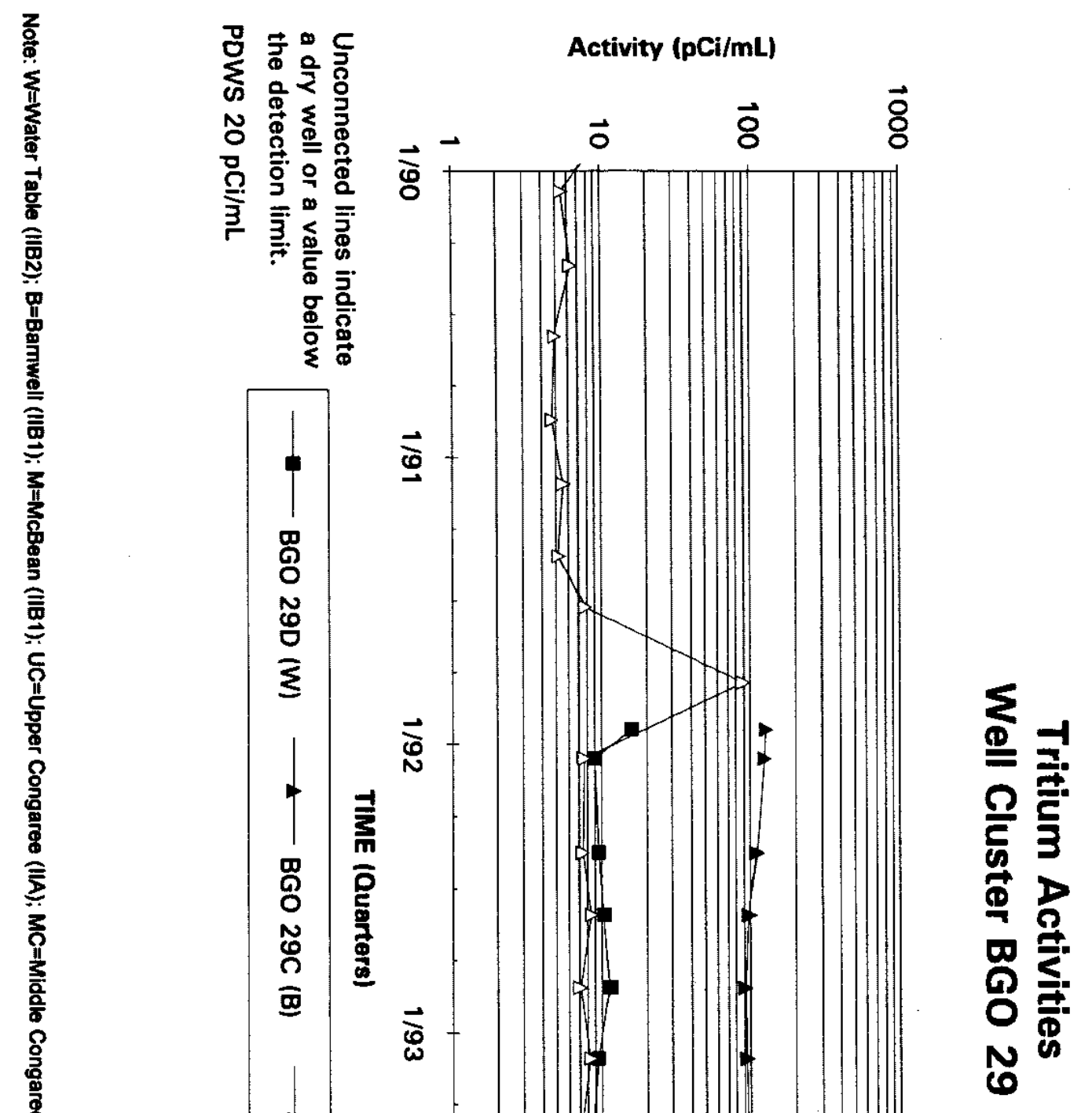

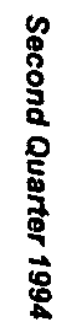


而

뭉

$\sum_{\text {in }}^{\text {in }}$

兽

总

界

羃

言

진

胥

등

ᄋ

흘

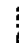

Iㅡㄴ

8

용

产

일

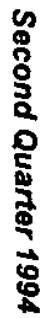

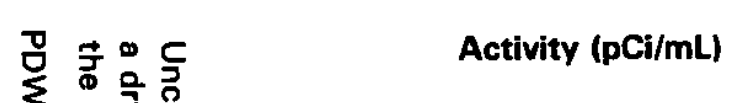

응 응

듕

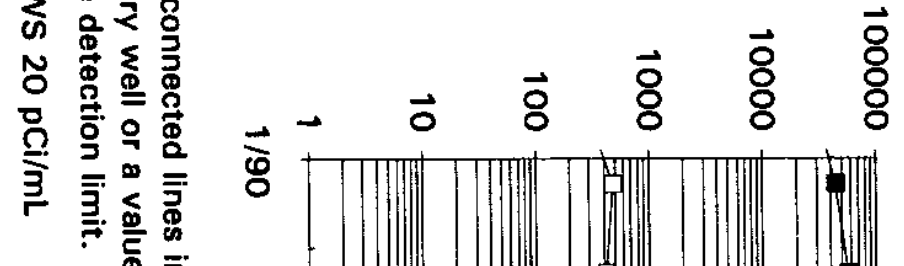

总

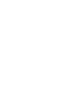

(

ज़

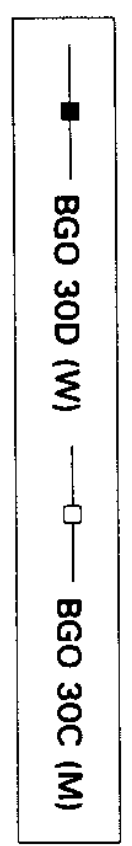

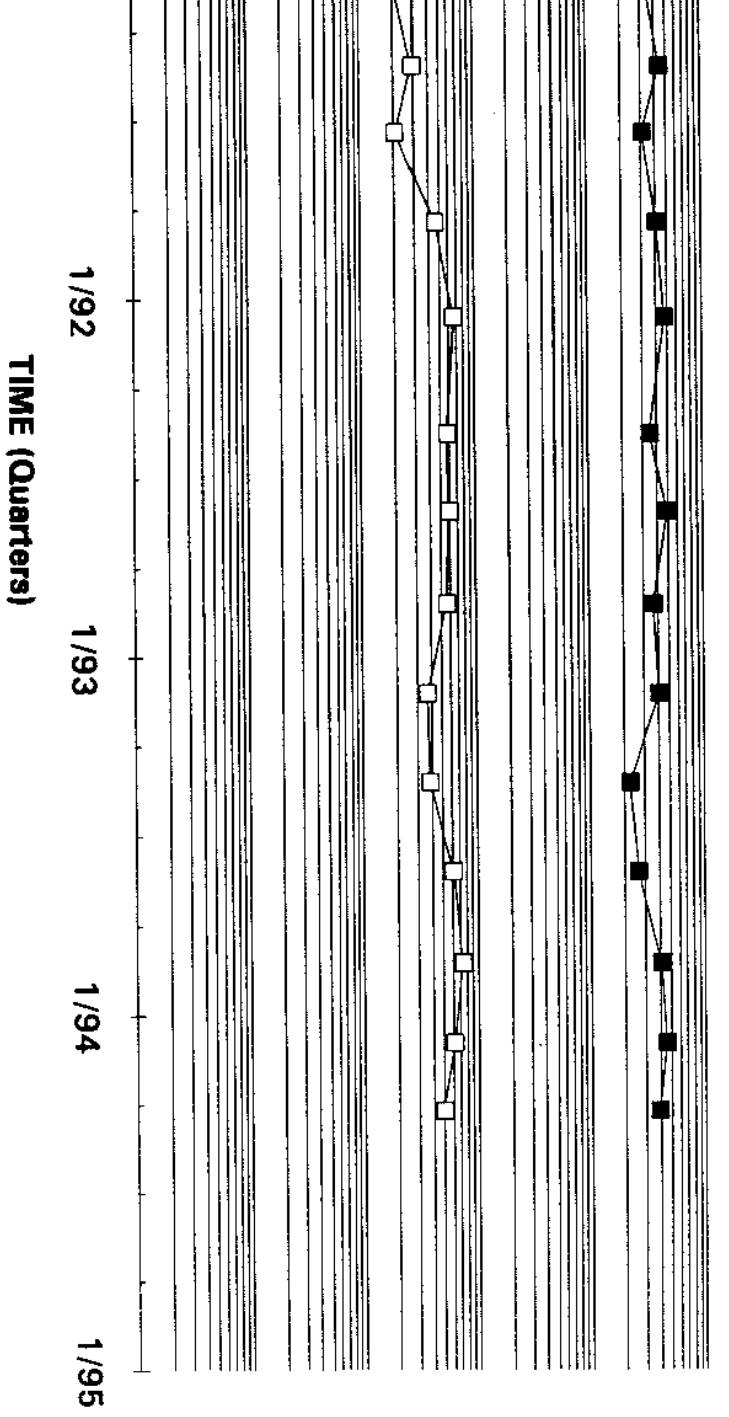

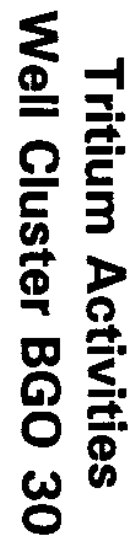

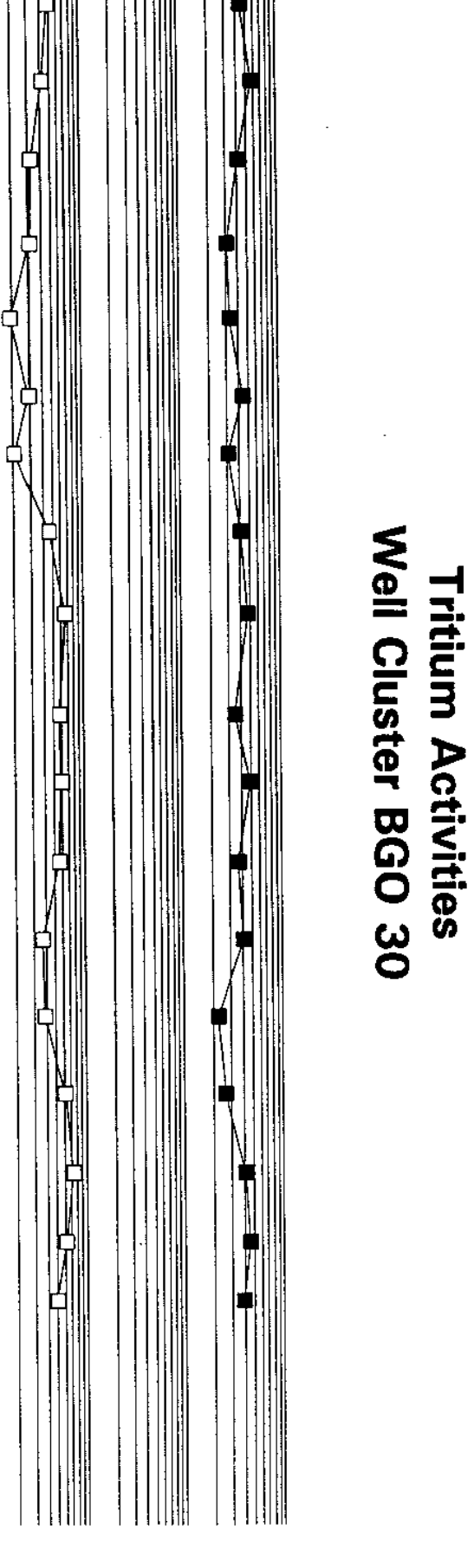

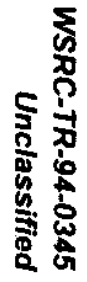


羿

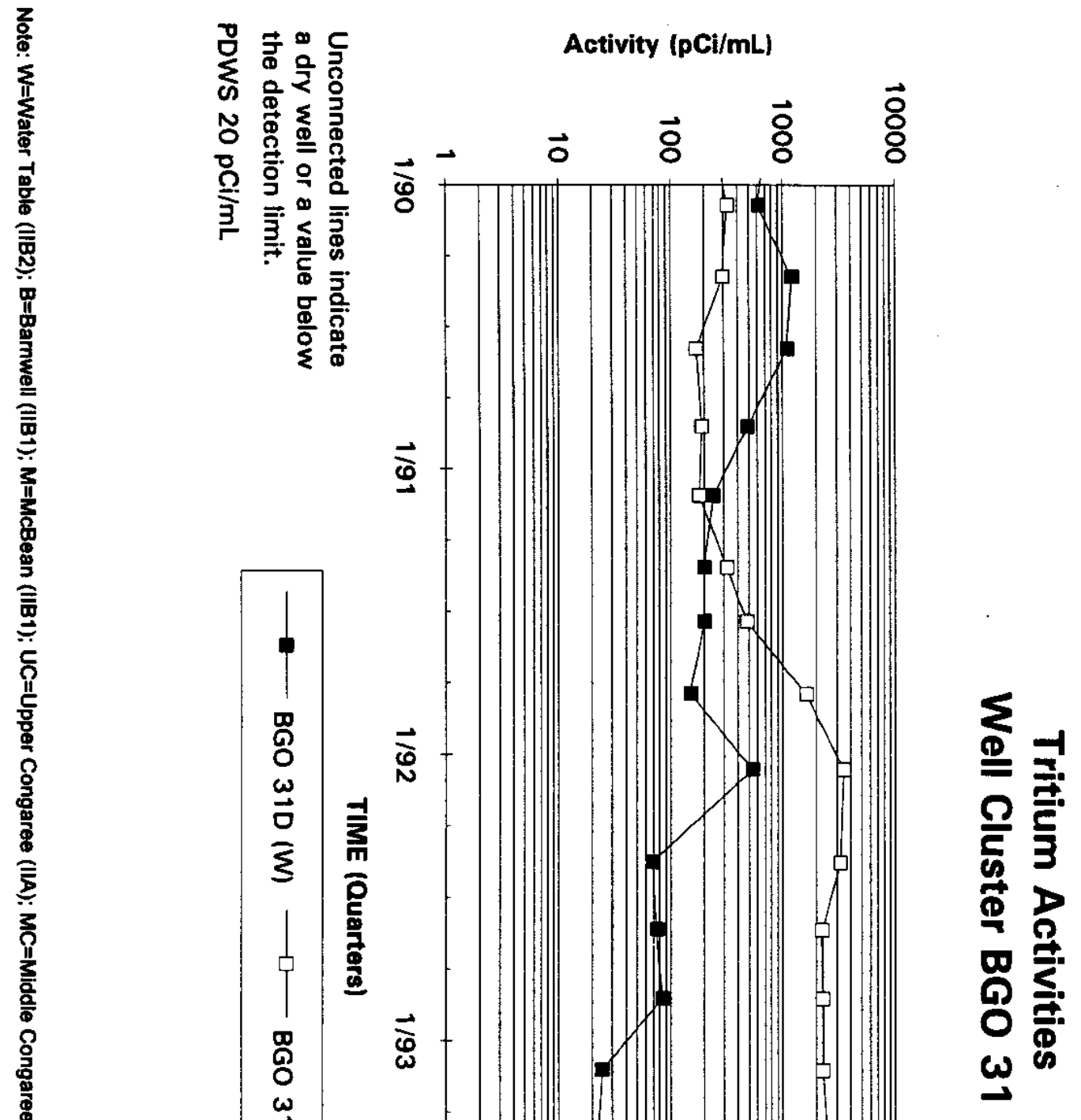

\begin{tabular}{l}
6 \\
8 \\
8 \\
0 \\
0 \\
5 \\
0 \\
0 \\
\hline \\
\hline
\end{tabular}

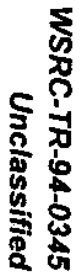


疍

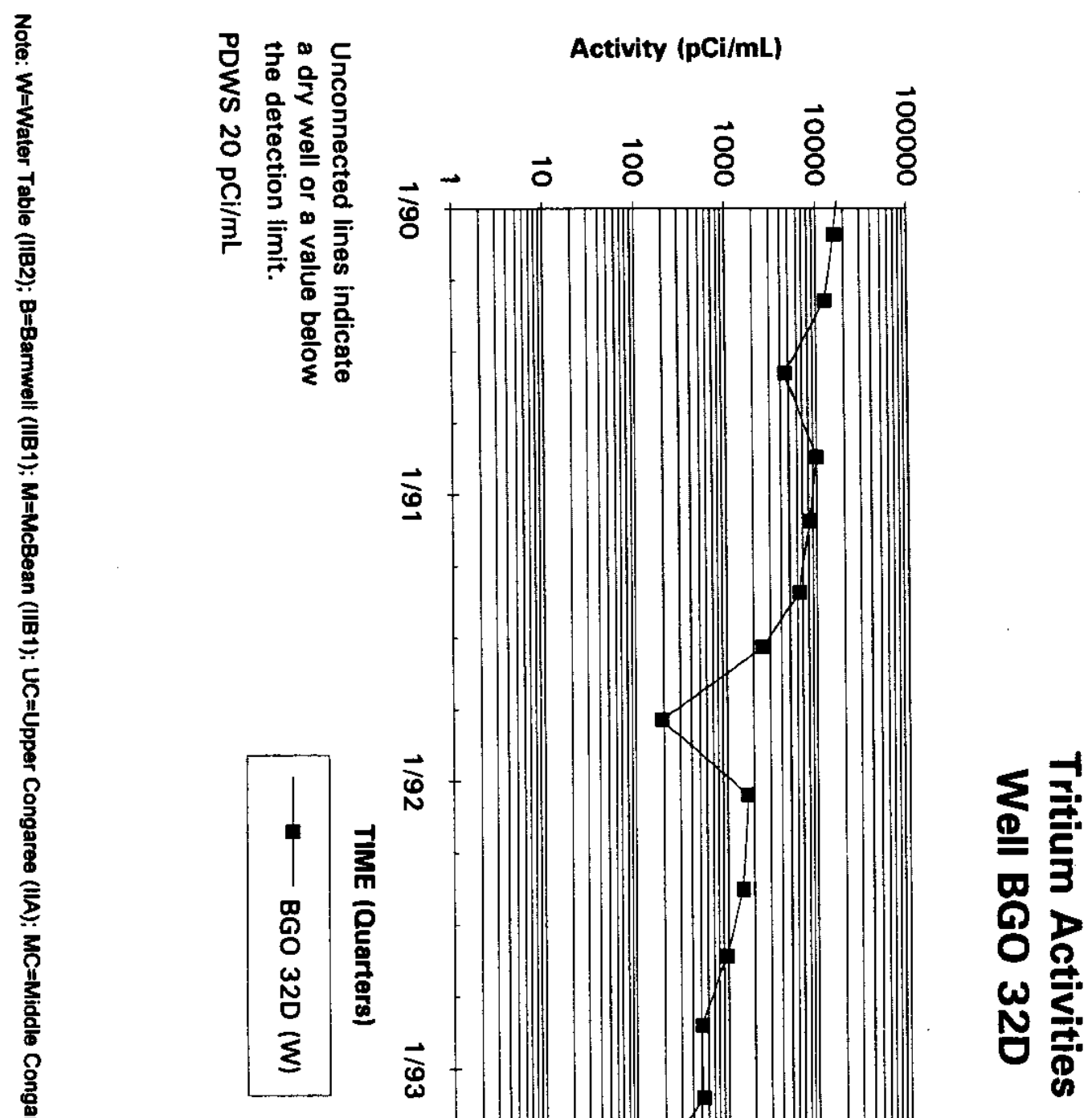

0
8
0
0
0
8
0
0
8
8

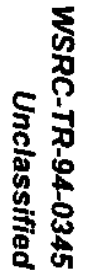


3

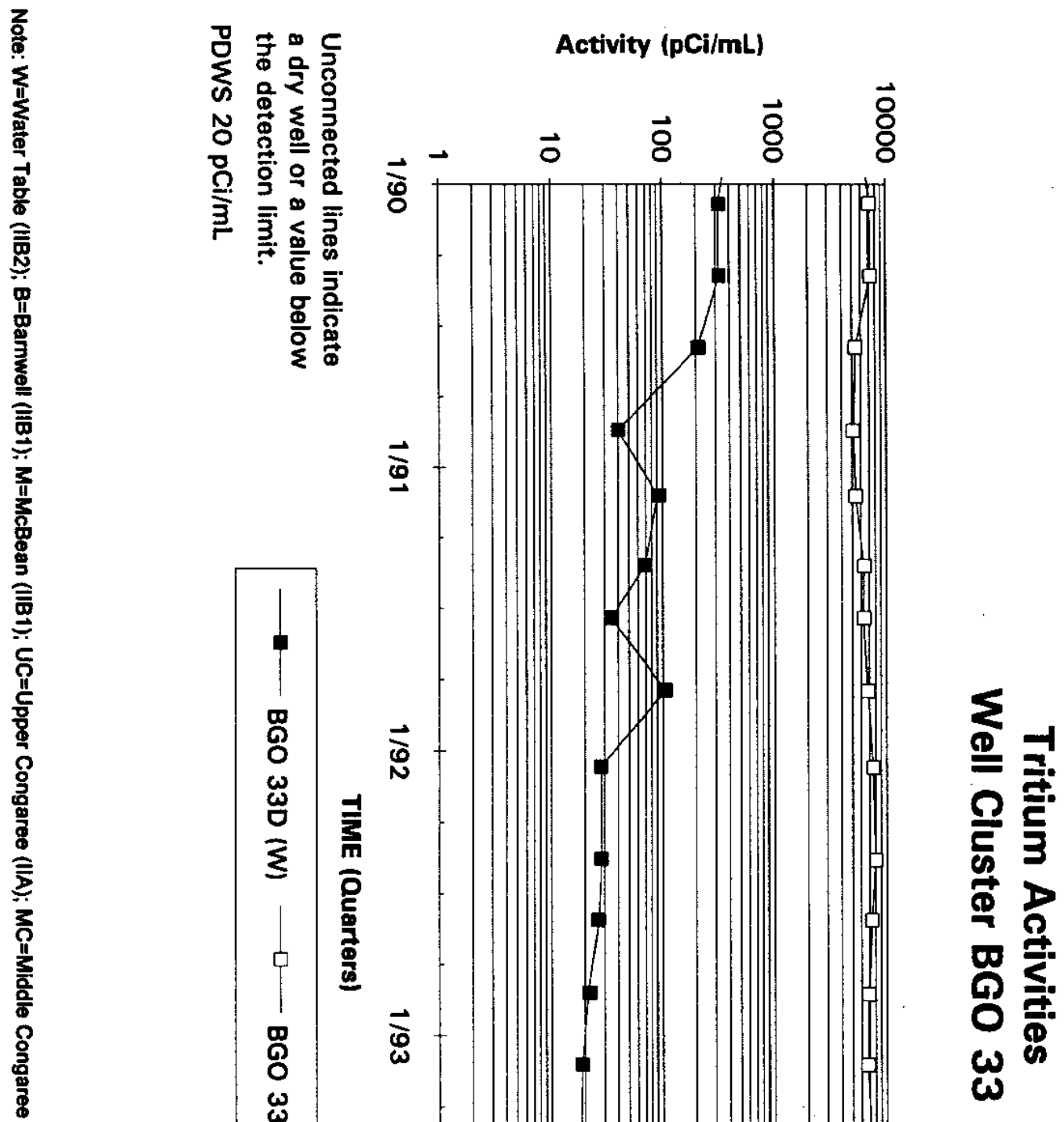


$\frac{5}{7}$

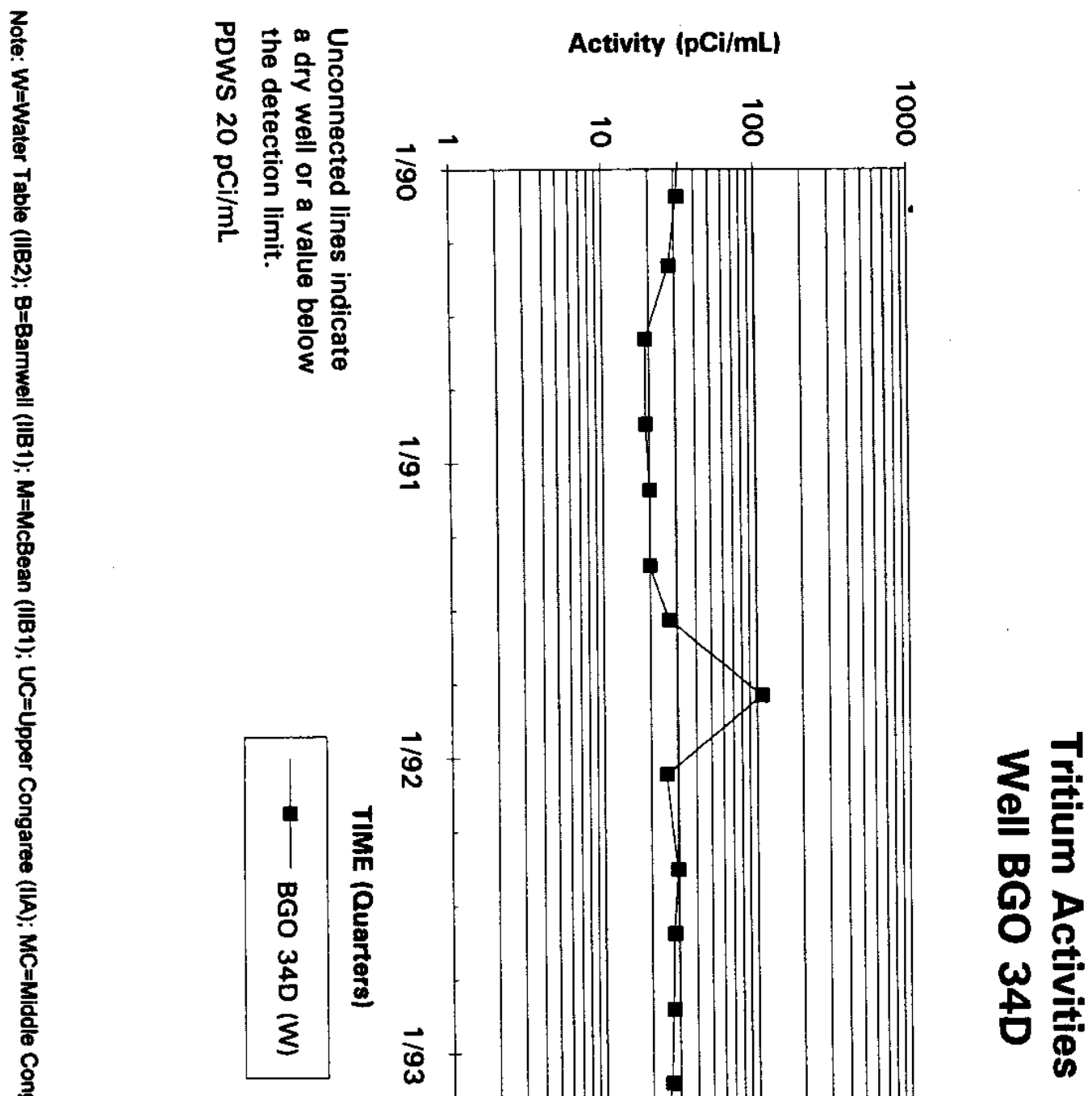

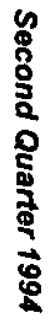

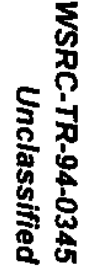


羿

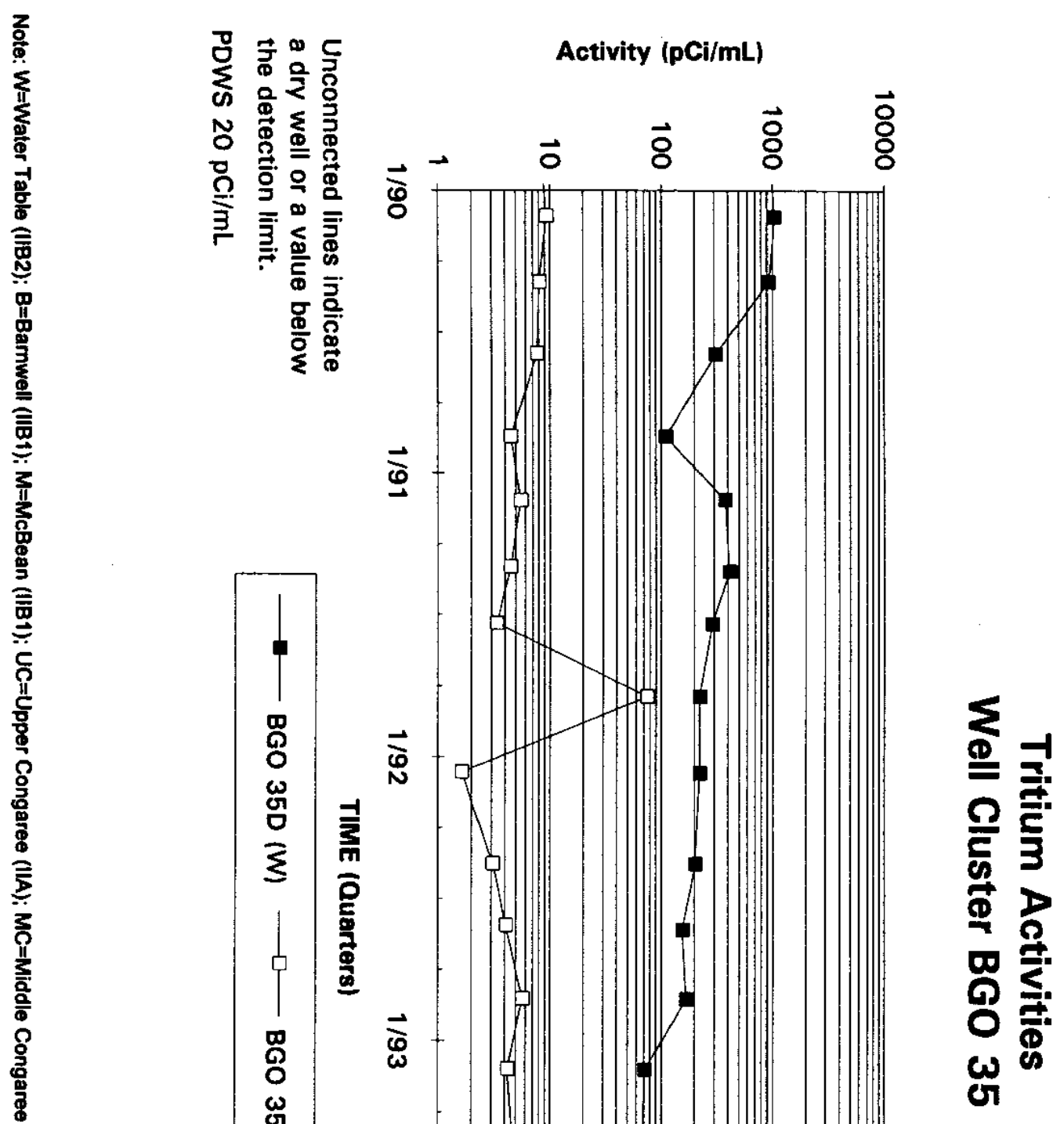

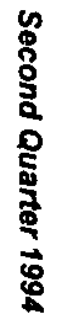


3

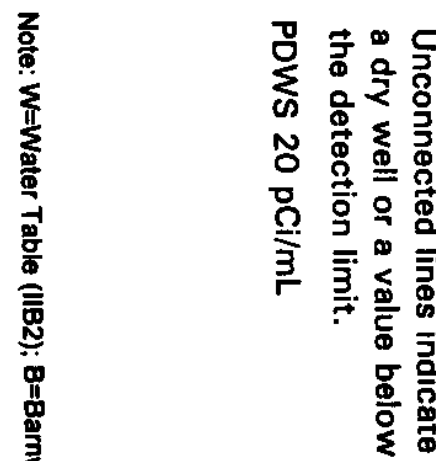

Activity (pCi/mL)
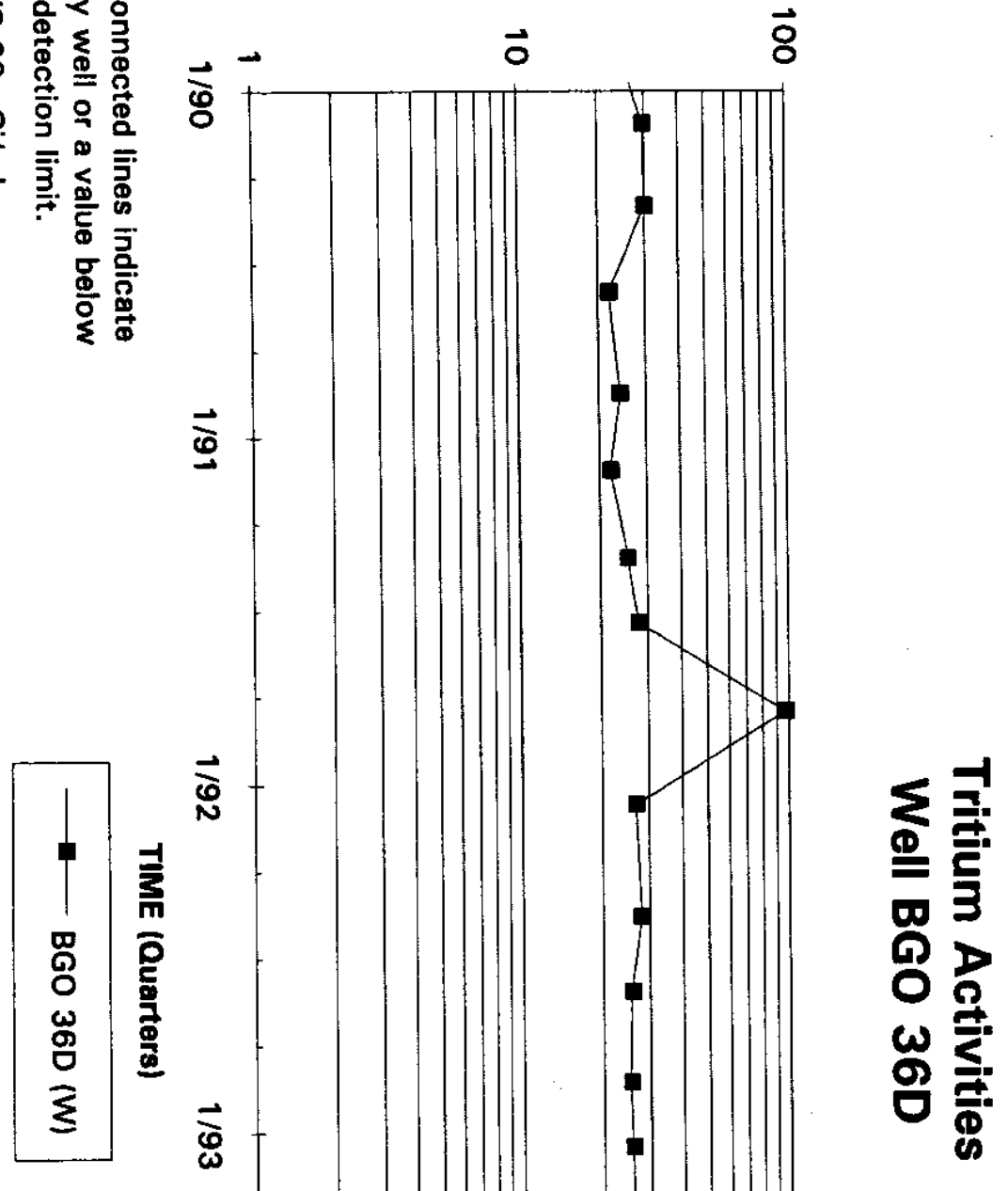

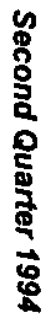

空 
3

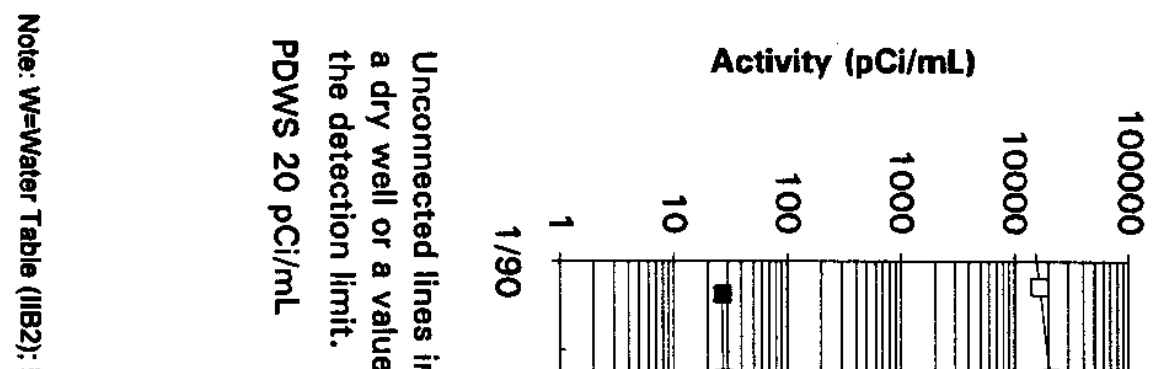

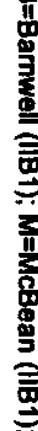

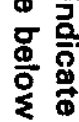

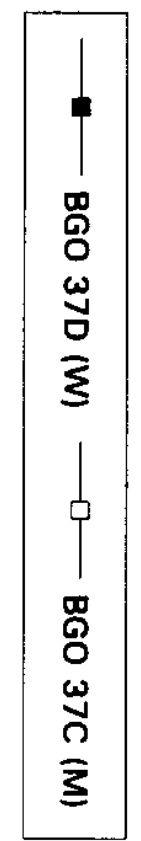

0
8
8
0
0
0
5
9
9
8
8

胥 
灵

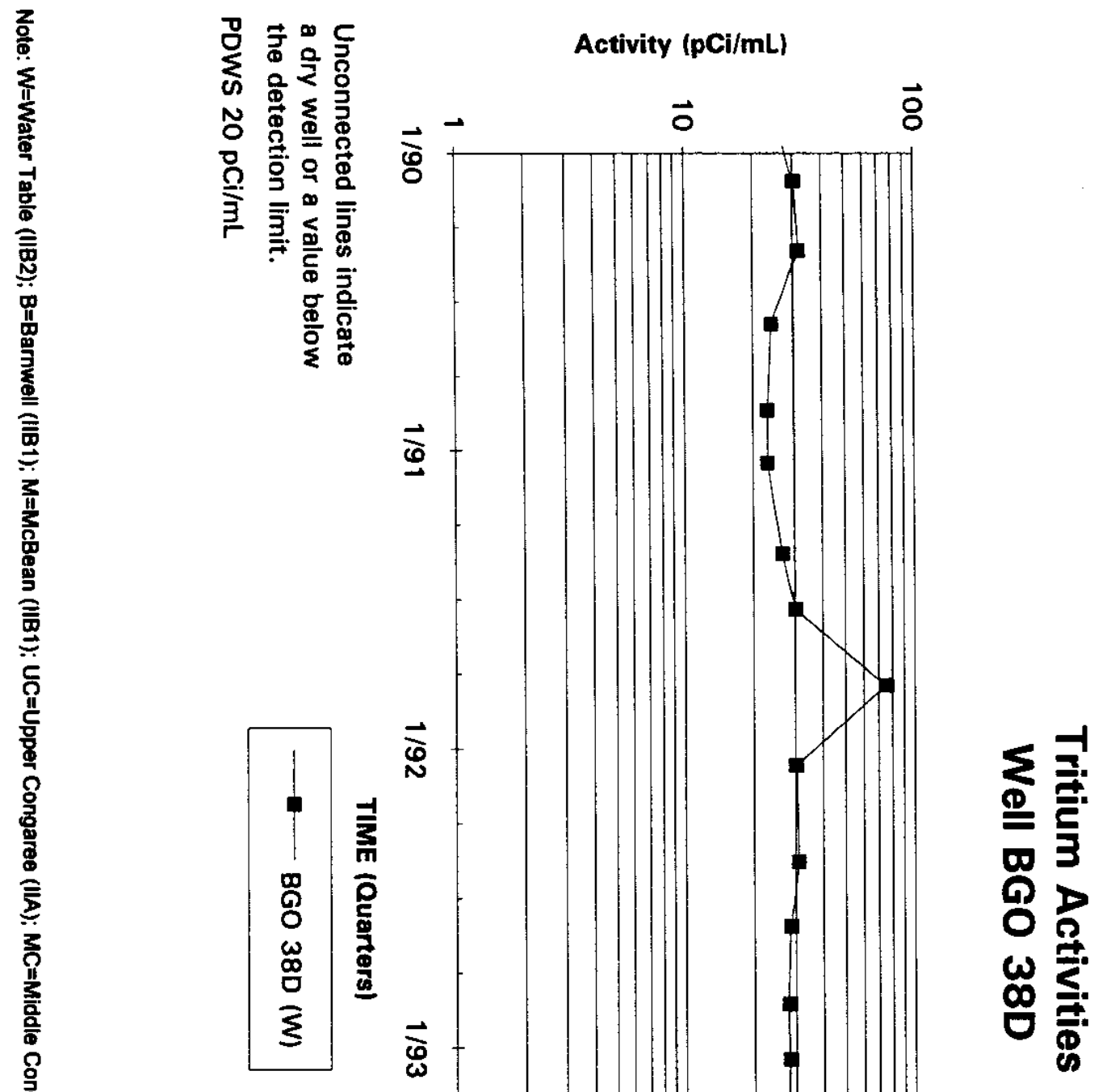

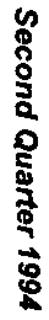

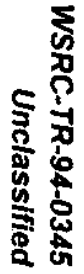




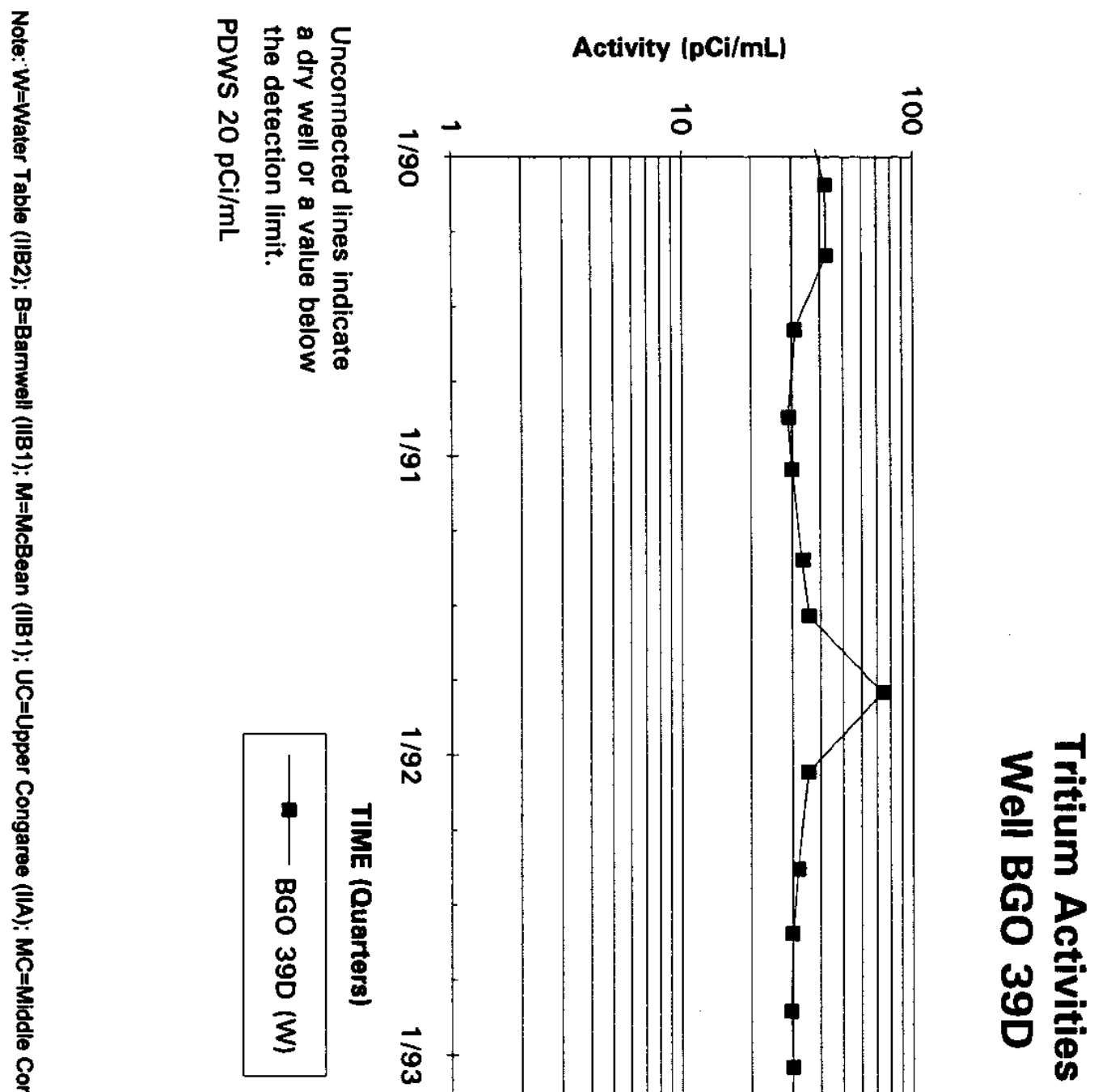




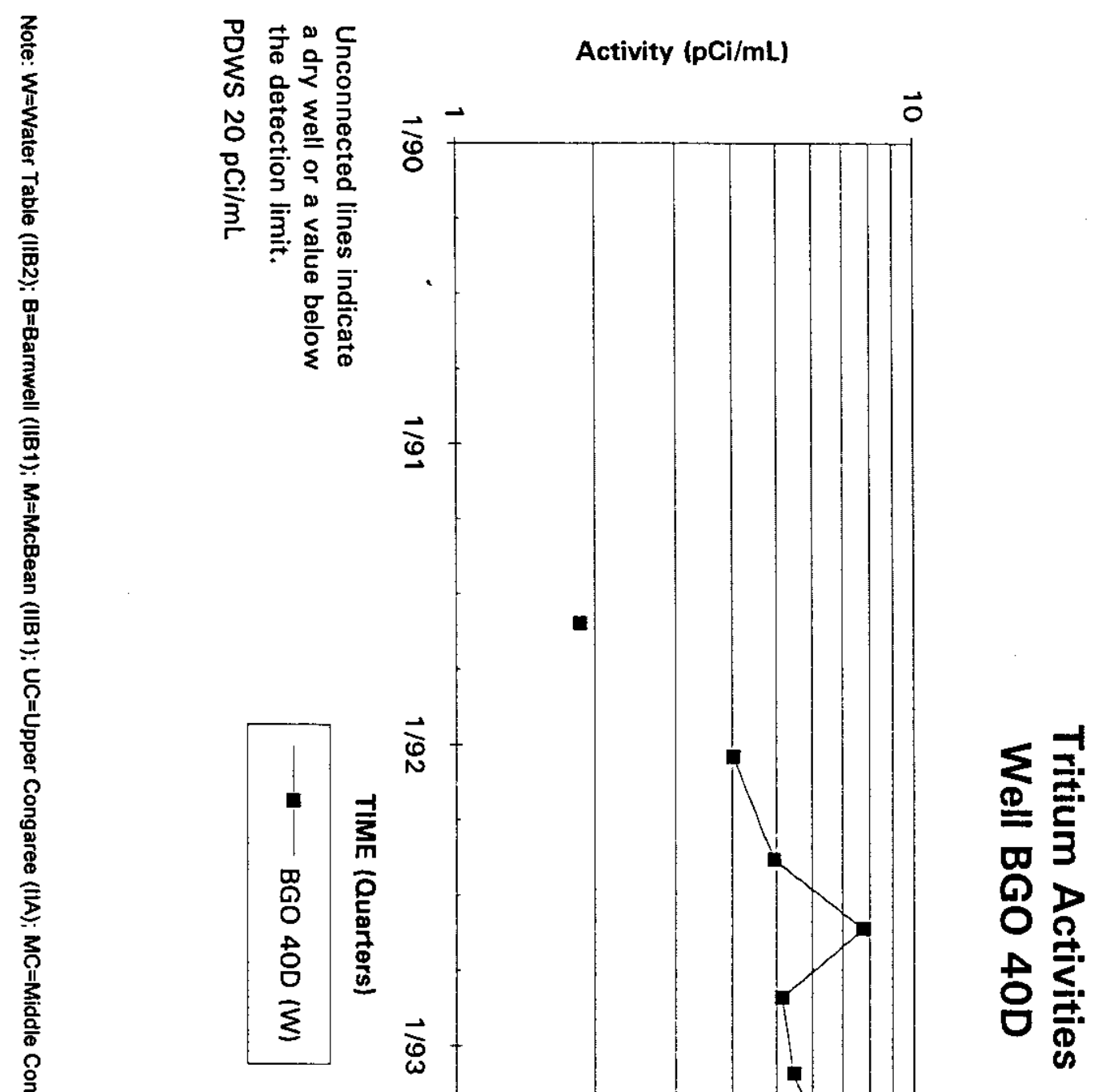


永

궁

\section{.}

.

.

.

苋

II

高

言

욤

음

言

중

흘

$\stackrel{\circ}{\circ}$

哀

产

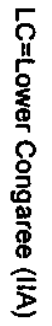

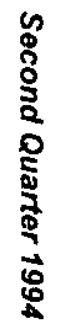

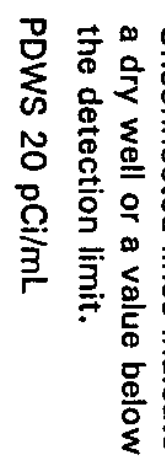

Activity (pCi/mL)

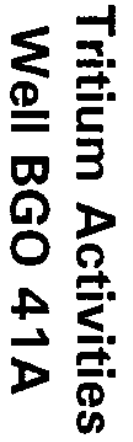


疍

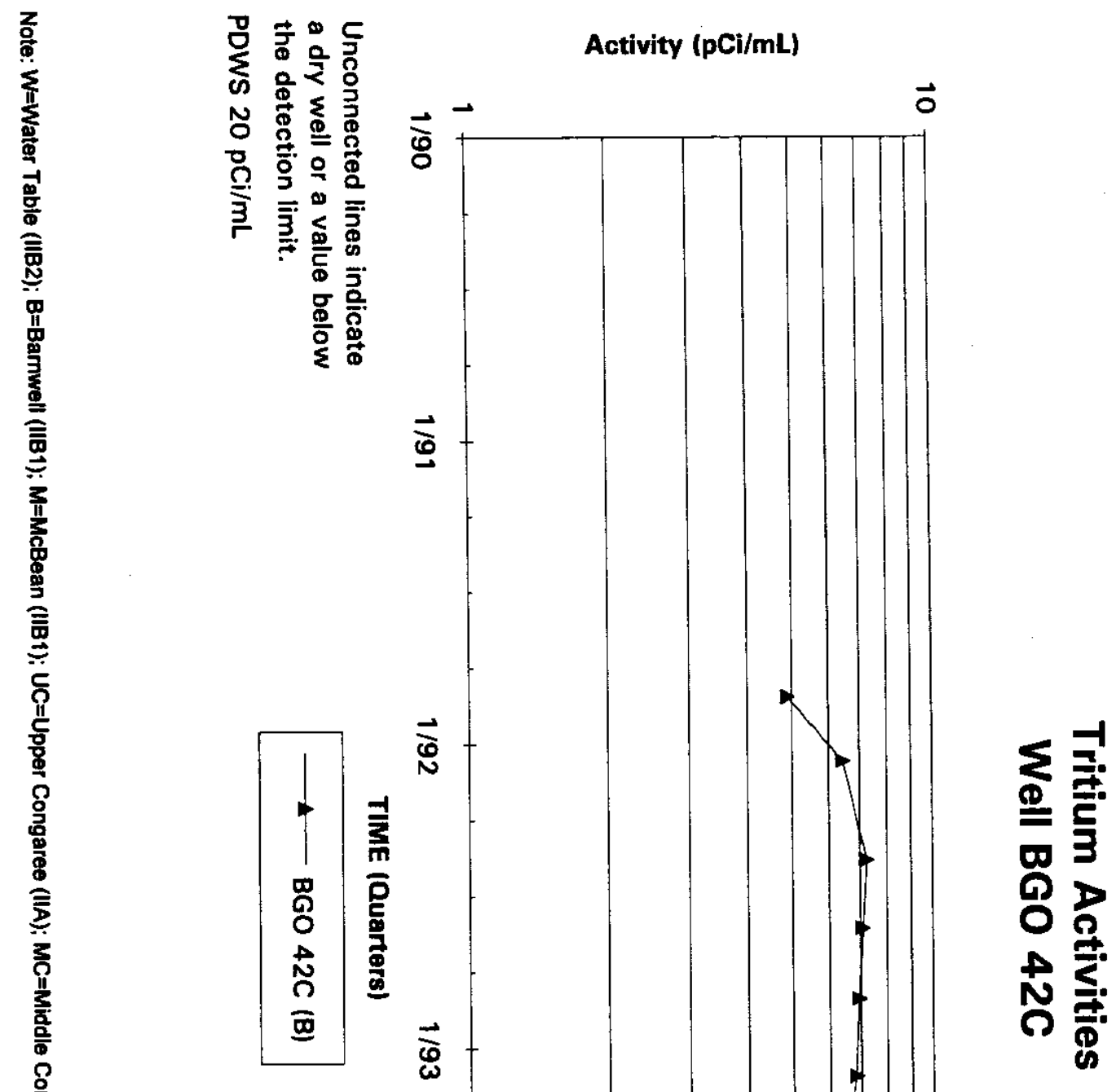

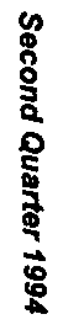

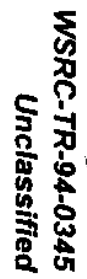


호음

능호옹

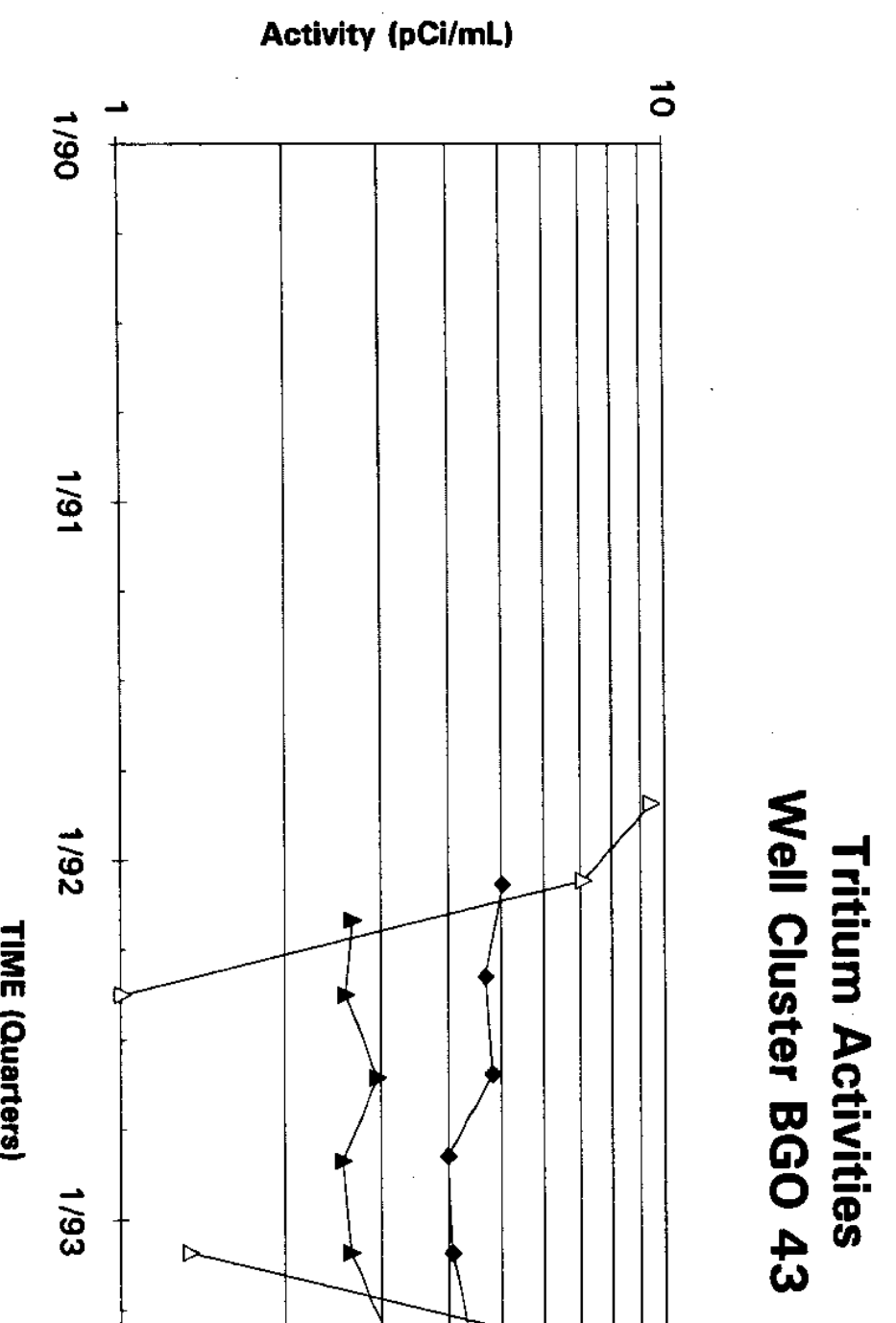

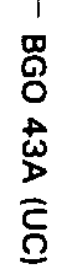

○

罗

ऐ $\vec{\nabla}$

ต 
$\sum_{1}^{3}$

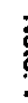

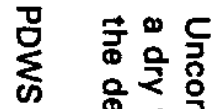

Activity (pCi/mL)

章

䍘

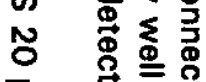

ํㅜ 홍용

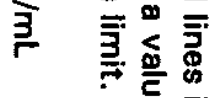

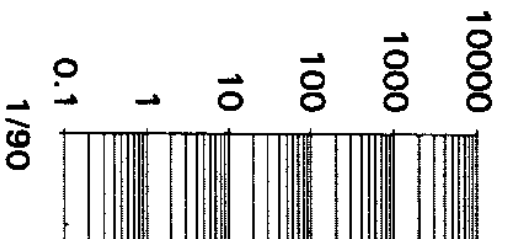

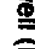

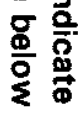

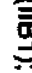

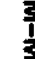

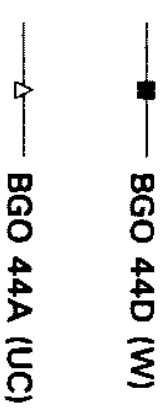

$\uparrow+$

등 兽

촌

言

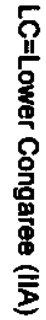

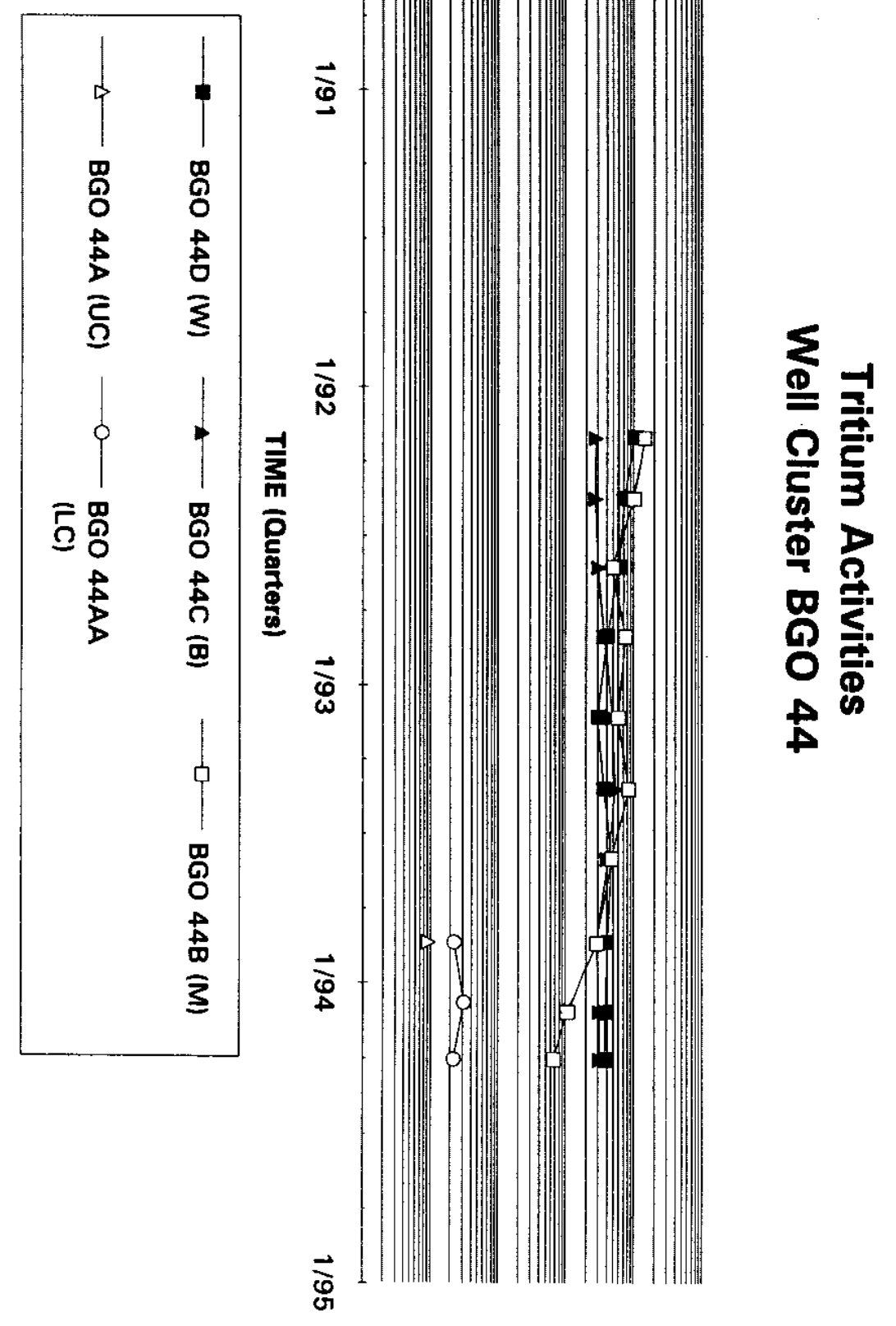

0
8
8
0
0
0
0
7
9
0
0

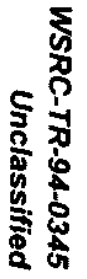


3

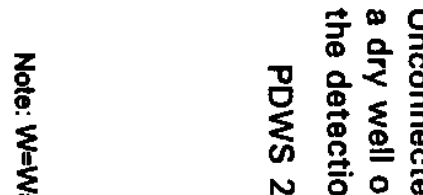

年

喜 旁

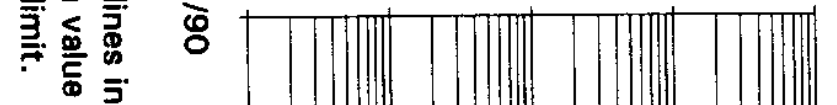

言

要

娄

罡

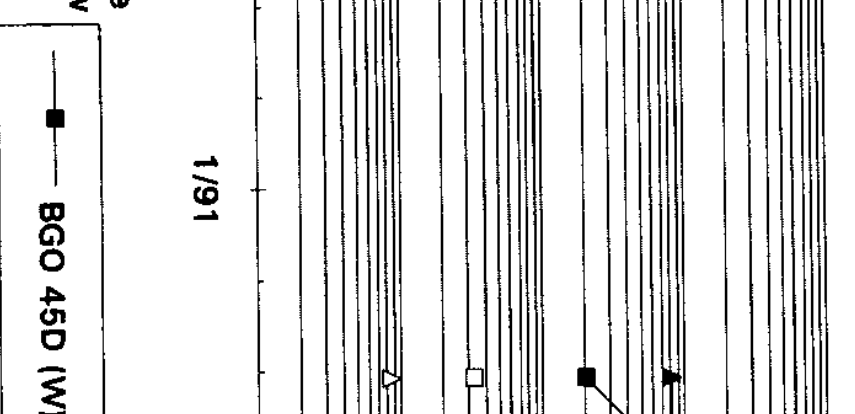

产

๙

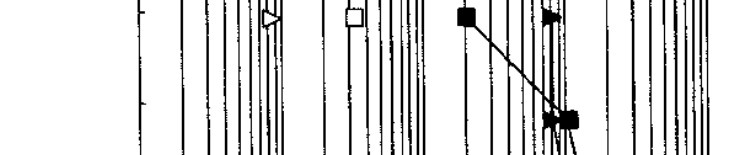

옹 
章

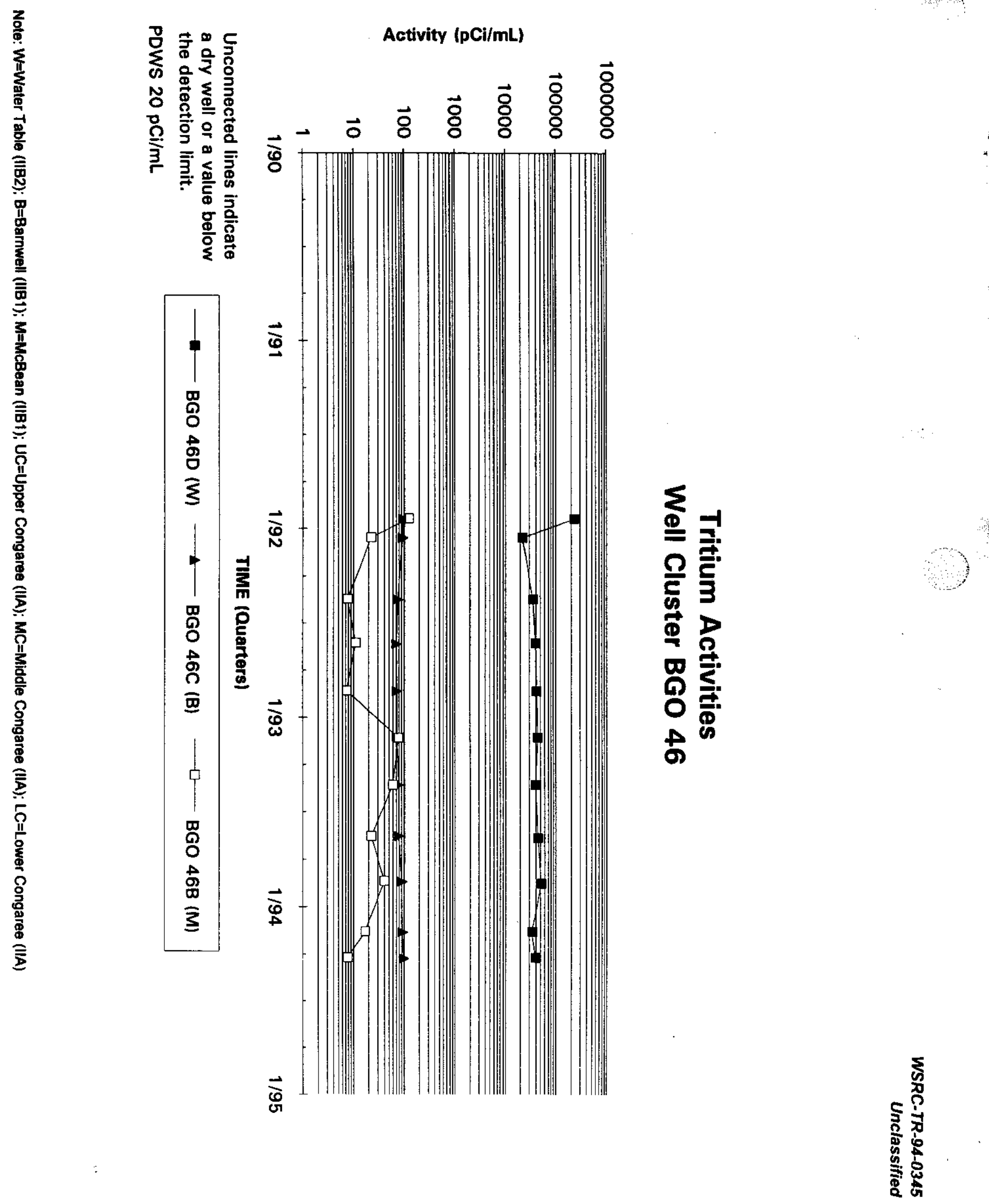


3

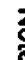

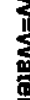

흠

兽

II

要

言

II

营

言

ธ

동

옹

.

흫

옹

$\stackrel{\$}{\overline{\bar{\Sigma}}}$

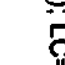

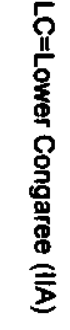

0
8
8
0
0
0
5
3
9
0
0
0

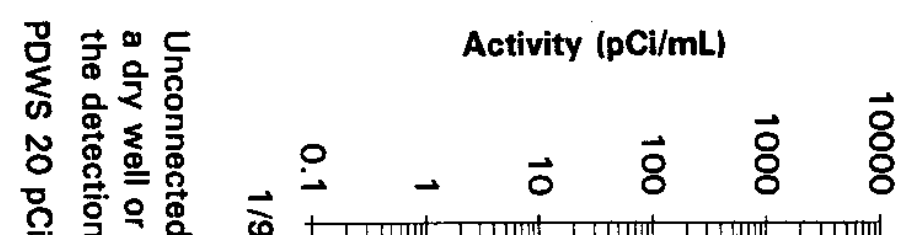

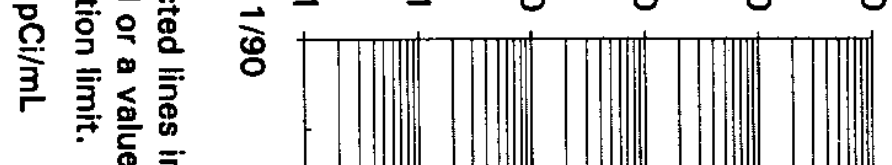

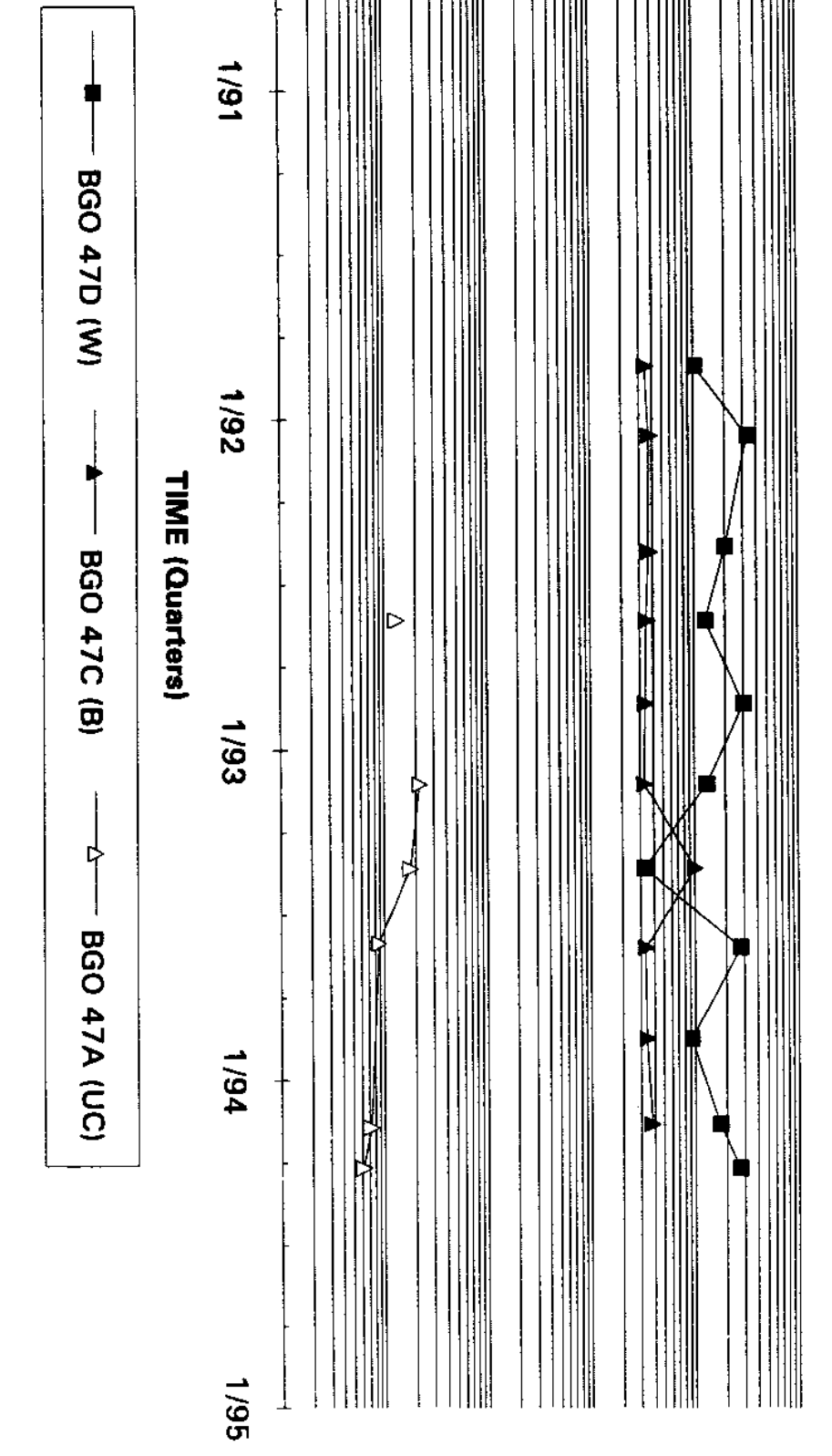

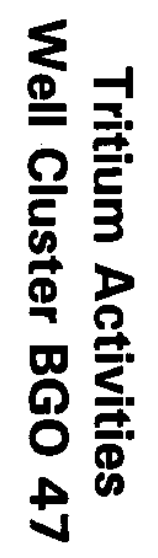

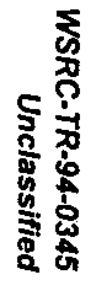


章

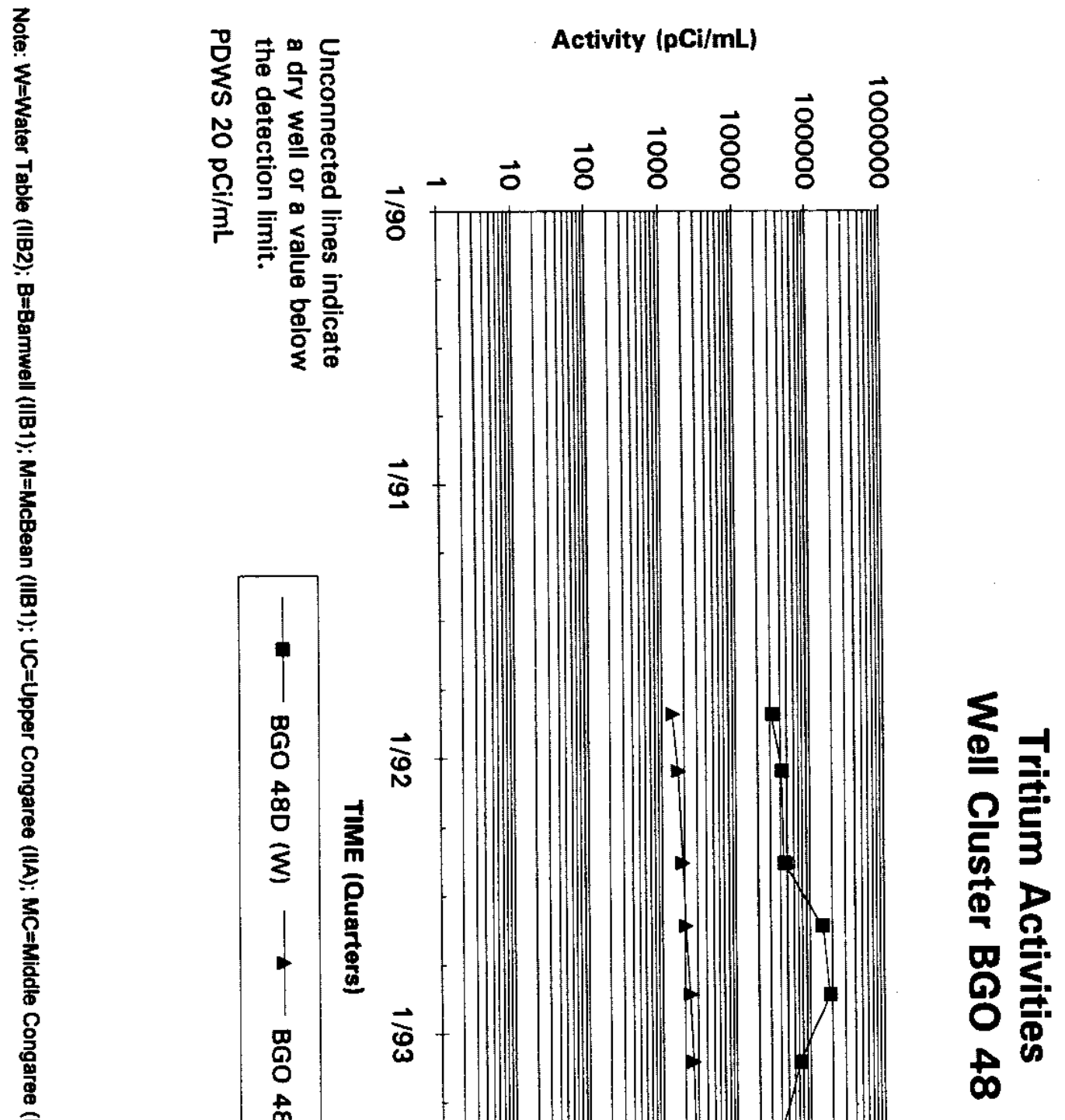

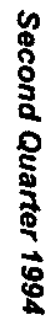

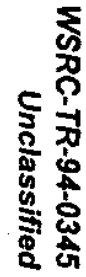


3

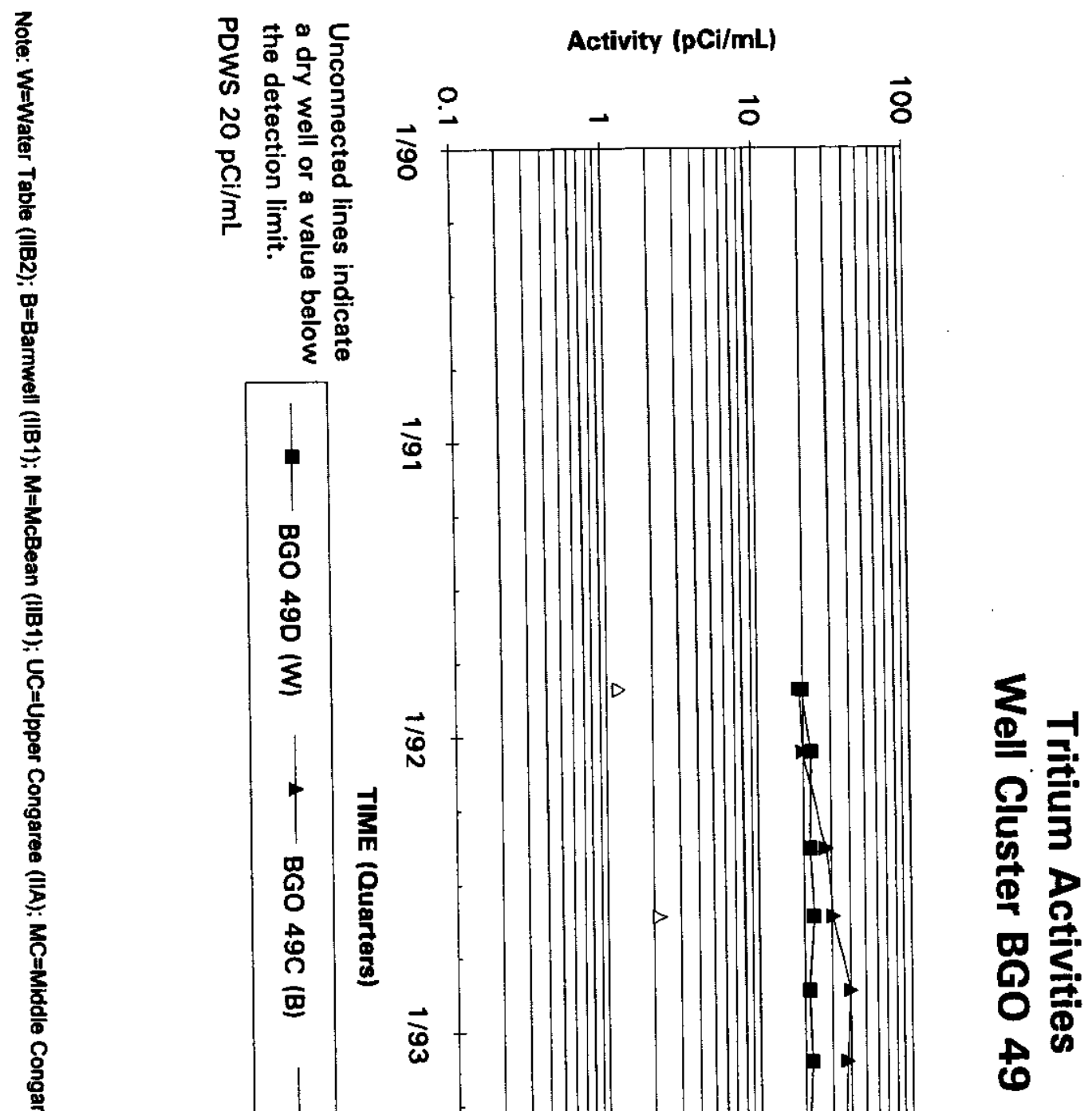

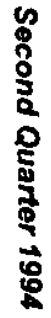

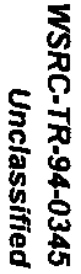


I

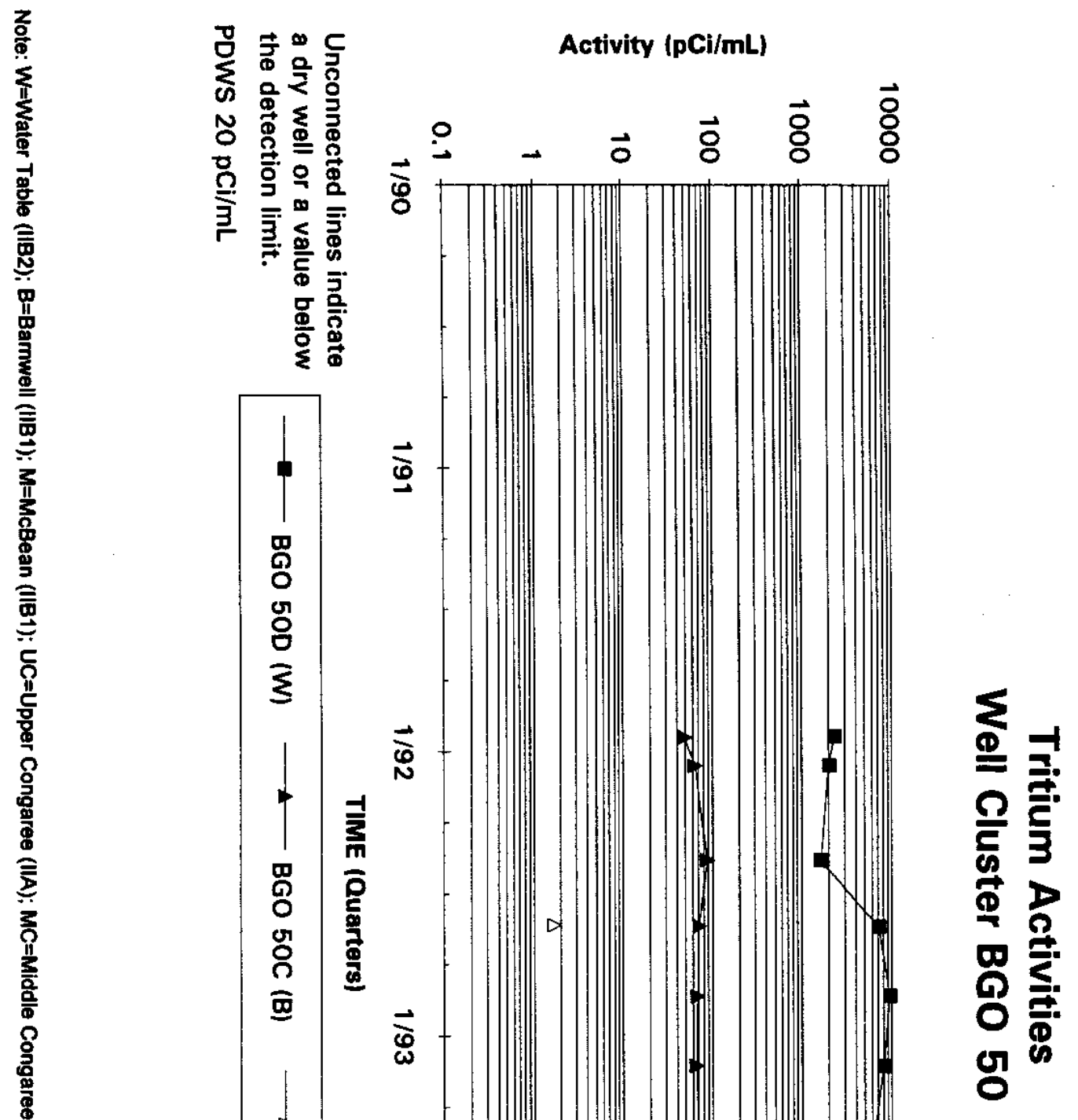

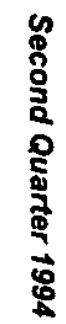

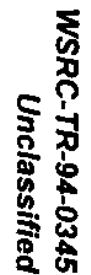


3

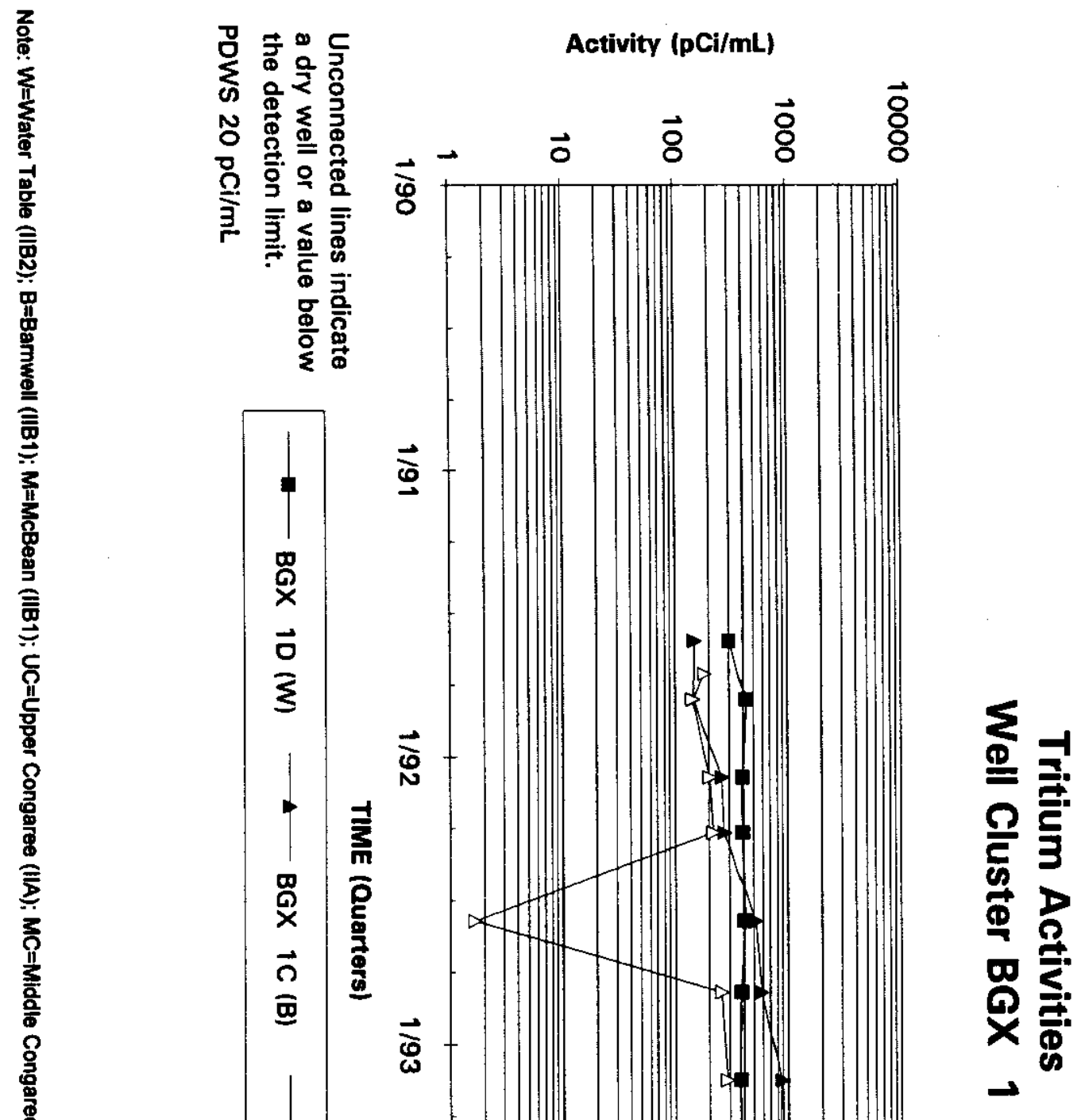


3

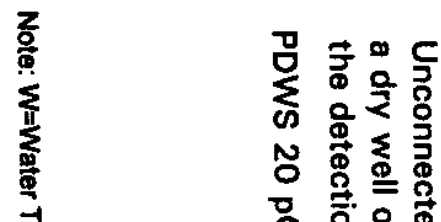

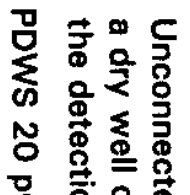

奂

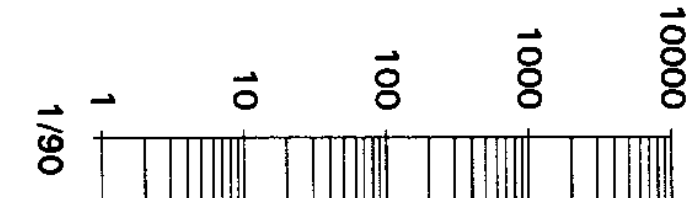

言

栗

兽 更 品 훙

言

il

$\frac{11}{8}$

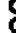

등

8

\%

를

z

응

8

产

产

iั

常

总
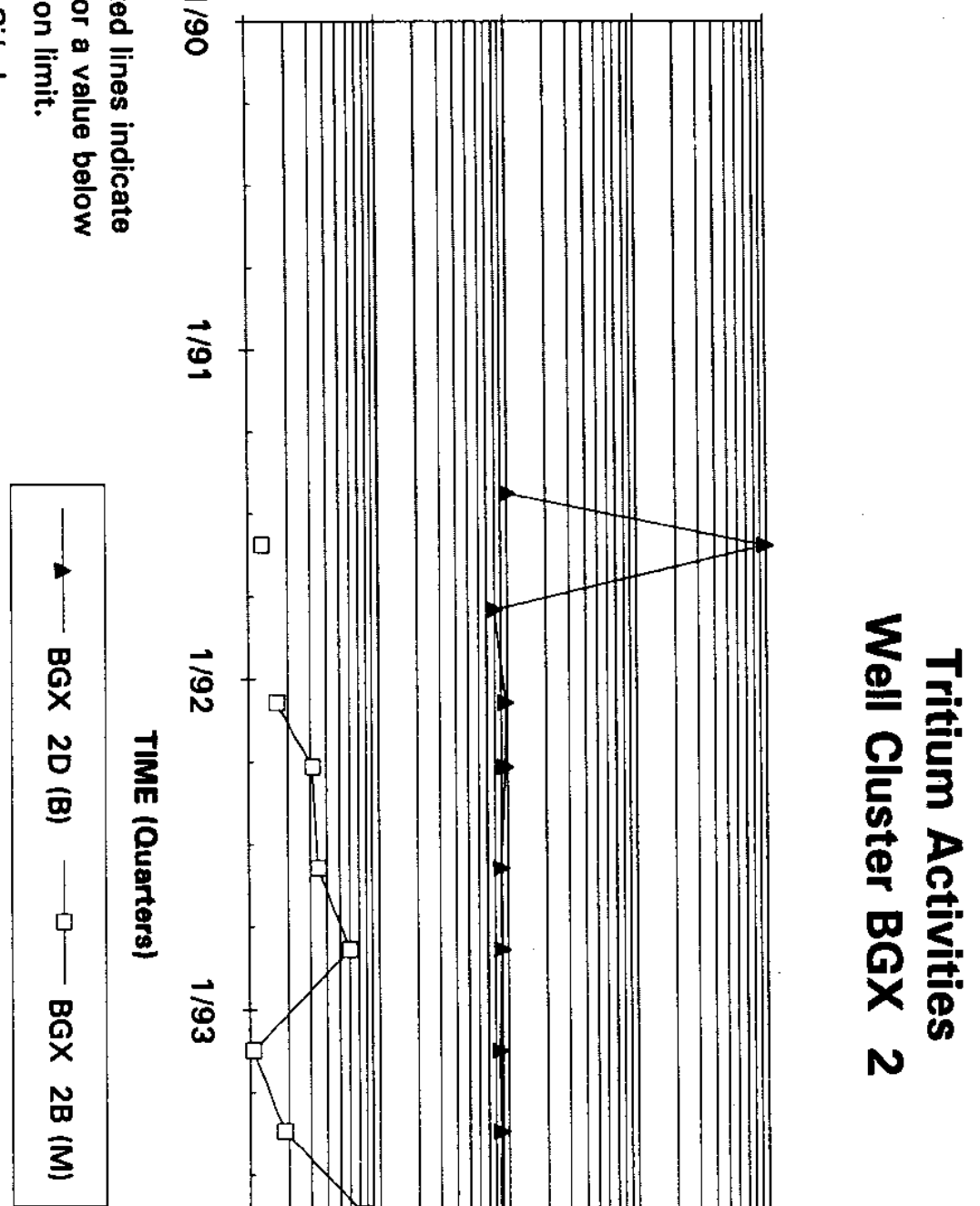
I

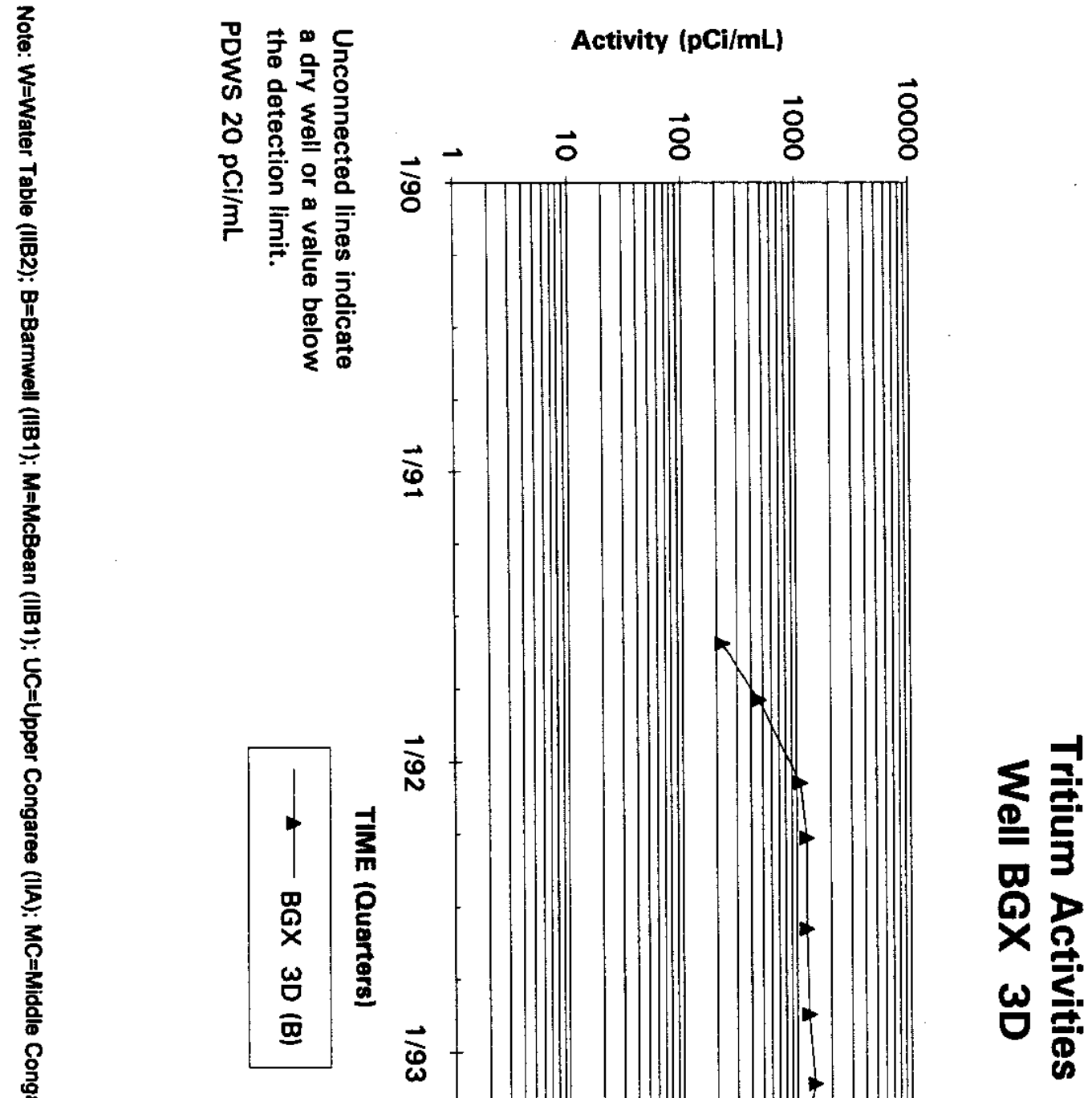

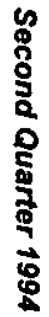

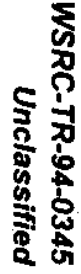


章

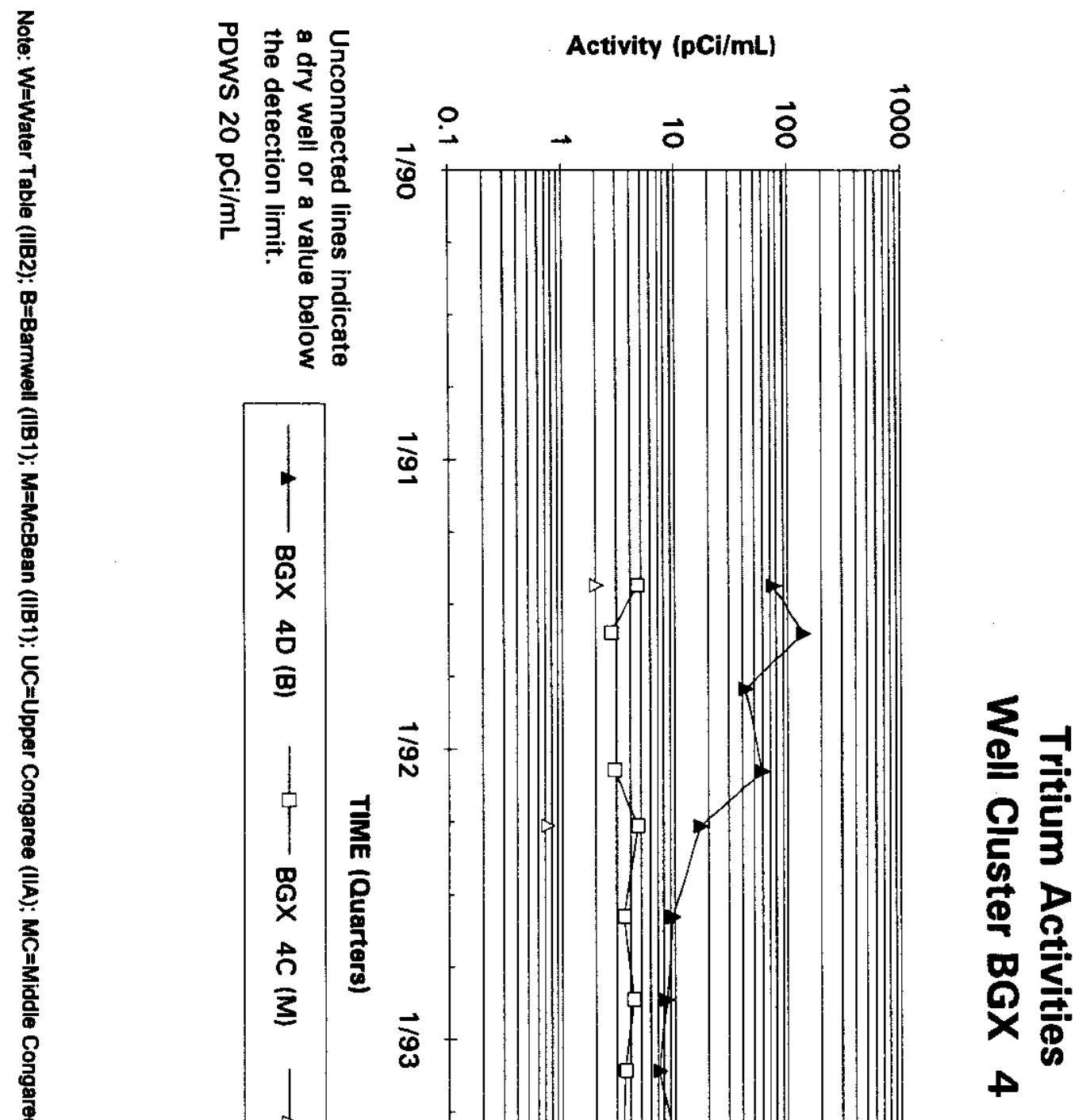

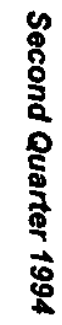

勇 
禎

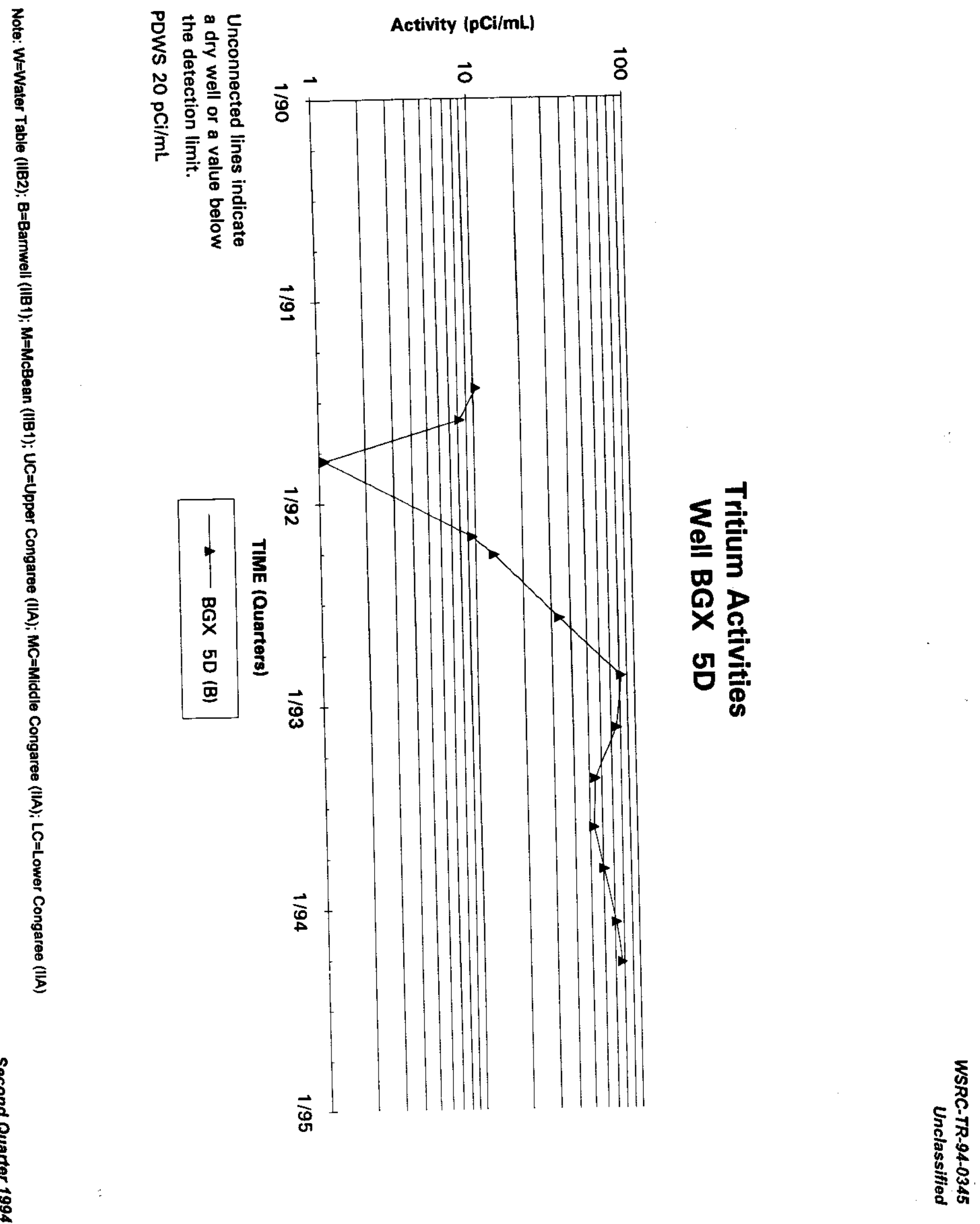


资

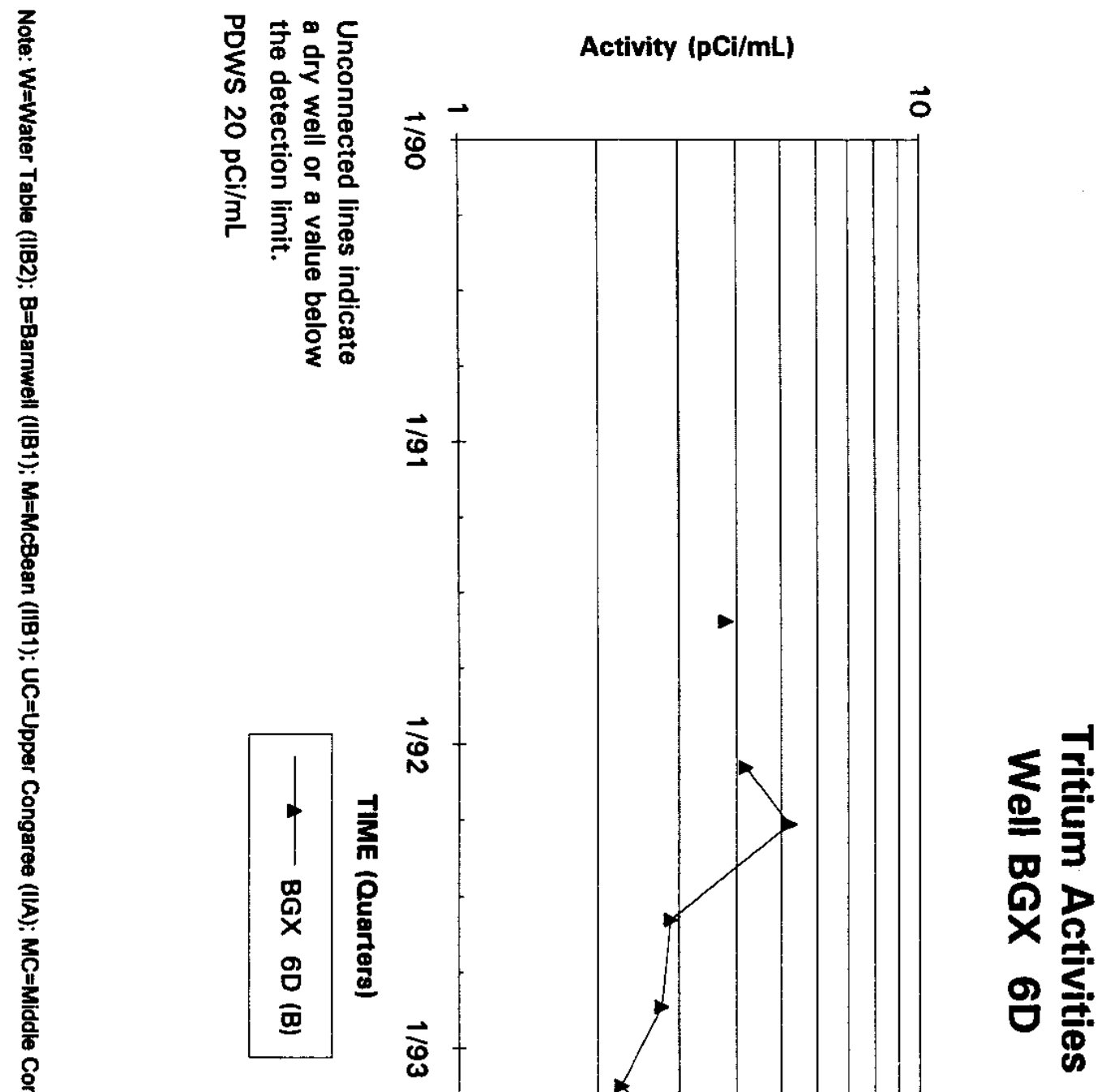

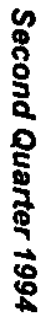

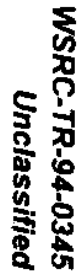


ti

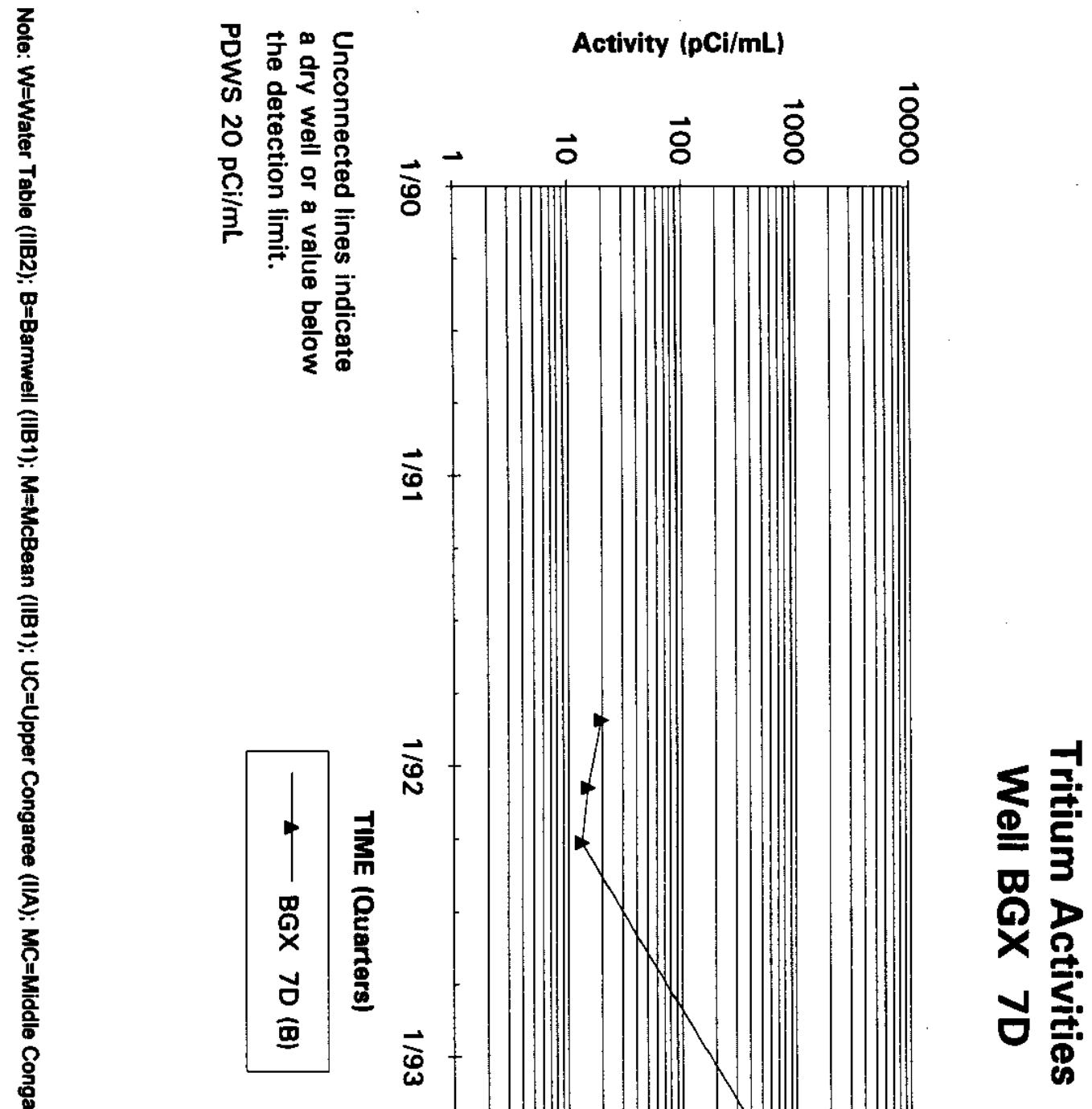

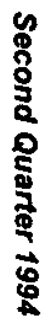

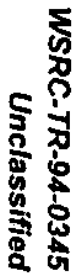


$\frac{3}{3}$

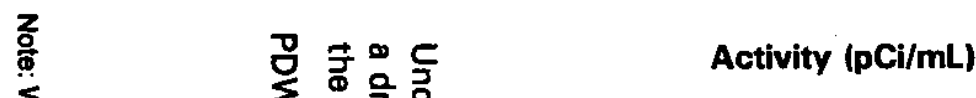

章

$\begin{array}{lllll} & & \overrightarrow{8} & \vec{\delta} & \delta\end{array}$

要

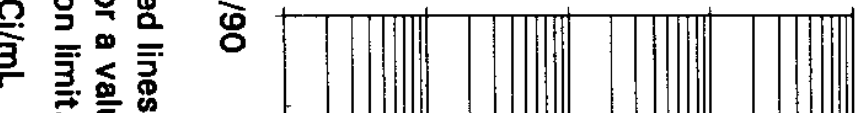

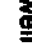

言 至

11 ₹ 
3

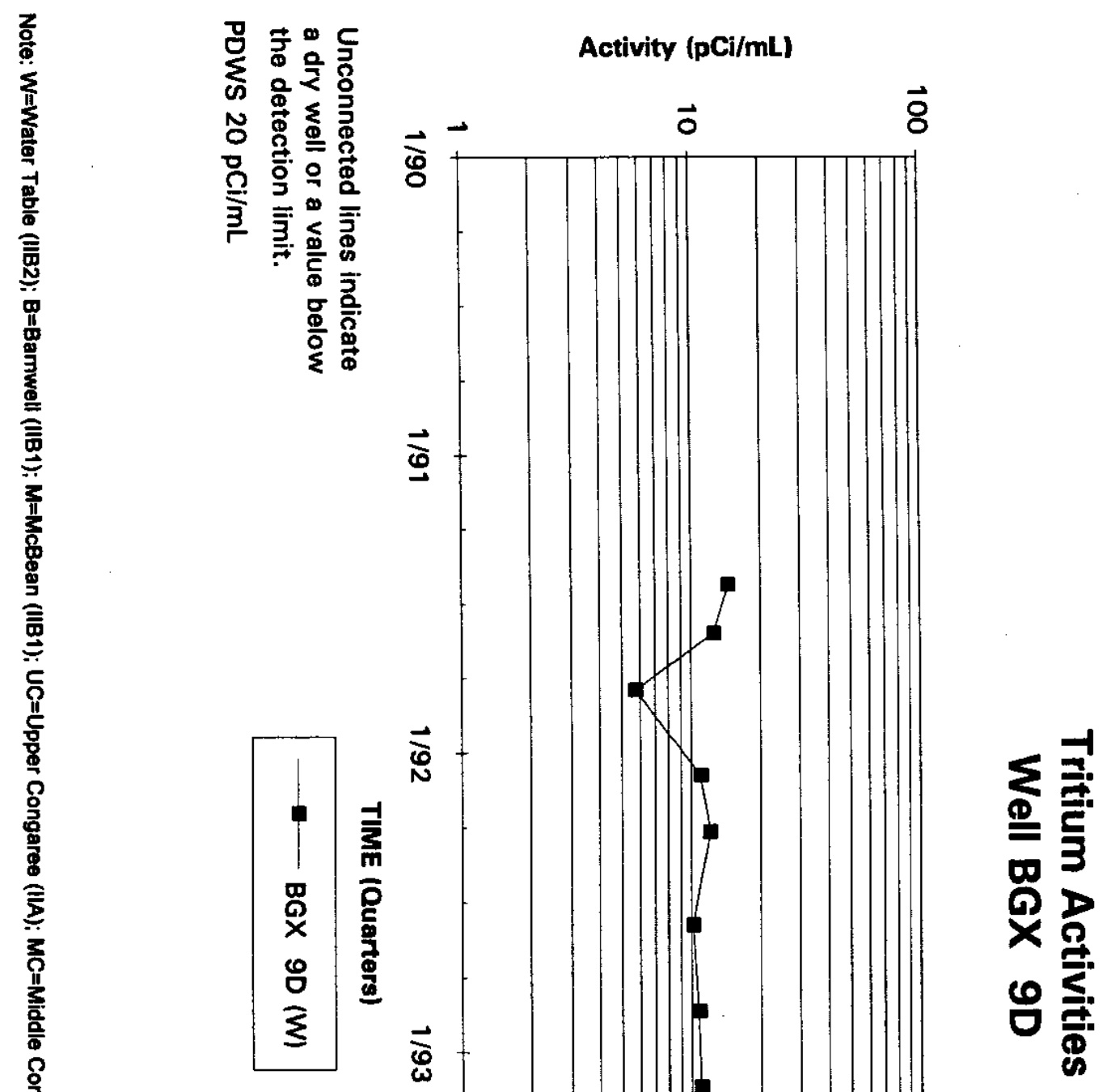


疍

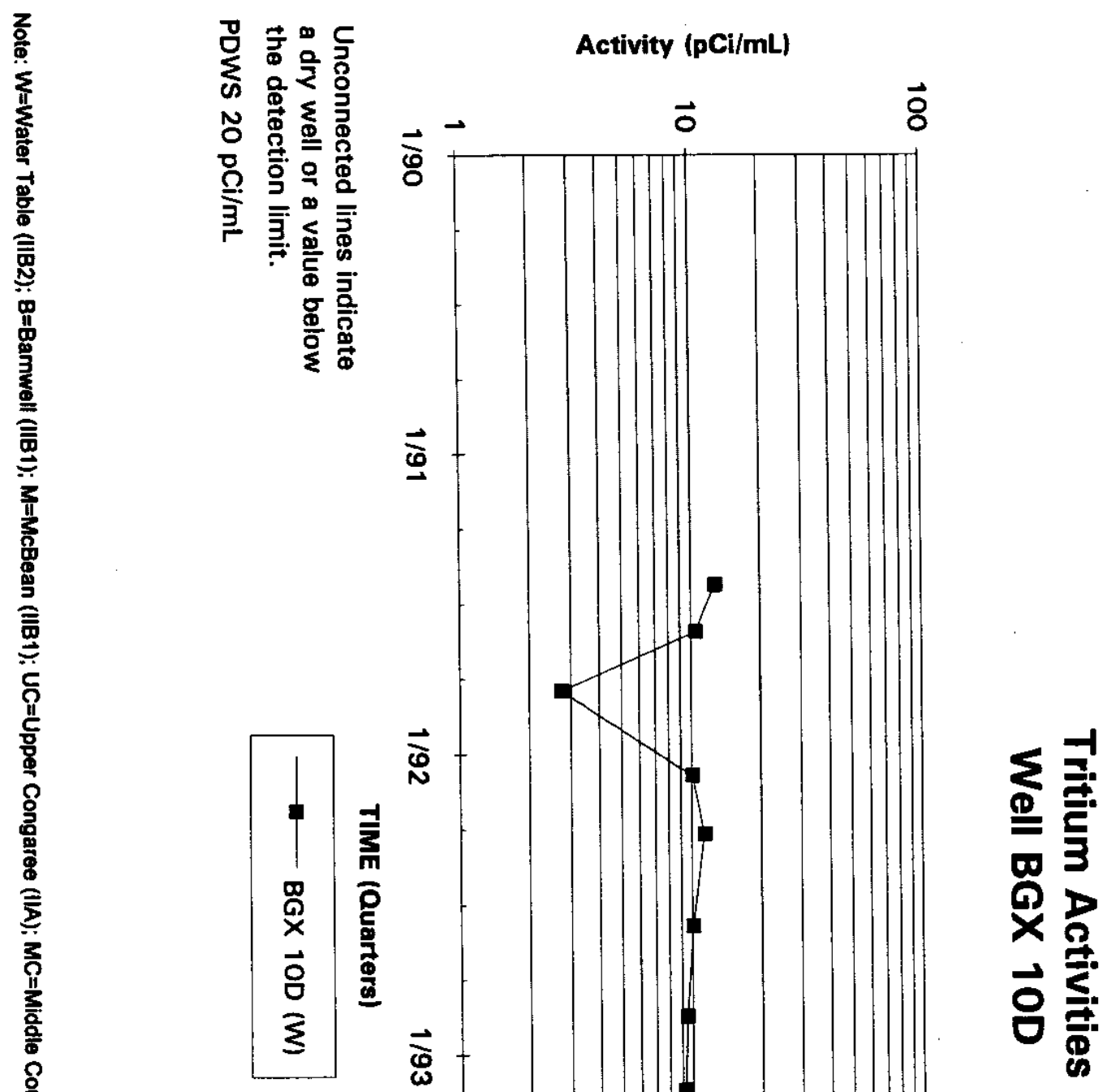

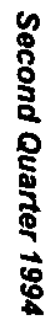


3

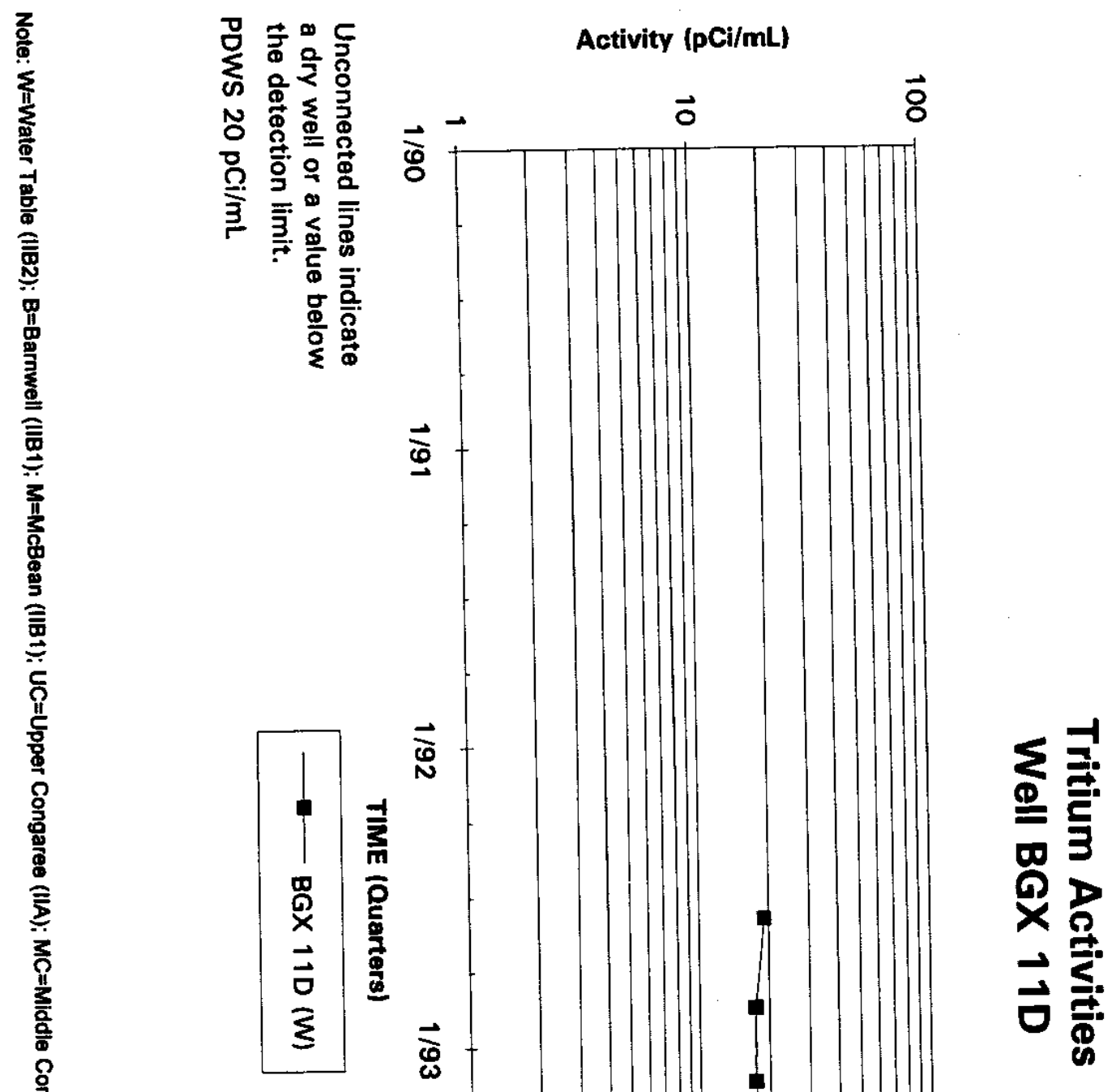



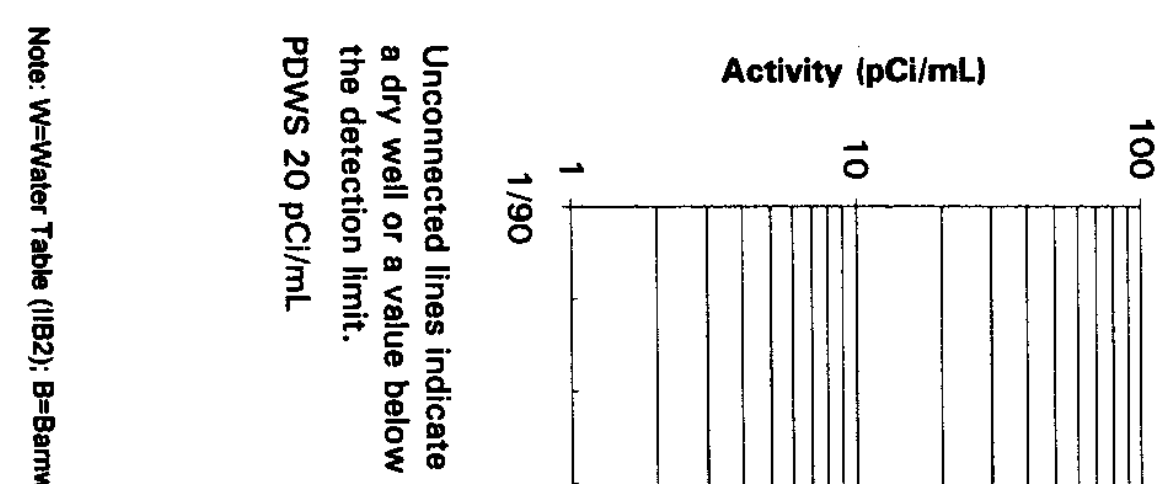

흥유융

产

$\frac{3}{\frac{3}{2}}$

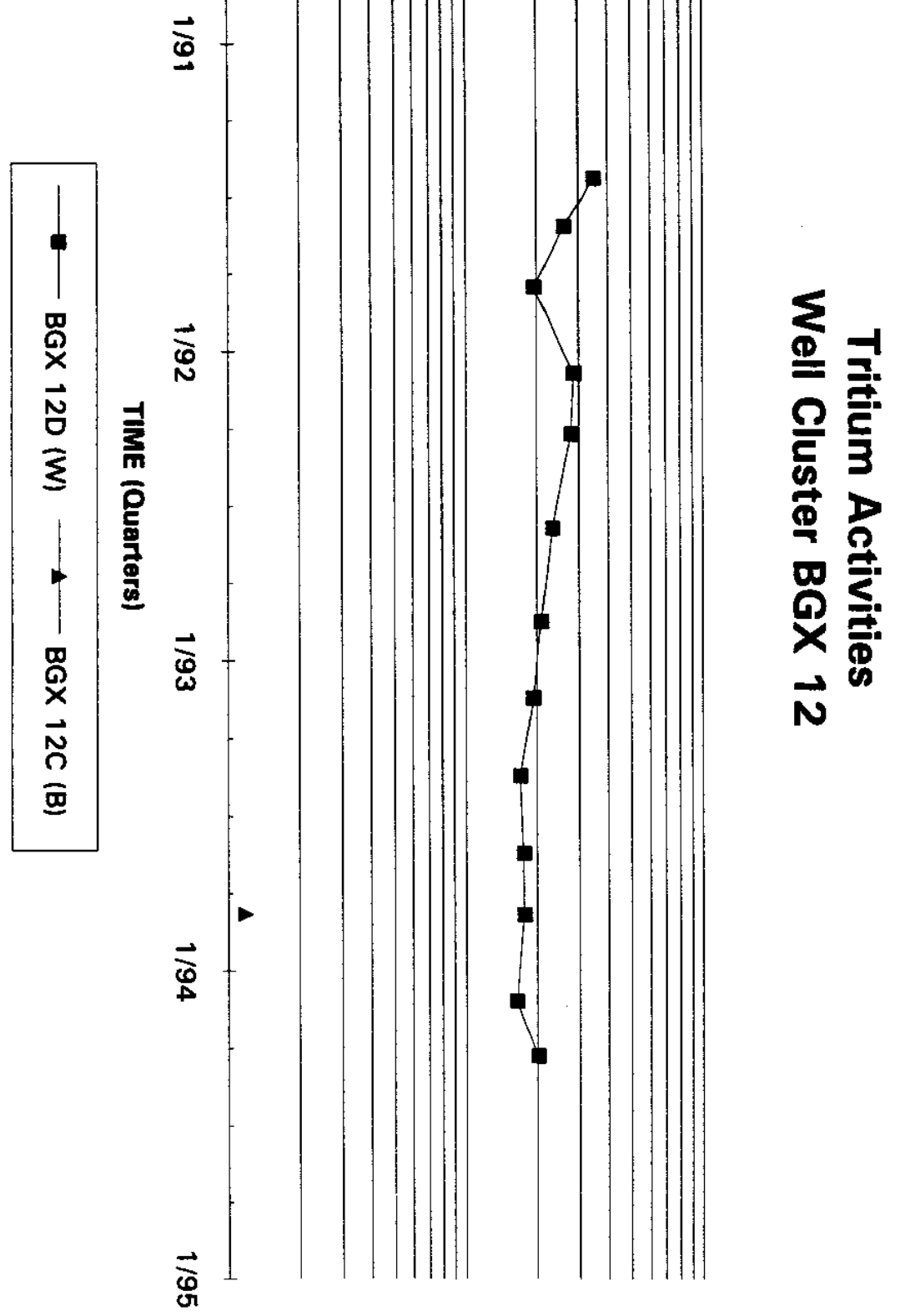

\begin{tabular}{l}
0 \\
8 \\
8 \\
0 \\
0 \\
5 \\
0 \\
\hline 0 \\
\hline 0
\end{tabular}

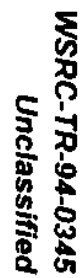


$\sum_{3}^{3}$

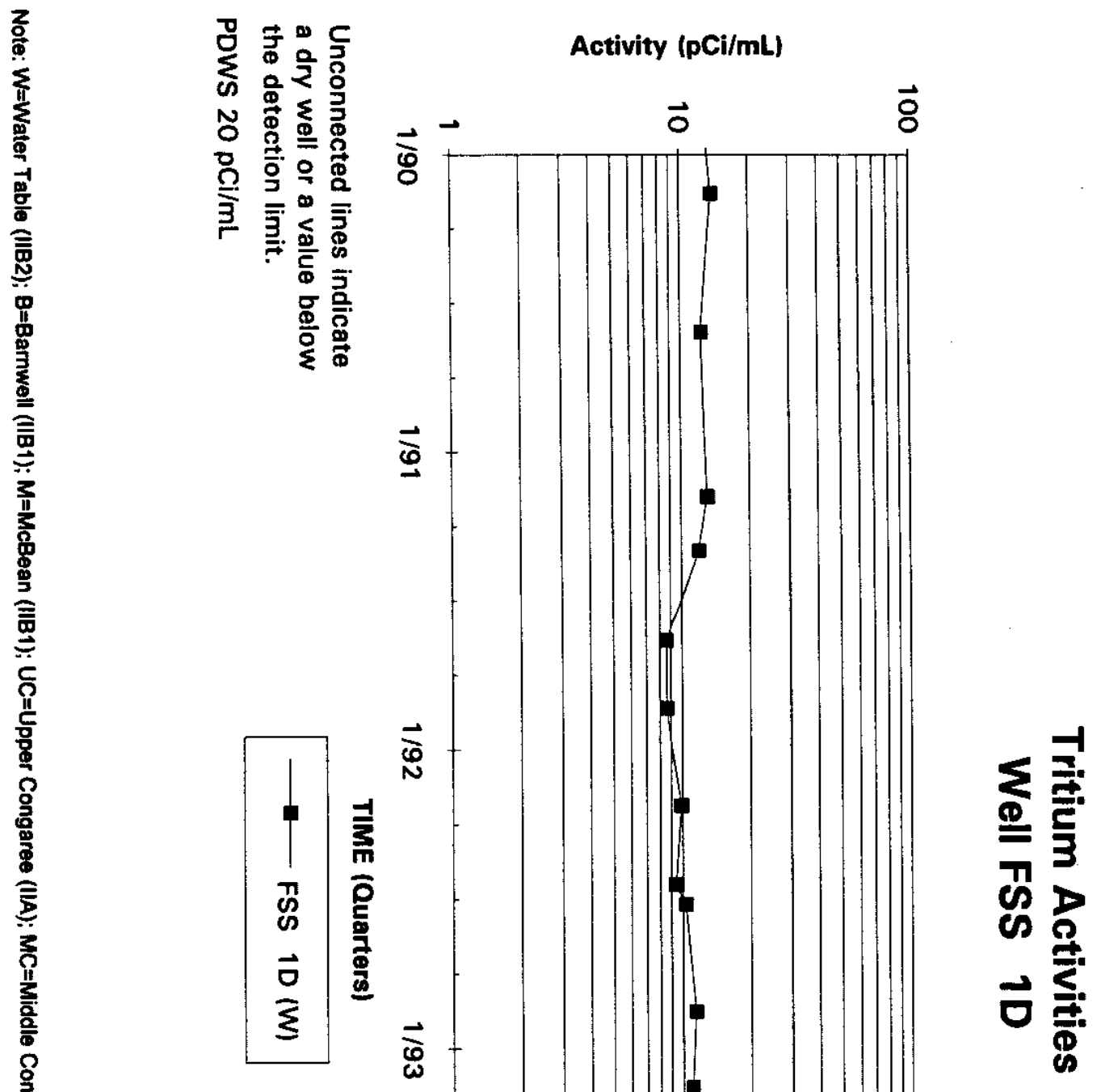

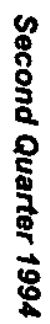

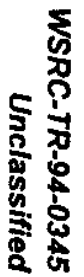


3

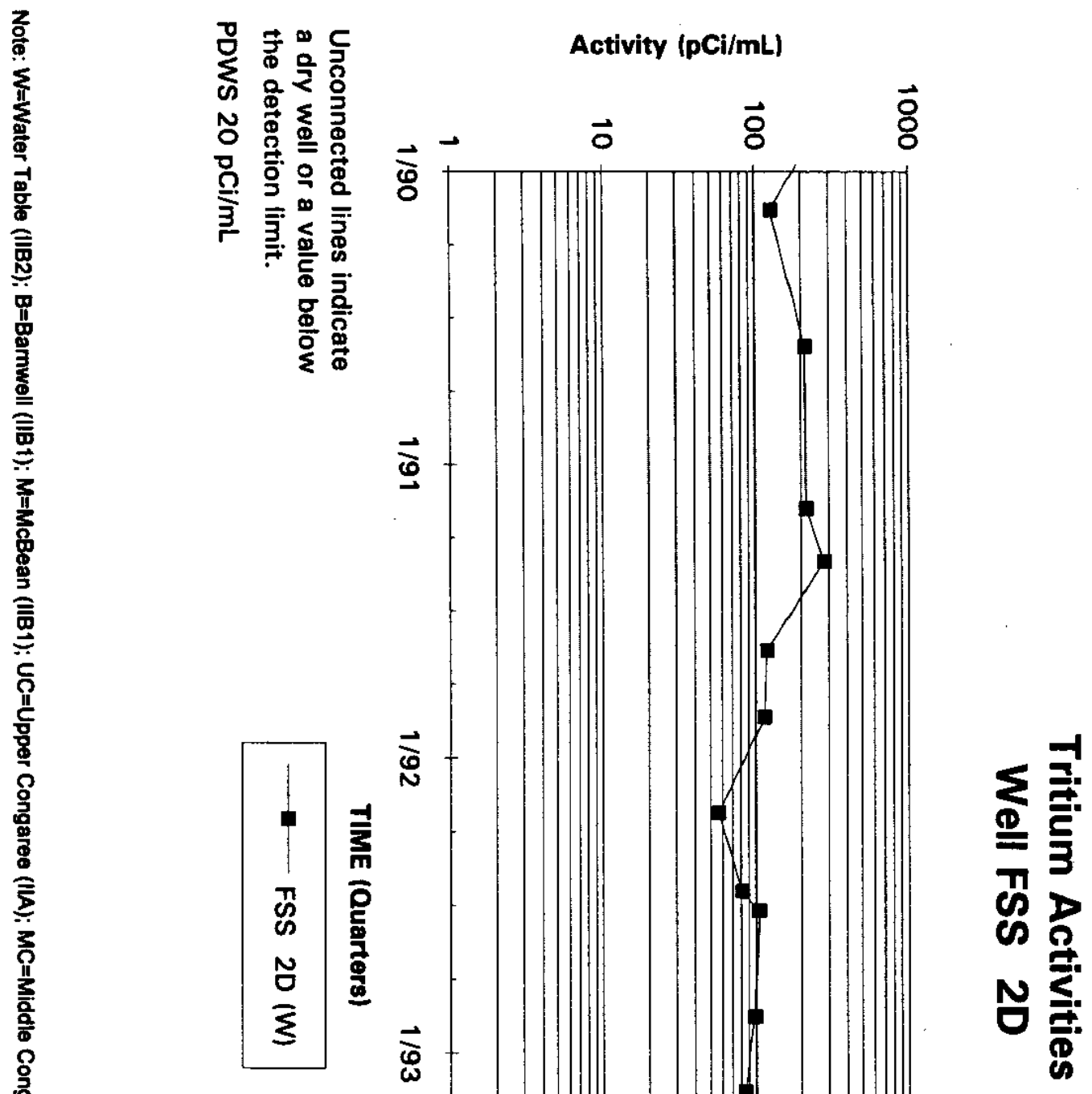


糸

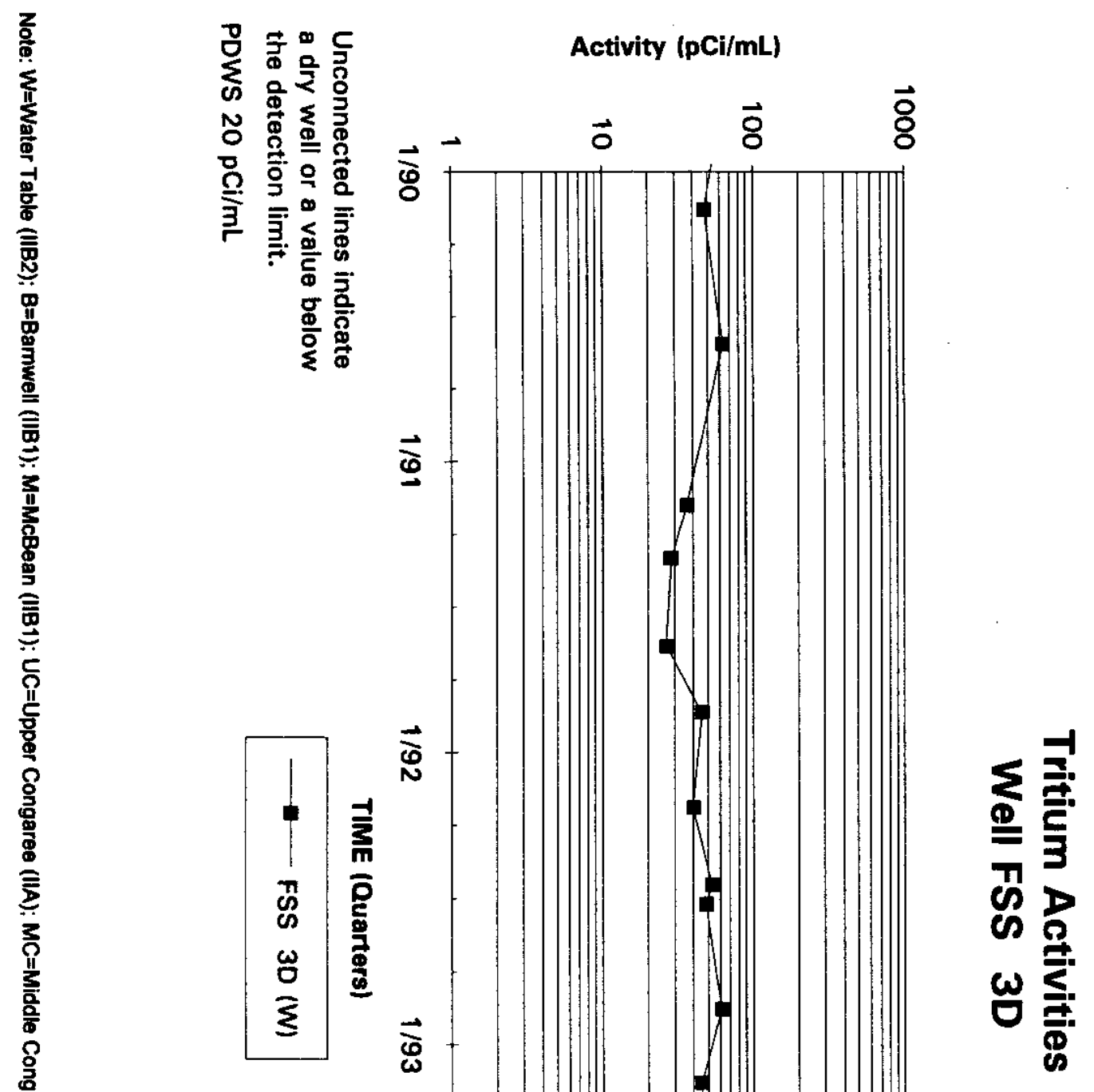

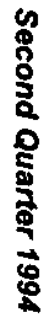

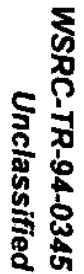


3

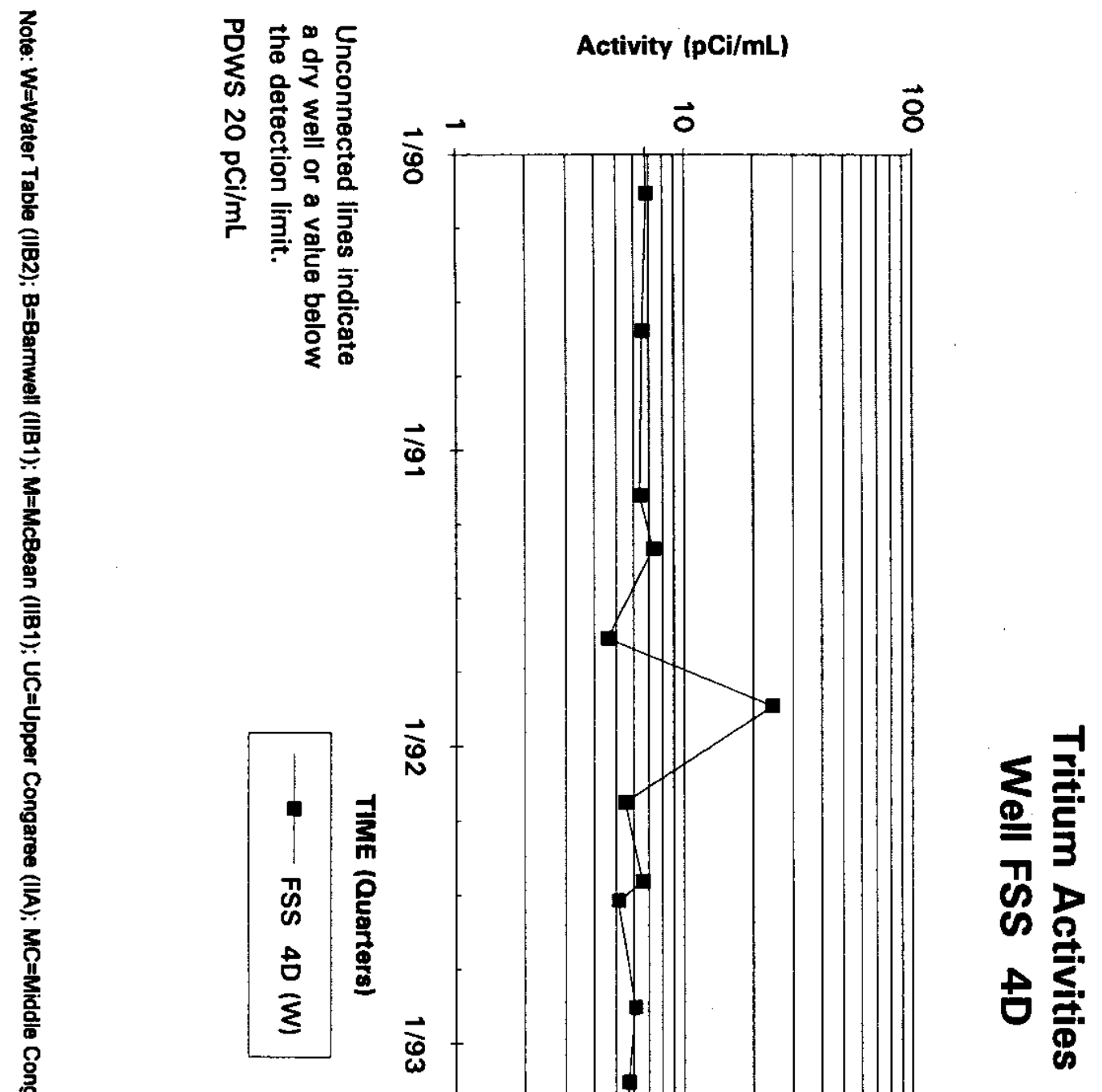

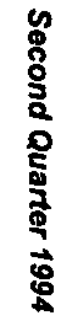

总 
3

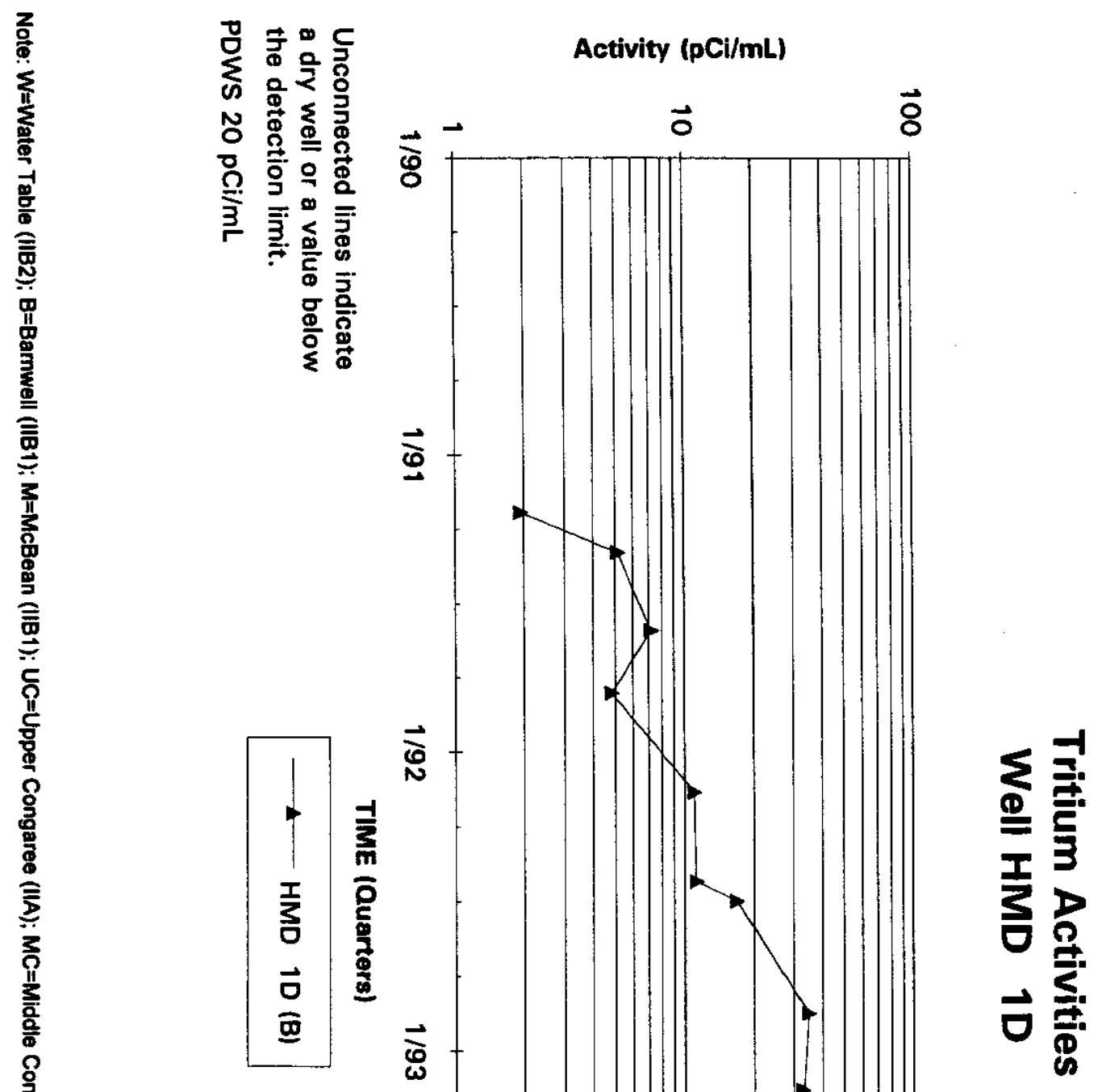

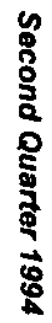

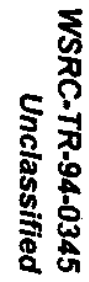


章

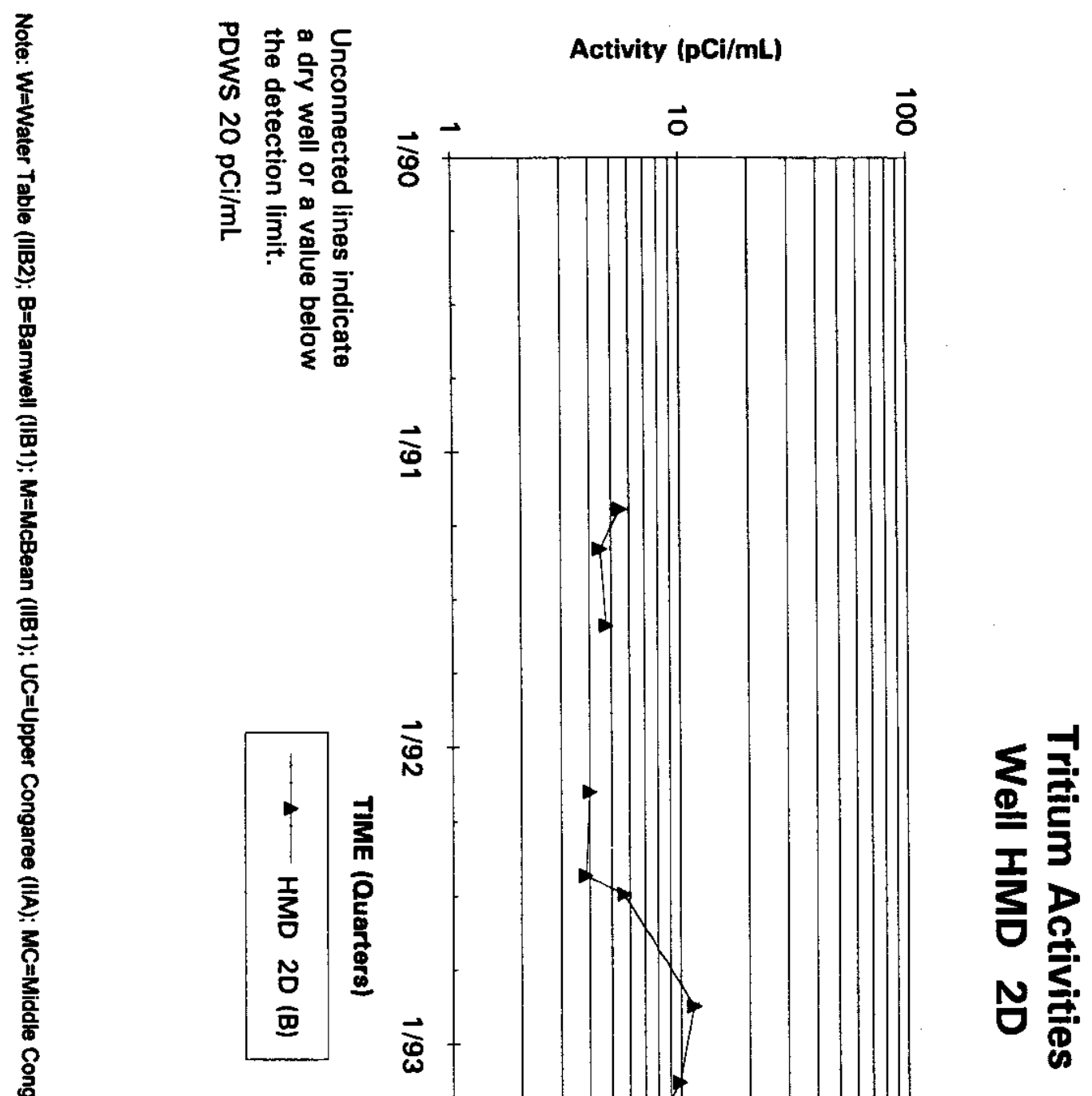

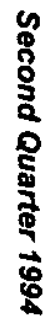


I

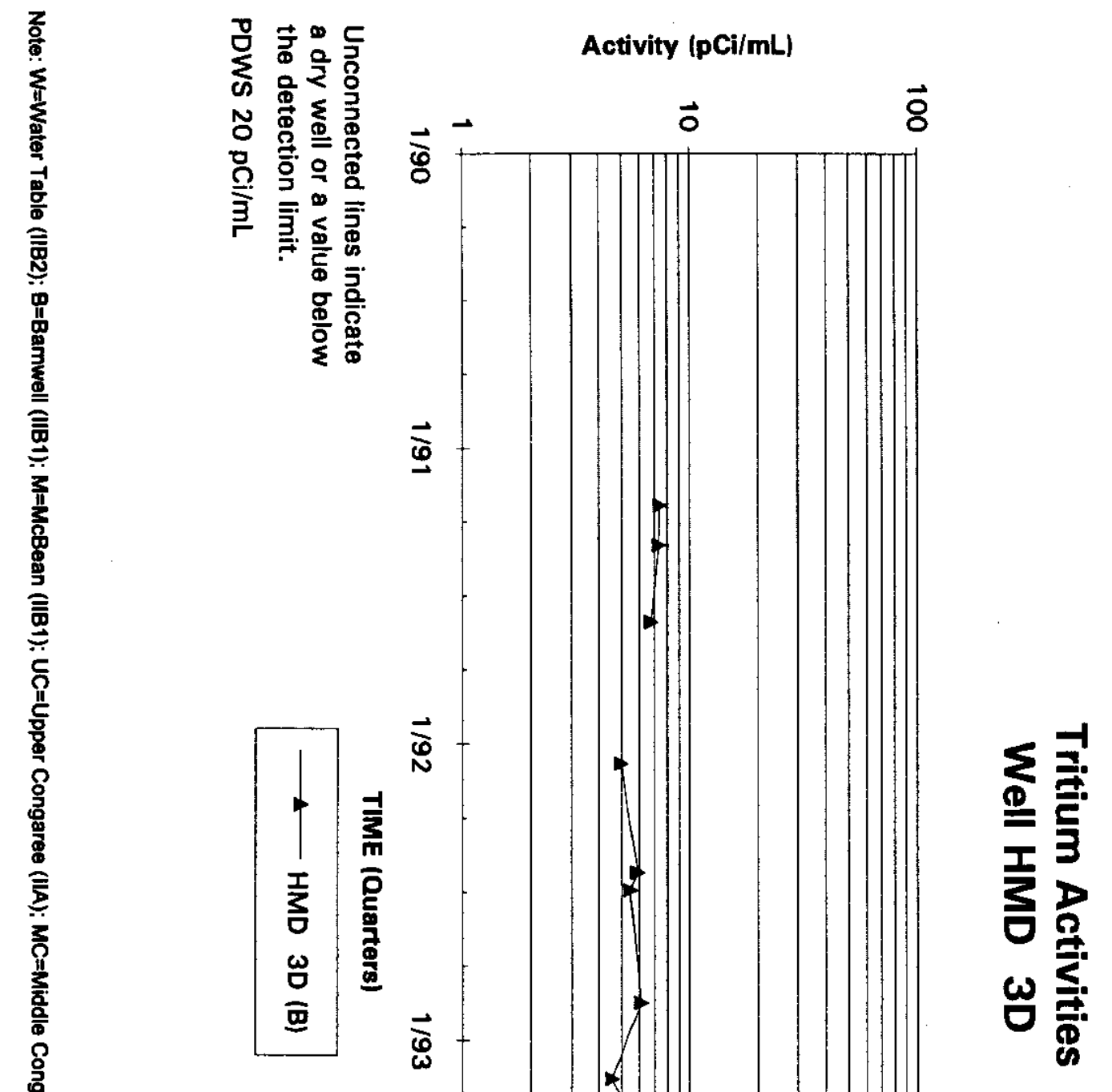




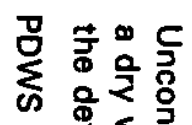

Activity (pCi/mL)

듀요

กุ

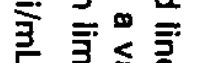

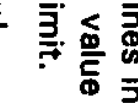

$\overrightarrow{0} \quad \overrightarrow{0}$

$\overrightarrow{8}$

흥 흥

言

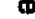
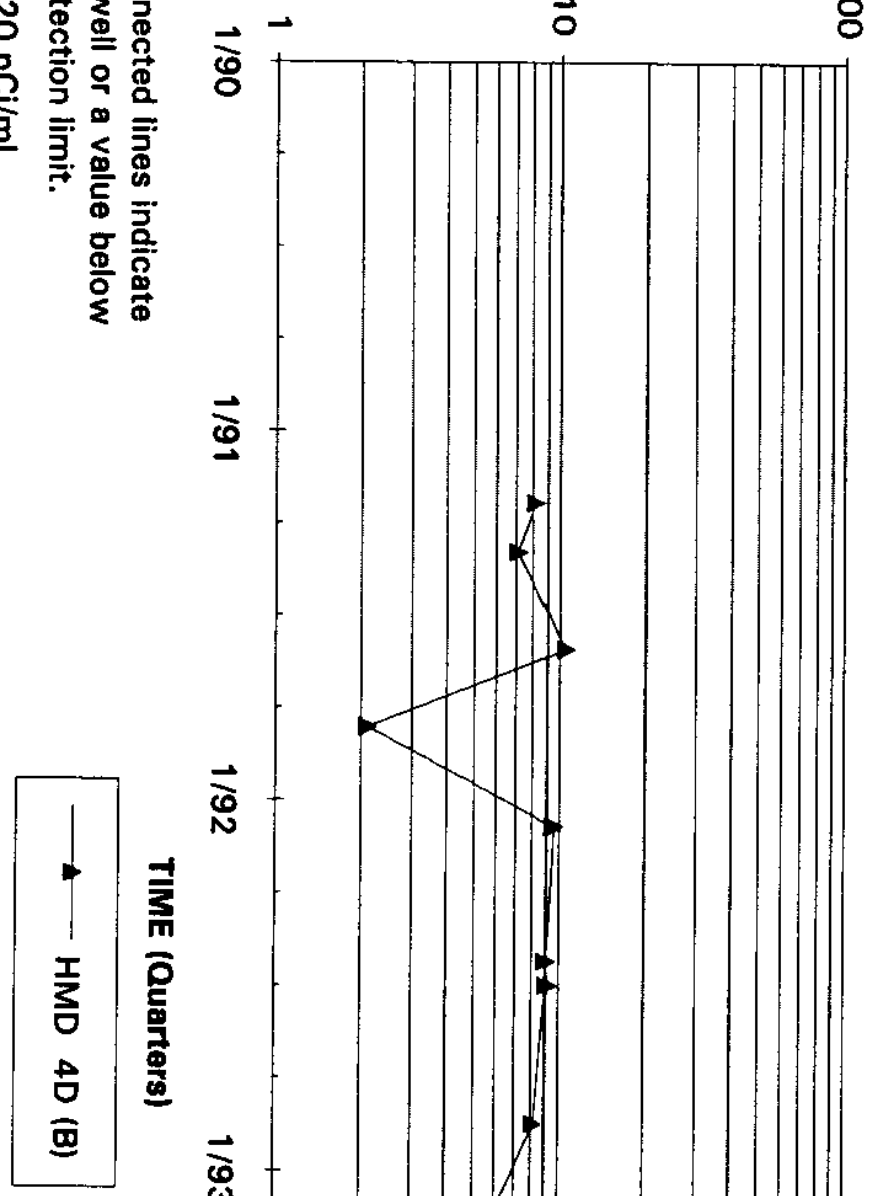

$\grave{\Xi}$

高

产

동

ᄋ

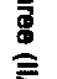

총

흥

兴

㐫

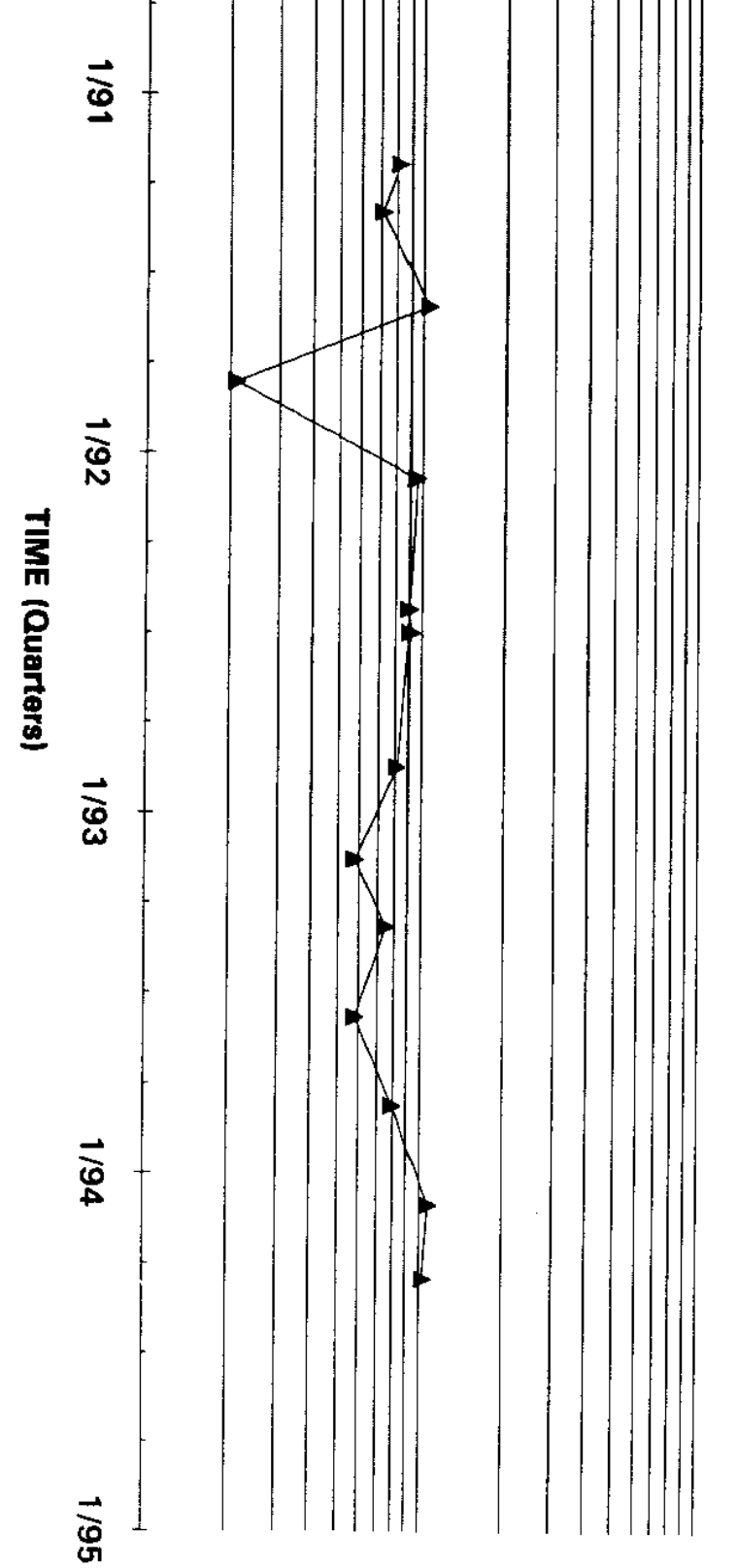

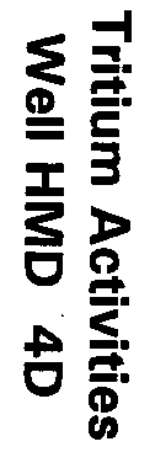


3

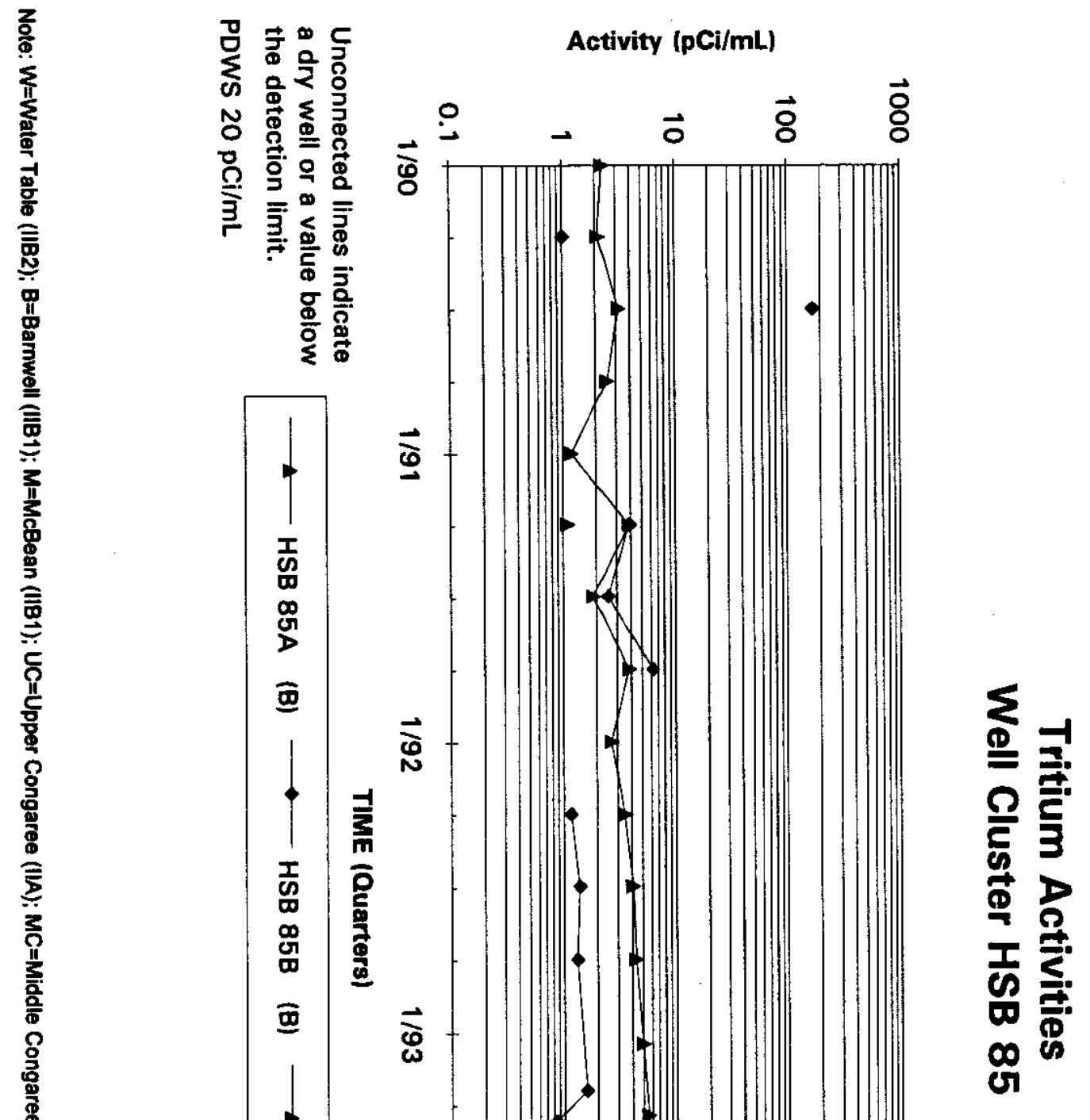

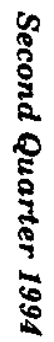

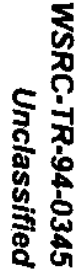


WSRC-TR-94-0345

Unclassified

\section{Appendix G}

\section{Hydrographs}


THIS PAGE LEFT BLANK INTENTIONALLY. 
㞼

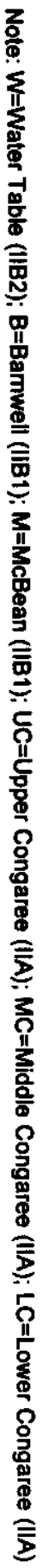

Whest

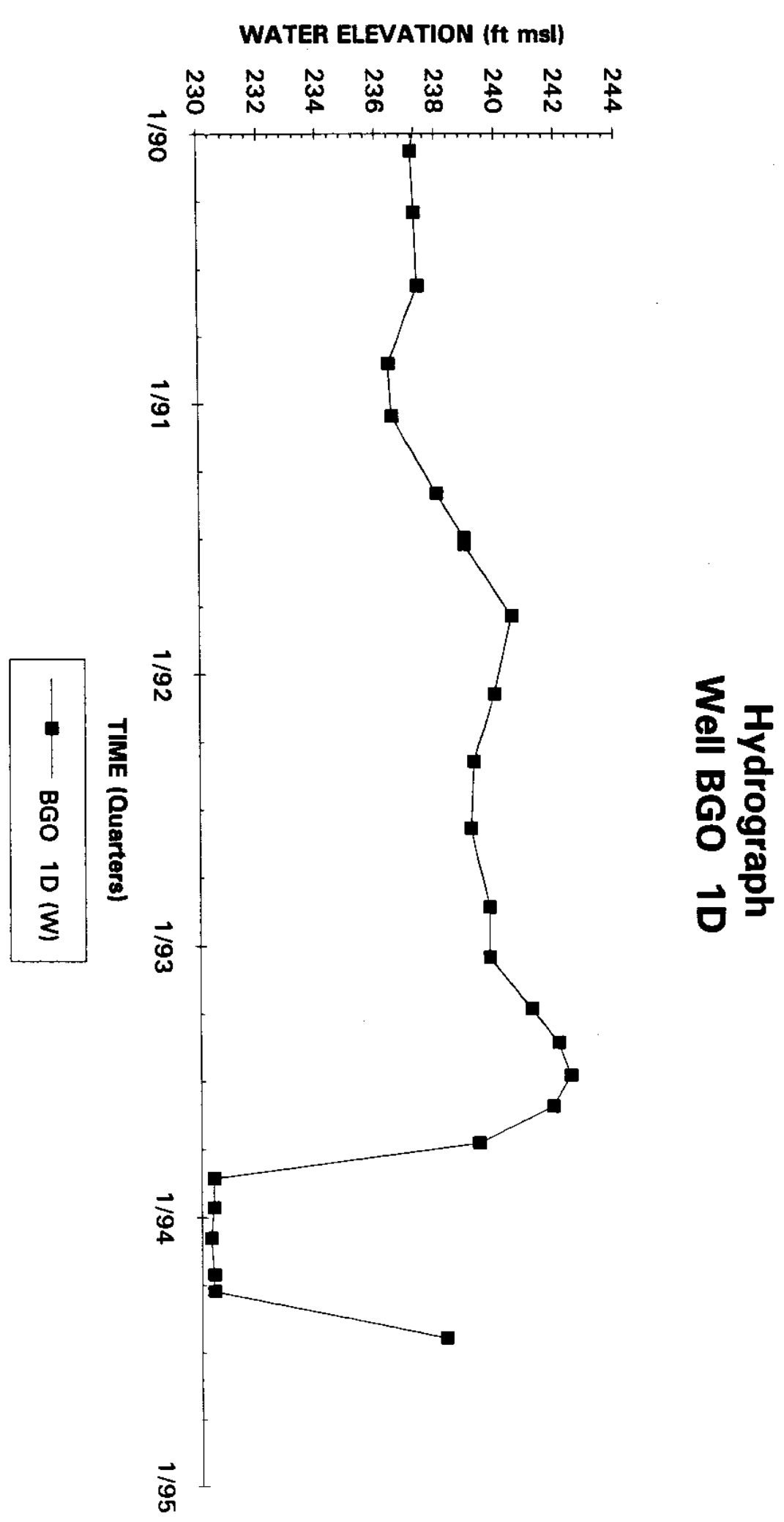

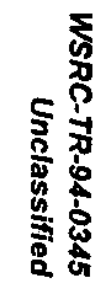



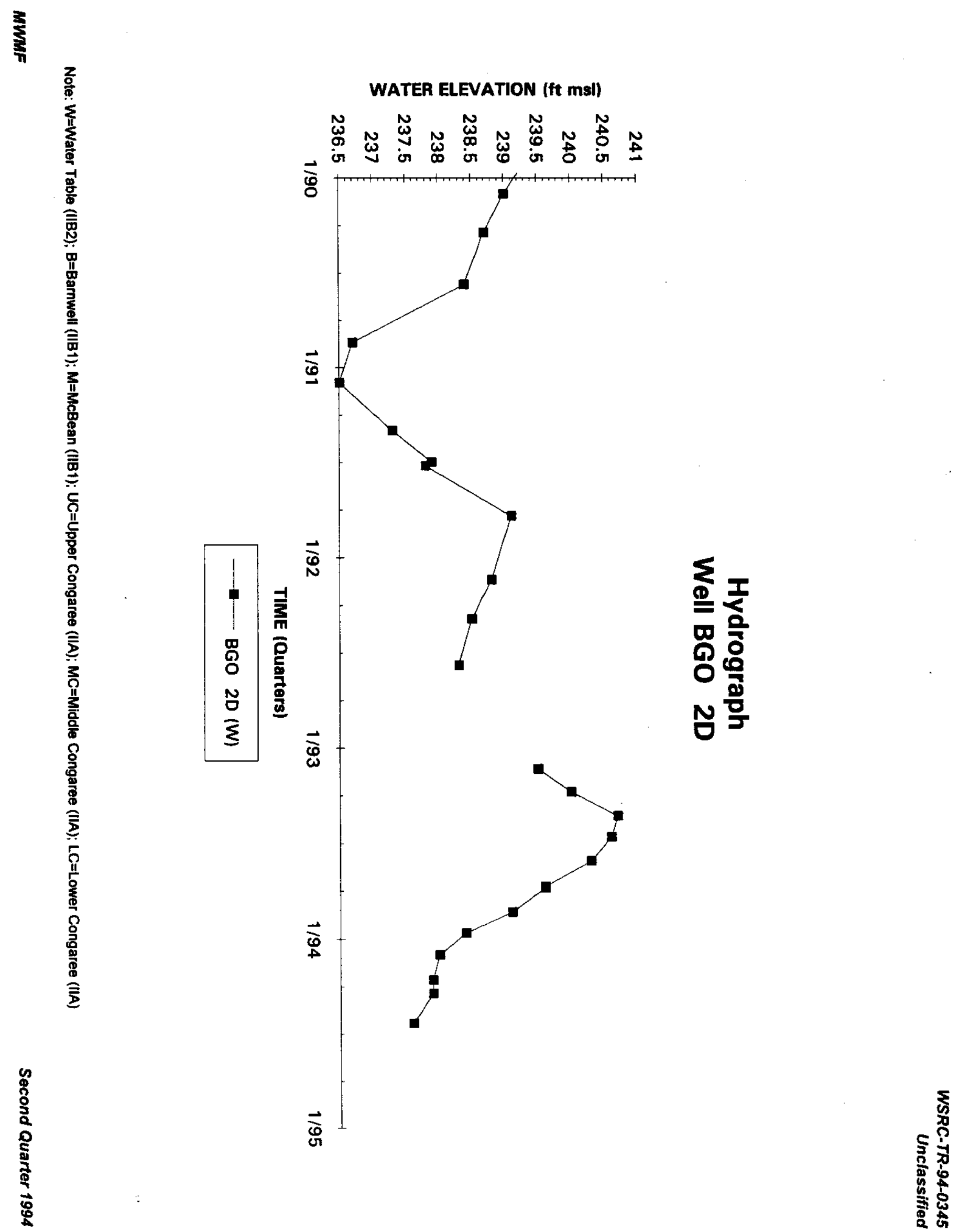


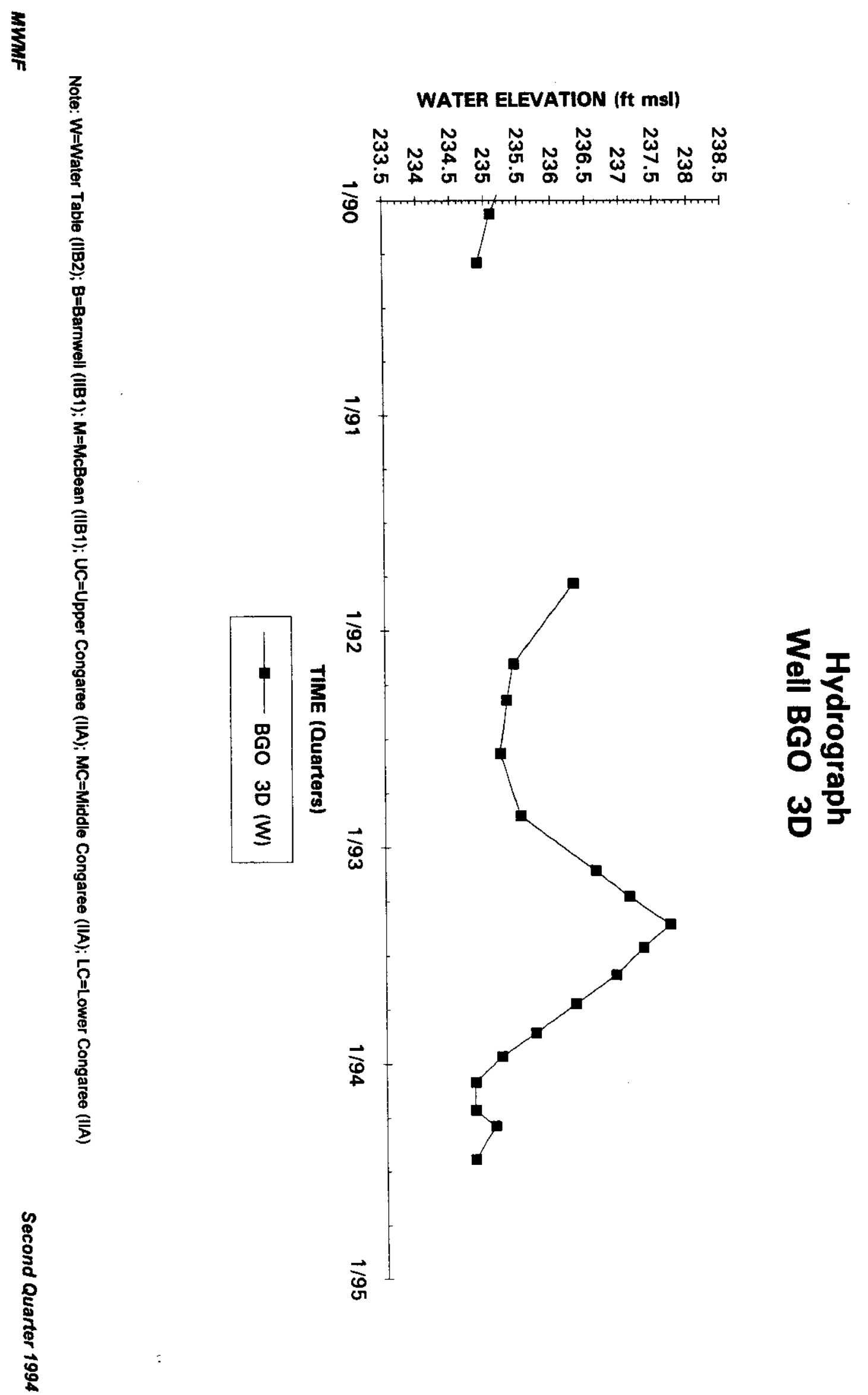

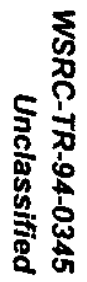




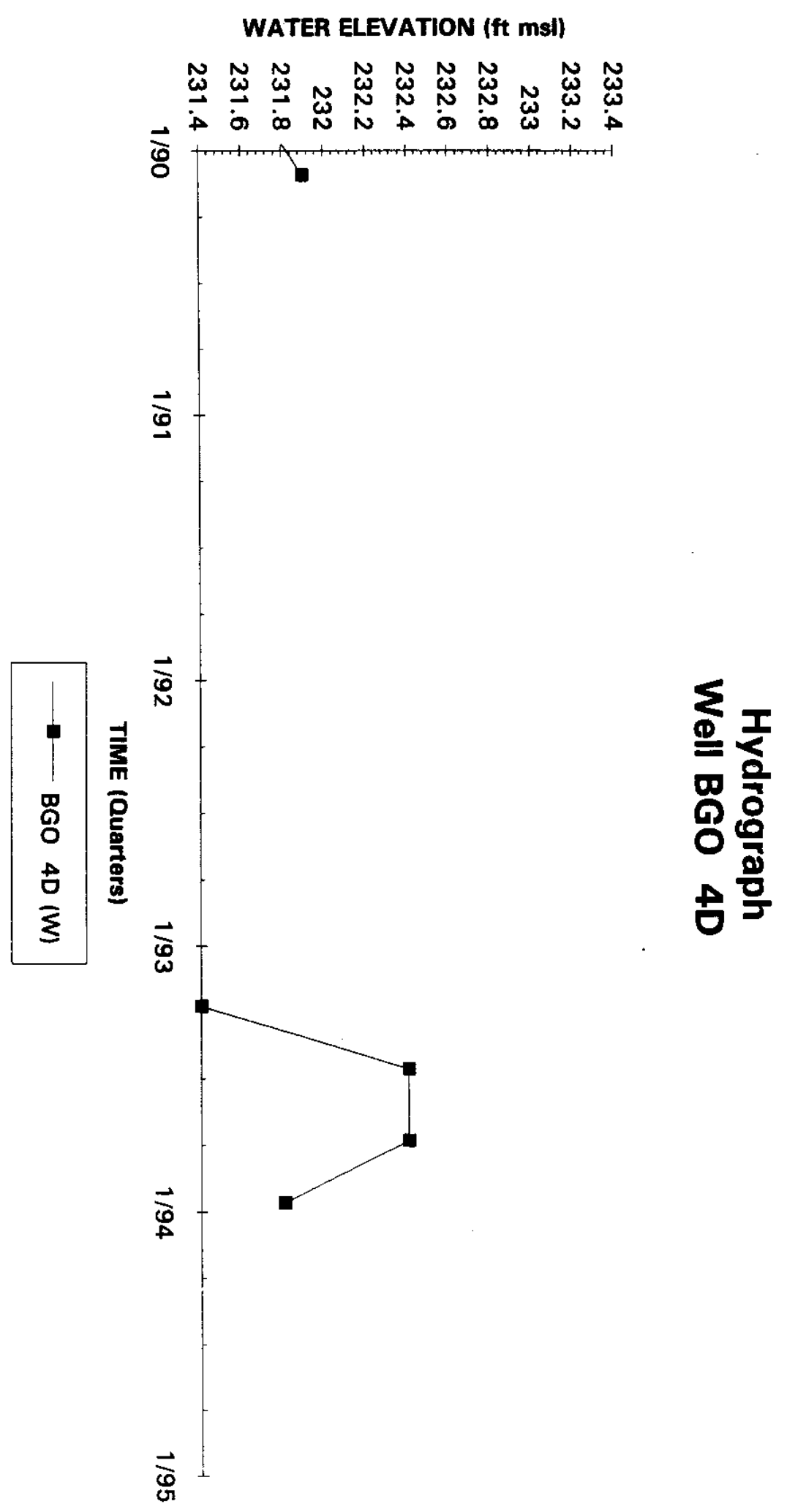




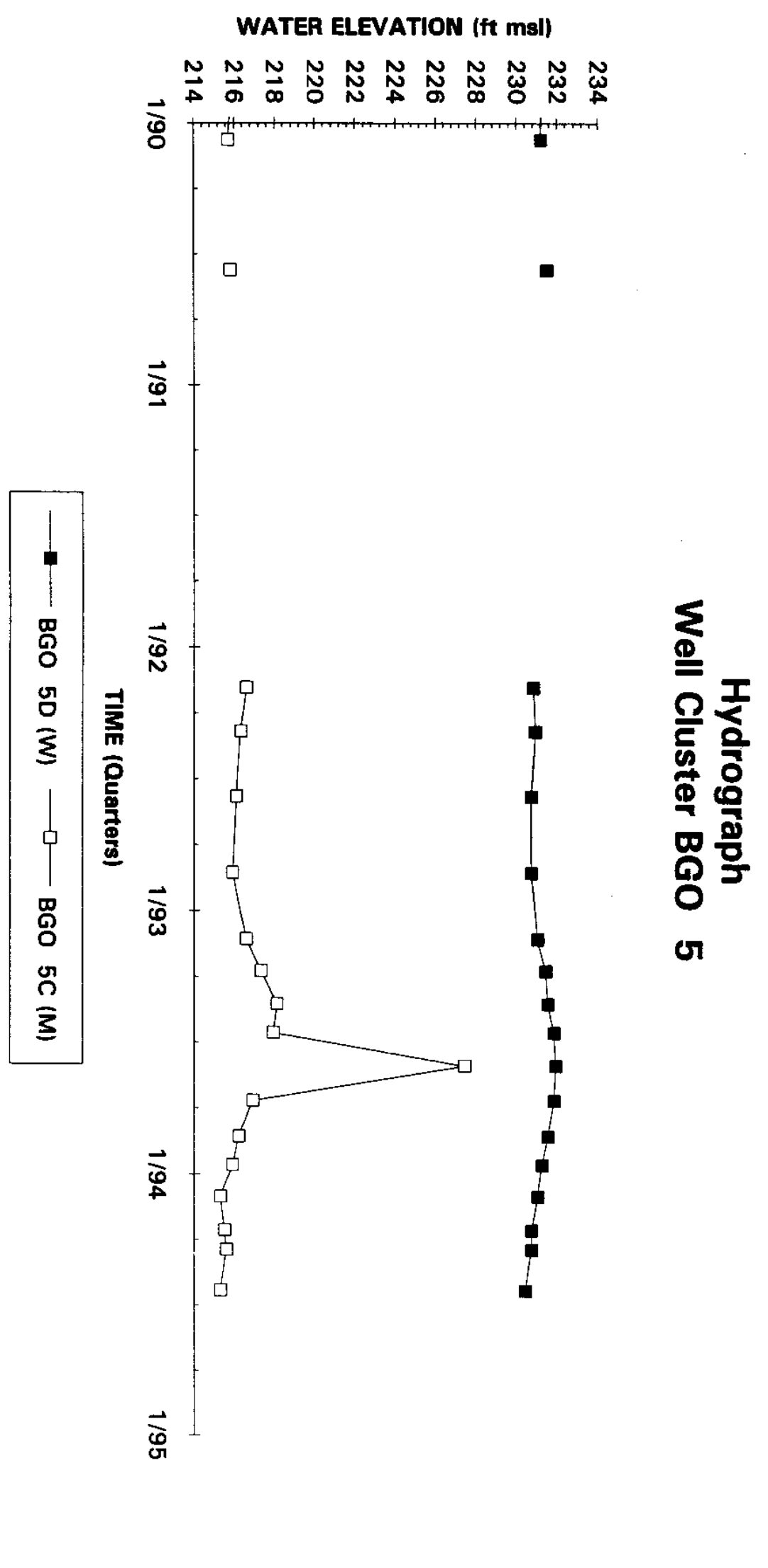

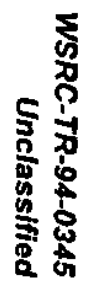



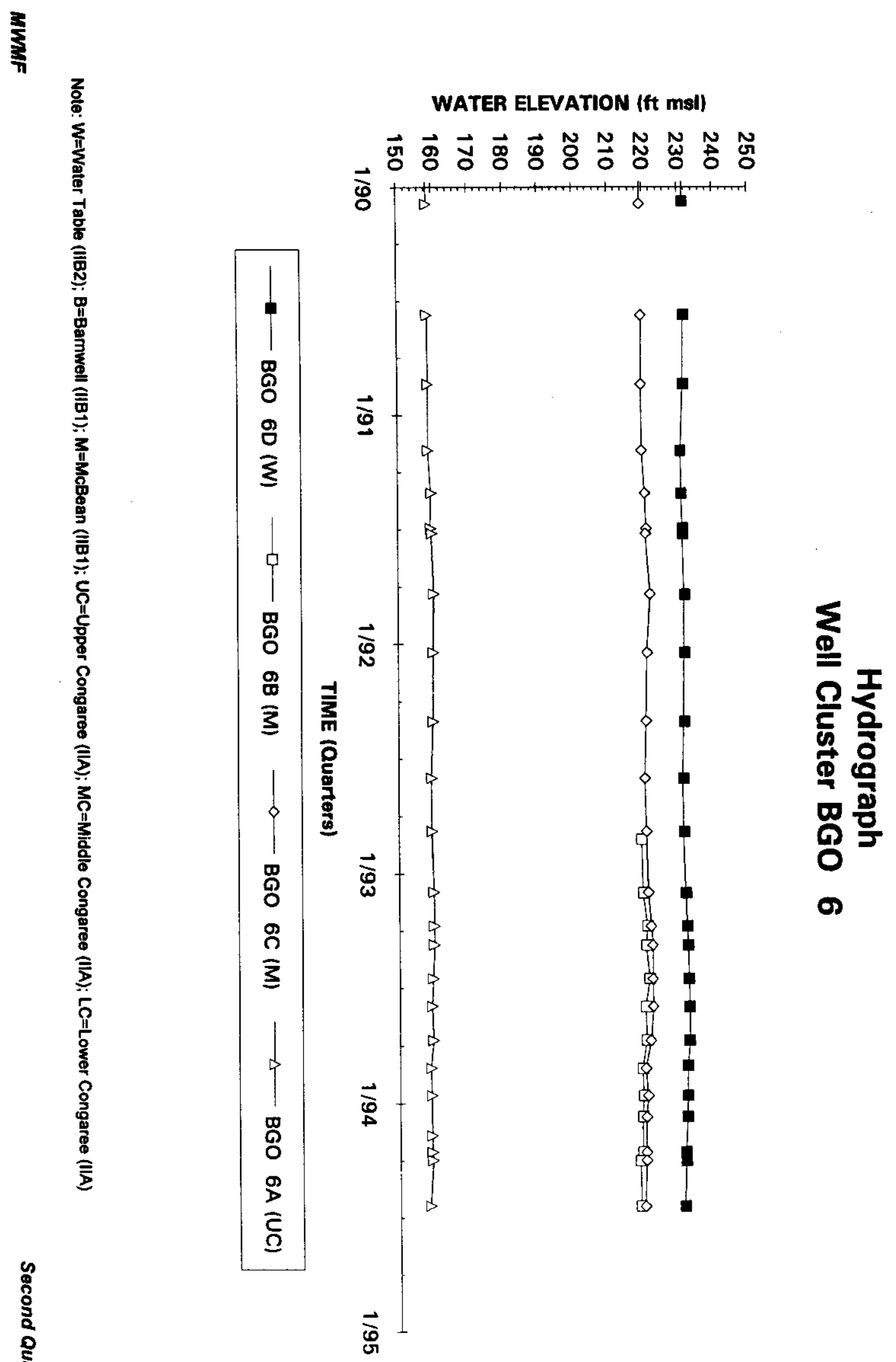

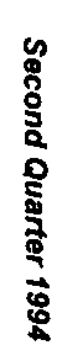

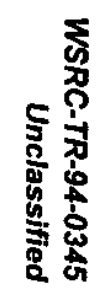




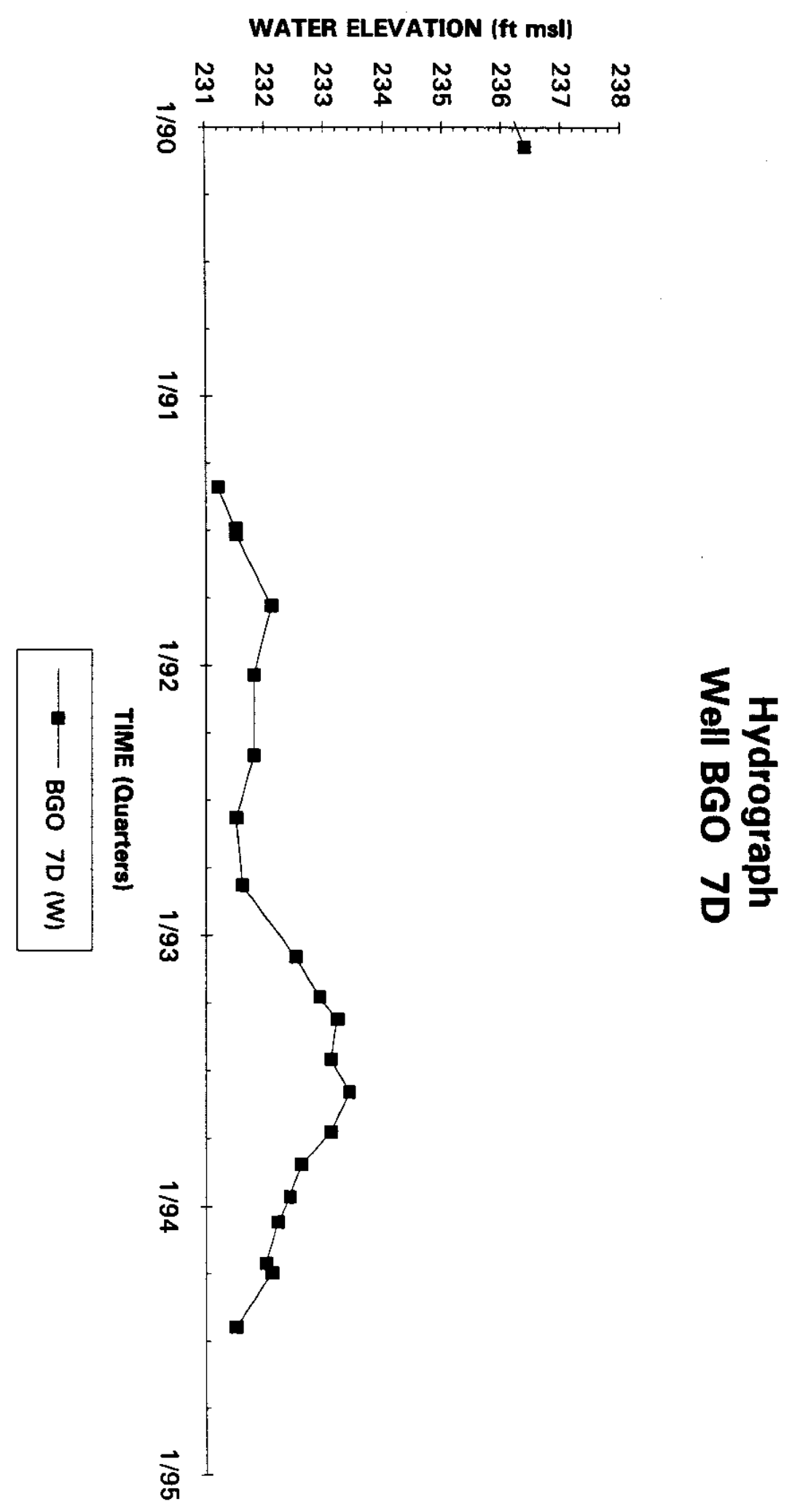

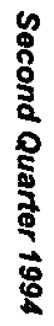




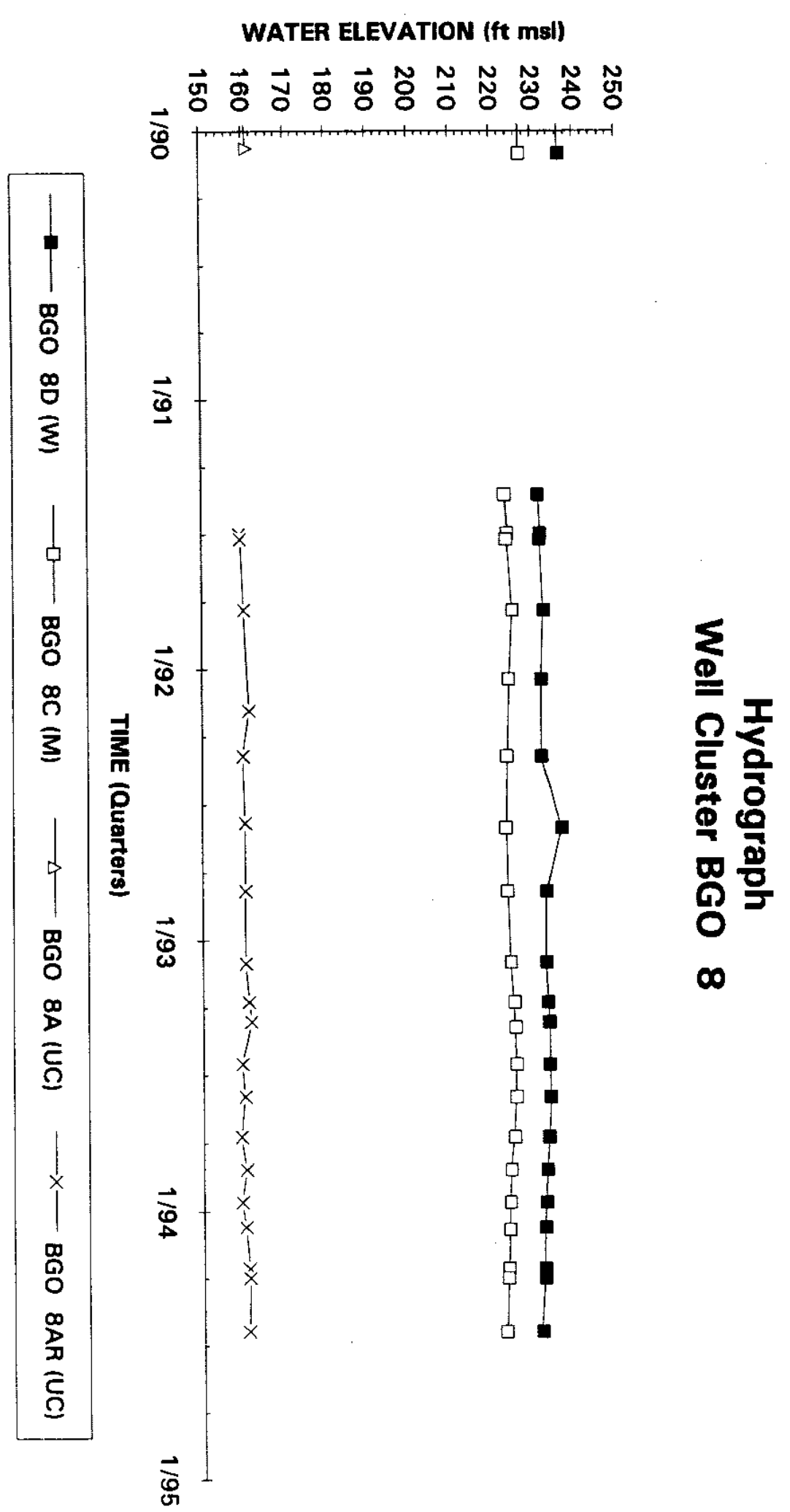




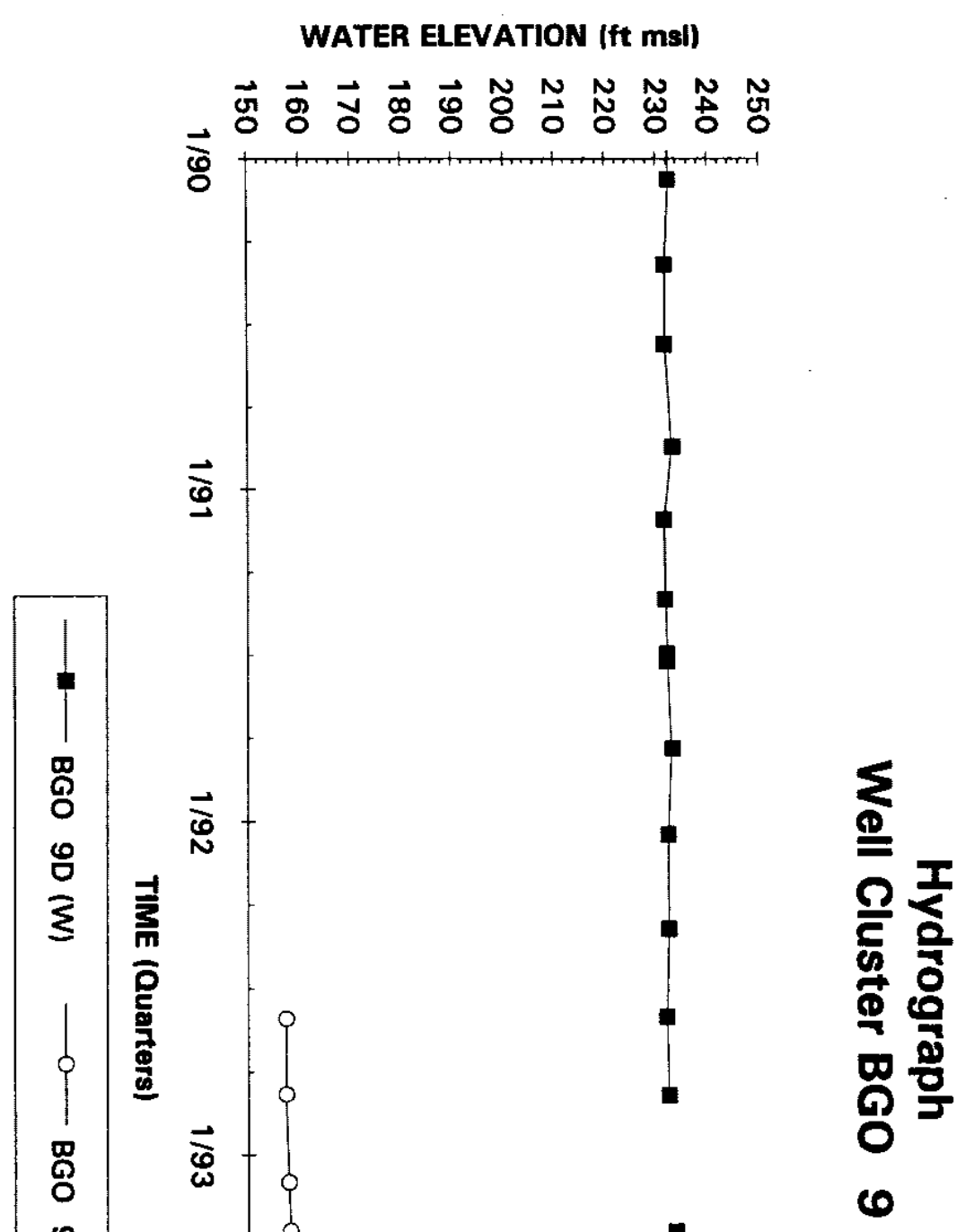




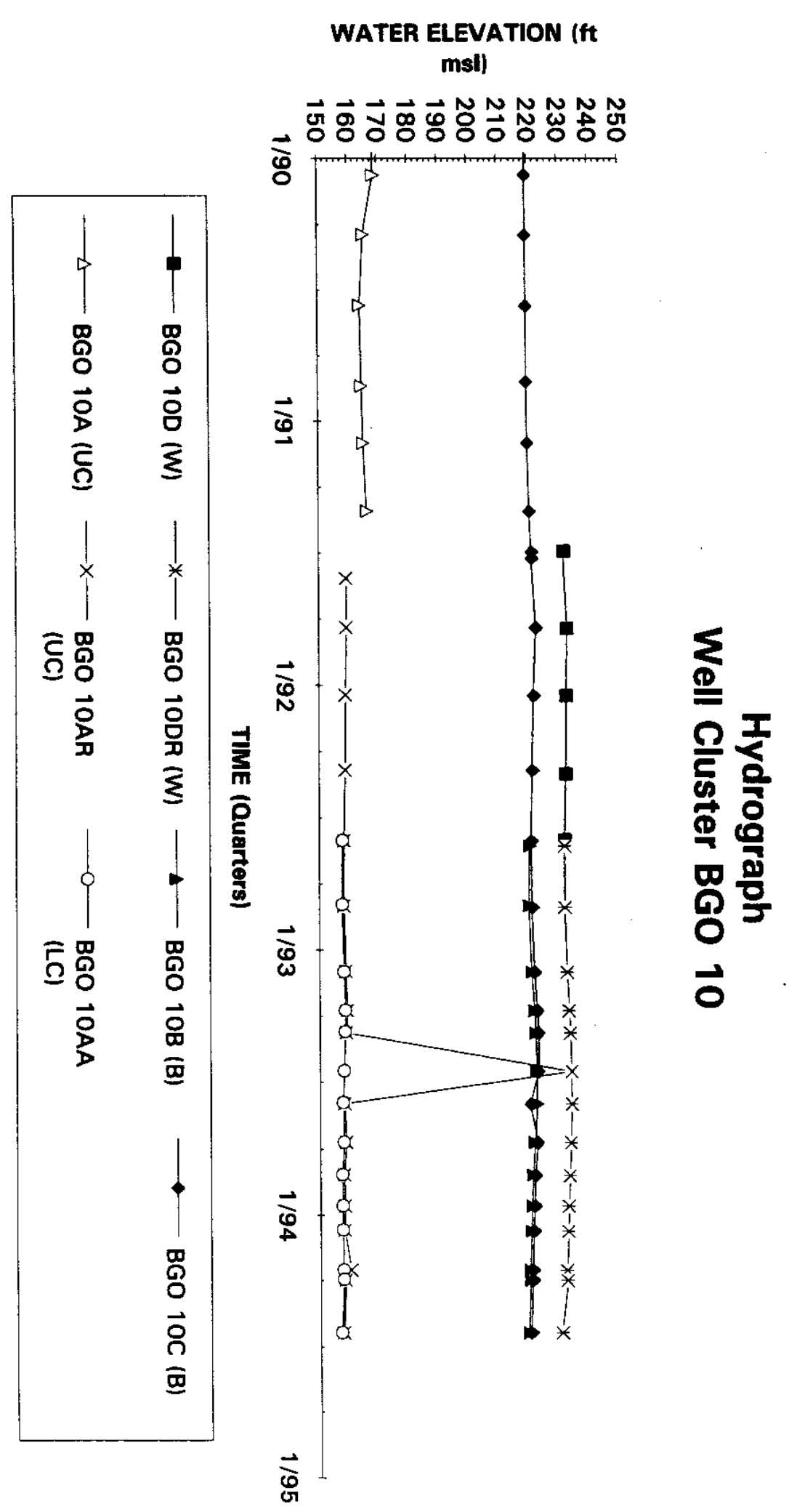



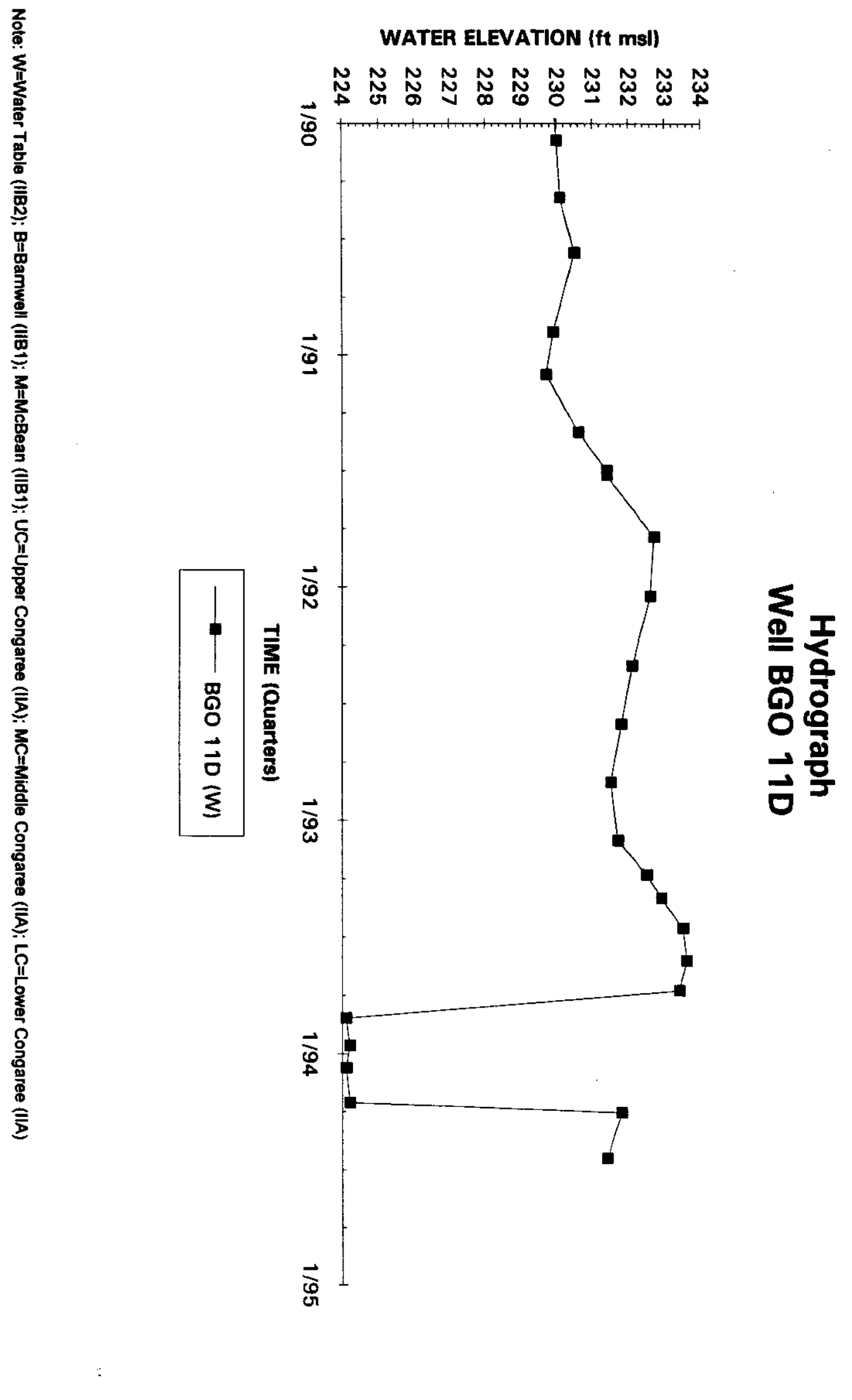

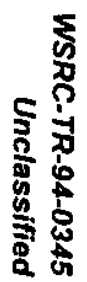




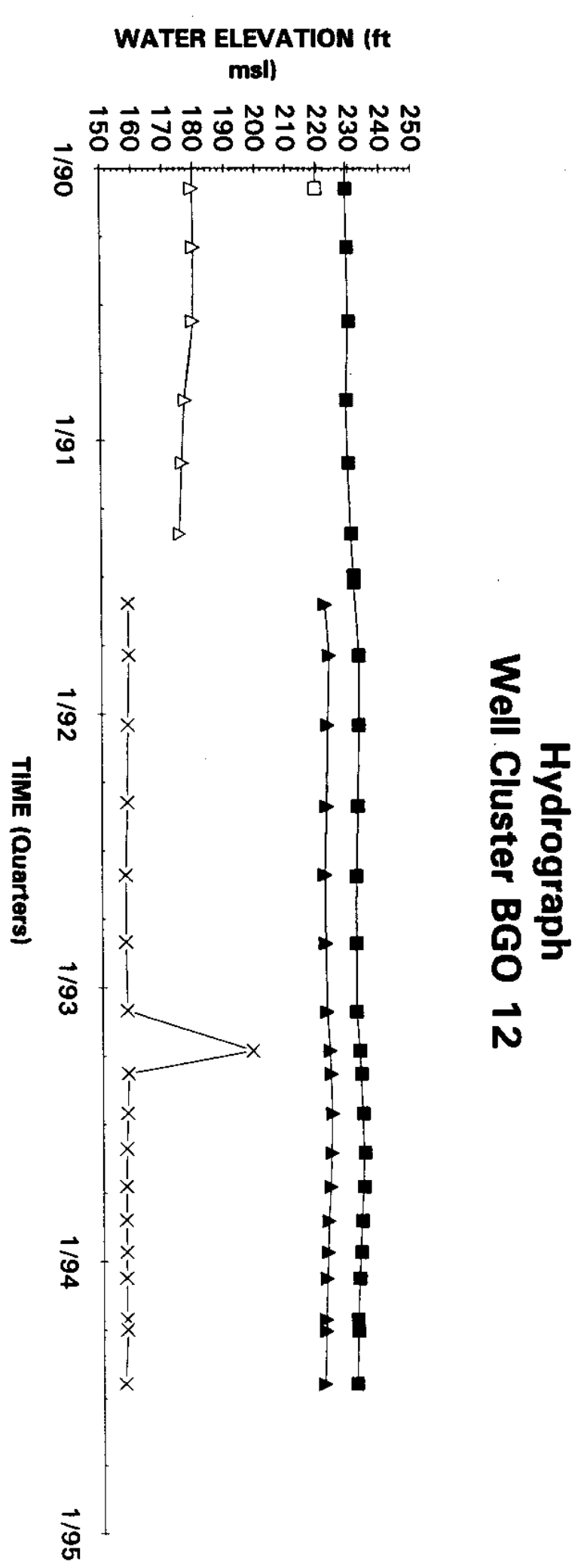

$\infty$
8
0
0
0
5
0
0
0

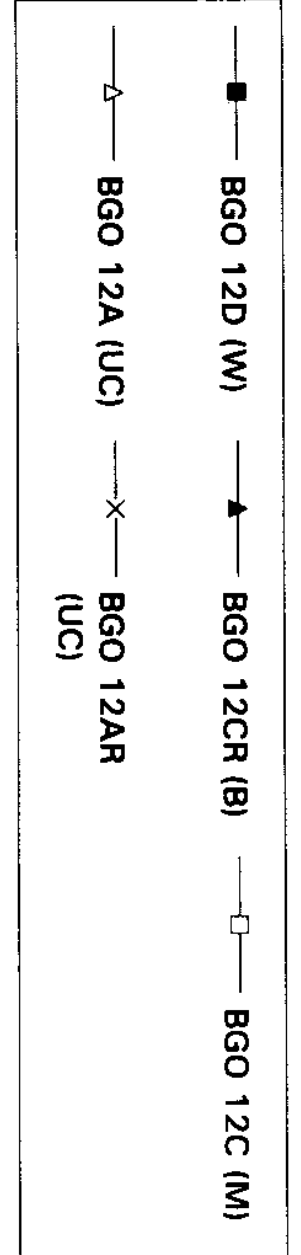



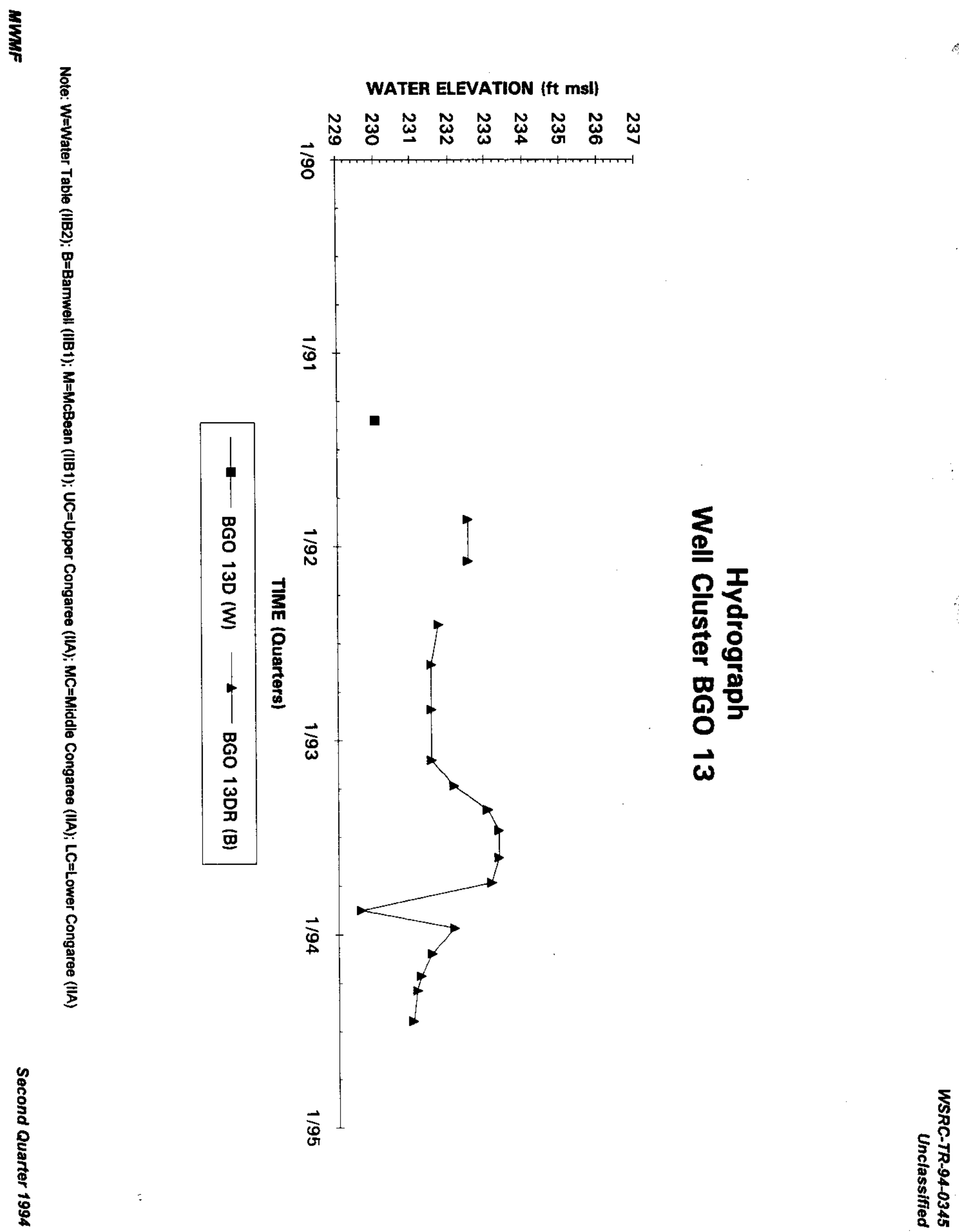
WATER ELEVATION (ft msl)

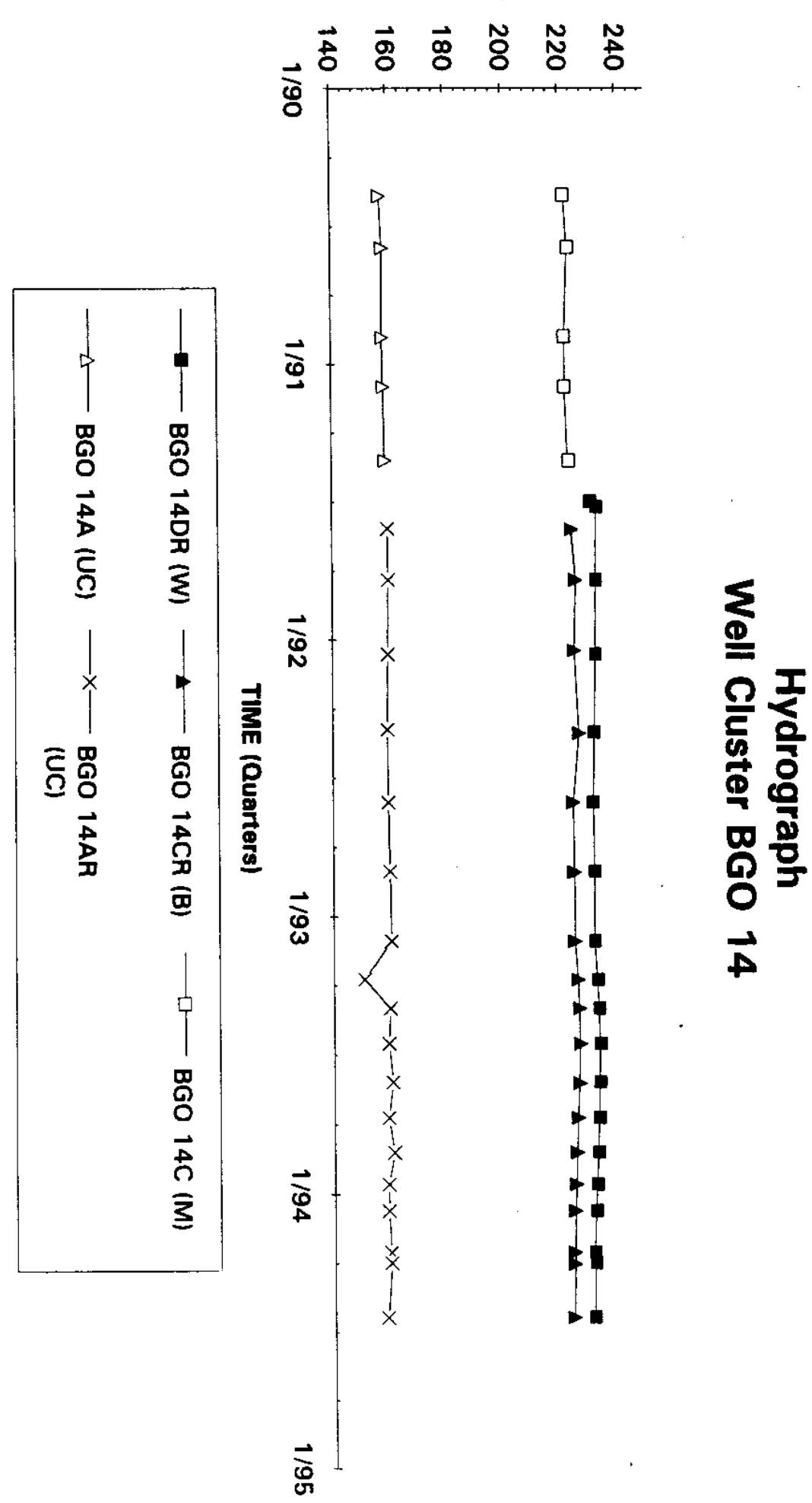


$\underset{3}{3}$
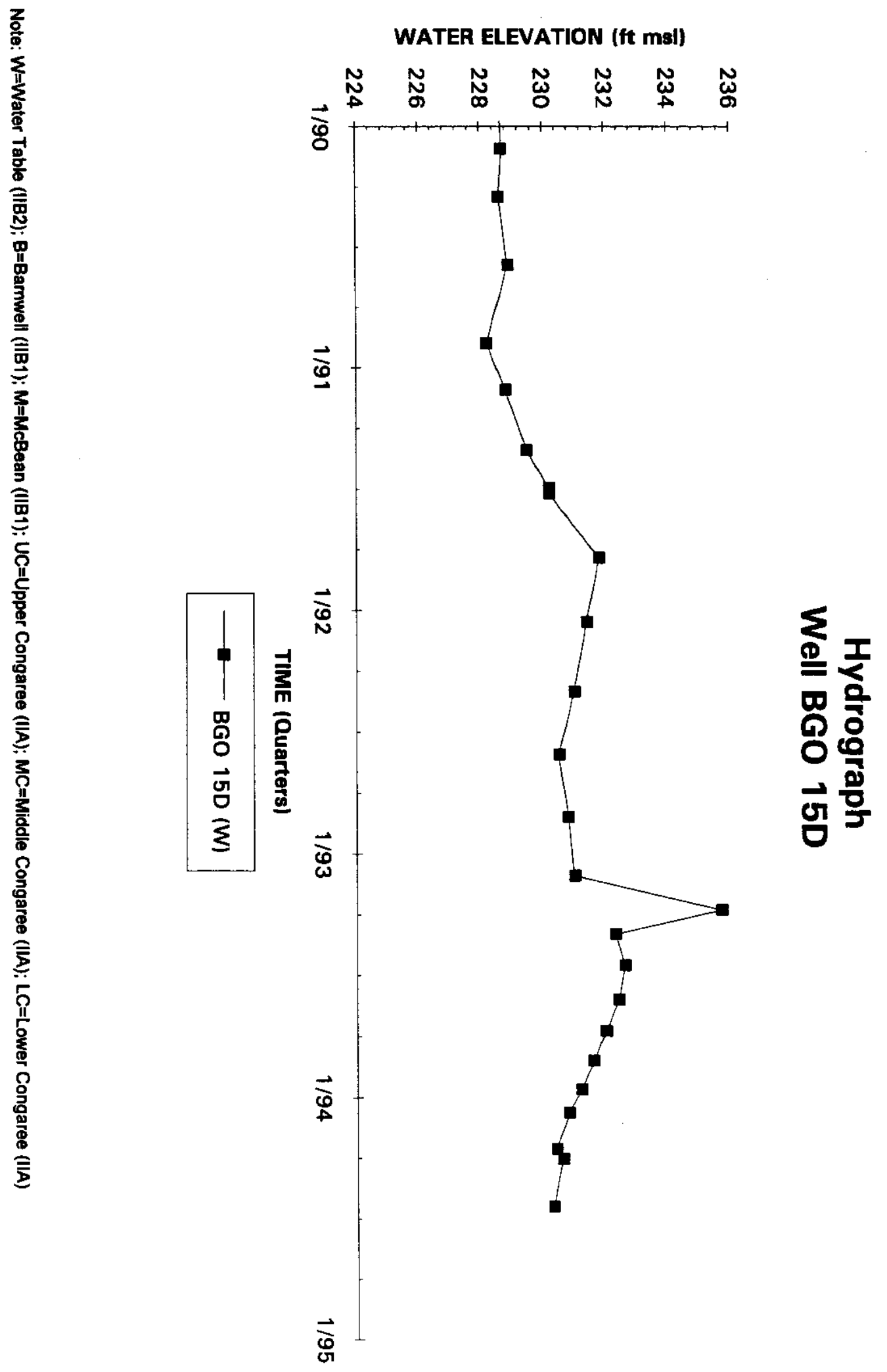

\begin{tabular}{l}
0 \\
8 \\
0 \\
0 \\
0 \\
5 \\
7 \\
\hline \\
\hline
\end{tabular}

勇 


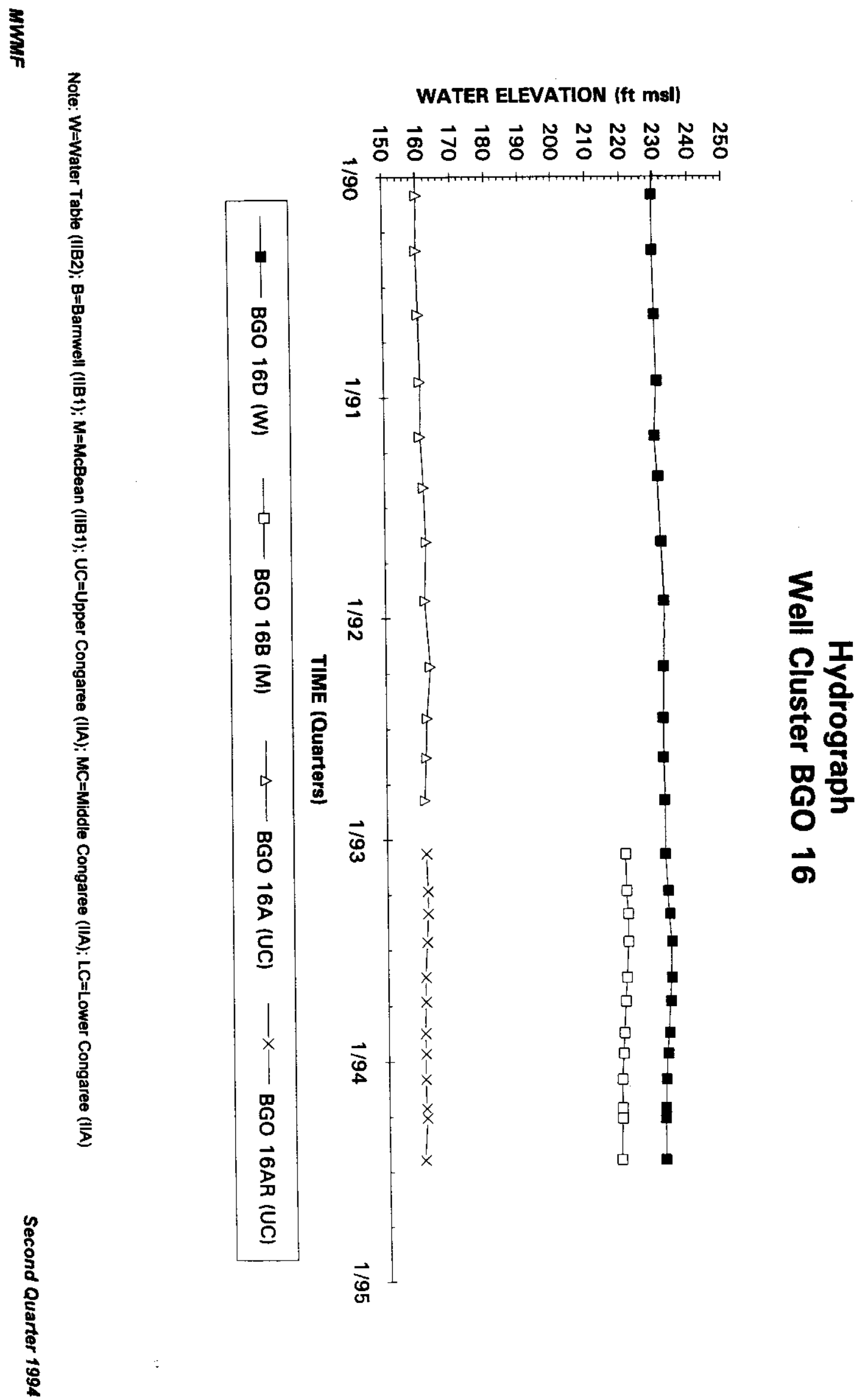

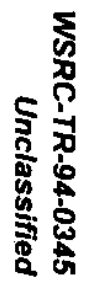


WATER ELEVATION (ft msl)

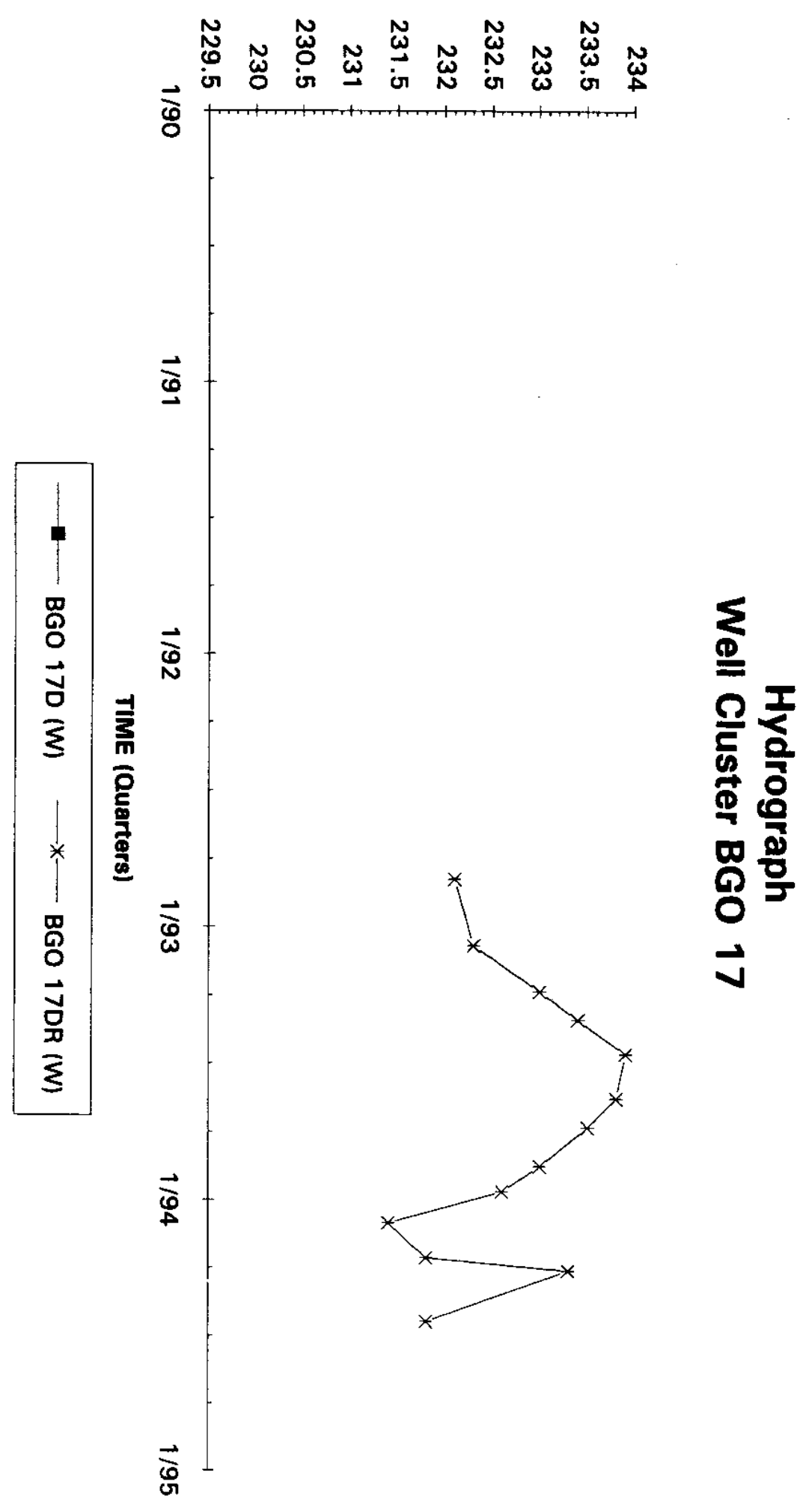



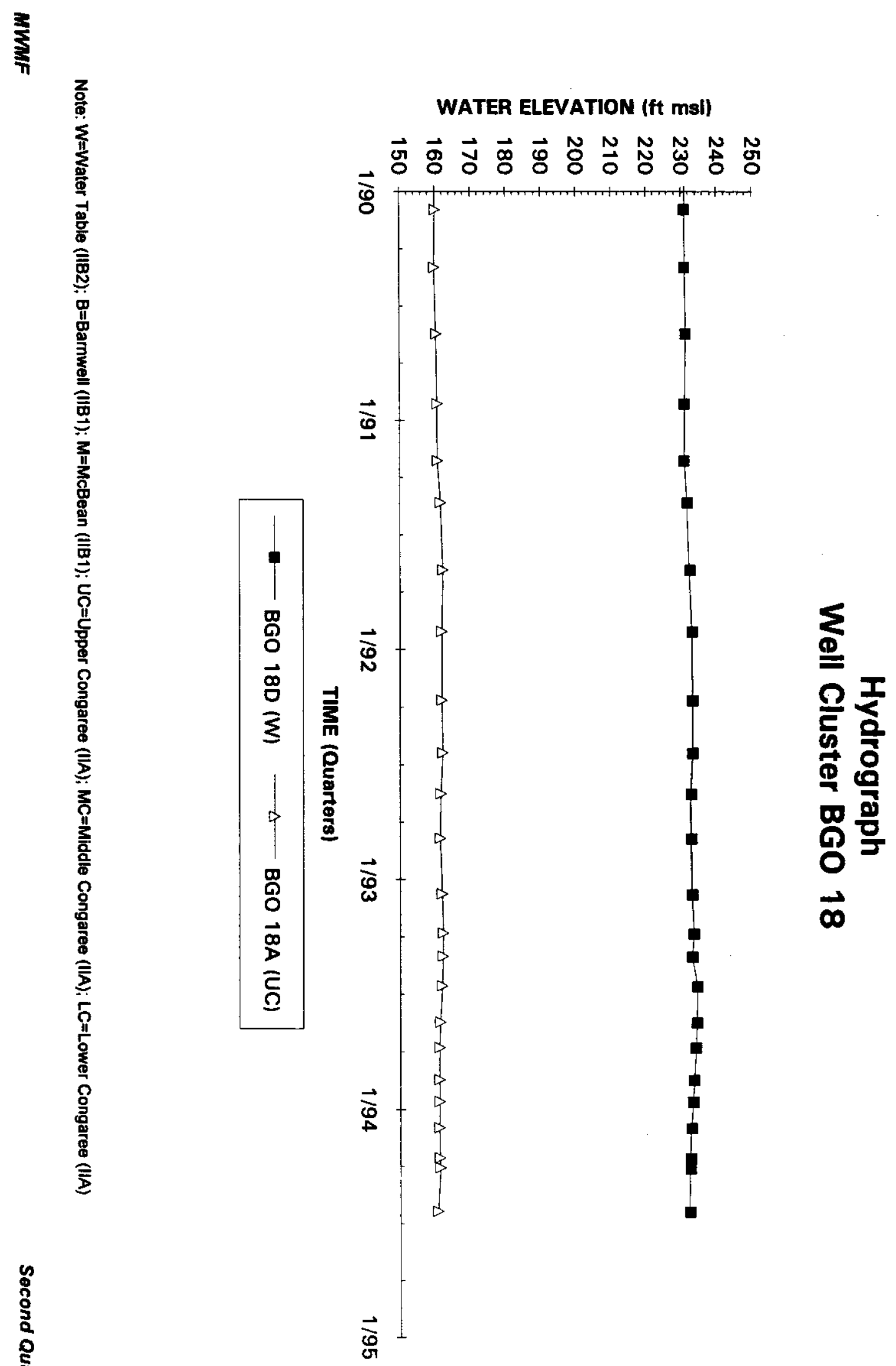

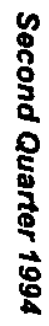

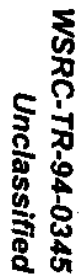


WATER ELEVATION (ft msl)

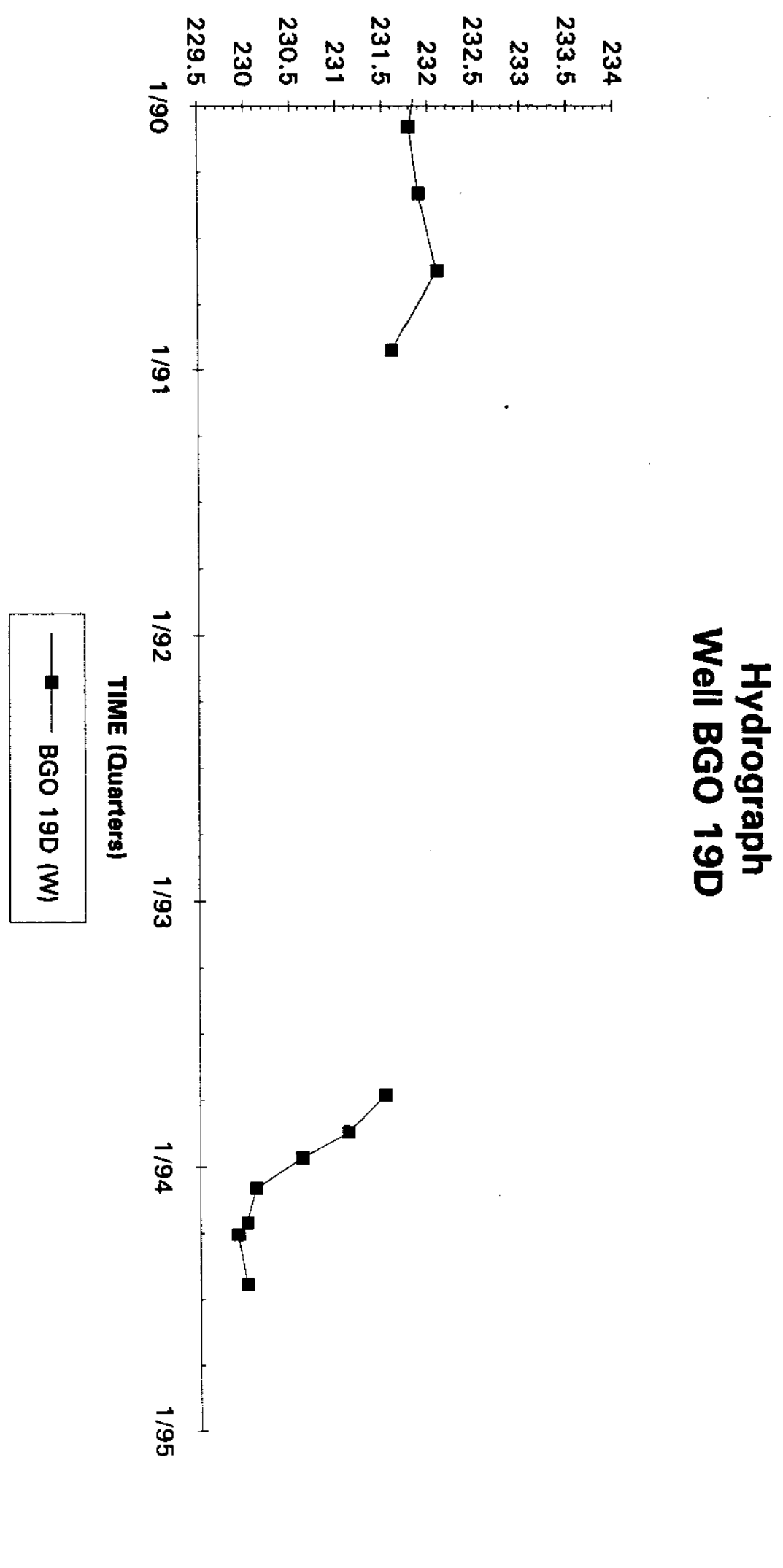



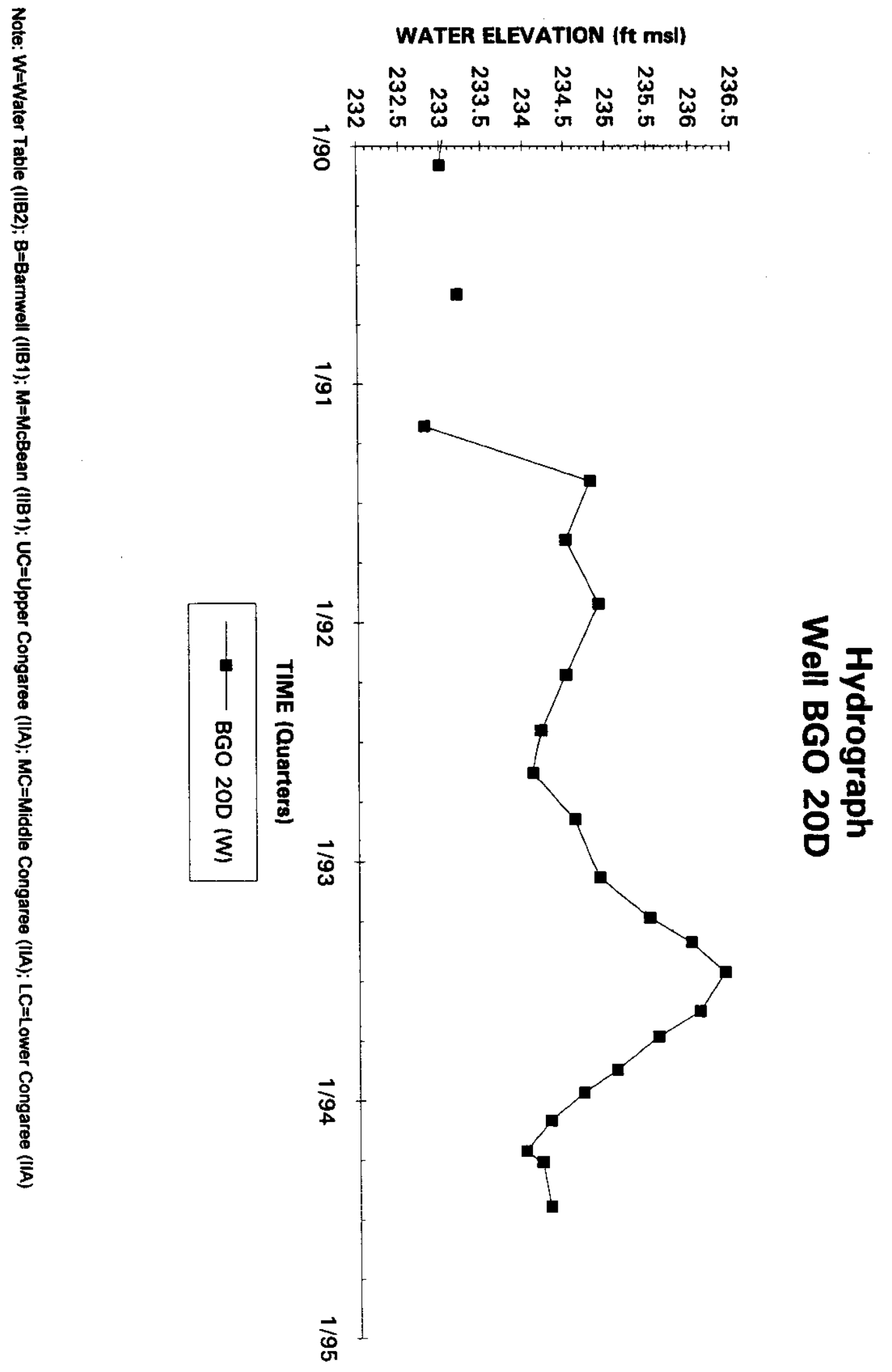

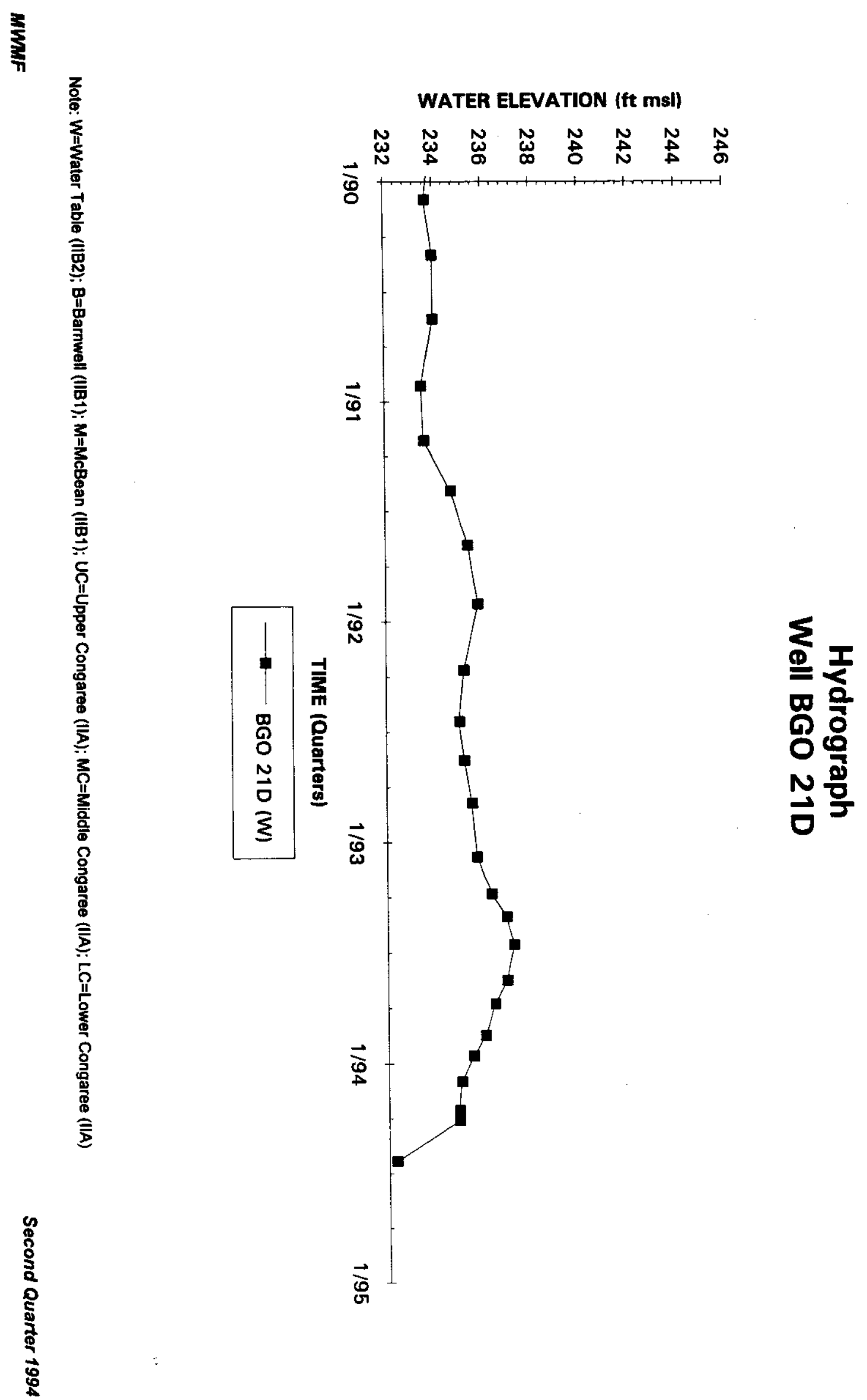

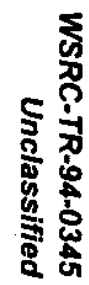



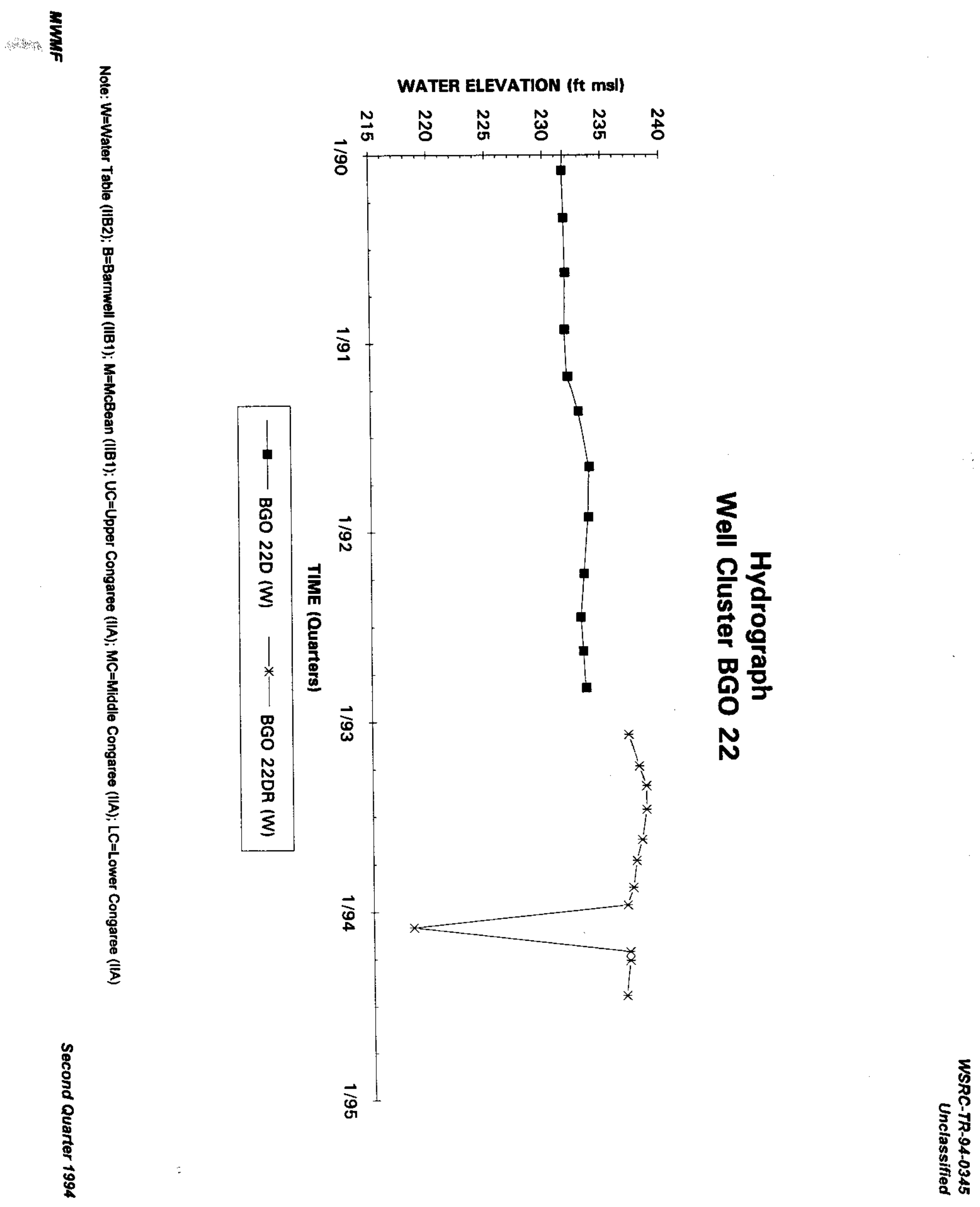


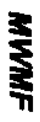

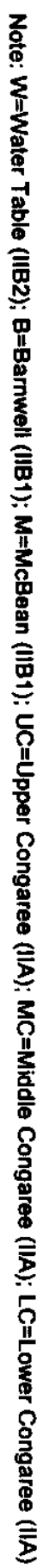

WATER ELEVATION (ft msl)

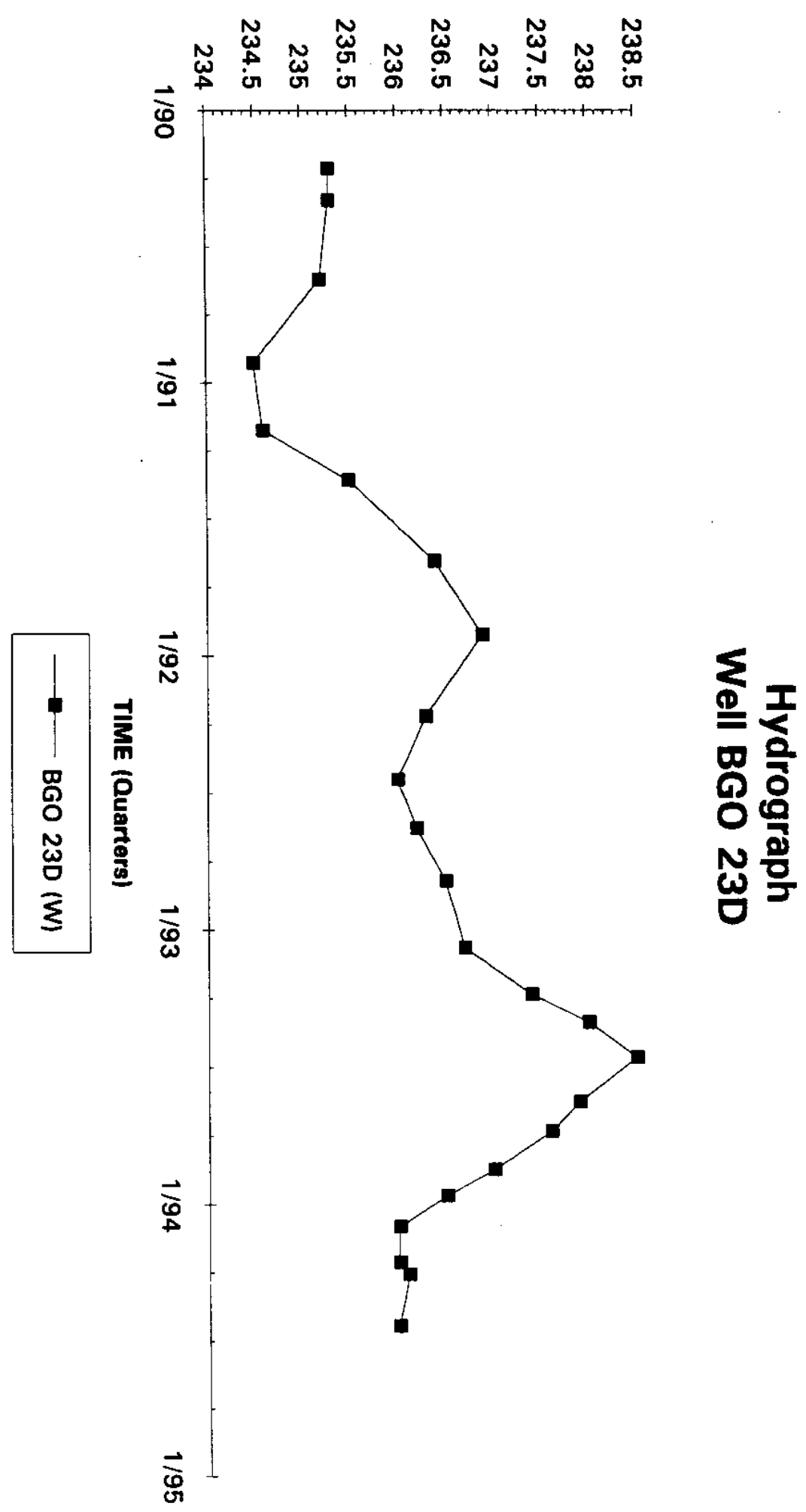

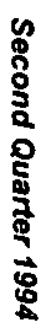

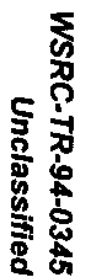



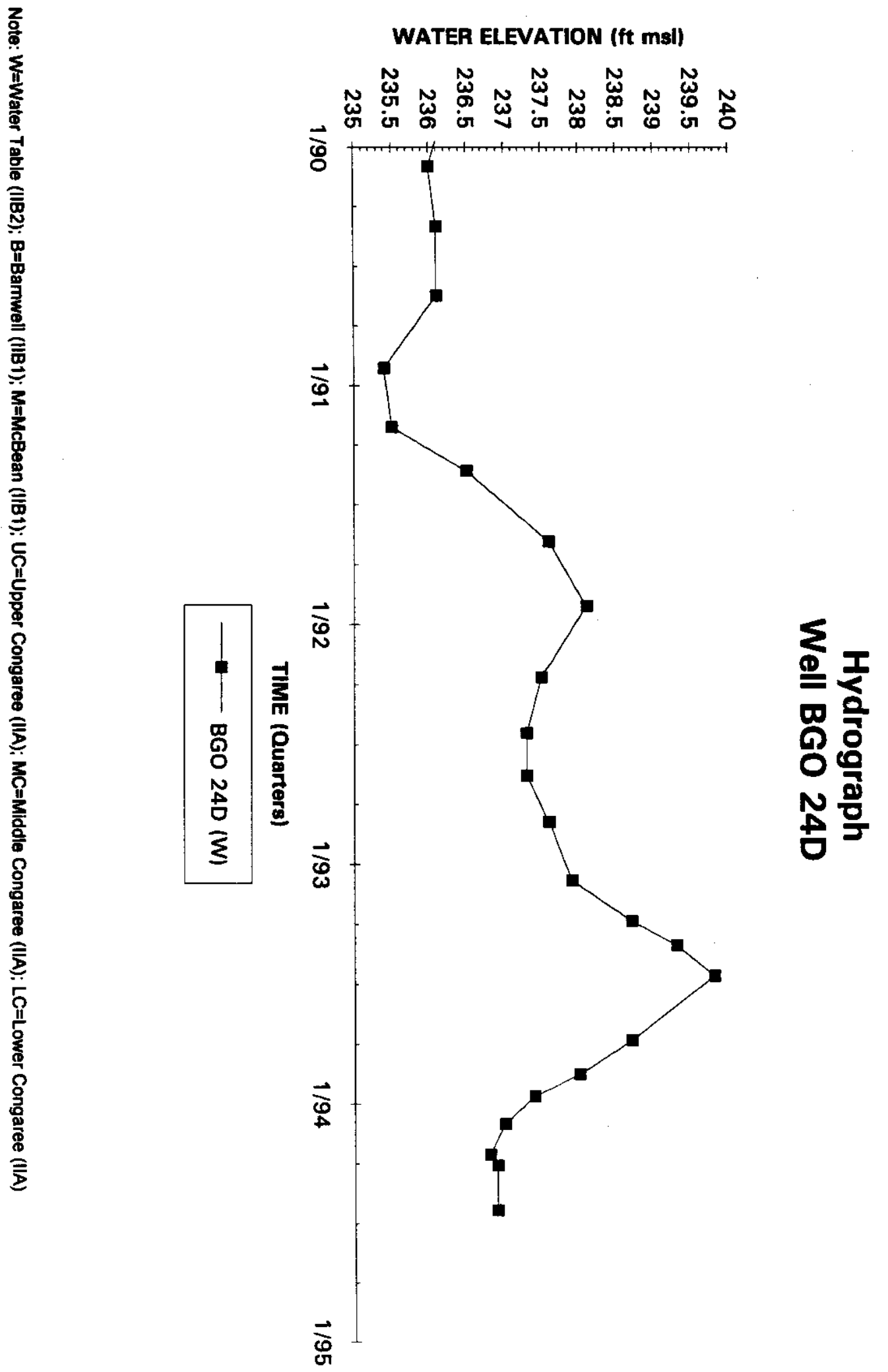

0
8
8
0
0
5
0
0
8
8

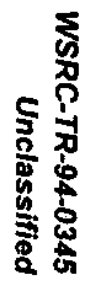



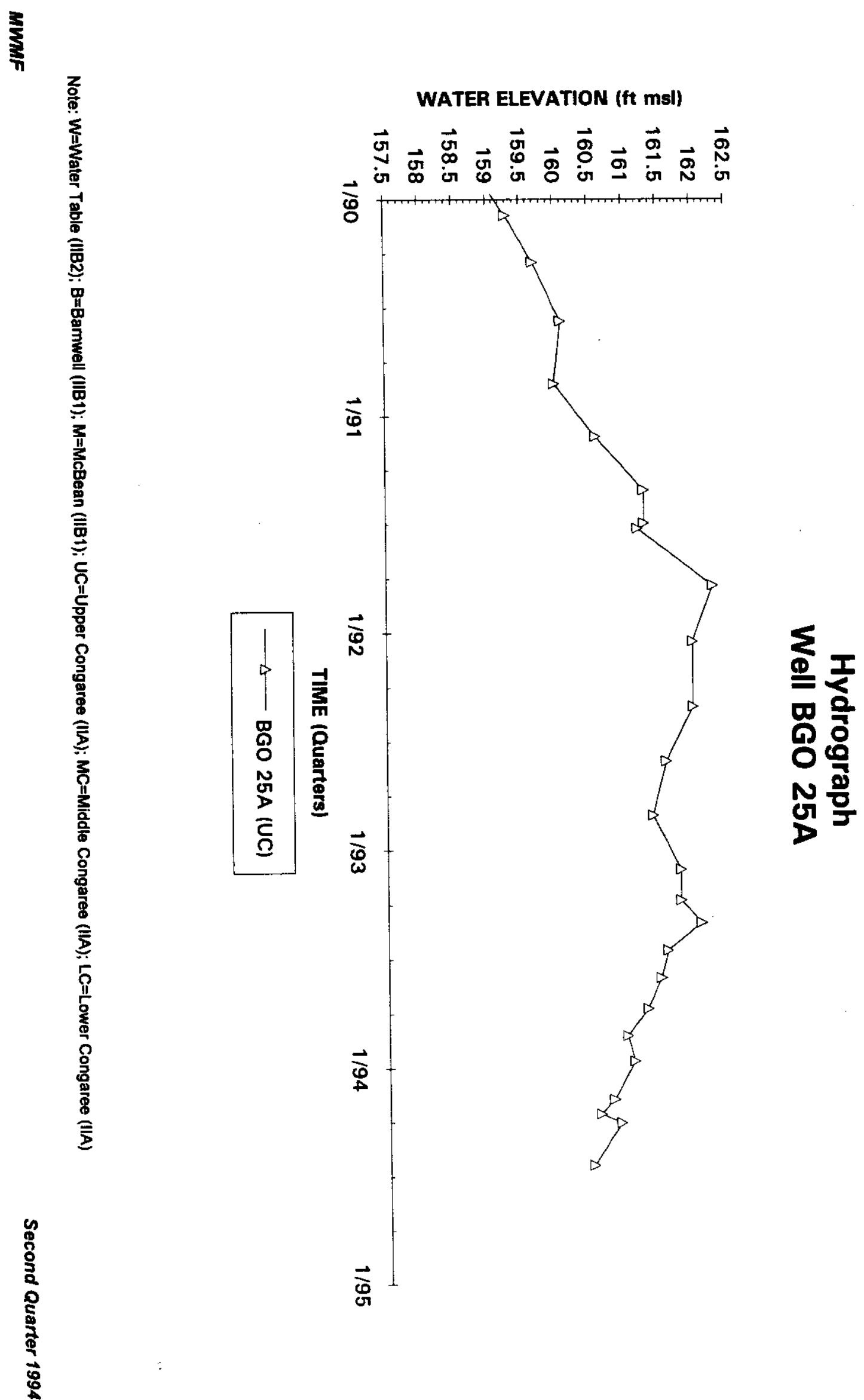

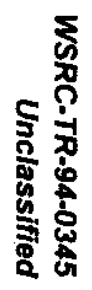




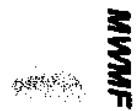

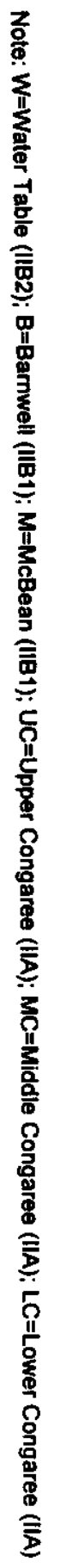

0
8
8
0
0
0
9
0
8
8

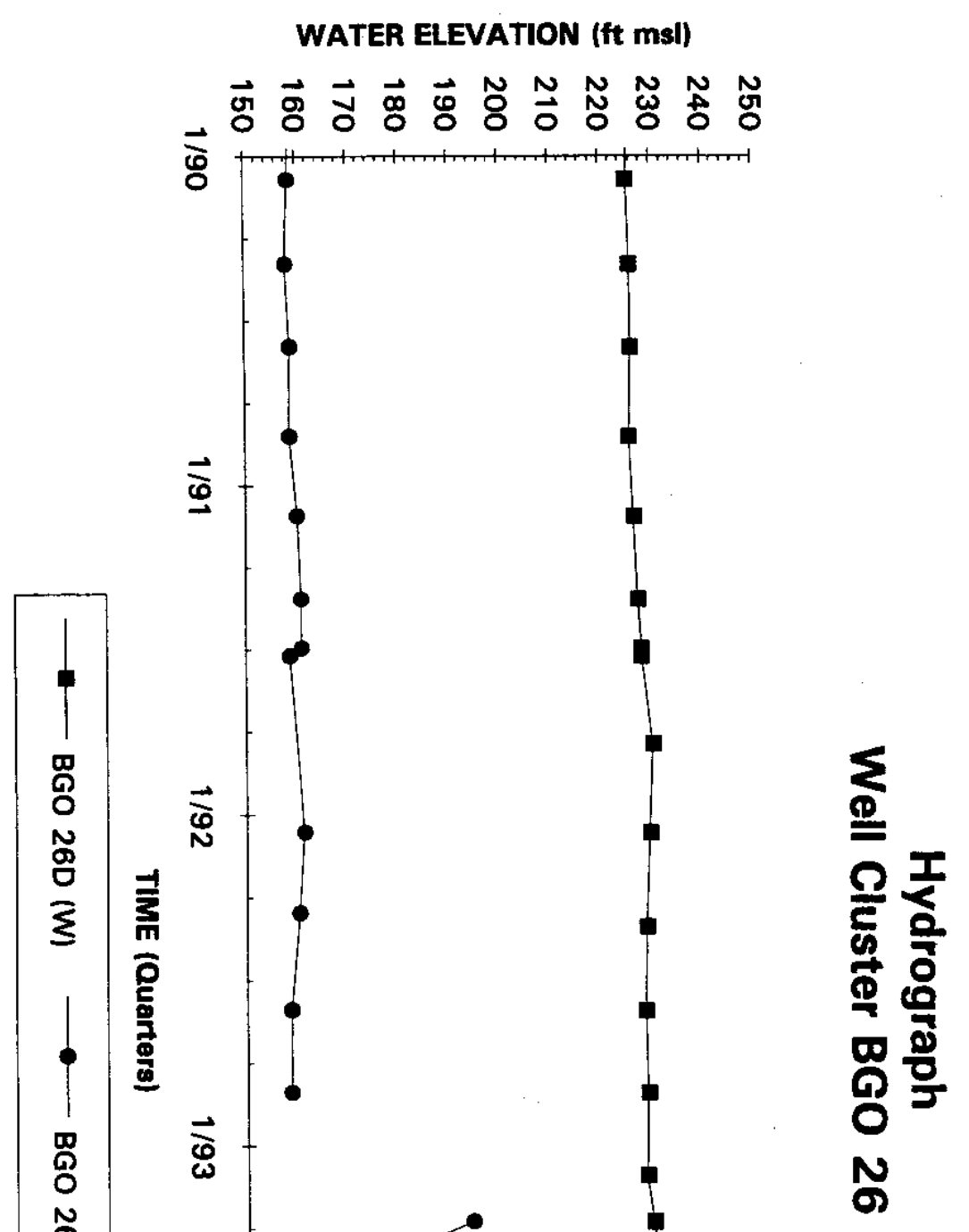

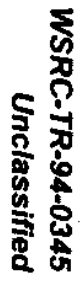




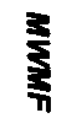

WATER ELEVATION (ft msi)

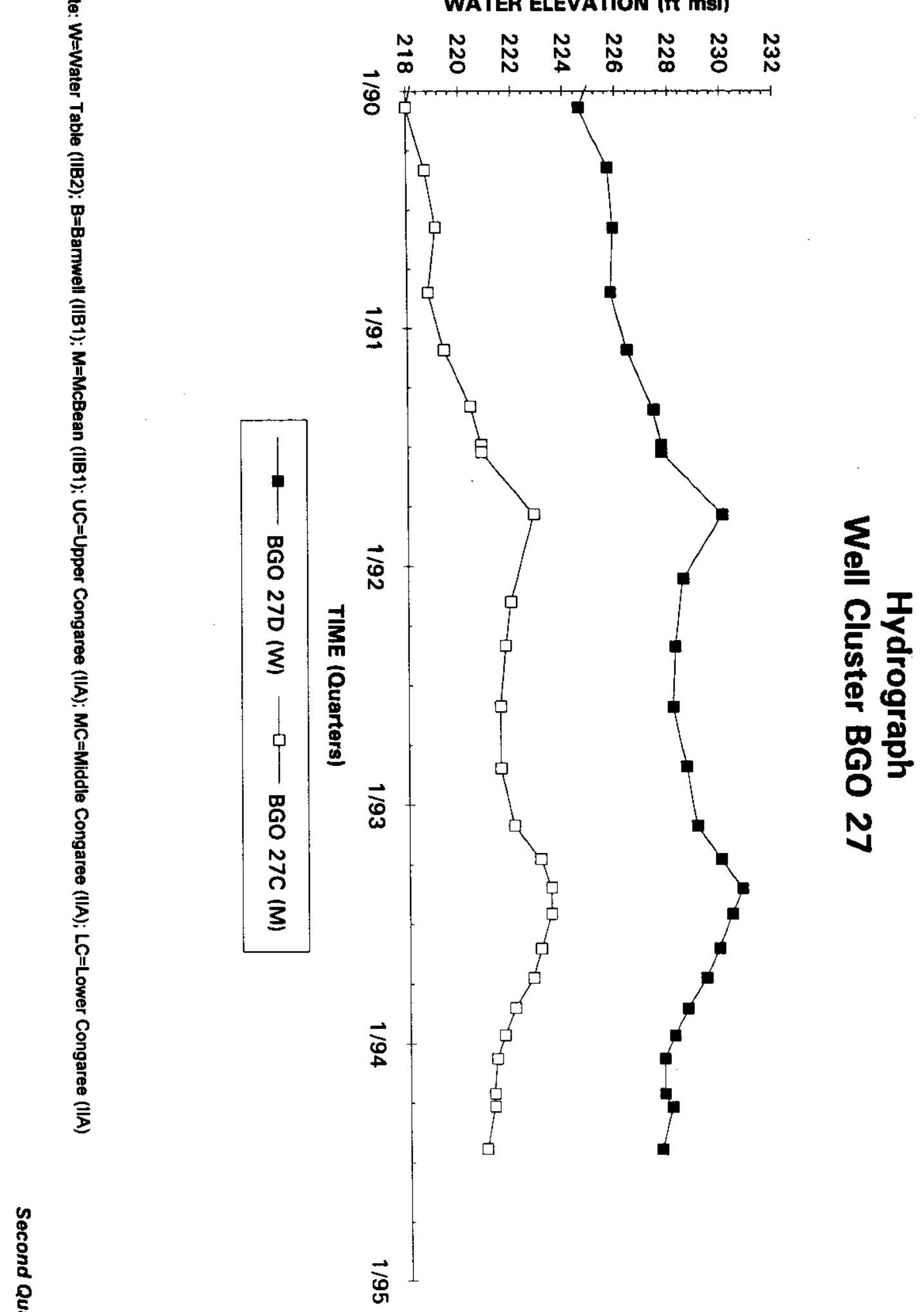


$\operatorname{Arm}$

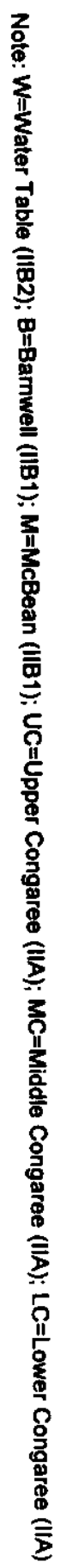

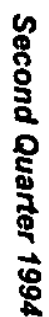

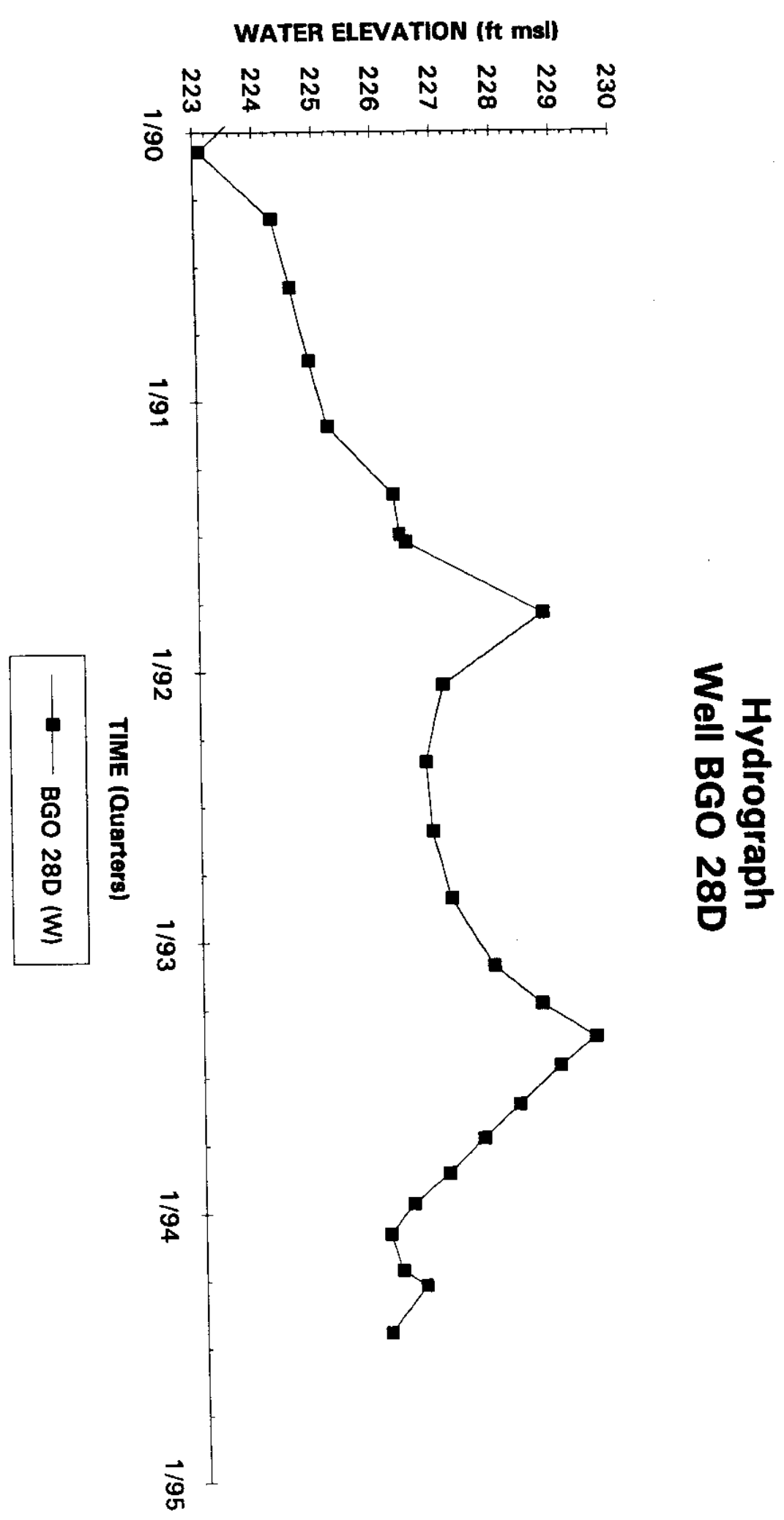

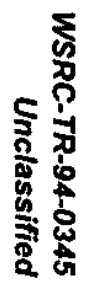


涌

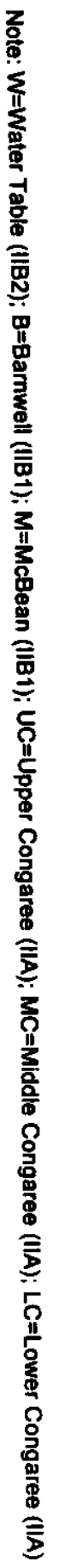

WATER ELEVATION (ft msl)

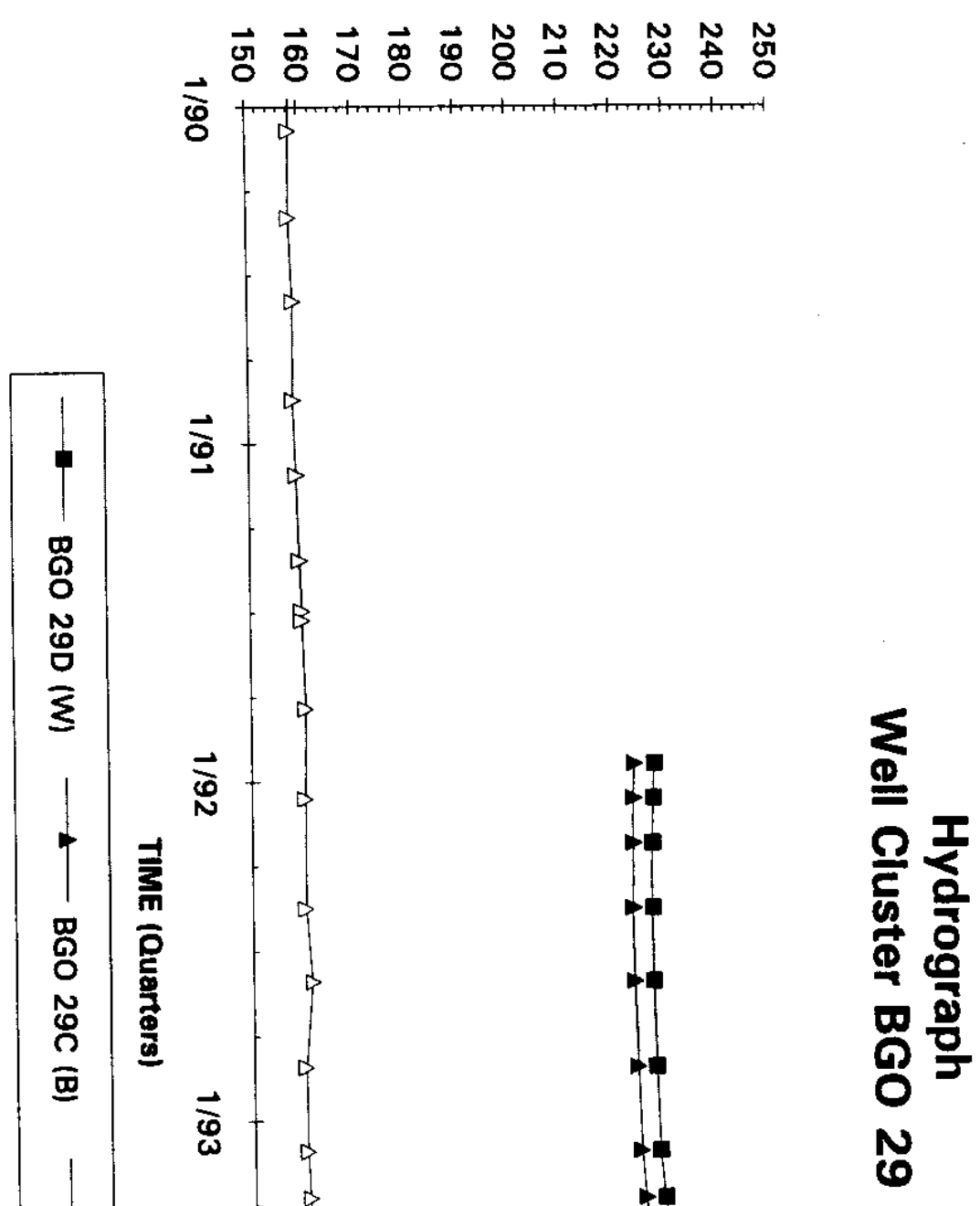


I

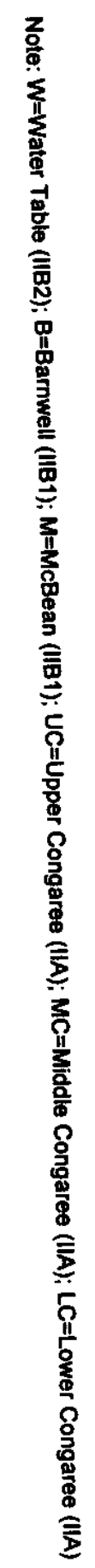

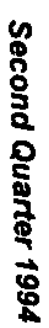

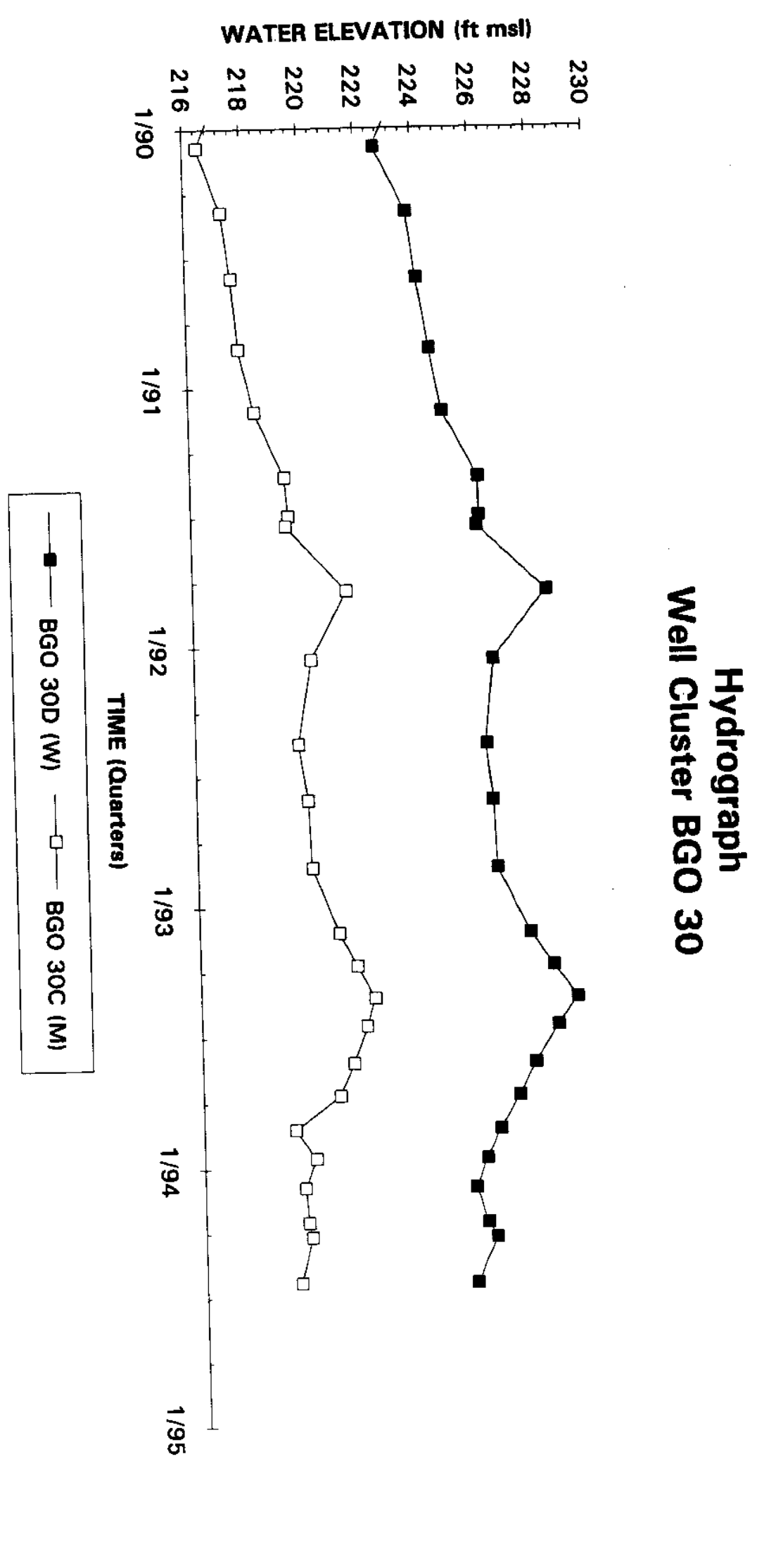

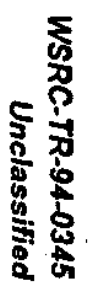



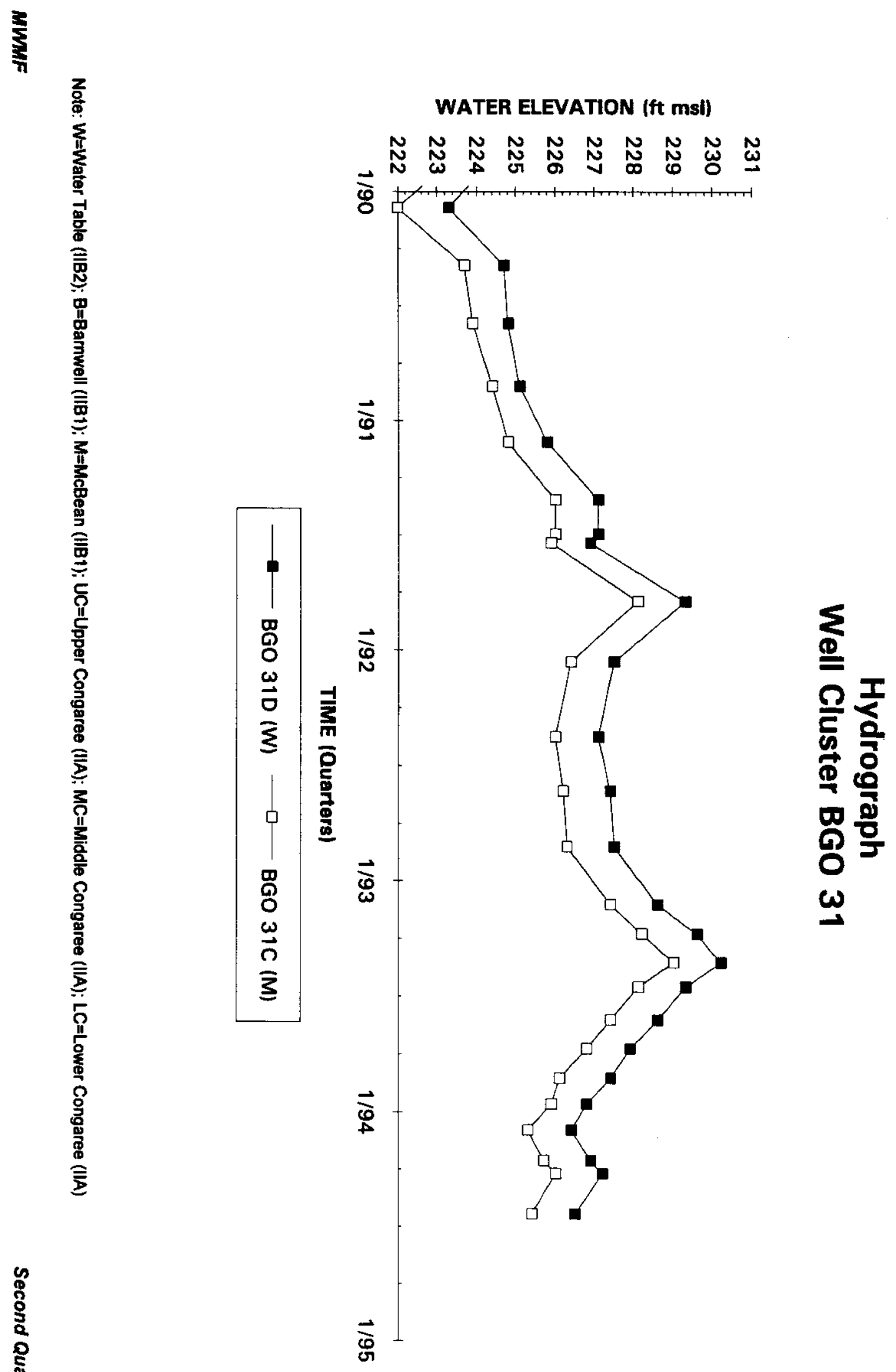

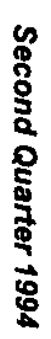


mant

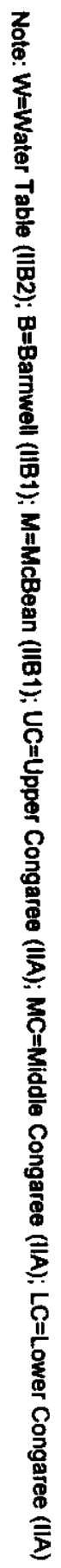

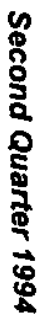

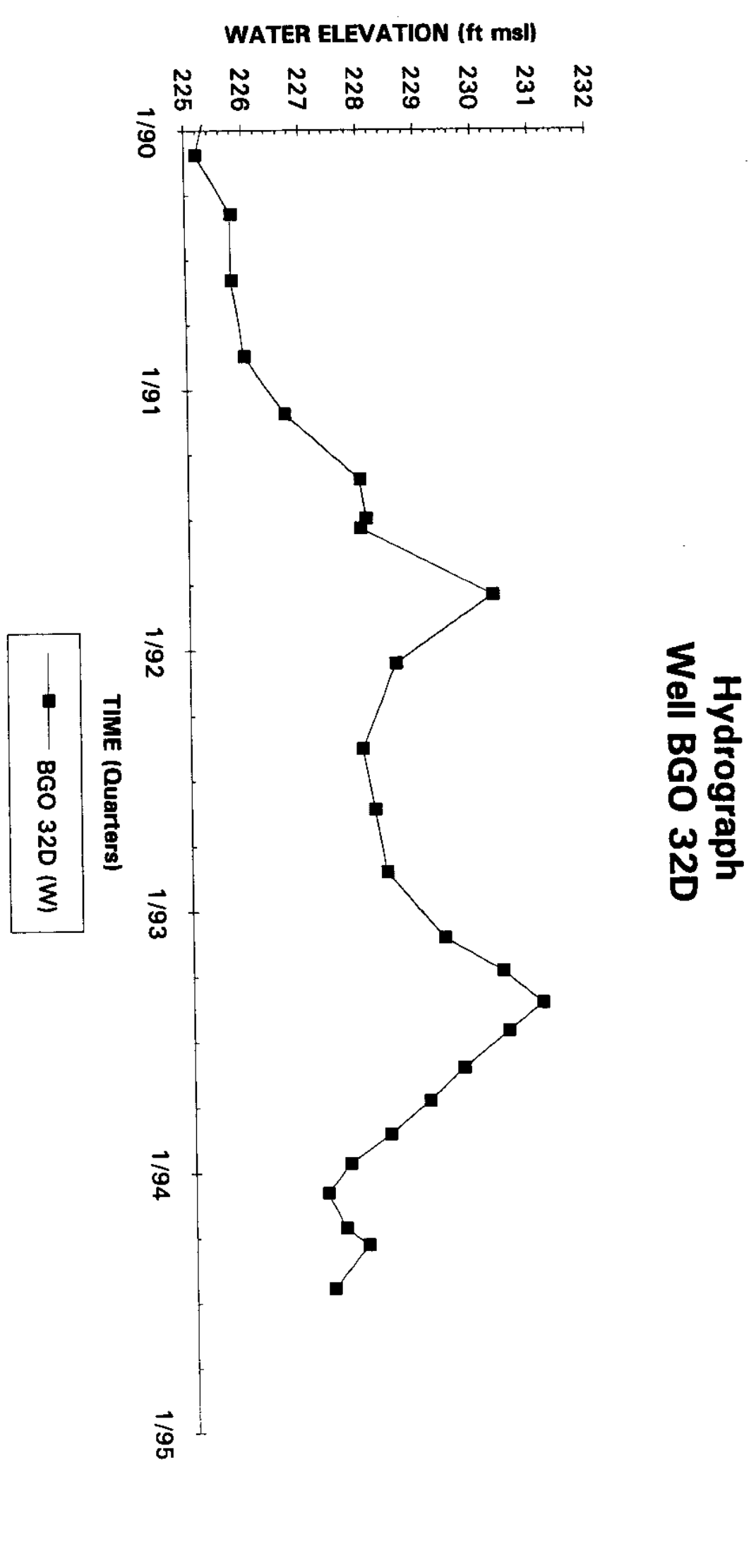

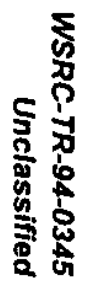



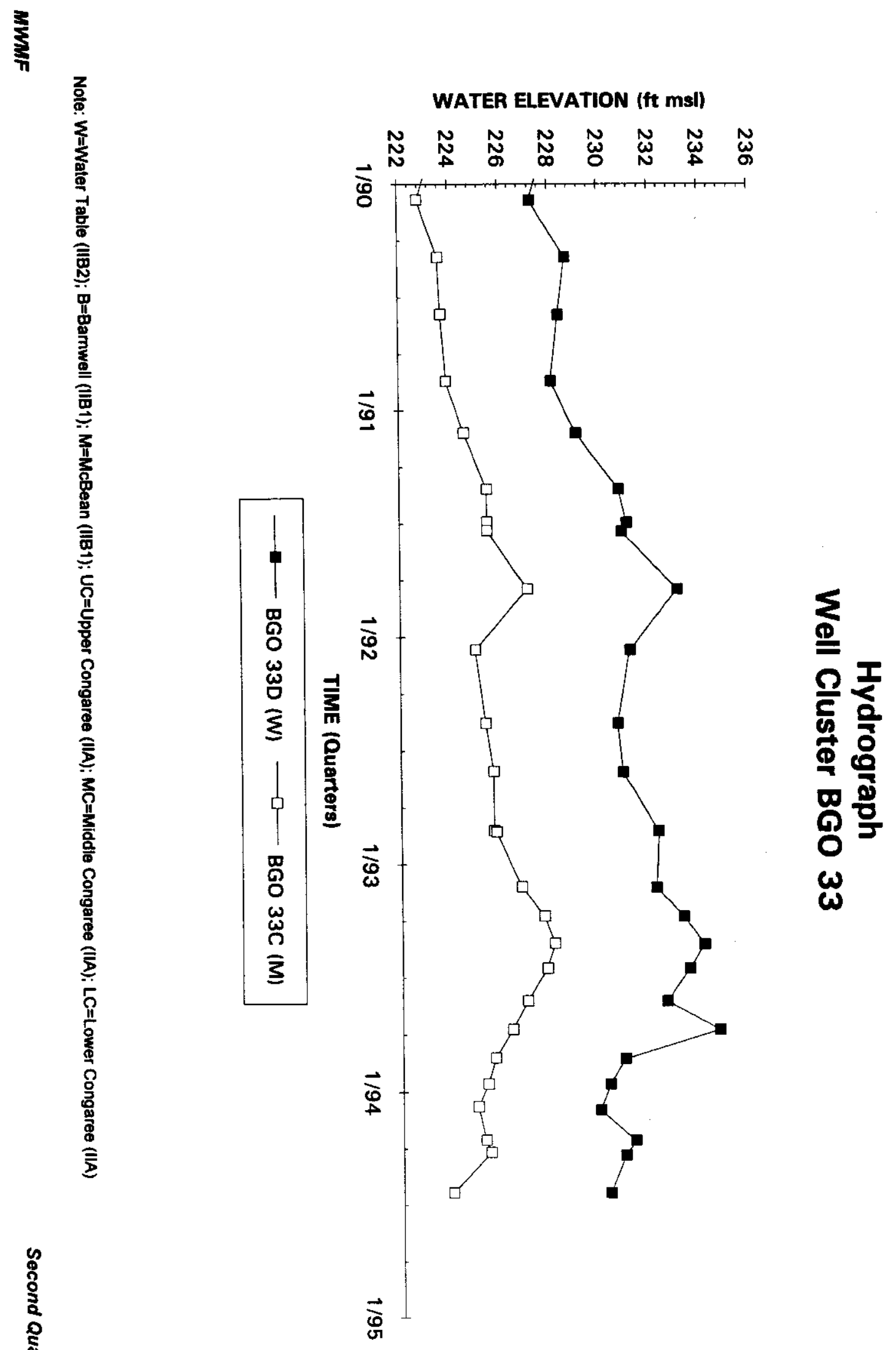

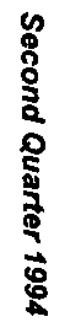

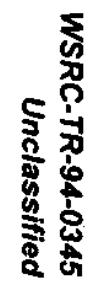


अ

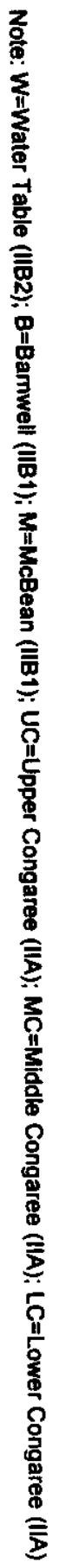

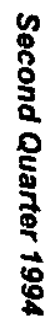

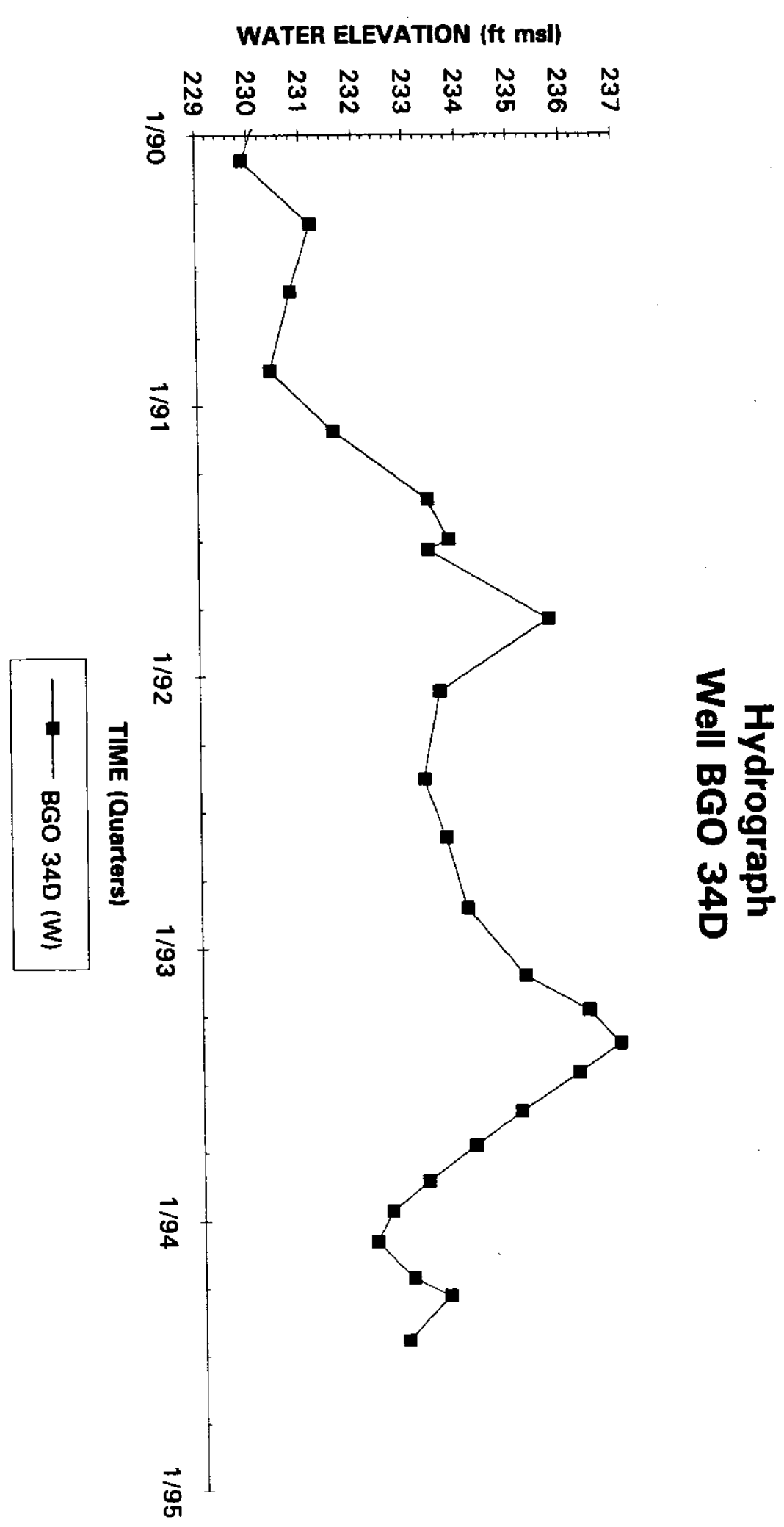

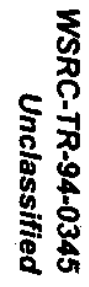




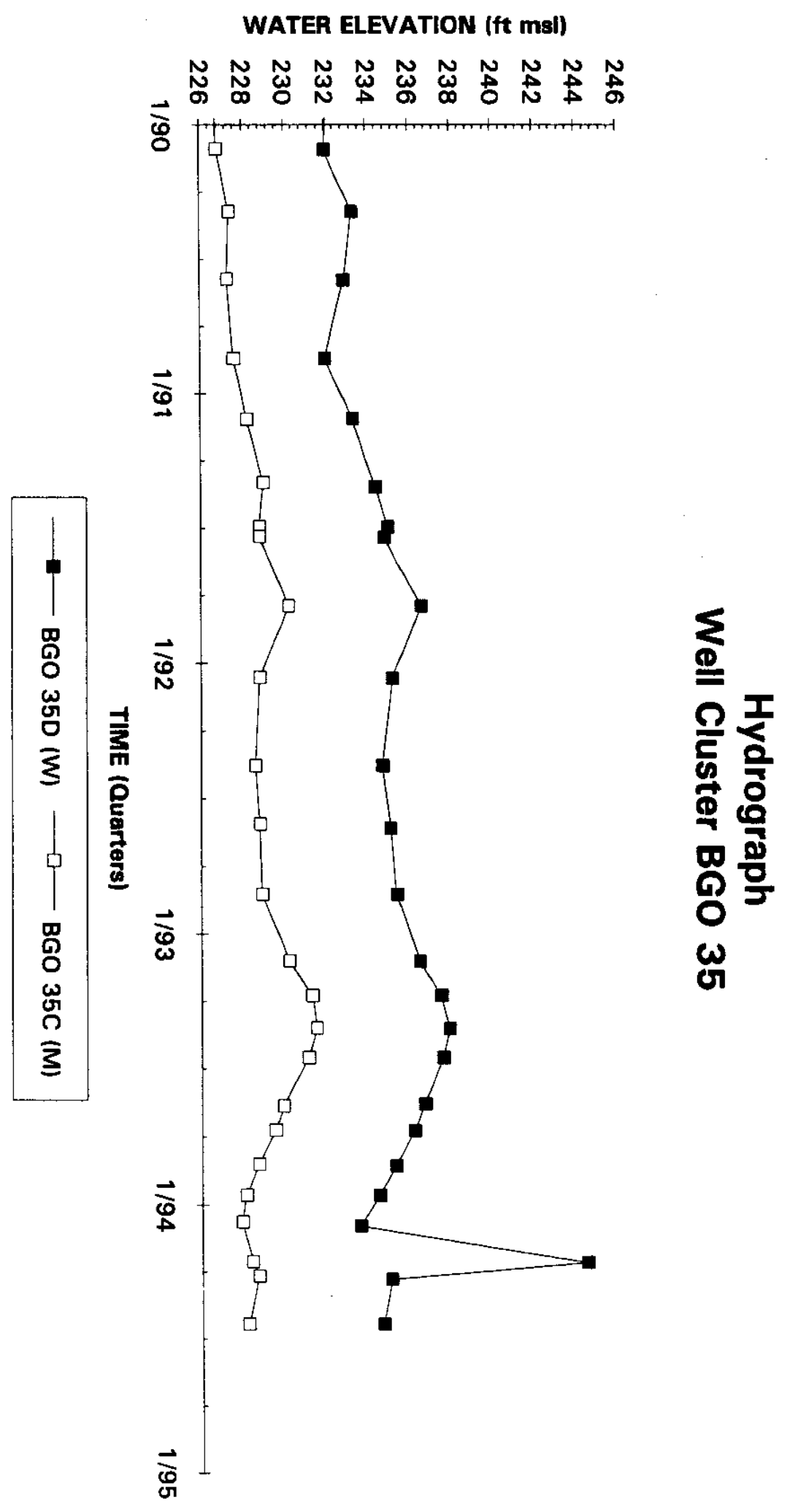




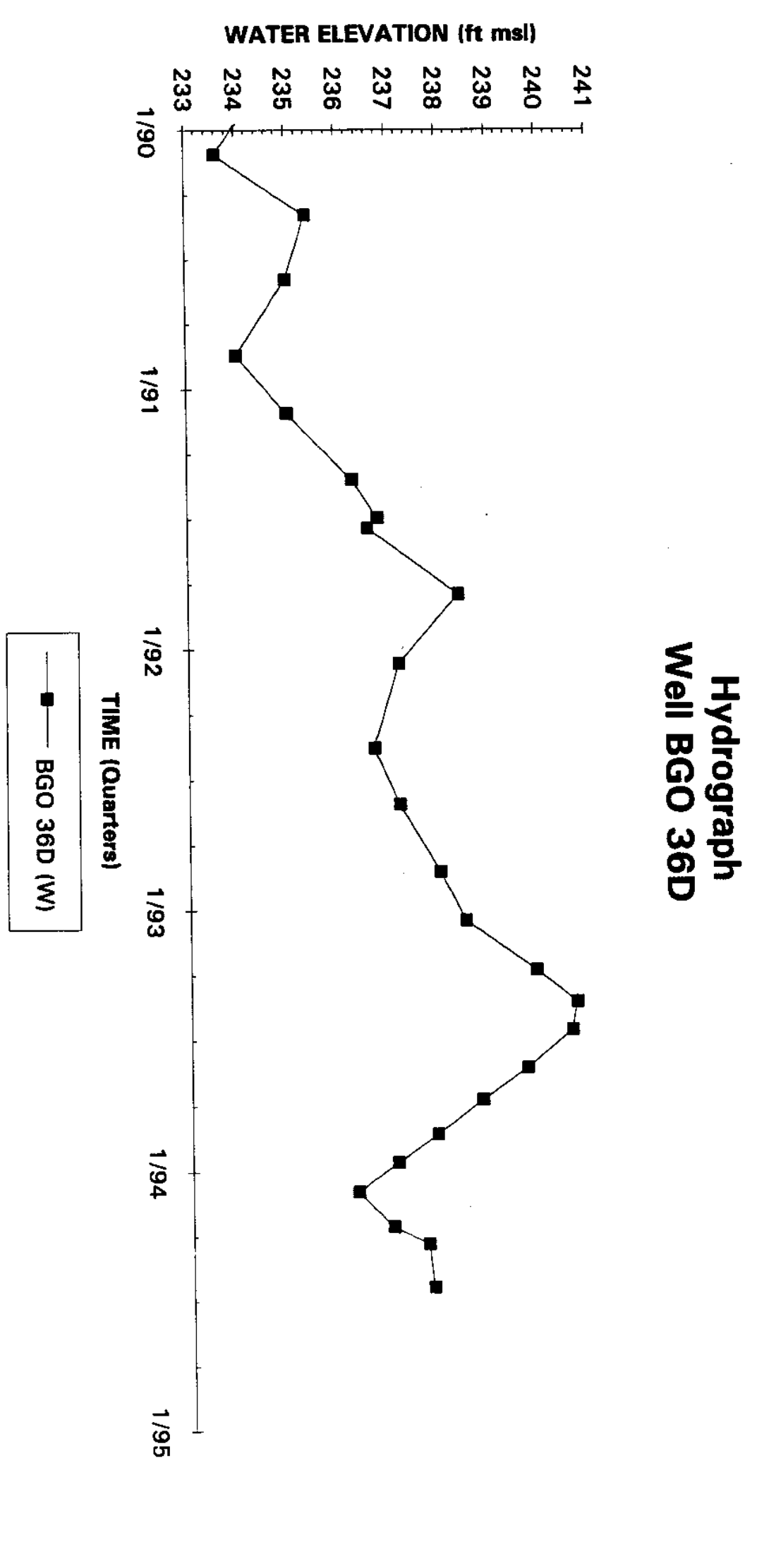




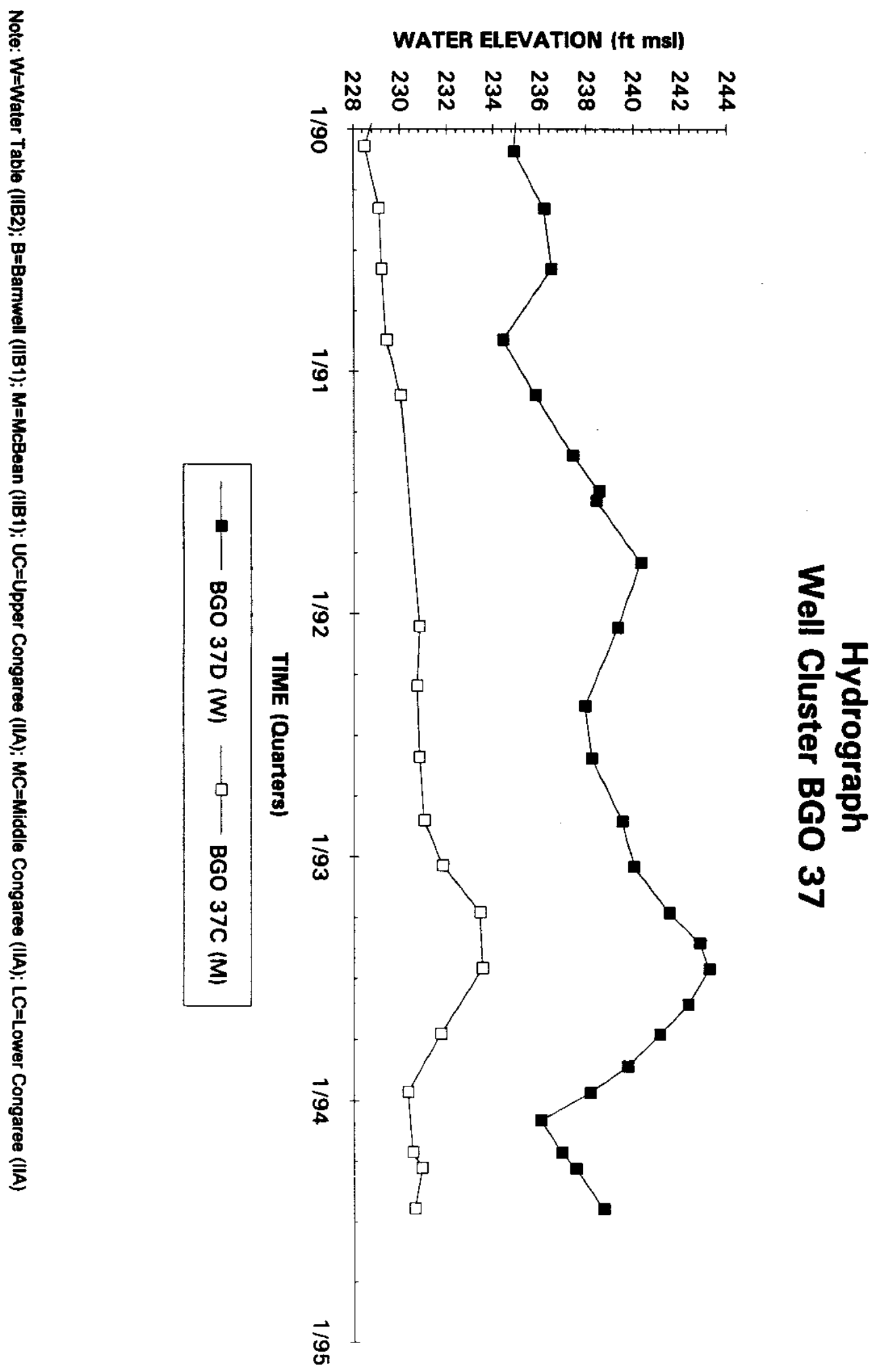



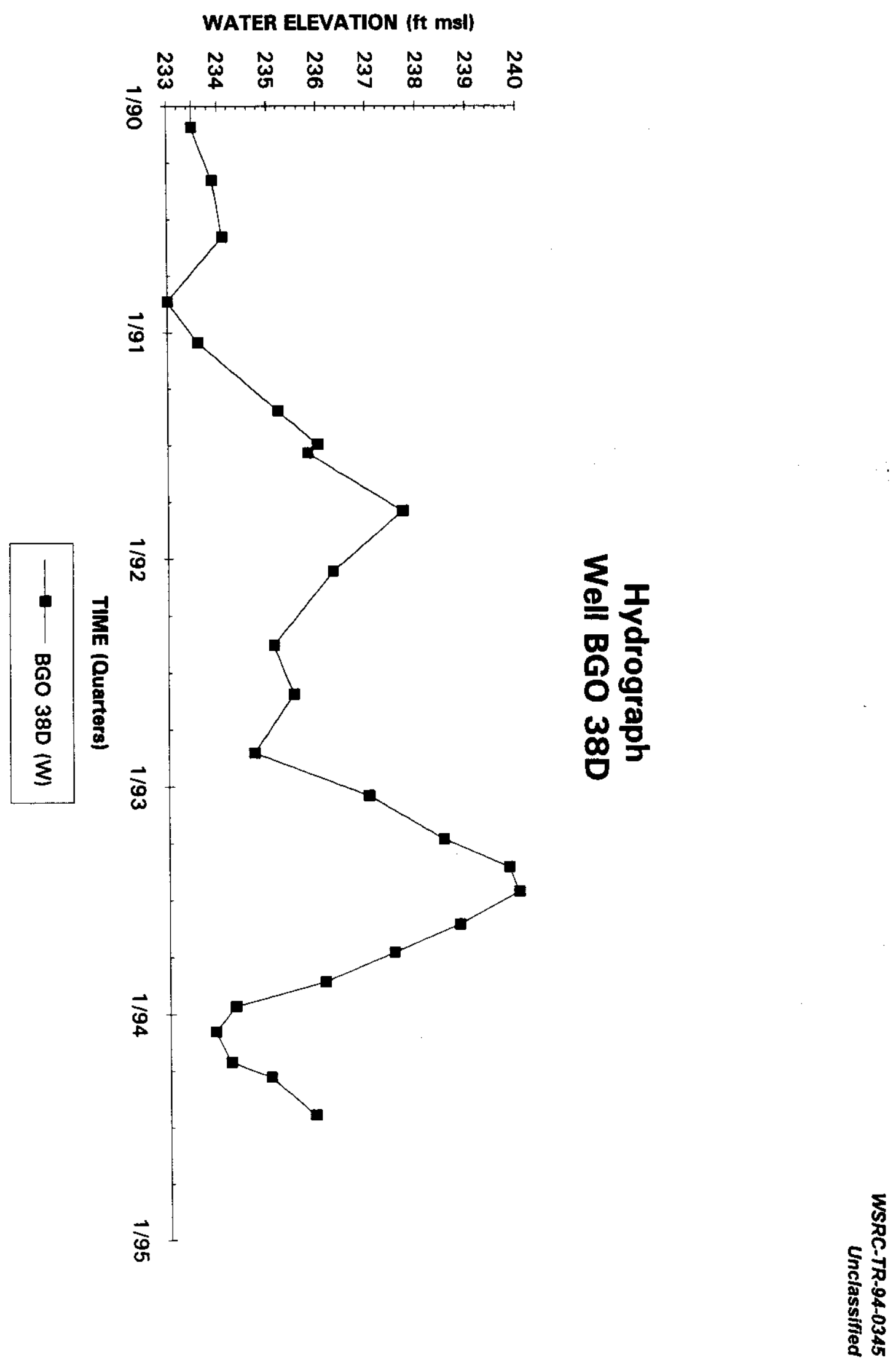


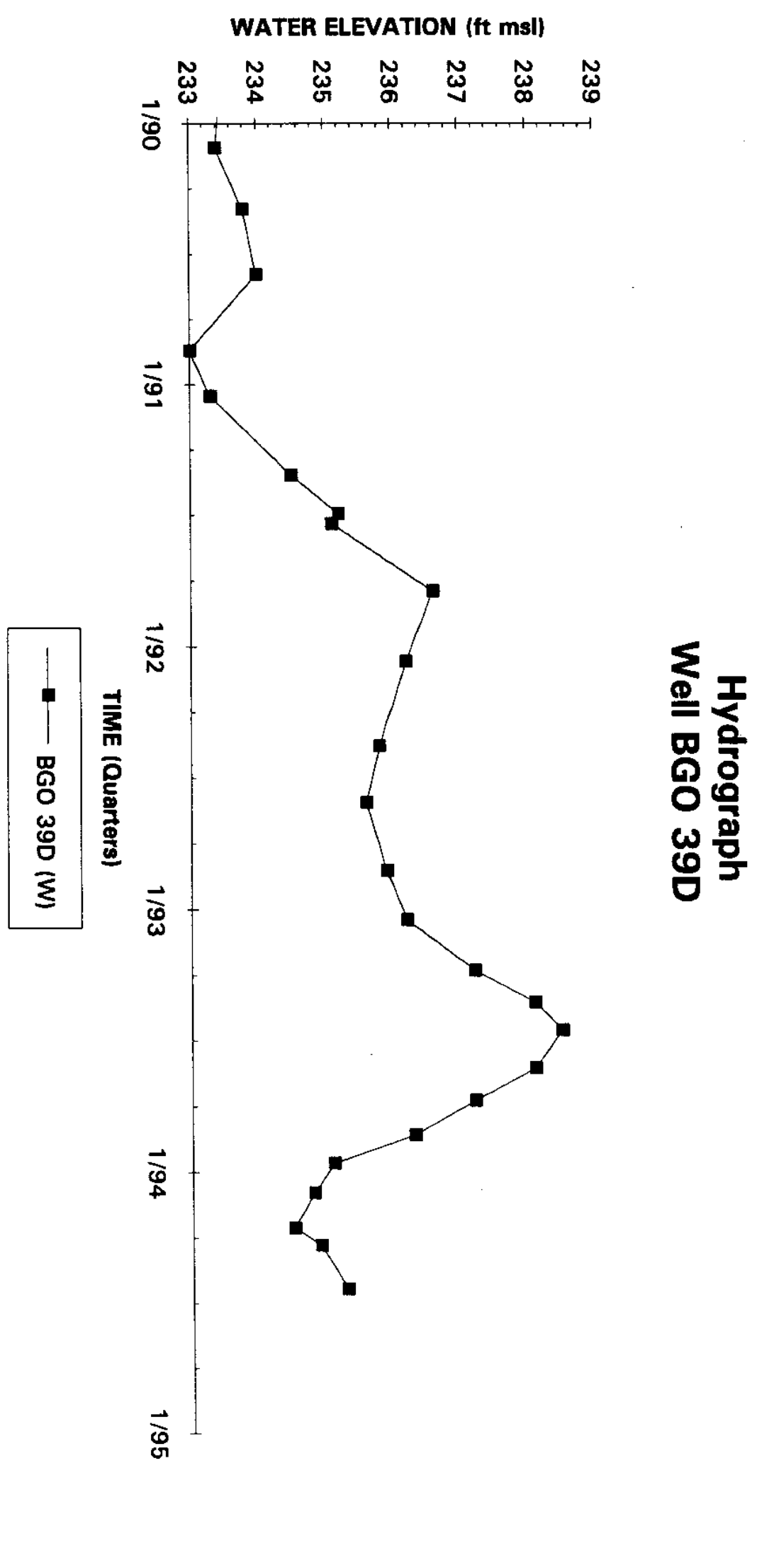

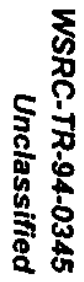



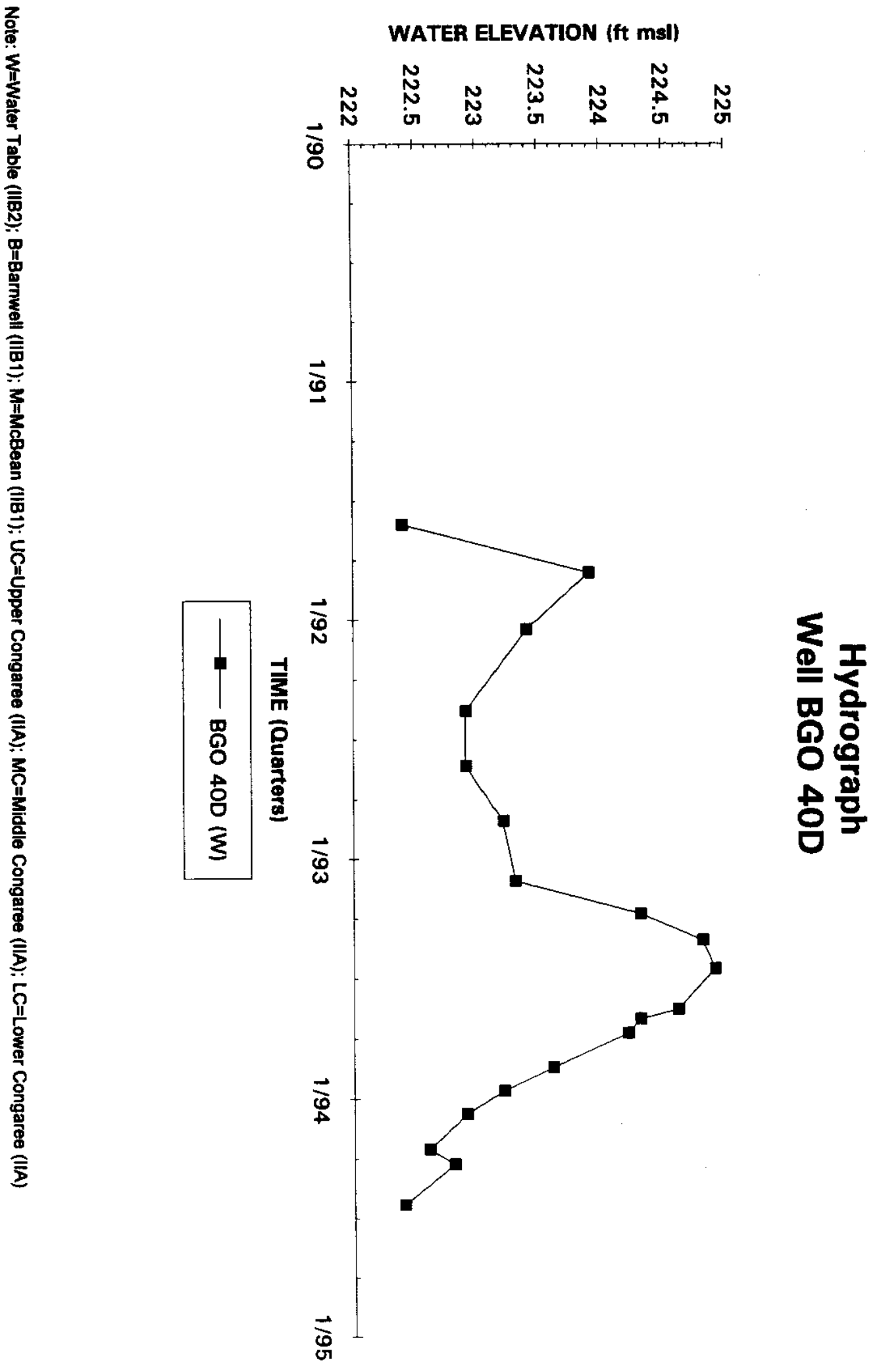

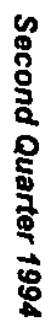

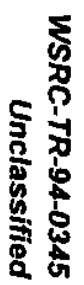



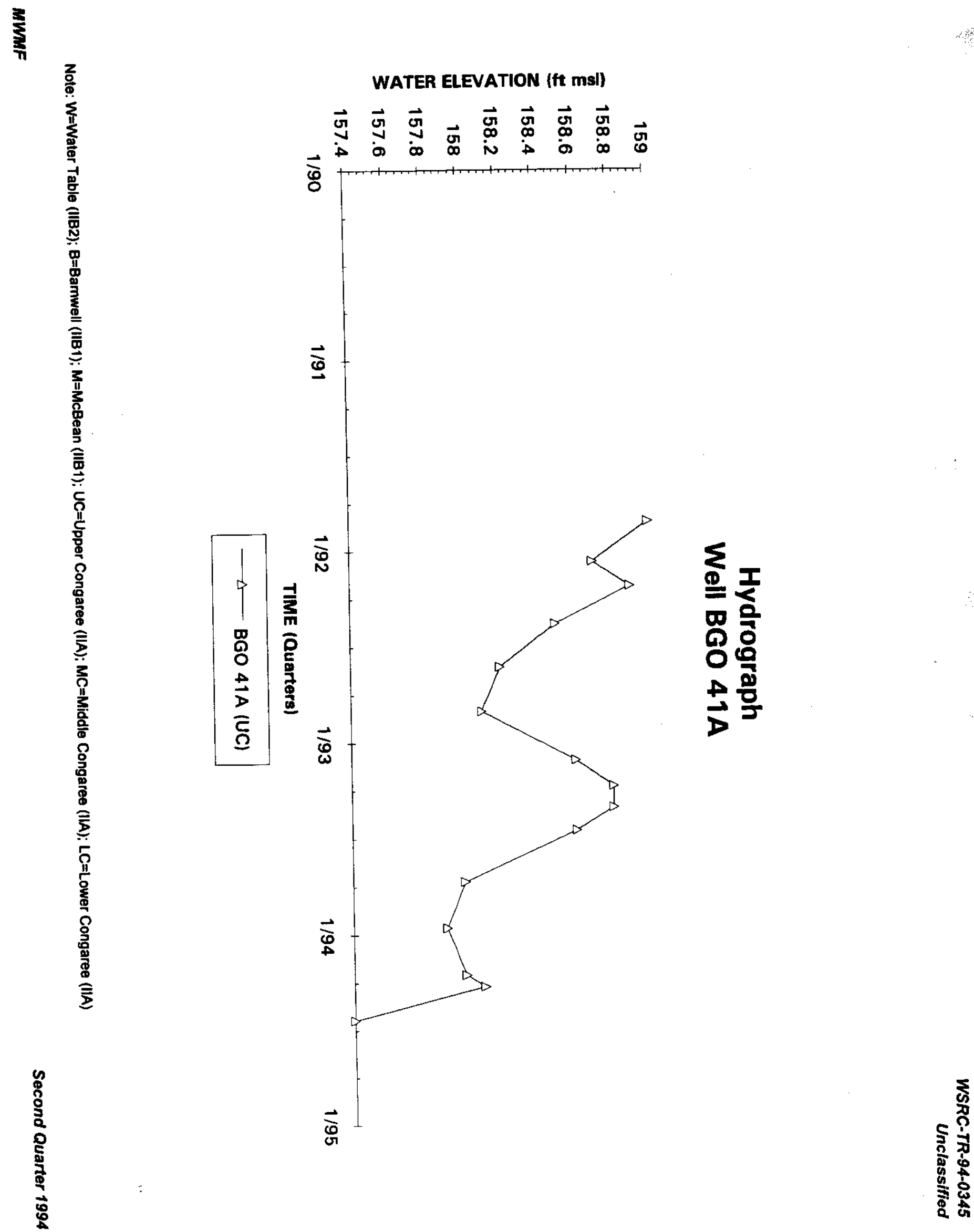
I

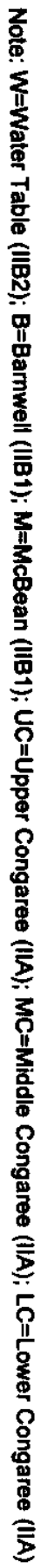

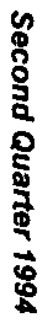

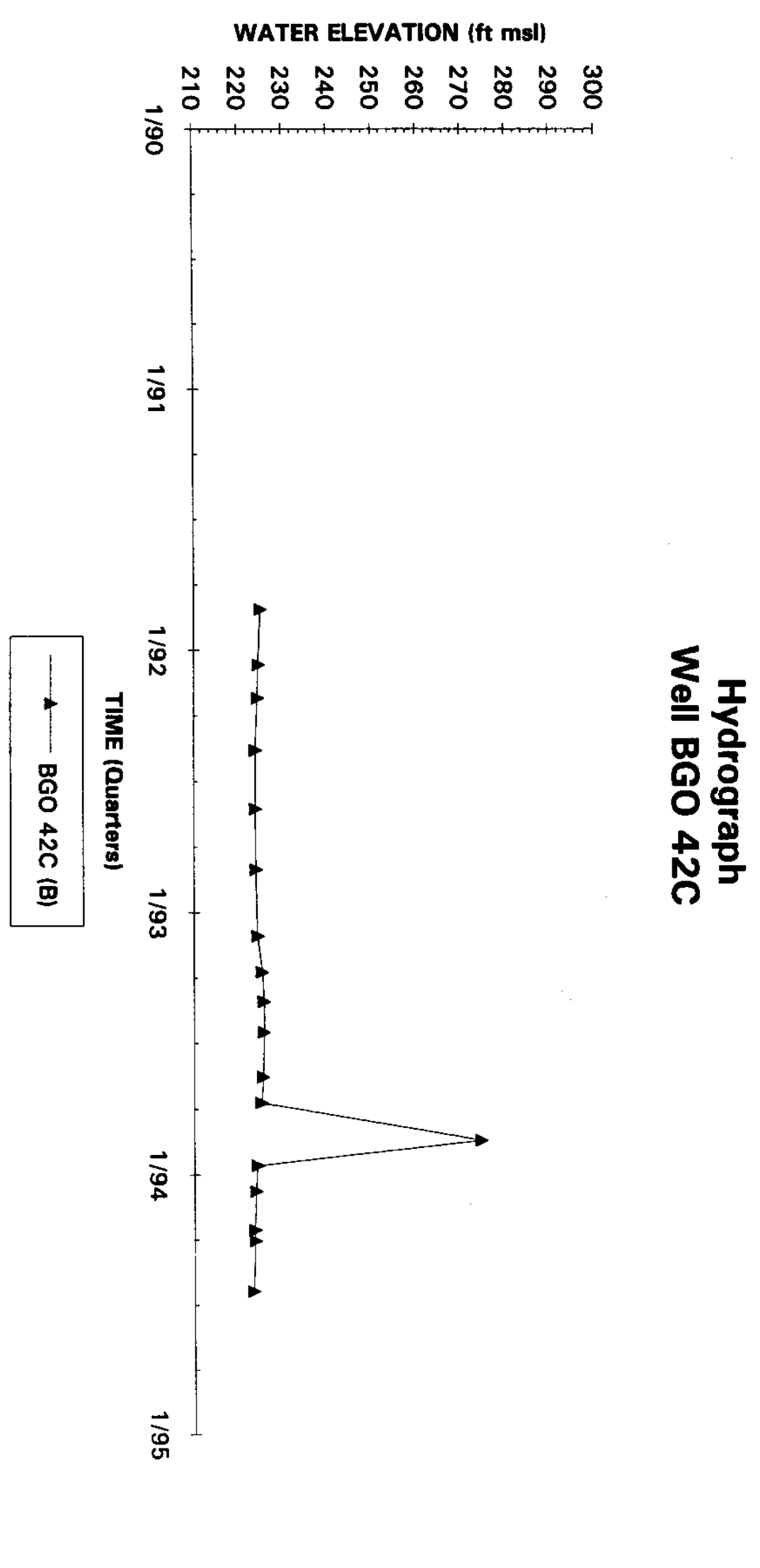




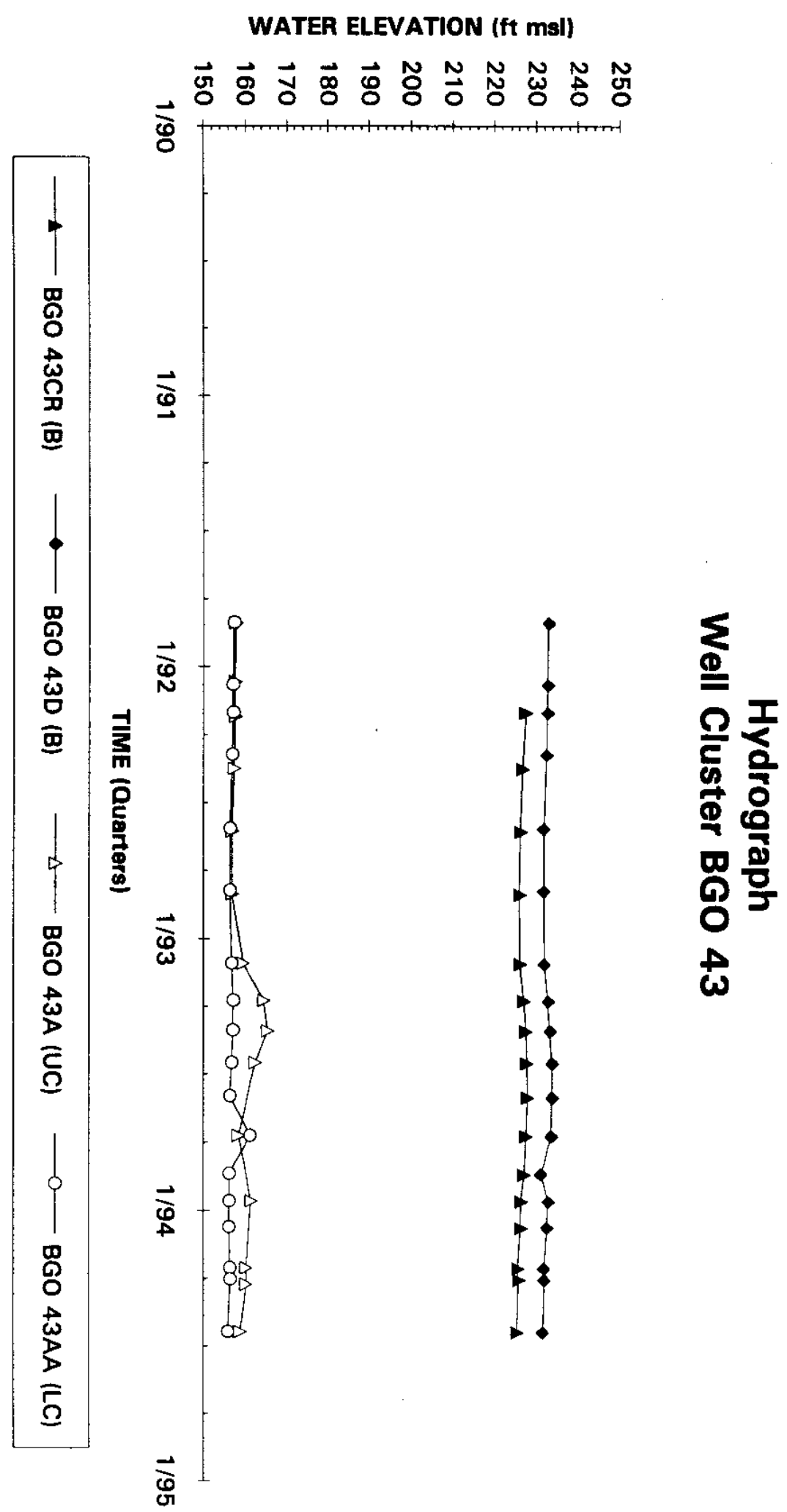



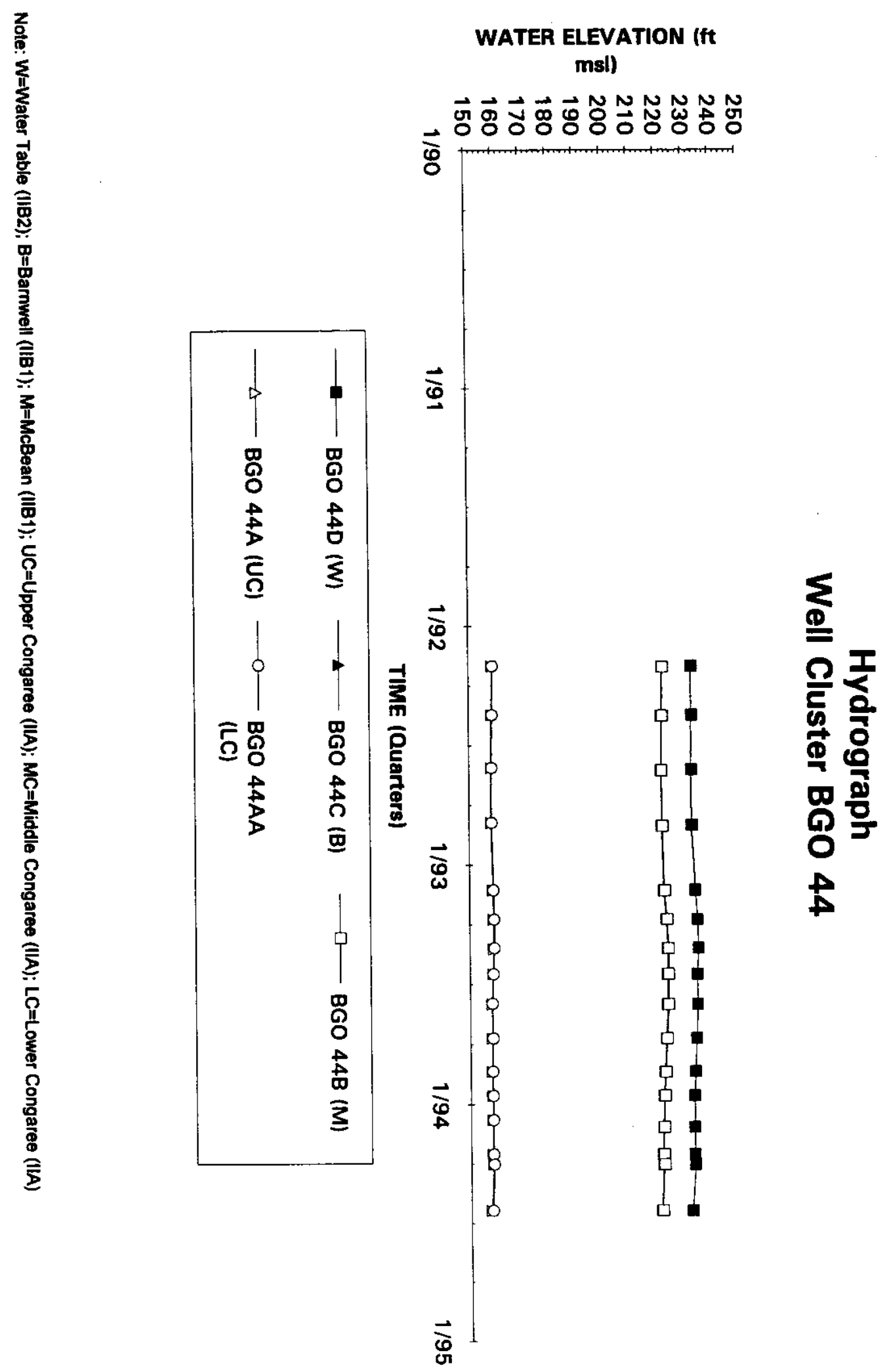

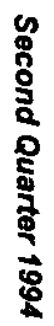

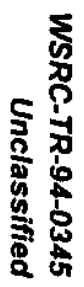


촊

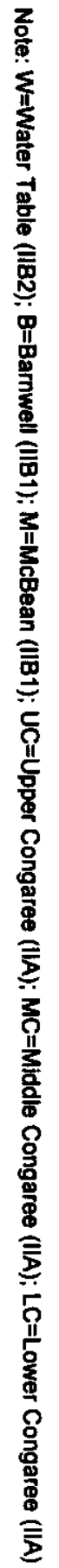

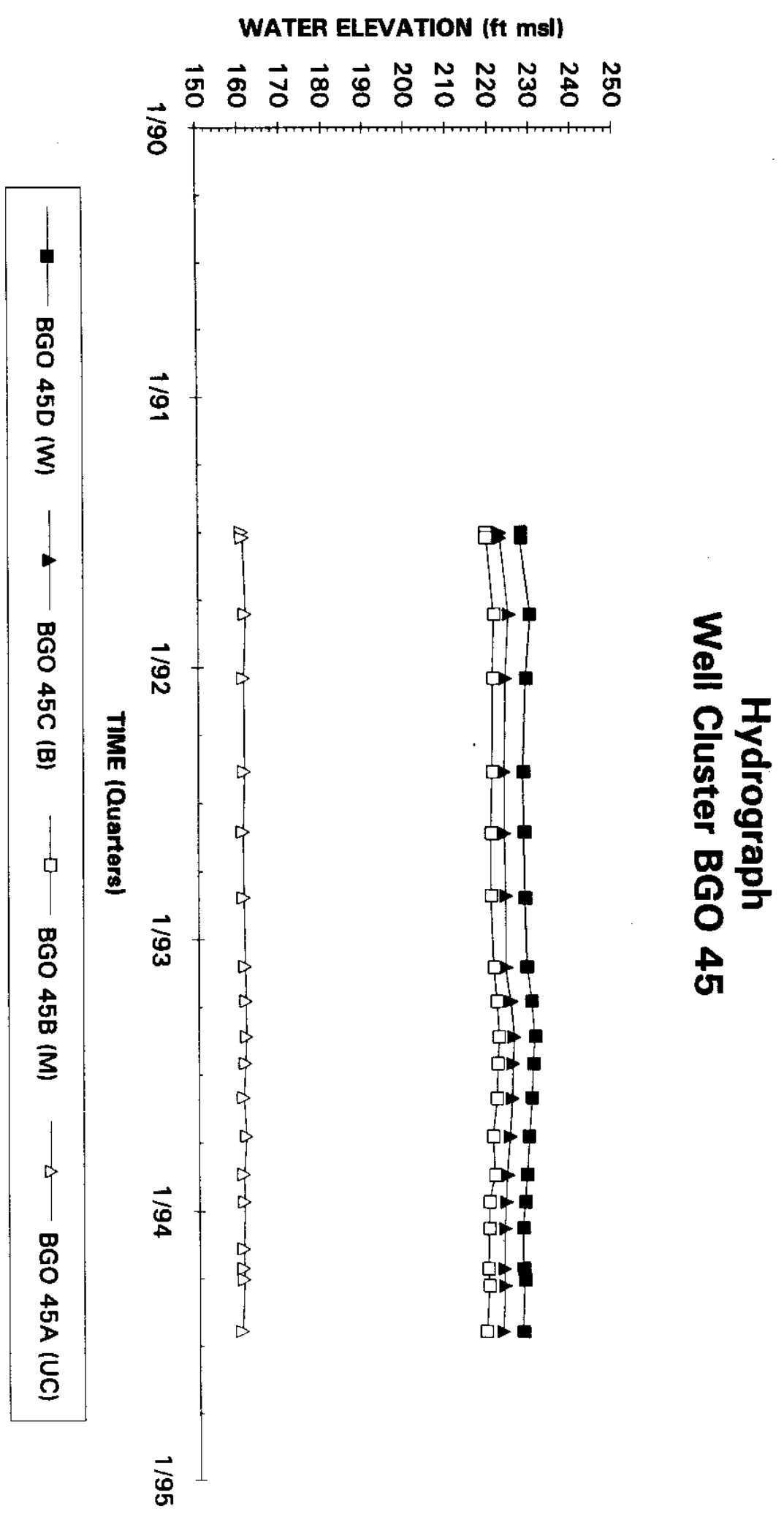

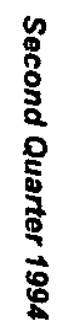

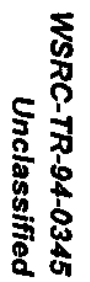




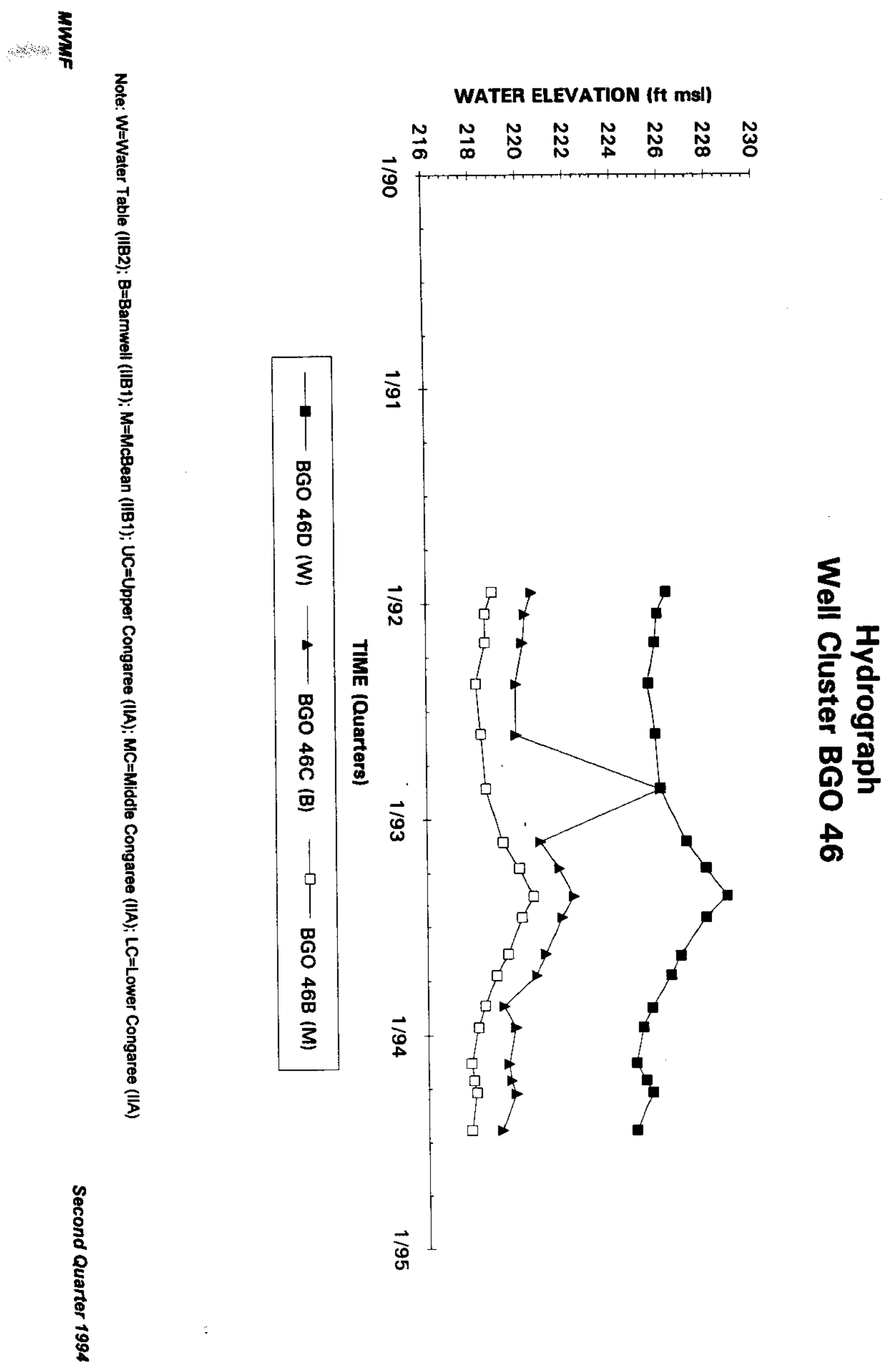

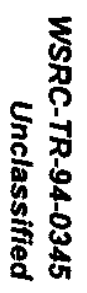



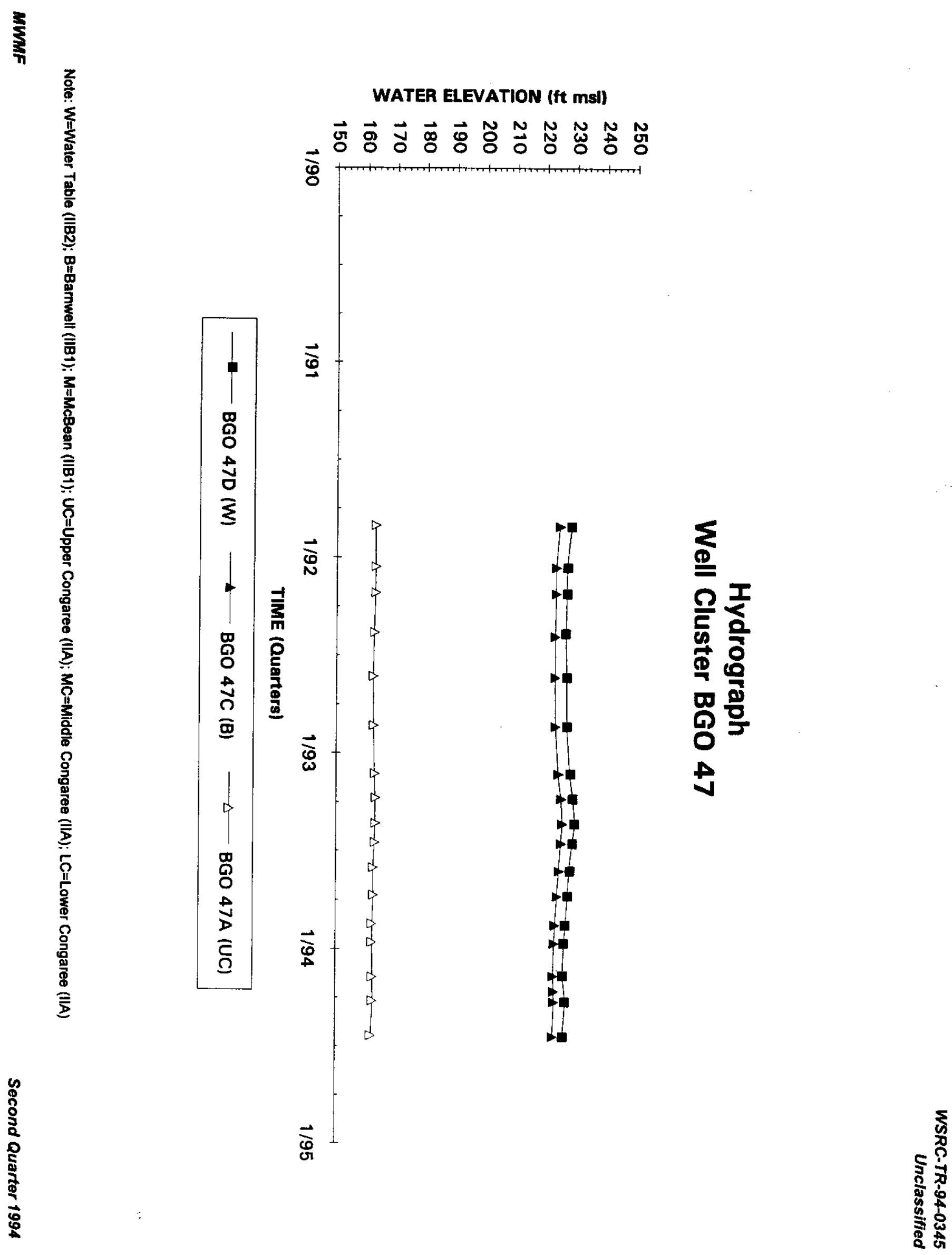
疍

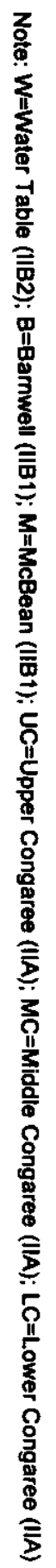

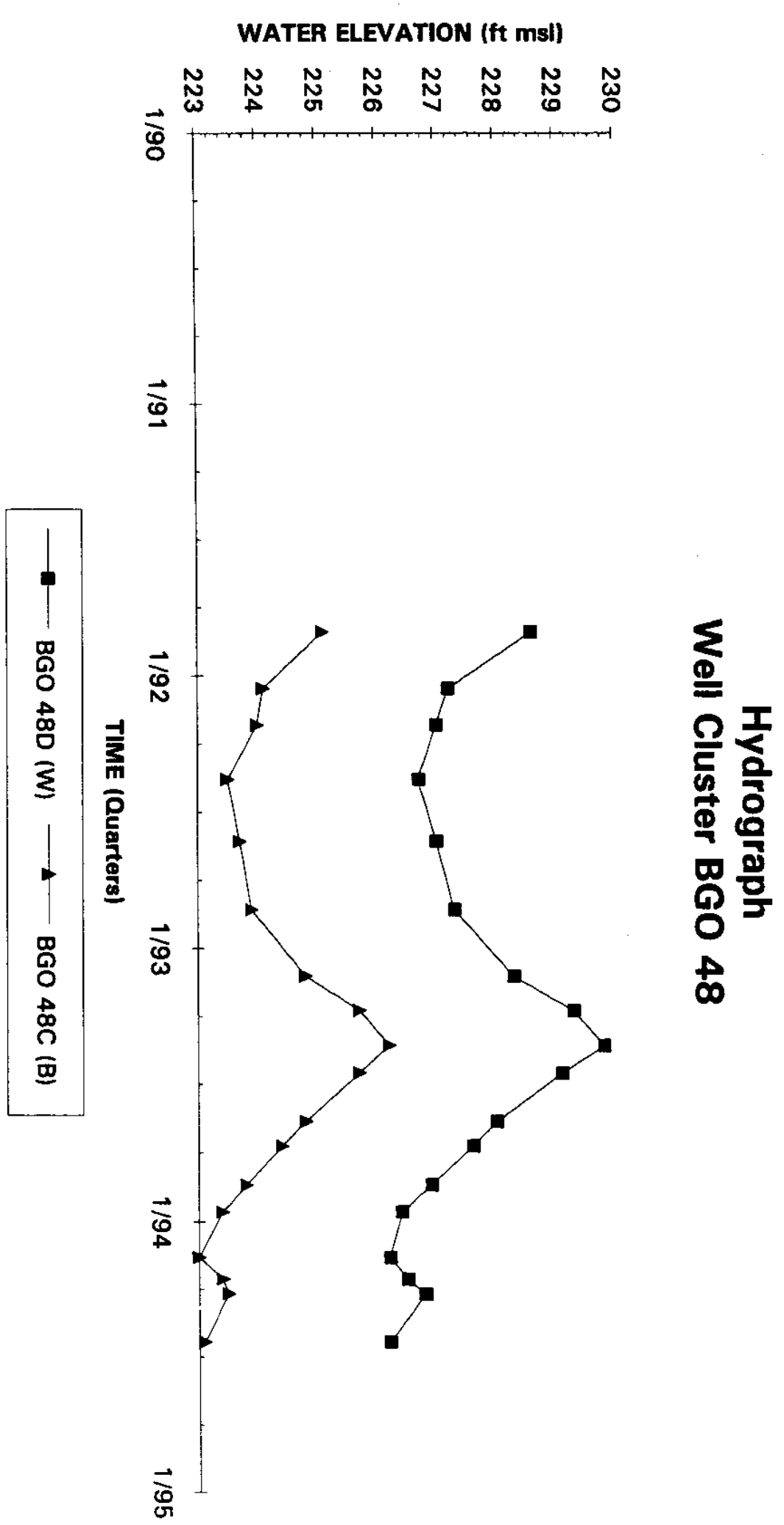

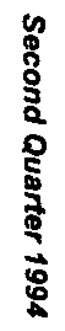




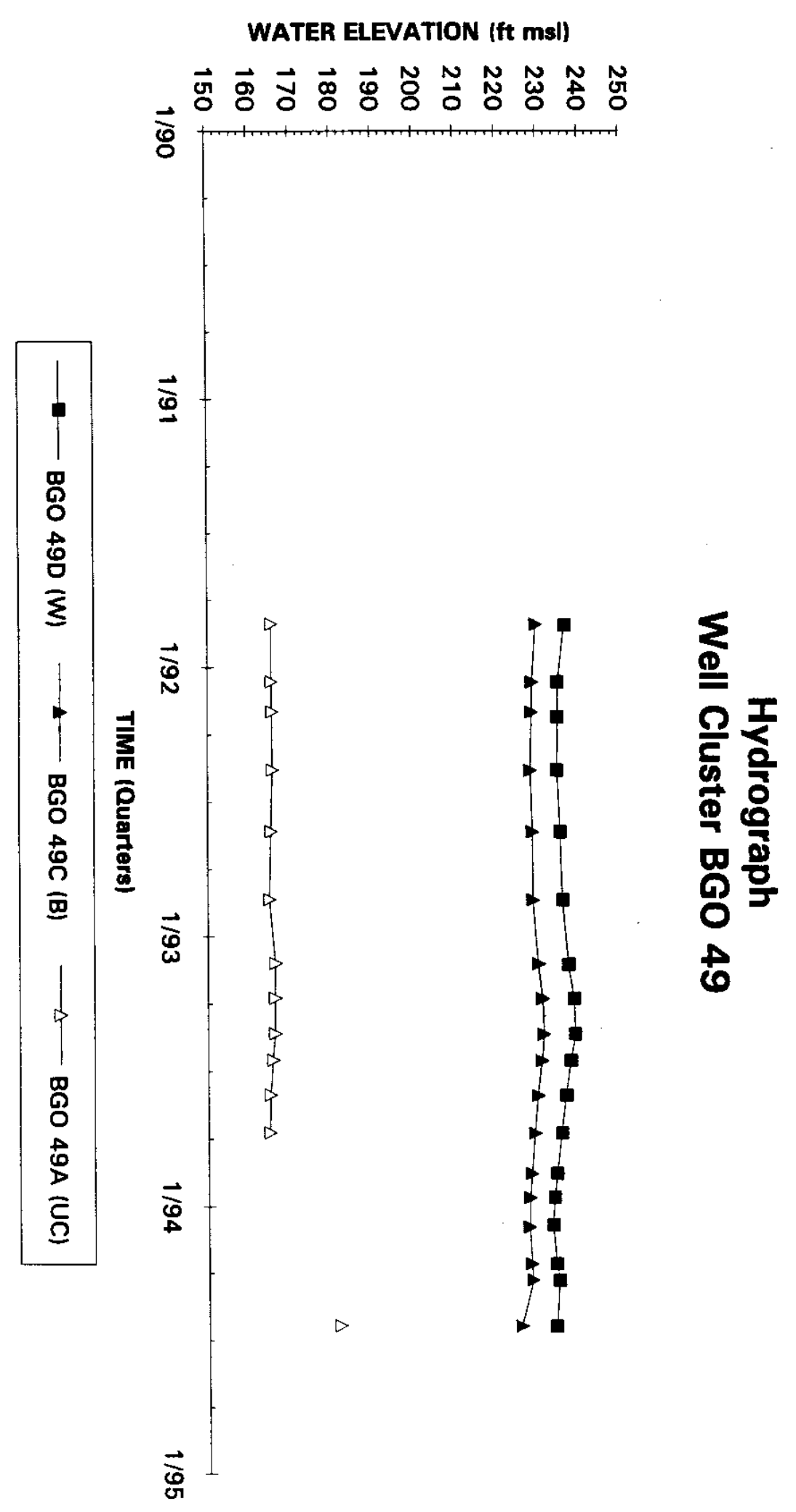


WATER ELEVATION (ft msl)

点

兽

苗

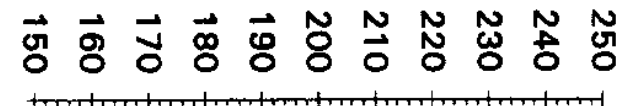

乌

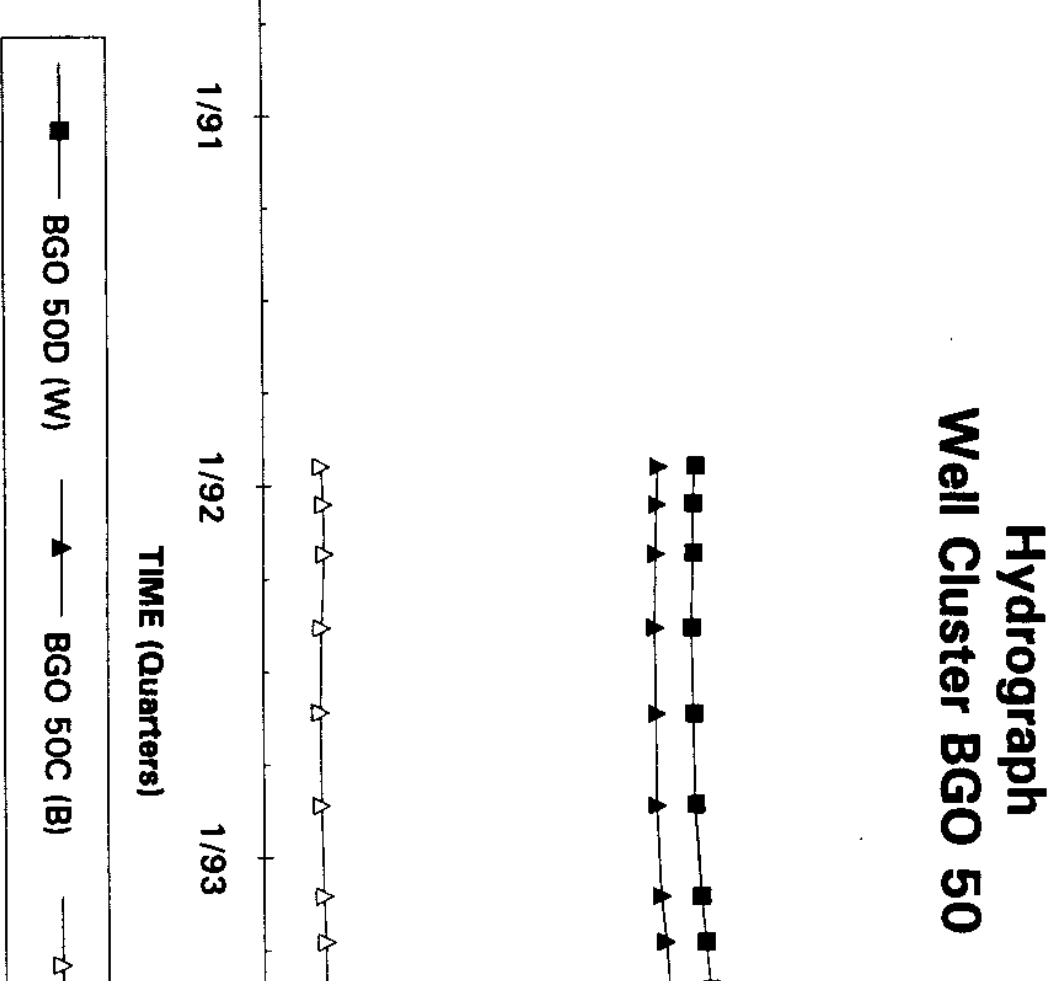

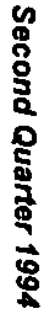

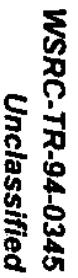


3

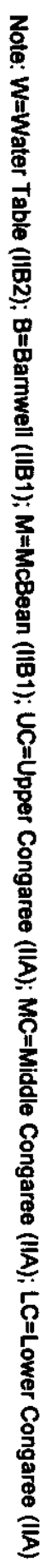

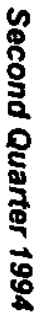

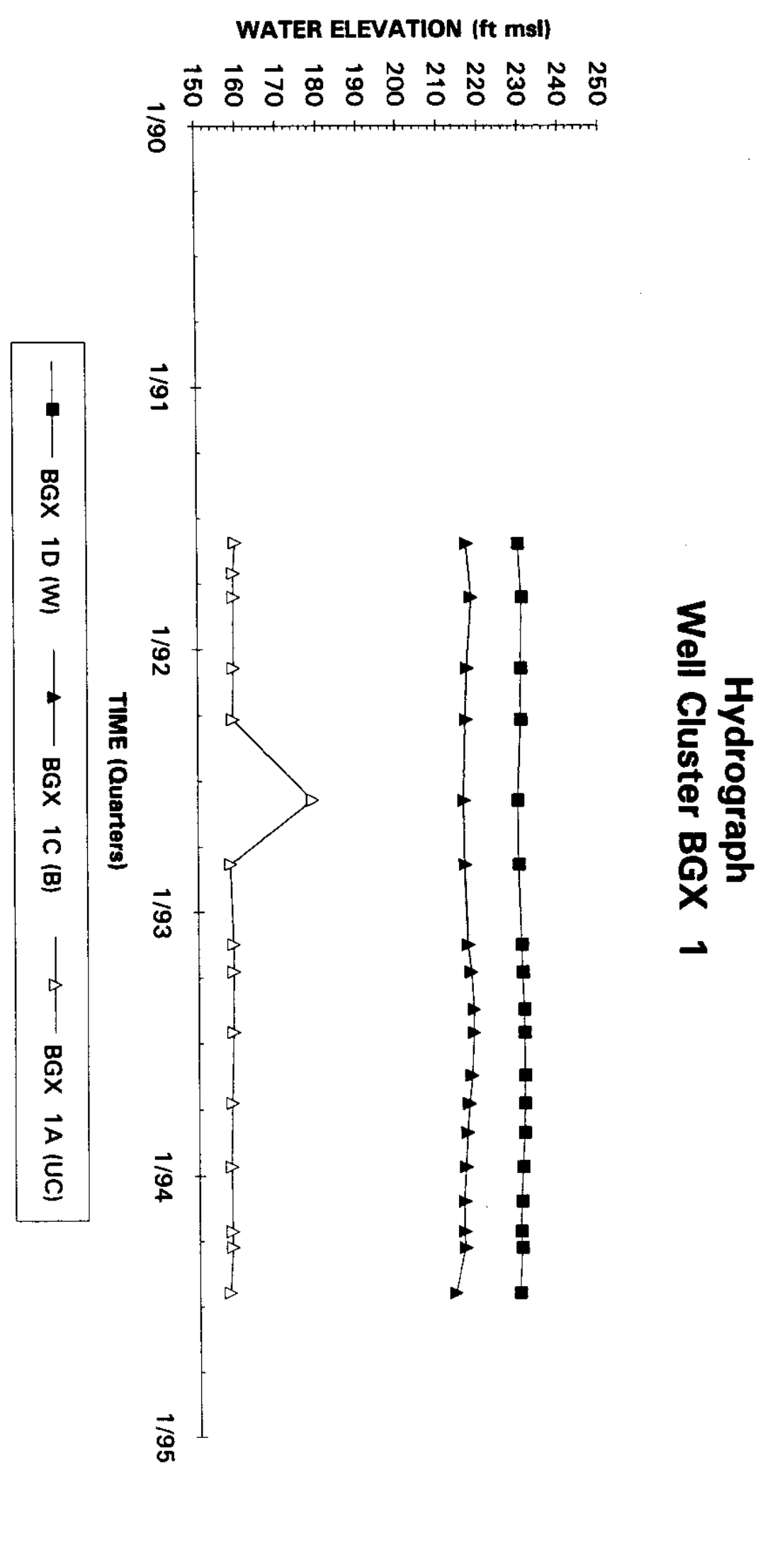

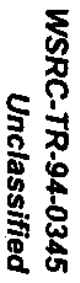



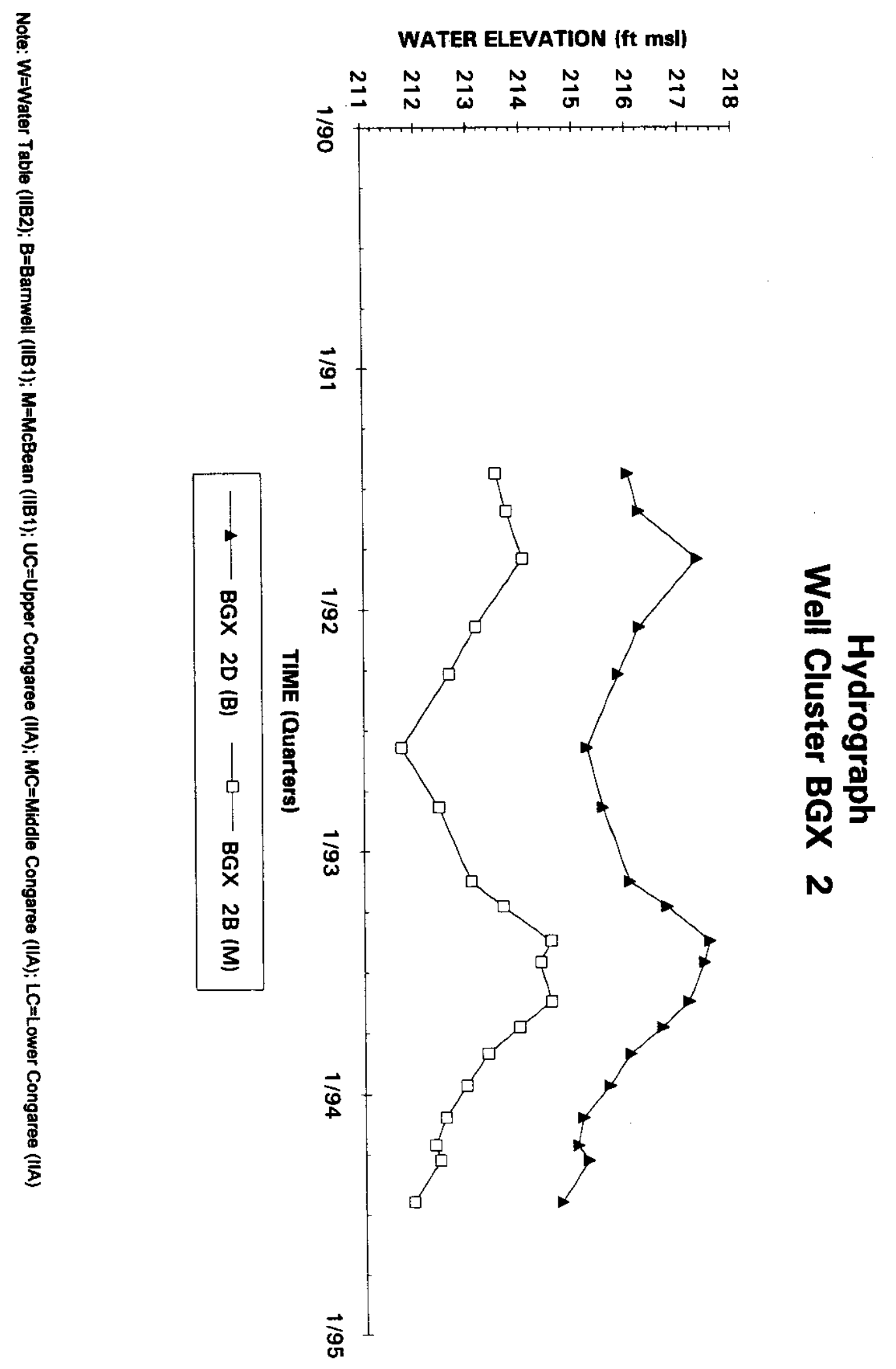

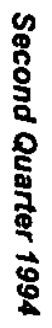

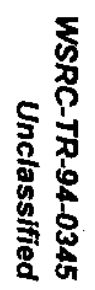



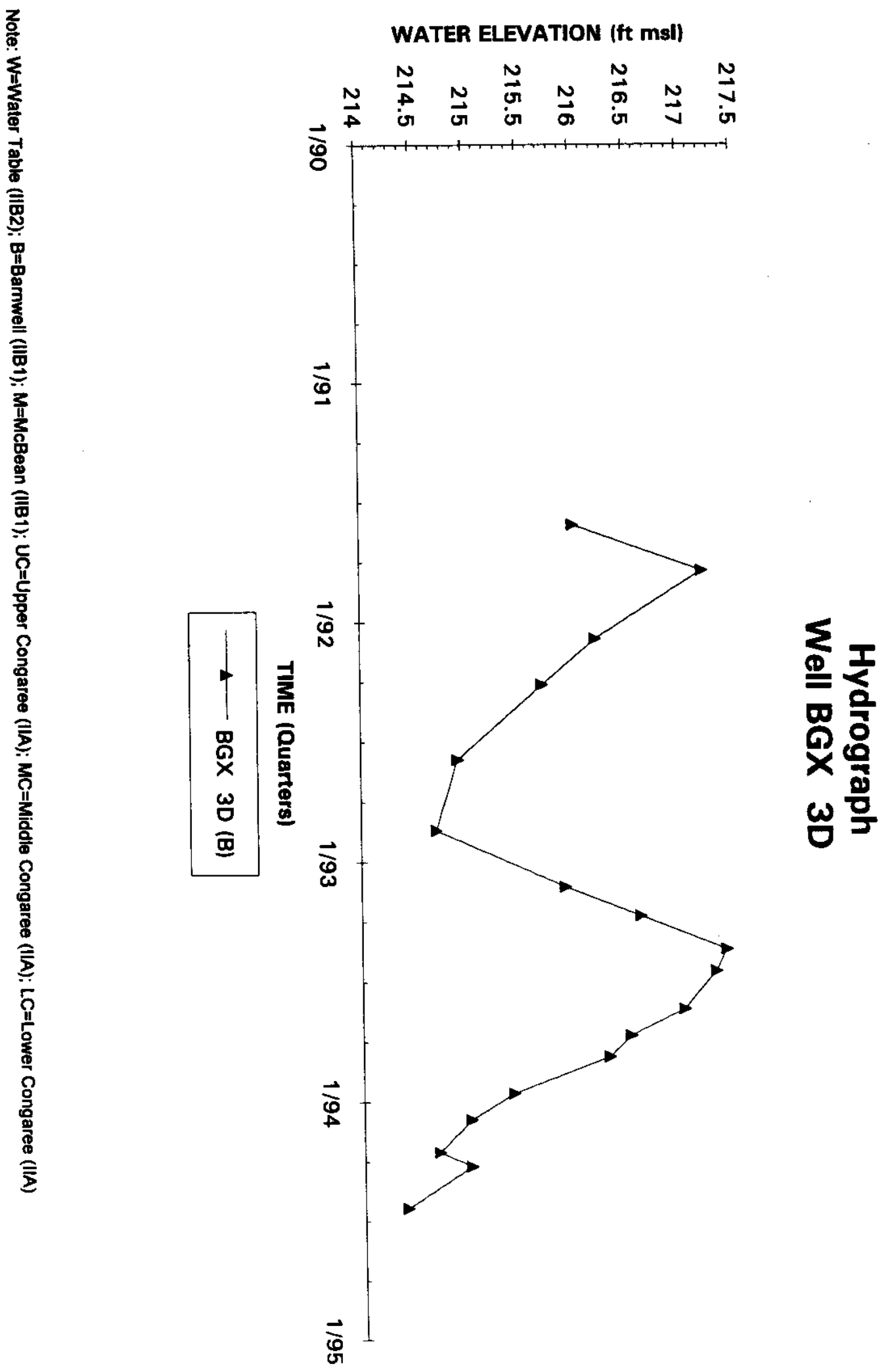


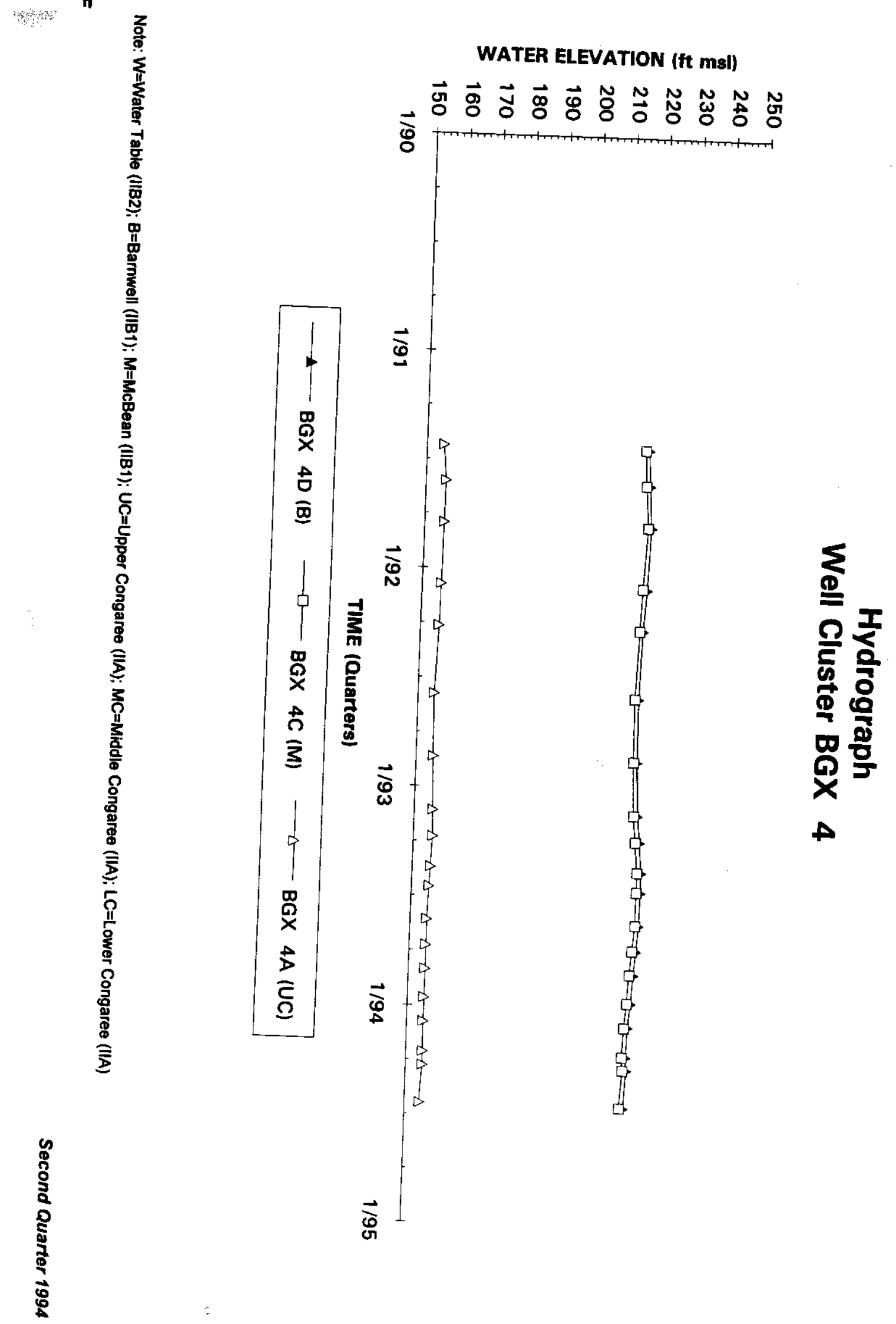



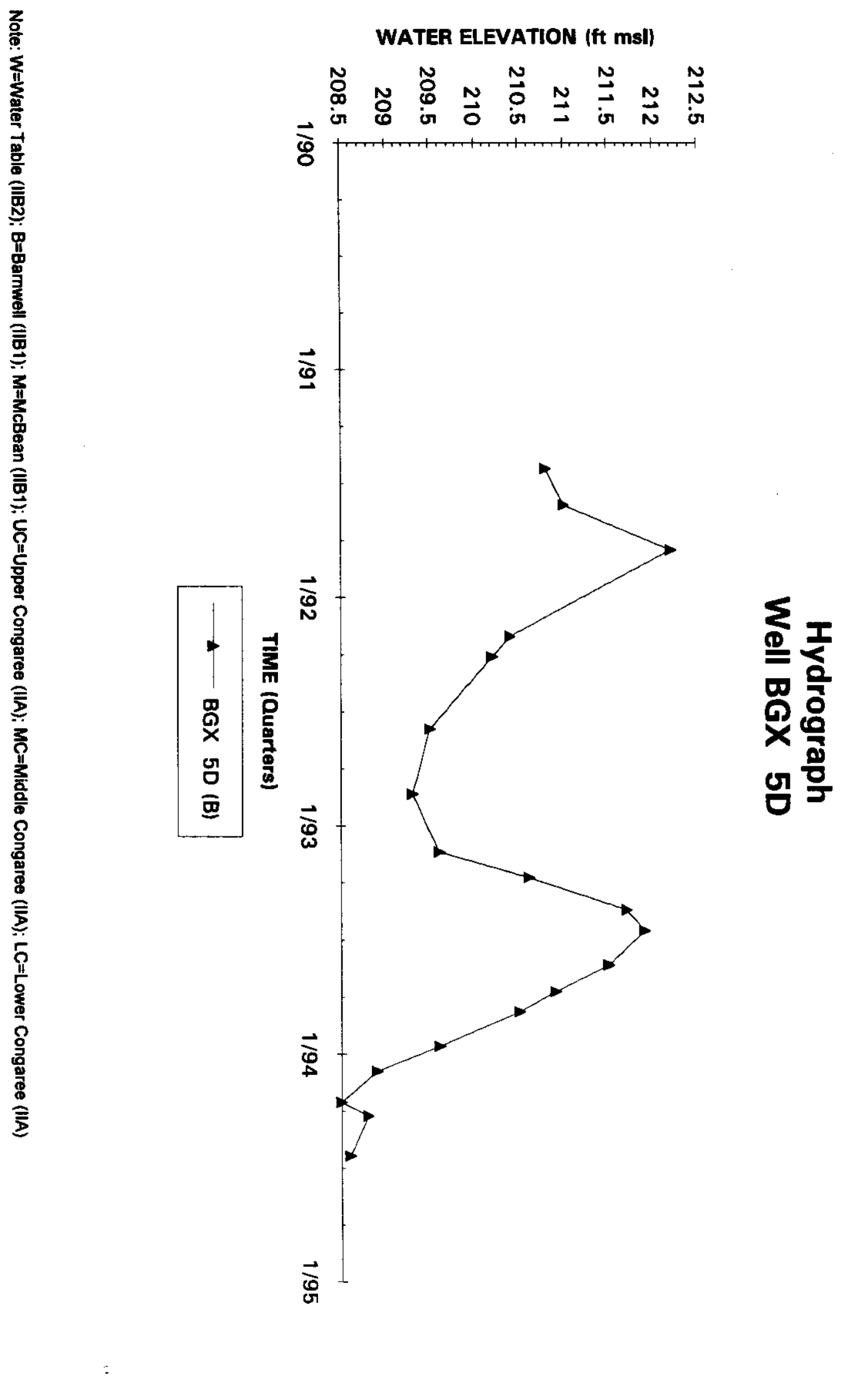

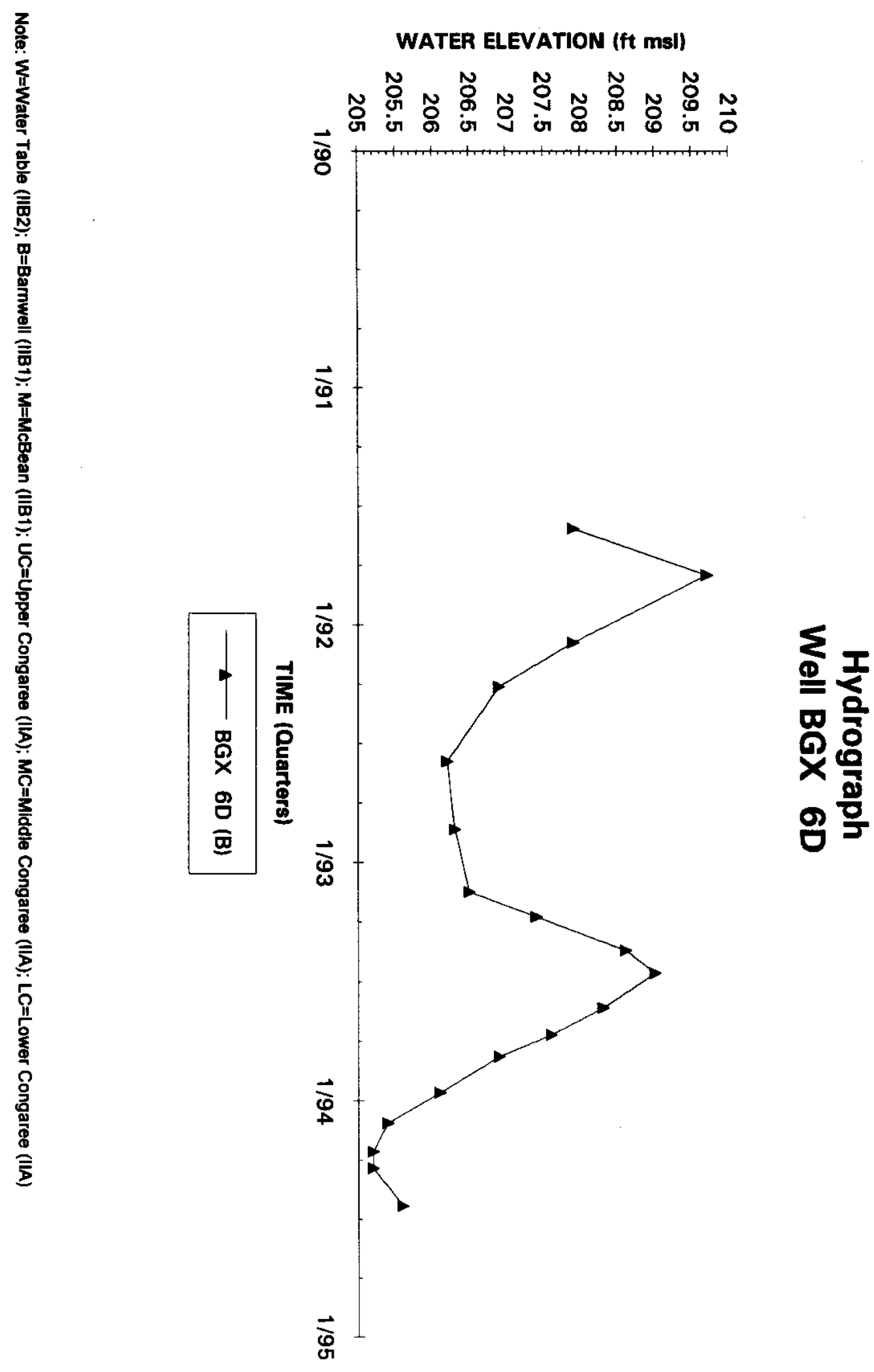
WATER ELEVATION (ft msl)

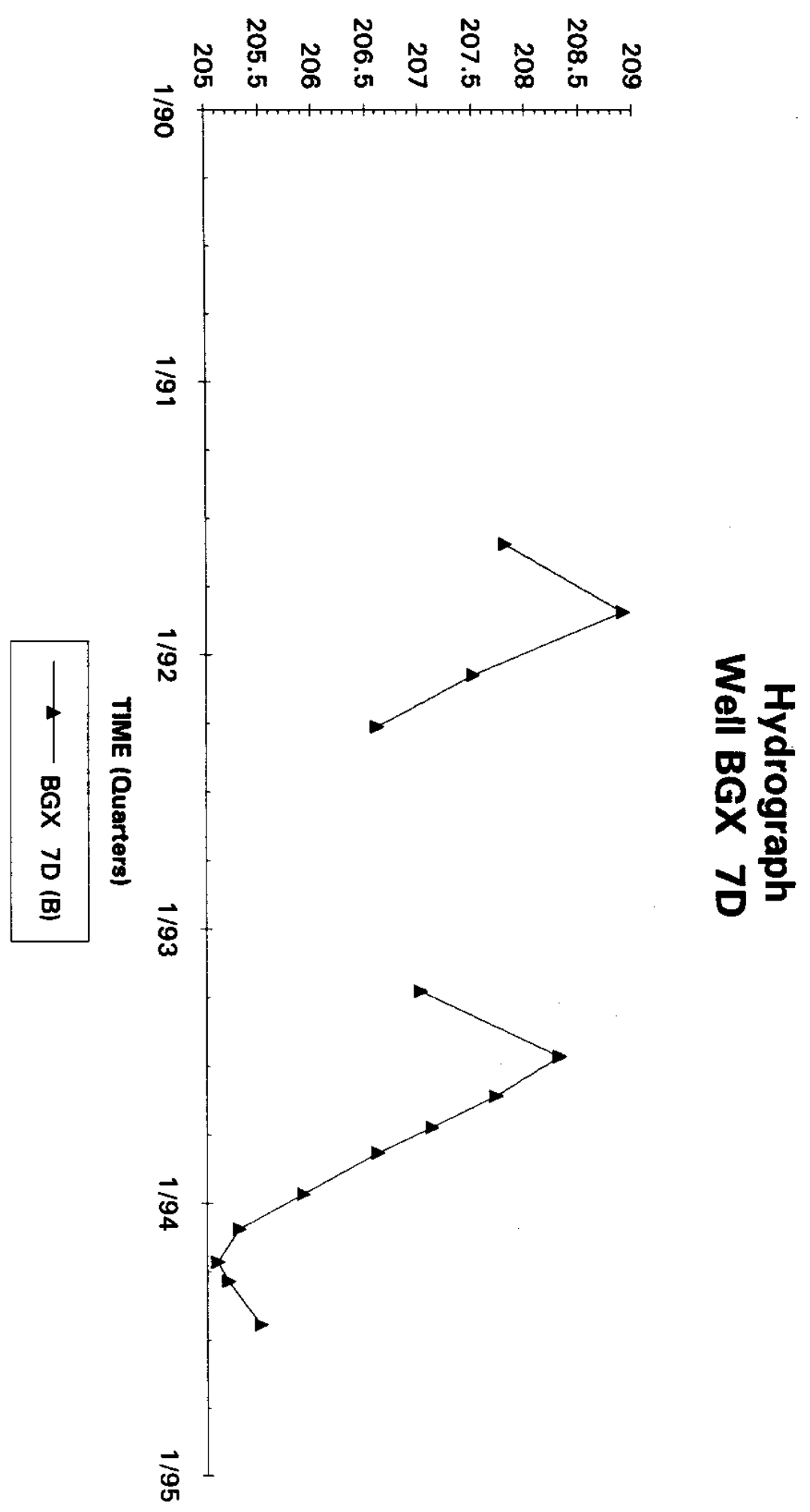




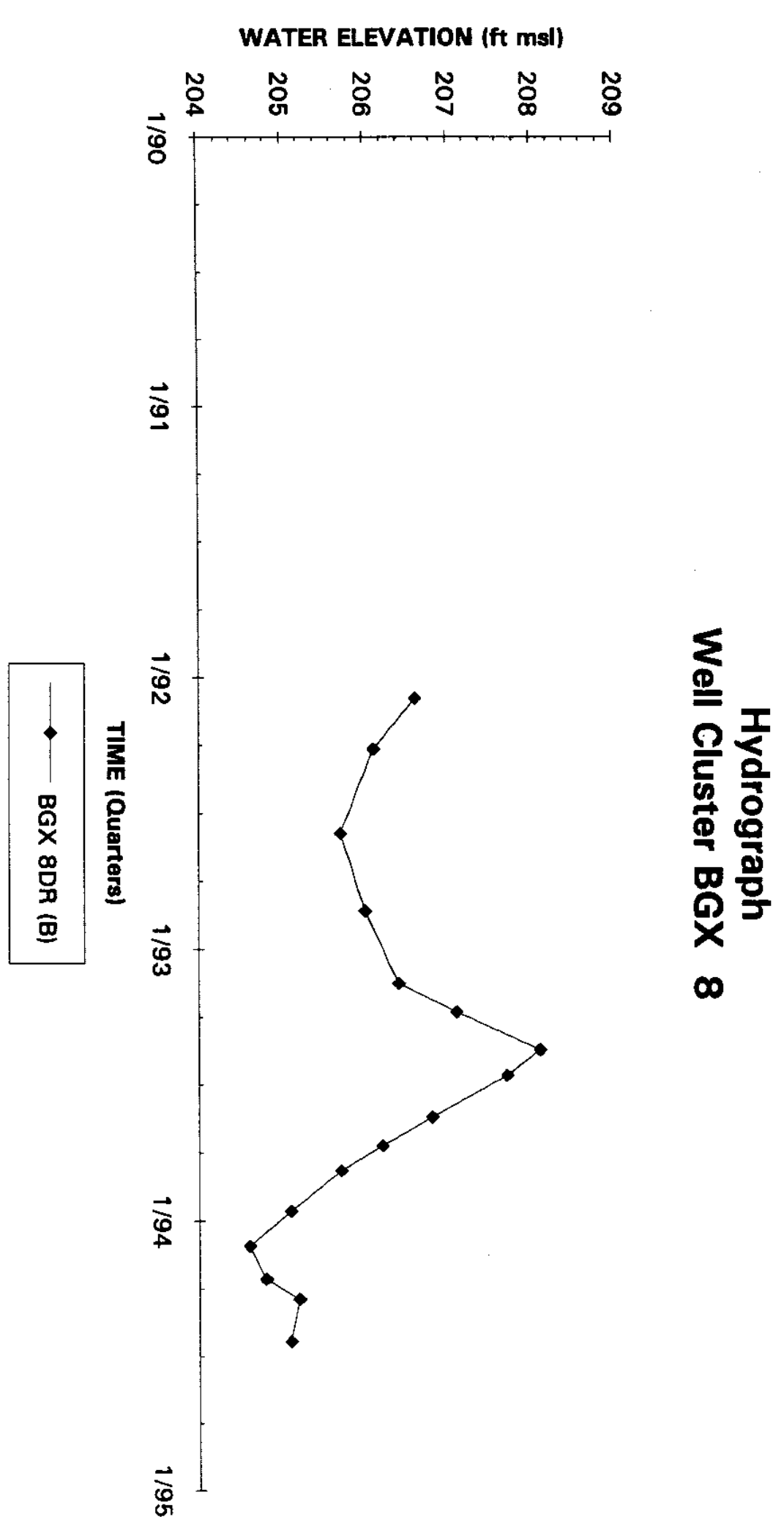

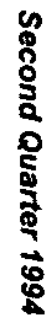



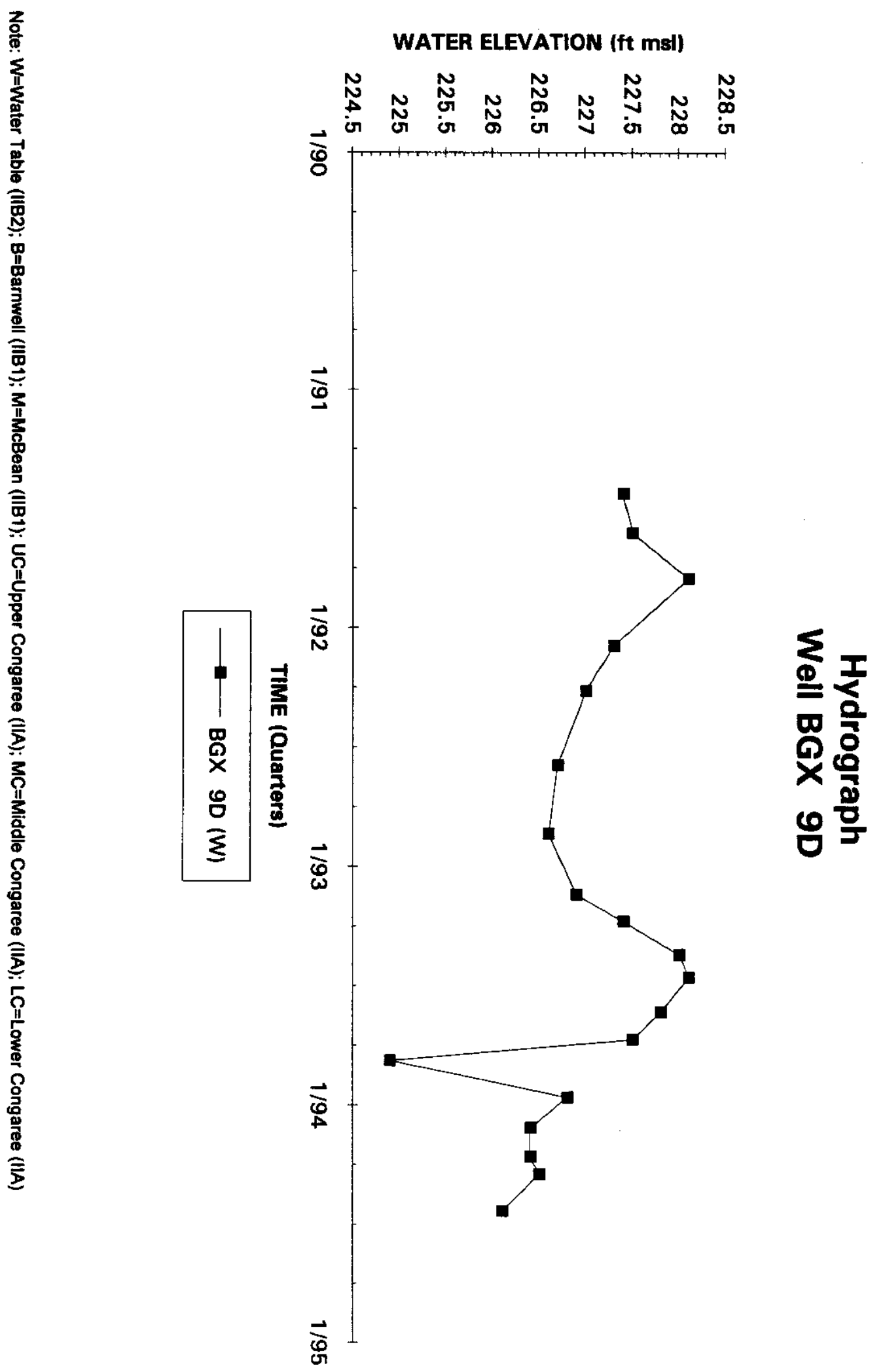

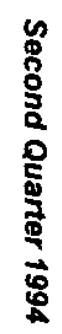

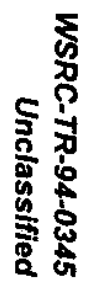




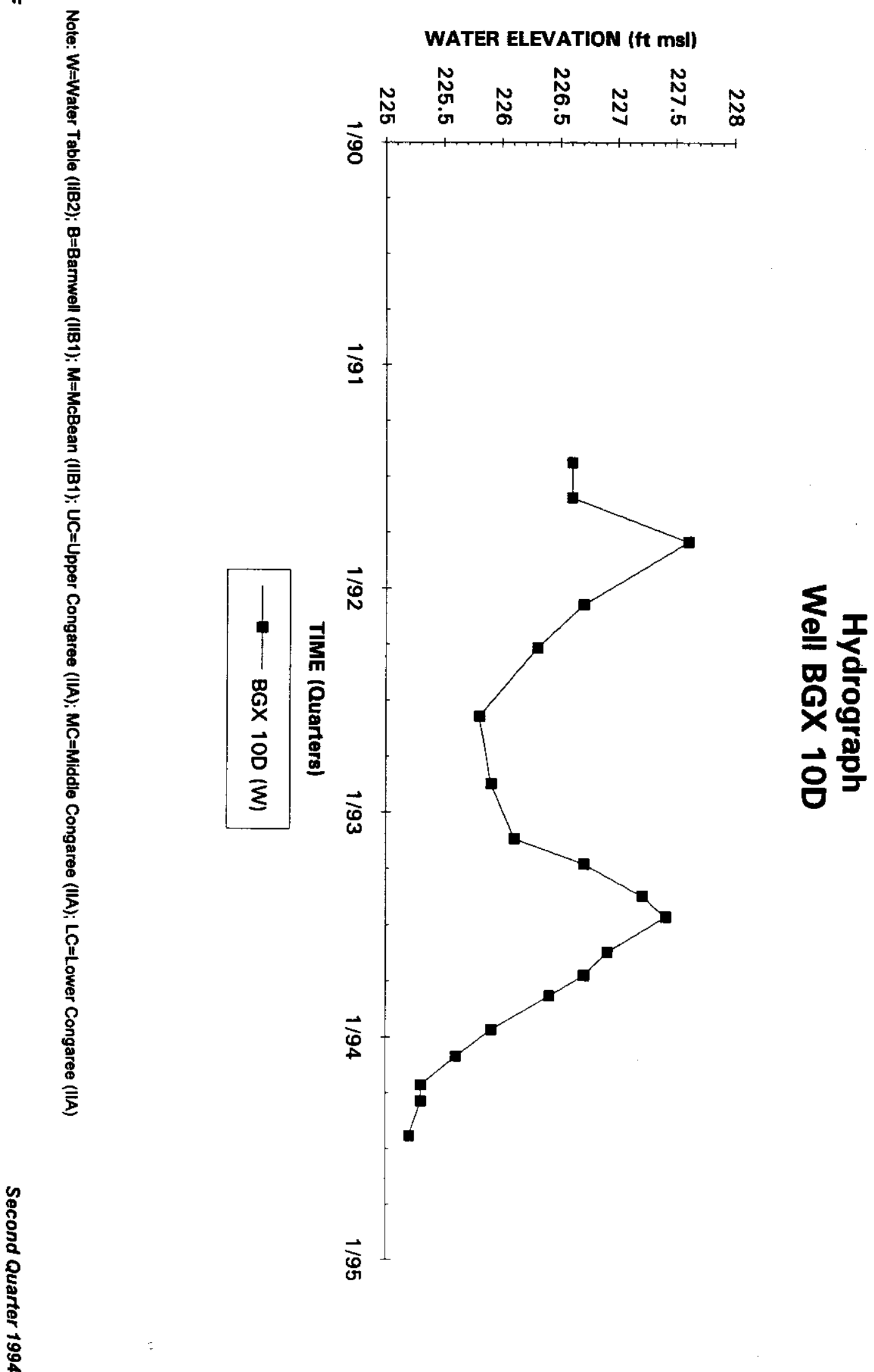


零

응 WATER ELEVATION (ft msI)

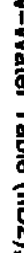

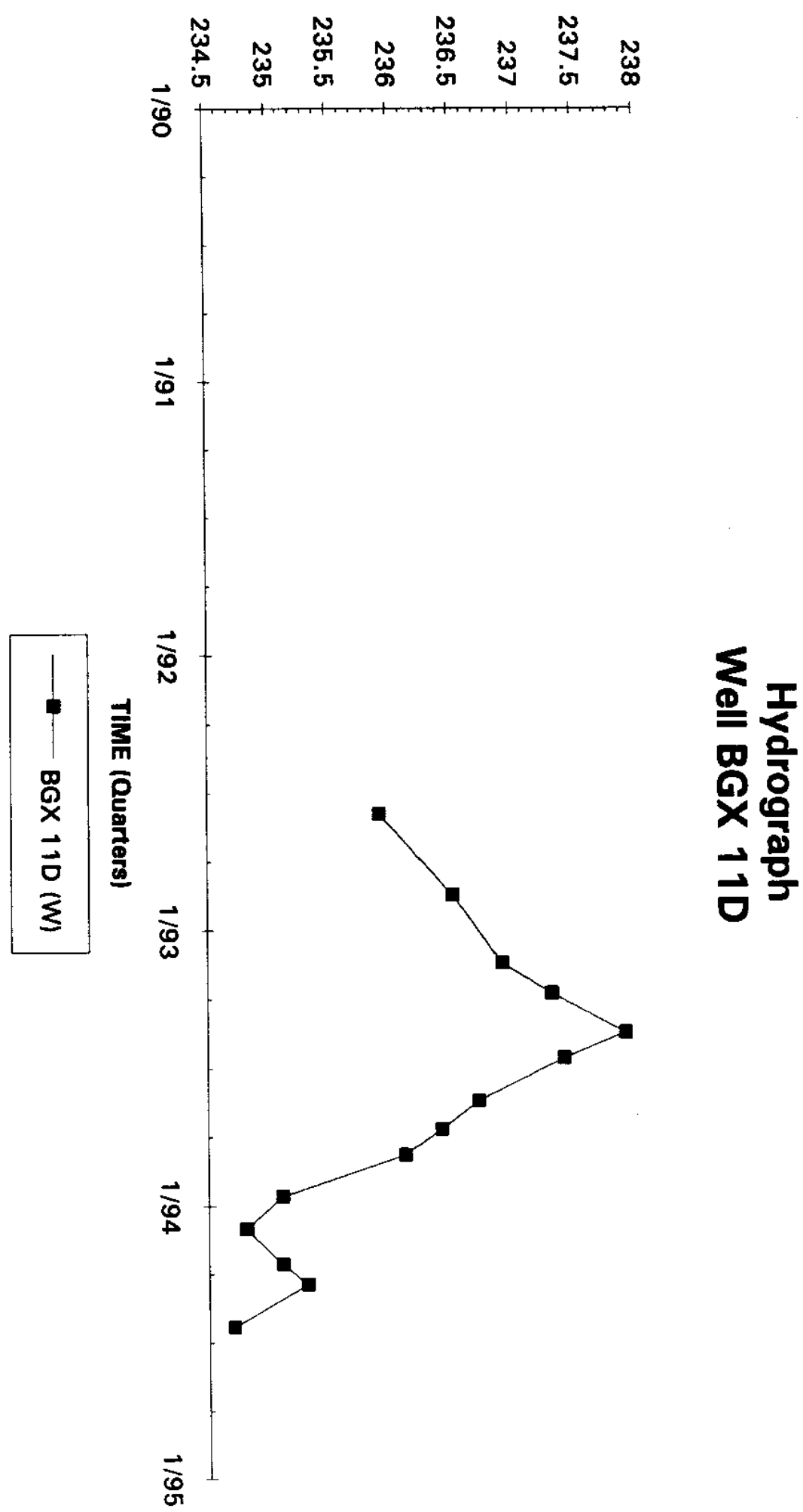

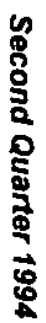

敢 


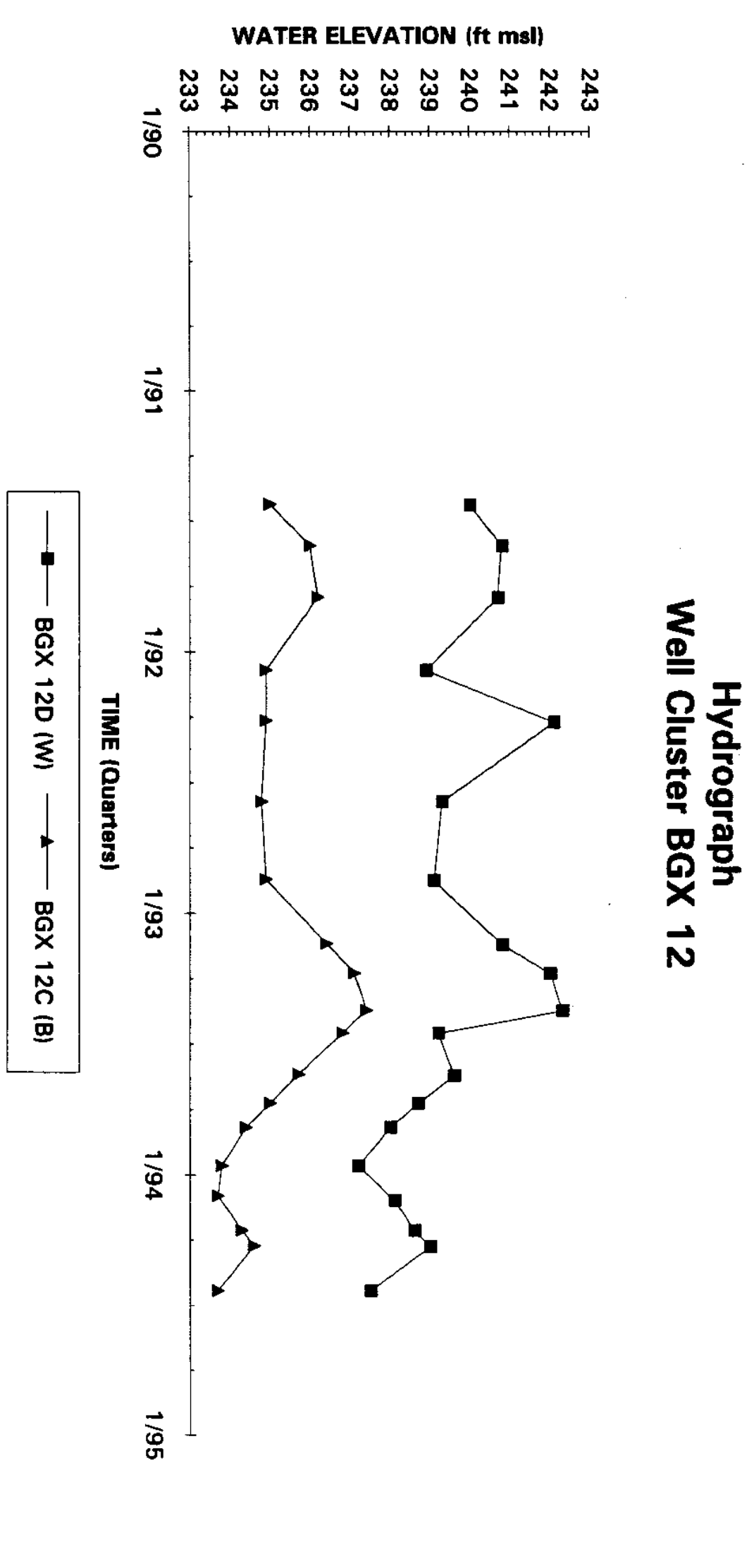

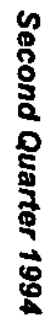




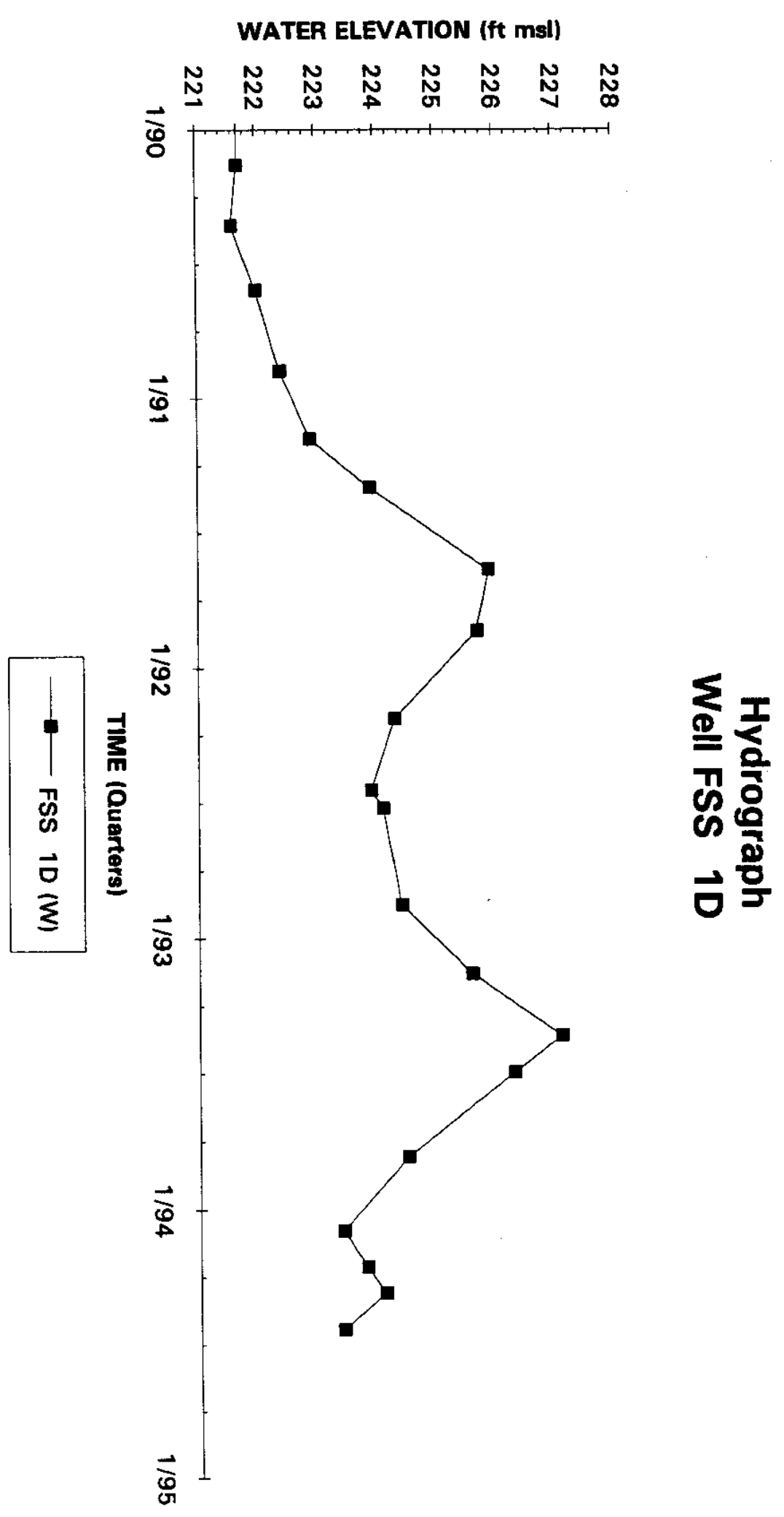

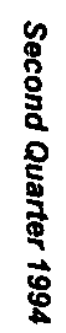

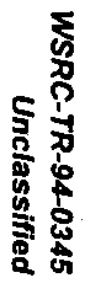



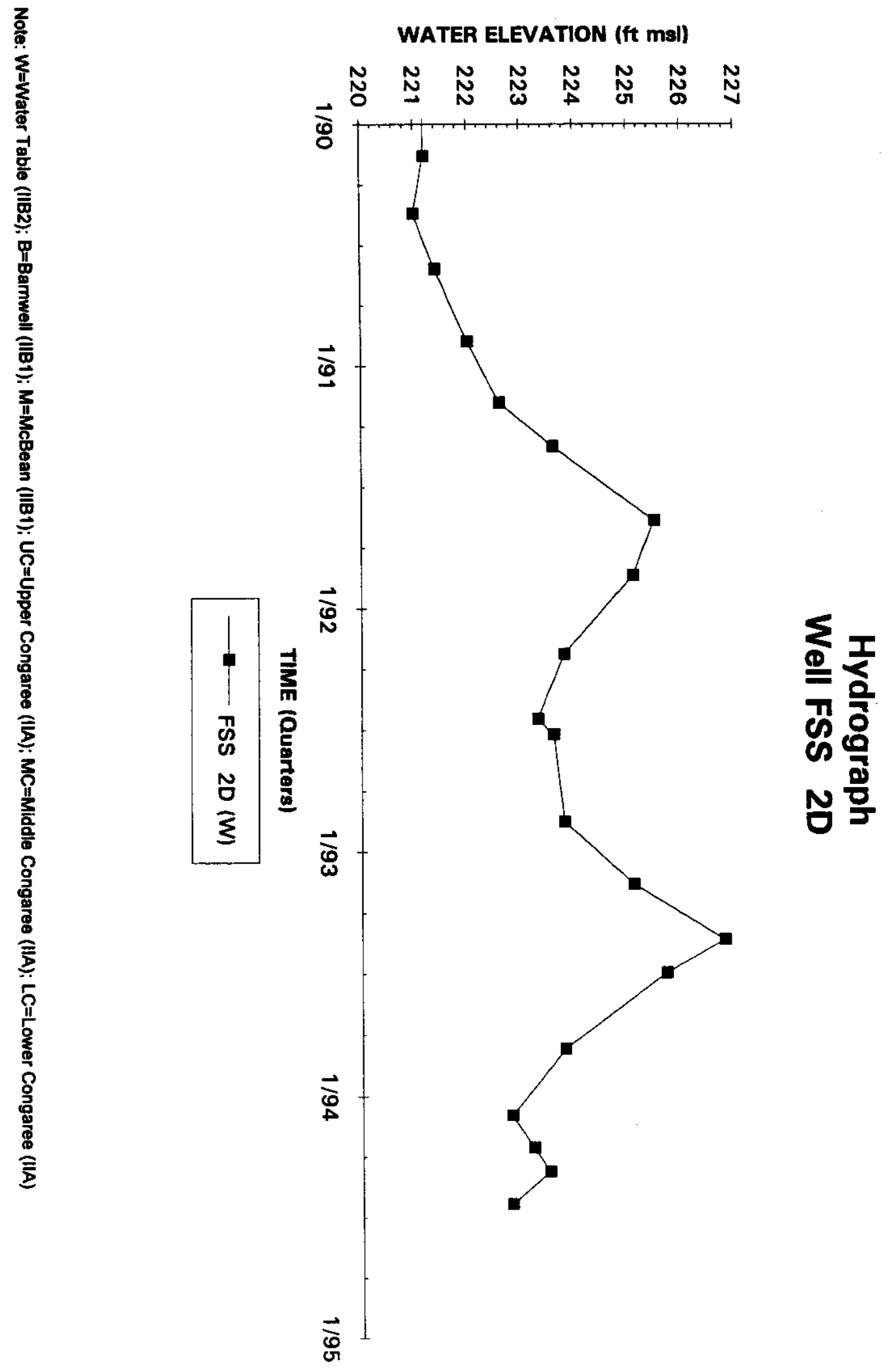

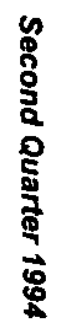

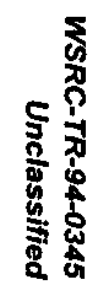


翠
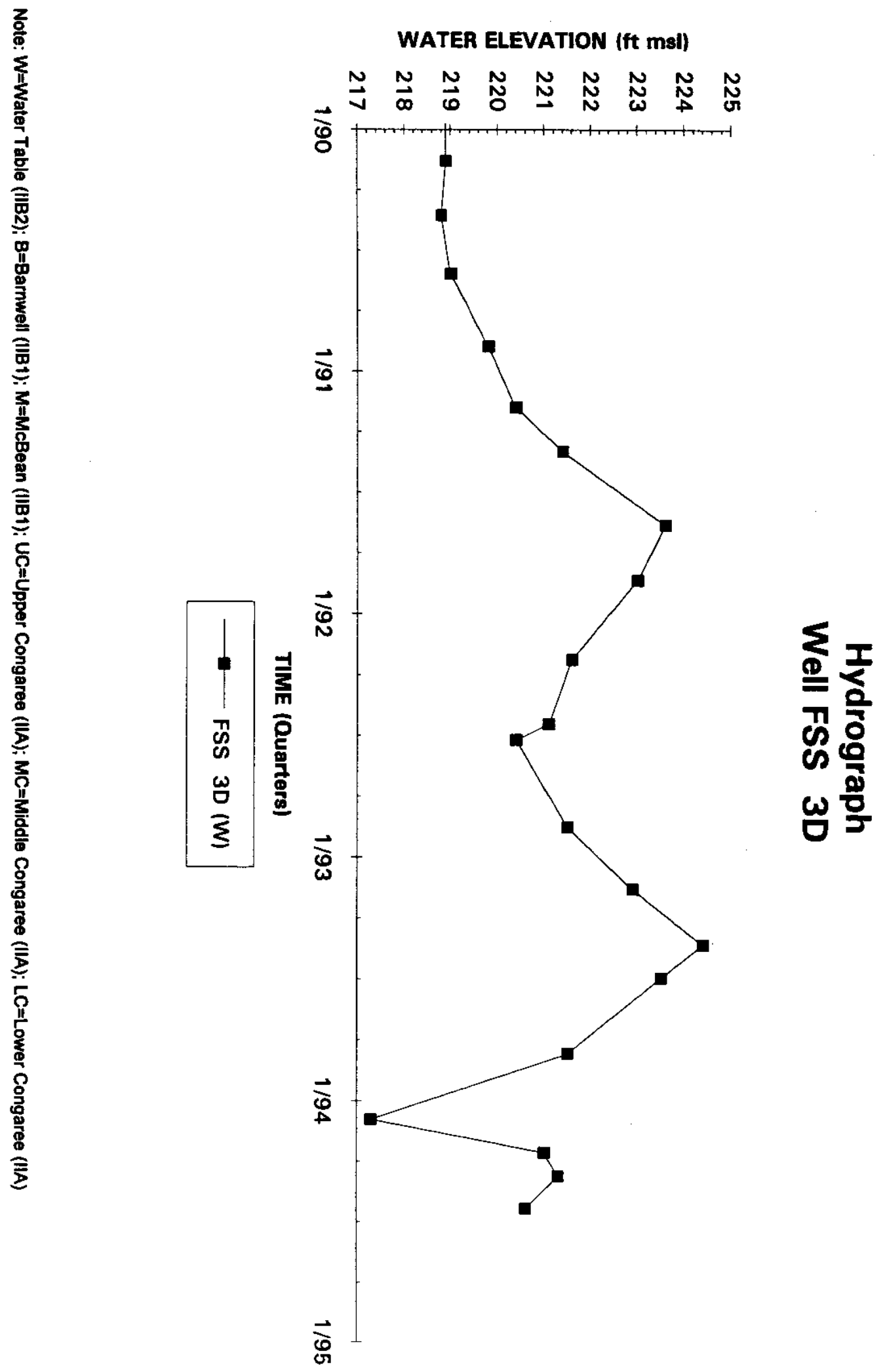

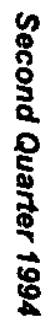

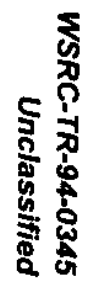




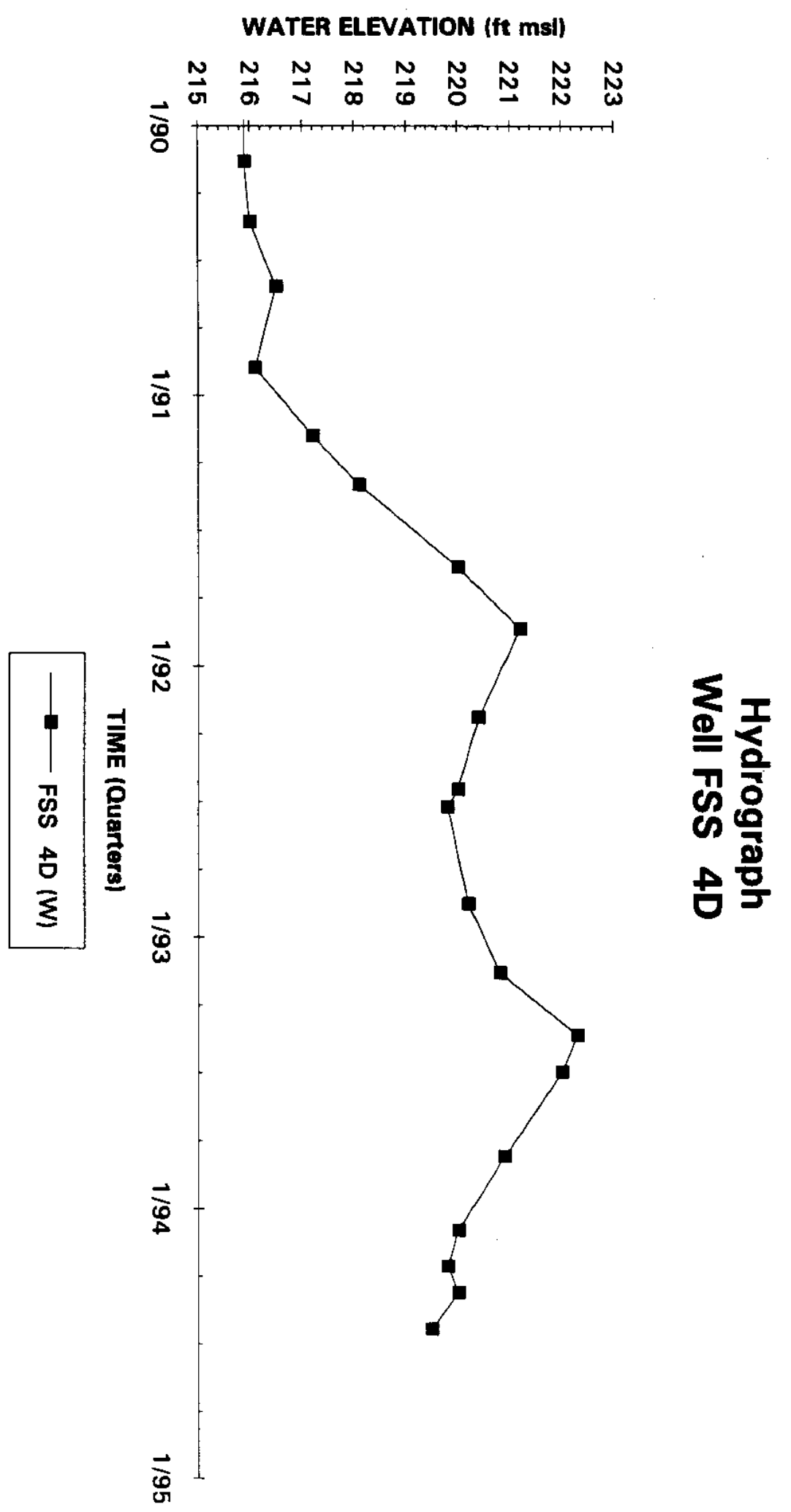

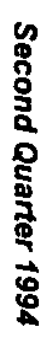

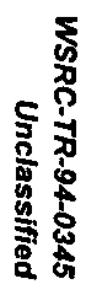



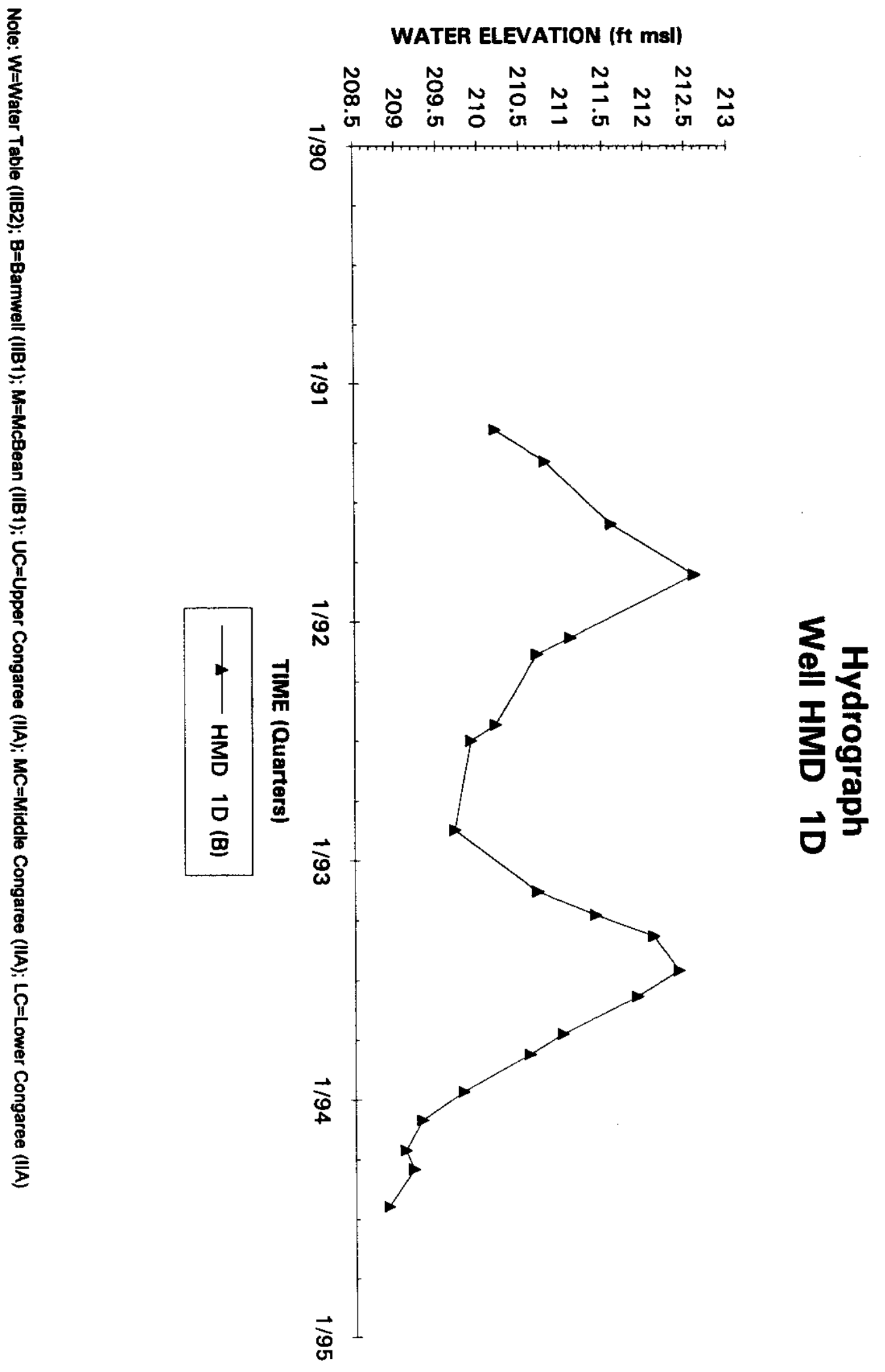

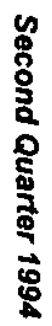




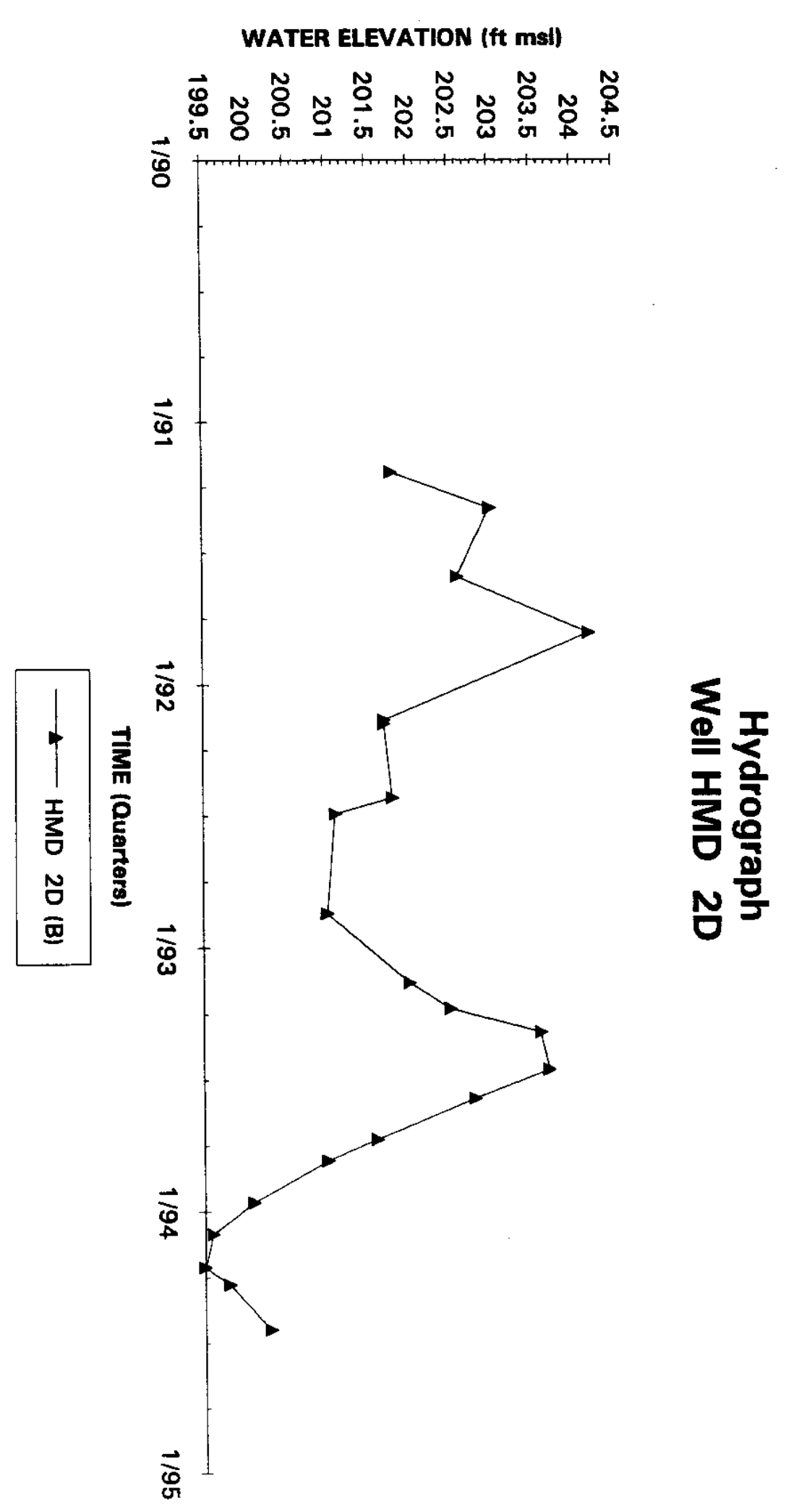


z



龺

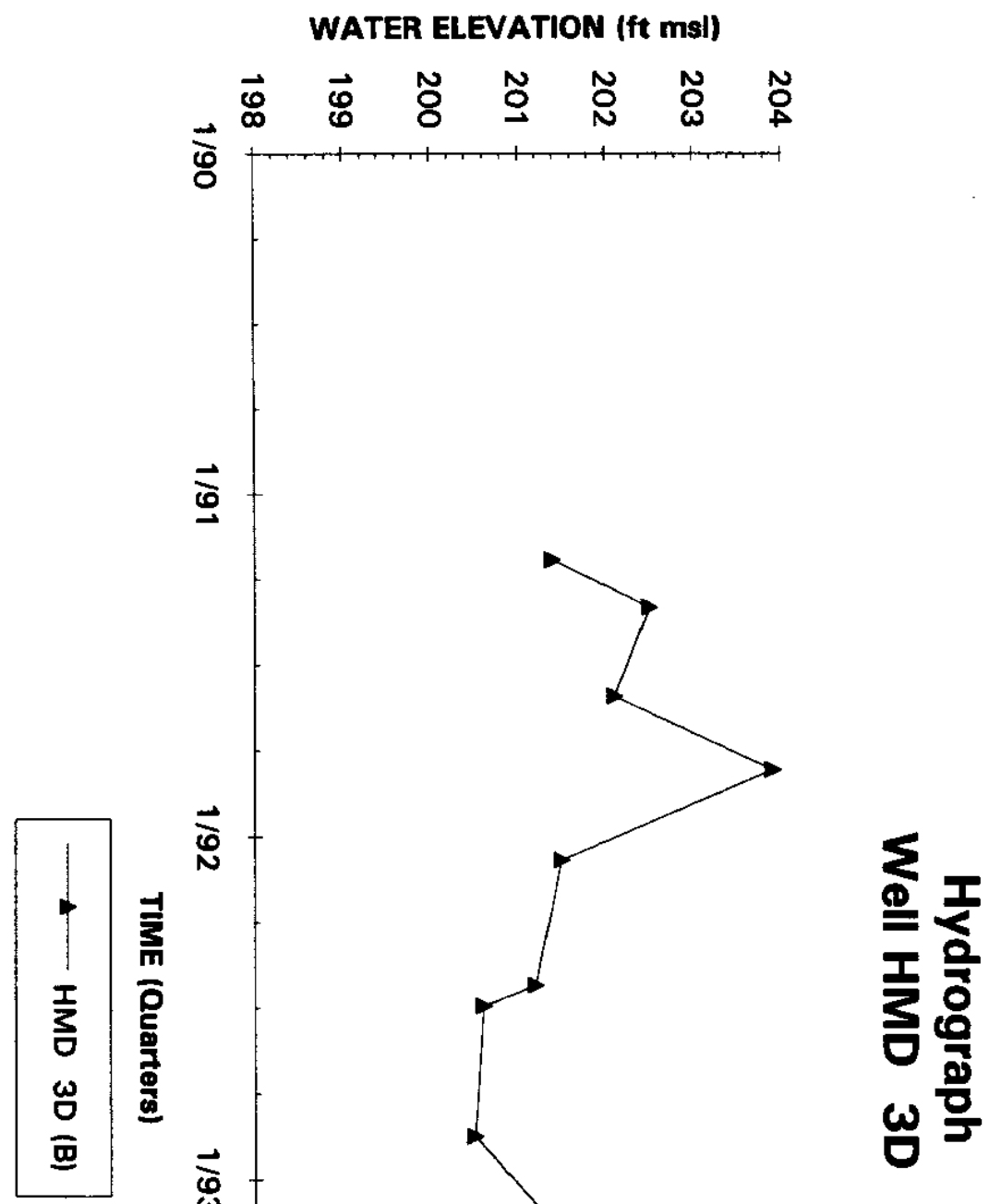

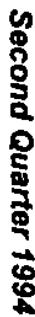

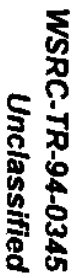


w

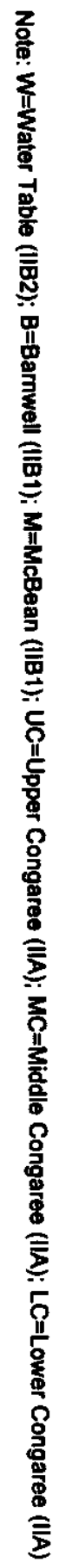

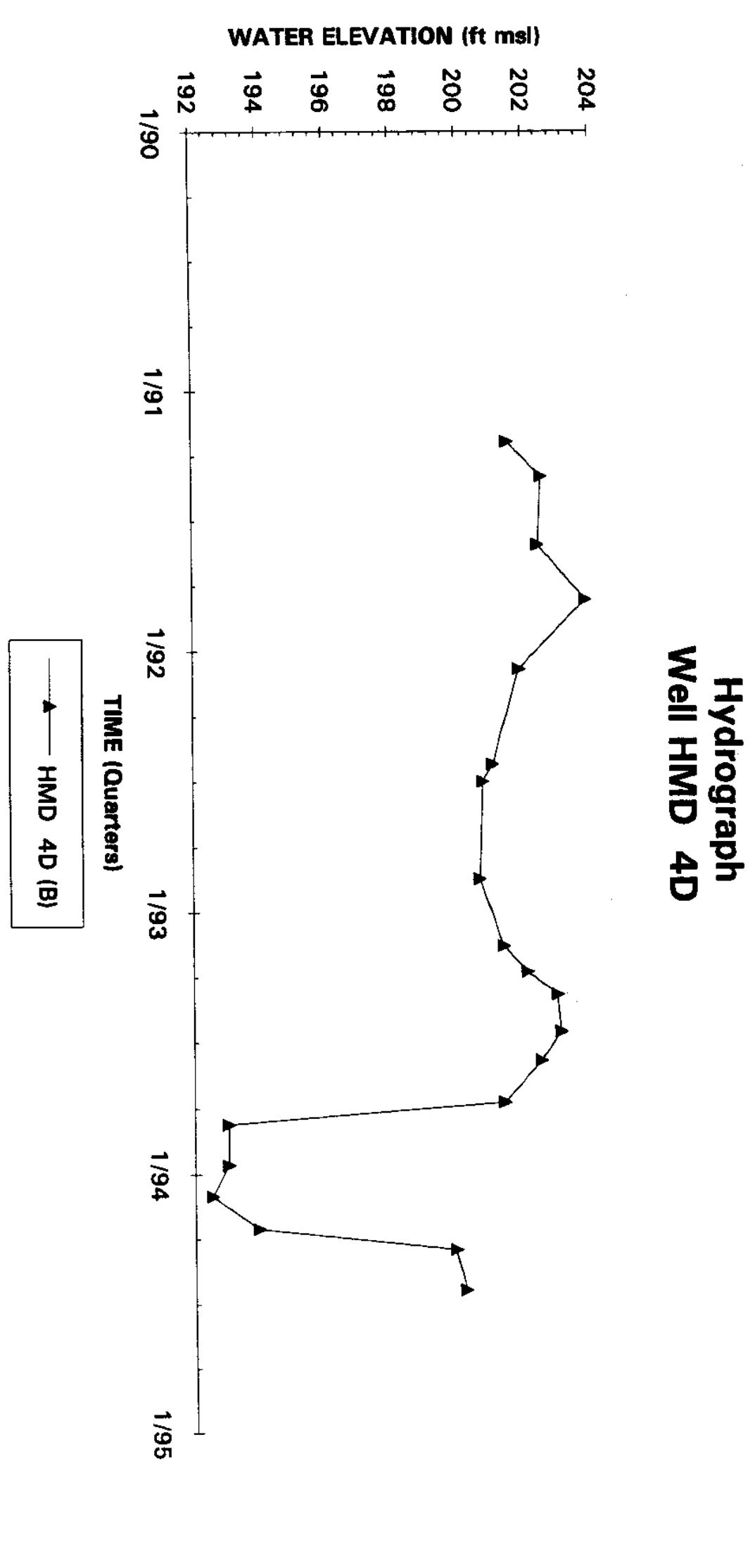

敢 
$z$

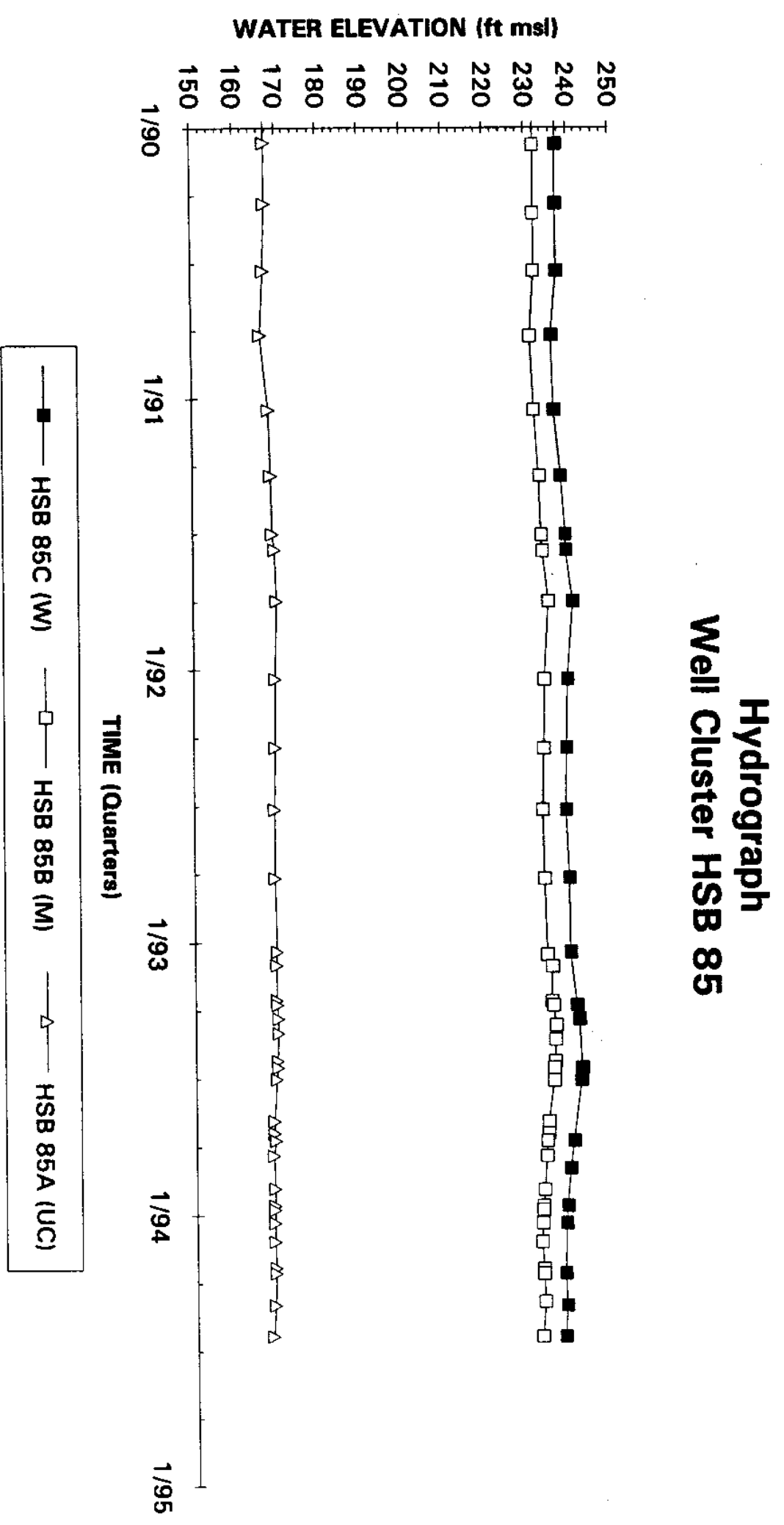

